\title{
Care Interventions for People Living With Dementia and Their Caregivers
}




\section{Comparative Effectiveness Review}

Number 231

\section{Care Interventions for People Living With Dementia and Their Caregivers}

Prepared for:

Agency for Healthcare Research and Quality

U.S. Department of Health and Human Services

5600 Fishers Lane

Rockville, MD 20857

www.ahrq.gov

Contract No. 290-2015-00008-I

Prepared by:

Minnesota Evidence-based Practice Center

Minneapolis, MN

Investigators:

Mary Butler, Ph.D., M.B.A.

Joseph E. Gaugler, Ph.D.

Kristine M.C. Talley, Ph.D., R.N., G.N.P.-B.C., F.G.S.A.

Hamdi I. Abdi, M.P.H.

Priyanka J. Desai, Ph.D., M.S.P.H.

Susan Duval, Ph.D.

Mary L. Forte, Ph.D., D.C.

Victoria A. Nelson, M.Sc.

Weiwen Ng, M.P.H.

Jeannine M. Ouellette, M.F.A.

Edward Ratner, M.D.

Jayati Saha, Ph.D., M.P.H.

Tetyana Shippee, Ph.D.

Brittin L. Wagner, Ph.D.

Timothy J. Wilt, M.D., M.P.H.

Lobsang Yeshi, M.Sc.

AHRQ Publication No. 20-EHC023

August 2020

Errata December 2020 
This report is based on research conducted by the Minnesota Evidence-based Practice Center (EPC) under contract to the Agency for Healthcare Research and Quality (AHRQ), Rockville, MD (Contract No. 290-2015-00008-I). The findings and conclusions in this document are those of the authors, who are responsible for its contents; the findings and conclusions do not necessarily represent the views of AHRQ. Therefore, no statement in this report should be construed as an official position of AHRQ or of the U.S. Department of Health and Human Services.

\section{None of the investigators have any affiliations or financial involvement that conflicts with the material presented in this report.}

The information in this report is intended to help healthcare decisionmakers-patients and clinicians, health system leaders, and policymakers, among others-make well-informed decisions and thereby improve the quality of healthcare services. This report is not intended to be a substitute for the application of clinical judgment. Anyone who makes decisions concerning the provision of clinical care should consider this report in the same way as any medical reference and in conjunction with all other pertinent information, i.e., in the context of available resources and circumstances presented by individual patients.

This report is made available to the public under the terms of a licensing agreement between the author and the Agency for Healthcare Research and Quality. This report may be used and reprinted without permission except those copyrighted materials that are clearly noted in the report. Further reproduction of those copyrighted materials is prohibited without the express permission of copyright holders.

AHRQ or U.S. Department of Health and Human Services endorsement of any derivative products that may be developed from this report, such as clinical practice guidelines, other quality enhancement tools, or reimbursement or coverage policies, may not be stated or implied.

This report may periodically be assessed for the currency of conclusions. If an assessment is done, the resulting surveillance report describing the methodology and findings will be found on the Effective Health Care Program Web site at www.effectivehealthcare.ahrq.gov. Search on the title of the report.

\section{Suggested citation:}

Butler M, Gaugler JE, Talley KMC, Abdi HI, Desai PJ, Duval S, Forte ML, Nelson VA, Ng W, Ouellette JM, Ratner E, Saha J, Shippee T, Wagner BL, Wilt TJ, Yeshi L. Care Interventions for People Living With Dementia and Their Caregivers. Comparative Effectiveness Review No. 231. (Prepared by the Minnesota Evidence-based Practice Center under Contract No. 290-2015-00008-I.) AHRQ Publication No. 20-EHC023. Rockville, MD: Agency for Healthcare Research and Quality; August 2020. Errata December 2020. Posted final reports are located on the Effective Health Care Program search page. DOI: https://doi.org/10.23970/ AHRQEPCCER231. 


\section{Errata}

In the original version of the report, the strength of evidence grade for intensive multicomponent interventions (i.e. discrete adaptations of REACH II) on informal caregiver quality of life at 6 months was inconsistently reported. The strength of evidence grade for this finding was erroneously reported as "low" rather than "insufficient” in five instances. These instances have been removed from the Evidence Summary key points and the full report Results and Discussion sections (pp. 77, 78, 81, and 107).

Additional references were added in two instances:

- $\quad$ Page 78: Reference 528 was added to the following sentence: “This result was consistent with a second study that found no difference between groups but improvement over baseline in both intervention and control groups."

- Page 95: The reference in row 2 of Table 8.16 was corrected to read "616" and not "641." 


\section{Preface}

The Agency for Healthcare Research and Quality (AHRQ), through its Evidence-based Practice Centers (EPCs), sponsors the development of systematic reviews to assist public- and private-sector organizations in their efforts to improve the quality of healthcare in the United States. These reviews provide comprehensive, science-based information on common, costly medical conditions, and new healthcare technologies and strategies. The National Institute on Aging (NIA) of the National Institutes of Health (NIH) requested this report from the AHRQ Evidence-based Practice Center (EPC) Program. The report was presented April 15, 2020, at the Health and Medicine Division, National Academies of Sciences, Engineering, and Medicine public meeting on Care Interventions for Individuals With Dementia and Their Caregivers.

The reports and assessments provide organizations with comprehensive, evidence-based information on common medical conditions and new healthcare technologies and strategies. They also identify research gaps in the selected scientific area, identify methodological and scientific weaknesses, suggest research needs, and move the field forward through an unbiased, evidence-based assessment of the available literature. The EPCs systematically review the relevant scientific literature on topics assigned to them by AHRQ and conduct additional analyses when appropriate prior to developing their reports and assessments.

To bring the broadest range of experts into the development of evidence reports and health technology assessments, AHRQ encourages the EPCs to form partnerships and enter into collaborations with other medical and research organizations. The EPCs work with these partner organizations to ensure that the evidence reports and technology assessments they produce will become building blocks for healthcare quality improvement projects throughout the Nation. The reports undergo peer review and public comment prior to their release as a final report.

AHRQ expects that these systematic reviews will be helpful to health plans, providers, purchasers, government programs, and the healthcare system as a whole. Transparency and stakeholder input are essential to the Effective Health Care Program.

If you have comments on this systematic review, they may be sent by mail to the Task Order Officer named below at: Agency for Healthcare Research and Quality, 5600 Fishers Lane, Rockville, MD 20857, or by email to epc@ahrq.hhs.gov.

Gopal Khanna, M.B.A.

Director

Agency for Healthcare Research and Quality

Stephanie Chang, M.D., M.P.H.

Director

Evidence-based Practice Center Program

Center for Evidence and Practice Improvement Agency for Healthcare Research and Quality
Arlene S. Bierman M.D., M.S.

Director

Center for Evidence and Practice Improvement Agency for Healthcare Research and Quality

Kim Wittenberg, M.A.

Task Order Officer

Center for Evidence and Practice Improvement Agency for Healthcare Research and Quality 


\section{Acknowledgments}

Many people were instrumental to the process that led to this final report. In particular, we wish to thank Melinda Kelley, Elena Fazio, and Courtney Wallin from the NIA for their guidance and editorial skill; Kim Wittenberg from AHRQ for her unflagging support; Ken Tobacman, Mary Walters, Morgaine Butler, and Caroline Au-Yeung for help with abstracting studies for the evidence map; Michelle Brasure for her unparalleled skill in developing search algorithms; Michael Anderson for creating graphics; Cheryl Cole-Hill for helping to collate the draft report; and Carla Kahl for her technical skills that kept all our computers functional.

\section{Key Informants and Technical Expert Panel}

The role of the Key Informants was filled by the Health and Medicine Division (HMD) Committee of the National Academies of Sciences, Engineering, and Medicine (NASEM) that will use the report to help develop its own consensus report on which care interventions are supported by sufficient evidence to be widely disseminated and implemented to NASEM and the NIA. (An overview of the NASEM conflict of interest policies can be found at https://www.nationalacademies.org/about/institutional-policies-and-procedures/conflict-ofinterest-policies-and-procedures) Because the HMD committee would not see the draft Key Questions, the population, intervention, comparator, outcomes, and study timing and setting (PICOTS) to specify the Key Questions, and analytic framework until the KQs were posted for public comment, a panel of content experts from federal agencies acted as proxy Key Informants prior to posting. The proxy Key Informants disclosed any financial conflicts of interest greater than $\$ 10,000$ and any other relevant business or professional conflicts of interest.

The list of proxy Key Informants who provided input to this report follows:

*Susan Cooley, Ph.D.

Chief, Dementia Initiatives

U.S. Department of Veterans Affairs

Washington, DC

Valerie Edwards, Ph.D.

Alzheimer's Disease and Healthy Aging Program

Centers for Disease Control and Prevention

Atlanta, GA

Melissa Gerald, Ph,D.

Program Director

Division of Behavioral and Social Research

National Institute on Aging

Bethesda, MD 
Margaret Kabat, L.C.S.W.-C.

National Director Caregiver \& Family Support

U.S. Department of Veterans Affairs

Washington, DC

Rohini Khillan, M.P.H.

Office of Disability, Aging, and Long Term Care Policy

Office of the Assistant Secretary for Planning and Evaluation

Washington, DC

Greg Link, M.A.

Director, Office of Supportive and Caregiver Services

Administration for Community Living

Washington, DC

*Erin Long, M.S.W.

Aging Services Program Specialist, Dementia Team Lead

Administration for Community Living

Washington, DC

Lisa McGuire, Ph.D.

Lead, Alzheimer's Disease and Healthy Aging Program

Centers for Disease Control and Prevention

Atlanta, GA

*Anthony Pacifico, Ph.D.

Program Manager, Peer Reviewed Alzheimer’s Research Program

Congressionally Directed Medical Research Program

Fort Detrick, MD

*This TEP member also provided review of the draft report.

\section{Peer Reviewers}

Prior to publication of the final evidence report, EPCs sought input from independent Peer Reviewers without financial conflicts of interest. However, the conclusions and synthesis of the scientific literature presented in this report do not necessarily represent the views of individual reviewers.

Peer Reviewers must disclose any financial conflicts of interest greater than $\$ 10,000$ and any other relevant business or professional conflicts of interest. Because of their unique clinical or content expertise, individuals with potential nonfinancial conflicts may be retained. The Task Order Officer and the EPC work to balance, manage, or mitigate any potential nonfinancial conflicts of interest identified. 
The list of Peer Reviewers follows:

Richard H. Fortinsky, Ph.D.

Professor

University of Connecticut Center on Aging

University of Connecticut

Farmington, CT

Helen Lamont, Ph.D.

Long-Term Care Policy Analyst

Office of Disability, Aging, and Long Term Care Policy

Office of the Assistant Secretary for Planning and Evaluation

Washington, DC

Katherine Ornstein, Ph.D.

Associate Professor

Icahn School of Medicine at Mount Sinai

New York, NY

Tia Powell, M.D.

Professor

Albert Einstein College of Medicine

Montefiore Medical Center

Bronx, NY

Barbara Resnick, Ph.D., C.R.N.P., F.A.A.N.

Professor

University of Maryland

College Park, MD 


\section{Care Interventions for People Living With Dementia and their Caregivers}

\section{Structured Abstract}

Objective. To understand the evidence base for care interventions for people living with dementia (PLWD) and their caregivers, and to assess the potential for broad dissemination and implementation of that evidence.

Data sources. We searched Ovid Medline, Ovid Embase, Ovid PsycINFO, CINAHL, and the Cochrane Central Register of Controlled Trials (CENTRAL) to identify randomized controlled trials, nonrandomized controlled trials, and quasi-experimental designs published and indexed in bibliographic databases through March 2020.

Review methods. We searched for nondrug interventions targeting PLWD, their informal or formal caregivers, or health systems. Two investigators screened abstracts and full-text articles of identified references for eligibility. Eligible studies included randomized controlled trials and quasi-experimental observational studies enrolling people with Alzheimer's disease or related dementias or their informal or formal caregivers. We extracted basic study information from all eligible studies. We assessed risk of bias and summarized results for studies not judged to be NIH Stage Model 0 to 2 (pilot or small sample size studies) or to have high risk of bias. We grouped interventions into categories based on intervention target.

Results. We identified 9,217 unique references, of which 627 unique studies with an additional 267 companion articles were eligible. We classified interventions into 37 major categories. With few exceptions, we did not combine data quantitatively due to variability of interventions, comparison groups, outcomes measured, and study timing. Low-strength evidence shows that an intensive multicomponent intervention for informal caregiver support, with education, group discussion, in-home and phone support, and caregiver feedback (i.e., discrete adaptations of REACH II), may improve informal caregiver depression at 6 months. Low-strength evidence also shows that collaborative care models (i.e., Care Ecosystems or discrete adaptations of the ACCESS models) may improve quality of life for PLWD and health system-level markers, including improvements in guideline-based quality indicators and reducing emergency room visits. The literature does not allow for further determination of whether the very small to small average effects in quality of life applied to all enrolled PLWD or if larger effects were concentrated in an unidentified subgroup. For all other interventions and outcomes, we found the evidence insufficient to draw conclusions. Insufficient evidence does not mean that the intervention is determined to be of no value to PLWD or their caregivers. Rather, it means that, due to the uncertainty of the evidence, we cannot draw meaningful conclusions at this time.

Conclusions. Despite hundreds of studies, very little evidence supports widespread dissemination of any general care approaches for PLWD or caregivers. This review demonstrates the need for larger, longer-term, and more-rigorous studies of interventions. 


\section{Contents}

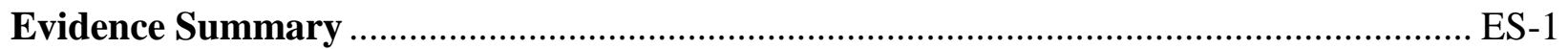

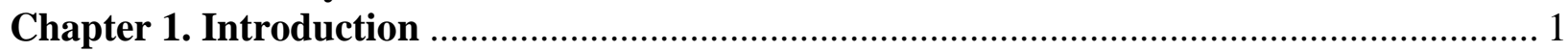

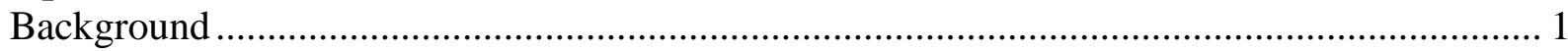

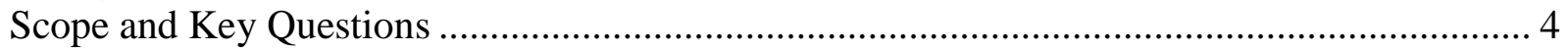

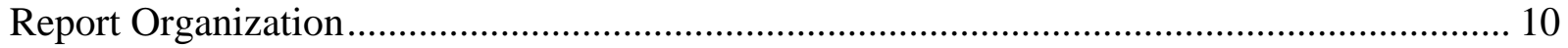

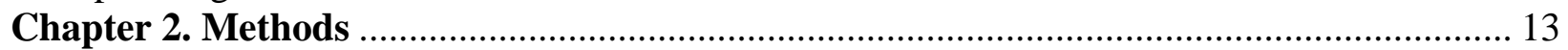

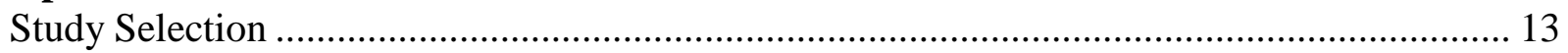

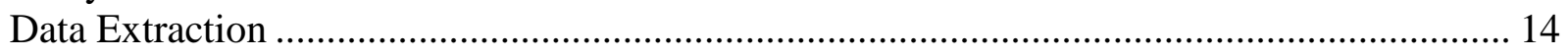

Assessing Methodological Risk of Bias and NIH Stage of Individual Studies ...................... 16

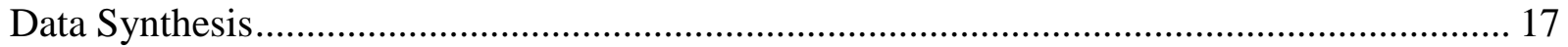

Grading the Strength of Evidence for Major Comparisons and Outcomes ........................... 18

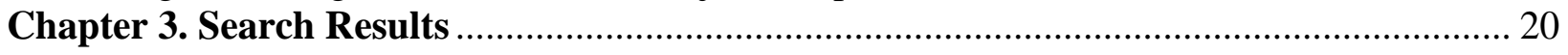

Chapter 4. Care Interventions for Managing BPSD in PLWD ...................................... 23

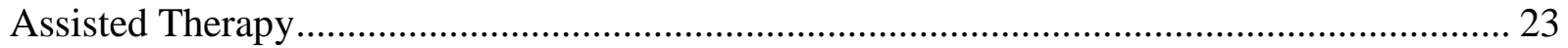

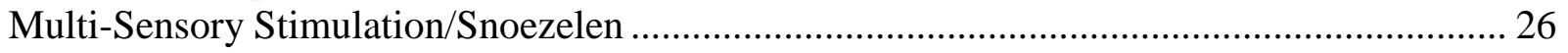

Complementary and Alternative Medicine (CAM) Therapies ........................................... 28

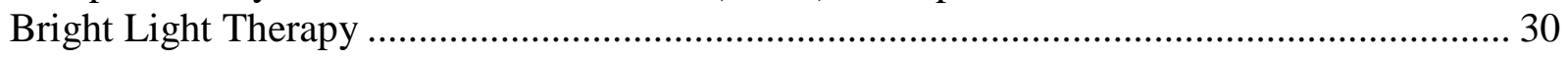

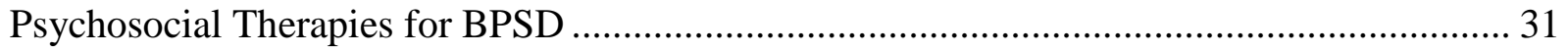

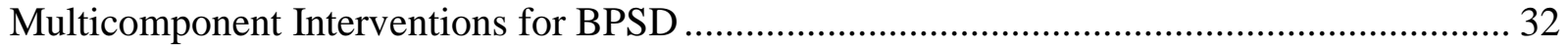

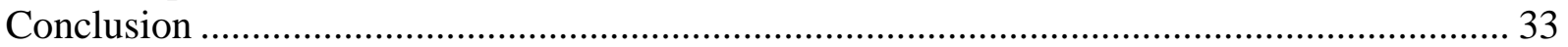

Chapter 5. Care Interventions for PLWD Well-Being................................................ 34

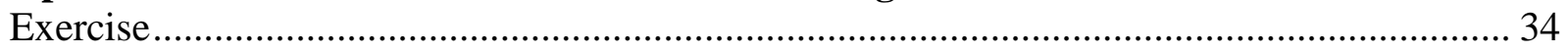

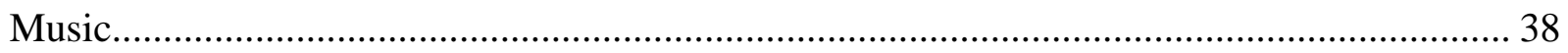

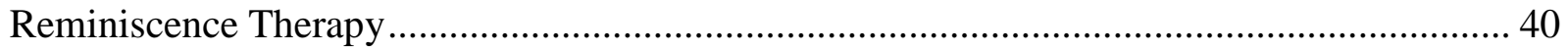

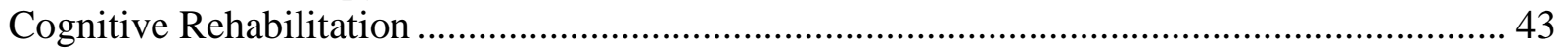

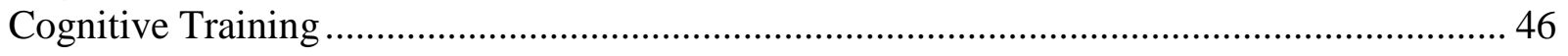

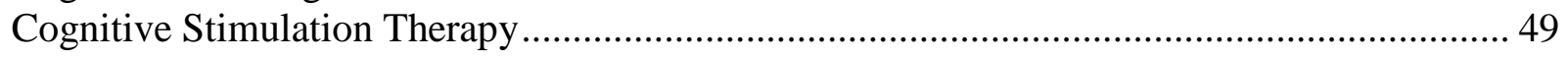

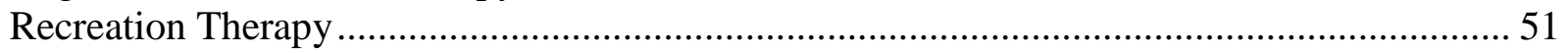

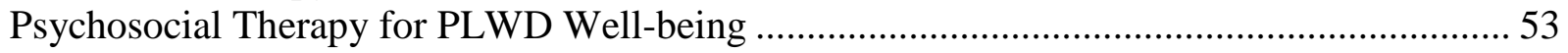

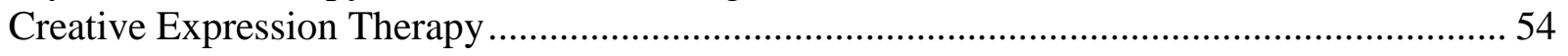

Multicomponent Interventions for PLWD Well-being ..................................................... 55

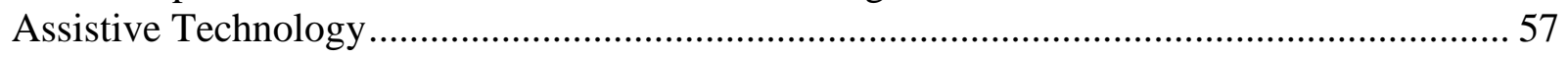

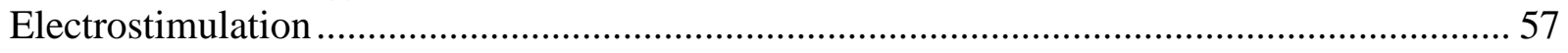

Other Interventions for PLWD Well-being ................................................................. 59

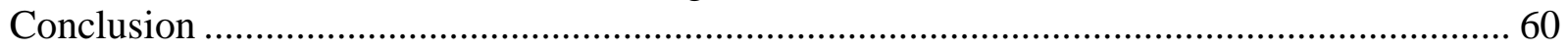

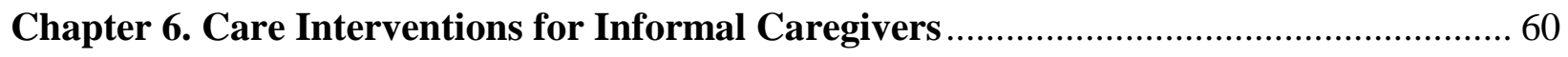

Psychosocial Interventions to Support Informal Caregiver Well-being ................................ 60

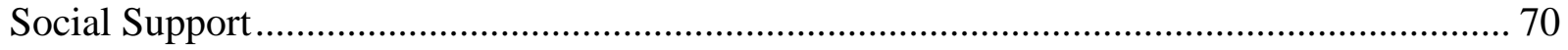

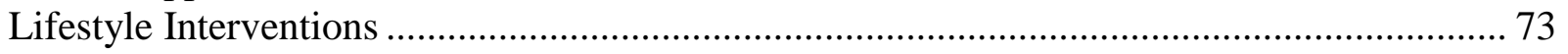

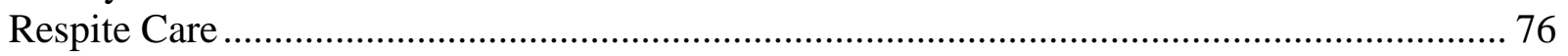

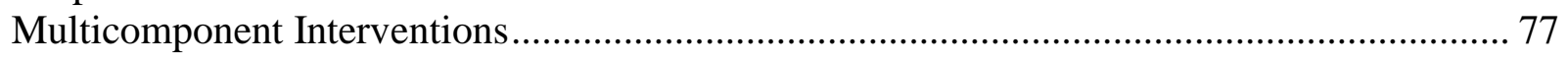

Other Interventions for Caregiver Well-being ............................................................ . 79

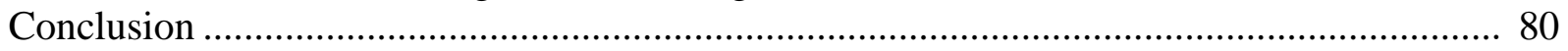

Chapter 7. Care Interventions for Formal Caregivers.................................................. 81 


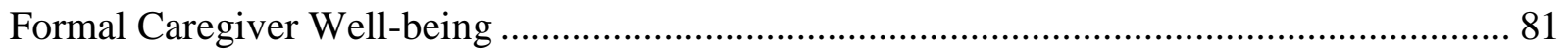

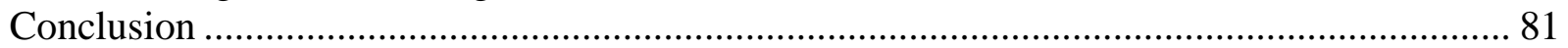

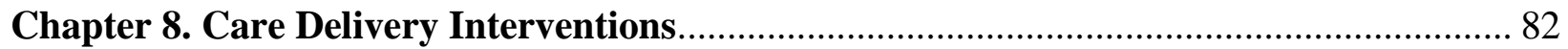

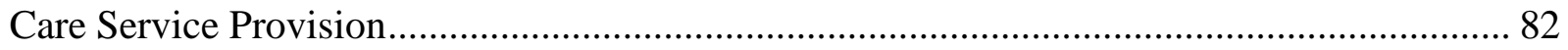

Care Delivery Models and Programs ......................................................................................... 93

Care Staff Education and Support Needs.......................................................................... 98

Conclusion ..................................................................................................................... 103

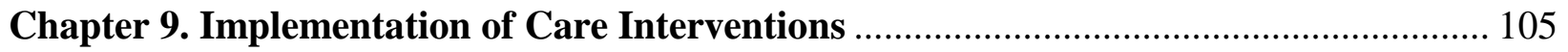

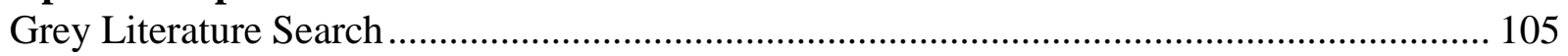

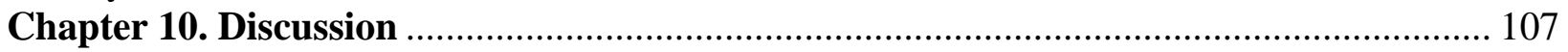

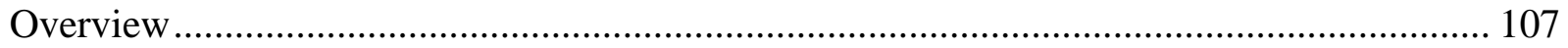

Strengths and Weaknesses of the Review..................................................................... 107

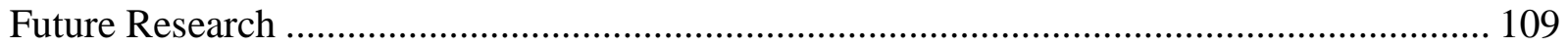

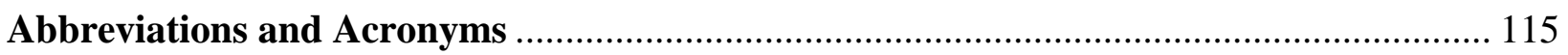

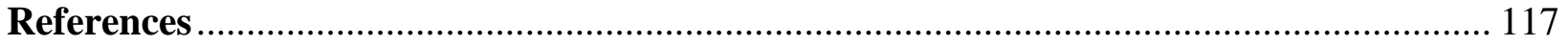

\section{Tables}

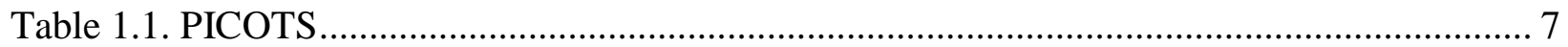

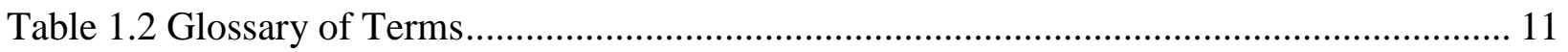

Table 2.1. Study inclusion criteria ............................................................................................ 12

Table 3.1 Identified unique eligible studies bt intervention category, by results chapter ............. 21

Table 4.1. Basic characteristics of literature set: robot-assisted therapy ....................................... 24

Table 4.2. Summary of findings for PLWD outcomes: robot-assisted therapy............................... 25

Table 4.3. Basic characteristics of literature set: live animal- and doll-assisted therapy .............. 25

Table 4.4. Basic characteristics of literature set: multisensory stimulation/Snoezelen .............. 26

Table 4.5. Summary of findings for PLWD outcomes: multisensory stimulation/Snoezelen...... 27

Table 4.6. Basic characteristics of literature set: complementary and alternative medicine ........ 28

Table 4.7. Summary of findings for PLWD outcomes: complementary and alternative medicine

Table 4.8. Basic characteristics of literature set: bright light therapy ………………………...... 30

Table 4.9. Basic characteristics of literature set: psychosocial therapies for PLWD .................... 31

Table 4.10 Basic characteristics of literature set: multicomponent interventions for BPSD......... 32

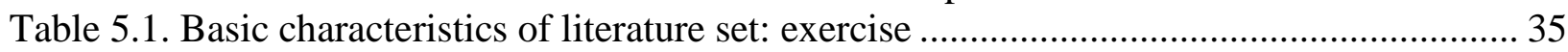

Table 5.2. Summary of findings for PLWD outcomes: exercise .................................................. 37

Table 5.3. Basic characteristics of literature set: music............................................................. 38

Table 5.4 Summary of findings for PLWD outcomes: music ........................................................ 39

Table 5.5 Summary of findings for caregiver outcomes: music ................................................... 40

Table 5.6 Basic characteristics of literature set: reminiscence therapy …...................................... 41

Table 5.7 Summary of findings for PLWD outcomes: reminiscence therapy …………................. 42

Table 5.8 Summary of findings for caregiver outcomes: reminiscence therapy ........................... 43

Table 5.9 Basic characteristics of literature set: cognitive rehabilitation ........................................ 44

Table 5.10: Summary of findings for PLWD outcomes: cognitive rehabilitation ......................... 45

Table 5.11. Basic characteristics of literature set: cognitive training ............................................... 46

Table 5.12 Summary of findings for PLWD outcomes: cognitive training................................... 47

Table 5.13 Summary of findings for caregiver outcomes: cognitive training ................................ 48

Table 5.14. Basic characteristics of literature set: cognitive stimulation therapy ......................... 49 
Table 5.15 Summary of findings for PLWD outcomes: cognitive stimulation therapy .............. 50

Table 5.16 Basic characteristics of literature set: recreational therapy ................................... 51

Table 5.17 Summary of findings for PLWD outcomes: recreational therapy ............................ 52

Table 5.18 Basic characteristics of literature set: psychosocial interventions for PLWD........... 53

Table 5.19 Basic characteristics of literature set: creative expression therapy.......................... 55

Table 5.20 Basic characteristics of literature set: multicomponent for PLWD well-being .......... 55

Table 5.21 Summary of findings for PLWD outcomes: multicomponent for PLWD well-being 56

Table 5.22 Basic characteristics of literature set: assistive home technology .......................... 57

Table 5.23 Basic characteristics of literature set:electrostimulation ......................................... 58

Table 5.24 Basic characteristics of literature set:other interventions for BPSD....................... 58

Table 6.1 Basic characteristics of literature set: psychosocial interventions for informal

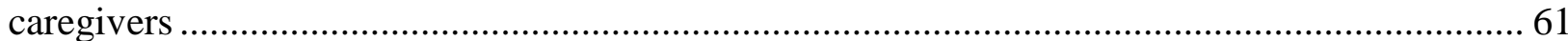

Table 6.2 Summary of findings for PLWD outcomes: psychosocial interventions for CG/P ..... 64

Table 6.3 Summary of findings for caregiver outcomes: psychosocial interventions for CG/P .. 67

Table 6.4 Basic characteristics of literature set:in-person social support ............................... 70

Table 6.5 Summary of findings for caregiver outcomes: social support ................................ 71

Table 6.6 Basic characteristics of literature set: mindfulness, meditation, and spiritually-focused

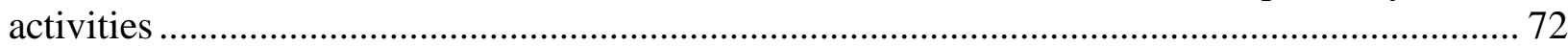

Table 6.7 Summary of findings for caregiver outcomes: mindfulness, meditation, and spiritually-

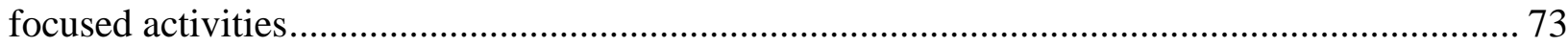

Table 6.8 Basic characteristics of literature set:physical activity and other lifestyle interventions

Table 6.9 Basic characteristics of literature set: respite care ............................................... 75

Table 6.10 Basic characteristics of literature set: multicomponent for CG/P............................ 76

Table 6.11 Summary of findings for caregiver outcomes:multicomponent for CG/P............... 77

Table 6.12 Basic characteristics of literature set: other interventions for CG/P......................... 78

Table 7.1 Basic characteristics of literature set: formal caregiver well-being............................. 81

Table 8.1 Basic characteristics of literature set: consultation services ..................................... 83

Table 8.2 Summary of findings for PLWD outcomes: consultation services............................ 83

Table 8.3 Summary of findings for caregiver outcomes: consultation services ........................ 82

Table 8.4 Basic characteristics of literature set: case management ...................................... 85

Table 8.5 Summary of findings for PLWD outcomes: case management............................... 85

Table 8.6 Summary of findings for caregiver outcomes: case management ............................. 86

Table 8.7 Basic characteristics of literature set: care protocols for PLWD ............................... 87

Table 8.8. Basic characteristics of literature set: advance care planning.................................. 88

Table 8.9. Summary of findings for PLWD outcomes: advance care planning ........................ 88

Table 8.10 Summary of findings for caregiver outcomes: advance care planning...................... 89

Table 8.11. Basic characteristics of literature set: palliative care ........................................... 89

Table 8.12 Basic characteristics of literature set: other service provision interventions .............. 90

Table 8.13 Summary of findings for PLWD outcomes: other service provision interventions.... 91

Table 8.14 Summary of findings for caregiver outcomes: other service provision interventions 91

Table 8.15. Basic characteristics of literature set: collaborative care models .......................... 93

Table 8.16 Summary of findings for PLWD outcomes: collaborative care models .................... 94

Table 8.17. Summary of findings for caregiver outcomes: collaborative care models ................ 95

Table 8.18. Basic characteristics of literature set: individualized person-centered care (non-

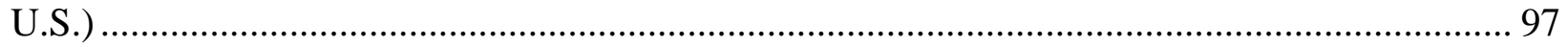


Table 8.19. Basic characteristics of literature set: formal caregiver staff training ..................... 98

Table 8.20 Basic characteristics of literature set: CG/P training ............................................. 99

Table 8.21 Basic characteristics of literature set: family education and partnering .................. 100

Table 8.22 Basic characteristics of literature set: multitier training ....................................... 101

Table 8.23 Summary of findings for PLWD outcomes: multitier training.............................. 101

Table 8.24 Summary of findings for caregiver outcomes: multitier training .......................... 102

\section{Figures}

Figure 1.1. Framework for care interventions ................................................................... 3

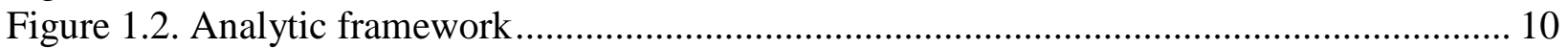

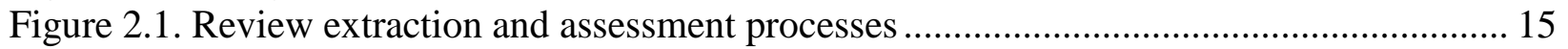

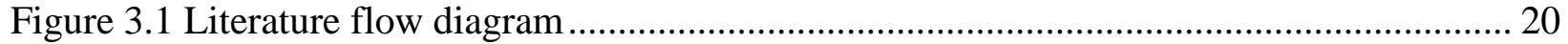

Figure 6.1 Collaboration network visualization of informal caregiver psychosocial intervention

components...............................................................61

\section{Appendixes}

Appendix A. Methods

Appendix B. Excluded Studies

Appendix C. Chapter 4 Treatment for BPSD

Appendix D. Chapter 5 Interventions for PLWD Well-Being

Appendix E. Chapter 6 and 7 Interventions for Caregiver Well-Being

Appendix F. Chapter 8 Care Delivery Interventions Consultation 


\section{Evidence Summary}

\section{Main Points}

- An intensive multicomponent intervention with education, group discussion, in-home and phone support sessions, and caregiver feedback for informal caregiver support (i.e. discrete adaptations of REACH II), may improve informal caregiver depression at 6 months. (low-strength evidence)

- Collaborative care models (i.e. Care Ecosystems or discrete adaptations of the ACCESS models) may improve people living with dementia (PLWD) quality of life. (low-strength evidence) The literature does not allow for further determination of whether the very small to small average effects applied to all enrolled PLWD or if larger effects were concentrated in an unidentified subgroup.

- Collaborative care models (i.e. discrete adaptations of the ACCESS model) may improve system-level markers, including guideline-based quality indicators and reduction in emergency department visits. (low-strength evidence)

- For all other outcomes and interventions, we found the evidence was insufficient because the uncertainty of the evidence was too high to draw conclusions.

- We found little information to determine whether interventions are equally appropriate for or have been successfully adapted to other race/ethnic cultures within the United States, rural communities/communities with low resources, or specific populations of PLWD, such as people with Down syndrome or complex presentations of dementias.

\section{Background and Purpose}

The aging of the U.S. population and the concurrent rise in the number of adults living with dementia underscore the urgent need for a systematic review of the available evidence for care interventions for PLWD and their formal and informal caregivers. The National Institute on Aging commissioned such a review from the Evidence-based Practice Center Program at the Agency for Healthcare Research and Quality. Dementia affects about 5 million U.S. adults 65 years and older (with disparities by race and ethnicity), and that number may grow to almost 14 million by 2060.,

The goal is to understand the evidence base for effective care interventions, and to assess the potential for broad dissemination and implementation of that evidence. Subsequently, a National Academies of Sciences, Engineering, and Medicine committee of experts, established at the request of the National Institute on Aging, will use the evidence findings delivered in this report to help develop its own independent recommendations regarding which care interventions are supported by sufficient evidence to be widely disseminated and implemented, as well as to identify research gaps.

\section{Methods}

The methods for this systematic review follow the Agency for Healthcare Research and Quality Methods Guide for Effectiveness and Comparative Effectiveness Reviews. ${ }^{9}$ See the review protocol (https://effectivehealthcare.ahrq.gov/products/care-interventions-pwd/protocol) and the full report of the review for additional details. We searched Ovid Medline, Ovid Embase, Ovid PsycINFO, CINAHL, and the Cochrane Central Register of Controlled Trials (CENTRAL) 
to identify randomized controlled trials, nonrandomized controlled trials, and quasi-experimental designs published and indexed in bibliographic databases through October, 2019.

\section{Results}

We identified 9217 unique references, 894 of which were eligible for our review, comprising 627 unique studies with an additional 267 companion publications. We sorted eligible studies into 37 major intervention categories. Approximately 60 percent of the literature emerged from research conducted outside of the United States.

While the literature was highly diverse, we found little information to determine whether interventions are equally appropriate for or have been successfully adapted to other race/ethnic cultures within the United States, with only a handful of studies providing sufficient inclusion of African-American or Hispanic/Latino populations. Interventions for rural communities were found to be even more rare in the literature. Additionally, the many countries in which care interventions were evaluated were almost exclusively high-resource, with very few low-resource countries represented. Finally, few studies gathered the granular detail necessary for a deeper understanding of the applicability of the interventions. Many important groups were rarely studied, including people with Down syndrome, who are living longer and who overall experience higher rates of dementia than the general population, and individuals with complex presentations of dementia.

We found low-strength evidence that one multicomponent intervention for informal caregiver support, REACH II (comprised of education, group discussion, in-home and phone support sessions, and caregiver feedback), may improve caregiver depression. ${ }^{3-5}$ Collaborative care models based on the ACCESS or Care Ecosystems models may improve quality of life for $\mathrm{PLWD}^{6-9}$ and health system-level markers, including improvements in guideline-based quality indicators ${ }^{7,8}$ and reducing emergency room visits. ${ }^{6}$ For all other interventions and outcomes, we found the uncertainty of the evidence was too high to draw conclusions. The vast majority of studies had small sample size, were pilots that had not undergone traditional efficacy testing, and/or had high risk of bias, and provided insufficient evidence to draw conclusions.

\section{Limitations}

As stated, the goal of the review was to understand the evidence base for effective care interventions for PLWD and their caregivers in order to assess the potential for broad dissemination and implementation of that evidence. All decisions about the review methodology followed from this goal, which has implications for our findings. Therefore, some specific approaches for a particular intervention, or even whole classes of interventions, may not have been captured. Similarly, we may have missed some community services and support approaches such as tool kits, referral services and links, or awareness-raising outreach.

Because we excluded studies with fewer than 10 participants per study arm, we may not have identified some interventions with only very preliminary research. Our approach to risk of bias assessment was generous, relative to how risk of bias is assessed in more targeted systematic review topics. This is in part due to the unusually varied studies included in this review as well as the complexity of the condition and the care approaches.

Furthermore, included studies had methodological problems such as low subject retention, widely varying measures of success, and relatively small size trials that may have lacked the power to detect benefits. These problems stem from well-recognized challenges in researching these populations; therefore, we erred on the side of assessing a body of research as insufficient 
rather than ineffective. Additionally, given the progressive nature of dementia and the anticipated increase in care needs over time, it may be difficult to identify improvement in caregiver burden. The goal may in fact be to slow the rate of burden - and studies may be underpowered to detect such a small effect.

Finally, given the breadth of the topic, our systematic review is naturally reductionist in nature. That is, small but true differences may exist between many of the categories we summarized into our outcomes.

\section{Implications and Conclusions}

Ultimately, we uncovered very little evidence to support interventions and programs for active, widespread dissemination because evidence was insufficient to draw conclusions about the effects of the vast majority of interventions studied.

Dementia care research has been slow to incorporate key elements of rigorous intervention design. Until relatively recently, many dementia care intervention studies were not held to reporting standards (e.g. the Consolidated Standards of Reporting Trials statement), preregistration of trials, data safety and monitoring boards, or other standards more common in other areas of clinical science. As a direct result, despite a few positive findings, our global conclusions, largely similar to past reviews, ${ }^{10}$ are that the amount of high-quality evidence is insufficient to draw firm conclusions about interventions.

In order for Federal funders and stakeholders to expedite the translational pipeline of idea development to implementation, as they aim to do, critical improvements are needed in dementia care research. Only with such improvements will we be able to draw clearer, less ambiguous conclusions related to efficacy.

\section{References}

1. Matthews KA, Xu W, Gaglioti AH, et al. Racial and ethnic estimates of Alzheimer's disease and related dementias in the United States (2015-2060) in adults aged $\geq 65$ years. Alzheimers Dement. 2019 Jan;15(1):17-24. doi: 10.1016/j.jalz.2018.06.3063. PMID: 30243772.

2. Hebert LE, Weuve J, Scherr PA, et al. Alzheimer disease in the United States (2010-2050) estimated using the 2010 census. Neurology. 2013 May 7;80(19):1778-83. doi: 10.1212/WNL.0b013e31828726f5. PMID: 23390181.

3. Belle SH, Burgio L, Burns R, et al. Enhancing the quality of life of dementia caregivers from different ethnic or racial groups: a randomized, controlled trial.[Summary for patients in Ann Intern Med. 2006 Nov 21;145(10):I39; PMID: 17116914]. Annals of Internal Medicine. $2006 \mathrm{Nov}$ 21;145(10):727-38. PMID: 17116917.
4. Berwig M, Heinrich S, Spahlholz J, et al. Individualized support for informal caregivers of people with dementia effectiveness of the German adaptation of REACH II. BMC Geriatrics. 2017 Dec 12;17(1):286. doi: https://dx.doi.org/10.1186/s12877-0170678-y. PMID: 29233097.

5. Luchsinger JA, Burgio L, Mittelman M, et al. Comparative Effectiveness of 2 Interventions for Hispanic Caregivers of Persons with Dementia. Journal of the American Geriatrics Society. 2018 Sep;66(9):1708-15. doi: https://dx.doi.org/10.1111/jgs.15450. PMID: 30084133. 
6. Possin KL, Merrilees JJ, Dulaney S, et al. Effect of Collaborative Dementia Care via Telephone and Internet on Quality of Life, Caregiver Well-being, and Health Care Use: The Care Ecosystem Randomized Clinical Trial. JAMA Internal Medicine. 2019 Sep 30;30:30. doi: https://dx.doi.org/10.1001/jamainternmed.20 19.4101. PMID: 31566651.

7. Chodosh J, Colaiaco BA, Connor KI, et al. Dementia Care Management in an Underserved Community: The Comparative Effectiveness of Two Different Approaches. Journal of Aging \& Health. 2015 Aug;27(5):864-93. doi: https://dx.doi.org/10.1177/08982643155694 54. PMID: 25656074.
8. Vickrey BG, Mittman BS, Connor KI, et al. The effect of a disease management intervention on quality and outcomes of dementia care: a randomized, controlled trial.[Summary for patients in Ann Intern Med. 2006 Nov 21;145(10):I31; PMID: 17116913]. Annals of Internal Medicine. 2006 Nov 21;145(10):713-26. PMID: 17116916.

9. Thyrian JR, Hertel J, Wucherer D, et al. Effectiveness and Safety of Dementia Care Management in Primary Care: A Randomized Clinical Trial. JAMA Psychiatry. 2017 Oct 01;74(10):996-1004. doi:

https://dx.doi.org/10.1001/jamapsychiatry.2 017.2124. PMID: 28746708.

10. Gaugler JE, Jutkowitz E, Gitlin L. NonPharmacological Interventions for Persons Living with Alzheimer's Disease: Decadal Review and Recommendations. National Academies of Sciences, Engineering, and Medicine: Washington DC. 2020. 


\section{Chapter 1. Introduction}

\section{Background}

The aging of the U.S. population and the concurrent rise in the number of adults living with dementia underscore the urgent need for a systematic review of the available evidence for care interventions for people living with dementia (PLWD) and their formal and informal caregivers. ${ }^{1}$ The National Institute on Aging (NIA) has commissioned such a review from the Evidencebased Practice Center Program at the Agency for Healthcare Research and Quality (AHRQ). The goal is to understand the evidence base for effective care interventions, and to assess the potential for broad dissemination and implementation of that evidence.

Dementia affects about 5 million U.S. adults 65 years and older (with disparities by race and ethnicity), and that number may grow to almost 14 million by $2060 .{ }^{1-3}$ A further 200,000 individuals under age 65 have some form of early-onset dementia. ${ }^{1}$ As a clinical syndrome and a disability, dementia is characterized by an acquired cognitive deficit that interferes with independence in daily activities. ${ }^{4}$ Alzheimer's disease is the most common form of progressive dementia and, grouped with Lewy body, frontotemporal, vascular, and mixed forms, it has been referred to as AD/ADRD (i.e., Alzheimer's disease [AD] and Alzheimer's disease related dementias [ADRD]). ${ }^{5}$ Dementia can lower an individual's quality of life, burden caregivers (even those who find caregiving very rewarding), increase institutionalization, and increase costs to families and society. ${ }^{6}$ Agitation, aggression, and other behavioral disturbances are common, especially late in the disease course. ${ }^{7}$

The significant public health implications of dementia led to the 2011 passage of the National Alzheimer's Project Act, an effort to create a national research strategy to accelerate scientific discovery of curative treatments, preventive approaches, and effective strategies to manage and alleviate the many clinical symptoms of AD/ADRD. The National Alzheimer's Project Act has spurred considerable Federal investment; the National Institutes of Health (NIH) funding for $\mathrm{AD} / \mathrm{ADRD}$ research has more than tripled since $2015 .^{8}$ (See https://report.nih.gov/categorical_spending.aspx.)

In addition to attracting new investigators, the NIA and other Federal agencies have leveraged funding increases to initiate and/or contribute to several important, complementary, large-scale efforts to improve the design and delivery of care for PLWD. Among these are the first ever National Research Summit on Care, Services, and Supports for Persons with Dementia and Their Caregivers. This 2017 summit generated a number of recommendations to advance the science of dementia care and catalyzed several important actions, including a substantial investment in several Funding Opportunity Announcements, one of which resulted in award of the NIA IMbedded Pragmatic Alzheimer's disease (AD) and AD Related Dementias (AD/ADRD) Clinical Trials (IMPACT) Collaboratory. The IMPACT Collaboratory is designed to provide technical assistance and pilot grant support to "embed” dementia care trials within healthcare systems across the United States, a decadal review of the state of behavioral and social science research (including but not limited to AD/ADRD care intervention research) commissioned by the National Academies of Sciences, Engineering and Medicine (NASEM), as well as other efforts.

While these initiatives are designed for rapid advancement of the science of dementia, this systematic review of the available evidence for care interventions for PLWD and their formal and informal caregivers will provide valuable information about the efficacy or effectiveness of certain types of strategies/interventions. This review also pinpoints areas that require greater 
attention when evaluating, disseminating, or implementing certain dementia care and caregiver interventions.

Dementia has no known cure, but both drug and nondrug interventions are available to treat symptoms, support function, and improve quality of life. Nondrug interventions have been recommended as first-line treatments for behavioral and psychological symptoms of dementia (BPSD), but pharmacological treatment options such as antipsychotics are also available. ${ }^{9}$ And although nondrug interventions are generally presumed safe, few trials have reported information on their harms or other unintended consequences. (Drugs and over-the-counter supplements to treat clinical Alzheimer's-type dementia and BPSD are being addressed by a separate AHRQ systematic review; please see https://effectivehealthcare.ahrq.gov/products/alzheimers-typedementia/research.)

Dementia care is costly, and more than 83 percent of community-residing older adults who need it rely on help from family members. ${ }^{10}$ In 2017, informal (unpaid) caregivers for PLWD provided an estimated 17 billion hours of care at an economic value of \$232.1 billion, and about two-thirds of informal caregivers are women. ${ }^{11}$ Caregiving for dementia is multifaceted and can be both rewarding and burdensome, sometimes simultaneously. Many surveys suggest that, for some, caregiving instills confidence, provides lessons on dealing with difficult situations, and increases feelings of closeness to the care recipient. ${ }^{12}$ However, evidence also suggests that caregivers have lower self-ratings of physical health, elevated levels of stress hormones, higher rates of chronic disease, and impaired health behaviors. Therefore, many research teams have developed and tested interventions for supporting the health and well-being of informal caregivers. Some examples include social support, therapeutic counseling, skills training, respite, and combined approaches. ${ }^{13}$ Additionally, many frontline paid caregivers, such as home health aides in home-based settings or certified nursing assistants in institutional settings, lack adequate training and support for this difficult work. ${ }^{12}$ A recent NASEM report recommended an increase in Federal requirements for training of direct care workers — from 75 hours to 120 hours—along with more focus on knowledge and skills related to caring for PLWD. ${ }^{14}$

Care interventions for PLWD encompass a broad range of activities that support, enhance, or otherwise help the care recipient. Likewise, care interventions comprise an array of options that, as noted by the NASEM committee's feedback on the framing and parameters for this review [https://www.nationalacademies.org/our-work/care-interventions-for-individuals-with-dementiaand-their-caregivers], “contribute to a person’s well-being, happiness, identity, privacy, capacity, autonomy, or authority. They can be supports, services, programs, accommodations, or practices that include behavioral, environmental, technological, and psychological methods or approaches. They may be delivered by healthcare, social services, and other community organizations or caregivers with the intention of having a direct impact on either a person with dementia or their caregiver or both."

Necessarily, then, interventions addressing care for PLWD and their caregivers can be complicated and multifaceted. Unfortunately, no consensus has been reached on classification systems for types of interventions, and investigators are left to categorize interventions themselves, based on varied criteria. In our own effort to categorize interventions for this review, we identified two basic intervention groups: 1) interventions testing a type of care that is aimed at improving the health and well-being of PLWD and/or their caregivers (e.g., interventions that use music or essential oils to help calm the care recipient, or respite care that provides a break for the caregiver and 2) interventions testing the manner in which care is delivered in order to improve effectiveness, efficiency, and/or accessibility and availability of care (e.g., staff training 
for caregivers, coordination of care). This review refers to interventions testing a type of care as care interventions, and interventions testing the manner in which care is delivered as care delivery interventions.

Intervention complexity also stems from the diversity of PLWD (e.g., younger adults with Down syndrome or other genetic risk factors, younger and middle-aged adults with frontotemporal dementia, and older adults with AD, from very early to advanced stages) as well as different caregiver populations (e.g., spousal caregivers, adult child caregivers, paid caregivers). Intervention designs may be straightforward and aimed at supporting a single, welldefined group, such as formal or informal caregivers, or they may be very complex and target several levels of a system simultaneously, from a care system (e.g., healthcare or social services) to family units or caregiver/PLWD dyads to individual formal or informal caregivers. (Figure 1.1) Furthermore, complexity in outcomes may arise because interventions targeting one level of a system, such as PLWD, may benefit other individuals, such as caregivers, or other levels of the system, such as reduced use of healthcare services for an accountable care organization.

Figure 1.1. Framework for care interventions

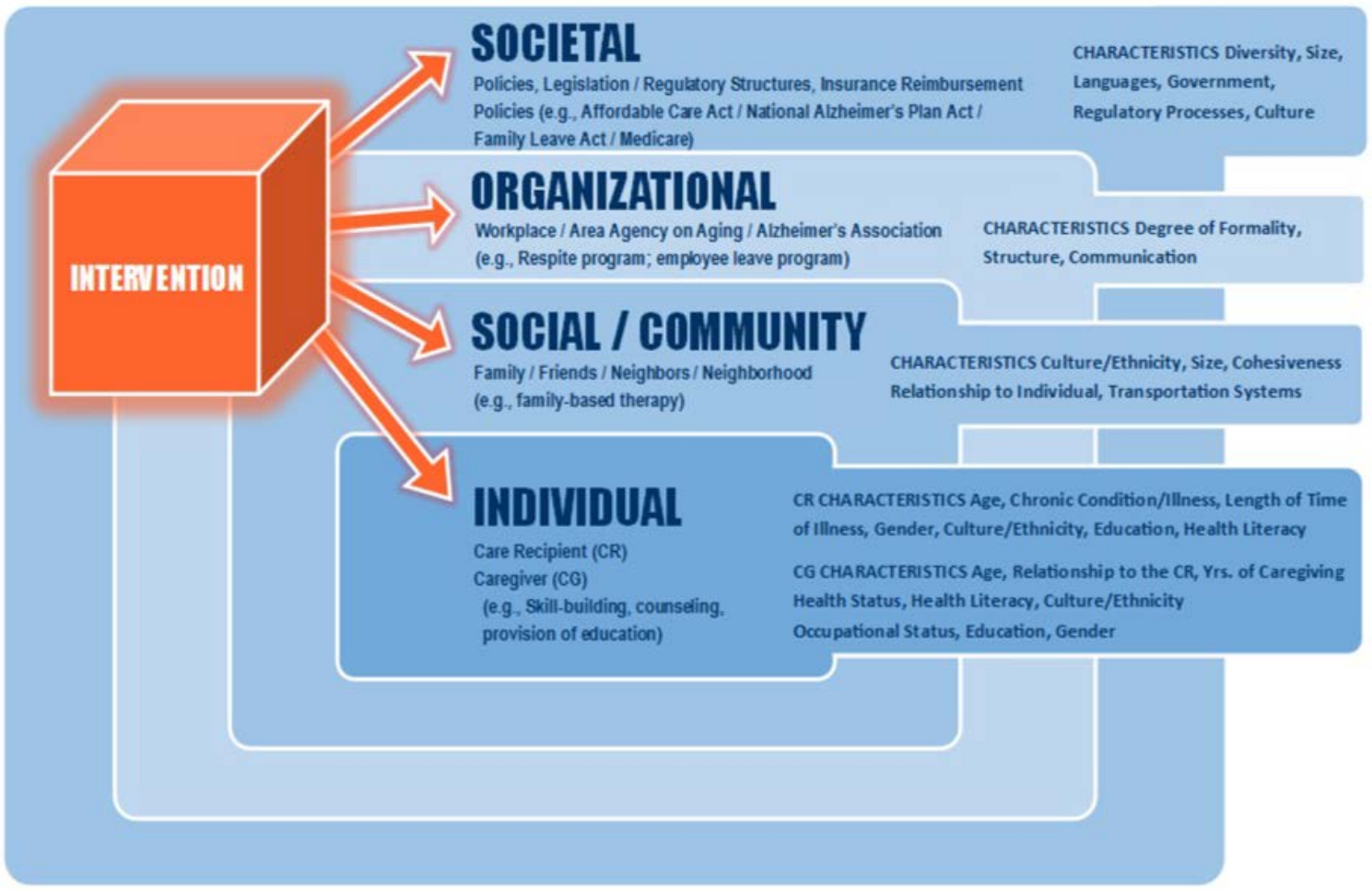

Abbreviations: $\mathrm{CG}=$ caregiver; $\mathrm{CR}=$ care recipient

Source: NASEM, 2016, Families caring for an aging America. p. 163. Used with permission.

Given these complexities, our review aims to specify intervention characteristics that link to benefits. Unfortunately, informal and formal caregivers may not always be easily characterized according to the levels outlined in Figure 1.1; paid caregivers may be hired as independent contractors by family of a PLWD, whereas unpaid volunteers may be affiliated with a larger organization. Nonetheless, information regarding relationships between PLWD and caregiver characteristics and outcomes will help clinicians, care providers, and other stakeholders make decisions about the best interventions for their specific circumstances or PLWD. 
Assessing whether a care intervention is ready for broad implementation is challenging. For this review, we were guided by the NIH Stage Model for Behavioral Interventions. ${ }^{15}$ This model provides a conceptual framework of intervention research development, ranging from basic science research (Stage 0) to new intervention creation (Stage 1), research-setting efficacy (Stage 2), "real-world" community-clinic efficacy (Stage 3), broad community-based effectiveness (Stage 4), to eventually dissemination and implementation research (Stage 5). This model not only describes the stages of behavioral intervention development, but also supports eventual implementation. While the stages are not a direct assessment of implementation readiness, the model suggests that interventions at Stage 3 or higher are more likely to be ready for broad dissemination. Interventions at Stage 4 that use pragmatic study designs move research closer to "real world" conditions and population levels.

\section{Scope and Key Questions}

This review examines a large number of nondrug care interventions targeted at PLWD, their informal and formal caregivers, and the larger health systems, including collaborative or integrated care. The intended audience is similarly broad, from PLWD and their families, to care services and support providers, to research organizations and policymakers at national, local, state, tribal, and Federal levels.

Given such a wide range of stakeholders, we have prioritized the readability and usability of our review by striving for plain language and avoiding technical and field-specific jargon as much as possible throughout this report. We acknowledge that the wide readership for these findings makes communication challenging. Stakeholder groups differ in how they define and address terms and concepts related to care interventions for PLWD and their caregivers, and some of these differences may be philosophical. Additionally, individual members of stakeholder groups may differ on these matters. This report cannot resolve the rich and nuanced discussions that would be needed to come to a wide-ranging consensus about which terms to use and where. Therefore, we have opted to use the terms most commonly found within the studies we identified for this review. Our choice reflects our priority to communicate the evidence base in the clearest and most accessible way to the largest number people. In no way does our choice of terms reflect any particular philosophical position. We recognize that the various perspectives among readers may lead to different interpretations of our report. Our intention is to honor all perspectives and value all audiences.

The review specifically focuses on AD/ADRD, informal and formal caregivers, and the effect of interventions on outcomes for people or systems beyond the intended intervention target. We did not exclude any care setting. However, because the purpose is to inform readiness for dissemination, the review does not include education interventions conducted in educational settings. Because of the interest in daily caregiving, we also did not include workplace-based training programs that targeted professional staff (such as physicians and registered nurses) rather than auxiliary staff.

\section{Key Questions}

The Key Questions (KQs) are structured to organize the literature by the intervention target and grouped such that outcomes for PLWD and caregivers were examined regardless of the intervention target. The KQs are further specified by the populations, interventions, comparators, outcomes, timing, and settings (PICOTS) laid out in Table 1.1. 
Care Interventions for Behavioral and Psychological Symptoms of Dementia (BPSD) in People Living With Dementia (PLWD):

- KQ1: For people living with dementia (PLWD), what are the benefits and harms of care interventions aimed at treating the behavioral and psychological symptoms of dementia (BPSD) in PLWD?

o KQ1a: What evidence is available on how outcomes differ by PLWD characteristics?

o KQ1b: What evidence is available on how outcomes differ by informal and/or formal PLWD Caregiver characteristics?

o KQ1c: Which intervention characteristics or components are associated with effectiveness?

- KQ2: For informal and/or formal PLWD Caregivers, what are the benefits and harms for care interventions aimed at treating BPSD in PLWD?

o KQ2a: What evidence is available on how outcomes differ by $\underline{\text { PLWD }}$ characteristics?

o KQ2b: What evidence is available on how outcomes differ by informal and/or formal PLWD Caregiver characteristics?

o KQ2c: Which intervention characteristics or components are associated with effectiveness?

Care Interventions for Quality of Life, Function, or Non-BPSD Symptoms in PLWD:

- KQ3: For people living with dementia (PLWD), what are the benefits and harms for care interventions aimed at improving quality of life, function, or non-BPSD symptoms in PLWD?

o KQ3a: What evidence is available on how outcomes differ by $\underline{\text { PLWD }}$ characteristics?

o KQ3b: What evidence is available on how outcomes differ by informal and/or formal PLWD Caregiver characteristics?

o KQ3c: Which intervention characteristics or components are associated with effectiveness?

- KQ4: For informal and/or formal PLWD Caregivers, what are the benefits and harms for care interventions aimed at improving quality of life, function, or non-BPSD symptoms in PLWD?

o KQ4a: What evidence is available on how outcomes differ by PLWD characteristics?

o KQ4b: What evidence is available on how outcomes differ by informal and/or formal PLWD Caregiver characteristics?

o KQ4c: Which intervention characteristics or components are associated with effectiveness?

\section{Care Interventions for Quality of Life and Health Outcomes for Informal and Formal PLWD Caregivers:}

- KQ5: For people living with dementia (PLWD), what are the benefits and harms for care interventions aimed at supporting the quality of life and health outcomes of the informal PLWD Caregivers?

o KQ5a: What evidence is available on how quality of life and outcomes differ by PLWD characteristics?

o KQ5b: What evidence is available on how quality of life and outcomes differ by informal or formal PLWD Caregiver characteristics? 
o KQ5c: Which intervention characteristics or components are associated with effectiveness?

- KQ6: For informal and/or formal PLWD Caregivers, what are the benefits and harms for care interventions aimed at supporting the quality of life and health outcomes of the informal PLWD Caregivers?

o KQ6a: What evidence is available on how quality of life and outcomes differ by PLWD characteristics?

o KQ6b: What evidence is available on how quality of life and outcomes differ by informal and/or formal PLWD Caregiver characteristics?

o KQ6c: Which intervention characteristics or components are associated with effectiveness?

- KQ7: For people living with dementia (PLWD), what are the benefits and harms for care interventions aimed at supporting the quality of life and health outcomes of the formal PLWD Caregivers?

o KQ7a: What evidence is available on how quality of life and outcomes differ by PLWD characteristics?

o KQ7b: What evidence is available on how quality of life and outcomes differ by informal and/or formal PLWD Caregiver characteristics?

o KQ7c: Which intervention characteristics or components are associated with effectiveness?

- KQ8: For informal and/or formal PLWD Caregivers, what are the benefits and harms for care interventions aimed at supporting the quality of life and health outcomes of the formal PLWD Caregivers?

o KQ8a: What evidence is available on how quality of life and outcomes differ by PLWD characteristics?

o KQ8b: What evidence is available on how quality of life and outcomes differ by informal and/or formal PLWD Caregiver characteristics?

o KQ8c: Which intervention characteristics or components are associated with effectiveness?

\section{Interventions for How Care Is Delivered:}

- KQ9: For people living with dementia (PLWD), what are the benefits and harms for care delivery interventions?

o KQ9a: What evidence is available on how outcomes differ by PLWD characteristics?

o KQ9b: What evidence is available on how outcomes differ by informal and/or formal PLWD Caregiver characteristics?

o KQ9c: Which intervention characteristics or components are associated with effectiveness?

- KQ10: For informal and formal PLWD Caregivers, what are the benefits and harms for care delivery interventions?

o KQ10a: What evidence is available on how outcomes differ by $\underline{\text { PLWD }}$ characteristics?

o KQ10b: What evidence is available on how outcomes differ by informal and/or formal PLWD Caregiver characteristics?

o KQ10c: Which intervention characteristics or components are associated with effectiveness? 


\section{Dissemination and Implementation Research:}

- Guiding Question 1: What is the state of the empirical evidence on implementation of interventions that have at least low-strength evidence for "real-world" benefits and harms (i.e., NIH Stage Model for Behavioral Intervention Development Stages 3-5)?

Note that in Table 1.1, outcomes are loosely organized to correspond with the levels shown in Figure 1.1. Importantly, we based our final organization of outcomes according to the outcomes examined and measures used in eligible studies, as well as the intentions of the authors.

Table 1.1. PICOTS

\begin{tabular}{|c|c|c|}
\hline Element & PLWD & PLWD Caregiver \\
\hline Population & $\begin{array}{l}\text { PLWD, including individuals with possible } \\
\text { or diagnosed AD/ADRD. } \\
\text { PLWD Subgroups: } \\
\text { Age, sex, sexual orientation/gender } \\
\text { identity, race/ethnicity, education, } \\
\text { socioeconomic status, prior disability, age } \\
\text { at diagnosis, dementia type, dementia } \\
\text { severity [e.g. stage of dementia (early } \\
\text { stage, moderate, or severe), level of } \\
\text { cognitive impairment rate of cognitive } \\
\text { decline], family/household characteristics, } \\
\text { health insurance, geographic location (e.g. } \\
\text { urban, rural), setting type }\end{array}$ & $\begin{array}{l}\text { Informal PLWD Caregivers, such as spouses, } \\
\text { family, friends, and volunteers } \\
\text { Informal PLWD Caregiver Subgroups, including } \\
\text { age, sex, sexual orientation/gender identity, } \\
\text { race/ethnicity, family history of dementia, } \\
\text { education, socioeconomic status, employment } \\
\text { status, relationship with PLWD, living distance } \\
\text { from PLWD, dementia care training, general health } \\
\text { status, caregiving networks, setting type } \\
\text { Formal PLWD Caregivers, such as certified } \\
\text { nursing assistants (CNAs), home health aides, } \\
\text { auxiliary workers, personal care aides, hospice } \\
\text { aides, promotoras or promotores, and community } \\
\text { health workers } \\
\text { Formal PLWD Caregiver Subgroups, including } \\
\text { age, sex, race/ethnicity, education, job position, } \\
\text { skill, training, general health status, setting type }\end{array}$ \\
\hline Intervention & $\begin{array}{l}\text { KQ 1-4. Any nondrug care intervention } \\
\text { intended to benefit PLWD except } \\
\text { interventions to treat conditions other than } \\
\text { dementia, including but not limited to } \\
\text { CPAP, and those that use } \\
\text { supplements/natural products. } \\
\text { (See list of example intervention types in } \\
\text { Appendix A.) } \\
\text { Guiding Question: Any quality improvement } \\
\text { or implementation science study that } \\
\text { informs the dissemination or } \\
\text { implementation of a care intervention at } \\
\text { least low-strength evidence for "real-world" } \\
\text { benefits and harms (i.e., NIH Stage Model } \\
\text { for Behavioral Intervention Development } \\
\text { Stages 3-5) }\end{array}$ & $\begin{array}{l}\text { KQ 5-6. Any care intervention intended to support } \\
\text { informal PLWD caregivers' well-being except } \\
\text { interventions to treat health conditions unrelated to } \\
\text { providing care to PLWD. } \\
\text { KQ 7-8. Any care intervention intended to support } \\
\text { formal PLWD caregivers' well-being except } \\
\text { interventions to treat health conditions unrelated to } \\
\text { providing care to PLWD. } \\
\text { KQ 9-10. Any care delivery intervention to improve } \\
\text { how care is delivered IF the training intervention is } \\
\text { incorporated as on-going operational procedures } \\
\text { into the structure or processes of the organization. } \\
\text { Interventions carried out by higher education } \\
\text { organizations or professional organizations to } \\
\text { provide training toward licensed professionals, and } \\
\text { continuing education for degreed health } \\
\text { professionals are also excluded. } \\
\text { (See list of example intervention types in Appendix } \\
\text { A.) } \\
\text { Guiding Question: Any quality improvement or } \\
\text { implementation science study that informs the } \\
\text { dissemination or implementation of a care } \\
\text { intervention at least low-strength evidence for }\end{array}$ \\
\hline
\end{tabular}




\begin{tabular}{|c|c|c|}
\hline Element & PLWD & PLWD Caregiver \\
\hline Population & $\begin{array}{l}\text { PLWD, including individuals with possible } \\
\text { or diagnosed AD/ADRD. } \\
\text { PLWD Subgroups: } \\
\text { Age, sex, sexual orientation/gender } \\
\text { identity, race/ethnicity, education, } \\
\text { socioeconomic status, prior disability, age } \\
\text { at diagnosis, dementia type, dementia } \\
\text { severity [e.g. stage of dementia (early } \\
\text { stage, moderate, or severe), level of } \\
\text { cognitive impairment rate of cognitive } \\
\text { decline], family/household characteristics, } \\
\text { health insurance, geographic location (e.g. } \\
\text { urban, rural), setting type }\end{array}$ & $\begin{array}{l}\text { Informal PLWD Caregivers, such as spouses, } \\
\text { family, friends, and volunteers } \\
\text { Informal PLWD Caregiver Subgroups, including } \\
\text { age, sex, sexual orientation/gender identity, } \\
\text { race/ethnicity, family history of dementia, } \\
\text { education, socioeconomic status, employment } \\
\text { status, relationship with PLWD, living distance } \\
\text { from PLWD, dementia care training, general health } \\
\text { status, caregiving networks, setting type } \\
\text { Formal PLWD Caregivers, such as certified } \\
\text { nursing assistants (CNAs), home health aides, } \\
\text { auxiliary workers, personal care aides, hospice } \\
\text { aides, promotoras or promotores, and community } \\
\text { health workers } \\
\text { Formal PLWD Caregiver Subgroups, including } \\
\text { age, sex, race/ethnicity, education, job position, } \\
\text { skill, training, general health status, setting type }\end{array}$ \\
\hline & & $\begin{array}{l}\text { "real-world" benefits and harms (i.e., NIH Stage } \\
\text { Model for Behavioral Intervention Development 3- } \\
\text { 5) }\end{array}$ \\
\hline Comparator & $\begin{array}{l}\text { Inactive Comparator: No intervention, usual } \\
\text { care, waitlist, attention control } \\
\text { Active Comparator: Different intervention }\end{array}$ & $\begin{array}{l}\text { Inactive Comparator: No intervention, usual care, } \\
\text { waitlist, attention control } \\
\text { Active Comparator: Different intervention }\end{array}$ \\
\hline $\begin{array}{l}\text { Outcomes } \\
\text { (Generally } \\
\text { organized to } \\
\text { correspond } \\
\text { with Figure } \\
1.1 \\
\text { Framework } \\
\text { for care } \\
\text { inter- } \\
\text { ventions) }\end{array}$ & $\begin{array}{l}\text { Quality of life and subjective well-being } \\
\text { Burden of care* } \\
\text { Satisfaction with care } \\
\text { Perceived Support } \\
\text { Expenditures/financial burden (informal } \\
\text { caregivers) } \\
\text { Health-related outcomes: } \\
\text { Psychological health (e.g., depression, } \\
\text { anxiety) } \\
\text { Neuropsychiatric symptoms (including } \\
\text { apathy, aggression, and agitation) } \\
\text { Function (e.g., ADL, IADL, ability to care for } \\
\text { one's self, ability to recreate/socialize } \\
\text { Weight loss } \\
\text { Sleep problems } \\
\text { Use of restraints } \\
\text { Use of anti-psychotics } \\
\text { Harm reduction (e.g. driving, firearms) } \\
\text { Palliative care/hospice outcomes: } \\
\text { Completion of advanced directives } \\
\text { Comfort during dying process } \\
\text { Concordance with preferred location of } \\
\text { death } \\
\text { Social/Community level outcomes: } \\
\text { Engagement in community activities, } \\
\text { Perceived inclusion } \\
\text { Safety/perceived safety } \\
\text { Utilization of healthcare service outcomes: }\end{array}$ & $\begin{array}{l}\text { Quality of life and subjective well-being } \\
\text { Burden of care* } \\
\text { Satisfaction with care for PLWD (informal } \\
\text { caregivers) } \\
\text { Perceived Support } \\
\text { Expenditures/financial burden (informal caregivers) } \\
\text { Health-related outcomes: } \\
\text { Psychological health (e.g., depression, anxiety) } \\
\text { Immune function (e.g., inflammation or cortisol) } \\
\text { Sleep problems } \\
\text { Weight loss due to stress } \\
\text { Health behaviors (e.g., exercise, substance use) } \\
\text { Caregiving self-efficacy } \\
\text { Confidence to manage caregiver tasks } \\
\text { Social/Community level outcomes (informal } \\
\text { caregivers): } \\
\text { Engagement in community activities, } \\
\text { Perceived inclusion } \\
\text { Safety/perceived safety } \\
\text { Turnover and retention (formal caregivers) } \\
\text { Utilization of healthcare service (e.g., physician } \\
\text { visits, antidepressant or antianxiety medication } \\
\text { usage) } \\
\text { Societal costs including caregiving time/time spent } \\
\text { on activities } \\
\text { Harms, including isolation, loneliness, perceived } \\
\text { stigma, caregiver PTSD }\end{array}$ \\
\hline
\end{tabular}




\begin{tabular}{|c|c|c|}
\hline Element & PLWD & PLWD Caregiver \\
\hline Population & $\begin{array}{l}\text { PLWD, including individuals with possible } \\
\text { or diagnosed AD/ADRD. } \\
\text { PLWD Subgroups: } \\
\text { Age, sex, sexual orientation/gender } \\
\text { identity, race/ethnicity, education, } \\
\text { socioeconomic status, prior disability, age } \\
\text { at diagnosis, dementia type, dementia } \\
\text { severity [e.g. stage of dementia (early } \\
\text { stage, moderate, or severe), level of } \\
\text { cognitive impairment rate of cognitive } \\
\text { decline], family/household characteristics, } \\
\text { health insurance, geographic location (e.g. } \\
\text { urban, rural), setting type }\end{array}$ & $\begin{array}{l}\text { Informal PLWD Caregivers, such as spouses, } \\
\text { family, friends, and volunteers } \\
\text { Informal PLWD Caregiver Subgroups, including } \\
\text { age, sex, sexual orientation/gender identity, } \\
\text { race/ethnicity, family history of dementia, } \\
\text { education, socioeconomic status, employment } \\
\text { status, relationship with PLWD, living distance } \\
\text { from PLWD, dementia care training, general health } \\
\text { status, caregiving networks, setting type } \\
\text { Formal PLWD Caregivers, such as certified } \\
\text { nursing assistants (CNAs), home health aides, } \\
\text { auxiliary workers, personal care aides, hospice } \\
\text { aides, promotoras or promotores, and community } \\
\text { health workers } \\
\text { Formal PLWD Caregiver Subgroups, including } \\
\text { age, sex, race/ethnicity, education, job position, } \\
\text { skill, training, general health status, setting type }\end{array}$ \\
\hline & $\begin{array}{l}\text { Admission to nursing home } \\
\text { Access to care and services } \\
\text { ICU and ED usage } \\
\text { Hospital admission and readmission } \\
\text { Primary, Specialty, Long-term Care usage } \\
\text { Quality of care and services (e.g., } \\
\text { overutilization of unnecessary antibiotics, } \\
\text { other quality care metrics.) } \\
\text { Societal costs, including caregiving } \\
\text { time/time spent on activities } \\
\text { Harms, including isolation, loneliness, } \\
\text { perceived stigma, suicidal ideation or } \\
\text { suicide, elder abuse (e.g., physical harm, } \\
\text { abuse, neglect, exploitation, family } \\
\text { violence) }\end{array}$ & \\
\hline Timing & No minimum duration or followup & No minimum duration or followup \\
\hline Setting & $\begin{array}{l}\text { Any setting; no exclusion based on } \\
\text { geographic location or setting. Includes } \\
\text { home, home health care, adult day care, } \\
\text { acute care settings, social service } \\
\text { agencies, nursing homes, assisted living, } \\
\text { memory care units, hospice, rehabilitation } \\
\text { centers/ skilled nursing facilities, long- } \\
\text { distance caregiving, and nonplace-based } \\
\text { settings }\end{array}$ & $\begin{array}{l}\text { Any setting; no exclusion based on geographic } \\
\text { locations or setting. Includes home, home health } \\
\text { care, adult day care, acute care settings, social } \\
\text { service agencies, nursing homes, assisted living, } \\
\text { memory care units, hospice, rehabilitation centers/ } \\
\text { skilled nursing facilities, long-distance caregiving, } \\
\text { and nonplace-based settings }\end{array}$ \\
\hline
\end{tabular}

*We are obligated to use the proper names of measurement tools, such as the Zarit Caregiver Burden Scale, when used in a specific study.

Abbreviations: $\mathrm{AD}=$ Alzheimer's disease; $\mathrm{ADL}=$ activities of daily living; $\mathrm{ADRD}=$ Alzheimer's disease-related dementias; $\mathrm{CNA}=$ certified nursing assistant; $\mathrm{CPAP}=$ continuous positive airway pressure; $\mathrm{ED}=$ =mergency department; IADL=instrumental activities of daily living; ICU=intensive care unit; KQ=Key Question; NIH=National Institutes of Health; PICOTS=population, intervention, comparator, outcome, timing, setting; PLWD=person/people living with dementia; PTSD=post-traumatic stress disorder 


\section{Analytic Framework}

Figure 1.2 is a traditional analytic framework, illustrating the relationships between interventions and outcomes. Due to limited space, not all baseline characteristics or outcomes listed in Table 1.1 are specifically listed in Figure 1.2.

\section{Figure 1.2. Analytic framework}

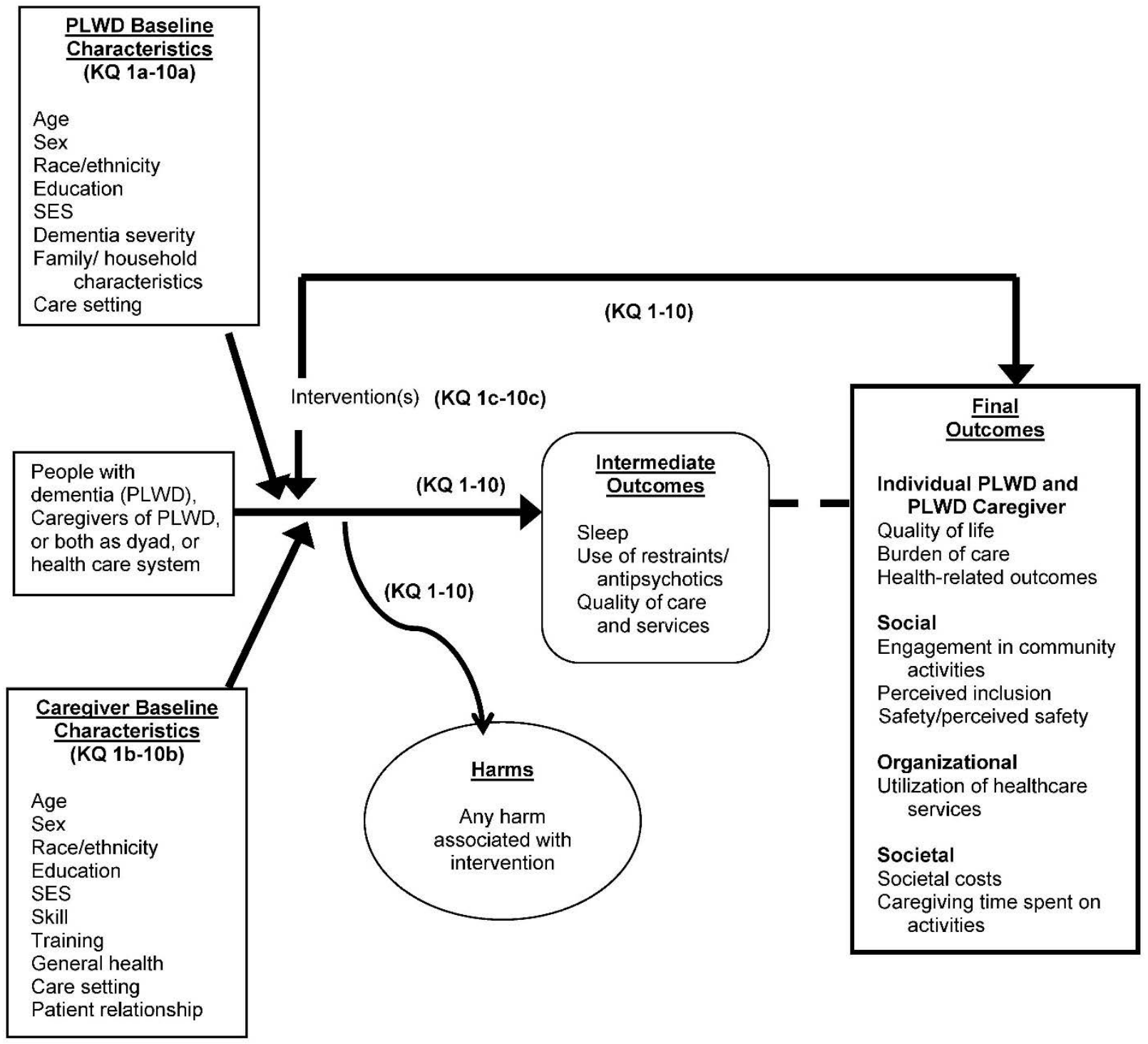

Abbreviations: KQ=Key Question; PLWD=people living with dementia; SES=socioeconomic status

\section{Report Organization}

This report provides, in Chapter 2, details intended to familiarize readers with the methods used to conduct this systematic review. Chapter 3 presents the overall results of the search for the review's eligible studies. Beginning in Chapter 4, results are organized by the intervention target, then by outcome. Due to the breadth and complexity of the interventions, we also present for each result section a brief description of the intervention as well as the intervention's research context, especially regarding how the eligible studies may or may not represent how that 
particular body of research has progressed along the NIH Stage Model. A glossary of terms for the report is provided in Table 1.2.

Results Chapters are structured to present each pair of KQs, keeping the PLWD and caregiver outcomes together for each intervention. And, as discussed earlier, we have categorized interventions as either care interventions (which test the effects of a specific type of care) or care delivery interventions (which test the effects of different manners in which care is delivered). We present the care interventions addressed in KQs 1-8 in Chapters 4-7, and the care delivery interventions addressed in KQs 8 - 9 in Chapter 8. Readers interested in considering potential implementation costs and investments (of technologies, support materials, and personnel required for intervention implementation) to suit their unique settings and contexts may find this separation helpful. Chapter 9 provides a brief response to the Guiding Question 1. The report then concludes with the Discussion in Chapter 10.

Table 1.2. Glossary of terms

\begin{tabular}{|c|c|}
\hline Term & Description \\
\hline Analytic set & $\begin{array}{l}\text { For the purposes of this review, the analytic set is the set of studies that underwent } \\
\text { synthesis. It consists of the studies not judged to be pilots or have a high potential for bias } \\
\text { that might have interfered with the ability of the study to answer its research question. }\end{array}$ \\
\hline $\begin{array}{l}\text { Care delivery } \\
\text { intervention }\end{array}$ & $\begin{array}{l}\text { Care delivery interventions aim to improve the manner in which care is delivered, } \\
\text { including the scheduling of staff and tasks as well as the ways in which staff and tasks are } \\
\text { interdependent. Care delivery interventions can change the tasks that are performed, the } \\
\text { set of staff who perform the tasks, or the way the staff work together. While much of this } \\
\text { change is implemented through education and training, care delivery interventions differ } \\
\text { from the type of education and training targeted at improving already established roles } \\
\text { and tasks. }\end{array}$ \\
\hline Care intervention & $\begin{array}{l}\text { Care interventions contribute to a person's well-being, happiness, identity, privacy, } \\
\text { capacity, autonomy, or authority. They can be supports, services, programs, } \\
\text { accommodations, or practices that include behavioral, environmental, technological, and } \\
\text { psychological methods or approaches. They may be delivered by healthcare, social } \\
\text { services, and other community organizations or caregivers with the intention of having a } \\
\text { direct impact on either a person with dementia or their caregiver or both. }\end{array}$ \\
\hline Eligible study & $\begin{array}{l}\text { An eligible study is one that meets the initial study criteria that were defined in advance } \\
\text { regarding the type of study that would be included in the systematic or comparative } \\
\text { effectiveness review. }\end{array}$ \\
\hline Evidence map & $\begin{array}{l}\text { An evidence map is the result of a systematic search of a defined topic area that can } \\
\text { facilitate evidence-informed decision making or identify gaps in knowledge and future } \\
\text { research needs. }\end{array}$ \\
\hline Explanatory studies & Explanatory studies aim to test whether an intervention works under optimal situations. \\
\hline Exploratory study & $\begin{array}{l}\text { Exploratory studies are preliminary research designed to clarify the exact nature of the } \\
\text { problem to be solved. }\end{array}$ \\
\hline Formal caregiver & $\begin{array}{l}\text { Formal caregivers are paid caregivers, such as certified nursing assistants (CNAs), } \\
\text { home health aides, auxiliary workers, personal care aides, hospice aides, promotoras or } \\
\text { promotores, and community health workers. }\end{array}$ \\
\hline Heterogeneity & $\begin{array}{l}\text { Heterogeneity is a word that signifies diversity in something. A classroom consisting of } \\
\text { people from lots of different backgrounds would be considered having the quality } \\
\text { of heterogeneity. Likewise, a wide range of study designs in a group of studies would be } \\
\text { considered heterogeneous. }\end{array}$ \\
\hline $\begin{array}{l}\text { Informal caregiver or } \\
\text { Caregiver/Care Partner } \\
\text { (CGIP) }\end{array}$ & $\begin{array}{l}\text { For the purposes of this review, Informal caregivers are spouses, family, friends, and } \\
\text { volunteers providing care to one or more PLWD. Informal caregivers are typically unpaid. } \\
\text { While caregiver has been a term commonly used in the literature, some people prefer the } \\
\text { term "care partner." }\end{array}$ \\
\hline $\begin{array}{l}\text { People Living With } \\
\text { Dementia (PLWD) }\end{array}$ & $\begin{array}{l}\text { For the purposes of this review, People Living With Dementia (PLWD) is a term/ } \\
\text { abbreviation that refers to individuals living with Alzheimer's disease or Alzheimer's } \\
\text { disease related dementias. }\end{array}$ \\
\hline Person-centered & $\begin{array}{l}\text { Person-centered, in this context, is a way of designing interventions with consideration } \\
\text { for the needs of the people using health and social services in planning, developing and } \\
\text { monitoring care. }\end{array}$ \\
\hline
\end{tabular}




\begin{tabular}{|l|l|}
\hline Term & \multicolumn{1}{|c|}{ Description } \\
\hline Analytic set & $\begin{array}{l}\text { For the purposes of this review, the analytic set is the set of studies that underwent } \\
\text { synthesis. It consists of the studies not judged to be pilots or have a high potential for bias } \\
\text { that might have interfered with the ability of the study to answer its research question. }\end{array}$ \\
\hline Pragmatic studies & $\begin{array}{l}\text { Pragmatic studies or trials are designed to evaluate the effectiveness of interventions in } \\
\text { real-life routine practice conditions. }\end{array}$ \\
\hline Preliminary studies & A preliminary study is an initial exploration of issues related to a proposed intervention. \\
\hline Protocol & $\begin{array}{l}\text { A protocol is set of steps or procedures for health systems or units providing the care. } \\
\text { They can also specify the tools and tasks that need to be carried out, and they help } \\
\text { processes to be understood by staff regardless of staff's tenure or experience. }\end{array}$ \\
\hline Pilot study & $\begin{array}{l}\text { A pilot study is a small-scale test of the methods and procedures to be used on a larger } \\
\text { scale in a future study. }\end{array}$ \\
\hline Risk of bias & $\begin{array}{l}\text { Risk of bias is the extent to which the design and conduct of a study are likely to have } \\
\text { prevented bias in the results. }\end{array}$ \\
\hline Small sample & $\begin{array}{l}\text { Sample size is a count of the individual people or observations in any statistical setting, } \\
\text { such as a scientific experiment or a public opinion survey. Too small a sample yields } \\
\text { unreliable results, while an overly large sample requires a significant commitment of time } \\
\text { and resources. }\end{array}$ \\
\hline
\end{tabular}

Abbreviations: $\mathrm{CG} / \mathrm{P}=$ caregiver/care partner; $\mathrm{CNA}=$ certified nursing assistant; $\mathrm{PLWD}=$ person/people living with dementia 


\section{Chapter 2. Methods}

The methods for this systematic review followed the Agency for Healthcare Research and Quality (AHRQ) Methods Guide for Effectiveness and Comparative Effectiveness Reviews (available at https://effectivehealthcare.ahrq.gov/topics/cer-methods-guide/overview). This systematic review also reports in accordance with the Preferred Items for Reporting in Systematic Reviews and Meta-Analyses (PRISMA), ${ }^{16}$ A Measurement Tool to Assess Systematic Reviews (AMSTAR 2), ${ }^{17}$ and any relevant extension statements.

The topic of this review was initially developed by the National Institute on Aging (NIA) in consultation with AHRQ. The role of the Key Informants was filled by the NASEM committee, which, as noted earlier, will use the review to help develop its own recommendations regarding which interventions are ready for dissemination and implementation on a broad scale. However, the NASEM committee did not see the draft Key Questions (KQs), PICOTS, and analytic framework until the KQs were posted for public comment; therefore, a panel of content experts from Federal agencies acted as proxy Key Informants, providing input on the KQs to be examined. Federal content experts were drawn from the NIA, the Department of Veterans Affairs, The Department of Defense, the Center for Disease Control and Prevention, the Office of the Assistant Secretary for Planning and Evaluation, and the Administration for Community Living within the Department of Health and Human Services. The NASEM committee also served as Technical Experts, providing high-level content and methodological expertise throughout development of the review protocol. The final protocol is posted on the Effective Health Care website at https://effectivehealthcare.ahrq.gov/products/care-interventionspwd/protocol.

\section{Study Selection}

Studies were included in the review based on the PICOTS framework outlined above in Table 1.1 and the study-specific inclusion criteria described in Table 2.1.

Table 2.1. Study inclusion criteria

\begin{tabular}{|l|l|}
\hline \multicolumn{1}{|c|}{ Category } & \multicolumn{1}{|c|}{ Criteria for Inclusion } \\
\hline Study Enrollment & $\begin{array}{l}\text { Adults with possible or diagnosed AD/ADRD. No age requirement is made, that is, } \\
\text { early onset disease that may be experienced by people with Down syndrome or other } \\
\text { genetic risk factors are included. Study populations may include adults with mild } \\
\text { cognitive impairment (MCI) if 15\% or less of total sample, or must report results for } \\
\text { dementia population separately. }\end{array}$ \\
\hline Study Objective & $\begin{array}{l}\text { KQ 1-2: Evaluate benefits and harms of care interventions for BPSD symptoms in } \\
\text { PLWD } \\
\text { KQ 3-4: Evaluate benefits and harms of care interventions for quality of life, function, } \\
\text { or non-BPSD symptoms in PLWD } \\
\text { KQ 5-6: Evaluate benefits and harms of care interventions for quality of life and health } \\
\text { outcomes of informal caregivers for PLWD } \\
\text { KQ 7-8: Evaluate benefits and harms of care interventions for quality of life and health } \\
\text { outcomes of formal caregivers for PLWD } \\
\text { KQ 9-10: Evaluate benefits and harms of care delivery interventions that address how } \\
\text { care is delivered } \\
\text { KQ subquestions: Evaluate possible effect modifiers of intervention benefits and } \\
\text { harms }\end{array}$ \\
\hline $\begin{array}{l}\text { RCTs, and prospective studies with concurrent comparator arms, and at least 10 } \\
\text { participants per arm at study analysis. Interrupted time series with at least 3 } \\
\text { measures both pre- and post-intervention (therefore excluding simple controlled } \\
\text { before/after studies without comparator arm). }\end{array}$ \\
\hline Study Design
\end{tabular}




\begin{tabular}{|l|l|}
\hline \multicolumn{1}{|c|}{ Category } & \multicolumn{1}{|c|}{ Criteria for Inclusion } \\
\hline Study Enrollment & $\begin{array}{l}\text { Adults with possible or diagnosed AD/ADRD. No age requirement is made, that is, } \\
\text { early onset disease that may be experienced by people with Down syndrome or other } \\
\text { genetic risk factors are included. Study populations may include adults with mild } \\
\text { cognitive impairment (MCI) if 15\% or less of total sample, or must report results for } \\
\text { dementia population separately. }\end{array}$ \\
\hline Outcomes & $\begin{array}{l}\text { Outcomes listed in Table 1. Actual outcome measures will be defined by study } \\
\text { authors. Common measures are provided in Appendix A. We will only include studies } \\
\text { with immune function, turnover, or retention of caregivers if the study also includes } \\
\text { another PLWD or quality outcomes; that is, we will not include the study if it only } \\
\text { examines turnover or retention as an intermediate outcome in isolation. }\end{array}$ \\
\hline Publication type & $\begin{array}{l}\text { Published in peer-reviewed journals and grey literature with full text available (if } \\
\text { sufficient information to assess eligibility and risk of bias are provided). Letters and } \\
\text { conference abstracts are excluded due to the inability of such short publications to } \\
\text { provide the information needed to fully describe the interventions. }\end{array}$ \\
\hline $\begin{array}{l}\text { Language of } \\
\text { Publication }\end{array}$ & \begin{tabular}{l} 
English only, due to resource limitations \\
\hline
\end{tabular}
\end{tabular}

Abbreviations: $\mathrm{AD}=$ Alzheimer's disease; ADRD=Alzheimer's disease-related dementias; BPSD=behavioral and psychological symptoms of dementia; KQ=Key Question; MCI=mild cognitive impairment; PLWD=person/people living with dementia; $\mathrm{RCT}=$ randomized controlled trial

The following discussion about the review search processes is organized by type of research question - first the KQs, then the guiding question.

For the KQs, we searched Ovid Medline ${ }^{\circledR}$, Ovid $\AA$ Embase, Ovid PsycInfo ${ }^{\circledR}$, CINAHL, and the Cochrane Central Register of Controlled Trials (CENTRAL) to identify studies published and indexed in bibliographic databases. The search algorithm included relevant controlled vocabulary and natural language terms for the concepts of Alzheimer's disease (AD) and related dementias (ADRD) (Appendix A).

We reviewed bibliographic database search results for studies relevant to our PICOTS framework and study-specific criteria. Search results were downloaded to EndNote. Two reviewers independently reviewed titles and abstracts to identify studies meeting PICOTS framework and inclusion/exclusion criteria. Two reviewers independently performed full-text screening to determine if inclusion criteria were met. Differences in screening decisions were resolved by consultation between reviewers, and, if necessary, consultation with a third reviewer. We documented the inclusion and exclusion status of citations that underwent full-text screening. Throughout the screening process, team members met regularly to discuss training material and issues as they arose to ensure consistent application of inclusion criteria.

We conducted limited additional searching of grey literature (research or other written material produced outside of traditional academic publishing) to identify relevant completed and ongoing studies that met the study design inclusion criteria. Grey literature search results were used to identify studies, outcomes, and analyses not reported in the peer-reviewed published literature to assess publication and reporting bias and inform future research needs. We also tracked published protocols for studies that have not published results in the public domain.

For the guiding question, we conducted forward citation searching of studies with low to moderate strength of evidence for companion articles describing implementation processes.

Lastly, to provide resources for care interventions which may not have been empirically studied using study designs that met inclusion criteria, we searched websites of relevant governmental agencies, professional associations, and AD or ADRD nongovernmental groups for curated lists of known interventions. An example list of organizations is provided in Appendix A. 
We will update searches while the draft report is under public/peer review.

\section{Data Extraction}

Studies that met inclusion criteria were distributed to EPC reviewers for data extraction. Data extraction used a two-stage process: (1) we first used an evidence map table for basic data extraction, and (2) we then created a series of analytic set tables including comprehensive evidence and assessment tables for those studies that went on to further analysis. Figure 2.1 provides a graphic illustration of the flow of studies through the review processes. Data fields for both the evidence map and analytic set included author, year of publication, population of interest (including a granular checklist of PLWD and caregiver characteristics), intervention, comparison, setting, outcomes cited, intervention duration, and study followup.

As mentioned in Chapter 1, we were guided by the National Institutes of Health (NIH) Stage Model for Behavioral Interventions. ${ }^{15}$ This model provides a conceptual framework of intervention research development, ranging from basic science research (Stage 0) to new intervention creation (Stage 1), research-setting efficacy (Stage 2), "real-world" communityclinic efficacy (Stage 3), broad community-based effectiveness (Stage 4), to eventually dissemination and implementation research (Stage 5). This model not only describes the stages of behavioral intervention development, but also supports eventual implementation. While the stages are not a direct assessment of implementation readiness, the model suggests that interventions at Stage 3 or higher are more likely to be ready for broad dissemination. Interventions at Stage 4 that use pragmatic study designs move research closer to "real world" conditions and population levels.

For studies that appeared to be at NIH Stage 0 to 2 (pilot, feasibility, and small sample size studies), extraction was complete at the evidence map stage; these studies did not advance to further extraction or outcome assessment. For NIH Stage 3 (efficacy or explanatory studies) and NIH Stage 4 studies (effectiveness, or pragmatic studies), if a study was assessed as high risk of bias or over threshold risk of bias (see section below on risk of bias assessment for further details), these studies did not advance to further extraction or outcome assessment, however, we do present details of the risk of bias assessment. 
Figure 2.1. Review assessment and extraction processes

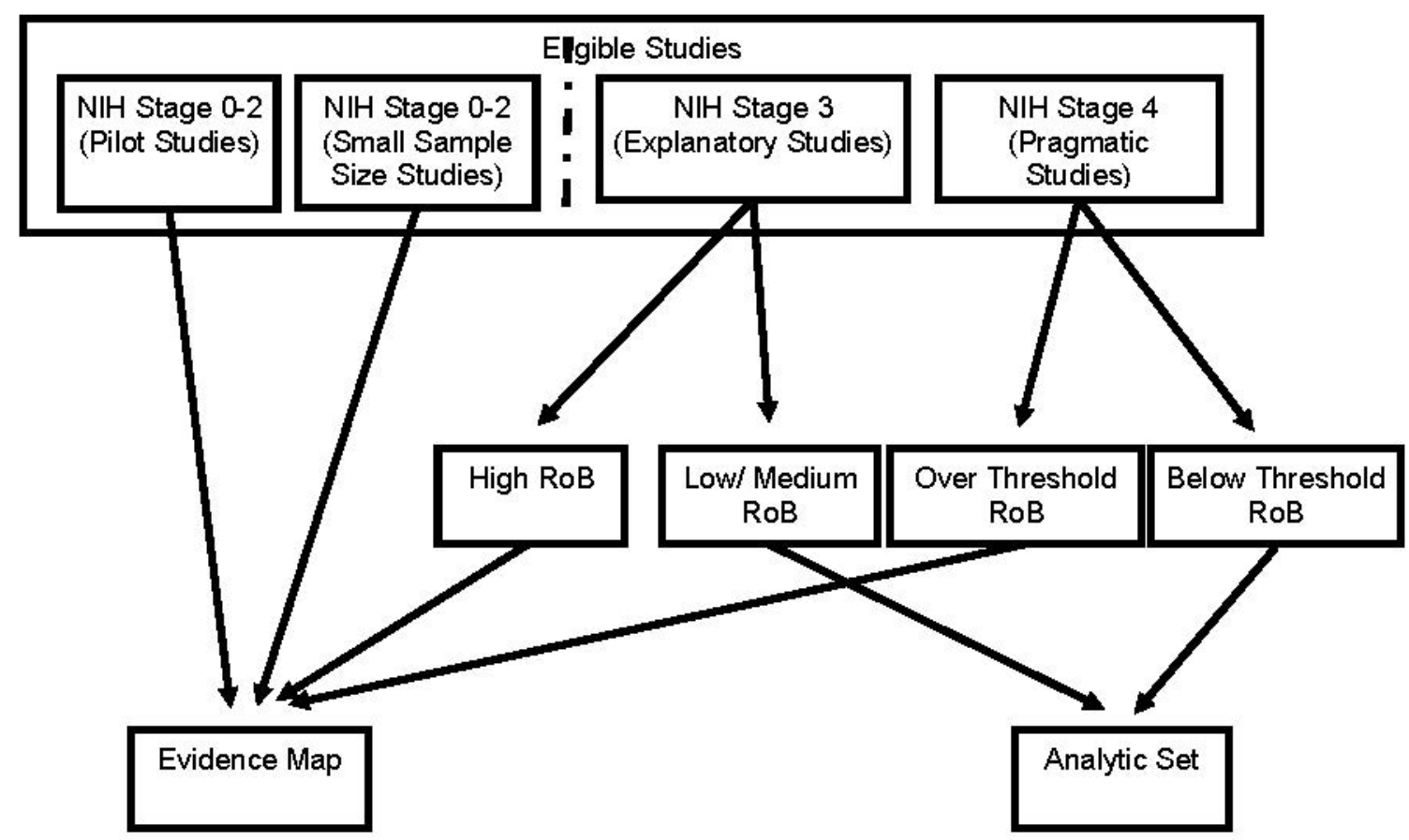

Abbreviations: $\mathrm{NIH}=$ National Institutes of Health; RoB= risk of bias

The remaining studies that were assessed as having low to moderate risk of bias and appearing to be NIH Stage 3 to 5 comprise the analytic set. We extracted additional data fields including subject inclusion criteria, more detailed PLWD and caregiver characteristics, intervention and comparison characteristics, descriptions and results of included outcomes and harms, risk of bias elements, elements to distinguish NIH Stage 3 or 4 (where appropriate), and study funding source. Intervention characteristics included theory base, components and activities, timing, frequency, duration, use of technology, training, delivery approach (prescriptive or manualized vs. tailored), other delivery modalities, and use of cultural adaptations or modifications. We noted the point on the disease continuum (i.e. stage of dementia) for which the intervention was intended and methods for targeting the interventions to PLWD and/or caregivers and their identified goals and priorities.

We extracted relevant data into Microsoft Excel. Evidence map tables were verified for accuracy by a second EPC reviewer. For the analytic set, one reviewer extracted data to evidence and outcomes tables, and a second reviewer reviewed and verified the data for accuracy. Given the number of included studies, we did not contact study authors for missing data.

\section{Assessing Methodological Risk of Bias and NIH Stage of Individual Studies}

Based on AHRQ guidance, ${ }^{18}$ two EPC reviewers independently assessed risk of bias for all eligible studies. Reviewers consulted to reconcile discrepancies in overall risk of bias. Overall risk of bias assessments for each study were classified as low, moderate, or high based on the collective risk of bias inherent in each domain and the level of confidence that the results were believable given the study's limitations. However, the approach differed based on the KQ and 
study NIH Stage mode; these differences are detailed below. We began with an initial sorting into NIH Stages 0 to 2 versus NIH Stages 3 to 5 by simple examination of the study aims.

For KQ 1-8: For studies of individual care interventions, we used a modified Cochrane risk of bias tool to assess them as high, medium, or low for each of the following domains: (1) selection bias (adequacy of randomization method [RCTs], accounting for imbalance in prognostic variables [observational studies]); (2) attrition bias (differentiated by mortality versus loss to followup); (3) detection bias (outcome measurement quality, outcome assessor masking); (4) performance bias (intention to treat or test analysis, adjustment for potential confounding variables, participant masking to treatment assignment); (5) reporting bias (selective reporting of outcomes). (Appendix A) While we were not expressly looking for studies identified as quality improvement interventions, we recognize that complex care delivery interventions use multicomponent approaches similar to quality improvement interventions. Therefore, for these complex interventions, risk of bias included domains similar to those outlined in a risk of bias tool for quality improvement, e.g., fidelity to the program. ${ }^{19}$

For KQs 9-10: We anticipated that care delivery studies would generally fall in the range of NIH Stage 3 to 4 effectiveness trials, with the possibility that a few were carried out as quality improvements and thus Stage 5. Along with categorizing studies by NIH Stage Model, we also broadly labeled study designs as explanatory or pragmatic. Explanatory studies test whether an intervention works under optimal conditions, similar to Stage 3, while pragmatic studies evaluate effectiveness of interventions in real-life practice conditions, similar to Stage $4 .{ }^{20}$ Since study designs exist on a continuum, rather than as discreet categories, we included a "balanced" category for study designs that appeared poised between explanatory and pragmatic. Because both the higher NIH stages and pragmatic trials are explicitly designed to balance, or trade off, internal and external validity, we approached risk of bias assessment as a threshold requirement rather than a continuum for suspected pragmatic design studies. We targeted studies selfidentifying as pragmatic and studies using advanced study designs such as cluster or stepped wedge designs. We assessed whether such studies were below the threshold of high risk of bias based on selection bias, level of attrition, and fidelity to the intervention. If a study was over threshold risk of bias, we abstracted it into the evidence map with no further action. If a study was determined to be below the threshold, we then assessed it for NIH stage. To assess NIH stage, we used a modified PRECIS-2 tool, ${ }^{21}$ initially developed to help interventionists design pragmatic trials. Because explanatory and pragmatic classifications fall along a continuum rather than being discrete categories, ${ }^{20}$ and because reporting details for fine distinctions are often lacking in publications, we used the three categories outlined above (explanatory, balanced, and pragmatic) rather than the 5-point scale of the PRECIS-2 tool. Appendix A provides the modified tool. (Advanced study designs such as cluster trials were considerably less prevalent in KQ $1-8$ and often readily identified as explanatory based on the stated purpose of the study and the reason for using a cluster design. We therefore found it essentially unnecessary to use this staged assessment process for KQ $1-8$.)

\section{Data Synthesis}

We summarized results in evidence maps or analytic set tables and synthesized evidence for each unique population, comparison, and outcome or harm. Evidence maps provide a quick synthesis of what the identified literature has studied. Analytic set tables gather a more comprehensive set of data, allowing the reviewer to attempt to go further and answer "what did the included studies find.” For this review, we organized analytic set tables by intervention 
targets, interventions, comparators, and PLWD, caregiver, or other system-level outcomes. We reported descriptive information regarding numbers of studies reporting more granular PLWD or caregiver characteristics.

Because we could not identify a consensus taxonomy of interventions to apply to the literature, we categorized interventions empirically by intervention and comparator pairs. Because splitting into very narrow categories can make drawing conclusions impossible (due to few studies of often questionable risk of bias), we sought to balance two competing concerns: (1) a need to group broadly conceptual ideas together as much as possible in order to have sufficient studies informing the synthesis, and (2) avoiding excessive heterogeneity in the studies grouped together because that makes interpretation difficult. We used the explanatory or pragmatic classifications along with the NIH Stage Model to inform our qualitative synthesis of the intervention's research context (i.e., a brief representation of the current state of the research and its development over time, including a summary and description of the eligible studies). The intervention research context sections function as the main results reporting for the evidence maps, and as contextual information for outcomes reported for the analytic sets.

For the KQs, we assessed the effects of outcomes using clinically important differences if well-established, but for many outcomes this was not the case. Because of the very wide range of outcomes of interest across the panel of potential interventions, we did not list specific priority outcomes beyond those noted in Table 1.1. For any individual study, we examined no more than five to seven outcomes per PLWD or caregiver population, prioritizing person-centered outcomes, (e.g., quality of life, function, and harms), over intermediate outcomes (e.g., laboratory test values, subscales of outcome measurement tools). Our rationale for this decision is that excessive reporting of outcomes generally happens with the latter type of outcome.

When pooling outcomes across studies was possible, we used random effects models. For continuous outcomes, we calculated weighted mean differences and/or standardized mean differences with the corresponding 95 percent confidence intervals. We assessed the clinical and methodological heterogeneity and variation in effect size to determine appropriateness of pooling data. We assessed statistical heterogeneity with Cochran's Q test and measure magnitude with I2 statistic.

\section{Grading the Strength of Evidence for Major Comparisons and Outcomes}

The overall strength of evidence for select outcomes for KQs 1 - 10 were evaluated based on five required domains: (1) study limitations (risk of bias); (2) consistency (similarity of effect direction and size); (3) directness (single, direct link between intervention and outcome); (4) precision (degree of certainty around an estimate); and (5) reporting bias. ${ }^{22}$ An outcome with an overall rating of "high strength of evidence" implies that the included contributing studies were randomized controlled trial studies with both a low risk of bias, and with consistent, direct, and precise domains. We assessed strength of evidence for key final health outcomes measured with validated scales.

Based on study design and risk of bias, we rated study limitations as low, medium, or high. Consistency was rated as consistent, inconsistent, or unknown/not applicable (e.g., single study) based on whether intervention effects were similar in direction and magnitude, and statistical significance of all studies. Directness was rated as either direct or indirect based on the need for indirect comparisons when inference requires observations across studies (i.e., more than one step was needed to reach the conclusion). Precision was rated as precise or imprecise based on 
the degree of certainty surrounding each effect estimate or qualitative finding. An imprecise estimate is one for which the confidence interval is wide enough to include clinically distinct conclusions. If we had found any outcome to have at least moderate or high strength of evidence, we would have evaluated reporting bias by the potential for publication bias, selective outcome reporting bias, and selective analysis reporting bias. We would have done this by comparing reported results with those mentioned in the methods section and an assessment of the grey literature to assess potentially unpublished studies. However, no findings rose to this level. Other factors considered in assessing strength of evidence included weighting by strength of study design to address broad dissemination (thus pragmatic trials hold stronger weight), dose-response relationship, the presence of confounders, and strength of association.

Based on these factors, we rated the overall strength of evidence for each outcome as:

High: Very confident that estimate of effect lies close to true effect. Few or no deficiencies in body of evidence, findings are believed to be stable.

Moderate: Moderately confident that estimate of effect lies close to true effect. Some deficiencies in body of evidence; findings likely to be stable, but some doubt.

Low: Limited confidence that estimate of effect lies close to true effect; major or numerous deficiencies in body of evidence. Additional evidence necessary before concluding that findings are stable or that estimate of effect is close to true effect.

Insufficient: No evidence, unable to estimate an effect, or no confidence in estimate of effect. Available evidence or lack of evidence precludes judgment.

Notably, an assessment of insufficient evidence does not mean that the intervention is ineffective. Rather, it means that due to the uncertainty of the evidence, we could not draw meaningful conclusions about its effectiveness at this time. 


\section{Chapter 3. Search Results}

Our search identified 9,217 publications for screening. Based on inclusion criteria, we identified 595 unique eligible studies discussed in 850 publications. We list studies excluded at full text screening, by exclusion category, in Appendix B. See Figure 3.1 for details of the screening process.

\section{Figure 3.1. Literature flow diagram}

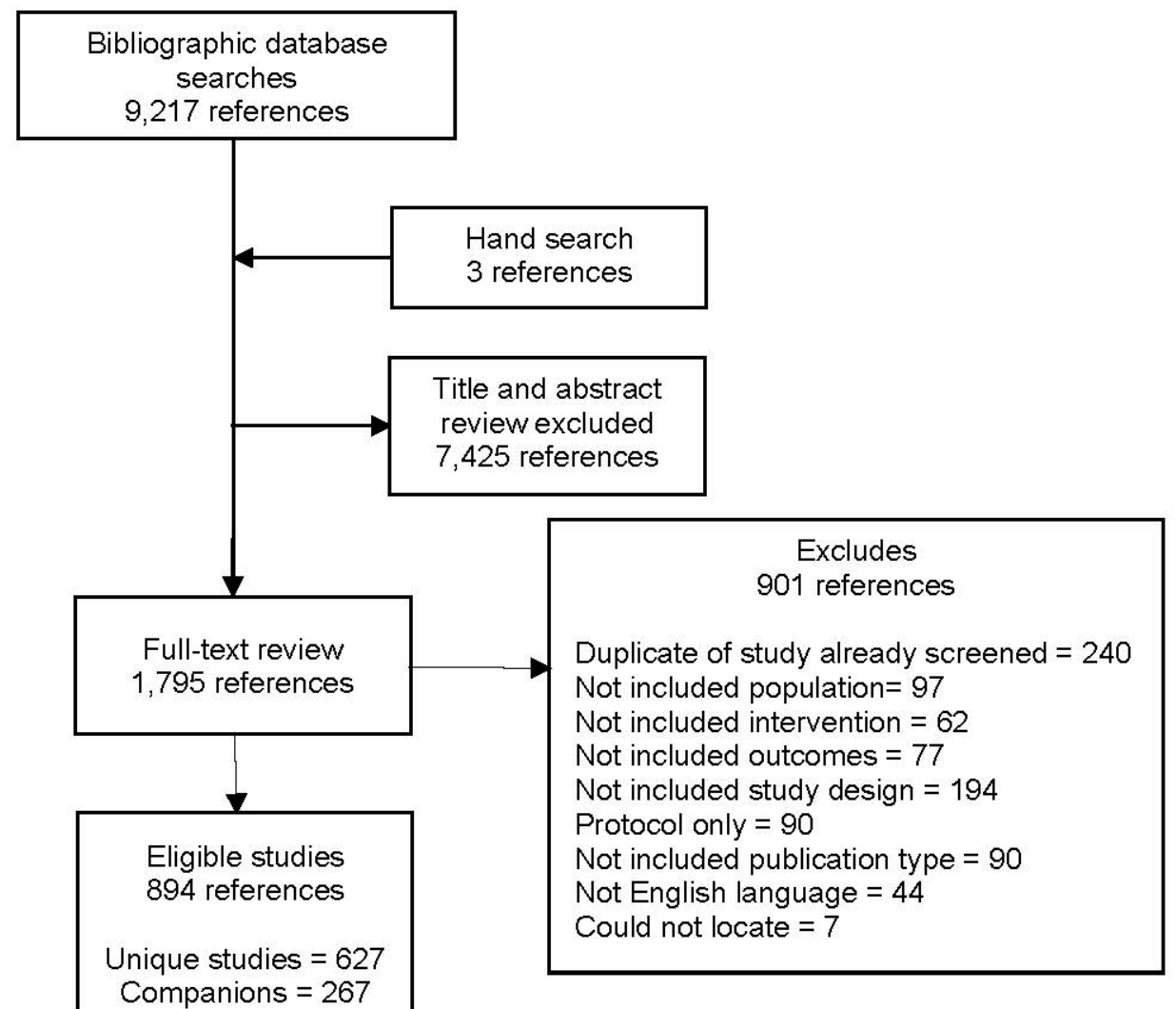

Of the eligible 627 unique studies, we categorized 409 studies as pilot or small sample studies which thus appeared to be National Institutes of Health (NIH) Stage Model Stage 0 - 2 (i.e., for newer interventions that would not be appropriate to address readiness for implementation). A further 218 were assessed as high risk of bias, most commonly due to issues with selection bias, attrition, or intervention fidelity. This left 100 low to moderate risk of bias studies for the analytic set. (Table 3.1) Non-U.S. studies were a large portion of the eligible studies. Overall, approximately 67 percent of the literature emerged from research conducted outside of the United States. This was heavily weighted by care interventions for treating or managing behavioral or psychological symptoms of dementia for people living with dementia (PLWD) (Chapter 4) or PLWD well-being (Chapter 5).

Unfortunately, many important groups warrant further inclusion in research, including people with Down syndrome, who are living longer and who overall experience higher rates of dementia than the general population. Individuals with complex presentations of dementias, e.g., earlyonset, amnestic forms, also need further consideration. We also found little information to determine whether interventions are equally appropriate for or have been successfully adapted to other race/ethnic cultures within the United States, with only a handful of studies providing 
sufficient inclusion of African-American or Hispanic/Latino populations. Interventions for rural communities were found to be even more rare in the literature. Additionally, the many countries in which these care interventions were evaluated were almost exclusively high-resource, with very few low-resource countries represented. Finally, few studies gathered the granular detail necessary for a deeper understanding of the applicability of the interventions. For example, the minority of studies that measured PLWD disability used medical approaches, like the Charlson score, rather than functional approaches that note disability prior to dementia onset.

Based on the structure of the Key Questions and the eligible studies identified in the search process, we developed categories and assigned each study (or comparison within a multi-arm study) to one of 37 major intervention categories. A few interventions exhibited characteristics that could be ascribed to more than one category. Further, some care interventions are variably defined and described within the literature, making categorization more challenging. We attempted to keep clearly defined interventions together and classified less clearly defined interventions into more general categories such as psychosocial therapies or multicomponent interventions. If an intervention's characteristics were balanced between a care intervention and a care delivery intervention, we tended to classify the intervention into the care intervention category and present it in Chapters $4-7$.

Table 3.1. Identified unique eligible studies by intervention category, by results chapter

\begin{tabular}{|c|c|c|c|c|c|}
\hline Location & Intervention & $\begin{array}{c}\text { Total } \\
\text { Unique } \\
\text { Studies }\end{array}$ & $\begin{array}{l}\text { \# Analytic } \\
\text { Set }\end{array}$ & $\begin{array}{l}\text { \# Evidence } \\
\text { map }\end{array}$ & $\begin{array}{l}\text { \# non- } \\
\text { U.S. }\end{array}$ \\
\hline \multirow{7}{*}{$\begin{array}{l}\text { Chapter } 4 \\
\text { Managing } \\
\text { PLWD BPSD }\end{array}$} & Assisted Therapy & 16 & 1 & 15 & 15 \\
\hline & Multisensory Stimulation/Snoezelen & 9 & 2 & 7 & 6 \\
\hline & $\begin{array}{l}\text { Complementary and Alternative } \\
\text { Medicine (CAM) Therapies }\end{array}$ & 21 & 5 & 16 & 19 \\
\hline & Bright Light Therapy & 9 & 0 & 9 & 4 \\
\hline & Psychosocial Therapies for BPSD & 6 & 0 & 6 & 1 \\
\hline & $\begin{array}{l}\text { Multicomponent Interventions for } \\
\text { BPSD }\end{array}$ & 9 & 0 & 9 & 5 \\
\hline & Chapter 4 TOTAL & 70 & 8 & 62 & 50 \\
\hline \multirow{14}{*}{$\begin{array}{l}\text { Chapter } 5 \\
\text { PLWD } \\
\text { Wellbeing }\end{array}$} & Exercise & 53 & 10 & 43 & 48 \\
\hline & Music & 35 & 5 & 30 & 26 \\
\hline & Reminiscence Therapy & 25 & 4 & 21 & 22 \\
\hline & Cognitive Rehabilitation & 23 & 3 & 20 & 19 \\
\hline & Cognitive Training & 18 & 5 & 13 & 15 \\
\hline & Cognitive Stimulation Therapy & 12 & 2 & 10 & 11 \\
\hline & Recreation Therapy & 14 & 3 & 11 & 11 \\
\hline & $\begin{array}{l}\text { Psychosocial Interventions for } \\
\text { PLWD well-being }\end{array}$ & 7 & 0 & 7 & 4 \\
\hline & Creative Expression Therapy & 5 & 0 & 5 & 2 \\
\hline & Multicomponent Interventions & 24 & 3 & 21 & 14 \\
\hline & Assistive Technology & 4 & 0 & 4 & 2 \\
\hline & Electrostimulation & 14 & 0 & 11 & 12 \\
\hline & $\begin{array}{l}\text { Other Interventions for PLWD well- } \\
\text { being }\end{array}$ & 7 & 0 & 7 & 4 \\
\hline & Chapter 5 TOTAL & 241 & 35 & 207 & 190 \\
\hline \multirow{5}{*}{$\begin{array}{l}\text { Chapter } \mathbf{6} \\
\text { Informal } \\
\text { Caregivers }\end{array}$} & $\begin{array}{l}\text { Psychosocial Interventions for } \\
\text { Informal Caregiver Wellbeing }\end{array}$ & 122 & 29 & 93 & 74 \\
\hline & Social Support & 13 & 2 & 11 & 10 \\
\hline & Lifestyle Interventions & 19 & 1 & 18 & 10 \\
\hline & Respite Care & 3 & 0 & 3 & 0 \\
\hline & $\begin{array}{l}\text { Multicomponent for Informal } \\
\text { Caregivers }\end{array}$ & 22 & 7 & 15 & 13 \\
\hline
\end{tabular}




\begin{tabular}{|c|c|c|c|c|c|}
\hline Location & Intervention & $\begin{array}{c}\text { Total } \\
\text { Unique } \\
\text { Studies }\end{array}$ & $\begin{array}{l}\text { \# Analytic } \\
\text { Set }\end{array}$ & $\begin{array}{l}\text { \# Evidence } \\
\text { map }\end{array}$ & $\begin{array}{l}\text { \# non- } \\
\text { U.S. }\end{array}$ \\
\hline & $\begin{array}{l}\text { Other Interventions for Informal } \\
\text { Caregiver Wellbeing }\end{array}$ & 6 & 0 & 6 & 4 \\
\hline & Chapter 6 TOTAL & 185 & 39 & 146 & 111 \\
\hline \multirow{2}{*}{$\begin{array}{l}\text { Chapter } 7 \\
\text { Formal } \\
\text { Caregivers }\end{array}$} & Formal Caregiver Wellbeing & 3 & 0 & 3 & 3 \\
\hline & Chapter 7 TOTAL & 3 & 0 & 3 & 3 \\
\hline \multirow{14}{*}{$\begin{array}{l}\text { Chapter } 8 \\
\text { Care Delivery }\end{array}$} & Care Service Provision & 50 & 6 & 44 & 29 \\
\hline & Consultation Services & 5 & 1 & 4 & 4 \\
\hline & Case Management & 9 & 3 & 6 & 6 \\
\hline & Care Protocols for PLWD & 17 & 0 & 17 & 11 \\
\hline & Advance Care Planning & 9 & 1 & 8 & 3 \\
\hline & Palliative Care & 5 & 0 & 5 & 3 \\
\hline & $\begin{array}{l}\text { Other Service Provision } \\
\text { Interventions }\end{array}$ & 5 & 1 & 4 & 2 \\
\hline & $\begin{array}{l}\text { Care Delivery Models or } \\
\text { Programs }\end{array}$ & 31 & 12 & 18 & 18 \\
\hline & $\begin{array}{l}\text { Care Delivery Staff Education and } \\
\text { Support Needs }\end{array}$ & 46 & 1 & 45 & 27 \\
\hline & Caregiver Staff Training & 22 & 0 & 22 & 18 \\
\hline & Informal Caregiver Staff Training & 12 & 0 & 12 & 6 \\
\hline & Family Education and Partnering & 5 & 0 & 5 & 1 \\
\hline & Mutitier Training & 7 & 1 & 6 & 2 \\
\hline & Chapter 8 TOTAL & 127 & 19 & 107 & 74 \\
\hline
\end{tabular}

Abbreviations: BPSD=behavioral and psychological symptoms of dementia; CAM=complementary and alternative medicine; PLWD=person/people living with dementia 


\section{Chapter 4. Care Interventions for Managing BPSD in PLWD}

This chapter includes care interventions that aimed to address behavioral or psychological symptoms of dementia (BPSD) in people living with dementia (PLWD). Studies in this chapter enrolled PLWD currently identified as experiencing BPSD, the term most commonly used in this literature set. In contrast, Chapter 5 includes studies of interventions aimed at improving the general well-being of PLWD (e.g., exercise, music) and tested with PLWD regardless of presence of BPSD. Care delivery interventions specifically designed to address BPSD in PLWD are presented in Chapter 8.

For each intervention, we present Key Points followed by results in three general sections: Intervention Description, Eligible Studies, and Intervention Research Context. For interventions with no eligible studies assessed as low to medium risk of bias, we present the studies from the evidence map with a brief discussion of what has been examined and the research context. For interventions for which low- to medium-risk-of-bias studies were available for an analytic set, we present Outcomes sections by PLWD, by caregiver, and by variation in outcomes when available. Because differences in outcome measures and intervention complexity prohibited combining outcomes for a statistical meta-analysis, we present summary findings as brief statements of how many studies reported statistically significant benefit or no difference between the intervention and the comparator. Detailed information on all eligible studies can be found in Appendix C.

\section{Assisted Therapy}

Assisted therapy interventions aim to reduce BPSD in PLWD with the assistance of an animal or an object that represents a living being. Eligible studies examined therapy with robots, dogs, and dolls. While the use of such interventions does not depend on the setting, all studies examined the use of assisted therapy in nursing homes or other long-term care settings.

\section{Robot-Assisted Therapy}

\section{Key Point}

- Evidence was insufficient to draw conclusions about the effect of robot-assisted therapy on PLWD and their caregivers.

\section{Intervention Description}

Robot-assisted therapy studies primarily focused on the use of PARO, an autonomous, robotic baby harp seal.

\section{Eligible Studies}

Table 4.1 summarizes the characteristics of the literature set. We identified eight unique studies from 13 publications that examined robot-assisted therapy for the treatment of dementia. ${ }^{23-33}$ Four studies were pilots or small sample studies ${ }^{30,31,33,34}$ and another three were assessed as high risk of bias; ${ }^{27-29,} 32$ therefore, these six studies were excluded from the analytic set. ${ }^{27-29,32}$ We present information on all pilot studies and high risk of bias studies in the evidence map in Appendix C. 
The remaining study $(\mathrm{n}=415)$ was a three-arm cluster randomized trial comparing PARO versus usual care and PARO with the robotic features deactivated in PLWD in Australian nursing homes. ${ }^{23-26}$ The study was assessed as medium risk of bias and categorized as explanatory, or Stage 3 of the National Institutes of Health Stage Model. It enrolled individuals living in long-term care facilities with a documented dementia diagnosis (all types and severities). Appendix C provides evidence tables, summary risk of bias assessments, and strength of evidence for key comparisons and outcomes.

Table 4.1. Basic characteristics of literature set: robot-assisted therapy

\begin{tabular}{|l|l|}
\hline \multicolumn{1}{|c|}{ Characteristic } & \multicolumn{1}{c|}{ Information } \\
\hline Total Studies & 8 studies \\
\hline Non-U.S. studies & 7 studies \\
\hline Evidence map studies & 2 pilot studies \\
& 2 small sample studies \\
& 3 high risk of bias studies \\
\hline Analytic set studies & 1 cluster randomized controlled trial \\
\hline Risk of bias of analytic set & 1 medium \\
\hline Number of PLWD in analytic set & 415 \\
\hline Dementia type/definition & All dementia types and severities \\
\hline Caregiver type (number) & Not reported \\
\hline
\end{tabular}

Abbreviations: PLWD=person/people living with dementia

\section{Intervention Research Context}

The literature on robot-assisted therapy generally follows the NIH Stage Model, and consists primarily of pilots. With the exception of one U.S. study, ${ }^{32}$ the studies were conducted in Australia, ${ }^{31,}{ }^{34}$ New Zealand, ${ }^{30}$ the Netherlands, ${ }^{27}$ Norway, ${ }^{28}$ and Spain. ${ }^{33}$ We identified two pilots comparing PARO versus usual care or an attention control. ${ }^{30,31,34}$ We also identified a pilot comparing PARO versus live animal therapy, a human-like robot, and usual care. ${ }^{33}$ The PARO study included in our analytic set appears to be one of the first larger cluster RCTs of sufficient methodological rigor that compares PARO versus usual care. ${ }^{23-26}$ The study also appears to be one of the first larger cluster RCTs to compare PARO versus PARO with robotic features deactivated. While the study also included outcomes related to motor activity and sleep patterns, these outcomes were exploratory and data were available for less than half the study population. ${ }^{25}$

\section{PLWD Outcomes}

Evidence was insufficient to draw conclusions about either PARO versus usual care $(\mathrm{n}=257)$ or PARO versus PARO with robotic features deactivated $(n=278) .{ }^{23-26}$ Table 4.2 summarizes the primary findings. Results for reduction in agitation were mixed. PLWD showed more visual and verbal engagement with PARO than with PARO with robotic features deactivated. The two groups did not differ for positive behavioral engagement or social engagement. No harms were assessed. ${ }^{23-26}$ An assessment of insufficient evidence does not mean that the intervention is determined to be of no value. Rather, it means that due to the uncertainty of the evidence we could not draw meaningful conclusions at this time. 
Table 4.2. Summary of findings for PLWD outcomes: robot-assisted therapy

\begin{tabular}{|c|c|c|c|c|}
\hline $\begin{array}{l}\text { Outcome } \\
\text { Comparison }\end{array}$ & $\begin{array}{l}\text { \# Studies/Design } \\
\text { (n analyzed) } \\
\text { Timing }\end{array}$ & Population & Findings & $\begin{array}{l}\text { Strength of } \\
\text { Evidence* }^{*}\end{array}$ \\
\hline $\begin{array}{l}\text { Agitation measures } \\
\text { PARO vs. usual } \\
\text { care }\end{array}$ & $\begin{array}{l}1 \text { cluster } \mathrm{RCT}^{26} \\
(\mathrm{n}=257) \\
10 \text { weeks }\end{array}$ & $\begin{array}{l}\text { All dementia } \\
\text { severities and } \\
\text { types in Australia }\end{array}$ & $\begin{array}{l}1 \text { found benefit } \\
1 \text { found no difference }\end{array}$ & Insufficient \\
\hline $\begin{array}{l}\text { Agitation measures } \\
\text { PARO vs. } \\
\text { deactivated PARO }\end{array}$ & $\begin{array}{l}1 \text { cluster } \mathrm{RCT}^{26} \\
(\mathrm{n}=278) \\
10 \text { weeks }\end{array}$ & $\begin{array}{l}\text { All dementia } \\
\text { severities and } \\
\text { types in Australia }\end{array}$ & $\begin{array}{l}0 \text { found benefit } \\
2 \text { found no difference }\end{array}$ & Insufficient \\
\hline $\begin{array}{l}\text { Engagement } \\
\text { measures } \\
\text { PARO vs. } \\
\text { deactivated PARO }\end{array}$ & $\begin{array}{l}1 \text { cluster } \mathrm{RCT}^{26} \\
(\mathrm{n}=278) \\
10 \text { weeks }\end{array}$ & $\begin{array}{l}\text { All dementia } \\
\text { severities and } \\
\text { types in Australia }\end{array}$ & $\begin{array}{l}2 \text { found benefit } \\
2 \text { found no difference }\end{array}$ & Insufficient \\
\hline
\end{tabular}

*Insufficient ratings due to few studies and imprecision in the findings.

Abbreviations: $\mathrm{n}=$ number; $\mathrm{RCT}=$ randomized controlled trials

\section{Caregiver Outcomes}

The analytic study set did not report outcomes related to informal or formal caregivers.

\section{Variation in Outcomes}

In the PARO group, lower levels of agitation at baseline were associated with greater behavioral positive engagement and visual engagement at 10 weeks. ${ }^{26}$ No similar analysis was conducted for the PARO with robotic features deactivated group. The analytic study set did not report variation in outcomes by caregiver or intervention characteristics.

\section{Live Animal- and Doll-Assisted Therapy}

\section{Key Point}

- Studies of live animal- and doll-assisted therapy were described in the evidence map but not considered for analysis due to limitations in study designs.

\section{Intervention Description}

Live animal-assisted therapy involves treatment sessions that include animals with a focus on specific goals (e.g., mental, emotional and/or social). Doll-assisted therapy involves offering dolls to PLWD in order to improve their comfort, engagement, and quality of life.

\section{Eligible Studies}

We identified five unique studies from five publications that examined animal-assisted therapy (with dogs) in the treatment of dementia. (Table 4.3) Two were pilot or small sample studies and the remaining three studies were assessed as high risk of bias. ${ }^{35-39}$ Three unique pilot studies examined doll-assisted therapy. ${ }^{40-42}$ We present information on all pilot studies and high risk of bias studies in the evidence map in Appendix C.

Table 4.3. Basic characteristics of literature set: live animal- and doll-assisted therapy

\begin{tabular}{|l|l|}
\hline \multicolumn{1}{|c|}{ Characteristic } & \multicolumn{1}{c|}{ Information } \\
\hline Total Studies & 8 studies \\
\hline Non-U.S. studies & 8 studies \\
\hline
\end{tabular}




\begin{tabular}{|l|l|}
\hline \multicolumn{1}{|c|}{ Characteristic } & \multicolumn{1}{c|}{ Information } \\
\hline Evidence map studies & 5 pilot studies \\
& 3 high risk of bias \\
\hline Analytic set studies & 0 studies \\
\hline
\end{tabular}

\section{Intervention Research Context}

One study compared the use of doll-therapy versus an active control of hand warmers, which would mimic the sensory characteristics of holding a doll, in PLWD residing in an Italian nursing home. ${ }^{42} \mathrm{~A}$ second compared doll-therapy to teaching PLWD to use gestures to improve communication in Italian nursing homes. ${ }^{40}$ An third study compared doll-therapy to usual care in Australian nursing homes. ${ }^{41}$ One study compared individual animal therapy versus usual care in PLWD in German nursing homes, ${ }^{35}$ while another used group animal therapy in Italian adult daycare centers. ${ }^{36}$ Two others compared group animal therapy versus usual care in PLWD in nursing homes in Norway, ${ }^{37,38}$ while the third compared group animal therapy versus group therapy without an animal in Australian nursing homes. ${ }^{39}$

\section{Multisensory Stimulation/Snoezelen}

\section{Key Point}

- Evidence was insufficient to draw conclusions about the effect of multisensory stimulation (MSS) on PLWD and their caregivers.

\section{Intervention Description}

MSS is intended to have both relaxing and activating effects that promote calm engagement for PLWD in nursing homes through an experiential process that includes light, sound, scents, and music, usually with the accompaniment of an aide or therapist. The "Snoezelen rooms" often used for this intervention were developed in the Netherlands in the 1970s, and are designed to deliver stimuli to various senses, including through different tactile materials and floors that may be adjusted to stimulate the sense of balance.

\section{Eligible Studies}

Table 4.4 summarizes the characteristics of the literature set. We identified nine unique studies from 11 publications. ${ }^{43-50}$ Two studies $(\mathrm{N}=256)$ were assessed as medium risk of bias and classified as explanatory, or Stage 3 of the NIH Stage Model. ${ }^{44,45}$ One was a two-arm randomized trial comparing Snoezelen versus activity sessions, which were treated as an active control. ${ }^{44}$ The other was a three-arm randomized trial comparing Sonas, a different type of MSS intervention, to reading sessions and to usual care. ${ }^{45}$ Appendix $C$ provides evidence tables, summary risk of bias assessments, and strength of evidence for key comparisons and outcomes. ${ }^{46-52}$ Pilot studies, small sample studies, or studies assessed as high risk of bias were excluded from the analytic set. We present information on these studies in the evidence map in Appendix C.

Table 4.4. Basic characteristics of literature set: multisensory stimulation/Snoezelen

\begin{tabular}{|l|l|}
\hline \multicolumn{1}{|c|}{ Characteristic } & \multicolumn{1}{|c|}{ Information } \\
\hline Total studies & 9 studies \\
\hline Non-U.S. studies & 6 studies \\
\hline
\end{tabular}




\begin{tabular}{|l|l|}
\hline \multicolumn{1}{|c|}{ Characteristic } & \multicolumn{1}{c|}{ Information } \\
\hline Evidence map studies & 2 pilot studies \\
& 4 small sample studies \\
& 1 high risk of bias studies \\
\hline Analytic set studies & 2 randomized controlled trials \\
\hline Risk of bias of analytic set & 2 medium \\
\hline Number of PLWD in analytic set & 256 \\
\hline Dementia type/definition & Diagnoses of Alzheimer's, vascular, or mixed dementia \\
\hline Caregiver type (number) & Not reported \\
\hline
\end{tabular}

Abbreviation: PLWD=people living with dementia

\section{Intervention Research Context}

This literature set did not clearly follow progression along the NIH Stage Model. Earlier studies were either pilots ${ }^{50}$ or full studies that reported a pilot component ${ }^{43}$, ${ }^{44}$ However, later studies did not appear to progress to pragmatic trials. The studies generally used active controls, such as activity or reminiscence sessions.

\section{PLWD Outcomes}

Evidence was insufficient to draw conclusions about the effect of MSS on BPSD compared with an active control. ${ }^{44}$ There was no significant mean change on several measures of behavioral problems in the study. Table 4.5 summarizes these findings. Similarly, evidence was insufficient to draw conclusions about the effect of MSS on social function versus both an attention control and usual care. ${ }^{45}$ There was a statistically significant benefit for MSS versus both comparators on improvements in PLWD communication.

Table 4.5. Summary of findings for PLWD outcomes: multisensory stimulation/Snoezelen

\begin{tabular}{|c|c|c|c|c|}
\hline $\begin{array}{l}\text { Outcome } \\
\text { Comparison }\end{array}$ & $\begin{array}{c}\text { \# Studies/Design } \\
\text { (n analyzed) } \\
\text { Timing }\end{array}$ & Population & Findings & $\begin{array}{l}\text { Strength of } \\
\text { Evidence* }^{*}\end{array}$ \\
\hline $\begin{array}{l}\text { Neuropsychiatric } \\
\text { symptoms } \\
\text { MSS vs. } \\
\text { attention control }\end{array}$ & $\begin{array}{l}1 \mathrm{RCT}^{44} \\
(\mathrm{n}=136) \\
4 \text { weeks, } 8 \text { weeks }\end{array}$ & $\begin{array}{l}\text { Persons with } \\
\text { Alzheimer's, vascular, } \\
\text { or mixed dementia in } \\
\text { Norway }\end{array}$ & $\begin{array}{l}0 \text { found benefit } \\
1 \text { found no difference }\end{array}$ & Insufficient \\
\hline $\begin{array}{l}\text { Social function } \\
\text { MSS vS. } \\
\text { attention control }\end{array}$ & $\begin{array}{l}1 \mathrm{RCT}^{45} \\
(\mathrm{n}=120) \\
24 \text { weeks }\end{array}$ & $\begin{array}{l}\text { Persons with } \\
\text { Alzheimer's, vascular, } \\
\text { or mixed dementia in } \\
\text { Norway }\end{array}$ & $\begin{array}{l}1 \text { found benefit } \\
0 \text { found no difference }\end{array}$ & Insufficient \\
\hline $\begin{array}{l}\text { Social function } \\
\text { MSS vs. usual } \\
\text { care }\end{array}$ & $\begin{array}{l}1 \mathrm{RCT}^{45} \\
(\mathrm{n}=120) \\
24 \text { weeks }\end{array}$ & $\begin{array}{l}\text { Persons with } \\
\text { Alzheimer's, vascular, } \\
\text { or mixed dementia in } \\
\text { Norway }\end{array}$ & $\begin{array}{l}1 \text { found benefit } \\
0 \text { found no difference }\end{array}$ & Insufficient \\
\hline
\end{tabular}

*Insufficient ratings due to few studies and imprecision in the findings.

Abbreviations: MSS=multisensory stimulation; n=number; RCT=randomized controlled trial

\section{Caregiver Outcomes}

No studies in the analytic set reported caregiver outcomes.

\section{Variation in Outcomes}

In PLWD with severe cognitive impairment (Mini-Mental State Examination (MMSE) scores 0 to 9), MSS appeared slightly more effective than the activity control at improving behavior at 4 weeks (mean difference -1.0 points, $\mathrm{p}<0.05$ ). ${ }^{44}$ For PLWD with moderate cognitive impairment, 
activity sessions appeared to improve the behavior more than MSS (mean difference 0.8 points, p-value not significant). ${ }^{44}$ However, this trend was not seen with other outcomes.

In PLWD with severe cognitive impairment (MMSE scores 0 to 10), MSS was reported more effective than the active control group at improving HCS scores at 12 weeks, but not at 24 weeks. In addition, MSS did not show a significant advantage in improving HCS scores when compared with usual care. ${ }^{45}$

\section{Complementary and Alternative Medicine (CAM) Therapies}

\section{Key Point}

- Evidence was insufficient to draw conclusions about the effects of aromatherapy or foot massage on PLWD and their caregivers.

\section{Intervention Description}

CAM is a set of therapies that are intended to achieve health effects, but are not part of conventional medical practice. While this category has the potential to be very broad, only a few interventions were examined in the eligible literature, mainly aromatherapy, massage, acupressure, and healing touch. Interventions that involve physical movement, such as yoga or tai chi/taiji, are presented with the section on exercise in Chapter 5.

\section{Eligible Studies}

Table 4.6 summarizes the characteristics of the literature set. We identified 21 unique studies from 23 publications that examined CAM for the treatment of dementia. ${ }^{53-71}$ Five studies were assessed as low to moderate risk of bias and included in the analytic set. ${ }^{57,67,72-74}$ Four studies $(n=278)$ evaluated the effects of aromatherapy in the form of lavender and lemon-balm oils versus either sunflower oil or usual care in the United Kingdom, ${ }^{72,73}$ Hong Kong, ${ }^{74}$ or Taiwan. ${ }^{67}$ The fifth study $(n=55)$ assessed the effects of foot massage for PLWD versus attention control. ${ }^{57}$ No study in the analytic set was conducted in the United States. Appendix C provides evidence tables, summary risk of bias assessments, and strength of evidence for key comparisons and outcomes. The evidence map in Appendix C provides information on all other studies. ${ }^{53-56,58-66,}$ $68,71,75$

Table 4.6. Basic characteristics of literature set: complementary and alternative medicine

\begin{tabular}{|l|l|}
\hline \multicolumn{1}{|c|}{ Characteristic } & \multicolumn{1}{c|}{ Information } \\
\hline Total studies & 21 studies \\
\hline Non-U.S. studies & 19 studies \\
\hline Evidence map studies & 6 pilot studies \\
& 8 small samples \\
& 2 high risk of bias studies \\
\hline Analytic set studies & 5 randomized controlled trials \\
\hline Risk of bias of analytic set & 4 medium (aromatherapy) \\
& 1 low (foot massage) \\
\hline Number of PLWD in analytic set & 278 (aromatherapy) \\
& 5 (foot massage) \\
\hline Dementia type/definition & All dementia types and severity \\
\hline Caregiver type (number) & Not reported \\
\hline
\end{tabular}

Abbreviation: PLWD=people living with dementia 


\section{Intervention Research Context}

As evaluated against the NIH Stage Model, this literature is overall preliminary and based on small sample studies that have not developed beyond a basic explanatory stage, or Stage 3 . Evidence map studies examined various CAM techniques such as healing touch and body talk cortices, ${ }^{60}$ acupressure/acupuncture, ${ }^{61,64}$ mixed aroma exposure, ${ }^{62}$ back and leg massage with moisturizing cream, ${ }^{70}$ aromatherapy, ${ }^{53,54,58,66,67,71,76}$ aromatherapy and hand massage, ${ }^{55}$ aromatherapy massage, ${ }^{56}$ aromatherapy massage plus acupressure, ${ }^{59,67}$ and therapeutic touch. ${ }^{65}$, ${ }^{68}$ Evidence map trials are from Hong Kong, ${ }^{59,}{ }^{64}$ China, ${ }^{61}$ Japan $^{53,} 62$ Taiwan, ${ }^{67}$ Canada, ${ }^{65,} 68$ Australia, ${ }^{55,}{ }^{58}$ Spain, ${ }^{70}$ United States, ${ }^{54,58-60}$ the United Kingdom, ${ }^{56}$ and Israel. ${ }^{66}$

\section{PLWD Outcomes}

Evidence was insufficient for all outcomes. The most commonly investigated outcomes were agitation and neuropsychiatric symptoms. Table 4.7 summarizes the number of studies investigating each outcome and the number of studies that found a benefit and no difference between PLWD and comparison groups.

Agitation was investigated by three of the four included studies. One found that lavender oil versus sunflower oil resulted in short-term improvements (3 weeks). ${ }^{74}$ Another compared lavender plus orange oil versus usual care and found no improvement, ${ }^{67}$ while two other studies found mixed results, with lemon balm oil versus sunflower oil reducing agitation at 4 weeks, ${ }^{72}$ but not 12 weeks. ${ }^{73}$

Depression was investigated in one study that compared lavender plus orange oil versus usual care, and reported improved depression scores with the intervention at 9 weeks. ${ }^{67}$

Neuropsychiatric symptoms were investigated in two studies comparing lavender oil and lemon balm oil versus sunflower oil. Lavender oil seemed to improve neuropsychiatric symptoms at 3 weeks, ${ }^{74}$ but not at 12 weeks. ${ }^{73}$

Quality of life and activities of daily living were examined in one study that evaluated lemon balm oil versus sunflower oil and reported that quality of life seemed to improve over 12 weeks, but activities of daily living did not. ${ }^{73}$

One study examined foot massage versus attention control. ${ }^{57}$ Both increased agitation in PLWD, but the increase was greater in the comparator group than in the intervention group $(p=0.03) .^{57}$

Table 4.7. Summary of findings for PLWD outcomes: complementary and alternative medicine

\begin{tabular}{|l|l|l|l|l|}
\hline \multicolumn{1}{|c|}{$\begin{array}{c}\text { Outcome } \\
\text { Comparison }\end{array}$} & $\begin{array}{c}\text { \# Studies/ } \\
\text { Design } \\
\text { (n analyzed) } \\
\text { Timing }\end{array}$ & \multicolumn{1}{|c|}{ Population } & \multicolumn{1}{|c|}{ Finding } & $\begin{array}{c}\text { Strength of } \\
\text { Evidence* }\end{array}$ \\
\hline $\begin{array}{l}\text { Agitation } \\
\text { Lavender vs } \\
\text { sunflower oil }\end{array}$ & $\begin{array}{l}1 \mathrm{RCT}^{74}(\mathrm{n}=70) \\
3 \text { weeks }\end{array}$ & $\begin{array}{l}\text { PLWD with significant } \\
\text { agitation in Hong Kong }\end{array}$ & $\begin{array}{l}1 \text { found benefit } \\
0 \text { found no difference }\end{array}$ & Insufficient \\
\hline $\begin{array}{l}\mathrm{NPI} \\
\text { Lavender vs } \\
\text { sunflower oil }\end{array}$ & $\begin{array}{l}1 \mathrm{RCT}^{74}(\mathrm{n}=70) \\
3 \text { weeks }\end{array}$ & $\begin{array}{l}\text { PLWD with significant } \\
\text { agitation in Hong Kong }\end{array}$ & $\begin{array}{l}1 \text { found benefit } \\
0 \text { found no difference }\end{array}$ & Insufficient \\
$\begin{array}{l}\text { Agitation } \\
\text { Lavender and } \\
\text { orange oil vs. } \\
\text { Usual care }\end{array}$ & $\begin{array}{l}1 \mathrm{RCT}^{67}(\mathrm{n}=59) \\
9 \text { weeks }\end{array}$ & $\begin{array}{l}\text { PLWD with mild to severe } \\
\text { dementia in Taiwan }\end{array}$ & $\begin{array}{l}0 \text { found benefit } \\
1 \text { found no difference }\end{array}$ & Insufficient \\
\hline $\begin{array}{l}\text { Depression } \\
\text { Lavender and }\end{array}$ & $\begin{array}{l}1 \mathrm{RCT}^{67}(\mathrm{n}=59) \\
9 \text { weeks }\end{array}$ & $\begin{array}{l}\text { PLWD with mild to severe } \\
\text { dementia in Taiwan }\end{array}$ & $\begin{array}{l}1 \text { found benefit } \\
0 \text { found no difference }\end{array}$ & Insufficient \\
\hline
\end{tabular}




\begin{tabular}{|c|c|c|c|c|}
\hline $\begin{array}{l}\text { Outcome } \\
\text { Comparison }\end{array}$ & $\begin{array}{c}\text { \# Studies/ } \\
\text { Design } \\
\text { (n analyzed) } \\
\text { Timing } \\
\end{array}$ & Population & Finding & $\begin{array}{l}\text { Strength of } \\
\text { Evidence* }\end{array}$ \\
\hline \multicolumn{5}{|l|}{$\begin{array}{l}\text { orange oil vs. } \\
\text { usual care }\end{array}$} \\
\hline $\begin{array}{l}\text { Agitation } \\
\text { Lemon Balm oil vs } \\
\text { sunflower oil }\end{array}$ & $\begin{array}{l}2 \mathrm{RCT}^{72,73} \\
(\mathrm{n}=149) \\
4-12 \text { weeks }\end{array}$ & $\begin{array}{l}\text { PLWD with agitation in the } \\
\text { United Kingdom }\end{array}$ & $\begin{array}{l}1 \text { of } 2 \text { found benefit } \\
1 \text { of } 2 \text { found no difference }\end{array}$ & Insufficient \\
\hline $\begin{array}{l}\text { NPI } \\
\text { Lemon Balm oil vs } \\
\text { sunflower oil }\end{array}$ & $\begin{array}{l}1 \mathrm{RCT}^{73}(\mathrm{n}=77) \\
12 \text { weeks }\end{array}$ & $\begin{array}{l}\text { PLWD with agitation in the } \\
\text { United Kingdom }\end{array}$ & $\begin{array}{l}0 \text { found benefit } \\
1 \text { found no difference }\end{array}$ & Insufficient \\
\hline $\begin{array}{l}\text { QoL } \\
\text { Lemon Balm oil vs } \\
\text { sunflower oil }\end{array}$ & $\begin{array}{l}1 \mathrm{RCT}^{73}(\mathrm{n}=77) \\
12 \text { weeks }\end{array}$ & $\begin{array}{l}\text { PLWD with agitation in the } \\
\text { United Kingdom }\end{array}$ & $\begin{array}{l}1 \text { found benefit } \\
0 \text { found no difference }\end{array}$ & Insufficient \\
\hline $\begin{array}{l}\text { Caregiver Burden } \\
\text { Lemon Balm oil vs } \\
\text { sunflower oil }\end{array}$ & $\begin{array}{l}1 \mathrm{RCT}^{73}(\mathrm{n}=77) \\
12 \text { weeks }\end{array}$ & $\begin{array}{l}\text { PLWD with agitation in the } \\
\text { United Kingdom }\end{array}$ & $\begin{array}{l}0 \text { found benefit } \\
1 \text { found no difference }\end{array}$ & Insufficient \\
\hline $\begin{array}{l}\text { Agitation } \\
\text { Foot massage vs } \\
\text { attention control }\end{array}$ & $\begin{array}{l}1 \mathrm{RCT}^{57}(n=55) \\
3 \text { weeks }\end{array}$ & $\begin{array}{l}\text { Moderate to late stage } \\
\text { PLWD in Australia with } \\
\text { history of agitation }\end{array}$ & $\begin{array}{l}1 \text { found benefit for control } \\
\text { group }\end{array}$ & Insufficient \\
\hline
\end{tabular}

*Insufficient ratings due to few studies and imprecision in the findings.

Abbreviations: n=number; NPI=Neuropsychiatric Inventory; PLWD=people living with dementia; QoL=quality of life; RCT=randomized controlled trial

\section{Caregiver Outcomes}

The analytic study set did not report outcomes related to informal or formal caregivers.

\section{Variation in Outcomes}

The analytic set did not report variations in outcomes by PLWD, caregiver, or intervention characteristics.

\section{Bright Light Therapy}

\section{Key Point}

- Studies on bright light therapy were described in the evidence map but not considered for analysis due to limitations in study designs.

\section{Intervention Description}

Bright light therapy interventions focus on technology to influence the circadian rhythm of PLWD in order to improve a range of psychiatric symptoms and behavioral disturbances including sleep disruption, agitation, or depression.

\section{Eligible Studies}

We identified nine unique studies from 12 publications that examined the use of bright light therapy interventions for individuals with dementia. ${ }^{77-85}$ (Table 4.8) Excluded from the analytic set are five pilot or small sample studies and four studies assessed as high risk of bias. We present information on all studies in the evidence map in Appendix C. 
Table 4.8. Basic characteristics of literature set: bright light therapy

\begin{tabular}{|l|l|}
\hline \multicolumn{1}{|c|}{ Characteristics } & \multicolumn{1}{c|}{ Information } \\
\hline Total unique studies & 9 studies \\
\hline Non-U.S. studies & 4 studies \\
\hline Evidence map studies & 1 pilot \\
& 4 small sample studies \\
& 4 high risk of bias studies \\
\hline Analytic set studies & 0 \\
\hline
\end{tabular}

\section{Intervention Research Context}

This literature set is preliminary. Studies examined bright light therapy on PLWD ${ }^{77-79,81-83,86}$ tailored lighting, ${ }^{85}$ or dawn-dusk simulation, a "naturalistic" form of light therapy. ${ }^{80}$ Publications date from 1998, but U.S.-based studies continued until 2019, while the last non-U.S. study was published in 2009. Non-U.S. settings included Japan, ${ }^{82}$ Switzerland, ${ }^{80}$ and the Netherlands. ${ }^{86}$ Nursing home or long-term care facilities were the most common setting with the exception of one study that took place in the geriatric unit of a psychiatric hospital. ${ }^{81}$

\section{Psychosocial Therapies for BPSD}

\section{Key Point}

- Studies on psychosocial therapies were described in the evidence map but not considered for analysis due to limitations in study designs.

\section{Intervention Description}

Psychosocial therapies for PLWD include a diverse set of interventions, ranging from cognitive behavioral training for anxiety to individualized social activities to improve sleep to validation group therapy.

\section{Eligible Studies}

We identified six unique studies from seven publications that examined the use of psychosocial interventions for individuals with dementia. ${ }^{87-92}$ (Table 4.9) Excluded from the analytic set are four pilot or small sample studies and two studies assessed as high risk of bias. ${ }^{87-}$ ${ }^{92}$ We present information on all studies in the evidence map in Appendix C.

Table 4.9. Basic characteristics of literature set: psychosocial therapies for PLWD

\begin{tabular}{|l|l|}
\hline \multicolumn{1}{|c|}{ Characteristics } & \multicolumn{1}{c|}{ Information } \\
\hline Total unique studies & 6 studies \\
\hline Non-U.S. studies & 1 study \\
\hline Evidence map studies & 1 pilot \\
& 3 small sample studies \\
\hline Analytic set studies & 2 high risk of bias studies \\
\hline
\end{tabular}

Abbreviation: PLWD=person/people living with dementia

\section{Intervention Research Context}

This literature set is preliminary. One study examined problem adaptation therapy for depression and suicidal ideation in U.S. PLWD with early stage dementia. ${ }^{91}$ One study examined the effect of individualized social activity on disruptive behaviors, ${ }^{90}$ while another examined on sleep patterns in PLWD in U.S. nursing homes. ${ }^{92}$ Two studies examined the use of cognitive 
behavioral training to control anxiety in PLWD living in the community with mild to moderate dementia in the United Kingdom ${ }^{88}$ and United States. ${ }^{89}$ An early 1997 publication examined validation therapy in PLWD in U.S. nursing homes. ${ }^{87}$

\section{Multicomponent Interventions for BPSD}

\section{Key Point}

- Studies of various unique multicomponent interventions were described in the evidence map but not considered for analysis due to limitations in study designs.

\section{Intervention Description}

This section encompasses a small and diverse set of interventions combining several components targeted at improving BPSD.

\section{Eligible Studies}

We identified nine unique studies from 10 publications that examined the use of various multicomponent interventions among PLWD. ${ }^{90,}$ 93-100 (Table 4.10) All the studies were either small; sample studies or assessed as high risk of bias; we describe them in the evidence map in Appendix C.

Table 4.10. Basic characteristics of literature set: multicomponent interventions for BPSD

\begin{tabular}{|l|l|}
\hline \multicolumn{1}{|c|}{ Characteristics } & \multicolumn{1}{c|}{ Information } \\
\hline Total unique studies & 9 studies \\
\hline Non-U.S. studies & 5 studies \\
\hline Evidence map studies & 1 pilot \\
& 3 small sample studies \\
\hline Analytic set studies & 5 high risk of bias \\
\hline
\end{tabular}

Abbreviation: BPSD=behavioral and psychological symptoms of dementia

\section{Intervention Research Context}

This research is generally preliminary, consisting of a small set of distinct and mostly unrelated studies. One study used question-asking, reading, reminiscence, and cognitive behavioral techniques, as well as environmental supports and individualized behavioral activity for depression in PLWD in U.S. nursing homes. ${ }^{94}$ One small study examined an intervention in U.S. nursing homes to balance periods of high and low arousal in PLWD throughout the day. ${ }^{96}$ An earlier 1998 study used a somewhat similar stimulation-retreat model. ${ }^{97}$ One study conducted in Taiwan long-term care facilities examined a combined acupressure and Montessori-based activity to address anxiety in PLWD. ${ }^{98}$ One community-based study in Hong Kong examined self-management support plus therapeutic exercise for knee osteoarthritis for PLWD. ${ }^{93}$ One study in a German nursing home modified a cognitive engagement program for people with autism and combined it with music therapy for PLWD with moderate dementia. ${ }^{95}$

One set of two studies on emotion-oriented care were conducted in the Netherlands. ${ }^{99,100}$ Emotion-oriented care is a combination of validation therapy and sensory stimulation (discussed briefly in the sections above) and reminiscence therapy, which we present in Chapter 5. Although publications did not clarify whether authors of the separate studies collaborated on study development and purpose, the study by Schrijnemaeker and colleagues tested an 
intervention with more elements aimed at broader intervention implementation. ${ }^{99}$ Reported results between the two studies were mixed.

The study by Beck and colleagues examined several groups of PLWD in U.S. nursing homes. ${ }^{90}$ It is also included this chapter's section on psychosocial interventions and Chapter 5's section on cognitive rehabilitation. We present it here because one study group used a combination of ADL-focused cognitive rehabilitation plus the psychosocial engagement activity. ${ }^{90}$

\section{Conclusion}

We found 63 unique studies from 76 publications that coalesced into six categories of care interventions aimed at managing BPSD in PLWD. Applying the framework for care interventions from the NASEM Families Caring for an Aging America 2016 report (Figure 1.1 in Chapter 1), the vast majority of the care interventions were delivered at the individual level. Almost 90 percent of the studies were pilots or small sample studies, Stage 0 to 2 according to the NIH Stage Model, or assessed as high risk of bias. Of the three care interventions that had low to moderate risk of bias studies - robot-assisted therapy, multisensory stimulation/Snoezelen, and CAM - we found the uncertainty of the evidence was too high to draw conclusions. However, our being unable to draw a conclusion does not mean that the intervention has no effect. Research on interventions to change behavioral and psychological outcomes is challenging, and many factors can influence the outcomes. Future research may reduce uncertainty enough to allow for conclusions about the effect of these interventions to be made with greater confidence. 


\section{Chapter 5. Care Interventions for PLWD Well-Being}

This chapter includes care interventions intended to improve the quality of life and wellbeing of people living with dementia (PLWD). Interventions use a wide range of approaches from physical to cognitive to environmental, both alone and in combination. Most studies did not base study enrollment on the absence or presence of behavioral or psychological symptoms of dementia (BPSD).

We organized this chapter by the most commonly studied interventions first, followed by whole person, multisensory stimulation, and various “other” intervention categories, including assistive technology. For each intervention, we present Key Points followed by results in three general sections: Intervention Description, Eligible Studies, and Intervention Research Context. For interventions with no studies assessed as low to medium risk of bias, we present the studies from the evidence map with a brief discussion of what has been examined and the research context. For interventions for which low- to medium-risk-of-bias studies were available for an analytic set, we present Outcomes sections by PLWD, by caregiver, and by variation in outcomes when available. Because we were generally unable to pool outcomes for any given intervention and comparison group, we synthesized the information qualitatively; therefore, we present summary findings as brief statements of how many studies reported statistically significant benefits or no difference between the intervention and the comparator. We present detailed information on all eligible studies in Appendix D.

\section{Exercise}

\section{Key Point}

- For both community-dwelling PLWD and PLWD living in residential care facilities, evidence was insufficient to draw conclusions about the effects of exercise interventions on PLWD and their informal caregivers or care partners (CG/P).

\section{Intervention Description}

Exercise interventions are used to improve daily function, aerobic conditioning, strength, gait and balance. While exercise can be done in a wide range of activities and intensity, most exercise interventions compared moderate- to high-intensity aerobic plus strength training exercise with usual care or seated group activities. The type and duration of exercise varied widely. Aerobic training usually involved walking or stationary cycling. Strength training involved repetitive functional maneuvers that relied on body weight, or standard weight training via gym or therapy equipment. Specific balance training was uncommon and involved functional maneuvers conducted with a therapist. ${ }^{101}$ More often, improvements in balance and gait speed were anticipated outcomes from general aerobic and lower extremity strengthening interventions. Exercise programs commonly included a build-up phase. Settings varied from unidentified areas of nursing homes to gyms, adult daycare, outpatient rehabilitation, homes, or church halls. Most exercises were supervised and conducted in small groups. Those supervising the interventions had varied training and experience. Only two studies also involved incorporating CG/P in exercise training activities. ${ }^{102,103}$ 


\section{Eligible Studies}

Below, we provide a summary of exercise intervention studies for adults with dementia (Table 5.1). We identified 53 unique studies from 69 eligible publications that reported the effects of exercise interventions on quality of life and functional outcomes in PLWD. Of these, eight randomized trials (RCT) with low or medium risk of bias were included in the analytic set. ${ }^{101,102,104-111}$ (Table 5.1). Information on all pilot, small sample, and high risk of bias studies is provided as part of the evidence map in Appendix D. ${ }^{75,103,112-150}$ Appendix D provides evidence tables, summary risk of bias assessments, and strength of evidence for key comparisons and outcomes of the analytic set.

Table 5.1. Basic characteristics of literature set: exercise

\begin{tabular}{|l|l|}
\hline \multicolumn{1}{|c|}{ Characteristic } & \multicolumn{1}{c|}{ Information } \\
\hline Total studies & 53 studies \\
\hline Non-U.S. studies & 48 studies \\
\hline Evidence map studies & 13 pilot \\
& 21 small sample studies \\
& 9 high risk of bias \\
\hline Analytic set studies & 7 randomized controlled trials \\
& 3 cluster randomized controlled trials \\
\hline Risk of bias of analytic set & 2 low, 8 medium \\
\hline Number of PLWD in analytic set & 1,706 \\
\hline Dementia type/definition & Mostly mild to moderate Alzheimer's disease, although the range \\
& included mild to severe and type included vascular and mixed \\
\hline Caregiver type (number) & dementias \\
\hline
\end{tabular}

Abbreviations: NA=not available; PLWD=people living with dementia

\section{Intervention Research Context}

Exercise was the most commonly examined care intervention for PLWD. Most studies assessed the effects of exercise on functional and cognitive outcomes in adults with mild or moderate dementia. Enrolled adults typically had few mobility restrictions, could follow directions, were healthy enough to exercise moderately, and were dependent in at least one activity of daily living (ADL). Few studies included adults with severe dementia, and when included, their outcomes were not separately reported.

Overall, this literature is broad but dominated by pilot and small sample studies, reflecting that preliminary investigations for PLWD are more common. All eligible trials were published within the last two decades, and most (85\%) within the past 10 years. The higher quality, analytic set studies were all published within the past decade, yet small sample and pilot studies are scattered throughout the publication dates.

Outcome domains included quality of life, function, neuropsychiatric symptoms, intervention adherence, adverse events, health or social service use, costs, and cognition. Daily functioning, measured with various ADL scales, was the most common outcome reported. The interpretability and clinical utility of reported outcomes varied considerably. Assessed outcomes were highly heterogeneous, even within domains and residential sites. Most outcomes were proxy-reported, except quality of life. Adverse events were proxy reported or chart-identified.

\section{PLWD Outcomes}

Evidence was insufficient to address any PLWD outcome. Table 5.2 summarizes the primary findings from 10 exercise intervention studies. We could not pool data for any outcome due to the wide variety of outcome measures, outcomes timing, and idiosyncratic reporting that 
rendered some outcomes uninterpretable or noncomparable. Given these and other study limitations, the exercise intervention literature was insufficient for all intervention-outcome comparisons. An assessment of insufficient evidence does not mean that the intervention is determined to be of no value. Rather, it means that due to the uncertainty of the evidence we could not draw meaningful conclusions at this time.

Three exercise trials $(n=864)$ assessed quality of life. ${ }^{104,105,107}$ Two trials $(n=694)$ tested moderate to high intensity aerobic conditioning plus strength training exercises in community settings compared with usual care. ${ }^{104,105}$ One trial compared high-intensity functional exercise with seated group activities in 18 nursing homes $(n=170) .{ }^{107}$ Intervention duration was $3^{107}$ to 4 months, ${ }^{104,105}$ and final outcomes were reported from 4 to 12 months. Studies used four quality of life measures, and one study reported dual quality of life measures. ${ }^{104}$ Self-reported quality of life did not differ significantly between group aerobic with strength training exercises versus usual care in community-dwelling PLWD with mild to moderate dementia. Similarly, no meaningful between-group differences were seen for quality of life with high-intensity functional exercise compared with seated group activities in nursing home residents with mild to moderate dementia. ${ }^{107}$

Eight randomized trials $(\mathrm{n}=1,518)$ assessed daily functioning using nine different outcome measures. ${ }^{101,102,104-107,110,111}$ Five of these were conducted in nursing facilities ${ }^{101,106,107,110,111}$ Six studies found no benefit for any outcome from exercise versus an inactive comparator, while two found some benefit on activities of daily living. The use of multiple daily functioning measures over varied followup time frames precluded pooling of data.

Balance was reported in two nursing home-based RCTs that examined high-intensity functional exercise versus seated group activities, and both reported balance outcomes with the Berg Balance Scale ${ }^{101,107}$ in adults with mild to moderate dementia. One RCT $(n=186)$ reported a post-intervention benefit of exercise on balance at 4 months, but improvement was not sustained at 7 months; ${ }^{101}$ the other trial found no difference at 3 or 6 months. ${ }^{107}$ Other mobility-related outcomes were varied, incompletely reported, or shown only as percent change from baseline. ${ }^{102,}$ 107,108 Worse baseline motor function was associated with greater functional gains in one RCT. ${ }^{108}$

Although neuropsychiatric symptoms were not required for study enrollment, half of analytic set RCTs reported at least one neuropsychiatric outcome. ${ }^{105,107,109,111,151}$ Group Tai Chi improved neuropsychiatric symptoms and depression in one RCT ( $\mathrm{n}=80)$, while all other exercise interventions had no significant impact on the neuropsychiatric symptoms, depression, or anxiety.

Exercise-related adverse events, when reported, were most often musculoskeletal, such as muscle soreness. Serious adverse events were uncommon, variably reported, questionably related to the intervention, and similar between groups. ${ }^{101,102,105,107,108,111,151}$

Other outcomes included exercise intervention adherence, attendance, or compliance, which ranged from 65 percent to 94 percent in studies that reported it.

Estimated costs of healthcare services in adults enrolled in a community-based group exercise intervention were statistically significantly lower than those of the usual care group at 24 months, ${ }^{102}$ but home exercise and usual care costs did not differ. 
Table 5.2. Summary of findings for PLWD outcomes: exercise

\begin{tabular}{|c|c|c|c|c|}
\hline $\begin{array}{l}\text { Outcome } \\
\text { Comparison }\end{array}$ & $\begin{array}{c}\text { \# Studies (\# NH) } \\
\text { n Analyzed } \\
\text { Outcome Timing }\end{array}$ & Population & Finding & $\begin{array}{l}\text { Strength o } \\
\text { Evidence* }^{*}\end{array}$ \\
\hline $\begin{array}{l}\text { Quality of Life } \\
\text { Group exercise vs usual } \\
\text { care or seated group } \\
\text { activity }\end{array}$ & $\begin{array}{l}\text { 3 RCTs. }{ }^{104,105,107}(1 \\
\mathrm{NH}) \\
\mathrm{n}=864 \\
\text { 3-12 months }\end{array}$ & $\begin{array}{l}\text { Mild to } \\
\text { Moderate }\end{array}$ & $\begin{array}{l}0 \text { of } 3 \text { found benefit } \\
3 \text { of } 3 \text { found no difference }\end{array}$ & Insufficient \\
\hline $\begin{array}{l}\text { Daily function } \\
\text { Group exercise vs usual } \\
\text { care or home exercise }\end{array}$ & $\begin{array}{l}3 \text { RCTs }^{102,104,105}(0 \\
\mathrm{NH}) \\
\mathrm{n}=904 \\
4-12 \text { months }\end{array}$ & $\begin{array}{l}1 \text { Mild } \\
1 \text { Mild- } \\
\text { Moderate } \\
1 \text { Mild-Severe }\end{array}$ & $\begin{array}{l}1 \text { of } 3 \text { found benefit for } \\
\text { group exercise vs UC, and } \\
\text { in-home exercise vs UC; } \\
2 \text { of } 3 \text { found no difference }\end{array}$ & Insufficient \\
\hline $\begin{array}{l}\text { Daily function } \\
\text { Group exercise vs } \\
\text { seated group activity }\end{array}$ & $\begin{array}{l}3 \text { RCTs }^{101,106,107}(3 \\
\mathrm{NH}) \\
\mathrm{n}=474 \\
3-7 \text { months }\end{array}$ & $\begin{array}{l}2 \text { Mild- } \\
\text { Moderate } \\
1 \text { Mild-Severe }\end{array}$ & $\begin{array}{l}\text { 1of } 3 \text { found benefit for } \\
\text { combined group exercise vs } \\
\text { social activity, and walking } \\
\text { vs social activity; } \\
2 \text { of } 3 \text { found no difference }\end{array}$ & Insufficient \\
\hline $\begin{array}{l}\text { Daily function } \\
\text { Group Tai Chi vs usual } \\
\text { care }\end{array}$ & $\begin{array}{l}1 \mathrm{RCT}^{111}(1 \mathrm{NH}) \\
\mathrm{n}=80 \\
10 \text { months }\end{array}$ & Mild & $\begin{array}{l}0 \text { found benefit } \\
1 \text { found no difference }\end{array}$ & Insufficient \\
\hline $\begin{array}{l}\text { Daily function: Eating } \\
\text { help needed. Hand } \\
\text { exercise program vs } \\
\text { usual care }\end{array}$ & $\begin{array}{l}1 \mathrm{RCT}^{110}(1 \mathrm{NH}) \\
\mathrm{n}=60 \\
6 \text { months }\end{array}$ & $\begin{array}{l}\text { Mild to } \\
\text { Moderate }\end{array}$ & $\begin{array}{l}1 \text { found benefit } \\
0 \text { found no difference }\end{array}$ & Insufficient \\
\hline $\begin{array}{l}\text { Daily function: } \\
\text { Autonomous eating time. } \\
\text { Hand exercise program } \\
\text { vs usual care }\end{array}$ & $\begin{array}{l}1 \mathrm{RCT}^{110}(1 \mathrm{NH}) \\
\mathrm{n}=60 \\
6 \text { months }\end{array}$ & $\begin{array}{l}\text { Mild to } \\
\text { Moderate }\end{array}$ & $\begin{array}{l}1 \text { found benefit } \\
0 \text { found no difference }\end{array}$ & Insufficient \\
\hline $\begin{array}{l}\text { Balance } \\
\text { HIFE (strength + } \\
\text { balance) vs. seated } \\
\text { group activity }\end{array}$ & $\begin{array}{l}2 \mathrm{RCT}^{101,107}(2 \mathrm{NH}) \\
\mathrm{n}=356 \\
3-7 \text { months }\end{array}$ & $\begin{array}{l}\text { Mild to } \\
\text { moderate }\end{array}$ & $\begin{array}{l}1 \text { of } 2 \text { found early benefit } \\
1 \text { of } 2 \text { found no difference }\end{array}$ & Insufficient \\
\hline $\begin{array}{l}\text { Neuropsychiatric } \\
\text { symptoms } \\
\text { Group exercise vs usual } \\
\text { care (2) or seated group } \\
\text { activity (1) }\end{array}$ & $\begin{array}{l}3 \text { RCTs. }{ }^{104,105,107}(1 \\
\mathrm{NH}) \\
\mathrm{n}=864 \\
3-12 \text { months }\end{array}$ & $\begin{array}{l}\text { Mild to } \\
\text { Moderate }\end{array}$ & $\begin{array}{l}1 \text { of } 3 \text { found benefit (non- } \\
\mathrm{NH} \text { ) } \\
2 \text { of } 3 \text { found no difference (1 } \\
\mathrm{NH} \text { ) }\end{array}$ & Insufficient \\
\hline $\begin{array}{l}\text { Neuropsychiatric } \\
\text { symptoms: } \\
\text { Group Tai Chi vs usual } \\
\text { care }\end{array}$ & $\begin{array}{l}1 \mathrm{RCT}^{111}(1 \mathrm{NH}) \\
\mathrm{n}=80 \\
10 \text { months }\end{array}$ & Mild & 1 found benefit & Insufficient \\
\hline $\begin{array}{l}\text { Depression } \\
\text { Group exercise vs usual } \\
\text { care (1) or seated group } \\
\text { activity (2) }\end{array}$ & $\begin{array}{l}3 \text { RCTs. }{ }^{101,104,107} \\
(2 \mathrm{NH}) \mathrm{n}=436 \\
\text { 3-6 months }\end{array}$ & $\begin{array}{l}\text { Mild to } \\
\text { Moderate }\end{array}$ & $\begin{array}{l}0 \text { of } 3 \text { found benefit } \\
3 \text { of } 3 \text { found no difference }\end{array}$ & Insufficient \\
\hline $\begin{array}{l}\text { Anxiety } \\
\text { Group hand movement } \\
\text { program vs. attention } \\
\text { control }\end{array}$ & $\begin{array}{l}1 \mathrm{RCT}^{107}(\mathrm{NH}) \\
\mathrm{n}=66 \\
3 \text { months }\end{array}$ & Moderate & $\begin{array}{l}0 \text { found benefit } \\
1 \text { found no difference }\end{array}$ & Insufficient \\
\hline
\end{tabular}

*Insufficient ratings due to few studies and imprecision in the findings.

Abbreviations: HIFE= high-intensity functional exercise (repetitive strength, balance, and mobility moves that mimic daily activities, such as rising from a chair); $\mathrm{n}=$ number; $\mathrm{NH}=$ nursing home; PLWD=person/people living with dementia; $\mathrm{RCT}=$ randomized controlled trial; UC=usual care 


\section{Caregiver Outcomes}

Caregiver quality of life and burden did not differ over 6 and 12 months in the one RCT that measured it. ${ }^{151}$

\section{Variation in Outcomes}

Two of eight RCTs reported subgroup outcomes for adults with Alzheimer's disease (AD) versus other dementias. ${ }^{101,102}$ Adults with AD showed higher healthcare costs in all groups (group exercise in adult daycare versus home exercise versus usual care), but a similar cost pattern per intervention group to those with other dementias. ${ }^{102}$ Compared with adults with AD, adults with non-AD dementia showed greater balance improvements with high-intensity functional exercise versus seated group activity. ${ }^{101}$ Men were more likely to enroll in ${ }^{101}$ and adhere to ${ }^{151}$ exercise interventions than women. Session attendance was higher for exercise than for control group activities.

\section{Music}

\section{Key Point}

- Evidence was insufficient to draw conclusions about the effects of music interventions for PLWD or CG/P.

\section{Intervention Description}

Music interventions are intended to be calming or to provide pleasure or cognitive and sensory stimulation. They can be delivered as individual or group activities, often with music choices based on familiarity or preferences of PLWD. PLWD may be encouraged to move with the music (ranging from small hand movements to dancing) or listen passively (e.g., background music at mealtimes).

\section{Eligible Studies}

Table 5.3 summarizes the effect of music interventions for PLWD and caregivers. We identified 35 unique studies from 38 publications of music interventions for PLWD and caregivers. Five studies, all RCTs, were assessed as medium risk of bias and included in the analytic set. ${ }^{152-159}$ Four used group music as an intervention compared with usual care. ${ }^{153-156}$ One compared music-with-movement with music listening combined with social activity. ${ }^{152}$ The analytic set studies were conducted in either Hong Kong or Taiwan. We describe the 10 pilot, 13 small sample, and seven high risk of bias studies in the evidence map in Appendix D. ${ }^{153,155,160-}$ ${ }^{184}$ Appendix D also includes an evidence table, summary of risk of bias assessments, and strength of evidence.

Table 5.3. Basic characteristics of literature set: music

\begin{tabular}{|l|l|}
\hline \multicolumn{1}{|c|}{ Characteristic } & \multicolumn{1}{c|}{ Information } \\
\hline Total unique studies & 35 studies \\
\hline Non-U.S. studies & 26 studies \\
\hline Total Evidence map studies & 10 pilot studies \\
& 13 small sample studies \\
& 7 high risk of bias studies \\
\hline Analytic set studies & 5 randomized controlled trials \\
\hline Risk of bias of analytic set & Medium \\
\hline Number of PLWD in analytic set & 404 \\
\hline
\end{tabular}




\begin{tabular}{|l|l|}
\hline \multicolumn{1}{|c|}{ Characteristic } & \multicolumn{1}{c|}{ Information } \\
\hline Dementia type/definition & All types of dementia with all stages of severity \\
\hline Caregiver type (number) & 1 randomized controlled trial with 59 family, 30 nurses \\
\hline
\end{tabular}

Abbreviation: PLWD=people living with dementia

\section{Intervention Research Context}

Using the National Institutes of Health (NIH) Stage Model, about 65 percent of the studies were categorized as preliminary pilot or small sample studies and the rest were categorized as explanatory, or Stage 3. The analytic set consisted of basic explanatory designs; the largest study enrolled 165 PLWD. We did not see the evolution of research on any particular intervention over time; instead, each study tested a different music intervention approach. Interventions in the evidence map were similar to those in the analytic set. Interventions were administered in groups $^{115,162,168,170,172,174,176,178,180-182,185-188}$ or individually ${ }^{160,165-167,175,177,183,189}$ and included watching live music ${ }^{164,169,190}$ or listening to familiar songs from recordings, ${ }^{182,}{ }^{183}$ playing with musical instruments, ${ }^{95,173}$ and singing along or dancing to music. ${ }^{171,174}$ Only one study used background music as an intervention. ${ }^{161}$ Non-U.S settings include: Australia, ${ }^{164,165,172}$ France, ${ }^{167}$, ${ }^{182}$ Germany, ${ }^{183}$ Hong Kong, ${ }^{184}$ Iceland, ${ }^{178}$ Italy, ${ }^{160 ., ~ 174, ~ 191, ~} 192$ Japan, ${ }^{173}$ the Netherlands, ${ }^{187}$ Norway, ${ }^{175}$ Singapore, ${ }^{168}$ South Korea, ${ }^{162}$ Taiwan, ${ }^{161,176,} 188$ Turkey, ${ }^{177}$ and the United Kingdom. ${ }^{169}$

\section{PLWD Outcomes}

Evidence was insufficient to draw conclusions about music therapy for agitation, anxiety, depression, mood, and quality of life. Table 5.4 provides a summary of findings.

One study ( $\mathrm{n}=84$ ) found the intervention improved quality of life in PLWD. ${ }^{155}$

Three studies examined agitation in PLWD; two compared $(n=155)$ group music versus usual care over a period of 6 weeks, ${ }^{154,156}$ and one $(n=165)$ compared music-with-movement versus music listening combined with social activity for 6 weeks. ${ }^{152}$ Results were inconsistent. While one study reported a significant effect of group music on agitation, ${ }^{154}$ the other two reported no statistical difference between intervention and control groups.

One study ( $\mathrm{n}=55$ ) found a group music intervention decreased anxiety compared with usual care. ${ }^{156}$ One study reported that a group music intervention significantly decreased depression after 6 weeks. ${ }^{158}$ A three-armed study ( $\mathrm{n}=84$ ) found music improved mood. ${ }^{155}$

Table 5.4. Summary of findings for PLWD outcomes: music

\begin{tabular}{|c|c|c|c|c|}
\hline $\begin{array}{l}\text { Outcome } \\
\text { Comparison }\end{array}$ & $\begin{array}{l}\text { \# Studies/ } \\
\text { Design } \\
\text { (n analyzed) } \\
\text { Timing }\end{array}$ & Population & Findings & $\begin{array}{l}\text { Strength of } \\
\text { Evidence* }\end{array}$ \\
\hline $\begin{array}{l}\text { Quality of Life } \\
\text { Group music vs. } \\
\text { usual care }\end{array}$ & $\begin{array}{l}1 \mathrm{RCT}^{155}(\mathrm{n}=84) \\
10 \text { weeks }\end{array}$ & $\begin{array}{l}\text { Dementia (all } \\
\text { types) with mild- } \\
\text { moderate }\end{array}$ & $\begin{array}{l}1 \text { found benefit } \\
0 \text { found no difference }\end{array}$ & Insufficient \\
\hline $\begin{array}{l}\text { Agitation } \\
\text { Group music vs. } \\
\text { Standard/usual } \\
\text { care }\end{array}$ & $\begin{array}{l}3 \mathrm{RCT}^{152,154,156} \\
(\mathrm{n}=320) \\
6 \text { weeks }\end{array}$ & $\begin{array}{l}\text { All dementia types } \\
\text { with mild to severe } \\
\text { severity }\end{array}$ & $\begin{array}{l}1 \text { of } 3 \text { found benefit } \\
2 \text { of } 3 \text { found no difference }\end{array}$ & Insufficient \\
\hline $\begin{array}{l}\text { Agitation } \\
\text { Music with } \\
\text { movement vs. } \\
\text { music listening \& } \\
\text { social activity }\end{array}$ & $\begin{array}{l}1 \mathrm{RCT}^{152}(\mathrm{n}=165) \\
6 \text { weeks }\end{array}$ & $\begin{array}{l}\text { Any type of } \\
\text { dementia with } \\
\text { moderate severity }\end{array}$ & $\begin{array}{l}0 \text { found benefit } \\
1 \text { found no difference }\end{array}$ & Insufficient \\
\hline
\end{tabular}




\begin{tabular}{|c|c|c|c|c|}
\hline $\begin{array}{l}\text { Outcome } \\
\text { Comparison }\end{array}$ & $\begin{array}{c}\text { \# Studies/ } \\
\text { Design } \\
\text { (n analyzed) } \\
\text { Timing }\end{array}$ & Population & Findings & $\begin{array}{l}\text { Strength of } \\
\text { Evidence* }\end{array}$ \\
\hline $\begin{array}{l}\text { Anxiety } \\
\text { Group music vs. } \\
\text { usual care }\end{array}$ & $\begin{array}{l}1 \mathrm{RCT}^{156} \quad(n=55) \\
6 \text { weeks }\end{array}$ & $\begin{array}{l}\text { Any type of } \\
\text { dementia with } \\
\text { moderate severity }\end{array}$ & $\begin{array}{l}0 \text { found benefit } \\
1 \text { found no difference }\end{array}$ & Insufficient \\
\hline $\begin{array}{l}\text { Depression } \\
\text { Group music vs. } \\
\text { usual care }\end{array}$ & $\begin{array}{l}1 \mathrm{RCT}^{156}(n=55) \\
6 \text { weeks }\end{array}$ & $\begin{array}{l}\text { Any type of } \\
\text { dementia with } \\
\text { moderate severity }\end{array}$ & $\begin{array}{l}0 \text { found benefit } \\
1 \text { found no difference }\end{array}$ & Insufficient \\
\hline $\begin{array}{l}\text { Mood } \\
\text { Group music vs. } \\
\text { usual care }\end{array}$ & $\begin{array}{l}1 \mathrm{RCT}^{155}(\mathrm{n}=84) \\
10 \text { weeks }\end{array}$ & $\begin{array}{l}\text { Dementia (all } \\
\text { types) with mild- } \\
\text { moderate }\end{array}$ & $\begin{array}{l}1 \text { found benefit } \\
0 \text { found no difference }\end{array}$ & Insufficient \\
\hline
\end{tabular}

*Insufficient ratings due to few studies and imprecision in the findings.

Abbreviations: n=number; PLWD=person/people living with dementia; RCT=randomized controlled trial

\section{Caregiver Outcomes}

Evidence was insufficient for the effect of music on stress and caregiver burden for family caregivers. One study reported psychological well-being outcomes (stress and burden) for family caregivers and found benefit at 9 months. ${ }^{155}$ Table 5.5 summarizes the primary findings. No significant reduction in stress was reported.

Table 5.5. Summary of findings for caregiver outcomes: music

\begin{tabular}{|l|l|l|l|l|}
\hline $\begin{array}{c}\text { Outcome } \\
\text { Comparison }\end{array}$ & $\begin{array}{c}\text { Studiesl } \\
\text { Design } \\
\text { (n=analyzed) } \\
\text { Timing }\end{array}$ & \multicolumn{1}{|c|}{ Population } & Findings & $\begin{array}{c}\text { Strength of } \\
\text { Evidence* }^{*}\end{array}$ \\
\hline $\begin{array}{l}\text { Stress \& } \\
\text { burden } \\
\begin{array}{l}\text { Group music } \\
\text { vs. usual care }\end{array}\end{array}$ & $\begin{array}{l}\mathrm{RCT}^{155} \\
(\mathrm{n}=84)\end{array}$ & $\begin{array}{l}\text { Informal } \\
\text { caregivers }\end{array}$ & One benefit & Insufficient \\
\hline
\end{tabular}

*Insufficient ratings due to few studies and imprecision in the findings.

Abbreviations: n=number; RCT=randomized controlled trial

\section{Variation in Outcomes}

A single study reported that both singing and listening were more effective in improving mood in PLWD with mild dementia and AD, while listening-only interventions were more beneficial for PLWD with moderate non-AD dementia (e.g., vascular and mixed dementias, frontotemporal dementia). ${ }^{155}$

\section{Reminiscence Therapy}

\section{Key Point}

- Evidence was insufficient to draw conclusions about the effect of reminiscence therapy versus usual care for PLWD and CG/P.

\section{Intervention Description}

Reminiscence therapy for PLWD involves a discussion of past life events and experiences with the goal of improving memory and well-being. These discussions can be held in groups, 
individually, or as a combination of both. Sessions may incorporate sentimental objects or photographs and include family members/caregivers.

\section{Eligible Studies}

We identified 25 unique studies from 27 publications examining reminiscence therapy for PLWD. ${ }^{47,193-217}$ Table 5.6 summarizes the characteristics of the literature set. Four studies were included in the analytic set. ${ }^{193-198}$ All studies in the analytic set were RCTs comparing group reminiscence therapy to usual care for PLWD. One study enrolled PLWD-CG/P dyads. ${ }^{197,198}$ Three studies examined the use of reminiscence therapy for PLWD with mild to moderate dementia. ${ }^{193,194,197,198}$. One study examined the use of reminiscence therapy for PLWD with mild to severe dementia. ${ }^{195,196}$ Appendix D provides evidence tables, summary risk of bias assessments, and strength of evidence for key comparisons and outcomes.

Eleven studies were pilots or had fewer than 50 participants and were not included as part of the analytic study set. ${ }^{47,199-207,218}$ Ten additional studies were assessed as high risk of bias. ${ }^{208-217}$, ${ }^{219}$ We present information on all pilot studies and high risk of bias in the evidence map in Appendix D.

Table 5.6. Basic characteristics of literature set: reminiscence therapy

\begin{tabular}{|l|l|}
\hline \multicolumn{1}{|c|}{ Characteristic } & \multicolumn{1}{c|}{ Information } \\
\hline Total Studies & 25 studies \\
\hline Non-U.S. studies & 22 studies \\
\hline Evidence map studies & 7 pilot studies \\
& 4 small sample size \\
& 10 high risk of bias \\
\hline Analytic set studies & 4 randomized controlled trials \\
\hline Risk of bias of analytic set & Medium \\
\hline Number of PLWD in analytic set & 904 \\
\hline Dementia type/definition & Predominantly mild to moderate dementia \\
\hline Caregiver type (number) & Informal caregivers (n=904) \\
\hline
\end{tabular}

Abbreviations: $n=$ number; PLWD=people living with dementia

\section{Intervention Research Context}

This literature set comprises mostly pilot studies and small RCTs. Included studies were published between 1999 and 2019. Only three studies were conducted in the United States. and none of these were in the analytic set. ${ }^{203,205,208}$ Eleven studies, including the four in the analytic set, examined group reminiscence therapy. ${ }^{193-199,}$ 209, 210, 212, 213, 217, 218 Ten studies examined individual reminiscence therapy, including life-story approaches. ${ }^{47,201,205-207,211,214-216}$ Four studies examined reminiscence therapy using technology such as audio recordings and webbased videos. $^{200,202,203,208}$ We observed no clear trend in the publication of pilot studies or larger RCTs. Most of the studies that examined technology in reminiscence therapy were pilots, and we identified a mix of both pilots and small to large RCTs for group and individual reminiscence published across a two-decade span. The continuation of pilot studies might be partially attributed to study quality, as we found that a large proportion of nonpilot studies had high risk of bias. Potential detection and performance bias were significant concerns in nonpilot studies rated as high risk of bias.

\section{PLWD Outcomes}

Evidence was insufficient to draw conclusions about the effects of reminiscence therapy for PLWD. Four studies in the analytic study set reported outcomes for PLWD. ${ }^{193-198}$ Two studies 
enrolled PLWD with mild to moderate AD, ${ }^{193,} 194$ one study enrolled PLWD with mild to moderate dementia of any type, ${ }^{197,} 198$ and one study enrolled PLWD with dementia of any type or severity. ${ }^{195,196}$ Studies measured a range of outcomes for PLWD. We were able to quantitatively analyze and pool PLWD outcomes in four domains: activities of daily living, BPSD, depression, and quality of life. However, we assessed the evidence as insufficient due to the variety of measures used within domains, lack of minimally important differences for measures, and variation in analytic methods across studies.

Table 5.7 summarizes the primary outcomes for PLWD. Three studies with a total of 802 PLWD reported measures of activities of daily living. ${ }^{193,194,197,198}$ At 3 months, groups did not differ significantly across all three studies. One study also reported outcomes at 6 months, finding no difference between groups. ${ }^{194}$ Two studies with a total of 416 PLWD reported measures of BPSD. ${ }^{193,194}$ Reminiscence therapy and usual care groups did not differ at 3 months. ${ }^{193,194}$ Four studies with a total of 1,006 PLWD reported measures of depression. ${ }^{193-198}$ Individual study findings were mixed; however the pooled effect size showed no difference between groups at 2 to 3 months. Two studies with a total of 814 PLWD reported measures quality of life. Both found no difference between groups at 3 months. ${ }^{193,}$ 197, 198

Two studies reported additional PLWD outcomes. One $(n=488)$ reported a measure of anxiety and a PLWD assessment of the PLWD-caregiver relationship. ${ }^{197,} 198$ The second ( $\left.\mathrm{n}=326\right)$ reported a measure of apathy and a measure of dependency. ${ }^{193}$ Groups did not differ at 3 months for either measure.

Table 5.7. Summary of findings for PLWD outcomes: reminiscence therapy

\begin{tabular}{|c|c|c|c|c|}
\hline $\begin{array}{l}\text { Outcome } \\
\text { Comparison }\end{array}$ & $\begin{array}{l}\text { \# Studies/Design } \\
\text { (n analyzed) } \\
\text { Timing }\end{array}$ & Population & Findings & $\begin{array}{l}\text { Strength of } \\
\text { Evidence* }\end{array}$ \\
\hline $\begin{array}{l}\text { Activities of Daily Living } \\
\text { Reminiscence vs usual } \\
\text { care }\end{array}$ & $\begin{array}{l}3 \text { RCTs } 193,194,197, \\
198(n=802) \\
3 \text { months }\end{array}$ & $\begin{array}{l}\text { Mild to moderate } \\
\text { dementia }\end{array}$ & $\begin{array}{l}0 \text { of } 3 \text { found benefit } \\
3 \text { of } 3 \text { found no difference }\end{array}$ & Insufficient \\
\hline $\begin{array}{l}\text { BPSD } \\
\text { Reminiscence vs usual } \\
\text { care }\end{array}$ & $\begin{array}{l}2 \text { RCTs } 193,194 \\
(n=416) \\
3 \text { months } \\
\end{array}$ & $\begin{array}{l}\text { Mild to moderate } \\
\text { AD }\end{array}$ & $\begin{array}{l}0 \text { of } 2 \text { found benefit } \\
2 \text { of } 2 \text { found no difference }\end{array}$ & Insufficient \\
\hline $\begin{array}{l}\text { Depression } \\
\text { Reminiscence vs usual } \\
\text { care }\end{array}$ & $\begin{array}{l}\text { 4 RCTs }^{193-198} \\
(\mathrm{n}=1,006) \\
2 \text { to } 3 \text { months }\end{array}$ & $\begin{array}{l}\text { All dementia types } \\
\text { and severities }\end{array}$ & $\begin{array}{l}0 \text { of } 4 \text { found benefit } \\
4 \text { of } 4 \text { found no difference }\end{array}$ & Insufficient \\
\hline $\begin{array}{l}\text { Quality of Life } \\
\text { Reminiscence vs usual } \\
\text { care }\end{array}$ & $\begin{array}{l}2 \text { RCTs } 193,197,198 \\
(n=814) \\
3 \text { months }\end{array}$ & $\begin{array}{l}\text { Mild to moderate } \\
\text { dementia }\end{array}$ & $\begin{array}{l}0 \text { of } 2 \text { found benefit } \\
2 \text { of } 2 \text { found no difference }\end{array}$ & Insufficient \\
\hline $\begin{array}{l}\text { Anxiety } \\
\text { Reminiscence vs usual } \\
\text { care }\end{array}$ & $\begin{array}{l}1 \mathrm{RCT} \mathrm{197,198} \\
(\mathrm{n}=488) \\
3 \text { months }\end{array}$ & $\begin{array}{l}\text { Mild to moderate } \\
\text { dementia }\end{array}$ & $\begin{array}{l}0 \text { found benefit } \\
1 \text { found no difference }\end{array}$ & Insufficient \\
\hline $\begin{array}{l}\text { PLWD-Caregiver } \\
\text { Reminiscence vs usual } \\
\text { care Relationship }\end{array}$ & $\begin{array}{l}1 \text { RCT 197, } 198 \\
(\mathrm{n}=488) \\
3 \text { months } \\
\end{array}$ & $\begin{array}{l}\text { Mild to moderate } \\
\text { dementia }\end{array}$ & $\begin{array}{l}0 \text { found benefit } \\
1 \text { found no difference }\end{array}$ & Insufficient \\
\hline $\begin{array}{l}\text { Apathy } \\
\text { Reminiscence vs usual } \\
\text { care }\end{array}$ & $\begin{array}{l}1 \text { RCT } 193 \\
(n=326) \\
3 \text { months } \\
\end{array}$ & $\begin{array}{l}\text { Mild to moderate } \\
\text { AD }\end{array}$ & $\begin{array}{l}0 \text { found benefit } \\
1 \text { found no difference }\end{array}$ & Insufficient \\
\hline $\begin{array}{l}\text { Dependency } \\
\text { Reminiscence vs usual } \\
\text { care }\end{array}$ & $\begin{array}{l}1 \mathrm{RCT} 193 \\
(\mathrm{n}=326) \\
3 \text { months } \\
\end{array}$ & $\begin{array}{l}\text { Mild to moderate } \\
\text { AD }\end{array}$ & $\begin{array}{l}0 \text { found benefit } \\
1 \text { found no difference }\end{array}$ & Insufficient \\
\hline
\end{tabular}

*Insufficient ratings due to few studies and imprecision in the findings.

Abbreviations: $\mathrm{AD}=$ Alzheimer's disease; $\mathrm{BPSD}=$ behavioral and psychological symptoms of dementia; $\mathrm{n}=$ number; PLWD=people living with dementia; RCT=randomized controlled trial 


\section{Caregiver Outcomes}

Evidence was insufficient to draw conclusions about the effects of PLWD reminiscence therapy for CG/P. Three studies in the analytic set reported a range of outcomes for CG/P of PLWD. 193, 194, 197, 198 Overlap between outcome measures was insufficient to assess the potential benefit of PLWD reminiscence therapy for CG/P. Table 5.8 summarizes the primary outcomes for CG/P.

Two studies enrolled PLWD with mild to moderate AD. ${ }^{193,194}$ One study ( $\left.n=326\right)$ measured caregiver burden, finding no difference between the reminiscence therapy group and the usual care group at 3 months. ${ }^{193}$ Another study ( $\left.n=90\right)$ measured CG/P distress, with no difference between groups at 3 months or 6 months. ${ }^{194}$

One study ( $n=488$ ) enrolled PLWD with mild to moderate dementia of any type. The study reported measures of mental health, quality of life, stress, anxiety, and depression in CG/Ps. ${ }^{197}$, 198 The study also measured CG/Ps' ratings of their relationship with the PLWD. At 3 months, groups did not differ in any of these measures. ${ }^{197,198}$

Table 5.8. Summary of findings for caregiver outcomes: reminiscence therapy

\begin{tabular}{|c|c|c|c|c|}
\hline $\begin{array}{l}\text { Outcome } \\
\text { Comparison }\end{array}$ & $\begin{array}{c}\text { \# Studies/Design } \\
\text { (n analyzed) } \\
\text { Timing }\end{array}$ & Population & Findings & $\begin{array}{l}\text { Strength of } \\
\text { Evidence* }^{*}\end{array}$ \\
\hline $\begin{array}{l}\text { Caregiver Burden } \\
\text { Reminiscence vs } \\
\text { usual care }\end{array}$ & $\begin{array}{l}1 \mathrm{RCT}^{193}(\mathrm{n}=326) \\
3 \text { months }\end{array}$ & Informal caregivers & 1 found no benefit. & Insufficient \\
\hline $\begin{array}{l}\text { Caregiver } \\
\text { Distress } \\
\text { Reminiscence vs } \\
\text { usual care }\end{array}$ & $\begin{array}{l}1 \mathrm{RCT}^{194}(\mathrm{n}=90) \\
3 \text { months, } 6 \text { months }\end{array}$ & Informal caregivers & 1 found no benefit. & Insufficient \\
\hline $\begin{array}{l}\text { Mental Health } \\
\text { Reminiscence vs } \\
\text { usual care }\end{array}$ & $\begin{array}{l}1 \text { RCT 197, } 198 \\
(n=488) \\
3 \text { months }\end{array}$ & Informal caregivers & 1 found no benefit. & Insufficient \\
\hline $\begin{array}{l}\text { Quality of Life } \\
\text { Reminiscence vs } \\
\text { usual care }\end{array}$ & $\begin{array}{l}1 \text { RCT } 197,198 \\
(n=488) \\
3 \text { months }\end{array}$ & Informal caregivers & 1 found no benefit. & Insufficient \\
\hline $\begin{array}{l}\text { Stress } \\
\text { Reminiscence vs } \\
\text { usual care }\end{array}$ & $\begin{array}{l}1 \text { RCT 197, } 198 \\
(n=488) \\
3 \text { months }\end{array}$ & Informal caregivers & 1 found no benefit. & Insufficient \\
\hline $\begin{array}{l}\text { Anxiety } \\
\text { Reminiscence vs } \\
\text { usual care }\end{array}$ & $\begin{array}{l}1 \text { RCT 197, } 198 \\
(n=488) \\
3 \text { months }\end{array}$ & Informal caregivers & 1 found no benefit. & Insufficient \\
\hline $\begin{array}{l}\text { Depression } \\
\text { Reminiscence vs } \\
\text { usual care }\end{array}$ & $\begin{array}{l}1 \text { RCT 197, } 198 \\
(n=488) \\
3 \text { months }\end{array}$ & Informal caregivers & 1 found no benefit. & Insufficient \\
\hline $\begin{array}{l}\text { PLWD-Caregiver } \\
\text { Relationship } \\
\text { Reminiscence vs } \\
\text { usual care }\end{array}$ & $\begin{array}{l}1 \mathrm{RCT} 197,198 \\
(\mathrm{n}=488) \\
3 \text { months }\end{array}$ & Informal caregivers & 1 found no benefit. & Insufficient \\
\hline
\end{tabular}

*Insufficient ratings due to few studies and imprecision in the findings.

Abbreviations: n=number; PLWD=people living with dementia; RCT=randomized controlled trial

\section{Variation in Outcomes}

The analytic study set did not report variation in outcomes by PLWD, caregiver, or intervention characteristics. 


\section{Cognitive Rehabilitation}

\section{Key Point}

- Evidence was insufficient to draw conclusions about the effects of cognitive rehabilitation for PLWD and their CG/P.

\section{Intervention Description}

Cognitive rehabilitation, originally developed for people experiencing cognitive impairment from brain injury, was later adapted for use among PLWD with the goal of enabling them to maintain as much independent function as possible in areas they care most about. ${ }^{220,221} \mathrm{We}$ classified studies as cognitive rehabilitation when interventions focused on cognitive activities required for daily living (such as memory and executive function), and/or were aimed at recovery ${ }^{22}$ or "reactivation of retained daily skills."223 Therefore, studies in this section range from cognitive process activities in PLWD with mild dementia, to specific, task-based guidance to complete single or multiple ADLs in adults with more advanced dementia.

\section{Eligible Studies}

We identified 23 unique studies from 28 publications that examined various cognitive rehabilitation interventions for adults with dementia (Table 5.9). ${ }^{90,193,221,222,224-243}$ Three randomized trials were assessed as low or medium risk of bias and are included in the analytic set. ${ }^{193,224,244}$ The remaining 23 studies were pilot, small sample trials, or assessed as high risk of bias. ${ }^{90,154,221,222,225-230,232-234,239-245}$ We present information on all eligible studies in the evidence map in Appendix D.

Table 5.9. Basic characteristics of literature set: cognitive rehabilitation

\begin{tabular}{|l|l|}
\hline \multicolumn{1}{|c|}{ Characteristics } & \multicolumn{1}{c|}{ Information } \\
\hline Total unique studies & 23 studies \\
\hline Non-U.S. studies & 19 studies \\
\hline Evidence map studies & 2 pilot studies \\
& 15 small samples studies \\
& 3 high risk of bias studies \\
\hline Analytic set studies & 3 studies \\
\hline Risk of bias of analytic set & Low/medium \\
\hline Number of PLWD in analytic set & 890 \\
\hline Dementia type/definition & Mild to moderate dementia \\
\hline Caregiver type (number) & Informal caregivers (n=474) \\
\hline
\end{tabular}

Abbreviations: n=number; PLWD=people living with dementia

\section{Intervention Research Context}

The cognitive rehabilitation literature largely comprises unique interventions that have been tested only in small sample studies, thus the literature is largely preliminary. Trials included small group or individually tailored activities that engaged a variety of cognitive functions to improve the completion of daily activities in PLWD. Interventions were diverse, and details about both cognitive exercises and task guidance were often lacking, especially for individual rehabilitation of basic functional tasks.

Most included studies compared cognitive rehabilitation to other active cognitive approaches or usual care. The longest study lasted 2 years, ${ }^{193}$ although most ranged from 3 to 6 months. Some studies reported both task-based outcomes and measures of positive affect surrounding task accomplishment, such as satisfaction, quality of life, or reduction in neuropsychiatric 
symptoms. Studies were community, ${ }^{193,} 220,221,224,225,228,230,232$ hospital, ${ }^{239}$ or nursing home-

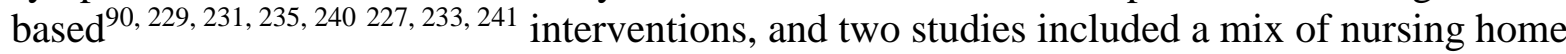
and community residents. ${ }^{222,}{ }^{228}$ Most studies (83\%) took place outside of the United States.

\section{PLWD Outcomes}

Evidence was insufficient to draw conclusions about the effects of cognitive rehabilitation to improve quality of life, function, neuropsychiatric symptoms, mood, health service use, institutionalization, and harms for adults with dementia. We could not combine studies because the three trials tested very different interventions and assessed different outcomes at varying time points. The three analytic set trials $(n=890)$ were conducted in noninstitutionalized adults with mild to moderate dementia. ${ }^{193,224,244}$ Table 5.10 summarizes the primary findings.

The largest, medium risk of bias RCT of 653 community-dwelling adults found no benefit of individual cognitive rehabilitation versus group usual care ( $\mathrm{n}=311$ for these study arms) on function, ADLs, behavioral issues, quality of life, depression, or caregiver burden over 24 months. ${ }^{193}$ However, authors reported greater 24-month clinical benefits, including slower functional declines, improved survival, and marginal benefits on behavior, caregiver burden and resource use. ${ }^{193}$

A medium risk of bias RCT of 475 community-dwelling adults found a statistically but not clinically significant benefit of individual cognitive rehabilitation compared with usual care on function assessed as self-rated goal attainment, at three and nine months. ${ }^{244}$ The mean Mini Mental State Exam scores of enrolled adults was 24 (range 18 to 30), which marginally met criteria for mild dementia. There was no benefit of cognitive rehabilitation on quality of life, depression, or anxiety, compared with usual care. ${ }^{244}$

One RCT found no benefit of errorless learning with feed-forward (continuous verbal) training over trial and error learning on ADLs in adults with AD and mixed dementia who were living at home. ${ }^{224}$ 
Table 5.10. Summary of findings for PLWD outcomes: cognitive rehabilitation

\begin{tabular}{|c|c|c|c|c|}
\hline $\begin{array}{l}\text { Outcome } \\
\text { Comparison }\end{array}$ & $\begin{array}{c}\text { \# Studies/Design } \\
\text { (n analyzed) } \\
\text { Timing }\end{array}$ & Population & Findings & $\begin{array}{l}\text { Strength of } \\
\text { Evidence* }^{*}\end{array}$ \\
\hline $\begin{array}{l}\text { Quality of Life } \\
\text { Individual cognitive } \\
\text { rehabilitation vs } \\
\text { usual care }\end{array}$ & $\begin{array}{l}1 \mathrm{RCT}^{193} \\
(\mathrm{n}=311) \\
24 \text { months }\end{array}$ & $\begin{array}{l}\text { Mild to } \\
\text { moderate } \\
\text { dementia }\end{array}$ & $\begin{array}{l}0 \text { foun benefit } \\
1 \text { found no difference }\end{array}$ & Insufficient \\
\hline $\begin{array}{l}\text { Quality of Life } \\
\text { Individual cognitive } \\
\text { rehabilitation vs } \\
\text { usual care }\end{array}$ & $\begin{array}{l}1 \mathrm{RCT}^{244} \\
(\mathrm{n}=475) \\
9 \text { months }\end{array}$ & $\begin{array}{l}\text { Mild } \\
\text { dementia }\end{array}$ & $\begin{array}{l}0 \text { found benefit } \\
1 \text { found no difference }\end{array}$ & Insufficient \\
\hline $\begin{array}{l}\text { Daily function } \\
\text { Individual cognitive } \\
\text { rehabilitation vs } \\
\text { usual care }\end{array}$ & $\begin{array}{l}1 \mathrm{RCT}^{193} \\
(\mathrm{n}=311) \\
24 \text { months }\end{array}$ & $\begin{array}{l}\text { Mild to } \\
\text { moderate } \\
\text { dementia }\end{array}$ & $\begin{array}{l}0 \text { found benefit } \\
1 \text { found no difference }\end{array}$ & Insufficient \\
\hline $\begin{array}{l}\text { Daily function } \\
\text { Errorless Learning } \\
\text { (feed-forward } \\
\text { instructions) vs Trial } \\
\text { \& Error Learning }\end{array}$ & $\begin{array}{l}1 \mathrm{RCT}^{224} \\
(\mathrm{n}=161) \\
6 \text { months }\end{array}$ & $\begin{array}{l}\text { Mild to } \\
\text { moderate } \\
\text { dementia }\end{array}$ & $\begin{array}{l}0 \text { found benefit } \\
1 \text { found no difference }\end{array}$ & Insufficient \\
\hline $\begin{array}{l}\text { Self-rated goal } \\
\text { attainment } \\
\text { Individual cognitive } \\
\text { rehabilitation vs } \\
\text { usual care }\end{array}$ & $\begin{array}{l}1 \mathrm{RCT}^{244} \\
(\mathrm{n}=475) \\
9 \text { months }\end{array}$ & $\begin{array}{l}\text { Mild } \\
\text { dementia }\end{array}$ & $\begin{array}{l}1 \text { found benefit } \\
0 \text { found no difference }\end{array}$ & Insufficient \\
\hline $\begin{array}{l}\text { Dependency } \\
\text { Individual cognitive } \\
\text { rehabilitation vs } \\
\text { usual care }\end{array}$ & $\begin{array}{l}1 \mathrm{RCT}^{193} \\
(\mathrm{n}=311) \\
24 \text { months }\end{array}$ & $\begin{array}{l}\text { Mild to } \\
\text { moderate } \\
\text { dementia }\end{array}$ & $\begin{array}{l}1 \text { found benefit } \\
0 \text { found no difference }\end{array}$ & Insufficient \\
\hline $\begin{array}{l}\text { Neuropsychiatric } \\
\text { symptoms } \\
\text { Individual cognitive } \\
\text { rehabilitation vs } \\
\text { usual care }\end{array}$ & $\begin{array}{l}1 \mathrm{RCT}^{193} \\
(\mathrm{n}=311) \\
24 \text { months }\end{array}$ & $\begin{array}{l}\text { Mild to } \\
\text { moderate } \\
\text { dementia }\end{array}$ & $\begin{array}{l}0 \text { found benefit } \\
1 \text { found no difference }\end{array}$ & Insufficient \\
\hline $\begin{array}{l}\text { Neuropsychiatric } \\
\text { symptoms } \\
\text { Errorless Learning } \\
\text { (feed-forward } \\
\text { instructions) vs Trial } \\
\text { \& Error Learning }\end{array}$ & $\begin{array}{l}1 \mathrm{RCT}^{224} \\
(\mathrm{n}=161) \\
6 \text { months }\end{array}$ & $\begin{array}{l}\text { Mild to } \\
\text { moderate } \\
\text { dementia }\end{array}$ & $\begin{array}{l}0 \text { found benefit } \\
1 \text { found no difference }\end{array}$ & Insufficient \\
\hline $\begin{array}{l}\text { Depression } \\
\text { Individual cognitive } \\
\text { rehabilitation vs } \\
\text { usual care }\end{array}$ & $\begin{array}{l}1 \mathrm{RCT}^{244} \\
(\mathrm{n}=475) \\
9 \text { months }\end{array}$ & $\begin{array}{l}\text { Mild } \\
\text { dementia }\end{array}$ & $\begin{array}{l}0 \text { found benefit } \\
1 \text { found no difference }\end{array}$ & Insufficient \\
\hline $\begin{array}{l}\text { Anxiety } \\
\text { Individual cognitive } \\
\text { rehabilitation vs } \\
\text { usual care }\end{array}$ & $\begin{array}{l}1 \mathrm{RCT}^{244} \\
(\mathrm{n}=475) \\
9 \text { months }\end{array}$ & $\begin{array}{l}\text { Mild } \\
\text { dementia }\end{array}$ & $\begin{array}{l}0 \text { found benefit } \\
1 \text { found no difference }\end{array}$ & Insufficient \\
\hline
\end{tabular}

*Insufficient ratings due to few studies and imprecision in the findings.

Abbreviations: n-number; PLWD=person/people living with dementia; RCT=randomized controlled trial 


\section{Caregiver Outcomes}

Cognitive rehabilitation offered no significant benefit over reminiscence on CG/P burden or apathy in the one trial that measured it. ${ }^{193}$ Cognitive rehabilitation offered no significant benefit over usual care on CG/P stress, quality of life, or health-related quality of life over 9 months. ${ }^{244}$

\section{Variation in Outcomes}

No analytic set studies reported variations in outcomes by PLWD, caregiver, or intervention characteristics.

\section{Cognitive Training}

\section{Key Point}

- Evidence was insufficient to draw conclusions about the effect of cognitive training interventions for PLWD and their CG/P.

\section{Intervention Description}

Cognitive Training interventions involve repetitive or progressive drill-type exercises aimed at improving various domains of cognitive function (e.g., memory or executive functions). ${ }^{193}$ The cognitive training interventions included in eligible studies incorporated some form of cognitive drills, whether paper-pencil tests or computer-based.

\section{Eligible Studies}

We identified 18 unique studies from 18 publications that examined cognitive training interventions among PLWD (Table 5.11). ${ }^{189,191,193,246-260}$ Five studies were assessed as medium risk of bias and were included in the analytic set. ${ }^{189,193,246-249}$ Appendix D provides evidence tables, summary risk of bias assessments, and strength of evidence for key comparisons and outcomes. Ten studies had small sample sizes, ${ }^{191,250-252,254-257,259-261}$ and two were assessed as high risk of bias. ${ }^{253,258}$ We present information on all pilot studies and high risk of bias studies as part of the evidence map in Appendix D.

Table 5.11. Basic characteristics of literature set: cognitive training

\begin{tabular}{|l|l|}
\hline \multicolumn{1}{|c|}{ Characteristics } & \multicolumn{1}{c|}{ Information } \\
\hline Total unique studies & 18 studies \\
\hline Non-U.S. studies & 15 studies \\
\hline Evidence map studies & 3 pilot \\
& 9 small sample studies \\
& 1 high risk of bias \\
\hline Analytic set studies & 5 randomized controlled trials \\
\hline Risk of bias of analytic set & Medium \\
\hline Number of PLWD in analytic set & 748 \\
\hline Dementia type/definition & All types and severities \\
\hline Caregiver type (number) & Informal $(\mathrm{n}=324)$, Formal $(\mathrm{n}=\mathrm{NR})$ \\
\hline
\end{tabular}

Abbreviations: n-number; NR=not reported; PLWD=people living with dementia

\section{Intervention Research Context}

The majority of eligible studies were small sample size trials involving fewer than 50 total participants each. Studies were published between 2001 and 2019, and were mostly conducted outside the United States. Intervention mode ranged from paper-and-pencil tasks to software or 
web-based cognitive training. Cognitive training tasks targeted a range of functions that included memory, language, and executive function. Interventions were delivered in community-based (e.g., adult day care centers) or institutional settings (e.g., nursing homes, veterans homes). Three of the studies included in the analytic set took place at nursing homes, dementia special care units, or assisted-living residences, ${ }^{189,}$ 246, 249 while two took place at adult day care or memory clinics. ${ }^{193,247,248}$

\section{PLWD Outcomes}

Evidence was insufficient to draw conclusions about the effects of cognitive therapy for PLWD. Five studies reported outcomes for PLWD. Four studies compared cognitive training interventions to usual care. ${ }^{189,193,247-249}$ One study compared cognitive training with an attention control. ${ }^{246}$ Table 5.12 summarizes the primary outcomes for PLWD. Two studies enrolling a total of 471 PLWD reported measures of quality of life and found no difference between groups at 3 months. ${ }^{193,247,248}$ Two studies enrolling a total of 404 PLWD reported measures of depression found no difference between groups from 3 to 6 months. ${ }^{193,246}$

Two studies enrolling a total of 197 PLWD with excessive eating, or hyperphagia, reported outcome measures of hyperphagic behavior. ${ }^{189,} 249$ Both studies found that hyperphagia behaviors improved in the cognitive training group versus usual care at 6 weeks. ${ }^{189,}{ }^{249}$ One study measured hyperphagia behavior up to 6 months post-intervention (30 weeks total), finding that this benefit was sustained in the cognitive training group. ${ }^{249}$ Both studies reported a variety of additional measures related to excessive eating. One study $(n=97)$ reported food intake, finding a reduction in average food intake for the cognitive training group versus usual care at 6 weeks. ${ }^{189}$ The other study ( $n=100)$ reported measures of short meal frequency, pica behavior, and change in eating habits from post-intervention (6 weeks) to 6-months post-intervention (30 weeks). ${ }^{249}$ Short-meal frequency improved in the cognitive training group versus the usual care group at all measured time points. ${ }^{249}$ Pica behavior improved in the cognitive training group immediately postintervention as well as at 1 and 3 months post-intervention, but the benefit was not sustained 6 months post-intervention. ${ }^{249}$ Groups did not differ for change in eating habits. ${ }^{249}$

One study ( $n=324)$ also reported measures of BPSD, function/ADLs, apathy, and dependence, and found no difference between groups at 3 months. ${ }^{193}$ Another study ( $\left.\mathrm{n}=80\right)$ reported a measure of anxiety and found no difference between groups at 3 and 6 months. ${ }^{246}$ Appendix D provides evidence tables, summary risk of bias assessments, and strength of evidence for key comparisons and outcomes.

Table 5.12. Summary of findings for PLWD outcomes: cognitive training

\begin{tabular}{|c|c|c|c|c|}
\hline $\begin{array}{l}\text { Outcome } \\
\text { Comparison }\end{array}$ & $\begin{array}{l}\text { \# Studies/Design } \\
\text { (n analyzed) } \\
\text { Timing }\end{array}$ & Population & Findings & $\begin{array}{l}\text { Strength of } \\
\text { Evidence* }\end{array}$ \\
\hline $\begin{array}{l}\text { Quality of Life } \\
\text { Cognitive training } \\
\text { vs usual care }\end{array}$ & $\begin{array}{l}2 \text { RCTs 193, 247, } 248 \\
(\mathrm{n}=471) \\
3 \text { months }\end{array}$ & $\begin{array}{l}\text { Mild to } \\
\text { moderate AD } \\
\text { or other } \\
\text { dementia }\end{array}$ & $\begin{array}{l}0 \text { of } 2 \text { found benefit } \\
2 \text { of } 2 \text { found no difference }\end{array}$ & Insufficient \\
\hline $\begin{array}{l}\text { Depression } \\
\text { Cognitive training } \\
\text { vs usual care }\end{array}$ & $\begin{array}{l}2 \text { RCTs }{ }^{193,246} \\
(\mathrm{n}=404) \\
3 \text { to } 6 \text { months }\end{array}$ & $\begin{array}{l}\text { Early-stage to } \\
\text { Moderate AD }\end{array}$ & $\begin{array}{l}0 \text { of } 2 \text { found benefit } \\
2 \text { of } 2 \text { found no difference }\end{array}$ & Insufficient \\
\hline $\begin{array}{l}\text { Hyperphagia } \\
\text { Behavior } \\
\text { Cognitive training } \\
\text { vs usual care }\end{array}$ & $\begin{array}{l}2 \text { RCTs } 189,249 \\
(n=197) \\
6 \text { to } 30 \text { weeks }\end{array}$ & $\begin{array}{l}\text { Dementia with } \\
\text { hyperphagia }\end{array}$ & $\begin{array}{l}2 \text { of } 2 \text { found benefit } \\
0 \text { of } 2 \text { found no difference }\end{array}$ & Insufficient \\
\hline
\end{tabular}




\begin{tabular}{|c|c|c|c|c|}
\hline $\begin{array}{l}\text { Outcome } \\
\text { Comparison }\end{array}$ & $\begin{array}{l}\text { \# Studies/Design } \\
\text { (n analyzed) } \\
\text { Timing }\end{array}$ & Population & Findings & $\begin{array}{l}\text { Strength of } \\
\text { Evidence* }\end{array}$ \\
\hline $\begin{array}{l}\text { Food Intake } \\
\text { Cognitive training } \\
\text { vs usual care }\end{array}$ & $\begin{array}{l}1 \mathrm{RCT}^{189}(\mathrm{n}=97) \\
6 \text { weeks }\end{array}$ & $\begin{array}{l}\text { Dementia with } \\
\text { hyperphagia }\end{array}$ & $\begin{array}{l}1 \text { found benefit } \\
0 \text { found no difference }\end{array}$ & Insufficient \\
\hline $\begin{array}{l}\text { Short meal } \\
\text { frequency } \\
\text { Cognitive training } \\
\text { vs usual care }\end{array}$ & $\begin{array}{l}1 \mathrm{RCT}^{249}(\mathrm{n}=100) \\
6 \text { to } 30 \text { weeks }\end{array}$ & $\begin{array}{l}\text { Dementia with } \\
\text { hyperphagia }\end{array}$ & $\begin{array}{l}1 \text { found benefit at all time points } \\
0 \text { found no difference }\end{array}$ & Insufficient \\
\hline $\begin{array}{l}\text { Pica behavior } \\
\text { Cognitive training } \\
\text { vs usual care }\end{array}$ & $\begin{array}{l}1 \text { RCT }^{249}(n=100) \\
6 \text { to } 30 \text { weeks }\end{array}$ & $\begin{array}{l}\text { Dementia with } \\
\text { hyperphagia }\end{array}$ & $\begin{array}{l}1 \text { found benefit favoring } \\
\text { intervention post-intervention, but } \\
\text { not sustained } 6 \text {-months post- } \\
\text { intervention } \\
0 \text { found no difference }\end{array}$ & Insufficient \\
\hline $\begin{array}{l}\text { Change in eating } \\
\text { habits } \\
\text { Cognitive training } \\
\text { vs usual care }\end{array}$ & $\begin{array}{l}1 \mathrm{RCT}^{249}(\mathrm{n}=100) \\
6 \text { to } 30 \text { weeks }\end{array}$ & $\begin{array}{l}\text { Dementia with } \\
\text { hyperphagia }\end{array}$ & $\begin{array}{l}0 \text { found benefit } \\
0 \text { found no difference }\end{array}$ & Insufficient \\
\hline $\begin{array}{l}\text { BPSD } \\
\text { Cognitive training } \\
\text { vs attention } \\
\text { control }\end{array}$ & $\begin{array}{l}1 \mathrm{RCT}(\mathrm{n}=324) \\
3 \text { months }\end{array}$ & $\begin{array}{l}\text { Mild to } \\
\text { moderate AD }\end{array}$ & $\begin{array}{l}0 \text { found benefit } \\
1 \text { found no difference }\end{array}$ & Insufficient \\
\hline $\begin{array}{l}\text { Function/ADLs } \\
\text { Cognitive training } \\
\text { vs attention } \\
\text { control }\end{array}$ & $\begin{array}{l}1 \mathrm{RCT}^{193}(\mathrm{n}=324) \\
3 \text { months }\end{array}$ & $\begin{array}{l}\text { Mild to } \\
\text { moderate AD }\end{array}$ & $\begin{array}{l}0 \text { found benefit } \\
1 \text { found no difference }\end{array}$ & Insufficient \\
\hline $\begin{array}{l}\text { Apathy } \\
\text { Cognitive training } \\
\text { vs attention } \\
\text { control }\end{array}$ & $\begin{array}{l}1 \mathrm{RCT}^{193}(\mathrm{n}=324) \\
3 \text { months }\end{array}$ & $\begin{array}{l}\text { Mild to } \\
\text { moderate AD }\end{array}$ & $\begin{array}{l}0 \text { found benefit } \\
1 \text { found no difference }\end{array}$ & Insufficient \\
\hline $\begin{array}{l}\text { Dependence } \\
\text { Cognitive training } \\
\text { vs attention } \\
\text { control }\end{array}$ & $\begin{array}{l}1 \mathrm{RCT}^{193}(\mathrm{n}=324) \\
3 \text { months }\end{array}$ & $\begin{array}{l}\text { Mild to } \\
\text { moderate AD }\end{array}$ & $\begin{array}{l}0 \text { found benefit } \\
1 \text { found no difference }\end{array}$ & Insufficient \\
\hline $\begin{array}{l}\text { Anxiety } \\
\text { Cognitive training } \\
\text { vs attention } \\
\text { control }\end{array}$ & $\begin{array}{l}1 \mathrm{RCT}^{246}(\mathrm{n}=80) \\
3 \text { and } 6 \text { months }\end{array}$ & Early-stage AD & $\begin{array}{l}0 \text { found benefit } \\
1 \text { found no difference }\end{array}$ & Insufficient \\
\hline
\end{tabular}

*Insufficient ratings due to few studies and imprecision in the findings.

Abbreviations: $\mathrm{AD}=\mathrm{Alzheimer}$ 's disease; $\mathrm{ADL}=$ activities of daily living; $\mathrm{BPSD}=$ =hehavioral and psychological symptoms of dementia; $n=$ number; PLWD=people living with dementia; RCT=randomized controlled trial

\section{Caregiver Outcomes}

Evidence was insufficient to draw conclusions about the effects of cognitive therapy for informal and formal caregivers of PLWD. Two studies reported outcomes for caregivers and compared cognitive training interventions compared with usual care. ${ }^{193,249}$ Table 5.13 summarizes primary outcomes for caregivers. One study $(n=324)$ reported a measure of caregiver distress for CG/P, and found no difference between groups at 3 months. ${ }^{193}$ The second study reported a measure of formal caregiver distress specific to PLWD hyperphagic behavior, and found benefit for formal caregivers of the cognitive training group versus the usual care group post-intervention (6 weeks). ${ }^{249}$ However, the benefit was not sustained at 6 months. ${ }^{249}$ 
Table 5.13. Summary of findings for caregiver outcomes: cognitive training

\begin{tabular}{|c|c|c|c|c|}
\hline $\begin{array}{l}\text { Outcome } \\
\text { Comparator }\end{array}$ & $\begin{array}{c}\text { \# Studies/Design } \\
\text { (n analyzed) } \\
\text { Timing }\end{array}$ & Population & Findings & $\begin{array}{c}\text { Strength } \\
\text { of } \\
\text { Evidence* }\end{array}$ \\
\hline $\begin{array}{l}\text { Caregiver } \\
\text { Distress } \\
\text { Cognitive training } \\
\text { vs usual care }\end{array}$ & $\begin{array}{l}1 \mathrm{RCT}^{193}(\mathrm{n}=324) \\
3 \text { months }\end{array}$ & $\begin{array}{l}\text { Informal } \\
\text { caregivers }\end{array}$ & $\begin{array}{l}0 \text { found benefit } \\
1 \text { found no difference }\end{array}$ & Insufficient \\
\hline $\begin{array}{l}\text { Caregiver } \\
\text { Distress, PLWD } \\
\text { hyperphagic } \\
\text { behavior } \\
\text { Cognitive training } \\
\text { vs usual care }\end{array}$ & $\begin{array}{l}1 \mathrm{RCT}^{249}(\mathrm{n}=\mathrm{NR}) \\
6 \text { to } 30 \text { weeks }\end{array}$ & $\begin{array}{l}\text { Formal } \\
\text { caregivers }\end{array}$ & $\begin{array}{l}1 \text { found benefit favoring intervention } \\
\text { group immediately post-intervention ( } 6 \\
\text { weeks); however, difference was not } \\
\text { sustained over the } 6 \text {-month period } \\
\text { post-intervention. }\end{array}$ & Insufficient \\
\hline
\end{tabular}

*Insufficient ratings due to few studies and imprecision in the findings.

Abbreviations: $n=$ number; NR=not reported; PLWD=people living with dementia; RCT=randomized controlled trial

\section{Variation in Outcomes}

Studies in the analytic set did not examine variation in outcomes by PLWD, caregiver, or intervention characteristics.

\section{Cognitive Stimulation Therapy}

\section{Key Point}

- Evidence was insufficient to draw conclusions about the effect of cognitive stimulation therapy (CST) for PLWD and their CG/P.

\section{Intervention Description}

Cognitive stimulation is an intervention for people with mild to moderate dementia that offers engagement in various activities and discussions (usually in a group) aimed at general enhancement of cognitive and social functioning. Activities and material are presented in a game-like manner rather than as schooling.

\section{Eligible Studies}

Table 5.14 summarizes the characteristics of the literature set. We identified 12 unique studies from 15 publications. ${ }^{44,212,262-272}$ One of these was a pilot study, four had small samples, and two were assessed as high risk of bias; these seven studies were not part of the analytic set but information about them is provided as part of the evidence map in Appendix D. One study $(n=201)$ was assessed as low risk of bias, ${ }^{262,} 264$ and the other $(n=236)$ was assessed as medium risk of bias. ${ }^{263}$ The first compared CST versus usual care. ${ }^{262,264}$ The second compared CST maintenance therapy versus withdrawal of CST (in both cases after an initial period of CST). ${ }^{263}$ Neither study reported caregiver outcomes. Appendix D provides evidence tables, summary risk of bias assessments, and strength of evidence for key comparisons and outcomes.

Table 5.14. Basic characteristics of literature set: cognitive stimulation therapy

\begin{tabular}{|l|l|}
\hline \multicolumn{1}{|c|}{ Characteristic } & \multicolumn{1}{c|}{ Information } \\
\hline Total studies & 12 studies \\
\hline Non-U.S. studies & 11 studies \\
\hline
\end{tabular}




\begin{tabular}{|l|l|}
\hline \multicolumn{1}{|c|}{ Characteristic } & \multicolumn{1}{c|}{ Information } \\
\hline Evidence map studies & $\begin{array}{l}1 \text { pilot study } \\
5 \text { small sample studies } \\
\end{array}$ \\
\hline Analytic set studies & 2 randomized controlled trials \\
\hline Risk of bias of analytic set & 1 low, 1 medium \\
\hline Number of PLWD in analytic set & 437 \\
\hline Dementia type/definition & Mild to moderate severity \\
\hline Caregiver type (number) & Not reported \\
\hline
\end{tabular}

Abbreviation: PLWD=people living with dementia

\section{Intervention Research Context}

The literature set remains mostly preliminary, using the NIH Stage Model, with pilot or small sample studies. One study reported a pilot component, ${ }^{264}$ but the other did not. One study in the literature set described itself as a pilot study to adapt CST to the Hong Kong context. ${ }^{273}$ Three studies had an active control, ${ }^{265,268,272}$ and another had both an active control and a usual care $\mathrm{arm}^{212}$ The remaining studies used usual care or some variation thereof as the control.

\section{PLWD Outcomes}

Evidence was insufficient to determine the effect of CST on quality of life, function, neuropsychiatric symptoms, and mood. Table 5.15 summarizes the primary outcomes for PLWD. Only one study addressed the effect of CST versus usual care on quality of life, cognition, neuropsychiatric symptoms, anxiety, and depression $\left(n=201\right.$ subjects analyzed). ${ }^{44}$ The other study took place after an initial session of CST, and examined the effect of CST maintenance therapy versus withdrawal of CST on quality of life, cognition, and neuropsychiatric symptoms. ${ }^{263}$

Table 5.15. Summary of findings for PLWD outcomes: cognitive stimulation therapy

\begin{tabular}{|c|c|c|c|c|}
\hline $\begin{array}{l}\text { Outcome } \\
\text { Comparison }\end{array}$ & $\begin{array}{c}\text { \# Studies/Design } \\
\text { (n analyzed) } \\
\text { Timing }\end{array}$ & Population & Findings & $\begin{array}{l}\text { Strength of } \\
\text { Evidence* }\end{array}$ \\
\hline $\begin{array}{l}\text { Quality of life } \\
\text { CST vs. usual care }\end{array}$ & $\begin{array}{l}1 \text { RCT }^{44} \\
(\mathrm{n}=201) \\
8 \text { weeks }\end{array}$ & PLWD & $\begin{array}{l}1 \text { found benefit } \\
0 \text { found no difference }\end{array}$ & Insufficient \\
\hline $\begin{array}{l}\text { Function } \\
\text { CST vs. usual care }\end{array}$ & $\begin{array}{l}1 \text { RCT }^{44} \\
(n=201) \\
8 \text { weeks }\end{array}$ & PLWD & $\begin{array}{l}0 \text { found benefit } \\
1 \text { found no difference }\end{array}$ & Insufficient \\
\hline $\begin{array}{l}\text { Neuropsychiatric symptoms } \\
\text { CST vs. usual care }\end{array}$ & $\begin{array}{l}1 \mathrm{RCT}^{44} \\
(\mathrm{n}=201) \\
8 \text { weeks }\end{array}$ & PLWD & $\begin{array}{l}0 \text { found benefit } \\
1 \text { found no difference }\end{array}$ & Insufficient \\
\hline $\begin{array}{l}\text { Anxiety } \\
\text { CST vs. usual care }\end{array}$ & $\begin{array}{l}1 \text { RCT }^{44} \\
(n=201) \\
8 \text { weeks }\end{array}$ & PLWD & $\begin{array}{l}0 \text { found benefit } \\
1 \text { found no difference }\end{array}$ & Insufficient \\
\hline $\begin{array}{l}\text { Depression } \\
\text { CST vs. usual care }\end{array}$ & $\begin{array}{l}1 \mathrm{RCT}^{44} \\
(\mathrm{n}=201) \\
8 \text { weeks }\end{array}$ & PLWD & $\begin{array}{l}0 \text { found benefit } \\
1 \text { found no difference }\end{array}$ & Insufficient \\
\hline $\begin{array}{l}\text { Quality of life } \\
\text { CST maintenance therapy } \\
\text { after initial CST vS. } \\
\text { withdrawal of CST after initial } \\
\text { CST }\end{array}$ & $\begin{array}{l}1 \mathrm{RCT}^{263} \\
(\mathrm{n}=236) \\
12 \text { weeks } \\
24 \text { weeks }\end{array}$ & PLWD & $\begin{array}{l}1 \text { found benefit } \\
0 \text { found no difference }\end{array}$ & Insufficient \\
\hline
\end{tabular}




\begin{tabular}{|c|c|c|c|c|}
\hline $\begin{array}{l}\text { Outcome } \\
\text { Comparison }\end{array}$ & $\begin{array}{l}\text { \# Studies/Design } \\
\text { (n analyzed) } \\
\text { Timing }\end{array}$ & Population & Findings & $\begin{array}{l}\text { Strength of } \\
\text { Evidence* }\end{array}$ \\
\hline $\begin{array}{l}\text { Function } \\
\text { CST maintenance therapy } \\
\text { after initial CST vs. } \\
\text { withdrawal of CST after initial } \\
\text { CST }\end{array}$ & $\begin{array}{l}1 \mathrm{RCT}^{263} \\
(\mathrm{n}=236) \\
12 \text { weeks } \\
24 \text { weeks }\end{array}$ & PLWD & $\begin{array}{l}1 \text { found benefit } \\
0 \text { found no difference }\end{array}$ & Insufficient \\
\hline $\begin{array}{l}\text { Neuropsychiatric symptoms } \\
\text { CST maintenance therapy } \\
\text { after initial CST vs. } \\
\text { withdrawal of CST after initial } \\
\text { CST }\end{array}$ & $\begin{array}{l}1 \mathrm{RCT}^{263} \\
(\mathrm{n}=236) \\
12 \text { weeks } \\
24 \text { weeks }\end{array}$ & PLWD & $\begin{array}{l}1 \text { found benefit } \\
0 \text { found no difference }\end{array}$ & Insufficient \\
\hline
\end{tabular}

*Insufficient ratings due to few studies and imprecision in the findings.

Abbreviations: $\mathrm{CST}=$ cognitive stimulation therapy; $\mathrm{n}=$ number; PLWD=people living with dementia: $\mathrm{RCT}=$ randomized controlled trial

\section{Caregiver Outcomes}

No studies reported caregiver outcomes.

\section{Variation in Outcomes}

One study reported that women appeared to benefit more than men for quality of life, neuropsychiatric symptoms, and function. The study did not report the magnitude of the differences, nor did it report p-values. ${ }^{264}$ Neither study reported variation in outcomes by caregiver or intervention characteristics.

\section{Recreation Therapy}

\section{Key Point}

- Evidence was insufficient to draw conclusions about recreation therapy interventions for PLWD or their CG/Ps.

\section{Intervention Description}

Recreation therapy interventions may involve art, games, music, or exercise. They are usually structured and facilitated, and may be conducted in groups or individually. Most of these interventions aim to involve participants actively in the chosen activity, rather than letting them participate passively (e.g. listen to music or read).

\section{Eligible Studies}

Table 5.16 summarizes the effect of recreation therapy for PLWD. We identified 14 unique studies from 14 publications that examined the effect of recreation therapy for PLWD. Three studies were assessed as low or medium risk of bias and included in the analytic set. All studies were randomized controlled trials (RCTs; $n=552$ ), ${ }^{274-276}$ and all used usual care as the comparator. The studies were conducted in the United States, Australia, and Italy.. All pilot, small sample, and high-risk-or-bias studies are described as part of the evidence map in Appendix D. ${ }^{276-287}$ An evidence table, summary of risk of bias assessments, and strength of evidence is provided in Appendix D. 
Table 5.16. Basic characteristics of literature set: recreational therapy

\begin{tabular}{|l|l|}
\hline \multicolumn{1}{|c|}{ Characteristic } & \multicolumn{1}{c|}{ Information } \\
\hline Total unique studies & 14 studies \\
\hline Non-U.S. studies & 11 studies \\
\hline Total Evidence map studies & 1 pilot study \\
& 5 small sample studies \\
5 high risk of bias studies
\end{tabular}

Abbreviation: PLWD=people living with dementia

\section{Intervention Research Context}

The literature for recreational therapy interventions is still quite preliminary. Using the NIH Stage Model, large majority of studies were assessed as pilot and small sample studies. The largest study enrolled 389 PLWD. All but two studies were conducted outside of the United States. We did not see evolution of a particular intervention over time; instead, each study tested a different recreational therapy approach. Two studies involved activity sessions conducted one- - on-one with an aide, a wheelchair tandem bicycle ride, ${ }^{277}$ and individualized activities such as singing or working with clay. ${ }^{288}$ The remainder involved larger group activities. The studies involved a diverse set of activities, and some studies involved more than one type of activity. One involved some form of group sporting activity. ${ }^{277,}{ }^{279}$ Four involved different types of games: GO, ${ }^{281}$ chess, ${ }^{286}$ dhakonan, ${ }^{287}$ or party games. ${ }^{285}$ One based activities on Chinese folk art, music, and games. ${ }^{280}$ Three involved art in some form, such as singing in a choir or visits to art museum plus art-making. ${ }^{278,280,} 283$ Of the evidence map studies, only four used usual care as a comparator. ${ }^{277,279-281}$

\section{PLWD Outcomes}

Evidence was insufficient to draw conclusions about PLWD outcomes, including neuropsychological symptoms, depression, agitation, social function, physical function, quality of life, and antipsychotic medication use. Table 5.17 provides a summary of findings. For the most part, each outcome was only covered in one study.

Two studies $(n=552)$ compared neuropsychiatric symptoms using the NPI, with one finding a benefit and the other showing no difference. The studies also reported markedly different baseline levels of neuropsychiatric symptoms. ${ }^{274,275}$ Two other studies $(n=517){ }^{274,276}$ compared agitation; neither found a difference.

At both 13 and 26 weeks, the humor therapy study $(n=389)$ reported quality of life, depression and agitation symptoms, and social function, and found no difference between groups at either time point. ${ }^{274}$

The indoor garden study ( $\mathrm{n}=163$ ) reported a small but statistically insignificant benefit for physical function. However, the study reported a statistically significant reduction in antipsychotic use at 24 weeks. ${ }^{275}$ 
Table 5.17. Summary of findings for PLWD outcomes: recreational therapy

\begin{tabular}{|c|c|c|c|c|}
\hline Outcome Comparison & $\begin{array}{c}\text { \# Studies/Design } \\
\text { (n=analyzed) } \\
\text { Timing }\end{array}$ & Population & Findings & $\begin{array}{l}\text { Strength of } \\
\text { Evidence* }^{*}\end{array}$ \\
\hline $\begin{array}{l}\text { Neuropsychiatric } \\
\text { symptoms } \\
\text { Recreational therapy } \\
\text { vs. usual care }\end{array}$ & $\begin{array}{l}2 \text { RCTs } 274,275 \\
(n=552) \\
24-26 \text { weeks }\end{array}$ & $\begin{array}{l}\text { Dementia type } \\
\text { and severity not } \\
\text { reported }\end{array}$ & $\begin{array}{l}1 \text { of } 2 \text { found benefit } \\
1 \text { of } 2 \text { found no difference }\end{array}$ & Insufficient \\
\hline $\begin{array}{l}\text { Depression } \\
\text { Recreational therapy } \\
\text { vs. usual care }\end{array}$ & $\begin{array}{l}1 \text { RCT } 274 \\
(n=389) \\
13 \text { and } 26 \text { weeks }\end{array}$ & $\begin{array}{l}\text { Dementia type } \\
\text { and severity not } \\
\text { reported }\end{array}$ & $\begin{array}{l}1 \text { found benefit } \\
1 \text { found no difference }\end{array}$ & Insufficient \\
\hline $\begin{array}{l}\text { Agitation } \\
\text { Recreational therapy } \\
\text { vs. usual care }\end{array}$ & $\begin{array}{l}2 \text { RCTs } 274,276 \\
(\mathrm{n}=517) \\
\text { Up to } 13 \text { weeks }\end{array}$ & $\begin{array}{l}\text { Dementia type } \\
\text { and severity not } \\
\text { reported }\end{array}$ & $\begin{array}{l}0 \text { found benefit } \\
2 \text { no difference }\end{array}$ & Insufficient \\
\hline $\begin{array}{l}\text { Function, social } \\
\text { Recreational therapy } \\
\text { vs. usual care }\end{array}$ & $\begin{array}{l}1 \mathrm{RCT} 274 \\
(\mathrm{n}=389) \\
13 \text { and } 26 \text { weeks }\end{array}$ & $\begin{array}{l}\text { Dementia type } \\
\text { and severity not } \\
\text { reported }\end{array}$ & $\begin{array}{l}0 \text { found benefit } \\
1 \text { found no difference }\end{array}$ & Insufficient \\
\hline $\begin{array}{l}\text { Function, physical } \\
\text { Recreational therapy } \\
\text { vs. usual care }\end{array}$ & $\begin{array}{l}1 \mathrm{RCT}^{275} \\
(\mathrm{n}=163) \\
24 \text { weeks }\end{array}$ & $\begin{array}{l}\text { Dementia type } \\
\text { and severity not } \\
\text { reported }\end{array}$ & $\begin{array}{l}1 \text { found benefit } \\
0 \text { found no difference }\end{array}$ & Insufficient \\
\hline $\begin{array}{l}\text { Quality of life } \\
\text { Recreational therapy } \\
\text { vs. usual care }\end{array}$ & $\begin{array}{l}1 \mathrm{RCT} 274 \\
(\mathrm{n}=389) \\
13 \text { and } 26 \text { weeks }\end{array}$ & $\begin{array}{l}\text { Dementia type } \\
\text { and severity not } \\
\text { reported }\end{array}$ & $\begin{array}{l}0 \text { found benefit } \\
1 \text { found no difference }\end{array}$ & Insufficient \\
\hline $\begin{array}{l}\text { Antipsychotic use } \\
\text { Recreational therapy } \\
\text { vs. usual care }\end{array}$ & $\begin{array}{l}1 \mathrm{RCT}^{275} \\
(\mathrm{n}=163) \\
24 \text { weeks }\end{array}$ & $\begin{array}{l}\text { Dementia type } \\
\text { and severity not } \\
\text { reported }\end{array}$ & $\begin{array}{l}1 \text { found benefit } \\
0 \text { found no difference }\end{array}$ & Insufficient \\
\hline
\end{tabular}

*Insufficient ratings due to few studies and imprecision in the findings.

Abbreviations: $\mathrm{n}=$ number; PLWD=person/people living with dementia; RCT=randomized controlled trial

\section{Caregiver Outcomes}

No studies in the analytic set reported caregiver outcomes.

\section{Variation in Outcomes}

No studies in the analytic set reported any variation in outcomes.

\section{Psychosocial Interventions for PLWD Well-Being}

\section{Key Point}

- Studies of psychosocial interventions to improve PLWD well-being were described in the evidence map but not considered for analysis due to limitations in study designs.

\section{Intervention Description}

Psychosocial interventions include components such as group counseling, use of an assigned nursing home resident buddy, or approaches to improve effective communication in order to help psychosocial and behavioral aspects by improving communication, memory, and interpersonal skills among PLWD.

\section{Eligible Studies}

We identified seven unique studies from eight publications that examined various psychosocial session interventions among PLWD. ${ }^{289-296}$ (Table 5.18) All studies were either 
pilots or small sample studies, and we provide information about them in the evidence map in Appendix D.

Table 5.18. Basic characteristics of literature set: psychosocial interventions for PLWD

\begin{tabular}{|l|l|}
\hline \multicolumn{1}{|c|}{ Characteristics } & \multicolumn{1}{c|}{ Information } \\
\hline Total unique studies & 7 studies \\
\hline Non-U.S. studies & 4 studies \\
\hline Evidence map studies & 1 pilot study \\
& 4 small sample studies \\
\hline Analytic set studies & 2 high risk of bias studies \\
\hline
\end{tabular}

Abbreviation: PLWD=person/people living with dementia

\section{Intervention Research Context}

The research is largely preliminary with small sample or pilot trials. Psychosocial session topics included psychodynamic interpersonal therapy for community-dwelling PLWD in the United Kingdom, ${ }^{289}$ using noncognitively impaired nursing home residents as "buddies" for PLWD in the United States, ${ }^{290}$ a self-management group intervention for PLWD in the early stages of dementia in the United Kingdom, ${ }^{291,} 292$ therapeutic conversation counseling sessions for community-dwelling PLWD in the United States, ${ }^{293}$ and peer support group sessions for community-dwelling PLWD in Hong Kong. ${ }^{294}$ Lastly, two studies examined the use of reality orientation therapy among PLWD in the United States in $1997^{295}$ and Italy in $2005 .^{296}$

\section{Creative Expression Therapy}

\section{Key Point}

- Studies to assess the effect of creative expression therapy were described in the evidence map but not considered for analysis due to limitations in study designs.

\section{Intervention Description}

Creative expression programs are emerging interventions targeted at improving quality of care and life for PLWD in long-term care facilities. Creative expression interventions, such as storytelling or theatrical improvisation, do not require PLWD to access or use memories. They instead focus on creativity in the moment.

\section{Eligible Studies}

We identified five unique studies from five publications that examined creative expression interventions for PLWD and formal caregivers. (Table 5.19) Three studies were assessed as high risk of bias and two were pilot studies; all five were therefore excluded from the analytic set. We present information on all studies as part of the evidence map in Appendix D.

Table 5.19. Basic characteristics of literature set: creative expression therapy

\begin{tabular}{|l|l|}
\hline \multicolumn{1}{|c|}{ Characteristics } & \multicolumn{1}{c|}{ Information } \\
\hline Total unique studies & 5 studies \\
\hline Non-U.S. studies & 2 studies \\
\hline Evidence map studies & 2 pilot studies \\
& 3 high risk of bias studies \\
\hline Analytic set studies & 0 \\
\hline
\end{tabular}




\section{Intervention Research Context}

Four interventions examined “TimeSlips (TS)," a group storytelling method, ${ }^{297-300}$ while one study investigated the "Veder Method" which integrates theater improvisation techniques with elements from other communication methods. ${ }^{301}$ These interventions are relatively new, and the research is emerging (published between 2009 and 2019). Three studies were conducted in the United States, ${ }^{297,}$ 298, 300 and the other two were conducted in China ${ }^{299}$ and the Netherlands. ${ }^{301}$ All five studies focused mainly on the outcomes of behavior, mood, and quality of life for PLWD, although one study examined the caregiver outcomes of job satisfaction, attitude towards residents, and burnout. ${ }^{297}$

\section{Multicomponent Interventions for PLWD Well-Being}

\section{Key Point}

- Evidence is insufficient to draw conclusion about the effects of multicomponent care interventions in PLWD or their caregivers.

\section{About the Interventions}

The multicomponent studies in this set are largely unrelated. Earlier in this chapter we reported on another multicomponent intervention, CST. Since CST had a relatively distinct literature set, we chose to report it in a separate section.

Multicomponent interventions aimed at improving PLWD include a combination of components used simultaneously to support cognitive function, quality of life, and other health outcomes for PLWD. Intervention components may include cognitive and/or motor stimulation, physical activity using daily living activities, strategies adapted from cognitive training and neurorehabilitation, reminiscence therapy, and reality orientation, and exposure to bright light. Multicomponent interventions are intended to improve PLWD outcomes such as functional ability (to engage in activities of daily living) and depression. Multicomponent may or may not use special personnel such as nurses, aides, and therapists, and are delivered in varied settings including the PLWD's home, day care centers, and nursing homes.

\section{Eligible Studies}

Table 5.20 summarizes the characteristics of the literature set. We identified 24 unique studies from 39 publications. Three studies were assessed as low to medium risk of bias and were included in the analytic set. Multicomponent interventions targeting quality of life, function, and/or non-BPSD symptoms in PLWD included three unique studies across four publications. One study examined an intervention in Germany including motor stimulation, activities of daily living, and cognitive stimulation components, delivered by two therapists, one aide, and nurses when necessary. ${ }^{302,303}$ One study examined walking, light therapy, and combination exercise, light therapy, and education in the United. States. ${ }^{304}$ One Japanese study examined reminiscence and reality orientation therapy care methods, in addition to a routine daycare service. ${ }^{305}$ Excluded from the analytic set are 21 pilot, small sample, and high risk of bias studies and are described as part of the evidence map in Appendix D. ${ }^{44,}$ 94, 114, 116, 303, 306-324 Appendix D provides an evidence table, summary of risk of bias assessments, and strength of evidence for the analytic set. 
Table 5.20. Basic characteristics of literature set: multicomponent for PLWD well-being

\begin{tabular}{|l|l|}
\hline \multicolumn{1}{|c|}{ Characteristics } & \multicolumn{1}{c|}{ Information } \\
\hline Total unique studies & 24 studies \\
\hline Non-U.S. studies & 14 studies \\
\hline Evidence map studies & 3 pilot studies \\
& 7 small sample studies \\
& 11 high risk of bias \\
\hline Analytic set studies & 3 randomized controlled trials \\
\hline Risk of bias of analytic set & Medium \\
\hline Number of PLWD in analytic set & 333 \\
\hline Dementia type/definition & Varied across studies \\
\hline Caregiver type (number) & NA \\
\hline
\end{tabular}

Abbreviations: NA=not available; PLWD=people living with dementia

\section{Intervention Research Context}

Evidence map studies published between 2004 and 2020 tended to be largely preliminary or early Stage 3 of the NIH Stage Model. Studies in the analytic set were likewise explanatory in nature and as a whole, they did not exhibit much progression along the NIH Stage Model. Study duration ranged from 6 weeks to 18 months, targeted highly varied PLWD populations, and focused primarily on PLWD with mild and moderate dementia residing in various communitybased residences and nursing homes. Two of the trials targeting PLWD with mild to moderate dementia focused on an intervention for motor stimulation, activities of daily living, and cognitive stimulation. ${ }^{302,325}$ These trials represented the most pragmatic approach to multicomponent interventions for PLWD health outcomes.

\section{PLWD Outcomes}

Evidence was insufficient for drawing conclusions about the effects of multicomponent interventions for PLWD well-being. The findings are summarized in Table 5.21. One study reported small benefit for PLWD quality of life (social behavior), cognitive function, and instrumental activity of daily living for PLWD with mild to moderate dementia living in nursing homes. ${ }^{302,303}$ One study reported a small reduction of sleep disturbances. ${ }^{304}$ and the other reported small improvements in overall cognitive and social function. ${ }^{305}$

Table 5.21 Summary of findings for PLWD outcomes: multicomponent for PLWD well-being

\begin{tabular}{|c|c|c|c|c|}
\hline $\begin{array}{l}\text { Outcome } \\
\text { Comparison }\end{array}$ & $\begin{array}{l}\text { \# Studies/Design } \\
\text { ( } \mathrm{n=analyzed)} \\
\text { Timing }\end{array}$ & Population & Findings & $\begin{array}{l}\text { Strength of } \\
\text { Evidence* }^{*}\end{array}$ \\
\hline $\begin{array}{l}\text { Instrumental Activities of Daily } \\
\text { Living } \\
\text { Multicomponent: motor } \\
\text { stimulation, activities of daily } \\
\text { living, and cognitive stimulation } \\
\text { components contact control }\end{array}$ & $\begin{array}{l}1 \text { RCT } 302,303 \\
\mathrm{n}=98 \\
6 \text { months }\end{array}$ & ADRD & $\begin{array}{l}1 \text { found benefit } \\
0 \text { found no difference }\end{array}$ & Insufficient \\
\hline $\begin{array}{l}\text { Sleep } \\
\text { Walking, light, combination } \\
\text { treatment versus contact control }\end{array}$ & $\begin{array}{l}1 \text { RCT } 304 \\
n=66 \\
6 \text { months }\end{array}$ & $\begin{array}{l}\text { People with } \\
\text { AD } \\
\text { and their in- } \\
\text { home } \\
\text { caregivers. }\end{array}$ & $\begin{array}{l}0 \text { found benefit } \\
1 \text { found no difference. }\end{array}$ & Insufficient \\
\hline $\begin{array}{l}\text { Cognitive and Social Function } \\
\text { Reminiscence and reality } \\
\text { orientation care methods, } \\
\text { routine day-care service versus } \\
\text { usual care }\end{array}$ & $\begin{array}{l}1 \mathrm{RCT} 305 \\
\mathrm{n}=60 \\
10 \text { weeks }\end{array}$ & $\begin{array}{l}\text { Community- } \\
\text { dwelling } \\
\text { older PLWD. }\end{array}$ & $\begin{array}{l}1 \text { found benefit } \\
0 \text { found no difference }\end{array}$ & Insufficient \\
\hline
\end{tabular}


*Insufficient ratings due to few studies and imprecision in the findings.

Abbreviations: $\mathrm{AD}=$ Alzheimer's disease; $\mathrm{ADRD}=$ Alzheimer's disease-related dementias; $\mathrm{n}=$ number; PLWD=people living with dementia; RCT=randomized controlled trial

\section{Caregiver Outcomes}

Where studies may have included caregiver outcomes, these lacked sufficient or clear reporting for caregiver outcomes.

\section{Variation in Outcomes}

Studies did not report variation in PLWD outcomes by PLWD characteristics, caregiver characteristics, or setting.

\section{Assistive Technology}

We classified as assistive technology interventions include technology that controls house functions such as lights and/or systems that alert caregivers when a PLWD is at risk.

Additionally, these interventions evaluated caregiver outcomes such as job satisfaction, relieving worry, and sleep quality.

\section{Key Point}

- Studies of assistive technology interventions were described in the evidence map but not considered for analysis due to limitations in study designs.

\section{Eligible Studies}

We identified four unique studies from four publications that examined the use of assistive technology for PLWD and informal/formal caregivers. (Table 5.22) All included studies were pilot studies. Information on all studies is provided as part of the evidence map in Appendix D. ${ }^{320,326-329}$

Table 5.22. Basic characteristics of literature set: assistive home technology

\begin{tabular}{|l|l|}
\hline \multicolumn{1}{|c|}{ Characteristics } & \multicolumn{1}{c|}{ Information } \\
\hline Total unique studies & 4 studies \\
\hline Non-U.S. studies & 2 studies \\
\hline Evidence map studies & 4 pilot studies \\
\hline Analytic set studies & 0 \\
\hline
\end{tabular}

\section{Intervention Research Context}

Assistive technology interventions identified are relatively novel and thus these studies were preliminary in design, spanning nine years from 2010 to 2019. Intervention topics include the effectiveness of implementation of assistive technology in group homes in Amsterdam on the quality of life of PLWD and on the job satisfaction of caregivers. ${ }^{326}$ The effectiveness of homebased technologies coupled with teleassistance service was also assessed in order to prevent indoor falls of individuals with dementia in France. ${ }^{328}$ Additionally, a home monitoring system designed to track the movements of PLWD was assessed to see if it would relieve worry and improve sleep in caregivers in the United States. ${ }^{330}$ A second U.S. study examined wearable technology to alert caregivers of activities, generating reports of activity patterns over time. ${ }^{329}$ 


\section{Electrostimulation}

\section{Key Point}

- Studies of electrostimulation were described in the evidence map but not considered for analysis due to limitations in study designs.

\section{Intervention Description}

Electrostimulation interventions encompass a variety of techniques using either electric current or electromagnetic pulses of different intensity in various of parts of brain to improve cognitive and behavioral functioning for PLWD.

\section{Eligible Studies}

We identified 14 unique studies from 15 publications that examined electrostimulation among PLWD. (Table 5.23) All studies were either pilots or had small sample sizes. ${ }^{50,320,326-328,}$ 331-343 We present information on all studies as part of the evidence map in Appendix D.

Table 5.23. Basic characteristics of literature set: electrostimulation

\begin{tabular}{|l|l|}
\hline \multicolumn{1}{|c|}{ Characteristics } & \multicolumn{1}{c|}{ Information } \\
\hline Total unique studies & 14 studies \\
\hline Non-U.S. studies & 12 studies \\
\hline Evidence map studies & 5 pilot \\
& 9 small sample studies \\
\hline Analytic set studies & 0 \\
\hline
\end{tabular}

\section{Intervention Research Context}

The research is early in development and based on small sample sizes, thus preliminary. Nursing home or other long-term care facilities were the most common settings. Non-U.S. locations included Brazil, Canada, China, Egypt, Japan, Italy, The Netherlands, and Spain. Electrostimulation topics are highly technical neuroscience research. Please refer to the evidence map for more details. ${ }^{331-345}$

\section{Other Interventions for PLWD Well-Being}

\section{Key Point}

- No studies of other care interventions for PLWD well-being advanced from the evidence map to further analysis.

\section{Intervention Description}

This section encompasses a wide range of interventions. These included passive exercise (movement therapy or finger movement performed by a formal caregiver on PLWD), suicide prevention programs to pain assessments to verbal cues, and handfeeding techniques to help improve various (physical, mental, cognitive and behavioral) aspects of functioning.

\section{Eligible Studies}

We identified seven unique studies from eight publications that examined the various interventions among PLWD. ${ }^{133,302,325,346-352}$ (Table 5.24) All the studies were either pilot or small sample studies. We present information on all studies in the evidence map in Appendix D. 
Table 5.24. Basic characteristics of literature set: other interventions for BPSD

\begin{tabular}{|l|l|}
\hline \multicolumn{1}{|c|}{ Characteristics } & \multicolumn{1}{|c|}{ Information } \\
\hline Total unique studies & 7 studies \\
\hline Non-U.S. studies & 4 studies \\
\hline Evidence map studies & 4 pilot \\
& 1 small sample studies \\
\hline Analytic set studies & 1 high risk of bias \\
\hline
\end{tabular}

Abbreviation: BPSD=behavioral and psychological symptoms of dementia

\section{Intervention Research Context}

This research is preliminary. Publications included a suicide prevention program tested in South Korea for older adults with early-stage dementia, ${ }^{351}$ pain assessment in Norwegian nursing homes, ${ }^{352}$ passive finger movement exercise to improve grip strength in Chinese PLWD in residential facilities, ${ }^{133}$ various handfeeding techniques in PLWD with advanced dementia in the United States, ${ }^{346}$ and passive movement therapy for severe paratonia, or muscle stiffness, in latestage dementia. ${ }^{349}$ One study from 1997 examined directed verbal prompts and positive reinforcements for eating independence in PLWD in U.S. nursing homes. ${ }^{347}$

\section{Conclusion}

We found 223 unique studies that we grouped into 13 categories of care interventions aimed at improving PLWD quality of life and well-being. The large majority of studies, 85 percent, were conducted in non-U.S. settings. Eighty-five percent of the studies were pilots or small sample studies, Stage 0 to 2 according to the NIH Stage Model, or assessed as high risk of bias. Of those interventions with an analytic set of low to moderate risk of bias studies, we found the uncertainty of the evidence was too high to draw conclusions. Challenges with clear definitions of classes of interventions, and variability both in outcomes and how they were measured, hampered our ability to combine studies. Loss of PLWD participants after study enrollment was a frequent cause of increased risk of bias. However, evidence that is insufficient does not mean that none of the individual interventions described are potentially useful for individual PLWD or their caregivers. It simply means the uncertainty of the evidence is too high for us to draw conclusions, at present. 


\section{Chapter 6. Care Interventions for Informal Caregivers}

This chapter includes care interventions for informal caregivers, with the goal of improving their quality of life and well-being. While the large majority of studies use the term "informal caregiver," the term care partner is also occasionally present; we use the term caregiver/partner, or CG/P. The interventions include psychosocial interventions, social support, lifestyle interventions, respite care, multicomponent interventions, and other interventions that did not fall into these previous categories. We also present in this chapter interventions that target dyads of people living with dementia (PLWD) and their CG/P.

For each intervention, we present Key Points followed by results in three general sections: Intervention Description, Eligible Studies, and Intervention Research Context. For interventions with no studies assessed as low to medium risk of bias, we present the studies from the evidence map with a brief discussion of what has been examined and research context. For interventions for which low- to medium-risk-of-bias studies were available for an analytic set, we present Outcomes sections by PLWD, by caregiver, and by variation in outcomes when available.

We present a qualitative synthesis of the findings, because differences in outcome measures and intervention complexity prohibited the pooling of outcomes for a statistical analysis, such as a meta-analysis. We present summary findings as brief statements of how many studies reported statistically significant benefits or no difference between the intervention and comparator. Effect sizes and the data to calculate them were rarely reported in this literature. In studies where an effect size was reported, we present the study's originally reported effect size where it may be helpful for interpreting the study's findings. We present detailed information on eligible studies in Appendix E.

\section{Psychosocial Interventions To Support Informal Caregiver Well-Being}

\section{Key Point}

- For both PLWD and CG/Ps, evidence is insufficient to draw conclusions about the effects of psychosocial interventions targeting CG/P.

- For CG/P, we found no studies that assessed harms for psychosocial interventions targeting CG/P well-being.

\section{Intervention Descriptions}

As an umbrella term, we use "psychosocial interventions" to capture a wide range of psychoeducational and psychotherapeutic interventions that seek to improve the well-being of caregivers. Newly formulated conceptual definitions for psychoeducational and psychotherapeutic caregiver interventions do exist. However, overlap in intervention components made it impossible for us to use these newer definitions to categorize our eligible interventions. ${ }^{353}$ Figure 6.1 provides a visual representation of the overlap of intervention components drawn from the analytic set (for which results are provided below). No clear pattern distinguished interventions as either psychoeducational or psychotherapeutic. 
Figure 6.1. Collaboration network visualization of CG/P psychosocial intervention components

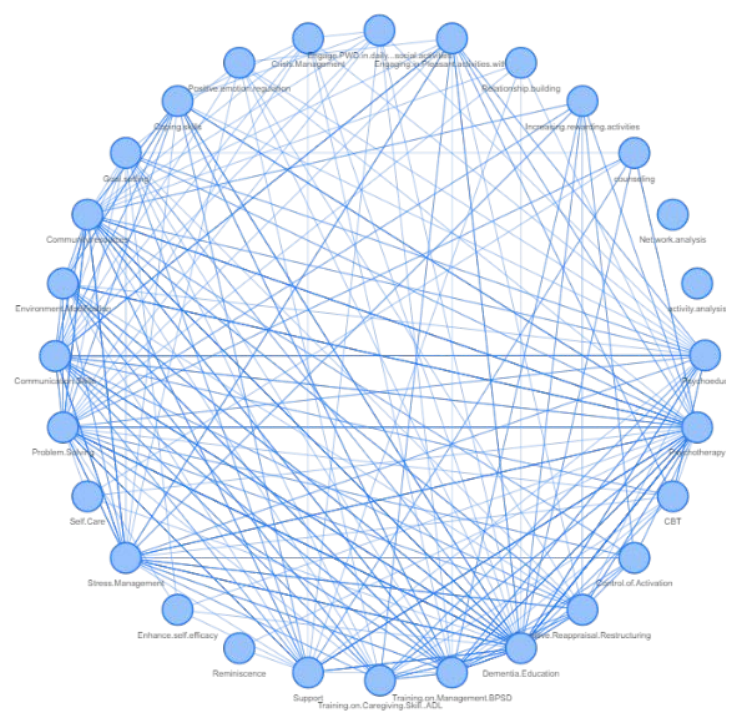

Abbreviations: BPSD=behavioral and psychological symptoms of dementia; $\mathrm{CBT}=$ Cognitive Behavioral Therapy; $\mathrm{CG} / \mathrm{P}=$ caregiver/care partner; $\mathrm{PLWD}=$ person/people living with dementia

We also could not categorize interventions based on duration, dose, delivery elements, or the interventionists. In almost all of the studies, interventionists were highly trained healthcare professionals or graduate level trainees, such as psychologists, social workers, registered nurses, occupational therapists, and licensed therapists. Interventions lasted anywhere from 6 weeks to 10 months, and participants spent anywhere from 5.5 to 32 hours receiving education, skills training, or counseling. Interventions were delivered to groups, individuals, and caregiver/ PLWD dyads. Most sessions occurred in person, a few by phone, and one online. We summarize intervention components, interventionists, duration, dose, and delivery format in Appendix E.

\section{Eligible Studies}

Table 6.1 summarizes the characteristics of the literature set on psychosocial interventions for CG/P. We identified 122 unique studies from 161 publications. Excluded from the analytic set are 46 pilot and small sample studies and 47 studies assessed as high risk of bias. The analytic dataset includes 29 studies with medium to low risk of bias, and represents 5054 caregiver/ PLWD dyads. ${ }^{354-381}$ The majority of PLWD had mild to moderate dementia; however, studies infrequently reported dementia severity. We provide information on all pilot studies and high risk of bias studies as part of the evidence map in Appendix E. ${ }^{382-476}$ Appendix E also provides evidence tables, summary risk of bias assessments, and strength of evidence for key comparisons and outcomes.

Table 6.1. Basic characteristics of literature set: psychosocial interventions for CG/P

\begin{tabular}{|l|l|}
\hline \multicolumn{1}{|c|}{ Characteristic } & \multicolumn{1}{|c|}{ Information } \\
\hline Total unique studies & 122 studies \\
\hline Non-U.S. studies & 74 studies (17 analytic set non-U.S.) \\
\hline
\end{tabular}




\begin{tabular}{|l|l|}
\hline \multicolumn{1}{|c|}{ Characteristic } & \multicolumn{1}{c|}{ Information } \\
\hline Evidence map studies & 28 pilot studies \\
& 18 small sample studies \\
& 47 high risk of bias studies \\
\hline Analytic set studies & 27 randomized controlled trials \\
& 1 cluster randomized controlled trial \\
\hline Risk of bias of analytic set & 29 medium/low \\
\hline Number of PLWD in analytic set & 5054 \\
\hline Dementia type & Predominantly mild to moderate dementia, commonly not defined \\
\hline Caregiver type (number) & Informal caregivers (4952) \\
\hline
\end{tabular}

Abbreviations: $\mathrm{CG} / \mathrm{P}=$ caregiver/care partner; $\mathrm{PLWD}=$ people living with dementia

\section{Intervention Research Context}

Using the National Institutes of Health (NIH) Stage Model, about 40 percent of the studies were categorized as pilot studies and the rest were categorized as explanatory, or Stage 3. Most of the randomized controlled trials had high risk of bias. All of the 28 medium to low risk of bias studies in the analytic dataset were categorized as explanatory studies using the NIH Stage Model. None were categorized as a pragmatic trial, or Stage 4. All but two of the studies occurred in high-resource countries including the United States, the United Kingdom, France, Hong Kong, Finland, Spain, Germany, Denmark, Norway, and the Netherlands. These interventions tended to be costly and resource intensive. Two smaller studies occurred in Peru and Iran, low-resource countries. The Peruvian intervention was a modification of the 10/66 Helping Carer's to Care program from India that was designed for diverse low to middle income countries with limited health and social care resources. The Iranian intervention promoted resilience training. Few studies investigated diverse caregivers (three investigated Latino caregivers, and one investigated African American caregivers). About half of the studies used attention control groups of generic psychoeducational programs, reminiscence, or support. A single comparative effectiveness study compared two cognitive behavioral therapy programs, one conducted by phone and one in person.

Modifications of the REACH Palo Alto Coping with Caregiving program were investigated in several countries including the United States, ${ }^{371,372}$ the United Kingdom, ${ }^{363}$ Spain, ${ }^{375} 364$ and Hong Kong. ${ }^{354}$ Over time, the Coping with Caregiving group program ${ }^{371}$ was adapted for individual sessions occurring in the caregiver's home or by phone and for shorter sessions.

A research program of occupational therapy interventions were also investigated in the United States, ${ }^{359,}{ }^{373}$ Germany, ${ }^{224}$ and the Netherlands. ${ }^{374}$ The occupational therapy interventions added components of environmental adaptations and compensatory actions for the PLWD's daily activities to common psychoeducational activities.

\section{PLWD Outcomes}

Only 16 of the 29 medium to low risk of bias studies reported outcomes related to PLWD. We synthesized results qualitatively because differences in outcome reporting, outcome measures, time to outcomes, and comparison groups prohibited quantitative pooling. The most commonly investigated outcomes were function, neuropsychiatric symptoms, and quality of life, and the least commonly investigated were depression, healthcare use, social support, harms, and institutionalization. Table 6.2 summarizes the number of studies investigating each outcome and the number of studies that found a statistically significant benefit or no difference in outcomes between PLWD and controls.

Evidence was insufficient to draw conclusions about the effects of psychosocial interventions for any PLWD outcome. The inability to combine outcomes from multiple studies made it 
impossible to show precision in any outcome. Several outcomes were only investigated by a single study, making the consistency of findings unknown. Many PLWD outcomes were deemed indirect because the researchers relied on proxy respondents. Although a few comparisons had reasonably large numbers of participants (500 to 900+) and several studies contributing, the studies often failed to report effect sizes or the data needed to calculate them. The uncertainty regarding a true "no difference between groups" finding versus an "inability to show a difference” finding remained too high.

Depression was investigated by four studies using usual care and one study using attention control groups, with only one study reporting benefit for the psychosocial intervention in the short term and one study reporting benefit in the long term. One RCT reported improvements in PLWD depression at 6 weeks for the psychosocial treatment group. ${ }^{374}$ Three RCTs reported no group differences in depression scores over 12 to 52 weeks between psychosocial groups and usual care, ${ }^{224,355,367}$ with one study reporting a Cohen's d effect size of $0 .{ }^{367}$ One RCT reported improved depression scores over 1 year for their psychosocial treatment group versus attention control. ${ }^{369}$

Function was investigated by three studies using usual care and five using attention control groups, with mixed benefit for the psychosocial interventions. Two ${ }^{373,374}$ found short-term improvements in function for the psychosocial treatment group versus usual care, but one did not. ${ }^{355}$ Three studies found no differences in long-term function between psychosocial intervention groups and usual care comparisons. ${ }^{224,356,367}$ Outcomes were mixed for psychosocial groups versus attention control groups; one study found improved functioning for the treatment group, ${ }^{359}$ but a second found no difference. ${ }^{369}$

Neuropsychiatric symptoms were investigated by three studies using usual care and five studies with attention control groups. For studies using usual care comparisons, only one found improved neuropsychiatric symptoms for the psychosocial group, ${ }^{377}$ while three found no difference over 3 to 6 months. ${ }^{355,361,373}$ For studies using an attention control group, two found short-term benefits in neuropsychiatric symptoms for their psychosocial treatment groups, ${ }^{359,} 360$ but three found no long-term differences in neuropsychiatric symptoms. ${ }^{356,369,370}$

Quality of life was investigated by six studies using usual care and two using attention control groups. For studies using usual care comparisons, only one found improved PLWD quality of life at 6 weeks, ${ }^{377}$ while five found no difference in quality of life over 6 to 24 months. ${ }^{224,356,362,363,367}$ For studies using an attention control group, one study found benefits in quality of life for their psychosocial treatment group, ${ }^{369}$ but another ${ }^{359}$ found no difference.

Social support was investigated by one study using usual care and another using an attention control. Both found no difference in PLWD social support over 6 months. ${ }^{367,} 368$

Unmet needs was investigated by one study using education and information support over 6 months. No difference was found between groups. ${ }^{477}$

Healthcare use was investigated by four studies using usual care and one using an attention control group. One English study found that participants in the psychosocial group spent 436 fewer pounds sterling over 2 years $(p=0.035)$ on healthcare services than their usual care group. ${ }^{362}$ However, four other studies found no difference in healthcare use over 4 to 24 months. ${ }^{363,375,378}$

Harms were investigated by one study using a usual care control group. It found zero adverse events for participants in both the psychosocial treatment and control groups. ${ }^{378}$

Nursing home placement was investigated by two studies using usual care and two using attention control groups. One of the two studies using a usual care control group found a benefit 
for the psychosocial intervention, ${ }^{376}$ and the other found no difference. ${ }^{355}$ The studies using an attention control group found no difference between groups. ${ }^{368,}, 370$

Table 6.2. Summary of findings for PLWD outcomes: psychosocial interventions for CG/P

\begin{tabular}{|c|c|c|c|c|}
\hline $\begin{array}{l}\text { Outcome } \\
\text { Comparison }\end{array}$ & $\begin{array}{c}\text { \# Studies/Design } \\
\text { (n analyzed) } \\
\text { Timing }\end{array}$ & Population & Findings & $\begin{array}{l}\text { Strength of } \\
\text { Evidence* }^{*}\end{array}$ \\
\hline $\begin{array}{l}\text { Depression } \\
\text { sychosocial vs } \\
\text { Usual care }\end{array}$ & $\begin{array}{l}\text { 4 RCTs } 224,355,367,374 \\
(n=592) \\
\text { 6-52 weeks }\end{array}$ & CG/P & $\begin{array}{l}1 \text { of } 4 \text { found benefit } \\
3 \text { of } 4 \text { found no difference }\end{array}$ & Insufficient \\
\hline $\begin{array}{l}\text { Depression } \\
\text { Psychosocial vs } \\
\text { Attention control }\end{array}$ & $\begin{array}{l}1 \mathrm{RCT}^{369} \\
\mathrm{n}=330 \\
52 \text { weeks }\end{array}$ & CG/P & $\begin{array}{l}1 \text { found benefit } \\
0 \text { found no difference }\end{array}$ & Insufficient \\
\hline $\begin{array}{l}\text { Function } \\
\text { Psychosocial vs } \\
\text { Usual care }\end{array}$ & $\begin{array}{l}\text { 3 RCTs } 355,373,374 \\
(n=545) \\
6-12 \text { weeks }\end{array}$ & CG/P & $\begin{array}{l}2 \text { of } 3 \text { found benefit } \\
1 \text { of } 3 \text { found no difference }\end{array}$ & Insufficient \\
\hline $\begin{array}{l}\text { Function } \\
\text { Psychosocial vs } \\
\text { Usual care }\end{array}$ & $\begin{array}{l}\text { 3 RCTs } 224,356,367 \\
(n=540) \\
6-12 \text { months }\end{array}$ & CG/P & $\begin{array}{l}0 \text { of } 3 \text { found benefit } \\
3 \text { of } 3 \text { found no difference }\end{array}$ & Insufficient \\
\hline $\begin{array}{l}\text { Function } \\
\text { Psychosocial vs } \\
\text { Attention control }\end{array}$ & $\begin{array}{l}2 \text { RCTs }{ }^{359,369} \\
(n=490) \\
4-12 \text { months }\end{array}$ & $\mathrm{CG} / \mathrm{P}$ & $\begin{array}{l}1 \text { of } 2 \text { found benefit } \\
1 \text { of } 2 \text { found no difference }\end{array}$ & Insufficient \\
\hline $\begin{array}{l}\text { Neuropsychiatric } \\
\text { symptoms } \\
\text { Psychosocial vs } \\
\text { Usual care }\end{array}$ & 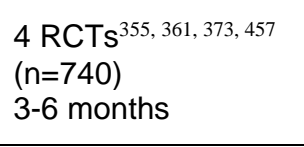 & CG/P & $\begin{array}{l}1 \text { of } 4 \text { found benefit } \\
3 \text { of } 4 \text { found no difference }\end{array}$ & Insufficient \\
\hline $\begin{array}{l}\text { Neuropsychiatric } \\
\text { symptoms } \\
\text { Psychosocial vs } \\
\text { Attention control }\end{array}$ & $\begin{array}{l}2 \text { RCTs }{ }^{359,360} \\
(n=227) \\
4-6 \text { months }\end{array}$ & CG/P & $\begin{array}{l}2 \text { of } 2 \text { found benefit } \\
0 \text { of } 2 \text { found no difference }\end{array}$ & Insufficient \\
\hline $\begin{array}{l}\text { Neuropsychiatric } \\
\text { symptoms } \\
\text { Psychosocial vs } \\
\text { Attention control }\end{array}$ & $\begin{array}{l}3 \text { RCTs } 356,369,370 \\
(n=916) \\
12-18 \text { months }\end{array}$ & $\mathrm{CG} / \mathrm{P}$ & $\begin{array}{l}0 \text { of } 3 \text { found benefit } \\
3 \text { of } 3 \text { found no difference }\end{array}$ & Insufficient \\
\hline $\begin{array}{l}\text { Quality of life } \\
\text { Psychosocial vs } \\
\text { Usual care }\end{array}$ & $\begin{array}{l}1 \mathrm{RCT}^{457} \\
(\mathrm{n}=135) \\
6 \text { weeks }\end{array}$ & CG/P & $\begin{array}{l}1 \text { found benefit } \\
0 \text { found no difference }\end{array}$ & Insufficient \\
\hline $\begin{array}{l}\text { Quality of life } \\
\text { Psychosocial vs } \\
\text { Usual care }\end{array}$ & $\begin{array}{l}\text { 5 RCTs 224, 356, 362, 363, } \\
367 \\
(n=936) \\
6-24 \text { months }\end{array}$ & CG/P & $\begin{array}{l}0 \text { of } 5 \text { found benefit } \\
5 \text { of } 5 \text { found no difference }\end{array}$ & Insufficient \\
\hline $\begin{array}{l}\text { Quality of life } \\
\text { Psychosocial vs } \\
\text { Attention control }\end{array}$ & $\begin{array}{l}\text { 2 RCTs }{ }^{359,369} \\
(n=490) \\
4-12 \text { months }\end{array}$ & $\mathrm{CG} / \mathrm{P}$ & $\begin{array}{l}1 \text { of } 2 \text { found benefit } \\
1 \text { of } 2 \text { found no difference }\end{array}$ & Insufficient \\
\hline $\begin{array}{l}\text { Social support } \\
\text { Psychosocial vs } \\
\text { Usual care }\end{array}$ & $\begin{array}{l}1 \mathrm{RCT}^{367} \\
(\mathrm{n}=108) \\
6 \text { months }\end{array}$ & CG/P & $\begin{array}{l}0 \text { found benefit } \\
1 \text { found no difference }\end{array}$ & Insufficient \\
\hline $\begin{array}{l}\text { Social support } \\
\text { Psychosocial vs } \\
\text { Attention control }\end{array}$ & $\begin{array}{l}1 \text { RCT } 368 \\
(n=250) \\
6 \text { months } \\
\end{array}$ & CG/P & $\begin{array}{l}0 \text { found benefit } \\
1 \text { found no difference }\end{array}$ & Insufficient \\
\hline $\begin{array}{l}\text { Healthcare usage } \\
\text { Psychosocial vs } \\
\text { Usual care }\end{array}$ & $\begin{array}{l}\text { 4 RCTs } 224,362,363,375 \\
(n=652) \\
\text { 4-24 months }\end{array}$ & CG/P & $\begin{array}{l}1 \text { of } 4 \text { found benefit } \\
3 \text { of } 4 \text { found no difference }\end{array}$ & Insufficient \\
\hline $\begin{array}{l}\text { Healthcare usage } \\
\text { Psychosocial vs } \\
\text { Attention control }\end{array}$ & $\begin{array}{l}1 \text { RCT } 368 \\
(n=250) \\
6 \text { months }\end{array}$ & CG/P & $\begin{array}{l}0 \text { found benefit } \\
1 \text { found no difference }\end{array}$ & Insufficient \\
\hline
\end{tabular}




\begin{tabular}{|c|c|c|c|c|}
\hline $\begin{array}{l}\text { Outcome } \\
\text { Comparison }\end{array}$ & $\begin{array}{l}\text { \# Studies/Design } \\
\text { (n analyzed) } \\
\text { Timing }\end{array}$ & Population & Findings & $\begin{array}{l}\text { Strength of } \\
\text { Evidence* }\end{array}$ \\
\hline $\begin{array}{l}\text { Harms - adverse } \\
\text { events } \\
\text { Psychosocial vs } \\
\text { Usual care }\end{array}$ & $\begin{array}{l}1 \mathrm{RCT}^{224} \\
(\mathrm{n}=141) \\
52 \text { weeks }\end{array}$ & CG/P & $\begin{array}{l}0 \text { found benefit } \\
1 \text { found no difference }\end{array}$ & Insufficient \\
\hline $\begin{array}{l}\text { Institutionalization } \\
\text { Psychosocial vs } \\
\text { Usual care }\end{array}$ & $\begin{array}{l}2 \text { RCTs } 355,376 \\
(\mathrm{n}=288) \\
6-12 \text { months }\end{array}$ & CG/P & $\begin{array}{l}1 \text { of } 2 \text { found benefit } \\
1 \text { of } 2 \text { found no difference }\end{array}$ & Insufficient \\
\hline $\begin{array}{l}\text { Institutionalization } \\
\text { Psychosocial vs } \\
\text { Attention control }\end{array}$ & $\begin{array}{l}2 \mathrm{RCT} 368,370 \\
(\mathrm{n}=545) \\
6-18 \text { months }\end{array}$ & CG/P & $\begin{array}{l}0 \text { of } 2 \text { found benefit } \\
2 \text { of } 2 \text { found no difference }\end{array}$ & Insufficient \\
\hline
\end{tabular}

*Insufficient ratings due to study limitations and imprecision in the findings.

Abbreviations: $\mathrm{CG} / \mathrm{P}=$ =caregiver/care partner; $\mathrm{n}=$ number; $\mathrm{PLWD}=$ person/people living with dementia; $\mathrm{RCT}$ =randomized controlled trial

\section{Caregiver Outcomes}

All 29 medium to low risk of bias studies reported caregiver outcomes related to the caregivers' psychological health, quality of life, social support, healthcare use, and their perception of caregiving burden, bother or distress, confidence, knowledge, and skills. We synthesized results qualitatively because differences in outcome measures, time to outcomes, and comparison groups prohibited quantitative pooling. The most commonly investigated outcomes were depression, quality of life, caregiver burden, and caregiver bother or distress, and the least common were coping, caregiving knowledge, social support, healthcare use, and relationship with PLWD. No studies looked for caregiver harms associated with the psychosocial intervention. Table 6.3 summarizes the number of studies investigating each outcome and the number of studies that found a statistically significant benefit or no difference in outcomes for caregivers enrolled in the psychosocial intervention group and those enrolled in the comparison group.

Evidence was insufficient for all caregiver outcomes. The insufficient ratings were attributed to several limitations across the bodies of evidence. The inability to quantitatively combine outcomes from multiple studies made it impossible to show precision for any outcome. Several outcomes were only investigated by a single study, making the consistency of findings unknown. Even though a few comparisons had reasonably large numbers of PLWD (500 to 900+) combined from several studies, those studies rarely reported effect sizes or the data to calculate them. The uncertainty regarding a true no difference between groups versus an inability to show a difference remained too high.

Anxiety was investigated by two studies using usual care and two using attention control groups, and only one study reported benefit for the psychosocial intervention. For studies using usual care comparisons, just one reported short-term improvements at 6 weeks for the psychosocial group with a Cohen's d effect size of $-0.32(-.63,-.02) .{ }^{366}$ However, another study found no group difference in anxiety over the long term. ${ }^{363}$ Both of the two studies using attention control comparisons found no group differences in anxiety over 6 to 12 months. ${ }^{356,360}$

Depression was investigated by 10 studies using usual care and 10 others using attention control groups; most found no statistically significant difference between the psychosocial and comparison groups. For studies using usual care comparisons, two found short-term benefits for the psychosocial groups, ${ }^{366,}, 377$ while three found no short-term group differences in depression. ${ }^{358,366,367}$ Two studies found long-term benefits for the psychosocial group, ${ }^{355,379}$ 
while three found no group differences in depression over 1 to 2 years. ${ }^{224,356,363}$ For studies using attention control groups, six found short-term benefits for the psychosocial groups, 354,357 , $360,368,372,380$ with one reporting a moderate Cohen's d effect size of $-0.66(-1.04,-0.74){ }^{354}$ However, one study reported no short-term group differences in depression. ${ }^{359}$ We calculated the standardized mean difference (SMD) and 95 percent confidence intervals for each of these studies and found a nonsignificant overall SMD of $0.207(-0.117,0.531)(p=0.15)$. See forest plots in Appendix E. Depression in the long-term studies was investigated by three studies that all reported no group differences in depression at 12 or 18 months. ${ }^{356,369,370}$

Stress was investigated by three studies using usual care and one using attention control groups, with only one study reporting benefit for the psychosocial intervention. Three studies using usual care comparisons found no group differences in caregiver stress in the short or long term, ${ }^{355,366,367}$ with one study reporting a nonsignificant Cohen's d effect size of $-0.20(-0.50$, 0.11). ${ }^{366}$ A single study using an attention control comparison found improvements in stress for the psychosocial group. ${ }^{372}$

Caregiving burden was investigated by six studies using usual care and four using attention control groups with no apparent pattern of benefit for the psychosocial interventions. For studies using usual care comparisons, three found no short-term group differences, ${ }^{364,366,377}$ with one reporting a nonsignificant Cohen's d effect size of $-0.16(-0.46,0.14) .{ }^{366}$ Results for long-term caregiver burden were mixed, with two studies finding benefit for the psychosocial intervention $^{361,376}$ and three finding no group differences. ${ }^{224,358,375}$ For studies using attention control groups, results were mixed, with three studies finding benefit for the psychosocial group, ${ }^{354,357,380}$ and two finding no group differences in caregiving burden. ${ }^{359,} 368$

Caregiving bother/distress was investigated by four studies using usual care and five using attention control groups, with most studies finding no group differences. For studies using usual care comparisons, only one study found benefit for the psychosocial group, ${ }^{377}$ while three studies found no group differences in caregiver bother. ${ }^{361,366,373}$ For studies using attention control comparisons, results were mixed, with three studies finding benefit for the psychosocial interventions, ${ }^{359,360,372}$ but two finding no group differences for caregiving burden. ${ }^{356,368}$

Caregiving knowledge was investigated by a single study using a usual care comparison. It found that the psychosocial group had greater improvements in their caregiving knowledge than the usual care comparison group at 6 months. ${ }^{358}$

Caregiving confidence was investigated by three studies using usual care and three using attention control groups, and more studies found no group differences than benefit for the psychosocial intervention. For studies using a usual care comparison, only one study found a benefit for the psychosocial group, ${ }^{377}$ while two found no group differences at 12 to 24 weeks. $^{367,373}$ For studies using attention controls, two studies found a benefit for the psychosocial interventions ${ }^{354,360}$ one of which reported moderate Cohen's d effect size of 0.86 $(1.24,0.46),{ }^{354}$ while another study found no group differences. ${ }^{368}$

Caregiving skill was investigated by five studies using usual care and two using attention control groups, with six of the seven reporting no long-term group differences, and a single study reporting short-term benefit for the psychosocial intervention. For studies using usual care comparisons, one found short-term benefit at 6 weeks for the psychosocial group, ${ }^{374}$ one found no short-term benefit, ${ }^{457}$ and three found no difference in caregiving skill long term. ${ }^{224,358,362}$ An additional two studies using attention control comparisons also found no long-term group differences in caregiving skill. ${ }^{368,370}$ 
Coping was investigated by a single study using a usual care comparison. This study reported higher coping skills in the psychosocial group at 6 months. ${ }^{358}$

Quality of life was investigated by 11 studies using usual care and three using attention control groups. Eight studies found no group differences while six found benefit for the psychosocial group. For studies using usual comparison groups, four found short-term benefits for the psychosocial groups, ${ }^{366,374,376,381}$ and three found no group differences. ${ }^{361,364,367}$ In the long term, only one study found a benefit for the psychosocial group, ${ }^{375}$ while three did not. ${ }^{224}$, 362, 363 For studies using attention control comparisons, a single study found short-term benefits for the psychosocial group; ${ }^{357}$ however, two found no group differences long term. ${ }^{356,368}$

The caregiver/PLWD relationship was investigated by three studies using attention control groups. One found a benefit for the psychosocial group and reported a moderate Cohen's $\mathrm{d}$ effect size of $0.44(0.82,0.07),{ }^{354}$ while two found no group differences. ${ }^{356,368}$

Social support was investigated by two studies using usual care comparisons and one using attention control groups. The two studies using usual care comparisons found no group differences in social support. ${ }^{367,376}$ A single study using an attention control group found a benefit for the psychosocial group at 6 months. ${ }^{368}$

Healthcare use was investigated by two studies using usual care and two using attention control groups. Only one study using an attention control group found a benefit for the psychosocial group, ${ }^{368}$ while all the others found no group differences. ${ }^{362,365,375}$

No studies reported outcomes on caregiver harms for psychosocial interventions.

Table 6.3. Summary of findings for caregiver outcomes: psychosocial interventions for CG/P

\begin{tabular}{|c|c|c|c|c|}
\hline $\begin{array}{l}\text { Outcome } \\
\text { Comparison }\end{array}$ & $\begin{array}{l}\text { \# Studies/ Design } \\
\text { (n analyzed) } \\
\text { Timing }\end{array}$ & Population & Findings & $\begin{array}{l}\text { Strength of } \\
\text { Evidence* }^{*}\end{array}$ \\
\hline $\begin{array}{l}\text { Anxiety } \\
\text { Psychosocial vs } \\
\text { Attention control }\end{array}$ & $\begin{array}{l}2 \mathrm{RCT}^{356,360} \\
\mathrm{n}=358 \\
24-52 \text { weeks }\end{array}$ & CG/P & $\begin{array}{l}0 \text { of } 2 \text { found benefit } \\
2 \text { of } 2 \text { found no difference }\end{array}$ & Insufficient \\
\hline $\begin{array}{l}\text { Anxiety } \\
\text { Psychosocial vs } \\
\text { Usual care }\end{array}$ & $\begin{array}{l}1 \mathrm{RCT}^{366} \\
\mathrm{n}=170 \\
6 \text { weeks }\end{array}$ & CG/P & $\begin{array}{l}1 \text { found benefit } \\
0 \text { found no difference }\end{array}$ & Insufficient \\
\hline $\begin{array}{l}\text { Anxiety } \\
\text { Psychosocial vs } \\
\text { Usual care }\end{array}$ & $\begin{array}{l}1 \mathrm{RCT}^{363} \\
\mathrm{n}=260 \\
2 \text { years } \\
\end{array}$ & CG/P & $\begin{array}{l}0 \text { found benefit } \\
1 \text { found no difference }\end{array}$ & Insufficient \\
\hline $\begin{array}{l}\text { Burden of care } \\
\text { Psychosocial vs } \\
\text { Attention control }\end{array}$ & $\begin{array}{l}5 \mathrm{RCT}^{354,357,359,368,380} \\
\mathrm{n}=725 \\
8-24 \text { weeks }\end{array}$ & CG/P & $\begin{array}{l}3 \text { of } 5 \text { found benefit } \\
2 \text { of } 5 \text { found no difference }\end{array}$ & Insufficient \\
\hline $\begin{array}{l}\text { Burden of care } \\
\text { Psychosocial vs } \\
\text { Usual care }\end{array}$ & $\begin{array}{l}3 \text { RCT 364, 366, } 457 \\
n=680 \\
6-16 \text { weeks }\end{array}$ & CG/P & $\begin{array}{l}0 \text { of } 3 \text { found benefit } \\
3 \text { of } 3 \text { found no difference }\end{array}$ & Insufficient \\
\hline $\begin{array}{l}\text { Burden of care } \\
\text { Psychosocial vs } \\
\text { Usual care }\end{array}$ & $\begin{array}{l}5 \mathrm{RCT}^{224,358,361,375,376} \\
\mathrm{n}=561 \\
24-52 \text { weeks }\end{array}$ & CG/P & $\begin{array}{l}2 \text { of } 5 \text { found benefit } \\
3 \text { of } 5 \text { found no difference }\end{array}$ & Insufficient \\
\hline $\begin{array}{l}\text { Depressive } \\
\text { symptoms } \\
\text { Psychosocial vs } \\
\text { Attention control }\end{array}$ & $\begin{array}{l}\text { 7 RCT } 354,357,359,360,368, \\
372,380 \\
n=976 \\
8-24 \text { weeks }\end{array}$ & CG/P & $\begin{array}{l}6 \text { of } 7 \text { found benefit } \\
1 \text { of } 7 \text { found no difference }\end{array}$ & Insufficient \\
\hline $\begin{array}{l}\text { Depressive } \\
\text { symptoms } \\
\text { Psychosocial vs } \\
\text { Attention control }\end{array}$ & $\begin{array}{l}3 \mathrm{RCT} \text { 356, 369, } 370 \\
n=916 \\
52-72 \text { weeks }\end{array}$ & CG/P & $\begin{array}{l}0 \text { of } 3 \text { found benefit } \\
3 \text { of } 3 \text { found no difference }\end{array}$ & Insufficient \\
\hline
\end{tabular}




\begin{tabular}{|c|c|c|c|c|}
\hline $\begin{array}{l}\text { Outcome } \\
\text { Comparison }\end{array}$ & $\begin{array}{c}\text { \# Studies/ Design } \\
\text { (n analyzed) } \\
\text { Timing }\end{array}$ & Population & Findings & $\begin{array}{l}\text { Strength of } \\
\text { Evidence* }\end{array}$ \\
\hline $\begin{array}{l}\text { Depressive } \\
\text { symptoms } \\
\text { Psychosocial vs } \\
\text { Usual care } \\
\end{array}$ & $\begin{array}{l}5 \mathrm{RCT} 358,366,367,374,457 \\
\mathrm{n}=852 \\
6-24 \text { weeks }\end{array}$ & CG/P & $\begin{array}{l}2 \text { of } 5 \text { found benefit } \\
3 \text { of } 5 \text { found no difference }\end{array}$ & Insufficient \\
\hline $\begin{array}{l}\text { Depressive } \\
\text { symptoms } \\
\text { Psychosocial vs } \\
\text { Usual care } \\
\end{array}$ & $\begin{array}{l}5 \mathrm{RCT} 224,355,356,363 \\
\mathrm{n}=1073 \\
52-104 \text { weeks }\end{array}$ & CG/P & $\begin{array}{l}2 \text { of } 5 \text { found benefit } \\
3 \text { of } 5 \text { found no difference }\end{array}$ & Insufficient \\
\hline $\begin{array}{l}\text { Caregiving } \\
\text { bother/distress/affect } \\
\text { Psychosocial vs } \\
\text { Attention control } \\
\end{array}$ & $\begin{array}{l}5 \text { RCT } 356,359,360,368,372 \\
n=952 \\
4-12 \text { months }\end{array}$ & CG/P & $\begin{array}{l}3 \text { of } 5 \text { found benefit } \\
2 \text { of } 5 \text { found no difference }\end{array}$ & Insufficient \\
\hline $\begin{array}{l}\text { Caregiving } \\
\text { bother/distress/affect } \\
\text { Psychosocial vs } \\
\text { Usual care } \\
\end{array}$ & $\begin{array}{l}\begin{array}{l}4 \mathrm{RCT}^{361,366,373,457} \\
\mathrm{n}=702 \\
\text { 6-24 weeks }\end{array}\end{array}$ & CG/P & $\begin{array}{l}1 \text { of } 4 \text { found benefit } \\
3 \text { of } 4 \text { found no difference }\end{array}$ & Insufficient \\
\hline $\begin{array}{l}\text { Healthcare usage } \\
\text { Psychosocial vs } \\
\text { Attention control }\end{array}$ & $\begin{array}{l}2 \text { RCT } 365,368 \\
\mathrm{n}=359 \\
3-6 \text { months }\end{array}$ & CG/P & $\begin{array}{l}1 \text { of } 2 \text { found benefit } \\
1 \text { of } 2 \text { found no difference }\end{array}$ & Insufficient \\
\hline $\begin{array}{l}\text { Healthcare usage } \\
\text { Psychosocial vs } \\
\text { Usual care }\end{array}$ & $\begin{array}{l}2 \mathrm{RCT}^{362,375} \\
\mathrm{n}=251 \\
4-24 \text { months }\end{array}$ & CG/P & $\begin{array}{l}0 \text { of } 2 \text { found benefit } \\
2 \text { of } 2 \text { found no difference }\end{array}$ & Insufficient \\
\hline $\begin{array}{l}\text { Caregiving } \\
\text { knowledge } \\
\text { Psychosocial vs } \\
\text { Usual care } \\
\end{array}$ & $\begin{array}{l}1 \mathrm{RCT}^{358} \\
\mathrm{n}=167 \\
24 \text { weeks }\end{array}$ & CG/P & $\begin{array}{l}1 \text { found benefit } \\
0 \text { found no difference }\end{array}$ & Insufficient \\
\hline $\begin{array}{l}\text { Quality of life } \\
\text { Psychosocial vs } \\
\text { Attention control }\end{array}$ & $\begin{array}{l}1 \mathrm{RCT}^{357} \\
\mathrm{n}=132 \\
8 \text { weeks }\end{array}$ & CG/P & $\begin{array}{l}1 \text { found benefit } \\
0 \text { found no difference }\end{array}$ & Insufficient \\
\hline $\begin{array}{l}\text { Quality of life } \\
\text { Psychosocial vs } \\
\text { Attention control }\end{array}$ & $\begin{array}{l}2 \text { RCT } 356,368 \\
\mathrm{n}=541 \\
24-52 \text { weeks }\end{array}$ & CG/P & $\begin{array}{l}0 \text { of } 2 \text { found benefit } \\
2 \text { of } 2 \text { found no difference }\end{array}$ & Insufficient \\
\hline $\begin{array}{l}\text { Quality of life } \\
\text { Psychosocial vs } \\
\text { Usual care }\end{array}$ & $\begin{array}{l}7 \mathrm{RCT} 361,364,366,367,374, \\
376,381 \mathrm{n}=843 \\
6-24 \text { weeks }\end{array}$ & CG/P & $\begin{array}{l}4 \text { of } 7 \text { found benefit } \\
3 \text { of } 7 \text { found no difference }\end{array}$ & Insufficient \\
\hline $\begin{array}{l}\text { Quality of life } \\
\text { Psychosocial vs } \\
\text { Usual care }\end{array}$ & $\begin{array}{l}4 \mathrm{RCT}^{224,362,363,375} \\
\mathrm{n}=652 \\
9 \text { months to } 2 \text { years }\end{array}$ & CG/P & $\begin{array}{l}1 \text { of } 4 \text { found benefit } \\
3 \text { of } 4 \text { found no difference }\end{array}$ & Insufficient \\
\hline $\begin{array}{l}\text { Relationship with } \\
\text { person with } \\
\text { dementia } \\
\text { Psychosocial vs } \\
\text { Attention control }\end{array}$ & $\begin{array}{l}4 \mathrm{RCT} 354,356,368,477 \\
\mathrm{n}=652 \\
20-52 \text { weeks }\end{array}$ & CG/P & $\begin{array}{l}1 \text { of } 4 \text { found benefit } \\
3 \text { of } 4 \text { found no difference }\end{array}$ & Insufficient \\
\hline $\begin{array}{l}\text { Caregiving } \\
\text { confidence } \\
\text { Psychosocial vs } \\
\text { Attention control } \\
\end{array}$ & $\begin{array}{l}3 \mathrm{RCT} 354,360,368 \\
\mathrm{n}=428 \\
20-24 \text { weeks }\end{array}$ & CG/P & $\begin{array}{l}2 \text { of } 3 \text { found benefit } \\
1 \text { of } 3 \text { found no difference }\end{array}$ & Insufficient \\
\hline $\begin{array}{l}\text { Caregiving } \\
\text { confidence } \\
\text { Psychosocial vs } \\
\text { Usual care } \\
\end{array}$ & $\begin{array}{l}3 \mathrm{RCT}^{367,373,457} \\
\mathrm{n}=582 \\
12-24 \text { weeks }\end{array}$ & CG/P & $\begin{array}{l}1 \text { of } 3 \text { found benefit } \\
2 \text { of } 3 \text { found no difference }\end{array}$ & Insufficient \\
\hline $\begin{array}{l}\text { Caregiving skill } \\
\text { Psychosocial vs } \\
\text { Attention control }\end{array}$ & $\begin{array}{l}2 \text { RCT } 368,370 \\
n=545 \\
6-18 \text { months }\end{array}$ & CG/P & $\begin{array}{l}0 \text { of } 2 \text { found benefit } \\
2 \text { of } 2 \text { found no difference }\end{array}$ & Insufficient \\
\hline $\begin{array}{l}\text { Caregiving skill } \\
\text { Psychosocial vs } \\
\text { Usual care }\end{array}$ & $\begin{array}{l}2 \mathrm{RCT}^{374,457} \\
\mathrm{n}=407 \\
6-16 \text { weeks }\end{array}$ & CG/P & $\begin{array}{l}1 \text { of } 2 \text { found benefit } \\
1 \text { of } 2 \text { found no difference }\end{array}$ & Insufficient \\
\hline
\end{tabular}




\begin{tabular}{|c|c|c|c|c|}
\hline $\begin{array}{l}\text { Outcome } \\
\text { Comparison }\end{array}$ & $\begin{array}{c}\text { \# Studies/ Design } \\
\text { (n analyzed) } \\
\text { Timing }\end{array}$ & Population & Findings & $\begin{array}{l}\text { Strength of } \\
\text { Evidence* }^{*}\end{array}$ \\
\hline $\begin{array}{l}\text { Caregiving skill } \\
\text { Psychosocial vs } \\
\text { Usual care }\end{array}$ & $\begin{array}{l}3 \mathrm{RCT}^{224,358,362} \\
\mathrm{n}=444 \\
24-52 \text { weeks }\end{array}$ & CG/P & $\begin{array}{l}0 \text { of } 3 \text { found benefit } \\
3 \text { of } 3 \text { found no difference }\end{array}$ & Insufficient \\
\hline $\begin{array}{l}\text { Stress } \\
\text { Psychosocial vs } \\
\text { Attention }\end{array}$ & $\begin{array}{l}1 \text { RCT }^{372} \\
\mathrm{n}=184 \\
6 \text { months }\end{array}$ & CG/P & $\begin{array}{l}1 \text { found benefit } \\
0 \text { found no difference }\end{array}$ & Insufficient \\
\hline $\begin{array}{l}\text { Stress } \\
\text { Psychosocial vs } \\
\text { Usual care }\end{array}$ & $\begin{array}{l}\text { 3 RCT 355, 366, } 367 \\
n=486 \\
6-52 \text { weeks }\end{array}$ & CG/P & $\begin{array}{l}0 \text { of } 3 \text { found benefit } \\
3 \text { of } 3 \text { found no difference }\end{array}$ & Insufficient \\
\hline $\begin{array}{l}\text { Social support } \\
\text { Psychosocial vs } \\
\text { Attention control }\end{array}$ & $\begin{array}{l}1 \mathrm{RCT}^{368} \\
\mathrm{n}=250 \\
6 \text { months }\end{array}$ & CG/P & $\begin{array}{l}1 \text { found benefit } \\
0 \text { found no difference }\end{array}$ & Insufficient \\
\hline $\begin{array}{l}\text { Social support } \\
\text { Psychosocial vs } \\
\text { Usual care }\end{array}$ & $\begin{array}{l}2 \text { RCT }^{367,376} \\
\mathrm{n}=188 \\
12-24 \text { weeks }\end{array}$ & CG/P & $\begin{array}{l}0 \text { of } 2 \text { found benefit } \\
2 \text { of } 2 \text { found no difference }\end{array}$ & Insufficient \\
\hline $\begin{array}{l}\text { Caregiving coping } \\
\text { Psychosocial vs } \\
\text { Usual care }\end{array}$ & $\begin{array}{l}1 \mathrm{RCT}^{358} \\
\mathrm{n}=167 \\
24 \text { weeks }\end{array}$ & CG/P & $\begin{array}{l}1 \text { found benefit } \\
0 \text { found no difference }\end{array}$ & Insufficient \\
\hline
\end{tabular}

*Insufficient ratings due to study limitations and imprecision in the findings.

Abbreviations: $\mathrm{CG} / \mathrm{P}=$ caregiver/care partner; $\mathrm{n}=$ =number; $\mathrm{RCT}=$ =randomized controlled trial

\section{Variation in Outcomes}

\section{By PLWD Characteristics}

Only one study identified how PLWD characteristics may influence outcomes. A subgroup analysis for the Farran study (2004) found that data from 143 caregivers of family members with baseline agitated behaviors indicated more reduction of emotional distress with the skill-building intervention than with an information- and support-oriented comparison over an 18-month period. These researchers suggested their findings indicate that dementia caregivers exposed to agitated behaviors can benefit from psychosocial interventions, particularly those aimed at building behavioral management skills. ${ }^{370}$

\section{By Caregiver Characteristics}

Only a few studies identified how caregiver characteristics may influence outcomes. Gallagher-Thompson and colleagues (2003) found no differences between Hispanic and nonHispanic white female caregivers $(\mathrm{n}=213)$ in caregiver coping, depression, social support, and caregiver burden 3 months after completing the Coping with Caregiving intervention. ${ }^{371}$

Gallagher-Thompson and colleagues in 2008 investigated differences in caregiver coping, depression, stress, support, burden, and bother between Hispanic and nonHispanic white female caregivers $(\mathrm{n}=184) .{ }^{372}$ The only differences they found were that nonHispanic white female caregivers reported higher bother managing neuropsychiatric symptoms at baseline $(\mathrm{p}=0.03)$ and post intervention $(\mathrm{p}=0.010)$ than Hispanic female caregivers. The two groups did not differ in depressive symptoms or perceived stress before or 6 months after completing a psychosocial intervention.

Gitlin and colleagues (2001) reported that intervention spouses reported reduced upset $(\mathrm{p}=.049)$, women reported enhanced self-efficacy in managing PLWD behaviors $(\mathrm{p}=.038)$, and women $(\mathrm{p}=.049)$ and racial/ethnic minorities $(\mathrm{p}=.037)$ reported enhanced self-efficacy in managing PLWD functional dependency. ${ }^{373}$ 
A moderator analysis of the Gallagher-Thompson (2003) intervention ${ }^{371}$ found that caregiver self-efficacy predicted differential outcomes in a randomized trial comparing a cognitive behavior psychoeducational intervention versus an enhanced support group. The four key outcomes were depression, anxiety, social support, and coping. The findings showed that low baseline self-efficacy scores better predicted positive response to treatment in the psychosocial intervention than in the enhanced support group intervention. This study supports the use of selfefficacy as a screening tool for appropriate caregiver intervention assignment. ${ }^{478}$

Otero and colleagues (2015) reported that caregivers younger than 65 years and with higher emotional distress at baseline were more likely than those receiving usual care to improve their depressive symptoms after completing five weeks of a group-based cognitive behavioral program focused on problem solving. ${ }^{379}$

\section{By Intervention Characteristics}

No studies investigated whether intervention characteristics modified the intervention effects.

\section{Social Support}

\section{Key Point}

- Evidence was insufficient to draw conclusions about the effect of in-person social support on PLWD and their CG/P.

- Phone-based social support for CG/P were described in the evidence map but not considered for analysis due to limitations in study designs.

\section{Intervention Description}

We defined social support interventions as those targeted to provide information, resources, and a form of social interaction to caregivers of PLWD. Intervention delivery modes could involve talking to peers or other supports in-person or over the phone, or automated support programs that were phone or web-based. Phone and web-based platforms provided some form of social interaction either through automated conversations, voicemail, or chat groups.

\section{Eligible Studies}

We identified 13 unique studies from 16 publications (Table 6.4). ${ }^{479-493}$ Two studies were assessed as medium risk of bias for the analytic set. ${ }^{479-481}$ We provide information on all pilot studies and high risk of bias studies as part of the evidence map in Appendix E.

The two studies in the analytic set were categorized as being in the explanatory stage, or Stage 3 of the NIH Stage Model. One $(n=78)$ was a randomized trial comparing in-person, peerled mutual support groups for CG/P with usual care. ${ }^{481}$ Support groups met bi-weekly over 24 months. The other $(\mathrm{n}=100)$ was a randomized trial comparing an automated phone support system for CG/P with usual care. ${ }^{479,480}$ The phone support system included an activity-respite module that engaged PLWD in an 18-minute automated conversation, weekly automated conversations to check stress levels, a personal voice mailbox, and a phone support group "bulletin board" (which functioned like an internet chat group). The support system was available for 22 hours a day over 12 months. ${ }^{479,480}$ Appendix E provides evidence tables, summary risk of bias assessments, and strength of evidence for key comparisons and outcomes. 
Table 6.4. Basic characteristics of literature set: in-person social support

\begin{tabular}{|l|l|}
\hline \multicolumn{1}{|c|}{ Characteristic } & \multicolumn{1}{c|}{ Information } \\
\hline Total Studies & 13 studies \\
\hline Non-U.S. studies & 10 studies \\
\hline Evidence map studies & $\begin{array}{l}4 \text { pilot studies } \\
7 \text { high risk of bias studies }\end{array}$ \\
\hline Analytic set studies & 2 randomized controlled trials \\
\hline Risk of bias of analytic set & 2 medium \\
\hline Number of PLWD in analytic set & 178 \\
\hline Dementia type/definition & $\begin{array}{l}\text { All dementia types and severities (primarily AD and cerebrovascular) } \\
\text { Probable AD with functional impairments and AD-related disturbing } \\
\text { behavior }\end{array}$ \\
\hline Caregiver type (number) & Informal caregivers (n=178) \\
\hline
\end{tabular}

Abbreviations: $\mathrm{AD}=$ Alzheimer's disease; $n=$ number; $\mathrm{PLWD}=$ people living with dementia

\section{Intervention Research Context}

The eligible literature on social support services for caregivers generally comprises pilot or explanatory research. Studies were published between 1990 and 2019 and tended to be pilot, small sample, or high risk of bias studies. One intervention did show studies progressing along the NIH Stage Model. ${ }^{489,490}$

In-person social support approaches included a befriending intervention where volunteers were recruited and trained to befriend CG/Ps. ${ }^{481,482,485,487}$ One study examined social support groups that engaged CG/P and PLWD dyads separately and together. ${ }^{485}$ The automated social support for CG/P comprised phone-based automated support and web-based automated support. We identified only one study that examined the use of phone-based automated support, which was included in our analytic study set. ${ }^{479,480}$ The lack of additional studies on phone-based automated support may be due to a shift towards web-based platforms as technology has evolved. Four web-based approaches were piloted in Europe. ${ }^{486,488,489}$ One piloted a tool allowing caregivers to assess their needs for care and support and providing tailored advice as well as links to local support organizations. Two piloted platforms providing information, relaxation exercises, a social networking platform, and allowing caregivers to assess their health and reach out to clinical sites. An additional pilot examining the use of a website to provide stress reduction, support, and information for caregivers was conducted in the United States. ${ }^{494}$ The pilots suggested some benefit to web-based social support tools. However, a later small study was assessed as high risk of bias. ${ }^{490}$ One study examined email contacts with a specialist dementia nurse with online videos and e-bulletins. ${ }^{493}$

\section{PLWD Outcomes}

No reportable PLWD outcomes were available from the analytic set.

\section{Caregiver Outcomes}

Evidence was insufficient to draw conclusions about in-person social support versus usual care for CG/P. Table 6.5 summarizes the primary outcomes for CG/P. At 28 weeks, findings showed statistically significant differences in Neuropsychiatric Inventory-Caregiver Distress Scale scores between the peer support and usual care groups, with the peer support group reporting lower levels of distress. ${ }^{481}$ The peer support group also had statistically significant improvements in quality of life at 28 weeks compared with the usual care group. Groups did not differ in the use of mental health services. No harms were reported. 
Evidence was also insufficient to draw conclusions about automated social support versus usual care for CG/P. At 6 months, findings showed no difference between the automated phonebased support group and usual care in depressive symptoms. ${ }^{479,480}$ Additionally, no difference was found between groups in anxiety. ${ }^{479,480}$ No study assessed harms.

Table 6.5. Summary of findings for caregiver outcomes: social support

\begin{tabular}{|c|c|c|c|c|}
\hline $\begin{array}{l}\text { Outcome } \\
\text { Comparison }\end{array}$ & $\begin{array}{c}\text { \# Studies/ Design } \\
\text { (n analyzed) } \\
\text { Timing }\end{array}$ & Population & Findings & $\begin{array}{l}\text { Strength of } \\
\text { Evidence* }^{*}\end{array}$ \\
\hline $\begin{array}{l}\text { Quality of Life } \\
\text { In person social } \\
\text { support vs. Usual care }\end{array}$ & $\begin{array}{l}1 \mathrm{RCT}^{481}(\mathrm{n}=78) \\
28 \text { weeks }\end{array}$ & $\begin{array}{l}\text { CG/P, respite } \\
\text { centers in China }\end{array}$ & $\begin{array}{l}1 \text { found benefit } \\
0 \text { found no difference }\end{array}$ & Insufficient \\
\hline $\begin{array}{l}\text { Caregiver Distress } \\
\text { In person social } \\
\text { support vs. Usual care }\end{array}$ & $\begin{array}{l}1 \mathrm{RCT}^{481}(\mathrm{n}=78) \\
28 \text { weeks }\end{array}$ & $\begin{array}{l}\text { CG/P, respite } \\
\text { centers in China }\end{array}$ & $\begin{array}{l}1 \text { found benefit } \\
0 \text { found no difference }\end{array}$ & Insufficient \\
\hline $\begin{array}{l}\text { Depression } \\
\text { Automated social } \\
\text { support vs. Usual care }\end{array}$ & $\begin{array}{l}1 \mathrm{RCT}^{479}(\mathrm{n}=100) \\
6 \text { months }\end{array}$ & $\mathrm{CG} / \mathrm{P}$ & $\begin{array}{l}0 \text { found benefit } \\
1 \text { found no difference }\end{array}$ & Insufficient \\
\hline $\begin{array}{l}\text { Anxiety } \\
\text { Automated social } \\
\text { support vs. Usual care }\end{array}$ & $\begin{array}{l}1 \mathrm{RCT}^{479}(\mathrm{n}=100) \\
6 \text { months }\end{array}$ & CG/P & $\begin{array}{l}0 \text { found benefit } \\
1 \text { found no difference }\end{array}$ & Insufficient \\
\hline
\end{tabular}

*Insufficient ratings due to study limitations and imprecision in the findings.

Abbreviations: $\mathrm{CG} / \mathrm{P}=$ caregiver/care partner; $\mathrm{n}=$ =number; $\mathrm{RCT}=$ =randomized controlled trial

\section{Variation in Outcomes}

No studies reported variation in outcomes by PLWD, caregiver, or intervention characteristics.

\section{Lifestyle Interventions}

Lifestyle interventions include a range of interventions targeted at improving the health and well-being of participants, including physical activity, leisure activities, visual arts, and mindfulness, meditation, or spiritually focused activities.

\section{Mindfulness, Meditation, or Spiritually Focused Activities}

\section{Key Point}

- Evidence was insufficient to draw conclusions about the effect of mindfulness-based stress reduction on PLWD and their CG/P.

\section{Eligible Studies}

We identified 10 unique studies from 10 publications examining mindfulness, meditation, or spiritually focused activities. Table 6.6 summarizes the characteristics of the literature set. Only one study was included in the analytic study set. ${ }^{495}$ The study was assessed as medium risk of bias and categorized as explanatory, or Stage 3 of the NIH stage model. Three studies were pilot studies and four studies had fewer than 50 participants. ${ }^{496-502}$ Two studies were assessed as high risk of bias due to potential performance bias. ${ }^{503,504}$ We provide information on all pilot studies and high risk of bias studies in the evidence map in Appendix E. Appendix E also provides evidence tables, summary risk of bias assessments, and strength of evidence for key comparisons and outcomes. 
Table 6.6. Basic characteristics of literature set: mindfulness, meditation, and spiritually focused activities

\begin{tabular}{|l|l|}
\hline \multicolumn{1}{|c|}{ Characteristic } & \multicolumn{1}{c|}{ Information } \\
\hline Total Studies & 10 studies \\
\hline Non-U.S. studies & 5 studies \\
\hline Evidence map studies & 3 pilot studies \\
& 4 small sample size studies \\
\hline Analytic set studies & 2 high risk of bias studies \\
\hline Risk of bias of analytic set & 1 randomized controlled trial \\
\hline Number of PLWD in analytic set & Medium \\
\hline Dementia type/definition & 31 \\
\hline Caregiver type (number) & Progressive dementia diagnosis \\
\hline
\end{tabular}

Abbreviation: PLWD=people living with dementia

\section{Intervention Research Context}

The literature set follows the NIH Stage Model, with most research consisting of pilots and small RCTs. Studies were published between 2010 and 2019, reflecting the relatively recent interest in these types of interventions for CG/P of PLWD. These studies generally used group sessions as the intervention delivery mode, and they focused on therapy or education using mindfulness, religion, or meditation as a main feature of the intervention. Interventions lasted from 5 to 12 weeks. Five studies specifically examined the effect of mindfulness therapy or mindfulness-based stress reduction for CG/P. 495, 496, 498, 501, 502 Two studies examined spiritually based therapy or education. ${ }^{503,504}$ Three studies examined meditation. ${ }^{497,499,500}$

\section{PLWD Outcomes}

The analytic study set did not report outcomes related to PLWD.

\section{Caregiver Outcomes}

One study was included in the analytic set and reported outcomes for CG/P. ${ }^{495}$ The study $(n=78)$ randomized CG/P to either mindfulness-based stress reduction or education and support. Evidence was insufficient to draw conclusions about the impact of mindfulness-based stress reduction versus education and support. In both arms, participants attended in-person group sessions over 8 weeks. The education and support group also received support calls by phone. The study reported outcomes for caregiver stress, burden, depression, anxiety, mental health, physical health, and social support at 2 months (immediately post-intervention) and 6 months. ${ }^{495}$

Table 6.7 summarizes the outcomes for CG/P. Groups did not differ significantly at either time point for measures of caregiver burden, social support, or physical health. ${ }^{495}$ Participants in the mindfulness group showed statistically significant improvements in mental health versus the education and support group at 2 and 6 months. ${ }^{495}$ Participants in the mindfulness group also showed a reduction in anxiety at 2 and 6 months versus the education and support group. At 2 months, participants in the mindfulness group showed a statistically significant reduction in stress versus those in the education and support group. This difference was not sustained at 6 months. ${ }^{495}$ However, this was also true for depression, where the mindfulness group showed a statistically significant improvement in depressive symptoms versus the education and support group at 2 months, but not 6 months. ${ }^{495}$ 
Table 6.7. Summary of findings for caregiver outcomes: mindfulness, meditation, and spiritually focused activities

\begin{tabular}{|c|c|c|c|c|}
\hline $\begin{array}{l}\text { Outcome } \\
\text { Comparison }\end{array}$ & $\begin{array}{c}\text { \# Studies/Design } \\
\text { (n analyzed) } \\
\text { Timing }\end{array}$ & Population & Findings & $\begin{array}{l}\text { Strength of } \\
\text { Evidence* }^{*}\end{array}$ \\
\hline $\begin{array}{l}\text { Caregiver Stress } \\
\text { Mindfulness vs } \\
\text { Active Control }\end{array}$ & $\begin{array}{l}1 \mathrm{RCT}(\mathrm{n}=78)^{495} \\
2 \text { months, } 6 \text { months }\end{array}$ & CG/P & $\begin{array}{l}1 \text { found benefit } \\
0 \text { found no difference }\end{array}$ & Insufficient \\
\hline $\begin{array}{l}\text { Caregiver Burden } \\
\text { Mindfulness vs } \\
\text { Active Control }\end{array}$ & $\begin{array}{l}1 \mathrm{RCT}(\mathrm{n}=78)^{495} \\
2 \text { months, } 6 \text { months }\end{array}$ & CG/P & $\begin{array}{l}0 \text { found benefit } \\
1 \text { found no difference }\end{array}$ & Insufficient \\
\hline $\begin{array}{l}\text { Depression } \\
\text { Mindfulness vs } \\
\text { Active Control }\end{array}$ & $\begin{array}{l}1 \mathrm{RCT}(\mathrm{n}=78)^{495} \\
2 \text { months, } 6 \text { months }\end{array}$ & CG/P & $\begin{array}{l}1 \text { found benefit at } 2 \text { months but } \\
\text { no difference at } 6\end{array}$ & Insufficient \\
\hline $\begin{array}{l}\text { Anxiety } \\
\text { Mindfulness vs } \\
\text { Active Control }\end{array}$ & $\begin{array}{l}1 \mathrm{RCT}(\mathrm{n}=78)^{495} \\
2 \text { months, } 6 \text { months }\end{array}$ & CG/P & $\begin{array}{l}1 \text { found benefit at } 2 \text { months but } \\
\text { no difference at } 6\end{array}$ & Insufficient \\
\hline $\begin{array}{l}\text { Mental Health } \\
\text { Mindfulness vs } \\
\text { Active Control }\end{array}$ & $\begin{array}{l}1 \mathrm{RCT}(\mathrm{n}=78)^{495} \\
2 \text { months, } 6 \text { months }\end{array}$ & $\mathrm{CG} / \mathrm{P}$ & $\begin{array}{l}1 \text { found benefit } \\
0 \text { found no difference }\end{array}$ & Insufficient \\
\hline $\begin{array}{l}\text { Physical Health } \\
\text { Mindfulness vs } \\
\text { Active Control }\end{array}$ & $\begin{array}{l}1 \mathrm{RCT}(\mathrm{n}=78)^{495} \\
2 \text { months, } 6 \text { months }\end{array}$ & CG/P & $\begin{array}{l}0 \text { found benefit } \\
1 \text { found no difference }\end{array}$ & Insufficient \\
\hline $\begin{array}{l}\text { Social Support } \\
\text { Mindfulness vs } \\
\text { Active Control }\end{array}$ & $\begin{array}{l}1 \mathrm{RCT}(\mathrm{n}=78)^{495} \\
2 \text { months, } 6 \text { months }\end{array}$ & $\mathrm{CG} / \mathrm{P}$ & $\begin{array}{l}0 \text { found benefit } \\
1 \text { found no difference }\end{array}$ & Insufficient \\
\hline
\end{tabular}

*Insufficient ratings due to study limitations and imprecision in the findings.

Abbreviations: $\mathrm{CG} / \mathrm{P}=$ caregiver/care partner; $\mathrm{n}=$ number; $\mathrm{RCT}=$ randomized controlled trial

\section{Variation in Outcomes}

The analytic study set did not report variation in outcomes by PLWD, caregiver, or intervention characteristics.

\section{Physical Activity and Other Lifestyle Interventions}

\section{Key Point}

- No physical activity or other lifestyle interventions advanced from the evidence map for further analysis.

\section{Intervention Description}

Physical activity interventions may involve caregivers alone or caregiver/PLWD dyads, and often involve an interventionist encouraging (in person or via phone or through written materials) physical activity, helping with goal setting, and/or supervising the implementation of assigned physical activity.

\section{Eligible Studies}

We identified five unique studies from six publications on physical activity interventions ${ }^{505-}$ 509 and four unique studies from four publications focused on other various lifestyle interventions for CG/P. ${ }^{510-513}$ Table 6.8 summarizes the characteristics of the literature set. No studies were eligible for the analytic set. Four studies were assessed as high risk of bias due to potential performance bias. ${ }^{505-507,509}$ The remaining study was a pilot. ${ }^{508}$ Appendix E presents information on all studies as part of the evidence map. 
Table 6.8. Basic characteristics of literature set: physical activity and other lifestyle interventions

\begin{tabular}{|l|l|}
\hline \multicolumn{1}{|c|}{ Characteristic } & \multicolumn{1}{c|}{ Information } \\
\hline Total Studies & 9 studies \\
\hline Non-U.S. studies & 5 studies \\
\hline Evidence map studies & 2 pilot study \\
& 7 high risk of bias studies \\
\hline Analytic set studies & 0 studies \\
\hline
\end{tabular}

\section{Intervention Research Context}

Studies were published between 2002 and 2018. Generally, this literature did not progress along the NIH Stage Model, as we identified only two pilot studies. One study enrolled CG/P and PLWD dyads to jointly participate in physical activity. ${ }^{509}$ Of the five studies that examined physical activity, three focused on phone-based physical activity interventions. ${ }^{505-507}$ Phone contact was used to encourage physical activity, set goals, and/or supervise the implementation of assigned physical activity. The remaining two physical activity studies assigned CG/P a physical activity regimen without the use of phone support. ${ }^{508,509}$ Of four studies that examined leisure interventions, two focused on leisure activities or leisure education for CG/P, ${ }^{510,513}$ while two others enrolled CG/P and PLWD dyads. ${ }^{511,512}$ Of the dyad studies, one study compared a structured visual arts education program (with visual arts projects of increasing difficulty) for CG/P and PLWD versus painting and discussion about art. ${ }^{511}$ The second study compared providing assistance to female spouse caregivers and PLWD while on vacation versus a waitlist control. ${ }^{512}$

\section{Respite Care}

\section{Key Point}

- No respite care interventions advanced from the evidence map for further analysis.

\section{Intervention Description}

Respite care interventions provide temporary breaks to caregivers of PLWD. Interventions include in-home care for PLWD, adult day care programs, and institutional respite services. These interventions provide breaks for a few hours a day or allow individuals to take a full break from caregiving for a short-term period of time.

\section{Eligible Studies}

We identified three unique studies from four publications (Table 6.9). ${ }^{514-517}$ All three were assessed as high risk of bias due to potential attrition bias, and were not included in the analytic set. Two studies used a quasi-experimental study design. ${ }^{515-517}$ The remaining study was an RCT. ${ }^{514}$ We present information on all high risk of bias studies as part of the evidence map in Appendix E.

Table 6.9. Basic characteristics of literature set: respite care

\begin{tabular}{|l|l|}
\hline \multicolumn{1}{|c|}{ Characteristic } & \multicolumn{1}{c|}{ Information } \\
\hline Total Studies & 3 studies \\
\hline Non-U.S. studies & 1 study \\
\hline Evidence map studies & 3 high risk of bias \\
\hline Analytic set studies & 0 studies \\
\hline
\end{tabular}




\section{Intervention Research Context}

The studies were published between 1989 and 2019 and compared a range of respite care activities for CG/P versus usual care. One study examined in-home 24-hour respite care for 5 to 14 days, ${ }^{515,} 516$ one study examined adult day care for PLWD (twice or more per week), ${ }^{517}$ and one study examined a program offering in-home day care versus institutional respite services used at the discretion of CG/P. ${ }^{514}$

\section{Multicomponent Interventions}

\section{Key Point}

- Intensive multicomponent intervention with education, group discussion, in-home and phone support sessions, and caregiver feedback for CG/P support (i.e. discrete adaptations of REACH II), improved CG/P depression at 6 months.(low-strength evidence)

- Evidence was insufficient to draw conclusions about the effect of other forms of multicomponent interventions on PLWD and their CG/P.

\section{Intervention Description}

Multicomponent interventions include more than one care technique or delivery method to foster support, expertise, information, or skills for caregivers in order to improve caregiver quality of life and health outcomes. Care techniques and delivery methods found in multicomponent interventions include therapy and support such as counseling (in groups and/or one-on-one, phone-based and/or in person), training (in person with key personnel, one-on-one and/or paired, group, or classroom style, and/or via written materials), supportive feedback, goalsetting, and planning (in any setting, with family members, in groups, and in person or remote/via phone), as well as relaxation and physical exercises. Offered as structured, programmatic approaches to strengthen communication, develop resources and skills, and/or create or maintain relationships for caregivers and PLWD, multicomponent interventions may benefit informal (family) caregivers.

\section{Eligible Studies}

Multicomponent interventions targeting quality of life and other outcomes for informal and formal caregivers included seven studies of three different multicomponent interventions across 22 publications on multicomponent interventions (Table 6.10). ${ }^{383,518-540}$ All seven multicomponent interventions in the analytic set targeted outcomes for caregivers by offering multiple intervention components within a structured, programmatic approach for communitydwelling caregivers living with PLWD. We provide information on all pilot studies and high risk of bias studies as part of the evidence map in Appendix E. Appendix E also provides evidence tables, summary risk of bias assessments, and strength of evidence for key comparisons and outcomes.

Table 6.10. Basic characteristics of literature set: multicomponent for CG/P

\begin{tabular}{|l|l|}
\hline \multicolumn{1}{|c|}{ Characteristic } & \multicolumn{1}{c|}{ Information } \\
\hline Total unique studies & 22 studies \\
\hline Non-U.S. studies & 13 studies \\
\hline
\end{tabular}




\begin{tabular}{|c|c|}
\hline Characteristic & Information \\
\hline Total Evidence map studies & $\begin{array}{l}4 \text { pilot studies } \\
2 \text { small sample studies } \\
9 \text { high risk of bias studies }\end{array}$ \\
\hline Analytic set studies & $\begin{array}{l}6 \text { randomized controlled trials } \\
1 \text { pragmatic trial }\end{array}$ \\
\hline Risk of bias of analytic set & 7 medium/low \\
\hline Number of PLWD in analytic set & 1688 \\
\hline Dementia type/definition & All types of dementia with all stages of severity \\
\hline Caregiver type (number) & Informal caregivers (1688) \\
\hline
\end{tabular}

Abbreviation: PLWD=people living with dementia

\section{Intervention Research Context}

Studies published from 1993 to 2017 tended to be small or high risk of bias, and largely based on pilot or explanatory research. More trials of structured multicomponent interventions, focusing on how treatments correspond to improvement in outcomes for caregivers (via intervention for caregiver and PLWD dyads) appeared in six of the studies.

The literature in the evidence map and the analytic set contains a considerable amount of preliminary work. However, we observed more development along the NIH Stage Model in this set than in most other intervention categories. This literature set demonstrates growth over time toward the development of both pragmatic trials as well as dissemination/implementation research. Specific examples include the REACH II intervention ${ }^{519,520}$ and the RDAD dualcomponent intervention. ${ }^{535,537}$

\section{PLWD Outcomes}

One study reported benefits for PLWD physical role functioning and depression. ${ }^{541}$ Evidence was insufficient to draw conclusions about PLWD outcomes.

\section{Caregiver Outcomes}

Table 6.11 provides a summary of findings. Structured multicomponent interventions presented low-strength evidence for improving caregiver depression. ${ }^{519,520,528}$

Three studies tested an intensive multicomponent intervention aimed at improving outcomes for community-dwelling informal (family) caregivers. The intervention incorporated education, group discussion, in-home and phone support sessions, and caregiver feedback, four components, for CG/P support across a range of characteristics. ${ }^{519,520,528}$ This intervention was developed to manage behavioral problems, reduce functional dependence, and prevent functional impairment. Low-strength evidence showed reduced depression for CG/P. One U.S.-based study found moderate effect size for depression at 6 months. ${ }^{482}$ This result was consistent with a second study that found no difference between groups but improvement over baseline in both intervention and control groups. ${ }^{528}$ For outcomes including caregiver quality of life, stress, burden, caregiver support, and nursing home placement, we found too much variation in outcomes definition, measurement, and reporting within ${ }^{482}$ and across studies ${ }^{482,483,491}$ to draw conclusions. An assessment of insufficient evidence does not mean that the intervention is determined to be of no value. Rather, it means that due to the uncertainty of the evidence we could not draw meaningful conclusions at this time.

Evidence was insufficient from three studies to assess one intervention that used a structured, three-component approach comprising in-person counseling sessions, family counseling sessions 
involving family members whom caregivers invited, and phone counseling sessions for caregivers on-demand across three countries. Evidence was also insufficient from two studies for one intervention examining combined exercise and CG/P support.

Table 6.11. Summary of findings for caregiver outcomes: multicomponent for CG/P

\begin{tabular}{|c|c|c|c|c|}
\hline $\begin{array}{l}\text { Outcome } \\
\text { Comparison }\end{array}$ & $\begin{array}{l}\text { \# Studies/ Design } \\
\text { (n analyzed) } \\
\text { Timing }\end{array}$ & Population & Findings & $\begin{array}{c}\text { Strength } \\
\text { of } \\
\text { Evidence* }^{*}\end{array}$ \\
\hline $\begin{array}{l}\text { Health } \\
\text { Combined exercise \& support } \\
\text { vs. monthly phone calls \& } \\
\text { mailed bulletins }\end{array}$ & $\begin{array}{l}1 \mathrm{RCT}^{535,541} \\
(\mathrm{n}=153) \\
12 \text { weeks }\end{array}$ & $\begin{array}{l}\text { Community-dwelling } \\
\text { PLWD and Informal } \\
\text { (family) caregivers }\end{array}$ & $\begin{array}{l}1 \text { found benefit } \\
0 \text { found no } \\
\text { difference }\end{array}$ & Insufficient \\
\hline $\begin{array}{l}\text { Depression } \\
\text { Counseling sessions (in- } \\
\text { person, family, and phone) for } \\
\text { caregivers vs. usual care }\end{array}$ & $\begin{array}{l}1 \mathrm{RCT}^{528,531,533} \\
(\mathrm{n}=371) \\
\text { 4-6 months }\end{array}$ & $\begin{array}{l}\text { Spouse caregivers } \\
\text { living at home with } \\
\text { PLWD }\end{array}$ & $\begin{array}{l}1 \text { found benefit } \\
0 \text { found no } \\
\text { difference }\end{array}$ & Insufficient \\
\hline $\begin{array}{l}\text { Caregiver stress perception } \\
\text { Counseling sessions (in- } \\
\text { person, family, and phone) for } \\
\text { caregivers vs. usual care }\end{array}$ & $\begin{array}{l}1 \mathrm{RCT}^{528,531,533} \\
(\mathrm{n}=158) \\
\text { 4-6 months }\end{array}$ & $\begin{array}{l}\text { Spouse caregivers } \\
\text { living at home with } \\
\text { PLWD }\end{array}$ & $\begin{array}{l}1 \text { found benefit } \\
0 \text { found no } \\
\text { difference }\end{array}$ & Insufficient \\
\hline $\begin{array}{l}\text { Depression } \\
\text { Education, group discussion, } \\
\text { in-home and phone support } \\
\text { sessions, and caregiver } \\
\text { feedback vs usual care }^{\star \star}\end{array}$ & $\begin{array}{l}3 \text { RCT 519, 520, } 528 \\
(n=895) \\
6 \text { months }\end{array}$ & $\begin{array}{l}\text { Family caregivers } \\
\text { (living with or sharing } \\
\text { cooking) with PLWD }\end{array}$ & $\begin{array}{l}2 \text { of } 3 \text { found } \\
\text { benefit } \\
1 \text { of } 3 \text { found no } \\
\text { difference }\end{array}$ & $\begin{array}{l}\text { Low } \\
\text { discrete } \\
\text { adaptations } \\
\text { of REACH } \\
\text { II }\end{array}$ \\
\hline $\begin{array}{l}\text { Caregiver stress, burden } \\
\text { Education, group discussion, } \\
\text { in-home and phone support } \\
\text { sessions, and caregiver } \\
\text { feedback vs usual care }\end{array}$ & $\begin{array}{l}3 \mathrm{RCT}^{519,520,528} \\
(\mathrm{n}=895) \\
6 \text { months }\end{array}$ & $\begin{array}{l}\text { Family caregivers } \\
\text { (living with or sharing } \\
\text { cooking) with PLWD }\end{array}$ & $\begin{array}{l}1 \text { of } 3 \text { found } \\
\text { benefit } \\
2 \text { of } 3 \text { found no } \\
\text { difference }\end{array}$ & Insufficient \\
\hline
\end{tabular}

*Insufficient ratings due to study limitations and imprecision in the findings.

**Luchsinger et al. compares REACH II to New York University Caregiver interventions and finds no difference between groups but improvement over baseline in both groups.

Abbreviations: $n=$ number; PLWD=person/people living with dementia; RCT=randomized controlled trial; REACH II=Resources for Enhancing Alzheimer's Caregiver Health II

\section{Variation in Outcome}

Studies reported variation in benefit across racial and ethnic groups studied. ${ }^{519,528}$ In one study, spouse caregivers in the intervention experienced significantly greater improvement in quality of life than those in the control group for all included racial and ethnic groups (Hispanic or Latino, white, and black or African-American). ${ }^{519}$ Another study reported benefits for both a four-component intervention and a three-component intervention ${ }^{533}$ for Hispanic caregivers. ${ }^{528}$

\section{Other Interventions for Caregiver Well-Being}

\section{Key Point}

- Studies of several other types of interventions were described in the evidence map but not considered for analysis due to limitations in study designs.

\section{Intervention Description}

This set of diverse interventions included approaches such as support meetings, decision aids, virtual reality experience to increase empathy, and cranial electrical stimulation of the CG/P. 


\section{Eligible Studies}

We identified six unique studies from seven publications, each examining a unique intervention. ${ }^{542-547}$ We provide characteristics of the literature set in Table 6.12, and information on all studies in the evidence map in Appendix E.

Table 6.12. Basic characteristics of literature set: other interventions for CG/P

\begin{tabular}{|l|l|}
\hline \multicolumn{1}{|c|}{ Characteristic } & Information \\
\hline Total Studies & 6 studies \\
\hline Non-U.S. studies & 4 studies \\
\hline Evidence map studies & 3 pilot studies \\
& 3 high risk of bias studies \\
\hline Analytic set studies & 0 studies \\
\hline
\end{tabular}

\section{Intervention Research Context}

The literature set was highly varied, comprising unrelated studies that represented earlier stages of the NIH Stage Model. Two studies were conducted in the United States. One study examined support meetings for CG/P to assist in conversations with PLWD about driving cessation. ${ }^{546}$ The other study examined cranial electrical stimulation for CG/P to improve sleep. ${ }^{544}$ Four studies were conducted in non-U.S. settings. One study examined an aid for CG/P in Australia to make decisions about community services including respite care. ${ }^{547}$ Another study examined an aid for CG/P in the United Kingdom to make decisions about place of residence for PLWD. ${ }^{543}$ One study in the Netherlands examined virtual reality devices to give CG/P a simulated experience of having dementia. ${ }^{542}$ The last study, conducted in the United Kingdom, examined the use of a caregiver-held record folder that included sections for caregiver/family and professional feedback as well as a caregiver diary. ${ }^{545}$

\section{Conclusion}

We identified many studies with a large number of participants that investigated the efficacy of care interventions to improve the well-being of CG/P. Applying the framework for care interventions from the NASEM Families Caring for an Aging America 2016 report (Figure 1.1 in Chapter 1), this category included interventions delivered at two levels: the individual level (i.e., psychosocial programs, lifestyle interventions, and multicomponent interventions) and the social or community level (i.e., social support and respite care). The vast majority of studies investigated psychosocial interventions delivered at the individual level. Fewer investigated multicomponent and lifestyle interventions delivered at the individual level. Very few investigated social support and respite care delivered at the social or community level.

Using the NIH Stage Model as a guide, about one-third of studies were pilot studies, and almost all of the others were explanatory, or Stage 3. Only a few multicomponent interventions were Stage 4 pragmatic trials. Of the explanatory studies, two thirds were rated as high risk of bias and excluded from the analytic set. Ultimately, just over 20 percent of the literature was eligible for the analytic set.

Included studies investigated many types of PLWD and caregiver outcomes. Only psychosocial studies examined PLWD outcomes; the most common were function, neuropsychiatric symptoms, and proxy-rated quality of life. Several important PLWD outcomes, such as depression, healthcare use, social support, and nursing home admission, were infrequently investigated. All of the studies investigated outcomes for the CG/P; the most common were depression, quality of life, caregiving burden, and caregiving bother and distress. 
Several important CG/P outcomes, such as coping, caregiving knowledge, social support, and healthcare use, were infrequently investigated. Other important outcomes, such as those related to resilience and the positive aspects of caregiving, and social health outcomes, such as social isolation, were also missing from the literature.

While the literature set was large, the overwhelming majority of the evidence was insufficient to draw conclusions about whether or not these interventions work. We found, however, that one intensive multicomponent intervention (i.e. discrete adaptations of REACH II) did have low-strength evidence for improving depression among CG/P. Of all included interventions to improve caregiver well-being, multicomponent interventions used the most targeted components. Possibly, these comprehensive interventions addressed at least one critical need (across a wide range of individual caregiver needs), thus improving outcomes.

Multicomponent interventions were structured to provide the same components to all participants. Very few studies were delivered at the social and community level. Only 12 studies investigated social support, and just two of these had low to medium risk of bias. The three studies that examined respite care were all assessed as high risk of bias.

About half of the studies were conducted outside of the United States, and all but one of these occurred in high-resource countries. The vast majority of studies conducted in the United States enrolled white urban or suburban dwelling caregivers. Only a few studies investigated Hispanic and African American caregivers and caregivers living in rural and underserved urban areas.

Our assessment of the evidence as insufficient should not be interpreted to mean that we concluded these interventions do not work. Rather, it means that the findings were too uncertain to draw conclusions about their combined effect. Research on interventions to change behavioral and psychological outcomes is challenging, and many factors can influence the outcomes. The insufficient ratings were driven mainly by the inconsistency and imprecision of study findings. For almost every outcome investigated, findings from several trials were inconsistent; some studies found improvement for the intervention group, and some found no difference in outcomes between the intervention and comparison groups. For studies that found no difference, often the comparison groups scores improved more than the intervention group (although not enough to be statistically significant). The findings were also generally imprecise. Our ability to combine results from several studies was hampered by the heterogeneity of the intervention components, duration and intensity of treatment, and comparison groups. Our ability to combine results from several studies statistically was generally not possible, because studies rarely reported the data necessary to calculate effect sizes. 


\section{Chapter 7. Care Interventions for Formal Caregivers}

This chapter includes care interventions with elements designed to address the well-being of formal caregivers in the workplace. We present Key Points followed by results in three general sections: Intervention Description, Eligible Studies, and Intervention Research Context. Since no studies were assessed as low to medium risk of bias, we present the studies from the evidence map with a brief discussion of what has been examined, and research context. We present detailed information on all eligible studies in Appendix E.

\section{Formal Caregiver Well-Being}

\section{Key Point}

- Studies of interventions to improve formal caregiver well-being were described in the evidence map but not considered for analysis due to limitations in study designs.

\section{Intervention Description}

Interventions categorized as targeting formal caregiver well-being include components such as peer support, stress management, and relaxation techniques.

\section{Eligible Studies}

We identified three unique studies from five publications that examined interventions to improve the well-being of formal caregiving staff in long-term care facilities. ${ }^{548-552}$ (Table 7.1) All were pilot studies. We provide information on all studies in the evidence map in Appendix E.

Table 7.1. Basic characteristics of literature set: formal caregiver well-being

\begin{tabular}{|l|l|}
\hline \multicolumn{1}{|c|}{ Characteristics } & \multicolumn{1}{|c|}{ Information } \\
\hline Total unique studies & 3 studies \\
\hline Non-U.S. studies & 3 studies \\
\hline Evidence map studies & 3 pilot studies \\
\hline Analytic set studies & 0 \\
\hline
\end{tabular}

\section{Intervention Research Context}

The research is very preliminary, with small pilot studies conducted in non-U.S. settings from 2003 to 2015. Similarly to research conducted to support informal caregivers, the Canadian 2003 study tested the premise that education and training to improve skills will reduce formal caregiver stress (in this case as measured by burnout). ${ }^{551}$ Later studies examined more direct interventions such as peer support in Australia ${ }^{550,552}$ and stress management and relaxation techniques to reduce job-related stress and burnout in Portugal. ${ }^{548,549}$

\section{Conclusion}

The literature for formal caregivers is preliminary. Possibly, our search algorithm (designed to maximize the ability to find therapies for PLWD) limited our ability to locate related literature. We chose not to include the workforce literature, since this review is focused on the well-being of both PLWD and caregiver. We anticipated that this association would be more prominent in the informal caregiver literature, but not well-studied in the workforce literature. 


\section{Chapter 8. Care Delivery Interventions}

This chapter includes care delivery interventions intended to improve how care is delivered. These interventions target the organization of care, including the scheduling of staff and tasks as well as the ways in which staff and tasks are interdependent. While care delivery interventions may be implemented through education and training, they differ from most education and training in that they do not aim to improve already established roles and tasks. Instead, they aim to change the tasks that are performed, the set of staff who perform the tasks, or the way the staff work together. This chapter is organized into three categories: care service provision, care delivery models or programs, and care staff education and support needs.

For each intervention, we present Key Points followed by results in three general sections: Intervention Description, Eligible Studies, and Intervention Research Context. For interventions with no studies assessed as low to medium risk of bias, we present the studies from the evidence map with a brief discussion of what has been examined as well as the research context. For interventions for which low to medium risk of bias studies were available for an analytic set, we present Outcomes sections by people living with dementia (PLWD), by caregiver, and by variation in outcomes when available. We use the term caregiver/partner, or CG/P, for informal caregiver outcomes. Because we generally could not pool outcomes for any given intervention and comparison group, we synthesized the information qualitatively; therefore, we present summary findings as brief statements of how many studies reported statistically significant benefits or no statistically significant difference between the intervention and the comparator. We present detailed information on all eligible studies in Appendix F.

\section{Care Service Provision}

Care service provision refers to the act of providing care for the PLWD. In general business terms, specialized service provision usually requires qualified staff members to be regularly available to perform the services offered. The qualifications needed for staff will depend on the care being provided or supported.

\section{Consultation Services}

\section{Key Point}

- Evidence was insufficient to draw conclusions about the effect of consultation services on PLWD and their caregivers.

\section{Intervention Description}

Consultation services include individualized and multidisciplinary approaches targeting PLWD as well as their caregivers. Most of the consultants interacted with CG/P not only to improve well-being among PLWD, but also to improve the health of CG/P by improving their overall knowledge for managing dementia and reducing their burden and depression. While this category of interventions has the potential to be very broad, we identified only a few forms examined in the eligible literature, namely a dementia outreach service, individualized consultations, multidisciplinary care consultations, and a needs assessment for older adults. 


\section{Eligible Studies}

Table 8.1 summarizes the characteristics of the literature set. We identified five unique studies that examined the use of consultation interventions for improving quality of life and reducing hospitalizations and depression among PLWD, and improving efficiency of response among CG/P. ${ }^{553-557}$ Four studies were assessed as high risk of bias. ${ }^{553,}{ }^{555-557}$ Only one study was included in the analytic set. ${ }^{554}$ We present information on the high risk of bias studies in the evidence map in Appendix E.

The included study $(\mathrm{n}=84)$ was a randomized controlled trial targeted at family (informal) caregivers. It compared consultation services versus an attention control. ${ }^{554}$ The study was assessed as medium risk of bias and categorized as explanatory, or Stage 3 of the National Institutes of Health (NIH) Stage Model. Appendix C Tables provide evidence tables, summary risk of bias assessments, and strength of evidence for key comparisons and outcomes.

Table 8.1. Basic characteristics of literature set: consultation services

\begin{tabular}{|l|l|}
\hline \multicolumn{1}{|c|}{ Characteristics } & \multicolumn{1}{c|}{ Information } \\
\hline Total unique studies & 5 studies \\
\hline Non-U.S. studies & 4 studies \\
\hline Evidence map studies & 4 high risk of bias studies \\
\hline Analytic set studies & 1 randomized controlled trial \\
\hline Risk of bias of analytic set & 1 medium \\
\hline Number of PLWD in analytic set & 84 \\
\hline Dementia type/definition & All dementia types and severities \\
\hline Caregiver type (number) & Informal caregivers (84) \\
\hline
\end{tabular}

Abbreviation: PLWD=people living with dementia

\section{Intervention Research Context}

The literature on consultation services comprises mostly high risk of bias studies in non-U.S. locations. We identified a dementia outreach study led by nurse practitioners in Australia; the intervention aimed to assist staff at residential care facilities to better manage behavioral and psychological symptoms of dementia (BPSD). ${ }^{553}$ We also identified two other Australian studies that used multidisciplinary consultation approaches to help nursing home staff improve care in order to reduce PLWD depression ${ }^{555}$ and BPSD. ${ }^{556}$ An additional study conducted in the United Kingdom examined a liaison-mediated intervention to reduce unmet needs and improve quality of life among community-dwelling PLWD. ${ }^{557}$

\section{PLWD Outcomes}

Evidence was insufficient to draw conclusions about nursing home admission among PLWD who received individualized consultation versus attention control interventions ( $n=84)^{554}$ Table 8.2 summarizes the findings.

Table 8.2. Summary of findings for PLWD outcomes: consultation services

\begin{tabular}{|l|l|l|l|l|}
\hline \multicolumn{1}{|c|}{$\begin{array}{c}\text { Outcomes } \\
\text { Comparison }\end{array}$} & \multicolumn{1}{|c|}{$\begin{array}{c}\text { \# Studies/ Design } \\
\text { (n analyzed) } \\
\text { Timing }\end{array}$} & Population & Findings & $\begin{array}{c}\text { Strength of } \\
\text { Evidence* }\end{array}$ \\
\hline $\begin{array}{l}\text { Nursing home } \\
\text { admission } \\
\begin{array}{l}\text { Consultation vs Usual } \\
\text { Care }\end{array}\end{array}$ & $\begin{array}{l}1 \mathrm{RCT}^{554} \text { (n=84) } \\
12 \text { months }\end{array}$ & PLWD & $\begin{array}{l}\text { 0 found benefit } \\
1 \text { found no difference }\end{array}$ & Insufficient \\
\hline
\end{tabular}

*Insufficient ratings due to study limitations and imprecision in the findings. 
Abbreviations: $\mathrm{n}=$ number; PLWD=person/people living with dementia; RCT=randomized controlled trial

\section{Caregiver Outcomes}

Evidence was insufficient to draw conclusions about individualized consultation versus attention control ( $\mathrm{n}=84) .{ }^{554}$ Table 8.3 summarizes the findings. The study reported no statistically significant improvement among caregivers in physical health symptoms or self-efficacy for managing dementia, and no reduction in caregiver depression or care burden.

Table 8.3. Summary of findings for caregiver outcomes: consultation services

\begin{tabular}{|c|c|c|c|c|}
\hline $\begin{array}{l}\text { Outcome } \\
\text { Comparison }\end{array}$ & $\begin{array}{l}\text { \# Studies/ Design } \\
\text { (n analyzed) } \\
\text { Timing }\end{array}$ & Population & Findings & $\begin{array}{l}\text { Strength of } \\
\text { Evidence* }^{*}\end{array}$ \\
\hline $\begin{array}{l}\text { Physical health } \\
\text { Consultation vs } \\
\text { Usual Care }\end{array}$ & $\begin{array}{l}1 \mathrm{RCT}^{554}(\mathrm{n}=84) \\
12 \text { months }\end{array}$ & $\begin{array}{l}\text { Primary family } \\
\text { caregivers }\end{array}$ & $\begin{array}{l}0 \text { found benefit } \\
1 \text { found no difference }\end{array}$ & Insufficient \\
\hline $\begin{array}{l}\text { Self-efficacy score } \\
\text { Consultation vs } \\
\text { Usual Care }\end{array}$ & $\begin{array}{l}1 \mathrm{RCT}^{554}(\mathrm{n}=84) \\
12 \text { months }\end{array}$ & $\begin{array}{l}\text { Primary family } \\
\text { caregivers }\end{array}$ & $\begin{array}{l}0 \text { found benefit } \\
1 \text { found no difference }\end{array}$ & Insufficient \\
\hline $\begin{array}{l}\text { Depression } \\
\text { Consultation vs } \\
\text { Usual Care } \\
\end{array}$ & $\begin{array}{l}1 \mathrm{RCT}^{554}(\mathrm{n}=84) \\
12 \text { months }\end{array}$ & $\begin{array}{l}\text { Primary family } \\
\text { caregivers }\end{array}$ & $\begin{array}{l}0 \text { found benefit } \\
1 \text { found no difference }\end{array}$ & Insufficient \\
\hline $\begin{array}{l}\text { Burden } \\
\text { Consultation vs } \\
\text { Usual Care }\end{array}$ & $\begin{array}{l}1 \mathrm{RCT}^{554}(\mathrm{n}=84) \\
12 \text { months }\end{array}$ & $\begin{array}{l}\text { Primary family } \\
\text { caregivers }\end{array}$ & $\begin{array}{l}0 \text { found benefit } \\
1 \text { found no difference }\end{array}$ & Insufficient \\
\hline
\end{tabular}

*Insufficient ratings due to study limitations and imprecision in the findings.

Abbreviations: n=number; PLWD=person/people living with dementia; RCT=randomized controlled trial

\section{Variation in Outcomes}

Studies in the analytic set did not examine variation in outcomes by PLWD, caregiver, or intervention characteristics.

\section{Case Management}

\section{Key Point}

- Evidence was insufficient to draw conclusions about the effect of case management for PLWD and their caregivers.

\section{Intervention Description}

Case management is a service to support CG/P and their PLWD care recipient. Case managers help coordinate health and social services to support PLWD and their caregivers. Case managers commonly work with CG/P while the PLWD is still living in the community, although case management can also be engaged in nursing homes or other long-term care facilities. We categorized an intervention as case management if the study did not report the case or care manager as being embedded in a team-based care approach.

\section{Eligible Studies}

Table 8.4 summarizes the characteristics of the literature set. We identified nine unique studies from 12 publications that examined the use of case management for improving health outcomes for PLWD and their caregivers. Three studies, all non-U.S. settings, were assessed as 
low to medium risk of bias and included in the analytic set. ${ }^{558-561}$ Excluded from the analytic set were high risk of bias studies, ${ }^{562-567}$ which we describe in the evidence map in Appendix F.

Evidence tables, summary risk of bias assessments, and strength of evidence for key

comparisons and outcomes for the analytic set are also in Appendix F.

Table 8.4. Basic characteristics of literature set: case management

\begin{tabular}{|l|l|}
\hline \multicolumn{1}{|c|}{ Characteristics } & \multicolumn{1}{c|}{ Information } \\
\hline Total unique studies & 9 studies \\
\hline Non-U.S. studies & 6 studies \\
\hline Evidence map studies & $\begin{array}{l}\text { 1 pilot/demonstration study } \\
5 \text { high risk of bias studies }\end{array}$ \\
\hline Analytic set studies & 3 (non-U.S.) studies \\
\hline Risk of bias of analytic set & Medium \\
\hline Number of PLWD in analytic set & 294 \\
\hline Dementia type/definition & Alzheimer's disease or dementia unspecified \\
\hline Caregiver type (number) & Informal caregivers (294) \\
\hline
\end{tabular}

Abbreviation: PLWD=people living with dementia

\section{Intervention Research Context}

Eligible studies on case management were published between 1993 and 2016, although the analytic set was published between 2001 and 2011. The majority were non-U.S. settings, including Australia, ${ }^{562}$ Hong Kong, ${ }^{558,} 559,561$ Finland, ${ }^{560}$ and the Netherlands. ${ }^{564}$ One study compared case management versus consultation services in a nursing home. ${ }^{562}$ The remaining studies were community-based. No particular research program in this literature set showed evolution over time, although the majority of studies were explanatory, or Stage 3 of the NIH Stage Model.

\section{PLWD Outcomes}

Evidence was insufficient to assess the effect of case management on PLWD outcomes. Table 8.5 provides the summary findings. All studies reported outcomes for PLWD. ${ }^{558-561}$ Studies reported mixed results for rate of institutionalization ${ }^{558-560}$ and change in dementia symptoms. ${ }^{558,559,561}$ Groups showed no statistically significant difference for quality of life or depression. ${ }^{561}$

Table 8.5. Summary of findings for PLWD outcomes: case management

\begin{tabular}{|c|c|c|c|c|}
\hline $\begin{array}{l}\text { Outcome } \\
\text { Comparison }\end{array}$ & $\begin{array}{l}\text { \# Studies/Design } \\
\text { (n analyzed) } \\
\text { Timing }\end{array}$ & Population & Findings & $\begin{array}{l}\text { Strength of } \\
\text { Evidence* }\end{array}$ \\
\hline $\begin{array}{l}\text { Institutionalization } \\
\text { Case management vs } \\
\text { usual care }\end{array}$ & $\begin{array}{l}2 \mathrm{RCTs}^{558,560} \\
(\mathrm{n}=192) \\
18-24 \text { months }\end{array}$ & $\begin{array}{l}\text { PLWD dementia } \\
\text { unspecified }\end{array}$ & $\begin{array}{l}1 \text { of } 2 \text { found benefit } \\
1 \text { of } 2 \text { found no difference }\end{array}$ & Insufficient \\
\hline $\begin{array}{l}\mathrm{NPI} \\
\text { Case management vs } \\
\text { usual care }\end{array}$ & $\begin{array}{l}2 \mathrm{RCT}^{558,561}(\mathrm{n}=194) \\
12-18 \text { months }\end{array}$ & $\begin{array}{l}\text { PLWD dementia } \\
\text { unspecified }\end{array}$ & $\begin{array}{l}1 \text { of } 2 \text { found benefit } \\
1 \text { of } 2 \text { found no difference }\end{array}$ & Insufficient \\
\hline $\begin{array}{l}\text { Quality of Life } \\
\text { Case management vs } \\
\text { usual care }\end{array}$ & $\begin{array}{l}1 \mathrm{RCT}^{561}(\mathrm{n}=102) \\
12 \text { months }\end{array}$ & $\begin{array}{l}\text { PLWD dementia } \\
\text { unspecified }\end{array}$ & $\begin{array}{l}0 \text { found benefit } \\
1 \text { found no difference }\end{array}$ & Insufficient \\
\hline $\begin{array}{l}\text { Depression } \\
\text { Case management vs } \\
\text { usual care }\end{array}$ & $\begin{array}{l}1 \mathrm{RCT}^{561}(\mathrm{n}=102) \\
12 \text { months }\end{array}$ & $\begin{array}{l}\text { PLWD dementia } \\
\text { unspecified }\end{array}$ & $\begin{array}{l}0 \text { found benefit } \\
1 \text { found no benefit }\end{array}$ & Insufficient \\
\hline
\end{tabular}

*Insufficient ratings due to study limitations and imprecision in the findings. 
Abbreviations: n=number; NPI=Neuropsychiatric Inventory; PLWD=person/people living with dementia; RCT=randomized controlled trial

\section{Caregiver Outcomes}

Evidence was insufficient to assess the effect of case management on CG/P outcomes. Table 8.6 summarizes the primary outcomes for CG/P. Two studies reported outcomes for CG/P. ${ }^{558,559 \text {, }}$ ${ }^{561}$ The studies found mixed results for quality of life and caregiving burden. One study reported no statistically significant difference between groups for caregiver health status. ${ }^{561}$

Table 8.6. Summary of findings for caregiver outcomes: case management

\begin{tabular}{|c|c|c|c|c|}
\hline $\begin{array}{l}\text { Outcome } \\
\text { Comparison }\end{array}$ & $\begin{array}{c}\text { \# Studies/Design } \\
\text { (n analyzed) } \\
\text { Timing }\end{array}$ & Population & Findings & $\begin{array}{l}\text { Strength of } \\
\text { Evidence* }^{*}\end{array}$ \\
\hline $\begin{array}{l}\text { Quality of Life } \\
\text { Case management } \\
\text { vs usual care }\end{array}$ & $\begin{array}{l}2 \mathrm{RCT}^{558,561}(\mathrm{n}=194) \\
12-18 \text { months }\end{array}$ & CG/P & $\begin{array}{l}1 \text { found benefit } \\
1 \text { found no } \\
\text { difference }\end{array}$ & Insufficient \\
\hline $\begin{array}{l}\text { Caregiver Burden } \\
\text { Case management } \\
\text { vs usual care }\end{array}$ & $\begin{array}{l}2 \mathrm{RCT}^{558,561}(\mathrm{n}=194) \\
12-18 \text { months }\end{array}$ & CG/P & $\begin{array}{l}1 \text { found benefit } \\
1 \text { found no } \\
\text { difference }\end{array}$ & Insufficient \\
\hline $\begin{array}{l}\text { Caregiver health } \\
\text { status } \\
\text { Case management } \\
\text { vs usual care }\end{array}$ & $\begin{array}{l}1 \mathrm{RCT}(\mathrm{n}=102) \\
12 \text { months }\end{array}$ & CG/P & $\begin{array}{l}0 \text { found benefit } \\
1 \text { found no } \\
\text { difference }\end{array}$ & Insufficient \\
\hline
\end{tabular}

*Insufficient ratings due to study limitations and imprecision in the findings.

Abbreviations: CG/P=caregiver/care partner; $\mathrm{n}=$ number; $\mathrm{RCT}=$ randomized controlled trial

\section{Variation in Outcomes}

Studies in the analytic set did not examine variation in outcomes by PLWD, caregiver, or intervention characteristics.

\section{Care Protocols for PLWD}

\section{Key Point}

- Studies of care protocols for PLWD were described in the evidence map but not considered for analysis due to limitations in study designs.

\section{Intervention Description}

Interventions in this section targeted the health system level and aimed to improve quality of care through the implementation of protocols. Generally speaking, protocols are rules and procedures for providing care in a health system or in units within an organization. They also specify the tools and tasks to be carried out, and can therefore help formal caregiving staff, regardless of their length of service or experience, understand the processes. Protocols can be based on published guidelines or other sources for what are considered best practices. Staff training is an essential process for implementing protocols. Care protocols can be wide ranging, from decision trees to help formal caregivers systematize care decisions to protocols to improve nutrition care. Many protocols addressed agitation in nursing home settings. 


\section{Eligible Studies}

We identified 17 unique studies from 25 publications that examined the impact of training and delivery of evidence-based guidelines/protocols targeted toward PLWD. (Table 8.7) We provide information on all studies in the evidence map in Appendix Tables F. ${ }^{568-583}$

Table 8.7. Basic characteristics of literature set: care protocols for PLWD

\begin{tabular}{|l|l|}
\hline \multicolumn{1}{|c|}{ Characteristics } & \multicolumn{1}{c|}{ Information } \\
\hline Total unique studies & 17 studies \\
\hline Non-U.S. studies & 11 studies \\
\hline Evidence map studies & 6 pilot or feasibility studies \\
& 11 high risk of bias \\
\hline Analytic set studies & 0 \\
\hline
\end{tabular}

\section{Intervention Research Context}

Eligible studies on case management were published between 2006 and 2019. The majority were non-U.S. settings, including Australia, ${ }^{575}$ Spain, ${ }^{580}$ Germany, ${ }^{579}$ Norway, ${ }^{584}$ Taiwan, ${ }^{261}$ and the Netherlands. ${ }^{568,581,582}$ Using the NIH Stage Model as a framework for assessment, this literature set comprised Stage 3 explanatory studies. A few studies built directly upon a prior publication, showing some evolution of the research over time. With the exception of the one nutrition protocol, ${ }^{580}$ the non-U.S. studies were published in the last 6 years and focused on preventing or addressing BPSD in nursing home settings. While also focused on nursing home settings, U.S.-based studies tended to be older publications, published between 2006 and 2012. However, one U.S.-based study tested a decision algorithm to help care managers create care plans to reduce burden and depression among CG/P in the community. ${ }^{576}$

\section{Advance Care Planning}

\section{Key Point}

- Evidence was insufficient to draw conclusions about the effect of decision support tools for advance care planning on PLWD and their caregivers.

\section{Intervention Description}

Advance care planning is a process that broadly involves individuals making healthcare decisions for themselves or for others about future healthcare needs. Within this section, studies focused on family members/caregivers participating in advance care planning as decisionmakers for PLWD unable to make their own decisions. Advance care planning interventions can include a range of tools such as decision support tools or treatment plans, and they usually include some form of discussion with healthcare professionals. Studies in our analytic set focused on the use of decision support tools for advance care planning.

\section{Eligible Studies}

We identified nine unique studies from 12 publications. ${ }^{585-594}$ Table 8.8 summarizes the characteristics of the literature set. Three studies were pilots $587,589,593,595$ and an additional three were assessed as high risk of bias, therefore these six studies are not part of the analytic set. ${ }^{588 \text {, }}$ 590-592 We provide information about them in the evidence map in Appendix F.

One study examining the use of decision support tools in advance care planning was assessed as medium risk of bias and is the analytic set. ${ }^{585,586,596-598}$ This study enrolled PLWD with 
advanced dementia and their surrogates, and it examined a brief video decision support tool focused on advance directives, goals of care, and burdensome treatments. ${ }^{585,586}$ Appendix F provides evidence tables, summary risk of bias assessments, and strength of evidence for key comparisons and outcomes.

Table 8.8. Basic characteristics of literature set: advance care planning

\begin{tabular}{|l|l|}
\hline \multicolumn{1}{|c|}{ Characteristic } & \multicolumn{1}{c|}{ Information } \\
\hline Total Studies & 9 studies \\
\hline Non-U.S. studies & 3 studies \\
\hline Evidence map studies & $\begin{array}{l}5 \text { pilot studies } \\
\text { 3 high risk of bias studies }\end{array}$ \\
\hline Analytic set studies & 1 cluster randomized controlled trial \\
\hline Risk of bias of analytic set & Medium \\
\hline Number of PLWD in analytic set & 402 \\
\hline Dementia type/definition & Advanced dementia (type not specified) \\
\hline Caregiver type (number) & Informal caregivers (402) \\
\hline
\end{tabular}

Abbreviation: PLWD=people living with dementia

\section{Intervention Research Context}

Eligible studies on advance care planning were published between 2011 and 2019 and were primarily conducted in the United States. One study took place in an adult-day care setting among African American family caregivers. ${ }^{587}$ The remaining studies took place in nursing homes. The fairly recent publication dates of this literature set and the prevalence of pilot studies may reflect increased interest over the last decade in person-centered care and decision support tools. Four of eight eligible studies examined the use of decision support tools. ${ }^{585,586,589-591}$ The other four studies examined advance care planning conversations between health professionals and CG/P or decision-makers incorporating education, treatment plans, and other tools. ${ }^{587,588,592,}$ 593, 595

\section{PLWD Outcomes}

Evidence was insufficient to assess the effect of advance care planning decision support tools on PLWD outcomes. Table 8.9 summarizes the primary outcomes for PLWD. One study (n=402) reported hospitalization rates at 12 months, finding them similar between the decision support and usual care groups. ${ }^{586}$

Table 8.9. Summary of findings for PLWD outcomes: advance care planning

\begin{tabular}{|l|l|l|l|l|}
\hline \multicolumn{1}{|c|}{$\begin{array}{c}\text { Outcome } \\
\text { Comparator }\end{array}$} & \multicolumn{1}{|c|}{$\begin{array}{c}\text { \# Studies/Design } \\
\text { (n analyzed) } \\
\text { Timing }\end{array}$} & Population & \multicolumn{1}{|c|}{ Findings } & $\begin{array}{c}\text { Strength of } \\
\text { Evidence* }\end{array}$ \\
\hline $\begin{array}{l}\text { Hospitalizations } \\
\text { Advance care planning } \\
\text { decision support tool vs } \\
\text { usual care }\end{array}$ & $\begin{array}{l}1 \text { cluster RCT } \mathrm{RC}^{586} \\
(\mathrm{n}=402) \\
12 \text { months }\end{array}$ & $\begin{array}{l}\text { Advanced } \\
\text { dementia }\end{array}$ & $\begin{array}{l}0 \text { found benefit } \\
1 \text { found no difference }\end{array}$ & Insufficient \\
\hline
\end{tabular}

*Insufficient ratings due to study limitations and imprecision in the findings.

Abbreviations: $\mathrm{n}=$ number; PLWD=person/people living with dementia; RCT=randomized controlled trial

\section{Caregiver Outcomes}

Evidence was insufficient to assess the effect of advance care planning decision support tools on CG/P outcomes. Table 8.10 summarizes the primary outcomes for CG/P. One study (n=402) reported outcomes for whether CG/P completed an advance directive for no hospitalization on 
behalf of PLWD, participated in a goals of care discussion, or stated a preference for comfort care (over basic or intensive care). Groups did not differ for any outcome at 6 or 12 months. ${ }^{586}$

Table 8.10. Summary of findings for caregiver outcomes: advance care planning

\begin{tabular}{|c|c|c|c|c|}
\hline Outcome & $\begin{array}{c}\text { \# Studies/Design } \\
\text { (n analyzed) } \\
\text { Timing }\end{array}$ & Population & Findings & $\begin{array}{l}\text { Strength of } \\
\text { Evidence* }^{*}\end{array}$ \\
\hline $\begin{array}{l}\text { Documented Do Not } \\
\text { Hospitalize Directive } \\
\text { ACP decision } \\
\text { support tool vs usual } \\
\text { care }\end{array}$ & $\begin{array}{l}1 \text { cluster } \mathrm{RCT}^{586}(\mathrm{n}=402) \\
6 \text { months, } 12 \text { months }\end{array}$ & Advanced dementia & 1 found no benefit. & Insufficient \\
\hline $\begin{array}{l}\text { Goal of care } \\
\text { discussions } \\
\text { ACP decision } \\
\text { support tool vs usual } \\
\text { care }\end{array}$ & $\begin{array}{l}1 \text { cluster } \mathrm{RCT}^{586}(\mathrm{n}=402) \\
6 \text { months, } 12 \text { months }\end{array}$ & Advanced dementia & 1 found no benefit. & Insufficient \\
\hline $\begin{array}{l}\text { Proxies Preferring } \\
\text { Comfort Care } \\
\text { ACP decision } \\
\text { support tool vs usual } \\
\text { care }\end{array}$ & $\begin{array}{l}1 \text { cluster } \mathrm{RCT}^{586}(\mathrm{n}=402) \\
6 \text { months, } 12 \text { months }\end{array}$ & Advanced dementia & 1 found no benefit. & Insufficient \\
\hline
\end{tabular}

Abbreviations: $\mathrm{ACP}=$ advance care planning; $\mathrm{n}=$ number; $\mathrm{RCT}=$ randomized controlled trial

\section{Variation in Outcomes}

The analytic set study did not examine variation in outcomes by PLWD, caregiver, or intervention characteristics.

\section{Palliative Care}

\section{Key Point}

- Studies of palliative care for PLWD were described in the evidence map but not considered for analysis due to limitations in study designs.

\section{Intervention Description}

Palliative care interventions aim improve symptom relief, distress, hospitalization burden, and comfort with death among PLWD and their caregivers.

\section{Eligible Studies}

We identified five unique studies from six publications that examined the use of palliative care interventions among PLWD. ${ }^{599-604}$ (Table 8.11) All studies were either pilots or assessed as high risk of bias. We present information on all studies in the evidence map in Appendix F.

Table 8.11. Basic characteristics of literature set: palliative care

\begin{tabular}{|l|l|}
\hline \multicolumn{1}{|c|}{ Characteristics } & \multicolumn{1}{c|}{ Information } \\
\hline Total unique studies & 5 studies \\
\hline Non-U.S. studies & 3 studies \\
\hline Evidence map studies & 1 pilot study \\
& 4 high risk of bias studies \\
\hline Analytic set studies & 0 \\
\hline
\end{tabular}




\section{Intervention Research Context}

Eligible studies on palliative care were published between 2000 and 2019. The three nonU.S. studies were set in nursing homes. ${ }^{599,600,602,604}$ One examined case conferencing in Australia, ${ }^{599,} 600$ one examined a multicomponent intervention that included training, communication, routine palliative care tasks, and a nurse facilitator in Canada, ${ }^{604}$ and one compared general versus personalized feedback for end-of-life care in the Netherlands. ${ }^{602}$ The U.S.-based studies examined palliative care triggered by acute hospital stays for PLWD. ${ }^{601,603}$

\section{Other Service Provision Interventions}

\section{Key Point}

- Evidence was insufficient to draw conclusions about the effect of other service provision interventions on PLWD or their caregivers.

\section{Intervention Description}

This set of diverse interventions included approaches such as decision aids, technology to assist CG/P, and a tool-kit to assist CG/P in improving the safety of the home for PLWD.

\section{Eligible Studies}

We identified five unique studies from seven publications. ${ }^{596-598,605-608}$ Table 8.12 summarizes the characteristics of the literature set. Two studies were a pilot ${ }^{605,608}$ and two were assessed as high risk of bias; ${ }^{606,607}$ these studies were therefore excluded from the analytic set. We provide information about them in the evidence map in Appendix F.

One study was assessed as medium risk of bias and was classified as an NIH Stage 3 explanatory study. This study enrolled PLWD with advanced dementia and feeding issues, along with their surrogates, to compare a print decision aid for feeding options versus usual care. ${ }^{\text {596-598 }}$ Appendix F provides evidence tables, summary risk of bias assessments, and strength of evidence for key comparisons and outcomes.

Table 8.12. Basic characteristics of literature set: other service provision interventions

\begin{tabular}{|l|l|}
\hline \multicolumn{1}{|c|}{ Characteristic } & \multicolumn{1}{c|}{ Information } \\
\hline Total Studies & 5 studies \\
\hline Non-U.S. studies & 2 studies \\
\hline Evidence map studies & 2 pilot studies \\
& 2 high risk of bias studies \\
\hline Analytic set studies & 1 cluster randomized controlled trial \\
\hline Risk of bias of analytic set & Medium \\
\hline Number of PLWD in analytic set & 256 \\
\hline Dementia type/definition & Advanced dementia (type not specified) \\
\hline Caregiver type (number) & Informal caregivers $(\mathrm{n}=256)$ \\
\hline
\end{tabular}

Abbreviations: $n=$ number; PLWD=people living with dementia

\section{Intervention Research Context}

All studies were unrelated to each other and U.S.-based. Two studies examined video-based telehealth technology to support community-based CG/P. ${ }^{605,607}$ Another study examined a selfdirected educational program and home safety tool-kit to improve home safety for PLWD. ${ }^{606}$ Another examined a decision aid to help CG/P make decisions about feeding care for their 
PLWD with advanced dementia and swallowing difficulties. ${ }^{596}$ A fourth study examined including PLWD living in nursing homes in care planning meetings. ${ }^{608}$

\section{PLWD Outcomes}

Evidence was insufficient to address PLWD outcomes for a decision aid for PLWD feeding. Table 8.13 provides a summary of the findings. At 3 months, the decision aid study $(n=256)$ found a statistically significant benefit with the intervention in the number of PLWD receiving a specialized dysphagia diet. ${ }^{596}$ However, groups did not differ for other types of feeding interventions (e.g., specialized utensils, specialized staff assistance, high-calorie diet). ${ }^{596}$

Table 8.13. Summary of findings for PLWD outcomes: other service provision interventions

\begin{tabular}{|l|l|l|l|l|}
\hline \multicolumn{1}{|c|}{$\begin{array}{c}\text { Outcome } \\
\text { Comparison }\end{array}$} & $\begin{array}{c}\text { \# Studies/Design } \\
\text { (n analyzed) } \\
\text { Timing }\end{array}$ & Population & \multicolumn{1}{|c|}{ Findings } & $\begin{array}{c}\text { Strength of } \\
\text { Evidence* }^{*}\end{array}$ \\
\hline $\begin{array}{l}\text { Assisted Feeding } \\
\begin{array}{l}\text { Intervention-Specialized } \\
\text { Dysphagia Diet } \\
\text { Decision aid vs usual care }\end{array}\end{array}$ & $\begin{array}{l}1 \text { cluster RCT } \\
\text { (n=256) } \\
3 \text { months }\end{array}$ & $\begin{array}{l}\text { Advance } \\
\text { dementia with } \\
\text { feeding issues }\end{array}$ & $\begin{array}{l}1 \text { found benefit } \\
0 \text { found no difference }\end{array}$ & Insufficient \\
\hline
\end{tabular}

*Insufficient ratings due to study limitations and imprecision in the findings.

Abbreviations: $\mathrm{n}=$ number; PLWD=person/people living with dementia; RCT=randomized controlled trial

\section{Caregiver Outcomes}

Evidence was insufficient to address CG/P outcomes for a decision aid for PLWD feeding. Table 8.14 provides a summary of the findings. One study $(n=256)$ reported outcomes for CG/P decisional conflict, satisfaction with decisions, decisional regret, and feeding discussions with nursing home staff. At 3 months, CG/P who reviewed the decision support tool reported less decisional conflict than the usual care group. ${ }^{596}$ Informal caregivers who reviewed the decision support tool also reported more conversations about feeding issues with doctors, nurse practitioners, and physician's assistants than usual care. ${ }^{596}$ However, the groups did not differ in number of conversations with other nursing home staff. ${ }^{596}$ Nor did groups differ in measures of decision satisfaction and regret. ${ }^{596}$

Table 8.14. Summary of findings for caregiver outcomes: other service provision interventions

\begin{tabular}{|c|c|c|c|c|}
\hline $\begin{array}{l}\text { Outcome } \\
\text { Comparison }\end{array}$ & $\begin{array}{c}\text { \# Studies/Design } \\
\text { (n analyzed) } \\
\text { Timing }\end{array}$ & Population & Findings & $\begin{array}{l}\text { Strength of } \\
\text { Evidence* }^{*}\end{array}$ \\
\hline $\begin{array}{l}\text { Decisional conflict } \\
\text { Decision aid vs usual } \\
\text { care }\end{array}$ & $\begin{array}{l}1 \text { cluster } \operatorname{RCT}^{596}(n=256) \\
3 \text { months }\end{array}$ & $\begin{array}{l}\text { Advance dementia } \\
\text { with feeding issues }\end{array}$ & $\begin{array}{l}1 \text { found benefit } \\
0 \text { found no difference }\end{array}$ & Insufficient \\
\hline $\begin{array}{l}\text { Satisfaction with } \\
\text { Decisions } \\
\text { Decision aid vs usual } \\
\text { care }\end{array}$ & $\begin{array}{l}1 \text { cluster } \mathrm{RCT}^{596}(\mathrm{n}=256) \\
3 \text { months }\end{array}$ & $\begin{array}{l}\text { Advance dementia } \\
\text { with feeding issues }\end{array}$ & $\begin{array}{l}0 \text { found benefit } \\
1 \text { found no difference }\end{array}$ & Insufficient \\
\hline $\begin{array}{l}\text { Decisional Regret } \\
\text { Decision aid vs usual } \\
\text { care }\end{array}$ & $\begin{array}{l}1 \text { cluster } \operatorname{RCT}^{596}(n=256) \\
3 \text { months }\end{array}$ & $\begin{array}{l}\text { Advance dementia } \\
\text { with feeding issues }\end{array}$ & $\begin{array}{l}0 \text { found benefit } \\
1 \text { found no difference }\end{array}$ & Insufficient \\
\hline $\begin{array}{l}\text { Feeding Discussions } \\
\text { Decision aid vs usual } \\
\text { care }\end{array}$ & $\begin{array}{l}1 \text { cluster } \operatorname{RCT}^{596}(n=256) \\
3 \text { months }\end{array}$ & $\begin{array}{l}\text { Advance dementia } \\
\text { with feeding issues }\end{array}$ & $\begin{array}{l}1 \text { found benefit for } \\
\text { discussions with } \\
\text { doctors, PA, or NP. No } \\
\text { benefit for other nursing } \\
\text { home staff }\end{array}$ & Insufficient \\
\hline
\end{tabular}

*Insufficient ratings due to study limitations and imprecision in the findings. 
Abbreviations: $\mathrm{n}=$ number; $\mathrm{NP}=$ nurse practitioner; $\mathrm{PA}=$ physician assistant; $\mathrm{RCT}=$ randomized controlled trial

\section{Care Delivery Models and Programs}

We use the term "model" in a generic manner to refer to developed conceptual approaches. We use the term "program” for more clearly identified sets of components and/or protocols. To further distinguish between interventions presented in Chapters $4-7$ and the care delivery interventions in this chapter, we use the term intervention in a slightly different manner than in previous chapters. Here, we reserve the term intervention only for discretely identified interventions that, if supported by research, could be incorporated into an evidence-based program or model. Person-centered or individualized programs are interventions that are evidence-based, manualized or algorithmic, and ideally built from studies of individual components that may be incorporated into an individualized plan or approach. This differs from the many tailored interventions presented in Chapters $4-7$, which rely on care staff to use their knowledge and experience to adapt the intervention to the individual receiving care.

\section{Collaborative Care and Care Coordination Models}

\section{Key Points}

- Collaborative care models (i.e. Care Ecosystems or discrete adaptations of the ACCESS models) may improve PLWD quality of life. (low-strength evidence) This improvement may be very small to small, or it may be larger but concentrated in some not yet identified subgroup of people.

- Collaborative care models (i.e. discrete adaptations of the ACCESS model) may improve system-level markers, including guideline-based quality indicators and reduction in emergency department visits. (low-strength evidence)

- Evidence was insufficient to draw conclusions about all other outcomes for both PLWD and CG/P.

\section{Intervention Description}

Collaborative care models use multidisciplinary teams to integrate medical and psychosocial approaches to healthcare for PLWD. Care coordination functions are usually assigned to specific care coordinators. Team members may be co-located or spread across locations with a designated hub. Since care coordination is central to collaborative care models, we include care coordination studies in this section. Collaborative care is organized at the healthcare system level and may be provided through services either in the community or in residential facilities. As such, these approaches try to leverage local care and support resources. Most are aimed at providing CG/P support, along with coordinating care for PLWD. Often, studies required care coordinators or navigators to have a minimum of one contact per month with CG/P, or with PLWD directly. However, contact could be more frequent if necessary for caregivers to accomplish the care plans. Contact took place by phone or internet except in one German study that used in-home visits. Studies ran from 6 months to almost 2 years, but most outcomes were measured at 12 months.

\section{Eligible Studies}

We identified 13 unique studies from 32 publications that examined collaborative care. (Table 8.15) We provide information on all pilot and high risk of bias studies as part of the 
evidence map in Appendix F. ${ }^{609-614}$ The analytic set includes seven low to medium bias of bias studies and represents care for 2,597 PLWD. ${ }^{615-640}$ Given the pragmatic nature of most of the studies, information on dementia type and severity is less available. Appendix F provides evidence tables, summary risk of bias assessments, and strength of evidence for key comparisons and outcomes.

Table 8.15. Basic characteristics of literature set: collaborative care models

\begin{tabular}{|l|l|}
\hline \multicolumn{1}{|c|}{ Characteristics } & \multicolumn{1}{c|}{ Information } \\
\hline Total unique studies & 13 studies \\
\hline Non-U.S. studies & 4 studies \\
\hline Evidence map studies & $\begin{array}{l}4 \text { pilot studies } \\
2 \text { high risk of bias studies }\end{array}$ \\
\hline Analytic set studies & 5 pragmatic, 2 explanatory studies \\
\hline Risk of bias of analytic set & 6 medium \\
\hline Number of PLWD in analytic set & 2,641 \\
\hline Dementia type/definition & Generally dementia unspecified, usually more mild to moderate \\
\hline Caregiver type (number) & Informal caregivers $(2,405)$ \\
\hline
\end{tabular}

Abbreviation: PLWD=people living with dementia

\section{Intervention Research Context}

This literature falls mainly into Stage 4 of the NIH Stage Model, or mostly pragmatic trial designs, although we did identify a few pilot studies. The pragmatic studies either cited previous evidence-based research or related pilot studies, but we identified no literature showing any collaborative care approach as progressing through all stages of the model. The pragmatic trials appeared along a spectrum of balanced explanatory/pragmatic to fully pragmatic. Most studies were conducted in high-resource locations, including the United States, Germany, Singapore, and the Netherlands. Few studies investigated diverse caregivers. However, one study enrolled 50 percent urban African-American caregivers, ${ }^{616}$ and one study used the ACCESS protocol for Latino/immigrant populations in an underserved urban setting. ${ }^{617}$ Two high risk of bias studies of multidisciplinary teams, reported in the evidence map, were conducted in nursing homes. One was a relatively large pragmatic trial of 793 PLWD, but only 40 percent of participants received the intervention, leaving it difficult to understand what drove the lack of difference between the intervention and the control groups. ${ }^{613}$

\section{PLWD Outcomes}

Six of the seven medium to low risk of bias studies reported outcomes related to PLWD. We synthesized results qualitatively because differences in outcome measures, time to outcomes, and analytic methods prohibited quantitative pooling. The most commonly investigated outcome was quality of life. We also include quality indicators derived from guideline recommendations as PLWD-related outcomes. Table 8.16 summarizes the number of studies investigating each outcome and the number of studies that found a statistically significant benefit or no difference in outcomes for PLWD in the intervention group versus the comparison group.

Quality of life improvement was supported by low-strength evidence from four studies using usual care controls. One large pragmatic study found statistically significant but very small to small benefit using the QoL-AD. ${ }^{618}$ Another large pragmatic trial found benefit greater than the established minimally important difference for the health utility index, which indicates the improvement may have been large enough for the study participants to notice the change. ${ }^{620}$ Two other trials found no difference for QoL-AD ${ }^{619}$ and the health utility index. ${ }^{617}$ However, when 
assessing the strength of evidence, we gave less weight to the findings from these studies due to the smaller sample sizes and other concerns with the statistical analyses they used.

Quality indicators were supported by low-strength evidence drawn from two studies. These indicators were based on adherence to 19 to 23 dementia guideline recommendations in four domains: assessment, treatment, education and support, and safety. Both studies found benefit of about the same magnitude across the quality indicators. ${ }^{617,620}$

Low-strength evidence also showed a decrease in rate of emergency department visits over a 12-month period. ${ }^{618}$ Five PLWD needed to participate in collaborative care to achieve a decrease of one emergency room visit.

Evidence was insufficient for the remaining outcomes. Some outcomes were only investigated by a single small study, making the consistency in findings unknown. Also, the uncertainty regarding a true "no difference between groups" finding versus an "inability to show a difference" finding remained too high. Two studies investigated neuropsychiatric symptoms, but the outcome was assessed as high risk of bias in one, which was therefore not included. One explanatory study found benefit for the neuropsychiatric symptom inventory. ${ }^{616}$ Groups did not differ significantly for daily activities of living, ${ }^{616,619}$ depression as measured by the CSDD, ${ }^{616}$ or hospitalization or ambulance. ${ }^{618}$ Nursing home placement had mixed results. ${ }^{616,619}$

Table 8.16. Summary of findings for PLWD outcomes: collaborative care models

\begin{tabular}{|c|c|c|c|}
\hline $\begin{array}{l}\text { Outcome } \\
\text { Comparison }\end{array}$ & $\begin{array}{l}\text { \# Studies/ Design } \\
\text { (n analyzed) } \\
\text { Timing }\end{array}$ & Findings & $\begin{array}{l}\text { Strength of } \\
\text { Evidence* }^{*}\end{array}$ \\
\hline $\begin{array}{l}\text { Quality of Life } \\
\text { Collaborative care vs } \\
\text { usual care }\end{array}$ & $\begin{array}{l}\text { 4 Pragmatic trials }{ }^{617-620} \\
(\mathrm{n}=1,746) \\
6-18 \text { months }\end{array}$ & $\begin{array}{l}2 \text { of } 4 \text { found benefit } \\
2 \text { of } 4 \text { found no difference }\end{array}$ & $\begin{array}{l}\text { Low for benefit } \\
\text { (Weighted to larger } \\
\text { pragmatic trials) }\end{array}$ \\
\hline $\begin{array}{l}\text { Neuropsychiatric } \\
\text { symptoms } \\
\text { Collaborative care vs } \\
\text { usual care }\end{array}$ & $\begin{array}{l}1 \text { Explanatory cluster trial }{ }^{616} \\
(\mathrm{n}=152) \\
12 \text { months }\end{array}$ & $\begin{array}{l}1 \text { found benefit } \\
0 \text { found no difference }\end{array}$ & Insufficient \\
\hline $\begin{array}{l}\text { Function } \\
\text { Collaborative care vs } \\
\text { usual care }\end{array}$ & $\begin{array}{l}1 \text { Pragmatic trial }{ }^{619} \\
1 \text { Explanatory cluster trial } \\
(\mathrm{n}=560) \\
6-12 \text { months }\end{array}$ & $\begin{array}{l}0 \text { found benefit } \\
2 \text { found no difference }\end{array}$ & Insufficient \\
\hline $\begin{array}{l}\text { Depression } \\
\text { Collaborative care vs } \\
\text { usual care }\end{array}$ & $\begin{array}{l}1 \text { Explanatory cluster trial }{ }^{616} \\
(\mathrm{n}=152) \\
12 \text { months }\end{array}$ & $\begin{array}{l}0 \text { found benefit } \\
1 \text { found no difference }\end{array}$ & Insufficient \\
\hline $\begin{array}{l}\text { Quality indicators } \\
\text { Collaborative care vs } \\
\text { usual care }\end{array}$ & $\begin{array}{l}2 \text { Pragmatic trials }{ }^{617,620} \\
(n=559) \\
6-18 \text { months }\end{array}$ & $\begin{array}{l}2 \text { of } 2 \text { found benefit } \\
0 \text { of } 2 \text { found no difference }\end{array}$ & $\begin{array}{l}\text { Low for benefit } \\
\text { (Weighted to larger } \\
\text { pragmatic trials) }\end{array}$ \\
\hline $\begin{array}{l}\text { Emergency room visits } \\
\text { Collaborative care vs } \\
\text { usual care }\end{array}$ & $\begin{array}{l}1 \text { Pragmatic trial }^{618} \\
(\mathrm{n}=780) \\
12 \text { months }\end{array}$ & $\begin{array}{l}1 \text { found benefit } \\
0 \text { found no difference } \\
\text { (no difference for hospitalization } \\
\text { or ambulance use) }\end{array}$ & $\begin{array}{l}\text { Low for benefit } \\
\text { (Weighted to larger } \\
\text { pragmatic trials) }\end{array}$ \\
\hline $\begin{array}{l}\text { Nursing home } \\
\text { placement } \\
\text { Collaborative care vs } \\
\text { usual care }\end{array}$ & $\begin{array}{l}1 \text { Pragmatic trial } \\
1 \text { Explanatory cluster trial }{ }^{616} \\
1 \mathrm{RCT}^{640} \\
(\mathrm{n}=794) \\
6-18 \text { months }\end{array}$ & $\begin{array}{l}0 \text { found benefit } \\
2 \text { found no difference } \\
1 \text { found benefit at } 1.6 \text { years but } \\
\text { not at } 2 \text { years }\end{array}$ & Insufficient \\
\hline
\end{tabular}

*Insufficient ratings due to study limitations and imprecision in the findings.

Abbreviations: $\mathrm{n}=$ number; $\mathrm{RCT}=$ randomized controlled trial 


\section{Caregiver Outcomes}

Six of the seven medium- to low-risk-of-bias studies reported CG/P outcomes. Again, we synthesized results qualitatively because differences in outcome measures, time to outcomes, and analytic methods prohibited quantitative pooling. The most commonly investigated outcomes were caregiver burden and depression. Table 8.17 summarizes the number of studies investigating each outcome and the number of studies that found a statistically significant benefit or no difference in outcomes for CG/P.

Evidence was insufficient to draw conclusions about collaborative care versus usual care for CG/P. The inability to combine outcomes from multiple studies made it impossible to show precision in any outcome. Several outcomes were only investigated by a single study, making the consistency of findings unknown. The uncertainty regarding a true "no difference between groups" finding versus an "inability to show a difference" finding remained too high. One study found no statistical difference between groups for quality of life as measured by the EuroQual$5 \mathrm{D} .{ }^{620}$ Caregiver burden was variably measured with mixed findings. One large pragmatic trial found very small benefit, ${ }^{618}$ while the other study reported mixed findings ${ }^{617,619,620}$ Caregiver depression was slightly reduced in one large study ${ }^{618}$ but two other studies found no difference. ${ }^{615-617}$ One trial reported no difference between groups for caregiver self-efficacy. ${ }^{618}$ Another trial found mixed results in quality measures. ${ }^{615}$

Table 8.17. Summary of findings for caregiver outcomes: collaborative care models

\begin{tabular}{|c|c|c|c|}
\hline $\begin{array}{l}\text { Outcome } \\
\text { Comparison }\end{array}$ & $\begin{array}{l}\text { \# Studies/Design } \\
\text { (n analyzed) } \\
\text { Timing }\end{array}$ & Findings & $\begin{array}{l}\text { Strength of } \\
\text { Evidence* }\end{array}$ \\
\hline $\begin{array}{l}\text { Quality of Life } \\
\text { Collaborative care vs usual } \\
\text { care }\end{array}$ & $\begin{array}{l}1 \text { Pragmatic trial }{ }^{620} \\
(\mathrm{n}=408) \\
18 \text { months }\end{array}$ & $\begin{array}{l}0 \text { found benefit } \\
1 \text { found no difference }\end{array}$ & Insufficient \\
\hline $\begin{array}{l}\text { Caregiver burden } \\
\text { Collaborative care vs usual } \\
\text { care }\end{array}$ & $\begin{array}{l}\text { 4 Pragmatic trials }{ }^{615} \text {, } \\
617-620 \\
(n=1,719) \\
6-18 \text { months }\end{array}$ & $\begin{array}{l}2 \text { of } 4 \text { found benefit } \\
2 \text { of } 4 \text { found no difference } \\
\text { (also no difference in several related } \\
\text { measures such as role captivity or } \\
\text { physical health strain) }\end{array}$ & Insufficient \\
\hline $\begin{array}{l}\text { Depression } \\
\text { Collaborative care vs usual } \\
\text { care }\end{array}$ & $\begin{array}{l}3 \text { Pragmatic trials }{ }^{615} \\
617,6181 \text { Explanatory } \\
\text { cluster trial }{ }^{616} \\
(n=1,570) \\
6-18 \text { months }\end{array}$ & $\begin{array}{l}1 \text { of } 4 \text { found benefit } \\
3 \text { of } 4 \text { found no difference }\end{array}$ & Insufficient \\
\hline $\begin{array}{l}\text { Self-efficacy } \\
\text { Collaborative care vs usual } \\
\text { care }\end{array}$ & $\begin{array}{l}1 \text { Pragmatic trial }{ }^{618} \\
(\mathrm{n}=780) \\
12 \text { months }\end{array}$ & $\begin{array}{l}0 \text { found benefit } \\
1 \text { found no difference }\end{array}$ & Insufficient \\
\hline $\begin{array}{l}\text { Quality measures } \\
\text { Collaborative care vs usual } \\
\text { care }\end{array}$ & $\begin{array}{l}1 \text { Pragmatic trial } \\
(\mathrm{n}=486) \\
12 \text { months }\end{array}$ & $\begin{array}{l}1 \text { found benefit in unmet needs but no } \\
\text { difference in use of support services or } \\
\text { informal helpers }\end{array}$ & Insufficient \\
\hline
\end{tabular}

*Insufficient ratings due to study limitations and imprecision in the findings.

Abbreviation: $n=$ number

\section{Variation in Outcomes}

\section{By PLWD Characteristics}

One pragmatic study, the ACCESS study, enrolled primarily white urban caregivers and found variation in outcomes by caregiver education. Those who had not graduated from high school showed greater improvement than college graduates in the quality indicators (44.4 vs 29.5 
for the assessment dimension, 36.9 vs. 15.7 for the treatment dimension, and 52.7 vs. 40.9 for the safety dimension $\left[\mathrm{P}<.001\right.$ for all three]). ${ }^{632}$

The ACCESS protocol was also later tested with a more intensive in-person protocol in an urban Latino population with a large immigrant population. This study also found improvement in quality indicators, further supporting the ACCESS trial. However, they were unable to confirm that more intensive in-person delivery further improved scores. Since the adapted model was also tested with a different population, we could not separate the potential effects of the new model from those of the new population.

\section{By Caregiver Characteristics}

The VA Partners in Dementia Care program reported that benefits were larger among CG/P of PLWD with higher baseline cognitive impairment (unmet needs: $B=-0.97, p<0.001$ ), caregivers with higher baseline conflict with caregiving (role captivity: $B=-0.23, p=0.02$ ), and caregivers of PLWD with higher baseline behavior problems (physical health strain: $\mathrm{B}=-0.09$, $\mathrm{p}=0.06$; number informal helpers: $\mathrm{B}=0.61, \mathrm{p}=0.005){ }^{615}$

\section{By Intervention Characteristics}

The ACCESS study also reported increased benefit with increased contact between CG/P and providers. For every additional monthly encounter between a health organization care manager and a caregiver, the mean percentage of quality care indicators rose between 10 and 16 percentage points across four quality domains. ${ }^{631}$

\section{Nursing Home-Based Interdisciplinary Individualized Care/Person-Centered Care and Dementia Care Mapping}

\section{Intervention Description}

Similar to Collaborative Care models for community-dwelling PLWD and their CG/P, models and programs to improve care have also been used in nursing home settings.

Collaborative Care models incorporate working with CG/P, which means care is personalized to the informal caregivers' PLWD. Programs for nursing home settings focus more on providing individualized care through training formal caregivers to foster PLWD personhood and attending to unmet needs through individualized care plans. These programs support person-centered care structurally through training, embedding caregivers in care teams, and/or providing tools to support systematic observation of factors that enhance person-centered care.

This form of individualized care for nursing homes has been explored predominantly by nonU.S. countries. Only one recent small pilot ${ }^{642}$ and one study from 1997 were based in the United States; these studies examined interdisciplinary team approaches to provide individualized care. ${ }^{643}$ The remaining locations in which these programs were investigated include the United Kingdom, ${ }^{644-647}$ Australia, ${ }^{648-650}$ Norway, ${ }^{641,651}$ Germany, ${ }^{652}$ and the Netherlands. ${ }^{653}$ Because the programs have been tested in non-U.S. long-term care systems, we could not determine their adaptability to U.S. healthcare and support systems. Interpreting the usefulness of the findings would be challenging. For this reason, we did not further analyze these studies, but we briefly describe them in the Intervention Research Context subsection without performing further analysis. 


\section{Eligible Studies}

We identified 16 unique studies from 22 publications that examined individualized personcentered care in nursing homes. (Table 8.18) We provide information on all pilot and high risk of bias studies as part of the evidence map in Appendix F. 608, 642, 644, 645, 648, 649, 651, 652, 654, 655 Five studies were rated as low to medium risk of bias. ${ }^{641,643,647,650,653,656-659}$ These studies enrolled 1,722 PLWD with generally moderate to severe dementia. Appendix F provides evidence tables and summary risk of bias assessments.

Table 8.18. Basic characteristics of literature set: individualized person-centered care (non-U.S.)

\begin{tabular}{|l|l|}
\hline \multicolumn{1}{|c|}{ Characteristics } & \multicolumn{1}{c|}{ Information } \\
\hline Total unique studies & 16 studies \\
\hline Non-U.S. studies & 13 studies \\
\hline Evidence map studies & $\begin{array}{l}4 \text { pilot studies } \\
\text { high risk of bias studies }\end{array}$ \\
\hline Analytic set studies & 1 pragmatic, 2 balanced, and 2 explanatory studies \\
\hline Risk of bias of analytic set & 5 medium \\
\hline
\end{tabular}

\section{Intervention Research Context}

With the exception of one study from 1996, this literature is relatively recent, with the eight studies published within the last 5 years. The predominant research design involved assigning care facilities to treatment or control study arms. Using the NIH Stage Model, these studies were assessed as Stage 4 and more pragmatic in study design. Problems with ensuring the intervention was actually delivered to the PLWD residents was a common cause of high risk of bias. Study authors were frequently very transparent regarding reporting fidelity to the interventions.

The largest set of literature examined person-centered care based on Kitwood's framework, generally coupled with Dementia Care Mapping ${ }^{\mathrm{TM}}\left(\mathrm{DCM}^{\mathrm{TM}}\right) .{ }^{644,653} \mathrm{DCM}^{\mathrm{TM}}$ is an intervention that promotes care practice development. It involves training formal caregivers to apply a systematic approach to observing factors associated with PLWD well-being, and then share the information with staff to support care planning. DCM ${ }^{\mathrm{TM}}$ is a trade-marked tool that has been used in several countries including Europe, the United Kingdom, and Australia. Over 60 percent of published trials of DCM ${ }^{\mathrm{TM}}$ are in the evidence map as pilots or high risk of bias studies, and results were mixed. ${ }^{649,651,652,654}$ Recently, two large pragmatic trials both found no benefit for $\mathrm{DCM}^{\mathrm{TM}}$, but these trials also reported challenges with ensuring that the intervention was fully and widely implemented. ${ }^{644,653}$

A few studies in the analytic set used a different structural approach and supported personcentered care within interdisciplinary teams. ${ }^{641,647}$ These larger studies, published in 2018, stemmed from prior work presented in the evidence map, and showed indications that the research was developing along a distinct line of inquiry. These studies noted small improvement in PLWD quality of life and reduction in agitation over 8 weeks to 9 months. However, these program approaches will require further investigation into their sustainability.

\section{Care Staff Education and Support Needs}

As noted above, specialized service provision requires qualified staff members to be regularly available to perform the services offered to PLWD or their CG/P. Interventions addressing staff education and support needs include approaches targeted at helping informal and formal caregivers work together to support PLWD well-being. 


\section{Caregiver Staff Training}

We considered training programs as being at the pilot stage if the training was not embedded in the organizational structure, such that the knowledge or skills developed by the training will sustain across staff turnover.

\section{Key Point}

- Studies of training interventions to improve formal caregiver staff knowledge and skills were described in the evidence map but not considered for analysis due to limitations in study designs.

\section{Intervention Description}

Formal staff training is intended to improve staff knowledge and facilitate skill-building.

\section{Eligible Studies}

We identified 22 unique studies from 23 publications that examined the use of training interventions for formal caregiver staff. ${ }^{214,660-680}$ (Table 8.19) No studies were assessed as low or moderate risk of bias, while two were assessed as high risk of bias. Remaining studies were all pilot or small sample studies. We provide information on all studies in the evidence map in Appendix F.

Table 8.19. Basic characteristics of literature set: formal caregiver staff training

\begin{tabular}{|l|l|}
\hline \multicolumn{1}{|c|}{ Characteristics } & \multicolumn{1}{c|}{ Information } \\
\hline Total unique studies & 22 studies \\
\hline Non-U.S. studies & 12 studies \\
\hline Evidence map studies & 12 pilot studies \\
& 3 small sample studies \\
& 4 training pilot studies (without evidence of sustained training) \\
\hline Analytic set studies & 3 high risk of bias studies \\
\hline
\end{tabular}

\section{Intervention Research Context}

Training topics in the evidence map included general education about dementia and dementia care, ${ }^{668,669,678,680}$ communication techniques, ${ }^{660,663,671,672,679}$ bathing techniques, ${ }^{667,676}$ identifying and addressing pain, ${ }^{666,680}$ morning care, ${ }^{677}$ integrating physical activity into daily routines, ${ }^{665}$ sleep, ${ }^{673}$ and feeding skills. ${ }^{661}$ Several used activities or taught skills targeted at improving behavioral issues, ${ }^{664,674}$ including apathy. ${ }^{670}$ One intervention examined training to identify signs of awareness in PLWD with severe dementia. ${ }^{662}$ While nursing homes or other long-term care facilities were the most common settings, one study examined education on dementia for acute care hospital staff, ${ }^{675}$ and another study examined education for communitybased care staff. ${ }^{663}$ One examined an online training portal on dementia care available to both formal and CG/P. ${ }^{668}$ Non-U.S. locations included Australia, Canada, France, Germany, Taiwan, the United Kingdom, and the European Union.

The research is largely preliminary across a varied set of training interventions. Most interventions stopped at the pilot stage or did not embed the training intervention into the care organization in order that new skills and knowledge would be sustained over time even with staff turnover. Research activity grew between 1999 and 2017, with new unrelated pilot studies scattered throughout the whole period and across locations. We found, however, one small indication of research progressing through the NIH Stage Model. One training intervention 
(Bathing Without a Battle) published both a small sample initial study ${ }^{676}$ and a later larger multisite study that enrolled PLWD with a broader range of dementia severity. ${ }^{667}$

\section{Informal Caregiver Staff Training}

\section{Key Point}

- Studies of training interventions to improve CG/Ps' knowledge and skills were described in the evidence map but not considered for analysis due to limitations in study designs.

\section{Intervention Description}

Training interventions for CG/P differ from interventions in Chapter 6 because they do not incorporate any psychoeducation or therapy to support CG/P' well-being. They consist, instead, of dementia education and training focused on skill-building with an emphasis on role-training.

\section{Eligible Studies}

We identified 12 unique studies from 12 publications that examined training interventions to improve CG/P skills. ${ }^{668,681-691}$ (Table 8.20) Three pilot studies and five small sample studies were excluded from the analytic study set. Four additional studies were assessed as high risk of bias. We provide information on all pilot studies and high risk of bias studies as part of the evidence map in Appendix F.

Table 8.20. Basic characteristics of literature set: CG/P training

\begin{tabular}{|l|l|}
\hline \multicolumn{1}{|c|}{ Characteristics } & \multicolumn{1}{c|}{ Information } \\
\hline Total unique studies & 12 studies \\
\hline Non-U.S. studies & 6 studies \\
\hline Evidence map studies & 3 pilot studies \\
& 5 small sample studies \\
& 4 high risk of bias studies \\
\hline Analytic set studies & 0 \\
\hline
\end{tabular}

Abbreviation: $\mathrm{CG} / \mathrm{P}=$ caregiver/care partner

\section{Intervention Research Context}

The research is largely preliminary and includes studies examining a varied set of training interventions. Most interventions in the evidence map stopped at the small sample or pilot stage. Research spanned over 20 years, from 1994 to 2015. Studies conducted in the United States were older, from 1994 to 2007, and mostly focused on the role of caregiving, building general dementia caregiver skills, or skills for behavioral management. ${ }^{681,684-686,689,691}$ Non-U.S.-based studies, conversely, were published from 2001 to 2015 and were balanced across general care ${ }^{683 \text {, }}$ 690 and communication-specific skills. ${ }^{682,687,688}$ Studies also examined portable training materials in the United States ${ }^{684}$ and internet-accessible training materials in the European Union. ${ }^{668}$

\section{Family Education and Partnering}

\section{Key Point}

- Studies of training interventions to improve formal caregiver staff knowledge and skills were described in the evidence map but not considered for analysis due to limitations in study designs. 


\section{Intervention Description}

Family involvement interventions provide education, training, and other supports to establishing a collaborative relationship between family members and formal caregivers of PLWD in nursing homes. These interventions seek to improve PLWD well-being through: 1) improving the partnership between the staff and family members to identify unmet needs among PLWD, and 2) helping family members structure visits to avoid triggering behavioral symptoms in PLWD. Training in communication techniques is prominent.

\section{Eligible Studies}

We identified five unique studies from five publications that examined family education and partnership interventions. ${ }^{76,692-695}$ (Table 8.21) Three studies were pilots and two were assessed as high risk of bias, thus none were included in the analytic set. We provide information on all studies as part of the evidence map in Appendix F.

Table 8.21. Basic characteristics of literature set: family education and partnering

\begin{tabular}{|l|l|}
\hline \multicolumn{1}{|c|}{ Characteristics } & \multicolumn{1}{c|}{ Information } \\
\hline Total unique studies & 5 studies \\
\hline Non-U.S. studies & 1 study (Australia) \\
\hline Evidence map studies & 3 pilot studies \\
& 2 high risk of bias studies \\
\hline Analytic set studies & 0 \\
\hline
\end{tabular}

\section{Intervention Research Context}

The research is preliminary, comprising pilot or explanatory studies with design or conduct challenges. Studies conducted in the United States were published from 1999 to 2007. Family Involvement in Care was examined in several studies, ${ }^{692-694}$ including a 2011 study in Australia. ${ }^{76}$ The 1999 study was a foundation for the Family Involvement in Care program. ${ }^{694}$ The Partners in Caregiving adaptation for Special Care Units in the 2007 publication added concurrent staff and family training, thereby broadening the intervention focus beyond primarily family members. ${ }^{695}$ PLWD outcomes were not prominent in this literature set; outcomes primarily applied to family members and staff.

\section{Multitier Training}

Multitier training interventions involve formal caregivers being trained to provide training to CG/P for specific care needs of the PLWD.

\section{Key Point}

- Evidence was insufficient to draw conclusions about the effect of multi-tier training on PLWD and their caregiver.

\section{Intervention Description}

This literature set comprises mostly practice guideline-based interventions encompassing multidisciplinary care interventions. These interventions aimed to improve communication between PLWD and their caregivers, and used nutrition and activity planning to help improve various aspects (psychosocial, physical, and behavioral) related to better functioning among PLWD and their caregivers. 


\section{Eligible Studies}

Table 8.22 summarizes the characteristics of the literature set. We identified seven unique studies from nine publications that examined the use of training plus delivery of evidence-based guidelines/protocols for caregivers, with the goal in improving their own health outcomes as well as the health outcomes of PLWD. ${ }^{696-702}$ One study was assessed as high risk of bias ${ }^{697}$ and five were pilots. ${ }^{696,698,700-702}$ Only one study was included in the analytic set. ${ }^{699}$ We provide information on the high risk of bias studies in the evidence map in Appendix F.

The included study ( $n=95)$ was a randomized controlled trial targeted toward family (informal) caregivers. The trial compared STAR-C (interactive sessions between community consultants and caregivers) with routine medical care. ${ }^{699}$ It was assessed as medium risk of bias and categorized as explanatory. PLWD were eligible if they were community dwellers living with their caregivers and had a probable diagnosis of AD (with moderate cognitive impairment). Family caregivers were also enrolled in the study. Appendix F provide evidence tables, summary risk of bias assessments, and strength of evidence for key comparisons and outcomes.

Table 8.22. Basic characteristics of literature set: multitier training

\begin{tabular}{|l|l|}
\hline \multicolumn{1}{|c|}{ Characteristics } & \multicolumn{1}{c|}{ Information } \\
\hline Total unique studies & 7 studies \\
\hline Non-U.S. studies & 2 studies \\
\hline Evidence map studies & 5 pilot studies \\
& 1 high risk of bias studies \\
\hline Analytic set studies & 1 randomized controlled trial \\
\hline Risk of bias of analytic set & 1 medium \\
\hline Number of PLWD in analytic set & 95 \\
\hline Dementia type/definition & Moderate cognitive impaired probable Alzheimer's disease \\
\hline Caregiver type (number) & Informal caregivers (95) \\
\hline
\end{tabular}

Abbreviation: PLWD=people living with dementia

\section{Intervention Research Context}

The literature on multitier training interventions comprises mostly pilot and high risk of bias studies. Four studies were conducted in United States ${ }^{697,699,701,702}$ while the others occurred in the United Kingdom ${ }^{696,700}$ and Finland. ${ }^{698}$ We identified a manual-based intervention targeted at improving caregiver's understanding of sleep and dementia. ${ }^{696}$ We also identified a study that provided caregivers with instruction on how to recognize pain among PLWD. ${ }^{697}$ Also evaluated was an intervention that used tailored nutritional guidance on the basis of the food diaries to prevent weight gain among PLWD. ${ }^{698}$

\section{PLWD Outcomes}

Evidence was insufficient to draw conclusions on quality of life and memory or behavioral problems of PLWD using the STAR-C intervention versus routine medical care $(n=95) .{ }^{699}$ Table 8.23 summarizes the primary findings.

Table 8.23. Summary of findings for PLWD outcomes: multitier training

\begin{tabular}{|l|l|l|l|l|}
\hline $\begin{array}{c}\text { Outcome } \\
\text { Comparison }\end{array}$ & $\begin{array}{c}\text { \# Studiesl } \\
\text { Design } \\
\text { (n analyzed) } \\
\text { Timing }\end{array}$ & \multicolumn{1}{|c|}{ Population } & \multicolumn{1}{c|}{ Findings } & $\begin{array}{c}\text { Strength of } \\
\text { Evidence* }\end{array}$ \\
\hline $\begin{array}{l}\text { RMB-PC } \\
\text { STAR-C vs } \\
\text { RMC }\end{array}$ & $\begin{array}{l}1 \mathrm{RCT}^{699} \\
(\mathrm{n}=95) \\
2 \text { months }\end{array}$ & $\begin{array}{l}\text { Probable Alzheimer's } \\
\text { disease }\end{array}$ & $\begin{array}{l}0 \text { found benefit } \\
1 \text { found no difference }\end{array}$ & Insufficient \\
\hline
\end{tabular}




\begin{tabular}{|l|l|l|l|l|}
\hline $\begin{array}{c}\text { Outcome } \\
\text { Comparison }\end{array}$ & $\begin{array}{c}\text { \# Studies/ } \\
\text { Design } \\
\text { (n analyzed) } \\
\text { Timing }\end{array}$ & \multicolumn{1}{|c|}{ Population } & \multicolumn{1}{c|}{ Findings } & $\begin{array}{c}\text { Strength of } \\
\text { Evidence* }\end{array}$ \\
\hline $\begin{array}{l}\text { Quality of life } \\
\text { STAR-C vs } \\
\text { RMC }\end{array}$ & $\begin{array}{l}1 \mathrm{RCT}^{699} \\
(\mathrm{n}=95) \\
2 \text { months }\end{array}$ & $\begin{array}{l}\text { Probable Alzheimer's } \\
\text { disease }\end{array}$ & $\begin{array}{l}0 \text { found benefit } \\
1 \text { found no difference }\end{array}$ & Insufficient \\
\hline
\end{tabular}

*Insufficient ratings due to study limitations and imprecision in the findings.

Abbreviations: $\mathrm{n}=$ number; PLWD=person/people living with dementia; RCT=randomized controlled trial; RMB-PC=Revised Memory and Behavior Problem Checklist; RMC=routine medical care

\section{Caregiver Outcomes}

Evidence was insufficient to draw conclusions about using the STAR-C intervention versus RMC ( $\mathrm{n}=95$ ) training plus delivery of evidence-based guidelines/protocols targeted toward caregivers. (Table 8.24)

Table 8.24. Summary of findings for caregiver outcomes: multitier training

\begin{tabular}{|c|c|c|c|c|}
\hline $\begin{array}{l}\text { Outcome } \\
\text { Comparison }\end{array}$ & $\begin{array}{l}\text { \# Studies/ } \\
\text { Design } \\
\text { (n analyzed) } \\
\text { Timing }\end{array}$ & Population & Findings & $\begin{array}{l}\text { Strength of } \\
\text { Evidence* }^{*}\end{array}$ \\
\hline $\begin{array}{l}\text { CES-D } \\
\text { STAR-C vs } \\
\text { RMC }\end{array}$ & $\begin{array}{l}1 \mathrm{RCT} \\
(\mathrm{n}=95) \\
2 \text { months } \\
\end{array}$ & Primary family caregivers & $\begin{array}{l}1 \text { found benefit } \\
0 \text { found no difference }\end{array}$ & Insufficient \\
\hline $\begin{array}{l}\text { HDRS } \\
\text { STAR-C vs } \\
\text { RMC }\end{array}$ & $\begin{array}{l}1 \mathrm{RCT} \\
(\mathrm{n}=95) \\
2 \text { months } \\
\end{array}$ & Primary family caregivers & $\begin{array}{l}0 \text { found benefit } \\
1 \text { found no difference }\end{array}$ & Insufficient \\
\hline $\begin{array}{l}\text { Caregiver } \\
\text { burden } \\
\text { STAR-C vs } \\
\text { RMC }\end{array}$ & $\begin{array}{l}1 \mathrm{RCT} \\
(\mathrm{n}=95) \\
2 \text { months }\end{array}$ & Primary family caregivers & $\begin{array}{l}1 \text { found benefit } \\
0 \text { found no difference }\end{array}$ & Insufficient \\
\hline $\begin{array}{l}\text { Caregiver } \\
\text { reaction } \\
\text { STAR-C vs } \\
\text { RMC }\end{array}$ & $\begin{array}{l}1 \mathrm{RCT} \\
(\mathrm{n}=95) \\
2 \text { months }\end{array}$ & Primary family caregivers & $\begin{array}{l}1 \text { found benefit } \\
0 \text { found no difference }\end{array}$ & Insufficient \\
\hline $\begin{array}{l}\text { Caregiver } \\
\text { sleep } \\
\text { questionnaire } \\
\text { STAR-C vs } \\
\text { RMC }\end{array}$ & $\begin{array}{l}1 \mathrm{RCT} \\
(\mathrm{n}=95) \\
2 \text { months }\end{array}$ & Primary family caregivers & $\begin{array}{l}0 \text { found benefit } \\
1 \text { found no difference }\end{array}$ & Insufficient \\
\hline
\end{tabular}

*Insufficient ratings due to study limitations and imprecision in the findings.

Abbreviations: CES-D=Center for Epidemiological Studies Depression Inventory; HDRS=Hamilton Depression Rating scale; $\mathrm{n}=$ number; $\mathrm{RCT}=$ randomized controlled trial; $\mathrm{RMC}=$ routine medical care; STAR-C=staff training in assisted living residencescaregivers

\section{Conclusion}

We found 123 unique studies that investigated 11 care delivery interventions to improve how care is delivered. We grouped the 11 intervention categories into three main themes of care service delivery, care delivery models and programs, and care staff education and support needs. These care delivery interventions conform well to the framework for care interventions from the NASEM Families Caring for an Aging America 2016 report (Figure 1.1 in Chapter 1), delivering the interventions at the system level. 
We found collaborative care models (i.e. discrete adaptations of the ACCESS ${ }^{620}$ or Care Ecosystem $^{618}$ ) may improve PLWD quality of life. It is difficult to estimate an effect size from a qualitative synthesis of study results. The largest, rigorously designed study found a statistically significant improvement in quality of life, but the effect size was very small. Based on reported data, we could not determine whether the average effect was broadly distributed across the full study population, but too small of a benefit to be noticeable by PLWD, or if the benefits were largely concentrated in some not yet identified subgroup of PLWD for whom the improvement would be noticeable. The other pragmatic trial also found improvement in health-related quality of life, with the average change being greater than what is considered a minimally detectable difference. These two studies may represent the range of possible effect sizes.

We also found collaborative care models may improve system-level markers, including guideline-based quality indicators and reduction in emergency department visits. Cost was not presented as an outcome. Cost data for the interventions were provided, but a cost-effectiveness analysis is outside the scope of this review.

For the remainder of the PLWD and CG/P outcomes for collaborative care, and for all other care delivery interventions, we found the uncertainty of the evidence was too high to draw conclusions. However, our being unable to draw a conclusion does not mean that the intervention has no effect. Research on interventions to change behavioral and psychological outcomes is challenging, and many factors can influence the outcomes. Future research may reduce uncertainty enough to allow for conclusions about the effect of these interventions, including potential benefits. 


\section{Chapter 9. Implementation of Care Interventions}

This chapter addresses Guiding Question 1 on providing context for implementation and dissemination of care interventions. We present the results of the grey literature search conducted to provide resources for care interventions which may not have been empirically studied using study designs required by the review inclusion criteria. Because so few interventions were assessed to have at least low-strength evidence to support our review findings, we were concerned that providing implementation and dissemination information based only on those studies would leave readers with an unbalanced or skewed view of implementation and dissemination. Therefore, we do not report on this evidence here.

\section{Grey Literature Search}

We searched 15 different sources from the grey literature (i.e., research or other written material produced outside of traditional academic publishing) during April, 2019 to identify repositories of care interventions and criteria for evaluating and/or categorizing care interventions for people living with dementia (PLWD) and their caregivers. Most of these sources provided educational materials, webinars, and information on research and policy. However, three housed lists or libraries of interventions: the Benjamin Rose Institute on Aging, Family Caregiver Alliance, and Rosalynn Carter Institute for Caregiving. Subsequently, the Benjamin Rose Institute on Aging, in partnership with the Family Caregiver Alliance and the Gerontological Society of America, has formally launched their online resource for caregiving for dementia, the Best Practice Caregiving resource (see https://www.benrose.org/best-practicecaregiving). The Rosalynn Carter Institute has since discontinued their online resource.

The Best Practice Caregiving resource, a free online searchable database, provides information about programs for informal caregivers. The standard of evidence used by this resource differs substantially from that of this review. To be listed in the Best Practice database, a program needed to have at least one published statistically significant benefit for a caregiver outcome from a study of any empirical research design, plus have been implemented in at least one organization's regular service portfolio. The published research needed to be U.S.-based and have at least 50 percent of the informal caregivers providing care to PLWD.

The Family Caregiver Alliance provides a list of interventions (formerly their "Innovations Clearinghouse") for family caregivers of people with chronic disabling conditions. Housed under an umbrella of "program development," this list groups interventions into three categories: evidence-based practices, emerging practices, and model programs. The list is partially searchable, and the options under the program development umbrella can be narrowed by caregiver role or specific topics. Again, the standard of evidence used by this resource differs substantially from that of this review. The website does not provide uniform criteria to evaluate implementation readiness of level of evidence. Practices categorized as evidence-based are published in the literature after 1990, and must provide credible evidence for improved caregiver outcomes. Additionally, included publications must provide adequate information on methodology to allow for replication (see https://www.caregiver.org/evidence-based-practices). Emerging practices must use innovative methods and/or focus on underserved populations in diverse settings and populations (see https://www.caregiver.org/emerging-practices). Model programs are selected based on expert input, and must have been proven effective, replicated, or adapted, and provide training materials (see https://www.caregiver.org/model-programs). Both 
emerging practices and model programs must be from a credible source and remain available for the foreseeable future.

A fourth source, the Administration for Community Living (ACL), through its National Alzheimer's and Dementia Resource Center (NADRC) (see https://nadrc.acl.gov/node/140) provides a report compendium listing dementia specific evidence-based and evidence-informed interventions that have been implemented through its grant programs. The interventions meet the ACL criteria and have been implemented by Alzheimer's Disease Supportive Services Program (ADSSP), Alzheimer's Disease Initiative Specialized Supportive Services (ADI-SSS) and Alzheimer's Disease Program Initiative (ADPI) grantees from 2007 to 2018. 


\section{Chapter 10. Discussion}

\section{Overview}

This systematic review sought to assess the evidence base for effective care interventions for people living with dementia (PLWD) and their caregivers. Our findings were intended to support the task of identifying which approaches are ready for wider dissemination and implementation. In a society experiencing unprecedented population longevity, this is a crucial task. To accomplish it, we tried to identify interventions and programs supported by evidence that met a minimum threshold of quality. We identified 595 unique eligible studies discussed in 850 publications, in which we found a remarkably diverse set of interventions. We used the National Institutes of Health (NIH) Stage Model framework to classify the interventions into pilot, explanatory, and "real world" pragmatic trials. This classification method allowed us to focus on the studies within this literature set that were best designed to look for real-world effects. We also removed from consideration studies with the potential to bias the outcomes due to concerns with study design or conduct.

Ultimately, we uncovered no moderate- or high-strength evidence to support care interventions and programs for active, widespread dissemination. We found low-strength evidence that collaborative care models (i.e. Care Ecosystems or discrete adaptations of the ACCESS models), may improve quality of life for PLWD and health system-level markers, including improvements in guideline-based quality indicators and reduction of emergency room visits, but the evidence was insufficient for informal caregiver outcomes. We also found lowstrength evidence that an intensive multicomponent intervention, REACH II or discrete adaptations, improved informal caregiver depression at 6 months.

For all other interventions and outcomes, we found the evidence was insufficient. This does not mean that none of the individual interventions described are potentially useful for individual PLWD, their caregivers, or healthcare systems. Rather, it means that current available evidence cannot yet provide clear answers about which interventions offer consistent benefits. Therefore, the uncertainty of the evidence is too high for us to draw conclusions, at present. Further, when the evidence overall does not find a difference between groups, uncertainty is even higher about whether the lack of difference is truly because the interventions being compared did not differ in effect, or because the studies were designed to detect differences rather than no difference.

The lack of sufficient evidence to support widespread dissemination of all other interventions analyzed in this review leaves PLWD, caregivers, programs that support PLWD and caregivers, funders, and policymakers without clear answers. These groups, when deciding whether to disseminate or implement these interventions, will continue to depend on subjective observations, low-quality evidence, economics, and local and institutional policies. For individual PLWD and caregivers, trial and error with interventions, either one at a time or in combination, will likely continue as the norm.

\section{Strengths and Weaknesses of the Review}

We determined the methods for this review in order to best answer the question of readiness for broad dissemination. Decision making through this lens has implications for our findings.

We also adopted a review scope based on concerns about whether results from a literature search would give a biased view of interventions to address symptoms, safety, or quality of life, all of which overlap with frailty in older adults. For example, if falls risk and prevention differs 
greatly between older adults with and without dementia, then research that specifically targets dementia would be useful. Without a big difference in outcomes, study populations likely remained mixed and these studies were screened out. Therefore, some specific approaches for a particular intervention, or even whole classes of interventions, may not have been captured. Similarly, we may have missed some community services and support approaches such as tool kits, referral services and links, or awareness-raising outreach. The case management literature revealed some of these studies, but search terms are diffuse and may have resulted in some studies being overlooked.

Our approach not to advance pilot, small sample, and high risk of bias studies to full analysis resulted in a very high-level assessment of the state of the science. It is possible that in many instances, the inclusion of the preliminary literature may have provided enough data for quantitative pooling for specific outcomes. Systematic reviews of specific interventions can investigate and report very fine details. This review was not intended for that purpose. Our use of the NIH Stage Model as a framework to focus on studies that would best support broad dissemination precluded reporting deeply on pilot studies. Additionally, current guidance for Evidence-based Practice Center (EPC) program methods supports not looking to studies assessed as high risk of bias to fortify what is already at best low-strength evidence. ${ }^{703}$ Lastly, small-study bias in reporting large effect sizes presents an important issue in a literature set where the majority of research is preliminary. ${ }^{704}$

Because we excluded studies with fewer than 10 participants per study arm, we may not have identified some interventions with very preliminary research supporting them. We accepted this limitation because exact precision for the research context findings was not feasible due to the wide range of care approaches and large literature set. Likewise, our decision not to include single-arm pre/post or evaluation studies limited our ability to address practices supported through evaluation studies.

Our approach to risk of bias assessment was generous, compared with how risk of bias is assessed in more targeted systematic review topics. In part, we based this decision on the unusually varied studies included in this review as well as the complexity of dementia and its associated care approaches. We allowed attrition to reach relatively high levels before assigning high risk of bias. Likewise, we treated fidelity generously, giving credit based on relatively brief mention. Concurrent treatments for specific interventions aimed at PLWD, especially for behavioral and psychiatric symptoms of dementia, were given a secondary position in the assessment. Unfortunately, studies rarely presented such information in a way that would allow for a sound evaluation of the implication for bias.

We also used the truncated risk of bias approach for studies assessed as pragmatic. The use of the PRagmatic Explanatory Continuum Indicator Summary-2 (PRECIS-2) tool in systematic reviews is preliminary for classifying and understanding the relative pragmatism in research design. We conservatively used a threshold approach, classifying studies as mostly explanatory, pragmatic, or balanced. We further labeled studies that were mostly pragmatic as good, fair, or marginal based on the analytic techniques. All of this represents modifications to EPC systematic review methods as we expand the boundaries of the topics systematic reviews are being asked to address. We cannot say with certainty how the practices used here affect the findings, regardless of whether we used more or less conservative methods for any particular finding. Nonetheless, as systematic reviews attempt to answer increasingly complex questions, we must find novel ways to answer them. The experiences from this review process may help inform future efforts. 
Finally, given the already expansive breadth of this topic, our systematic review and metaanalysis is naturally reductionist in nature. That is, small but true differences may exist between many of the interventions within the various intervention categories we created for summarizing outcomes.

\section{Future Research}

The questions of "what works" in dementia care and how to deliver that care greatly interests researchers, funders, care providers, healthcare systems, and PLWD and their families. The intensive investment in dementia funding at the Federal level reflects the increasing public health importance not only of finding a way to prevent and treat dementia, but also of developing and eventually disseminating optimal dementia care and caregiver programs. While our review offers no firm conclusions, our findings provide valuable insights for the further development and improvement of dementia care science.

Current practice regarding how PLWD are diagnosed, treated, and supported throughout the disease trajectory are underpinned by concurrent and sometimes overlapping streams of research on dementia diagnosis and medical treatment, geriatric and chronic disease models of care, and dementia care support. ${ }^{705}$ Together, these streams of research have informed best practice recommendations for dementia care, ${ }^{706,707}$ including the central idea that support for needs of family caregivers should be incorporated into any care plan. ${ }^{707}$

\section{Methodological Rigor}

Dementia care research has been slow to incorporate key elements of rigorous intervention design. Until relatively recently, many dementia care and caregiver intervention studies were not held to pre-registration of trials, data safety and monitoring boards, or other standards more common in other areas of clinical science including reporting standards required by journals (e.g., the Consolidated Standards of Reporting Trials [CONSORT] statement). As a direct result, despite a few positive findings, we reached the global conclusion that the evidence is insufficient to say with certainty that interventions were beneficial or not.

Federal funding requirements have instigated change in favor of oversight and reporting mechanisms that will likely lead to more transparent and reproducible research. However, many of these improvements in rigor did not occur until late 2015 and 2016, which coincided with increased investment in research on dementia care interventions. Therefore, many of the trials initially subject to more stringent data monitoring oversight and reporting are just now ending, and their findings have yet to be captured in reviews such as this one. This new, more rigorous research base will hopefully propel future comprehensive reviews to draw conclusions beyond insufficient evidence. Indeed, we did note an improvement in rigor from 2016 to 2017 in the published eligible studies.

In order for Federal funders and stakeholders to fulfill their goal of expediting the translational pipeline of idea development to implementation, critical improvements must be made in dementia care and caregiver research. Only with such improvements will we be able to draw clearer, less ambiguous conclusions related to efficacy.

\section{Populations}

During the topic refinement period for this review, we received many requests to ensure that certain groups that experience dementia were included as research participants in the examined literature. However, the published research rarely included many important populations. For 
example, people with Down syndrome aging into dementia were absent, despite our later ad hoc literature searches to assure no relevant studies were missed. People with frontotemporal dementia were rarely included, and the few identified studies were limited to pilot or small sample studies. Few studies addressed racial or ethnic differences, and those that did were limited to major race/ethnic categories of Black/African American or Hispanic/Latinx. No study specifically studied LGBTQ populations. Likewise, culturally sensitive or culturally adapted interventions were rare. Some identified non-U.S. based research may help inform future intervention adaptations for PLWD or caregivers with immigrant or related racial/ethnic heritages.

Lastly, we identified very few studies of interventions specifically designed for low-resource areas (including rural and/or tribal communities) beyond pilot or small sample studies, which could not reach any level of certainty of the finding. All of these populations represent areas for future research.

\section{Outcomes}

Ultimately, care interventions aim to support quality of life and well-being and prevent harm for PLWD and caregivers, while enabling both to continue in their roles within their families and society. Unfortunately, quality of life was often not measured, and rarely as the outcome of primary interest. Further, PLWD and caregivers exist in relationships with one another, and this literature offers little to help us understand how they change in concert within their dyadic relationship. We attempted to address this issue by placing PLWD and caregiver outcomes adjacent to one another within the results section for each intervention; however, no clear patterns emerged. However, research would be improved by better measures for psychosocial outcomes in PLWD, and better methods of measurement.

Harms were rarely assessed. Although studies frequently measured caregiver burden, they far more often sought to observe reduction in burden than to check for increased burden. Other harms, such as elder abuse, were completely absent.

Additionally, the progressive nature of dementia and the anticipated increase in care needs complicates this research. Studies may be challenged to identify improvement in caregiver burden scales, because the goal of the intervention may in fact be to slow the rate of burden. Studies may be underpowered to detect such a small effect, or small benefits may be overwhelmed by the larger context, including social and financial implications, of caring for a person with a progressive condition.

Lastly, even within intervention categories, outcomes were variably measured and reported. More consistency in the outcomes measured would make it easier to assess bodies of evidence for specific interventions, or to understand how outcomes may differ by setting.

\section{Interventions}

The wide range of identified interventions, and the relationships between PLWD and caregivers, highlight the importance of understanding potential intervention mechanisms. We found low-strength evidence that multicomponent interventions may improve select outcomes, but the underlying question of what drives the benefit (i.e., the specific set of components, the mere presence of a multicomponent approach, or both) remains unanswered.

One anomaly of note in our findings is that while evidence was insufficient for all individual interventions, low-strength evidence showed that multicomponent interventions (i.e. discrete adaptations of REACH II) or collaborative care models (i.e. Care Ecosystems or discrete adaptations of the ACCESS model) could improve some outcomes. On the surface, this finding 
appears contradictory to our global finding of insufficient evidence for all individual interventions. After all, theoretically, multicomponent interventions incorporate individual intervention components that have been shown to work. This discrepancy in our findings suggests two possibilities to explore with further research. One is that adequate support structures at the health system level, such as those provided by the REACH II ${ }^{519}$ or Care Ecosystems interventions, ${ }^{618}$ are crucial factors in addressing the needs of PLWD and their caregivers. Another possibility is that the particular set of interventions may matter less than whether PLWD and caregivers feel supported adequately at the health-system level. Perhaps such structural supports, although not specifically examined or measured in this literature, add significant value to the interventions applied within them.

Lack of consensus about taxonomies to classify interventions hampers the work of assessing this evidence base. We found a lack of precision in how individual interventions were described within specific intervention classifications, and this imprecision inhibits understanding. Many research publications used vague and inconsistent terminology for what constituted a specific intervention, especially given the broad range of baseline dementia severity. For example, we found lack of clarity in the differentiation between cognitive rehabilitation (aimed at restoring daily activity function) and cognitive training activities (cognitive drills that lack direct relationship to completion of daily activities). Authors often used both terms to describe intervention components within a single article. Clearly defining the classes of cognitive rehabilitation interventions would improve comparability across studies, as would specifying which interventions are suitable for PLWD with varying degrees of dementia-related impairment. This is made evident by the extent of misclassification across various systematic reviews and meta-analysis of the effectiveness of dementia caregiving interventions ${ }^{13}$ For example, in a synthesis of reviews, Gaugler and colleagues found that among eight metaanalyses and systematic reviews that considered psychoeducation/skills building, 45 individual interventions were inconsistently classified. One review classified them as psychoeducational/skills building interventions, another placed them in an entirely different category. ${ }^{13}$ Although taxonomies exist to improve the reporting of elements of dementia care and caregiver support interventions, ${ }^{708}$ studies often neglect to use them. Improved reporting of dementia care interventions through the use of taxonomy strategies, either in outcome evaluations or protocol reports, would enhance the ability of reviews such as this one to better classify interventions and thus improve inferences of efficacy/effectiveness.

\section{Complex Interventions for Complex Systems}

Most importantly, the care approaches examined in this review represent complex interventions nested within complex systems. The framework for care interventions from the NASEM Families Caring for an Aging America 2016 report displayed in Figure 1.1 in the Introduction sought to display this idea graphically. Complex systems, by their nature, always encompass some level of uncertainty; indeed, such irreducible uncertainty is a defining element of complex systems. In this literature, the multiple levels of uncertainty are difficult or impossible to overcome. Therefore, we must emphasize again that low-strength evidence is already a difficult bar to reach. Insufficient evidence, places where the evidence is very uncertain, means we could not, with integrity, say that a care approach is beneficial or notwhich, as we have noted, is different from saying it does not work.

For example, even when a care approach focuses on a "lower" complex system level—such as the simple addition of aromatherapy to help a person feel calmer-the challenge is still steep to design a study that rules out all competing influences on that person's sense of calmness. And 
if the effect is small, or moderate but for only a very select group of people, that effect becomes difficult to see in the data, and uncertainty in the findings remains high.

A further problem with researching complex interventions is that complex systems tend to be self-preserving. ${ }^{709}$ Traditional research rests on an assumption that the way the overall process functions does not change as a result of being intervened upon. Yet, a defining feature of a complex system is its ability to adapt to change, even if it takes great effort to propel it from one state of homeostasis toward another, different one. And, when the system does arrive at a new state, it may not be the desired one, but instead an unanticipated adaptation. As a simple example, an intervention may teach staff how to do a new task intended to bring about a desired outcome. But the staff may look for ways to preserve the familiarity of their previous methods, and therefore devise work-arounds. Or they may feel the need to preserve efficiency overall, and make trade-offs in other areas of work. These modifications may multiply across the system as people adapt to the new approaches or requirements.

Many care approaches may actually be aimed, at least in part, at shifting the care culture. As the apex of complex systems, culture is highly diffuse. Yet, culture tends to shift through specific activities that gain traction over time. We see clear examples of this in Veteran-centered care in the United States, or person-centered care in non-U.S. settings. In both examples, training focuses on a relationship-based approach that reframes how caregivers perceive PLWD. Specific activities and tasks are considered secondary, and perhaps even a natural outcome, of this shift in perception and relationship.

Certainly, research is lacking in regard to how components in complex interventions interact to influence key outcomes. The prevailing approach in the dementia care literature is to develop an intervention with multiple components, and deliver it to determine efficacy on caregiver or PLWD outcomes; whether a single or select number of components are essential to an effect (if it exists) is generally unanswered. This has important and adverse implications for the field as a whole, and reduces the dissemination and implementation potential of dementia care interventions. This problem escalates as interventions increase in complexity due to the time, cost, and training requirements to deliver them successfully. In other domains of the intervention literature, approaches such as the Multiphase Optimization Strategy (MOST) have been applied to increase understanding of how singular intervention components interact to influence key outcomes prior to efficacy testing. ${ }^{710}$ Such approaches may expedite the timeline from intervention development to potential implementation by crafting and evaluating interventions that are distilled to their essential components. Additionally, these approaches may offer greater insight into not just whether an intervention works, but why. It would also be helpful if researchers were to conduct and publish process evaluations of dementia care interventions that better described the mechanisms of benefit. Relatedly, basing dementia care and caregiver interventions on theories or conceptual models to test such mechanisms is an essential component of the NIH Stage Model. These theories and conceptual models should inform the design and evaluation of future dementia care interventions.

\section{Intervention Fidelity}

Whether interventions are simple or complex, problems with fidelity are significant for this research. Many studies reported no differences between groups. If an intervention showed benefit, delivery of a sufficient dose is assumed. Conversely, if no difference could be demonstrated, the reader is left with uncertainty about whether the dose was large enough. We used a liberal approach to fidelity while assessing risk of bias. Had we imposed a firm restriction on some form of fidelity measure, much or most of the literature would have been excluded. 
Even so, problems with fidelity often contributed to high risk of bias. Unfortunately, fidelity assessment approaches lack consensus regarding both the components and how to measure them.

Further, we note that this research treats the concept of fidelity to intervention in training and formal caregiving differently than fidelity to intervention by informal caregivers. Informal caregivers have arguably the best incentives to help PLWD, which would motivate fidelity. However, many factors may inhibit fidelity even when desired. Although companion publications may use mixed-methods research to probe informal caregivers' views on an intervention, the question of whether interventions are delivered as designed and trained is rarely tracked for informal caregivers.

\section{Implementation}

Overall, the evidence we reviewed suggests that to consider questions related to dissemination and implementation at the outset of intervention design would result in more dementia care and caregiver interventions becoming ready for rapid implementation in realworld settings. For example, incorporating measures or indicators of implementation (e.g., appropriateness, feasibility, acceptability, cost) alongside clinical outcomes of efficacy/effectiveness would expedite the timeline from dementia care intervention development and evaluation to dissemination and implementation. That, in turn, would help the interventions that demonstrate efficacy to reach and benefit those who need and desire them. ${ }^{711}$

Threats to scalability in dementia care include reliance on interventions that require extensive training and fidelity monitoring; too great a need to rely on the original developers of the intervention; a requirement for highly trained and skilled professionals to deliver the intervention; highly complex, intense, and costly interventions; lack of implementation manuals; and lack of payment mechanisms to ensure sustainability. ${ }^{712}$

Further, implementation will also differ by setting. Future attempts to transfer or modify interventions to assisted living facilities could be helped by a better understanding of implementation factors. Assisted living facilities were among the least studied settings in the included literature.

Another important question pertains to the evidentiary standard that should apply to the complex interventions so overwhelmingly represented in this literature set. EPC guidance provides, as does the Grading of Recommendations Assessment, Development, and Evaluation (GRADE) working group, clear principles and guidelines to apply to medical treatment or therapies that come with benefits weighed against potential harms. Complex interventions applied to complex whole-person and community systems in varied and often complex settings can be exceedingly difficult to implement with fidelity and potential for replication and dissemination. In addition, the study of these interventions is challenged by the appropriateness of basic assumptions that underlie research designs and statistical tools.

One approach to many of these issues is better use of community-based research methods. Involving PLWD and caregivers in planning, implementing, and disseminating research is increasingly recognized as valuable for addressing the populations for which interventions are intended. Applying this approach to PLWD and their caregivers was recognized at a Health and Human Services Summit in $2017 .{ }^{713}$

\section{Broader Research Context}

Another concern is how informal caregivers are perceived, and the way in which this perception informs research designs. While caregiving for PLWD presents challenges, burdens, and risks to the health of caregivers, it is not a pathological condition. Interventions aimed at 
mitigating burdens for informal caregivers can also recognize and build on the rewards of caregiving and the bonds it nurtures between caregivers/partners and care recipients.

Additional questions to consider pertain to how dementia care science might be conducted more efficiently and effectively. Perhaps it is possible to create an environment that encourages experimenting with care solutions while maximizing the ability to learn from those efforts. One avenue for growth may lie in more collaborative, open science with collective impact approaches to its development. This represents a different form of "big science," in which resources such as Benjamin Rose Institute on Aging’s (BRI) Best Practice Caregiving resource (see https://www.benrose.org/best-practice-caregiving) could be an important asset, allowing us another way to close feedback loops and gain knowledge from real-world applications. Innovation requires something more than status quo behavior, as does the research needed to push toward paradigm growth or adoption. Innovation in research involves maximizing discovery while minimizing the risk of locking in solutions that are only currently relevant or partially accurate. Perhaps we can find easier ways for researchers and care systems to test new things efficiently, without overburdening coordination and/or oversight. Many researchers are already thinking deeply about these kinds of questions, including the Center for Open Science (see https://cos.io/ or http://www.unesco.org/new/en/communication-and-information/portalsand-platforms/goap/) or MetaScience (see https://www.metascience2019.org/) initiatives.

Much research has limited impact because it is conducted in isolation, among small groups, and with variable timing for study endpoints to measure outcomes. However, demonstration projects have been conducted to examine specific research questions being crowdsourced by the research community, with peer review processes built in prior to and during analysis, rather than through an extensive research and publication process. ${ }^{714}$ This type of collaborative research can prioritize outcome measurement tools and timing in a way that leads to science that others can measure and replicate.

Many aspects of care interventions for PLWD and their caregivers need more thorough exploration. We hesitated to give an exhaustive list for fear of overwhelming the readers. We were instead guided by peer and public comments on the draft version of this report to specifically mention the following areas: functional and health status limitations, access to care and intervention services and supports as well as accessibility, transportation, culture, racial/ethnic, and related factors. Public commentators to this report who are living with dementia noted an urgent need for more research on interventions that support personhood, purpose and meaning, social and peer supports, proactive approaches to living with a chronic, progressive illness, and lifestyle and spirituality interventions.

Importantly, we do note a gap in the literature for interventions that address the early stages of dementia and being an informal caregiver, just at the time of diagnosis, when problems associated with disconnection, denial, and misinformation may be most urgent.

In the end, high-level discussions of how future research might be structured should not distract us from the primary need to provide research that is relevant to all of the populations that matter. With the exception of a handful of studies, the current research is silent for many social groups, whether by race/ethnicity, citizen status, geographic locations, or dementia types, to name some of the larger categories. Much crucial work remains to be done. 


\section{Abbreviations and Acronyms}

ACCESS

ACP

$\mathrm{AD}$

ADL

ADRD

AHRQ

AMSTAR

BPSD

CAM

CONSORT

CST

DCM $^{\mathrm{TM}}$

EPC

HHS

HMD

IMPACT

KQ

MMSE

MOST

MSS

$\mathrm{N}$

NASEM

NIA

$\mathrm{NIH}$

NPI

PICOTS

PRECIS-2

PRISMA

PLWD

QoL

QOL-AD

RCT

RDAD

REACH II

RMC

SMD
Alzheimer's Disease Coordinated Care for San Diego Seniors

Advance Care Planning

Alzheimer's Disease

Activities of Daily Living

Alzheimer's Disease and Related Dementias

Agency for Healthcare Research and Quality

Assessment of Multiple Systematic Reviews

Behavioral and Psychological Symptoms of Dementia

Complementary and Alternative Medicine

Consolidated Standards of Reporting Trials

Cognitive Stimulation Therapy

Dementia Care Mapping ${ }^{\mathrm{TM}}$

Evidence-based Practice Center

Health and Human Services

Health and Medicine Division

IMbedded Pragmatic Alzheimer’s disease and Related Dementias Clinical Trials

Key Question

Mini Mental State Examination

Multiphase Optimization Strategy

Multisensory Stimulation

Number

National Academies of Sciences, Engineering, and Medicine

National Institute on Aging

National Institutes of Health

Neuropsychiatric Inventory

Population, Intervention, Comparison, Outcomes and Timing

PRagmatic Explanatory Continuum Indicator Summary-2 tool

Preferred Reporting Items for Systematic Review and Meta-Analysis

People Living With Dementia

Quality of Life

Quality of Life-Alzheimer's Disease

Randomized Controlled Trial

Reducing Disability in Alzheimer's Disease

Resources for Enhancing Alzheimer’s Caregivers Health

Routine Medical Care

Standardized Mean Difference 
STAR-C

UK

US
Social learning theory and principles of behavior analysis of caregivers United Kingdom

United States 


\section{References}

1. Alzheimer's Association. 2018 Alzheimer's disease facts and figures. Alzheimer's \& Dementia: The Journal of the Alzheimer's Association. 2018;14:367429.

2. Matthews KA, Xu W, Gaglioti AH, et al. Racial and ethnic estimates of Alzheimer's disease and related dementias in the United States (20152060 ) in adults aged $\geq 65$ years. Alzheimers Dement. 2019 Jan;15(1):1724. doi: 10.1016/j.jalz.2018.06.3063. PMID: 30243772.

3. Hebert LE, Weuve J, Scherr PA, et al. Alzheimer disease in the United States (2010-2050) estimated using the 2010 census. Neurology. 2013 May 7;80(19):1778-83. doi: 10.1212/WNL.0b013e31828726f5. PMID: 23390181.

4. McKhann GM, Knopman DS, Chertkow H, et al. The diagnosis of dementia due to Alzheimer's disease: recommendations from the National Institute on AgingAlzheimer's Association workgroups on diagnostic guidelines for Alzheimer's disease. Alzheimer's \& Dementia. The journal of the Alzheimer's Association. 2011 May;7(3):263-9. doi: 10.1016/j.jalz.2011.03.005. PMID: 21514250.

5. Office of the Assistant Secretary for Planning and Evaluation. National Alzheimer's Project Act. https://aspe.hhs.gov/nationalalzheimers-project-act https://aspe.hhs.gov/nationalalzheimers-project-act. Accessed on February 222019.

6. Hurd MD, Martorell P, Delayande A, et al. Monetary costs of dementia in the United States. New England Journal of Medicine. 2013 Apr 4;368(14):1326-34. doi: 10.1056/NEJMsa1204629. PMID: 23550670.
7. Zhao QF, Tan L, Wang HF, et al. The prevalence of neuropsychiatric symptoms in Alzheimer's disease: Systematic review and meta-analysis. Journal of Affective Disorders. 2016;190:264-71. doi: 10.1016/j.jad.2015.09.069. PMID: 26540080.

8. National Alzheimer's Project Act. 124 STAT. 4100 111th Congress. United States of America; 2011.

9. Reus VI, Fochtmann LJ, Eyler AE, et al. The American Psychiatric Association Practice Guideline on the Use of Antipsychotics to Treat Agitation or Psychosis in Patients With Dementia. American Journal of Psychiatry. 2016 May 1;173(5):543-6. doi: 10.1176/appi.ajp.2015.173501. PMID: 27133416.

10. Corbett A, Stevens J, Aarsland D, et al. Systematic review of services providing information and/or advice to people with dementia and/or their caregivers. International Journal of Geriatric Psychiatry. 2012 June 27;27(6):628-36. doi: 10.1002/gps.2762. PMID: 22038644.

11. Centers for Disease Control and Prevention. Caregiving for Person with Alzheimer's Disease or a related Dementia. https://www.cdc.gov/aging/caregiving/a lzheimer.htm. Accessed on Feb 22.

12. Committee on Family Caregiving for Older Adults. Families Caring for an Aging America. In: Shulz R, Eden J, eds. Washington (DC): National Academies Press (US); 2016.

13. Gaugler JE, Jutkowitz E, Shippee TP, et al. Consistency of dementia caregiver intervention classification: an evidencebased synthesis. International psychogeriatrics / IPA. 2017;29(1):1930. doi: 10.1017/S1041610216001514. PMID: 27671663. 
14. Warshaw GA, Bragg EJ. Preparing The Health Care Workforce To Care For Adults With Alzheimer's Disease And Related Dementias. Health Affairs. 2014;33(4):633-41. doi: 10.1377/hlthaff.2013.1232. PMID: 24711325.

15. Onken LS, Carroll KM, Shoham V, et al. Reenvisioning clinical science: Unifying the discipline to improve the public health. Clinical Psychological Science. 2014;2(1):22-34. doi: 10.1177/2167702613497932. PMID: 25821658.

16. Moher D, Altman DG, Liberati A, et al. PRISMA statement. Epidemiology. 2011 Jan;22(1):128; author reply doi: http://dx.doi.org/10.1097/EDE.0b013e3 181fe7825. PMID: 21150360.

17. Shea BJ, Reeves BC, Wells G, et al. AMSTAR 2: a critical appraisal tool for systematic reviews that include randomised or non-randomised studies of healthcare interventions, or both. BMJ. 2017;358. doi: 10.1136/bmj.j4008.

18. Viswanathan M, Ansari M, Berkman N, et al. Assessing the Risk of Bias of Individual Studies in Systematic Reviews of Health Care Interventions AHRQ. 2012.

19. Hempel S, Shekelle PG, Liu JL, et al. Development of the quality improvement minimum quality criteria set (QI-MOCS): a tool for critical appraisal of quality improvement intervention publications. BMJ Quality and Safety. 2015;24:796-804.

20. Patsopoulos N. A pragmatic view on pragmatic trials. Dialogues Clin Neurosci. 2011;13(2):217-24. PMID: 21842619.

21. Loudon K, Treweek S, Sullivan F, et al. The PRECIS-2 tool: designing trials that are fit for purpose. Bmj. 2015 May 8;350:h2147. doi: 10.1136/bmj.h2147. PMID: 25956159.
22. Berkman ND, Lohr K, Ansari M, et al. Grading the strength of a body of evidence when assessing health care interventions for the effective health care program of the Agency for Healthcare Research and Quality: An update. Rockville, MD: Agency for Healthcare Research and Quality; 2013. (vol. AHRQ Publication No. 13(14)EHC 130-EF).

23. Jones C, Moyle W, Murfield J, et al. Does Cognitive Impairment and Agitation in Dementia Influence Intervention Effectiveness? Findings From a ClusterRCT With the Therapeutic Robot, PARO. Journal of the American Medical Directors Association. 2018 Apr 13;13:13. doi: https://dx.doi.org/10.1016/j.jamda.2018. 02.014. PMID: 29656838.

24. Mervin MC, Moyle W, Jones C, et al. The Cost-Effectiveness of Using PARO, a Therapeutic Robotic Seal, to Reduce Agitation and Medication Use in Dementia: Findings from a ClusterRandomized Controlled Trial. Journal of the American Medical Directors Association. 2018 Jan 09;09:09. doi: https://dx.doi.org/10.1016/j.jamda.2017. 10.008. PMID: 29325922.

25. Moyle W, Jones C, Murfield J, et al. Effect of a robotic seal on the motor activity and sleep patterns of older people with dementia, as measured by wearable technology: A cluster-randomised controlled trial. Maturitas. 201801 Apr;110:10-7. doi: http://dx.doi.org/10.1016/j.maturitas.20 18.01.007. PMID: 621060108.

26. Moyle W, Jones CJ, Murfield JE, et al. Use of a Robotic Seal as a Therapeutic Tool to Improve Dementia Symptoms: A Cluster-Randomized Controlled Trial. Journal of the American Medical Directors Association. 2017 Sep 01;18(9):766-73. doi: https://dx.doi.org/10.1016/j.jamda.2017. 03.018. PMID: 28780395. 
27. Bemelmans R, Gelderblom GJ, Jonker P, et al. Effectiveness of Robot Paro in Intramural Psychogeriatric Care: A Multicenter Quasi-Experimental Study. Journal of the American Medical Directors Association. 2015 Nov 01;16(11):946-50. doi: https://dx.doi.org/10.1016/j.jamda.2015. 05.007. PMID: 26115817.

28. Joranson N, Pedersen I, Rokstad AM, et al. Effects on Symptoms of Agitation and Depression in Persons With Dementia Participating in Robot-Assisted Activity: A Cluster-Randomized Controlled Trial. Journal of the American Medical Directors Association. 2015 Oct 01;16(10):86773. doi: https://dx.doi.org/10.1016/j.jamda.2015. 05.002. PMID: 26096582.

29. Joranson N, Pedersen I, Rokstad AM, et al. Change in quality of life in older people with dementia participating in Paroactivity: a cluster-randomized controlled trial.[Erratum appears in J Adv Nurs. 2017 Jun;73(6):1531; PMID: 28485533]. Journal of Advanced Nursing. 2016 Dec;72(12):3020-33. doi: https://dx.doi.org/10.1111/jan.13076. PMID: 27434512.

30. Liang A, Piroth I, Robinson $\mathrm{H}$, et al. A Pilot Randomized Trial of a Companion Robot for People With Dementia Living in the Community. Journal of the American Medical Directors Association. 2017 Oct 01;18(10):871-8. doi:

https://dx.doi.org/10.1016/j.jamda.2017. 05.019. PMID: 28668664.

31. Moyle W, Cooke M, Beattie E, et al. Exploring the effect of companion robots on emotional expression in older adults with dementia: a pilot randomized controlled trial. Journal of Gerontological Nursing. 2013 May;39(5):46-53. doi: https://dx.doi.org/10.3928/0098913420130313-03. PMID: 23506125.

32. Petersen S, Houston S, Qin H, et al. The Utilization of Robotic Pets in Dementia Care. Journal of Alzheimer's Disease. 2017;55(2):569-74. PMID: 27716673.
33. Valenti SM, Aguera-Ortiz L, Olazaran RJ, et al. Social robots in advanced dementia. Frontiers in aging neuroscience. 2015;7(Jun). doi: 10.3389/fnagi.2015.00133. PMID: CN01103263.

34. Pu L, Moyle W, Jones C, et al. The Effect of Using PARO for People Living With Dementia and Chronic Pain: A Pilot Randomized Controlled Trial. Journal of the American Medical Directors Association. 2020 Feb 28;28:28. doi: https://dx.doi.org/10.1016/j.jamda.2020. 01.014. PMID: 32122797.

35. Majic T, Gutzmann H, Heinz A, et al. Animal-assisted therapy and agitation and depression in nursing home residents with dementia: a matched case-control trial. American Journal of Geriatric Psychiatry. 2013 Nov;21(11):1052-9. doi: https://dx.doi.org/10.1016/j.jagp.2013.0 3.004. PMID: 23831177.

36. Menna LF, Santaniello A, Gerardi F, et al. Efficacy of animal-assisted therapy adapted to reality orientation therapy: measurement of salivary cortisol. Psychogeriatrics:The Official Journal of the Japanese Psychogeriatric Society. 2019 Sep;19(5):510-2. doi: https://dx.doi.org/10.1111/psyg.12418. PMID: 30740833.

37. Olsen C, Pedersen I, Bergland A, et al. Effect of animal-assisted activity on balance and quality of life in home-dwelling persons with dementia. Geriatric Nursing. 2016 Jul-Aug;37(4):284-91. doi:

https://dx.doi.org/10.1016/j.gerinurse.20 16.04.002. PMID: 27155968.

38. Olsen C, Pedersen I, Bergland A, et al. Effect of animal-assisted interventions on depression, agitation and quality of life in nursing home residents suffering from cognitive impairment or dementia: a cluster randomized controlled trial. International Journal of Geriatric Psychiatry. 2016 Dec;31(12):1312-21. doi: https://dx.doi.org/10.1002/gps.4436. PMID: 26807956. 
39. Travers C, Perkins J, Rand J, et al. An evaluation of dog-assisted therapy for residents of aged care facilities with dementia. Anthrozoos. 2013;26(2):21325. PMID: CN-00871982.

40. Balzotti A, Filograsso M, Altamura C, et al. Comparison of the efficacy of gestureverbal treatment and doll therapy for managing neuropsychiatric symptoms in older patients with dementia. International Journal of Geriatric Psychiatry. 2019 09;34(9):1308-15. doi: https://dx.doi.org/10.1002/gps.4961. PMID: 30136743.

41. Moyle W, Murfield J, Jones C, et al. Can lifelike baby dolls reduce symptoms of anxiety, agitation, or aggression for people with dementia in long-term care? Findings from a pilot randomised controlled trial. Aging \& Mental Health. 2019;23(10):1442-50. doi: 10.1080/13607863.2018.1498447. PMID: 138051539. Language: English. Entry Date: 20190817. Revision Date: 20190823. Publication Type: Article.

42. Cantarella A, Borella E, Faggian S, et al. Using dolls for therapeutic purposes: A study on nursing home residents with severe dementia. International Journal of Geriatric Psychiatry. 2018 July;33(7):915-25. doi: http://dx.doi.org/10.1002/gps.4872. PMID: 622559552.

43. Baker R, Bell S, Baker E, et al. A randomized controlled trial of the effects of multi-sensory stimulation (MSS) for people with dementia. British Journal of Clinical Psychology. 2001 Mar;40(Pt 1):81-96. PMID: 11317951.

44. Baker R, Holloway J, Holtkamp CCM, et al. Effects of multi-sensory stimulation for people with dementia. Journal of Advanced Nursing. 2003;43(5):465-77. doi: 10.1046/j.1365-2648.2003.02744.x. PMID: 106688835. Language: English. Entry Date: 20050425. Revision Date: 20150819. Publication Type: Journal Article.
45. Strom BS, Engedal K, Benth JS, et al. Effect of the Sonas Programme on Communication in People with Dementia: A Randomized Controlled Trial. Dementia and Geriatric Cognitive Disorders Extra. 2017 JanApr;7(1):122-35. doi: https://dx.doi.org/10.1159/000468147. PMID: 28553314.

46. van Weert JC, van Dulmen AM, Spreeuwenberg PM, et al. The effects of the implementation of snoezelen on the quality of working life in psychogeriatric care. International Psychogeriatrics. 2005 Sep;17(3):40727. PMID: 16252374.

47. Baillon S, Van Diepen E, Prettyman R, et al. A comparison of the effects of Snoezelen and reminiscence therapy on the agitated behaviour of patients with dementia. International Journal of Geriatric Psychiatry. 2004 Nov;19(11):1047-52. PMID: 15481068.

48. Collier L, McPherson K, Ellis-Hill C, et al. Multisensory stimulation to improve functional performance in moderate to severe dementia-interim results. American Journal of Alzheimer's Disease \& Other Dementias. 2010;25(8):698-703. doi: 10.1177/1533317510387582. PMID: 104960420. Language: English. Entry Date: 20101214. Revision Date: 20150820. Publication Type: Journal Article.

49. Staal JA, Sacks A, Matheis R, et al. The effects of Snoezelen (multi-sensory behavior therapy) and psychiatric care on agitation, apathy, and activities of daily living in dementia patients on a short term geriatric psychiatric inpatient unit. International Journal of Psychiatry in Medicine. 2007;37(4):357-70. PMID: 18441625.

50. Van Diepen E, Baillon SF, Redman J, et al. A pilot study of the physiological and behavioural effects of snoezelen in dementia. British Journal of Occupational Therapy. 2002 February;65(2):61-6. PMID: 35396447. 
51. Baker R, Dowling Z, Wareing LA, et al. Snoezelen: its Long-Term and ShortTerm Effects on Older People with Dementia. British Journal of Occupational Therapy. 1997;60(5):2138. PMID: 104158278. Language: English. Entry Date: 20131114. Revision Date: 20150820. Publication Type: Journal Article.

52. Maseda A, Cibeira N, Lorenzo-Lopez L, et al. Multisensory Stimulation and Individualized Music Sessions on Older Adults with Severe Dementia: Effects on Mood, Behavior, and Biomedical Parameters. Journal of Alzheimer's Disease. 2018;63(4):1415-25. doi: https://dx.doi.org/10.3233/JAD-180109. PMID: 29843244.

53. Kouzuki M, Kitao S, Kaju T, et al. Evaluation of the effect of aroma oil as a bath salt on cognitive function. Psychogeriatrics : the official journal of the Japanese Psychogeriatric Society. 2019;02. doi: http://dx.doi.org/10.1111/psyg.12481. PMID: 629513763.

54. Moorman Li R, Gilbert B, Orman A, et al. Evaluating the effects of diffused lavender in an adult day care center for patients with dementia in an effort to decrease behavioral issues: a pilot study. Journal of Drug Assessment. 2017;6(1):1-5. doi: https://dx.doi.org/10.1080/21556660.20 16.1278545. PMID: 28265482.

55. Chieh-Yu F, Moyle W, Cooke M. A randomised controlled trial of the use of aromatherapy and hand massage to reduce disruptive behaviour in people with dementia. BMC Complementary \& Alternative Medicine. 2013;13(1):16573. doi: 10.1186/1472-6882-13-165. PMID: 104229104. Language: English. Entry Date: 20130205. Revision Date: 20150711. Publication Type: Journal Article.

56. Smallwood J, Brown R, Coulter F, et al. Aromatherapy and behaviour disturbances in dementia: a randomized controlled trial. International Journal of Geriatric Psychiatry. 2001 Oct;16(10):1010-3. PMID: 11607948.
57. Moyle W, Cooke ML, Beattie E, et al. Foot massage versus quiet presence on agitation and mood in people with dementia: a randomised controlled trial. International Journal of Nursing Studies. 2014 Jun;51(6):856-64. doi: https://dx.doi.org/10.1016/j.ijnurstu.201 3.10.019. PMID: 24216598.

58. Fu CY, Moyle W, Cooke M. A randomised controlled trial of the use of aromatherapy and hand massage to reduce disruptive behaviour in people with dementia. BMC Complementary \& Alternative Medicine. 2013 Jul 10;13:165. doi: https://dx.doi.org/10.1186/1472-688213-165. PMID: 23837414.

59. Fung JKK, Tsang HW. Management of behavioural and psychological symptoms of dementia by an aromamassage with acupressure treatment protocol: A randomised clinical trial. Journal of Clinical Nursing. 2018 May;27(9-10):1812-25. doi: https://dx.doi.org/10.1111/jocn.14101. PMID: 28986942.

60. Lu DF, Hart LK, Lutgendorf SK, et al. Slowing progression of early stages of AD with alternative therapies: a feasibility study. Geriatric Nursing. 2013 Nov-Dec;34(6):457-64. doi: https://dx.doi.org/10.1016/j.gerinurse.20 13.07.003. PMID: 23972540.

61. Jia Y, Zhang X, Yu J, et al. Acupuncture for patients with mild to moderate Alzheimer's disease: a randomized controlled trial. BMC Complementary \& Alternative Medicine. 2017 Dec 29;17(1):556. doi: https://dx.doi.org/10.1186/s12906-0172064-X. PMID: 29284465.

62. Jimbo D, Kimura Y, Taniguchi M, et al. Effect of aromatherapy on patients with Alzheimer's disease. Psychogeriatrics. 2009;9(4):173-9. doi: 10.1111/j.14798301.2009.00299.x. PMID: CN00750884. 
63. Rodriguez-Mansilla J, Gonzalez-Lopez-Arza MV, Varela-Donoso E, et al. Ear therapy and massage therapy in the elderly with dementia: a pilot study. Journal of Traditional Chinese Medicine. 2013 Aug;33(4):461-7. PMID: 24187866.

64. Kwan RYC, Leung MCP, Lai CKY. A Randomized Controlled Trial Examining the Effect of Acupressure on Agitation and Salivary Cortisol in Nursing Home Residents with Dementia. Dementia \& Geriatric Cognitive Disorders. 2017;44(1-2):92104. doi: https://dx.doi.org/10.1159/000478739. PMID: 28768251.

65. Woods DL, Craven RF, Whitney J. The effect of therapeutic touch on behavioral symptoms of persons with dementia. Alternative Therapies in Health \& Medicine. 2005 JanFeb;11(1):66-74. PMID: 15712768.

66. Zalomonson S, Freud T, Punchik B, et al. The results of a cross-over placebocontrolled study of the effect of lavender oil on behavioral and psychological symptoms of dementia. Rejuvenation Research. 2018 Oct 17;17:17. doi: https://dx.doi.org/10.1089/rej.2018.212 3. PMID: 30328781.

67. Yang YP, Wang CJ, Wang JJ. Effect of Aromatherapy Massage on Agitation and Depressive Mood in Individuals With Dementia. Journal of Gerontological Nursing. 2016 Sep 01;42(9):38-46. doi: https://dx.doi.org/10.3928/0098913420160615-03. PMID: 27319407.

68. Hawranik P, Johnston P, Deatrich J. Therapeutic touch and agitation in individuals with Alzheimer's disease. Western Journal of Nursing Research. 2008 Jun;30(4):417-34. doi: https://dx.doi.org/10.1177/01939459073 05126. PMID: 18272750.
69. Kwan RY, Leung MC, Lai CK. Acupressure for agitation in nursing home residents with dementia: study protocol for a randomized controlled trial. Trials [Electronic Resource]. 2014 Oct 27;15:410. doi: https://dx.doi.org/10.1186/1745-621515-410. PMID: 25346026.

70. Rodriguez-Mansilla J, Gonzalez Lopez-Arza MV, Varela-Donoso E, et al. The effects of ear acupressure, massage therapy and no therapy on symptoms of dementia: a randomized controlled trial. Clinical Rehabilitation. 2015 Jul;29(7):683-93. doi: https://dx.doi.org/10.1177/02692155145 54240. PMID: 25322869.

71. Takahashi Y, Shindo S, Kanbayashi T, et al. Examination of the influence of cedar fragrance on cognitive function and behavioral and psychological symptoms of dementia in Alzheimer type dementia. Neuropsychopharmacology reports. 2020. PMID: CN-02084429.

72. Ballard CG, O’Brien JT, Reichelt K, et al. Aromatherapy as a safe and effective treatment for the management of agitation in severe dementia: the results of a double-blind, placebo-controlled trial with Melissa. Journal of Clinical Psychiatry. 2002 Jul;63(7):553-8. PMID: 12143909.

73. Burns A, Perry E, Holmes C, et al. A doubleblind placebo-controlled randomized trial of Melissa officinalis oil and donepezil for the treatment of agitation in Alzheimer's disease. Dementia \& Geriatric Cognitive Disorders. 2011;31(2):158-64. doi: https://dx.doi.org/10.1159/000324438. PMID: 21335973.

74. Lin PW, Chan WC, Ng BF, et al. Efficacy of aromatherapy (Lavandula angustifolia) as an intervention for agitated behaviours in Chinese older persons with dementia: a cross-over randomized trial. International Journal of Geriatric Psychiatry. 2007 May;22(5):405-10. PMID: 17342790. 
75. Yang SY, Shan CL, Qing H, et al. The Effects of Aerobic Exercise on Cognitive Function of Alzheimer's Disease Patients. CNS \& Neurological Disorders Drug Targets. 2015;14(10):1292-7. PMID: 26556080.

76. Bramble M, Moyle W, Shum D. A quasiexperimental design trial exploring the effect of a partnership intervention on family and staff well-being in long-term dementia care. Aging \& Mental Health. 2011 Nov;15(8):995-1007. doi: https://dx.doi.org/10.1080/13607863.20 11.583625. PMID: 21702706.

77. Ancoli-Israel S, Martin JL, Gehrman P, et al. Effect of light on agitation in institutionalized patients with severe Alzheimer disease. American Journal of Geriatric Psychiatry. 2003 MarApr;11(2):194-203. PMID: 12611749.

78. Burns A, Allen H, Tomenson B, et al. Bright light therapy for agitation in dementia: a randomized controlled trial. International Psychogeriatrics. 2009 Aug;21(4):711-21. doi: https://dx.doi.org/10.1017/S104161020 9008886. PMID: 19323872.

79. Dowling GA, Graf CL, Hubbard EM, et al. Light treatment for neuropsychiatric behaviors in Alzheimer's disease. Western Journal of Nursing Research. 2007 Dec;29(8):961-75. PMID: 17596638.

80. Fontana Gasio P, Krauchi K, Cajochen C, et al. Dawn-dusk simulation light therapy of disturbed circadian rest-activity cycles in demented elderly. Experimental Gerontology. 2003 JanFeb;38(1-2):207-16. PMID: 12543279.

81. Hickman SE, Barrick AL, Williams CS, et al. The effect of ambient bright light therapy on depressive symptoms in persons with dementia. Journal of the American Geriatrics Society. 2007 Nov;55(11):1817-24. PMID: 17944896.
82. Mishima K, Hishikawa Y, Okawa M. Randomized, dim light controlled, crossover test of morning bright light therapy for rest-activity rhythm disorders in patients with vascular dementia and dementia of Alzheimer's type. Chronobiology International. 1998 Nov;15(6):647-54. PMID: 9844752.

83. Onega LL, Pierce TW, Epperly L. Effect of Bright Light Exposure on Depression and Agitation in Older Adults with Dementia. Issues in Mental Health Nursing. 2016 Sep;37(9):660-7. PMID: 27254531.

84. Riemersma-van der Lek RF, Swaab DF, Twisk J, et al. Effect of bright light and melatonin on cognitive and noncognitive function in elderly residents of group care facilities: a randomized controlled trial. JAMA: Journal of the American Medical Association. 2008;299(22):2642-55. doi: 10.1001/jama.299.22.2642. PMID: 105752996. Language: English. Entry Date: 20080627. Revision Date: 20161112. Publication Type: journal article.

85. Figueiro MG, Plitnick B, Roohan C, et al. Effects of a Tailored Lighting Intervention on Sleep Quality, RestActivity, Mood, and Behavior in Older Adults With Alzheimer Disease and Related Dementias: A Randomized Clinical Trial. Journal of Clinical Sleep Medicine. 2019 Dec 15;15(12):1757-67. doi:

https://dx.doi.org/10.5664/jcsm.8078. PMID: 31855161.

86. Riemersma-van der Lek RF, Swaab DF, Twisk J, et al. Effect of bright light and melatonin on cognitive and noncognitive function in elderly residents of group care facilities: a randomized controlled trial. JAMA. 2008 Jun 11;299(22):2642-55. doi: https://dx.doi.org/10.1001/jama.299.22. 2642. PMID: 18544724. 
87. Toseland RW, Diehl M, Freeman K, et al. The impact of validation group therapy on nursing home residents with dementia. Journal of Applied Gerontology. 1997 March;16(1):31-50. doi:

http://dx.doi.org/10.1177/07334648970 1600102. PMID: 27097884.

88. Spector A, Charlesworth G, King M, et al. Cognitive-behavioural therapy for anxiety in dementia: pilot randomised controlled trial. British Journal of Psychiatry. 2015 Jun;206(6):509-16. doi: https://dx.doi.org/10.1192/bjp.bp.113.1 40087. PMID: 25698766.

89. Stanley MA, Calleo J, Bush AL, et al. The peaceful mind program: a pilot test of a cognitive-behavioral therapy-based intervention for anxious patients with dementia. American Journal of Geriatric Psychiatry. 2013 Jul;21(7):696-708. doi: https://dx.doi.org/10.1016/j.jagp.2013.0 1.007. PMID: 23567399.

90. Beck CK, Vogelpohl TS, Rasin JH, et al. Effects of behavioral interventions on disruptive behavior and affect in demented nursing home residents. Nursing Research. 2002 JulAug;51(4):219-28. PMID: 12131234.

91. Kiosses DN, Rosenberg PB, McGovern A, et al. Depression and Suicidal Ideation During Two Psychosocial Treatments in Older Adults with Major Depression and Dementia. Journal of Alzheimer's Disease. 2015;48(2):453-62. doi: https://dx.doi.org/10.3233/JAD-150200. PMID: 26402009.

92. Richards KC, Beck C, O'Sullivan PS, et al. Effect of individualized social activity on sleep in nursing home residents with dementia. Journal of the American Geriatrics Society. 2005

Sep;53(9):1510-7. PMID: 16137280.
93. Law W, Kwok TCY. Impacts of a multicomponent intervention programme on neuropsychiatric symptoms in people with dementia and psychological health of caregivers: A feasibility pilot study. International Journal of Geriatric Psychiatry. 2019 Aug 07;07:07. doi: https://dx.doi.org/10.1002/gps.5191. PMID: 31390090.

94. Bailey EM, Stevens AB, LaRocca MA, et al. A Randomized Controlled Trial of a Therapeutic Intervention for Nursing Home Residents With Dementia and Depressive Symptoms. Journal of Applied Gerontology. 2017 Jul;36(7):895-908. doi: https://dx.doi.org/10.1177/07334648156 27956. PMID: 26912731.

95. Fischer-Terworth C, Probst P. Evaluation of a TEACCH-and music therapy-based psychological intervention in mild to moderate dementia. In Geropsych: the journal of gerontopsychology and geriatric psychiatry

96. Kovach CR, Taneli Y, Dohearty P, et al. Effect of the BACE intervention on agitation of people with dementia. Gerontologist. 2004 Dec;44(6):797-806. PMID: 15611216.

97. Lawton MP, Van Haitsma K, Klapper J, et al. A stimulation-retreat special care unit for elders with dementing illness. International Psychogeriatrics. 1998 Dec;10(4):379-95. PMID: 9924833.

98. Lin LC, Yang MH, Kao CC, et al. Using acupressure and Montessori-based activities to decrease agitation for residents with dementia: a cross-over trial. Journal of the American Geriatrics Society. 2009 Jun;57(6):1022-9. PMID: 19507295.

99. Schrijnemaekers V, van Rossum E, Candel $\mathrm{M}$, et al. Effects of emotion-oriented care on elderly people with cognitive impairment and behavioral problems. International Journal of Geriatric Psychiatry. 2002 Oct;17(10):926-37. PMID: 12325052. 
100. Finnema E, Droes RM, Ettema T, et al. The effect of integrated emotion-oriented care versus usual care on elderly persons with dementia in the nursing home and on nursing assistants: a randomized clinical trial. International Journal of Geriatric Psychiatry. 2005 Apr;20(4):330-43. PMID: 15799079.

101. Toots A, Littbrand H, Lindelof N, et al. Effects of a High-Intensity Functional Exercise Program on Dependence in Activities of Daily Living and Balance in Older Adults with Dementia. Journal of the American Geriatrics Society. 2016 Jan;64(1):55-64. doi: https://dx.doi.org/10.1111/jgs.13880. PMID: 26782852.

102. Pitkala KH, Poysti MM, Laakkonen ML, et al. Effects of the Finnish Alzheimer disease exercise trial (FINALEX): a randomized controlled trial. JAMA Internal Medicine. 2013 May 27;173(10):894-901. doi: https://dx.doi.org/10.1001/jamainternme d.2013.359. PMID: 23589097.

103. Nyman SR, Ingram W, Sanders J, et al. Randomised Controlled Trial Of The Effect Of Tai Chi On Postural Balance Of People With Dementia. Clinical Interventions In Aging. 2019;14:201729. doi: https://dx.doi.org/10.2147/CIA.S22893 1. PMID: 31819385.

104. Lamb SE, Sheehan B, Atherton N, et al. Dementia And Physical Activity (DAPA) trial of moderate to high intensity exercise training for people with dementia: randomised controlled trial. BMJ. 2018;361:k1675. PMID: 29769247.

105. Hoffmann K, Sobol NA, Frederiksen KS, et al. Moderate-to-High Intensity Physical Exercise in Patients with Alzheimer's Disease: A Randomized Controlled Trial. Journal of Alzheimer's Disease. 2016;50(2):443-53. doi: 10.3233/JAD150817. PMID: 112439259. Language: English. Entry Date: 20161122. Revision Date: 20180627. Publication Type: journal article.
106. Bossers WJ, van der Woude LH, Boersma F, et al. Comparison of Effect of Two Exercise Programs on Activities of Daily Living in Individuals with Dementia: A 9-Week Randomized, Controlled Trial. Journal of the American Geriatrics Society. 2016 Jun;64(6):1258-66. doi: https://dx.doi.org/10.1111/jgs.14160. PMID: 27321604.

107. Telenius EW, Engedal K, Bergland A. Long-term effects of a 12 weeks highintensity functional exercise program on physical function and mental health in nursing home residents with dementia: a single blinded randomized controlled trial. BMC Geriatrics. 2015 Dec 03;15:158. doi: https://dx.doi.org/10.1186/s12877-0150151-8. PMID: 26630910.

108. Hauer K, Schwenk M, Zieschang T, et al. Physical training improves motor performance in people with dementia: a randomized controlled trial. Journal of the American Geriatrics Society. 2012 Jan;60(1):8-15. doi: https://dx.doi.org/10.1111/j.15325415.2011.03778.x. PMID: 22211512.

109. Eggermont LH, Knol DL, Hol EM, et al. Hand motor activity, cognition, mood, and the rest-activity rhythm in dementia: a clustered RCT. Behavioural Brain Research. 2009 Jan 23;196(2):271-8. doi: https://dx.doi.org/10.1016/j.bbr.2008.09 .012. PMID: 18926856.

110. Chen LL, Li H, Chen XH, et al. Effects of Hand Exercise on Eating Action in Patients With Alzheimer's Disease. American Journal of Alzheimer's Disease \& Other Dementias. 2019 02;34(1):57-62. doi: https://dx.doi.org/10.1177/15333175188 03722. PMID: 30301358.

111. Huang N, Li W, Rong X, et al. Effects of a Modified Tai Chi Program on Older People with Mild Dementia: A Randomized Controlled Trial. Journal of Alzheimer's Disease. 2019;72(3):947-56. doi: https://dx.doi.org/10.3233/JAD-190487. PMID: 31743998. 
112. Cancela JM, Ayan C, Varela S, et al. Effects of a long-term aerobic exercise intervention on institutionalized patients with dementia. Journal of Science \& Medicine in Sport. 2016 Apr;19(4):2938. doi: https://dx.doi.org/10.1016/j.jsams.2015. 05.007. PMID: 26087884.

113. Chen KM, Kuo CC, Chang YH, et al. Resistance Band Exercises Reduce Depression and Behavioral Problems of Wheelchair-Bound Older Adults with Dementia: A Cluster-Randomized Controlled Trial. Journal of the American Geriatrics Society. 2017 Feb;65(2):356-63. doi: https://dx.doi.org/10.1111/jgs.14526. PMID: 27879982.

114. Henskens M, Nauta IM, Drost KT, et al. The effects of movement stimulation on activities of daily living performance and quality of life in nursing home residents with dementia: a randomized controlled trial. Clinical Interventions In Aging. 2018;13:805-17. doi: https://dx.doi.org/10.2147/CIA.S16003 1. PMID: 29750023.

115. Ho RTH, Fong TCT, Chan WC, et al. Psychophysiological effects of Dance Movement Therapy and physical exercise on older adults with mild dementia: A randomized controlled trial. Journals of Gerontology Series B Psychological Sciences \& Social Sciences. 2018 Nov 28;28:28. doi: https://dx.doi.org/10.1093/geronb/gby1 45. PMID: 30496547.

116. Masayuki S, Jun-ichi O, Tomoko T, et al. Physical Exercise with Music Maintains Activities of Daily Living in Patients with Dementia: Mihama-Kiho Project Part 21. Journal of Alzheimer's Disease. 2017;57(1):85-96. doi: 10.3233/JAD161217. PMID: 121747018. Language: English. Entry Date: 20180302. Revision Date: 20180619. Publication Type: journal article.
117. Roach KE, Tappen RM, Kirk-Sanchez N, et al. A randomized controlled trial of an activity specific exercise program for individuals with Alzheimer disease in long-term care settings. Journal of Geriatric Physical Therapy. 2011 AprJun;34(2):50-6. doi: https://dx.doi.org/10.1519/JPT.0b013e3 1820aab9c. PMID: 21937893.

118. Rolland Y, Pillard F, Klapouszczak A, et al. Exercise program for nursing home residents with Alzheimer's disease: a 1year randomized, controlled trial. Journal of the American Geriatrics Society. 2007 Feb;55(2):158-65. PMID: 17302650.

119. Bossers WJ, Scherder EJ, Boersma F, et al. Feasibility of a combined aerobic and strength training program and its effects on cognitive and physical function in institutionalized dementia patients. A pilot study. PLoS ONE [Electronic Resource]. 2014;9(5):e97577. doi: https://dx.doi.org/10.1371/journal.pone. 0097577. PMID: 24844772.

120. Bossers WJ, van der Woude LH, Boersma F, et al. A 9-Week Aerobic and Strength Training Program Improves Cognitive and Motor Function in Patients with Dementia: A Randomized, Controlled Trial. American Journal of Geriatric Psychiatry. 2015 Nov;23(11):1106-16. doi:

https://dx.doi.org/10.1016/j.jagp.2014.1 2.191. PMID: 25648055.

121. Burge E, Berchtold A, Maupetit C, et al. Does physical exercise improve adl capacities in people over 65 years with moderate or severe dementia hospitalized in an acute psychiatric setting? A multisite randomized clinical trial. International psychogeriatrics. 2016. PMID: CN-01308751.

122. de Souto Barreto P, Cesari M, Denormandie $\mathrm{P}$, et al. Exercise or Social Intervention for Nursing Home Residents with Dementia: A Pilot Randomized, Controlled Trial. Journal of the American Geriatrics Society. 2017 Sep;65(9):E123-E9. doi: https://dx.doi.org/10.1111/jgs.14947. PMID: 28542742. 
123. Holthoff VA, Marschner K, Scharf M, et al. Effects of physical activity training in patients with Alzheimer's dementia: results of a pilot RCT study. PLoS ONE [Electronic Resource]. 2015;10(4):e0121478. doi: https://dx.doi.org/10.1371/journal.pone. 0121478. PMID: 25884637.

124. Morris JK, Vidoni ED, Johnson DK, et al. Aerobic exercise for Alzheimer's disease: A randomized controlled pilot trial. PLoS ONE [Electronic Resource]. 2017;12(2):e0170547. doi: https://dx.doi.org/10.1371/journal.pone. 0170547. PMID: 28187125.

125. Padala KP, Padala PR, Lensing SY, et al. Home-Based Exercise Program Improves Balance and Fear of Falling in Community-Dwelling Older Adults with Mild Alzheimer's Disease: A Pilot Study. Journal of Alzheimer's Disease. 2017;59(2):565-74. doi: https://dx.doi.org/10.3233/JAD-170120. PMID: 28655135.

126. Stevens J, Killeen M. A randomised controlled trial testing the impact of exercise on cognitive symptoms and disability of residents with dementia. Contemporary Nurse. 2006 FebMar;21(1):32-40. PMID: 16594879.

127. Suttanon P, Hill KD, Said CM, et al. Feasibility, safety and preliminary evidence of the effectiveness of a homebased exercise programme for older people with Alzheimer's disease: a pilot randomized controlled trial. Clinical Rehabilitation. 2013 May;27(5):427-38. doi:

https://dx.doi.org/10.1177/02692155124 60877. PMID: 23117349.

128. Yu F, Thomas W, Nelson NW, et al. Impact of 6-month aerobic exercise on Alzheimer's symptoms. Journal of Applied Gerontology. 2015 Jun;34(4):484-500. doi: https://dx.doi.org/10.1177/07334648135 12895. PMID: 24652914.
129. Canonici P, Andrade LP, Gobbi S, et al. Functional dependence and caregiver burden in Alzheimer's disease: a controlled trial on the benefits of motor intervention. Psychogeriatrics. 2012;12(3):186-92. doi: 10.1111/j.1479-8301.2012.00407.x. PMID: CN-00863385.

130. Dawson N, Judge KS, Gerhart H. Improved Functional Performance in Individuals With Dementia After a ModerateIntensity Home-Based Exercise Program: A Randomized Controlled Trial. Journal of Geriatric Physical Therapy. 2019 Jan/Mar;42(1):18-27. doi:

https://dx.doi.org/10.1519/JPT.0000000 000000128. PMID: 28252473.

131. Henwood T, Neville C, Baguley C, et al. Aquatic exercise for residential aged care adults with dementia: benefits and barriers to participation. International psychogeriatrics. 2017;29(9):1439-49. doi: 10.1017/S104161021700028X. PMID: CN-01403352.

132. Kwak YS, Um SY, Son TG, et al. Effect of regular exercise on senile dementia patients. International Journal of Sports Medicine. 2008 Jun;29(6):471-4. PMID: 18050054.

133. Liu B, Chen X, Li Y, et al. Effect of passive finger exercises on grip strength and the ability to perform activities of daily living for older people with dementia: a 12-week randomized controlled trial. Clinical Interventions In Aging. 2018;13:2169-77. doi: https://dx.doi.org/10.2147/CIA.S17475 6. PMID: 30464426.

134. Nascimento C, Teixeira C, Gobbi L, et al. A controlled clinical trial on the effects of exercise on neuropsychiatric disorders and instrumental activities in women with Alzheimer's disease. Revista brasileira de fisioterapia (sao carlos (sao paulo, brazil)). 2012;16(3):197-204. PMID: CN-00863837. 
135. Steinberg M, Leoutsakos JM, Podewils LJ, et al. Evaluation of a home-based exercise program in the treatment of Alzheimer's disease: the Maximizing Independence in Dementia (MIND) study. International Journal of Geriatric Psychiatry. 2009 Jul;24(7):680-5. doi: https://dx.doi.org/10.1002/gps.2175. PMID: 19089875.

136. Stella F, Canonici A, Gobbi S, et al. Attenuation of neuropsychiatric symptoms and caregiver burden in Alzheimer's disease by motor intervention: a controlled trial. Clinics (sao paulo, brazil). 2011;66(8):1353-60. PMID: CN-00860745.

137. Toulotte C, Fabre C, Dangremont B, et al. Effects of physical training on the physical capacity of frail, demented patients with a history of falling: a randomised controlled trial. Age \& Ageing. 2003 Jan;32(1):67-73. PMID: 12540351.

138. Van de Winckel A, Feys H, De Weerdt W, et al. Cognitive and behavioural effects of music-based exercises in patients with dementia. Clinical Rehabilitation. 2004 May;18(3):253-60. PMID: 15137556.

139. Venturelli M, Scarsini R, Schena F. Sixmonth walking program changes cognitive and ADL performance in patients with Alzheimer. American Journal of Alzheimer's Disease \& Other Dementias. 2011 Aug;26(5):381-8. doi: https://dx.doi.org/10.1177/15333175114 18956. PMID: 21852281.

140. Vreugdenhil A, Cannell J, Davies A, et al. A community-based exercise programme to improve functional ability in people with Alzheimer's disease: a randomized controlled trial. Scandinavian Journal of Caring Sciences. 2012 Mar;26(1):12-9. doi: https://dx.doi.org/10.1111/j.14716712.2011.00895.x. PMID: 21564154.
141. Brett L, Stapley P, Meedya S, et al. Effect of physical exercise on physical performance and fall incidents of individuals living with dementia in nursing homes: a randomized controlled trial. Physiotherapy Theory \& Practice. 2019 Mar 26:1-14. doi: https://dx.doi.org/10.1080/09593985.20 19.1594470. PMID: 30912690.

142. Fan J, Chen K. Using silver yoga exercises to promote physical and mental health of elders with dementia in long-term care facilities. In International psychogeriatrics / IPA

143. Karssemeijer EGA, Bossers WJR, Aaronson JA, et al. Exergaming as a Physical Exercise Strategy Reduces Frailty in People With Dementia: A Randomized Controlled Trial. Journal of the American Medical Directors Association. 2019 Aug 10;10:10. doi: https://dx.doi.org/10.1016/j.jamda.2019. 06.026. PMID: 31409559.

144. Lee SM, Joung J, Shin SH. Effects of Fumanet exercise on Korean older adults with mild dementia. Japan Journal of Nursing Science: JJNS. 2019 Jul 25:e12286. doi: https://dx.doi.org/10.1111/jjns.12286. PMID: 31347294.

145. Taylor ME, Close JCT, Lord SR, et al. Pilot feasibility study of a home-based fall prevention exercise program (StandingTall) delivered through a tablet computer (iPad) in older people with dementia. Australasian journal on ageing. 2019;19. doi: http://dx.doi.org/10.1111/ajag.12717. PMID: 629382297.

146. Enette L, Vogel T, Merle S, et al. Effect of 9 weeks continuous vs. interval aerobic training on plasma BDNF levels, aerobic fitness, cognitive capacity and quality of life among seniors with mild to moderate Alzheimer's disease: a randomized controlled trial. European Reviews of Aging \& Physical Activity. 2020;17:2. doi: https://dx.doi.org/10.1186/s11556-0190234-1. PMID: 31921371. 
147. Fleiner T, Dauth H, Zijlstra W, et al. A Structured Physical Exercise Program Reduces Professional Caregiver's Burden Caused by Neuropsychiatric Symptoms in Acute Dementia Care: Randomized Controlled Trial Results. Journal of Alzheimer's Disease. 2020;74(2):429-33. doi: https://dx.doi.org/10.3233/JAD-191102. PMID: 32039852.

148. Liu IT, Lee WJ, Lin SY, et al. Therapeutic Effects of Exercise Training on Elderly Patients With Dementia: A Randomized Controlled Trial. Archives of Physical Medicine \& Rehabilitation. 2020 Feb 19;19:19. doi: https://dx.doi.org/10.1016/j.apmr.2020. 01.012. PMID: 32084347.

149. Sanders LMJ, Hortobagyi T, Karssemeijer EGA, et al. Effects of low- and highintensity physical exercise on physical and cognitive function in older persons with dementia: a randomized controlled trial. Alzheimer's Research \& Therapy. 2020 Mar 19;12(1):28. doi: https://dx.doi.org/10.1186/s13195-02000597-3. PMID: 32192537.

150. Todri J, Lena O, Martinez Gil JL. A single blind randomized controlled trial of global postural re-education: Cognitive effects on Alzheimer disease patients. European Journal of Psychiatry. 2019 April - June;33(2):83-90.

151. Lamb SE, Mistry D, Alleyne S, et al. Aerobic and strength training exercise programme for cognitive impairment in people with mild to moderate dementia: The DAPA RCT. Health Technology Assessment. 2018 May;22(28):1-201. doi: http://dx.doi.org/10.3310/hta22280. PMID: 622601831.

152. Cheung DSK, Lai CKY, Wong FKY, et al. The effects of the music-withmovement intervention on the cognitive functions of people with moderate dementia: a randomized controlled trial. Aging \& Mental Health. 2018 Mar;22(3):306-15. doi: https://dx.doi.org/10.1080/13607863.20 16.1251571. PMID: 27819483.
153. Kwak J, Anderson K, O’Connell Valuch K. Findings From a Prospective Randomized Controlled Trial of an Individualized Music Listening Program for Persons With Dementia. Journal of Applied Gerontology. 2018 Jun 01:733464818778991. doi: https://dx.doi.org/10.1177/07334648187 78991. PMID: 29871544.

154. Lin Y, Chu H, Yang CY, et al. Effectiveness of group music intervention against agitated behavior in elderly persons with dementia. International Journal of Geriatric Psychiatry. 2011 Jul;26(7):670-8. doi: https://dx.doi.org/10.1002/gps.2580. PMID: 20672256.

155. Sarkamo T, Tervaniemi M, Laitinen S, et al. Cognitive, emotional, and social benefits of regular musical activities in early dementia: randomized controlled study. Gerontologist. 2014 Aug;54(4):634-50. doi: https://dx.doi.org/10.1093/geront/gnt10 ‥ PMID: 24009169.

156. Sung HC, Lee WL, Li TL, et al. A group music intervention using percussion instruments with familiar music to reduce anxiety and agitation of institutionalized older adults with dementia. International Journal of Geriatric Psychiatry. 2012 Jun;27(6):621-7. doi: https://dx.doi.org/10.1002/gps.2761. PMID: 21823174.

157. Cheung DSK, Lai CKY, Wong FKY, et al. Is music-with-movement intervention better than music listening and social activities in alleviating agitation of people with moderate dementia? A randomized controlled trial. Dementia. 2018 Sep 20:1471301218800195. doi: https://dx.doi.org/10.1177/14713012188 00195. PMID: 30235949.

158. Chu H, Yang CY, Lin Y, et al. The impact of group music therapy on depression and cognition in elderly persons with dementia: a randomized controlled study. Biological Research for Nursing. 2014 Apr;16(2):209-17. doi: https://dx.doi.org/10.1177/10998004134 85410. PMID: 23639952. 
159. Sarkamo T, Laitinen S, Numminen A, et al. Clinical and Demographic Factors Associated with the Cognitive and Emotional Efficacy of Regular Musical Activities in Dementia. Journal of Alzheimer’s Disease. 2016;49(3):76781. doi: https://dx.doi.org/10.3233/JAD150453. PMID: 26519435.

160. Ceccato E, Vigato G, Bonetto C, et al. STAM protocol in dementia: a multicenter, single-blind, randomized, and controlled trial. American Journal of Alzheimer's Disease \& Other Dementias. 2012 Aug;27(5):301-10. doi:

https://dx.doi.org/10.1177/15333175124 52038. PMID: 22815078.

161. Chang F, Huang H, Lin K, et al. The effect of a music programme during lunchtime on the problem behaviour of the older residents with dementia at an institution in Taiwan. Journal of clinical nursing. 2010;19(7-8):939-48. doi: 10.1111/j.1365-2702.2009.02801.x. PMID: CN-00752730.

162. Choi A, Lee M, Cheong K, et al. Effects of group music intervention on behavioral and psychological symptoms in patients with dementia: a pilot-controlled trial. International journal of neuroscience. 2009;119(4):471-81. doi: 10.1080/00207450802328136. PMID: CN-00688678.

163. Giovagnoli AR, Manfredi V, Schifano L, et al. Combining drug and music therapy in patients with moderate Alzheimer's disease: a randomized study. Neurological Sciences. $2018 \mathrm{Mar}$ 17;17:17. doi: https://dx.doi.org/10.1007/s10072-0183316-3. PMID: 29550981.

164. Cooke ML, Moyle W, Shum DH, et al. A randomized controlled trial exploring the effect of music on agitated behaviours and anxiety in older people with dementia. Aging \& Mental Health. 2010 Nov;14(8):905-16. doi: https://dx.doi.org/10.1080/13607861003 713190. PMID: 20635236.
165. Gerdner LA. Effects of individualized versus classical "relaxation" music on the frequency of agitation in elderly persons with Alzheimer's disease and related disorders. International Psychogeriatrics. 2000 Mar;12(1):4965. PMID: 10798453.

166. Groene RW. Effectiveness of music therapy 1:1 intervention with individuals having Senile Dementia of the Alzheimer's Type. Journal of Music Therapy. 1993 Fal;30(3):138-57. doi: http://dx.doi.org/10.1093/jmt/30.3.138. PMID: 1994-10861-001.

167. Guetin S, Portet F, Picot MC, et al. Effect of music therapy on anxiety and depression in patients with Alzheimer's type dementia: randomised, controlled study. Dementia \& Geriatric Cognitive Disorders. 2009;28(1):36-46. doi: https://dx.doi.org/10.1159/000229024. PMID: 19628939.

168. Han P, Kwan M, Chen D, et al. A controlled naturalistic study on a weekly music therapy and activity program on disruptive and depressive behaviors in dementia. Dementia and geriatric cognitive disorders. 2010;30(6):540-6. PMID: CN00856782.

169. Holmes C, Knights A, Dean C, et al. Keep music live: music and the alleviation of apathy in dementia subjects. International Psychogeriatrics. 2006 Dec;18(4):623-30. PMID: 16805928.

170. Lord TR, Garner JE. Effects of music on Alzheimer patients. Perceptual \& Motor Skills. 1993 Apr;76(2):451-5. PMID: 8483655.

171. McHugh L, Gardstrom S, Hiller J, et al. The effect of pre-meal, vocal re-creative music therapy on nutritional intake of residents with Alzheimer's disease and related dementias: a pilot study. Music ther perspect. 2012;30(1):32-42. PMID: CN-01140174.

172. Ledger A, Baker F. An investigation of long-term effects of group music therapy on agitation levels of people with Alzheimer's disease. Aging \& mental health. 2007;11(3):330-8. PMID: CN-00738375. 
173. Sakamoto M, Ando H, Tsutou A. Comparing the effects of different individualized music interventions for elderly individuals with severe dementia. International Psychogeriatrics. 2013 May;25(5):77584. doi: https://dx.doi.org/10.1017/S104161021 2002256. PMID: 23298693.

174. Raglio A, Oasi O, Gianotti M, et al. Effects of music therapy on psychological symptoms and heart rate variability in patients with dementia. A pilot study. Current Aging Science. 2010 Dec;3(3):242-6. PMID: 20735342.

175. Ridder HM, Stige B, Qvale LG, et al. Individual music therapy for agitation in dementia: an exploratory randomized controlled trial. Aging \& Mental Health. 2013;17(6):667-78. doi: https://dx.doi.org/10.1080/13607863.20 13.790926. PMID: 23621805.

176. Sung HC, Chang SM, Lee WL, et al. The effects of group music with movement intervention on agitated behaviours of institutionalized elders with dementia in Taiwan. Complementary Therapies in Medicine. 2006 Jun;14(2):113-9. PMID: 16765849.

177. Ugur HG, Orak OS, Aktas YY, et al. Effects of music therapy on the care burden of in-home caregivers and physiological parameters of their inhome dementia patients: a randomized controlled trial. Complementary medicine research. 2019;26(1):22-30. PMID: CN-01911571.

178. Svansdottir HB, Snaedal J. Music therapy in moderate and severe dementia of Alzheimer's type: a case-control study. International Psychogeriatrics. 2006 Dec;18(4):613-21. PMID: 16618375.

179. Ihara ES, Tompkins CJ, Inoue $\mathrm{M}$, et al. Results from a person-centered music intervention for individuals living with dementia. Geriatrics \& gerontology international. 2019 Jan;19(1):30-4. doi: https://dx.doi.org/10.1111/ggi.13563. PMID: 30460747.
180. Narme P, Clement S, Ehrle N, et al. Efficacy of musical interventions in dementia: evidence from a randomized controlled trial. Journal of Alzheimer's Disease. 2014;38(2):359-69. doi: https://dx.doi.org/10.3233/JAD-130893. PMID: 23969994.

181. Park J, Tolea MI, Sherman D, et al. Feasibility of Conducting Nonpharmacological Interventions to Manage Dementia Symptoms in Community-Dwelling Older Adults: A Cluster Randomized Controlled Trial. American Journal of Alzheimer's Disease \& Other Dementias. 2019 Sep 18:1533317519872635. doi: https://dx.doi.org/10.1177/15333175198 72635. PMID: 31533443.

182. Pongan E, Tillmann B, Leveque $Y$, et al. Can Musical or Painting Interventions Improve Chronic Pain, Mood, Quality of Life, and Cognition in Patients with Mild Alzheimer's Disease? Evidence from a Randomized Controlled Trial. Journal of Alzheimer's Disease. 2017;60(2):663-77. doi: https://dx.doi.org/10.3233/JAD-170410. PMID: 28922159.

183. Weise L, Töpfer NF, Deux J, et al. Feasibility and effects of individualized recorded music for people with dementia: A pilot RCT study. Nordic Journal of Music Therapy. 2020;29(1):39-56. doi: 10.1080/08098131.2019.1661507. PMID: 141134381. Language: English. Entry Date: 20200117. Revision Date: 20200117. Publication Type: Article.

184. Ho RT, Fong TC, Sing CY, et al. Managing behavioral and psychological symptoms in Chinese elderly with dementia via group-based music intervention: A cluster randomized controlled trial. Dementia. 2018 Jan 01:1471301218760023. doi: https://dx.doi.org/10.1177/14713012187 60023. PMID: 29468887. 
185. Raglio A, Bellelli G, Traficante D, et al. Efficacy of music therapy in the treatment of behavioral and psychiatric symptoms of dementia. Alzheimer Disease \& Associated Disorders. 2008 Apr-Jun;22(2):158-62. doi: https://dx.doi.org/10.1097/WAD.0b013 e3181630b6f. PMID: 18525288.

186. Raglio A, Bellelli G, Traficante D, et al. Addendum to 'Efficacy of music therapy treatment based on cycles of sessions: A randomised controlled trial' (Raglio et al., 2010). Aging \& Mental Health. 2012 Mar;16(2):265-7. doi: http://dx.doi.org/10.1080/13607863.201 1.630376. PMID: 2012-00529-017.

187. Vink AC, Zuidersma M, Boersma F, et al. The effect of music therapy compared with general recreational activities in reducing agitation in people with dementia: a randomised controlled trial. International Journal of Geriatric Psychiatry. 2013 Oct;28(10):1031-8. doi:

https://dx.doi.org/10.1002/gps.3924. PMID: 23280604.

188. Wang SC, Yu CL, Chang SH. Effect of music care on depression and behavioral problems in elderly people with dementia in Taiwan: a quasiexperimental, longitudinal study. Aging \& Mental Health. 2017 02;21(2):15662. doi: https://dx.doi.org/10.1080/13607863.20 15.1093602. PMID: 26443002.

189. Hsu CN, Lin LC, Wu SC. The effects of spaced retrieval training in improving hyperphagia of people living with dementia in residential settings. Journal of Clinical Nursing. 2017 Oct;26(1920):3224-31. doi: https://dx.doi.org/10.1111/jocn.13671. PMID: 27878873.

190. Cooke M, Moyle W, Shum D, et al. A randomized controlled trial exploring the effect of music on quality of life and depression in older people with dementia. Journal of Health Psychology. 2010 Jul;15(5):765-76. doi: https://dx.doi.org/10.1177/13591053103 68188. PMID: 20603300.
191. Giovagnoli AR, Manfredi V, Parente A, et al. Cognitive training in Alzheimer's disease: a controlled randomized study. Neurological Sciences. 2017

Aug;38(8):1485-93. doi: https://dx.doi.org/10.1007/s10072-0173003-9. PMID: 28577267.

192. Raglio A, Bellelli G, Traficante D, et al. Efficacy of music therapy treatment based on cycles of sessions: a randomised controlled trial. Aging \& Mental Health. 2010 Nov;14(8):900-4. doi:

https://dx.doi.org/10.1080/13607861003 713158. PMID: 21069596.

193. Amieva H, Robert PH, Grandoulier AS, et al. Group and individual cognitive therapies in Alzheimer's disease: the ETNA3 randomized trial. International Psychogeriatrics. 2016 May;28(5):70717. doi: https://dx.doi.org/10.1017/S104161021 5001830. PMID: 26572551.

194. Li M, Lyu JH, Zhang Y, et al. Efficacy of Group Reminiscence Therapy on Cognition, Depression, Neuropsychiatric Symptoms, and Activities of Daily Living for Patients With Alzheimer Disease. Journal of Geriatric Psychiatry \& Neurology. 2019 Oct 23:891988719882099. doi: https://dx.doi.org/10.1177/08919887198 82099. PMID: 31645180.

195. Wang J, Yen M, OuYang W. Group reminiscence intervention in Taiwanese elders with dementia. In Archives of gerontology and geriatrics

196. Wang JJ. Group reminiscence therapy for cognitive and affective function of demented elderly in Taiwan. International Journal of Geriatric Psychiatry. 2007 Dec;22(12):1235-40. PMID: 17503545.

197. Woods RT, Bruce E, Edwards RT, et al. Reminiscence groups for people with dementia and their family carers: pragmatic eight-centre randomised trial of joint reminiscence and maintenance versus usual treatment: a protocol. Trials [Electronic Resource]. $2009 \mathrm{Jul}$ 30;10:64. doi: https://dx.doi.org/10.1186/1745-621510-64. PMID: 19642992. 
198. Woods RT, Orrell M, Bruce E, et al. REMCARE: Pragmatic Multi-Centre Randomised Trial of Reminiscence Groups for People with Dementia and their Family Carers: Effectiveness and Economic Analysis. PLoS ONE [Electronic Resource]. 2016;11(4):e0152843. doi: https://dx.doi.org/10.1371/journal.pone. 0152843. PMID: 27093052.

199. Gonzalez J, Mayordomo T, Torres M, et al. Reminiscence and dementia: a therapeutic intervention. International Psychogeriatrics. 2015 Oct;27(10):1731-7. doi: https://dx.doi.org/10.1017/S104161021 5000344. PMID: 25765779.

200. Laird EA, Ryan A, McCauley C, et al. Using Mobile Technology to Provide Personalized Reminiscence for People Living With Dementia and Their Carers: Appraisal of Outcomes From a Quasi-Experimental Study. JMIR Mental Health. 2018 Sep 11;5(3):e57. doi: https://dx.doi.org/10.2196/mental.9684. PMID: 30206053.

201. Lalanne J, Gallarda T, Piolino P. “The Castle of Remembrance”: New insights from a cognitive training programme for autobiographical memory in Alzheimer's disease.

Neuropsychological Rehabilitation. 2015;25(2):254-82. doi: https://dx.doi.org/10.1080/09602011.20 14.949276. PMID: 25122521.

202. Manav AI, Simsek N. The effect of reminiscence therapy with internetbased videos on cognitive status and apathy of older people with mild dementia. Journal of geriatric psychiatry and neurology. 2019;32(2):104-13. PMID: CN-01707450.

203. Martinez N, Stutzman SE, Olson DM. Electronic interventions aimed at increasing self-worth in mild dementia may not be feasible. Journal of the American Association of Nurse Practitioners. 2019 Aug 22;22:22. doi: https://dx.doi.org/10.1097/JXX.000000 0000000280. PMID: 31453826.
204. Nakamae T, Yotsumoto K, Tatsumi E, et al. Effects of productive activities with reminiscence in occupational therapy for people with dementia: A pilot randomized controlled study. Hong Kong Journal of Occupational Therapy. 2014 June;24(1):13-9. doi: http://dx.doi.org/10.1016/j.hkjot.2014.0 1.003. PMID: 53104158.

205. Politis AM, Vozzella S, Mayer LS, et al. A randomized, controlled, clinical trial of activity therapy for apathy in patients with dementia residing in long-term care. International Journal of Geriatric Psychiatry. 2004 Nov;19(11):1087-94. PMID: 15481065.

206. Subramaniam P, Woods B, Whitaker C. Life review and life story books for people with mild to moderate dementia: a randomised controlled trial. Aging \& Mental Health. 2014;18(3):363-75. doi: https://dx.doi.org/10.1080/13607863.20 13.837144. PMID: 24063317.

207. Van Bogaert P, Van Grinsven R, Tolson D, et al. Effects of SolCos model-based individual reminiscence on older adults with mild to moderate dementia due to Alzheimer disease: a pilot study. Journal of the American Medical Directors Association. 2013 Jul;14(7):528.e9-13. doi: https://dx.doi.org/10.1016/j.jamda.2013. 01.020. PMID: 23583001.

208. Camberg L, Woods P, Ooi WL, et al. Evaluation of Simulated Presence: a personalized approach to enhance wellbeing in persons with Alzheimer's disease. Journal of the American Geriatrics Society. 1999 Apr;47(4):44652. PMID: 10203120.

209. Duru Asiret G, Kapucu S. The Effect of Reminiscence Therapy on Cognition, Depression, and Activities of Daily Living for Patients With Alzheimer Disease. Journal of Geriatric Psychiatry \& Neurology. 2016 Jan;29(1):31-7. doi: https://dx.doi.org/10.1177/08919887155 98233. PMID: 26251112. 
210. Hsieh C-J, Chang C, Su S-F, et al. Reminiscence group therapy on depression and apathy in nursing home residents with mild-to-moderate dementia. In Journal of experimental and clinical medicine

211. Lai CK, Chi I, Kayser-Jones J. A randomized controlled trial of a specific reminiscence approach to promote the well-being of nursing home residents with dementia. International Psychogeriatrics. 2004 Mar;16(1):3349. PMID: 15190995.

212. Lin H-C, Yang Y-P, Cheng W-Y, et al. Distinctive effects between cognitive stimulation and reminiscence therapy on cognitive function and quality of life for different types of behavioural problems in dementia. In Scandinavian journal of caring sciences

213. Lok N, Bademli K, Selcuk-Tosun A. The effect of reminiscence therapy on cognitive functions, depression, and quality of life in Alzheimer patients: Randomized controlled trial. International Journal of Geriatric Psychiatry. 2019 Jan;34(1):47-53. doi: https://dx.doi.org/10.1002/gps.4980. PMID: 30246408.

214. O’Shea E, Devane D, Cooney A, et al. The impact of reminiscence on the quality of life of residents with dementia in longstay care. International Journal of Geriatric Psychiatry. 2014 Oct;29(10):1062-70. doi: https://dx.doi.org/10.1002/gps.4099. PMID: 24633858.

215. Serrani Azcurra DJ. A reminiscence program intervention to improve the quality of life of long-term care residents with Alzheimer's disease: a randomized controlled trial. Revista Brasileira de Psiquiatria. 2012 Dec;34(4):422-33. PMID: 23429813.
216. Van Bogaert P, Tolson D, Eerlingen R, et al. SolCos model-based individual reminiscence for older adults with mild to moderate dementia in nursing homes: a randomized controlled intervention study. Journal of Psychiatric \& Mental Health Nursing. 2016 Nov;23(910):568-75. doi: https://dx.doi.org/10.1111/jpm.12336. PMID: 27511740.

217. Wu LF, Koo M. Randomized controlled trial of a six-week spiritual reminiscence intervention on hope, life satisfaction, and spiritual well-being in elderly with mild and moderate dementia. International Journal of Geriatric Psychiatry. 2016

Feb;31(2):120-7. doi: https://dx.doi.org/10.1002/gps.4300. PMID: 25965388.

218. Ching-Teng Y, Ya-Ping Y, Chia-Ju L, et al. Effect of group reminiscence therapy on depression and perceived meaning of life of veterans diagnosed with dementia at veteran homes. Social Work in Health Care. 2020;59(2):75-90. doi: 10.1080/00981389.2019.1710320. PMID: 141877576. Language: English. Entry Date: 20200227. Revision Date: 20200319. Publication Type: Article.

219. Inel Manav A, Simsek N. The Effect of Reminiscence Therapy With InternetBased Videos on Cognitive Status and Apathy of Older People With Mild Dementia. Journal of Geriatric Psychiatry \& Neurology. 2019 Mar;32(2):104-13. doi: https://dx.doi.org/10.1177/08919887188 19864. PMID: 30612511.

220. Seyun K. Cognitive rehabilitation for elderly people with early-stage Alzheimer's disease. Journal of Physical Therapy Science. 2015;27(2):543-6. doi: 10.1589/jpts.27.543. PMID: 103780951. Language: English. Entry Date: 20150327. Revision Date: 20150820. Publication Type: Journal Article. 
221. Clare L, Linden DE, Woods RT, et al. Goaloriented cognitive rehabilitation for people with early-stage Alzheimer disease: a single-blind randomized controlled trial of clinical efficacy. American Journal of Geriatric Psychiatry. 2010 Oct;18(10):928-39. doi:

https://dx.doi.org/10.1097/JGP.0b013e3 181d5792a. PMID: 20808145.

222. Thivierge S, Jean L, Simard M. A randomized cross-over controlled study on cognitive rehabilitation of instrumental activities of daily living in Alzheimer disease. American Journal of Geriatric Psychiatry. 2014 Nov;22(11):1188-99. doi: https://dx.doi.org/10.1016/j.jagp.2013.0 3.008. PMID: 23871120.

223. Rogers JC, Holm MB, Burgio LD, et al. Improving morning care routines of nursing home residents with dementia. Journal of the American Geriatrics Society. 1999:1049-57. PMID: 107127490. Language: English. Entry Date: 20000801. Revision Date: 20150711. Publication Type: Journal Article.

224. Voigt-Radloff S, de Werd MM, Leonhart R, et al. Structured relearning of activities of daily living in dementia: the randomized controlled REDALI-DEM trial on errorless learning. Alzheimer's Research \& Therapy. 2017 Mar 23;9(1):22. doi: https://dx.doi.org/10.1186/s13195-0170247-9. PMID: 28335810.

225. Kumar P, Tiwari SC, Goel A, et al. Novel occupational therapy interventions may improve quality of life in older adults with dementia. International archives of medicine. 2014;7:26. doi: https://dx.doi.org/10.1186/1755-7682-726. PMID: 24982692.

226. Quayhagen MP, Quayhagen M, Corbeil $\mathrm{RR}$, et al. A dyadic remediation program for care recipients with dementia. Nursing Research. 1995 May-Jun;44(3):153-9. PMID: 7761291.
227. Lin LC, Huang YJ, Su SG, et al. Using spaced retrieval and Montessori-based activities in improving eating ability for residents with dementia. International Journal of Geriatric Psychiatry. 2010 Oct;25(10):953-9. doi: https://dx.doi.org/10.1002/gps.2433. PMID: 20054841.

228. Lam LC, Lui VW, Luk DN, et al. Effectiveness of an individualized functional training program on affective disturbances and functional skills in mild and moderate dementia--a randomized control trial. International Journal of Geriatric Psychiatry. 2010 Feb;25(2):133-41. doi: https://dx.doi.org/10.1002/gps.2309. PMID: 19582757.

229. van der Ploeg ES, Eppingstall B, Camp CJ, et al. A randomized crossover trial to study the effect of personalized, one-toone interaction using Montessori-based activities on agitation, affect, and engagement in nursing home residents with Dementia. International Psychogeriatrics. 2013 Apr;25(4):56575. doi:

https://dx.doi.org/10.1017/S104161021 2002128. PMID: 23237211.

230. Loewenstein DA, Acevedo A, Czaja SJ, et al. Cognitive rehabilitation of mildly impaired Alzheimer disease patients on cholinesterase inhibitors. American Journal of Geriatric Psychiatry. 2004 Jul-Aug;12(4):395-402. PMID: 15249277.

231. Lin LC, Huang YJ, Watson R, et al. Using a Montessori method to increase eating ability for institutionalised residents with dementia: a crossover design. Journal of Clinical Nursing. 2011 Nov;20(21-22):3092-101. doi: https://dx.doi.org/10.1111/j.13652702.2011.03858.x. PMID: 21981704.

232. Silva AR, Pinho MS, Macedo L, et al. It is not only memory: effects of sensecam on improving well-being in patients with mild alzheimer disease. International Psychogeriatrics. 2017 May;29(5):741-54. doi: https://dx.doi.org/10.1017/S104161021 600243X. PMID: 28124633. 
233. Tanaka S, Honda S, Nakano H, et al. Comparison between group and personal rehabilitation for dementia in a geriatric health service facility: singleblinded randomized controlled study. Psychogeriatrics:The Official Journal of the Japanese Psychogeriatric Society. 2017 May;17(3):177-85. doi: https://dx.doi.org/10.1111/psyg.12212. PMID: 27612310.

234. Kim S. Cognitive rehabilitation for elderly people with early-stage Alzheimer's disease. Journal of Physical Therapy Science. 2015 Feb;27(2):543-6. doi: https://dx.doi.org/10.1589/jpts.27.543. PMID: 25729212.

235. Tappen RM. The effect of skill training on functional abilities of nursing home residents with dementia. Research in Nursing \& Health. 1994 Jun;17(3):15965. PMID: 8184127.

236. Silva AR, Pinho MS, Macedo L, et al. The cognitive effects of wearable cameras in mild Alzheimer disease-An experimental study. Current Alzheimer Research. 2017;14(12):1270-82. doi: http://dx.doi.org/10.2174/15672050146 66170531083015. PMID: 2018-01500004.

237. Brunelle-Hamann L, Thivierge S, Simard M. Impact of a cognitive rehabilitation intervention on neuropsychiatric symptoms in mild to moderate Alzheimer's disease. Neuropsychological Rehabilitation. 2015;25(5):677-707. doi: https://dx.doi.org/10.1080/09602011.20 14.964731. PMID: 25312605.

238. Voigt-Radloff S, Leonhart R, Rikkert MO, et al. Study protocol of the multi-site randomised controlled REDALI-DEM trial--the effects of structured relearning methods on daily living task performance of persons with dementia. BMC Geriatrics. 2011 Aug 18;11:44. doi: https://dx.doi.org/10.1186/14712318-11-44. PMID: 21851594.

239. Chen Z, Yu H. Effect of intervention guided by montessori method on improving feeding capacity of patients with dementia. International Journal of Clinical and Experimental Medicine. 2020;13(2):1148-55.
240. Mbakile-Mahlanza L, van der Ploeg ES, Busija L, et al. A cluster-randomized crossover trial of Montessori activities delivered by family carers to nursing home residents with behavioral and psychological symptoms of dementia. International Psychogeriatrics. 2020 Mar;32(3):347-58. doi: https://dx.doi.org/10.1017/S104161021 9001819. PMID: 31762434.

241. Wu HS, Lin LC, Wu SC, et al. The effectiveness of spaced retrieval combined with Montessori-based activities in improving the eating ability of residents with dementia. Journal of Advanced Nursing. 2014 Aug;70(8):1891-901. doi: https://dx.doi.org/10.1111/jan.12352. PMID: 24444172.

242. Kurz A, Thone-Otto A, Cramer B, et al. CORDIAL: cognitive rehabilitation and cognitive-behavioral treatment for early dementia in Alzheimer disease: a multicenter, randomized, controlled trial. Alzheimer Disease \& Associated Disorders. 2012 Jul-Sep;26(3):246-53. PMID: 21986341.

243. Tsuchiya K, Yamaguchi T, Fujita T, et al. A Quasi-Randomized Controlled Trial of Brain-Activating Rehabilitation in an Acute Hospital. American Journal of Alzheimer's Disease \& Other Dementias. 2016 Dec;31(8):612-7. PMID: 27303064.

244. Clare L, Kudlicka A, Oyebode JR, et al. Individual goal-oriented cognitive rehabilitation to improve everyday functioning for people with early-stage dementia: A multicentre randomised controlled trial (the GREAT trial). International Journal of Geriatric Psychiatry. 2019 May;34(5):709-21. doi: http://dx.doi.org/10.1002/gps.5076. PMID: 626609676.

245. Yuen I, Kwok T. Effectiveness of DementiAbility Methods: The Montessori Way on agitation in longterm care home residents with dementia in Hong Kong. International Journal of Geriatric Psychiatry. 2019;34(9):13528. doi: http://dx.doi.org/10.1002/gps.5063. PMID: 626609449. 
246. Cavallo M, Hunter EM, van der Hiele K, et al. Computerized Structured Cognitive Training in Patients Affected by EarlyStage Alzheimer's Disease is Feasible and Effective: A Randomized Controlled Study. Archives of Clinical Neuropsychology. 2016 Sep 06;06:06. PMID: 27600448.

247. Kallio EL, Ohman H, Carlson S, et al. Feasibility and baseline findings of a Finnish cognitive training (FINCOG) intervention in a randomised controlled trial among community-dwelling persons with dementia. European Geriatric Medicine. 2017 July;8(3):2459. doi: http://dx.doi.org/10.1016/j.eurger.2017. 04.008. PMID: 616108233.

248. Kallio EL, Ohman H, Hietanen M, et al. Effects of Cognitive Training on Cognition and Quality of Life of Older Persons with Dementia. Journal of the American Geriatrics Society. 2018 Apr;66(4):664-70. doi: https://dx.doi.org/10.1111/jgs.15196. PMID: 29345724.

249. Kao CC, Lin LC, Wu SC, et al. Effectiveness of different memory training programs on improving hyperphagic behaviors of residents with dementia: a longitudinal single-blind study. Clinical Interventions In Aging. 2016;11:707-20. doi: https://dx.doi.org/10.2147/CIA.S10202 7. PMID: 27307717.

250. Bergamaschi S, Arcara G, Calza A, et al. One-year repeated cycles of cognitive training (CT) for Alzheimer's disease. Aging-Clinical \& Experimental Research. 2013 Aug;25(4):421-6. doi: https://dx.doi.org/10.1007/s40520-0130065-2. PMID: 23784727.

251. Davis RN, Massman PJ, Doody RS. Cognitive intervention in Alzheimer disease: a randomized placebocontrolled study. Alzheimer Disease \& Associated Disorders. 2001 JanMar;15(1):1-9. PMID: 11236819.
252. De Luca R, Bramanti A, De Cola MC, et al. Cognitive training for patients with dementia living in a sicilian nursing home: a novel web-based approach. Neurological Sciences. 201601 Oct;37(10):1685-91. doi: http://dx.doi.org/10.1007/s10072-0162659-x. PMID: 611200073.

253. Giuli C, Papa R, Lattanzio F, et al. The Effects of Cognitive Training for Elderly: Results from My Mind Project. Rejuvenation Research. 2016 Dec;19(6):485-94. PMID: 26952713.

254. Kawashima R, Hiller D, Sereda S, et al. SAIDO Learning as a Cognitive Intervention for Dementia Care: aPreliminary Study. In Journal of the american medical directors association

255. Mapelli D, Di Rosa E, Nocita R, et al. Cognitive stimulation in patients with dementia: randomized controlled trial. Dementia and Geriatric Cognitive Disorders Extra. 2013;3(1):263-71. doi: https://dx.doi.org/10.1159/000353457. PMID: 24052800.

256. McGilton KS, Rivera TM, Dawson P. Can we help persons with dementia find their way in a new environment?.[Erratum appears in Aging Ment Health. 2003 Nov;7(6):following author index]. Aging \& Mental Health. 2003 Sep;7(5):363-71. PMID: 12959805.

257. Tarraga L, Boada M, Modinos G, et al. A randomised pilot study to assess the efficacy of an interactive, multimedia tool of cognitive stimulation in Alzheimer's disease. Journal of Neurology, Neurosurgery \& Psychiatry. 2006 Oct;77(10):1116-21. PMID: 16820420.

258. Tseng CF, Lee SH, Hsieh TC, et al. Smart Restored by Learning Exercise Alleviates the Deterioration of Cognitive Function in Older Adults with Dementia-A Quasi-Experimental Research. International Journal of Environmental Research \& Public Health [Electronic Resource]. 201904 09;16(7):09. doi: https://dx.doi.org/10.3390/ijerph160712 70. PMID: 30970666. 
259. Venturelli M, Sollima A, Ce E, et al. Effectiveness of Exercise- and Cognitive-Based Treatments on Salivary Cortisol Levels and Sundowning Syndrome Symptoms in Patients with Alzheimer's Disease. Journal of Alzheimer's Disease. 2016;53(4):1631-40. doi: http://dx.doi.org/10.3233/JAD-160392. PMID: 611692625.

260. Zarit SH, Zarit JM, Reever KE. Memory training for severe memory loss: Effects on senile dementia patients and their families. Gerontologist. 1982;22(4):373-7. PMID: 13238424.

261. Chen HM, Tsai LJ, Chao SY, et al. Study on the Effects of Individualized Learning Therapy on Cognitive Function and Behavioral and Psychological Symptoms of Dementia in the Institutionalized Older Adults. Journal of Nursing Research. 2016 Dec;24(4):300-10. PMID: 27846102.

262. Knapp M, Thorgrimsen L, Patel A, et al. Cognitive stimulation therapy for people with dementia: costeffectiveness analysis. British Journal of Psychiatry. 2006 Jun;188:574-80. PMID: 16738349.

263. Orrell M, Aguirre E, Spector A, et al. Maintenance cognitive stimulation therapy for dementia: single-blind, multicentre, pragmatic randomised controlled trial. British Journal of Psychiatry. 2014 Jun;204(6):454-61. doi: https://dx.doi.org/10.1192/bjp.bp.113.1 37414. PMID: 24676963.

264. Spector A, Thorgrimsen L, Woods B, et al. Efficacy of an evidence-based cognitive stimulation therapy programme for people with dementia: randomised controlled trial. British Journal of Psychiatry. 2003 Sep;183:248-54. PMID: 12948999.
265. Capotosto E, Belacchi C, Gardini S, et al. Cognitive stimulation therapy in the Italian context: Its efficacy in cognitive and non-cognitive measures in older adults with dementia. International Journal of Geriatric Psychiatry. 2017 Mar;32(3):331-40. doi: http://dx.doi.org/10.1002/gps.4521. PMID: 2017-05902-002.

266. Cove J, Jacobi N, Donovan H, et al. Effectiveness of weekly cognitive stimulation therapy for people with dementia and the additional impact of enhancing cognitive stimulation therapy with a carer training program. Clinical Interventions In Aging. 2014;9:214350. doi: https://dx.doi.org/10.2147/CIA.S66232. PMID: 25525349.

267. Matsuda O. Cognitive stimulation therapy for Alzheimer's disease: the effect of cognitive stimulation therapy on the progression of mild Alzheimer's disease in patients treated with donepezil. International psychogeriatrics. 2007;19(2):241-52. doi: 10.1017/S1041610206004194. PMID: CN-00640778.

268. Niu YX, Tan JP, Guan JQ, et al. Cognitive stimulation therapy in the treatment of neuropsychiatric symptoms in Alzheimer's disease: a randomized controlled trial. Clinical Rehabilitation. 2010 Dec;24(12):1102-11. doi: https://dx.doi.org/10.1177/02692155103 76004. PMID: 20713437.

269. Orgeta V, Leung P, Yates L, et al. Individual cognitive stimulation therapy for dementia: a clinical effectiveness and cost-effectiveness pragmatic, multicentre, randomised controlled trial. Health Technology Assessment (Winchester, England). 2015 Aug;19(64):1-108. doi: https://dx.doi.org/10.3310/hta19640. PMID: 26292178. 
270. Orrell M, Yates L, Leung P, et al. The impact of individual Cognitive Stimulation Therapy (iCST) on cognition, quality of life, caregiver health, and family relationships in dementia: A randomised controlled trial. PLoS Medicine / Public Library of Science. 2017 Mar;14(3):e1002269. doi:

https://dx.doi.org/10.1371/journal.pmed .1002269. PMID: 28350796.

271. Lok N, Buldukoglu K, Barcin E. Effects of the cognitive stimulation therapy based on Roy's adaptation model on Alzheimer's patients' cognitive functions, coping-adaptation skills, and quality of life: A randomized controlled trial. Perspectives in Psychiatric Care. 2020 Jan 12;12:12. doi: https://dx.doi.org/10.1111/ppc.12472. PMID: 31930518.

272. Cheung DSK, Li B, Lai DWL, et al. Cognitive Stimulating Play Intervention for Dementia: A Feasibility Randomized Controlled Trial. American Journal of Alzheimer's Disease \& Other Dementias. 2019 02;34(1):63-71. doi: https://dx.doi.org/10.1177/15333175188 08036. PMID: 30370782.

273. Wong G, Yek O, Zhang A, et al. Cultural adaptation of cognitive stimulation therapy (CST) for Chinese people with dementia: multicentre pilot study. In International journal of geriatric psychiatry

274. Low LF, Brodaty H, Goodenough B, et al. The Sydney Multisite Intervention of LaughterBosses and ElderClowns (SMILE) study: cluster randomised trial of humour therapy in nursing homes. BMJ Open. 2013 Jan 11;3(1):11. doi: https://dx.doi.org/10.1136/bmjopen2012-002072. PMID: 23315520.

275. Pedrinolla A, Tamburin S, Brasioli A, et al. An Indoor Therapeutic Garden for Behavioral Symptoms in Alzheimer's Disease: A Randomized Controlled Trial. Journal of Alzheimer's Disease. 2019;71(3):813-23. doi: https://dx.doi.org/10.3233/JAD-190394. PMID: 31424399.
276. Kolanowski A, Litaker M, Buettner L, et al. A randomized clinical trial of theorybased activities for the behavioral symptoms of dementia in nursing home residents. Journal of the American Geriatrics Society. 2011 Jun;59(6):1032-41. doi: https://dx.doi.org/10.1111/j.15325415.2011.03449.x. PMID: 21649633.

277. Buettner LL, Fitzsimmons S. AD-venture program: therapeutic biking for the treatment of depression in long-term care residents with dementia. American Journal of Alzheimer's Disease \& Other Dementias. 2002 Mar-Apr;17(2):121-7. PMID: 11954670.

278. Hattori H, Hattori C, Hokao C, et al. Controlled study on the cognitive and psychological effect of coloring and drawing in mild Alzheimer's disease patients. Geriatrics \& gerontology international. 2011 Oct;11(4):431-7. doi: https://dx.doi.org/10.1111/j.14470594.2011.00698.x. PMID: 21518170.

279. Hopman-Rock M, Staats PGM, Tak ECPM, et al. The effects of a psychomotor activation programme for use in groups of cognitively impaired people in homes for the elderly. International Journal of Geriatric Psychiatry. 1999

August;14(8):633-42. doi: http://dx.doi.org/10.1002/\%28SICI\%29 10991166\%28199908\%2914:8\%3C633::AI D-GPS996\%3E3.0.CO;2-W. PMID: 29403282.

280. Li D, Li X. The effect of folk recreation program in improving symptoms: a study of Chinese elder dementia patients. International journal of geriatric psychiatry. 2017;32(8):901-8. doi: 10.1002/gps.4543. PMID: CN01628262.

281. Lin Q, Cao Y, Gao J. The impacts of a gOgame (Chinese chess) intervention on alzheimer disease in a Northeast Chinese population. Frontiers in Aging Neuroscience. 2015;7 (AUG) (no pagination)(163). doi: http://dx.doi.org/10.3389/fnagi.2015.00 163. PMID: 607203463. 
282. Pongan E, Delphin-Combe F, KrolakSalmon P, et al. Immediate Benefit of Art on Pain and Well-Being in Community-Dwelling Patients with Mild Alzheimer's. American Journal of Alzheimer's Disease \& Other Dementias. 2019 Jul 09:1533317519859202. doi: https://dx.doi.org/10.1177/15333175198 59202. PMID: 31288544.

283. Schall A, Tesky VA, Adams AK, et al. Art museum-based intervention to promote emotional well-being and improve quality of life in people with dementia: The ARTEMIS project. Dementia. 2017 Jan 01:1471301217730451. doi: https://dx.doi.org/10.1177/14713012177 30451. PMID: 28914089.

284. Shalek M, Richeson NE, Buettner LL. Air mat therapy for treatment of agitated wandering: an evidence-based recreational therapy intervention. American Journal of Recreation Therapy. 20042004 Spring;3(2):18-26. PMID: 106658647. Language: English. Entry Date: 20041105. Revision Date: 20150818. Publication Type: Journal Article.

285. Tse MMY, Lau JL, Kwan R, et al. Effects of play activities program for nursing home residents with dementia on pain and psychological well-being: Cluster randomized controlled trial. Geriatrics \& gerontology international. 2018 Oct;18(10):1485-90. doi: https://dx.doi.org/10.1111/ggi.13509. PMID: 30255637.

286. Laksono WAW, Haryanto J, Bahiyah K. The impact of chess games towards comfortableness of cognitive mind on elderly. Indian Journal of Public Health Research and Development. 2019 September;10(9):873-7.

287. Lathifah MN, Haryanto J, Fauziningtyas R. The influence of dhakonan games to impede dementia in elders. Indian Journal of Public Health Research and Development. 2019 September;10(9):1440-4.
288. Van Haitsma KS, Curyto K, Abbott KM, et al. A randomized controlled trial for an individualized positive psychosocial intervention for the affective and behavioral symptoms of dementia in nursing home residents. Journals of Gerontology Series B-Psychological Sciences \& Social Sciences. 2015 Jan;70(1):35-45. doi: https://dx.doi.org/10.1093/geronb/gbt10 2. PMID: 24304555.

289. Burns A, Guthrie E, Marino-Francis F, et al. Brief psychotherapy in Alzheimer's disease: randomised controlled trial. British Journal of Psychiatry. 2005 Aug;187:143-7. PMID: 16055825.

290. Cohen CI, Hyland K, Devlin M. An evaluation of the use of the natural helping network model to enhance the well-being of nursing home residents. Gerontologist. 1999;39(4):426-33. PMID: 107227653. Language: English. Entry Date: 19991201. Revision Date: 20150711. Publication Type: Journal Article.

291. Quinn C, Anderson D, Toms G, et al. Selfmanagement in early-stage dementia: a pilot randomised controlled trial of the efficacy and cost-effectiveness of a selfmanagement group intervention (the SMART study). Trials [Electronic Resource]. 2014 Mar 08;15:74. doi: https://dx.doi.org/10.1186/1745-621515-74. PMID: 24606601.

292. Quinn C, Toms G, Jones C, et al. A pilot randomized controlled trial of a selfmanagement group intervention for people with early-stage dementia (The SMART study). International Psychogeriatrics. 2016 May;28(5):787800. doi: https://dx.doi.org/10.1017/S104161021 5002094. PMID: 26674087.

293. Tappen RM, Williams CL. Therapeutic conversation to improve mood in nursing home residents with Alzheimer's disease. Research in Gerontological Nursing. 2009 Oct;2(4):267-75. doi: https://dx.doi.org/10.3928/1940492120090428-02. PMID: 20077983. 
294. Young DK, Kwok TC, Ng PY. A single blind randomized control trial on support groups for Chinese persons with mild dementia. Clinical Interventions In Aging. 2014;9:2105-12. doi: https://dx.doi.org/10.2147/CIA.S68687. PMID: 25587218.

295. Wallis GG, Baldwin M, Higginbotham P. Reality orientation therapy--a controlled trial. British Journal of Medical Psychology. 1983 Sep;56(Pt 3):271-7. PMID: 6354248.

296. Onder G, Zanetti O, Giacobini E, et al. Reality orientation therapy combined with cholinesterase inhibitors in Alzheimer's disease: randomised controlled trial. British Journal of Psychiatry. 2005 Nov;187:450-5. PMID: 16260821.

297. Fritsch T, Kwak J, Grant S, et al. Impact of TimeSlips, a Creative Expression Intervention Program, on Nursing Home Residents with Dementia and their Caregivers. Gerontologist. 2009;49(1):117-27. doi: 10.1093/geront/gnp008. PMID: CN00755001.

298. Houser WS, George DR, Chinchilli VM. Impact of TimeSlips creative expression program on behavioral symptoms and psychotropic medication use in persons with dementia in long-term care: a cluster-randomized pilot study. American Journal of Geriatric Psychiatry. 2014 Apr;22(4):337-40. doi: https://dx.doi.org/10.1016/j.jagp.2012.1 2.005. PMID: 23602304.

299. Lin R, Chen HY, Li H, et al. Effects of creative expression therapy on Chinese elderly patients with dementia: an exploratory randomized controlled trial. Neuropsychiatric Disease \& Treatment. 2019;15:2171-80. doi: https://dx.doi.org/10.2147/NDT.S20004 5. PMID: 31440055.

300. Phillips L, Reid-Arndt S, Pak Y. Effects of a creative expression intervention on emotions, communication, and quality of life in persons with dementia. Nursing research. 2010;59(6):417-25. doi: 10.1097/NNR.0b013e3181faff52. PMID: CN-00770848.
301. van Dijk AM, van Weert JC, Droes RM. Does theatre improve the quality of life of people with dementia? International Psychogeriatrics. 2012 Mar;24(3):36781. doi: https://dx.doi.org/10.1017/S104161021 1001992. PMID: 22040605.

302. Luttenberger K, Donath C, Uter W, et al. Effects of multimodal nondrug therapy on dementia symptoms and need for care in nursing home residents with degenerative dementia: a randomizedcontrolled study with 6-month followup. Journal of the American Geriatrics Society. 2012 May;60(5):830-40. doi: https://dx.doi.org/10.1111/j.15325415.2012.03938.x. PMID: 22468985.

303. Graessel E, Stemmer R, Eichenseer B, et al. Non-pharmacological, multicomponent group therapy in patients with degenerative dementia: a 12-month randomizied, controlled trial. BMC Medicine. 2011 Dec 01;9:129. doi: https://dx.doi.org/10.1186/1741-7015-9129. PMID: 22133165.

304. McCurry SM, Pike KC, Vitiello MV, et al. Increasing walking and bright light exposure to improve sleep in community-dwelling persons with Alzheimer's disease: results of a randomized, controlled trial. Journal of the American Geriatrics Society. 2011 Aug;59(8):1393-402. doi: https://dx.doi.org/10.1111/j.15325415.2011.03519.x. PMID: 21797835.

305. Tadaka E, Kanagawa K. Randomized controlled trial of a group care program for community-dwelling elderly people with dementia. Japan Journal of Nursing Science. 2004 Aug;1(1):19-25. doi: http://dx.doi.org/10.1111/j.17427924.2004.00006.x. PMID: 200706056-002.

306. Brooker DJ, Argyle E, Scally AJ, et al. The enriched opportunities programme for people with dementia: a clusterrandomised controlled trial in 10 extra care housing schemes. Aging \& Mental Health. 2011 Nov;15(8):1008-17. doi: https://dx.doi.org/10.1080/13607863.20 11.583628. PMID: 21702705. 
307. Burgener SC, Marsh-Yant S, Nega KK. A Combined, Multimodal Intervention for Individuals with Dementia. Research in Gerontological Nursing. 2011;4(1):6475. doi: 10.3928/19404921-2010050401. PMID: 104897556. Language: English. Entry Date: 20110622. Revision Date: 20150711. Publication Type: Journal Article.

308. Burgener SC, Yang Y, Gilbert R, et al. The effects of a multimodal intervention on outcomes of persons with early-stage dementia. American Journal of Alzheimer's Disease \& Other Dementias. 2008 Aug-Sep;23(4):38294. doi: https://dx.doi.org/10.1177/15333175083 17527. PMID: 18453642.

309. Chapman SB, Weiner MF, Rackley A, et al. Effects of cognitive-communication stimulation for Alzheimer's disease patients treated with donepezil. Journal of Speech Language \& Hearing Research. 2004 Oct;47(5):1149-63. PMID: 15603468.

310. Chen YL, Pei YC. Musical dual-task training in patients with mild-tomoderate dementia: a randomized controlled trial. Neuropsychiatric Disease \& Treatment. 2018;14:1381-93. doi: https://dx.doi.org/10.2147/NDT.S15917 4. PMID: 29881275.

311. Ferrero-Arias J, Goni-Imizcoz M, Gonzalez-Bernal J, et al. The efficacy of nonpharmacological treatment for dementia-related apathy. Alzheimer Disease \& Associated Disorders. 2011 Jul-Sep;25(3):213-9. doi: https://dx.doi.org/10.1097/WAD.0b013 e3182087dbc. PMID: 21346517.

312. Kampragkou C, Iakovidis P, Kampragkou E, et al. Effects of a 12-week aerobic exercise program combined with music therapy and memory exercises on cognitive and functional ability in people with middle type of alzheimer's disease. 2017;4(5):262-8. doi: 10.15621/ijphy/2017/v4i5/159420. PMID: CN-01425536.
313. Kim H-J, Yang Y, Oh J-G, et al. Effectiveness of a community-based multidomain cognitive intervention program in patients with Alzheimer's disease. Geriatrics \& gerontology international. 2016;16(2):191-9. doi: 10.1111/ggi.12453. PMID: CN01132849.

314. Okamura H, Otani M, Shimoyama N, et al. Combined Exercise and Cognitive Training System for Dementia Patients: A Randomized Controlled Trial. Dementia \& Geriatric Cognitive Disorders. 2018;45(5-6):318-25. doi: https://dx.doi.org/10.1159/000490613. PMID: 30036871.

315. Tappen RM, Roach KE, Applegate EB, et al. Effect of a combined walking and conversation intervention on functional mobility of nursing home residents with Alzheimer disease. Alzheimer Disease \& Associated Disorders. 2000 OctDec;14(4):196-201. PMID: 11186596.

316. Wesson J, Clemson L, Brodaty H, et al. A feasibility study and pilot randomised trial of a tailored prevention program to reduce falls in older people with mild dementia. BMC Geriatrics. 2013 Sep 03;13:89. doi:

https://dx.doi.org/10.1186/1471-231813-89. PMID: 24004682.

317. Yamagami T, Takayama Y, Maki Y, et al. A randomized controlled trial of brainactivating rehabilitation for elderly participants with dementia in residential care homes. Dementia and Geriatric Cognitive Disorders Extra. 2012 Jan;2(1):372-80. doi: https://dx.doi.org/10.1159/000342614. PMID: 23300492.

318. Young DK, Ng PY, Kwok T, et al. The effects of an expanded cognitive stimulation therapy model on the improvement of cognitive ability of elderly with mild stage Dementia living in a community - a randomized waitlist controlled trial. Aging \& Mental Health. 2019 Jul;23(7):855-62. doi: https://dx.doi.org/10.1080/13607863.20 18.1471586. PMID: 29781725. 
319. Femia EE, Zarit SH, Stephens MA, et al. Impact of adult day services on behavioral and psychological symptoms of dementia. Gerontologist. 2007 Dec;47(6):775-88. PMID: 18192631.

320. Fernandez-Calvo B, Contador I, Ramos F, et al. Effect of unawareness on rehabilitation outcome in a randomised controlled trial of multicomponent intervention for patients with mild Alzheimer's disease.

Neuropsychological Rehabilitation. 2015;25(3):448-77. doi: https://dx.doi.org/10.1080/09602011.20 14.948461. PMID: 25121567.

321. Middelstadt J, Folkerts AK, Blawath S, et al. Cognitive Stimulation for People with Dementia in Long-Term Care Facilities: Baseline Cognitive Level Predicts Cognitive Gains, Moderated by Depression. Journal of Alzheimer's Disease. 201608 04;54(1):253-68. doi: https://dx.doi.org/10.3233/JAD-160181. PMID: 27497474.

322. Rokstad AMM, Engedal K, Kirkevold O, et al. The impact of attending day care designed for home-dwelling people with dementia on nursing home admission: a 24-month controlled study. BMC Health Services Research. 2018 Nov 16;18(1):864. doi: https://dx.doi.org/10.1186/s12913-0183686-5. PMID: 30445937.

323. Wimo A, Mattsson B, Adolfsson R, et al. Dementia day care and its effects on symptoms and institutionalization--a controlled Swedish study. Scandinavian Journal of Primary Health Care. 1993 Jun;11(2):117-23. PMID: 8356361.

324. Sado M, Funaki K, Ninomiya A, et al. Does the Combination of the Cognitive Interventions Improve the Function of Daily Living and Save the Long-Term Care Cost? A Pilot Study of Effectiveness and Cost Saving Analysis of "Learning Therapy" for People with Dementia. Journal of Alzheimer's Disease. 2020 Feb 27;27:27. doi: https://dx.doi.org/10.3233/JAD-190886. PMID: 32116248.
325. Luttenberger K, Hofner B, Graessel E. Are the effects of a non-drug multimodal activation therapy of dementia sustainable? Follow-up study 10 months after completion of a randomised controlled trial. BMC Neurology. 2012 Dec 05;12:151. doi: https://dx.doi.org/10.1186/1471-237712-151. PMID: 23217188.

326. Lauriks SM, Meiland FJMP, Oste JPM, et al. Effects of Assistive Home Technology on quality of life and falls of people with dementia and job satisfaction of caregivers; results from a pilot randomized controlled trial. Assistive Technology. 2018 Dec 28:1-8. doi:

https://dx.doi.org/10.1080/10400435.20 18.1531952. PMID: 30592439.

327. Rowe M, Kelly A, Horne C, et al. Reducing dangerous nighttime events in persons with dementia by using a nighttime monitoring system. In Alzheimer's \& dementia

328. Tchalla AE, Lachal F, Cardinaud N, et al. Preventing and managing indoor falls with home-based technologies in mild and moderate Alzheimer's disease patients: Pilot study in a community dwelling. Dementia and Geriatric Cognitive Disorders. 2013 September;36(3-4):251-61. doi: http://dx.doi.org/10.1159/000351863. PMID: 369900271.

329. Gaugler JE, Zmora R, Mitchell LL, et al. Six-Month Effectiveness of Remote Activity Monitoring for Persons Living With Dementia and Their Family Caregivers: An Experimental Mixed Methods Study. Gerontologist. 201901 09;59(1):78-89. doi: https://dx.doi.org/10.1093/geront/gny07 8. PMID: 29982413.

330. Rowe MA, Kairalla JA, McCrae CS. Sleep in dementia caregivers and the effect of a nighttime monitoring system. Journal of Nursing Scholarship. 2010 Sep 01;42(3):338-47. doi: https://dx.doi.org/10.1111/j.15475069.2010.01337.x. PMID: 20738745. 
331. Ahmed MA, Darwish ES, Khedr EM, et al. Effects of low versus high frequencies of repetitive transcranial magnetic stimulation on cognitive function and cortical excitability in Alzheimer's dementia. Journal of Neurology. 2012 Jan;259(1):83-92. doi: https://dx.doi.org/10.1007/s00415-0116128-4. PMID: 21671144.

332. Cotelli M, Manenti R, Brambilla M, et al. Anodal tDCS during face-name associations memory training in Alzheimer's patients. Frontiers in aging neuroscience. 2014;6:38. doi: https://dx.doi.org/10.3389/fnagi.2014.0 0038. PMID: 24678298.

333. Hozumi S, Hori H, Okawa M, et al. Favorable effect of transcranial electrostimulation on behavior disorders in elderly patients with dementia: a double-blind study. International Journal of Neuroscience. 1996 Nov;88(1-2):1-10. PMID: 9003961.

334. Khedr EM, Salama RH, Abdel Hameed M, et al. Therapeutic Role of Transcranial Direct Current Stimulation in Alzheimer Disease Patients: DoubleBlind, Placebo-Controlled Clinical Trial. Neurorehabilitation \& Neural Repair. 2019 05;33(5):384-94. doi: https://dx.doi.org/10.1177/15459683198 40285. PMID: 30940012.

335. Lozano AM, Fosdick L, Chakravarty MM, et al. A Phase II Study of Fornix Deep Brain Stimulation in Mild Alzheimer's Disease. Journal of Alzheimer's Disease. 201609 06;54(2):777-87. doi: https://dx.doi.org/10.3233/JAD-160017. PMID: 27567810.

336. Olazaran J, Gonzalez B, Lopez-Alvarez J, et al. Motor effects of REAC in advanced Alzheimer's disease: results from a pilot trial. Journal of Alzheimer's Disease. 2013;36(2):297302. doi:

https://dx.doi.org/10.3233/JAD-130077. PMID: 23603397.
337. Olazaran J, Gonzalez B, Osa-Ruiz E, et al. Motor effects of radio electric asymmetric conveyer in Alzheimer's disease: results from a cross-over trial. Journal of Alzheimer's Disease. 2014;42(1):325-32. doi: https://dx.doi.org/10.3233/JAD-140417. PMID: 24898637.

338. Scherder EJ, van Tol MJ, Swaab DF. Highfrequency cranial electrostimulation (CES) in patients with probable Alzheimer's disease. American Journal of Physical Medicine \& Rehabilitation. 2006 Jul;85(7):614-8. PMID: 16788393.

339. Suemoto CK, Apolinario D, NakamuraPalacios EM, et al. Effects of a nonfocal plasticity protocol on apathy in moderate Alzheimer's disease: a randomized, double-blind, shamcontrolled trial. Brain Stimulation. 2014 Mar-Apr;7(2):308-13. doi: https://dx.doi.org/10.1016/j.brs.2013.10. 003. PMID: 24262299.

340. Wu Y, Xu W, Liu X, et al. Adjunctive treatment with high frequency repetitive transcranial magnetic stimulation for the behavioral and psychological symptoms of patients with Alzheimer's disease: a randomized, double-blind, sham-controlled study. Shanghai Jingshen Yixue. 2015 Oct;27(5):280-8. doi:

https://dx.doi.org/10.11919/j.issn.10020829.215107. PMID: 26977125.

341. Elder GJ, Colloby SJ, Firbank MJ, et al. Consecutive sessions of transcranial direct current stimulation do not remediate visual hallucinations in Lewy body dementia: a randomised controlled trial. Alzheimer's Research \& Therapy. 201901 18;11(1):9. doi: https://dx.doi.org/10.1186/s13195-0180465-9. PMID: 30658705.

342. Sabbagh M, Sadowsky C, Tousi B, et al. Effects of a combined transcranial magnetic stimulation (TMS) and cognitive training intervention in patients with Alzheimer's disease. Alzheimer's \& Dementia. 2019 Dec 23;23:23. doi:

https://dx.doi.org/10.1016/j.jalz.2019.08 .197. PMID: 31879235. 
343. Zhang F, Qin Y, Xie L, et al. Highfrequency repetitive transcranial magnetic stimulation combined with cognitive training improves cognitive function and cortical metabolic ratios in Alzheimer's disease. Journal of Neural Transmission. 201901 Aug;126(8):1081-94.

344. van Dijk KR, Scheltens P, Luijpen MW, et al. Peripheral electrical stimulation in Alzheimer's disease. Dementia \& Geriatric Cognitive Disorders. 2005;19(5-6):361-8. PMID: 15802911.

345. Leoutsakos JS, Yan H, Anderson WS, et al. Deep Brain Stimulation Targeting the Fornix for Mild Alzheimer Dementia (the ADvance Trial): A Two Year Follow-up Including Results of Delayed Activation. Journal of Alzheimer's Disease. 2018;64(2):597-606. doi: https://dx.doi.org/10.3233/JAD-180121. PMID: 29914028.

346. Batchelor-Murphy M, McConnell E, Amella E, et al. Experimental Comparison of Efficacy for Three Handfeeding Techniques in Dementia. Journal of the american geriatrics society. 2017;65(4):E89-e94. doi: 10.1111/jgs.14728. PMID: CN01415879 .

347. Coyne ML, Hoskins L. Improving eating behaviors in dementia using behavioral strategies. Clinical Nursing Research. 1997 Aug;6(3):275-90. PMID: 9281930.

348. Henskens M, Nauta IM, Scherder EJA, et al. Implementation and effects of Movement-oriented Restorative Care in a nursing home - a quasi-experimental study. BMC Geriatrics. 2017;17:1-11. doi: 10.1186/s12877-017-0642-x. PMID: 125844550. Language: English. Entry Date: 20180724. Revision Date: 20181018. Publication Type: journal article.

349. Hobbelen JH, Tan FE, Verhey FR, et al. Passive movement therapy in severe paratonia: a multicenter randomized clinical trial. International Psychogeriatrics. 2012 May;24(5):83444. doi: https://dx.doi.org/10.1017/S104161021 1002468. PMID: 22185768.
350. Hobbelen JS, Verhey FR, Bor JH, et al. Passive movement therapy in patients with moderate to severe paratonia; study protocol of a randomised clinical trial (ISRCTN43069940). BMC Geriatrics. 2007 Dec 19;7:30. PMID: 18093298.

351. Kim JP, Yang J. Effectiveness of a community-based program for suicide prevention among elders with earlystage dementia: A controlled observational study. Geriatric Nursing. 2017 Mar - Apr;38(2):97-105. doi: https://dx.doi.org/10.1016/j.gerinurse.20 16.08.002. PMID: 27594544.

352. Rostad HM, Utne I, Grov EK, et al. The impact of a pain assessment intervention on pain score and analgesic use in older nursing home residents with severe dementia: A cluster randomised controlled trial. International Journal of Nursing Studies. 2018 Aug;84:52-60. doi: https://dx.doi.org/10.1016/j.ijnurstu.201 8.04.017. PMID: 29763832.

353. Cheng ST, Li KK, Losada A, et al. The effectiveness of nonpharmacological interventions for informal dementia caregivers: an updated systematic review and meta-analysis. Psychol Aging. 2020;35(1):55-77. doi: 10.1037/pag0000401. PMID: 31985249.

354. Au A, Yip HM, Lai S, et al. Telephonebased behavioral activation intervention for dementia family caregivers: Outcomes and mediation effect of a randomized controlled trial. Patient Education \& Counseling. 2019 Nov;102(11):2049-59. doi: https://dx.doi.org/10.1016/j.pec.2019.06 .009. PMID: 31279613.

355. Bjorge H, Kvaal K, Ulstein I. The effect of psychosocial support on caregivers' perceived criticism and emotional overinvolvement of persons with dementia: an assessor-blinded randomized controlled trial. BMC Health Services Research. 2019 Oct 24;19(1):744. doi: https://dx.doi.org/10.1186/s12913-0194551-x. PMID: 31651321. 
356. Charlesworth G, Burnell K, Crellin N, et al. Peer support and reminiscence therapy for people with dementia and their family carers: a factorial pragmatic randomised trial. Journal of Neurology, Neurosurgery \& Psychiatry. 2016 Nov;87(11):1218-28. doi: https://dx.doi.org/10.1136/jnnp-2016313736. PMID: 27521377.

357. Cheng ST, Fung HH, Chan WC, et al. Short-Term Effects of a Gain-Focused Reappraisal Intervention for Dementia Caregivers: A Double-Blind ClusterRandomized Controlled Trial. American Journal of Geriatric Psychiatry. 2016 Sep;24(9):740-50. doi: https://dx.doi.org/10.1016/j.jagp.2016.0 4.012. PMID: 27401052.

358. de Rotrou J, Cantegreil I, Faucounau V, et al. Do patients diagnosed with Alzheimer's disease benefit from a psycho-educational programme for family caregivers? A randomised controlled study. International Journal of Geriatric Psychiatry. 2011 Aug;26(8):833-42. doi: https://dx.doi.org/10.1002/gps.2611. PMID: 20922772.

359. Gitlin LN, Arthur P, Piersol C, et al. Targeting Behavioral Symptoms and Functional Decline in Dementia: A Randomized Clinical Trial. Journal of the American Geriatrics Society. 2018 Feb;66(2):339-45. doi: https://dx.doi.org/10.1111/jgs.15194. PMID: 29192967.

360. Gonyea JG, Lopez LM, Velasquez EH. The Effectiveness of a Culturally Sensitive Cognitive Behavioral Group Intervention for Latino Alzheimer's Caregivers. Gerontologist. 2016 Apr;56(2):292-302. doi: https://dx.doi.org/10.1093/geront/gnu04 5. PMID: 24855313.

361. Guerra M, Ferri CP, Fonseca M, et al. Helping carers to care: the 10/66 dementia research group's randomized control trial of a caregiver intervention in Peru. Revista Brasileira de Psiquiatria. 2011 Mar;33(1):47-54. PMID: 20602013.
362. Laakkonen ML, Kautiainen H, Holtta E, et al. Effects of Self-Management Groups for People with Dementia and Their Spouses--Randomized Controlled Trial. Journal of the American Geriatrics Society. 2016 Apr;64(4):752-60. doi: https://dx.doi.org/10.1111/jgs.14055. PMID: 27060101.

363. Livingston G, Barber J, Rapaport P, et al. START (STrAtegies for RelaTives) study: a pragmatic randomised controlled trial to determine the clinical effectiveness and cost-effectiveness of a manual-based coping strategy programme in promoting the mental health of carers of people with dementia. Health Technology Assessment (Winchester, England). 2014 Oct;18(61):1-242. doi: https://dx.doi.org/10.3310/hta18610. PMID: 25300037.

364. Martin-Carrasco M, Dominguez-Panchon AI, Gonzalez-Fraile E, et al. Effectiveness of a psychoeducational intervention group program in the reduction of the burden experienced by caregivers of patients with dementia: the EDUCA-II randomized trial. Alzheimer Disease \& Associated Disorders. 2014 Jan-Mar;28(1):79-87. doi:

https://dx.doi.org/10.1097/WAD.00000 00000000003 . PMID: 24113563.

365. Meng H, Marino VR, Conner KO, et al. Effects of in-person and telephonebased cognitive behavioral therapies on health services use and expenditures among African-American dementia caregivers with depressive symptoms. Ethnicity \& Health. 2019 Mar 19:1-14. doi:

https://dx.doi.org/10.1080/13557858.20 19.1590536. PMID: 30884961.

366. Moskowitz JT, Cheung EO, Snowberg KE, et al. Randomized controlled trial of a facilitated online positive emotion regulation intervention for dementia caregivers. Health Psychology. 2019 May;38(5):391-402. doi: https://dx.doi.org/10.1037/hea0000680. PMID: 31045422. 
367. Nordheim J, Hausler A, Yasar S, et al. Psychosocial Intervention in Couples Coping with Dementia Led by a Psychotherapist and a Social Worker: The DYADEM Trial. Journal of Alzheimer’s Disease. 2019;68(2):74555. doi: https://dx.doi.org/10.3233/JAD180812. PMID: 30775982.

368. Tremont G, Davis JD, Papandonatos GD, et al. Psychosocial telephone intervention for dementia caregivers: A randomized, controlled trial. Alzheimer's \& Dementia. 2015 May;11(5):541-8. doi: https://dx.doi.org/10.1016/j.jalz.2014.05 .1752. PMID: 25074341.

369. Waldorff FB, Buss DV, Eckermann A, et al. Efficacy of psychosocial intervention in patients with mild Alzheimer's disease: the multicentre, rater blinded, randomised Danish Alzheimer Intervention Study (DAISY). BMJ. 2012 Jul 17;345:e4693. doi: https://dx.doi.org/10.1136/bmj.e4693. PMID: 22807076.

370. Farran CJ, Gilley DW, McCann JJ, et al. Psychosocial interventions to reduce depressive symptoms of dementia caregivers: A randomized clinical trial comparing two approaches. Journal of Mental Health and Aging. 2004 Winter;10(4):337-50. PMID: 41552352.

371. Gallagher-Thompson D, Coon DW, Solano $\mathrm{N}$, et al. Change in indices of distress among Latino and Anglo female caregivers of elderly relatives with dementia: site-specific results from the REACH national collaborative study. Gerontologist. 2003 Aug;43(4):580-91. PMID: 12937336.

372. Gallagher-Thompson D, Gray HL, Dupart $\mathrm{T}$, et al. Effectiveness of Cognitive/Behavioral Small Group Intervention for Reduction of Depression and Stress in Non-Hispanic White and Hispanic/Latino Women Dementia Family Caregivers: Outcomes and Mediators of Change. Journal of Rational-Emotive \& Cognitive Behavior Therapy. 2008 Dec 01;26(4):286-303. PMID: 25067886.
373. Gitlin LN, Corcoran M, Winter L, et al. A randomized, controlled trial of a home environmental intervention: effect on efficacy and upset in caregivers and on daily function of persons with dementia. Gerontologist. 2001 Feb;41(1):4-14. PMID: 11220813.

374. Graff MJ, Vernooij-Dassen MJ, Thijssen $\mathrm{M}$, et al. Community based occupational therapy for patients with dementia and their care givers: randomised controlled trial. BMJ. 2006 Dec 09;333(7580):1196. PMID: 17114212.

375. Martin-Carrasco M, Martin MF, Valero CP, et al. Effectiveness of a psychoeducational intervention program in the reduction of caregiver burden in Alzheimer's disease patients' caregivers. International Journal of Geriatric Psychiatry. 2009 May;24(5):489-99. doi: https://dx.doi.org/10.1002/gps.2142. PMID: 18949763.

376. Wang LQ, Chien WT. Randomised controlled trial of a family-led mutual support programme for people with dementia. Journal of Clinical Nursing. 2011 Aug;20(15-16):2362-6. doi: https://dx.doi.org/10.1111/j.13652702.2011.03746.x. PMID: 21752121.

377. Gitlin LN, Winter L, Dennis MP, et al. Targeting and managing behavioral symptoms in individuals with dementia: a randomized trial of a nonpharmacological intervention. Journal of the American Geriatrics Society. 2010 Aug;58(8):1465-74. doi: https://dx.doi.org/10.1111/j.15325415.2010.02971.x. PMID: 20662955.

378. Voigt-Radloff S, Graff M, Leonhart R, et al. A multicentre RCT on community occupational therapy in Alzheimer's disease: 10 sessions are not better than one consultation. BMJ Open. 2011;1. doi:10.1136/bmjopen-2011-000096.

379. Otero P, Smit F, Cuijpers P, et al. Longterm efficacy of indicated prevention of depression in non-professional caregivers: randomized controlled trial. Psychol Med. 2015;45(7):1401-12. doi: 10.1017/S0033291714002505. PMID: 25331992. 
380. Au A, Lai DWL, Biggs S, et al. Perspective-taking interventions for intergenerational caregivers of alzheimer's diseases: a randomized controlled trial. Research on social work practice. 2020;30(3):306-19. PMID: CN-02084449.

381. Ghaffari F, Rostami M, Fotokian Z, et al. Effectiveness of resilience education in the mental health of family caregivers of elderly patients with Alzheimer's disease. Iranian Journal of Psychiatry and Behavioral Sciences. 2019;13 (3) (no pagination)(e69507).

382. Chang BL. Cognitive-behavioral intervention for homebound caregivers of persons with dementia. Nursing Research. 1999 May-Jun;48(3):173-82. PMID: 10337848.

383. Dias A, Dewey ME, D’Souza J, et al. The effectiveness of a home care program for supporting caregivers of persons with dementia in developing countries: a randomised controlled trial from Goa, India. PLoS ONE [Electronic

Resource]. 2008 Jun 04;3(6):e2333. doi: https://dx.doi.org/10.1371/journal.pone. 0002333. PMID: 18523642.

384. Marriott A, Donaldson C, Tarrier N, et al. Effectiveness of cognitive-behavioural family intervention in reducing the burden of care in carers of patients with Alzheimer's disease. British Journal of Psychiatry. 2000 Jun;176:557-62. PMID: 10974962.

385. Spijker A, Wollersheim H, Teerenstra S, et al. Systematic care for caregivers of patients with dementia: a multicenter, cluster-randomized, controlled trial. American Journal of Geriatric Psychiatry. 2011 Jun;19(6):521-31. doi: https://dx.doi.org/10.1097/JGP.0b013e3 182110599. PMID: 21358385.

386. Stolley JM, Reed D, Buckwalter KC. Caregiving appraisal and interventions based on the progressively lowered stress threshold model. American Journal of Alzheimer's Disease \& Other Dementias. 2002 Mar-Apr;17(2):11020. PMID: 11954669.
387. Wawrziczny E, Larochette C, Papo D, et al. A Customized Intervention for Dementia Caregivers: A QuasiExperimental Design. Journal of Aging \& Health. 2019 Aug;31(7):1172-95.

doi:

https://dx.doi.org/10.1177/08982643187 70056. PMID: 29665714.

388. Au A, Gallagher-Thompson D, Wong MK, et al. Behavioral activation for dementia caregivers: scheduling pleasant events and enhancing communications. Clinical Interventions In Aging. 2015;10:611-9. doi: https://dx.doi.org/10.2147/CIA.S72348. PMID: 25848237.

389. Chen HM, Huang MF, Yeh YC, et al. Effectiveness of coping strategies intervention on caregiver burden among caregivers of elderly patients with dementia. Psychogeriatrics:The Official Journal of the Japanese Psychogeriatric Society. 2015 Mar;15(1):20-5. doi: https://dx.doi.org/10.1111/psyg.12071. PMID: 25515800.

390. Cristancho-Lacroix V, Wrobel J, Cantegreil-Kallen I, et al. A web-based psychoeducational program for informal caregivers of patients with Alzheimer's disease: a pilot randomized controlled trial. Journal of Medical Internet Research. 2015 May 12;17(5):e117. doi:

https://dx.doi.org/10.2196/jmir.3717. PMID: 25967983.

391. Gallagher-Thompson D, Wang PC, Liu W, et al. Effectiveness of a psychoeducational skill training DVD program to reduce stress in Chinese American dementia caregivers: results of a preliminary study. Aging \& mental health. 2010 Apr;14(3):263-73. doi: http://dx.doi.org/10.1080/13607860903 420989. PMID: 359062574.

392. Gant JR, Steffen AM, Lauderdale SA. Comparative outcomes of two distancebased interventions for male caregivers of family members with dementia. American Journal of Alzheimer's Disease \& Other Dementias. 2007 AprMay;22(2):120-8. PMID: 17545139. 
393. Gonzalez EW, Polansky M, Lippa CF, et al. Enhancing resourcefulness to improve outcomes in family caregivers and persons with Alzheimer's disease: a pilot randomized trial. International journal of Alzheimer's disease. 2014;2014:323478. doi: https://dx.doi.org/10.1155/2014/323478 . PMID: 25328754.

394. Hebert R, Leclerc G, Bravo G, et al. Efficacy of a support group programme for care-givers of demented patients in the community: A randomized controlled trial. Archives of Gerontology and Geriatrics. 1994;18(1):1-14. doi: http://dx.doi.org/10.1016/01674943\%2894\%2990043-4. PMID: 24087060.

395. Kales HC, Gitlin LN, Stanislawski B, et al. Effect of the WeCareAdvisorTM on family caregiver outcomes in dementia: a pilot randomized controlled trial. BMC Geriatrics. 201805 10;18(1):113. doi: https://dx.doi.org/10.1186/s12877018-0801-8. PMID: 29747583.

396. Lok N, Bademli K. Pilot testing of the "First You Should Get Stronger" program among caregivers of older adults with dementia. Archives of Gerontology \& Geriatrics. 2017 Jan Feb;68:84-9. doi: https://dx.doi.org/10.1016/j.archger.201 6.09.006. PMID: 27689315.

397. Martin-Cook K, Remakel-Davis B, Svetlik $\mathrm{D}$, et al. Caregiver attribution and resentment in dementia care. American Journal of Alzheimer's Disease \& Other Dementias. 2003 Nov-Dec;18(6):36674. PMID: 14682086.

398. McCurry SM, Logsdon RG, Vitiello MV, et al. Successful behavioral treatment for reported sleep problems in elderly caregivers of dementia patients: a controlled study. Journals of Gerontology Series B-Psychological Sciences \& Social Sciences. 1998 Mar;53(2):P122-9. PMID: 9520929.
399. Nunez-Naveira L, Alonso-Bua B, de Labra C, et al. UnderstAID, an ICT Platform to Help Informal Caregivers of People with Dementia: A Pilot Randomized Controlled Study. BioMed Research International. 2016;2016:5726465. doi: https://dx.doi.org/10.1155/2016/572646 5. PMID: 28116300.

400. Steffen AM. Anger management for dementia caregivers: A preliminary study using video and telephone interventions. Behavior Therapy. 2000 Spr;31(2):281-99. doi: http://dx.doi.org/10.1016/S00057894\%2800\%2980016-7. PMID: 200016043-005.

401. Ta Park VM, Ton V, Tiet QQ, et al. Promising results from a pilot study to reduce distress in Vietnamese American dementia and memory loss caregivers. Alzheimer's \& Dementia : Translational Research \& Clinical Interventions. 2019;5:319-27. doi: https://dx.doi.org/10.1016/j.trci.2019.05 .006. PMID: 31384663.

402. Zauszniewski JA, Lekhak N, Burant CJ, et al. Preliminary Evidence for Effectiveness of Resourcefulness Training in Women Dementia Caregivers. Journal of Family Medicine. 2016;3(5). PMID: 27500286.

403. Aboulafia-Brakha T, Suchecki D, GouveiaPaulino F, et al. Cognitive-behavioural group therapy improves a psychophysiological marker of stress in caregivers of patients with Alzheimer's disease. Aging \& Mental Health. 2014;18(6):801-8. doi: https://dx.doi.org/10.1080/13607863.20 14.880406. PMID: 24499394.

404. Akkerman RL, Ostwald SK. Reducing anxiety in Alzheimer's disease family caregivers: the effectiveness of a nineweek cognitive-behavioral intervention. American Journal of Alzheimer's Disease \& Other Dementias. 2004 MarApr;19(2):117-23. PMID: 15106393.

405. Au A, Li S, Lee K, et al. The Coping with Caregiving Group Program for Chinese caregivers of patients with Alzheimer's disease in Hong Kong. In Patient education and counseling 
406. Brodaty H, Gresham M, Luscombe G. The Prince Henry Hospital dementia caregivers' training programme. International Journal of Geriatric Psychiatry. 1997 Feb;12(2):183-92. PMID: 9097211.

407. Davis JD, Tremont G, Bishop DS, et al. A telephone-delivered psychosocial intervention improves dementia caregiver adjustment following nursing home placement. International Journal of Geriatric Psychiatry. 2011 Apr;26(4):380-7. doi: https://dx.doi.org/10.1002/gps.2537. PMID: 20842759.

408. Dowling GA, Merrilees J, Mastick J, et al. Life enhancing activities for family caregivers of people with frontotemporal dementia. Alzheimer Disease \& Associated Disorders. 2014 Apr-Jun;28(2):175-81. doi: https://dx.doi.org/10.1097/WAD.0b013 e3182a6b905. PMID: 24113564.

409. Garand L, Buckwalter KC, Lubaroff D, et al. A pilot study of immune and mood outcomes of a community-based intervention for dementia caregivers: the PLST intervention. Archives of Psychiatric Nursing. 2002 Aug;16(4):156-67. PMID: 12143075.

410. Gaugler JE, Reese M, Sauld J. A Pilot Evaluation of Psychosocial Support for Family Caregivers of Relatives with Dementia in Long-Term Care: The Residential Care Transition Module. Research in Gerontological Nursing. 2015 Jul-Aug;8(4):161-72. doi: https://dx.doi.org/10.3928/1940492120150304-01. PMID: 25751083.

411. Gendron C, Poitras L, Dastoor DP, et al. Cognitive-behavioral group intervention for spousal caregivers: Findings and clinical considerations. Clinical Gerontologist. 1996;17(1):3-19. doi: http://dx.doi.org/10.1300/J018v17n01_0 2. PMID: 26250550.

412. Glueckauf R, Sharma D, Davis W, et al. Telephone-based cognitive-behavioral intervention for distressed rural dementia caregivers: initial findings. In Clinical gerontologist
413. Huang H-L, Shyu Y-IL, Chen M-C, et al. A pilot study on a home-based caregiver training program for improving caregiver self-efficacy and decreasing the behavioral problems of elders with dementia in Taiwan. International Journal of Geriatric Psychiatry. 2003;18(4):337-45. doi: 10.1002/gps.835. PMID: 104731281. Language: English. Entry Date: 20110610. Revision Date: 20150711. Publication Type: Journal Article.

414. Kamkhagi D, Costa A, Kusminsky S, et al. Benefits of psychodynamic group therapy on depression, burden and quality of life of family caregivers to Alzheimer's disease patients. Revista de psiquiatria clinica. 2015;42(6):157-60. doi: 10.1590/0101-60830000000067. PMID: CN-01162210.

415. Meichsner F, Theurer C, Wilz G. Acceptance and treatment effects of an internet-delivered cognitive-behavioral intervention for family caregivers of people with dementia: A randomizedcontrolled trial. Journal of Clinical Psychology. 2018 Dec 31;31:31. doi: https://dx.doi.org/10.1002/jclp.22739. PMID: 30597537.

416. Moniz-Cook E, Agar S, Gibson G, et al. A preliminary study of the effects of early intervention with people with dementia and their families in a memory clinic. Aging \& Mental Health. 1998;2(3):199211. PMID: 107163779. Language: English. Entry Date: 20070101. Revision Date: 20150711. Publication Type: Journal Article.

417. Nobili A, Riva E, Tettamanti M, et al. The effect of a structured intervention on caregivers of patients with dementia and problem behaviors: a randomized controlled pilot study. Alzheimer Disease \& Associated Disorders. 2004 Apr-Jun;18(2):75-82. PMID: 15249851. 
418. Orgeta V, Tuijt R, Leung P, et al. Behavioral Activation for Promoting Well-Being in Mild Dementia: Feasibility and Outcomes of a Pilot Randomized Controlled Trial. Journal of Alzheimer's Disease. 2019 Oct 08;08:08. doi:

https://dx.doi.org/10.3233/JAD-190696. PMID: 31609689.

419. Paun O, Farran CJ, Fogg L, et al. A chronic grief intervention for dementia family caregivers in long-term care. Western Journal of Nursing Research. 2015 Jan;37(1):6-27. doi: https://dx.doi.org/10.1177/01939459145 21040. PMID: 24510968.

420. de Oliveira AM, Radanovic M, Homem de Mello PC, et al. An intervention to reduce neuropsychiatric symptoms and caregiver burden in dementia: Preliminary results from a randomized trial of the tailored activity programoutpatient version. International Journal of Geriatric Psychiatry. 2018 Jul 23;23:23. doi: https://dx.doi.org/10.1002/gps.4958. PMID: 30035341.

421. Gitlin LN, Winter L, Burke J, et al. Tailored activities to manage neuropsychiatric behaviors in persons with dementia and reduce caregiver burden: a randomized pilot study. American Journal of Geriatric Psychiatry. 2008 Mar;16(3):229-39. doi: https://dx.doi.org/10.1097/JGP.0b013e3 18160da72. PMID: 18310553.

422. Kwok T, Wong B, Ip I, et al. Telephonedelivered psychoeducational intervention for Hong Kong Chinese dementia caregivers: a single-blinded randomized controlled trial. Clinical Interventions In Aging. 2013;8:1191-7. doi:

https://dx.doi.org/10.2147/CIA.S48264. PMID: 24072965.
423. Meichsner F, Theurer C, Wilz G.

Acceptance and treatment effects of an internet-delivered cognitive-behavioral intervention for family caregivers of people with dementia: A randomizedcontrolled trial. Journal of Clinical Psychology. 2019 Apr;75(4):594-613. doi:

https://dx.doi.org/10.1002/jclp.22739. PMID: 30597537.

424. Novelli M, Machado SCB, Lima GB, et al. Effects of the Tailored Activity Program in Brazil (TAP-BR) for Persons With Dementia: A Randomized Pilot Trial. Alzheimer Disease \& Associated Disorders. 2018 Apr 25;25:25. doi: https://dx.doi.org/10.1097/WAD.00000 00000000256. PMID: 29698251.

425. O’Connor CM, Clemson L, Brodaty H, et al. The tailored activity program (TAP) to address behavioral disturbances in frontotemporal dementia: a feasibility and pilot study. Disability \& Rehabilitation. 2017 Oct 15:1-12. doi: https://dx.doi.org/10.1080/09638288.20 17.1387614. PMID: 29034719.

426. Sepe-Monti M, Vanacore N, Bartorelli L, et al. The Savvy Caregiver Program: A Probe Multicenter Randomized Controlled Pilot Trial in Caregivers of Patients Affected by Alzheimer's Disease. Journal of Alzheimer's Disease. 201610 04;54(3):1235-46. PMID: 27567824.

427. Supiano KP, Haynes LB, Pond V. The Process of Change in Complicated Grief Group Therapy for Bereaved Dementia Caregivers: An Evaluation Using the Meaning of Loss Codebook. Journal of Gerontological Social Work. 2017 FebMar;60(2):155-69. doi: https://dx.doi.org/10.1080/01634372.20 16.1274930. PMID: 28051926.

428. Teri L, Logsdon RG, Uomoto J, et al. Behavioral treatment of depression in dementia patients: a controlled clinical trial. Journals of Gerontology Series BPsychological Sciences \& Social Sciences. 1997 Jul;52(4):P159-66. PMID: 9224439. 
429. Tremont G, Davis JD, Bishop DS, et al. Telephone-delivered psychosocial intervention reduces burden in dementia caregivers. Dementia: The International Journal of Social Research and Practice. 2008 Nov;7(4):503-20. doi: http://dx.doi.org/10.1177/14713012080 96632. PMID: 2008-16304-006.

430. van Knippenberg RJM, de Vugt ME, Ponds RW, et al. An Experience Sampling Method Intervention for Dementia Caregivers: Results of a Randomized Controlled Trial. American Journal of Geriatric Psychiatry. 2018 Dec;26(12):1231-43. doi: https://dx.doi.org/10.1016/j.jagp.2018.0 6.004. PMID: 30126766.

431. Yoo R, Yeom J, Kim GH, et al. A Multicenter, Randomized Clinical Trial to Assess the Efficacy of a Therapeutic Intervention Program for Caregivers of People with Dementia. Journal of Clinical Neurology. 2019 Apr;15(2):235-42. doi: https://dx.doi.org/10.3988/jcn.2019.15.2 .235. PMID: 30938110.

432. Boots LM, de Vugt ME, Kempen GI, et al. Effectiveness of a Blended Care SelfManagement Program for Caregivers of People With Early-Stage Dementia (Partner in Balance): Randomized Controlled Trial. Journal of Medical Internet Research. 2018 Jul 13;20(7):e10017. doi: https://dx.doi.org/10.2196/10017. PMID: 30006327.

433. Huang HL, Shyu YI, Chen MC, et al. A pilot study on a home-based caregiver training program for improving caregiver self-efficacy and decreasing the behavioral problems of elders with dementia in Taiwan. International Journal of Geriatric Psychiatry. 2003 Apr;18(4):337-45. PMID: 12673611.

434. Spalding-Wilson KN, Guzman-Velez E, Angelica J, et al. A novel two-day intervention reduces stress in caregivers of persons with dementia. Alzheimer's and Dementia: Translational Research and Clinical Interventions. 201801 Jan;4:450-60. doi: http://dx.doi.org/10.1016/j.trci.2018.08. 004. PMID: 2001115918.
435. Whitlatch CJ, Heid AR, Femia EE, et al. The Support, Health, Activities, Resources, and Education program for early stage dementia: Results from a randomized controlled trial. Dementia. 2019 08;18(6):2122-39. doi: https://dx.doi.org/10.1177/14713012177 43033. PMID: 29171296.

436. Wilz G, Reder M, Meichsner F, et al. The Tele.TAnDem Intervention: Telephonebased CBT for Family Caregivers of People With Dementia. Gerontologist. 201803 19;58(2):e118-e29. doi: https://dx.doi.org/10.1093/geront/gnx18 3. PMID: 29190357.

437. Au A. Developing Volunteer-Assisted Behavioral Activation Teleprograms to Meet the Needs of Chinese Dementia Caregivers. Clinical gerontologist. 2015;38(3):190-202. PMID: CN01071414.

438. Blom MM, Zarit SH, Groot Zwaaftink RB, et al. Effectiveness of an Internet intervention for family caregivers of people with dementia: results of a randomized controlled trial. PLoS ONE [Electronic Resource]. 2015;10(2):e0116622. doi: https://dx.doi.org/10.1371/journal.pone. 0116622. PMID: 25679228.

439. Callahan CM, Boustani MA, Schmid AA, et al. Targeting Functional Decline in Alzheimer Disease: A Randomized Trial. Annals of Internal Medicine. 2017 Feb 07;166(3):164-71. doi: https://dx.doi.org/10.7326/M16-0830. PMID: 27893087.

440. Chiu M, Pauley T, Wesson V, et al. Evaluation of a problem-solving (PS) techniques-based intervention for informal carers of patients with dementia receiving in-home care. International psychogeriatrics. 2015;27(6):937-48. doi: 10.1017/S1041610214002798. PMID: CN-01102329. 
441. Taati F, Bahramnezhad F, Seyedfatemi N, et al. The effect of participation in support groups on depression, anxiety and stress in family caregivers of people with Alzheimer's: randomized clinical trail. International journal of medical research \& health sciences. 2016;5(7):256-62. PMID: CN01308282 .

442. Whitlatch CJ, Heid AR, Femia EE, et al. The Support, Health, Activities, Resources, and Education program for early stage dementia: Results from a randomized controlled trial. Dementia. 2017 Jan 01:1471301217743033. doi: https://dx.doi.org/10.1177/14713012177 43033. PMID: 29171296.

443. Arango-Lasprilla JC, Panyavin I, Merchan $\mathrm{EJH}$, et al. Evaluation of a group cognitive-behavioral dementia caregiver intervention in Latin America. American Journal of Alzheimer's Disease and Other Dementias. 2014 Sep;29(6):548-55. doi: http://dx.doi.org/10.1177/15333175145 23668. PMID: 2014-38564-010.

444. Bruvik FK, Allore HG, Ranhoff AH, et al. The effect of psychosocial support intervention on depression in patients with dementia and their family caregivers: an assessor-blinded randomized controlled trial. Dementia and Geriatric Cognitive Disorders Extra. 2013;3(1):386-97. doi: https://dx.doi.org/10.1159/000355912. PMID: 24348500.

445. Gallagher-Thompson D, Tzuang M, Hinton L, et al. Effectiveness of a fotonovela for reducing depression and stress in Latino dementia family caregivers. Alzheimer Disease \& Associated Disorders. 2015 Apr-Jun;29(2):146-53. doi:

https://dx.doi.org/10.1097/WAD.00000 00000000077. PMID: 25590939.
446. Huang HL, Kuo LM, Chen YS, et al. A home-based training program improves caregivers' skills and dementia patients' aggressive behaviors: a randomized controlled trial. American Journal of Geriatric Psychiatry. 2013 Nov;21(11):1060-70. doi: https://dx.doi.org/10.1016/j.jagp.2012.0 9.009. PMID: 23933422.

447. Losada A, Marquez-Gonzalez M, RomeroMoreno R, et al. Cognitive-behavioral therapy (CBT) versus acceptance and commitment therapy (ACT) for dementia family caregivers with significant depressive symptoms: Results of a randomized clinical trial. Journal of Consulting \& Clinical Psychology. 2015 Aug;83(4):760-72. doi: https://dx.doi.org/10.1037/ccp0000028. PMID: 26075381.

448. Otero P, Smit F, Cuijpers P, et al. Differential response to depression prevention among a sample of informal caregivers: Moderator analysis of longer-term follow-up trial data. Psychiatry Research. 2015 Dec 15;230(2):271-8. doi: https://dx.doi.org/10.1016/j.psychres.20 15.09.005. PMID: 26456895.

449. Passoni S, Moroni L, Toraldo A, et al. Cognitive behavioral group intervention for Alzheimer caregivers. Alzheimer Disease \& Associated Disorders. 2014;28(3):275-82. doi: 10.1097/WAD.0000000000000033. PMID: 107799151. Language: English. Entry Date: 20150508. Revision Date: 20150712. Publication Type: Journal Article.

450. Chu H, Yang CY, Liao YH, et al. The effects of a support group on dementia caregivers' burden and depression. Journal of aging and health. 2011;23(2):228-41. PMID: CN00858523. 
451. Joling KJ, van Marwijk HW, Smit F, et al. Does a family meetings intervention prevent depression and anxiety in family caregivers of dementia patients? A randomized trial. PLoS ONE [Electronic Resource]. 2012;7(1):e30936. doi: https://dx.doi.org/10.1371/journal.pone. 0030936. PMID: 22303473.

452. Judge KS, Yarry SJ, Looman WJ, et al. Improved Strain and Psychosocial Outcomes for Caregivers of Individuals with Dementia: Findings from Project ANSWERS. Gerontologist. 2013 Apr;53(2):280-92. doi: https://dx.doi.org/10.1093/geront/gns07 6. PMID: 22899427.

453. Kajiyama B, Thompson LW, Eto-Iwase T, et al. Exploring the effectiveness of an internet-based program for reducing caregiver distress using the iCare Stress Management e-Training Program.[Erratum appears in Aging Ment Health. 2013;17(5):c1]. Aging \& Mental Health. 2013;17(5):544-54. doi: https://dx.doi.org/10.1080/13607863.20 13.775641. PMID: 23461355.

454. Kuo LM, Huang HL, Huang HL, et al. A home-based training program improves Taiwanese family caregivers' quality of life and decreases their risk for depression: a randomized controlled trial. International Journal of Geriatric Psychiatry. 2013 May;28(5):504-13. doi: https://dx.doi.org/10.1002/gps.3853. PMID: 22778053.

455. Moore RC, Chattillion EA, Ceglowski J, et al. A randomized clinical trial of Behavioral Activation (BA) therapy for improving psychological and physical health in dementia caregivers: results of the Pleasant Events Program (PEP). Behaviour Research \& Therapy. 2013 Oct;51(10):623-32. doi: https://dx.doi.org/10.1016/j.brat.2013.0 7.005. PMID: 23916631.
456. Gavrilova SI, Ferri CP, Mikhaylova N, et al. Helping carers to care--the 10/66 dementia research group's randomized control trial of a caregiver intervention in Russia. International Journal of Geriatric Psychiatry. 2009 Apr;24(4):347-54. doi: https://dx.doi.org/10.1002/gps.2126. PMID: 18814197.

457. Gitlin LN, Winter L, Dennis MP, et al. A biobehavioral home-based intervention and the well-being of patients with dementia and their caregivers: the COPE randomized trial. JAMA. 2010 Sep 01;304(9):983-91. doi: https://dx.doi.org/10.1001/jama.2010.12 53. PMID: 20810376.

458. Kurz A, Wagenpfeil S, Hallauer J, et al. Evaluation of a brief educational program for dementia carers: the AENEAS study. International Journal of Geriatric Psychiatry. 2010 Aug;25(8):861-9. doi: https://dx.doi.org/10.1002/gps.2428. PMID: 19946869.

459. Losada A, Marquez-Gonzalez M, RomeroMoreno R. Mechanisms of action of a psychological intervention for dementia caregivers: effects of behavioral activation and modification of dysfunctional thoughts. International Journal of Geriatric Psychiatry. 2011 Nov;26(11):1119-27. doi: https://dx.doi.org/10.1002/gps.2648. PMID: 21061414.

460. Marquez-Gonzalez M, Losada A, Izal M, et al. Modification of dysfunctional thoughts about caregiving in dementia family caregivers: description and outcomes of an intervention programme. Aging \& Mental Health. 2007 Nov;11(6):616-25. PMID: 18074249.

461. Williams V, Bishop-Fitzpatrick L, Lane J, et al. Video-Based Coping Skills to Reduce Health Risk and Improve Psychological and Physical Well-Being in Alzheimer's Disease Family Caregivers. Psychosomatic medicine. 2010;72(9):897-904. doi: 10.1097/PSY.0b013e3181fc2d09. PMID: CN-00892462. 
462. Wilz G, Schinkothe D, Soellner R. Goal attainment and treatment compliance in a cognitive-behavioral telephone intervention for family caregivers of persons with dementia. GeroPsych: The Journal of Gerontopsychology and Geriatric Psychiatry. 201101 Sep;24(3):115-25. doi: http://dx.doi.org/10.1024/16629647/a000043. PMID: 362501128.

463. Beauchamp N, Irvine AB, Seeley J, et al. Worksite-based internet multimedia program for family caregivers of persons with dementia. Gerontologist. 2005 Dec;45(6):793-801. PMID: 16326661.

464. Burgio L, Stevens A, Guy D, et al. Impact of two psychosocial interventions on white and African American family caregivers of individuals with dementia. Gerontologist. 2003 Aug;43(4):568-79. PMID: 12937335.

465. Burns R, Nichols LO, Martindale-Adams J, et al. Primary care interventions for dementia caregivers: 2-year outcomes from the REACH study. Gerontologist. 2003 Aug;43(4):547-55. PMID: 12937333.

466. Coon DW, Thompson L, Steffen A, et al. Anger and depression management: psychoeducational skill training interventions for women caregivers of a relative with dementia. Gerontologist. 2003 Oct;43(5):678-89. PMID: 14570964.

467. Gitlin LN, Winter L, Corcoran M, et al. Effects of the home environmental skillbuilding program on the caregiver-care recipient dyad: 6-month outcomes from the Philadelphia REACH Initiative. Gerontologist. 2003 Aug;43(4):532-46. PMID: 12937332.

468. Gonyea JG, O’Connor MK, Boyle PA. Project CARE: a randomized controlled trial of a behavioral intervention group for Alzheimer's disease caregivers. Gerontologist. 2006 Dec;46(6):827-32. PMID: 17169938.
469. Ulstein ID, Sandvik L, Wyller TB, et al. A one-year randomized controlled psychosocial intervention study among family carers of dementia patients-effects on patients and carers. Dementia \& Geriatric Cognitive Disorders. 2007;24(6):469-75. PMID: 17986818.

470. Buckwalter KC, Gerdner L, Kohout F, et al. A nursing intervention to decrease depression in family caregivers of persons with dementia. Archives of Psychiatric Nursing. 1999 Apr;13(2):80-8. PMID: 10222636.

471. Fung W, Chien W. The effectiveness of a mutual support group for family caregivers of a relative with dementia. Archives of Psychiatric Nursing. 2002;16(3):134-44. PMID: 106975399. Language: English. Entry Date: 20021108. Revision Date: 20150819. Publication Type: Journal Article.

472. Hebert R, Levesque L, Vezina J, et al. Efficacy of a psychoeducative group program for caregivers of demented persons living at home: a randomized controlled trial. Journals of Gerontology Series B-Psychological Sciences \& Social Sciences. 2003 Jan;58(1):S58-67. PMID: 12496309.

473. Ostwald SK, Hepburn KW, Caron W, et al. Reducing caregiver burden: a randomized psychoeducational intervention for caregivers of persons with dementia. Gerontologist. 1999 Jun;39(3):299-309. PMID: 10396888.

474. Wright LK, Litaker M, Laraia MT, et al. Continuum of care for Alzheimer's disease: a nurse education and counseling program. Issues in Mental Health Nursing. 2001 AprMay;22(3):231-52. PMID: 11885210.

475. Terracciano A, Artese A, Yeh J, et al. Effectiveness of Powerful Tools for Caregivers on Caregiver Burden and on Care Recipient Behavioral and Psychological Symptoms of Dementia: A Randomized Controlled Trial. Journal of the American Medical Directors Association. 2019 Dec 19;19:19. doi: https://dx.doi.org/10.1016/j.jamda.2019. 11.011. PMID: 31866419. 
476. Uyar F, Ozmen D, Mavioglu H, et al. Assessment of the impact of dementia care and support program in both patient and caregiver outcomes: An intervention study. Turk Geriatri Dergisi. 2019;22(4):482-93.

477. Kunik ME, Stanley MA, Shrestha S, et al. Aggression Prevention Training for Individuals With Dementia and Their Caregivers: A Randomized Controlled Trial. American Journal of Geriatric Psychiatry. 2020 Feb 03;03:03. doi: https://dx.doi.org/10.1016/j.jagp.2020.0 1.190. PMID: 32115311.

478. Rabinowitz YG, Mausbach BT, Coon DW, et al. The moderating effect of selfefficacy on intervention response in women family caregivers of older adults with dementia. American Journal of Geriatric Psychiatry. 2006 Aug;14(8):642-9. PMID: 16861368.

479. Mahoney DF, Tarlow BJ, Jones RN. Effects of an automated telephone support system on caregiver burden and anxiety: findings from the REACH for TLC intervention study. Gerontologist. 2003 Aug;43(4):556-67. PMID: 12937334.

480. Mahoney DM, Tarlow B, Jones RN, et al. Factors affecting the use of a telephonebased intervention for caregivers of people with Alzheimer's disease. Journal of Telemedicine \& Telecare. 2001;7(3):139-48. PMID: 11346473.

481. Wang LQ, Chien WT, Lee IY. An experimental study on the effectiveness of a mutual support group for family caregivers of a relative with dementia in mainland China. Contemporary Nurse. 2012 Feb;40(2):210-24. doi: https://dx.doi.org/10.5172/conu.2012.40 .2.210. PMID: 22554214 .

482. Charlesworth G, Shepstone L, Wilson E, et al. Befriending carers of people with dementia: randomised controlled trial. BMJ. 2008 Jun 07;336(7656):1295-7. doi: https://dx.doi.org/10.1136/bmj.39549.5 48831.AE. PMID: 18505757.
483. Charlesworth G, Shepstone L, Wilson E, et al. Does befriending by trained lay workers improve psychological wellbeing and quality of life for carers of people with dementia, and at what cost? A randomised controlled trial. Health Technology Assessment (Winchester, England). 2008 Mar;12(4):iii, v-ix, 178. PMID: 18284895.

484. Goodman C. Evaluation of a model selfhelp telephone program: impact on natural networks. Social Work. 1990 Nov;35(6):556-62. PMID: 2284602.

485. Logsdon RG, Pike KC, McCurry SM, et al. Early-stage memory loss support groups: outcomes from a randomized controlled clinical trial. Journals of Gerontology Series B: Psychological Sciences \& Social Sciences. 2010;65B(6):691-7. doi: geronb/gbq054. PMID: 104963362. Language: English. Entry Date: 20110128. Revision Date: 20150820. Publication Type: Journal Article.

486. Metcalfe A, Jones B, Mayer J, et al. Online information and support for carers of people with young-onset dementia: A multi-site randomised controlled pilot study. International Journal of Geriatric Psychiatry. 201901 Oct;34(10):145564. doi: http://dx.doi.org/10.1002/gps.5154. PMID: 2002845656.

487. Pillemer K, Suitor JJ. Peer support for Alzheimer's caregivers: Is it enough to make a difference? Research on Aging. 2002;24(2):171-92. PMID: 34163761.

488. Torkamani M, McDonald L, Saez Aguayo I, et al. A randomized controlled pilot study to evaluate a technology platform for the assisted living of people with dementia and their carers. Journal of Alzheimer's Disease. 2014;41(2):51523. doi: https://dx.doi.org/10.3233/JAD132156. PMID: 24643137. 
489. van der Roest HG, Meiland FJ, Jonker C, et al. User evaluation of the DEMentiaspecific Digital Interactive Social Chart (DEM-DISC). A pilot study among informal carers on its impact, user friendliness and, usefulness. Aging \& Mental Health. 2010 May;14(4):461-70. doi:

https://dx.doi.org/10.1080/13607860903 311741. PMID: 20455122.

490. Van Mierlo LD, Meiland FJ, Van de Ven PM, et al. Evaluation of DEM-DISC, customized e-advice on health and social support services for informal carers and case managers of people with dementia; a cluster randomized trial. International Psychogeriatrics. 2015 Aug;27(8):1365-78. doi: https://dx.doi.org/10.1017/S104161021 5000423. PMID: 25872457.

491. Wilson E, Thalanany M, Shepstone L, et al. Befriending carers of people with dementia: a cost utility analysis. International Journal of Geriatric Psychiatry. 2009 Jun;24(6):610-23. doi: https://dx.doi.org/10.1002/gps.2164. PMID: 19101921.

492. Winter L, Gitlin LN. Evaluation of a telephone-based support group intervention for female caregivers of community-dwelling individuals with dementia. American Journal of Alzheimer's Disease \& Other Dementias. 2006 Dec-2007 Jan;21(6):391-7. PMID: 17267370.

493. Huis In Het Veld JG, Willemse BM, van Asch IF, et al. Online Self-Management Support for Family Caregivers Dealing With Behavior Changes in Relatives With Dementia (Part 2): Randomized Controlled Trial. Journal of Medical Internet Research. 2020 Feb 25;22(2):e13001. doi: https://dx.doi.org/10.2196/13001. PMID: 32130142.

494. Gustafson DH, Cody OJ, Chih MY, et al. Pilot Test of a Computer-Based System to Help Family Caregivers of Dementia Patients. Journal of Alzheimer's Disease. 2019;70(2):539-50. doi: http://dx.doi.org/10.3233/JAD-190052. PMID: 628746285.
495. Whitebird RR, Kreitzer M, Crain AL, et al. Mindfulness-based stress reduction for family caregivers: a randomized controlled trial. Gerontologist. 2013 Aug;53(4):676-86. doi: https://dx.doi.org/10.1093/geront/gns12 6. PMID: 23070934.

496. Brown KW, Coogle CL, Wegelin J. A pilot randomized controlled trial of mindfulness-based stress reduction for caregivers of family members with dementia. Aging \& Mental Health. 2016 Nov;20(11):1157-66. PMID: 26211415.

497. Danucalov MA, Kozasa EH, Afonso RF, et al. Yoga and compassion meditation program improve quality of life and self-compassion in family caregivers of Alzheimer's disease patients: A randomized controlled trial. Geriatrics \& gerontology international. 2017 Jan;17(1):85-91. doi: https://dx.doi.org/10.1111/ggi.12675. PMID: 26685923.

498. Kor PPK, Liu JYW, Chien WT. Effects of a modified mindfulness-based cognitive therapy for family caregivers of people with dementia: A pilot randomized controlled trial. International Journal of Nursing Studies. 2019 Oct;98:107-17. doi: https://dx.doi.org/10.1016/j.ijnurstu.201 9.02.020. PMID: 30922609.

499. Lavretsky H, Epel ES, Siddarth P, et al. A pilot study of yogic meditation for family dementia caregivers with depressive symptoms: effects on mental health, cognition, and telomerase activity. International Journal of Geriatric Psychiatry. 2013 Jan;28(1):5765. doi: https://dx.doi.org/10.1002/gps.3790. PMID: 22407663.

500. Leach MJ, Francis A, Ziaian T. Transcendental Meditation for the improvement of health and wellbeing in community-dwelling dementia caregivers [TRANSCENDENT]: a randomised wait-list controlled trial. BMC Complementary \& Alternative Medicine. 2015 May 08;15:145. doi: https://dx.doi.org/10.1186/s12906-0150666-8. PMID: 25952550. 
501. Oken BS, Fonareva I, Haas M, et al. Pilot controlled trial of mindfulness meditation and education for dementia caregivers. Journal of Alternative \& Complementary Medicine. 2010 Oct;16(10):1031-8. doi: https://dx.doi.org/10.1089/acm.2009.07 33. PMID: 20929380.

502. Waelde LC, Meyer H, Thompson JM, et al. Randomized Controlled Trial of Inner Resources Meditation for Family Dementia Caregivers. Journal of Clinical Psychology. 2017 Dec;73(12):1629-41. doi: https://dx.doi.org/10.1002/jclp.22470. PMID: 28263398.

503. Mahdavi B, Fallahi-Khoshknab M, Mohammadi F, et al. Effects of spiritual group therapy on caregiver strain in home caregivers of the elderly with Alzheimer's disease. Archives of psychiatric nursing. 2016. PMID: CN01308784.

504. Salamizadeh AMS, Mirzaei TP, Ravari AP. The Impact of Spiritual Care Education on the Self-Efficacy of the Family Caregivers of Elderly People with Alzheimer's Disease. International Journal of Community Based Nursing \& Midwifery. 2017 Jul;5(3):231-8. PMID: 28670585.

505. Castro CM, Wilcox S, O’Sullivan P, et al. An exercise program for women who are caring for relatives with dementia. Psychosomatic Medicine. 2002 MayJun;64(3):458-68. PMID: 12021419.

506. Connell CM, Janevic MR. Effects of a Telephone-Based Exercise Intervention for Dementia Caregiving Wives: A Randomized Controlled Trial. Journal of Applied Gerontology. 2009 Apr;28(2):171-94. PMID: 21709757.

507. Farran CJ, Etkin CD, Eisenstein A, et al. Effect of Moderate to Vigorous Physical Activity Intervention on Improving Dementia Family Caregiver Physical Function: A Randomized Controlled Trial. Journal of Alzheimer's Disease \& Parkinsonism. 2016 Aug;6(4). doi: https://dx.doi.org/10.4172/21610460.1000253. PMID: 28752016.
508. Hirano A, Suzuki Y, Kuzuya M, et al. Influence of regular exercise on subjective sense of burden and physical symptoms in community-dwelling caregivers of dementia patients: a randomized controlled trial. Archives of Gerontology \& Geriatrics. 2011 SepOct;53(2):e158-63. doi: https://dx.doi.org/10.1016/j.archger.201 0.08.004. PMID: 20850878.

509. Lowery D, Cerga-Pashoja A, Iliffe S, et al. The effect of exercise on behavioural and psychological symptoms of dementia: the EVIDEM-E randomised controlled clinical trial. International Journal of Geriatric Psychiatry. 2014 Aug;29(8):819-27. doi: https://dx.doi.org/10.1002/gps.4062. PMID: 24338799.

510. Hirano A, Umegaki H, Suzuki Y, et al. Effects of leisure activities at home on perceived care burden and the endocrine system of caregivers of dementia patients: a randomized controlled study. International Psychogeriatrics. 2016 Feb;28(2):261-8. doi: https://dx.doi.org/10.1017/S104161021 5001295. PMID: 26289496.

511. Richards AG, Tietyen AC, Jicha GA, et al. Visual Arts Education improves selfesteem for persons with dementia and reduces caregiver burden: A randomized controlled trial. Dementia. 2018 Jan 01:1471301218769071. doi: https://dx.doi.org/10.1177/14713012187 69071. PMID: 29653492.

512. Wilz G, Fink-Heitz M. Assisted vacations for men with dementia and their caregiving spouses: evaluation of health-related effects. Gerontologist. 2008 Feb;48(1):115-20. PMID: 18381838.

513. Carbonneau H, Caron CD, Desrosiers J. Effects of an adapted leisure education program as a means of support for caregivers of people with dementia. Archives of Gerontology \& Geriatrics. 2011 Jul-Aug;53(1):31-9. doi: https://dx.doi.org/10.1016/j.archger.201 0.06.009. PMID: 20598758. 
514. Lawton MP, Brody EM, Saperstein A, et al. Respite services for caregivers: research findings for service planning. Home Health Care Services Quarterly. 1989;10(1-2):5-32. PMID: 10318337.

515. Vandepitte S, Putman K, Van Den Noortgate N, et al. Effectiveness of an in-home respite care program to support informal dementia caregivers: A comparative study. International Journal of Geriatric Psychiatry. 2019 Oct;34(10):1534-44. doi: https://dx.doi.org/10.1002/gps.5164. PMID: 31243801.

516. Vandepitte S, Van Den Noortgate N, Putman K, et al. Effectiveness and costeffectiveness of an in-home respite care program in supporting informal caregivers of people with dementia: design of a comparative study. BMC Geriatrics. 201612 02;16(1):207. PMID: 27912740.

517. Zarit SH, Stephens MA, Townsend A, et al. Stress reduction for family caregivers: effects of adult day care use. Journals of Gerontology Series B-Psychological Sciences \& Social Sciences. 1998 Sep;53(5):S267-77. PMID: 9750575.

518. Baglio F, Griffanti L, Saibene FL, et al. Multistimulation group therapy in Alzheimer's disease promotes changes in brain functioning. Neurorehabilitation \& Neural Repair. 2015 Jan;29(1):13-24. doi: https://dx.doi.org/10.1177/15459683145 32833. PMID: 24788581.

519. Belle SH, Burgio L, Burns R, et al. Enhancing the quality of life of dementia caregivers from different ethnic or racial groups: a randomized, controlled trial.[Summary for patients in Ann Intern Med. 2006 Nov 21;145(10):I39; PMID: 17116914]. Annals of Internal Medicine. 2006 Nov 21;145(10):727-38. PMID: 17116917.

520. Berwig M, Heinrich S, Spahlholz J, et al. Individualized support for informal caregivers of people with dementia effectiveness of the German adaptation of REACH II. BMC Geriatrics. 2017 Dec 12;17(1):286. doi: https://dx.doi.org/10.1186/s12877-0170678-y. PMID: 29233097.
521. Brijoux T, Kricheldorff C, M HL, et al. Supporting Families Living With Dementia in Rural Areas. Deutsches Arzteblatt International. 2016 Oct 14;113(41):681-7. doi: https://dx.doi.org/10.3238/arztebl.2016. 0681. PMID: 27839534.

522. Czaja SJ, Loewenstein D, Schulz R, et al. A videophone psychosocial intervention for dementia caregivers. American Journal of Geriatric Psychiatry. 2013 Nov;21(11):1071-81. doi: https://dx.doi.org/10.1016/j.jagp.2013.0 2.019. PMID: 23831174.

523. Ducharme F, Levesque L, Giroux F, et al. Follow-up of an intervention program for caregivers of a relative with dementia living in a long-term care setting: Are there any persistent and delayed effects? Aging \& Mental Health. 2005 Sep;9(5):461-9. PMID: 16024406.

524. Eisdorfer C, Czaja SJ, Loewenstein DA, et al. The effect of a family therapy and technology-based intervention on caregiver depression. Gerontologist. 2003 Aug;43(4):521-31. PMID: 12937331.

525. Finkel S, Czaja SJ, Schulz R, et al. E-care: a telecommunications technology intervention for family caregivers of dementia patients. American Journal of Geriatric Psychiatry. 2007 May;15(5):443-8. PMID: 17463195.

526. Gaugler JE, Reese M, Mittelman MS. Effects of the NYU caregiver intervention-adult child on residential care placement. Gerontologist. 2013 Dec;53(6):985-97. doi: https://dx.doi.org/10.1093/geront/gns19 3. PMID: 23339050.

527. Koivisto AM, Hallikainen I, Valimaki T, et al. Early psychosocial intervention does not delay institutionalization in persons with mild Alzheimer disease and has impact on neither disease progression nor caregivers' well-being: ALSOVA 3-year follow-up. International Journal of Geriatric Psychiatry. 2016 Mar;31(3):273-83. doi: https://dx.doi.org/10.1002/gps.4321. PMID: 26177825. 
528. Luchsinger JA, Burgio L, Mittelman M, et al. Comparative Effectiveness of 2 Interventions for Hispanic Caregivers of Persons with Dementia. Journal of the American Geriatrics Society. 2018 Sep;66(9):1708-15. doi: https://dx.doi.org/10.1111/jgs.15450. PMID: 30084133.

529. Marshall A, Spreadbury J, Cheston R, et al. A pilot randomised controlled trial to compare changes in quality of life for participants with early diagnosis dementia who attend a â€ Living Well with Dementiaâ€ $€^{\mathrm{TM}}$ group compared to waiting-list control. Aging \& mental health. 2015;19(6):526-35. PMID: CN01071410.

530. Martindale-Adams J, Nichols LO, Burns R, et al. A trial of dementia caregiver telephone support. Canadian Journal of Nursing Research. 2013 Dec;45(4):3048. PMID: 24617278.

531. Mittelman MS, Brodaty H, Wallen AS, et al. A three-country randomized controlled trial of a psychosocial intervention for caregivers combined with pharmacological treatment for patients with Alzheimer disease: effects on caregiver depression. American Journal of Geriatric Psychiatry. 2008;16(11):893-904. doi: 10.1097/JGP.0b013e3181898095. PMID: 105627901. Language: English. Entry Date: 20090130. Revision Date: 20161207. Publication Type: journal article.

532. Mittelman MS, Ferris SH, Steinberg G, et al. An intervention that delays institutionalization of Alzheimer's disease patients: treatment of spousecaregivers. Gerontologist. 1993 Dec;33(6):730-40. PMID: 8314099.

533. Mittelman MS, Roth DL, Haley WE, et al. Effects of a caregiver intervention on negative caregiver appraisals of behavior problems in patients with Alzheimer's disease: results of a randomized trial. Journals of Gerontology Series B-Psychological Sciences \& Social Sciences. 2004 Jan;59(1):P27-34. PMID: 14722336.
534. Mohide EA, Pringle DM, Streiner DL, et al. A randomized trial of family caregiver support in the home management of dementia. Journal of the American Geriatrics Society. 1990 Apr;38(4):44654. PMID: 2184186.

535. Prick AE, de Lange J, Twisk J, et al. The effects of a multi-component dyadic intervention on the psychological distress of family caregivers providing care to people with dementia: a randomized controlled trial. International Psychogeriatrics. 2015 Dec;27(12):2031-44. doi: https://dx.doi.org/10.1017/S104161021 500071X. PMID: 26004290.

536. Signe A, Elmståhl S. Psychosocial intervention for family caregivers of people with dementia reduces caregiver's burden: development and effect after 6 and 12 months. Scandinavian journal of caring sciences. 2008;22(1):98-109. doi: 10.1111/j.1471-6712.2007.00498.x. PMID: CN-00630033.

537. Teri L, Gibbons LE, McCurry SM, et al. Exercise plus behavioral management in patients with Alzheimer disease: a randomized controlled trial. JAMA. 2003 Oct 15;290(15):2015-22. PMID: 14559955.

538. Zhang SY, Wu F, Tang DL, et al. Pilot testing the caregiver self-management intervention for caregivers of relatives with dementia. Geriatric Nursing. 2019 Aug 26;26:26. doi: https://dx.doi.org/10.1016/j.gerinurse.20 19.08.006. PMID: 31466807.

539. Correction: northern Manhattan Hispanic Caregiver Intervention Effectiveness Study: protocol of a pragmatic randomised trial comparing the effectiveness of two established interventions for informal caregivers of persons with dementia (BMJ Open (2016) 6 (e014082) DOI: 10.1136/bmjopen-2016-014082). In BMJ open 
540. Mazurek J, Szczesniak D, Lion KM, et al. Does the Meeting Centres Support Programme reduce unmet care needs of community-dwelling older people with dementia? A controlled, 6-month follow-up Polish study. Clinical Interventions In Aging. 2019;14:11322. doi: https://dx.doi.org/10.2147/CIA.S18568 3. PMID: 30666097.

541. Teri L, Gibbons LE, McCurry SM, et al. Exercise plus behavioral management in patients with Alzheimer disease: a randomized controlled trial. JAMA : the journal of the American Medical Association. 2003;290(15):2015-22. doi: 10.1001/jama.290.15.2015.

542. Jutten LH, Mark RE, Sitskoorn MM. Can the Mixed Virtual Reality Simulator Into D'mentia Enhance Empathy and Understanding and Decrease Burden in Informal Dementia Caregivers? Dementia and Geriatric Cognitive Disorders Extra. 2018 SepDec;8(3):453-66. doi: https://dx.doi.org/10.1159/000494660. PMID: 30631337.

543. Lord K, Livingston G, Cooper C. A feasibility randomised controlled trial of the DECIDE intervention: dementia carers making informed decisions. Bjpsych open. 2017;3(1):12-4. PMID: CN-01415827.

544. Rose KM, Taylor AG, Bourguignon C. Effects of cranial electrical stimulation on sleep disturbances, depressive symptoms, and caregiving appraisal in spousal caregivers of persons with Alzheimer's disease. Applied Nursing Research. 2009 May;22(2):119-25. doi: https://dx.doi.org/10.1016/j.apnr.2007.0 6.001. PMID: 19427574.

545. Simpson R, Wakefield P, Spiers N, et al. Carer-held records for dementia: a controlled trial. International Psychogeriatrics. 2006 Jun;18(2):25968. PMID: 16734920.
546. Stern RA, D’Ambrosio LA, Mohyde M, et al. At the crossroads: development and evaluation of a dementia caregiver group intervention to assist in driving cessation. Gerontology \& Geriatrics Education. 2008;29(4):363-82. doi: https://dx.doi.org/10.1080/02701960802 497936. PMID: 19064472.

547. Stirling C, Leggett S, Lloyd B, et al. Decision aids for respite service choices by carers of people with dementia: development and pilot RCT. BMC Medical Informatics \& Decision Making. 2012 Mar 19;12:21. doi: https://dx.doi.org/10.1186/1472-694712-21. PMID: 22429384.

548. Barbosa A, Nolan M, Sousa L, et al. Supporting direct care workers in dementia care: effects of a psychoeducational intervention. American Journal of Alzheimer's Disease \& Other Dementias. 2015 Mar;30(2):130-8. doi: https://dx.doi.org/10.1177/15333175145 50331. PMID: 25237132.

549. Barbosa A, Nolan M, Sousa L, et al. Effects of a Psychoeducational Intervention for Direct Care Workers Caring for People With Dementia: Results From a 6Month Follow-Up Study. American Journal of Alzheimer's Disease \& Other Dementias. 2016 Mar;31(2):144-55. doi:

https://dx.doi.org/10.1177/15333175156 03500. PMID: 26399485.

550. Davison T, McCabe M, Visser S, et al. Controlled trial of dementia training with a peer support group for aged care staff. International journal of geriatric psychiatry. 2007;22(9):868-73. doi: 10.1002/gps.1754. PMID: CN00627010.

551. Mackenzie CS, Peragine G. Measuring and enhancing self-efficacy among professional caregivers of individuals with dementia. American Journal of Alzheimer's Disease \& Other Dementias. 2003 Sep-Oct;18(5):291-9. PMID: 14569646. 
552. Visser SM, McCabe MP, Hudgson C, et al. Managing behavioural symptoms of dementia: effectiveness of staff education and peer support. Aging \& Mental Health. 2008 Jan;12(1):47-55. doi:

https://dx.doi.org/10.1080/13607860701 366012. PMID: 18297478.

553. Borbasi S, Emmanuel E, Farrelly B, et al. Report of an evaluation of a nurse-led dementia outreach service for people with the behavioural and psychological symptoms of dementia living in residential aged care facilities. Perspectives in public health. 2011;131(3):124-30. PMID: CN00850682.

554. Fortinsky RH, Kulldorff M, Kleppinger A, et al. Dementia care consultation for family caregivers: collaborative model linking an Alzheimer's association chapter with primary care physicians. Aging \& Mental Health. 2009 Mar;13(2):162-70. doi: https://dx.doi.org/10.1080/13607860902 746160. PMID: 19347683.

555. McSweeney K, Jeffreys A, Griffith J, et al. Specialist mental health consultation for depression in Australian aged care residents with dementia: a cluster randomized trial. International Journal of Geriatric Psychiatry. 2012 Nov;27(11):1163-71. doi: https://dx.doi.org/10.1002/gps.3762. PMID: 22344753.

556. Opie J, Doyle C, O’Connor DW. Challenging behaviours in nursing home residents with dementia: a randomized controlled trial of multidisciplinary interventions. International Journal of Geriatric Psychiatry. 2002 Jan;17(1):6-13. PMID: 11802224.

557. Orrell M, Hancock G, Hoe J, et al. A cluster randomised controlled trial to reduce the unmet needs of people with dementia living in residential care. International Journal of Geriatric Psychiatry. 2007 Nov;22(11):1127-34. PMID: 17394129.
558. Chien WT, Lee IY. Randomized controlled trial of a dementia care programme for families of home-resided older people with dementia. Journal of Advanced Nursing. 2011 Apr;67(4):774-87. doi: https://dx.doi.org/10.1111/j.13652648.2010.05537.x. PMID: 21198803.

559. Chien WT, Lee YM. A disease management program for families of persons in Hong Kong with dementia. Psychiatric Services. 2008 Apr;59(4):433-6. doi: https://dx.doi.org/10.1176/ps.2008.59.4. 433. PMID: 18378844.

560. Eloniemi-Sulkava U, Notkola IL, Hentinen $\mathrm{M}$, et al. Effects of supporting community-living demented patients and their caregivers: a randomized trial. Journal of the American Geriatrics Society. 2001 Oct;49(10):1282-7. PMID: 11890485.

561. Lam LC, Lee JS, Chung JC, et al. A randomized controlled trial to examine the effectiveness of case management model for community dwelling older persons with mild dementia in Hong Kong. International Journal of Geriatric Psychiatry. 2010 Apr;25(4):395-402.

doi:

https://dx.doi.org/10.1002/gps.2352. PMID: 19606455.

562. Brodaty H, Draper BM, Millar J, et al. Randomized controlled trial of different models of care for nursing home residents with dementia complicated by depression or psychosis. Journal of Clinical Psychiatry. 2003 Jan;64(1):6372. PMID: 12590626.

563. Challis D, von Abendorff R, Brown P, et al. Care management, dementia care and specialist mental health services: an evaluation. International Journal of Geriatric Psychiatry. 2002 Apr;17(4):315-25. PMID: 11994884.

564. MacNeil VJ, Bosmans J, Ven P, et al. Community-dwelling patients with dementia and their informal caregivers with and without case management: 2year outcomes of a pragmatic trial. In Journal of the American Medical Directors Association 
565. Newcomer R, Spitalny M, Fox P, et al. Effects of the Medicare Alzheimer's Disease Demonstration on the use of community-based services. Health Services Research. 1999 Aug;34(3):645-67. PMID: 10445896.

566. Weinberger M, Gold DT, Divine GW, et al. Social service interventions for caregivers of patients with dementia: impact on health care utilization and expenditures. Journal of the American Geriatrics Society. 1993 Feb;41(2):1536. PMID: 8426038.

567. Xiao LD, De Bellis A, Kyriazopoulos H, et al. The Effect of a Personalized Dementia Care Intervention for Caregivers From Australian Minority Groups. American Journal of Alzheimer's Disease \& Other Dementias. 2016 Feb;31(1):57-67. doi: https://dx.doi.org/10.1177/15333175155 78256. PMID: 25805891.

568. Appelhof B, Bakker C, de Vugt ME, et al. Effects of a Multidisciplinary Intervention on the Presence of Neuropsychiatric Symptoms and Psychotropic Drug Use in Nursing Home Residents WithYoung-Onset Dementia: Behavior and Evolution of Young-Onset Dementia Part 2 (BEYOND-II) Study. American Journal of Geriatric Psychiatry. 2019 Jan 04;04:04. doi: https://dx.doi.org/10.1016/j.jagp.2018.1 2.032. PMID: 30799167.

569. Chen YH, Lin LC. Ability of the Pain Recognition and Treatment (PRT) Protocol to Reduce Expressions of Pain among Institutionalized Residents with Dementia: A Cluster Randomized Controlled Trial. Pain Management Nursing. 2016 Feb;17(1):14-24. doi: https://dx.doi.org/10.1016/j.pmn.2015.0 8.003. PMID: 26584896.

570. Cohen-Mansfield J, Libin A, Marx MS. Nonpharmacological treatment of agitation: a controlled trial of systematic individualized intervention. Journals of Gerontology Series A-Biological Sciences \& Medical Sciences. 2007 Aug;62(8):908-16. PMID: 17702884.
571. Cohen-Mansfield J, Thein K, Marx MS, et al. Efficacy of nonpharmacologic interventions for agitation in advanced dementia: a randomized, placebocontrolled trial. Journal of Clinical Psychiatry. 2012 Sep;73(9):1255-61. doi: https://dx.doi.org/10.4088/JCP.12m079 18. PMID: 23059151.

572. Kovach C, Simpson M, Joosse L, et al. Comparison of the effectiveness of two protocols for treating nursing home residents with advanced dementia. In Research in gerontological nursing

573. Kovach CR, Hekel B, Rababa M. Feasibility Testing of a Protocol to Stop Ineffective Drug and Nondrug Treatments. Western Journal of Nursing Research. 2015 Nov;37(11):1404-22. doi:

https://dx.doi.org/10.1177/01939459155 99070. PMID: 26250849.

574. Kovach CR, Logan BR, Noonan PE, et al. Effects of the Serial Trial Intervention on discomfort and behavior of nursing home residents with dementia.

American Journal of Alzheimer's Disease \& Other Dementias. 2006 JunJul;21(3):147-55. PMID: 16869334.

575. McCabe MP, Bird M, Davison TE, et al. An RCT to evaluate the utility of a clinical protocol for staff in the management of behavioral and psychological symptoms of dementia in residential aged-care settings. Aging \& Mental Health. 2015;19(9):799-807. doi: https://dx.doi.org/10.1080/13607863.20 14.967659. PMID: 25319535.

576. Montgomery RJ, Kwak J, Kosloski K, et al. Effects of the TCARE intervention on caregiver burden and depressive symptoms: preliminary findings from a randomized controlled study. Journals of Gerontology Series B-Psychological Sciences \& Social Sciences. 2011 Sep;66(5):640-7. doi: https://dx.doi.org/10.1093/geronb/gbr08 ‥ PMID: 21840840. 
577. Nakanishi M, Endo K, Hirooka K, et al. Psychosocial behaviour management programme for home-dwelling people with dementia: A cluster-randomized controlled trial. International Journal of Geriatric Psychiatry. 2018 March;33(3):495-503. doi: http://dx.doi.org/10.1002/gps.4784. PMID: 620640680 Pubmed 28857263.

578. Pieper MJ, Francke AL, van der Steen JT, et al. Effects of a Stepwise Multidisciplinary Intervention for Challenging Behavior in Advanced Dementia: A Cluster Randomized Controlled Trial.[Erratum appears in J Am Geriatr Soc. 2016 Jun;64(6):1383; PMID: 27321637]. Journal of the American Geriatrics Society. 2016 Feb;64(2):261-9. doi: https://dx.doi.org/10.1111/jgs.13868. PMID: 26804064.

579. Rapp MA, Mell T, Majic T, et al. Agitation in nursing home residents with dementia (VIDEANT trial): effects of a cluster-randomized, controlled, guideline implementation trial. Journal of the American Medical Directors Association. 2013 Sep;14(9):690-5. doi: https://dx.doi.org/10.1016/j.jamda.2013. 05.017. PMID: 23827658.

580. Salva A, Andrieu S, Fernandez E, et al. Health and nutrition promotion program for patients with dementia (NutriAlz): Cluster randomized trial. Journal of Nutrition, Health \& Aging. 2011;15(10):822-30. doi: 10.1007/s12603-011-0363-3. PMID: 104625164. Language: English. Entry Date: 20120530. Revision Date: 20150818. Publication Type: Journal Article.

581. Verkaik R, Francke AL, van Meijel B, et al. The effects of a nursing guideline on depression in psychogeriatric nursing home residents with dementia. International Journal of Geriatric Psychiatry. 2011 Jul;26(7):723-32. doi: https://dx.doi.org/10.1002/gps.2586. PMID: 21495077.
582. Zwijsen SA, Smalbrugge M, Eefsting JA, et al. Coming to grips with challenging behavior: a cluster randomized controlled trial on the effects of a multidisciplinary care program for challenging behavior in dementia. Journal of the American Medical Directors Association. 2014 Jul;15(7):531.e1-.e10. doi: https://dx.doi.org/10.1016/j.jamda.2014. 04.007. PMID: 24878214.

583. Livingston G, Barber J, Marston L, et al. Clinical and cost-effectiveness of the Managing Agitation and Raising Quality of Life (MARQUE) intervention for agitation in people with dementia in care homes: a single-blind, cluster-randomised controlled trial. The Lancet. Psychiatry. 2019 04;6(4):293304. doi: https://dx.doi.org/10.1016/S22150366(19)30045-8. PMID: 30872010.

584. Husebo BS, Ballard C, Aarsland D, et al. The Effect of a Multicomponent Intervention on Quality of Life in Residents of Nursing Homes: A Randomized Controlled Trial (COSMOS). Journal of the American Medical Directors Association. 2019. doi:

http://dx.doi.org/10.1016/j.jamda.2018. 11.006. PMID: 2001439020.

585. Cohen SM, Volandes AE, Shaffer ML, et al. Concordance Between Proxy Level of Care Preference and Advance Directives Among Nursing Home Residents With Advanced Dementia: A Cluster Randomized Clinical Trial. Journal of Pain \& Symptom Management. 2019 Jan;57(1):37-46.e1. doi:

https://dx.doi.org/10.1016/j.jpainsymma n.2018.09.018. PMID: 30273717.

586. Mitchell SL, Shaffer ML, Cohen S, et al. An Advance Care Planning Video Decision Support Tool for Nursing Home Residents With Advanced Dementia: A Cluster Randomized Clinical Trial. JAMA Internal Medicine. 2018 Jul 01;178(7):961-9. doi:

https://dx.doi.org/10.1001/jamainternme d.2018.1506. PMID: 29868778. 
587. Bonner GJ, Wang E, Wilkie DJ, et al. Advance care treatment plan (ACTPlan) for African American family caregivers: a pilot study. Dementia. 2014 Jan;13(1):79-95. doi: https://dx.doi.org/10.1177/14713012124 49408. PMID: 24381040.

588. Brazil K, Carter G, Cardwell C, et al. Effectiveness of advance care planning with family carers in dementia nursing homes: A paired cluster randomized controlled trial. Palliative Medicine. 2018 Mar;32(3):603-12. doi: https://dx.doi.org/10.1177/02692163177 22413. PMID: 28786323.

589. Einterz SF, Gilliam R, Lin FC, et al. Development and testing of a decision aid on goals of care for advanced dementia. Journal of the American Medical Directors Association. 2014 Apr;15(4):251-5. doi: https://dx.doi.org/10.1016/j.jamda.2013. 11.020. PMID: 24508326.

590. Hanson LC, Song MK, Zimmerman S, et al. Fidelity to a behavioral intervention to improve goals of care decisions for nursing home residents with advanced dementia. Clinical Trials. 2016 Dec;13(6):599-604. PMID: 27271683.

591. Hanson LC, Zimmerman S, Song MK, et al. Effect of the Goals of Care Intervention for Advanced Dementia: A Randomized Clinical Trial. JAMA Internal Medicine. 2017 Jan 01;177(1):24-31. doi: https://dx.doi.org/10.1001/jamainternme d.2016.7031. PMID: 27893884.

592. Reinhardt JP, Chichin E, Posner L, et al. Vital conversations with family in the nursing home: preparation for end-stage dementia care. Journal Of Social Work In End-Of-Life \& Palliative Care. 2014;10(2):112-26. doi: https://dx.doi.org/10.1080/15524256.20 14.906371. PMID: 24835382.

593. Sampson EL, Jones L, Thune-Boyle IC, et al. Palliative assessment and advance care planning in severe dementia: an exploratory randomized controlled trial of a complex intervention. Palliative Medicine. 2011 Apr;25(3):197-209. doi: https://dx.doi.org/10.1177/02692163103 91691. PMID: 21228087.
594. Song MK, Ward SE, Hepburn K, et al. Can Persons with Dementia Meaningfully Participate in Advance Care Planning Discussions? A Mixed-Methods Study of SPIRIT. Journal of Palliative Medicine. 2019 Nov;22(11):1410-6. doi:

https://dx.doi.org/10.1089/jpm.2019.00 88. PMID: 31373868.

595. Goossens B, Sevenants A, Declercq A, et al. 'We DECide optimized' - training nursing home staff in shared decisionmaking skills for advance care planning conversations in dementia care: protocol of a pretest-posttest cluster randomized trial. BMC Geriatrics. 2019 02 04;19(1):33. doi: https://dx.doi.org/10.1186/s12877-0191044-z. PMID: 30717700.

596. Hanson LC, Carey TS, Caprio AJ, et al. Improving decision-making for feeding options in advanced dementia: a randomized, controlled trial. Journal of the American Geriatrics Society. 2011 Nov;59(11):2009-16. doi: https://dx.doi.org/10.1111/j.15325415.2011.03629.x. PMID: 22091750.

597. Hanson LC, Gilliam R, Lee TJ. Successful clinical trial research in nursing homes: the Improving Decision-Making Study. Clinical Trials. 2010 Dec;7(6):735-43. doi:

https://dx.doi.org/10.1177/17407745103 80241. PMID: 20729251.

598. Snyder EA, Caprio AJ, Wessell K, et al. Impact of a decision aid on surrogate decision-makers' perceptions of feeding options for patients with dementia. Journal of the American Medical Directors Association. 2013 Feb;14(2):114-8. doi: https://dx.doi.org/10.1016/j.jamda.2012. 10.011. PMID: 23273855.

599. Agar M, Luckett T, Luscombe G, et al. Effects of facilitated family case conferencing for advanced dementia: A cluster randomised clinical trial. PLoS ONE [Electronic Resource]. 2017;12(8):e0181020. doi: https://dx.doi.org/10.1371/journal.pone. 0181020. PMID: 28786995. 
600. Agar M, Beattie E, Luckett T, et al. Pragmatic cluster randomised controlled trial of facilitated family case conferencing compared with usual care for improving end of life care and outcomes in nursing home residents with advanced dementia and their families: the IDEAL study protocol. BMC Palliative Care. 2015 Nov 21;14:63. doi: https://dx.doi.org/10.1186/s12904-0150061-8. PMID: 26589957.

601. Ahronheim JC, Morrison RS, Morris J, et al. Palliative care in advanced dementia: a randomized controlled trial and descriptive analysis. Journal of Palliative Medicine. 2000;3(3):265-73. PMID: 15859668.

602. Boogaard JA, de Vet HC, van SoestPoortvliet MC, et al. Effects of two feedback interventions on end-of-life outcomes in nursing home residents with dementia: A cluster-randomized controlled three-armed trial. Palliative Medicine. 2018 Mar;32(3):693-702. doi: https://dx.doi.org/10.1177/02692163177 50071. PMID: 29343173.

603. Hanson LC, Kistler CE, Lavin K, et al. Triggered Palliative Care for Late-Stage Dementia: A Pilot Randomized Trial. Journal of Pain \& Symptom Management. 2019 Jan;57(1):10-9. doi: https://dx.doi.org/10.1016/j.jpainsymma n.2018.10.494. PMID: 30342242.

604. Verreault R, Arcand M, Misson L, et al. Quasi-experimental evaluation of a multifaceted intervention to improve quality of end-of-life care and quality of dying for patients with advanced dementia in long-term care institutions. Palliative Medicine. 2018 03;32(3):61321. doi: https://dx.doi.org/10.1177/02692163177 19588. PMID: 28731379.

605. Williams KN, Perkhounkova Y, Shaw CA, et al. Supporting Family Caregivers With Technology for Dementia Home Care: A Randomized Controlled Trial. Innovation in Aging. 2019 Jul;3(3):igz037. doi: https://dx.doi.org/10.1093/geroni/igz03 7. PMID: 31660443.
606. Horvath KJ, Trudeau SA, Rudolph JL, et al. Clinical trial of a home safety toolkit for Alzheimer's disease. International journal of Alzheimer's disease. 2013;2013:913606. doi: https://dx.doi.org/10.1155/2013/913606 . PMID: 24195007.

607. Laver K, Liu E, Clemson L, et al. Does Telehealth Delivery of a Dyadic Dementia Care Program Provide a Noninferior Alternative to Face-ToFace Delivery of the Same Program? A Randomized, Controlled Trial. American Journal of Geriatric Psychiatry. 2020 Mar 02;02:02. doi: https://dx.doi.org/10.1016/j.jagp.2020.0 2.009. PMID: 32234275.

608. Villars H, Dupuy C, Soler P, et al. A follow-up intervention in severely demented patients after discharge from a special Alzheimer acute care unit: impact on early emergency room rehospitalization rate. International Journal of Geriatric Psychiatry. 2013 Nov;28(11):1131-40. doi: https://dx.doi.org/10.1002/gps.3932. PMID: 23348897.

609. Mavandadi S, Wright EM, Graydon MM, et al. A randomized pilot trial of a telephone-based collaborative care management program for caregivers of individuals with dementia.

Psychological Services. 2017

Feb;14(1):102-11. doi: https://dx.doi.org/10.1037/ser0000118. PMID: 28134558.

610. Chapman DG, Toseland RW. Effectiveness of advanced illness care teams for nursing home residents with dementia. Social Work. 2007 Oct;52(4):321-9. PMID: 18232242.

611. Samus QM, Johnston D, Black BS, et al. A multidimensional home-based care coordination intervention for elders with memory disorders: the maximizing independence at home (MIND) pilot randomized trial. American Journal of Geriatric Psychiatry. 2014 Apr;22(4):398-414. doi: https://dx.doi.org/10.1016/j.jagp.2013.1 2.175. PMID: 24502822. 
612. Saxena N, George PP, Teo KW, et al. Evaluation of an integrated primary care-led dementia shared care program in Singapore: An effectiveness and costeffectiveness study. Geriatrics \& gerontology international. 2018 Mar;18(3):479-86. doi: https://dx.doi.org/10.1111/ggi.13196. PMID: 29193721.

613. Leontjevas R, Gerritsen DL, Smalbrugge $\mathrm{M}$, et al. A structural multidisciplinary approach to depression management in nursing-home residents: a multicentre, stepped-wedge cluster-randomised trial. Lancet. 2013 Jun 29;381(9885):225564. doi: https://dx.doi.org/10.1016/S01406736(13)60590-5. PMID: 23643110.

614. Chen C, Huang Y, Liu C, et al. Effects of an Interdisciplinary Care Team on the Management of Alzheimer's Disease in China. Journal of gerontological nursing. 201901 May;45(5):39-45. doi: http://dx.doi.org/10.3928/0098913420190318-01. PMID: 627579646.

615. Bass D, Judge K, Snow A, et al. Caregiver outcomes of partners in dementia care: effect of a care coordination program for veterans with dementia and their family members and friends. Journal of the american geriatrics society. 2013;61(8):1377-86. doi: 10.1111/jgs.12362. PMID: CN00875084.

616. Callahan CM, Boustani MA, Unverzagt FW, et al. Effectiveness of collaborative care for older adults with Alzheimer disease in primary care: a randomized controlled trial. JAMA. 2006 May 10;295(18):2148-57. PMID: 16684985.

617. Chodosh J, Colaiaco BA, Connor KI, et al. Dementia Care Management in an Underserved Community: The Comparative Effectiveness of Two Different Approaches. Journal of Aging \& Health. 2015 Aug;27(5):864-93. doi: https://dx.doi.org/10.1177/08982643155 69454. PMID: 25656074.
618. Possin KL, Merrilees JJ, Dulaney S, et al. Effect of Collaborative Dementia Care via Telephone and Internet on Quality of Life, Caregiver Well-being, and Health Care Use: The Care Ecosystem Randomized Clinical Trial. JAMA Internal Medicine. 2019 Sep 30;30:30. doi:

https://dx.doi.org/10.1001/jamainternme d.2019.4101. PMID: 31566651.

619. Thyrian JR, Hertel J, Wucherer D, et al. Effectiveness and Safety of Dementia Care Management in Primary Care: A Randomized Clinical Trial. JAMA Psychiatry. 2017 Oct 01;74(10):9961004. doi: https://dx.doi.org/10.1001/jamapsychiat ry.2017.2124. PMID: 28746708.

620. Vickrey BG, Mittman BS, Connor KI, et al. The effect of a disease management intervention on quality and outcomes of dementia care: a randomized, controlled trial.[Summary for patients in Ann Intern Med. 2006 Nov 21;145(10):I31; PMID: 17116913]. Annals of Internal Medicine. 2006 Nov 21;145(10):71326. PMID: 17116916.

621. Eichler T, Thyrian JR, Dreier A, et al. Dementia care management: going new ways in ambulant dementia care within a GP-based randomized controlled intervention trial. International Psychogeriatrics. 2014 Feb;26(2):24756. doi: https://dx.doi.org/10.1017/S104161021 3001786. PMID: 24152974.

622. Eichler T, Thyrian JR, Fredrich D, et al. The benefits of implementing a computerized interventionmanagement-system (IMS) on delivering integrated dementia care in the primary care setting. International Psychogeriatrics. 2014 Aug;26(8):137785. doi: https://dx.doi.org/10.1017/S104161021 4000830. PMID: 24811145. 
623. Fis T, Thyrian JR, Wucherer D, et al. Medication management for people with dementia in primary care: description of implementation in the DelpHi study. BMC Geriatrics. 2013 Nov 13;13:121. doi: https://dx.doi.org/10.1186/1471-231813-121. PMID: 24225205.

624. Thyrian JR, Winter P, Eichler T, et al. Relatives' burden of caring for people screened positive for dementia in primary care : Results of the DelpHi study. Zeitschrift fur Gerontologie und Geriatrie. 2017 Jan;50(1):4-13. doi: https://dx.doi.org/10.1007/s00391-0161119-9. PMID: 27534949.

625. Thyrian JR, Fis T, Dreier A, et al. Life- and person-centred help in MecklenburgWestern Pomerania, Germany (DelpHi): study protocol for a randomised controlled trial. Trials [Electronic Resource]. 2012 May 10;13:56. doi: https://dx.doi.org/10.1186/1745-621513-56. PMID: 22575023.

626. Zwingmann I, Hoffmann W, Michalowsky $\mathrm{B}$, et al. Supporting family dementia caregivers: testing the efficacy of dementia care management on multifaceted caregivers' burden. Aging \& Mental Health. 2017 Nov 20:1-8. doi: https://dx.doi.org/10.1080/13607863.20 17.1399341. PMID: 29156941.

627. Michalowsky B, Xie F, Eichler T, et al. Cost-effectiveness of a collaborative dementia care management-Results of a cluster-randomized controlled trial. Alzheimer's \& Dementia. 2019 Oct;15(10):1296-308. doi: https://dx.doi.org/10.1016/j.jalz.2019.05 .008. PMID: 31409541.

628. Bass DM, Judge KS, Maslow K, et al. Impact of the care coordination program "partners in Dementia Care" on veterans' hospital admissions and emergency department visits. Alzheimer's and Dementia: Translational Research and Clinical Interventions. 201514 Oct;1(1):13-22. doi:

http://dx.doi.org/10.1016/j.trci.2015.03. 003. PMID: 606362402.
629. Morgan RO, Bass DM, Judge KS, et al. A break-even analysis for dementia care collaboration: Partners in Dementia Care. JGIM: Journal of General Internal Medicine. 2015;30(6):804-9. doi: 10.1007/s11606-015-3205-x. PMID: 109738468. Language: English. Entry Date: 20150923. Revision Date: 20160604. Publication Type: journal article.

630. Duru OK, Ettner SL, Vassar SD, et al. Cost evaluation of a coordinated care management intervention for dementia. American Journal of Managed Care. 2009 Aug;15(8):521-8. PMID: 19670955.

631. Chodosh J, Pearson ML, Connor KI, et al. A dementia care management intervention: which components improve quality? American Journal of Managed Care. 2012 Feb;18(2):85-94. PMID: 22435836.

632. Brown AF, Vassar SD, Connor KI, et al. Collaborative care management reduces disparities in dementia care quality for caregivers with less education. Journal of the American Geriatrics Society. 2013 Feb;61(2):243-51. doi: https://dx.doi.org/10.1111/jgs.12079. PMID: 23320655.

633. Bass DM, Clark PA, Looman WJ, et al. The Cleveland Alzheimer's managed care demonstration: outcomes after 12 months of implementation. Gerontologist. 2003 Feb;43(1):73-85. PMID: 12604748.

634. Clark PA, Bass DM, Looman WJ, et al. Outcomes for patients with dementia from the Cleveland Alzheimer's Managed Care Demonstration. Aging \& Mental Health. 2004 Jan;8(1):40-51. PMID: 14690867.

635. Bernstein A, Harrison KL, Dulaney S, et al. The Role of Care Navigators Working with People with Dementia and Their Caregivers. Journal of Alzheimer's Disease. 2019;71(1):45-55. doi: https://dx.doi.org/10.3233/JAD-180957. PMID: 31322558. 
636. Possin KL, Merrilees J, Bonasera SJ, et al. Development of an adaptive, personalized, and scalable dementia care program: Early findings from the Care Ecosystem. PLoS Medicine / Public Library of Science. 2017 03;14(3):e1002260. doi: https://dx.doi.org/10.1371/journal.pmed 1002260. PMID: 28323819.

637. Rosa TD, Possin KL, Bernstein A, et al. Variations in Costs of a Collaborative Care Model for Dementia. Journal of the American Geriatrics Society. 2019 Dec;67(12):2628-33. doi: https://dx.doi.org/10.1111/jgs.16076. PMID: 31317539.

638. Guterman EL, Allen IE, Josephson SA, et al. Association Between Caregiver Depression and Emergency Department Use Among Patients With Dementia. JAMA Neurology. 2019 Jul 08;08:08. doi: https://dx.doi.org/10.1001/jamaneurol.2 019.1820. PMID: 31282955.

639. Merrilees JJ, Bernstein A, Dulaney S, et al. The Care Ecosystem: Promoting selfefficacy among dementia family caregivers. Dementia. 2018 Nov 29:1471301218814121. doi: https://dx.doi.org/10.1177/14713012188 14121. PMID: 30497302.

640. Eloniemi-Sulkava U, Saarenheimo M, Laakkonen ML, et al. Family care as collaboration: effectiveness of a multicomponent support program for elderly couples with dementia. Randomized controlled intervention study. Journal of the American Geriatrics Society. 2009 Dec;57(12):2200-8. doi: https://dx.doi.org/10.1111/j.15325415.2009.02564.x. PMID: 20121986.

641. Lichtwarck B, Selbaek G, Kirkevold O, et al. Targeted Interdisciplinary Model for Evaluation and Treatment of Neuropsychiatric Symptoms: A Cluster Randomized Controlled Trial. American Journal of Geriatric Psychiatry. 2018 January;26(1):25-38. doi: http://dx.doi.org/10.1016/j.jagp.2017.05 .015. PMID: 617024611.
642. Li J, Grandner M, Chang Y, et al. Personcentered dementia care and sleep in assisted living residents with dementia: a pilot study. Behavioral sleep medicine. 2017(2):97-113. doi: 10.1080/15402002.2015.1104686. PMID: CN-01380345.

643. Rovner BW, Steele CD, Shmuely Y, et al. A randomized trial of dementia care in nursing homes. Journal of the American Geriatrics Society. 1996 Jan;44(1):7-13. PMID: 8537594.

644. Surr CA, Griffiths AW, Kelley R, et al. The Implementation of Dementia Care Mapping in a Randomized Controlled Trial in Long-Term Care: Results of a Process Evaluation. American Journal of Alzheimer's Disease \& Other Dementias. 2019 Sep;34(6):390-8. doi: https://dx.doi.org/10.1177/15333175198 45725. PMID: 31056923.

645. Ballard C, Orrell M, YongZhong S, et al. Impact of Antipsychotic Review and Nonpharmacological Intervention on Antipsychotic Use, Neuropsychiatric Symptoms, and Mortality in People With Dementia Living in Nursing Homes: A Factorial ClusterRandomized Controlled Trial by the Well-Being and Health for People With Dementia (WHELD) Program. American Journal of Psychiatry. 2016 Mar 01;173(3):252-62. doi: https://dx.doi.org/10.1176/appi.ajp.2015 .15010130. PMID: 26585409.

646. Fossey J, Ballard C, Juszczak E, et al. Effect of enhanced psychosocial care on antipsychotic use in nursing home residents with severe dementia: cluster randomised trial. BMJ (Clinical research ed.). 20061 Apr;332(7544):756-61. PMID: 43509632. 
647. Ballard C, Corbett A, Orrell M, et al. Impact of person-centred care training and person-centred activities on quality of life, agitation, and antipsychotic use in people with dementia living in nursing homes: A cluster-randomised controlled trial. PLoS Medicine / Public Library of Science. 2018 02;15(2):e1002500. doi: https://dx.doi.org/10.1371/journal.pmed .1002500. PMID: 29408901.

648. Moyle W, Venturato L, Cooke M, et al. Evaluating the capabilities model of dementia care: A non-randomized controlled trial exploring resident quality of life and care staff attitudes and experiences. International Psychogeriatrics. 201601 Jul;28(7):1091-100. doi: http://dx.doi.org/10.1017/S1041610216 000296. PMID: 608901615.

649. Chenoweth L, Forbes I, Fleming R, et al. PerCEN: a cluster randomized controlled trial of person-centered residential care and environment for people with dementia. International Psychogeriatrics. 2014 Jul;26(7):114760. doi: https://dx.doi.org/10.1017/S104161021 4000398. PMID: 24666667.

650. Chenoweth L, King MT, Jeon YH, et al. Caring for Aged Dementia Care Resident Study (CADRES) of personcentred care, dementia-care mapping, and usual care in dementia: a clusterrandomised trial.[Erratum appears in Lancet Neurol. 2009 May;8(5):419]. Lancet Neurology. 2009 Apr;8(4):31725. doi: https://dx.doi.org/10.1016/S14744422(09)70045-6. PMID: 19282246.

651. Rokstad AM, Rosvik J, Kirkevold O, et al. The effect of person-centred dementia care to prevent agitation and other neuropsychiatric symptoms and enhance quality of life in nursing home patients: a 10-month randomized controlled trial. Dementia \& Geriatric Cognitive Disorders. 2013;36(5-6):34053. doi: https://dx.doi.org/10.1159/000354366. PMID: 24022375.
652. Dichter M, Quasdorf T, Schwab C, et al. Dementia care mapping: effects on residents' quality of life and challenging behavior in German nursing homes. A quasi-experimental trial. International psychogeriatrics. 2015;27(11):1875-92. doi: 10.1017/S1041610215000927. PMID: CN-01215190.

653. van de Ven G, Draskovic I, Adang EM, et al. Effects of dementia-care mapping on residents and staff of care homes: a pragmatic cluster-randomised controlled trial. PLoS ONE [Electronic Resource]. 2013;8(7):e67325. doi: https://dx.doi.org/10.1371/journal.pone. 0067325. PMID: 23844003.

654. Fossey J, Ballard C, Juszczak E, et al. Effect of enhanced psychosocial care on antipsychotic use in nursing home residents with severe dementia: cluster randomised trial.[Erratum appears in BMJ. 2006 Apr 1;332(7544):61]. BMJ. 2006 Apr 01;332(7544):756-61. PMID: 16543297.

655. Froggatt K, Best A, Bunn F, et al. A group intervention to improve quality of life for people with advanced dementia living in care homes: The namaste feasibility cluster RCT. Health Technology Assessment. 2020 January;24(6):vii-139.

656. Lichtwarck B, Selbaek G, Kirkevold O, et al. TIME - Targeted interdisciplinary model for evaluation and treatment of neuropsychiatric symptoms: protocol for an effectiveness-implementation cluster randomized hybrid trial. BMC Psychiatry. 2016 Jul 12;16:233. doi: https://dx.doi.org/10.1186/s12888-0160944-0. PMID: 27406242.

657. Lichtwarck B, Myhre J, Selbaek G, et al. TIME to reduce agitation in persons with dementia in nursing homes. A process evaluation of a complex intervention. BMC Health Services Research. 2019 May 31;19(1):349. doi: https://dx.doi.org/10.1186/s12913-0194168-0. PMID: 31151437. 
658. Fossey J, Garrod L, Tolbol Froiland C, et al. What influences the sustainability of an effective psychosocial intervention for people with dementia living in care homes? A 9 to 12-month follow-up of the perceptions of staff in care homes involved in the WHELD randomised controlled trail. International Journal of Geriatric Psychiatry. 2019

May;34(5):674-82. doi: http://dx.doi.org/10.1002/gps.5066.

659. Romeo R, Zala D, Knapp M, et al. Improving the quality of life of care home residents with dementia: Costeffectiveness of an optimized intervention for residents with clinically significant agitation in dementia.

Alzheimer's \& Dementia. 2019

Feb;15(2):282-91. doi: https://dx.doi.org/10.1016/j.jalz.2018.08 .010. PMID: 30470592.

660. Bourgeois MS, Dijkstra K, Burgio L, et al. Memory aids as an augmentative and alternative communication strategy for nursing home residents with dementia. AAC: Augmentative and Alternative Communication. 2001 Sep;17(3):196210. doi:

http://dx.doi.org/10.1080/714043383. PMID: 2001-11280-006.

661. Chang CC, Lin LC. Effects of a feeding skills training programme on nursing assistants and dementia patients. Journal of Clinical Nursing. 2005

Nov;14(10):1185-92. PMID: 16238764.

662. Clare L, Whitaker R, Woods RT, et al. AwareCare: a pilot randomized controlled trial of an awareness-based staff training intervention to improve quality of life for residents with severe dementia in long-term care settings. International Psychogeriatrics. 2013 Jan;25(1):128-39. doi: https://dx.doi.org/10.1017/S104161021 2001226. PMID: 22840185.
663. Conway ER, Chenery HJ. Evaluating the MESSAGE Communication Strategies in Dementia training for use with community-based aged care staff working with people with dementia: a controlled pretest-post-test study. Journal of Clinical Nursing. 2016 Apr;25(7-8):1145-55. doi: https://dx.doi.org/10.1111/jocn.13134. PMID: 26821868.

664. Deudon A, Maubourguet N, Gervais X, et al. Non-pharmacological management of behavioural symptoms in nursing homes. International Journal of Geriatric Psychiatry. 2009 Dec;24(12):1386-95. doi: https://dx.doi.org/10.1002/gps.2275. PMID: 19370714.

665. Galik E, Resnick B, Hammersla M, et al. Optimizing function and physical activity among nursing home residents with dementia: testing the impact of function-focused care. Gerontologist. 2014 Dec;54(6):930-43. doi: https://dx.doi.org/10.1093/geront/gnt10 8. PMID: 24092822.

666. Ghandehari OO, Hadjistavropoulos T, Williams J, et al. A controlled investigation of continuing pain education for long-term care staff. Pain Research \& Management. 2013;18(1):11-8. PMID: 107988179. Language: English. Entry Date: 20130426. Revision Date: 20161117. Publication Type: journal article.

667. Gozalo P, Prakash S, Qato DM, et al. Effect of the bathing without a battle training intervention on bathing-associated physical and verbal outcomes in nursing home residents with dementia: a randomized crossover diffusion study. Journal of the American Geriatrics Society. 2014 May;62(5):797-804. doi: https://dx.doi.org/10.1111/jgs.12777. PMID: 24697702. 
668. Hattink B, Meiland F, van der Roest H, et al. Web-Based STAR E-Learning Course Increases Empathy and Understanding in Dementia Caregivers: Results from a Randomized Controlled Trial in the Netherlands and the United Kingdom. Journal of Medical Internet Research. 2015 Oct 30;17(10):e241. doi:

https://dx.doi.org/10.2196/jmir.4025. PMID: 26519106.

669. Kuske B, Luck T, Hanns S, et al. Training in dementia care: a cluster-randomized controlled trial of a training program for nursing home staff in Germany. International Psychogeriatrics. 2009 Apr;21(2):295-308. doi: https://dx.doi.org/10.1017/S104161020 8008387. PMID: 19193252.

670. Leone E, Deudon A, Bauchet M, et al. Management of apathy in nursing homes using a teaching program for care staff: the STIM-EHPAD study. International Journal of Geriatric Psychiatry. 2013 Apr;28(4):383-92. doi: https://dx.doi.org/10.1002/gps.3836. PMID: 22700526.

671. Magai C, Cohen CI, Gomberg D. Impact of training dementia caregivers in sensitivity to nonverbal emotion signals. International Psychogeriatrics. 2002 Mar;14(1):25-38. PMID: 12094904.

672. McCallion P, Toseland RW, Lacey D, et al. Educating nursing assistants to communicate more effectively with nursing home residents with dementia. Gerontologist. 1999 Oct;39(5):546-58. PMID: 10568079.

673. McCurry SM, LaFazia DM, Pike KC, et al. Development and evaluation of a sleep education program for older adults with dementia living in adult family homes. American Journal of Geriatric Psychiatry. 2012 Jun;20(6):494-504. doi:

https://dx.doi.org/10.1097/JGP.0b013e3 18248ae79. PMID: 22367233.

674. Proctor R, Burns A, Powell HS, et al. Behavioural management in nursing and residential homes: a randomised controlled trial. Lancet. 1999 Jul 03;354(9172):26-9. PMID: 10406361.
675. Schindel ML, Gillies L, Coker E, et al. An Education Intervention to Enhance Staff Self-Efficacy to Provide Dementia Care in an Acute Care Hospital in Canada: a Nonrandomized Controlled Study. In American journal of alzheimer's disease and other dementias

676. Sloane PD, Hoeffer B, Mitchell CM, et al. Effect of person-centered showering and the towel bath on bathingassociated aggression, agitation, and discomfort in nursing home residents with dementia: a randomized, controlled trial. Journal of the American Geriatrics Society. 2004 Nov;52(11):1795-804. PMID: 15507054.

677. Wells DL, Dawson P, Sidani S, et al. Effects of an abilities-focused program of morning care on residents who have dementia and on caregivers. Journal of the American Geriatrics Society. 2000 Apr;48(4):442-9. PMID: 10798473.

678. Wenborn J, Challis D, Head J, et al. Providing activity for people with dementia in care homes: a cluster randomised controlled trial. International Journal of Geriatric Psychiatry. 2013 Dec;28(12):1296-304. doi:

https://dx.doi.org/10.1002/gps.3960. PMID: 23637069.

679. Williams KN, Perkhounkova Y, Herman R, et al. A Communication Intervention to Reduce Resistiveness in Dementia Care: A Cluster Randomized Controlled Trial. Gerontologist. 2017 Aug 01;57(4):707-18. doi: https://dx.doi.org/10.1093/geront/gnw0 47. PMID: 27048705.

680. Zimmerman S, Mitchell CM, Reed D, et al. Outcomes of a dementia care training program for staff in nursing homes and residential care/assisted living settings. Alzheimer's Care Today. 2010;11(2):83-99. PMID: 105194596. Language: English. Entry Date: 20100625. Revision Date: 20150711. Publication Type: Journal Article.

681. Burgener SC, Bakas T, Murray C, et al. Effective caregiving approaches for patients with Alzheimer's disease. Geriatric Nursing. 1998 MayJun;19(3):121-6. PMID: 9708136. 
682. Done DJ, Thomas JA. Training in communication skills for informal carers of people suffering from dementia: a cluster randomized clinical trial comparing a therapist led workshop and a booklet. International Journal of Geriatric Psychiatry. 2001 Aug;16(8):816-21. PMID: 11536349.

683. Gormley N, Lyons D, Howard R. Behavioural management of aggression in dementia: a randomized controlled trial. Age \& Ageing. 2001 Mar;30(2):141-5. PMID: 11395344.

684. Hepburn K, Lewis M, Tornatore J, et al. The Savvy Caregiver program: the demonstrated effectiveness of a transportable dementia caregiver psychoeducation program. Journal of Gerontological Nursing. 2007 Mar;33(3):30-6. PMID: 17378189.

685. Hepburn KW, Lewis M, Narayan S, et al. Partners in caregiving: A psychoeducation program affecting dementia family caregivers' distress and caregiving outlook. Clinical Gerontologist. 2005;29(1):53-69. doi: http://dx.doi.org/10.1300/J018v29n01_0 5. PMID: 43539927.

686. Hepburn KW, Tornatore J, Center B, et al. Dementia family caregiver training: affecting beliefs about caregiving and caregiver outcomes. Journal of the American Geriatrics Society. 2001 Apr;49(4):450-7. PMID: 11347790.

687. Klodnicka Kouri K, Ducharme FC, Giroux F. A psycho-educational intervention focused on communication for caregivers of a family member in the early stage of Alzheimer's disease: Results of an experimental study. Dementia: The International Journal of Social Research and Practice. 2011 Aug;10(3):435-53. doi: http://dx.doi.org/10.1177/14713012114 08124. PMID: 2011-16142-011.
688. Liddle J, Smith-Conway ER, Baker R, et al. Memory and communication support strategies in dementia: effect of a training program for informal caregivers. International Psychogeriatrics. 2012 Dec;24(12):1927-42. doi: https://dx.doi.org/10.1017/S104161021 2001366. PMID: 23092595.

689. Martin-Cook K, Davis BA, Hynan LS, et al. A randomized, controlled study of an Alzheimer's caregiver skills training program. American Journal of Alzheimer's Disease \& Other Dementias. 2005 Jul-Aug;20(4):204-10. PMID: 16136843.

690. Neely A, Vikstrom S, Josephsson S. Collaborative memory intervention in dementia: caregiver participation matters. In Neuropsychological rehabilitation

691. Robinson K, Yates K. Effects of two caregiver-training programs on burden and attitude toward help. Archives of Psychiatric Nursing. 1994 Oct;8(5):3129. PMID: 7993133.

692. Jablonski RA, Reed D, Maas ML. Care intervention for older adults with Alzheimer's disease and related dementias: effect of family involvement on cognitive and functional outcomes in nursing homes. Journal of Gerontological Nursing. 2005 Jun;31(6):38-48. PMID: 16138529.

693. Maas ML, Reed D, Park M, et al. Outcomes of family involvement in care intervention for caregivers of individuals with dementia. Nursing Research. 2004 Mar-Apr;53(2):76-86. PMID: 15084992.

694. McCallion P, Toseland RW, Freeman K. An evaluation of a family visit education program. Journal of the American Geriatrics Society. 1999 Feb;47(2):203-14. PMID: 9988292.

695. Robison J, Curry L, Gruman C, et al. Partners in caregiving in a special care environment: cooperative communication between staff and families on dementia units. Gerontologist. 2007 Aug;47(4):504-15. PMID: 17766671. 
696. Kinnunen KM, Rapaport P, Webster L, et al. A manual-based intervention for carers of people with dementia and sleep disturbances: An acceptability and feasibility RCT. Health Technology Assessment. 2018 December;22(71):I408. doi: http://dx.doi.org/10.3310/hta22710. PMID: 626134933.

697. Kunik ME, Snow AL, Wilson N, et al. Teaching Caregivers of Persons with Dementia to Address Pain. American Journal of Geriatric Psychiatry. 2017 Feb;25(2):144-54. doi: https://dx.doi.org/10.1016/j.jagp.2016.0 4.009. PMID: 27743840.

698. Suominen MH, Puranen TM, Jyvakorpi SK, et al. Nutritional Guidance Improves Nutrient Intake and Quality of Life, and May Prevent Falls in Aged Persons with Alzheimer Disease Living with a Spouse (NuAD Trial). Journal of Nutrition, Health \& Aging. 2015 Nov;19(9):901-7. doi: https://dx.doi.org/10.1007/s12603-0150558-0. PMID: 26482691.

699. Teri L, McCurry SM, Logsdon R, et al. Training community consultants to help family members improve dementia care: a randomized controlled trial. Gerontologist. 2005 Dec;45(6):802-11. PMID: 16326662.

700. Livingston G, Barber JA, Kinnunen KM, et al. DREAMS-START (Dementia RElAted Manual for Sleep; STrAtegies for RelaTives) for people with dementia and sleep disturbances: a single-blind feasibility and acceptability randomized controlled trial. International Psychogeriatrics. 2019 02;31(2):251-65. doi: https://dx.doi.org/10.1017/S104161021 8000753. PMID: 30221615.

701. McCurry SM, Gibbons LE, Logsdon RG, et al. Training caregivers to change the sleep hygiene practices of patients with dementia: the NITE-AD project. Journal of the American Geriatrics Society. 2003 Oct;51(10):1455-60. PMID: 14511168.
702. McCurry SM, Gibbons LE, Logsdon RG, et al. Nighttime insomnia treatment and education for Alzheimer's disease: a randomized, controlled trial. Journal of the American Geriatrics Society. 2005 May;53(5):793-802. PMID: 15877554.

703. Morton S, Murad M, O’Connor E, et al. Quantitative Synthesis-An Update. Methods Guide for Comparative Effectiveness Reviews. (Prepared by the Scientific Resource Center under Contract No. 290-2012-0004-C). AHRQ Publication No. 18-EHC007-EF. Rockville, MD: Agency for Healthcare Research and Quality; 2018.

704. Mills EJ, Ayers D, Chou R, et al. Are current standards of reporting quality for clinical trials sufficient in addressing important sources of bias? Contemporary Clinical Trials. 2015;45:2-7. doi: 10.1016/j.cct.2015.07.019. PMID: 26232560.

705. Callahan CM, Sachs GA, LaMantia MA, et al. Redesigning systems of care for older adults with Alzheimer's disease. 2014;33(4):626-32.

706. Schultz SK, Llorente MD, Sanders AE, et al. Quality improvement in dementia care: Dementia Management Quality Measurement Set 2018 Implementation Update. 2020;94(5):210-6.

707. Odenheimer G, Borson S, Sanders AE, et al. Quality improvement in neurology: dementia management quality measures. 2013;81(17):1545-9.

708. Czaja SI, Schulz R, Lee CC, et al. A methodology for describing and decomposing complex psychosocial and behavioral interventions. Psychology \& Aging. 2003;18:385-95. doi: 10.1037/0882-7974.18.3.385 PMID: 14518802.

709. Jackson JW, Arah OA. Making causal inference more social and (social) epidemiology more causal. American Journal of Epidemiology. 2019 October 1, 2019;kwz199. doi: 10.1093/aje/kwz199. 
710. Collins LM. Optimization of behavioral, biobehavioral, and biomedical interventions: the multiphase optimization strategy (MOST). New York: Springer; 2018.

711. Gitlin LNB, R.; Jutkowitz, E.; Gustavosn, A. M.; Sefcik, J. S.; Hodgson, N. A.; Keouth, S.,; Gaugler, J. E. Critical factors for successful dissemination and implementation of interventions designed to improve care for people living with dementia and care partners in health care systems. Journal of the American Geriatrics Society. under review.

712. Gitlin LNH, N.; Choi, S.; Marx, K. Interventions to address functional decline in persons with dementia: Closing the gap between what a person "does do" and what they "can do." In: Jefferson PZB, ed Neuropsychology of Alzheimer's Disease and Other Dementias. New York, NY: Oxford University Press; in press.

713. Office of Assistant Secretary of Health and Human Services. Including People with Dementia and Their Caregivers as CoResearchers in Studies of Dementia Care and Services. 2018.

https:/aspe.hhs.gov/basicreport/including-people-with-dementiaand-their-caregivers-as-co-Researchersin-studies-of-dementia-care-andservices. Accessed on March 1, 2020.

714. Silberzahn R, Uhlmann EL, Martin DP, et al. Many analysts, one data set: making transparent how variations in analytic choices affect results. Advances in Mehtods and Practices in Psychological Science. 2018;1(3):337-56. doi: $10.1177 / 2515245917747646$. 


\section{Appendixes}

Appendix A. Methods

I. Example Interventions

II. Common Outcome Measures

III. Search Strategy

IV. Risk of Bias - Traditional Studies

V. Risk of Bias - Cluster Trials

VI. PRECIS-2 Tool for Care Interventions

Appendix B. Excluded Studies

Appendix C. Chapter 4 Treatment for BPSD

I. Assisted Therapy

II. Multi-Sensory Stimulation/Snoezelen

III. Complementary and Alternative Medicine (CAM) Therapy

IV. Bright Light Therapy

V. Psychosocial for BPSD

VI. Multicomponent Interventions

Appendix D. Chapter 5 Interventions for PWD wellbeing

I. Exercise

II. Music Intervention

III. Reminiscence Therapy

IV. Cognitive Rehabilitation

V. Cognitive Training

VI. Cognitive Stimulation Therapy

VII. Recreation Therapy

VIII. Psychosocial for PWD Well-Being

IX. Creative-Expression Therapy

X. Multicomponent for PWD Well-Being

$\mathrm{XI}$. Assistive Technology

XII. Electrostimulation

XIII. Other Interventions for PWD Well-Being

Appendix E. Chapter 6 and 7 Interventions for Caregiver Well-Being

I. Psychosocial Interventions to Support Caregiver's Well-Being

II. Social Support

III. Lifestyle Interventions

IV. Respite Care

V. Multicomponent for Informal Caregiver Well-Being

VI. Other Interventions for Caregiver Well-Being

VII. Interventions for Formal Caregiver Well-Being

Appendix F. Chapter 8 Care delivery interventions

I. Care Service Provision
a. Consultation
b. Case Management
c. Care Protocols for PWD
d. Advance Care Planning
e. Palliative Care
f. Other Service Provision Interventions 
II. Care Delivery Models and Programs - Collaborative Care

III. Care Staff Education and Support Needs
a. Caregiver Staff Training
b. Informal Caregiver Staff Training
c. Family Education and Partnering
d. Multitier Training 


\section{Appendix A. Methods}

\section{Example Interventions}

Essentially, interventions are automatically included unless specifically stated as excluded. Note that the list is not divided by KQs 1-10. Some interventions may be aimed at both PWD and PWD Caregivers; some may be aimed at one or the other. The list is not intended to be exhaustive, and is a simple categorization based on what may be a more likely classification. The actual distinction between whether an intervention is examining what care is delivered or how to deliver care would be determined by the study purpose.

- Memory evaluation

- Driving evaluation or encouraging driving cessation

- Meaningful activities

- Advance care planning

- Behavior management

- ADL support

- Home modifications

- Wandering and fall risk management

- Palliative care

- Caregiver support and support groups

- Sensory-based interventions

- Changing the physical environment/environmental modification across settings (e.g., in hospitals, in people's homes)

- Mindfulness training

- Interventions focused on the development of Dementia Friendly Training (e.g., training of police officers in local communities)

- Wandering and Wayfinding

- Reminiscence Therapy

- Prompts and Multicomponent Interventions

- Engagement Interventions

- Exercise Interventions

- Psychoeducational

- Art therapy

- Dance movement therapy

- Music therapy

- Cognitive behavior therapy

- Counseling/care management (including emotionally focused couples therapy)

- General support

- Respite

- Training of PWD

- Psychosocial interventions/studies

- Caregiver support groups

- Therapeutic counseling 
- Support interventions, including involving informal caregiver social network to support the primary caregiver

- Cognitive reframing (changing caregivers' maladaptive behaviors or beliefs)

- Web-based multimedia intervention

- Caregiver-therapist e-mail support

- Educational and peer-support website

- Bereavement support

- Improving acute care systems

- Skill training, including for CNAs, home health aides, and/or informal caregivers

- Training for CNAs, home health aides, and/or informal caregivers

- Improving care transitions

- Care coordination

- Multicomponent interventions 


\section{Common Outcome Measures}

Table A-1. Common outcome measures

\begin{tabular}{|c|c|c|c|}
\hline Test Name & Domain & Data Source & Reference \\
\hline BEHAVE-AD & $\begin{array}{l}\text { General behavior } \\
\text { scales \& global } \\
\text { BPSD }\end{array}$ & & Reisberg et al. 1987 \\
\hline Neuropsychiatric Inventory (NPI) & $\begin{array}{l}\text { General behavior } \\
\text { scales \& global } \\
\text { BPSD }\end{array}$ & informant & Cummings et al 1994 \\
\hline Cohen-Mansfield Agitation Inventory (CMAI) & $\begin{array}{l}\text { Agitation/ } \\
\text { aggression }\end{array}$ & informant & $\begin{array}{l}\text { Cohen-Mansfield, } \\
1986\end{array}$ \\
\hline Cornell Scale & Depression & patient or informant & $\begin{array}{l}\text { Alexopoulos et al. } \\
1988\end{array}$ \\
\hline Patient Health Questionnaire (PHQ-9) & Depression & patient & Spitzer et al., 1999 \\
\hline Geriatric Depression Scale (GDS) 30-item & Depression & patient & Yesavage et al. 1983 \\
\hline Geriatric Depression Scale (GDS) 15-item & Depression & patient & \\
\hline $\begin{array}{l}\text { Montgomery Asberg Depression Rating } \\
\text { Scale (MADRS) }\end{array}$ & Depression & & $\begin{array}{l}\text { Montgomery \& } \\
\text { Asberg, } 1979\end{array}$ \\
\hline Hamilton Depression Rating Scale (HDRS) & Depression & patient & Hamilton, 1960 \\
\hline Beck Anxiety Inventory (BAl) & Anxiety & patient & \\
\hline Brief Psychiatric Rating Scale (BPRS) & Mood; Psychosis & $\begin{array}{l}\text { clinician } \\
\text { administered } \\
\text { interview }\end{array}$ & $\begin{array}{l}\text { Overall 1962; Beller } \\
1984\end{array}$ \\
\hline $\begin{array}{l}\text { Schedule for Affective Disorders and } \\
\text { Schizophrenia (SADS) }\end{array}$ & Mood; Psychosis & $\begin{array}{l}\text { clinician } \\
\text { administered } \\
\text { interview }\end{array}$ & Endicott 1978 \\
\hline $\begin{array}{l}\text { Schedule for Affective Disorders and } \\
\text { Schizophrenia -Lifetime version (SADS-L) }\end{array}$ & Mood; Psychosis & $\begin{array}{l}\text { clinician } \\
\text { administered } \\
\text { interview }\end{array}$ & Endicott 1978 \\
\hline $\begin{array}{l}\text { Schedule for Affective Disorders and } \\
\text { Schizophrenia -Change version (SADS-C) }\end{array}$ & Mood; Psychosis & $\begin{array}{l}\text { clinician } \\
\text { administered } \\
\text { interview }\end{array}$ & Endicott 1978 \\
\hline $\begin{array}{l}\text { Behavioral Syndromes Scale for Dementia } \\
\text { (BSSD) }\end{array}$ & $\begin{array}{l}\text { General behavior } \\
\text { scales \& global } \\
\text { BPSD }\end{array}$ & informant & Devanand 1992 \\
\hline Barthel index & ADLs & informant & $\begin{array}{l}\text { Mahoney and } \\
\text { Barthel, } 1965 \\
\end{array}$ \\
\hline $\begin{array}{l}\text { Bristol Activities of Daily Living Scale } \\
\text { (BADLS) }\end{array}$ & & & Bucks et al. 1996 \\
\hline Direct Assessment of Functional Status & ADLs + IADLs & performance-based & $\begin{array}{l}\text { Loewenstein, Amigo, } \\
\& \text { Duara, } 1989\end{array}$ \\
\hline $\begin{array}{l}\text { Disability Assessment for Dementia (DAD) } \\
\text { Scale }\end{array}$ & & informant & \\
\hline Functional Activities Questionnaire (FAQ) & & informant & Pfeffer et al 1982 \\
\hline Functional Independence Measure (FIM) & $\begin{array}{l}\text { ADLs + (social, } \\
\text { cogn, etc) }\end{array}$ & informant & Keith et al. 1987 \\
\hline Health Assessment Questionnaire (HAQ) & & & \\
\hline $\begin{array}{l}\text { Informant Questionnaire on Cognitive } \\
\text { Decline in the Elderly (IQCODE) }\end{array}$ & & informant & $\begin{array}{l}\text { Jorm and } \\
\text { Jacomb,1989 }\end{array}$ \\
\hline Instrumental Activities of Daily Living scale & IADLS & & $\begin{array}{l}\text { Lawton and Brody, } \\
1969\end{array}$ \\
\hline Katz Index of Independence in ADLs & ADLs & & Katz et al. 1963 \\
\hline $\begin{array}{l}\text { Modified Health Assessment Questionnaire } \\
\text { (MHAQ) }\end{array}$ & & & \\
\hline $\begin{array}{l}\text { Older Americans Resources and Services } \\
\text { (OARS) }\end{array}$ & ADLs + IADLs & self-report & $\begin{array}{l}\text { George \& } \\
\text { Fillenbaum, } 1985\end{array}$ \\
\hline
\end{tabular}




\begin{tabular}{|l|l|l|l|}
\hline Test Name & Domain & Data Source & Reference \\
\hline $\begin{array}{l}\text { Physical Self-Maintenance Scale (PSMS) } \\
\text { Pinimum Data Set (MDS)-ADL Self }\end{array}$ & ADLs & & $\begin{array}{l}\text { Lawton and Brody, } \\
1969\end{array}$ \\
\hline Progressive Deterioration Scale (PDS) & ADLs & & \\
\hline AD-related Quality of Life Scale (QoL-AD) & & informant & DeJong 1989 \\
\hline DEMQOL & & patient or informant & Logsdon et al. 1999 \\
\hline DEMQOL & & patient & Smith et al. 2007 \\
\hline EuroQol measure & & informant & Smith et al. 2007 \\
\hline Short Form-36 (SF-36) & & patient or informant & EuroQol Group, 1990 \\
\hline General Health Questionnaire (GHQ) & Global Distress & $\begin{array}{l}\text { Ware \& Sherbourne, } \\
1992\end{array}$ \\
\hline Zarit Burden Interview & Caregiver Burden & & $\begin{array}{l}\text { Goldberg \& Williams } \\
1988\end{array}$ \\
\hline Neuropsychiatric Inventory - Distress Scale & Caregiver Distress & & Zarit et al. 1980 \\
\hline $\begin{array}{l}\text { Revised Memory and Behavior Problem } \\
\text { Checklist (RMBPC) }\end{array}$ & & informant & Terie et al 1992 \\
\hline
\end{tabular}




\section{Search Strategy}

Database: Ovid MEDLINE(R) Epub Ahead of Print, In-Process \& Other Non-Indexed Citations, Ovid MEDLINE(R) Daily, Ovid MEDLINE and Versions(R) <1946

Search Strategy:

1 exp Alzheimer Disease/

2 Dementia/

3 (dementia or alzheimer*).ti.

41 or 2 or 3

5 limit 4 to "therapy (best balance of sensitivity and specificity)"

6 limit 5 to english language

7 limit 6 to (addresses or autobiography or bibliography or biography or case reports or clinical conference or comment or comparative study or congresses or consensus development conference or consensus development conference, nih or dataset or dictionary or directory or editorial or evaluation studies or "expression of concern" or festschrift or government publications or guideline or historical article or interactive tutorial or interview or lectures or legal cases or legislation or letter or news or newspaper article or observational study or patient education handout or periodical index or personal narratives or portraits or "review" or "scientific integrity review" or validation studies or video-audio media or webcasts)

8 limit 7 to (adaptive clinical trial or clinical study or clinical trial, all or clinical trial, phase i or clinical trial, phase ii or clinical trial, phase iii or clinical trial, phase iv or clinical trial or controlled clinical trial or randomized controlled trial)

$9 \quad 6$ not 7

108 or 9

11 limit 10 to ("all child (0 to 18 years)"

12 limit 11 to ("middle age (45 to 64 years)" or "middle aged (45 plus years)" or "all aged (65 and over)" or "aged (80 and over)")

1310 not 11

1412 or 13

Database: Embase Classic+Embase $<1947$

Search Strategy:

1 exp *Alzheimer disease/

2 *dementia/

3 (alzheimer* or dementia*).ti.)

41 or 2 or 3

5 limit 4 to english language

6 limit 5 to "therapy (best balance of sensitivity and specificity)"

7 limit 6 to "reviews (best balance of sensitivity and specificity)"

86 not 7

9 limit 8 to (embryo $<$ first trimester $>$ or infant $<$ to one year $>$ or child $<$ unspecified age $>$ or preschool child $<1$ to 6 years $>$ or school child $<7$ to 12 years $>$ or adolescent $<13$ to 17 years $>$ )

10 limit 9 to (adult $<18$ to 64 years $>$ or aged $<65+$ years $>$ )

118 not 9 
1210 or 11

13 limit 12 to (book or book series or conference proceeding or trade journal)

1412 not 13

15 limit 14 to conference abstracts

1614 not 15

17 limit 16 to (abstract report or books or "book review" or chapter or conference abstract or "conference review" or editorial or letter or note or patent or reports or "review" or short survey or tombstone)

1816 not 17

19 limit 18 to (amphibia or ape or bird or cat or cattle or chicken or dog or "ducks and geese" or fish or "frogs and toads" or goat or guinea pig or "hamsters and gerbils" or horse or monkey or mouse or "pigeons and doves" or "rabbits and hares" or rat or reptile or sheep or swine)

2018 not 19

Database: PsycINFO <1806

Search Strategy:

$1 \exp *$ ALZHEIMER'S DISEASE/

$2 *$ dementia/

3 (dementia* or alzheimer*).ti.

41 or 2 or $3(64340)$

5 limit 4 to "therapy (best balance of sensitivity and specificity)"

6 limit 5 to (childhood <birth to 12 years $>$ or adolescence $<13$ to 17 years $>$ )

7 limit 6 to adulthood $<18+$ years $>$

85 not 6

97 or 8

10 limit 9 to animal

119 not 10

12 limit 11 to (100 childhood <birth to age 12 yrs $>$ or 120 neonatal <birth to age 1 mo $>$ or 140 infancy $<2$ to $23 \mathrm{mo}>$ or 160 preschool age <age 2 to 5 yrs $>$ or 180 school age <age 6 to 12 yrs $>$ or 200 adolescence <age 13 to 17 yrs > or 320 young adulthood <age 18 to 29 yrs $>$ or 340 thirties $<$ age 30 to 39 yrs $>$ )

13 limit 12 to (360 middle age <age 40 to 64 yrs> or "380 aged <age 65 yrs and older>" or "390 very old <age 85 yrs and older $>$ ")

1411 not 12

1513 or 14

16 limit 15 to (abstract collection or bibliography or chapter or clarification or

"column/opinion" or "comment/reply" or dissertation or editorial or encyclopedia entry or interview or letter or obituary or poetry or publication information or review-book or reviewmedia or review-software \& other or reviews)

1715 not 16

18 limit 17 to ("0200 book" or "0240 authored book" or "0280 edited book" or "0300

encyclopedia" or "0400 dissertation abstract")

1917 not 18

20 limit 19 to english language

21 limit 20 to "therapy (maximizes specificity)" 


\section{Risk of Bias Assessment Guide - Traditional Studies}

INSTRUCTIONS: Review the methods of each trial and assess each risk of bias component as described in these instructions. You may need to have separate assessments for different outcomes (i.e. different measures; different time points may have different attrition rates).

\section{1) ATTRITION Bias}

Table A-2. Attrition bias

\begin{tabular}{|l|l|}
\hline Description/Guiding Questions & Notes \\
\hline Systematic differences in the loss of & - $\begin{array}{l}\text { Attrition assessment is dependent on } \\
\text { participants from the study and how }\end{array}$ \\
they were accounted for in the results & Report attrition rate in spreadsheet. \\
(e.g., incomplete follow-up, differential & If a study reports outcomes at multiple \\
attrition). Those who drop out of the & intervals (e.g., 6 months, 12 months, \\
study or who are lost to follow-up may & 18 months) assess attrition at the first \\
be systematically different from those & relevant time point and the last time- \\
who remain in the study. Attrition bias & point separately, you do not need to \\
can potentially change the collective & do every time point. \\
(group) characteristics of the relevant & Analysis should be done with \\
groups and their observed outcomes & appropriate method (i.e. sensitivity \\
in ways that affect study results by & analysis with various scenarios); last \\
confounding and spurious & value forward would only be \\
associations. & appropriate for interventions that are \\
& supposed to improve the outcomes \\
Reasons for incomplete/missing data & (i.e. memory training that intends to \\
adequately explained? & improve memory). \\
Do the author's attempt to address & \\
attrition in the analysis? & \\
\hline
\end{tabular}


Figure A-1. Attrition bias assessment guidance

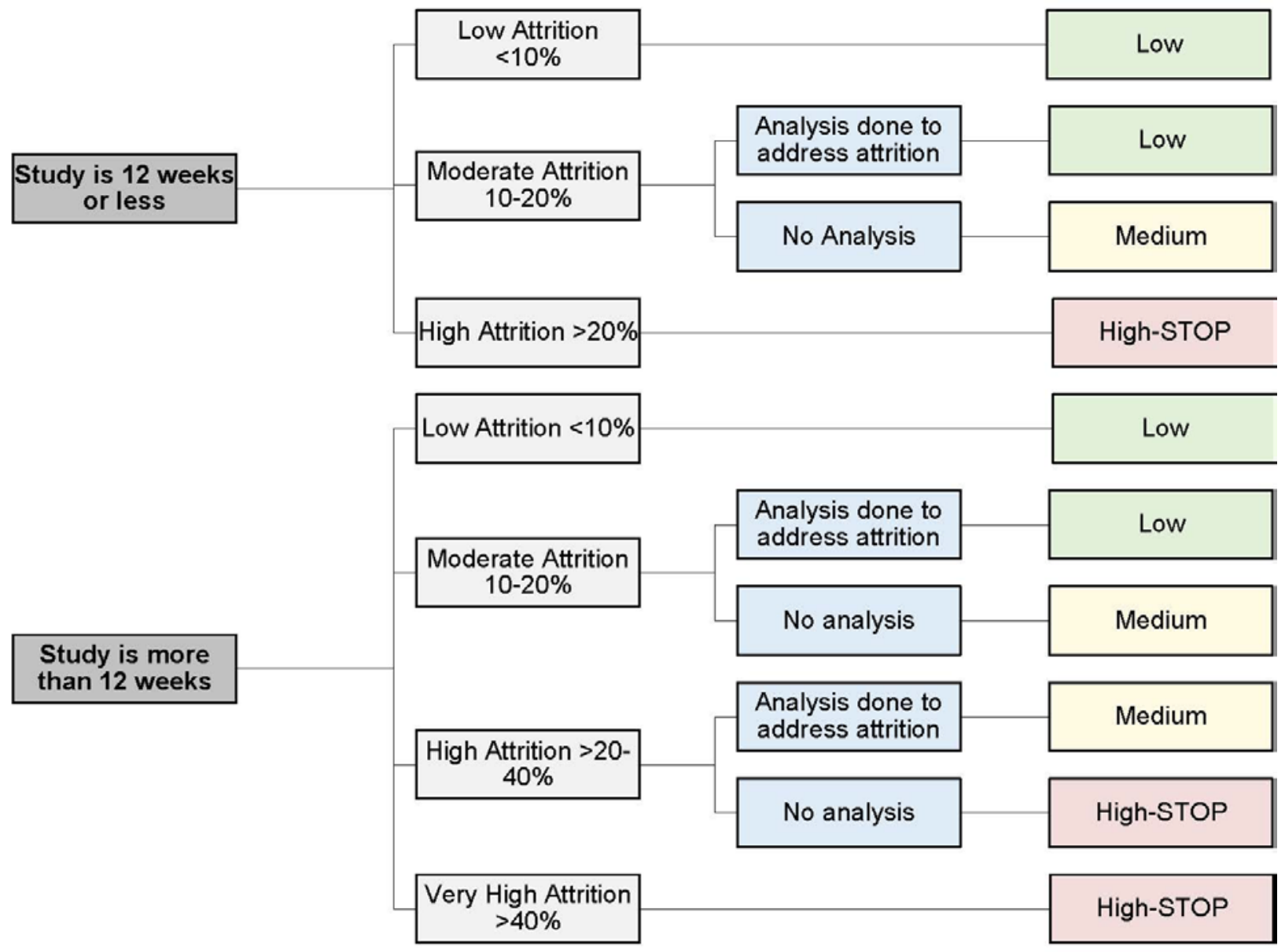




\section{2) SELECTION BIAS}

Table A-3. Selection bias

\begin{tabular}{|l|l|}
\hline Description/Guiding Questions & Notes \\
\hline - Systematic differences between & "Good" Randomization: Detailed \\
baseline characteristics of the groups & $\begin{array}{l}\text { methodology would include providing } \\
\text { that arise from self-selection of }\end{array}$ \\
treatments, physician-directed & of a random numbers table, or \\
selection of treatments, or association & computer random number generator. \\
of treatment assignments with & Limited methodology would be the \\
demographic, clinical, or social & study saying simply saying they \\
characteristics. & randomized in the methods or provided \\
& limited detail such as randomizing by a \\
Did method of randomization create & $2: 1$ ratio. \\
biased allocation to interventions & "Poor"/No Randomization: \\
(inadequate randomization)? & Randomized based on week of the \\
& month of birthday or a non-randomized \\
& clinical trial, observational study. \\
\hline
\end{tabular}

Figure A-2. Selection bias assessment guidance

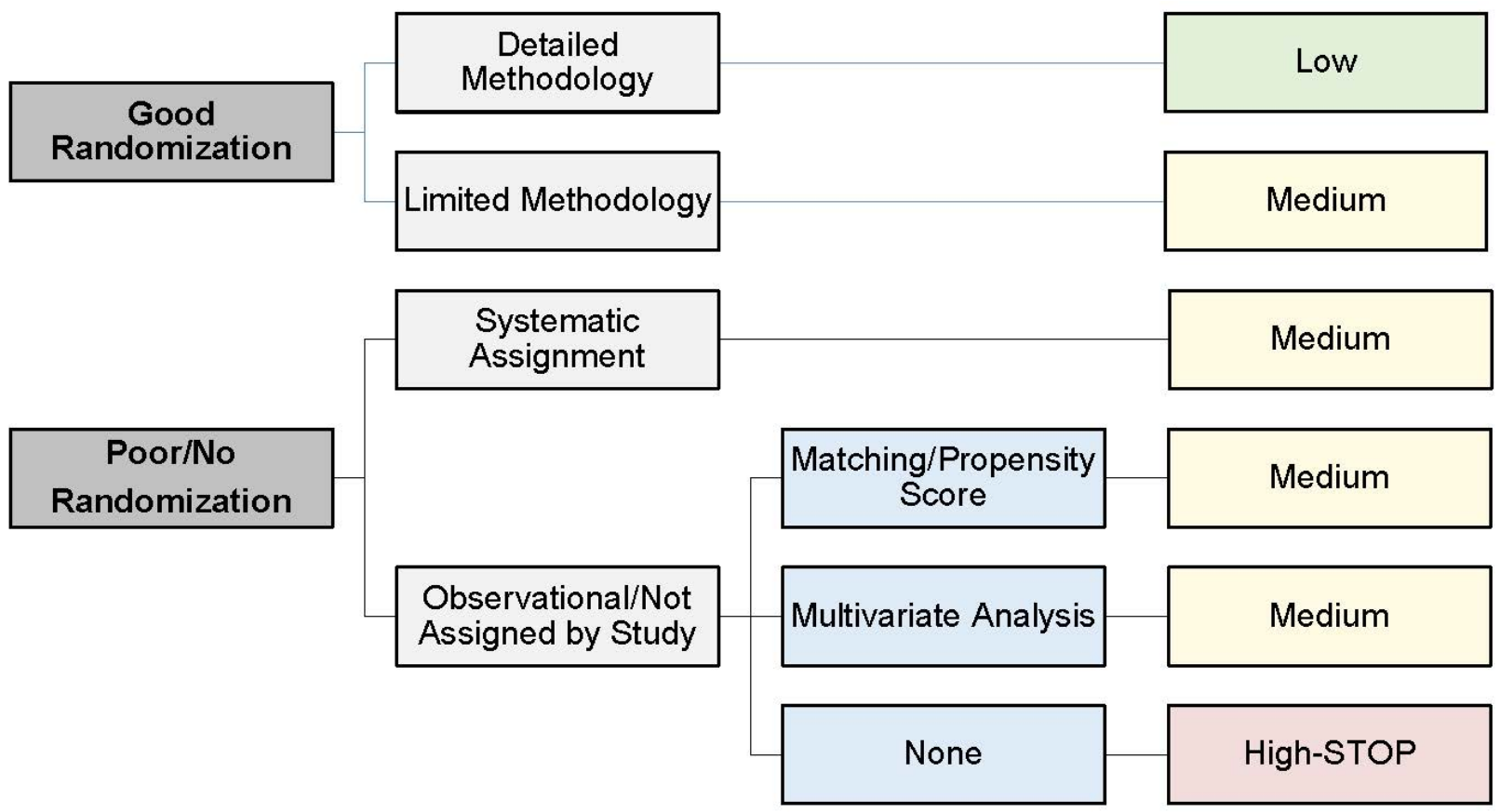


3) ATTRITION AND SELECTION BIAS OVERALL

Assess joint selection and attrition bias. If either selection or attrition bias is high, the risk of bias is $\mathrm{HIGH}$.

Table A-4. Attrition and selection bias overall

\begin{tabular}{|c|c|c|c|c|c|c|c|}
\hline Attrition Bias & Low & Low & Medium & Low & Medium & Medium & High \\
\hline Selection Bias & Low & Medium & Low & High & Medium & High \\
\hline Action & Assess & Assess & Assess & STOP* & Assess \\
& other & other & other \\
biases & biases & & STOP* \\
& biases & biases & & \\
\end{tabular}

*Send to evidence map

***PRIOR TO ASSESSING OTHER BIASES, CHECK PUBLICATION TO MAKE SURE OUTCOMES ARE ABSTRACTABLE. IF OUTCOMES ARE NOT ABSTRACTABLE (e.g., DATA IS PRESENTED AS GRAPHS ONLY), STOP ASSESSMENT AND CHECK WITH TEAM TO CONFIRM THAT PUBLICATION BELONGS IN EVIDENCE MAP*** 


\section{4) OTHER BIASES}

\section{A. DETECTION BIAS}

Table A-5. Detection bias

\begin{tabular}{|l|l|}
\hline Description/Guiding Questions & Notes \\
\hline - Systematic differences in outcomes & $-\mathrm{X}$ \\
assessment among groups being & \\
compared, including systematic & \\
misclassification of the exposure or & \\
intervention, covariates, or outcomes & \\
because of variable definitions and & \\
timings, diagnostic thresholds, recall from & \\
memory, inadequate assessor blinding, & \\
and faulty measurement techniques. & \\
Erroneous statistical analysis might also & \\
affect the validity of effect estimates. & \\
& \\
Were the outcome assessors blinded to & \\
the intervention ("outcome assessor & \\
blinded")? & \\
Was the timing of the outcome & \\
assessment similar in all groups & \\
(“comparable timing outcomes & \\
assessment")? & \\
Was the scale used to measure & \\
outcomes validated, reliable? & \\
Were outcomes measured in clinically & \\
meaningful ways? & \\
\hline
\end{tabular}


Figure A-3. Detection bias assessment guidance

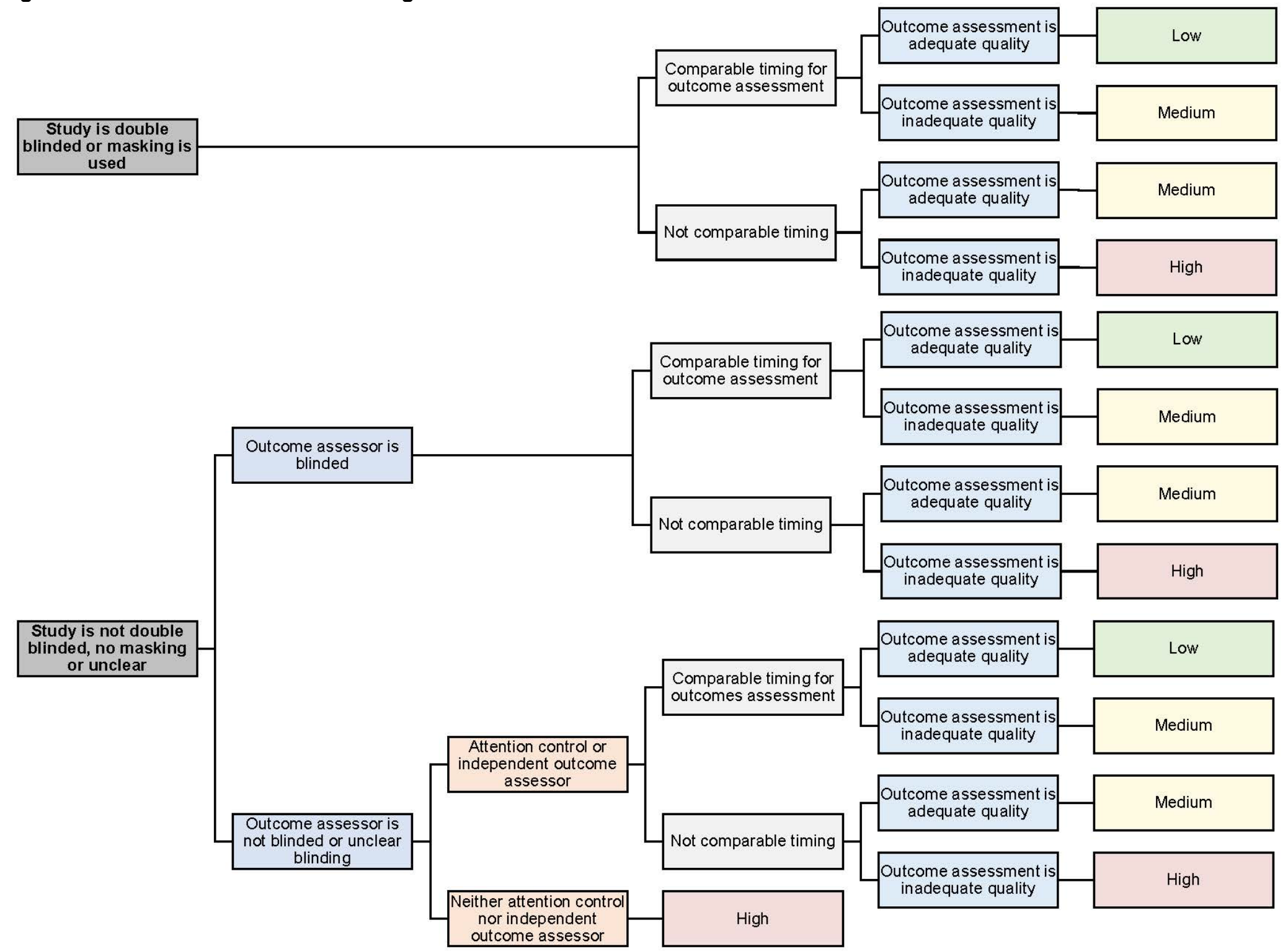




\section{B. PERFORMANCE BIAS}

Table A-6. Performance bias

\begin{tabular}{|l|l|}
\hline Description/Guiding Questions & Notes \\
\hline Systematic differences in the care & - \\
provided to participants and protocol & every subject according to \\
deviation. Examples include & randomized treatment assignment. \\
contamination of the control group with & Ignores noncompliance, protocol \\
the exposure or intervention, problems & deviations, withdrawal, and anything \\
with fidelity to the intervention, & that happens after randomization. \\
unbalanced provision of additional & Concurrent Intervention: Study \\
interventions or co-interventions, & participants are receiving another \\
difference in co-interventions, and & intervention (i.e., treatment) that is not \\
inadequate blinding of providers and & part of the intervention being tested. \\
participants. & Example: Participants are \\
& randomized to a physical activity \\
& intervention (or no intervention), but \\
& are also dieting. \\
\hline
\end{tabular}


Figure A-4. Performance Bias Assessment Guidance

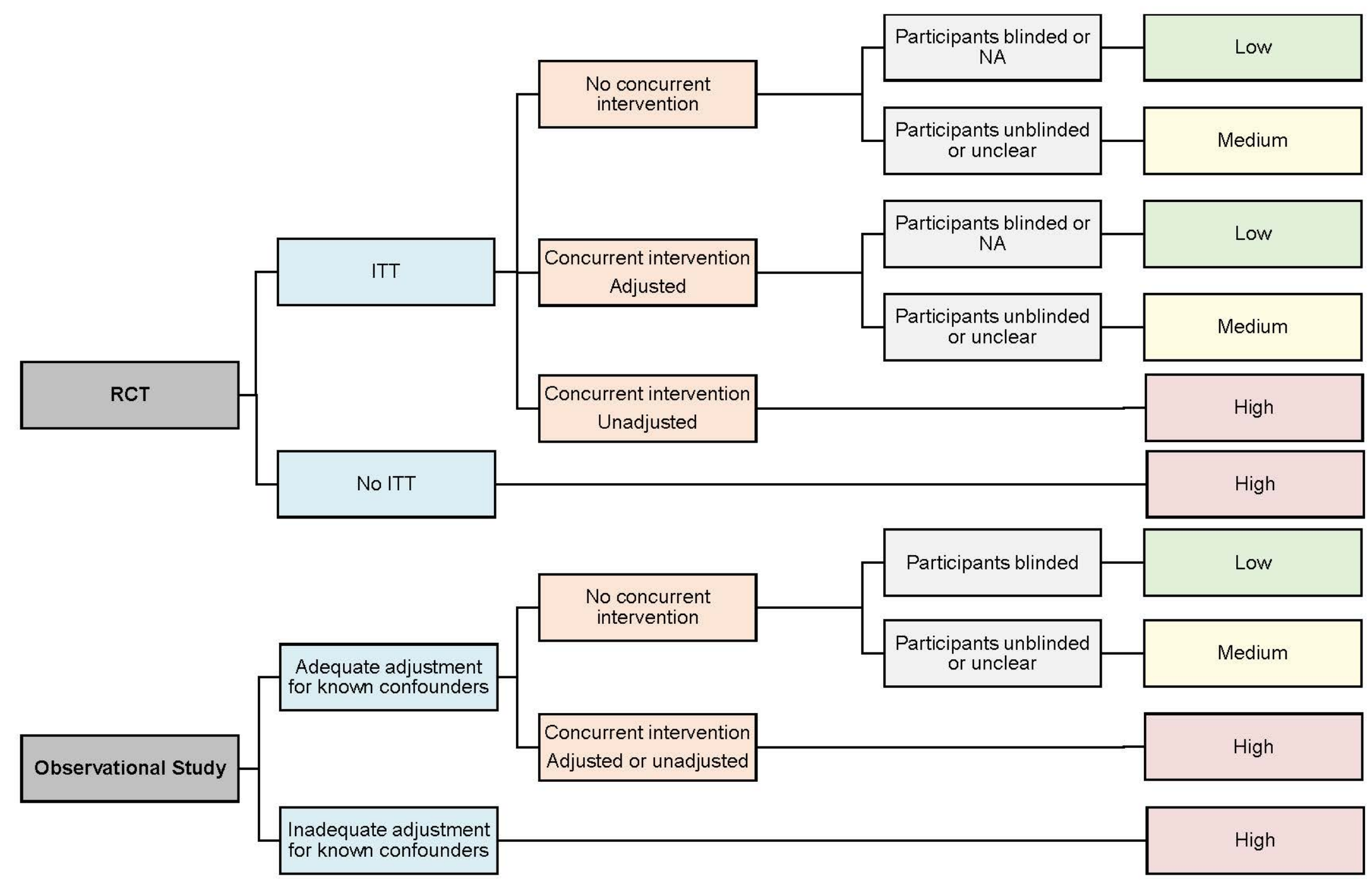




\section{REPORTING BIAS}

Table A-7. Reporting bias

\begin{tabular}{|l|l}
\hline Description/Guiding Questions & Notes \\
\hline
\end{tabular}

- Systematic differences between reported and unreported findings (e.g., differential reporting of outcomes or harms, incomplete reporting of study findings, potential for bias in reporting through source of funding).

Was a select group of outcomes reported?

- Compare results to methods section and/ or protocol.

Check if some results are reported in a different publication.

REPORTING BIAS ASSESSMENT GUIDANCE

Table A-8. Reporting bias assessment guidance

\begin{tabular}{|l|l|l|}
\hline \multicolumn{1}{|c|}{ Domain } & \multicolumn{1}{|c|}{ Options } & \multicolumn{1}{c|}{ Rating } \\
\hline \multirow{3}{*}{ All outcomes reported } & Yes & Low \\
\cline { 2 - 3 } & No & Medium \\
\cline { 2 - 3 } & Not Reported & Medium \\
\hline
\end{tabular}




\section{***CHECK OVERALL ROB RATING BEFORE ASSESSING FIDELITY TO INTERVENTION. IF THE COMBINATION OF PREVIOUS DOMAINS INDICATES HIGH RISK OF BIAS, YOU DO NOT NEED TO ASSESS FIDELITY***}

\section{FIDELITY TO INTERVENTION}

Table A-9. Fidelity to intervention Description/Guiding Questions
- We anticipate that care delivery studies will generally fall in the range of $\mathrm{NIH}$ Stage 3 to 4 , with the possibility that one or a few may be carried out as quality improvement and thus Stage 5. Since the Stage Model is explicitly designed to balance, or trade off, internal and external validity, we will approach risk of bias assessment as a threshold requirement rather than a continuum.

Look for reporting on intervention compliance, any data reported on consistency of intervention use, or any mechanisms used to ensure compliance (e.g., reminders, guides, manuals).

FIDELITY TO INTERVENTION ASSESSMENT GUIDANCE

Table A-10. Fidelity to intervention assessment guidance

\begin{tabular}{|l|c|c|}
\hline \multicolumn{1}{|c|}{ Domain } & Options & Rating \\
\hline Fidelity to & Yes (at least 70\%) & Low \\
\cline { 2 - 3 } & Yes-adaptation planned/ replicable & Medium \\
\cline { 2 - 3 } & No-adaptation not planned & High \\
\cline { 2 - 3 } & Unclear/Not Reported & Medium \\
\hline
\end{tabular}


Figure A-5. Overall risk of bias assessment guidance

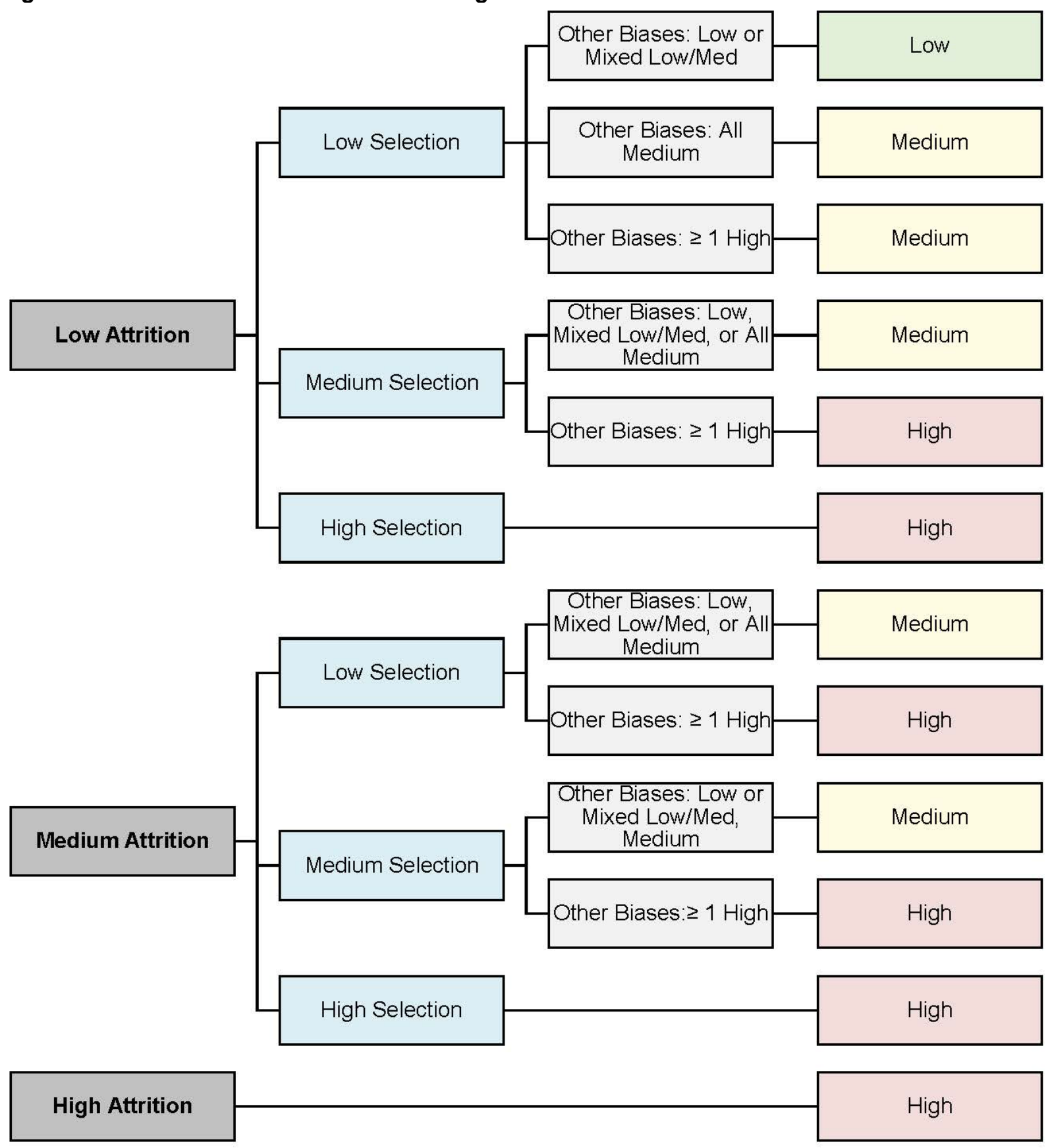




\section{Risk of Bias Assessment Guide - Cluster Trials 1) ATTRITION Bias}

Table A-11. Attrition bias

\begin{tabular}{|c|c|}
\hline Description/Guiding Questions & Notes \\
\hline $\begin{array}{l}\text { Systematic differences in the loss of } \\
\text { participants from the study and how } \\
\text { they were accounted for in the results } \\
\text { (e.g., incomplete follow-up, differential } \\
\text { attrition). Those who drop out of the } \\
\text { study or who are lost to follow-up may } \\
\text { be systematically different from those } \\
\text { who remain in the study. Attrition bias } \\
\text { can potentially change the collective } \\
\text { (group) characteristics of the relevant } \\
\text { groups and their observed outcomes in } \\
\text { ways that affect study results by } \\
\text { confounding and spurious associations. } \\
\\
\text { Reasons for incomplete/missing data } \\
\text { adequately explained? } \\
\text { Do the author's attempt to address } \\
\text { attrition in the analysis? }\end{array}$ & $\begin{array}{l}\text { Attrition assessment is dependent on } \\
\text { overall study duration (see flowchart) } \\
\text { Report attrition rate in spreadsheet. } \\
\text { If a study reports outcomes at } \\
\text { multiple intervals (e.g., } 6 \text { months, } 12 \\
\text { months, } 18 \text { months) assess attrition } \\
\text { at the first relevant time point and the } \\
\text { last time-point separately, you do not } \\
\text { need to do every time point. } \\
\text { Analysis should be done with } \\
\text { appropriate method (i.e. sensitivity } \\
\text { analysis with various scenarios); last } \\
\text { value forward would only be } \\
\text { appropriate for interventions that are } \\
\text { supposed to improve the outcomes } \\
\text { (i.e. memory training that intends to } \\
\text { improve memory). }\end{array}$ \\
\hline
\end{tabular}


Figure A-6. Attrition bias assessment guidance

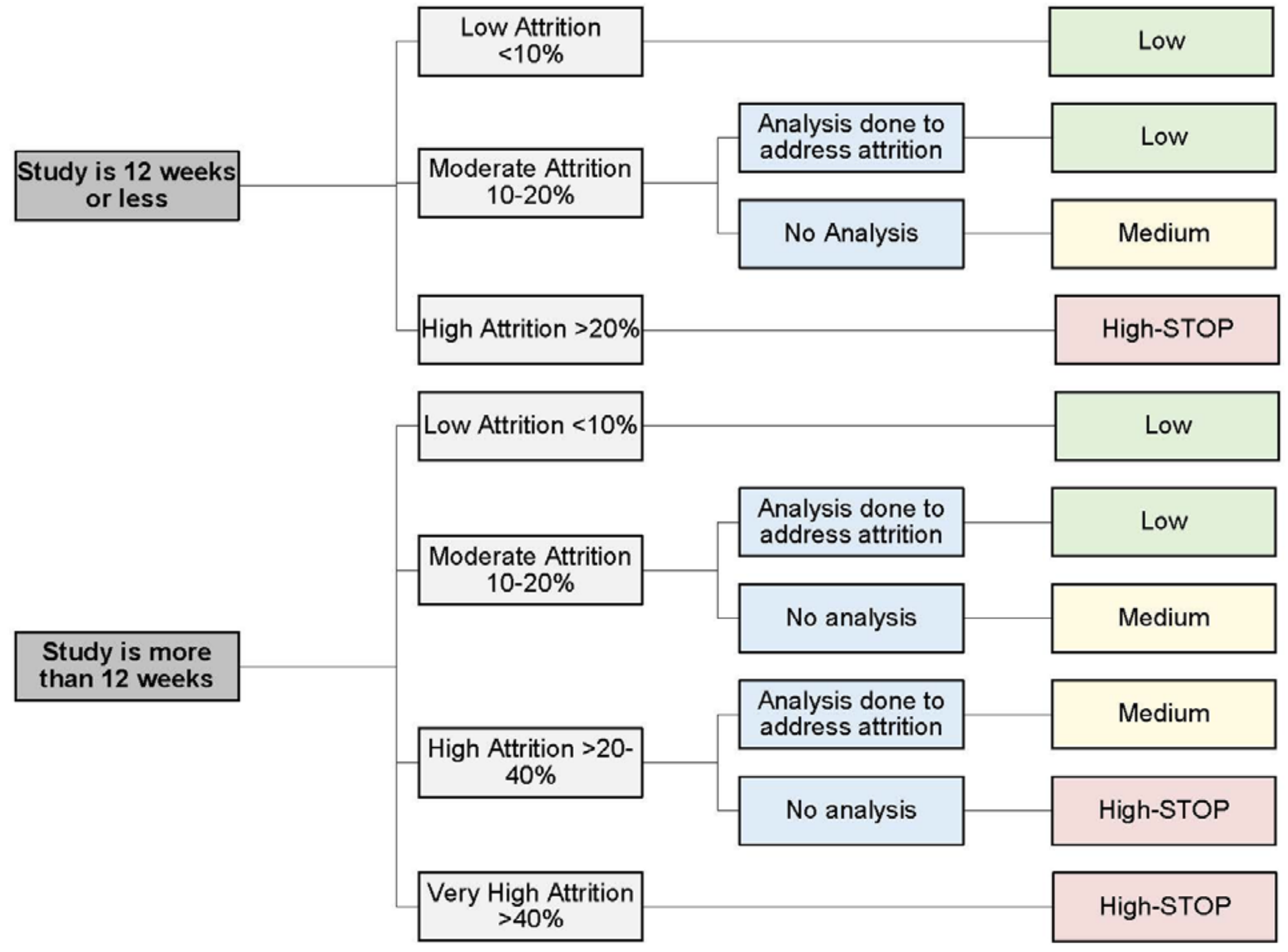




\section{3) SELECTION BIAS}

Table A-12. Selection bias

\begin{tabular}{|l|l|}
\hline Description/Guiding Questions & Notes \\
\hline $\begin{array}{l}\text { Systematic differences between } \\
\text { baseline characteristics of the groups } \\
\text { that arise from self-selection of } \\
\text { treatments, physician-directed }\end{array}$ & $\begin{array}{l}\text { "Good" Randomization: Random } \\
\text { numbers table, computer random } \\
\text { number generator }\end{array}$ \\
$\begin{array}{l}\text { selion of treatments, or association } \\
\text { of treatment assignments with } \\
\text { demographic, clinical, or social } \\
\text { characteristics. }\end{array}$ & $\begin{array}{l}\text { based on week of the month of } \\
\text { birthday }\end{array}$ \\
$\begin{array}{l}\text { No Randomization: Non-randomized } \\
\text { clinical trial, observational study }\end{array}$ \\
$\begin{array}{l}\text { Did method of randomization create } \\
\text { biased allocation to interventions } \\
\text { (inadequate randomization)? }\end{array}$ & \\
\hline
\end{tabular}

Figure A-7. Selection bias assessment guidance

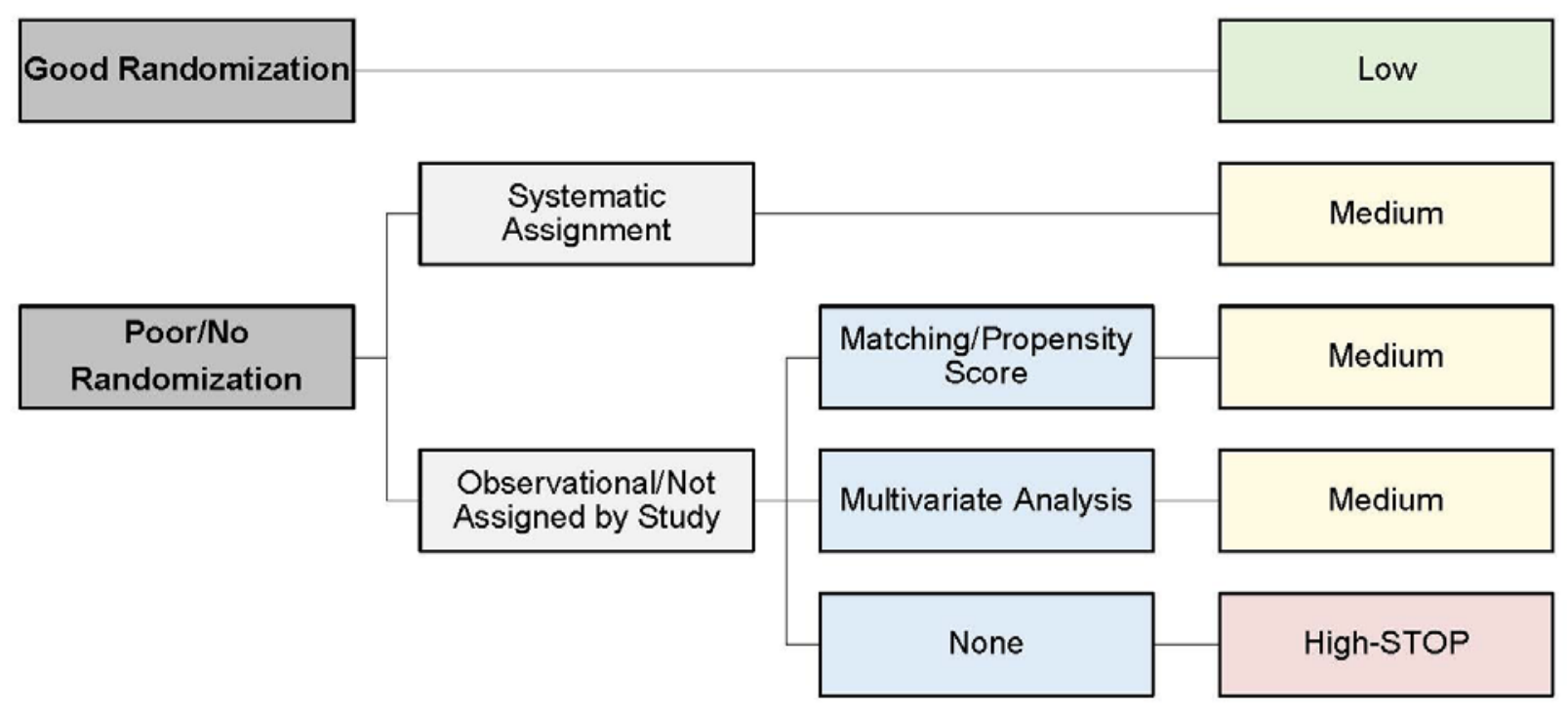


3) ATTRITION AND SELECTION BIAS OVERALL

Assess joint selection and attrition bias. If either selection or attrition bias is high, the risk of bias is $\mathrm{HIGH}$.

Table A-13. Attrition and selection bias overall

\begin{tabular}{|c|c|c|c|c|c|c|c|}
\hline Attrition Bias & Low & Low & Medium & Low & Medium & Medium & High \\
\hline Selection Bias & Low & Medium & Low & High & Medium & High \\
\hline Action & $\begin{array}{c}\text { Assess } \\
\text { other } \\
\text { biases }\end{array}$ & $\begin{array}{c}\text { Assess } \\
\text { other } \\
\text { biases }\end{array}$ & $\begin{array}{c}\text { Assess } \\
\text { other } \\
\text { biases }\end{array}$ & STOP* & $\begin{array}{c}\text { Assess } \\
\text { other } \\
\text { biases }\end{array}$ & STOP* $^{*}$ & STOP* $^{*}$ \\
\end{tabular}

*Send to evidence map

***PRIOR TO ASSESSING OTHER BIASES, CHECK PUBLICATION TO MAKE SURE OUTCOMES ARE ABSTRACTABLE. IF OUTCOMES ARE NOT ABSTRACTABLE (e.g., DATA IS PRESENTED AS GRAPHS ONLY), STOP ASSESSMENT AND CHECK WITH TEAM TO CONFIRM THAT PUBLICATION BELONGS IN EVIDENCE MAP*** 


\section{4) FIDELITY TO INTERVENTION}

Table A-14. Fidelity to intervention

\begin{tabular}{|l|l|}
\hline Description/Guiding Questions & Notes \\
\hline We anticipate that care delivery studies will & - X \\
generally fall in the range of NIH Stage 3 to & \\
4, with the possibility that one or a few may & \\
be carried out as quality improvement and & \\
thus Stage 5. Since the Stage Model is & \\
explicitly designed to balance, or trade off, & \\
internal and external validity, we will & \\
approach risk of bias assessment as a & \\
threshold requirement rather than a & \\
continuum. & \\
\hline
\end{tabular}

\section{FIDELITY TO INTERVENTION ASSESSMENT GUIDANCE}

Table A-15. Fidelity to intervention assessment guidance

\begin{tabular}{|l|c|c|}
\hline \multicolumn{1}{|c|}{ Domain } & Options & Rating \\
\hline Fidelity to & Yes (at least 70\%) & Low \\
\cline { 2 - 3 } & Yes-adaptation planned/ replicable & Medium \\
\cline { 2 - 3 } & No-adaptation not planned & High \\
\cline { 2 - 3 } & No/Not Reported & NR \\
\hline
\end{tabular}


Figure A-8. Overall risk of bias assessment guidance

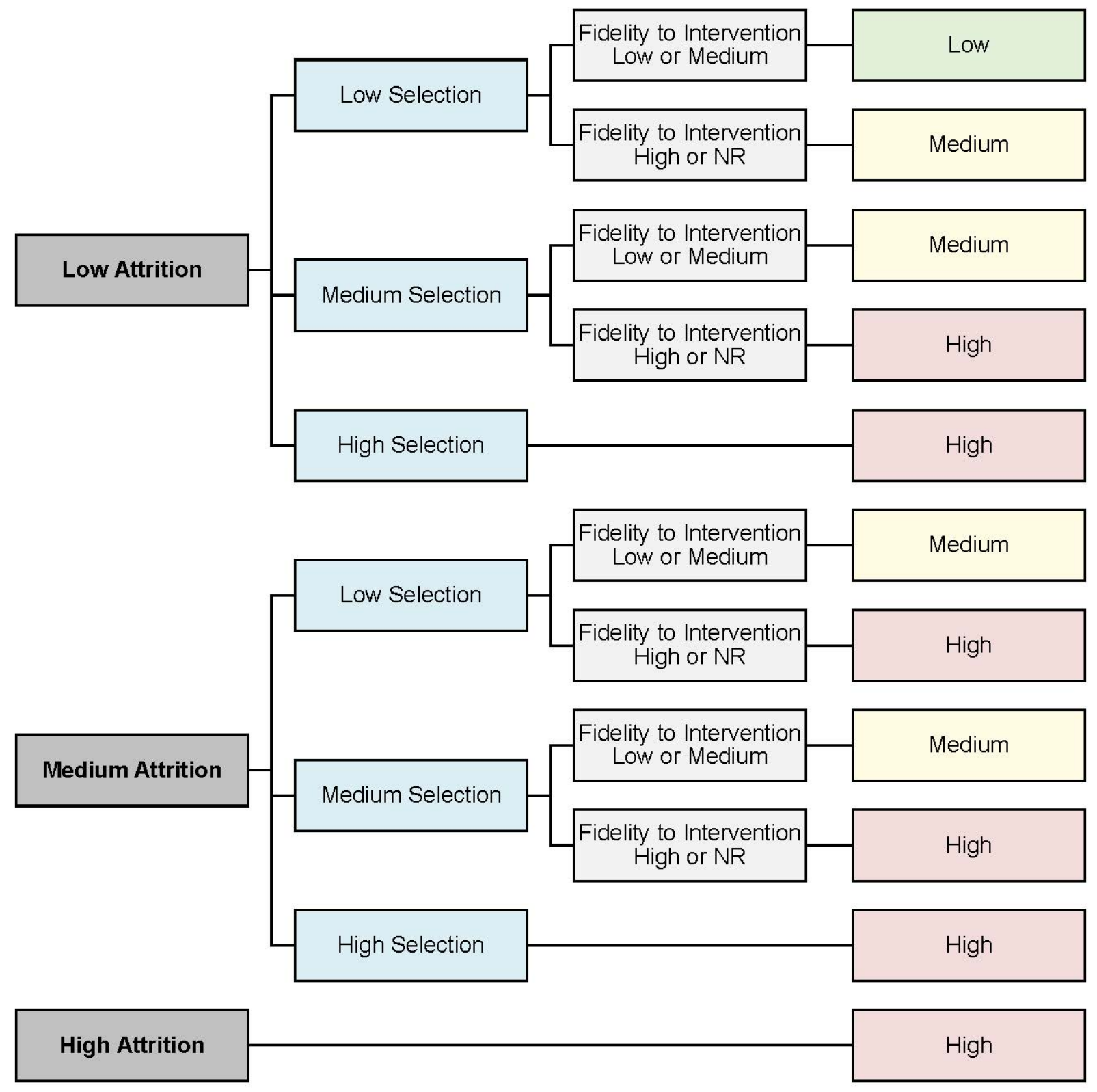




\section{PRECIS-2 - Modified for Care Delivery Intervention Literature}

Table A-16. PRECIS-2-modified for care delivery intervention literature

\begin{tabular}{|c|c|c|c|}
\hline Domain & $\begin{array}{l}\text { Score } \\
(1-5)\end{array}$ & Rationale for Score & Modified Prompts, With Examples \\
\hline $\begin{array}{l}\text { Eligibility } \\
\text { criteria }\end{array}$ & & & $\begin{array}{l}\text { To what extent are trial participants similar to PWD who } \\
\text { would receive the intervention as part of usual care? [5= } \\
\text { identical to usual care; } 1=\text { many exclusions (highly selected } \\
\text { sample, uncommon tests used, exclude noncompliant or } \\
\text { non-responders, etc.)] } \\
\text { Example considerations: } \\
\text { PWD: Other comorbidities allowed? Health or behavior } \\
\text { restrictions? Mobility or language restrictions? Dementia } \\
\text { severity range? Small percentage of eligible chose to } \\
\text { participate. Insurance restrictions? Participant had to opt in? } \\
\text { Caregiver: Level of mobility/health/cognition necessary? } \\
\text { How much time/work loss required? }\end{array}$ \\
\hline $\begin{array}{l}\text { Recruitment } \\
\text { path }\end{array}$ & & & $\begin{array}{l}\text { How much extra effort is required to recruit participants over } \\
\text { usual care? [5=pragmatic, usual care (appt. or clinic); } \\
\text { 1=targeted invitation letters, public media announcements, } \\
\text { incentives] } \\
\text { Example possible scores: } \\
\text { 5: Invited during routine clinic visit } \\
\text { 4: Invitation letter/call from doctor } \\
\text { 3: Identified PWD via diagnosis/billing code(s) } \rightarrow \text { sent letter } \\
\text { 2: Incentive(s) for participation } \\
\text { 1: Worker hired to find participants (clinic, health plan) }\end{array}$ \\
\hline Setting & & & $\begin{array}{l}\text { How different is the trial setting from usual care for PWD? } \\
\text { [5=identical to usual care; } 1=\text { single center, special trial or } \\
\text { academic center, etc. ] } \\
\text { Example considerations (if setting not part of study } \\
\text { question): } \\
\text { Urban only, or likely available in rural settings? } \\
\text { Multiple settings included (private group practice, academic, } \\
\text { HMO) } \\
\text { Components: training for PWD/Caregiver on-site, but } \\
\text { implemented at home via case manager? }\end{array}$ \\
\hline $\begin{array}{l}\text { Intervention } \\
\text { organization }\end{array}$ & & & $\begin{array}{l}\text { How different are intervention resources, provider expertise, } \\
\text { and care organization from those available in usual care? } \\
\text { How easy to implement without major changes (new staff, } \\
\text { funding, policy)? } \\
\text { Example possible scores: } \\
\text { 4: Multicomponent + requires community partners } \\
\text { 3: Multicomponent + requires new software } \\
\text { 2-4: Requires new staff and funding (some) } \\
\text { 1-2: Requires new or proprietary software (1+ sites), policy } \\
\text { change, major new staff and funding }\end{array}$ \\
\hline $\begin{array}{l}\text { Flexibility of } \\
\text { intervention: } \\
\text { delivery }\end{array}$ & & & $\begin{array}{l}\text { How different is flexibility of intervention delivery from usual } \\
\text { care for PWD? } \\
\text { Example possible scores: }\end{array}$ \\
\hline
\end{tabular}




\begin{tabular}{|c|c|c|c|}
\hline Domain & $\begin{array}{l}\text { Score } \\
(1-5)\end{array}$ & Rationale for Score & Modified Prompts, With Examples \\
\hline & & & $\begin{array}{l}\text { 5: Suggested services obtained based on ability to pay } \\
\text { 4-5: Care manager calls/care coordination per care } \\
\text { manager/participant discretion } \\
\text { 2-3: short training required of PWD/Caregiver (at clinic) } \\
\text { 1: lengthy/intensive training required of PWD/Caregiver, or at } \\
\text { academic center } \\
2-3: \text { Scheduled calls from case manager (1-2 if frequent; } 1 \text { if } \\
\text { frequent + case manager calls when needed) }\end{array}$ \\
\hline $\begin{array}{l}\text { Flexibility of } \\
\text { intervention: } \\
\text { adherence }\end{array}$ & & & $\begin{array}{l}\text { How different is the flexibility of intervention adherence } \\
\text { requirement from usual care? How rigorous are measures to } \\
\text { increase adherence? (Note: rate adherence studies too) } \\
\text { Example possible scores: } \\
\text { 5: usual encouragement; } \\
\text { 1-2: prompts/measures to improve adherence }\end{array}$ \\
\hline Follow-up & & & $\begin{array}{l}\text { How different is trial follow-up or measurement intensity from } \\
\text { usual care? Does trial follow-up (frequency, intensity, } \\
\text { content) result in care that differs from usual care? } \\
\text { Example possible scores } \\
\text { 5: measurement from usual follow up. } \\
\text { 3-4: in home assessment every } 6 \text { months by case manager } \\
\text { 1: extensive data collection, longer/more frequent clinic } \\
\text { visits, event(s) triggered visits }\end{array}$ \\
\hline $\begin{array}{l}\text { Primary } \\
\text { outcome }\end{array}$ & & & $\begin{array}{l}\text { To what extent is the primary outcome relevant to } \\
\text { participants? [5=obviously important; } 1=\text { =intermediate or } \\
\text { physiologic outcome, requires expert assessment, outcome } \\
\text { timing/measure differs from usual care] } \\
\text { Example possible scores: } \\
\text { 5: important to PWD and routinely assessed in usual care } \\
\text { 4-5: important to PWD and longer term } \\
\text { 3-4: composite primary outcome, some elements } \\
\text { unimportant to PWD } \\
\text { 2-3: important to PWD but measured earlier than usual } \\
\text { care/short-term } \\
\text { 1-2: assessment expertise differs from usual care; surrogate, } \\
\text { intermediate outcomes. }\end{array}$ \\
\hline $\begin{array}{l}\text { Primary } \\
\text { analysis }\end{array}$ & & & $\begin{array}{l}\text { To what extent are all data included in the analysis of the } \\
\text { primary outcome? } \\
\text { Example possible scores: } \\
\text { 4-5: ITT or modified ITT } \\
\text { 1-2: exclude PWD with low intervention adherence (when } \\
\text { adherence } \neq \text { an outcome) } \\
\text { 1-2: post hoc-derived subgroup analysis; secondary } \\
\text { endpoints } \\
\text { 1-2: data merged from > } 1 \text { study } \\
\text { 1: compliant completer analysis }\end{array}$ \\
\hline Applicability & & & $\begin{array}{l}\text { Population: PWD and/or PWD Caregivers: } \\
\text {-narrow or broadly generalizable for PWD? }\end{array}$ \\
\hline Qualifier(s) & & & $\begin{array}{l}\text { Setting/implementation: } \\
\text {-urban setting, practices with linked electronic health records } \\
\text {-health plan level with trained case managers } \\
\text {-modest vs. intensive electronic health record data extraction } \\
\text { required? }\end{array}$ \\
\hline
\end{tabular}




\begin{tabular}{|l|l|l|l|}
\hline Domain & $\begin{array}{l}\text { Score } \\
\mathbf{( 1 - 5 )}\end{array}$ & Rationale for Score & Modified Prompts, With Examples \\
\hline & & $\begin{array}{l}\text {-needs proprietary software } \\
\text {-costs not reported but startup likely intensive } \\
\end{array}$ & \\
& & $\begin{array}{l}\text {-costs not reported but likely feasible addition to usual care } \\
\text {-not likely feasible in US health system } \\
\text {-not likely feasible in (some) rural areas }\end{array}$ \\
\hline
\end{tabular}




\section{Appendix B. Excluded Studies}

\section{Could not locate}

1. Cameron H, du Toit S, Richard G, et al. Using lemon balm oil to reduce aggression and agitation in dementia: results of a pilot study. Journal of Dementia Care. 2011;19(5):36-8. PMID: 108258536. Language: English. Entry Date: 20111104. Revision Date: 20150712.

Publication Type: Journal Article.

2. Essence A. A double blind, placebo controlled trial of aromatherapy using melissa/lavender compared to aricept for the treatment of significant agitation in people with severe dementia.

3. L $\tilde{A}^{3}$ pez-Almela As, GÃ $\tilde{A}^{3}$ mez-Conesa A. Intervention in dementias by multisensory stimulation (snoezelen). In Fisioterapia

4. Lukaszewska B, Tomaszewski W, Mirski A, et al. An evaluation of social isolation of people with dementia of the alzheimer type in the process of neurorehabilitation. In Psychogeriatria polska

5. Lyu J, Gao T, Li M, et al. The effect of music therapy on memory, language and psychological symptoms of patients with mild Alzheimer's disease. In Chinese journal of neurology

6. Reuster T, Jurjanz L, Schutzwohl M, et al. A randomized controlled trial on occupational therapy for patients with dementia and their caregivers (ERGODEM). In Zeitschrift fur gerontopsychologie \& -psychiatrie 7. Yang D-L, Li X, Su N, et al. Family medical intervention model of senile dementia with behavioral and psychological symptoms. In Journal of shanghai jiaotong university (medical science)

\section{Not English language}

8. Correction: northern Manhattan Hispanic Caregiver Intervention Effectiveness Study: protocol of a pragmatic randomised trial comparing the effectiveness of two established interventions for informal caregivers of persons with dementia (BMJ Open (2016) 6 (e014082) DOI: 10.1136/bmjopen-2016-014082). In BMJ Open 9. Ban S-h, Jun S-S. Spaced Retrieval Effects in Older Adults with Mild Alzheimer's Disease. Korean Journal of Adult Nursing. 2012;24(4):398-405. PMID: 104400737. Language: English. Entry Date: 20130125. Revision Date: 20180305. Publication Type: Journal Article.

10. Bang M, Kim O. Effect of the Family Resilience Reinforcement Program for Family Caregivers of the Elderly with Dementia. In Korean j adult nurs 11. Chae K. Effects of Laughing and Music Therapy on Depression and Activities of the Autonomic Nervous System in the Elderly with Dementia. In J korean biol nurs sci

12. Chu J, Bao Y, Zou C. Effect of long-time retention of scalp needle on the abilities of cognition, daily living activity and P300 in vascular dementia patients. In Zhen CI yan jiu = acupuncture research

13. Delphin-Combe F, Rouch I, Martin-Gaujard G, et al. Effect of a non-pharmacological intervention, Voix d'Or( $\left({ }^{\circledR}\right)$, on behavior disturbances in Alzheimer disease and associated disorders. In Geriatrie ET psychologie neuropsychiatrie du vieillissement
14. Dijk A, Weert J, Droes R. Theatre as communication method in psychogeriatric care: effects on behaviour, mood and quality of life of people with dementia ]. [Dutch. In Tijdschrift voor gerontologie en geriatrie

15. Etxeberria AI, Garcia SA, Iglesias DSA, et al. Effects of training in emotional regulation strategies on the wellbeing of carers of Alzheimer patients]. [Spanish. In Revista espanola de geriatria y gerontologia

16. Fernández-Calvo B, Rodríguez-Pérez R, Contador I, et al. Efficacy of cognitive training programs based on new software technologies in patients with Alzheimer-type dementia. In Psicothema

17. Fischer-Terworth C, Probst P. Effects of a psychological group intervention on neuropsychiatric symptoms and communication in Alzheimer's dementia. In Z Gerontol Geriatr

18. Fleiner T, Dauth H, Zijlstra W, et al. Effects of a Daysstructured Training Program on mental and Behavioral Symptoms in Dementia Patients (BPSD) - Results of a randomized controlled Study. In Z Gerontol Geriatr 19. Garzon-Maldonado FJ, Gutierrez-Bedmar M, SerranoCastro V, et al. An assessesment of telephone assistance systems for caregivers of patients with Alzheimer's disease. Neurologia. 2017 Nov - Dec;32(9):595-601. doi: https://dx.doi.org/10.1016/j.nrl.2016.03.009. PMID: 27293022

20. Geschke K, Scheurich A, Schermuly I, et al. Effectivity of early psychosocial counselling for family caregivers in general practioner based dementia care. In Deutsche medizinische wochenschrift (1946) 21. Gok Ugur H, Orak OS, Yaman Aktas Y, et al. Effects of Music Therapy on the Care Burden of In-Home Caregivers and Physiological Parameters of Their In-Home Dementia Patients: A Randomized Controlled Trial. Complementary Med. 2018 Nov 30;30:30. doi: https://dx.doi.org/10.1159/000490348. PMID: 30497077. 22. Goudour A, Samson S, Bakchine S, et al. Semantic memory training in Alzheimer's disease. In Geriatrie ET psychologie neuropsychiatrie du vieillissement 23. Graff M, Vernooy-Dassen M, Thijssen M, et al. Home based ergotherapy in patients with dementia. In Nederlands tijdschrift ergotherapie

24. Grassel E, Behrndt E, Straubmeier M. Resourcesustaining therapy in dementia: the MAKS study. In Public health forum

25. Haberstroh J, Neumeyer K, Schmitz B, et al. Development and evaluation of a training program for nursing home professionals to improve communication in dementia care. In Z Gerontol Geriatr

26. Hong S. Effects of Multi-Component Exercise and Retraining after Detraining on Functional Fitness and Cognitive Function in Elder People with Mild Dementia. In Korean j health promot

27. Hori M, Kubota M, Ando K, et al. The effect of videophone communication (with skype and webcam)for elderly patients with dementia and their caregivers. In Gan to kagaku ryoho Cancer \& chemotherapy

28. Knight S, Singh I. Profile of inpatient falls in patients with dementia: a prospective comparative study between 
$100 \%$ single rooms and traditional multibedded wards. In Journal of clinical gerontology and geriatrics 29. Li X, Sun Y, Yan Y. Observation on effect of application of sleep restriction therapy for Alzheimer's disease patients with sleep disorders. In Chinese nursing research

30. Lopez N, Veliz A, Soto-Anari M, et al. Effects of a combined program of physical activity and cognitive training in Chilean patients with mild Alzheimer. In Neurologia argentina

31. Lukas A, Kilian R, Hay B, et al. Maintenance of health and relief for caregivers of elderly with dementia by using

"initial case management". Experiences from the lighthouse project on dementia, ulm, ultdem-study. Z Gerontol Geriatr. 2012 June;45(4):298-309. doi:

http://dx.doi.org/10.1007/s00391-012-0337-z. PMID: 51981666.

32. Menezes A, Aguiar A, Alves E, et al. Effectiveness of a cognitive-motorphysiotherapeutical therapy intervention in institutionalized older adults with mild cognitive impairment and mild dementia. In Ciencia \& saude coletiva 33. Morgenstern U, Ketelhut K, Rösler D. Concentration enhancement for retaining daily living competence in dementia. In Z Gerontol Geriatr

34. Muller B, Staudinger B. Evaluation of an inpatient multimodal treatment concept for people with dementia. In Pflegewissenschaft

35. Negovanska V, Hergueta T, Guichart-Gomez E, et al. Beneficial effect of a cognitive behavioral and multidisciplinary program in Alzheimer Disease on spouse caregiver anxiety: french study ELMMA. In Revue neurologique

36. Pickel S, Grässel E, Luttenberger K. Efficacy of an occupational group therapy in degenerative dementias: a controlled study in the nursing home setting. In Psychiatrische praxis

37. Pitteri F, Soulas T, Essertaise A, et al. Contribution of relaxation to a psychoeducational intervention program for family carers of persons with Alzheimer's disease. In Geriatrie ET psychologie neuropsychiatrie du vieillissement

38. Quibel C, Bonin M, Bonnet M, et al. Evaluation of the animal-assisted therapy in Alzheimer's disease. In Soins Gerontologie

39. Ravari A, Mirzaei T, Salamizadeh A, et al. Effect of the spiritual care training on anxiety reduction in home caregivers of the elderly with alzheimer disease. In Koomesh

40. Rexhausen E. Psychosocial care assistants caring for residents with dementia according to section 87b SGB XI. Emotional and cognitive effects. In Pflege zeitschrift 41. SeungHyun C, Young-ae Y. The Effect of Occupational Therapy Based Multimodal Cognitive Rehabilitation Therapy on Cognitive Function in Elderly People With Mild Dementia: a Randomized Controlled Trial.

42. Song J. Effects of a robot pet-assisted program for elderly people with dementia. In Journal of korean academy of nursing

43. Steinert T, Bohnet U, Flammer E, et al. Effects of a training of power and balance on the use of mechanical restraint among in-patients with dementia. In

Psychiatrische praxis

44. Stemmer R, Grassel E, Schmid M. Individual activation of dementia sufferers in a home setting. A randomized controlled study. Z Gerontol Geriatr. 2019;52(3):256-63. PMID: CN-01954578.

45. Tannen A. Effectiveness of a case management program for the elderly in early stage dementia and their caregivers. In Pflege zeitschrift

46. Todri J, Lena O, Martinez Gil JL. A single blind randomized controlled trial of global postural re-education: Cognitive effects on Alzheimer disease patients. European Journal of Psychiatry. 2019. doi:

http://dx.doi.org/10.1016/j.ejpsy.2019.01.001. PMID: 2001581733.

47. Wan C, Hu Z, Sun Y. Application of acupoint massage in nursing care of community dementia patients. In Chinese nursing research

48. Wang Y, Chiu C, Chen Y, et al. The efficacy of multisensory environment therapy on psychological, behavioral symptoms among elderly patients with dementia]. [Chinese. In Hu li za zhi [journal of nursing] 49. Zhang HZLHCHKLJ. Clinically multi-central randomized controlled study on scalp electroacupuncture for treatment of vascular dementia. In Zhongguo zhen jiu [Chinese acupuncture \& moxibustion]

50. Zhou Y, Han H, Jia J. Correlation analysis on changes between cognitive ability and brain fMRI after acupoint thread embedding in Alzheimer's disease patients. In Zhongguo zhong xi yi jie he za zhi zhongguo zhongxiyi jiehe zazhi $=$ chinese journal of integrated traditional and western medicine

\section{Ineligible Intervention}

51. Aasmul I, Husebo BS, Flo E. Staff Distress Improves by Treating Pain in Nursing Home Patients With Dementia: Results From a Cluster-Randomized Controlled Trial. J Pain Symptom Manage. 2016 Dec;52(6):795-805. doi: https://dx.doi.org/10.1016/j.jpainsymman.2016.07.004. PMID: 27524403.

52. Adrait A, Perrot X, Nguyen MF, et al. Do Hearing

Aids Influence Behavioral and Psychological Symptoms of Dementia and Quality of Life in Hearing Impaired Alzheimer's Disease Patients and Their Caregivers? J Alzheimers Dis. 2017;58(1):109-21. doi: https://dx.doi.org/10.3233/JAD-160792. PMID: 28269769. 53. Allen V, Methven L, Gosney M. The influence of nutritional supplement drinks on providing adequate calorie and protein intake in older adults with dementia. In J Nutr Health Aging

54. Atri A, Shaughnessy LWLJJGJH. Long-term course and effectiveness of combination therapy in Alzheimer disease. In Alzheimer disease and associated disorders 55. Ayalon L, Ancoli-Israel S, Stepnowsky C, et al. Adherence to continuous positive airway pressure treatment in patients with Alzheimer's disease and obstructive sleep apnea. Am J Geriatr Psychiatry. 2006 Feb;14(2):176-80. PMID: 16473983.

56. Beer LE, Hutchinson SR, Skala-Cordes KK. Communicating with patients who have advanced dementia: training nurse aide students. Gerontol Geriatr 
Educ. 2012;33(4):402-20. doi: https://dx.doi.org/10.1080/02701960.2012.702165. PMID: 23095223.

57. Bellantonio S, Kenny AM, Fortinsky RH, et al. Efficacy of a geriatrics team intervention for residents in dementia-specific assisted living facilities: effect on unanticipated transitions. J Am Geriatr Soc. 2008 Mar;56(3):523-8. doi: https://dx.doi.org/10.1111/j.15325415.2007.01591.x. PMID: 18179497.

58. Chan A, Paskavitz J, Remington R, et al. Efficacy of a vitamin/nutriceutical formulation for early-stage Alzheimer's disease: a 1-year, open-label pilot study with an 16-month caregiver extension. Am J Alzheimers Dis Other Demen. 2008 Dec-2009 Jan;23(6):571-85. doi: https://dx.doi.org/10.1177/1533317508325093. PMID: 19047474.

59. Chodosh J, Berry E, Lee M, et al. Effect of a dementia care management intervention on primary care provider knowledge, attitudes, and perceptions of quality of care. J Am Geriatr Soc. 2006 Feb;54(2):311-7. PMID: 43419419. 60. Cooke JR, Ayalon L, Palmer BW, et al. Sustained use of CPAP slows deterioration of cognition, sleep, and mood in patients with Alzheimer's disease and obstructive sleep apnea: a preliminary study. J Clin Sleep Med. 2009 Aug 15;5(4):305-9. PMID: 19968005.

61. Culo S, Mulsant BH, Rosen J, et al. Treating neuropsychiatric symptoms in dementia with Lewy bodies: a randomized controlled-trial. Alzheimer Dis Assoc Disord. 2010 Oct-Dec;24(4):360-4. doi: https://dx.doi.org/10.1097/WAD.0b013e3181e6a4d7. PMID: 20625270.

62. de Boer B, Hamers JP, Zwakhalen SM, et al. Green Care Farms as Innovative Nursing Homes, Promoting Activities and Social Interaction for People With Dementia. J Am Med Dir Assoc. 2017 01;18(1):40-6. doi: https://dx.doi.org/10.1016/j.jamda.2016.10.013. PMID: 28012503.

63. Donath C, Grassel E, Grossfeld-Schmitz M, et al. Effects of general practitioner training and family support services on the care of home-dwelling dementia patients-results of a controlled cluster-randomized study. BMC Health Serv Res. 2010 Nov 18;10:314. doi: https://dx.doi.org/10.1186/1472-6963-10-314. PMID: 21087474.

64. Dopp CM, Graff MJ, Teerenstra S, et al. A new combined strategy to implement a community occupational therapy intervention: designing a cluster randomized controlled trial. BMC geriatr. 2011 Mar 30;11:13. doi: https://dx.doi.org/10.1186/1471-2318-11-13. PMID: 21450063.

65. Dopp CM, Graff MJ, Teerenstra S, et al. Effectiveness of a multifaceted implementation strategy on physicians' referral behavior to an evidence-based psychosocial intervention in dementia: a cluster randomized controlled trial. BMC Fam Pract. 2013 May 30;14:70. doi: https://dx.doi.org/10.1186/1471-2296-14-70. PMID: 23718565.

66. Dopp CM, Graff MJ, Teerenstra S, et al. Effectiveness of a training package for implementing a community-based occupational therapy program in dementia: a cluster randomized controlled trial. Clin Rehabil. 2015 Oct;29(10):974-86. doi: https://dx.doi.org/10.1177/0269215514564699. PMID: 25547113.

67. Fabris F, Molaschi M, Aimonino N, et al. Home care for demented subjects: new models of care and home-care allowance. Arch Gerontol Geriatr Suppl. 2004(9):155-62. PMID: 15207409.

68. Gil Gregorio P, Ramirez Diaz SP, Ribera Casado JM, et al. Dementia and Nutrition. Intervention study in institutionalized patients with Alzheimer disease. J Nutr Health Aging. 2003;7(5):304-8. PMID: 12917745.

69. Gustavsson A, Cattelin F, Jonsson L. Costs of care in a mild-to-moderate Alzheimer clinical trial sample: key resources and their determinants. Alzheimer's dement. 2011 Jul;7(4):466-73. doi:

https://dx.doi.org/10.1016/j.jalz.2010.06.002. PMID: 21784355.

70. Hinchliffe AC, Katona C, Livingston G. The assessment and management of behavioural manifestations of dementia: a review and results of a controlled trial. International Journal of Psychiatry in Clinical Practice. 1997;1(3):157-68. doi:

https://dx.doi.org/10.3109/13651509709024722. PMID: 24940831.

71. Hincliffe AC, Hyman IL, Blizard B, et al. Behavioural complications of dementia - Can they be treated? Int J Geriatr Psychiatry. 1995;10(10):839-47. doi: http://dx.doi.org/10.1002/gps.930101005. PMID: 25311941.

72. Holle D, Roes M, Buscher I, et al. Process evaluation of the implementation of dementia-specific case conferences in nursing homes (FallDem): study protocol for a randomized controlled trial. Trials. 2014 Dec 11;15:485. doi: https://dx.doi.org/10.1186/1745-6215-15485. PMID: 25496425.

73. Huizing AR, Hamers JP, Gulpers MJ, et al. Short-term effects of an educational intervention on physical restraint use: a cluster randomized trial. BMC geriatr. 2006 Oct 26;6:17. PMID: 17067376.

74. Iliffe S, Wilcock J, Griffin M, et al. Evidence-based interventions in dementia: A pragmatic cluster-randomised trial of an educational intervention to promote earlier recognition and response to dementia in primary care (EVIDEM-ED). Trials. 2010 Feb 10;11:13. doi: https://dx.doi.org/10.1186/1745-6215-11-13. PMID: 20146803.

75. Jordan S, Gabe-Walters ME, Watkins A, et al. NurseLed Medicines' Monitoring for Patients with Dementia in Care Homes: A Pragmatic Cohort Stepped Wedge Cluster Randomised Trial. PLoS ONE. 2015;10(10):e0140203. doi: https://dx.doi.org/10.1371/journal.pone.0140203. PMID: 26461064.

76. Judge TG, Urquhart A. Naftidrofuryl--a double blind cross-over study in the elderly. Curr Med Res Opin. 1972;1(3):166-72. PMID: 4589711.

77. Kok J, Berg I, Blankevoort G, et al. Rest-activity rhythms in small scale homelike care and traditional care for residents with dementia. In BMC geriatr 78. Kotynia-English R, McGowan H, Almeida OP. A randomized trial of early psychiatric intervention in residential care: impact on health outcomes. Int Psychogeriatr. 2005 Sep;17(3):475-85. PMID: 16252379. 
79. Lea E, Marlow A, Bramble M, et al. Learning opportunities in a residential aged care facility: the role of supported placements for first-year nursing students. J Nurs Educ. 2014 Jul 01;53(7):410-4. doi:

https://dx.doi.org/10.3928/01484834-20140620-02. PMID: 24972402.

80. Lee K, Cho S-J, Kim B, et al. Caregiver Preference and Treatment Compliance in Patients with Mild-to-Moderate Alzheimer's Disease in South Korea: RECAP Study Results. In Adv Ther

81. Livingston G, Baio G, Sommerlad A, et al. Effectiveness of an intervention to facilitate prompt referral to memory clinics in the United Kingdom: Cluster randomised controlled trial. PLoS Medicine. 2017 March;14 (3) (no pagination)(e1002252). doi: http://dx.doi.org/10.1371/journal.pmed.1002252. PMID: 615141552.

82. Logiudice D, Waltrowicz W, Brown K, et al. Do memory clinics improve the quality of life of carers? A randomized pilot trial. Int J Geriatr Psychiatry. 1999 Aug;14(8):626-32. PMID: 10489653.

83. Maguire A, Rosato M, O'Reilly D. Mental health and morbidity of caregivers and co-residents of individuals with dementia: a quasi-experimental design. Int J Geriatr Psychiatry. 2017 Oct;32(10):1104-13. doi: https://dx.doi.org/10.1002/gps.4573. PMID: 27594205. 84. Meeuwsen EJ, Melis RJ, Van Der Aa GC, et al. Effectiveness of dementia follow-up care by memory clinics or general practitioners: randomised controlled trial. Bmj. 2012 May 15;344:e3086. doi: https://dx.doi.org/10.1136/bmj.e3086. PMID: 22589500. 85. Menn P, Holle R, Kunz S, et al. Dementia care in the general practice setting: a cluster randomized trial on the effectiveness and cost impact of three management strategies. Value Health. 2012 Sep-Oct;15(6):851-9. doi: https://dx.doi.org/10.1016/j.jval.2012.06.007. PMID: 22999135.

86. Nourhashemi F, Andrieu S, Gillette-Guyonnet S, et al. Effectiveness of a specific care plan in patients with Alzheimer's disease: cluster randomised trial (PLASA study). Bmj. 2010 Jun 03;340:c2466. doi: https://dx.doi.org/10.1136/bmj.c2466. PMID: 20522656. 87. Pai M-C, Aref H, Bassil N, et al. Real-world evaluation of compliance and preference in Alzheimer's disease treatment. In Clin Interv Aging

88. Peng X, Dong K. Clinical observation on acupuncture combined with Yizhi Jiannao granules for treatment of Alzheimer's disease. In Zhongguo zhen jiu [Chinese acupuncture \& moxibustion]

89. Pivi GA, da Silva RV, Juliano Y, et al. A prospective study of nutrition education and oral nutritional supplementation in patients with Alzheimer's disease. Nutr J. 2011 Sep 26;10:98. doi: https://dx.doi.org/10.1186/14752891-10-98. PMID: 21943331.

90. Pond D, Mate K, Stocks N, et al. Effectiveness of a peer-mediated educational intervention in improving general practitioner diagnostic assessment and management of dementia: A cluster randomised controlled trial. BMJ Open. 201801 Aug;8 (8) (no pagination)(e021125). doi: http://dx.doi.org/10.1136/bmjopen-2017-021125. PMID: 623793422.
91. Shikiar R, Shakespeare A, Sagnier PP, et al. The impact of metrifonate therapy on caregivers of patients with Alzheimer's disease: Results from the MALT clinical trial. J Am Geriatr Soc. 2000 March;48(3):268-74. PMID: 30164209.

92. Silverberg GD, Levinthal E, Sullivan EV, et al. Assessment of low-flow CSF drainage as a treatment for AD: results of a randomized pilot study. Neurology. 2002 Oct 22;59(8):1139-45. PMID: 12391340.

93. Silverberg GD, Mayo M, Saul T, et al. Continuous CSF drainage in AD: results of a double-blind, randomized, placebo-controlled study. Neurology. 2008 Jul 15;71(3):202-9. doi:

https://dx.doi.org/10.1212/01.wnl.0000316197.04157.6f. PMID: 18525029.

94. Smeets CH, Smalbrugge M, Gerritsen DL, et al. Improving psychotropic drug prescription in nursing home patients with dementia: design of a cluster randomized controlled trial. BMC Psychiatry. 2013 Nov 01;13:280. doi: https://dx.doi.org/10.1186/1471-244X-13-280. PMID: 24180295.

95. Sobów T, K?oszewska I, Flirski M, et al. Predictors of long-term treatment effect of rivastigmine in Alzheimer's disease: a role for beta-amyloid plasma levels? In Neurologia i neurochirurgia polska

96. Steen J, Ribbe M. Pneumonia mortality risk in patients with dementia: nursing home physicians' use and evaluation of a prognostic score. In Tijdschrift voor gerontologie en geriatrie

97. Swanson EA, Maas ML, Buckwalter KC. Alzheimer's residents' cognitive and functional measures: special and traditional care unit comparison. Clin Nurs Res. 1994 Feb;3(1):27-41. PMID: 8167575.

98. Testad I, Aasland AM, Aarsland D. The effect of staff training on the use of restraint in dementia: a single-blind randomised controlled trial. Int J Geriatr Psychiatry. 2005 Jun;20(6):587-90. PMID: 15920716.

99. Testad I, Ballard C, Bronnick K, et al. The effect of staff training on agitation and use of restraint in nursing home residents with dementia: a single-blind, randomized controlled trial. J Clin Psychiatry. 2010 Jan;71(1):80-6. doi: https://dx.doi.org/10.4088/JCP.09m05486oli. PMID: 20129008.

100. Testad I, Mekki TE, Forland O, et al. Modeling and evaluating evidence-based continuing education program in nursing home dementia care (MEDCED)--training of care home staff to reduce use of restraint in care home residents with dementia. A cluster randomized controlled trial. Int J Geriatr Psychiatry. 2016 Jan;31(1):24-32. doi: https://dx.doi.org/10.1002/gps.4285. PMID: 25845462. 101. Tian JZ, Shi J, Ni JN, et al. Sequential Therapy Based on Evolvement of Patterns: A New Model for Treatment of Alzheimer's Disease. Chin J Integr Med. 2019 Aug;25(8):565-73. doi: https://dx.doi.org/10.1007/s11655019-3066-y. PMID: 31069693.

102. van Uffelen JG, Chin APMJ, Hopman-Rock M, et al. The effect of walking and vitamin B supplementation on quality of life in community-dwelling adults with mild cognitive impairment: a randomized, controlled trial. Qual Life Res. 2007 Sep;16(7):1137-46. PMID: 17616840. 103. Viola S, Viola P, Buongarzone $M$, et al. New brain reperfusion rehabilitation therapy improves cognitive 
impairment in mild alzheimer's disease: a prospective, controlled, open-label 12-month study with nirs correlates. In Aging clinical and experimental research

104. Vollmar H, Grassel E, Lauterberg J, et al. Multimodal training of general practitioners--evaluation and knowledge increase within the framework of the dementia management initiative in general medicine (IDA)]. [German. In Zeitschrift fur arztliche fortbildung und qualitatssicherung

105. Vollmar H, Mayer H, Ostermann T, et al. Knowledge transfer for the management of dementia: a clusterrandomised trial of blended learning in general practice. In Implement Sci

106. Vollmar HC, Butzlaff ME, Lefering R, et al. Knowledge translation on dementia: a cluster randomized trial to compare a blended learning approach with a "classical" advanced training in GP quality circles. BMC Health Serv Res. 2007 Jun 22;7:92. PMID: 17587452. 107. Waldorff FB, Siersma V, Nielsen B, et al. The effect of reminder letters on the uptake of an e-learning programme on dementia: a randomized trial in general practice. Fam Pract. 2009 Dec;26(6):466-71. doi: https://dx.doi.org/10.1093/fampra/cmp072. PMID: 19858123.

108. Wang F, Xiao LD, Wang K, et al. Evaluation of a WeChat-based dementia-specific training program for nurses in primary care settings: A randomized controlled trial. Appl Nurs Res. 2017 Dec;38:51-9. doi: https://dx.doi.org/10.1016/j.apnr.2017.09.008. PMID: 29241520.

109. Weiner MF, Tractenberg RE, Sano M, et al. No longterm effect of behavioral treatment on psychotropic drug use for agitation in Alzheimer's disease patients. J Geriatr Psychiatry Neurol. 2002;15(2):95-8. PMID: 12083600. 110. Wimo A, Winblad B, Shah SN, et al. Impact of donepezil treatment for Alzheimer's disease on caregiver time. Curr Med Res Opin. 2004 Aug;20(8):1221-5. PMID: 15324524.

111. Winblad B, Kawata AK, Beusterien KM, et al. Caregiver preference for rivastigmine patch relative to capsules for treatment of probable Alzheimer's disease. Int J Geriatr Psychiatry. 2007 May;22(5):485-91. PMID: 17407176.

112. Wolfs CA, Kessels A, Dirksen CD, et al. Integrated multidisciplinary diagnostic approach for dementia care: randomised controlled trial. Br J Psychiatry. 2008 Apr;192(4):300-5. doi:

https://dx.doi.org/10.1192/bjp.bp.107.035204. PMID: 18378994.

\section{No Eligible Outcomes}

113. Ancoli-Israel S, Gehrman P, Martin JL, et al. Increased light exposure consolidates sleep and strengthens circadian rhythms in severe Alzheimer's disease patients. Behav Sleep Med. 2003;1(1):22-36. PMID: 15600135. 114. Ancoli-Israel S, Martin JL, Kripke DF, et al. Effect of light treatment on sleep and circadian rhythms in demented nursing home patients. J Am Geriatr Soc. 2002

Feb;50(2):282-9. PMID: 12028210.

115. Andrade L, Gobbi L, Coelho F, et al. Benefits of multimodal exercise intervention for postural control and frontal cognitive functions in individuals with Alzheimer's disease: a controlled trial. In J Am Geriatr Soc 116. Aukner C, Eide HD, Iversen PO. Nutritional status among older residents with dementia in open versus special care units in municipal nursing homes: an observational study. BMC geriatr. 2013 Mar 14;13:26. doi: https://dx.doi.org/10.1186/1471-2318-13-26. PMID: 23496975.

117. Black DS, Cole SW, Irwin MR, et al. Yogic meditation reverses NF-kappaB and IRF-related transcriptome dynamics in leukocytes of family dementia caregivers in a randomized controlled trial.

Psychoneuroendocrinology. 2013 Mar;38(3):348-55. doi: https://dx.doi.org/10.1016/j.psyneuen.2012.06.011. PMID: 22795617.

118. Bossers WJR, van der Woude LHV, Boersma F, et al. A 9-Week Aerobic and Strength Training Program Improves Cognitive and Motor Function in Patients with Dementia: A Randomized, Controlled Trial. Am J Geriatr Psychiatry. 2015;23(11):1106-16. doi:

10.1016/j.jagp.2014.12.191. PMID: 111672087. Language: English. Entry Date: 20160911. Revision Date: 20180629. Publication Type: journal article.

119. Brennan PF. Computer networks promote caregiving collaboration: the ComputerLink Project. Proc Annu Symp Comput Appl Med Care. 1992:156-60. PMID: 1482859.

120. Camargo CH, Justus FF, Retzlaff G. The

effectiveness of reality orientation in the treatment of Alzheimer's disease. Am J Alzheimers Dis Other Demen. 2015 Aug;30(5):527-32. doi:

https://dx.doi.org/10.1177/1533317514568004. PMID: 25588408 .

121. Cavallo M, Angilletta C. Long-Lasting Neuropsychological Effects of a Computerized Cognitive Training in Patients Affected by Early Stage Alzheimer's Disease: Are They Stable Over Time? J Appl Gerontol. 2018 Jan 01:733464817750276. doi: https://dx.doi.org/10.1177/0733464817750276. PMID: 29295652.

122. Cheng ST, Chan AC, Yu EC. An exploratory study of the effect of mahjong on the cognitive functioning of persons with dementia. Int J Geriatr Psychiatry. 2006 Jul;21(7):611-7. PMID: 16779765.

123. Cheng ST, Chow PK, Song YQ, et al. Mental and physical activities delay cognitive decline in older persons with dementia. Am J Geriatr Psychiatry. 2014

Jan;22(1):63-74. doi:

https://dx.doi.org/10.1016/j.jagp.2013.01.060. PMID: 23582750 .

124. Cheng ST, Chow PK, Song YQ, et al. Can leisure activities slow dementia progression in nursing home residents? A cluster-randomized controlled trial. Int Psychogeriatr. 2014 Apr;26(4):637-43. doi: https://dx.doi.org/10.1017/S1041610213002524. PMID: 24411480 .

125. Cheung DSK, Li B, Lai DWL, et al. Cognitive Stimulating Play Intervention for Dementia: A Feasibility Randomized Controlled Trial. Am J Alzheimers Dis Other Demen. 2019 Feb;34(1):63-71. doi: https://dx.doi.org/10.1177/1533317518808036. PMID: 30370782. 
126. Chiverton P, Caine ED. Education to assist spouses in coping with Alzheimer's disease: a controlled trial. J Am Geriatr Soc. 1989:593-8. PMID: 107540167. Language: English. Entry Date: 19891201. Revision Date: 20150712. Publication Type: Journal Article.

127. Christofoletti G, Oliani M, Gobbi S, et al. A controlled clinical trial on the effects of motor intervention on balance and cognition in institutionalized elderly patients with dementia. In Clin Rehabil 128. Coelho FGdM, Andrade LP, Pedroso RV, et al. Multimodal exercise intervention improves frontal cognitive functions and gait in Alzheimer's disease: A controlled trial. Geriatr Gerontol Int. 2013;13(1):198-203. doi: 10.1111/j.1447-0594.2012.00887.x. PMID: 104404885. Language: English. Entry Date: 20130110. Revision Date: 20150711. Publication Type: Journal Article. 129. Cotelli M, Adenzato M, Cantoni V, et al. Enhancing theory of mind in behavioural variant frontotemporal dementia with transcranial direct current stimulation. Cogn Affect Behav Neurosci. 2018 Dec;18(6):1065-75. doi: https://dx.doi.org/10.3758/s13415-018-0622-4. PMID: 29995274.

130. Cotelli M, Manenti R, Cappa S, et al. Transcranial magnetic stimulation improves naming in Alzheimer disease patients at different stages of cognitive decline. In Eur J Neurol

131. Dijkstra K, Bourgeois M, Burgio L, et al. Effects of a communication intervention on the discourse of nursing home residents with dementia and their nursing assistants. Journal of Medical Speech-Language Pathology.

2002;10(2):143-57. PMID: 106787750. Language: English. Entry Date: 20031212. Revision Date: 20150711.

Publication Type: Journal Article.

132. Eggermont LH, Swaab DF, Hol EM, et al. Walking the line: a randomised trial on the effects of a short term walking programme on cognition in dementia. J Neurol Neurosurg Psychiatry. 2009 Jul;80(7):802-4. doi: https://dx.doi.org/10.1136/jnnp.2008.158444. PMID: 19531688.

133. Eggermont LH, Swaab DF, Hol EM, et al. Walking the line: a randomised trial on the effects of a short term walking programme on cognition in dementia. J Neurol Neurosurg Psychiatry. 2009;80(7):802-4. doi:

10.1136/jnnp.2008.158444. PMID: 105369423. Language: English. Entry Date: 20090814. Revision Date: 20171118. Publication Type: journal article.

134. Eggermont LHP, Blankevoort CG, Scherder EJA. Walking and night-time restlessness in mild-to-moderate dementia: a randomized controlled trial. Age Ageing. 2010;39(6):746-9. PMID: 105008895. Language: English. Entry Date: 20101027. Revision Date: 20150711. Publication Type: Journal Article.

135. Elder GJ, Colloby SJ, Firbank MJ, et al. Consecutive sessions of transcranial direct current stimulation do not remediate visual hallucinations in Lewy body dementia: a randomised controlled trial. Alzheimers Res Ther. 2019 Jan 18;11(1):9. doi: https://dx.doi.org/10.1186/s13195-0180465-9. PMID: 30658705.

136. Epstein C, Auclair U, Mittelman M. Couples Counseling in Alzheimer's Disease: First Observations of a Novel Intervention Study. Clinical Gerontologist: The
Journal of Aging and Mental Health. 2007;30(2):21-35. doi: http://dx.doi.org/10.1300/J018v30n02_03. PMID: 2007-01997-003.

137. Eritz H, Hadjistavropoulos T, Williams J, et al. A life history intervention for individuals with dementia: a randomised controlled trial examining nursing staff empathy, perceived patient personhood and aggressive behaviours. In Ageing and society

138. Finnema E, de Lange J, Dröes R, et al. The quality of nursing home care: do the opinions of family members change after implementation of emotion-oriented care? J Adv Nurs. 2001;35(5):728-40. doi: 10.1046/j.13652648.2001.01905.x. PMID: 107069044. Language: English. Entry Date: 20050425. Revision Date: 20151008. Publication Type: Journal Article.

139. Frederiksen KS, Larsen CT, Hasselbalch SG, et al. A 16-Week Aerobic Exercise Intervention Does Not Affect Hippocampal Volume and Cortical Thickness in Mild to Moderate Alzheimer's Disease. Front Aging Neurosci. 2018;10:293. doi: https://dx.doi.org/10.3389/fnagi.2018.00293. PMID: 30319397.

140. Gallagher-Thompson D, Singer LS, Depp C, et al. Effective recruitment strategies for Latino and Caucasian dementia family caregivers in intervention research. Am J Geriatr Psychiatry. 2004 Sep-Oct;12(5):484-90. PMID: 15353386.

141. Gao H, Yan L, Liu B, et al. Clinical study on treatment of senile vascular dementia by acupuncture. J Tradit Chin Med. 2001 Jun;21(2):103-9. PMID: 11498896. 142. Garland K, Beer E, Eppingstall B, et al. A comparison of two treatments of agitated behavior in nursing home residents with dementia: simulated family presence and preferred music. Am J Geriatr Psychiatry. 2007

Jun;15(6):514-21. PMID: 17293386.

143. Garrido S, Stevens CJ, Chang E, et al. Musical Features and Affective Responses to Personalized Playlists in People With Probable Dementia. American Journal of Alzheimer's Disease and other Dementias. 2018. doi: http://dx.doi.org/10.1177/1533317518808011. PMID: 625754432.

144. Gaugler JE, Zmora R, Mitchell LL, et al. Six-Month Effectiveness of Remote Activity Monitoring for Persons Living With Dementia and Their Family Caregivers: An Experimental Mixed Methods Study. The Gerontologist. 201909 Jan;59(1):78-89. doi: http://dx.doi.org/10.1093/geront/gny078. PMID: 625875732.

145. Graf A, Wallner C, Schubert V, et al. The effects of light therapy on mini-mental state examination scores in demented patients. Biol Psychiatry. 2001 Nov 01;50(9):725-7. PMID: 11704081.

146. Hahm SC, Suh HR, Cho HY. The effect of transcutaneous electrical nerve stimulation on pain, muscle strength, balance, and gait in individuals with dementia: A double blind, pilot randomized controlled trial. European Journal of Integrative Medicine. 2019 August;29 (no pagination)(100932). doi: http://dx.doi.org/10.1016/j.eujim.2019.100932. PMID: 2002272126.

147. Harris M, Richards KC, Grando VT. The effects of slow-stroke back massage on minutes of nighttime sleep in 
persons with dementia and sleep disturbances in the nursing home: a pilot study. J Holist Nurs. 2012 Dec;30(4):255-63. doi: https://dx.doi.org/10.1177/0898010112455948. PMID: 23007716.

148. Heesterbeek M, van der Zee EA, van Heuvelen MJG. Feasibility of Three Novel Forms of Passive Exercise in a Multisensory Environment in Vulnerable Institutionalized Older Adults with Dementia. J Alzheimers Dis. 2019;70(3):681-90. doi: https://dx.doi.org/10.3233/JAD190309. PMID: 31256137.

149. Hoeffer B, Talerico KA, Rasin J, et al. Assisting cognitively impaired nursing home residents with bathing: effects of two bathing interventions on caregiving. Gerontologist. 2006 Aug;46(4):524-32. PMID: 16921006. 150. Innes KE, Selfe TK, Brundage K, et al. Effects of Meditation and Music-Listening on Blood Biomarkers of Cellular Aging and Alzheimer's Disease in Adults with Subjective Cognitive Decline: An Exploratory Randomized Clinical Trial. J Alzheimers Dis. 2018;66(3):947-70. doi: https://dx.doi.org/10.3233/JAD-180164. PMID: 30320574. 151. Ismail S, Christopher G, Dodd E, et al. Psychological and Mnemonic Benefits of Nostalgia for People with Dementia. J Alzheimers Dis. 2018;65(4):1327-44. doi: https://dx.doi.org/10.3233/JAD-180075. PMID: 30149444. 152. Jelcic N, Agostini M, Meneghello F, et al. Feasibility and efficacy of cognitive telerehabilitation in early Alzheimer's disease: a pilot study. Clin Interv Aging. 2014;9:1605-11. doi:

https://dx.doi.org/10.2147/CIA.S68145. PMID: 25284993. 153. Jelcic N, Cagnin A, Meneghello F, et al. Effects of lexical-semantic treatment on memory in early Alzheimer disease: an observer-blinded randomized controlled trial. Neurorehabil Neural Repair. 2012 Oct;26(8):949-56. doi: https://dx.doi.org/10.1177/1545968312440146. PMID: 22460609.

154. Johnson D, Niedens M, Wilson J, et al. Treatment outcomes of a crisis intervention program for dementia with severe psychiatric complications: the Kansas bridge project. In Gerontologist

155. Karssemeijer EGA, Aaronson JA, Bossers WJR, et al. The quest for synergy between physical exercise and cognitive stimulation via exergaming in people with dementia: a randomized controlled trial. Alzheimers Res Ther. 2019 Jan 05;11(1):3. doi:

https://dx.doi.org/10.1186/s13195-018-0454-z. PMID: 30611286.

156. Kawashima R. Mental exercises for cognitive function: clinical evidence. J Prev Med Pub Health. 2013 Jan;46 Suppl 1:S22-7. doi: https://dx.doi.org/10.3961/jpmph.2013.46.S.S22. PMID: 23412645.

157. Kemoun G, Thibaud M, Roumagne N, et al. Effects of a physical training programme on cognitive function and walking efficiency in elderly persons with dementia. Dement Geriatr Cogn Disord. 2010;29(2):109-14. doi: 10.1159/000272435. PMID: 105169833. Language: English. Entry Date: 20100604. Revision Date: 20150711. Publication Type: Journal Article.

158. Khedr E, Foly EGN, Abo E-FN, et al. Transcranial direct current stimulation and Alzheimer's disease. In Clin Neurophysiol
159. Kidd LI, Zauszniewski JA, Morris DL. Benefits of a poetry writing intervention for family caregivers of elders with dementia. Issues Ment Health Nurs. 2011;32(9):598604. doi:

https://dx.doi.org/10.3109/01612840.2011.576801. PMID: 21859411.

160. Kim MJ, Han CW, Min KY, et al. Physical Exercise with Multicomponent Cognitive Intervention for Older Adults with Alzheimer's Disease: A 6-Month Randomized Controlled Trial. Dement Geriatr Cogn Dis Extra. 2016 May-Aug;6(2):222-32. doi: https://dx.doi.org/10.1159/000446508. PMID: 27403134. 161. Kuhn J, Hardenacke K, Lenartz D, et al. Deep brain stimulation of the nucleus basalis of Meynert in Alzheimer's dementia. Mol Psychiatry. 201512 Mar;20(3):353-60. doi: http://dx.doi.org/10.1038/mp.2014.32. PMID: 53127239. 162. Laffan A, Metzler-Baddeley C, Walker I, et al. Making errorless learning more active: self-generation in an error free learning context is superior to standard errorless learning of face-name associations in people with Alzheimer's disease. In Neuropsychol

163. Lee M, Cho B, Min G, et al. Effects of therapeutic recreation on the brain quotient in the elderly dementia patients. In J Phys Ther Sci

164. Lemke NC, Werner C, Wiloth S, et al. Transferability and Sustainability of Motor-Cognitive Dual-Task Training in Patients with Dementia: A Randomized Controlled Trial. Gerontology. 2019;65(1):68-83. doi:

https://dx.doi.org/10.1159/000490852. PMID: 30041173.

165. Mortimer JA, Ding D, Borenstein AR, et al. Changes in brain volume and cognition in a randomized trial of exercise and social interaction in a community-based sample of non-demented Chinese elders. J Alzheimers Dis. 2012;30(4):757-66. doi: https://dx.doi.org/10.3233/JAD2012-120079. PMID: 22451320.

166. Moyle W, Cooke ML, Beattie E, et al. Foot Massage and Physiological Stress in People with Dementia: A Randomized Controlled Trial. J Altern Complement Med. 2014;20(4):305-11. doi: 10.1089/acm.2013.0177. PMID: 103928995. Language: English. Entry Date: 20140418. Revision Date: 20171010. Publication Type: Journal Article.

167. Nousia A, Siokas V, Aretouli E, et al. Beneficial Effect of Multidomain Cognitive Training on the Neuropsychological Performance of Patients with EarlyStage Alzheimer's Disease. Neural Plast.

2018;2018:2845176. doi:

https://dx.doi.org/10.1155/2018/2845176. PMID: 30123243.

168. Pahlavanzadeh S, Heidari FG, Maghsudi J, et al. The effects of family education program on the caregiver burden of families of elderly with dementia disorders. Iran J Nurs Midwifery Res. 2010;15(3):102-8. PMID: 21589771.

169. Pedrinolla A, Venturelli M, Fonte C, et al. Exercise Training on Locomotion in Patients with Alzheimer's Disease: A Feasibility Study. J Alzheimers Dis. 2018;61(4):1599-609. doi: https://dx.doi.org/10.3233/JAD170625. PMID: 29376858.

170. Perren S, Schmid R, Wettstein A. Caregivers' adaptation to change: the impact of increasing impairment 
of persons suffering from dementia on their caregivers' subjective well-being. Aging Ment Health. 2006 Sep;10(5):539-48. PMID: 16938689.

171. Pomeroy VM, Warren CM, Honeycombe C, et al. Mobility and dementia: is physiotherapy treatment during respite care effective? Int J Geriatr Psychiatry. 1999 May;14(5):389-97. PMID: 10389044.

172. Pot AM, Willemse BM, Horjus S. A pilot study on the use of tracking technology: feasibility, acceptability, and benefits for people in early stages of dementia and their informal caregivers. Aging Ment Health. 2012;16(1):12734. doi: https://dx.doi.org/10.1080/13607863.2011.596810. PMID: 21780960.

173. Puranen T, Pitkala K, Suominen M. Tailored nutritional guidance for home-dwelling $\mathrm{AD}$ families: The feasibility of and elements promoting positive changes in diet (NuAD-trial). J Nutr Health Aging. 2015;19(4):454-9. doi: 10.1007/s12603-014-0567-4. PMID: 103787980.

Language: English. Entry Date: 20150414. Revision Date: 20160331. Publication Type: Journal Article.

174. Rosen J, Mulsant BH, Kollar M, et al. Mental health training for nursing home staff using computer-based interactive video: a 6-month randomized trial. J Am Med Dir Assoc. 2002;3(5):291-6. PMID: 106804693. Language: English. Entry Date: 20030207. Revision Date: 20150711. Publication Type: Journal Article.

175. Rosvik J, Engedal K, Kirkevold O. Factors to make the VIPS practice model more effective in the treatment of neuropsychiatric symptoms in nursing home residents with dementia. Dement Geriatr Cogn Disord. 2014;37(5-6):33546. doi: https://dx.doi.org/10.1159/000357773. PMID: 24503517.

176. Shelton P, Schraeder C, Dworak D, et al. Caregivers' utilization of health services: results from the Medicare Alzheimer's Disease Demonstration, Illinois site. J Am Geriatr Soc. 2001 Dec;49(12):1600-5. PMID: 11843991. 177. Spector A, Orrell M, Woods B. Cognitive Stimulation Therapy (CST): effects on different areas of cognitive function for people with dementia. Int J Geriatr Psychiatry. 2010 Dec;25(12):1253-8. doi:

https://dx.doi.org/10.1002/gps.2464. PMID: 20069533.

178. Stocking CB, Hougham GW, Danner DD, et al.

Empirical assessment of a research advance directive for persons with dementia and their proxies. J Am Geriatr Soc. 2007 Oct;55(10):1609-12. PMID: 17714461.

179. Swanson EA, Maas ML, Buckwalter KC. Catastrophic reactions and other behaviors of Alzheimer's residents: Special unit compared with traditional units. Arch Psychiatr Nurs. 1993 Oct;7(5):292-9. doi: http://dx.doi.org/10.1016/0883-9417\%2893\%2990007-J. PMID: 1994-11146-001.

180. Thyrian JR, Eichler T, Michalowsky B, et al. Community-Dwelling People Screened Positive for Dementia in Primary Care: A Comprehensive, Multivariate Descriptive Analysis Using Data from the DelpHi-Study. J Alzheimers Dis. 201603 30;52(2):609-17. doi: https://dx.doi.org/10.3233/JAD-151076. PMID: 27031481. 181. Trebbastoni A, Imbriano L, Podda L, et al. Cognitive Training in Patients with Alzheimer's Disease: Findings of a 12-month Randomized Controlled Trial. Curr Alzheimer Res. 2018 Mar 14;15(5):452-61. doi: https://dx.doi.org/10.2174/1567205014666171113105044. PMID: 29141548.

182. Tsantali E, Economidis D, Rigopoulou S. Testing the benefits of cognitive training vs. cognitive stimulation in mild Alzheimer's disease: A randomised controlled trial. Brain Impairment. 201701 Sep;18(2):188-96. doi: http://dx.doi.org/10.1017/BrImp.2017.6. PMID: 615666653.

183. van Weert JC, Janssen BM, van Dulmen AM, et al. Nursing assistants' behaviour during morning care: effects of the implementation of snoezelen, integrated in 24-hour dementia care. J Adv Nurs. 2006 Mar;53(6):656-68. PMID: 16553674.

184. Werner C, Wiloth S, Lemke NC, et al. People with Dementia Can Learn Compensatory Movement Maneuvers for the Sit-to-Stand Task: A Randomized Controlled Trial. J Alzheimers Dis. 2017;60(1):107-20. doi: https://dx.doi.org/10.3233/JAD-170258. PMID: 28759967. 185. Yaguez L, Shaw KN, Morris R, et al. The effects on cognitive functions of a movement-based intervention in patients with Alzheimer's type dementia: a pilot study. Int J Geriatr Psychiatry. 2011 Feb;26(2):173-81. doi: https://dx.doi.org/10.1002/gps.2510. PMID: 20878665. 186. Young DK. Multicomponent intervention combining a cognitive stimulation group and tai chi to reduce cognitive decline among community-dwelling older adults with probable dementia: A multi-center, randomized controlled trial. Dementia. 2018 Nov

28:1471301218814637. doi: https://dx.doi.org/10.1177/1471301218814637. PMID: 30486656.

187. Zhang D, Zhou Z, Wu W, et al. Influence of electroencephalograph bionic electrical stimulation on neuronal activities in patients with Alzheimer's disease: A functional magnetic resonance imaging study. Radiology of Infectious Diseases. 2018 March;5(1):26-32. doi: http://dx.doi.org/10.1016/j.jrid.2018.02.005. PMID: 620922742.

188. Zhao J, Li Z, Cong Y, et al. Repetitive transcranial magnetic stimulation improves cognitive function of Alzheimer's disease patients. Oncotarget. 2017 May 16;8(20):33864-71. doi:

https://dx.doi.org/10.18632/oncotarget.13060. PMID: 27823981.

\section{Ineligible Population}

189. Allen VJ, Methven L, Gosney M. Impact of serving method on the consumption of nutritional supplement drinks: randomized trial in older adults with cognitive impairment. J Adv Nurs. 2014;70(6):1323-33. doi: 10.1111/jan.12293. PMID: 103928802. Language: English. Entry Date: 20140418. Revision Date: 20150819.

Publication Type: Journal Article.

190. Alves J, Alves-Costa F, Magalhaes R, et al. Cognitive stimulation for Portuguese older adults with cognitive impairment: a randomized controlled trial of efficacy, comparative duration, feasibility, and experiential relevance. Am J Alzheimers Dis Other Demen. 2014 Sep;29(6):503-12. doi:

https://dx.doi.org/10.1177/1533317514522541. PMID: 24526760 . 
191. Bakker TJ, Duivenvoorden HJ, van der Lee J, et al. Prognostic factors for a favourable long-term outcome from an integrative psychotherapeutic nursing home programme. Dement Geriatr Cogn Disord. 2011;32(5):318-31. doi: https://dx.doi.org/10.1159/000334969. PMID: 22286593. 192. Bakker TJ, Duivenvoorden HJ, van der Lee J, et al. Integrative psychotherapeutic nursing home program to reduce multiple psychiatric symptoms of cognitively impaired patients and caregiver burden: randomized controlled trial. Am J Geriatr Psychiatry. 2011;19(6):50720. doi: 10.1097/JGP.0b013e3181eafdc6. PMID: 108240694. Language: English. Entry Date: 20110930. Revision Date: 20171129. Publication Type: journal article. 193. Ball K, Berch DB, Helmers KF, et al. Effects of cognitive training interventions with older adults: a randomized controlled trial. JAMA: Journal of the American Medical Association. 2002;288(18):2271-81. PMID: 106804253. Corporate Author: Advanced Cognitive Training for Independent and Vital Elderly Study Group. Language: English. Entry Date: 20030207. Revision Date: 20161125. Publication Type: journal article.

194. Barban F, Annicchiarico R, Pantelopoulos S, et al. Protecting cognition from aging and Alzheimer's disease: a computerized cognitive training combined with reminiscence therapy. Int J Geriatr Psychiatry. 2016 Apr;31(4):340-8. doi: https://dx.doi.org/10.1002/gps.4328. PMID: 26205305.

195. Bateman C, Anderson K, Bird M, et al. Volunteers improving person-centred dementia and delirium care in a rural Australian hospital. Rural Remote Health. 2016 AprJun;16(2):3667. PMID: 27303861.

196. Bautmans I, Demarteau J, Cruts B, et al. Dysphagia in elderly nursing home residents with severe cognitive impairment can be attenuated by cervical spine mobilization. Journal of Rehabilitation Medicine (Stiftelsen Rehabiliteringsinformation). 2008;40(9):755-60. PMID: 105703943. Language: English. Entry Date: 20081205. Revision Date: 20150819. Publication Type: Journal Article.

197. Beer C, Horner B, Flicker L, et al. A clusterrandomised trial of staff education to improve the quality of life of people with dementia living in residential care: the DIRECT study. PLoS ONE. 2011;6(11):e28155. doi: https://dx.doi.org/10.1371/journal.pone.0028155. PMID: 22140531.

198. Beer C, Lowry R, Horner B, et al. Development and evaluation of an educational intervention for general practitioners and staff caring for people with dementia living in residential facilities. Int Psychogeriatr. 2011 Mar;23(2):221-9. doi: https://dx.doi.org/10.1017/S104161021000195X. PMID: 20950507.

199. Bier N, Grenier S, Brodeur C, et al. Measuring the impact of cognitive and psychosocial interventions in persons with mild cognitive impairment with a randomized single-blind controlled trial: rationale and design of the MEMO+ study. Int Psychogeriatr. 2015 Mar;27(3):511-25. doi: https://dx.doi.org/10.1017/S1041610214001902. PMID: 25268968.

200. Bird M, Jones RH, Korten A, et al. A controlled trial of a predominantly psychosocial approach to BPSD: treating causality. Int Psychogeriatr. 2007;19(5):874-91.
PMID: 105828624. Language: English. Entry Date: 20080307. Revision Date: 20161117. Publication Type: journal article.

201. Blair A, Anderson K, Bateman C. The "Golden Angels": effects of trained volunteers on specialling and readmission rates for people with dementia and delirium in rural hospitals. Int Psychogeriatr. 2018 Nov;30(11):170716. doi: https://dx.doi.org/10.1017/S1041610218000911. PMID: 30099973.

202. Brown LJE, Blake D, Berry K, et al. A pilot and feasibility study of the effectiveness of care mapping on person-centred care in neurorehabilitation settings.

Neuropsychol. 201908 Apr:1-18. doi: http://dx.doi.org/10.1080/09602011.2019.1598446. PMID: 627288558.

203. Buettner LL, Fitzsimmons S, Atav S, et al. Cognitive stimulation for apathy in probable early-stage Alzheimer's. J Aging Res. 2011 Apr 28;2011:480890. doi: https://dx.doi.org/10.4061/2011/480890. PMID: 21584241. 204. Burgio LD, Allen-Burge R, Roth DL, et al. Come talk with me: improving communication between nursing assistants and nursing home residents during care routines. Gerontologist. 2001;41(4):449-60. PMID: 107066936. Language: English. Entry Date: 20011109. Revision Date: 20150711. Publication Type: Journal Article.

205. Buschert VC, Friese U, Teipel SJ, et al. Effects of a newly developed cognitive intervention in amnestic mild cognitive impairment and mild Alzheimer's disease: a pilot study. J Alzheimers Dis. 2011;25(4):679-94. doi: https://dx.doi.org/10.3233/JAD-2011-100999. PMID: 21483095.

206. Catic A, Mattison M, Bakaev I, et al. ECHO-AGE: an Innovative Model of Geriatric Care for Long-Term Care Residents With Dementia and Behavioral Issues. In J Am Med Dir Assoc

207. Chiu wu CH, Perng SJ, Shi CK, et al. Advance Care Planning and Advance Directives: A Multimedia Education Program in Community-Dwelling Older Adults. Journal of Applied Gerontology. 2019. doi: http://dx.doi.org/10.1177/0733464819831596.

208. Conradsson M, Littbrand H, Lindelöf N, et al. Effects of a high-intensity functional exercise programme on depressive symptoms and psychological well-being among older people living in residential care facilities: a clusterrandomized controlled trial. Aging Ment Health. 2010;14(5):565-76. doi: 10.1080/13607860903483078. PMID: 105049096. Language: English. Entry Date: 20100827. Revision Date: 20150711. Publication Type: Journal Article.

209. Corbett A, Owen A, Hampshire A, et al. The Effect of an Online Cognitive Training Package in Healthy Older Adults: An Online Randomized Controlled Trial. J Am Med Dir Assoc. 2015 Nov 01;16(11):990-7. doi: https://dx.doi.org/10.1016/j.jamda.2015.06.014. PMID: 26543007.

210. Cox KL, Cyarto EV, Ellis KA, et al. A Randomized Controlled Trial of Adherence to a 24-Month Home-Based Physical Activity Program and the Health Benefits for Older Adults at Risk of Alzheimer's Disease: The AIBL Active-Study. J Alzheimers Dis. 2018 Oct 26;26:26. doi: https://dx.doi.org/10.3233/JAD-180521. PMID: 30372680. 
211. Dimitriou TD, Verykouki E, Papatriantafyllou J, et al. Non-pharmacological interventions for agitation/aggressive behaviour in patients with dementia: a randomized controlled crossover trial. Funct Neurol. 2018 Jul/Sept;33(3):143-7. PMID: 30457967.

212. Folkerts AK, Dorn ME, Roheger M, et al. Cognitive Stimulation for Individuals with Parkinson's Disease Dementia Living in Long-Term Care: Preliminary Data from a Randomized Crossover Pilot Study. Parkinson's dis. 2018;2018:8104673. doi:

https://dx.doi.org/10.1155/2018/8104673. PMID: 30631420.

213. Gaitan A, Garolera M, Cerulla N, et al. Efficacy of an adjunctive computer-based cognitive training program in amnestic mild cognitive impairment and Alzheimer's disease: a single-blind, randomized clinical trial. Int J Geriatr Psychiatry. 2013 Jan;28(1):91-9. doi: https://dx.doi.org/10.1002/gps.3794. PMID: 22473855. 214. Garand L, Morse JQ, ChiaRebecca L, et al. Problemsolving therapy reduces subjective burden levels in caregivers of family members with mild cognitive impairment or early-stage dementia: Secondary analysis of a randomized clinical trial. Int J Geriatr Psychiatry. 2019 07;34(7):957-65. doi: https://dx.doi.org/10.1002/gps.5095. PMID: 30868641.

215. Garand L, Rinaldo DE, Alberth MM, et al. Effects of problem solving therapy on mental health outcomes in family caregivers of persons with a new diagnosis of mild cognitive impairment or early dementia: a randomized controlled trial. Am J Geriatr Psychiatry. 2014;22(8):77181. doi: 10.1016/j.jagp.2013.07.007. PMID: 107797083. Language: English. Entry Date: 20150417. Revision Date: 20161207. Publication Type: journal article.

216. Goldberg SE, Bradshaw LE, Kearney FC, et al. Care in specialist medical and mental health unit compared with standard care for older people with cognitive impairment admitted to general hospital: randomised controlled trial (NIHR TEAM trial). Bmj. 2013 Jul 02;347:f4132. doi: https://dx.doi.org/10.1136/bmj.f4132. PMID: 23819964. 217. Gossink F, Pijnenburg Y, Scheltens P, et al. An intervention programme for caregivers of dementia patients with frontal behavioural changes: an explorative study with controlled effect on sense of competence. Psychogeriatrics. 2018 Nov;18(6):451-9. doi:

https://dx.doi.org/10.1111/psyg.12351. PMID: 30066463. 218. Gregory MA, Gill DP, Shellington EM, et al. Groupbased exercise and cognitive-physical training in older adults with self-reported cognitive complaints: The Multiple-Modality, Mind-Motor (M4) study protocol. BMC geriatr. 2016 Jan 16;16:17. doi:

https://dx.doi.org/10.1186/s12877-016-0190-9. PMID: 26772171.

219. Gustafsson M, Sjolander M, Pfister B, et al. Pharmacist participation in hospital ward teams and hospital readmission rates among people with dementia: a randomized controlled trial. Eur J Clin Pharmacol. 2017 Jul;73(7):827-35. doi: https://dx.doi.org/10.1007/s00228017-2249-8. PMID: 28391409.

220. Gustafsson M, Sjolander M, Pfister B, et al. Effects of Pharmacists' Interventions on Inappropriate Drug Use and Drug-Related Readmissions in People with Dementia-A Secondary Analysis of a Randomized Controlled Trial.
Pharmacy (Basel). 2018 Jan 16;6(1):16. doi: https://dx.doi.org/10.3390/pharmacy6010007. PMID: 29337859.

221. Han JW, Lee H, Hong JW, et al. Multimodal Cognitive Enhancement Therapy for Patients with Mild Cognitive Impairment and Mild Dementia: A MultiCenter, Randomized, Controlled, Double-Blind, Crossover Trial. J Alzheimers Dis. 2017;55(2):787-96. PMID: 120361850. Language: English. Entry Date: 20180725. Revision Date: 20180731. Publication Type: journal article. 222. Herrera C, Chambon C, Michel BF, et al. Positive effects of computer-based cognitive training in adults with mild cognitive impairment. Neuropsychologia. 2012 Jul;50(8):1871-81. doi: https://dx.doi.org/10.1016/j.neuropsychologia.2012.04.012. PMID: 22525705.

223. Hindle J, Watermeyer T, Roberts J, et al. Cognitive rehabiliation for Parkinson's disease dementia: a study protocol for a pilot randomised controlled trial. In Trials 224. Holmes D, Teresi JA, Ramirez M, et al. An evaluation of a monitoring system intervention: falls, injuries, and affect in nursing homes. Clin Nurs Res. 2007 Nov;16(4):317-35. PMID: 17991911.

225. Ito T, Meguro K, Akanuma K, et al. A randomized controlled trial of the group reminiscence approach in patients with vascular dementia. Dement Geriatr Cogn Disord. 2007;24(1):48-54. PMID: 17565213.

226. Jansen AP, van Hout HP, Nijpels G, et al.

Effectiveness of case management among older adults with early symptoms of dementia and their primary informal caregivers: a randomized clinical trial. Int J Nurs Stud. 2011 Aug;48(8):933-43. doi: https://dx.doi.org/10.1016/j.ijnurstu.2011.02.004. PMID: 21356537.

227. Jansen AP, van Hout HP, van Marwijk HW, et al. (Cost)-effectiveness of case-management by district nurses among primary informal caregivers of older adults with dementia symptoms and the older adults who receive informal care: design of a randomized controlled trial [ISCRTN83135728]. BMC Public Health. 2005 Dec 12;5:133. PMID: 16343336.

228. Jha A, Jan F, Gale T, et al. Effectiveness of a recovery-orientated psychiatric intervention package on the wellbeing of people with early dementia: a preliminary randomised controlled trial. Int J Geriatr Psychiatry. 2013 Jun;28(6):589-96. doi: https://dx.doi.org/10.1002/gps.3863. PMID: 22847712.

229. Jin Z. Efficacy of acupuncture combined with Nimodipine in the treatment of vascular dementia. In China and foreign medical treatment [zhong wai yi liao] 230. Keller HH, Gibbs AJ, Boudreau LD, et al. Prevention of weight loss in dementia with comprehensive nutritional treatment. J Am Geriatr Soc. 2003 Jul;51(7):945-52. PMID: 12834514.

231. Kiosses DN, Ravdin LD, Gross JJ, et al. Problem adaptation therapy for older adults with major depression and cognitive impairment: a randomized clinical trial. JAMA Psychiatry. 2015;72(1):22-30. doi: 10.1001/jamapsychiatry.2014.1305. PMID: 103874772. Language: English. Entry Date: 20150410. Revision Date: 20161222. Publication Type: journal article. 
232. Lam LC, Chau RC, Wong BM, et al. Interim followup of a randomized controlled trial comparing Chinese style mind body (Tai Chi) and stretching exercises on cognitive function in subjects at risk of progressive cognitive decline. Int J Geriatr Psychiatry. 2011 Jul;26(7):733-40. doi: https://dx.doi.org/10.1002/gps.2602. PMID: 21495078.

233. Larouche E, Hudon C, Goulet S. Mindfulness mechanisms and psychological effects for aMCI patients: A comparison with psychoeducation. Complementary therapies in clinical practice. 201901 Feb;34:93-104. doi: http://dx.doi.org/10.1016/j.ctcp.2018.11.008. PMID: 626243559 .

234. Lautenschlager NT, Cox KL, Flicker L, et al. Effect of physical activity on cognitive function in older adults at risk for Alzheimer disease: a randomized trial.[Erratum appears in JAMA. 2009 Jan 21;301(3):276]. Jama. 2008 Sep 03;300(9):1027-37. doi:

https://dx.doi.org/10.1001/jama.300.9.1027. PMID: 18768414.

235. Lee GJ, Bang HJ, Lee KM, et al. A comparison of the effects between 2 computerized cognitive training programs, Bettercog and COMCOG, on elderly patients with MCI and mild dementia: A single-blind randomized controlled study. Medicine (Baltimore). 2018

Nov;97(45):e13007. doi: https://dx.doi.org/10.1097/MD.0000000000013007. PMID: 30407291.

236. Lin HC, Yang YP, Cheng WY, et al. Distinctive effects between cognitive stimulation and reminiscence therapy on cognitive function and quality of life for different types of behavioural problems in dementia. Scand J Caring Sci. 2018 Jun;32(2):594-602. doi: https://dx.doi.org/10.1111/scs.12484. PMID: 28881430. 237. Littbrand H, Rosendahl E, Lindelöf N, et al. A highintensity functional weight-bearing exercise program for older people dependent in activities of daily living and living in residential care facilities: evaluation of the applicability with focus on cognitive function. Phys Ther. 2006;86(4):489-98. PMID: 106471666. Language: English. Entry Date: 20060714. Revision Date: 20150820. Publication Type: Journal Article.

238. Liu-Ambrose T, Best JR, Davis JC, et al. Aerobic exercise and vascular cognitive impairment: A randomized controlled trial. Neurology. 2016;87(20):2082-90. PMID: 119524521. Language: English. Entry Date: 20180723. Revision Date: 20180723. Publication Type: journal article. 239. Low L-F, Goodenough B, Fletcher J, et al. The Effects of Humor Therapy on Nursing Home Residents Measured Using Observational Methods: The SMILE Cluster Randomized Trial. J Am Med Dir Assoc. 2014;15(8):564-9. doi: 10.1016/j.jamda.2014.03.017. PMID: 103984346. Language: English. Entry Date: 20140805. Revision Date: 20150710. Publication Type: Journal Article.

240. Man DW, Chung JC, Lee GY. Evaluation of a virtual reality-based memory training programme for Hong Kong Chinese older adults with questionable dementia: a pilot study. Int J Geriatr Psychiatry. 2012 May;27(5):513-20. doi: https://dx.doi.org/10.1002/gps.2746. PMID: 21681818. 241. Meinzer M, Lindenberg R, Phan MT, et al. Transcranial direct current stimulation in mild cognitive impairment: Behavioral effects and neural mechanisms. Alzheimer's \& Dementia: The Journal of the Alzheimer's Association. 2015 Sep;11(9):1032-40. doi: http://dx.doi.org/10.1016/j.jalz.2014.07.159. PMID: 201452911-001.

242. Middleton LE, Ventura MI, Santos-Modesitt W, et al. The Mental Activity and eXercise (MAX) trial: Effects on physical function and quality of life among older adults with cognitive complaints. Contemp Clin Trials. 2018 01;64:161-6. doi:

https://dx.doi.org/10.1016/j.cct.2017.10.009. PMID: 29066293.

243. Muniz R, Serra CM, Reisberg B, et al. Cognitivemotor intervention in Alzheimer's disease: long-term results from the Maria Wolff trial. J Alzheimers Dis. 2015;45(1):295-304. doi: https://dx.doi.org/10.3233/JAD142364. PMID: 25547632.

244. Nakatsuka M, Nakamura K, Hamanosono R, et al. A Cluster Randomized Controlled Trial of Nonpharmacological Interventions for Old-Old Subjects with a Clinical Dementia Rating of 0.5: The Kurihara Project. Dement Geriatr Cogn Dis Extra. 2015 MayAug;5(2):221-32. doi: https://dx.doi.org/10.1159/000380816. PMID: 26195978. 245. Okamoto T, Hashimoto Y, Kobayashi R. Effects of interval walking training compared to normal walking training on cognitive function and arterial function in older adults: a randomized controlled trial. Aging Clinical and Experimental Research. 2018. doi: http://dx.doi.org/10.1007/s40520-018-1093-8. PMID: 625589391.

246. Olazaran J, Muniz R, Reisberg B, et al. Benefits of cognitive-motor intervention in MCI and mild to moderate Alzheimer disease. Neurology. 2004 Dec 28;63(12):234853. PMID: 15623698.

247. Padala PR, Padala KP, Lensing SY, et al. Repetitive transcranial magnetic stimulation for apathy in mild cognitive impairment: A double-blind, randomized, shamcontrolled, cross-over pilot study. Psychiatry Res. 2018 03;261:312-8. doi: https://dx.doi.org/10.1016/j.psychres.2017.12.063. PMID: 29331848.

248. Park JE, Jeon SY, Kim SA, et al. A Multidomain Intervention for Modifying Lifestyle Habits Reduces the Dementia Risk in Community-Dwelling Older Adults: A Single-Blinded Randomized Controlled Pilot Study. J Alzheimers Dis. 2019;70(1):51-60. doi: https://dx.doi.org/10.3233/JAD-190016. PMID: 31127782. 249. Quayhagen MP, Quayhagen M, Corbeil RR, et al. Coping with dementia: evaluation of four nonpharmacologic interventions. Int Psychogeriatr. 2000 Jun;12(2):249-65. PMID: 10937544.

250. Regan B, Wells Y, Farrow M, et al. MAXCOGmaximizing cognition: a randomized controlled trial of the efficacy of goal-oriented cognitive rehabilitation for people with mild cognitive impairment and early Alzheimer disease. In Am J Geriatr Psychiatry

251. Resnick B, Gruber-Baldini AL, Zimmerman S, et al. Nursing home resident outcomes from the Res-Care intervention. J Am Geriatr Soc. 2009;57(7):1156-65. doi: 10.1111/j.1532-5415.2009.02327.x. PMID: 105377579. 
Language: English. Entry Date: 20090904. Revision Date: 20150711. Publication Type: Journal Article.

252. Rovio S, Kareholt I, Helkala EL, et al. Leisure-time physical activity at midlife and the risk of dementia and Alzheimer's disease. Lancet neurol. 2005 Nov;4(11):70511. PMID: 16239176.

253. Sakamoto Y, Ebihara S, Ebihara T, et al. Fall Prevention Using Olfactory Stimulation with Lavender Odor in Elderly Nursing Home Residents: A Randomized Controlled Trial...[corrected][published erratum appears in J AM GERIATR SOC 2012 Nov; 60(11): 2193]. J Am Geriatr Soc. 2012;60(6):1005-11. doi: 10.1111/j.15325415.2012.03977.x. PMID: 104462071. Language: English. Entry Date: 20120615. Revision Date: 20150711. Publication Type: Journal Article.

254. Schaap FD, Dijkstra GJ, Stewart RE, et al. Effects of Dementia Care Mapping on well-being and quality of life of older people with intellectual disability: A quasiexperimental study. J Appl Res Intellect Disabil. 2019 Jul;32(4):849-60. doi: https://dx.doi.org/10.1111/jar.12576. PMID: 30868692.

255. Schaap FD, Finnema EJ, Stewart RE, et al. Effects of Dementia Care Mapping on job satisfaction and caring skills of staff caring for older people with intellectual disabilities: A quasi-experimental study. J Appl Res Intellect Disabil. 2019 Sep;32(5):1228-40. doi: https://dx.doi.org/10.1111/jar.12615. PMID: 31087472. 256. Schindel-Martin L, Morden P, Cetinski G, et al. Teaching staff to respond effectively to cognitively impaired residents who display self-protective behaviors. Am J Alzheimers Dis Other Demen. 2003;18(5):273-81. PMID: 106741786. Language: English. Entry Date: 20040604. Revision Date: 20150711. Publication Type: Journal Article.

257. Seltzer MM, Litchfield LC, Kapust LR, et al. Professional and family collaboration in case management: a hospital-based replication of a community-based study. Soc Work Health Care. 1992;17(1):1-22. PMID: 1440105. 258. Shelton JT, Lee JH, Scullin MK, et al. Improving Prospective Memory in Healthy Older Adults and Individuals with Very Mild Alzheimer's Disease. J Am Geriatr Soc. 2016 Jun;64(6):1307-12. doi: https://dx.doi.org/10.1111/jgs.14134. PMID: 27321610. 259. Sink KM, Espeland MA, Castro CM, et al. Effect of a 24-Month Physical Activity Intervention vs Health Education on Cognitive Outcomes in Sedentary Older Adults: The LIFE Randomized Trial. Jama. 2015 Aug 25;314(8):781-90. doi:

https://dx.doi.org/10.1001/jama.2015.9617. PMID: 26305648.

260. Sink KM, Espeland MA, Rushing J, et al. The LIFE Cognition Study: design and baseline characteristics. Clin Interv Aging. 2014;9:1425-36. doi: https://dx.doi.org/10.2147/CIA.S65381. PMID: 25210447. 261. Tappen R, Hain D. The effect of in-home cognitive training on functional performance of individuals with mild cognitive impairment and early-stage Alzheimer's disease. In Res

262. Thodberg K, Sorensen LU, Christensen JW, et al. Therapeutic effects of dog visits in nursing homes for the elderly. Psychogeriatrics. 2016 Sep;16(5):289-97. doi: https://dx.doi.org/10.1111/psyg.12159. PMID: 26510632.
263. Ukawa S, Yuasa M, Ikeno T, et al. Randomised controlled pilot study in Japan comparing a home visit program using a Functioning Improvement Tool with a home visit with conversation alone. Australas J Ageing. 2012 Sep;31(3):187-9. doi:

https://dx.doi.org/10.1111/j.1741-6612.2012.00589.x. PMID: 22950591.

264. Unverzagt FW, Smith DM, Rebok GW, et al. The Indiana Alzheimer Disease Center's Symposium on Mild Cognitive Impairment. Cognitive training in older adults: lessons from the ACTIVE Study. Curr Alzheimer Res. 2009 Aug;6(4):375-83. PMID: 19689237.

265. Van Der Lee J, Bakker TJEM, Droes RM. Recovery from burden: Informal caregiver profiles that predict treatment success. Int Psychogeriatr. 2019:317-29. doi: http://dx.doi.org/10.1017/S1041610218000613. PMID: 622667094.

266. Wang S-C, Yu C-L, Chang S-H. Effect of music care on depression and behavioral problems in elderly people with dementia in Taiwan: A quasi-experimental, longitudinal study. Aging Ment Health. 2017

Feb;21(2):156-62. doi:

http://dx.doi.org/10.1080/13607863.2015.1093602. PMID: 2017-03789-005.

267. Wang Y. Study of treatment for post stroke dementia. 268. Wang Y, Xiao LD, Ullah S, et al. Evaluation of a nurse-led dementia education and knowledge translation programme in primary care: A cluster randomized controlled trial. Nurse Educ Today. 2017 Feb;49:1-7. doi: https://dx.doi.org/10.1016/j.nedt.2016.10.016. PMID: 27855297.

269. Wilcock J, Iliffe S, Griffin M, et al. Tailored educational intervention for primary care to improve the management of dementia: the EVIDEM-ED cluster randomized controlled trial. Trials. 2013 Nov 20;14:397. doi: https://dx.doi.org/10.1186/1745-6215-14-397. PMID: 24257429.

270. Wilson KC, Scott M, Abou-Saleh M, et al. Long-term effects of cognitive-behavioural therapy and lithium therapy on depression in the elderly. Br J Psychiatry. 1995 Nov;167(5):653-8. PMID: 8564323.

271. Yang BF, Zeng XH, Liu Y, et al. Effect of acupuncture treatment on vascular cognitive impairment without dementia: Study protocol for a randomized controlled trial. Trials. 2015;13. doi:

http://dx.doi.org/10.1186/1745-6215-15-442. PMID: 605062793.

272. Yang JW, Shi GX, Zhang S, et al. Effectiveness of acupuncture for vascular cognitive impairment no dementia: a randomized controlled trial. Clin Rehabil. 2019 Jan 23:269215518819050. doi:

https://dx.doi.org/10.1177/0269215518819050. PMID: 30672317.

273. Young DK, Ng PY, Kwok T, et al. The effects of an expanded cognitive stimulation therapy model on the improvement of cognitive ability of elderly with mild stage Dementia living in a community - a randomized waitlist controlled trial. Aging Ment Health. 2018 May 21:1-8. doi: https://dx.doi.org/10.1080/13607863.2018.1471586. PMID: 29781725. 
274. Yu J, Zhang X, Liu C, et al. Effect of acupuncture treatment on vascular dementia. Neurol Res. 2006 Jan;28(1):97-103. PMID: 16464371.

275. Zenthofer A, Meyer-Kuhling I, Hufeland A-L, et al. Carers' education improves oral health of older people suffering from dementia - Results of an intervention study. In Clin Interv Aging

276. Zhao J, Li H, Lin R, et al. Effects of creative expression therapy for older adults with mild cognitive impairment at risk of Alzheimer's disease: a randomized controlled clinical trial. Clin Interv Aging. 2018;13:131320. doi: https://dx.doi.org/10.2147/CIA.S161861. PMID: 30087557.

277. Zhu Y, Wu H, Qi M, et al. Effects of a specially designed aerobic dance routine on mild cognitive impairment. Clin Interv Aging. 2018;13:1691-700. doi: https://dx.doi.org/10.2147/CIA.S163067. PMID: 30237705.

\section{Ineligible publication type}

278. Abalan F, Manciet G, Dartigues JF, et al. Nutrition and SDAT. Biol Psychiatry. 1992 Jan 01;31(1):103-5. PMID: 1543795

279. Agar M, Chenoweth L, Mitchell G, et al. Implementing facilitated case conferencing for aged care residents with advanced dementia-development of a palliative care planning coordinator role. In Palliat Med 280. Agar M, Chenoweth L, Mitchell G, et al. Cluster randomised controlled trial of facilitated case conferencing for aged care residents with advanced dementia. In Palliat Med

281. Amieva H, Dartigues JF. ETNA3, a clinical randomized study assessing three cognitive-oriented therapies in dementia: Rationale and general design. Revue Neurologique. 2013 October;169(10):752-6. doi: http://dx.doi.org/10.1016/j.neurol.2013.07.011. PMID: 52760976.

282. Ballard C. Video decision support affects patients' care choices in dementia care planning. In Evidence-based mental health

283. Brennan PF, Moore SM, Smyth KA. ComputerLink: electronic support for the home caregiver. ANS Adv Nurs Sci. 1991 Jun;13(4):14-27. PMID: 2059002.

284. Brodaty H, Arasaratnam C. Meta-analysis of nonpharmacological interventions for neuropsychiatric symptoms of dementia.[Erratum appears in Am J Psychiatry. 2013 Feb 1;170(2):227]. Am J Psychiatry. 2012 Sep;169(9):946-53. doi:

https://dx.doi.org/10.1176/appi.ajp.2012.11101529. PMID: 22952073.

285. Brodaty H, Green A, Koschera A. Meta-analysis of psychosocial interventions for caregivers of people with dementia. J Am Geriatr Soc. 2003 May;51(5):657-64. PMID: 12752841.

286. Capisizu A, Zamfirescu A, Aurelian SM, et al. Post intervention outcomes of physical exercise training in elderly with mild to moderate dementia. Eur J Neurol. 2019;26:113-. doi: 10.1111/ene.14018. PMID: CN01979194.

287. Casper GR, Calvitti A, Brennan PF, et al. ComputerLink: the impact of a computer network on
Alzheimer's caregivers' decision-making confidence and skill. Medinfo. 1995;8 Pt 2:1546. PMID: 8591497.

288. Chan WC, Lautenschlager N, Dow B, et al. A homebased exercise intervention for caregivers of persons with dementia: Study protocol for a randomised controlled trial. Trials. 201621 Sep;17 (1) (no pagination)(460). doi: http://dx.doi.org/10.1186/s13063-016-1582-z. PMID: 612244535.

289. Clark ME, Lipe AW, Bilbrey M. Use of music to decrease aggressive behaviors in people with dementia. $\mathrm{J}$ Gerontol Nurs. 1998 Jul;24(7):10-7. PMID: 9801526. 290. Collier. The use of multi-sensory stimulation to improve functional performance in older people with dementia: a randomised single blind trial. In British and irish theses

[http://ezproxyoulsoxacuk:2514/idx/scripts/itasp? Xml=f: \indexlidx\docs $\backslash a l l \backslash 56 \backslash$ it00525859htm\&subfolder=/s earch]

291. Crawford SE, Leaver VW, Mahoney SD. Using Reiki to decrease memory and behavior problems in mild cognitive impairment and mild Alzheimer's disease. J Altern Complement Med. 2006 Nov;12(9):911-3. PMID: 17109583.

292. Curto PD, Sole SC, Castro M, et al. Effects of music therapy in institutionalized elderly with dementia. In European geriatric medicine

293. Dam AEH, de Vugt ME, van Boxtel MPJ, et al. Effectiveness of an online social support intervention for caregivers of people with dementia: The study protocol of a randomised controlled trial. Trials. 201729 Aug;18 (1) (no pagination)(395). doi: http://dx.doi.org/10.1186/s13063017-2097-y. PMID: 618042762.

294. DeGregory C. The effects of multiple gratitude interventions among informal caregivers of persons with dementia and Alzheimer's disease.

295. Eggermont LH, Blankevoort CG, Scherder EJ.

Walking and night-time restlessness in mild-to-moderate dementia: a randomized controlled trial. Age Ageing. 2010 Nov;39(6):746-9. doi:

https://dx.doi.org/10.1093/ageing/afq115. PMID: 20823127.

296. Finnegan S, Nichols V, Sheehan B, et al. A randomised controlled trial of moderate to high intensity exercise training for people with dementia: dementia And Physical Activity (DAPA)...The Chartered Society of Physiotherapy UK Conference 2018, Birmingham, UK, 1920 October 2018. Physiotherapy. 2019;105:e104-e. PMID: CN-01690752.

297. Fleiner T, Zijlstra W, Dauth H, et al. Evaluation of a hospital-based day-structuring exercise programme on exacerbated behavioural and psychological symptoms in dementia - the exercise carrousel: Study protocol for a randomised controlled trial. Trials. 2015 May 26;16 (1) (no pagination)(228). doi: http://dx.doi.org/10.1186/s13063015-0758-2. PMID: 604800524.

298. Fortinsky RH, Gitlin LN, Pizzi LT, et al. Translation of the Care of Persons with Dementia in their Environments (COPE) intervention in a publicly-funded home care context: Rationale and research design. Contemp Clin Trials. 2016 07;49:155-65. doi: https://dx.doi.org/10.1016/j.cct.2016.07.006. PMID: 27394383. 
299. Fujii M, Hatakeyama R, Fukuoka Y, et al. Lavender aroma therapy for behavioral and psychological symptoms in dementia patients. Geriatr Gerontol Int. 2008

Jun;8(2):136-8. doi: https://dx.doi.org/10.1111/j.14470594.2008.00461.x. PMID: 18713168.

300. Gerlach LB, Kales HC. Learning their language: the importance of detecting and managing pain in dementia.

301. Goeman D, Comans T, Enticott JC, et al. Evaluating the Efficacy of the "Support for Life" Program for People with Dementia and Their Families and Carers' to Enable Them to Live Well: A Protocol for a Cluster Stepped Wedge Randomized Controlled Trial. Front. 2016;4:245. PMID: 27843893.

302. Griffiths AW, Surr CA, Creese B, et al. The development and use of the assessment of dementia awareness and person-centred care training tool in longterm care. Dementia. 2018 Jan 01:1471301218768165. doi: https://dx.doi.org/10.1177/1471301218768165. PMID: 29631493.

303. Hamilton G, Gallagher M, Pierson K, et al. Palliative care for dementia: a randomized controlled study of a replicable and financially viable model for organizations providing caregiver support. In Journal of pain and symptom management

304. Hatakeyama R, Fukushima K, Fukuoka Y, et al. Personal home made digital video disk for patients with behavioral psychological symptoms of dementia. Geriatr Gerontol Int. 2010 Jul;10(3):272-4. doi:

https://dx.doi.org/10.1111/j.1447-0594.2010.00623.x. PMID: 20629763.

305. Hayden LJ, Glynn SM, Hahn TJ, et al. The use of Internet technology for psychoeducation and support with dementia caregivers. Psychol Serv. 2012 May;9(2):215-8. doi: https://dx.doi.org/10.1037/a0027056. PMID: 22662739.

306. Heinrich S, Berwig M, Simon A, et al. German adaptation of the Resources for Enhancing Alzheimer's Caregiver Health II: study protocol of a single-centred, randomised controlled trial. BMC geriatr. $2014 \mathrm{Feb}$ 12;14:21. doi: https://dx.doi.org/10.1186/1471-2318-14-21. PMID: 24520910.

307. Hirazakura A, Hatakeyama R, Fukuoka Y, et al. Emotional therapy for patients with dementia. Geriatr Gerontol Int. 2008 Dec;8(4):303-6. doi: https://dx.doi.org/10.1111/j.1447-0594.2008.00486.x. PMID: 19149844.

308. Ho RT, Cheung JK, Chan WC, et al. A 3-arm randomized controlled trial on the effects of dance movement intervention and exercises on elderly with early dementia. BMC geriatr. 2015 Oct 19;15:127. doi: https://dx.doi.org/10.1186/s12877-015-0123-z. PMID: 26481870.

309. Hokkanen L, Rantala L, Remes AM, et al. Dance and movement therapeutic methods in management of dementia: a randomized, controlled study. J Am Geriatr Soc. 2008 Apr;56(4):771-2. doi:

https://dx.doi.org/10.1111/j.1532-5415.2008.01611.x. PMID: 18380687.

310. Holle R, Grassel E, Ruckdaschel S, et al. Dementia care initiative in primary practice: study protocol of a cluster randomized trial on dementia management in a general practice setting. BMC Health Serv Res. 2009 Jun
06;9:91. doi: https://dx.doi.org/10.1186/1472-6963-9-91. PMID: 19500383.

311. Hooghiemstra AM, Eggermont LH, Scheltens P, et al. Study protocol: EXERcise and cognition in sedentary adults with early-ONset dementia (EXERCISE-ON). BMC Neurol. 2012 Aug 16;12:75. doi: https://dx.doi.org/10.1186/1471-2377-12-75. PMID: 22897903.

312. Huis In Het Veld JG, Willemse BM, van Asch IF, et al. Online Self-Management Support for Family Caregivers to Help Them Manage Behavior Changes in Their Relative With Dementia: Study Protocol for a Randomized Controlled Trial and a Process Evaluation. JMIR Res Protoc. 2017 Nov 28;6(11):e239. doi: https://dx.doi.org/10.2196/resprot.8365. PMID: 29183870. 313. Huttemann D. Alzheimer's treatment: bearer of hope fails in phase III. In Pharmazeutische zeitung 314. Jablonski RA, Kolanowski A, Therrien B, et al. Reducing care-resistant behaviors during oral hygiene in persons with dementia. BMC Oral Health. 2011 Nov 19;11:30. doi: https://dx.doi.org/10.1186/1472-6831-11-30. PMID: 22100010.

315. Jablonski-Jaudon RA, Kolanowski AM, Winstead V, et al. Maturation of the MOUTh Intervention: From

Reducing Threat to Relationship-Centered Care. J Gerontol Nurs. 2016 Mar;42(3):15-23; quiz 4-5. doi: https://dx.doi.org/10.3928/00989134-20160212-05. PMID: 26934969.

316. Jutkowitz E, Pizzi LT, Popp J, et al. PND38 EVALUATION OF DEMENTIA INFORMAL CAREGIVERS WILLINGNESS TO PAY FOR A NONPHARMACOLOGIC INTERVENTION TO REDUCE BEHAVIORAL SYMPTOMS. Value Health. 2019;22:S277-. doi: 10.1016/j.jval.2019.04.1311. PMID: CN-01963978.

317. Kikutani T, Yoneyama T, Nishiwaki K, et al. Effect of oral care on cognitive function in patients with dementia. Geriatr Gerontol Int. 2010 Oct;10(4):327-8. doi: https://dx.doi.org/10.1111/j.1447-0594.2010.00637.x. PMID: 20887627.

318. Kohler L, Meinke-Franze C, Hein J, et al. Does an interdisciplinary network improve dementia care? Results from the IDemUck-study. Curr Alzheimer Res.

2014;11(6):538-48. PMID: 24938504.

319. Korn L, Logsdon R, Polissar N, et al. P02.84. A randomized trial of Polarity therapy for stress and pain reduction in American Indian and Alaska Native family dementia caregivers. BMC Altern Med. 2012;12(Suppl 1):1-. doi: 10.1186/1472-6882-12-S1-P140. PMID: 104481508. Language: English. Entry Date: 20121129. Revision Date: 20150711. Publication Type: Journal Article.

320. Kudoh H, Hatakeyama A, Yamamoto T, et al. Foot care using green tea paste for behavioral and psychological symptoms in dementia patients. Geriatr Gerontol Int. 2009 Dec;9(4):399-401. doi: https://dx.doi.org/10.1111/j.14470594.2009.00545.x. PMID: 20002761.

321. Kuo LM, Huang HL, Liang J, et al. Trajectories of health-related quality of life among family caregivers of individuals with dementia: A home-based caregivertraining program matters. Geriatr Nurs. 2017 Mar Apr;38(2):124-32. doi: 
https://dx.doi.org/10.1016/j.gerinurse.2016.08.017. PMID: 27720499.

322. Kwak J, Valuch K, Brondino M. Randomized crossover study of music \& memory intervention with nursing home residents with dementia. In Gerontologist 323. Kwok T, Lam L, Chung J. Case management to improve quality of life of older people with early dementia and to reduce caregiver burden. Hong Kong Med. 2012 Dec;18 Suppl 6:4-6. PMID: 23249844.

324. Lai CK, Lai DL, Ho JS, et al. Interdisciplinary collaboration in the use of a music-with-movement intervention to promote the wellbeing of people with dementia and their families: Development of an evidencebased intervention protocol. Nurs Health Sci. 2016 Mar;18(1):79-84. doi: https://dx.doi.org/10.1111/nhs.12238. PMID: 26354593. 325. Lai CKY, Wong LF, Liu K-H, et al. Online and onsite training for family caregivers of people with dementia: results from a pilot study. In Int J Geriatr Psychiatry 326. Lauterberg J, Grossfeld-Schmitz M, Ruckdäschel S, et al. IDA project--concept and implementation of a cluster-randomized trial on dementia management in a general practice setting. In Zeitschrift fur arztliche fortbildung und qualitatssicherung 327. Li J, Chang Y, Jungquist C, et al. Sleep in long-term care residents with dementia: pilot of a person-centered care intervention. In Sleep

328. Liu W, Wang P, Gray H, et al. Client satisfaction with a stress reduction program for Chinese dementia caregivers. Hallym International Journal of Aging. 2008;10(2):91-110. doi: 10.2190/HA.10.2.c. PMID: 105203686. Language: English. Entry Date: 20100625. Revision Date: 20150711. Publication Type: Journal Article.

329. Logsdon RG. Dementia: psychosocial interventions for family caregivers. Lancet. 2008 Jul 19;372(9634):1823. doi: https://dx.doi.org/10.1016/S0140-6736(08)61048-X. PMID: 18640438.

330. Logsdon RG, McCurry SM, Teri L. A home health care approach to exercise for persons with Alzheimer's disease. Care Manag J. 2005;6(2):90-7. PMID: 16544870. 331. Luk KY, Lai KY, Li CC, et al. The effect of horticultural activities on agitation in nursing home residents with dementia. Int J Geriatr Psychiatry. 2011 Apr;26(4):435-6. doi: https://dx.doi.org/10.1002/gps.2493. PMID: 21412848.

332. Mahoney DF, Tarlow B, Sandaire J. A computermediated intervention for Alzheimer's caregivers. Comput Nurs. 1998 Jul-Aug;16(4):208-16; quiz 17-8. PMID: 9675988.

333. Mahoney EK, Trudeau SA, Penyack SE, et al. Challenges to intervention implementation: lessons learned in the Bathing Persons with Alzheimer's Disease at Home study. Nurs Res. 2006 Mar-Apr;55(2 Suppl):S10-6. PMID: 16601628.

334. Mariko A, Matsuda H, Takahashi M, et al. Touch on the acupoint of shinchuu of Alzheimer's disease patients. In Geriatr Gerontol Int

335. Miskelly F. Electronic tracking of patients with dementia and wandering using mobile phone technology. Age Ageing. 2005 Sep;34(5):497-9. PMID: 16107453.
336. Moore J. Familiar physical activity to familiar music: the effects on apathy, agitation, eating ability, and dietary intake in institutionalized older adults with dementia. 337. Nguyen MF, Bonnefoy M, Adrait A, et al. Efficacy of Hearing Aids on the Cognitive Status of Patients with Alzheimer's Disease and Hearing Loss: A Multicenter Controlled Randomized Trial. J Alzheimers Dis. 2017;58(1):123-37. doi: https://dx.doi.org/10.3233/JAD160793. PMID: 28387664.

338. Ochmann S, Kasper E, Hoffmann W, et al. Feasibility of a cognitive rehabilitation group program for patients with mild dementia in Alzheimer's disease: a randomized, controlled, single blinded pilot study. In Alzheimer's dement

339. Panca M. PMH15 COST-EFFECTIVENESS OF THE MANAGING AGITATION AND RAISING QUALITY OF LIFE IN DEMENTIA (MARQUE) INTERVENTION FOR AGITATION SYMPTOMS IN PEOPLE WITH DEMENTIA IN CARE HOMES. Value Health. 2019;22:S228-. doi: 10.1016/j.jval.2019.04.1060. PMID: CN-01963980.

340. Parra-Vidales E, Soto-Perez F, Perea-Bartolome MV, et al. Online interventions for caregivers of people with dementia: a systematic review. Actas Esp Psiquiatr. 2017 May;45(3):116-26. PMID: 28594057.

341. Poon P, Hui E, Dai D, et al. Cognitive intervention for community-dwelling older persons with memory problems: telemedicine versus face-to-face treatment. Int $\mathrm{J}$ Geriatr Psychiatry. 2005 Mar;20(3):285-6. PMID: 15717335.

342. Poptsi E, Kounti F, Samakouri M, et al. Longitudinal cognitive training program in people with mild Alzheimer's disease: A Randomized Controlled Trial. Hellenic Journal of Nuclear Medicine. 2017 May-August;20(Supplement 1):233-43. PMID: 621578557.

343. Rollin-Sillaire A, Breuilh L, Salleron J, et al. Reasons that prevent the inclusion of Alzheimer's disease patients in clinical trials. Br J Clin Pharmacol. 2013 Apr;75(4):1089-

97. doi: https://dx.doi.org/10.1111/j.1365-

2125.2012.04423.x. PMID: 22891847.

344. Sarkamo T, Laitinen S, Numminen A, et al. Pattern of Emotional Benefits Induced by Regular Singing and Music Listening in Dementia. J Am Geriatr Soc. 2016

Feb;64(2):439-40. doi: https://dx.doi.org/10.1111/jgs.13963. PMID: 26889849.

345. Satoh M, Ogawa J, Tokita T, et al. Physical exercise with music maintains activities of daily living in patients with dementia: mihama-kiho project part 2. In Journal of alzheimers disease

346. Schmid AA, Spangler-Morris C, Beauchamp RC, et al. The Home-Based Occupational Therapy Intervention in the Alzheimer's Disease Multiple Intervention Trial (ADMIT). Occup Ther Ment Health. 2015 Jan 01;31(1):1934. PMID: 26997685.

347. Schulz R, Belle SH, Czaja SJ, et al. Introduction to the special section on Resources for Enhancing Alzheimer's Caregiver Health (REACH). Psychol Aging. 2003

Sep;18(3):357-60. PMID: 14518799.

348. Sithinamsuwan P, Saengwanitch S, Pinidbunjerdkool A, et al. The effect of Thai traditional music on cognitive function, psychological health and quality of sleep among older Thai individuals with dementia. In J Am Geriatr Soc 
349. Slomski A. Short therapy reduces depression in family caregivers of patients with dementia. Jama. 2015 Jan 27;313(4):347. doi:

https://dx.doi.org/10.1001/jama.2014.17991. PMID: 25626027.

350. Snowdon J. Tailored treatment for depression with co-morbid dementia. In Australian and new zealand journal of psychiatry

351. Soto M, Abushakra S, Cummings J, et al. Progress in Treatment Development for Neuropsychiatric Symptoms in Alzheimer's Disease: Focus on Agitation and Aggression. A Report from the EU/US/CTAD Task Force. Jpad. 2015 Sep;2(3):184-8. PMID: 26413494.

352. Spector A, Woods B, Orrell M. Cognitive stimulation for the treatment of Alzheimer's disease. In Expert rev 353. Tanner J, Student M, Black B, et al. Effectiveness of a multicomponent care coordination intervention on dementia caregivers in the community-a randomized control trial Jeremy A. Tanner. In Am J Geriatr Psychiatry 354. Thompson CA, Spilsbury K, Hall J, et al. Systematic review of information and support interventions for caregivers of people with dementia. BMC geriatr. 2007;7 (no pagination)(18). doi: http://dx.doi.org/10.1186/14712318-7-18. PMID: 47329461.

355. Thornley J, Hirjee H, Vasudev A. Music therapy in patients with dementia and behavioral disturbance on an inpatient psychiatry unit: results from a pilot randomized controlled study. Int Psychogeriatr. 2016 May;28(5):86971. doi: https://dx.doi.org/10.1017/S1041610215001866. PMID: 26572722.

356. Tibaldi V, Aimonino N, Ponzetto M, et al. A randomized controlled trial of a home hospital intervention for frail elderly demented patients: behavioral disturbances and caregiver's stress. Arch Gerontol Geriatr Suppl. 2004(9):431-6. PMID: 15207444.

357. Vernooij-Dassen M, Huygen F, Felling A, et al. Home care for dementia patients. J Am Geriatr Soc. 1995 Apr;43(4):456-7. PMID: 7706643.

358. Viana J, Vickers J, Cook M, et al. Currents of memory: recent progress, translational challenges, and ethical considerations in fornix deep brain stimulation trials for Alzheimer's disease. In Neurobiology of aging (no pagination), 2017

359. Vink A, Zuidersma M, Boersma F, et al. Effect of music therapy versus recreational activities on neuropsychiatric symptoms in elderly adults with dementia: an exploratory randomized controlled trial. In J Am Geriatr Soc

360. Voigt-Radloff S, Hüll M. Practice example: neurodegenerative disorders: randomised controlled multicentre trial of home-based occupational therapy for patients with dementia. In Zeitschrift fur evidenz, fortbildung und qualitat IM gesundheitswesen 361. Wells D, Dawson P, Sidani S, et al. The benefits of abilities-focused morning care for residents with dementia and their caregivers. Perspectives. 2000;24(1):17. PMID: 12026330.

362. Willemse BM, Smit D, de Lange J, et al. Nursing home care for people with dementia and residents' quality of life, quality of care and staff well-being: design of the Living Arrangements for people with Dementia (LAD)study. BMC geriatr. 2011 Mar 17;11:11. doi: https://dx.doi.org/10.1186/1471-2318-11-11. PMID: 21414185.

363. Williams V, Bishop-Fitzpatrick L, Lane J, et al. "Video-based coping skills to reduce health risk and improve psychological and physical well-being in Alzheimer's disease family caregivers": erratum. In Psychosom Med

364. Woods B. Promoting well-being and independence for people with dementia. Int J Geriatr Psychiatry. 1999 Feb;14(2):97-105; discussion -9. PMID: 10885861. 365. Yoo R, Yeom J, Kim GH, et al. Erratum to: A Multicenter, Randomized Clinical Trial to Assess the Efficacy of a Therapeutic Intervention Program for Caregivers of People with Dementia. J Clin Neurol. 2019 Jul;15(3):428. doi: https://dx.doi.org/10.3988/jcn.2019.15.3.428. PMID: 31286725 .

\section{Ineligible study design}

366. The tailored activity program (TAP) to address behavioral disturbances in frontotemporal dementia: a feasibility and pilot study. Disability and rehabilitation. 2019;41(3):299-310. doi: 10.1080/09638288.2017.1387614. PMID: CN-01966803. 367. Ahn N, Kim K. Effects of an elastic band resistance exercise program on lower extremity muscle strength and gait ability in patients with Alzheimer's disease. In J Phys Ther Sci

368. Alcala-Lozano R, Morelos-Santana E, Cortes-Sotres $\mathrm{JF}$, et al. Similar clinical improvement and maintenance after rTMS at $5 \mathrm{~Hz}$ using a simple vs. complex protocol in Alzheimer's disease. Brain Stimul. 2018 May Jun;11(3):625-7. doi:

https://dx.doi.org/10.1016/j.brs.2017.12.011. PMID: 29326021.

369. Anderson K, Bird M, Macpherson S, et al. Findings from a pilot investigation of the effectiveness of a snoezelen room in residential care: should we be engaging with our residents more? Geriatr Nurs. 2011 MayJun;32(3):166-77. doi:

https://dx.doi.org/10.1016/j.gerinurse.2010.12.011. PMID: 21306798.

370. Arcoverde C, Deslandes A, Rangel A, et al. Role of physical activity on the maintenance of cognition and activities of daily living in elderly with Alzheimer's disease: papel da atividade fã?sica na manutengPo da cognigPo e atividades de vida dibria em idosos com doenga de Alzheimer. In Arq Neuropsiquiatr

371. Bach D, Bach M, Bohmer F, et al. Reactivating occupational therapy: a method to improve cognitive performance in geriatric patients. Age Ageing. 1995 May;24(3):222-6. PMID: 7645443.

372. Ballard C, Brown R, Fossey J, et al. Brief psychosocial therapy for the treatment of agitation in Alzheimer disease (the CALM-AD trial). Am J Geriatr Psychiatry. 2009 Sep;17(9):726-33. doi:

https://dx.doi.org/10.1097/JGP.0b013e3181b0f8c0. PMID: 19700946.

373. Barnes DE, Mehling W, Wu E, et al. Preventing loss of independence through exercise (PLIE): a pilot clinical trial in older adults with dementia. PLoS ONE. 
2015;10(2):e0113367. doi: https://dx.doi.org/10.1371/journal.pone.0113367. PMID: 25671576.

374. Bass DM, Hornick T, Kunik M, et al. Findings From a Real-World Translation Study of the Evidence-Based "Partners in Dementia Care". Innov Aging. 2019 Jul;3(3):igz031. doi:

https://dx.doi.org/10.1093/geroni/igz031. PMID: 31660442.

375. Boulay M, Benveniste S, Boespflug S, et al. A pilot usability study of MINWii, a music therapy game for demented patients. Technology \& Health Care.

2011;19(1):233-46. PMID: 104577441. Language: English. Entry Date: 20120323. Revision Date: 20150711. Publication Type: Journal Article.

376. Brooker DJ, Snape M, Johnson E, et al. Single case evaluation of the effects of aromatherapy and massage on disturbed behaviour in severe dementia. Br J Clin Psychol. 1997 May;36(Pt 2):287-96. PMID: 9167869.

377. Cadore E, Moneo A, Mensat M, et al. Positive effects of resistance training in frail elderly patients with dementia after long-term physical restraint. In Age (Dordr)

378. Callahan CM, Boustani MA, Weiner M, et al. Implementing dementia care models in primary care settings: The Aging Brain Care Medical Home. Aging Ment Health. 2011 Jan;15(1):5-12. doi: https://dx.doi.org/10.1080/13607861003801052. PMID: 20945236.

379. Carmody J, Potter J, Lewis K, et al. Development and pilot testing of a decision aid for drivers with dementia. BMC Med Inf Decis Mak. 2014 Mar 19;14:19. doi: https://dx.doi.org/10.1186/1472-6947-14-19. PMID: 24642051.

380. Chao HC, Kaas M, Su YH, et al. Effects of the Advanced Innovative Internet-Based Communication Education Program on Promoting Communication Between Nurses and Patients With Dementia. J Nurs Res. 2016 Jun;24(2):163-72. doi:

https://dx.doi.org/10.1097/jnr.0000000000000109. PMID: 26376065.

381. Chenoweth L, Jeon Y-H. Determining the efficacy of dementia care mapping as an outcome measure and a process for change: a pilot study. In Aging Ment Health 382. Chew J, Chong MS, Fong YL, et al. Outcomes of a multimodal cognitive and physical rehabilitation program for persons with mild dementia and their caregivers: a goaloriented approach. Clin Interv Aging. 2015;10:1687-94. doi: https://dx.doi.org/10.2147/CIA.S93914. PMID: 26543358.

383. Chew J, Chong M-S, Fong Y-L, et al. Outcomes of a multimodal cognitive and physical rehabilitation program for persons with mild dementia and their caregivers: a goaloriented approach. In Clin Interv Aging

384. Churchill M, Safaoui J, McCabe BW, et al. Using a therapy dog to alleviate the agitation and desocialization of people with Alzheimer's disease. J Psychosoc Nurs Ment Health Serv. 1999 Apr;37(4):16-22. PMID: 10218187. 385. Clarke A, Chan J, Stott J, et al. An adapted mindfulness intervention for people with dementia in care homes: feasibility pilot study. In Int J Geriatr Psychiatry 386. Cohen-Mansfield J, Marx MS, Dakheel-Ali M, et al. Can agitated behavior of nursing home residents with dementia be prevented with the use of standardized stimuli? J Am Geriatr Soc. 2010 Aug;58(8):1459-64. doi: https://dx.doi.org/10.1111/j.1532-5415.2010.02951.x. PMID: 20579167.

387. Cohen-Mansfield J, Thein K, Marx MS, et al. The relationships of environment and personal characteristics to agitated behaviors in nursing home residents with dementia. J Clin Psychiatry. 2012 Mar;73(3):392-9. doi: https://dx.doi.org/10.4088/JCP.10m06605. PMID: 22152398.

388. Corbeil RR, Quayhagen MP, Quayhagen M. Intervention effects on dementia caregiving interaction: a stress-adaptation modeling approach. J Aging Health. 1999 Feb;11(1):79-95. PMID: 10848143.

389. Cotelli M, Calabria M, Manenti R, et al. Improved language performance in Alzheimer disease following brain stimulation. J Neurol Neurosurg Psychiatry. 2011 Jul;82(7):794-7. doi: https://dx.doi.org/10.1136/jnnp.2009.197848. PMID: 20574108.

390. Cott CA, Dawson P, Sidani S, et al. The effects of a walking/talking program on communication, ambulation, and functional status in residents with Alzheimer disease. Alzheimer Dis Assoc Disord. 2002 Apr-Jun;16(2):81-7. PMID: 12040303.

391. Custodio N, Herrera-Perez E, Lira D, et al. Evaluation of the INECO Frontal Screening and the Frontal

Assessment Battery in Peruvian patients with Alzheimer's

disease and behavioral variant Frontotemporal dementia. In Eneurologicalsci

392. Czaja SJ, Gitlin LN, Schulz R, et al. Development of the risk appraisal measure: a brief screen to identify risk areas and guide interventions for dementia caregivers. $\mathrm{J}$

Am Geriatr Soc. 2009 Jun;57(6):1064-72. doi:

https://dx.doi.org/10.1111/j.1532-5415.2009.02260.x. PMID: 19453305

393. Dabelko-Schoeny H, Phillips G, Darrough E, et al. Equine-assisted intervention for people with dementia. In Anthrozoos

394. Davison TE, Nayer K, Coxon S, et al. A personalized multimedia device to treat agitated behavior and improve mood in people with dementia: A pilot study. Geriatr Nurs. 2016 Jan-Feb;37(1):25-9. doi:

https://dx.doi.org/10.1016/j.gerinurse.2015.08.013. PMID: 26412509.

395. de Oliveira AM, Radanovic M, Homem de Mello PC, et al. An intervention to reduce neuropsychiatric symptoms and caregiver burden in dementia: Preliminary results from a randomized trial of the tailored activity programoutpatient version. Int J Geriatr Psychiatry. 2019 Sep;34(9):1301-7. doi: https://dx.doi.org/10.1002/gps.4958. PMID: 30035341.

396. de Oliveira Silva F, Ferreira JV, Placido J, et al. Three months of multimodal training contributes to mobility and executive function in elderly individuals with mild cognitive impairment, but not in those with Alzheimer's disease: A randomized controlled trial. Maturitas. 2019 Aug;126:28-33. doi: https://dx.doi.org/10.1016/j.maturitas.2019.04.217. PMID: 31239114.

397. de Souto Barreto P, Cadroy Y, Kelaiditi E, et al. The prognostic value of body-mass index on mortality in older 
adults with dementia living in nursing homes. Clin Nutr. 2017 Apr;36(2):423-8. doi: https://dx.doi.org/10.1016/j.clnu.2015.12.009. PMID: 26724185.

398. Deal JA, Betz J, Yaffe K, et al. Hearing Impairment and Incident Dementia and Cognitive Decline in Older Adults: The Health ABC Study. J Gerontol A Biol Sci Med Sci. 2017 May 01;72(5):703-9. doi: https://dx.doi.org/10.1093/gerona/glw069. PMID: 27071780.

399. Deponte A, Missan R. Effectiveness of validation therapy (VT) in group: preliminary results. Arch Gerontol Geriatr. 2007 Mar-Apr;44(2):113-7. PMID: 16712981. 400. Der-Fa L, Hart LK, Lutgendorf SK, et al. Slowing progression of early stages of $\mathrm{AD}$ with alternative therapies: A feasibility study. Geriatr Nurs.

2013;34(6):457-64. doi: 10.1016/j.gerinurse.2013.07.003. PMID: 107924864. Language: English. Entry Date: 20131224. Revision Date: 20150818. Publication Type: Journal Article.

401. Drummond MF, Mohide EA, Tew M, et al. Economic evaluation of a support program for caregivers of demented elderly. Int J Technol Assess Health Care. 1991;7(2):20919. PMID: 1907599.

402. Duggleby W, Ploeg J, McAiney C, et al. A Comparison of Users and Nonusers of a Web-Based Intervention for Carers of Older Persons With Alzheimer Disease and Related Dementias: Mixed Methods Secondary Analysis. J Med Internet Res. 2019 Oct 17;21(10):e14254. doi: https://dx.doi.org/10.2196/14254. PMID: 31625947. 403. Duggleby W, Ploeg J, McAiney C, et al. Web-Based Intervention for Family Carers of Persons with Dementia and Multiple Chronic Conditions (My Tools 4 Care): Pragmatic Randomized Controlled Trial. J Med Internet Res. 2018 Jun 29;20(6):e10484. doi: https://dx.doi.org/10.2196/10484. PMID: 29959111. 404. Dunne TE, Neargarder SA, Cipolloni PB, et al. Visual contrast enhances food and liquid intake in advanced Alzheimer's disease. Clin Nutr. 2004 Aug;23(4):533-8. PMID: 15297089.

405. Edvardsson D, Sandman PO, Borell L. Implementing national guidelines for person-centered care of people with dementia in residential aged care: effects on perceived person-centeredness, staff strain, and stress of conscience. Int Psychogeriatr. 2014 Jul;26(7):1171-9. doi: https://dx.doi.org/10.1017/S1041610214000258. PMID: 24576607.

406. Edwards N, Gardiner M, Ritchie D, et al. Effect of exercise on negative affect in residents in special care units with moderate to severe dementia. In Alzheimer disease and associated disorders

407. Ferrucci R, Mrakic-Sposta S, Gardini S, et al. Behavioral and Neurophysiological Effects of Transcranial Direct Current Stimulation (tDCS) in Fronto-Temporal Dementia. Front Behav Neurosci. 2018;12:235. doi: https://dx.doi.org/10.3389/fnbeh.2018.00235. PMID: 30420799.

408. Forester BP, Mellen E, Mathias L, et al. Electroconvulsive therapy for the treatment of acute agitation and aggression in alzheimer's dementia (ect-ad). Am J Geriatr Psychiatry. 2019;27 (3 Supplement):S125-S6. PMID: CN-01911574.
409. Fusar-Poli L, Bieleninik L, Brondino N, et al. The effect of music therapy on cognitive functions in patients with dementia: a systematic review and meta-analysis. Aging Ment Health. 2017 Jul 10:1-10. doi: https://dx.doi.org/10.1080/13607863.2017.1348474. PMID: 28691506.

410. Galante E, Venturini G, Fiaccadori C. Computerbased cognitive intervention for dementia: preliminary results of a randomized clinical trial. G Ital Med Lav Ergon. 2007 Jul-Sep;29(3 Suppl B):B26-32. PMID: 18575355.

411. Garcia-Alberca JM. Cognitive-behavioral treatment for depressed patients with Alzheimer's disease. An open trial. Arch Gerontol Geriatr. 2017 Jul;71:1-8. doi: https://dx.doi.org/10.1016/j.archger.2017.02.008. PMID: 28237746.

412. George DR, George DR. Intergenerational volunteering and quality of life: mixed methods evaluation of a randomized control trial involving persons with mild to moderate dementia. Qual Life Res. 2011;20(7):987-95. doi: 10.1007/s11136-010-9837-8. PMID: 104578344.

Language: English. Entry Date: 20120323. Revision Date: 20171109. Publication Type: journal article.

413. George DR, Whitehouse PJ. Intergenerational volunteering and quality of life for persons with mild-tomoderate dementia: results from a 5-month intervention study in the United States. J Am Geriatr Soc. 2010;58(4):796-7. doi: 10.1111/j.1532-5415.2010.02790.x. PMID: 105169520. Language: English. Entry Date: 20100521. Revision Date: 20150711. Publication Type: Journal Article.

414. Germain S, Wojtasik V, Lekeu F, et al. Efficacy of Cognitive Rehabilitation in Alzheimer Disease: A 1-Year Follow-Up Study. J Geriatr Psychiatry Neurol. 2019 Jan;32(1):16-23. doi: https://dx.doi.org/10.1177/0891988718813724. PMID: 30477375.

415. Glueckauf RL, Davis WS, Willis F, et al. Telephonebased, cognitive-behavioral therapy for African American dementia caregivers with depression: initial findings.

Rehabil Psychol. 2012 May;57(2):124-39. doi: https://dx.doi.org/10.1037/a0028688. PMID: 22686551. 416. Goyder J, Orrell M, Wenborn J, et al. Staff training using STAR: a pilot study in UK care homes. Int Psychogeriatr. 2012 Jun;24(6):911-20. doi: https://dx.doi.org/10.1017/S1041610211002559. PMID: 22217445.

417. Gray SG, Clair AA. Influence of aromatherapy on medication administration to residential-care residents with dementia and behavioral challenges. Am J Alzheimers Dis Other Demen. 2002 May-Jun;17(3):169-74. PMID: 12083347.

418. Greer KL, Pustay KA, Zaun TC, et al. A comparison of the effects of toys versus live animals on the communication of patients with dementia of the Alzheimer's type. Clinical Gerontologist: The Journal of Aging and Mental Health. 2001;24(3-4):157-82. doi: http://dx.doi.org/10.1300/J018v24n03_13. PMID: 200218267-012.

419. Guo Y, Shi X, Uchiyama H, et al. A study on the rehabilitation of cognitive function and short-term memory in patients with Alzheimer's disease using transcutaneous 
electrical nerve stimulation. Front Med Biol Eng. 2002;11(4):237-47. PMID: 12735425.

420. Guzman A, Wenborn J, Swinson T, et al. Evaluation of the 'Ladder to the Moon, Culture Change Studio Engagement Programme' staff training: Two quasiexperimental case studies. Int J Older People Nurs. 2017 Sep;12(3). doi: https://dx.doi.org/10.1111/opn.12147. PMID: 28185441.

421. Haffmans PM, Sival RC, Lucius SA, et al. Bright light therapy and melatonin in motor restless behaviour in dementia: a placebo-controlled study. Int J Geriatr Psychiatry. 2001 Jan;16(1):106-10. PMID: 11180494. 422. Harris JB. The impact of physical versus social activity on the physical and cognitive functioning of seniors with dementia.

423. Hassan S, Aguirre E, Betz A, et al. Evaluating the effect of Brainfood groups for people with mild cognitive impairment and mild dementia: Preliminary mixedmethodology study. BJPsych Open. 201801 Jul;4(4):20814. doi: http://dx.doi.org/10.1192/bjo.2018.29. PMID: 624229678.

424. Haupt M, Karger A, Jänner M. Improvement of agitation and anxiety in demented patients after psychoeducative group intervention with their caregivers. Int J Geriatr Psychiatry. 2000;15(12):1125-9. doi: 10.1002/1099-1166(200012)15:12<1125::AIDGPS257>3.0.CO;2-F. PMID: 104723571. Language: English. Entry Date: 20110610. Revision Date: 20150711. Publication Type: Journal Article.

425. Heyn P, Abreu BC, Ottenbacher KJ. The effects of exercise training on elderly persons with cognitive impairment and dementia: a meta-analysis. Arch Phys Med Rehabil. 2004 Oct;85(10):1694-704. PMID: 15468033. 426. Hicks-Moore SL, Robinson BA. Favorite music and hand massage: Two interventions to decrease agitation in residents with dementia. Dementia: The International Journal of Social Research and Practice. 2008 Feb;7(1):95108. doi: http://dx.doi.org/10.1177/1471301207085369. PMID: 2008-01605-006.

427. Hilgeman MM, Allen RS, Snow AL, et al. Preserving Identity and Planning for Advance Care (PIPAC): preliminary outcomes from a patient-centered intervention for individuals with mild dementia. Aging Ment Health. 2014 May;18(4):411-24. doi: https://dx.doi.org/10.1080/13607863.2013.868403. PMID: 24359036.

428. Hindle JV, Watermeyer TJ, Roberts J, et al. Goalorientated cognitive rehabilitation for dementias associated with parkinson's disease-a pilot randomised controlled trial. Int J Geriatr Psychiatry. 2018;33(5):718-28. doi: http://dx.doi.org/10.1002/gps.4845. PMID: 623403793. 429. Holm A, Michel M, Stern GA, et al. The outcomes of an inpatient treatment program for geriatric patients with dementia and dysfunctional behaviors. Gerontologist. 1999;39(6):668-76. PMID: 107097417. Language: English. Entry Date: 20000301. Revision Date: 20150711. Publication Type: Journal Article. 430. Holm AK, Lepp M, Ringsberg KC. Dementia: involving patients in storytelling--a caring intervention. A pilot study. J Clin Nurs. 2005 Feb;14(2):256-63. PMID: 15669935.
431. Holmes C, Hopkins V, Hensford C, et al. Lavender oil as a treatment for agitated behaviour in severe dementia: a placebo controlled study. Int J Geriatr Psychiatry. 2002 Apr;17(4):305-8. PMID: 11994882.

432. Hooghiemstra AM, Eggermont LH, Scheltens P, et al. Exercise and early-onset Alzheimer's disease: theoretical considerations. Dement Geriatr Cogn Dis Extra. 2012 Jan;2:132-45. doi: https://dx.doi.org/10.1159/000335493. PMID: 22590474.

433. Hsu MH, Flowerdew R, Parker M, et al. Individual music therapy for managing neuropsychiatric symptoms for people with dementia and their carers: a cluster randomised controlled feasibility study. BMC geriatr. $2015 \mathrm{Jul}$

18;15:84. doi: https://dx.doi.org/10.1186/s12877-015-00824. PMID: 26183582.

434. Hwang HR, Choi SH, Yoon DH, et al. The effect of cognitive training in patients with mild cognitive impairment and early Alzheimer's disease: a preliminary study. J Clin Neurol. 2012 Sep;8(3):190-7. doi: https://dx.doi.org/10.3988/jcn.2012.8.3.190. PMID: 23091528.

435. Ibarria M, Alegret M, Valero S, et al. Beneficial Effects of an Integrated Psychostimulation Program in Patients with Alzheimer's Disease. J Alzheimers Dis. 2016;50(2):559-66. doi: https://dx.doi.org/10.3233/JAD150455. PMID: 26757182

436. Iliffe S, Robinson L, Bamford C, et al. Introducing case management for people with dementia in primary care: a mixed-methods study. In Br J Gen Pract

437. Iso-Markku $P$, Waller K, Kujala UM, et al. Physical activity and dementia: long-term follow-up study of adult twins. Ann Med. 2015 Mar;47(2):81-7. doi: https://dx.doi.org/10.3109/07853890.2014.994675. PMID: 25613168.

438. Jayalath D, Ashaye K, Kvavilashvili L. Carers' diaries in dementia: Is there a role in clinical practice? Alzheimer's and Dementia: Diagnosis, Assessment and Disease Monitoring. 2016;4:94-8. doi: http://dx.doi.org/10.1016/j.dadm.2016.03.003. PMID: 612393378.

439. Jia J, Zuo X, Jia XF, et al. Diagnosis and treatment of dementia in neurology outpatient departments of general hospitals in China. Alzheimer's and Dementia. 201601 Apr;12(4):446-53. doi: http://dx.doi.org/10.1016/j.jalz.2015.06.1892. 440. Johannessen A, Povlsen L, Bruvik Fy, et al. Implementation of a multicomponent psychosocial programme for persons with dementia and their families in Norwegian municipalities: experiences from the perspective of healthcare professionals who performed the intervention. In Scand J Caring Sci

441. Johnson L, Deatrick EJ, Oriel K. The use of music to improve exercise participation in people with dementia: a pilot study. In Physical \& occupational therapy in geriatrics 442. Jones C, Moyle W, Murfield J, et al. Does Cognitive Impairment and Agitation in Dementia Influence Intervention Effectiveness? Findings From a ClusterRandomized-Controlled Trial With the Therapeutic Robot, PARO. J Am Med Dir Assoc. 2018 Jul;19(7):623-6. doi: https://dx.doi.org/10.1016/j.jamda.2018.02.014. PMID: 29656838. 
443. Kales HC, Gitlin LN, Stanislawski B, et al. WeCareAdvisorTM: The Development of a Caregiverfocused, Web-based Program to Assess and Manage Behavioral and Psychological Symptoms of Dementia. Alzheimer Dis Assoc Disord. 2017 Jul-Sep;31(3):263-70. doi: https://dx.doi.org/10.1097/WAD.0000000000000177. PMID: 27849639.

444. Kang HS, Ahn IS, Kim JH, et al. Neuropsychiatric symptoms in korean patients with Alzheimer's disease: exploratory factor analysis and confirmatory factor analysis of the neuropsychiatric inventory. Dement Geriatr Cogn Disord. 2010;29(1):82-7. doi: https://dx.doi.org/10.1159/000264629. PMID: 20130406. 445. Kerkhof YJ, Graff MJ, Bergsma A, et al. Better selfmanagement and meaningful activities thanks to tablets? Development of a person-centered program to support people with mild dementia and their carers through use of hand-held touch screen devices. Int Psychogeriatr. 2016 11;28(11):1917-29. PMID: 27425002.

446. Kessels RPC, Olde Hensken LMG. Effects of errorless skill learning in people with mild-to-moderate or severe dementia: a randomized controlled pilot study. NeuroRehabilitation. 2009;25(4):307-12. doi: 10.3233/NRE-2009-0529. PMID: 105277868. Language: English. Entry Date: 20100205. Revision Date: 20150711. Publication Type: Journal Article.

447. Klages K, Zecevic A, Orange JB, et al. Potential of Snoezelen room multisensory stimulation to improve balance in individuals with dementia: a feasibility randomized controlled trial. Clin Rehabil. 2011

Jul;25(7):607-16. doi:

https://dx.doi.org/10.1177/0269215510394221. PMID: 21382864.

448. Kolanowski AM, Litaker M, Buettner L. Efficacy of theory-based activities for behavioral symptoms of dementia. Nurs Res. 2005 Jul-Aug;54(4):219-28. PMID: 16027564 .

449. Koltai DC, Welsh-Bohmer KA, Schmechel DE. Influence of anosognosia on treatment outcome among dementia patients. Neuropsychol. 2001 Jul;11(3-4):455-75. doi: http://dx.doi.org/10.1080/09602010042000097. PMID: 2001-07363-012.

450. La Garce M. Daylight interventions and Alzheimer's behaviors: A twelve-month study. Journal of Architectural and Planning Research. 2004 Fal;21(3):257-69. PMID: 2004-19048-003.

451. Lamotte G, Shah RC, Lazarov O, et al. Exercise Training for Persons with Alzheimer's Disease and Caregivers: A Review of Dyadic Exercise Interventions. J Mot Behav. 2017 Jul-Aug;49(4):365-77. doi: https://dx.doi.org/10.1080/00222895.2016.1241739. PMID: 27870597.

452. Lancioni GE, O'Reilly MF, Singh NN, et al. Assessing the impact and social perception of selfregulated music stimulation with patients with Alzheimer's disease. Research in Developmental Disabilities. 2013;34(1):139-46. doi: 10.1016/j.ridd.2012.07.026. PMID: 104401179. Language: English. Entry Date: 20130607. Revision Date: 20150711. Publication Type: Journal Article.

453. Lancioni GE, Singh NN, O'Reilly MF, et al. Persons with mild or moderate Alzheimer's disease learn to use urine alarms and prompts to avoid large urinary accidents. Research in Developmental Disabilities. 2011;32(5):19982004. doi: 10.1016/j.ridd.2011.04.011. PMID: 104645817. Language: English. Entry Date: 20111028. Revision Date: 20150711. Publication Type: Journal Article. 454. Lancioni GE, Singh NN, O'Reilly MF, et al. Technology-aided verbal instructions to help persons with mild or moderate Alzheimer's disease perform daily activities. Research in Developmental Disabilities. 2010;31(6):1240-50. doi: 10.1016/j.ridd.2010.07.021. PMID: 104927845. Language: English. Entry Date: 20110204. Revision Date: 20150711. Publication Type: Journal Article.

455. Lanza G, Centonze SS, Destro G, et al. Shiatsu as an adjuvant therapy for depression in patients with

Alzheimer's disease: A pilot study. Complement Ther Med. 2018 Jun;38:74-8. doi:

https://dx.doi.org/10.1016/j.ctim.2018.04.013. PMID: 29857884.

456. Laporte UF, Graske J, Grill S, et al. Regional dementia care networks in Germany: changes in caregiver burden at one-year follow-up and associated factors. In Int Psychogeriatr

457. Lee GY, Yip CC, Yu EC, et al. Evaluation of a computer-assisted errorless learning-based memory training program for patients with early Alzheimer's disease in Hong Kong: a pilot study. Clin Interv Aging. 2013;8:62333. doi: https://dx.doi.org/10.2147/CIA.S45726. PMID: 23766638.

458. Lee J, Choi BH, Oh E, et al. Treatment of Alzheimer's Disease with Repetitive Transcranial Magnetic Stimulation Combined with Cognitive Training: A Prospective, Randomized, Double-Blind, Placebo-Controlled Study. J Clin Neurol. 2016 Jan;12(1):57-64. doi:

https://dx.doi.org/10.3988/jcn.2016.12.1.57. PMID: 26365021.

459. Lee MM, Camp CJ, Malone ML. Effects of intergenerational Montessori-based activities programming on engagement of nursing home residents with dementia. Clin Interv Aging. 2007;2(3):477-83. PMID: 18044197. 460. Libin A, Cohen-Mansfield J. Therapeutic robocat for nursing home residents with dementia: preliminary inquiry. Am J Alzheimers Dis Other Demen. 2004 Mar-

Apr;19(2):111-6. PMID: 15106392.

461. Lin S, Yang Y, Qi Q, et al. The Beneficial Effect of Physical Exercise on Cognitive Function in a Nondementia Aging Chinese Population. Front Aging Neurosci. 2019;11:238. doi:

https://dx.doi.org/10.3389/fnagi.2019.00238. PMID: 31555125 .

462. Livingston G, Lewis-Holmes E, Pitfield C, et al. Improving the end-of-life for people with dementia living in a care home: an intervention study. Int Psychogeriatr. 2013 Nov;25(11):1849-58. doi: https://dx.doi.org/10.1017/S1041610213001221. PMID: 23924580.

463. Low LF, Carroll S, Merom D, et al. We think you can dance! A pilot randomised controlled trial of dance for nursing home residents with moderate to severe dementia. Complement Ther Med. 2016 Dec;29:42-4. doi: https://dx.doi.org/10.1016/j.ctim.2016.09.005. PMID: 27912955. 
464. Lyketsos CG, Lindell Veiel L, Baker A, et al. A randomized, controlled trial of bright light therapy for agitated behaviors in dementia patients residing in longterm care. Int J Geriatr Psychiatry. 1999 Jul;14(7):520-5. PMID: 10440971.

465. Maci T, Pira FL, Quattrocchi G, et al. Physical and cognitive stimulation in Alzheimer Disease. the GAIA Project: a pilot study. Am J Alzheimers Dis Other Demen. 2012 Mar;27(2):107-13. doi:

https://dx.doi.org/10.1177/1533317512440493. PMID: 22495338.

466. Manckoundia P, Taroux M, Kubicki A, et al. Impact of ambulatory physiotherapy on motor abilities of elderly subjects with Alzheimer's disease. In Geriatr Gerontol Int 467. Maseda A, Sanchez A, Marante MP, et al. Effects of Multisensory Stimulation on a Sample of Institutionalized Elderly People With Dementia Diagnosis: A Controlled Longitudinal Trial. Am J Alzheimers Dis Other Demen. 2014 Aug;29(5):463-73. doi:

https://dx.doi.org/10.1177/1533317514522540. PMID: 24604894.

468. Matthews J, Lingler J, Campbell G, et al. Usability of a Wearable Camera System for Dementia Family Caregivers. In Journal of healthcare engineering 469. McCaffrey R, Park J, Newman D, et al. The effect of chair yoga in older adults with moderate and severe Alzheimer's disease. Res. 2014 Jul-Aug;7(4):171-7. doi: https://dx.doi.org/10.3928/19404921-20140218-01. PMID: 24568209.

470. McCreedy EM, Mor V, Thomas K, et al. Effects of music and memory on agitated and aggressive behaviors in dementia: results from nursing home pilot study. J Am Geriatr Soc. 2019;67:S91-. doi: 10.1111/jgs.15898. PMID: CN-01940453.

471. Mihailidis A, Boger J, Craig T, et al. The COACH prompting system to assist older adults with dementia through handwashing: an efficacy study. In BMC geriatr 472. Milev RV, Kellar T, McLean M, et al. Multisensory stimulation for elderly with dementia: a 24-week singleblind randomized controlled pilot study. Am J Alzheimers Dis Other Demen. 2008 Aug-Sep;23(4):372-6. doi: https://dx.doi.org/10.1177/1533317508316681. PMID: 18453647.

473. Mioshi E, McKinnon C, Savage S, et al. Improving burden and coping skills in frontotemporal dementia caregivers: a pilot study. Alzheimer Dis Assoc Disord. 2013;27(1):84-6. doi: 10.1097/WAD.0b013e31824a7f5b. PMID: 108031647. Language: English. Entry Date: 20130823. Revision Date: 20150712. Publication Type: Journal Article.

474. Mittelman MS, Bartels SJ. Translating research into practice: case study of a community-based dementia caregiver intervention. Health Aff (Millwood). 2014 Apr;33(4):587-95. doi:

https://dx.doi.org/10.1377/hlthaff.2013.1334. PMID: 24711319.

475. Moyle W, Cooke M, Beattie E, et al. Exploring the effect of companion robots on emotional expression in older adults with dementia: a pilot randomized controlled trial. J Gerontol Nurs. 2013 May;39(5):46-53. doi: https://dx.doi.org/10.3928/00989134-20130313-03. PMID: 23506125.
476. Moyle W, Cooke M, O'Dwyer ST, et al. The effect of foot massage on long-term care staff working with older people with dementia: a pilot, parallel group, randomized controlled trial. BMC Nurs. 2013 Feb 18;12:5. doi: https://dx.doi.org/10.1186/1472-6955-12-5. PMID: 23414448.

477. Nair BK, Heim C, Krishnan C, et al. The effect of Baroque music on behavioural disturbances in patients with dementia. Australas J Ageing. 2011 Mar;30(1):11-5. doi: https://dx.doi.org/10.1111/j.1741-6612.2010.00439.x. PMID: 21395934.

478. Nakanishi M, Hirooka K, Imai Y, et al. Dementia Care Competence Among Care Professionals and Reduced Challenging Behavior of Home-Dwelling Persons with Dementia: A Pre- and Post-Intervention Data Analysis. J Alzheimers Dis. 2018;64(2):515-23. doi: https://dx.doi.org/10.3233/JAD-171077. PMID: 29914021. 479. Nakrem S, Stensvik GT, Skjong RJ, et al. Staff experiences with implementing a case conferencing care model in nursing homes: a focus group study. BMC Health Serv Res. 2019 Mar 27;19(1):191. doi: https://dx.doi.org/10.1186/s12913-019-4034-0. PMID: 30917815.

480. Narme P, Tonini A, Khatir F, et al. Non pharmacological treatment for Alzheimer's disease: comparison between musical and non-musical interventions. In Geriatrie ET psychologie neuropsychiatrie du vieillissement

481. Okumura Y, Tanimukai S, Asada T. Effects of shortterm reminiscence therapy on elderly with dementia: a comparison with everyday conversation approaches. In Psychogeriatrics

482. Onor ML, Trevisiol M, Negro C, et al. Impact of a multimodal rehabilitative intervention on demented patients and their caregivers. Am J Alzheimers Dis Other Demen. 2007 Aug-Sep;22(4):261-72. PMID: 17712157.

483. Orrell M, Spector A, Thorgrimsen L, et al. A pilot study examining the effectiveness of maintenance Cognitive Stimulation Therapy (MCST) for people with dementia. Int J Geriatr Psychiatry. 2005;20(5):446-51. PMID: 106207035. Language: English. Entry Date: 20070105. Revision Date: 20150711. Publication Type: Journal Article.

484. Ott CH, Kelber ST, Blaylock M. "Easing the way" for spouse caregivers of individuals with dementia: a pilot feasibility study of a grief intervention. Res. 2010 Apr;3(2):89-99. doi: https://dx.doi.org/10.3928/1940492120100302-01. PMID: 20415358.

485. Ozdemir L, Akdemir N. Effects of multisensory stimulation on cognition, depression and anxiety levels of mildly-affected Alzheimer's patients. J Neurol Sci. 2009 Aug 15;283(1-2):211-3. doi: https://dx.doi.org/10.1016/j.jns.2009.02.367. PMID: 19289242.

486. Paddick SM, Mkenda S, Mbowe G, et al. Cognitive stimulation therapy as a sustainable intervention for dementia in sub-Saharan Africa: feasibility and clinical efficacy using a stepped-wedge design. Int Psychogeriatr. 2017 Jun;29(6):979-89. doi: https://dx.doi.org/10.1017/S1041610217000163. PMID: 28222815. 
487. Parker LJ, Gaugler JE, Samus Q, et al. Adult Day Service Use Decreases Likelihood of a Missed Physician's Appointment Among Dementia Caregivers. J Am Geriatr Soc. 2019 Jul;67(7):1467-71. doi: https://dx.doi.org/10.1111/jgs.15995. PMID: 31219175. 488. Paun O, Farran CJ, Perraud S, et al. Successful caregiving of persons with Alzheimer's disease: skill development over time. Alzheimer's Care Quarterly. 2004;5(3):241-51. PMID: 106657982. Language: English. Entry Date: 20041105. Revision Date: 20150711. Publication Type: Journal Article. 489. Ploeg J, Northwood M, Duggleby W, et al. Caregivers of older adults with dementia and multiple chronic conditions: Exploring their experiences with significant changes. Dementia. 2019 Mar 06:1471301219834423. doi: https://dx.doi.org/10.1177/1471301219834423. PMID: 30841745.

490. Pomeroy VM. The effect of physiotherapy input on mobility skills of elderly people with severe dementing illness. Clin Rehabil. 1993;7(2):163-70. PMID: 23161041. 491. Pomykala KL, Silverman DH, Geist CL, et al. A pilot study of the effects of meditation on regional brain metabolism in distressed dementia caregivers. Aging health. 2012 Oct 01;8(5):509-16. PMID: 23378856. 492. Rapp T, Grand A, Cantet C, et al. Public financial support receipt and non-medical resource utilization in Alzheimer's disease results from the PLASA study. Soc Sci Med. 2011 Apr;72(8):1310-6. doi: https://dx.doi.org/10.1016/j.socscimed.2011.02.039. PMID: 21463914.

493. Resnick B, Galik E, Kolanowski A, et al. Reliability and Validity of the Care Plan Checklist for Evidence of Person-Centered Approaches for Behavioral and Psychological Symptoms Associated With Dementia. J Am Med Dir Assoc. 2017 Nov 28;28:28. doi: https://dx.doi.org/10.1016/j.jamda.2017.10.021. PMID: 29191763.

494. Rokstad AM, Engedal K, Kirkevold O, et al. The association between attending specialized day care centers and the quality of life of people with dementia. Int Psychogeriatr. 2017 04;29(4):627-36. doi: https://dx.doi.org/10.1017/S1041610216002015. PMID: 27852334.

495. Romo ML, Schooling CM. Examining the Causal Role of Leptin in Alzheimer Disease: A Mendelian Randomization Study. Neuroendocrinology. 201701 Aug;105(2):182-8. doi: http://dx.doi.org/10.1159/000475713. PMID: 615575945. 496. Rosenberg PB, Mielke MM, Lyketsos CG. Caregiver assessment of patients' depression in Alzheimer disease: longitudinal analysis in a drug treatment study. Am J Geriatr Psychiatry. 2005 Sep;13(9):822-6. PMID: 16166413.

497. Rosewarne R, Bruce A, McKenna M. Dementia programme effectiveness in long-term care. Int J Geriatr Psychiatry. 1997 Feb;12(2):173-82. PMID: 9097210. 498. Rostad H, Puts M, Cvancarova SM, et al. Associations between Pain and Quality of Life in Severe Dementia: a Norwegian Cross-Sectional Study. In Dement Geriatr Cogn Dis Extra
499. Rouch I, Pongan E, Trombert B, et al. One-Year Evolution of Behavioral and Psychological Symptoms of Dementia in Patients Initially Hospitalized in Cognitive Behavioral Units: the EVITAL Prospective Cohort. In J Alzheimers Dis

500. Rouse HJ, Small BJ, Faust ME. Assessment of Cognitive Training \& Social Interaction in People with Mild to Moderate Dementia: A Pilot Study. Clin Gerontol. 201901 Jul;42(4):421-34. doi:

http://dx.doi.org/10.1080/07317115.2019.1590489. PMID: 626971925.

501. Rutherford G, Lithgow B, Moussavi Z. Short and long-term effects of rTMS treatment on Alzheimer's disease at different stages: a pilot study. In Journal of experimental neuroscience

502. Salotti P, Sanctis B, Clementi A, et al. Evaluation of the efficacy of a cognitive rehabilitation treatment on a group of Alzheimer's patients with moderate cognitive impairment: a pilot study. In Aging clinical and experimental research

503. Sanchez A, Marante-Moar MP, Sarabia C, et al. Multisensory Stimulation as an Intervention Strategy for Elderly Patients With Severe Dementia: A Pilot Randomized Controlled Trial. Am J Alzheimers Dis Other Demen. 2016 Jun;31(4):341-50. doi:

https://dx.doi.org/10.1177/1533317515618801. PMID: 26631687.

504. Sánchez A, Maseda A, Marante-Moar MP, et al. Comparing the Effects of Multisensory Stimulation and Individualized Music Sessions on Elderly People with Severe Dementia: A Randomized Controlled Trial. J Alzheimers Dis. 2016;52(1):303-15. doi: 10.3233/JAD151150. PMID: 114968532. Language: English. Entry Date: 20170126. Revision Date: 20180615. Publication Type: journal article.

505. Sanchez-Valdeon L, Fernandez-Martinez E, LomaRamos S, et al. Canine-Assisted Therapy and Quality of Life in People With Alzheimer-Type Dementia: Pilot Study. Front Psychol. 2019;10:1332. doi: https://dx.doi.org/10.3389/fpsyg.2019.01332. PMID: 31244731.

506. Santana-Sosa E, Barriopedro MI, López-Mojares LM, et al. Exercise training is beneficial for Alzheimer's patients. Int J Sports Med. 2008;29(10):845-50. PMID: 105568866. Language: English. Entry Date: 20090206. Revision Date: 20150711. Publication Type: Journal Article.

507. Scharre DW, Weichart E, Nielson D, et al. Deep Brain Stimulation of Frontal Lobe Networks to Treat Alzheimer's Disease. J Alzheimers Dis. 2018;62(2):621-33. doi: https://dx.doi.org/10.3233/JAD-170082. PMID: 29400666.

508. Scherder EJ, Bouma A. Effects of transcutaneous electrical nerve stimulation on memory and behavior in Alzheimer's disease may be stage-dependent. Biol Psychiatry. 1999 Mar 15;45(6):743-9. PMID: 10188004. 509. Scherder EJ, Van Someren EJ, Swaab DF. Transcutaneous electrical nerve stimulation (TENS) improves the rest-activity rhythm in midstage Alzheimer's disease. Behav Brain Res. 1999 May;101(1):105-7. PMID: 10342404. 
510. Schiffman SS, Graham BG, Sattely-Miller EA, et al. Taste, smell and neuropsychological performance of individuals at familial risk for Alzheimer's disease. Neurobiol Aging. 2002 May-Jun;23(3):397-404. PMID: 11959402.

511. Schinköthe D, Wilz G. The Assessment of Treatment Integrity in a Cognitive Behavioral Telephone Intervention Study With Dementia Caregivers. Clin Gerontol. 2014;37(3):211-34. doi: 10.1080/07317115.2014.886653. PMID: 104064713. Language: English. Entry Date: 20140418. Revision Date: 20151023. Publication Type: Journal Article.

512. Schulz R, Mendelsohn AB, Haley WE, et al. End-oflife care and the effects of bereavement on family caregivers of persons with dementia. N Engl J Med. 2003 Nov 13;349(20):1936-42. PMID: 14614169.

513. Schulze J, Bussche H, Kaduszkiewicz H, et al. Institutionalization in incident dementia cases in comparison to age- and sex- matched controls: a 5-year follow-up from Germany. In Social psychiatry and psychiatric epidemiology

514. Sedeno L, Piguet O, Abrevaya S, et al. Tackling variability: a multicenter study to provide a gold-standard network approach for frontotemporal dementia. In Human brain mapping

515. Siegel Jr BV, Buchsbaum MS, Starr A, et al. Glucose metabolic rate and progression of illness in Alzheimer's disease. Int J Geriatr Psychiatry. 1995;10(8):659-67. doi: http://dx.doi.org/10.1002/gps.930100806. PMID: 25262446.

516. Sindi S, Ngandu T, Hovatta I, et al. Baseline Telomere Length and Effects of a Multidomain Lifestyle Intervention on Cognition: The FINGER Randomized Controlled Trial. J Alzheimers Dis. 2017;59(4):1459-70. doi: https://dx.doi.org/10.3233/JAD-170123. PMID: 28777749.

517. Snow AL, Hovanec L, Brandt J. A controlled trial of aromatherapy for agitation in nursing home patients with dementia. J Altern Complement Med. 2004;10(3):431-7. doi: 10.1089/1075553041323696. PMID: 106568581. Language: English. Entry Date: 20050128. Revision Date: 20150820. Publication Type: Journal Article.

518. Sposito G, Barbosa A, Figueiredo D, et al. Effects of multisensory and motor stimulation on the behavior of people with dementia. Dementia. 2017 Apr;16(3):344-59. doi: https://dx.doi.org/10.1177/1471301215592080. PMID: 26112166.

519. Spring HJ, Rowe MA, Kelly A. Improving caregivers' well-being by using technology to manage nighttime activity in persons with dementia. Res. 2009;2(1):39-48. doi: 10.3928/19404921-20090101-10. PMID: 105631700 . Language: English. Entry Date: 20090306. Revision Date: 20150711. Publication Type: Journal Article.

520. Stevens AB, Lancer K, Smith ER, et al. Engaging communities in evidence-based interventions for dementia caregivers. Fam Community Health. 2009 Jan-Mar;32(1 Suppl):S83-92. doi:

https://dx.doi.org/10.1097/01.FCH.0000342843.28477.72. PMID: 19065098.

521. Sutcliffe C, Larner S. Counselling carers of the elderly at home: a preliminary study. Br J Clin Psychol. 1988 May;27(Pt 2):177-8. PMID: 3293671.
522. Svensson B, Hansson L. Mental health first aid for the elderly: A pilot study of a training program adapted for helping elderly people. Aging Ment Health. 2017 Jun;21(6):595-601. doi:

https://dx.doi.org/10.1080/13607863.2015.1135873. PMID: 26829477.

523. Teri L, Logsdon RG, Weiner MF, et al. Treatment for agitation in dementia patients: A behavior management approach. Psychotherapy. 1998 Winter;35(4):436-43. PMID: 29227715.

524. Thyrian JR, Eichler T, Hertel J, et al. Burden of Behavioral and Psychiatric Symptoms in People Screened Positive for Dementia in Primary Care: Results of the DelpHi-Study. J Alzheimers Dis. 2015;46(2):451-9. doi: https://dx.doi.org/10.3233/JAD-143114. PMID: 25765916. 525. Thyrian JR, Eichler T, Pooch A, et al. Systematic, early identification of dementia and dementia care management are highly appreciated by general physicians in primary care - results within a cluster-randomizedcontrolled trial (DelpHi). J Multidiscip Healthc. 2016;9:183-90. doi:

https://dx.doi.org/10.2147/JMDH.S96055. PMID: 27143912.

526. Thyrian JR, Eichler T, Reimann M, et al. Depressive symptoms and depression in people screened positive for dementia in primary care - results of the DelpHi-study. Int Psychogeriatr. 2016 06;28(6):929-37. doi:

https://dx.doi.org/10.1017/S1041610215002458. PMID: 26785854.

527. Travers C. Increasing enjoyable activities to treat depression in nursing home residents with dementia: A pilot study. Dementia. 2017 Feb;16(2):204-18. doi: https://dx.doi.org/10.1177/1471301215586069. PMID: 25972128.

528. van der Steen JT, Albers G, Licht-Strunk E, et al. A validated risk score to estimate mortality risk in patients with dementia and pneumonia: barriers to clinical impact. Int Psychogeriatr. 2011 Feb;23(1):31-43. doi: https://dx.doi.org/10.1017/S1041610210001079. PMID: 20653988.

529. van Mierlo LD, Meiland FJ, Dröes RM, et al. Dementelcoach: effect of telephone coaching on carers of community-dwelling people with dementia. Int

Psychogeriatr. 2012;24(2):212-22. doi: 10.1017/S1041610211001827. PMID: 104511872. Language: English. Entry Date: 20120511. Revision Date: 20161117. Publication Type: journal article. 530. van Paasschen J, Clare L, Yuen KS, et al. Cognitive rehabilitation changes memory-related brain activity in people with Alzheimer disease. Neurorehabil Neural Repair. 2013 Jun;27(5):448-59. doi:

https://dx.doi.org/10.1177/1545968312471902. PMID: 23369983.

531. Van Someren EJ, Scherder EJ, Swaab DF. Transcutaneous electrical nerve stimulation (TENS) improves circadian rhythm disturbances in Alzheimer disease. Alzheimer Dis Assoc Disord. 1998 Jun;12(2):1148. PMID: 9651141.

532. Vidoni ED, Watts AS, Burns JM, et al. Feasibility of a Memory Clinic-Based Physical Activity Prescription Program. J Alzheimers Dis. 2016;53(1):161-70. doi: 10.3233/JAD-160158. PMID: 116350716. Language: 
English. Entry Date: 20180129. Revision Date: 20180525. Publication Type: journal article.

533. Watson K, Hatcher D, Good A. A randomised controlled trial of Lavender (Lavandula Angustifolia) and Lemon Balm (Melissa Officinalis) essential oils for the treatment of agitated behaviour in older people with and without dementia. Complement Ther Med. 2019 Feb;42:366-73. doi: https://dx.doi.org/10.1016/j.ctim.2018.12.016. PMID: 30670268.

534. Wells Y, Jorm AF. Evaluation of a special nursing home unit for dementia sufferers: a randomised controlled comparison with community care. Aust N Z J Psychiatry. 1987 Dec;21(4):524-31. PMID: 3449047.

535. Werner C, Rosner R, Wiloth S, et al. Time course of changes in motor-cognitive exergame performances during task-specific training in patients with dementia: identification and predictors of early training response. $\mathrm{J}$ Neuroengineering Rehabil. 2018 Nov 08;15(1):100. doi: https://dx.doi.org/10.1186/s12984-018-0433-4. PMID: 30409202.

536. Wilks SE, Boyd PA, Bates SM, et al. Montessoribased activities among persons with late-stage dementia: Evaluation of mental and behavioral health outcomes. Dementia. 2017 Jan 01:1471301217703242. doi: https://dx.doi.org/10.1177/1471301217703242. PMID: 28449593.

537. Wiloth S, Werner C, Lemke NC, et al. Motorcognitive effects of a computerized game-based training method in people with dementia: a randomized controlled trial. Aging Ment Health. 2018 Sep;22(9):1124-35. doi: https://dx.doi.org/10.1080/13607863.2017.1348472. PMID: 28682124.
538. Woods DL, Dimond M. The effect of therapeutic touch on agitated behavior and cortisol in persons with Alzheimer's disease. Biol Res Nurs. 2002 Oct;4(2):104-14. PMID: 12408216.

539. Wu E, Barnes DE, Ackerman SL, et al. Preventing Loss of Independence through Exercise (PLIÉ): qualitative analysis of a clinical trial in older adults with dementia. Aging Ment Health. 2015;19(4):353-62. doi: 10.1080/13607863.2014.935290. PMID: 103879583. Language: English. Entry Date: 20150121. Revision Date: 20160922. Publication Type: Journal Article.

540. Yoon J, Lee S, Lim H, et al. The effects of cognitive activity combined with active extremity exercise on balance, walking activity, memory level and quality of life of an older adult sample with dementia. In J Phys Ther Sci 541. Yu F, Nelson NW, Savik K, et al. Affecting cognition and quality of life via aerobic exercise in Alzheimer's disease. West J Nurs Res. 2013 Jan;35(1):24-38. doi: https://dx.doi.org/10.1177/0193945911420174. PMID: 21911546.

542. Yu F, Savik K, Wyman JF, et al. Maintaining physical fitness and function in Alzheimer's disease: a pilot study. Am J Alzheimers Dis Other Demen. 2011 Aug;26(5):406-12. doi: https://dx.doi.org/10.1177/1533317511414861. PMID: 21750046.

543. Zhou Y, Jia J. Clinical observation on catgut implantation at acupoint for treatment of Alzheimer's disease. In Zhongguo zhen jiu [Chinese acupuncture \& moxibustion]

544. Zhu C, Cosentino S, Ornstein K, et al. Interactive Effects of Dementia Severity and Comorbidities on Medicare Expenditures. In J Alzheimers Dis 


\section{Appendix C. Treatment for BPSD}

\section{Assisted Therapy}

able C-1. Risk of bias assessment: assisted therapy

\begin{tabular}{|c|c|c|c|c|c|c|c|c|c|}
\hline \begin{tabular}{|l|} 
Study (PMID) \\
\end{tabular} & Outcome Timing & $\begin{array}{l}\text { Attrition Bias } \\
\text { Attrition \% }\end{array}$ & Selection Bias & Detection Bias & \begin{tabular}{|l} 
Performance Bias \\
\end{tabular} & Reporting Bias & Fidelity Bias & Funder & Overall Rating \\
\hline \begin{tabular}{|l|} 
Moyle 2017 $2(28780395)$ \\
Jones 2018 $(29656838)$ \\
Mervin 2018 $2018^{3}(29325922)$ \\
Moyle 2018 $(29563027)$ \\
\end{tabular} & 10 weeks & $\begin{array}{l}\text { Low } \\
10 \text { weeks: } 4.3 \% \\
15 \text { weeks: } 7.0 \%\end{array}$ & Low & Medium & Medium & Medium & Medium & Funder & Medium \\
\hline \begin{tabular}{|l} 
Peterson $2017^{5}$ (27716673) \\
\end{tabular} & 3 months & $\begin{array}{l}\text { Medium } \\
\text { NR }\end{array}$ & Medium & High & Medium & High & $x$ & NR & High \\
\hline \begin{tabular}{|l} 
Olsen $2016^{6}(27155968)$ \\
\end{tabular} & $\begin{array}{l}12 \text { weeks } \\
6 \text { months }\end{array}$ & $\begin{array}{l}\text { Low } \\
12 \text { weeks: } 1 \% \\
6 \text { months: } 1 \% \\
\end{array}$ & Low & High & Medium & Low & $\mathrm{x}$ & Foundation & High \\
\hline Olsen $2016^{7}(26807956)$ & $\begin{array}{l}12 \text { weeks } \\
6 \text { months }\end{array}$ & $\begin{array}{l}\text { Low } \\
12 \text { weeks: } 12 \% \\
6 \text { months: } 17 \% \\
\end{array}$ & Low & High & Medium & Low & $x$ & \begin{tabular}{|l|} 
Foundation \\
\end{tabular} & High \\
\hline \begin{tabular}{|l|l|l} 
Bemans $2015^{8}(26115817)$ \\
\end{tabular} & 4 months & $\begin{array}{l}\text { High } \\
21.9 \%\end{array}$ & $x$ & $\mathrm{x}$ & $x$ & $\mathrm{x}$ & $x$ & NR & High \\
\hline \begin{tabular}{|l|} 
Joranson 20159 $2(26096582)$ \\
Joranson 201610 (27434512)
\end{tabular} & $\begin{array}{l}12 \text { weeks } \\
6 \text { months }\end{array}$ & $\begin{array}{l}\text { Medium } \\
12 \text { weeks: } 10 \% \\
6 \text { months: } 16.7 \%\end{array}$ & Medium & High & High & Low & $\mathrm{x}$ & Government & High \\
\hline Travers $2013^{11}(\mathrm{NA})$ & 11 weeks & $\begin{array}{l}\text { Medium } \\
17.9 \%\end{array}$ & Medium & Low & High & Low & $x$ & \begin{tabular}{|l|} 
Foundation \\
\end{tabular} & High \\
\hline
\end{tabular}

$X$ indicates that domain was not assessed due to high risk of bias.

Abbreviations: NR=Not Reported; PMID=PubMed Identification Number 
Table C-2. Characteristics of included studies: assisted therapy

\begin{tabular}{|c|c|c|c|c|c|c|c|c|c|c|c|c|c|}
\hline $\begin{array}{l}\text { Study (PMID) } \\
\text { Country } \\
\text { RoB* } \\
\text { Type }\end{array}$ & \begin{tabular}{|l|} 
Intervention \\
Intervention \\
Focus \\
Theoretical \\
Model \\
Delivery \\
Person \\
Intervention \\
Target \\
Recipient \\
Mode \\
Components \\
Frequency \\
Duration \\
\end{tabular} & $\begin{array}{l}\text { Comparison } \\
\text { Target } \\
\text { Mode } \\
\text { Components } \\
\text { Frequency } \\
\text { Duration }\end{array}$ & $\begin{array}{l}\text { Setting } \\
\text { and Design } \\
\text { Setting } \\
\text { Design } \\
\text { Cluster N } \\
\text { Participants } \\
\text { Randomized } \\
\text { N }\end{array}$ & \begin{tabular}{|l} 
PLWD \\
Dementia \\
Characteristics \\
\\
Dementia Types \\
Dementia Severity \\
Diagnostic \\
Criteria \\
Age of Diagnosis
\end{tabular} & $\begin{array}{l}\text { PLWD } \\
\text { Non-Disease Char } \\
\text { PLWD N } \\
\text { PLWD Age (mean) } \\
\text { PLWD Sex (\% } \\
\text { female) } \\
\text { PLWD Race (\% } \\
\text { majority) } \\
\text { PLWD Education } \\
\text { (mean years) }\end{array}$ & \begin{tabular}{|l|} 
PLWD \\
Non-Disease \\
Char Reporting \\
Status (RS) \\
PLWD SES \\
PLWD Prior \\
Disability \\
PLWD \\
Household \\
Characteristics \\
PLWD Health \\
Insurance \\
PLWD Detailed \\
Race Information \\
\end{tabular} & $\begin{array}{l}\text { Informal Caregiver } \\
\text { (IC) } \\
\text { Characteristics } \\
\text { IC N } \\
\text { IC Age (mean) } \\
\text { IC Sex (\% female) } \\
\text { IC Race (\% } \\
\text { majority) } \\
\text { IC Education } \\
\text { (mean years) } \\
\text { IC Relation to } \\
\text { PLWD (\% majority) }\end{array}$ & \begin{tabular}{|l} 
Informal Caregiver \\
(IC) Char. RS \\
IC Duration \\
IC Living With \\
PLWD \\
IC Payment \\
IC Health Status \\
IC Dementia \\
Family History \\
IC Employment \\
Status \\
IC Training
\end{tabular} & $\begin{array}{l}\text { Formal Caregiver } \\
\text { (FC) } \\
\text { Characteristics } \\
\\
\text { FC N } \\
\text { FC Age (mean) } \\
\text { FC Sex (\% } \\
\text { female) } \\
\text { FC Race (\% } \\
\text { majority) } \\
\text { FC Education } \\
\text { (mean years) }\end{array}$ & $\begin{array}{l}\text { Formal } \\
\text { Caregiver (FC) } \\
\text { Char. RS } \\
\text { FC Health } \\
\text { Status } \\
\text { FC Training } \\
\text { FC Education } \\
\text { FC Position } \\
\text { FC Length of } \\
\text { Service }\end{array}$ & \begin{tabular}{|l|} 
Outcome \\
Timing(s)
\end{tabular} & $\begin{array}{l}\text { PLWD } \\
\text { Outcomes }\end{array}$ & $\begin{array}{l}\text { Caregiver } \\
\text { Outcomes } \\
\text { IC: } \\
\text { FC: }\end{array}$ \\
\hline \begin{tabular}{|l} 
Moyle 20171 \\
(28780395) \\
Australia \\
Medium \\
Explanatory \\
Jones 201812 \\
(29656838) \\
Mervin 2018 \\
(29325922) \\
Moyle 2018 \\
(29563027)
\end{tabular} & $\begin{array}{l}\text { Individual, non- } \\
\text { facilitated } \\
\text { sessions with } \\
\text { PARO (robotic } \\
\text { seal) for PLWD } \\
\text { over 10 weeks; } \\
\text { three afternoon } \\
\text { sessions per } \\
\text { week, 15 } \\
\text { minutes/session }\end{array}$ & $\begin{array}{l}\text { 1. Standard } \\
\text { care for } \\
\text { PLWD at } \\
\text { respective } \\
\text { facilities } \\
\\
\text { 2. Push toy } \\
\text { (PARO with } \\
\text { robotic } \\
\text { features } \\
\text { disabled) for } \\
\text { PLWD over } \\
10 \text { weeks; } \\
\text { three } \\
\text { afternoon } \\
\text { sessions per } \\
\text { week, 15 } \\
\text { minutes/sessi } \\
\text { on }\end{array}$ & $\begin{array}{l}\text { Long-term care } \\
\text { facilities } \\
\text { approved and } \\
\text { accredited by } \\
\text { the Australian } \\
\text { government } \\
\text { Cluster RCT } \\
\text { Multisite } \\
18 \text { Clusters } \\
415 \text { PLWD }\end{array}$ & $\begin{array}{l}\text { Any dementia type } \\
\text { or severity. } \\
\text { RUDAS } \leq 22 \text { and } \\
\text { documented } \\
\text { dementia diagnosis }\end{array}$ & \begin{tabular}{|l}
$\mathrm{N}=415$ \\
$76 \%$ Female \\
85 years \\
Race NR \\
Education NR
\end{tabular} & \begin{tabular}{|l|l} 
None \\
\end{tabular} & NA & NA & NA & NA & \begin{tabular}{|l|l|}
10 weeks \\
15 weeks
\end{tabular} & $\begin{array}{l}\text { Agitation } \\
\text { (observed) } \\
\text { CMAl-SF } \\
\text { Positive } \\
\text { behavioral } \\
\text { engagement } \\
\text { Using object } \\
\text { for social } \\
\text { engagement } \\
\text { Positive } \\
\text { verbal } \\
\text { engagement } \\
\text { Visual } \\
\text { engagement }\end{array}$ & NA \\
\hline
\end{tabular}

* High risk of bias studies included in evidence map. This table includes low and medium risk of bias studies only.

SES=socioeconomic status; RS=Reporting Status

Table C-3. Modified PRECIS-2: assisted therapy

\begin{tabular}{|c|c|c|c|c|c|c|c|c|c|c|}
\hline Study (PMID) & $\begin{array}{l}\text { Eligibility } \\
\text { Criteria }\end{array}$ & $\begin{array}{l}\text { Recruitment } \\
\text { Path }\end{array}$ & Setting & $\begin{array}{l}\text { Intervention } \\
\text { Organization }\end{array}$ & $\begin{array}{l}\text { Flexibility of } \\
\text { Intervention: } \\
\text { Delivery }\end{array}$ & \begin{tabular}{|l} 
Flexibility of \\
Intervention: \\
Adherence
\end{tabular} & Followup & Primary Outcome & Analysis & $\begin{array}{l}\text { Overall } \\
\text { Category } \\
\text { Applicability and/or Qualifiers }\end{array}$ \\
\hline $\begin{array}{l}\text { Moyle 2017' }(28780395) \\
\text { Jones 2018 }(29656838) \\
\text { Mervin 2018 } 2018^{3}(29325922) \\
\text { Moyle 2018 }(29563027)\end{array}$ & Balanced & $\begin{array}{l}\text { Mostly } \\
\text { Explanatory }\end{array}$ & $\begin{array}{l}\text { Mostly } \\
\text { Explanatory }\end{array}$ & Explanatory & Explanatory & Mostly Explanatory & Mostly Explanatory & Mostly Explanatory & Balanced & Explanatory \\
\hline
\end{tabular}

Abbreviations: NA=Not Assessed; PMID=PubMed Identification Number 
Table C-4. PLWD outcomes summary low and medium risk of bias studies: assisted therapy

\begin{tabular}{|c|c|c|c|c|c|}
\hline $\begin{array}{l}\text { Study (PMID) } \\
\text { Comparison } \\
\text { RoB } \\
\text { Category* }\end{array}$ & $\begin{array}{l}\text { Outcome } \\
\text { Timing }\end{array}$ & Summary Finding & Intervention & Comparator & $p$ Value \\
\hline $\begin{array}{l}\text { Moyle 2017 }{ }^{1}(28780395) \\
\text { PARO vs. usual care } \\
\text { Medium } \\
\text { Explanatory }\end{array}$ & $\begin{array}{l}\text { Adjusted Mean Difference }(95 \% \mathrm{Cl}) \\
\text { Reduction in agitation (observed via video recording) } \\
10 \text { weeks }\end{array}$ & $3.3(0.9,5.8)$ & $\mathrm{NR}$ & NR & $p=0.008$ \\
\hline $\begin{array}{l}\text { Moyle 2017 } 288780395) \\
\text { PARO vs. usual care } \\
\text { Medium } \\
\text { Explanatory }\end{array}$ & $\begin{array}{l}\text { Adjusted Mean Difference }(95 \% \mathrm{Cl}) \\
\text { CMAl-SF } \\
10 \text { weeks }\end{array}$ & $-1.9(-5.8,2.0)$ & NR & NR & $\mathrm{p}=0.34$ \\
\hline $\begin{array}{l}\text { Moyle } 2017^{1} \text { (28780395) } \\
\text { PARO vs. usual care } \\
\text { Medium } \\
\text { Explanatory }\end{array}$ & $\begin{array}{l}\text { Mortality } \\
\%(\mathrm{~N}) \\
15 \text { weeks }\end{array}$ & NR & $5.1 \%(7)$ & $3.6 \%(5)$ & NR \\
\hline $\begin{array}{l}\text { Moyle } 2017^{1}(28780395) \\
\text { PARO vs. plush toy } \\
\text { Medium } \\
\text { Explanatory }\end{array}$ & $\begin{array}{l}\text { Adjusted Mean Difference }(95 \% \mathrm{Cl}) \\
\text { Reduction in agitation (observed via video recording) } \\
10 \text { weeks }\end{array}$ & $1.3(-1.7,4.2)$ & $\mathrm{NR}$ & NR & $\mathrm{p}=0.39$ \\
\hline $\begin{array}{l}\text { Moyle 2017 }(28780395) \\
\text { PARO vs. plush toy } \\
\text { Medium } \\
\text { Explanatory }\end{array}$ & $\begin{array}{l}\text { Adjusted Mean Difference }(95 \% \mathrm{Cl}) \\
\text { CMAl-SF } \\
10 \text { weeks }\end{array}$ & $-0.99(-5.8,3.8)$ & $\mathrm{NR}$ & NR & $p=0.68$ \\
\hline $\begin{array}{l}\text { Moyle 2017 }(28780395) \\
\text { PARO vs. plush toy } \\
\text { Medium } \\
\text { Explanatory }\end{array}$ & $\begin{array}{l}\text { Adjusted Mean Difference }(95 \% \mathrm{Cl}) \\
\text { Positive behavioral engagement (observed via video recording) } \\
10 \text { weeks }\end{array}$ & $6.3(-0.8,13.5)$ & $\mathrm{NR}$ & $\mathrm{NR}$ & $\mathrm{p}=0.08$ \\
\hline $\begin{array}{l}\text { Moyle 2017 } 288780395) \\
\text { PARO vs. plush toy } \\
\text { Medium } \\
\text { Explanatory }\end{array}$ & $\begin{array}{l}\text { Adjusted Mean Difference }(95 \% \mathrm{Cl}) \\
\text { Using object for social engagement (observed via video recording) } \\
10 \text { weeks }\end{array}$ & $1.2(-0.6,3.0)$ & $\mathrm{NR}$ & $\mathrm{NR}$ & $p=0.18$ \\
\hline $\begin{array}{l}\text { Moyle } 2017^{1}(28780395) \\
\text { PARO vs. plush toy } \\
\text { Medium } \\
\text { Explanatory }\end{array}$ & $\begin{array}{l}\text { Adjusted Mean Difference }(95 \% \mathrm{Cl}) \\
\text { Positive verbal engagement (observed via video recording) } \\
10 \text { weeks }\end{array}$ & $3.6(0.81,6.40)$ & NR & NR & $p=0.01$ \\
\hline $\begin{array}{l}\text { Moyle 20171 (28780395) } \\
\text { PARO vs. plush toy } \\
\text { Medium } \\
\text { Explanatory }\end{array}$ & $\begin{array}{l}\text { Adjusted Mean Difference }(95 \% \mathrm{Cl}) \\
\text { Visual engagement (observed via video recording) } \\
10 \text { weeks }\end{array}$ & $13.1(9.06,17.05)$ & $\mathrm{NR}$ & NR & $p<0.0001$ \\
\hline $\begin{array}{l}\text { Moyle 2017' (28780395) } \\
\text { PARO vs. plush toy } \\
\text { Medium } \\
\text { Explanatory }\end{array}$ & $\begin{array}{l}\text { Mortality } \\
\%(\mathrm{~N}) \\
15 \text { weeks }\end{array}$ & $\mathrm{NR}$ & $5.1 \%(7)$ & $10 \%(14)$ & $\mathrm{NR}$ \\
\hline
\end{tabular}

Explanatory studies are listed first, followed by pragmatic studies.
Abbreviations: CI=Confidence interval; NR=Not Reported; PMID=PubMed Identification Number; RoB=Risk of Bias 
Table C-5. Summary of strength of evidence for PLWD outcomes: robot-assisted therapy

\begin{tabular}{|c|c|c|c|c|c|c|c|c|}
\hline $\begin{array}{l}\text { Comparison } \\
\text { Outcome }\end{array}$ & Timing & $\begin{array}{l}\text { \# Studies/ Design } \\
\text { ( } \mathrm{n} \text { analyzed) }\end{array}$ & Finding or Summary Statistic & Study Limitations & Consistency & Directness & Precision & Overall Grade/ Conclusion \\
\hline \begin{tabular}{|l} 
PARO vs. usual care \\
Agitation \\
\end{tabular} & 10 weeks & 1 clustered RCT $(n=257)$ & $\begin{array}{l}\text { Evidence was mixed for agitation reduction. Observation via } \\
\text { video recordings favored PARO; however, CMAI-SF showed } \\
\text { no difference between groups. }\end{array}$ & Moderate & Unknown & Direct & Imprecise & Insufficient \\
\hline $\begin{array}{l}\text { PARO vs. plush toy } \\
\text { Agitation }\end{array}$ & 10 weeks & 1 clustered RCT $(n=278)$ & No difference between groups in measures of agitation. & Moderate & Unknown & Direct & Imprecise & Insufficient \\
\hline $\begin{array}{l}\text { PARO vs. plush toy } \\
\text { Engagement }\end{array}$ & 10 weeks & 1 clustered RCT $(n=278)$ & $\begin{array}{l}\text { Observation via video recordings showed improvements in } \\
\text { PARO for positive verbal engagement and visual } \\
\text { engagement compared to plush toy; however, there was no } \\
\text { difference for positive behavioral engagement and using the } \\
\text { object for social engagement. }\end{array}$ & Moderate & Unknown & Direct & Imprecise & Insufficient \\
\hline
\end{tabular}

Abbreviations: n=Number, CMAI-SF=Cohen-Mansfield Agitation Inventory-Short Form; RCT=Randomized controlled trial. 


\section{Evidence Map: Assisted Therapy}

Table C-6. Characteristics of evidence map studies: assisted therapy 


\begin{tabular}{|c|c|c|c|c|c|c|c|c|c|c|c|c|c|}
\hline $\begin{array}{l}\text { Study (PMID) } \\
\text { Country } \\
\text { EM Reason }\end{array}$ & Intervention & Comparison & \begin{tabular}{|l|} 
Setting \\
and Design \\
\\
Setting \\
Design \\
Cluster N \\
Participants \\
Random- \\
ized N
\end{tabular} & \begin{tabular}{|l|} 
PLWD \\
Dementia \\
Characteristics
\end{tabular} & $\begin{array}{l}\text { PLWD } \\
\text { Non-Disease Char } \\
\text { PLWD N } \\
\text { PLWD Age (mean) } \\
\text { PLWD Sex (\% } \\
\text { female) } \\
\text { PLWD Race (\% } \\
\text { majority) } \\
\text { PLWD Education } \\
\text { (mean years) }\end{array}$ & \begin{tabular}{|l|} 
PLWD \\
Non-Disease \\
Char Reporting \\
Status (RS) \\
\\
PLWD SES \\
PLWD Prior \\
Disability \\
PLWD \\
Household \\
Characteristics \\
PLWD Health \\
Insurance \\
PLWD Detailed \\
Race Information \\
\end{tabular} & $\begin{array}{l}\text { Informal Caregiver } \\
\text { (IC) } \\
\text { Characteristics } \\
\text { IC N } \\
\text { IC Age (mean) } \\
\text { IC Sex (\% female) } \\
\text { IC Race (\% } \\
\text { majority) } \\
\text { IC Education } \\
\text { (mean years) } \\
\text { IC Relation to } \\
\text { PLWD (\% majority) }\end{array}$ & $\begin{array}{l}\text { Informal Caregiver } \\
\text { (IC) Char. RS } \\
\text { IC Duration } \\
\text { IC Living With } \\
\text { PLWD } \\
\text { IC Payment } \\
\text { IC Health Status } \\
\text { IC Dementia } \\
\text { Family History } \\
\text { IC Employment } \\
\text { Status } \\
\text { IC Training }\end{array}$ & 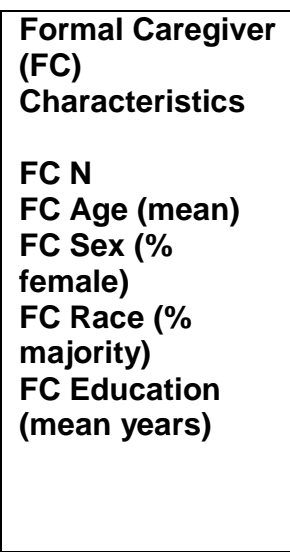 & $\begin{array}{l}\text { Formal } \\
\text { Caregiver (FC) } \\
\text { Char. RS } \\
\text { FC Health } \\
\text { Status } \\
\text { FC Training } \\
\text { FC Education } \\
\text { FC Position } \\
\text { FC Length of } \\
\text { Service }\end{array}$ & $\begin{array}{l}\text { Outcome } \\
\text { Timing(s) }\end{array}$ & $\begin{array}{l}\text { PLWD } \\
\text { Outcomes }\end{array}$ & $\begin{array}{l}\text { Caregiver } \\
\text { Outcomes } \\
\text { IC: } \\
\text { FC: }\end{array}$ \\
\hline $\begin{array}{l}\text { Balzotti 201915 } \\
(30136743) \\
\text { Italy } \\
\text { Small sample }\end{array}$ & $\begin{array}{l}\text { Gesture verbal } \\
\text { treatment: } \\
\text { develop PLWD } \\
\text { interactive use } \\
\text { of gestures with } \\
\text { other } \\
\text { communication } \\
\text { strategies. 2 1- } \\
\text { hour group } \\
\text { sessions weekly } \\
\text { for } 12 \text { weeks. } \\
\text { Doll therapy: } 1 \\
\text { hour daily for } 12 \\
\text { weeks. }\end{array}$ & $\begin{array}{l}\text { Usual } \\
\text { rehabilitative } \\
\text { care }\end{array}$ & \begin{tabular}{|l|} 
AD care \\
home \\
Quasi- \\
experimental \\
30 PLWDs \\
\end{tabular} & $\begin{array}{l}\text { Dementia } \\
\text { diagnosis based on } \\
\text { DSM-V criteria; }\end{array}$ & $\begin{array}{l}\mathrm{N}=30 \\
\text { Mean age: yes } \\
\% \text { Female: yes } \\
\% \text { majority race: no } \\
\text { Education: yes }\end{array}$ & None & NA & None & NA & NA & 12 weeks & NPI-Q & NA \\
\hline $\begin{array}{l}\text { Menna, 201916 } \\
\text { (30740833) } \\
\text { Italy } \\
\text { Pilot }\end{array}$ & $\begin{array}{l}\text { Dog-assisted } \\
\text { group therapy; } \\
12 \text { weekly } \\
\text { sessions }\end{array}$ & Unclear & $\begin{array}{l}\text { Adult } \\
\text { daycare } \\
\text { center } \\
\text { RCT } \\
\text { 22 PLWD } \\
\end{array}$ & $\begin{array}{l}\text { Mild to moderate } \\
\text { AD (per MMSE) }\end{array}$ & $\begin{array}{l}\mathrm{N}=22 \\
\text { Age: no } \\
\text { Sex: no } \\
\text { Race: no } \\
\text { Education: no } \\
\end{array}$ & None & NA & NA & NA & NA & 3 months & $\begin{array}{l}\text { Cortisol level } \\
\text { GDS }\end{array}$ & NA \\
\hline $\begin{array}{l}\text { Moyle, 201917 } \\
\text { (30474401) } \\
\text { Australia } \\
\text { Pilot }\end{array}$ & $\begin{array}{l}\text { Life-like baby } \\
\text { doll-assisted } \\
\text { therapy; } 330 \\
\text { minute sessions } \\
\text { per week for } 3 \\
\text { weeks } \\
\end{array}$ & Usual care & $\begin{array}{l}\text { Long-term } \\
\text { care } \\
\text { facilities } \\
\text { RCT } \\
35 \text { PLWD }\end{array}$ & $\begin{array}{l}\text { Dementia with } \\
\text { recent history of } \\
\text { BPSD }\end{array}$ & $\begin{array}{l}\mathrm{N}=35 \\
\text { Age: yes } \\
\text { Sex: yes } \\
\text { Race: no } \\
\text { Education: no }\end{array}$ & None & NA & NA & NA & NA & 3 months & $\begin{array}{l}\text { CMAI-SF } \\
\text { OERS }\end{array}$ & NA \\
\hline $\begin{array}{l}\text { Cantarella } \\
2018^{18} \\
(622559552) \\
\text { Italy } \\
\text { Pilot study }\end{array}$ & $\begin{array}{l}\text { Doll therapy, } 60 \\
\text { minute sessions } \\
5 \text { times/week for } \\
1 \text { month }\end{array}$ & $\begin{array}{l}\text { Hand warmers, } \\
60 \text {-minute } \\
\text { sessions } 5 \\
\text { times/week for } 1 \\
\text { month }\end{array}$ & \begin{tabular}{|l} 
Nursing \\
home \\
RCT, single \\
site \\
32 PLWD \\
\end{tabular} & \begin{tabular}{|l|} 
Severe AD or \\
vascular dementia \\
( $\geq 5$ Short Portable \\
Mental Status \\
Questionnaire) with \\
BPSD \\
\end{tabular} & $\begin{array}{l}\mathrm{N}=32 \\
\text { Age: yes } \\
\text { Sex: yes } \\
\text { Race: no } \\
\text { Education: yes }\end{array}$ & None & NA & NA & $\begin{array}{l}\mathrm{N}=\mathrm{NR} \\
\text { Age: no } \\
\text { Sex: no } \\
\text { \% majority race: } \\
\text { no } \\
\text { Education: no } \\
\end{array}$ & None & 1 month & $\begin{array}{l}\mathrm{NPI} \\
\text { Eating } \\
\text { Behavior } \\
\text { Scale }\end{array}$ & $\begin{array}{l}\mathrm{NPI}- \\
\text { Distress }\end{array}$ \\
\hline
\end{tabular}




\begin{tabular}{|c|c|c|c|c|c|c|c|c|c|c|c|c|c|}
\hline $\begin{array}{l}\text { Liang 201719 } \\
\text { (28668664) } \\
\text { New Zealand } \\
\text { Pilot }\end{array}$ & $\begin{array}{l}\text { PARO robotic } \\
\text { pet at day care } \\
\text { and at home, } \\
30-\text { minute group } \\
\text { sessions 2-3 } \\
\text { times a week for } \\
6 \text { weeks at day } \\
\text { care }\end{array}$ & $\begin{array}{l}\text { Facilities' } \\
\text { standard of care }\end{array}$ & \begin{tabular}{|l|} 
Dementia \\
day care \\
centers and \\
at home \\
RCT; \\
Multisite \\
30 PLWD \\
30 informal \\
caregivers \\
\end{tabular} & Not specified & $\begin{array}{l}\mathrm{N}=30 \\
\text { Age: yes } \\
\text { Sex: yes } \\
\text { Race: no } \\
\text { Education: no }\end{array}$ & None & $\begin{array}{l}\mathrm{N}=30 \\
\text { Age: yes } \\
\text { Sex: yes } \\
\text { \% majority race: no } \\
\text { Education: no }\end{array}$ & None & NA & NA & 12 weeks & $\begin{array}{l}\text { CMAI-SF } \\
\text { NPI Brief } \\
\text { Questionnaire } \\
\text { CSDD }\end{array}$ & NA \\
\hline $\begin{array}{l}\text { Petersen 20175 } \\
(27716673) \\
\text { US } \\
\text { High RoB }\end{array}$ & $\begin{array}{l}\text { PARO robotic } \\
\text { pet, } 20 \text { minutes } \\
3 \text { times a week } \\
\text { for } 3 \text { months }\end{array}$ & $\begin{array}{l}\text { Facilities' } \\
\text { standard of care }\end{array}$ & \begin{tabular}{|l|} 
Senior living \\
facilities \\
RCT; \\
Multisite \\
61 PLWD \\
\end{tabular} & $\begin{array}{l}\text { Mild to moderate } \\
\text { dementia according } \\
\text { to DSM or NIA } \\
\text { criteria }\end{array}$ & $\begin{array}{l}\mathrm{N}=61 \\
\text { Age: } \text { yes } \\
\text { Sex: yes } \\
\text { Race: yes } \\
\text { Education: no }\end{array}$ & None & NA & NA & NA & NA & 3 months & $\begin{array}{l}\text { RAID } \\
\text { CSDD } \\
\text { GDS }\end{array}$ & NA \\
\hline $\begin{array}{l}\text { Olsen 20166 } \\
\text { (27155968) } \\
\text { Norway } \\
\text { High RoB }\end{array}$ & $\begin{array}{l}\text { Group animal } \\
\text { therapy } \\
\text { sessions with a } \\
\text { dog, } 2 \\
\text { times/week for } \\
12 \text { weeks }\end{array}$ & Usual care & \begin{tabular}{|l|} 
Adult day \\
care centers \\
Cluster RCT \\
16 clusters \\
80 PLWD \\
\end{tabular} & $\begin{array}{l}\text { Dementia } \\
\text { diagnosis or MMSE } \\
<25\end{array}$ & $\begin{array}{l}\mathrm{N}=80 \\
\text { Age: yes } \\
\text { Sex: yes } \\
\text { Race: } \text { no } \\
\text { Education: yes }\end{array}$ & Prior disability: yes & NA & NA & NA & NA & $\begin{array}{l}12 \text { weeks } \\
6 \text { months }\end{array}$ & $\begin{array}{l}\text { Berg Balance } \\
\text { Scale } \\
\text { CDR } \\
\text { QUALID }\end{array}$ & NA \\
\hline $\begin{array}{l}\text { Olsen 20167 } \\
\text { (26807956) } \\
\text { Norway } \\
\text { High RoB }\end{array}$ & $\begin{array}{l}\text { Group animal } \\
\text { therapy } \\
\text { sessions with a } \\
\text { dog, } 2 \\
\text { times/week, } 30 \\
\text { minutes/session } \\
\text {, for } 12 \text { weeks }\end{array}$ & Usual care & \begin{tabular}{|l|} 
Nursing \\
homes \\
Cluster RCT \\
10 clusters \\
58 PLWD \\
\end{tabular} & $\begin{array}{l}\text { Dementia } \\
\text { diagnosis or MMSE } \\
<25\end{array}$ & $\begin{array}{l}\text { N=58 } \\
\text { Age: } \text { yes } \\
\text { Sex: yes } \\
\text { Race: no } \\
\text { Education: yes }\end{array}$ & Prior disability: yes & NA & NA & NA & NA & $\begin{array}{l}12 \text { weeks } \\
6 \text { months }\end{array}$ & $\begin{array}{l}\text { CSDD } \\
\text { QUALID } \\
\text { Brief Agitation } \\
\text { Rating Scale } \\
\text { CDR }\end{array}$ & NA \\
\hline $\begin{array}{l}\text { Bemelmans } \\
2015^{8} \\
(26115817) \\
\text { Netherlands } \\
\text { High RoB }\end{array}$ & $\begin{array}{l}\text { PARO robotic } \\
\text { pet to provide } \\
\text { comfort } \\
\text { individually to } \\
\text { distressed } \\
\text { PLWD; as } \\
\text { needed over } 4 \\
\text { months }\end{array}$ & $\begin{array}{l}\text { PARO robotic } \\
\text { pet incorporated } \\
\text { in daily activities } \\
\text { for care support; } \\
\text { as needed over } \\
4 \text { months }\end{array}$ & \begin{tabular}{|l|} 
Care \\
institutions \\
for \\
intramural \\
psychogeriat \\
ric care \\
Quasi \\
experimental \\
time series; \\
multisite \\
91 PLWD \\
\end{tabular} & \begin{tabular}{|l|} 
All dementia \\
severity
\end{tabular} & $\begin{array}{l}\mathrm{N}=91 \\
\text { Age: } n \text { no } \\
\text { Sex: yes } \\
\text { Race: no } \\
\text { Education: no }\end{array}$ & None & NA & NA & NA & NA & 4 months & $\begin{array}{l}\text { Individually } \\
\text { Prioritized } \\
\text { Problems } \\
\text { Assessment }\end{array}$ & NA \\
\hline $\begin{array}{l}\text { Joranson 201599 } \\
(26096582) \\
\text { Norway } \\
\text { High RoB } \\
\text { Joranson } \\
2016^{10} \\
(27434512)\end{array}$ & $\begin{array}{l}\text { PARO robotic } \\
\text { pet, } 30 \text {-minute } \\
\text { group sessions } \\
2 \text { times a week } \\
\text { for } 12 \text { weeks }\end{array}$ & Usual care & $\begin{array}{l}\text { Nursing } \\
\text { homes } \\
\text { Cluster } \\
\text { RCT; } \\
\text { Multisite } \\
60 \text { PLWD }\end{array}$ & \begin{tabular}{|l|} 
Dementia \\
diagnosis with \\
cognitive \\
impairment or \\
score lower than \\
$25 / 30$ on \\
Norwegian MMSE
\end{tabular} & $\begin{array}{l}\text { N=69 } \\
\text { Age: yes } \\
\text { Sex: yes } \\
\text { Race: no } \\
\text { Education: no }\end{array}$ & None & NA & NA & NA & NA & $\begin{array}{l}12 \text { weeks } \\
6 \text { months }\end{array}$ & $\begin{array}{l}\text { Norwegian } \\
\text { BARS } \\
\text { Norwegian } \\
\text { CSDD } \\
\text { QUALID }\end{array}$ & NA \\
\hline
\end{tabular}




\begin{tabular}{|c|c|c|c|c|c|c|c|c|c|c|c|c|c|}
\hline $\begin{array}{l}\text { Valenti Soler } \\
2015^{20} \\
(26388764) \\
\text { Spain } \\
\text { Pilot }\end{array}$ & $\begin{array}{l}\text { PARO robotic } \\
\text { pet, } 30-40- \\
\text { minute group } \\
\text { sessions } 2 \\
\text { times a week for } \\
3 \text { months }\end{array}$ & $\begin{array}{l}\text { 1. Facility's } \\
\text { standard of care } \\
\\
\text { 2. Humanoid } \\
\text { robot, 30-40- } \\
\text { minute group } \\
\text { sessions 2 } \\
\text { times a week for } \\
3 \text { months } \\
\text { 3. Animal } \\
\text { therapy with } \\
\text { dogs, 30-40- } \\
\text { minute group } \\
\text { sessions 2 } \\
\text { times a week for } \\
3 \text { months }\end{array}$ & \begin{tabular}{|l|} 
Public \\
nursing \\
home \\
RCT; Single \\
site \\
117 PLWD \\
\end{tabular} & \begin{tabular}{|l|} 
Diagnosis of \\
neurodegenerative \\
dementia, all \\
dementia types
\end{tabular} & $\begin{array}{l}\text { N=117 } \\
\text { Age: yes } \\
\text { Sex: yes } \\
\text { Race: no } \\
\text { Education: no }\end{array}$ & None & NA & NA & NA & NA & 3 months & $\begin{array}{l}\text { GDS } \\
\text { NPI } \\
\text { APADEM-NH } \\
\text { Apathy } \\
\text { Inventory } \\
\text { QUALID }\end{array}$ & NA \\
\hline $\begin{array}{l}\text { Moyle 201321 } \\
\text { (23506125) } \\
\text { Australia } \\
\text { Pilot }\end{array}$ & $\begin{array}{l}\text { PARO robotic } \\
\text { pet, } 45-\text {-minute } \\
\text { group sessions } \\
3 \text { times a week } \\
\text { for } 5 \text { weeks }\end{array}$ & $\begin{array}{l}\text { Reading group, } \\
\text { 45-minute group } \\
\text { sessions } 3 \\
\text { times a week for } \\
5 \text { weeks }\end{array}$ & $\begin{array}{l}\text { Residential } \\
\text { care facility } \\
\text { Crossover } \\
\text { RCT; Single } \\
\text { site } \\
18 \text { PLWD } \\
\end{array}$ & $\begin{array}{l}\text { Diagnosis of mid to } \\
\text { late stage dementia } \\
\text { or DSM-IV criteria } \\
\text { for probable } \\
\text { dementia }\end{array}$ & $\begin{array}{l}\text { N=18 } \\
\text { Age: yes } \\
\text { Sex: no } \\
\text { Race: no } \\
\text { Education: No }\end{array}$ & None & NA & NA & NA & NA & $\begin{array}{l}5 \text { weeks } \\
10 \text { weeks }\end{array}$ & $\begin{array}{l}\text { QoL-AD } \\
\text { RAID } \\
\text { AES } \\
\text { GDS } \\
\text { AWS } \\
\text { OERS }\end{array}$ & NA \\
\hline $\begin{array}{l}\text { Travers 2013 } \\
\text { (NA) } \\
\text { Australia } \\
\text { High RoB }\end{array}$ & $\begin{array}{l}\text { Group animal } \\
\text { therapy with a } \\
\text { dog, 40-50- } \\
\text { minute sessions } \\
2-3 \text { times/week } \\
\text { for } 11 \text { weeks }\end{array}$ & $\begin{array}{l}\text { Group therapy, } \\
40-50-\text { minute } \\
\text { sessions 2-3 } \\
\text { times/week for } \\
11 \text { weeks }\end{array}$ & $\begin{array}{l}\text { Nursing } \\
\text { home } \\
\text { RCT, } \\
\text { multisite } \\
67 \text { PLWD }\end{array}$ & \begin{tabular}{|l|} 
Mild to moderate \\
dementia (any \\
type)
\end{tabular} & $\begin{array}{l}\mathrm{N}=67 \\
\text { Age: } y \text { es } \\
\text { Sex: yes } \\
\text { Race: } \text { no } \\
\text { Education: yes }\end{array}$ & None & NA & NA & NA & NA & 11 weeks & $\begin{array}{l}\text { QoL-AD } \\
\text { SF-36 } \\
\text { GDS-SF } \\
\text { Multidimensio } \\
\text { nal } \\
\text { Observational } \\
\text { Scale for } \\
\text { Elderly } \\
\text { Subjects }\end{array}$ & NA \\
\hline $\begin{array}{l}\text { Majic 2013222 } \\
\text { (23831177) } \\
\text { Germany } \\
\text { Pilot }\end{array}$ & $\begin{array}{l}\text { Animal therapy } \\
\text { with a dog, one } \\
40-\text { minute } \\
\text { session/week } \\
\text { for } 10 \text { weeks }\end{array}$ & Usual Care & $\begin{array}{l}\text { Nursing } \\
\text { home } \\
\text { Case- } \\
\text { Control } \\
75 \text { PLWD }\end{array}$ & \begin{tabular}{|l|} 
Dementia \\
diagnosis (DSM-IV \\
criteria) and MMSE \\
$<25$
\end{tabular} & $\begin{array}{l}\mathrm{N}=75 \\
\text { Age: yes } \\
\text { Sex: yes } \\
\text { Race: no } \\
\text { Education: no }\end{array}$ & None & NA & NA & NA & NA & 10 weeks & $\begin{array}{l}\text { CMAl } \\
\text { Dementia } \\
\text { Mood } \\
\text { Assessment } \\
\text { Scale }\end{array}$ & NA \\
\hline
\end{tabular}

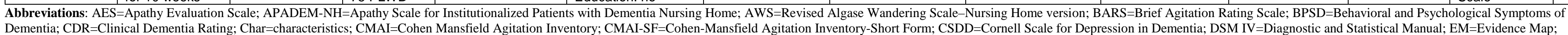

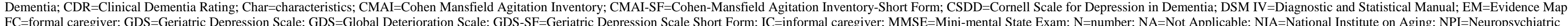
Inventory; OERS=Observed Emotion Rating Scale; PAINAD=Pain Assessment in Advanced Dementia; PMID=PubMed Identification Number PLWD=Persons Living with Dementia; QoL-AD=Quality of Life in Alzheimer's Disease; QUALID=Quality of Life in Late-stage Dementia; RAID=Rating for Anxiety in Dementia; RCT=Randomized controlled trial; RoB=Risk of Bias; RS=Reporting Status; SES=socioeconomic status; SF-36=Medical Outcomes Study 36-Item Short Form Health Survey;

\section{Multi-Sensory Stimulation/Snoezelen}

Table C-7. Risk of bias assessment: multisensory stimulation

\begin{tabular}{|c|c|c|c|c|c|c|c|c|c|}
\hline \begin{tabular}{|l} 
Study (PMID) \\
\end{tabular} & Outcome Timing & $\begin{array}{l}\text { Attrition Bias } \\
\text { Attrition \% }\end{array}$ & Selection Bias & \begin{tabular}{|l} 
Detection Bias \\
\end{tabular} & Performance Bias & Reporting Bias & Fidelity Bias & Funder & Overall Rating \\
\hline Strom 2017) $)^{23}$ (28553314) & 24 weeks & $\begin{array}{l}\text { Medium } \\
12.5 \%\end{array}$ & Medium & Medium & Medium & Medium & Medium & NR & Medium \\
\hline \begin{tabular}{|l} 
Baker $2003^{24}(12919265)$ \\
\end{tabular} & $\begin{array}{l}0 \text { weeks } \\
4 \text { weeks }\end{array}$ & $\begin{array}{l}\text { Low } \\
6.62 \%\end{array}$ & Low & Medium & Medium & Medium & High & $\mathrm{NR}$ & Medium \\
\hline \begin{tabular}{|l|l} 
Van Weert, $2005^{25}$ (15667372) \\
\end{tabular} & 72 weeks & $\begin{array}{l}\text { Medium } \\
27.61 \%\end{array}$ & Medium & High & High & Low & Low & Netherlands government & High \\
\hline
\end{tabular}

$\mathrm{X}$ indicates that domain was not assessed due to high risk of bias.

Abbreviations: NR=Not Reported; PMID=PubMed Identification Number 


\begin{tabular}{|c|c|c|c|c|c|c|c|c|c|c|c|c|c|}
\hline $\begin{array}{l}\text { Study (PMID) } \\
\text { Country } \\
\text { RoB* } \\
\text { Type }\end{array}$ & $\begin{array}{l}\text { Intervention } \\
\text { Intervention } \\
\text { Focus } \\
\text { Theoretical } \\
\text { Model } \\
\text { Delivery } \\
\text { Person } \\
\text { Intervention } \\
\text { Target } \\
\text { Recipient } \\
\text { Mode } \\
\text { Components } \\
\text { Frequency } \\
\text { Duration }\end{array}$ & \begin{tabular}{|l|} 
Comparison \\
\\
Target \\
Mode \\
Components \\
Frequency \\
Duration \\
\end{tabular} & $\begin{array}{l}\text { Setting } \\
\text { and Design } \\
\text { Setting } \\
\text { Design } \\
\text { Cluster N } \\
\text { Participants } \\
\text { Randomized } \\
\text { N }\end{array}$ & $\begin{array}{l}\text { PLWD } \\
\text { Dementia } \\
\text { Characteristics } \\
\text { Dementia Types } \\
\text { Dementia Severity } \\
\text { Diagnostic } \\
\text { Criteria } \\
\text { Age of Diagnosis }\end{array}$ & $\begin{array}{l}\text { PLWD } \\
\text { Non-Disease Char } \\
\text { PLWD N } \\
\text { PLWD Age (mean) } \\
\text { PLWD Sex (\% } \\
\text { female) } \\
\text { PLWD Race (\% } \\
\text { majority) } \\
\text { PLWD Education } \\
\text { (mean years) }\end{array}$ & $\begin{array}{l}\text { PLWD } \\
\text { Non-Disease } \\
\text { Char Reporting } \\
\text { Status (RS) } \\
\\
\text { PLWD SES } \\
\text { PLWD Prior } \\
\text { Disability } \\
\text { PLWD } \\
\text { Household } \\
\text { Characteristics } \\
\text { PLWD Health } \\
\text { Insurance } \\
\text { PLWD Detailed } \\
\text { Race Information }\end{array}$ & $\begin{array}{l}\text { Informal Caregiver } \\
\text { (IC) } \\
\text { Characteristics } \\
\text { IC N } \\
\text { IC Age (mean) } \\
\text { IC Sex (\% female) } \\
\text { IC Race (\% } \\
\text { majority) } \\
\text { IC Education } \\
\text { (mean years } \\
\text { IC Relation to } \\
\text { PLWD (\% majority) }\end{array}$ & $\begin{array}{l}\text { Informal Caregiver } \\
\text { (IC) Char. RS } \\
\text { IC Duration } \\
\text { IC Living With } \\
\text { PLWD } \\
\text { IC Payment } \\
\text { IC Health Status } \\
\text { IC Dementia } \\
\text { Family History } \\
\text { IC Employment } \\
\text { Status } \\
\text { IC Training }\end{array}$ & $\begin{array}{l}\text { Formal Caregiver } \\
\text { (FC) } \\
\text { Characteristics } \\
\text { FC N } \\
\text { FC Age (mean) } \\
\text { FC Sex (\% } \\
\text { female) } \\
\text { FC Race (\% } \\
\text { majority) } \\
\text { FC Education } \\
\text { (mean years) }\end{array}$ & $\begin{array}{l}\text { Formal } \\
\text { Caregiver (FC) } \\
\text { Char. RS } \\
\text { FC Health } \\
\text { Status } \\
\text { FC Training } \\
\text { FC Education } \\
\text { FC Position } \\
\text { FC Length of } \\
\text { Service }\end{array}$ & \begin{tabular}{|l|} 
Outcome \\
Timing(s)
\end{tabular} & $\begin{array}{l}\text { PLWD } \\
\text { Outcomes }\end{array}$ & $\begin{array}{l}\text { Caregiver } \\
\text { Outcomes } \\
\text { IC: } \\
\text { FC: }\end{array}$ \\
\hline $\begin{array}{l}\text { Strom, 201723 } \\
(28553314) \\
\text { Republic of } \\
\text { Ireland } \\
\text { Medium } \\
\text { Explanatory }\end{array}$ & $\begin{array}{l}\text { Group Sonas } \\
\text { sessions led by } \\
\text { a licensed } \\
\text { practitioner; two } \\
\text { 45-minute } \\
\text { sessions per } \\
\text { week for 24 } \\
\text { weeks }\end{array}$ & \begin{tabular}{|l|} 
Group \\
newspaper \\
reading \\
sessions; two \\
45-minute \\
sessions per \\
week for 24 \\
weeks \\
Usual nursing \\
facility care \\
\end{tabular} & $\begin{array}{l}\text { Nursing } \\
\text { facilities } \\
\text { approved and } \\
\text { accredited by } \\
\text { the Republic of } \\
\text { Ireland } \\
\text { RCT } \\
\text { Multisite } \\
120 \text { PLWD }\end{array}$ & $\begin{array}{l}\text { Diagnosis of } \\
\text { dementia, } \\
\text { moderate to severe } \\
\text { cognitive } \\
\text { impairment (MMSE } \\
\leq 20 \text { ) }\end{array}$ & \begin{tabular}{|l|}
$\mathrm{N}=120$ \\
Age: Mean age 84.8 \\
Sex: $77.5 \%$ female \\
Race: NR \\
Education NR
\end{tabular} & NR & NA & NA & NR & NR & \begin{tabular}{|l|}
12 weeks \\
post- \\
intervention \\
24 weeks \\
post- \\
intervention
\end{tabular} & Function & None \\
\hline $\begin{array}{l}\text { Baker 20032) } \\
(12919265) \\
(11317951)^{26} \\
\text { United } \\
\text { Kingdom, } \\
\text { Netherlands, } \\
\text { and Switzerland } \\
\text { Medium } \\
\text { Explanatory }\end{array}$ & $\begin{array}{l}\text { Individual, non- } \\
\text { directed } \\
\text { sessions in a } \\
\text { Snoezelen } \\
\text { room, facilitated } \\
\text { by a nursing } \\
\text { staff, } \\
\text { occupational } \\
\text { therapist, or } \\
\text { psychology } \\
\text { assistants; two } \\
\text { 30-minute } \\
\text { sessions per } \\
\text { week for } 4 \\
\text { weeks }\end{array}$ & \begin{tabular}{|l|} 
Individual \\
activity \\
sessions \\
chosen \\
according to \\
participant's \\
interests, e.g. \\
playing cards, \\
quizzes, \\
looking at \\
cards; \\
facilitated by \\
a nursing \\
staff, \\
occupational \\
therapist, or \\
psychology \\
assistants; \\
two sessions \\
per week for \\
4 weeks, 30 \\
minutes per \\
session \\
\end{tabular} & $\begin{array}{l}\text { Day hospital, } \\
\text { psycho- } \\
\text { geriatric ward } \\
\text { RCT } \\
\text { Multisite } \\
136 \text { PLWD } \\
\text { randomized, } \\
120 \text { analyzed }\end{array}$ & $\begin{array}{l}\text { Diagnosis of } \\
\text { Alzheimer's, } \\
\text { vascular, or mixed } \\
\text { dementia, } \\
\text { moderate to severe } \\
\text { cognitive } \\
\text { impairment (MMSE } \\
\leq 17 \text { ) }\end{array}$ & $\begin{array}{l}\mathrm{N}=136 \\
\text { Age: } 82 \text { years } \\
\text { Sex: not reported } \\
\text { Education: NR }\end{array}$ & NR & NA & NA & NR & NR & \begin{tabular}{|l}
0 weeks post \\
intervention \\
4 weeks post \\
intervention
\end{tabular} & $\begin{array}{l}\text { Neuropsychi } \\
\text { atric } \\
\text { symptoms }\end{array}$ & \begin{tabular}{|l|} 
None \\
\end{tabular} \\
\hline
\end{tabular}

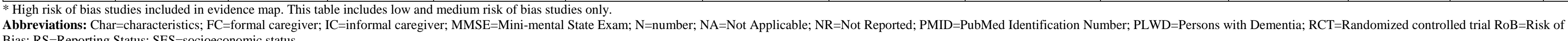


Table C-9. PLWD outcomes summary low and medium risk of bias studies: multisensory stimulation

\begin{tabular}{|c|c|c|c|c|c|}
\hline $\begin{array}{l}\text { Study (PMID) } \\
\text { Comparison } \\
\text { RoB } \\
\text { Category* }\end{array}$ & $\begin{array}{l}\text { Outcome } \\
\text { Timing }\end{array}$ & Summary Finding & Intervention & Comparator & p Value \\
\hline $\begin{array}{l}\text { Baker 200324 (12919265) } \\
\text { Explanatory }\end{array}$ & $\begin{array}{l}\text { Unadjusted Mean Difference } \\
\text { Reduction in neuropsychiatric symptoms (REHAB general behavior scale, UK participants only) } \\
4 \text { weeks (immediately post-intervention) }\end{array}$ & -1.9 points, $\mathrm{Cl}$ not reported & Snoezelen & Active control & $\begin{array}{l}\text { Not reported, but } \\
\text { described as no } \\
\text { difference }\end{array}$ \\
\hline $\begin{array}{l}\text { Baker 200324 (12919265) } \\
\text { Explanatory }\end{array}$ & $\begin{array}{l}\text { Unadjusted Mean Difference } \\
\text { Reduction in neuropsychiatric symptoms (REHAB deviant behavior subscale, UK participants } \\
\text { only) } \\
4 \text { weeks (immediately post-intervention) }\end{array}$ & 0.5 points, $\mathrm{Cl}$ not reported & Snoezelen & Active control & $\begin{array}{l}\text { Not reported, but } \\
\text { described as no } \\
\text { difference }\end{array}$ \\
\hline $\begin{array}{l}\text { Baker 200324(12919265) } \\
\text { Explanatory }\end{array}$ & $\begin{array}{l}\text { Unadjusted Mean Difference } \\
\text { Reduction in neuropsychiatric symptoms (GIP scale, Dutch participants only) } \\
4 \text { weeks (immediately post-intervention) }\end{array}$ & -2.4 points, $\mathrm{Cl}$ not reported & Snoezelen & Active control & $\begin{array}{l}\text { Not reported, but } \\
\text { described as no } \\
\text { difference }\end{array}$ \\
\hline $\begin{array}{l}\text { Strom 201723 (28553314) } \\
\text { Explanatory }\end{array}$ & $\begin{array}{l}\text { Unadjusted mean difference } \\
\text { Reduction in communication difficulty (HCS total score)(higher is worse) } \\
24 \text { weeks }\end{array}$ & 3.0 points, $\mathrm{Cl}$ not reported & Sonas & Active control & $\mathrm{P}=0.019$ \\
\hline $\begin{array}{l}\text { Strom } 20177^{23} \text { (28553314) } \\
\text { Explanatory }\end{array}$ & $\begin{array}{l}\text { Unadjusted mean difference } \\
\text { Reduction in communication difficulty (HCS total score) } \\
24 \text { weeks }\end{array}$ & 4.0 points, $\mathrm{Cl}$ not reported & Sonas & Inactive control & $P=0.001$ \\
\hline
\end{tabular}

Explanatory studies are listed first, followed by pragmatic studies.

Table C-10. Summary of strength of evidence for PLWD outcomes: multisensory stimulation

\begin{tabular}{|c|c|c|c|c|c|c|c|c|}
\hline $\begin{array}{l}\text { Comparison } \\
\text { Outcome }\end{array}$ & Timing & $\begin{array}{l}\text { \# Studies/ Design } \\
\text { (n analyzed) }\end{array}$ & Finding or Summary Statistic & Study Limitations & Consistency & Directness & Precision & $\begin{array}{l}\text { Overall Grade/ } \\
\text { Conclusion }\end{array}$ \\
\hline $\begin{array}{l}\text { MSS vs. Active control } \\
\text { Neuropsychiatric } \\
\text { symptoms }\end{array}$ & 4 weeks & $1 \mathrm{RCT}(\mathrm{n}=120)$ & $\begin{array}{l}\text { No significant difference in } \\
\text { measures of neuropsychiatric } \\
\text { symptoms (UK population) }\end{array}$ & Moderate & Unknown & $\begin{array}{l}\text { Indirect } \\
\text { (BPSD were assessed using instruments that are not } \\
\text { standard in the ADRD field (appear to be designed for } \\
\text { psychiatric settings) and that contain other domains } \\
\text { (e.g. mood, physical function)) }\end{array}$ & Imprecise & Insufficient \\
\hline
\end{tabular}

Abbreviations: $\mathrm{ADRD}=$ Alzheimer's disease and related disorders; $\mathrm{BPSD}=$ =behavioral and psychological symptoms of dementia; MSS=multisensory stimulation; n=number; RCT=randomized controlled trials

\section{Evidence Map: Multi-Sensory Stimulation}

Table C-11. Characteristics of evidence map studies: multisensory stimulation 


\begin{tabular}{|c|c|c|c|c|c|c|c|c|c|c|c|c|c|}
\hline $\begin{array}{l}\text { Study (PMID) } \\
\text { Country } \\
\text { EM Reason }\end{array}$ & Intervention & Comparison & \begin{tabular}{|l|} 
Setting \\
and Design \\
Setting \\
Design \\
Cluster N \\
Participants \\
Random- \\
ized N \\
\end{tabular} & \begin{tabular}{|l|} 
PLWD \\
Dementia \\
Characteristics
\end{tabular} & $\begin{array}{l}\text { PLWD } \\
\text { Non-Disease Char } \\
\text { PLWD N } \\
\text { PLWD Age (mean) } \\
\text { PLWD Sex (\% } \\
\text { female) } \\
\text { PLWD Race (\% } \\
\text { majority) } \\
\text { PLWD Education } \\
\text { (mean years) }\end{array}$ & \begin{tabular}{|l|} 
PLWD \\
Non-Disease \\
Char Reporting \\
Status (RS) \\
PLWD SES \\
PLWD Prior \\
Disability \\
PLWD \\
Household \\
Characteristics \\
PLWD Health \\
Insurance \\
PLWD Detailed \\
Race Information \\
\end{tabular} & $\begin{array}{l}\text { Informal Caregiver } \\
\text { (IC) } \\
\text { Characteristics } \\
\text { IC N } \\
\text { IC Age (mean) } \\
\text { IC Sex (\% female) } \\
\text { IC Race (\% } \\
\text { majority) } \\
\text { IC Education } \\
\text { (mean years) } \\
\text { IC Relation to } \\
\text { PLWD (\% majority) }\end{array}$ & $\begin{array}{l}\text { Informal Caregiver } \\
\text { (IC) Char. RS } \\
\text { IC Duration } \\
\text { IC Living With } \\
\text { PLWD } \\
\text { IC Payment } \\
\text { IC Health Status } \\
\text { IC Dementia } \\
\text { Family History } \\
\text { IC Employment } \\
\text { Status } \\
\text { IC Training }\end{array}$ & $\begin{array}{l}\text { Formal Caregiver } \\
\text { (FC) } \\
\text { Characteristics } \\
\text { FC N } \\
\text { FC Age (mean) } \\
\text { FC Sex (\% } \\
\text { female) } \\
\text { FC Race (\% } \\
\text { majority) } \\
\text { FC Education } \\
\text { (mean years) }\end{array}$ & $\begin{array}{l}\text { Formal } \\
\text { Caregiver (FC) } \\
\text { Char. RS } \\
\text { FC Health } \\
\text { Status } \\
\text { FC Training } \\
\text { FC Education } \\
\text { FC Position } \\
\text { FC Length of } \\
\text { Service }\end{array}$ & $\begin{array}{l}\text { Outcome } \\
\text { Timing(s) }\end{array}$ & $\begin{array}{l}\text { PLWD } \\
\text { Outcomes }\end{array}$ & $\begin{array}{l}\text { Caregiver } \\
\text { Outcomes } \\
\text { IC: } \\
\text { FC: }\end{array}$ \\
\hline $\begin{array}{l}\text { Maseda 201827 } \\
(29843244) \\
\text { Spain } \\
\text { Small sample }\end{array}$ & Snoezelen & $\begin{array}{l}\text { Individualized } \\
\text { music sessions }\end{array}$ & $\begin{array}{l}\text { Nursing } \\
\text { home; single } \\
\text { site } \\
21 \text { PLWD }\end{array}$ & \begin{tabular}{|l|}
$\mathrm{N}=21$ \\
Diagnosis of \\
dementia and \\
severe cognitive \\
impairment \\
(GDS $=6$ or 7 )
\end{tabular} & $\begin{array}{l}\mathrm{N}=21 \\
\text { Sex: yes } \\
\text { Age: yes } \\
\text { Race: no } \\
\text { Education: yes }\end{array}$ & None & NA & NA & NA & NA & 12 weeks & $\begin{array}{l}\text { No validated } \\
\text { outcomes }\end{array}$ & NR \\
\hline $\begin{array}{l}\text { Collier 200828 } \\
(21131677) \\
\text { United Kingdom } \\
\text { Small sample }\end{array}$ & $\begin{array}{l}\text { Multi-sensory } \\
\text { environment }\end{array}$ & Gardening & $\begin{array}{l}\text { Nursing } \\
\text { homes or } \\
\text { continuing } \\
\text { care wards; } \\
\text { multisite } \\
\text { 30 PLWD }\end{array}$ & $\begin{array}{l}\mathrm{N}=30 \\
\text { Moderate to severe } \\
\text { dementia } \\
\text { Mean MMSE } 10.0\end{array}$ & $\begin{array}{l}\mathrm{N}=30 \\
\text { Sex: yes } \\
\text { Age: yes } \\
\text { Race: no } \\
\text { Education: no }\end{array}$ & None & NA & NA & NA & NA & 4 weeks & $\begin{array}{l}\text { AMPS motor } \\
\text { and process } \\
\text { scales }\end{array}$ & NA \\
\hline $\begin{array}{l}\text { Staal 200729 } \\
(18441625) \\
\text { US } \\
\text { Pilot }\end{array}$ & Snoezelen & $\begin{array}{l}\text { Activity } \\
\text { sessions }\end{array}$ & $\begin{array}{l}\text { Geriatric } \\
\text { psychiatric } \\
\text { unit; single } \\
\text { site } \\
24 \text { PLWD }\end{array}$ & $\begin{array}{l}\mathrm{N}=24 \\
\text { Admitting diagnosis } \\
\text { of dementia, } \\
\text { criteria NR } \\
\text { Mean MMSE } 15.5\end{array}$ & $\begin{array}{l}\mathrm{N}=24 \\
\text { Sex: yes } \\
\text { Age: yes } \\
\text { Race: no } \\
\text { Education: no }\end{array}$ & None & NA & NA & NA & NA & Unclear & $\begin{array}{l}\text { PAS } \\
\text { MAl, } \\
\text { physical } \\
\text { health } \\
\text { domain } \\
\text { SANS-AD } \\
\text { Katz Index of } \\
\text { ADLs } \\
\text { RADL }\end{array}$ & NA \\
\hline $\begin{array}{l}\text { Van Weert } \\
2005^{25} \\
(15667372) \\
(15050851)^{30} \\
\text { Netherlands } \\
\text { High RoB }\end{array}$ & Snoezelen & Usual care & \begin{tabular}{|l|} 
Nursing \\
home \\
psychogeriat \\
ric wards; \\
multisite \\
125 PLWD \\
\end{tabular} & $\begin{array}{l}\mathrm{N}=125 \\
\text { Moderate to severe } \\
\text { dementia, DSM-III } \\
\text { criteria }\end{array}$ & $\begin{array}{l}\mathrm{N}=125 \\
\text { Sex: yes } \\
\text { Age: yes } \\
\text { Race: no } \\
\text { Education: no }\end{array}$ & None & NA & NA & NA & $\mathrm{NA}$ & 18 months & $\begin{array}{l}\text { BIP, } 8 \text { of } 14 \\
\text { subscales } \\
\text { CMAI } \\
\text { CSDD }\end{array}$ & NA \\
\hline $\begin{array}{l}\text { Baillon 200431 } \\
(15481068) \\
\text { United Kingdom } \\
\text { Small sample }\end{array}$ & Snoezelen & $\begin{array}{l}\text { Reminiscence } \\
\text { therapy }\end{array}$ & $\begin{array}{l}\text { Nursing } \\
\text { home; single } \\
\text { site }\end{array}$ & \begin{tabular}{|l|}
$\mathrm{N}=25$ \\
Median MMSE 4.0 \\
among completers
\end{tabular} & $\begin{array}{l}\mathrm{N}=25 \\
\text { Sex: yes } \\
\text { Age yes } \\
\text { Race: no } \\
\text { Education: no }\end{array}$ & None & NA & NA & NA & $\mathrm{NA}$ & $\begin{array}{l}1 \text { day (note: } \\
\text { comparisons } \\
\text { were done } \\
\text { before and } \\
\text { after each } \\
\text { individual } \\
\text { session) }\end{array}$ & ABMI & NA \\
\hline $\begin{array}{l}\text { Van Diepen } \\
2002^{32} \\
(35396447) \\
\text { United Kingdom } \\
\text { Pilot }\end{array}$ & Snoezelen & $\begin{array}{l}\text { Reminiscence } \\
\text { therapy }\end{array}$ & $\begin{array}{l}\text { Day } \\
\text { hospital; } \\
\text { single site } \\
15 \text { PLWD }\end{array}$ & \begin{tabular}{|l|}
$\mathrm{N}=15$ \\
Mean MMSE 7.7
\end{tabular} & $\begin{array}{l}\mathrm{N}=15 \\
\text { Sex: no } \\
\text { Age no } \\
\text { Race: no } \\
\text { Education: no }\end{array}$ & None & NA & NA & NA & $\mathrm{NA}$ & $\begin{array}{l}8 \text { weeks post } \\
\text { intervention }\end{array}$ & $\begin{array}{l}\text { CMAI } \\
\text { ABMII }\end{array}$ & $\mathrm{NA}$ \\
\hline
\end{tabular}




\begin{tabular}{|c|c|c|c|c|c|c|c|c|c|c|}
\hline $\begin{array}{l}\text { Baker 199733 } \\
\text { (12519587) } \\
\text { United Kingdom } \\
\text { Small sample }\end{array}$ & Snoezelen & \begin{tabular}{|l|} 
Activity \\
sessions
\end{tabular} & $\begin{array}{l}\text { Day } \\
\text { hospital; } \\
\text { multisite } \\
31 \text { PLWWD }\end{array}$ & $\begin{array}{l}\text { Mean MMSE } \\
\text { approximately } 6\end{array}$ & \begin{tabular}{|l|}
$\mathrm{N}=31$ \\
Sex: yes \\
Age: no \\
Race: no \\
Education: no
\end{tabular} & None & NA & NA & $\mathrm{N}$ & $\mathrm{N}$ \\
\hline
\end{tabular}

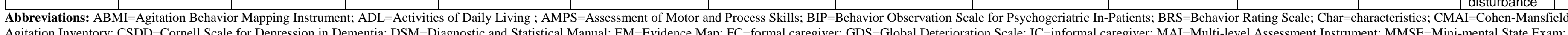

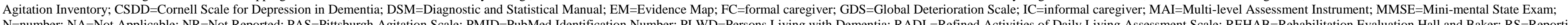

\section{Complementary and Alternative Medicine (CAM) Therapy}

\begin{tabular}{|c|c|c|c|c|c|c|c|c|}
\hline \begin{tabular}{|l|} 
Study (PMID) \\
\end{tabular} & Outcome Timing & $\begin{array}{l}\text { Attrition Bias } \\
\text { Attrition \% }\end{array}$ & Selection Bias & Detection Bias & Performance Bias & Reporting Bias & Fidelity Bias & Overall Rating \\
\hline Kwan $2017^{34}(25346026)$ & 8 weeks & $\begin{array}{l}\text { Low } \\
8 \text { weeks: } 0 \%\end{array}$ & Medium & High & High & Low & Medium & High \\
\hline Yang $2016^{35}$ (27319407) & 8 weeks & $\begin{array}{l}\text { Low } \\
8 \text { weeks: } 5 \%\end{array}$ & Low & Medium & High & Low & Medium & Medium \\
\hline Yang $2015^{36}(25880034)$ & 3 weeks & $\begin{array}{l}\text { High } \\
3 \text { weeks: 33\% }\end{array}$ & $\mathrm{x}$ & $\mathrm{x}$ & $\mathrm{x}$ & $\mathrm{x}$ & $\mathrm{x}$ & High \\
\hline 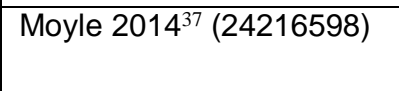 & 3 weeks & $\begin{array}{l}\text { Low } \\
3 \text { weeks: } 3.64 \%\end{array}$ & Low & Low & Low & Low & Low & Low \\
\hline Burns 2011 38 (21335973) & 4 weeks & $\begin{array}{l}\text { Medium } \\
\text { 4 weeks: } 16 \%\end{array}$ & Low & Medium & Low & Low & Low & Medium \\
\hline Lin $2007^{39}(17342790)$ & 8 weeks & $\begin{array}{l}\text { Low } \\
8 \text { weeks: } 0 \%\end{array}$ & Low & Medium & Medium & Low & Low & Medium \\
\hline Ballard 2002 $2^{40}(12143909)$ & 4 weeks & $\begin{array}{l}\text { Low } \\
4 \text { weeks: } 1.4 \%\end{array}$ & Low & Low & Medium & High & Low & Medium \\
\hline
\end{tabular}

X indicates that domain was not assessed due to high risk of bias.
Abbreviations: NA=Not Applicable; PMID $=$ PubMed Identification 
Table C-13. Characteristics of included studies: CAM therapy 


\begin{tabular}{|c|c|c|c|c|c|c|c|c|c|c|c|c|c|}
\hline $\begin{array}{l}\text { Study (PMID) } \\
\\
\text { Country } \\
\text { RoB* } \\
\text { Type }\end{array}$ & $\begin{array}{l}\text { Intervention } \\
\text { Intervention } \\
\text { Focus } \\
\text { Theoretical } \\
\text { Model } \\
\text { Delivery } \\
\text { Person } \\
\text { Intervention } \\
\text { Target } \\
\text { Recipient } \\
\text { Mode } \\
\text { Components } \\
\text { Frequency } \\
\text { Duration }\end{array}$ & $\begin{array}{l}\text { Comparison } \\
\text { Target } \\
\text { Mode } \\
\text { Components } \\
\text { Frequency } \\
\text { Duration }\end{array}$ & $\begin{array}{l}\text { Setting } \\
\text { and Design } \\
\text { Setting } \\
\text { Design } \\
\text { Cluster N } \\
\text { Participants } \\
\text { Randomized } \\
\text { N }\end{array}$ & \begin{tabular}{|l|} 
PLWD \\
Dementia \\
Characteristics \\
\\
Dementia Types \\
Dementia Severity \\
Diagnostic \\
Criteria \\
Age of Diagnosis \\
\end{tabular} & $\begin{array}{l}\text { PLWD } \\
\text { Non-Disease Char } \\
\text { PLWD N } \\
\text { PLWD Age (mean) } \\
\text { PLWD Sex (\% } \\
\text { female) } \\
\text { PLWD Race (\% } \\
\text { majority) } \\
\text { PLWD Education } \\
\text { (mean years) }\end{array}$ & $\begin{array}{l}\text { PLWD } \\
\text { Non-Disease } \\
\text { Char Reporting } \\
\text { Status (RS) } \\
\\
\text { PLWD SES } \\
\text { PLWD Prior } \\
\text { Disability } \\
\text { PLWD } \\
\text { Household } \\
\text { Characteristics } \\
\text { PLWD Health } \\
\text { Insurance } \\
\text { PLWD Detailed } \\
\text { Race Information }\end{array}$ & $\begin{array}{l}\text { Informal Caregiver } \\
\text { (IC) } \\
\text { Characteristics } \\
\text { IC N } \\
\text { IC Age (mean) } \\
\text { IC Sex (\% female) } \\
\text { IC Race (\% } \\
\text { majority) } \\
\text { IC Education } \\
\text { (mean years) } \\
\text { IC Relation to } \\
\text { PLWD (\% majority) }\end{array}$ & $\begin{array}{l}\text { Informal Caregiver } \\
\text { (IC) Char. RS } \\
\text { IC Duration } \\
\text { IC Living With } \\
\text { PLWD } \\
\text { IC Payment } \\
\text { IC Health Status } \\
\text { IC Dementia } \\
\text { Family History } \\
\text { IC Employment } \\
\text { Status } \\
\text { IC Training }\end{array}$ & $\begin{array}{l}\text { Formal Caregiver } \\
\text { (FC) } \\
\text { Characteristics } \\
\text { FC N } \\
\text { FC Age (mean) } \\
\text { FC Sex (\% } \\
\text { female) } \\
\text { FC Race (\% } \\
\text { majority) } \\
\text { FC Education } \\
\text { (mean years) }\end{array}$ & $\begin{array}{l}\text { Formal } \\
\text { Caregiver (FC) } \\
\text { Char. RS } \\
\text { FC Health } \\
\text { Status } \\
\text { FC Training } \\
\text { FC Education } \\
\text { FC Position } \\
\text { FC Length of } \\
\text { Service }\end{array}$ & $\begin{array}{l}\text { Outcome } \\
\text { Timing(s) }\end{array}$ & $\begin{array}{l}\text { PLWD } \\
\text { Outcomes }\end{array}$ & $\begin{array}{l}\text { Caregiver } \\
\text { Outcomes } \\
\text { IC: } \\
\text { FC: }\end{array}$ \\
\hline $\begin{array}{l}\text { Yang 201635 } \\
\text { (27319407) } \\
\text { Taiwan } \\
\text { Medium } \\
\text { Explanatory }\end{array}$ & $\begin{array}{l}\text { PLWD; got } 30 \\
\text { minutes of } \\
\text { aromatherapy } \\
\text { massage with } \\
\text { lavender oil and } \\
\text { orange oil once } \\
\text { per week for } 8 \\
\text { weeks } f\end{array}$ & $\begin{array}{l}\text { PLWD, Usual } \\
\text { Care }\end{array}$ & $\begin{array}{l}\text { Long-term care } \\
\text { facilities in } \\
\text { Taiwan } \\
\\
\text { RCT } \\
59 \text { PLWD }\end{array}$ & \begin{tabular}{|l|} 
Mild to severe \\
dementia based on \\
(score $\leq 8$ ) \\
(SPMSQ; or \\
(MMSE);
\end{tabular} & $\begin{array}{l}\mathrm{N}=29 \\
61 \% \text { Female } \\
92 \text { years } \\
\text { Race NR } \\
\text { High school } \\
\text { education or higher: } \\
38 \% \\
\text { Use of psychotropic } \\
\text { meds: } 70 \%\end{array}$ & None & NA & NA & NA & NA & $\begin{array}{l}5 \text { weeks } \\
9 \text { weeks }\end{array}$ & $\begin{array}{l}\text { CCMAI } \\
\text { CSDD-C }\end{array}$ & NA \\
\hline $\begin{array}{l}\text { Moyle 201437 } \\
\text { (24216598) } \\
\text { Australia } \\
\text { Low } \\
\text { Explanatory }\end{array}$ & $\begin{array}{l}\text { PLWD, Trained } \\
\text { massage } \\
\text { therapists } \\
\text { provided } 5 \\
\text { minutes } \\
\text { massage on } \\
\text { each foot using } \\
\text { unscented } \\
\text { sorbolene for } 3 \\
\text { weeks }\end{array}$ & $\begin{array}{l}\text { PLWD, } \\
\text { Trained } \\
\text { assistants sat } \\
\text { quietly near } \\
\text { the } \\
\text { participant's } \\
\text { feet for } 10 \\
\text { minutes } \\
\text { without } \\
\text { talking or } \\
\text { making } \\
\text { physical } \\
\text { contact or any } \\
\text { deliberate } \\
\text { touching or } \\
\text { conversation } \\
\text { with the } \\
\text { participant }\end{array}$ & $\begin{array}{l}\text { Long-term care } \\
\text { facilities in } \\
\text { South } \\
\text { Queensland } \\
\text { Crossover } \\
\text { RCT } \\
55 \text { PLWD }\end{array}$ & $\begin{array}{l}\text { Moderate to late } \\
\text { stage dementia, } \\
\text { MMSE score of } \\
\text { less than } 18 \text { with a } \\
\text { recent history of } \\
\text { agitation }(\text { PAS >3); }\end{array}$ & $\begin{array}{l}\mathrm{N}=55 \\
66 \% \text { Female } \\
86.5 \text { years } \\
\text { Race NR } \\
\text { Education NR }\end{array}$ & None & NA & NA & NA & NA & 3 weeks & $\begin{array}{l}\text { CMAI } \\
\text { OERS }\end{array}$ & NA \\
\hline
\end{tabular}




\begin{tabular}{|c|c|c|c|c|c|c|c|c|c|c|c|c|c|}
\hline $\begin{array}{l}\text { Burns 201138 } \\
\text { (21335973) } \\
\text { The United } \\
\text { Kingdom (UK) } \\
\text { Medium } \\
\text { Explanatory }\end{array}$ & $\begin{array}{l}\text { PLWD; 10\% } \\
\text { Melissa (lemon- } \\
\text { balm) oil mixed } \\
\text { with base lotion } \\
\text { was gently } \\
\text { massaged on } \\
\text { hands and } \\
\text { upper-arms } \\
\text { twice a day }\end{array}$ & $\begin{array}{l}\text { PLWD; } \\
\text { Sunflower oil } \\
\text { mixed with } \\
\text { base lotion } \\
\text { was gently } \\
\text { massaged on } \\
\text { hands and } \\
\text { upper arms } \\
\text { twice a day }\end{array}$ & $\begin{array}{l}\text { Clinical centers } \\
\text { - Manchester, } \\
\text { London and } \\
\text { Southampton, } \\
\text { UK } \\
\text { RCT } \\
77 \text { PLWD }\end{array}$ & \begin{tabular}{|l|} 
PLWD with \\
agitation for a \\
minimum of 4 \\
weeks, a score of \\
1399 on the CMAI, \\
and satisfied the \\
NINCDS/ADRDA \\
criteria for probable \\
or possible \\
Alzheimer's \\
disease; or was a \\
resident in a \\
nursing home or \\
NHS continuing \\
care facility, had a \\
clinical dementia \\
rating of 3, age \\
over 60 years and \\
were free of \\
psychotropic \\
medication \\
(antipsychotics \\
and/ or \\
cholinesterase \\
inhibitors) for at \\
least 2 weeks \\
\end{tabular} & $\begin{array}{l}\mathrm{N}=38 \\
43 \% \text { Female } \\
85 \text { years } \\
\text { Race NR } \\
\text { Education NR }\end{array}$ & None & NA & NA & NA & NA & $\begin{array}{l}4 \text { weeks } \\
12 \text { weeks }\end{array}$ & $\begin{array}{l}\text { PAS } \\
\text { NPI } \\
\text { Blau-QoL } \\
\text { Barthel index }\end{array}$ & $\overline{\mathrm{NA}}$ \\
\hline $\begin{array}{l}\text { Lin 200739 } \\
\text { (17342790) } \\
\text { Hong Kong } \\
\text { Medium } \\
\text { Explanatory }\end{array}$ & $\begin{array}{l}\text { PLWD, lavender } \\
\text { oil inhalation for } \\
3 \text { weeks. with a } \\
\text { washout period } \\
\text { of } 2 \text { weeks; } \\
2 \text { drops of oil } \\
\text { placed on } \\
\text { cotton using } \\
\text { dropper in an } \\
\text { aromatic } \\
\text { diffuser. Two } \\
\text { such diffusers } \\
\text { are placeed at } \\
\text { each side of } \\
\text { pillow for } 1 \mathrm{~h} \\
\text { every night }\end{array}$ & $\begin{array}{l}\text { PLWD, } \\
\text { Sunflower oil } \\
\text { inhalation for } \\
3 \text { weeks. with } \\
\text { a washout } \\
\text { period of } 2 \\
\text { weeks; } \\
2 \text { drops of oil } \\
\text { placed on } \\
\text { cotton using } \\
\text { dropper in an } \\
\text { aromatic } \\
\text { diffuser. Two } \\
\text { such diffusers } \\
\text { are placed at } \\
\text { each side of } \\
\text { pillow for } 1 \mathrm{~h} \\
\text { every nigh }\end{array}$ & \begin{tabular}{l|} 
Care and \\
attention \\
homes in Hong \\
Kong \\
Crossover \\
RCT \\
70 PLWD
\end{tabular} & \begin{tabular}{|l|} 
Dementia \\
diagnosis based on \\
DSM-IV APA A 1994 \\
with clinically \\
significant agitation \\
evaluated using \\
CCMAI
\end{tabular} & $\begin{array}{l}\mathrm{N}=35 \\
41 \% \text { Female } \\
82 \text { years } \\
\text { Race NR } \\
\text { Education NR } \\
\text { Use of psychotropic } \\
\text { meds: } 51 \%\end{array}$ & None & NA & NA & NA & NA & $\begin{array}{l}3 \text { weeks } \\
5 \text { weeks } \\
8 \text { weeks }\end{array}$ & $\begin{array}{l}\text { CCMAI } \\
\text { CNPI }\end{array}$ & $\mathrm{NA}$ \\
\hline $\begin{array}{l}\text { Ballard 200240 } \\
(12143909) \\
\\
\text { The United } \\
\text { Kingdom } \\
\text { Medium } \\
\text { Explanatory }\end{array}$ & $\begin{array}{l}\text { PLWD; 10\% } \\
\text { Melissa (lemon- } \\
\text { balm) oil mixed } \\
\text { with base lotion } \\
\text { was gently } \\
\text { applied (0.16- } \\
\text { o.17g) onto } \\
\text { PLWD face and } \\
\text { arms, twice a } \\
\text { day for } 6 \text { doses } \\
\text { per day by care } \\
\text { assistants }\end{array}$ & $\begin{array}{l}\text { PLWD; } \\
\text { Sunflower oil } \\
\text { mixed with } \\
\text { base lotion } \\
\text { was gently } \\
\text { applied (0.16- } \\
0.17 g \text { ) onto } \\
\text { PLWD face } \\
\text { and arms, } \\
\text { twice a day } \\
\text { for } 6 \text { doses } \\
\text { per day by } \\
\text { care } \\
\text { assistants }\end{array}$ & \begin{tabular}{l|} 
National \\
Health Service \\
nursing homes \\
in UK \\
RCT \\
72 PLWD
\end{tabular} & $\begin{array}{l}\text { Severe dementia } \\
\text { with agitation } \\
\text { characterized by } \\
\text { CMAI and also } \\
\text { screened by NPI } \\
\text { and CDR }\end{array}$ & $\begin{array}{l}\mathrm{N}=36 \\
60 \% \text { Female } \\
78.5 \text { years } \\
\text { Race NR } \\
\text { Education NR } \\
\text { Use of psychotropic } \\
\text { meds: } 92 \%\end{array}$ & None & NA & NA & NA & NA & 4 weeks & $\begin{array}{l}\text { CMAI } \\
\text { NPI }\end{array}$ & NA \\
\hline
\end{tabular}

* High risk of bias studies included in evidence map. This table includes low and medium risk of bias studies only.

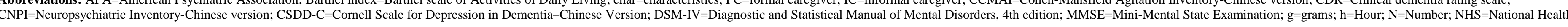



dentification Number; PLWD=Persons Living with Dementia; QoL=Quality of life, RCT=Randomized controlled trial; RoB=Risk of Bias; RS=Reporting Status; SPMSQ=Short Portable Mental Status Questionnaire

Table C-14. PLWD outcomes summary low and medium risk of bias studies: CAM therapy

\begin{tabular}{|c|c|c|c|c|c|c|c|}
\hline $\begin{array}{l}\text { Study (PMID) } \\
\text { Comparison } \\
\text { RoB } \\
\text { Category* }\end{array}$ & $\begin{array}{l}\text { Outcome } \\
\text { Timing }\end{array}$ & Summary Finding & Overall p-Value & Intervention & p-Value & Comparator & p-Value \\
\hline $\begin{array}{l}\text { Yang } 2016^{35}(27319407) \\
\text { Lavender and orange oil vs. Usual } \\
\text { care } \\
\text { Medium } \\
\text { Explanatory }\end{array}$ & $\begin{array}{l}\text { Mean Difference }(95 \% \mathrm{Cl}) \\
\text { CCMAI } \\
5 \text { weeks }\end{array}$ & $\mathrm{NR}$ & 0.316 & $3.65(\mathrm{NR})$ & NR & 7.08 (NR) & NR \\
\hline $\begin{array}{l}\text { Yang } 2016^{35}(27319407) \\
\text { Lavender and orange oil vs. Usual } \\
\text { care } \\
\text { Medium } \\
\text { Explanatory }\end{array}$ & $\begin{array}{l}\text { Mean Difference }(95 \% \mathrm{Cl}) \\
\text { CCMAI } \\
9 \text { weeks }\end{array}$ & $\mathrm{NR}$ & 0.316 & 3.41 (NR) & $\mathrm{NR}$ & $6.3(\mathrm{NR})$ & NR \\
\hline $\begin{array}{l}\text { Yang } 2016^{35}(27319407) \\
\text { Yavender and orange oil vs. Usual } \\
\text { care } \\
\text { Medium } \\
\text { Explanatory }\end{array}$ & $\begin{array}{l}\text { Mean Difference }(95 \% \mathrm{Cl}) \\
\text { CSDD-C } \\
5 \text { weeks }\end{array}$ & $\mathrm{NR}$ & $<0.001$ & 0.51 (NR) & NR & $3.04(N R)$ & NR \\
\hline $\begin{array}{l}\text { Yang } 2016^{35}(27319407) \\
\text { Lavender and orange oil vs. Usual } \\
\text { care } \\
\text { Medium } \\
\text { Explanatory }\end{array}$ & $\begin{array}{l}\text { Mean Difference }(95 \% \mathrm{Cl}) \\
\text { CSDD-C } \\
9 \text { weeks }\end{array}$ & NR & $<0.001$ & $0.62(N R)$ & NR & 6.45 (NR) & $\mathrm{NR}$ \\
\hline $\begin{array}{l}\text { Lin } 2007^{39}(17342790) \\
\text { Lavender oil vs. sunflower oil } \\
\text { Medium } \\
\text { Explanatory }\end{array}$ & $\begin{array}{l}\text { Mean Difference }(95 \% \mathrm{Cl}) \\
\text { CNPI } \\
3 \text { weeks }\end{array}$ & NR & $\mathrm{NR}$ & $6.91(\mathrm{NR})$ & $<0.001$ & $-0.08(N R)$ & $\mathrm{p}=0.24$ \\
\hline $\begin{array}{l}\text { Lin } 2007^{39} \text { (17342790) } \\
\text { Lavender oil vs. sunflower oil } \\
\text { Medium } \\
\text { Explanatory }\end{array}$ & $\begin{array}{l}\text { Mean Difference }(95 \% \mathrm{Cl}) \\
\text { CCMAI } \\
3 \text { weeks }\end{array}$ & $\mathrm{NR}$ & $\mathrm{NR}$ & $4.4(\mathrm{NR})$ & $<0.001$ & 0.04 (NR) & $\mathrm{p}=0.52$ \\
\hline $\begin{array}{l}\text { Burns } 2011^{38}(21335973) \\
\text { Melissa oil vs sunflower oil } \\
\text { Medium } \\
\text { Explanatory }\end{array}$ & $\begin{array}{l}\text { Median Difference }(95 \% \mathrm{Cl}) \\
\text { PAS } \\
4 \text { weeks }\end{array}$ & $\mathrm{NR}$ & 0.94 & $0.0(-1.3,0.3)$ & NR & $-0.3(-1.7,0)$ & NR \\
\hline $\begin{array}{l}\text { Burns } 2011^{38}(21335973) \\
\text { Melissa oil vs sunflower oil } \\
\text { Medium } \\
\text { Explanatory }\end{array}$ & $\begin{array}{l}\text { Median Difference }(95 \% \mathrm{Cl}) \\
\text { PAS } \\
12 \text { weeks }\end{array}$ & NR & 0.56 & $-0.7(-1.7,0)$ & $\mathrm{NR}$ & $-0.7(-1.7,0)$ & NR \\
\hline $\begin{array}{l}\text { Burns } 2011^{38}(21335973) \\
\text { Melissa oil vs sunflower oil } \\
\text { Medium } \\
\text { Explanatory }\end{array}$ & $\begin{array}{l}\text { Mean Difference }(95 \% \mathrm{Cl}) \\
\mathrm{NPI} \\
4 \text { weeks }\end{array}$ & NR & 0.54 & $-4.8(-11.3,1.6)$ & NR & $-9.8(-18.8,1.3)$ & NR \\
\hline $\begin{array}{l}\text { Burns } 2011^{38}(21335973) \\
\text { Melissa oil vs sunflower oil } \\
\text { Medium } \\
\text { Explanatory }\end{array}$ & $\begin{array}{l}\text { Mean Difference }(95 \% \mathrm{Cl}) \\
\mathrm{NPI} \\
12 \text { weeks }\end{array}$ & $\mathrm{NR}$ & 0.52 & $-7.2(-12.6,-1.7)$ & NR & $-10.0(-17.2,-3)$ & NR \\
\hline $\begin{array}{l}\text { Burns } 2011^{38}(21335973) \\
\text { Melissa oil vs sunflower oil } \\
\text { Medium } \\
\text { Explanatory }\end{array}$ & $\begin{array}{l}\text { Mean Difference }(95 \% \mathrm{Cl}) \\
\text { QoL } \\
4 \text { weeks }\end{array}$ & NR & 0.24 & $14(-15,42)$ & NR & $-12.0(-42,18)$ & NR \\
\hline $\begin{array}{l}\text { Burns } 2011^{38}(21335973) \\
\text { Melissa oil vs sunflower oil } \\
\text { Medium } \\
\text { Explanatory }\end{array}$ & $\begin{array}{l}\text { Mean Difference }(95 \% \mathrm{Cl}) \\
\text { QoL } \\
12 \text { weeks }\end{array}$ & NR & 0.033 & $17.0(-13,47)$ & NR & $-2.0(-34,30)$ & NR \\
\hline
\end{tabular}




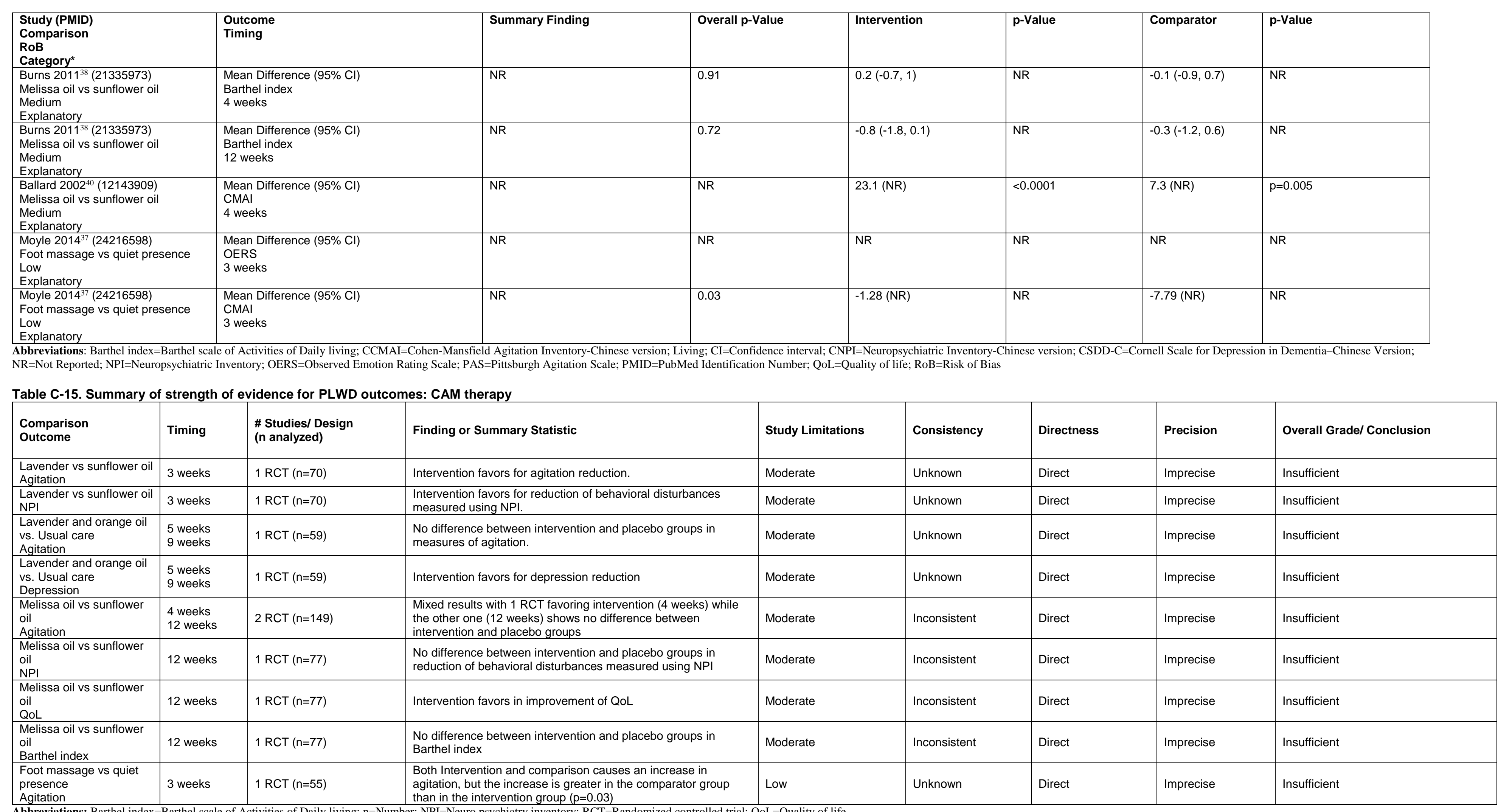




\section{Evidence Map: CAM Therapy}

\begin{tabular}{|c|c|c|c|c|c|c|c|c|c|c|c|c|c|}
\hline $\begin{array}{l}\text { Study (PMID) } \\
\text { Country } \\
\text { EM Reason }\end{array}$ & Intervention & $\begin{array}{l}\text { Compari- } \\
\text { son }\end{array}$ & $\begin{array}{l}\text { Setting } \\
\text { and Design } \\
\\
\text { Setting } \\
\text { Design } \\
\text { Cluster N } \\
\text { Participants } \\
\text { Randomized N }\end{array}$ & \begin{tabular}{|l|} 
PLWD \\
Dementia \\
Characteristics
\end{tabular} & $\begin{array}{l}\text { PLWD } \\
\text { Non-Disease Char } \\
\text { PLWD N } \\
\text { PLWD Age (mean) } \\
\text { PLWD Sex (\% } \\
\text { female) } \\
\text { PLWD Race (\% } \\
\text { majority) } \\
\text { PLWD Education } \\
\text { (mean years) }\end{array}$ & $\begin{array}{l}\text { PLWD } \\
\text { Non-Disease } \\
\text { Char Reporting } \\
\text { Status (RS) } \\
\text { PLWD SES } \\
\text { PLWD Prior } \\
\text { Disability } \\
\text { PLWD } \\
\text { Household } \\
\text { Characteristics } \\
\text { PLWD Health } \\
\text { Insurance } \\
\text { PLWD Detailed } \\
\text { Race Information }\end{array}$ & $\begin{array}{l}\text { Informal Caregiver } \\
\text { (IC) } \\
\text { Characteristics } \\
\text { IC N } \\
\text { IC Age (mean) } \\
\text { IC Sex (\% female) } \\
\text { IC Race (\% } \\
\text { majority) } \\
\text { IC Education } \\
\text { (mean years) } \\
\text { IC Relation to } \\
\text { PLWD (\% majority) }\end{array}$ & $\begin{array}{l}\text { Informal Caregiver } \\
\text { (IC) Char. RS } \\
\text { IC Duration } \\
\text { IC Living With } \\
\text { PLWD } \\
\text { IC Payment } \\
\text { IC Health Status } \\
\text { IC Dementia } \\
\text { Family History } \\
\text { IC Employment } \\
\text { Status } \\
\text { IC Training }\end{array}$ & 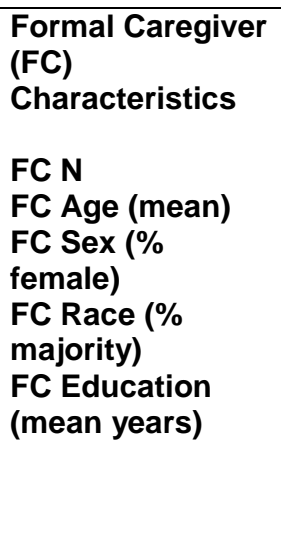 & $\begin{array}{l}\text { Formal } \\
\text { Caregiver (FC) } \\
\text { Char. RS } \\
\text { FC Health } \\
\text { Status } \\
\text { FC Training } \\
\text { FC Education } \\
\text { FC Position } \\
\text { FC Length of } \\
\text { Service }\end{array}$ & $\begin{array}{l}\text { Outcome } \\
\text { Timing(s) }\end{array}$ & $\begin{array}{l}\text { PLWD } \\
\text { Outcomes }\end{array}$ & $\begin{array}{l}\text { Caregiver } \\
\text { Outcomes } \\
\text { IC: } \\
\text { FC: }\end{array}$ \\
\hline $\begin{array}{l}\text { Kouzuki 201941 } \\
\text { (31578055) } \\
\text { Japan } \\
\text { Small sample }\end{array}$ & $\begin{array}{l}.1 \% \text { aroma } \\
\text { bath salts } \\
(\mathrm{n}=15) \\
0.5 \% \text { aroma } \\
\text { bath salts } \\
\text { ( } \mathrm{n}=14 \text { ) } \\
1 \% \text { aroma bath } \\
\text { salts }(\mathrm{n}=14) \\
\text { where aroma oil } \\
\text { added to the } \\
\text { bath salt was a } \\
2: 1 \text { blend of true } \\
\text { lavender and } \\
\text { sweet orange } \\
\text { and baths were } \\
\text { taken once daily } \\
\text { (>10 minutes) } \\
\text { for } 24 \text { weeks }\end{array}$ & NA & $\begin{array}{l}\text { Outpatient clinic } \\
\text { of Shinsei } \\
\text { Hospital } \\
\text { (Kurayoshi, } \\
\text { Japan) } \\
\text { 3-arm RCT } \\
\text { 43 PLWD }\end{array}$ & \begin{tabular}{|l|} 
AD diagnosis \\
according DSM-V \\
or MCI that met \\
Petersen's \\
diagnostic criteria
\end{tabular} & $\begin{array}{l}\mathrm{N}=43 \\
\text { Mean age: } 79 \text { years } \\
\text { Female: } 51 \% \\
\text { \% majority race: no } \\
\text { Education: no }\end{array}$ & None & NA & None & NA & NA & $\begin{array}{l}4 \text { weeks } \\
16 \text { weeks } \\
28 \text { weeks } \\
32 \text { weeks }\end{array}$ & $\begin{array}{l}\text { TDAS } \\
\text { OSIT-J } \\
\text { PSQI-J }\end{array}$ & NA \\
\hline $\begin{array}{l}\text { Takahashi } \\
2019^{42} \\
(32037737) \\
\text { Japan } \\
\text { Small samples }\end{array}$ & $\begin{array}{l}\text { Aromatherapy: } \\
\text { Low pressure, } \\
\text { eo\% ethanol } \\
\text { extract of Akita } \\
\text { cedar leaves } \\
\text { diffusdr in } \\
\text { rattan sticks } \\
\text { and as spray } \\
\text { for } 4 \text { weeks } \\
\text { (The reom } \\
\text { fragrance type } \\
\text { is placed in the } \\
\text { resident space } \\
\text { (living room } \\
\text { and bedroom), } \\
\text { and the spray } \\
\text { type is used to } \\
\text { mist the } \\
\text { patients' } \\
\text { clothing and } \\
\text { bedding.) }\end{array}$ & $\begin{array}{l}\text { Control } \\
\text { group: } \\
\text { Ethanol } \\
\text { without } \\
\text { cedar } \\
\text { leaves } \\
\text { extract }\end{array}$ & $\begin{array}{l}\text { PLWDs } \\
\text { RCT } \\
36 \text { PLWD }\end{array}$ & Diagnosis of AD & $\begin{array}{l}\mathrm{N}=36 \\
\text { Age: yes } \\
\text { Sex: yes } \\
\text { Race: no } \\
\text { Education: yes }\end{array}$ & None & NA & None & NA & NA & 8 weeks & $\begin{array}{l}\text { FAST } \\
\text { NPI } \\
\text { ADAS-cog } \\
\text { J-ZBI }\end{array}$ & NA \\
\hline
\end{tabular}




\begin{tabular}{|c|c|c|c|c|c|c|c|c|c|c|c|c|c|}
\hline $\begin{array}{l}\text { Study (PMID) } \\
\text { Country } \\
\text { EM Reason }\end{array}$ & Intervention & $\begin{array}{l}\text { Compari- } \\
\text { son }\end{array}$ & $\begin{array}{l}\text { Setting } \\
\text { and Design } \\
\\
\text { Setting } \\
\text { Design } \\
\text { Cluster N } \\
\text { Participants } \\
\text { Randomized N }\end{array}$ & \begin{tabular}{|l|} 
PLWD \\
Dementia \\
Characteristics
\end{tabular} & $\begin{array}{l}\text { PLWD } \\
\text { Non-Disease Char } \\
\text { PLWD N } \\
\text { PLWD Age (mean) } \\
\text { PLWD Sex (\% } \\
\text { female) } \\
\text { PLWD Race (\% } \\
\text { majority) } \\
\text { PLWD Education } \\
\text { (mean years) }\end{array}$ & $\begin{array}{l}\text { PLWD } \\
\text { Non-Disease } \\
\text { Char Reporting } \\
\text { Status (RS) } \\
\text { PLWD SES } \\
\text { PLWD Prior } \\
\text { Disability } \\
\text { PLWD } \\
\text { Household } \\
\text { Characteristics } \\
\text { PLWD Health } \\
\text { Insurance } \\
\text { PLWD Detailed } \\
\text { Race Information }\end{array}$ & $\begin{array}{l}\text { Informal Caregiver } \\
\text { (IC) } \\
\text { Characteristics } \\
\text { IC N } \\
\text { IC Age (mean) } \\
\text { IC Sex (\% female) } \\
\text { IC Race (\% } \\
\text { majority) } \\
\text { IC Education } \\
\text { (mean years) } \\
\text { IC Relation to } \\
\text { PLWD (\% majority) }\end{array}$ & $\begin{array}{l}\text { Informal Caregiver } \\
\text { (IC) Char. RS } \\
\text { IC Duration } \\
\text { IC Living With } \\
\text { PLWD } \\
\text { IC Payment } \\
\text { IC Health Status } \\
\text { IC Dementia } \\
\text { Family History } \\
\text { IC Employment } \\
\text { Status } \\
\text { IC Training }\end{array}$ & $\begin{array}{l}\text { Formal Caregiver } \\
\text { (FC) } \\
\text { Characteristics } \\
\text { FC N } \\
\text { FC Age (mean) } \\
\text { FC Sex (\% } \\
\text { female) } \\
\text { FC Race (\% } \\
\text { majority) } \\
\text { FC Education } \\
\text { (mean years) }\end{array}$ & $\begin{array}{l}\text { Formal } \\
\text { Caregiver (FC) } \\
\text { Char. RS } \\
\text { FC Health } \\
\text { Status } \\
\text { FC Training } \\
\text { FC Education } \\
\text { FC Position } \\
\text { FC Length of } \\
\text { Service }\end{array}$ & $\begin{array}{l}\text { Outcome } \\
\text { Timing(s) }\end{array}$ & $\begin{array}{l}\text { PLWD } \\
\text { Outcomes }\end{array}$ & $\begin{array}{l}\text { Caregiver } \\
\text { Outcomes } \\
\text { IC: } \\
\text { FC: }\end{array}$ \\
\hline $\begin{array}{l}\text { Watson 2019 } \\
\text { (30670268) } \\
\text { Australia } \\
\text { Small sample }\end{array}$ & $\begin{array}{l}\text { 1.Aromatherap } \\
\text { y with } \\
\text { Lavender oil } \\
\text { 2.Aromatherap } \\
\text { y with Lemon- } \\
\text { balm oil } \\
2 \text { drops of oil } \\
\text { was applied to } \\
\text { a cotton patch } \\
\text { and then } \\
\text { placed on the } \\
\text { participant's } \\
\text { collar area. } \\
\text { The patch was } \\
\text { removed after } \\
2 \text { hours for } 2 \\
\text { weeks. } \\
\text { Washout } \\
\text { period of } 2 \\
\text { weeks were } \\
\text { maintained }\end{array}$ & $\begin{array}{l}\text { 3. Same } \\
\text { procedure } \\
\text { was } \\
\text { followed } \\
\text { with } \\
\text { sunflower } \\
\text { oil }\end{array}$ & $\begin{array}{l}\text { Residents of } \\
\text { residential } \\
\text { aged care } \\
\text { facility } \\
\text { RCT } \\
39 \text { PLWD }\end{array}$ & $\begin{array}{l}\text { Diagnosis of } \\
\text { dementia and } \\
\text { MMSE score of } \\
\leq 10 ; \text { agitated } \\
\text { behaviors } \\
\text { recorded on at } \\
\text { least one of ACFI } \\
\text { domains; }\end{array}$ & $\begin{array}{l}\mathrm{N}=39 \\
\text { Mean age: no } \\
\text { \% Female: no } \\
\text { \% majority race: } \\
\text { no } \\
\text { Education: no }\end{array}$ & None & NA & None & NA & NA & $\begin{array}{l}0 \text { week } \\
2 \text { weeks } \\
4 \text { weeks } \\
6 \text { weeks } \\
8 \text { weeks } \\
10 \text { weeks }\end{array}$ & $\begin{array}{l}\text { CMAI } \\
\text { NPI }\end{array}$ & NA \\
\hline $\begin{array}{l}\text { Zalomonson } \\
2019^{43} \\
(30328781) \\
\text { Israel } \\
\text { Pilot }\end{array}$ & $\begin{array}{l}2 \text { drops of } \\
\text { lavender oil } \\
\text { were applied by } \\
\text { the study nurse } \\
\text { for no more } \\
\text { than } 1-2 \\
\text { minutes, } 3 \text { times } \\
\text { a day for } 16 \\
\text { weeks }\end{array}$ & $\begin{array}{l}\text { Same } \\
\text { procedure } \\
\text { using } \\
\text { sunflower oil }\end{array}$ & $\begin{array}{l}\text { Psychogeriatric } \\
\text { long-term care } \\
\text { departments at } \\
\text { the Center for } \\
\text { Mental Health of } \\
\text { the Iraee } \\
\text { Ministry of } \\
\text { Health, Beer- } \\
\text { Sheva } \\
\text { Crossover } \\
\text { Placebo- } \\
\text { Controlled } \\
\text { Study } \\
\text { 42 PLWD }\end{array}$ & $\begin{array}{l}\text { Diagnosis of } \\
\text { dementia of any } \\
\text { type }\end{array}$ & $\begin{array}{l}\mathrm{N}=42 \\
\text { Age: yes } \\
\text { Sex: yes } \\
\text { Race: no } \\
\text { Education: no }\end{array}$ & None & NA & NA & NA & $\mathrm{NA}$ & $\begin{array}{l}4 \text { weeks } \\
8 \text { weeks } \\
12 \text { weeks } \\
16 \text { weeks }\end{array}$ & $\mathrm{NPI}$ & $\mathrm{NA}$ \\
\hline $\begin{array}{l}\text { Fung 201844 } \\
\text { (28986942) } \\
\text { Hong-Kong } \\
\text { Small sample }\end{array}$ & $\begin{array}{l}\text { Aroma- } \\
\text { massage with } \\
\text { acupressure: } \\
\text { Trained carers } \\
\text { or therapists } \\
\text { provided no } \\
\text { more than } 20 \\
\text { minutes of } \\
\text { aromatherapy, } \\
\text { combining }\end{array}$ & $\begin{array}{l}\text { Cognitive } \\
\text { training: } \\
\text { Trained care } \\
\text { staff or } \\
\text { therapists } \\
\text { provided } 20 \\
\text { minutes of } \\
\text { cognitive } \\
\text { training } \\
(\mathrm{n}=20)\end{array}$ & $\begin{array}{l}\text { Long-term non- } \\
\text { government } \\
\text { geriatric care } \\
\text { facilities } \\
3 \text { arm RCT } \\
60 \text { PLWD }\end{array}$ & \begin{tabular}{|l} 
BPSD PLWD \\
scored below 18 \\
(illiterate), $19(1-2$ \\
years of education) \\
and $20(>2$ ( 2years \\
education) in \\
CMMSE
\end{tabular} & $\begin{array}{l}\mathrm{N}=20 \\
\text { Age: yes } \\
\text { Sex: yes } \\
\text { Race: yes } \\
\text { Education: yes }\end{array}$ & None & NA & NA & NA & NA & $\begin{array}{l}\text { Unclear } \\
\text { 3-months } \\
\text { Follow-up }\end{array}$ & $\begin{array}{l}\text { CMMSE } \\
\text { CCMAI } \\
\text { NPI } \\
\text { BI20 }\end{array}$ & NA \\
\hline
\end{tabular}




\begin{tabular}{|c|c|c|c|c|c|c|c|c|c|c|c|c|c|}
\hline $\begin{array}{l}\text { Study (PMID) } \\
\text { Country } \\
\text { EM Reason }\end{array}$ & Intervention & $\begin{array}{l}\text { Compari- } \\
\text { son }\end{array}$ & 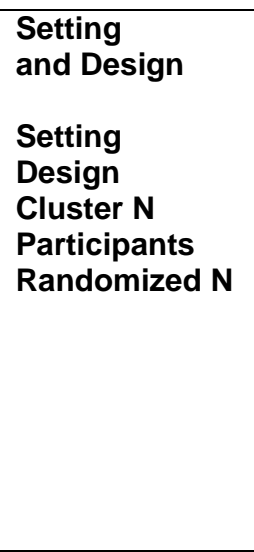 & \begin{tabular}{|l} 
PLWD \\
Dementia \\
Characteristics
\end{tabular} & $\begin{array}{l}\text { PLWD } \\
\text { Non-Disease Char } \\
\text { PLWD N } \\
\text { PLWD Age (mean) } \\
\text { PLWD Sex (\% } \\
\text { female) } \\
\text { PLWD Race (\% } \\
\text { majority) } \\
\text { PLWD Education } \\
\text { (mean years) }\end{array}$ & $\begin{array}{l}\text { PLWD } \\
\text { Non-Disease } \\
\text { Char Reporting } \\
\text { Status (RS) } \\
\\
\text { PLWD SES } \\
\text { PLWD Prior } \\
\text { Disability } \\
\text { PLWD } \\
\text { Household } \\
\text { Characteristics } \\
\text { PLWD Health } \\
\text { Insurance } \\
\text { PLWD Detailed } \\
\text { Race Information }\end{array}$ & $\begin{array}{l}\text { Informal Caregiver } \\
\text { (IC) } \\
\text { Characteristics } \\
\text { IC N } \\
\text { IC Age (mean) } \\
\text { IC Sex (\% female) } \\
\text { IC Race (\% } \\
\text { majority) } \\
\text { IC Education } \\
\text { (mean years) } \\
\text { IC Relation to } \\
\text { PLWD (\% majority) }\end{array}$ & $\begin{array}{l}\text { Informal Caregiver } \\
\text { (IC) Char. RS } \\
\text { IC Duration } \\
\text { IC Living With } \\
\text { PLWD } \\
\text { IC Payment } \\
\text { IC Health Status } \\
\text { IC Dementia } \\
\text { Family History } \\
\text { IC Employment } \\
\text { Status } \\
\text { IC Training }\end{array}$ & 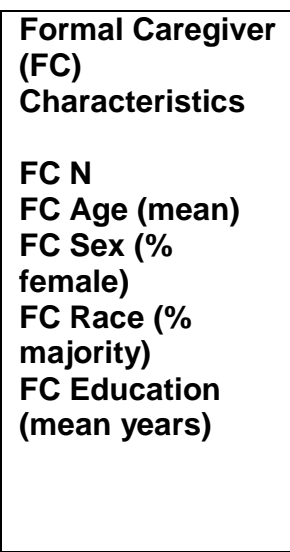 & $\begin{array}{l}\text { Formal } \\
\text { Caregiver (FC) } \\
\text { Char. RS } \\
\text { FC Health } \\
\text { Status } \\
\text { FC Training } \\
\text { FC Education } \\
\text { FC Position } \\
\text { FC Length of } \\
\text { Service }\end{array}$ & $\begin{array}{l}\text { Outcome } \\
\text { Timing(s) }\end{array}$ & $\begin{array}{l}\text { PLWD } \\
\text { Outcomes }\end{array}$ & $\begin{array}{l}\text { Caregiver } \\
\text { Outcomes } \\
\text { IC: } \\
\text { FC: }\end{array}$ \\
\hline $\begin{array}{l}\text { Kwan 201734 } \\
\text { (25346026) } \\
\text { Hong Kong } \\
\text { High RoB } \\
\text { Kwan 201445 } \\
\text { (25346026) } \\
\text { Hong Kong } \\
\text { Study Protocol }\end{array}$ & $\begin{array}{l}9 \text { minutes } \\
\text { acupressure } \\
\text { sessions }\end{array}$ & $\begin{array}{l}\text { Sham } \\
\text { acupressure; } \\
\text { Usual care }\end{array}$ & $\begin{array}{l}\text { Residential care } \\
\text { homes } \\
3 \text { arm RCT } \\
119 \text { PLWD }\end{array}$ & \begin{tabular}{|l|} 
PLWD showing \\
dementia in \\
medical records \\
and displayed \\
agitated behavior
\end{tabular} & $\begin{array}{l}\text { N=119 } \\
\text { Age: yes } \\
\text { Sex: yes } \\
\text { Race no } \\
\text { Education: no }\end{array}$ & None & NA & NA & NA & $\mathrm{NA}$ & 2 weeks & $\begin{array}{l}\text { CMAl } \\
\text { Salivary } \\
\text { cortisol }\end{array}$ & NA \\
\hline $\begin{array}{l}\text { Jia 201746 } \\
\text { (29284465) } \\
\text { China } \\
\text { Pilot }\end{array}$ & $\begin{array}{l}\text { Acupuncture } \\
\text { was performed } \\
\text { using needles of } \\
0.25 \mathrm{~mm} \text { and a } \\
\text { length of } 40 \mathrm{~mm} \\
\text { at various acu- } \\
\text { points for } 3 \\
\text { times a week for } \\
12 \text { weeks }\end{array}$ & $\begin{array}{l}5 \mathrm{mg} / \mathrm{dday} \text { of } \\
\text { donepezil } \\
\text { hydrochlorid } \\
\text { e for the first } \\
4 \text { weeks and } \\
10 \mathrm{mg} / \mathrm{day} \\
\text { thereafter }\end{array}$ & $\begin{array}{l}\text { Community } \\
\text { residents (older } \\
\text { PLWD with } \\
\text { dementia) who } \\
\text { participated in } \\
\text { survey from } \\
\text { Tianjin City, } \\
\text { China } \\
\text { RCT } \\
87 \text { PLWD }\end{array}$ & \begin{tabular}{|l|} 
Diagnosis based \\
on DSM-IV and \\
NIICDS-ADRDA \\
with MMSE score \\
between 10 and 23 \\
and HAMD \\
score 7 , a HIS \\
scores 4 , and have \\
a reliable caregiver \\
to accompany the \\
participant to all \\
studv visits
\end{tabular} & $\begin{array}{l}\mathrm{N}=43 \\
\text { Age: yes } \\
\text { Sex: yes } \\
\text { Race: no } \\
\text { Education: yes }\end{array}$ & None & NA & NA & NA & NA & $\begin{array}{l}16 \text { weeks } \\
28 \text { weeks }\end{array}$ & $\begin{array}{l}\text { ADAS-cog } \\
\text { CIBIC-Plus } \\
\text { ADCS-ADL23 } \\
\text { NPI }\end{array}$ & NA \\
\hline $\begin{array}{l}\text { Moorman Li } \\
2016^{47} \\
\text { (28265482) } \\
\text { US } \\
\text { Pilot }\end{array}$ & $\begin{array}{l}\text { Lavender } \\
\text { aromatherapy } \\
\text { twice a day for } \\
20 \text { min during a } \\
\text { two-month } \\
\text { period during } \\
\text { active clinic } \\
\text { days. }\end{array}$ & None & $\begin{array}{l}\text { Adult Day Care } \\
\text { Center } \\
\text { quasi- } \\
\text { experimental } \\
23 \text { PLWD }\end{array}$ & $\begin{array}{l}\text { Clinical diagnosis } \\
\text { of dementia }\end{array}$ & $\begin{array}{l}\mathrm{N}=23 \\
\text { Age: yes } \\
\text { Sex: yes } \\
\text { Race: yes } \\
\text { Education: no }\end{array}$ & None & NA & NA & NA & NA & $\begin{array}{l}\text { Daily for } 2 \\
\text { months pre- } \\
\text { observation } \\
\text { phase and } 2 \\
\text { months } \\
\text { intervention } \\
\text { period }\end{array}$ & $\begin{array}{l}\text { Behavior/Inter } \\
\text { vention } \\
\text { Monthly Flow } \\
\text { Record }\end{array}$ & NA \\
\hline $\begin{array}{l}\text { Yang 201548 } \\
\text { (26556080) } \\
\text { Taiwan } \\
\text { High RoB }\end{array}$ & $\begin{array}{l}\text { Aroma- } \\
\text { acupressure: } \\
\text { each acupoint } \\
\text { was pressed for }\end{array}$ & $\begin{array}{l}\text { Control: } \\
\text { Received } \\
\text { daily routine } \\
\text { as usual }\end{array}$ & $\begin{array}{l}\text { Retirement } \\
\text { homes for } \\
\text { veterans and } \\
\text { long-term care }\end{array}$ & \begin{tabular}{l|} 
Diagnosis \\
according to DSM- \\
IV criteria
\end{tabular} & $\begin{array}{l}\mathrm{N}=82 \\
\text { Age: yes } \\
\text { Sex: yes }\end{array}$ & None & $\mathrm{NA}$ & NA & NA & NA & weekly & $\begin{array}{l}\text { CCMAI } \\
\text { HRV index }\end{array}$ & $\mathrm{NA}$ \\
\hline
\end{tabular}




\begin{tabular}{|c|c|c|c|c|c|c|c|c|c|c|c|c|c|}
\hline $\begin{array}{l}\text { Study (PMID) } \\
\text { Country } \\
\text { EM Reason }\end{array}$ & Intervention & $\begin{array}{l}\text { Compari- } \\
\text { son }\end{array}$ & 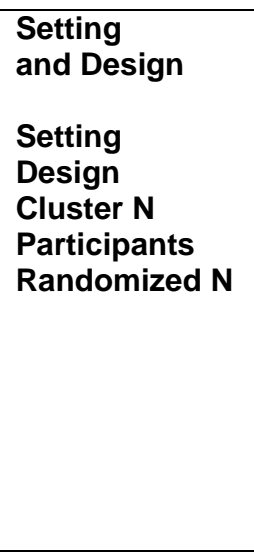 & \begin{tabular}{|l|} 
PLWD \\
Dementia \\
Characteristics
\end{tabular} & $\begin{array}{l}\text { PLWD } \\
\text { Non-Disease Char } \\
\text { PLWD N } \\
\text { PLWD Age (mean) } \\
\text { PLWD Sex (\% } \\
\text { female) } \\
\text { PLWD Race (\% } \\
\text { majority) } \\
\text { PLWD Education } \\
\text { (mean years) }\end{array}$ & \begin{tabular}{|l|} 
PLWD \\
Non-Disease \\
Char Reporting \\
Status (RS) \\
\\
PLWD SES \\
PLWD Prior \\
Disability \\
PLWD \\
Household \\
Characteristics \\
PLWD Health \\
Insurance \\
PLWD Detailed \\
Race Information \\
\end{tabular} & $\begin{array}{l}\text { Informal Caregiver } \\
\text { (IC) } \\
\text { Characteristics } \\
\text { IC N } \\
\text { IC Age (mean) } \\
\text { IC Sex (\% female) } \\
\text { IC Race (\% } \\
\text { majority) } \\
\text { IC Education } \\
\text { (mean years) } \\
\text { IC Relation to } \\
\text { PLWD (\% majority) }\end{array}$ & $\begin{array}{l}\text { Informal Caregiver } \\
\text { (IC) Char. RS } \\
\text { IC Duration } \\
\text { IC Living With } \\
\text { PLWD } \\
\text { IC Payment } \\
\text { IC Health Status } \\
\text { IC Dementia } \\
\text { Family History } \\
\text { IC Employment } \\
\text { Status } \\
\text { IC Training }\end{array}$ & $\begin{array}{l}\text { Formal Caregiver } \\
\text { (FC) } \\
\text { Characteristics } \\
\text { FC N } \\
\text { FC Age (mean) } \\
\text { FC Sex (\% } \\
\text { female) } \\
\text { FC Race (\% } \\
\text { majority) } \\
\text { FC Education } \\
\text { (mean years) }\end{array}$ & $\begin{array}{l}\text { Formal } \\
\text { Caregiver (FC) } \\
\text { Char. RS } \\
\text { FC Health } \\
\text { Status } \\
\text { FC Training } \\
\text { FC Education } \\
\text { FC Position } \\
\text { FC Length of } \\
\text { Service }\end{array}$ & $\begin{array}{l}\text { Outcome } \\
\text { Timing(s) }\end{array}$ & $\begin{array}{l}\text { PLWD } \\
\text { Outcomes }\end{array}$ & $\begin{array}{l}\text { Caregiver } \\
\text { Outcomes } \\
\text { IC: } \\
\text { FC: }\end{array}$ \\
\hline & $\begin{array}{l}2 \text { minutes with } \\
2.5 \% \text { lavender } \\
\text { oil and a warm- } \\
\text { up exercise was } \\
\text { completed for } 5 \\
\text { minutes, once } \\
\text { per day for } 5 \\
\text { days for } 4 \\
\text { weeks } \\
\text { Aromatherapy: } \\
2.5 \% \text { lavender } \\
\text { oil was applied } \\
\text { at five acupoints } \\
\text { with the same } \\
\text { operation time } \\
\text { as the aroma- } \\
\text { acupressure } \\
\text { group }(n=106)\end{array}$ & $\begin{array}{l}\text { without } \\
\text { intervention } \\
(n=88)\end{array}$ & $\begin{array}{l}\text { facilities } \\
3 \text { arm RCT } \\
276 \text { PLWD }\end{array}$ & \begin{tabular}{|l} 
CMAI >35 (extreme \\
agitation)
\end{tabular} & $\begin{array}{l}\text { Race: no } \\
\text { Education: no }\end{array}$ & & & & & & & & \\
\hline $\begin{array}{l}\text { Chieh-Yu } \\
2013^{49} \\
(23837414) \\
\text { Australia } \\
\text { Small sample }\end{array}$ & $\begin{array}{l}\text { Aromatherapy } \\
\text { and hand } \\
\text { massage } \\
\text { (n=22): Three } \\
\text { sprays of } \\
\text { lavender mist } \\
\text { (3\% lavender } \\
\text { mist) applied on } \\
\text { chest twice a } \\
\text { day, for } 6 \text { weeks } \\
\text { and } 5 \text { minutes } \\
\text { of hand } \\
\text { massage twice } \\
\text { a day for } 10 \\
\text { days: each } \\
\text { hand was } \\
\text { massaged for } \\
2.5 \text { minutes. } \\
\text { Aromatherapy } \\
\text { only (n=23): } \\
\text { Three sprays of } \\
\text { lavender mist } \\
\text { (3\% lavender } \\
\text { mist) applied on } \\
\text { chest twice a } \\
\text { day, for } 6 \text { weeks }\end{array}$ & $\begin{array}{l}\text { Placebo } \\
(\mathrm{n}=22) \\
\text { water spray } \\
\text { similar to } \\
\text { aromatherap } \\
\mathrm{y}\end{array}$ & $\begin{array}{l}\text { Long term care } \\
\text { facilities in } \\
\text { Brisbane } \\
3 \text { arm RCT } \\
67 \text { PLWD }\end{array}$ & $\begin{array}{l}\text { MMSE score } \leq 24 \\
\text { and AD diagnosis } \\
\text { according DSM-IV } \\
\text { with a documented } \\
\text { history of a } \\
\text { minimum of two } \\
\text { weeks of agitation } \\
\text { or aggression } \\
\text { within the past } \\
\text { three months, } \\
\text { requiring physical } \\
\text { and/or chemical } \\
\text { restraint }\end{array}$ & $\begin{array}{l}\mathrm{N}=67 \\
\text { Mean age: } 84 \text { years } \\
\text { Female: } 59 \% \\
\text { \% majority race: no } \\
\text { Education: no }\end{array}$ & \begin{tabular}{|l|} 
None \\
\end{tabular} & NA & None & NA & NA & 6 weeks & \begin{tabular}{|l|} 
MMSE \\
CMAI-SF
\end{tabular} & $\mathrm{NA}$ \\
\hline
\end{tabular}




\begin{tabular}{|c|c|c|c|c|c|c|c|c|c|c|c|c|c|}
\hline $\begin{array}{l}\text { Study (PMID) } \\
\text { Country } \\
\text { EM Reason }\end{array}$ & Intervention & $\begin{array}{l}\text { Compari- } \\
\text { son }\end{array}$ & $\begin{array}{l}\text { Setting } \\
\text { and Design } \\
\\
\text { Setting } \\
\text { Design } \\
\text { Cluster N } \\
\text { Participants } \\
\text { Randomized N }\end{array}$ & \begin{tabular}{|l|} 
PLWD \\
Dementia \\
Characteristics
\end{tabular} & $\begin{array}{l}\text { PLWD } \\
\text { Non-Disease Char } \\
\text { PLWD N } \\
\text { PLWD Age (mean) } \\
\text { PLWD Sex (\% } \\
\text { female) } \\
\text { PLWD Race (\% } \\
\text { majority) } \\
\text { PLWD Education } \\
\text { (mean years) }\end{array}$ & $\begin{array}{l}\text { PLWD } \\
\text { Non-Disease } \\
\text { Char Reporting } \\
\text { Status (RS) } \\
\text { PLWD SES } \\
\text { PLWD Prior } \\
\text { Disability } \\
\text { PLWD } \\
\text { Household } \\
\text { Characteristics } \\
\text { PLWD Health } \\
\text { Insurance } \\
\text { PLWD Detailed } \\
\text { Race Information }\end{array}$ & $\begin{array}{l}\text { Informal Caregiver } \\
\text { (IC) } \\
\text { Characteristics } \\
\text { IC N } \\
\text { IC Age (mean) } \\
\text { IC Sex (\% female) } \\
\text { IC Race (\% } \\
\text { majority) } \\
\text { IC Education } \\
\text { (mean years) } \\
\text { IC Relation to } \\
\text { PLWD (\% majority) }\end{array}$ & $\begin{array}{l}\text { Informal Caregiver } \\
\text { (IC) Char. RS } \\
\text { IC Duration } \\
\text { IC Living With } \\
\text { PLWD } \\
\text { IC Payment } \\
\text { IC Health Status } \\
\text { IC Dementia } \\
\text { Family History } \\
\text { IC Employment } \\
\text { Status } \\
\text { IC Training }\end{array}$ & $\begin{array}{l}\text { Formal Caregiver } \\
\text { (FC) } \\
\text { Characteristics } \\
\text { FC N } \\
\text { FC Age (mean) } \\
\text { FC Sex (\% } \\
\text { female) } \\
\text { FC Race (\% } \\
\text { majority) } \\
\text { FC Education } \\
\text { (mean years) }\end{array}$ & $\begin{array}{l}\text { Formal } \\
\text { Caregiver (FC) } \\
\text { Char. RS } \\
\text { FC Health } \\
\text { Status } \\
\text { FC Training } \\
\text { FC Education } \\
\text { FC Position } \\
\text { FC Length of } \\
\text { Service }\end{array}$ & \begin{tabular}{|l|} 
Outcome \\
Timing(s)
\end{tabular} & $\begin{array}{l}\text { PLWD } \\
\text { Outcomes }\end{array}$ & $\begin{array}{l}\text { Caregiver } \\
\text { Outcomes } \\
\text { IC: } \\
\text { FC: }\end{array}$ \\
\hline $\begin{array}{l}\text { Australia } \\
\text { Small sample }\end{array}$ & $\begin{array}{l}\text { (3\%) twice daily } \\
7 \text { days a week } \\
\text { for } 6 \text { weeks } \\
3 \text { sprays of } \\
\text { lavender mist } \\
\text { (3\%) Plus } 5 \\
\text { minutes of hand } \\
\text { massage ( } n=22)\end{array}$ & $\begin{array}{l}\text { the same } \\
\text { way }(n=22) .\end{array}$ & $\begin{array}{l}3 \text { arm RCT } \\
67 \text { PLWD }\end{array}$ & $\begin{array}{l}\text { medical practitioner } \\
\text { and a university } \\
\text { Alzheimer's clinic) } \\
\text { Moderate to } \\
\text { severe-MMSE }\end{array}$ & $\begin{array}{l}\text { Sex: yes } \\
\text { Race: yes } \\
\text { Education: no }\end{array}$ & & & & & & & $\begin{array}{l}\text { CMAI-SF } \\
\text { Cognition: } \\
\text { (MMSE) }\end{array}$ & \\
\hline $\begin{array}{l}\text { Lu 201351 } \\
\text { (23972540) } \\
\text { USA } \\
\text { Pilot }\end{array}$ & $\begin{array}{l}\text { Along with usual } \\
\text { medical and } \\
\text { care regimen, } \\
\text { HT and BTC } \\
\text { protocols were } \\
\text { given for the } 6 \\
\text { months } \\
\text { treatment period } \\
\text { on weekly basis }\end{array}$ & \begin{tabular}{|l|} 
PLWD got \\
usual \\
medical and \\
care \\
regimens
\end{tabular} & $\begin{array}{l}\text { Recruited from } \\
\text { long-term } \\
\text { nursing care } \\
\text { facilities and } \\
\text { from the } \\
\text { community } \\
\text { RCT } \\
22 \text { PLWD }\end{array}$ & $\begin{array}{l}\text { Early AD } \\
\text { (information } \\
\text { regarding their } \\
\text { doctor's diagnosis } \\
\text { was acquired from } \\
\text { the subjects, their } \\
\text { caregivers, or } \\
\text { nursing staff) }\end{array}$ & $\begin{array}{l}\mathrm{N}=12 \\
\text { Age: yes } \\
\text { Sex: yes } \\
\text { Race: yes } \\
\text { Education: no }\end{array}$ & None & NA & None & NA & NA & $\begin{array}{l}3 \text { months } \\
6 \text { months }\end{array}$ & $\begin{array}{l}\text { MoCA } \\
\text { POMS-BF } \\
\text { PHQ-9 }\end{array}$ & NA \\
\hline $\begin{array}{l}\text { Rodriguez- } \\
\text { Mansilla 201352 } \\
\text { (24187866) } \\
\text { Spain } \\
\text { Pilot } \\
\text { Rodriguez- } \\
\text { Mansilla 201553 } \\
\text { (25322869) } \\
\text { Spain } \\
\text { Pilot }\end{array}$ & $\begin{array}{l}20 \text { minutes of } \\
\text { back and lower } \\
\text { limb massage } \\
\text { with } \\
\text { moisturizing } \\
\text { cream; } \\
\text { Ear- } \\
\text { acupuncture at } \\
\text { various points } \\
(\mathrm{n}=40)\end{array}$ & $\begin{array}{l}\text { Control (did } \\
\text { not receive } \\
\text { any } \\
\text { experimental } \\
\text { treatment } \\
\mathrm{n}=40 \text { ) }\end{array}$ & $\begin{array}{l}\text { Residents } \\
\text { institutionalized } \\
\text { in 'CARE' } \\
\text { elderly } \\
\text { residential } \\
\text { homes in } \\
\text { Extremadura } \\
\text { 3-arm-RCT } \\
\text { 120 PLWWD }\end{array}$ & $\begin{array}{l}\text { PLWD were } \\
\text { diagnosed using } \\
\text { the DSM-VI criteria }\end{array}$ & $\begin{array}{l}\mathrm{N}=40 \\
\text { Age: yes } \\
\text { Sex: yes } \\
\text { Race: no } \\
\text { Education yes }\end{array}$ & None & NA & NA & NA & NA & 12 weeks & \begin{tabular}{|l} 
Behavioral \\
alteration and \\
sleep \\
disturbances: \\
Self-designed \\
structured \\
questionnaire \\
with closed \\
ended \\
questions \\
GDS \\
MMSE \\
DOLOPLUS2 \\
CSDD \\
Campbell \\
Scale
\end{tabular} & $\mathrm{NA}$ \\
\hline $\begin{array}{l}\text { Jimbo 200954 } \\
\text { (20377818) } \\
\text { Japan } \\
\text { Pilot }\end{array}$ & $\begin{array}{l}\text { Aroma } \\
\text { exposure of } \\
0.00 \mathrm{ml} \text { lemon } \\
\text { and } 0.08 \mathrm{ml} \\
\text { rosemary oil in } \\
\text { the morning for } \\
2 \text { hours. } 0.08 \mathrm{ml} \\
\text { Lavender and } \\
0.04 \mathrm{ml} \text { orange } \\
\text { oil in the } \\
\text { evening for } 1.5 \\
\end{array}$ & None & $\begin{array}{l}\text { Setting: NR } \\
\text { Quasi- } \\
\text { experimental } \\
\text { (crossover) } \\
28 \text { PLWD }\end{array}$ & $\begin{array}{l}\text { Diagnosis based } \\
\text { on DSM-IV and } \\
\text { NINCDS-ADRDA } \\
\text { or DSM-IV and } \\
\text { NINCDS-AIREN. }\end{array}$ & $\begin{array}{l}\mathrm{N}=28 \\
\text { Age: } \text { yes } \\
\text { Sex yes } \\
\text { Race: } \text { no } \\
\text { Education: No }\end{array}$ & None & NA & NA & NA & NA & $\begin{array}{l}4 \text { weeks } \\
9 \text { weeks }\end{array}$ & $\begin{array}{l}\text { HDS-R } \\
\text { GBSS-J } \\
\text { FAST } \\
\text { CT Scans } \\
\text { TDAS } \\
\text { Zarit } \\
\text { Blood and } \\
\text { biochemical } \\
\text { examination }\end{array}$ & NA \\
\hline
\end{tabular}




\begin{tabular}{|c|c|c|c|c|c|c|c|c|c|c|c|c|c|}
\hline $\begin{array}{l}\text { Study (PMID) } \\
\text { Country } \\
\text { EM Reason }\end{array}$ & Intervention & $\begin{array}{l}\text { Compari- } \\
\text { son }\end{array}$ & $\begin{array}{l}\text { Setting } \\
\text { and Design } \\
\text { Setting } \\
\text { Design } \\
\text { Cluster N } \\
\text { Participants } \\
\text { Randomized N }\end{array}$ & \begin{tabular}{|l|} 
PLWD \\
Dementia \\
Characteristics
\end{tabular} & $\begin{array}{l}\text { PLWD } \\
\text { Non-Disease Char } \\
\\
\text { PLWD N } \\
\text { PLWD Age (mean) } \\
\text { PLWD Sex (\% } \\
\text { female) } \\
\text { PLWD Race (\% } \\
\text { majority) } \\
\text { PLWD Education } \\
\text { (mean years) }\end{array}$ & \begin{tabular}{|l|} 
PLWD \\
Non-Disease \\
Char Reporting \\
Status (RS) \\
PLWD SES \\
PLWD Prior \\
Disability \\
PLWD \\
Household \\
Characteristics \\
PLWD Health \\
Insurance \\
PLWD Detailed \\
Race Information
\end{tabular} & $\begin{array}{l}\text { Informal Caregiver } \\
\text { (IC) } \\
\text { Characteristics } \\
\text { IC N } \\
\text { IC Age (mean) } \\
\text { IC Sex (\% female) } \\
\text { IC Race (\% } \\
\text { majority) } \\
\text { IC Education } \\
\text { (mean years) } \\
\text { IC Relation to } \\
\text { PLWD (\% majority) }\end{array}$ & $\begin{array}{l}\text { Informal Caregiver } \\
\text { (IC) Char. RS } \\
\text { IC Duration } \\
\text { IC Living With } \\
\text { PLWD } \\
\text { IC Payment } \\
\text { IC Health Status } \\
\text { IC Dementia } \\
\text { Family History } \\
\text { IC Employment } \\
\text { Status } \\
\text { IC Training }\end{array}$ & $\begin{array}{l}\text { Formal Caregiver } \\
\text { (FC) } \\
\text { Characteristics } \\
\text { FC N } \\
\text { FC Age (mean) } \\
\text { FC Sex (\% } \\
\text { female) } \\
\text { FC Race (\% } \\
\text { majority) } \\
\text { FC Education } \\
\text { (mean years) }\end{array}$ & $\begin{array}{l}\text { Formal } \\
\text { Caregiver (FC) } \\
\text { Char. RS } \\
\text { FC Health } \\
\text { Status } \\
\text { FC Training } \\
\text { FC Education } \\
\text { FC Position } \\
\text { FC Length of } \\
\text { Service }\end{array}$ & \begin{tabular}{|l|} 
Outcome \\
Timing(s)
\end{tabular} & \begin{tabular}{|l|} 
PLWD \\
Outcomes
\end{tabular} & $\begin{array}{l}\text { Caregiver } \\
\text { Outcomes } \\
\text { IC: } \\
\text { FC: }\end{array}$ \\
\hline & $\begin{array}{l}\text { hours for } 4 \\
\text { weeks }\end{array}$ & & & & & & & & & & & & \\
\hline $\begin{array}{l}\text { Hawranik } \\
2008^{55} \\
(18272750) \\
\text { Canada } \\
\text { Small sample }\end{array}$ & $\begin{array}{l}\text { TT once per day } \\
\text { for } 5 \text { days }\end{array}$ & $\begin{array}{l}\text { Simulated } \\
\text { TT once per } \\
\text { day for } 5 \\
\text { days }(n=16) \\
\text { Usual care } \\
\text { for } 5 \text { days } \\
(n=18)\end{array}$ & $\begin{array}{l}\text { Residents from } \\
\text { personal care } \\
\text { and special } \\
\text { needs units of } \\
\text { one long-term } \\
\text { care facility } \\
3 \text { arm RCT } \\
51 \text { PLWD }\end{array}$ & $\begin{array}{l}\text { Diagnosis of senile } \\
\text { dementia of the } \\
\text { Alzheimer type, } \\
\text { Score } 23 \text { or less in } \\
\text { MMSE; with a } \\
\text { history or } \\
\text { consistent agitated } \\
\text { behavior during last } \\
30 \text { days }\end{array}$ & $\begin{array}{l}\mathrm{N}=17 \\
\text { Age: yes } \\
\text { Sex: yes } \\
\text { Race: no } \\
\text { Education: no }\end{array}$ & None & NA & NA & NA & NA & $\begin{array}{l}2 \text { hours; } \\
24 \text { hours } \\
1 \text { week } \\
2 \text { weeks }\end{array}$ & $\begin{array}{l}\text { CMAI } \\
\text { MMSE }\end{array}$ & NA \\
\hline $\begin{array}{l}\text { Woods 200556 } \\
\text { (15712768) } \\
\text { Canada } \\
\text { Small sample }\end{array}$ & $\begin{array}{l}\text { TT was } \\
\text { performed with } \\
\text { contact on the } \\
\text { neck and } \\
\text { shoulders } \\
\text { through an } \\
\text { experienced } \\
\text { practitioner for } \\
5-7 \text { minutes } \\
\text { twice daily for } 3 \\
\text { days }\end{array}$ & $\begin{array}{l}\text { Sham TT } \\
\text { ( } \mathrm{n}=19) \\
\text { Routine care } \\
\text { ( } \mathrm{n}=19)\end{array}$ & $\begin{array}{l}\text { Long-term care } \\
\text { facilities } \\
\text { 3-arm-RCT } \\
57 \text { PLWD }\end{array}$ & $\begin{array}{l}\text { Diagnosis of } \\
\text { moderate to severe } \\
\text { AD according to } \\
\text { DSM-IV criteria; } \\
\text { MMSE<20; }\end{array}$ & $\begin{array}{l}\mathrm{N}=19 \\
\text { Age yes } \\
\text { Sex; yes } \\
\text { Race: yes } \\
\text { Education: yes }\end{array}$ & None & NA & NA & NA & NA & 3 days & \begin{tabular}{|l} 
RPMBC \\
ABRS
\end{tabular} & NA \\
\hline $\begin{array}{l}\text { Smallwood } \\
2001^{57} \\
\text { (11607948) } \\
\text { UK } \\
\text { Small sample }\end{array}$ & $\begin{array}{l}\text { Aromatherapy } \\
\text { massage } \\
\text { Conversation } \\
\text { and } \\
\text { aromatherapy } \\
\text { Massage only } \\
\text { for twice weekly }\end{array}$ & None & $\begin{array}{l}\text { PLWD were } \\
\text { recruited from } \\
\text { district general } \\
\text { hospital ward } \\
\text { 3-arm RCT } \\
\text { 21 PLWD }\end{array}$ & $\begin{array}{l}\text { Clinical diagnosis } \\
\text { of dementia }\end{array}$ & $\begin{array}{l}\mathrm{N}=21 \\
\text { Age: } \text { yes } \\
\text { Sex: yes } \\
\text { Race: no } \\
\text { Education: no }\end{array}$ & None & NA & NA & NA & NA & weekly & \begin{tabular}{|l|} 
Qualitative \\
assessment of \\
behavior using \\
video recorded \\
sessions
\end{tabular} & NA \\
\hline
\end{tabular}

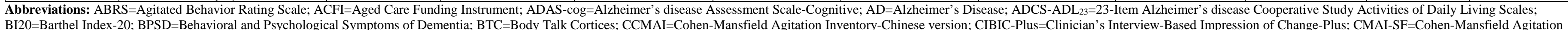

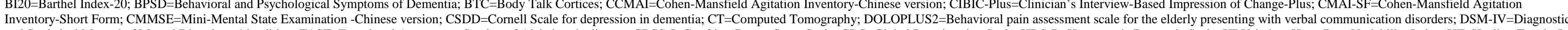

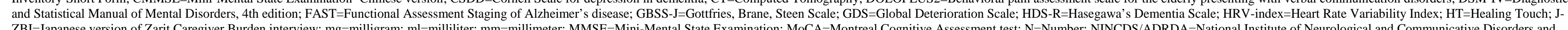

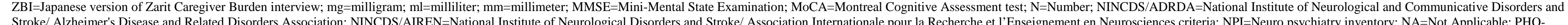

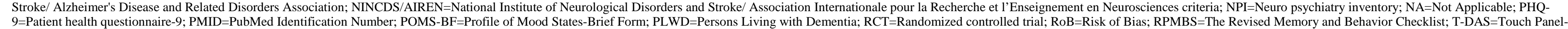
type Dementia Assessment Scale; TT=Therapeutic touch

\section{Bright Light Therapy}




\begin{tabular}{|c|c|c|c|c|c|c|c|c|c|}
\hline Study (PMID) & Outcome Timing & $\begin{array}{l}\text { Attrition Bias } \\
\text { Attrition \% }\end{array}$ & Selection Bias & Detection Bias & Performance Bias & Reporting Bias & Fidelity Bias & Funder & Overall Rating \\
\hline Onega $2016^{58}$ & 8 weeks & $\begin{array}{l}\text { Medium } \\
15 \%\end{array}$ & Low & High & Low & Medium & $x$ & Government & High \\
\hline $\begin{array}{l}\text { Rixt F. Riemersma-van der Lek } \\
2008(18544724)^{59}\end{array}$ & 6 weeks & $\begin{array}{l}\text { Low } \\
6 \text { weeks:7.4\% } \\
\text { High } \\
6 \text { months+: >21\% }\end{array}$ & Low & $\begin{array}{l}6 \text { weeks: Medium } \\
\text { 6+ Months: High }\end{array}$ & Low & High & Medium & Government & High \\
\hline $\begin{array}{l}\text { Hickman } 2007^{60} \\
\text { Barrick } 2010(20104513)^{61}\end{array}$ & 3 weeks & $\begin{array}{l}\text { Medium } \\
\text { NR }\end{array}$ & Medium & High & Medium & $\mathrm{x}$ & $\mathrm{x}$ & Government & High \\
\hline Ancoli-Israel $2003^{62}$ & 18 days & $\begin{array}{l}\text { High } \\
23.9 \%\end{array}$ & $\mathrm{x}$ & $\mathrm{x}$ & $\mathrm{x}$ & $\mathrm{x}$ & $\mathrm{x}$ & Government & High \\
\hline
\end{tabular}

$\mathrm{X}$ indicates that domain was not assessed due to high risk of bias.

\section{Evidence Map: Bright Light Therapy}




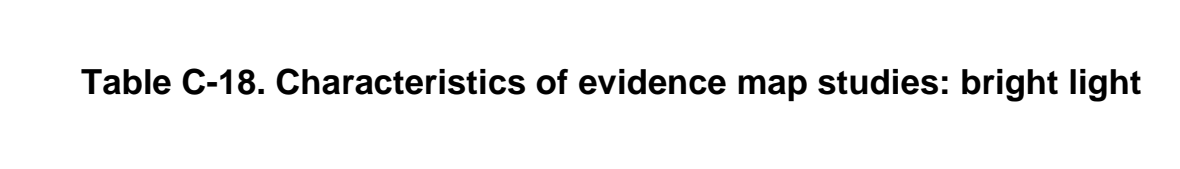




\begin{tabular}{|c|c|c|c|c|c|c|c|c|c|c|c|c|c|}
\hline $\begin{array}{l}\text { Study (PMID) } \\
\text { Country } \\
\text { EM Reason }\end{array}$ & \begin{tabular}{|l} 
Intervention \\
\end{tabular} & Comparison & \begin{tabular}{|l|} 
Setting \\
and Design \\
\\
Setting \\
Design \\
Cluster N \\
Participants \\
Randomized N
\end{tabular} & \begin{tabular}{|l|} 
PLWD \\
Dementia \\
Characteristics
\end{tabular} & \begin{tabular}{|l} 
PLWD \\
Non-Disease \\
Char \\
PLWD N \\
PLWD Age \\
(mean) \\
PLWD Sex (\% \\
female) \\
PLWD Race \\
(\% majority) \\
PLWDD \\
Education \\
(mean years)
\end{tabular} & \begin{tabular}{|l|} 
PLWD \\
Non-Disease \\
Char Reporting \\
Status (RS) \\
\\
PLWD SES \\
PLWD Prior \\
Disability \\
PLWD \\
Household \\
Characteristics \\
PLWD Health \\
Insurance \\
PLWD Detailed \\
Race \\
Information \\
\end{tabular} & $\begin{array}{l}\text { Informal } \\
\text { Caregiver (IC) } \\
\text { Characteristics } \\
\text { IC N } \\
\text { IC Age (mean) } \\
\text { IC Sex (\% } \\
\text { female) } \\
\text { IC Race (\% } \\
\text { majority) } \\
\text { IC Education } \\
\text { (mean years) } \\
\text { IC Relation to } \\
\text { PLWD (\% } \\
\text { majority) }\end{array}$ & \begin{tabular}{|l} 
Informal \\
Caregiver (IC) \\
Char. RS \\
IC Duration \\
IC Living With \\
PLWD \\
IC Payment \\
IC Health \\
Status \\
IC Dementia \\
Family History \\
IC \\
Employment \\
Status \\
IC Training
\end{tabular} & \begin{tabular}{|l|} 
Formal \\
Caregiver (FC) \\
Characteristics \\
FC N \\
FC Age (mean) \\
FC Sex (\% \\
female) \\
FC Race (\% \\
majority) \\
FC Education \\
(mean years)
\end{tabular} & 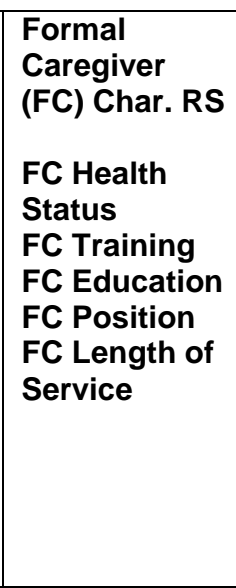 & \begin{tabular}{|l|} 
Outcome \\
Timing(s)
\end{tabular} & \begin{tabular}{|l|} 
PLWD \\
Outcomes
\end{tabular} & \begin{tabular}{|l} 
Caregiver \\
Outcomes \\
IC: \\
FC:
\end{tabular} \\
\hline $\begin{array}{l}\text { Figueiro, 201963 } \\
\text { (318551610 } \\
\text { United States } \\
\text { Small sample }\end{array}$ & \begin{tabular}{|l|} 
Tailored \\
lighting to \\
maximally \\
affect circadian \\
system \\
\end{tabular} & $\begin{array}{l}\text { Low intensity } \\
\text { lights }\end{array}$ & $\begin{array}{l}\text { 4 Assisted } \\
\text { Living Facilities } \\
\text { RCT } \\
52 \text { PLWD }\end{array}$ & $\begin{array}{l}\text { Dementia per } \\
\text { MMSE; unspecified }\end{array}$ & \begin{tabular}{|l|}
$\mathrm{N}=60$ \\
Age: Yes \\
Sex: Yes \\
Race: no \\
Education: no \\
\end{tabular} & None & NA & NA & NA & NA & & $\begin{array}{l}\text { Sleep and } \\
\text { circadian rhythms } \\
\text { CSDD } \\
\text { CMAI }\end{array}$ & NA \\
\hline $\begin{array}{l}\text { Lisa Onega 2016 } 6^{58} \\
\text { United States } \\
\text { High ROB }\end{array}$ & \begin{tabular}{|l}
$\begin{array}{l}\text { Bright light } \\
\text { exposure }\end{array}$ \\
\end{tabular} & $\begin{array}{l}\text { Low intensity } \\
\text { light }\end{array}$ & $\begin{array}{l}\text { Long-term care } \\
\text { facility ( } \mathrm{N}=4 \text { ) } \\
\mathrm{RCT} \text { (bright } \\
\text { light } \mathrm{N}=30 \text {; low } \\
\text { intensity light } \\
\mathrm{N}=30 \text { ) } \\
\text { PLWD }\end{array}$ & None & \begin{tabular}{|l|}
$\mathrm{N}=60$ \\
(treatment \\
$\mathrm{N}=30$ vs \\
control N=30) \\
Age: Yes \\
Sex: Yes \\
Race: Yes \\
Education: \\
Yes
\end{tabular} & \begin{tabular}{|l} 
SES \\
Health problems \\
Marital status
\end{tabular} & NA & NA & NA & NA & 8-weeks) & \begin{tabular}{|l|} 
Depression \\
(DSAOA; DMAS- \\
17; CSDD) \\
Agitation (CMAI- \\
F; CMAI-D; PAS; \\
BARS)
\end{tabular} & NA \\
\hline $\begin{array}{l}\text { Burns } 2009^{64} \\
\text { United Kingdom } \\
\text { Small sample }\end{array}$ & \begin{tabular}{|l|}
$\begin{array}{l}\text { Full spectrum } \\
\text { bright light } \\
\text { therapy (BLT) }\end{array}$ \\
\end{tabular} & Normal light & $\begin{array}{l}\text { Nursing homes } \\
R C T \text { (standard } \\
\text { light } N=26 ; B L T \\
N=22) \\
\text { PLWD }\end{array}$ & $\begin{array}{l}\text { Dementia and } \\
\text { behavioral } \\
\text { disturbances }\end{array}$ & \begin{tabular}{|l}
$\mathrm{N}=48(22$ \\
treatment vs \\
26 control) \\
Age: Yes \\
Sex: Yes
\end{tabular} & Cataracts & NA & NA & NA & NA & $\begin{array}{l}\text { Baseline week, } \\
\text { and in weeks } 4 \\
\text { and } 8\end{array}$ & \begin{tabular}{|l} 
Agitation (Cohen- \\
Mansfield \\
Agitation \\
Inventory; CMAI)
\end{tabular} & NA \\
\hline $\begin{array}{l}\text { Riemersma-van der } \\
\text { Lek 200859 } \\
(18544724) \\
\text { Netherlands } \\
\text { Medium } \\
\text { Explanatory }\end{array}$ & \begin{tabular}{|l|} 
Bright light \\
therapy, 8 \\
hours daily, \\
exposure of \\
\pm 1000 lux
\end{tabular} & \begin{tabular}{|l} 
Usual care \\
(double \\
placebo)
\end{tabular} & $\begin{array}{l}\text { Group care } \\
\text { facility } \\
\text { Cluster RCT } \\
12 \text { Long-term } \\
\text { care facilities } \\
\text { 94 PLWD }\end{array}$ & $\begin{array}{l}\text { Clinical diagnosis for } \\
\text { dementia and } \\
\text { dementia sub-types }\end{array}$ & \begin{tabular}{|l|}
$\mathrm{N}=94$ \\
Age: yes \\
Sex: yes \\
Race: no \\
Education: no
\end{tabular} & None & NA & NA & NA & NA & 6 weeks & \begin{tabular}{|l|} 
MMSE \\
CSDD \\
PGCMS \\
PGCARS \\
MOSES \\
Withdrawn \\
Behavior \\
NPI-Q \\
CMAI \\
NIADL \\
Total Sleep \\
Duration
\end{tabular} & NA \\
\hline $\begin{array}{l}\text { Dowling } 2007^{65} \\
\text { Dowling 2005 } \\
(16050432)^{66} \\
\text { Dowling 2005 } \\
(16035127)^{67} \\
\\
\text { United States } \\
\text { Small sample }\end{array}$ & \begin{tabular}{|l} 
Bright light \\
exposure
\end{tabular} & \begin{tabular}{|l} 
Usual indoor \\
light
\end{tabular} & $\begin{array}{l}\text { Nursing home } \\
\mathrm{RCT} \text { (morning } \\
\text { light } \mathrm{N}=29 ; \\
\text { afternoon light } \\
\mathrm{N}=24 ; \text { or } \\
\text { control } \mathrm{N}=17 \text { ) } \\
\mathrm{PLWD}\end{array}$ & $\begin{array}{l}\text { Rest-activity } \\
\text { disruption and were } \\
\text { diagnosis with } \\
\text { Alzheimer's disease }\end{array}$ & \begin{tabular}{|l}
$\mathrm{N}=71$ ( 54 \\
treatment vs \\
17 control) \\
Age: Yes \\
Sex: Yes \\
Race: Yes
\end{tabular} & None & NA & NA & NA & NA & $\begin{array}{l}\text { End of the } \\
\text { baseline week } \\
\text { and after the } \\
\text { last week of } \\
\text { intervention } \\
\text { (study protocol } \\
11 \text { weeks) }\end{array}$ & \begin{tabular}{|l|} 
Neuropsychiatric \\
behaviors \\
(Neuropsychiatric \\
Inventory nursing \\
Home version; \\
NPI-NH)
\end{tabular} & NA \\
\hline
\end{tabular}




\begin{tabular}{|c|c|c|c|c|c|c|c|c|c|c|c|c|c|}
\hline $\begin{array}{l}\text { Hickman 200760 } \\
20104513 \\
\text { Barrick 201061 } \\
\text { United States } \\
\text { High ROB }\end{array}$ & $\begin{array}{l}\text { Ambient bright } \\
\text { light (high- } \\
\text { intensity, low } \\
\text { glare) in public } \\
\text { areas, applied } \\
\text { morning, } \\
\text { evening, or all } \\
\text { day }\end{array}$ & \begin{tabular}{|l|} 
Standard \\
lighting
\end{tabular} & \begin{tabular}{|l|} 
Geriatric unit in \\
2 state- \\
operated \\
psychiatric \\
hospitals, \\
Cluster-unit \\
crossover \\
intervention \\
trial with 8 \\
study periods \\
in one, 22 in \\
the other ; \\
PLWD \\
\end{tabular} & $\begin{array}{l}\text { Severe or very } \\
\text { severe dementia }\end{array}$ & \begin{tabular}{|l|} 
N=66 \\
Age: Yes \\
Sex: Yes \\
Race: Yes \\
Education: \\
Yes
\end{tabular} & None & NA & NA & NA & NA & \begin{tabular}{|l|} 
Last week of \\
each 3-week \\
period (multiple \\
periods)
\end{tabular} & CSDD & NA \\
\hline $\begin{array}{l}\text { Ancoli-Irrael 200362 } \\
\text { United States } \\
\text { High ROB }\end{array}$ & \begin{tabular}{|l|} 
Bright light \\
exposure
\end{tabular} & $\begin{array}{l}\text { Morning dim } \\
\text { red light }\end{array}$ & \begin{tabular}{|l} 
Nursing home \\
Randomized to \\
treatment \\
groups \\
(morning bright \\
light N=30; \\
morning dim \\
red light $\mathrm{N}=31 ;$ \\
or evening \\
bright light \\
$\mathrm{N}=31$ \\
$\mathrm{PLWD}$
\end{tabular} & $\begin{array}{l}\text { Diagnosis of } \\
\text { possible or probable } \\
\text { Alzheimer's disease }\end{array}$ & $\begin{array}{l}\mathrm{N}=92(61 \\
\text { treatment vs } \\
31 \text { control) } \\
\text { Age: Yes } \\
\text { Sex: Yes } \\
\text { Education: No }\end{array}$ & \begin{tabular}{|l|} 
Current \\
diagnoses (e.g. \\
eye disease; \\
cataracts, \\
glaucoma)
\end{tabular} & NA & NA & NA & NA & $\begin{array}{l}3 \text { days of } \\
\text { baseline, } 10 \\
\text { days of } \\
\text { treatment } \\
\text { (treatment days } \\
1-5 \text { and } 6-10) \\
\text { and } 5 \text { days of } \\
\text { posttreatment } \\
\text { follow-up }\end{array}$ & $\begin{array}{l}\text { Sleep and } \\
\text { circadian activity } \\
\text { rhythms }\end{array}$ & NA \\
\hline $\begin{array}{l}\text { Paola Fontana } \\
\text { Gasio 2003 } \\
\\
\text { Switzerland } \\
\text { Pilot }\end{array}$ & $\begin{array}{l}\text { Low intensity } \\
\text { dawn-dusk } \\
\text { simulation } \\
\text { (DDS) }\end{array}$ & Dim red light & $\begin{array}{l}\text { Nursing homes } \\
\text { Randomly } \\
\text { assigned (DDS } \\
\mathrm{N}=9 \text {; Control } \\
\mathrm{N}=4 \text { ) } \\
\text { PLWD }\end{array}$ & $\begin{array}{l}\text { Symptoms/diagnosis } \\
\text { of dementia and } \\
\text { sleep disturbances }\end{array}$ & \begin{tabular}{|l|}
$\mathrm{N}=13$ \\
(treatment \\
$\mathrm{N}=9$ vs control \\
$\mathrm{N}=4$ ) \\
Age: Yes \\
Sex: Yes
\end{tabular} & None & NA & NA & NA & NA & $\begin{array}{l}\text { After } 3 \text { weeks } \\
\text { of baseline; } \\
\text { DDS or dim red } \\
\text { light treatment }\end{array}$ & $\begin{array}{l}\text { Circadian rest- } \\
\text { activity cycle, } \\
\text { nocturnal sleep, } \\
\text { and cognitive } \\
\text { function }\end{array}$ & NA \\
\hline $\begin{array}{l}\text { Kazuo Mishima } \\
1998^{69} \\
\text { Japan } \\
\text { Small sample }\end{array}$ & \begin{tabular}{|l} 
Bright light \\
therapy
\end{tabular} & None & \begin{tabular}{|l|} 
Facility \\
Randomized \\
crossover \\
PLWD
\end{tabular} & $\begin{array}{l}\text { Vascular dementia } \\
\text { (VD) and PLWD with } \\
\text { dementia of } \\
\text { Alzheimer's type } \\
\text { (DAT) with sleep } \\
\text { and behavioral } \\
\text { disturbances }\end{array}$ & $\begin{array}{l}\mathrm{N}=22 \text { (VD } \\
\mathrm{N}=12 ; \mathrm{DAT} \\
\mathrm{N}=10) \\
\text { Age: Yes } \\
\text { Sex: Yes }\end{array}$ & None & NA & NA & NA & NA & $\begin{array}{l}\text { Pretreatment, } \\
\text { week 1, week } \\
\text { 2, and } \\
\text { posttreatment }\end{array}$ & $\begin{array}{l}\text { Behavioral and } \\
\text { rest-activity (R-A) } \\
\text { rhythm disorders }\end{array}$ & NA \\
\hline
\end{tabular}

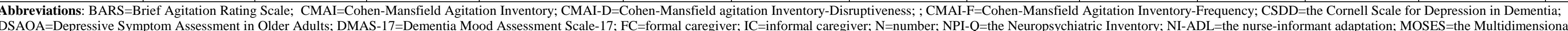

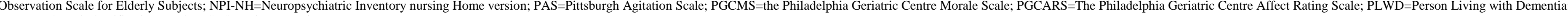

\section{Psychosocial Interventions for BPSD}

Table C-19. Risk of bias assessment: psychosocial interventions for BPSD

\begin{tabular}{|c|c|c|c|c|c|c|c|c|}
\hline Study (PMID) & Outcome Timing & $\begin{array}{l}\text { Attrition Bias } \\
\text { Attrition \% }\end{array}$ & Selection Bias & Detection Bias & Performance Bias & Reporting Bias & Fidelity Bias & Overall Rating \\
\hline \begin{tabular}{|l|l} 
Richards $2005(16137280)^{70}$ \\
\end{tabular} & 3 weeks & $\begin{array}{l}\text { Low } \\
5 \%\end{array}$ & Medium & Medium & Medium & Low & High & Medium \\
\hline \begin{tabular}{|l} 
Toseland $1997(27097884)^{71}$
\end{tabular} & 3 months, 1 year & $\begin{array}{l}\text { Medium } \\
25 \%\end{array}$ & Medium & $\begin{array}{l}\text { High } \\
\text { (30\% missing data for } \\
\text { bed/rise time) }\end{array}$ & Medium & Low & Low & High \\
\hline
\end{tabular}

Abbreviations: PMID=PubMed Identification Number 


\section{Evidence Map: Psychosocial Interventions for BPSD}

Table C-20. Characteristics of evidence map studies: psychosocial interventions for BPSD 


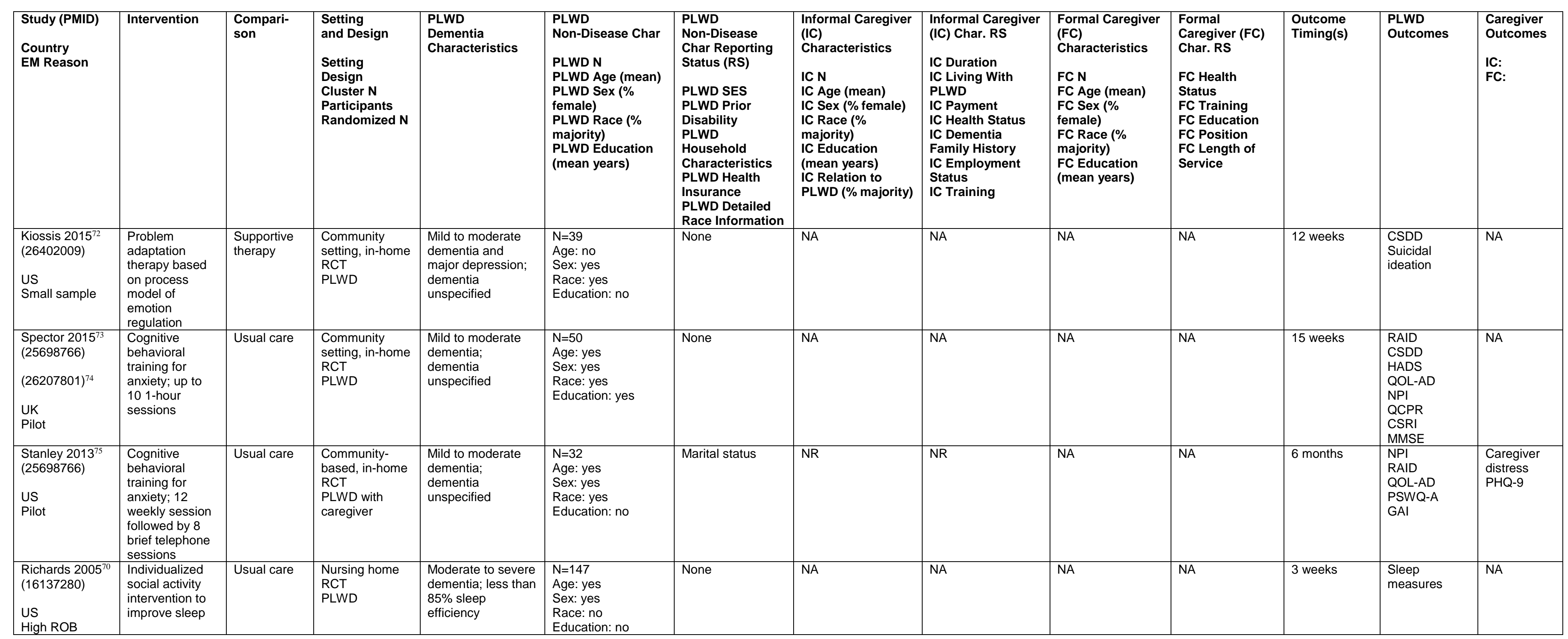




\begin{tabular}{|c|c|c|c|c|c|c|c|c|c|c|c|c|c|}
\hline $\begin{array}{l}\text { Beck 2002 } \\
(12131234) \\
\text { US } \\
\text { Small sample }\end{array}$ & $\begin{array}{l}\text { ADL: 45-60 } \\
\text { minutes of ADL } \\
\text { assistance } \\
\text { provided by } \\
\text { PNA which } \\
\text { catered to the } \\
\text { psychosocial, } \\
\text { cognitive and } \\
\text { physical abilities } \\
\text { of PLWD } \\
\text { PSA: } 30+ \\
\text { minutes of PSA } \\
\text { intervention } \\
\text { provided by } \\
\text { PNA, which } \\
\text { involved } 25 \\
\text { standardized } \\
\text { modules } \\
\text { designed to } \\
\text { meet the } \\
\text { psychosocial, } \\
\text { cognitive and } \\
\text { physical abilities } \\
\text { of PLWD } \\
\text { CB: consisted of } \\
\text { both the ADL } \\
\text { and PSA } \\
\text { interventions } \\
\text { and lasted } 90 \\
\text { minutes daily }\end{array}$ & \begin{tabular}{|l|} 
Placebo: \\
one-to-one \\
interaction \\
between the \\
participant \\
and PNA for \\
30 minutes \\
per day \\
\\
No \\
intervention: \\
routine care \\
with no \\
scheduled \\
contact \\
between \\
participants \\
and the PNA
\end{tabular} & $\begin{array}{l}\text { Nursing home } \\
5 \text { arm RCT } \\
\text { PLWD }\end{array}$ & \begin{tabular}{|l|} 
Dementia \\
diagnosis with a \\
MMSE score $\leq 20$ \\
and report of DB in \\
previous 2 weeks
\end{tabular} & $\begin{array}{l}\mathrm{N}=127 \\
\text { Age: yes } \\
\text { Sex: yes } \\
\text { Race: } \mathrm{n} \\
\text { Education: } \mathrm{no}\end{array}$ & None & NA & NA & NA & NA & $\begin{array}{l}\text { Videotape } \\
\text { analysis } \\
\text { 1-month } \\
\text { follow-up } \\
\text { 2-month } \\
\text { follow-up }\end{array}$ & \begin{tabular}{|l|} 
DBS \\
MMSE \\
ODAS \\
AARS \\
PVAS \\
\end{tabular} & NA \\
\hline $\begin{array}{l}\text { Toseland } \\
1997^{71} \\
(27097884) \\
\text { US } \\
\text { High ROB }\end{array}$ & $\begin{array}{l}\text { Validation } \\
\text { therapy or } \\
\text { social contact; } 4 \\
\text { 30-minute } \\
\text { sessions per } \\
\text { week for 52 } \\
\text { weeks }\end{array}$ & Usual care & $\begin{array}{l}\text { Nursing home } \\
3 \text { arm RCT } \\
\text { PLWD }\end{array}$ & $\begin{array}{l}\text { Moderate to severe } \\
\text { dementia with } \\
\text { behavioral } \\
\text { problems; } \\
\text { dementia } \\
\text { unspecified }\end{array}$ & $\begin{array}{l}\mathrm{N}=88 \\
\text { Age: yes } \\
\text { Sex: yes } \\
\text { Race: yes } \\
\text { Education: no }\end{array}$ & None & NA & NA & NA & NA & $\begin{array}{l}3 \text { months, } 1 \\
\text { year }\end{array}$ & \begin{tabular}{|l} 
MOSES \\
CMAI \\
GIPB \\
MDS+
\end{tabular} & NR \\
\hline
\end{tabular}

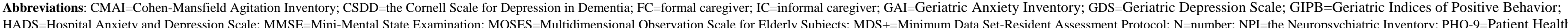

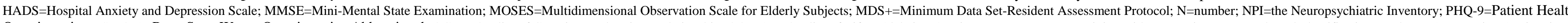

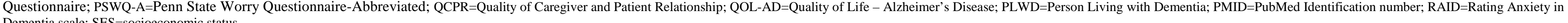




\section{Multicomponent Interventions for BPSD}

Table C-21. Risk of bias assessment: multicomponent interventions for BPSD

\begin{tabular}{|c|c|c|c|c|c|c|c|c|}
\hline Study (PMID) & Outcome Timing & $\begin{array}{l}\text { Attrition Bias } \\
\text { Attrition \% }\end{array}$ & Selection Bias & Detection Bias & Performance Bias & Reporting Bias & Fidelity Bias & Overall Rating \\
\hline Lin 200977 (19507295) & $\begin{array}{l}6 \text { weeks, } \\
14 \text { weeks; } \\
21-22 \text { weeks }\end{array}$ & $\begin{array}{l}\text { High } \\
\text { Unclear }\end{array}$ & Medium & High & High & Low & Medium & High \\
\hline Finnema $2005^{78}(15799079)$ & $\begin{array}{l}3 \text { months } \\
7 \text { months }\end{array}$ & $\begin{array}{l}\text { High } \\
25 \%\end{array}$ & Medium & $x$ & $x$ & $x$ & $x$ & High \\
\hline Kovach 2004 ${ }^{79}(15611216)$ & Unclear & $\begin{array}{l}\text { High } \\
\text { unclear 24\% }\end{array}$ & Low & $x$ & $\mathrm{x}$ & $\mathrm{x}$ & $x$ & High \\
\hline Schrijnemaekers 200280 (12325052) & 3,6, and 12 months & $\begin{array}{l}\text { Medium } \\
3 \text { month: } 9 \%, 6 \text { months: } \\
\text { 20.5\%, } 12 \text { months: } 38 \% \\
\end{array}$ & Medium & Medium & Medium & Low & High & High \\
\hline \begin{tabular}{|l} 
Lawton $1998^{81}$ (9924833) \\
\end{tabular} & $\begin{array}{l}6 \text { months } \\
12 \text { months }\end{array}$ & $\begin{array}{l}\text { Low } \\
12 \text { months: } 5 \%\end{array}$ & Medium & Medium & High & Low & Low & High \\
\hline
\end{tabular}

$X$ indicates that domain was not assessed due to high risk of bias.

Evidence Map: Multicomponent Interventions for BPSD 
- 


\begin{tabular}{|c|c|c|c|c|c|c|c|c|c|c|c|c|c|}
\hline $\begin{array}{l}\text { Study (PMID) } \\
\text { Country } \\
\text { EM Reason }\end{array}$ & Intervention & $\begin{array}{l}\text { Compari- } \\
\text { son }\end{array}$ & $\begin{array}{l}\text { Setting } \\
\text { and Design } \\
\\
\text { Setting } \\
\text { Design } \\
\text { Cluster N } \\
\text { Participants } \\
\text { Randomized N }\end{array}$ & \begin{tabular}{|l|} 
PLWD \\
Dementia \\
Characteristics
\end{tabular} & $\begin{array}{l}\text { PLWD } \\
\text { Non-Disease Char } \\
\text { PLWD N } \\
\text { PLWD Age (mean) } \\
\text { PLWD Sex (\% } \\
\text { female) } \\
\text { PLWD Race (\% } \\
\text { majority) } \\
\text { PLWD Education } \\
\text { (mean years) }\end{array}$ & \begin{tabular}{|l|} 
PLWD \\
Non-Disease \\
Char Reporting \\
Status (RS) \\
PLWD SES \\
PLWD Prior \\
Disability \\
PLWD \\
Household \\
Characteristics \\
PLWD Health \\
Insurance \\
PLWD Detailed \\
Race Information
\end{tabular} & $\begin{array}{l}\text { Informal Caregiver } \\
\text { (IC) } \\
\text { Characteristics } \\
\text { IC N } \\
\text { IC Age (mean) } \\
\text { IC Sex (\% female) } \\
\text { IC Race (\% } \\
\text { majority) } \\
\text { IC Education } \\
\text { (mean years) } \\
\text { IC Relation to } \\
\text { PLWD (\% majority) }\end{array}$ & $\begin{array}{l}\text { Informal Caregiver } \\
\text { (IC) Char. RS } \\
\text { IC Duration } \\
\text { IC Living With } \\
\text { PLWD } \\
\text { IC Payment } \\
\text { IC Health Status } \\
\text { IC Dementia } \\
\text { Family History } \\
\text { IC Employment } \\
\text { Status } \\
\text { IC Training }\end{array}$ & \begin{tabular}{|l} 
Formal Caregiver \\
(FC) \\
Characteristics \\
FC N \\
FC Age (mean) \\
FC Sex (\% \\
female) \\
FC Race (\% \\
majority) \\
FC Education \\
(mean years)
\end{tabular} & $\begin{array}{l}\text { Formal } \\
\text { Caregiver (FC) } \\
\text { Char. RS } \\
\\
\text { FC Health } \\
\text { Status } \\
\text { FC Training } \\
\text { FC Education } \\
\text { FC Position } \\
\text { FC Length of } \\
\text { Service }\end{array}$ & \begin{tabular}{|l|} 
Outcome \\
Timing(s)
\end{tabular} & $\begin{array}{l}\text { PLWD } \\
\text { Outcomes }\end{array}$ & $\begin{array}{l}\text { Caregiver } \\
\text { Outcomes } \\
\text { IC: } \\
\text { FC: }\end{array}$ \\
\hline $\begin{array}{l}\text { Law 201982 } \\
(31390090) \\
\\
\text { Hong Kong } \\
\text { Pilot }\end{array}$ & $\begin{array}{l}\text { Osteoarthritis- } \\
\text { specific knee } \\
\text { exercises plus } \\
\text { self- } \\
\text { management } \\
\text { support, } 8 \\
\text { weeks }\end{array}$ & $\begin{array}{l}\text { Routine } \\
\text { group } \\
\text { exercise }\end{array}$ & $\begin{array}{l}\text { Community } \\
\text { setting } \\
\text { RCT } \\
\text { PLWD }\end{array}$ & \begin{tabular}{|l|} 
Dementia \\
unspecified plus \\
knee osteoarthritis
\end{tabular} & $\begin{array}{l}\text { N=56 } \\
\text { Age: yes } \\
\text { Sex: yes } \\
\text { Race: no } \\
\text { Education: yes }\end{array}$ & \begin{tabular}{|l|} 
Marital status \\
\end{tabular} & $\begin{array}{l}\text { N=56 } \\
\text { Age: yes } \\
\text { Sex: yes } \\
\text { Race: no } \\
\text { Education: yes } \\
\text { Relation to PLWD: } \\
\text { yes }\end{array}$ & Duration & NA & NA & 8 weeks & NPI-Q & RSCSE \\
\hline $\begin{array}{l}\text { Bailey 201783 } \\
\text { (26912731) } \\
\text { US } \\
\text { Small sample }\end{array}$ & $\begin{array}{l}\text { Two } 30 \text { minutes } \\
\text { group activity } \\
\text { sessions, } \\
\text { including, CBT, } \\
\text { reminiscence, } \\
\text { environmental } \\
\text { supports and } \\
\text { individualized } \\
\text { activity for 6 } \\
\text { weeks }\end{array}$ & $\begin{array}{l}\text { Usual care } \\
(\mathrm{n}=25)\end{array}$ & $\begin{array}{l}\text { Privately } \\
\text { owned, for-profit } \\
\text { urban nursing } \\
\text { homes } \\
\text { Cluster RCT } \\
51 \text { PLWD }\end{array}$ & \begin{tabular}{|l|} 
Mild to moderate \\
cognitive \\
impairment (MMSE \\
score 10-24) and \\
symptoms of \\
depression (GDS)
\end{tabular} & $\begin{array}{l}\mathrm{N}=51 \\
\text { Age: } \text { yes } \\
\text { Sex: yes } \\
\text { Race: no } \\
\text { Education: no }\end{array}$ & None & NA & None & NA & NA & $\begin{array}{l}\text { Twice per } \\
\text { week for } 6 \\
\text { weeks }\end{array}$ & $\begin{array}{l}\text { ABC } \\
\text { CSDD } \\
\text { GDS } \\
\text { QOL-AD } \\
\text { Activity } \\
\text { enjoyment } \\
\text { rating } \\
\text { Barthel Scale } \\
\text { MMSE }\end{array}$ & NA \\
\hline $\begin{array}{l}\text { Fischer- } \\
\text { Terworth 2011 } \\
\text { (No PMID) } \\
\\
\text { Germany } \\
\text { Small sample } \\
\text { (<25 in each } \\
\text { arm) }\end{array}$ & $\begin{array}{l}\text { TEACCH- and } \\
\text { Music Therapy- } \\
\text { Based } \\
\text { Interventions } \\
\text { (TMI): modified } \\
\text { cognitive } \\
\text { engagement for } \\
\text { autism plus } \\
\text { group music } \\
\text { therapy for } 6 \\
\text { months. }\end{array}$ & $\begin{array}{l}\text { Nonspecific } \\
\text { occupational } \\
\text { therapy: } \\
\text { Participated } \\
\text { in } \\
\text { nonspecific } \\
\text { occupational } \\
\text { therapy }\end{array}$ & $\begin{array}{l}\text { Special } \\
\text { dementia care } \\
\text { unit } \\
\text { Non-RCT } \\
\text { 49 PLWD }\end{array}$ & $\begin{array}{l}\text { Mild or moderate } \\
\text { dementia (All } \\
\text { types) }\end{array}$ & $\begin{array}{l}\mathrm{N}=49 \\
\text { Age: No } \\
\text { Sex: Yes } \\
\text { Race: No } \\
\text { Education: No }\end{array}$ & None & NA & NA & NA & NA & 6 months & $\begin{array}{l}\text { NPI } \\
\text { GDS } \\
\text { ICEA-D }\end{array}$ & NA \\
\hline
\end{tabular}




\begin{tabular}{|c|c|c|c|c|c|c|c|c|c|c|c|c|c|}
\hline $\begin{array}{l}\text { Lin 200977 } \\
\text { (19507295) } \\
\text { Taiwan } \\
\text { High RoB }\end{array}$ & $\begin{array}{l}\text { I1: Acupressure: } \\
\text { acupressure at } \\
\text { each accupoint } \\
\text { for } 2 \text { minutes; } \\
15-\text { minute daily } \\
\text { sessions } \\
6 \text { days per } \\
\text { week; } 4 \text { weeks } \\
\text { 12: Montessori } \\
\text { based activity } \\
\text { programm five } \\
\text { categories } \\
\text { associated with } \\
\text { activities of daily } \\
\text { living: scooping, } \\
\text { pouring, } \\
\text { squeezing, fine } \\
\text { motor skills, } \\
\text { environmental } \\
\text { care, plus } \\
\text { personal care; } 6 \\
\text { days per week; } \\
4 \text { weeks }\end{array}$ & $\begin{array}{l}\text { Attention } \\
\text { control } \\
\text { (Presence): } \\
\text { for a 15- } \\
\text { minute } \\
\text { period each } \\
\text { day for } 6 \\
\text { days a week }\end{array}$ & $\begin{array}{l}\text { Long-term care } \\
\text { facilities } \\
3 \text { arm crossover } \\
\text { RCT } \\
133 \text { PLWD }\end{array}$ & \begin{tabular}{|l|} 
Diagnosis of \\
dementia, CMAI \\
score 35 or above
\end{tabular} & $\begin{array}{l}\mathrm{N}=23 \\
\text { Age: yes } \\
\text { Sex: yes } \\
\text { Race: yes } \\
\text { Education: yes }\end{array}$ & None & NA & NA & NA & NA & $\begin{array}{l}8 \text { weeks } \\
16 \text { weeks } \\
22 \text { weeks }\end{array}$ & $\begin{array}{l}\text { CMAI } \\
\text { Ease of care } \\
\text { AARS } \\
\text { Family-visit } \\
\text { restraint }\end{array}$ & $\mathrm{NA}$ \\
\hline $\begin{array}{l}\text { Kovach 2004 } \\
\text { (15611216) } \\
\text { US } \\
\text { High RoB }\end{array}$ & $\begin{array}{l}\text { BACE } \\
\text { intervention: } \\
\text { assessment; } \\
\text { diagnose and } \\
\text { plan a } \\
\text { correction of the } \\
\text { arousal } \\
\text { imbalance; } \\
\text { implement new } \\
\text { activity } \\
\text { schedule }\end{array}$ & Unclear & $\begin{array}{l}\text { Long-term care } \\
\text { facilities } \\
\text { Pretest posttest } \\
\text { RCT } \\
102 \text { PLWD }\end{array}$ & $\begin{array}{l}\text { MMSE score of } 15 \\
\text { or below and FAST } \\
\text { stage } 6 \text { or } 7 \text { with } \\
\text { some agitation but } \\
\text { no chronic } \\
\text { psychiatric } \\
\text { diagnosis other } \\
\text { than dementia }\end{array}$ & $\begin{array}{l}\mathrm{N}=78 \\
\text { Age: } y \text { es } \\
\text { Sex: yes } \\
\text { Race: no } \\
\text { Education: no }\end{array}$ & None & NA & NA & NA & NA & $\begin{array}{l}\text { Direct } \\
\text { observation of } \\
\text { participants }\end{array}$ & $\begin{array}{l}\text { MMSE } \\
\text { FAST } \\
\text { ASD } \\
\text { Arousal } \\
\text { Balance and } \\
\text { Imbalance } \\
\text { ABMI } \\
\text { Therapeutic } \\
\text { Activity }\end{array}$ & NA \\
\hline $\begin{array}{l}\text { Finnema 200578 } \\
(15799079) \\
(24337328)^{85} \\
\text { The } \\
\text { Netherlands } \\
\text { High ROB }\end{array}$ & $\begin{array}{l}\text { Emotion- } \\
\text { oriented care: } \\
\text { combination } \\
\text { validation } \\
\text { therapy, } \\
\text { reminiscence, } \\
\text { sensory } \\
\text { stimulation }\end{array}$ & Usual care & $\begin{array}{l}\text { Nursing homes } \\
\text { RCT with } \\
\text { matched } 16 \\
\text { psychogeriatric } \\
\text { wards } \\
\text { PLWD }\end{array}$ & $\begin{array}{l}\text { Moderate to severe } \\
\text { probably } \\
\text { Alzheimer-type } \\
\text { dementia, mixed } \\
\text { vascular dementia, } \\
\text { dementia or } \\
\text { amnestic syndrome }\end{array}$ & $\begin{array}{l}\mathrm{N}=146 \\
\text { Age: } \text { yes } \\
\text { Sex: yes } \\
\text { Race: no } \\
\text { Education: no }\end{array}$ & $\begin{array}{l}\text { Duration } \\
\text { institutionalized }\end{array}$ & NA & NA & $\begin{array}{l}\mathrm{N}=99 \\
\text { Age: } y \text { yes } \\
\text { Sex: yes } \\
\text { Race: } \text { no } \\
\text { Education: yes }\end{array}$ & Position & 7 months & $\begin{array}{l}\text { CSDD } \\
\text { GRGS } \\
\text { CMAI } \\
\text { PGCMS }\end{array}$ & $\begin{array}{l}\text { GHQ-28 } \\
\text { Organizatio } \\
\text { n and stress } \\
\text { scale } \\
\text { Absenteeis } \\
\mathrm{m}\end{array}$ \\
\hline
\end{tabular}




\begin{tabular}{|c|c|c|c|c|c|c|c|c|c|c|c|c|c|}
\hline $\begin{array}{l}\text { Beck 200276 } \\
(12131234) \\
\text { US } \\
\text { Small sample }\end{array}$ & $\begin{array}{l}\text { ADL: 45-60 } \\
\text { minutes of ADL } \\
\text { assistance from } \\
\text { PNA which } \\
\text { catered to the } \\
\text { psychosocial, } \\
\text { cognitive and } \\
\text { physical abilities } \\
\text { of PLWD } \\
\\
\text { PSA: } 30+ \\
\text { minutes of PSA } \\
\text { from PNA } ; 5 \\
\text { standardized } \\
\text { modules } \\
\text { designed to } \\
\text { meet the } \\
\text { psychosocial, } \\
\text { cognitive and } \\
\text { physical abilities } \\
\text { of PLWD } \\
\text { CB: both the } \\
\text { ADL and PSA } \\
\text { interventions; } \\
90 \text { minutes daily }\end{array}$ & $\begin{array}{l}\text { Placebo: } \\
\text { one-to-one } \\
\text { interaction } \\
\text { between the } \\
\text { participant } \\
\text { and PNA for } \\
30 \text { minutes } \\
\text { per day } \\
\text { No } \\
\text { intervention: } \\
\text { routine care } \\
\text { with no } \\
\text { scheduled } \\
\text { contact } \\
\text { between } \\
\text { participants } \\
\text { and the PNA }\end{array}$ & $\begin{array}{l}\text { Nursing home } \\
5 \text { arm RCT } \\
\text { PLWD }\end{array}$ & $\begin{array}{l}\text { Dementia } \\
\text { diagnosis with a } \\
\text { MMSE score } \leq 20 \\
\text { and report of DB in } \\
\text { previous } 2 \text { weeks }\end{array}$ & $\begin{array}{l}\mathrm{N}=127 \\
\text { Age: } \text { yes } \\
\text { Sex: yes } \\
\text { Race: yes } \\
\text { Education: no }\end{array}$ & None & $\mathrm{NA}$ & None & NA & NA & $\begin{array}{l}\text { Videotape } \\
\text { analysis } \\
\text { 1-month } \\
\text { follow-up } \\
\text { 2-month } \\
\text { follow-up }\end{array}$ & $\begin{array}{l}\text { DBS } \\
\text { MMSE } \\
\text { ODAS } \\
\text { AARS } \\
\text { PVAS }\end{array}$ & NR \\
\hline $\begin{array}{l}\text { Schrijnemaeker } \\
\text { s 2002 } \\
(12325052) \\
\text { The } \\
\text { Netherlands } \\
\text { High ROB }\end{array}$ & $\begin{array}{l}\text { Emotion- } \\
\text { oriented care: } \\
\text { combination } \\
\text { validation } \\
\text { therapy, } \\
\text { reminiscence, } \\
\text { sensory } \\
\text { stimulation }\end{array}$ & Usual care & $\begin{array}{l}\text { Residential } \\
\text { homes with } \\
\text { structured day } \\
\text { care units } \\
\text { Cluster RCT } \\
16 \text { units }\end{array}$ & $\begin{array}{l}\text { Moderate to severe } \\
\text { cognitive } \\
\text { impairment and } \\
\text { behavioral } \\
\text { problems; } \\
\text { dementia } \\
\text { unspecified }\end{array}$ & $\begin{array}{l}\mathrm{N}=151 \\
\text { Age: yes } \\
\text { Sex: yes } \\
\text { Race: } \mathrm{no} \\
\text { Education: no }\end{array}$ & $\begin{array}{l}\text { Duration } \\
\text { institutionalized }\end{array}$ & NA & NA & NR & NR & $\begin{array}{l}3,6, \text { and } 12 \\
\text { months }\end{array}$ & $\begin{array}{l}\text { Dutch } \\
\text { Behavior } \\
\text { Observation } \\
\text { Scale for } \\
\text { Psycho- } \\
\text { geriatric } \\
\text { Inpatients } \\
\text { ADL } \\
\text { CMAI } \\
\text { GRGS }\end{array}$ & NR \\
\hline $\begin{array}{l}\text { Lawton 1998 } \\
\text { (9924833) } \\
\text { US } \\
\text { High RoB }\end{array}$ & $\begin{array}{l}\text { Stimulation- } \\
\text { retreat model: } \\
\text { which } \\
\text { diagnosed, } \\
\text { prescribed, and } \\
\text { applieed a } \\
\text { package of care } \\
\text { according to } \\
\text { individual needs } \\
\text { for additional } \\
\text { stimulation or } \\
\text { relief from } \\
\text { stimulation } \\
\text { ("retreat") }\end{array}$ & Unclear & $\begin{array}{l}\text { Nursing home } \\
\text { residents } \\
\text { RCT } \\
102 \text { PLWD }\end{array}$ & NR & $\begin{array}{l}\text { N=49 } \\
\text { Age: no } \\
\text { Sex: no } \\
\text { Race: no } \\
\text { Education: yes }\end{array}$ & None & NA & NA & NA & NA & $\begin{array}{l}6 \text { months } \\
12 \text { months }\end{array}$ & $\begin{array}{l}\text { GDS } \\
\text { PSMS } \\
\text { BEHAVE-AD } \\
\text { CMAI } \\
\text { MOSES } \\
\text { TRS } \\
\text { AARS }\end{array}$ & NA \\
\hline
\end{tabular}

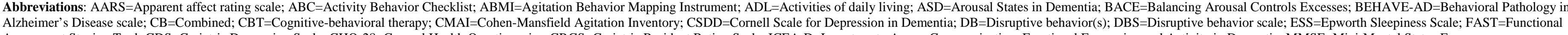
Assessment Staging Tool; GDS=Geriatric Depression Scale; GHQ-28=General Health Questionnaire; GRGS=Geriatric Resident Rating Scale; ICEA-D=Inventory to Assess Communication, Emotional Expression and Activity in Dementia; MMSE=Mini-Mental Status Exam;

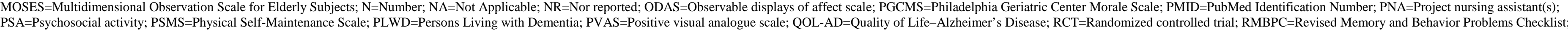
RSCSE=Revised Scale for Caregiving Self-Efficacy; RoB=Risk of Bias; SD=Standard deviation; TRS=Therapeutic Recreation Specialist 


\section{References for Appendix C}

1. Moyle W, Jones CJ, Murfield JE, et al. Use of a Robotic Seal as a Therapeutic Tool to Improve Dementia Symptoms: A Cluster-Randomize 2. Jones C, Moyle W, Murfield J, et al. Does Cognitive Impairment and Agitation in Dementia Influence Intervention Effectiveness? Finding From a Cluster-RCT With the Therapeutic Robot, PARO. J Am Med Dir Assoc. 2018 Apr 13;13:13. do

https://dx.doi.org/10.1016/j.jamda.2018.02.014. PMID: 29656838.

eness of Using PARO, a Therapeutic Robotic Seal, to Reduce Agitation and (1) hittps://dx.doi.org/10.1016/jijamda.2017.10.008. PMID: 29325922. measured by wearable technology: A cluster-randomised controlled trial. Maturitas. 201801 Apr;110:10-7. doi

itp://dx.doi.org/10.1016/j.maturitas.2018.01.007. PMID: 621060108.

(2017:55(2):569-74. PMID:

6. Olsen C, Pedersen I, Bergland A, et al. Effect of animal-assisted activity on balance and quality of life in home-dwelling persons with dementia. Geriatr Nurs. 2016 Jul-Aug;37(4):284-91. doi: https://dx.doi.org/10.1016/j.gerinurse.2016.04.002. PMID: 27155968. 7. Olsen C, Pedersen I, Bergland A, et al. Effect of animal-assisted interventions on depression, agitation and quality of life in nursing hom residents suffering from cognitive impairment or dementia: a cluster randomized controlled trial. Int J Geriatr Psychiatry. 2016 Dec;31(12):1312-21. doi: https:///dx.doi.org/10.1002/gps.4436. PMID: 26807956.

. 9. Joranson N, Pedersen I, Rokstad AM, et al. Effects on Symptoms of Agitation and Depression in Persons With Dementia Participating in Robot-Assisted Activity: A Cluster-Randomized Controlled Trial. J Am Med Dir Assoc. 2015 Oct 01;16(10):867-73. doi: itps://dx.doi.org/10.1016/i.jamda.2015.05.002. PMID: 26096582. A

0. Joranson N, Pedersen I, Rokstad AM, et al. Change in quality of life in older people with dementia participating in Paro-activity: a clusterdoi: https:///dx.doi.org/10.1111/jan.13076. PMID: 27434512. (2) 2013;26(2)::213-25. PMID: CN-00871982

12. Jones C, Moyle W, Murfield J, et al. Does Cognitive Impairment and Agitation in Dementia Influence Intervention Effectiveness? Findings rom a Cluster-Randomized-Controlled Trial With the Therapeutic Robot, PARO. J Am Med Dir Assoc. 2018 Jul;19(7):623-6. do:

13. Pu L, Moyle W, Jones C, et al. The Effect of Using PARO for People Living With Dementia and Chronic Pain: A Pilot Randomized Controlled Trial. J Am Med Dir Assoc. 2020 Feb 28;28:28. doi: https://dx.doi.org/10.1016/j.jamda.2020.01.014. PMID: 32122797. 14. Pu L, Moyle W, Jones C. How people with dementia perceive a therapeutic robot called PARO in relation to their pain and mood: $A$ qualitative study. J Clin Nurs. 2020 Feb;29(3-4):437-46. doi: https:///dx.doi.org/10.1111/jocn.15104. PMID: 31738463

doll therapy for managing europsychiatric symptoms in older patients with dementia. Int J Geriatr Psychiatry. 2019 09;34(9):1308-15. doi:

hitps:///dx.doi.org/10.1002/gps.4961. PMID: 30136743.

16. Menna LF, Santaniello A, Gerardi F, et al. Efficacy of animal-assisted therapy adapted to reality orientation therapy:
salivary cortisol. Psychogeriatrics. 2019 Sep;19(5):510-2. doi: https://dx.doi.org/10.1111/psyg.12418. PMID: 30740833 .

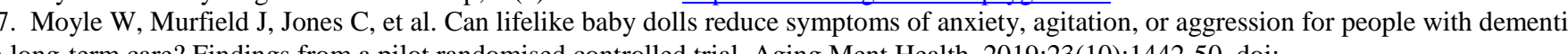

10.1080/136078632018.1498447. PMID: 138051539. Language: English. Entry Date: 20190817. Revision Date: 20190823. Publication Type: Article.

18. Cantarella A, Borella E, Faggian S, et al. Using dolls for therapeutic purposes: A study on nursing home residents with severe dementia. In
J Geriatr Psychiatry. 2018 July;33(7):915-25. doi: hhtp://dx.doi.org/10.1002/gps.4872. PMID: 622559552. . Liang A, Piroth I Robinson H, et al. A Pilot Randomized Trial of a Companion Robot for People With Dementia Living in the Community. J Am Med Dir Assoc. 2017 Oct 01;18(10):871-8. doi: https:///dx.doi.org/10.1016/j.jamda.2017.05.019. PMID: 28668664.

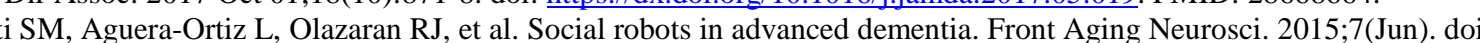

10.3389/fnagi.2015.00133. PMID: CN-01103263.

21. Moyle W, Cooke M, Beattie E, et al. Exploring the effect of companion robots on emotional expression in older adults with dementia: pilot randomized controlled trial. J Gerontol Nurs. 2013 May;39(5):46-53. doi: https://dx.doi.org/10.3928/00989134-20130313-03. PMID:

22. Majic T, Gutzmann H, Heinz A, et al. Animal-assisted therapy and agitation and depression in nursing home residents with dementia: a 23831177 .

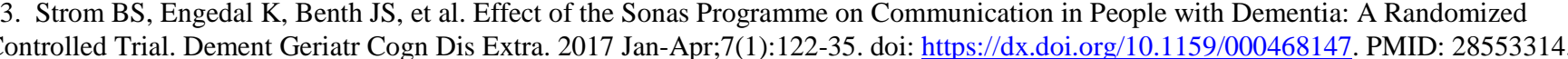

24. Baker R, Holloway J, Holtkamp CCM, et al. Effects of multi-sensory stimulation for people with dementia. J Adv Nurs. 2003;43(5):465-77. doi: 10.1046/j.1365-2

25. van Weert JC, van Dulmen AM, Spreeuwenberg PM, et al. Effects of snoezelen, integrated in $24 \mathrm{~h}$ den communication during morning care. Patient Educ Couns. 2005 Sep;58(3):312-26. PMID: 16054329.

26. Baker R, Bell S, Baker E, et al. A randomized controlled tria 0 ;

27. Maseda A Cibeira N, Lorenzo-Lopez L et al. Multisensory Stimulation and Individualized Music Sessions on Older Adults with Severe Dementia: Effects on Mood, Behavior, and Biomedical Parameters. J Alzheimers Dis. 2018;63(4):1415-25. doi: https://dx.doi.org/10.3233/JAD180109. PMID: 29843244.

28. Collier L, McPherson K, Ellis-Hill C, et al. Multisensory stimulation to improve functional performance in moderate to severe dementiaEnglish. Entry Date: 20101214. Devision Date: 20150820. Publication Type: Journal Article.

29. Staal JA, Sacks A, Matheis R, et al. The effects of Snoezelen (multi-sensory behavior therapy) and psychiatric care on agitation, apathy, and activities of daily living in dementia patients on a short term geriatric psychiatric inpatient unit. Int J Psychiatry Med. 2007;37(4):357-70. PMID: 18441625.

30. van Weert JC, Kerkstra A, van Dulmen AM, et al. The implementation of snoezelen in psychogeriatric care: an evaluation through the eyes of caregivers. Int J Nurs Stud. 2004 May;41(4):397-409. PMID: 1505085

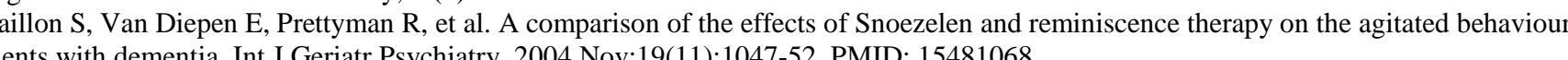

Journal of Occupational Therapy. 2002 February;65(2):61-6. PMID: 35396447 . 33. Baker R, Dowling Z, Wareing LA, et al. Snoezelen: its Long-Term and Short-Term Effects on Older People with Dementia. British Journa of Occupational Therapy. 1997;60(5)213-8. PMID: 104158278. Language: English. Entry Date: 20131114. Revision Date: 20150820.

34. Kwan RYC, Leung MCP, Lai CKY. A Randomized Controlled Trial Examining the Effect of Acupressure on Agitation and Salivary Cortisol in Nursing Home Residents with Dementia. Dement Geriatr Cogn Disord. 2017;44(1-2):92-104. doi

https://dx.doi.org/10.1159/000478739. PMID: 28768251

35. Yang YP, Wang CJ, Wang JJ. Effect of Aromatherapy Massage on Agitation and Depressive Mood in Individuals With Dementia. J Gerontol Nurs. 2016 Sep 01;42(9):38-46.

associated agitation. BMC Altern Med. 2015 Mar 29:15 efficacy of aroma-acupressure and aromatherapy for the treatment of dementi37. Moyle W, Cooke ML, Beattie E, et al. Foot massa:93. doi: https://dx.doi.org/10.1186//s12906-015-0612-9. PMID: 25880034. controlled trial. Int J Nurs Stud. 2014 Jun;51(6):856-64. doi: https://dx.doi.org/10.1016/jijijnurstu.2013.10.019. PMID: 24216598. 38. Burns A, Perry E, Holmes C, et al. A double-blind placebo-controlled randomized trial of Melissa officinalis oil and donepezil for the

39. Lin PW, Chan WC, Ng BF, et al. Efficacy of aromatherapy (Lavandula angustifolia) as an intervention for agitated behaviours in Chinese older persons with dementia: a cross-over randomized trial. Int J Geriatr Psychiatry. 2007 May;22(5):405-10. PMID: 17342790. 40. Ballard CG, O'Brien JT, Reichelt K, et al. Aromatherapy as a safe and effective treatment for the management of agitation in severe
dementia: the results of a double-blind, placebo-controlled trial with Melissa. J Clin Psychiatry. 2002 Jul:63(7):553-8. PMID: 12143909 41. Kouzuki M, Kitao S. Kaju T, et al. Evaluation of the effect of aroma oil as a bath salt on cognitive function. Psychogeriatrics : the officis

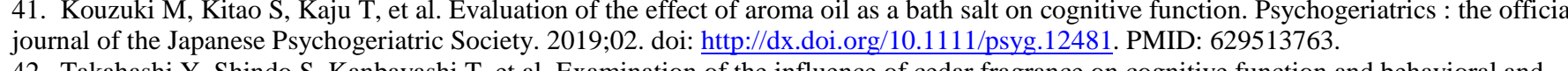
42. Takahashi Y, Shindo S, Kanbayashi T, et al. Examination of the influence of cedar fragrance on cognitive function and behavioral a psychological symptoms of dementia in Alzheimer type dementia. Neuropsychopharmacology reports. 2020. PMID: CN-02084429.
43. Zalomonson S, Freud T, Punchik B, et al. The results of a cross-over placebo-controlled study of the effect of lavender oil on behavioral and psychological symptoms of dementia. Rejuvenation Res. 2018 Oct 17,17:17. 44. Fung JKK, Tsang HW. Management of behavioural and psychological symptoms of dementia by an aroma-massage with acupressure
treatment protocol: A randomised clinical trial. J Clin Nurs. 2018 May;27(9-10):1812-25. doi: https://dx.doi.org/10.1111/jocn.14101. PMID 28986942.

45. Kwan RY, Leung MC, Lai CK. Acupressure for agitation in nursing home residents with dementia: study protocol for a randomized

46. Jia Y, Zhang X, Yu J, et al. Acupuncture for patients with mild to moderate Alzheimer's disease: a randomized controlled trial. BMC Altern Med. 2017 Dec 29;17(1):556. doi: https://dx.doi.org/10.1186/s12906-017-2064-X. PMID: 29284465. in an effort to decrease behavioral issues: a pilot study. J Drug Assess. 2017;6(1):1-5. doi: https://dx.doi.org/10.1080/21556660.2016.1278545. PMID: 28265482. 48. Yang SY, Shan CL, Qing H, et al. The Effects of Aerobic Exercise on Cognitive Function of Alzheimer's Disease Patients. CNS Neurol
Disord Drug Targets. 2015:14(10):1292-7. PMID: 26556080. 
49. Chieh-Yu F, Moyle W, Cooke M. A randomised controlled trial of the use of aromatherapy and hand massage to reduce disruptive tehaviour in people with dementia. BMC Altern Med. 2013;13(1):165-73. doi: 10.1186/14/2-682 English. Entry Date. 20130205 . Revis

Appy and hand massage to reduce disruptive behaviour in

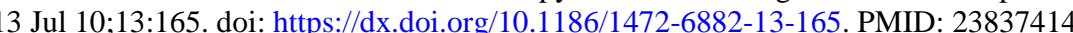
51. Lu DF, Hart LK, Lutgendorf SK, et al. Slowing progression of early stages of AD with alternative therapies: a feasibility study. Geriat Nurs. 2013 Nov-Dec;34(6):457-64. doi: https://dx.doi.org/10.1016/.gerinurse.2013.07.003. PMID: 23972540.

52. Rodriguez-Mansilla J, Gonzalez-Lopez-Arza MV, Varela-Donoso E, et al. Ear therapy and massage therapy in the elderly with dementia:

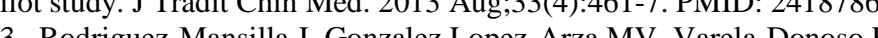

en et al. The effects of ear acupressure, massage therapy and no therapy

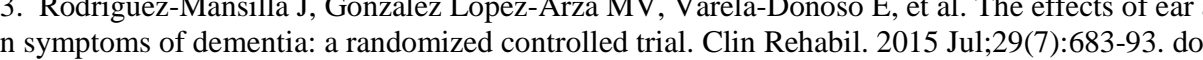

https://dx.doi.org/10.1177/0269215514554240. PMID: 25322869.

54. Jimbo D, Kimura Y, Taniguchi M, et al. Effect of aromatherapy on patients with Alzheimer's disease. Psychogeriatrics. 2009;9(4):173-9. doi: 10.1111/j.1479-8301.2009.00299.x. PMID: CN-0075088-

55. Hawranik P, Johnston P, Deatrich J. Therapeutic touch and agitation in individuals with Alzheimer's disease. West J Nurs Res. 2008

Con Med. 2005 Jan-Feb;11(1):66-74. PMID: 15712768.

57. Smallwood J, Brown R, Coulter F, et al. Aromatherapy and behaviour disturbances in dementia: a randomized controlled trial. Int J Geria sychiatry. 2001 Oct,16(10):1010-3. PMID: 11607948.

58. Onega LL, Pierce TW, Epperly L. Effect of Bright Light Exposure on Depression and Agitation in Older Adults with Dementia. Issues 59. Riemersma-van der Lek RF, Swaab DF, Twisk J, et al. Effect of bright light and melatonin on cognitive and noncognitive function in elderly residents of group care facilities: a randomized controlled trial. Jama. 2008 Jun 11;299(22):2642-55. do:

https://dx.doi.org/10.1001/jama.299.22.2642. PMID: 18544724,

60. Hickman SE, Barrick AL, Williams CS, et al. The effect of ambient bright light therapy on depressive symptoms in persons with dementia.

62. Ancoli-Israel S, Martin JL, Gehrman P, et al. Effect of light on agitation in institutionalized patients with severe Alzheimer disease. Am J Geriatr Psychiatry. 2003 Mar-Apr;11(2):194-203. PMID: 12611749.

3. Figueiro MG, Mitich Best-Activity, Mood, and Behavio in Older Adults With Alzheimer Disease and Related Dementias: A Randomized Clinical Trial. J Clin Sleep Med. 2019 Dec 15;15(12):1757-67. Aug;21(4):711-21. doi: https://dx.doi.org/10.1017//1041610209008886. PMID: 19323872.

65. Dowing GA, Graf CL, Hubbard EM, et al. Light treatment for neuropsychiatric behaviors in Alzheimer's disease. West J Nurs Res. 2007 D6. D(8).961-75. PMID: 17596638.

6. Dowling GA, Hubbard EM, Mastick J, et al. Effect of morning bright light treatment for rest-activity disruption in institutionalized patients

with Alzheing GA, Mastick J, Hubbard EM, et al. Effect of timed bright light treatment for rest-activity disruption in institutionalized patients with Alzheimer's disease. Int J Geriatr Psychiatry. 2005 Aug;20(8):738-43. PMID: 16035127.
68. Fontana Gasio P, Krauchi K, Cajochen C, et al. Dawn-dusk simulation light therapy of disturbed circadian rest-activity cycles in demented

69. Mishima K, Hishikawa Y, Okawa M. Randomized, dim light controlled, crossover test of morning bright light therapy for rest-activity

70. Richards KC, Beck C, O'Sullivan PS, et al. Effect of individualized social activity on sleep in nursing home residents with dementia. J Am Geriatr Soc. 2005 Sep;53(9):1510-7. PMID: 16137280.

71. Toseland RW, Diehl M, Freeman K, et al. The impact of validation group therapy on nursing home residents with dementia. J Appl

72. Kiosses DN, Ros, 16(1)31-

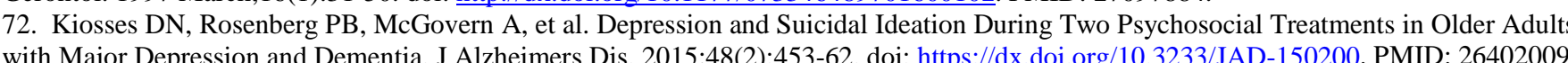
73. Spector A, Charlesworth G, King M, et al. Cognitive-behavioural therapy for anxiety in dementia: pilot randomised controlled trial. Br Psychiatry. 2015 Jun; 206(6):509-16. doi: https://dx.doi.org/10.1192/bjp.bp.113.140087. PMID: 25698766 .

74. Spector A, Orrell M, Charlesworth G, et al. Factors influencing the person-carer relationship in people with anxiety and dementia. In Aging

75. Stanley MA, Calleo J, Bush AL, et al. The peaceful mind program: a pilot test of a cognitive-behavioral therapy-based intervention for anxious patients with dementia. Am J Geriatr Psychiatry. 2013 Jul;21(7):696-708. doi: https://dx.doi.org/10.1016/j.jagp.2013.01.007. PMID: 76. Beck CK, Vogelpohl TS, Rasin JH, et al. Effects of behavioral interventions on disruptive behavior and affect in demented nursing hom residents. Nurs Res. 2002 Jul-Aug;51(4):219-28. PMID: 12131234

77. Lin LC, Yang MH, Kao CC, et al. Using acupressure and Montessori-based activities to decrease agitation for residents with dementia:

78. Finnema E, Droes RM, Ettema T, et al. The effect of integrated emotion-oriented care versus usual care on elderly persons with dementia in the nursing home and on nursing assistants: a randomized clinical trial. Int J Geriatr Psychiatry. 2005 Apr;20(4):330-43. PMID: 15799079. 79. Kovach CR, Taneli Y, Dohearty P, et al. Effect of the BACE intervention on agitation of people with dementia. Gerontologist. 2004

80. Schrijnemaekers V, van Rossum E, Candel M, et al. Effects of emotion-oriented care on elderly people with cognitive impairment and

列

1998 Dec;10(4):379-95. PMID: 9924833.

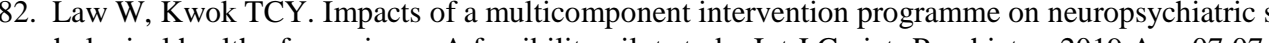
psychological health of caregivers: A feasibility pilot study. Int J Geriatr Psychiatry. 2019 Aug 07;07:07. do:

83. Bailey EM, Stevens AB, LARoce MA, et al, A Ravor

Controlled Trial of a Therapeutic Intervention for Nursing Home Residents PMID: 26912731. In Geropsych: the journal of gerontopsychology and geriatric psychiatry skills and time spent of Droes RM, de Lange J, et al. The implem:12(5):536-50, doi: https/ddv doi 00 110117711471301211435187. PMID: 24337328 . 
Appendix D. Interventions for PLWD Well-Being

\section{Exercise}

Table D-1. Risk of bias assessment: exercise

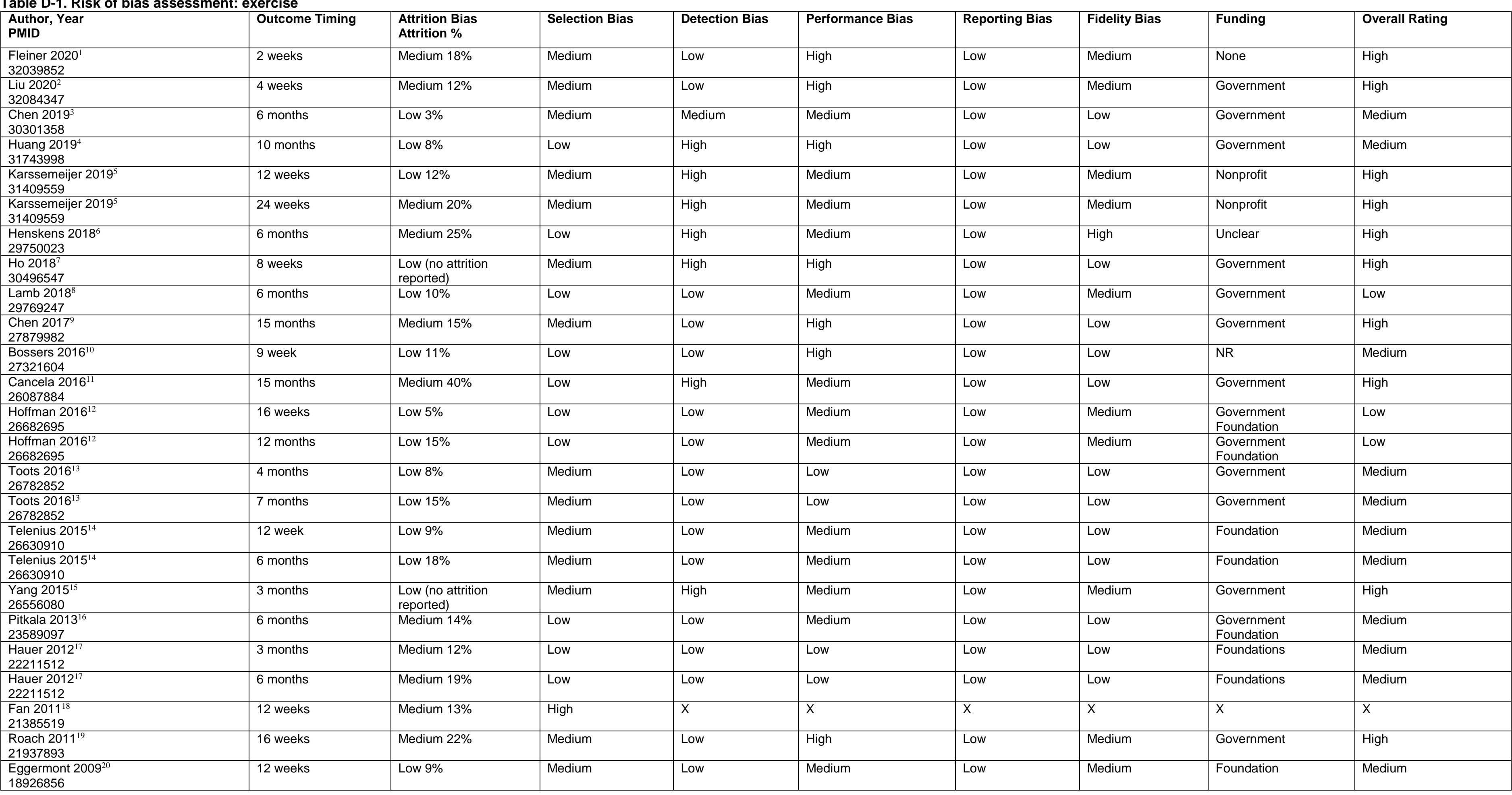




\begin{tabular}{|c|c|c|c|c|c|c|c|c|c|}
\hline $\begin{array}{l}\text { Rolland } 2007^{21} \\
17302650\end{array}$ & 6 months & Medium 13\% & Medium & Low & Medium & Low & High & Government & High \\
\hline $\begin{array}{l}\text { Rolland } 2007^{21} \\
17302650\end{array}$ & 12 months & Medium 18\% & Medium & Low & Medium & Low & High & Government & High \\
\hline
\end{tabular}

Abbreviations: NR=Not Reported; PMID=PubMed Identification Number

\begin{tabular}{|c|c|c|c|c|c|c|c|c|c|c|c|c|c|}
\hline $\begin{array}{l}\text { Author, Year } \\
\text { PMID } \\
\text { Country } \\
\text { RoB } \\
\text { Type }\end{array}$ & \begin{tabular}{|l|} 
Intervention: \\
Intervention Target \\
Mode \\
Components \\
Frequency \\
Duration \\
Delivery/Supervision
\end{tabular} & \begin{tabular}{|l|} 
Comparison: \\
Target \\
Mode \\
Components \\
Frequency \\
Duration \\
Delivery
\end{tabular} & \begin{tabular}{|l} 
Setting \\
Design \\
Clusters \\
$N$ \\
Randomized
\end{tabular} & \begin{tabular}{|l|} 
PLWD \\
Dementia \\
Characteristics \\
Type(s) \\
Severity \\
Diagnostic Criteria \\
Age Diagnosed
\end{tabular} & \begin{tabular}{|l|} 
PLWD \\
N \\
Age (mean) \\
Female \% \\
Race (\% \\
majority) \\
Detailed Race \\
Education \\
(mean years)
\end{tabular} & \begin{tabular}{|l} 
PLWD \\
SES \\
Prior Disability \\
Household \\
Characteristics \\
Health Insurance
\end{tabular} & $\begin{array}{l}\text { Informal } \\
\text { Caregiver } \\
\mathrm{N} \\
\text { Age (mean) } \\
\text { Female \% } \\
\text { Race (\% } \\
\text { majority) } \\
\text { Education } \\
\text { (mean years) } \\
\text { Relationship }\end{array}$ & \begin{tabular}{|l} 
Informal \\
Caregiver (IC) \\
Duration \\
Live With PLWD \\
IC Paid \\
Health Status \\
Dementia \\
Family History \\
Employment \\
IC Training
\end{tabular} & \begin{tabular}{|l} 
Formal \\
Caregiver (FC) \\
Characteristics \\
FC N \\
FC Age (mean) \\
FC Sex (\% \\
female) \\
FC Race (\% \\
majority) \\
FC Education \\
(mean \\
years)tics
\end{tabular} & \begin{tabular}{|l|} 
Formal \\
Caregiver \\
(FC) Char. \\
RS \\
\\
FC Health \\
Status \\
FC Training \\
FC \\
Education \\
FC Position \\
FC Length \\
of Service \\
\end{tabular} & \begin{tabular}{|l} 
Outcome \\
Timing
\end{tabular} & \begin{tabular}{|l} 
PLWD Outcomes \\
Quality of Life \\
Daily Function \\
Mobility \\
BPSD \\
Adverse Events \\
Services \\
Other
\end{tabular} & \begin{tabular}{|l|} 
Informal \\
Caregiver \\
Outcomes
\end{tabular} \\
\hline $\begin{array}{l}\text { Chen } 2019^{3} \\
30301358 \\
\text { China } \\
\text { Medium } \\
\text { Explanatory }\end{array}$ & \begin{tabular}{|l|} 
PLWD \\
Individual \\
30 min., 3x/week \\
6 months \\
Supervision: 1:1 with \\
trained Master's level RN
\end{tabular} & \begin{tabular}{|l} 
PLWD \\
Routine care
\end{tabular} & $\begin{array}{l}\text { Nursing Home } \\
\text { RCT } \\
\text { Clusters: NA } \\
\mathrm{N}=60\end{array}$ & $\begin{array}{l}\text { Type: } 100 \% \text { AD } \\
\text { Severity: mild- } \\
\text { moderate (mean } \\
\text { MMSE=18) } \\
\text { Diagnosis: ISCDRHP- } \\
\text { 10th edition } \\
\text { Age diagnosed: NR }\end{array}$ & \begin{tabular}{|l|}
$\mathrm{N}=60$ \\
82 years \\
$72 \%$ female \\
Race: NR \\
Education: NR
\end{tabular} & NR & NR & NR & NR & NR & 6 months & $\begin{array}{l}\text { QoL: NR } \\
\text { Function: EdFED, } \\
\text { Time autonomous } \\
\text { eating (min.) } \\
\text { Mobility: NR } \\
\text { BPSD: NR } \\
\text { AE: NR } \\
\text { Services: NR } \\
\text { Other: Eating } \\
\text { metrics } \\
\end{array}$ & NR \\
\hline $\begin{array}{l}\text { Huang } 2019^{4} \\
31743998 \\
\text { China } \\
\text { Medium } \\
\text { Explanatory }\end{array}$ & \begin{tabular}{|l|} 
PLWD \\
Group \\
Tai Chi \\
20 min., 3x/week \\
10 months \\
Supervision: professional \\
therapists in LTC \\
\end{tabular} & \begin{tabular}{|l|} 
PLWD \\
Routine care
\end{tabular} & $\begin{array}{l}\text { Nursing } \\
\text { Homes (3) } \\
\text { RCT } \\
\text { Clusters: NA } \\
\mathrm{N}=80\end{array}$ & \begin{tabular}{|l} 
Type: NR \\
Severity: mild (mean \\
MMSE=21) \\
Diagnosis: DSM-IV \\
Age diagnosed: NR
\end{tabular} & \begin{tabular}{|l|}
$\mathrm{N}=80$ \\
82 years \\
$68 \%$ female \\
Race: NR \\
Education: 72 \\
months
\end{tabular} & NR & NR & NR & NR & NR & $\begin{array}{l}5 \text { months, } \\
10 \text { months }\end{array}$ & $\begin{array}{l}\text { QoL: NR } \\
\text { Function: Barthel } \\
\text { Mobility: NR } \\
\text { BPSD: NPI, GDS } \\
\text { AE: AE, falls } \\
\text { Services: NR } \\
\text { Other: cognitive }\end{array}$ & NR \\
\hline $\begin{array}{l}\text { Lamb 2018 } \\
\text { 29769247 } \\
\text { UK } \\
\text { Low } \\
\text { Explanatory } \\
\text { Petrou 201922 } \\
\text { Lamb 2018 }\end{array}$ & $\begin{array}{l}\text { PLWD } \\
\text { Group sessions in gym: } \\
\text { moderate-high intensity } \\
\text { aerobic (cycling) \& strength } \\
\text { exercises (dumb bells) + } \\
\text { UC } \\
1-1.5 \text { hours, 2x/week } \\
4 \text { months; then advised } \\
\text { home exercise } \\
\text { Supervision: PT and } \\
\text { assistant }\end{array}$ & $\begin{array}{l}\text { Mostly PLWD } \\
\text { Individual } \\
\text { Usual care } \\
\text { (activity advice, } \\
\text { prescriptions for } \\
\text { symptoms; } \\
\text { counseling for IC) }\end{array}$ & $\begin{array}{l}\text { Community: } \\
\text { Groups in } \\
\text { gym; } \\
\text { Individual at } \\
\text { home. } \\
\text { RCT } \\
\text { Clusters: NA } \\
\mathrm{N}=494\end{array}$ & \begin{tabular}{|l|} 
Type: NR \\
Severity: mild- \\
moderate (mean \\
MMSE=22) \\
Diagnosis: DSM-IV \\
Age diagnosed: NR
\end{tabular} & \begin{tabular}{|l}
$\mathrm{N}=494$ \\
77 years \\
39\% female \\
97\% white \\
More race: no \\
Education: NR
\end{tabular} & NR & $\begin{array}{l}\mathrm{N}=459 \\
69 \text { years } \\
77 \% \text { female } \\
\text { Race: NR } \\
\text { Education: NR } \\
\text { Relation: } 78 \% \\
\text { spouse }\end{array}$ & NR & NA & NA & \begin{tabular}{|l}
6 months, \\
12 months
\end{tabular} & $\begin{array}{l}\text { QoL: EQ-5D-3L, } \\
\text { QOL-AD } \\
\text { Function: BADLS } \\
\text { Mobility: NR } \\
\text { BPSD: NPI } \\
\text { AE: AE, SAE (fall, } \\
\text { hospitalization, } \\
\text { fracture, death) } \\
\text { Services: health } \\
\text { service use } \\
\text { Other: ADAS-cog }\end{array}$ & $\begin{array}{l}\text { QOL: EQ- } \\
\text { 5D-3L } \\
\text { Burden: ZBI }\end{array}$ \\
\hline
\end{tabular}




\begin{tabular}{|c|c|c|c|c|c|c|c|c|c|c|c|c|c|}
\hline $\begin{array}{l}\text { Bossers 2016 } \\
27321604 \\
\text { Medium } \\
\text { Netherlands } \\
\text { Explanatory } \\
\text { Bossers, 201523, } \\
\text { Bossers 2014 }{ }^{24}\end{array}$ & $\begin{array}{l}\text { PLWD } \\
\text { Group } \\
\text { 1. Aerobic (walking) plus } \\
\text { strength training } \\
30 \text { min, 4x/week (2 weeks } \\
\text { strength, } 2 \text { walking) } \\
9 \text { weeks (total } 36 \text { ) } \\
\text { 2. Aerobic (walking) } \\
30 \text { min, 4x/week } \\
9 \text { weeks } \\
\text { Supervision: Trained RA } \\
\text { (HMS student) }\end{array}$ & $\begin{array}{l}\text { PLWD } \\
\text { Group } \\
\text { Social activity } \\
4 \times / \text { week } \\
9 \text { weeks }\end{array}$ & $\begin{array}{l}\text { Nursing } \\
\text { Homes; } \\
\text { Psycho- } \\
\text { geriatric } \\
\text { RCT } \\
\text { Clusters: NA } \\
\mathrm{N}=118\end{array}$ & $\begin{array}{l}\text { Type: AD, VD, mixed } \\
\text { AD-VD, NR } \\
\text { Severity: mild } 22 \%, \\
\text { moderate } 53 \% \text {, severe } \\
25 \% \text { (of } n=105 \text { ) } \\
\text { Diagnosis: Chart } \\
\text { diagnosis } \\
\text { Age diagnosed: NR }\end{array}$ & \begin{tabular}{l|}
$\mathrm{N}=118$ \\
86 years \\
$26 \%$ female \\
Race: NR \\
More race: no \\
Education: mean \\
NR; $60 \% \leq$ high \\
school
\end{tabular} & NR & $\mathrm{NR}$ & $\mathrm{NR}$ & NA & NA & 9 weeks & \begin{tabular}{l|} 
QoL: NR \\
Function: Katz ADL, \\
E-ADL, PPT-7 \\
Mobility: NR \\
BPSD: NR \\
AE: NR \\
Services: NR \\
Other: NR
\end{tabular} & NR \\
\hline $\begin{array}{l}\text { Hoffman 2016 } \\
\text { 26682695 } \\
\text { Denmark } \\
\text { Low } \\
\text { Explanatory } \\
\text { Sobol 201625 } \\
\text { Hoffman 2013 } 23^{26}\end{array}$ & $\begin{array}{l}\text { PLWD } \\
\text { Group } \\
\text { Supervised exercise } \\
\text { [strength } 1^{\text {st }} 4 \text { weeks; } \\
\text { aerobic } \times 16 \text { weeks (bike } \\
\text { ergometer, cross trainer, } \\
\text { treadmill)] } \\
60 \text { min, 3x/week } \\
16 \text { weeks } \\
\text { Suppervision: PT }\end{array}$ & $\begin{array}{l}\text { PLWD } \\
\text { Usual care } \\
\text { Individual } \\
\text { As needed } \\
\text { (access to } \\
\text { memory clinic } \\
\text { staff) }\end{array}$ & $\begin{array}{l}\text { NR (PLWD all } \\
\text { community- } \\
\text { dwelling) } \\
\text { Cluster: NA } \\
\mathrm{N}=200\end{array}$ & $\begin{array}{l}\text { 100\% probable AD } \\
\text { Severity: mild (MMSE } \\
\text { > 19) } \\
\text { Diagnosis: NINCDS- } \\
\text { ADRDA } \\
\text { Diagnosis age: NR }\end{array}$ & $\begin{array}{l}\mathrm{N}=200 \\
71 \text { years } \\
44 \% \text { female } \\
\text { Race: NR } \\
\text { More race: NA } \\
\text { Education: NR }\end{array}$ & NR & $\begin{array}{l}\mathrm{N}=\mathrm{NR}, \\
\text { assume } 200 \\
\text { (inclusion } \\
\text { criterion) } \\
\text { All: NR }\end{array}$ & $\begin{array}{l}\text { Live together: } \\
72 \% \\
\text { Other: NR }\end{array}$ & NA & NA & 16 weeks & \begin{tabular}{l|} 
QoL: EQ-5D-5L \\
Function: ADCS- \\
ADL \\
Mobility: NR \\
BPSD: NPI-12, \\
HAM-D \\
AE: AE, SAE \\
Services: NR \\
Other: ADAS-Cog, \\
MMSE, adherence
\end{tabular} & $\begin{array}{l}\text { NR } \\
\text { (ICG proxy } \\
\text { for NPI-12, } \\
\text { ADCSADL } \\
\text { and 1 EQ- } \\
\text { 5D) }\end{array}$ \\
\hline $\begin{array}{l}\text { Toots 201613 } \\
26782852 \\
\text { Sweden } \\
\text { Medium } \\
\text { Explanatory } \\
\text { Sondell 2018 } \\
\text { Toots } 2018^{28} \\
\text { Toots 201729 } \\
\text { Bostrom 2016 }\end{array}$ & $\begin{array}{l}\text { PLWD } \\
\text { Groups at RC facilities } \\
\text { Individualized high- } \\
\text { intensity functional (weight- } \\
\text { bearing) exercise (HIFE): } \\
\text { leg strength, balance, } \\
\text { mobility } \\
5 \times 45 \text { min per } 2 \text { weeks } \\
4 \text { months (40 sessions) } \\
\text { Supervision: } 2 \text { PTs }\end{array}$ & $\begin{array}{l}\text { PLWD } \\
\text { Seated group } \\
\text { activity per OT } \\
\text { Frequency: NR } \\
\text { Duration: NR } \\
\text { Supervision: OT } \\
\text { or OTA }\end{array}$ & \begin{tabular}{l|}
16 residential \\
care facilities \\
(9 NH units, 10 \\
dementia units \\
Cluster RCT \\
Clusters: 36 \\
$\mathrm{~N}=186$
\end{tabular} & $\begin{array}{l}\text { 86\% AD, VD, or mixed } \\
\text { AD-VD } \\
\text { Severity: mild- } \\
\text { moderate (mean } \\
\text { MMSE=15) } \\
\text { Diagnosis: DSM-IV-TR } \\
\text { Age diagnosed: NR }\end{array}$ & $\begin{array}{l}\mathrm{N}=186 \\
85 \text { years } \\
76 \% \text { female } \\
\text { Race: NR } \\
\text { More race: NA } \\
\text { Education: NR }\end{array}$ & NR & NA & NA & NA & NA & $\begin{array}{l}4 \text { months, } 7 \\
\text { months }\end{array}$ & $\begin{array}{l}\text { QoL: NR } \\
\text { Function: Barthel } \\
\text { ADL FIM } \\
\text { Mobility: BBS } \\
\text { BPSD: NR } \\
\text { AE: collected/NR } \\
\text { (except 1 death) } \\
\text { Services: NR } \\
\text { Other: adherence }\end{array}$ & NR \\
\hline $\begin{array}{l}\text { Telenius 2015 } \\
26630910 \\
\text { Norway } \\
\text { Medium } \\
\text { Explanatory }\end{array}$ & $\begin{array}{l}\text { PLWD } \\
\text { Group } \\
\text { Individualized high- } \\
\text { intensity functional (weight- } \\
\text { bearing) exercise (HIFE): } \\
50-60 \text { min, 2x/week } \\
12 \text { weeks } \\
\text { Supervision: } 1 \text { PT per } 3 \\
\text { PLWD }\end{array}$ & $\begin{array}{l}\text { PLWD } \\
\text { Group } \\
\text { Seated activity } \\
\text { (game, music, } \\
\text { read, stretch) } \\
\text { 50-60 min, } \\
\text { 2x/week } \\
12 \text { weeks } \\
\text { Supervision: OT } \\
\text { or nurse }\end{array}$ & \begin{tabular}{l|}
$18 \mathrm{NHs}$ \\
$\mathrm{RCT}$ \\
Block \\
randomized (6- \\
12 PLWD/site) \\
$\mathrm{N}=170$
\end{tabular} & $\begin{array}{l}\text { NR } \\
\text { Severity: Mild- } \\
\text { moderate (CDR } 1 \text { or } \\
\text { 2) } \\
\text { Diagnosis: NR } \\
\text { Age diagnosed: NR }\end{array}$ & $\begin{array}{l}\mathrm{N}=170 \\
87 \text { years } \\
74 \% \text { female } \\
\text { Race: NR } \\
\text { More race: NA } \\
\text { Education: NR }\end{array}$ & NR & $\mathrm{NA}$ & NA & $\mathrm{NA}$ & NA & $\begin{array}{l}12 \text { weeks, } 6 \\
\text { months }\end{array}$ & $\begin{array}{l}\text { QoL: QUALID } \\
\text { Function: Barthel } \\
\text { ADL } \\
\text { Mobility: BBS } \\
\text { BPSD: NPI, CSD } \\
\text { AE: AE } \\
\text { Services: NR } \\
\text { Other: MMSE, } \\
\text { attendance, CST,6- } \\
\text { meter walk }\end{array}$ & $\mathrm{NR}$ \\
\hline $\begin{array}{l}\text { Pitkala 201316 } \\
\text { 23589097 } \\
\text { Finland } \\
\text { Medium } \\
\text { Explanatory } \\
\text { Roitto 2018 } \\
\text { Ohman 2016 } \\
\text { Ohman 2016 } \\
\text { Pertilla 2016 } 2016^{34} \\
\text { Pitkala 2011 }\end{array}$ & $\begin{array}{l}\text { PLWD } \\
\text { 1. Individual tailored home- } \\
\text { based exercise (various) } \\
1 \text { hour, 2x/week } \\
12 \text { weeks } \\
\text { 2. Pre-determined group } \\
\text { exercise (balance strength, } \\
\text { endurance, executive } \\
\text { function) } \\
1 \text { hour, } 2 \times / \text { week } \\
12 \text { weeks } \\
\text { Supervision: PT; ICG } \\
\text { participation optional }\end{array}$ & $\begin{array}{l}\text { PLWD } \\
\text { Usual care plus } \\
\text { nutrition \& } \\
\text { exercise advice } \\
\text { per study nurses }\end{array}$ & \begin{tabular}{l|} 
Home \\
(individual), \\
adult day care \\
centers \\
(group) \\
RCT \\
Clusters: NA \\
$\mathrm{N}=210$
\end{tabular} & $\begin{array}{l}100 \% \text { AD } \\
67 \% \text { moderate-severe } \\
\text { Diagnosis: NINCDS- } \\
\text { ADRDA } \\
\text { Age diagnosed: NR }\end{array}$ & $\begin{array}{l}\mathrm{N}=210 \text { (dyads) } \\
78 \text { years } \\
39 \% \% \text { female } \\
100 \% \text { white } \\
\text { More race: NA } \\
\text { Education: mean } \\
\text { NR; } 41 \% \text { - } 12 \\
\text { years; } 38 \%<8 \\
\text { years }\end{array}$ & NR & $\begin{array}{l}\mathrm{N}=210 \\
76 \text { years } \\
61 \% \text { female } \\
100 \% \text { white } \\
\text { Education: } \\
\text { mean NR; } \\
54 \% \text { 8 }-12 \\
\text { years; } 26 \%<8 \\
\text { years } \\
\text { Relation: } \\
100 \% \text { spouse }\end{array}$ & $\begin{array}{l}\text { Duration: NR } \\
\text { Live together: } \\
100 \% \\
\text { Paid: NR } \\
\text { Health: count of } \\
\text { medications, } \\
\text { Charlson Index } \\
\text { Family history: } \\
\text { NR } \\
\text { Employed: NR } \\
\text { Training: NR }\end{array}$ & NA & NA & $\begin{array}{l}6 \text { months } \\
\text { (12 } \\
\text { months=high } \\
\text { RoB) }\end{array}$ & \begin{tabular}{l|} 
QoL: NR \\
Function: FIM \\
Mobility: SPPB \\
BPSD: NR \\
AE: falls, fractures, \\
hospitalizations \\
Services: health \& \\
social service use, \\
costs \\
Other: Adherence
\end{tabular} & NR \\
\hline
\end{tabular}




\begin{tabular}{|c|c|c|c|c|c|c|c|c|c|c|c|c|c|}
\hline $\begin{array}{l}\text { Hauer } 2012^{17} \\
22211512 \\
\text { Germany } \\
\text { Medium } \\
\text { Explanatory } \\
\text { Pomiersky } \\
2020^{36}\end{array}$ & \begin{tabular}{|l|} 
PLWD \\
Group \\
Progressive resistance \& \\
lower extremity functional \\
training (walk, stair climb, \\
sit to stand)) \\
2 hours, 2x/week \\
3 months \\
Supervision: "a qualified \\
instructor" \\
\end{tabular} & $\begin{array}{l}\text { PLWD } \\
\text { Group } \\
\text { Low-intensity } \\
\text { activity (stretch, } \\
\text { hand weights, } \\
\text { seated ball } \\
\text { games, etc.) } \\
1 \text { hour, 2x/week } \\
3 \text { months } \\
\text { Supervisor: Yes } \\
\text { but NR } \\
\end{array}$ & \begin{tabular}{|l|} 
Outpatient \\
geriatric \\
rehabilitation \\
RCT \\
Clusters: NA \\
$\mathrm{N}=122$
\end{tabular} & \begin{tabular}{|l|} 
Type: 50\% AD, 34\% \\
VD, 16\% other AD/VD \\
Severity: mean MMSE \\
22 (mild) \\
Diagnosis: NINCDS- \\
ADRDA or NINDS- \\
AlREN, CERAD, TMT \\
Diagnosis age: NR
\end{tabular} & \begin{tabular}{|l|}
$\mathrm{N}=122$ \\
83 years \\
$74 \%$ female \\
Race: NR \\
More race: NA \\
Education: 11 \\
years
\end{tabular} & $\begin{array}{l}\text { 84\% community- } \\
\text { dwelling } \\
\text { Other: NR }\end{array}$ & NR & NR & NA & NA & $\begin{array}{l}3 \text { months, } 6 \\
\text { months }\end{array}$ & \begin{tabular}{|l|} 
QOL: NR \\
Function: NR \\
Mobility: TUG, \\
POMA, modified \\
PAQE, modified \\
SPPB (SPPB NR) \\
AE: AE (text) \\
Services: NR \\
Other: strength (lab \\
metrics), \\
adherence \\
\end{tabular} & \begin{tabular}{|l|l|} 
NR \\
\end{tabular} \\
\hline \begin{tabular}{|l|} 
Eggermont \\
$2009^{20}$ \\
18926856 \\
Netherlands \\
Medium \\
Explanatory
\end{tabular} & \begin{tabular}{|l|} 
PLWD \\
Group (by living unit): Hand \\
movement program \\
(fingers, pinch ball, handle \\
rubber ring) \\
30 min, 5x/week \\
6 weeks \\
Supervision: RT or Master \\
students
\end{tabular} & $\begin{array}{l}\text { PLWD } \\
\text { Group } \\
\text { Stories read by } \\
\text { RT followed by } \\
\text { conversation } \\
30 \text { min, 5x/week } \\
6 \text { weeks } \\
\text { Supervision: RT } \\
\text { or student }\end{array}$ & $\begin{array}{l}\text { NHs (10 } \\
\text { psychogeriatric } \\
\text { units) } \\
\text { Cluster RCT } \\
\text { Clusters: } 10 \\
\text { N=66 }\end{array}$ & $\begin{array}{l}\text { Type: uncertain } \\
\text { Severity: mean } \\
\text { MMSE=16 } \\
\text { Diagnosis: DSM-IV } \\
\text { Age diagnosed: NR }\end{array}$ & \begin{tabular}{|l|}
$\mathrm{N}=66$ \\
85 years \\
Female\%: NR \\
Race: NR \\
Education: NR \\
but “NSD"
\end{tabular} & \begin{tabular}{|l|}
$N R$ \\
\end{tabular} & NA & NA & NA & NA & $\begin{array}{l}\begin{array}{l}6 \text { weeks, } 12 \\
\text { weeks }\end{array} \\
\end{array}$ & \begin{tabular}{|l} 
QoL: NR \\
Function: NR \\
Mobility: NR \\
BPSD: GDS, SCL- \\
90 (anxiety) \\
AE: NR \\
Services: NR \\
Other: cognition, \\
actigraphy
\end{tabular} & NR \\
\hline
\end{tabular}

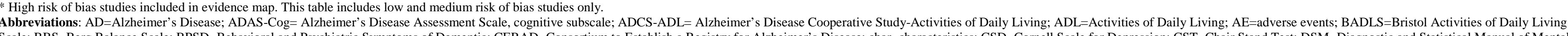
Disorders; E-ADL=Erlangen-ADL scale; EdFED=Edinburgh Feeding Evaluation in Dementia; EQ-5D-5L=EuroQol 5 dimension- 5 level; EQ-5D-3L=EuroQol 5 dimension-3 level; ET=exercise therapist; FC=formal caregiver; FIM=Functional Independence Measure; FINALEX=Finnish

Alzheimer's Disease Exercise Trial; GDS=Geriatric Depression Scale; GHQ=Global Health Questionnaire; Govt.=Government; HAM-D=Hamilton Depression Rating Scale; HIFE=high-intensity functional exercise; HMS=Human Movement Science; IC=informal caregiver:

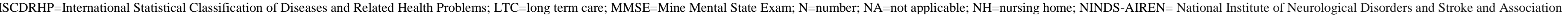

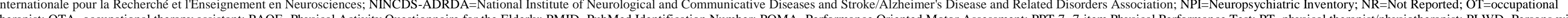

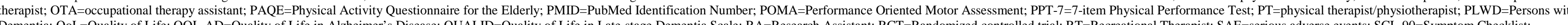
SPPB=Short Physical Performance Battery; TMT=Trail Making Test; TUG=Timed Up and Go; VD=vascular dementia; wk=week; yrs=years; ZBI=Zarit Burden Interview

Table D-3. PWD outcomes summary low and medium risk of bias studies: exercise

\begin{tabular}{|c|c|c|c|c|c|}
\hline $\begin{array}{l}\text { Study (PMID) } \\
\text { Comparison } \\
\text { RoB } \\
\text { Category }\end{array}$ & $\begin{array}{l}\text { Outcome } \\
\text { Timing }\end{array}$ & Summary Finding & Intervention & Comparator & $p$-Value \\
\hline $\begin{array}{l}\text { Chen } 2019^{3} \\
30301358 \\
\text { China } \\
\text { Medium } \\
\text { Explanatory }\end{array}$ & \begin{tabular}{|l|} 
EdFED (proxy) \\
Mean (SD) \\
Time of autonomous eating (minutes) \\
Mean (SD) \\
6 months \\
\end{tabular} & $\begin{array}{l}\text { Change from baseline } \\
\text { Change from baseline }\end{array}$ & \begin{tabular}{|l|}
$-0.63(0.8)$ \\
$2.13(3.5)$
\end{tabular} & $\begin{array}{l}-0.10(0.5) \\
0.29(0.4)\end{array}$ & $\begin{array}{l}<0.05 \\
<0.05\end{array}$ \\
\hline $\begin{array}{l}\text { Huang } 2019^{4} \\
31743998 \\
\text { Tai Chi vs. Usual Care } \\
\text { Medium. } \\
\text { Explanatory }\end{array}$ & \begin{tabular}{|l|} 
Barthel ADL \\
GDS \\
NPI \\
10 months
\end{tabular} & $\begin{array}{l}\text { Mean (SD) } \\
\text { Mean (SD) } \\
\text { Mean (SD) }\end{array}$ & \begin{tabular}{|l|}
$94.1(11.6)$ \\
$2.4(1.0)$ \\
$6.4(4.8)$
\end{tabular} & \begin{tabular}{|l|}
$92.6(13.3)$ \\
$5.4(1.9)$ \\
$10.2(5.7)$
\end{tabular} & \begin{tabular}{|l|} 
NR \\
$<0.05$ \\
$<0.05$
\end{tabular} \\
\hline $\begin{array}{l}\text { Lamb 2018 } \\
(29769247) \\
\text { Group Exercise (aerobic + strength } \\
\text { training) vs. Usual Care } \\
\text { Low } \\
\text { Explanatory }\end{array}$ & $\begin{array}{l}\text { QOL-AD (self-report) } \\
\text { Mean (SD) } \\
\text { EQ-5D-3L (self-report) } \\
\text { Mean (SD) } \\
\text { BADLS (proxy) } \\
\text { Mean (SD) } \\
\text { NPI (proxy) } \\
\text { Median (IQR) } \\
\text { 6 months, } 12 \text { months } \\
\end{array}$ & $\begin{array}{l}\text { Adjusted mean difference }(\mathrm{CI}) 0.7(-0.2,1.7) \\
\text { Adjusted mean difference }(\mathrm{CI})-0.002(-0.04,0.04) \\
\text { Adjusted mean difference }(\mathrm{CI}) 0.3(-1.7,1.2) \\
\text { Adjusted median difference }(\mathrm{Cl})-2.1(-4.8,0.7)\end{array}$ & \begin{tabular}{|l|}
12 months \\
$38.4(5.8)$ \\
$0.81(0.22)$ \\
$17.0(10.2)$ \\
$12(4$ to 23$)$
\end{tabular} & $\begin{array}{l}12 \text { months } \\
39.1(5.7) \\
0.82(0.25) \\
15.9(9.7) \\
9(3 \text { to } 20)\end{array}$ & $\begin{array}{l}0.13 \\
0.93 \\
0.70 \\
0.14\end{array}$ \\
\hline
\end{tabular}




\begin{tabular}{|c|c|c|c|c|c|}
\hline $\begin{array}{l}\text { Bossers } 2016^{10} \\
\text { (27321604) } \\
\text { Group (walking + strength training) } \\
\text { vs. Walking vs. Social Activity } \\
\text { Medium } \\
\text { Explanatory }\end{array}$ & $\begin{array}{l}\text { Katz ADL (proxy) } \\
\text { Between group mean change from baseline } \\
\text { E-ADL (RA) } \\
\text { Between group mean change from baseline } \\
\text { PPT-7 (RA) } \\
\text { Between group mean change from baseline } \\
9 \text { weeks }\end{array}$ & $\begin{array}{l}\text { Combined vs social }-2.79 \\
\text { Walking vs social }-0.99 \\
\text { Combined vs social }-3.83 \\
\text { Walking vs social }-2.92 \\
\text { Combined vs social }-3.38 \\
\text { Walking vs social }-1.66\end{array}$ & $\mathrm{NR}$ & NR & \begin{tabular}{|l|l}
0.01 \\
0.97 \\
$<0.001$ \\
0.01 \\
0.003 \\
0.29
\end{tabular} \\
\hline $\begin{array}{l}\text { Hoffman 201612 } \\
\text { (26682695) } \\
\text { Group Exercise (aerobic + initial } \\
\text { strength training) vs. Usual Care } \\
\text { Low } \\
\text { Explanatory }\end{array}$ & $\begin{array}{l}\text { EQ-5D-3L (self-report) } \\
\text { Mean (SD) } \\
\text { ADCS-ADL (proxy) } \\
\text { Mean (SD) } \\
\text { NPI-12 (proxy) } \\
\text { Mean (SD) } \\
\text { HAM-D: Depression } \\
\text { Mean (SD) } \\
16 \text { weeks }\end{array}$ & $\begin{array}{l}\text { Mean difference }(\mathrm{Cl})-0.1(-0.02,0.04) \\
\text { Mean difference }(\mathrm{Cl})-0.1(-1.8,1.5) \\
\text { Mean difference }(\mathrm{Cl})-3.5(-5.8,-1.3) \\
\text { Mean difference }(\mathrm{Cl})-0.1(-0.7,0.5)\end{array}$ & $\begin{array}{l}0.92(0.11) \\
64.4(9.4) \\
8.8(8.5) \\
1.7(2.5)\end{array}$ & $\begin{array}{l}0.92(0.09) \\
62.7(10.4) \\
11.4(11.0) \\
1.8(2.3\end{array}$ & $\begin{array}{l}0.40 \\
0.88 \\
0.002 \\
0.79\end{array}$ \\
\hline $\begin{array}{l}\text { Toots 201613 } \\
\text { (26782852) } \\
\text { HIFE (leg } \\
\text { strength, balance, mobility) vs. } \\
\text { seated group activities } \\
\text { Medium } \\
\text { Explanatory }\end{array}$ & $\begin{array}{l}\text { Barthel ADL } \\
\text { Mean (SE) } \\
\text { FIM } \\
\text { Mean (SE) } \\
\text { BBS (SE (SE) } \\
\text { Mean (SE) } \\
4 \text { month, 7 months }\end{array}$ & $\begin{array}{l}\text { Mean difference }(\mathrm{Cl}) 0.57(-0.30,1.43) \\
\text { Mean difference }(\mathrm{Cl}) 0.78(-2.21,3.77) \\
\text { Mean difference }(\mathrm{Cl})-0.02(-2.53,2.49)\end{array}$ & $\begin{array}{l}-1.56(0.32) \\
-6.77(1.09) \\
-2.08(0.91)\end{array}$ & $\begin{array}{l}-2.12(0.32) \\
-7.55(1.08) \\
-2.05(0.90)\end{array}$ & $\begin{array}{l}0.20 \\
0.61 \\
0.98\end{array}$ \\
\hline $\begin{array}{l}\text { Telenius 201514 } \\
(26630910) \\
\text { HIFE (strength + balance) vs. } \\
\text { seated group activity } \\
\text { Medium } \\
\text { Explanatory }\end{array}$ & $\begin{array}{l}\text { QUALID } \\
\text { Between group difference } \\
\text { Barthel ADL } \\
\text { Between group difference } \\
\text { Berg Balance Scale } \\
\text { Mean change from baseline } \\
\text { NPI } \\
\text { Mean (CI) } \\
\text { CSD } \\
\text { Between group difference } \\
3 \text { months, } 6 \text { months }\end{array}$ & $\begin{array}{l}-0.9 \text { points } \\
1.0 \text { points } \\
\text { NR } \\
\text { Between group difference }-1.6 \\
\text { Between group difference } 0.2 \text { points }\end{array}$ & $\begin{array}{l}\mathrm{NR} \\
\mathrm{NR} \\
+2.7 \text { points } \\
4.8(3.8,5.8) \\
\mathrm{NR}\end{array}$ & $\begin{array}{l}\text { NR } \\
\text { NR } \\
-1.4 \text { points } \\
6.4(5.1,7.7) \\
\text { NR }\end{array}$ & \begin{tabular}{|l}
$\mathrm{NR}$ \\
$\mathrm{NR}$ \\
0.031 \\
0.059 \\
$\mathrm{NR}$
\end{tabular} \\
\hline $\begin{array}{l}\text { Pitkala } 2013^{16} 23589097 \\
\text { Medium (6 months) } \\
\text { Explanatory }\end{array}$ & $\begin{array}{l}\text { FIM } \\
\text { Within-group mean change from baseline } \\
6 \text { months } \\
\text { FIM } \\
\text { Between-group change from baseline } \\
6 \text { months } \\
\text { Adverse events: } 12 \text { months } \\
\text { Falls } \\
\text { Incidence rate }(95 \% \mathrm{Cl}) \\
\text { Fractures }(\text { any }) \\
\text { Incidence rate }(95 \% \mathrm{Cl}) \\
\text { Hospitalizations } \\
\text { Incidence rate }(95 \% \mathrm{Cl}) \\
\text { Health service costs }(24 \text { months): } \\
\text { Adjusted mean }(95 \% \mathrm{Cl}) \text { per dyad per year, US dollars }\end{array}$ & $\begin{array}{l}\text { NR } \\
\text { NR } \\
\text { NR } \\
\text { NR } \\
\text { NR } \\
\text { NR }\end{array}$ & $\begin{array}{l}\text { Home exercise: } \\
-6.5(-4.4,-8.6) \\
\text { Group exercise: }-8.9 \\
(-6.7,-11.2) \\
\\
\text { Home: } 1.35(1.07,1.67) \\
\text { Group: } 1.86(1.51,2.26) \\
\text { Home: } 0.06(0.02,0.17) \\
\text { Group: } 0.09(0.03,0.21) \\
\text { Home: } 0.47(0.31,0.68) \\
\text { Group: } 0.54(0.37,077) \\
\text { Home exercise: } \\
\$ 25,112 \\
(\$ 17,642, \$ 32,581) \\
\text { Group exercise: } \\
\$ 22,066 \\
(\$ 15,931, \$ 28,199)\end{array}$ & $\begin{array}{l}-11.8(-9.7,-14.0) \\
\text { UC: } 3.07(2.63,3.57) \\
\text { UC: } 0.07(0.02,0.18) \\
\text { UC: } 0.65(0.46,0.90) \\
\text { UC: } \$ 34,121 \\
\text { (\$24,599, } \$ 43,681)\end{array}$ & $\begin{array}{l}\text { Mixed-effect } \\
\text { model } p=0.003 . \\
\text { Home exercise } \\
\text { vs. UC: } p=0.001 \\
\text { Group exercise } \\
\text { vs UC: } p=0.07 \\
p=0.005 \\
p=0.88 \\
\\
p=0.63 \\
\\
\text { Home vs. UC: } \\
p=0.13 \\
\text { Group vs. UC: } \\
p=0.03\end{array}$ \\
\hline $\begin{array}{l}\text { Hauer 201217 } \\
(22211512) \\
\text { Group resistance \& functional } \\
\text { training vs low intensity motor } \\
\text { activity } \\
\text { Medium } \\
\text { Explanatory }\end{array}$ & $\begin{array}{l}\text { TUG } \\
\text { Mean percent change from baseline (SD) } \\
\text { POMA } \\
\text { Mean percent change from baseline (SD) } \\
\text { Modified PAQE } \\
\text { Mean percent change from baseline (SD) } \\
3 \text { months, } 6 \text { months } \\
\end{array}$ & $\begin{array}{l}\text { Effect size } 0.07 \\
\text { Effect size } 0.15 \\
\text { Effect size } 0.002\end{array}$ & $\begin{array}{l}-11.2(28.1) \\
22.2(28.4) \\
134.5(274.5)\end{array}$ & \begin{tabular}{|l|}
$-1.4(26.3)$ \\
$0.8(23.5)$ \\
$101.1(178.6)$
\end{tabular} & $\begin{array}{l}0.009 \\
<0.001 \\
0.64\end{array}$ \\
\hline
\end{tabular}




\begin{tabular}{|c|c|c|c|c|c|}
\hline $\begin{array}{l}\text { Eggermont } 2009^{20} \\
(18926856) \\
\text { Group hand movement program vs. } \\
\text { read aloud control } \\
\text { Medium } \\
\text { Explanatory }\end{array}$ & $\begin{array}{l}\text { GDS } \\
\text { Change in group mean from baseline } \\
\text { SCL-90 } \\
\text { Change in group mean from baseline } \\
12 \text { weeks, } 6 \text { months }\end{array}$ & $\begin{array}{l}\mathrm{NR} \\
\mathrm{NR}\end{array}$ & $\begin{array}{l}-1.56 \\
-2.61\end{array}$ & $\begin{array}{l}-0.17 \\
-0.69\end{array}$ & $\begin{array}{l}\mathrm{NR} \\
\mathrm{NR}\end{array}$ \\
\hline
\end{tabular}

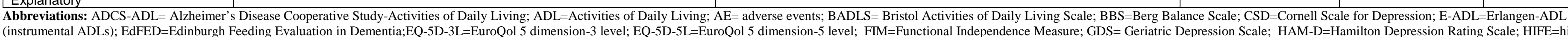

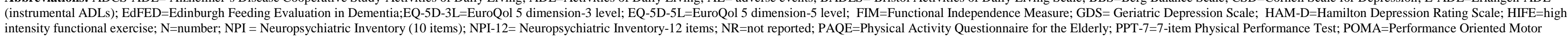
intensity functional exercise; N=number; NPI = Neuropsychiatric Inventory (10 items); NPI-12= Neuropsychiatric Inventory-12 items; NR=not reported; PAQE=Physical Activity Questionnaire for the Elderly; PPT-7=7-item Physical Performance Test; POMA=Performance Oriented Mo
Assessment (gait and balance); PMID=PubMed Identification Number; QOL-AD=Quality of Life in Alzheimer's Disease; QUALID= quality of life in late-stage dementia scale; SAE=serious adverse event; SCL-90=Symptom Checklist-90; SD=standard deviation; SPPB=Short Physical Performance Battery: TUG=Timed Up and Go; UC=usual care; vs=versus 
Table D-4. Summary of strength of evidence for PLWD outcomes: exercise

\begin{tabular}{|c|c|c|c|c|c|c|c|c|}
\hline $\begin{array}{l}\text { Outcome } \\
\text { Comparison }\end{array}$ & Timing & $\begin{array}{l}\text { \# Studies/ Design } \\
\text { ( } \mathrm{n} \text { analyzed) }\end{array}$ & Finding or Summary Statistic & Study Limitations & Consistency & Directness & \begin{tabular}{|l|} 
Precision \\
\end{tabular} & $\begin{array}{l}\text { Overall Gradel } \\
\text { Conclusion }\end{array}$ \\
\hline \begin{tabular}{|l|} 
Quality of Life: \\
Group exercise vs usual \\
care (2) or seated group \\
activity (1) \\
\end{tabular} & 3-12 months & $\begin{array}{l}\begin{array}{l}3 \mathrm{RCTs}^{8,12,14}(1 \mathrm{NH}) \\
\mathrm{n}=864\end{array} \\
\end{array}$ & $\begin{array}{l}\text { No significant difference in Quality of Life (QOL-AD, EQ-5D-5L, EQ- } \\
\text { 5D-3L or QUALID) for group exercise compared with usual care or } \\
\text { seated group activity. }\end{array}$ & Low & Inconsistent & Direct & Imprecise & Insufficient \\
\hline $\begin{array}{l}\text { Daily function: } \\
\text { Group exercise vs usual } \\
\text { care (3) or home exercise } \\
\text { (1) }\end{array}$ & 4-12 months & $\begin{array}{l}3 \mathrm{RCTS}^{8,12,16}(0 \mathrm{NH}) \\
\mathrm{n}=904\end{array}$ & $\begin{array}{l}\text { Inconsistent findings. Two RCTS found no significant difference in } \\
\text { daily functioning (BADLS, ADCS-ADL) for PLWD for group exercise } \\
\text { compared with usual care. One RCT found benefits of group } \\
\text { exercise vs usual care and for home exercise vs usual care on the } \\
\text { FIM at } 6 \text { months. }\end{array}$ & Low & Inconsistent & Direct & Imprecise & Insufficient \\
\hline \begin{tabular}{|l|} 
Daily function: \\
Group exercise vs seated \\
group activity (3)
\end{tabular} & 3-7 months & $\begin{array}{l}3 \mathrm{RCTs} \mathrm{s}^{10,13,14}(3 \mathrm{NH}) \\
\mathrm{n}=474\end{array}$ & $\begin{array}{l}\text { Inconsistent findings. Two RCTs found no significant difference in } \\
\text { daily functioning (Barthel ADL for group exercise compared with } \\
\text { seated group activity. One RCT found benefits of combined group } \\
\text { exercise vs social activity, and for walking vs. social activity on daily } \\
\text { functioning [Katz ADL, E-ADL, PPT-7 (combined only)] at } 9 \text { weeks. }\end{array}$ & Medium & Inconsistent & Direct & Imprecise & Insufficient \\
\hline \begin{tabular}{|l|} 
Daily function: \\
Tai Chi vs usual care
\end{tabular} & 10 months & $\begin{array}{l}1 \mathrm{RCT}^{4}(\mathrm{NH}) \\
\mathrm{n}=80\end{array}$ & $\begin{array}{l}\text { No significant difference in daily functioning (Barthel ADL) for Tai } \\
\text { Chi compared with usual care. }\end{array}$ & Medium & Unknown & Direct & Imprecise & Insufficient \\
\hline $\begin{array}{l}\text { Daily function: Eating } \\
\text { assistance needed. } \\
\text { Supervised } 1: 1 \text { hand } \\
\text { exercise program vs. } \\
\text { usual care }\end{array}$ & 6 months & $\begin{array}{l}1 \mathrm{RCT}^{3}(\mathrm{NH}) \\
\mathrm{n}=60\end{array}$ & $\begin{array}{l}\text { Statistically significant reduction ( }<1 \text { point) in feeding assistance } \\
\text { needed (EdFED) with individual hand exercise program vs. usual } \\
\text { care over } 6 \text { months. }\end{array}$ & Medium & Unknown & Direct & Imprecise & Insufficient \\
\hline $\begin{array}{l}\text { Daily function: Time of } \\
\text { autonomous eating (min.) } \\
\text { Supervised } 1: 1 \text { hand } \\
\text { exercise program vs. } \\
\text { usual care }\end{array}$ & 6 months & $\begin{array}{l}1 \mathrm{RCT}^{3}(\mathrm{NH}) \\
\mathrm{n}=60\end{array}$ & $\begin{array}{l}\text { Statistically significant improvement in minutes of autonomous } \\
\text { eating per meal with individual hand exercise program vs. usual } \\
\text { care over } 6 \text { months. }\end{array}$ & Medium & Unknown & Direct & Imprecise & Insufficient \\
\hline $\begin{array}{l}\text { Balance: } \\
\text { HIFE (strength + balance) } \\
\text { vs. seated group activity }\end{array}$ & 3-7 months & $\begin{array}{l}2 \mathrm{RCTS}^{13,14}(2 \mathrm{NH}) \\
\mathrm{n}=356\end{array}$ & $\begin{array}{l}\text { Inconsistent findings. One RCT found no significant difference in } \\
\text { balance (BBS) between HIFE compared with seated group activity } \\
\text { at } 3 \text { months, but beneficial effect at } 6 \text { months. One RCT found a } \\
\text { beneficial of HIFE vs. seated group activities at } 4 \text { months but not } 7 \\
\text { months. }\end{array}$ & Medium & Inconsistent & Direct & \begin{tabular}{|l|} 
Imprecise \\
\end{tabular} & Insufficient \\
\hline $\begin{array}{l}\text { Neuropsychiatric } \\
\text { symptoms: } \\
\text { Tai Chi vs usual care }\end{array}$ & 10 months & $\begin{array}{l}1 \mathrm{RCT}^{4}(\mathrm{NH}) \\
\mathrm{n}=80\end{array}$ & $\begin{array}{l}\text { Statistically significant improvement in NPI with group Tai Chi vs. } \\
\text { usual care over } 10 \text { months but not at } 5 \text { months. }\end{array}$ & Medium & Unknown & Direct & Imprecise & Insufficient \\
\hline $\begin{array}{l}\text { Neuropsychiatric } \\
\text { symptoms: } \\
\text { Group exercise vs usual } \\
\text { care (2) or seated group } \\
\text { activity (1) }\end{array}$ & 3-12 months & $\begin{array}{l}3 \mathrm{RCTS}^{8,12,14}(1 \mathrm{NH}) \\
\mathrm{n}=864\end{array}$ & $\begin{array}{l}\text { Inconsistent findings. Two RCTs found no significant difference in } \\
\text { neuropsychiatric symptoms (NPI) with group exercise vs. usual care } \\
(1 \mathrm{NH}) \text {, and one RCT reported a benefit of exercise vs. usual care } \\
\text { on the NPI-12 at } 4 \text { months. }\end{array}$ & Low & Inconsistent & Direct & Imprecise & Insufficient \\
\hline $\begin{array}{l}\text { Depression: } \\
\text { Tai Chi vs usual care }\end{array}$ & 10 months & $\begin{array}{l}1 \mathrm{RCT}^{4}(\mathrm{NH}) \\
\mathrm{n}=80\end{array}$ & $\begin{array}{l}\text { Statistically significant improvement in GDS with group Tai Chi vs. } \\
\text { usual care over } 10 \text { months but not at } 5 \text { months. }\end{array}$ & Medium & Unknown & Direct & \begin{tabular}{|l} 
Imprecise \\
\end{tabular} & Insufficient \\
\hline $\begin{array}{l}\text { Depression: } \\
\text { Group exercise vs usual } \\
\text { care (1) or seated group }\end{array}$ & 3-6 months & $\begin{array}{l}n \mathrm{RCTS}^{12,14,20}(2 \mathrm{NH}) \\
\mathrm{n}=436\end{array}$ & $\begin{array}{l}\text { No significant difference in depression (HAM-D, CSD, GDS) for } \\
\text { group exercise compared with usual care or seated group activity. }\end{array}$ & Medium & Inconsistent & Direct & Imprecise & Insufficient \\
\hline $\begin{array}{l}\text { Anxiety: } \\
\text { Group hand movement } \\
\text { program vs. attention } \\
\text { control }\end{array}$ & 3 months & $\begin{array}{l}1 \mathrm{RCT}^{20}(\mathrm{NH}) \\
\mathrm{n}=66\end{array}$ & $\begin{array}{l}\text { No significant difference in anxiety (SCL-90) with a group hand } \\
\text { movement program vs. attention control over } 3 \text { months. }\end{array}$ & Medium & Unknown & Direct & \begin{tabular}{|l|} 
Imprecise \\
\end{tabular} & Insufficient \\
\hline
\end{tabular}
program vs. attentio

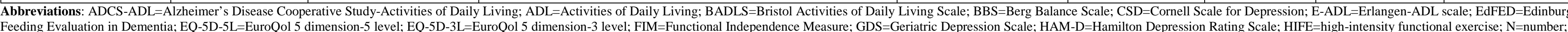

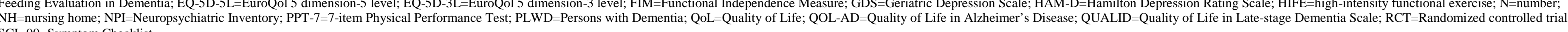

\section{Evidence Map: Exercise}




\begin{tabular}{|c|c|c|c|c|c|c|c|c|c|c|c|c|c|}
\hline $\begin{array}{l}\text { Study (PMID) } \\
\text { Country } \\
\text { EM Reason }\end{array}$ & Intervention & Comparison & \begin{tabular}{l|} 
Setting \\
and Design \\
Setting \\
Design \\
Cluster N \\
Participants \\
Random- \\
ized N
\end{tabular} & $\begin{array}{l}\text { PLWD } \\
\text { Dementia } \\
\text { Characteristics }\end{array}$ & $\begin{array}{l}\text { PLWD } \\
\text { Non-Disease Char } \\
\text { PLWD N } \\
\text { PLWD Age (mean) } \\
\text { PLWD Sex (\% } \\
\text { female) } \\
\text { PLWD Race (\% } \\
\text { majority) } \\
\text { PLWD Education } \\
\text { (mean years) }\end{array}$ & $\begin{array}{l}\text { PLWD } \\
\text { Non-Disease } \\
\text { Char Reporting } \\
\text { Status (RS) } \\
\text { PLWD SES } \\
\text { PLWD Prior } \\
\text { Disability } \\
\text { PLWD Household } \\
\text { Characteristics } \\
\text { PLWD Health } \\
\text { Insurance } \\
\text { PLWD Detailed } \\
\text { Race Information }\end{array}$ & $\begin{array}{l}\text { Informal Caregiver } \\
\text { (IC) } \\
\text { Characteristics } \\
\text { IC N } \\
\text { IC Age (mean) } \\
\text { IC Sex (\% female) } \\
\text { IC Race (\% } \\
\text { majority) } \\
\text { IC Education } \\
\text { (mean years) } \\
\text { IC Relation to } \\
\text { PLWD (\% majority) }\end{array}$ & $\begin{array}{l}\text { Informal Caregiver } \\
\text { (IC) Char. RS } \\
\text { IC Duration } \\
\text { IC Living With } \\
\text { PLWD } \\
\text { IC Payment } \\
\text { IC Health Status } \\
\text { IC Dementia } \\
\text { Family History } \\
\text { IC Employment } \\
\text { Status } \\
\text { IC Training }\end{array}$ & $\begin{array}{l}\text { Formal Caregiver } \\
\text { (FC) } \\
\text { Characteristics } \\
\\
\text { FC N } \\
\text { FC Age (mean) } \\
\text { FC Sex (\% } \\
\text { female) } \\
\text { FC Race (\% } \\
\text { majority) } \\
\text { FC Education } \\
\text { (mean years) }\end{array}$ & $\begin{array}{l}\text { Formal } \\
\text { Caregiver (FC) } \\
\text { Char. RS } \\
\text { FC Health } \\
\text { Status } \\
\text { FC Training } \\
\text { FC Education } \\
\text { FC Position } \\
\text { FC Length of } \\
\text { Service }\end{array}$ & $\begin{array}{l}\text { Outcome } \\
\text { Timing(s) }\end{array}$ & $\begin{array}{l}\text { PLWD } \\
\text { Outcomes }\end{array}$ & $\begin{array}{l}\text { Caregiver } \\
\text { Outcomes } \\
\text { IC: } \\
\text { FC: }\end{array}$ \\
\hline $\begin{array}{l}\text { Enette 202037 } \\
(31921371) \\
\text { France } \\
\text { Small sample }\end{array}$ & $\begin{array}{l}\text { 11. Continuous } \\
\text { aerobic training } \\
\text { (cycling) } \\
30 \text { minutes } \\
\text { 2x/week } \\
9 \text { weeks } \\
\text { 12. Interval } \\
\text { aerobic cycling } \\
30 \text { minutes } \\
\text { 2x/week } \\
9 \text { weeks }\end{array}$ & $\begin{array}{l}\text { Interactive } \\
\text { information } \\
\text { sessions: } \\
\text { supervised and } \\
\text { structured. } \\
\text { Multiple-choice } \\
\text { questionnaires } \\
\text { that PLWD } \\
\text { discussed } \\
\text { afterward }\end{array}$ & $\begin{array}{l}\text { Memory } \\
\text { clinic of a } \\
\text { university } \\
\text { hospital } \\
\text { RCT } \\
54 \text { PLWD } \\
\text { from NH or } \\
\text { home }\end{array}$ & $\begin{array}{l}\text { Mild to moderate } \\
\text { AD diagnosed } \\
\text { according to DSM- } \\
\text { IV and MMSE } \geq 16\end{array}$ & $\begin{array}{l}\mathrm{N}=54 \text { ( } 3 \text { groups) } \\
\text { Mean age: } 78 \text { years } \\
\text { Female: } 61 \% \\
\text { \% majority race: no } \\
\text { Education: } 7-10 \\
\text { years }\end{array}$ & NR & $\mathrm{NR}$ & NR & NR & NR & 10 weeks & $\begin{array}{l}\text { QoL-AD, 6 } \\
\text { meter walk, } \\
\text { cognitive, } \\
\text { blood tests }\end{array}$ & NR \\
\hline $\begin{array}{l}\text { Fleiner 20201 } \\
32039852 \\
\text { Germany } \\
\text { High RoB }\end{array}$ & $\begin{array}{l}\text { PLWD } \\
\text { Small group } \\
\text { exercise } \\
\text { (strength and } \\
\text { endurance) } \\
20 \text { minutes } \\
\text { 4x/day } \\
3 \text { days/week } \\
2 \text { weeks }\end{array}$ & $\begin{array}{l}\text { Social } \\
\text { stimulation } \\
\text { program (table } \\
\text { games per OT) } \\
120 \text { min./week } \\
2 \text { weeks }\end{array}$ & $\begin{array}{l}3 \text { acute } \\
\text { dementia } \\
\text { care wards } \\
\text { of a } \\
\text { psychiatric } \\
\text { hospital } \\
\text { RCT } \\
70 \text { PLWD }\end{array}$ & $\begin{array}{l}\text { PLWD with ICD-10 } \\
\text { diagnosis of } \\
\text { dementia (AD, VD, } \\
\text { mixed, LBD, PD). } \\
\text { Mean MMSE=18. } \\
\text { Able to do TUG. }\end{array}$ & $\begin{array}{l}\mathrm{N}=70 \\
\text { Mean age: } 80 \text { years } \\
\text { Female: } 47 \% \\
\text { \% majority race: no } \\
\text { Education: } 13 \text { years }\end{array}$ & NR & NA & NR & $\begin{array}{l}\text { All RNs } \\
\text { Other: NR }\end{array}$ & NA & 2 weeks & NR & NPI \\
\hline $\begin{array}{l}\text { Lee, 202038 } \\
\text { (31347294) } \\
\text { Korea } \\
\text { Small sample }\end{array}$ & $\begin{array}{l}\text { Fumanet } \\
\text { exercise } \\
\text { program } \\
1 \text { hour/week } \\
8 \text { weeks }\end{array}$ & $\begin{array}{l}\text { Non-equivalent } \\
\text { control group }\end{array}$ & $\begin{array}{l}\text { Community- } \\
\text { based } \\
\text { Quasi- } \\
\text { experimental } \\
\text { (segregated } \\
\text { by day at } \\
\text { center) } \\
\mathrm{N}=45\end{array}$ & $\begin{array}{l}\text { Persons with mild } \\
\text { dementia (CDR } 0.5 \\
\text { to } 1 \text { ). Not visually } \\
\text { impaired. Able to } \\
\text { ambulate with or } \\
\text { without assistance. } \\
\text { Age } 45 \text { or older. } \\
\text { Other exclusions }\end{array}$ & $\begin{array}{l}\mathrm{N}=45 \text { (22 exercise, } \\
23 \text { control) } \\
\text { Age: } \mathrm{Y} \\
\text { Sex: } \mathrm{Y} \\
\text { Education: } \mathrm{Y} \\
\text { Race \& majority: } \mathrm{N}\end{array}$ & $\begin{array}{l}\text { Dementia } \\
\text { diagnosis: } Y\end{array}$ & NA & NA & NR & Training: $Y$ & 8 weeks & $\begin{array}{l}\text { Cognitive } \\
\text { function, } \\
\text { gait, } \\
\text { depression }\end{array}$ & NA \\
\hline $\begin{array}{l}\text { Liu } 2020^{2} \\
(32084347) \\
\text { Taiwan } \\
\text { High RoB }\end{array}$ & $\begin{array}{l}\text { Isotonic strength } \\
\text { training (weight } \\
\text { machines) } \\
2 \text { sets of } 12 \\
5 \text { days/week } \\
4 \text { weeks }\end{array}$ & $\begin{array}{l}\text { Aerobic exercise } \\
\text { (stationary } \\
\text { cycling) } \\
30 \text { minutes } \\
5 \text { days/week } \\
4 \text { weeks }\end{array}$ & $\begin{array}{l}\text { PLWDs } \\
\text { Veterans NH } \\
\text { RCT } \\
80 \text { PLWDs }\end{array}$ & $\begin{array}{l}\text { MMSE 15-26 } \\
\text { Other: NR }\end{array}$ & $\begin{array}{l}\mathrm{N}=69 \text { analyzed } \\
\text { Mean age: } 86 \text { years } \\
\text { Female: } 16 \% \\
\text { \% majority race: no } \\
\text { Education: no }\end{array}$ & NR & NR & NR & NR & NR & 4 weeks & $\begin{array}{l}\text { Barthel } \\
\text { Index, GDS, } \\
\text { cognitive, } \\
\text { blood test }\end{array}$ & NR \\
\hline $\begin{array}{l}\text { Sanders 202039 } \\
\text { (32192537) } \\
\text { Netherlands } \\
\text { Pilot }\end{array}$ & $\begin{array}{l}\text { Outdoor walking } \\
\text { and lower limb } \\
\text { strength } \\
\text { training: } \\
12 \text { weeks low- } \\
\text { intensity, then } \\
12 \text { weeks high- } \\
\text { intensity training } \\
\text { 3x/week } \\
24 \text { weeks } \\
\end{array}$ & $\begin{array}{l}\text { Flexibility } \\
\text { exercises and } \\
\text { recreational } \\
\text { activities } \\
3 \times / \text { week } \\
24 \text { weeks }\end{array}$ & $\begin{array}{l}\text { Recruited } \\
\text { from daycare } \\
\text { or residential } \\
\text { care } \\
\text { RCT } \\
91 \text { PLWDs }\end{array}$ & $\begin{array}{l}\text { Dementia diagnosis } \\
\text { per physician using } \\
\text { DSM-IV; MMSE } \\
\text { score >10 (mild-to- } \\
\text { moderate } \\
\text { dementia) }\end{array}$ & $\begin{array}{l}\mathrm{N}=69 \\
\text { Mean age: } 82 \text { years } \\
\text { Female: } 62 \% \\
\text { \% majority race: no } \\
\text { Education: yes ( } 3 \\
\text { levels) }\end{array}$ & None & NA & None & NA & NA & $\begin{array}{l}12 \text { weeks } \\
24 \text { weeks } \\
6 \text { GWS, leg } \\
\text { strength and } \\
\text { STROOP (6 } \\
\text { weeks } \\
18 \text { weeks } \\
36 \text { weeks) }\end{array}$ & \begin{tabular}{l|}
$6 \mathrm{MWT}$ (6- \\
minute walk \\
test) \\
SPPB (Short \\
Physical \\
Performance \\
Battery) \\
Cognitive
\end{tabular} & NA \\
\hline
\end{tabular}




\begin{tabular}{|c|c|c|c|c|c|c|c|c|c|c|c|c|c|}
\hline $\begin{array}{l}\text { Brett 201940 } \\
(30912690) \\
\text { Australia } \\
\text { Small sample }\end{array}$ & $\begin{array}{l}\text { 11. 45-minute } \\
\text { exercise } \\
\text { program 1x per } \\
\text { week } \\
\text { 12. 15-minute } \\
\text { exercise } \\
\text { program } 3 x \text { per } \\
\text { week } \\
\end{array}$ & $\begin{array}{l}\text { Usual care } \\
\text { activities } \\
\text { (routine group } \\
\text { activities) }\end{array}$ & $\begin{array}{l}\text { nursing } \\
\text { homes } \\
\text { RCT } \\
\mathrm{N}=60\end{array}$ & $\begin{array}{l}\text { PLWD living in } \\
\text { nursing home, able } \\
\text { to participate in } \\
\text { intervention. } \\
\text { Stratified by able to } \\
\text { walk (71\%) vs. } \\
\text { participated sitting }\end{array}$ & \begin{tabular}{|l|} 
Reported $\mathrm{N}=55$ (17 \\
Group 1, 19 Group \\
2,19 usual care) \\
Mean age: 85 \\
Female: $66 \%$ \\
Race \% majority: $\mathrm{Y}$ \\
Education: $\mathrm{N}$
\end{tabular} & $\begin{array}{l}N \text { of comorbidities: } \\
\mathrm{Y}\end{array}$ & $\mathrm{NA}$ & NA & NR & $\begin{array}{l}\text { Length of service: } \\
Y\end{array}$ & 12 weeks & $\begin{array}{l}\text { Falls, Timed } \\
\text { Up \& Go, } \\
\text { Sit to Stand, } \\
\text { Gait speed, } \\
\text { Functional } \\
\text { reach }\end{array}$ & NA \\
\hline $\begin{array}{l}\text { Karssemeijer } \\
2019^{5} \\
\text { (31409559) } \\
\text { Netherlands } \\
\text { High RoB }\end{array}$ & $\begin{array}{l}\text { 11. Exergame } \\
\text { training } \\
\text { 12. Aerobic } \\
\text { training } \\
\text { Both: } 3 \text { x/week } \\
12 \text { weeks }\end{array}$ & $\begin{array}{l}\text { Active control } \\
12 \text { weeks }\end{array}$ & $\begin{array}{l}\text { Community } \\
\text { centers } \\
\mathrm{RCT} \\
\mathrm{N}=115(92 \\
\text { completed })\end{array}$ & $\begin{array}{l}\text { Diagnosed with VD, } \\
\text { AD or mixed } \\
\text { dementia, MMSE } \geq \\
17 \text {, age } \geq 60 \text { years. } \\
\text { Additional criteria } \\
\text { applied }\end{array}$ & $\begin{array}{l}\mathrm{N}=115 \text { (38 } \\
\text { exergame, } 38 \\
\text { aerobic, } 39 \text { controls) } \\
\text { Mean age: } 79 \\
\text { Female: } 54 \% \\
\text { Education: } \mathrm{N} \\
\text { Race \& majority: } \mathrm{N}\end{array}$ & Living situation: $\mathrm{Y}$ & NA & NA & NR & $\mathrm{NR}$ & 12 weeks & $\begin{array}{l}\text { Frailty, } \\
\text { adherence }\end{array}$ & NA \\
\hline $\begin{array}{l}\text { Nyman 201941 } \\
\text { (31819385) } \\
\text { UK } \\
\text { Pilot }\end{array}$ & $\begin{array}{l}\text { Group Tai Chi } \\
45 \text { min plus } \\
\text { discussion } \\
\text { Weekly } \\
20 \text { weeks }\end{array}$ & Usual care & $\begin{array}{l}\text { Community- } \\
\text { dwelling } \\
\text { dyads } \\
\text { (PLWD and } \\
\text { IC). Classes } \\
\text { in church } \\
\text { halls } \\
\mathrm{N}=85\end{array}$ & $\begin{array}{l}\text { Diagnosis of } \\
\text { dementia per MD } \\
\text { (VD, AD, mixed, } \\
\text { other), Mini } \\
\text { Addenbrooke's } \\
\text { Cognitive Exam } \geq \\
10 \text {, willing to attend } \\
\text { Tai Chi class, able } \\
\text { to do standing, } \\
\text { other exclusions }\end{array}$ & $\begin{array}{l}\mathrm{N}=85 \text { (70 complete } \\
\text { data) } \\
\text { Mean age: } 78 \text { years } \\
\text { Female: } 40 \% \\
\text { Education: } \mathrm{N} \\
\text { Race \& majority: } \mathrm{N} \\
\text { Time since } \\
\text { diagnosis: } \mathrm{Y}\end{array}$ & NR & $\begin{array}{l}\mathrm{N}=85 \\
\text { Mean age: } 71 \text { years } \\
\text { Female: } 79 \% \\
\text { Other: NR }\end{array}$ & $\begin{array}{l}\text { Living with PLWD: } \\
87 \%\end{array}$ & NA & NA & 6 months & \begin{tabular}{l|} 
Berg \\
Balance, \\
Timed Up \& \\
Go, falls, \\
cognitive
\end{tabular} & $\begin{array}{l}\text { IC: TUG, } \\
\text { other }\end{array}$ \\
\hline $\begin{array}{l}\text { Todri 201942 } \\
\text { (None) } \\
\text { Spain } \\
\text { Pilot }\end{array}$ & $\begin{array}{l}\text { Global Postural } \\
\text { Reeducation 30- } \\
40 \text { minutes } \\
2 \times / \text { week } \\
6 \text { months } \\
\text { (total } 48 \\
\text { sessions) }\end{array}$ & $\begin{array}{l}\text { Conventional } \\
\text { exercises } \\
\text { (proprioceptive, } \\
\text { equilibrium, } \\
\text { aerobic/walking) } \\
30-40 \text { minutes } \\
\text { 2x/week } \\
6 \text { months }\end{array}$ & $\begin{array}{l}\text { PLWDs were } \\
\text { recruited } \\
\text { from nursing } \\
\text { homes , } 2 \\
\text { countries } \\
174 \text { PLWD }\end{array}$ & $\begin{array}{l}\text { Dementia (mild to } \\
\text { moderate) per } \\
\text { NINCDS-ADRDA } \\
\text { criteria }\end{array}$ & $\begin{array}{l}\mathrm{N}=135 \text { analyzed } \\
\text { Mean age: } 81 \text { years } \\
\text { Female: } 66 \% \\
\text { \% majority race: no } \\
\text { Education: } \\
\text { "Medium" }\end{array}$ & NR & NA & NR & NR & NR & 2 weeks & $\begin{array}{l}\text { Qol-AD } \\
\text { GDS } \\
\text { Barthel } \\
\text { Index, NPI } \\
\text { Tinetti Scale, } \\
\text { MMSE }\end{array}$ & NR \\
\hline $\begin{array}{l}\text { Henskens 2018 } \\
(29750023) \\
\text { Netherlands } \\
\text { High RoB }\end{array}$ & $\begin{array}{l}\text { I1. [Exercise } \\
\text { (strength + } \\
\text { walking) + ADL } \\
\text { training] } \\
\text { 12. ADL training } \\
\text { only } \\
\text { 13. Exercise only } \\
\text { All: 3x/week } \\
6 \text { months }\end{array}$ & Social control & \begin{tabular}{|l|}
11 Nursing \\
homes \\
4 group RCT \\
Cluster: 11 \\
$\mathrm{~N}=87$
\end{tabular} & $\begin{array}{l}\text { Dementia (AD, VD, } \\
\text { Mixed, Unknown) } \\
\text { per DSM-IV, living } \\
\text { in NH, age } \geq 65 \\
\text { yrs., MMSE 1-24, } \\
\text { complete 6-minute } \\
\text { walk test }\end{array}$ & $\begin{array}{l}\mathrm{N}=87(66 \\
\text { completed) } \\
\text { Age: } \mathrm{Y} \\
\text { Sex: } \mathrm{Y} \\
\text { Race: } \mathrm{N} \\
\text { Education: } \mathrm{Y}\end{array}$ & NR & NA & NA & NR & NR & $\begin{array}{l}3 \text { months, } 6 \\
\text { months }\end{array}$ & $\begin{array}{l}\text { QoL, } \\
\text { function } \\
\text { (created } \\
\text { composite) }\end{array}$ & NA \\
\hline $\begin{array}{l}\text { Ho 2018 } \\
\text { (30496547) } \\
\text { Hong Kong } \\
\text { High RoB }\end{array}$ & $\begin{array}{l}\text { 11: Dance } \\
\text { movement } \\
\text { therapy } \\
\text { 12. Exercise } \\
\text { Both: 2x/week } \\
12 \text { weeks }\end{array}$ & Waitlist & $\begin{array}{l}\text { Outpatient } \\
\text { RCT } \\
\text { PLWD } \\
\text { N=204 }\end{array}$ & $\begin{array}{l}\text { Dementia per DSM- } \\
\text { IV or } \\
\text { neurocognitive } \\
\text { disorder (DSM V), } \\
\text { CDR } 0.5 \text { to } 1 \text {, age } \geq \\
65 \text { years. Other } \\
\text { exclusion criteria }\end{array}$ & $\begin{array}{l}\mathrm{N}=204(166 \\
\text { completed) }\end{array}$ & NR & NA & NA & NR & NR & $\begin{array}{l}3,6, \text { and } 12 \\
\text { months }\end{array}$ & $\begin{array}{l}\text { Depression, } \\
\text { mood, daily } \\
\text { function, } \\
\text { cortisol }\end{array}$ & NA \\
\hline $\begin{array}{l}\text { Liu, 201843 } \\
\text { (29969916) } \\
\text { Hong Kong/ } \\
\text { China } \\
\text { Pilot }\end{array}$ & $\begin{array}{l}\text { Simplified Tai- } \\
\text { chi program to } \\
\text { improve motor } \\
\text { skills } \\
16 \text { weeks }\end{array}$ & Social activities & $\begin{array}{l}\text { Community- } \\
\text { dwelling } \\
\text { PLWD; four } \\
\text { community } \\
\text { health } \\
\text { centers } \\
\text { RCT }\end{array}$ & $\begin{array}{l}\text { PLWD aged } 60 \text { or } \\
\text { older with Montreal } \\
\text { Cognitive } \\
\text { Assessment score } \\
<=20 \text {; capable of } \\
\text { exercise; caregiver } \\
\text { able to exercise }\end{array}$ & $\begin{array}{l}\mathrm{N}=26(13 \\
\text { intervention/, } 2 \\
\text { centers; } 13 \\
\text { controls/2 centers) } \\
\text { Age: } \mathrm{Y} \\
\text { Sex: } \mathrm{Y} \\
\text { \%majority race: } \mathrm{N} \\
\text { Education: } \mathrm{Y}\end{array}$ & $\begin{array}{l}\text { Prior disability: Y } \\
\text { Household } \\
\text { Characteristics: } Y\end{array}$ & $\begin{array}{l}\mathrm{N}=11 \text { exercise } \\
\text { group } \\
13 \text { control group } \\
\text { Age: } Y \\
\text { Sex: } Y \\
\text { Education: } Y \\
\text { Relation to PLWD: } \\
Y\end{array}$ & Duration: $\mathrm{Y}$ & NR & NR & 16 weeks & \begin{tabular}{l|} 
Motor \\
performance
\end{tabular} & \\
\hline
\end{tabular}




\begin{tabular}{|c|c|c|c|c|c|c|c|c|c|c|c|c|c|}
\hline $\begin{array}{l}\text { Chen 20179 } \\
\text { (27879982) } \\
\text { Taiwan } \\
\text { High RoB }\end{array}$ & $\begin{array}{l}\text { Resistance- } \\
\text { band exercise } \\
\text { program } \\
3 \times \text { /week } x \\
6 \text { mo., then } 9 \\
\text { mo. per DVD }\end{array}$ & $\begin{array}{l}\text { Usual daily } \\
\text { activities }\end{array}$ & \begin{tabular}{|l|} 
Nursing \\
homes \\
Cluster RCT \\
(8 NH) \\
N=150 \\
PLWD \\
\end{tabular} & \begin{tabular}{|l|} 
Wheelchair-bound \\
PLWD, lived in \\
nursing home 3 \\
months, dementia \\
per diagnosis or \\
MMSE (cutpoint by \\
education level) \\
\end{tabular} & $\begin{array}{l}\mathrm{N}=127 \text { completed } \\
\text { (65 intervention, } 62 \\
\text { controls) } \\
\text { Age: } Y \\
\text { Sex: } Y \\
\% \text { majority race: } N \\
\text { Education: } Y \\
Y\end{array}$ & $\begin{array}{l}100 \% \text { wheelchair- } \\
\text { bound } \\
\text { Other: NR }\end{array}$ & NR & NR & $\mathrm{NR}$ & NR & $\begin{array}{l}3,6,9,12 \text { and } \\
15 \text { months }\end{array}$ & $\begin{array}{l}\text { Depression, } \\
\text { behavioral } \\
\text { problems } \\
\text { (BRS) }\end{array}$ & NR \\
\hline $\begin{array}{l}\text { Dawson, } 2017^{44} \\
\text { (28252473) } \\
\text { USA } \\
\text { Small sample }\end{array}$ & $\begin{array}{l}\text { Moderate- } \\
\text { Intensity Home- } \\
\text { Based Exercise } \\
\text { Program }\end{array}$ & $\begin{array}{l}\text { Continuation of } \\
\text { current levels of } \\
\text { activity } \\
\text { Randomized } \\
\text { control trial }\end{array}$ & $\begin{array}{l}\text { Community- } \\
\text { based } \\
\text { RCT } \\
\text { PLWD }\end{array}$ & $\begin{array}{l}\text { Dementia } \\
\text { unspecified }\end{array}$ & $\begin{array}{l}\mathrm{N}=13 \text { intervention } \\
\text { group } \\
10 \text { control group } \\
\text { Age: } Y \\
\text { Sex: } Y \\
\% \text { majority race: } N \\
\text { Education: } Y\end{array}$ & NR & NA & NA & NR & NR & 12 weeks & $\begin{array}{l}\text { Measuremen } \\
\text { ts of walking } \\
\text { and balance } \\
\text { Assessment } \\
\text { of cognition }\end{array}$ & NA \\
\hline $\begin{array}{l}\text { De Souto, } \\
2017^{45} \\
(28542742) \\
\text { France } \\
\text { Pilot }\end{array}$ & $\begin{array}{l}\text { Multicomponent } \\
\text { training: } \\
\text { coordination, } \\
\text { balance, } \\
\text { muscle- } \\
\text { strengthening \& } \\
\text { aerobic }\end{array}$ & $\begin{array}{l}\text { Structured } \\
\text { social activity }\end{array}$ & $\begin{array}{l}\text { Nursing } \\
\text { Homes } \\
\text { RCT cluster } \\
\text { PLWD }\end{array}$ & $\begin{array}{l}\text { Dementia } \\
\text { unspecified }\end{array}$ & $\begin{array}{l}\mathrm{N}=47 \text { PLWD (4 } \\
\text { nursing homes) } \\
\text { exercise; 50 PLWD } \\
\text { (3 nursing homes) } \\
\text { social activity } \\
\text { Age: } Y \\
\text { Sex: } Y \\
\text { \% majority race: } N \\
\text { Education: } Y\end{array}$ & NR & NA & NA & NR & NR & 24 weeks & $\begin{array}{l}\text { MMSE } \\
\text { Physical } \\
\text { performance } \\
\text { evaluation }\end{array}$ & NA \\
\hline $\begin{array}{l}\text { Hamilton, } \\
2017^{46} \\
(27692024) \\
\text { Australia } \\
\text { Small sample }\end{array}$ & $\begin{array}{l}\text { Home-based } \\
\text { exercise } \\
\text { program }\end{array}$ & $\begin{array}{l}\text { Structured, } \\
\text { individually } \\
\text { tailored exercise } \\
\text { program }\end{array}$ & $\begin{array}{l}\text { Home-based } \\
\text { RCT }\end{array}$ & $\begin{array}{l}\text { PLWD diagnosed } \\
\text { with mild to } \\
\text { moderate dementia }\end{array}$ & $\begin{array}{l}\mathrm{N}=33 \text { completed } \\
\text { the study; } 9 \text { did not } \\
\text { Ae: } Y \\
\text { Sex: } Y \\
\text { \% majority race: } N \\
\text { Education: } Y\end{array}$ & NR & NA & NA & $\mathrm{NR}$ & NR & 6 months & $\begin{array}{l}\text { Psychologica } \\
\text { I assessment } \\
\text { Physical } \\
\text { assessment } \\
\text { fall risk } \\
\text { Adherence } \\
\end{array}$ & NA \\
\hline $\begin{array}{l}\text { Henwood, } \\
2017^{47} \\
\text { (28473006) } \\
\text { Australia } \\
\text { Small sample }\end{array}$ & $\begin{array}{l}\text { Aquatic exercise } \\
\text { for } \\
2 \times / \text { week } \\
12 \text { weeks }\end{array}$ & Usual care & $\begin{array}{l}\text { Non-RCT: } \\
\text { Facility } \\
\text { PLWD } \\
\text { allocated by } \\
\text { ability to } \\
\text { transport to } \\
\text { swimming } \\
\text { facility } \\
\mathrm{N}=56 \\
\end{array}$ & $\begin{array}{l}\text { PLWD living in } \\
\text { residential care } \\
\text { facility, moderate to } \\
\text { severe dementia }\end{array}$ & $\begin{array}{l}\mathrm{N}=56 \text { (analyzed 46; } \\
23 \text { each group) } \\
\text { Age: } \mathrm{Y} \\
\text { Sex: } \mathrm{Y} \\
\% \text { majority race: } \mathrm{N} \\
\text { Education: } \mathrm{N}\end{array}$ & NR & NA & NA & NR & NR & 12 weeks & $\begin{array}{l}\text { Assessment } \\
\text { of physical } \\
\text { condition, } \\
\text { balance and } \\
\text { physical } \\
\text { functions }\end{array}$ & NA \\
\hline $\begin{array}{l}\text { Lam, 201748 } \\
\text { (28094873) } \\
\text { Hong Kong } \\
\text { Small sample }\end{array}$ & $\begin{array}{l}\text { Whole-body } \\
\text { vibration plus } \\
\text { routine day } \\
\text { activity program } \\
\text { 2x/week } \\
9 \text { weeks }\end{array}$ & $\begin{array}{l}\text { Routine day } \\
\text { activity program } \\
\text { 2x/week } \\
9 \text { weeks }\end{array}$ & $\begin{array}{l}\text { PLWD with } \\
\text { mild or } \\
\text { moderate } \\
\text { dementia, } \\
\text { recruited } \\
\text { from } 2 \text { day- } \\
\text { care centers } \\
\text { RCT } \\
\end{array}$ & $\begin{array}{l}\text { PLWD aged } 65 \text { or } \\
\text { older with MMSE } \\
10-22 \text {; ability to } \\
\text { stand; living in the } \\
\text { community }\end{array}$ & $\begin{array}{l}\mathrm{N}=54(27 \\
\text { intervention, } 27 \\
\text { control) } \\
\text { Age: } \mathrm{Y} \\
\text { Sex: } \mathrm{Y} \\
\% \text { majority race: } \mathrm{N} \\
\text { Education: } \mathrm{N}\end{array}$ & NR & NA & NA & NR & NR & 9 weeks & $\begin{array}{l}\text { Ability to } \\
\text { stand; } \\
\text { balance; } \\
\text { quality of life; } \\
\text { balance } \\
\text { confidence }\end{array}$ & NA \\
\hline $\begin{array}{l}\text { Morris 201749 } \\
\text { (28187125) } \\
\text { USA } \\
\text { Pilot }\end{array}$ & $\begin{array}{l}\text { Supervised } \\
\text { aerobic exercise } \\
\text { program: } 150 \\
\text { minutes per } \\
\text { week for } 26 \\
\text { weeks }\end{array}$ & $\begin{array}{l}\text { Non-aerobic } \\
\text { stretching and } \\
\text { toning }\end{array}$ & $\begin{array}{l}\text { Community- } \\
\text { based } \\
\text { RCT } \\
\text { PLWD }\end{array}$ & \begin{tabular}{|l|} 
PLWD with \\
probable AD; age \\
55 or older; \\
sedentary; \\
community \\
dwelling, supportive \\
caregiver; other \\
exclusion criteria \\
\end{tabular} & $\begin{array}{l}\mathrm{N}=76 \\
\text { Age: yes } \\
\text { Sex: yes } \\
\% \text { majority race: no } \\
\text { Education: no }\end{array}$ & $\mathrm{NR}$ & NR & NR & NA & NA & 26 weeks & $\begin{array}{l}\text { Functional } \\
\text { ability and } \\
\text { depression }\end{array}$ & NA \\
\hline $\begin{array}{l}\text { Padala, 201750 } \\
\text { (28655135) } \\
\text { US } \\
\text { Pilot }\end{array}$ & $\begin{array}{l}\text { Home-based } \\
\text { Wii-Fit } \\
\text { interactive } \\
\text { video-game-led } \\
\text { physical } \\
\text { exercise } \\
\text { program, } 8 \\
\text { weeks } \\
\end{array}$ & $\begin{array}{l}\text { Walking } \\
\text { program }\end{array}$ & $\begin{array}{l}\text { Community- } \\
\text { based } \\
\text { RCT } \\
\text { N=30 }\end{array}$ & $\begin{array}{l}\text { PLWD } 60 \text { years } \\
\text { and older } \\
\text { diagnosed with AD; } \\
\text { MMSE score >=18 } \\
\text { with fear of falling; } \\
\text { exclusion criteria } \\
\text { applied }\end{array}$ & $\begin{array}{l}\mathrm{N}=15 \text { exercise } \\
\text { group } \\
15 \text { control group } \\
\text { Age: yes } \\
\text { Sex: yes } \\
\text { \% majority race: yes } \\
\text { Education: yes }\end{array}$ & $\begin{array}{l}\text { Comorbidities: yes } \\
\text { Medications: yes }\end{array}$ & NR & NR & NA & NA & $\begin{array}{l}8 \text { weeks, } 16 \\
\text { weeks }\end{array}$ & Balance & NA \\
\hline
\end{tabular}




\begin{tabular}{|c|c|c|c|c|c|c|c|c|c|c|c|c|c|}
\hline $\begin{array}{l}\text { Cancela 201611 } \\
(26087884) \\
\text { Spain } \\
\text { High RoB }\end{array}$ & $\begin{array}{l}\text { Exercise } \\
\text { (stationary } \\
\text { cycling) } \\
15 \text { minutes/day } \\
15 \text { months }\end{array}$ & $\begin{array}{l}\text { Sedentary } \\
\text { recreational } \\
\text { activities }\end{array}$ & $\begin{array}{l}\text { Institutional } \\
\text { residential } \\
\text { care } \\
\mathrm{RCT} \\
\mathrm{N}=189\end{array}$ & $\begin{array}{l}\text { PLWD age } \geq 65 \\
\text { yrs., dementia per } \\
\text { DSM-IV, able to } \\
\text { stand and walk } 30 \\
\text { meters without } \\
\text { assistance, resident } \\
\text { of facility }\end{array}$ & $\begin{array}{l}\mathrm{N}=114 \text { completed } \\
\text { Age: } \mathrm{Y} \\
\text { Sex: } \mathrm{Y} \\
\% \text { majority race: } \mathrm{N} \\
\text { Education: } \mathrm{Y}\end{array}$ & $\begin{array}{l}\text { Comorbidities: Y } \\
\text { Other: NR }\end{array}$ & $\mathrm{NR}$ & $\mathrm{NR}$ & $\mathrm{NR}$ & NR & 15 months & $\begin{array}{l}\text { Neuropsychi } \\
\text { atric } \\
\text { symptoms, } \\
\text { cognition, } \\
\text { Katz ADL, } \\
\text { mobility, } \\
\text { depression }\end{array}$ & NA \\
\hline $\begin{array}{l}\text { Burge, 201651 } \\
(27831462) \\
\text { Switzerland \& } \\
\text { Belgium } \\
\text { Pilot }\end{array}$ & $\begin{array}{l}\text { Group physical } \\
\text { exercise } \\
20 \text { sessions } \\
4 \text { weeks }\end{array}$ & $\begin{array}{l}\text { Social visits } \\
20 \text { sessions } \\
4 \text { weeks }\end{array}$ & $\begin{array}{l}5 \text { Hospitals, } \\
\text { acute } \\
\text { psychiatric } \\
\text { wards } \\
\text { RCT } \\
\text { PLWD }\end{array}$ & $\begin{array}{l}\text { Moderate to severe } \\
\text { dementia, on acute } \\
\text { psychiatric ward }\end{array}$ & $\begin{array}{l}\mathrm{N}=270 \\
\text { Age: yes } \\
\text { Sex: no } \\
\text { \% majority race: no } \\
\text { Education: no }\end{array}$ & $\mathrm{NR}$ & NA & $\mathrm{NA}$ & NA & NA & 4 weeks & $\begin{array}{l}\text { Barthel ADL, } \\
\text { FIM, } \\
\text { depression \& } \\
\text { behavior }\end{array}$ & NA \\
\hline $\begin{array}{l}\text { Venturelli 201652 } \\
\text { (27540967) } \\
\text { Italy } \\
\text { Small sample }\end{array}$ & $\begin{array}{l}11: \text { Aerobic } \\
\text { exercise and } \\
\text { cognitive } \\
\text { training } \\
\text { 12:aerobic } \\
\text { exercise only 13: } \\
\text { cognitive } \\
\text { training only }\end{array}$ & No training & $\begin{array}{l}\text { Nursing } \\
\text { home PLWD } \\
\text { Control trial }\end{array}$ & $\begin{array}{l}\text { PLWD diagnosed } \\
\text { with dementia, } \\
\text { MMSE between } 10 \\
\text { and 15; PLWD } \\
\text { screened for } \\
\text { physical limitations }\end{array}$ & $\begin{array}{l}\mathrm{N}=80 \text { (20 each } \\
\text { group) } \\
\text { Age: yes } \\
\text { Sex: yes } \\
\text { \% majority race: no } \\
\text { Education: no }\end{array}$ & Prior disability & NA & $\mathrm{NA}$ & NR & NR & 3 months & $\begin{array}{l}\text { Cortisol } \\
\text { level, level of } \\
\text { cognitive } \\
\text { function; } \\
\text { neuropsycho } \\
\text { logical } \\
\text { symptoms }\end{array}$ & NA \\
\hline $\begin{array}{l}\text { Bosser, 201523 } \\
\text { (25648055) } \\
\text { Netherlands } \\
\text { High RoB }\end{array}$ & $\begin{array}{l}\text { 11: Combined } \\
\text { aerobic and } \\
\text { strength } \\
\text { training: } 36 \\
\text { individualized } \\
\text { sessions over } 9 \\
\text { weeks } \\
\text { 12: Aerobic only: } \\
\text { participated in } \\
\text { four walking } \\
\text { sessions per } \\
\text { week, } 9 \text { weeks }\end{array}$ & $\begin{array}{l}\text { Four social visits } \\
\text { each week }\end{array}$ & $\begin{array}{l}\text { Nursing } \\
\text { homes } \\
\text { RCT } \\
\text { PLWD }\end{array}$ & $\begin{array}{l}\text { Mild-severe } \\
\text { vascular dementia }\end{array}$ & $\begin{array}{l}\mathrm{N}=123 \\
\text { Age: yes } \\
\text { Sex: yes } \\
\text { \% majority race: no } \\
\text { Education: yes }\end{array}$ & NR & NA & $\mathrm{NA}$ & NA & NA & 18 weeks & $\begin{array}{l}\text { MMSE } \\
6 \text {-minute } \\
\text { walk test, } \\
\text { the 30- } \\
\text { second sit- } \\
\text { to-stand test }\end{array}$ & NA \\
\hline $\begin{array}{l}\text { Holthoff, 201553 } \\
\text { (25884637) } \\
\text { Germany } \\
\text { Pillot }\end{array}$ & $\begin{array}{l}\text { Home-based } \\
\text { exercise; lower } \\
\text { body on a } \\
\text { movement } \\
\text { trainer with } \\
\text { computer } \\
\text { control } \\
12 \text { weeks }\end{array}$ & $\begin{array}{l}\text { Usual care } \\
\text { Monthly clinical } \\
\text { visits and a } \\
\text { counselling } \\
\text { including } \\
\text { specific advice } \\
\text { how to change } \\
\text { inactive habits } \\
\text { and increase the } \\
\text { exercise }\end{array}$ & $\begin{array}{l}\text { Community- } \\
\text { based } \\
\text { RCT } \\
\text { PLWD }\end{array}$ & $\begin{array}{l}\text { PLWD with mild to } \\
\text { moderate AD per } \\
\text { NINCDS-ADRA } \\
\text { criteria, age } 55 \text { or } \\
\text { older and their } \\
\text { family caregivers; } \\
\text { PLWD evaluated } \\
\text { for other conditions } \\
\text { that would rule out } \\
\text { AD }\end{array}$ & $\begin{array}{l}\mathrm{N}=30 \\
\text { Age: yes } \\
\text { Sex: yes } \\
\text { \% majority race: } \\
\text { Education: }\end{array}$ & Age at diagnosis & NR & NR & NR & NR & 12 weeks & $\begin{array}{l}\text { ADL; Neuro- } \\
\text { psychiatric } \\
\text { symptoms; } \\
\text { Executive } \\
\text { function, } \\
\text { language } \\
\text { ability; Motor } \\
\text { skills; } \\
\text { Caregiver } \\
\text { burden; } \\
\text { MMSE }\end{array}$ & NA \\
\hline $\begin{array}{l}\text { Yang 2015'15 } \\
\text { (26556080) } \\
\text { China } \\
\text { High RoB }\end{array}$ & $\begin{array}{l}\text { Moderate } \\
\text { aerobic exercise } \\
\text { (cycling) } \\
3 \times \text { week } \\
3 \text { months }\end{array}$ & $\begin{array}{l}\text { Health } \\
\text { education }\end{array}$ & $\begin{array}{l}\text { Outpatient } \\
\text { RCT } \\
\text { PLWD }\end{array}$ & $\begin{array}{l}\text { Mild AD (criteria } \\
\text { NR) or vascular } \\
\text { dementia, age 65- } \\
80 \text { years, MMSE } \\
\text { 10-24; other } \\
\text { exclusion criteria }\end{array}$ & $\begin{array}{l}\mathrm{N}=50 \\
\text { Age: } \mathrm{Y} \\
\text { Sex: } \mathrm{Y} \\
\text { \% majority race: } \mathrm{N} \\
\text { Education: } \mathrm{Y}\end{array}$ & NR & NR & NR & NR & NR & 3 months & $\begin{array}{l}\text { QoL, } \\
\text { cognition, } \\
\text { neuro- } \\
\text { psychiatric } \\
\text { symptoms }\end{array}$ & NA \\
\hline $\begin{array}{l}\text { Yu, 201554 } \\
\text { (24652914) } \\
\text { US } \\
\text { Pilot }\end{array}$ & $\begin{array}{l}\text { Cycling as } \\
\text { exercise }\end{array}$ & Single group & $\begin{array}{l}\text { Community- } \\
\text { based } \\
\text { Single group } \\
\text { Pilot study }\end{array}$ & $\begin{array}{l}\text { Older adults, mild } \\
\text { to moderate AD; } \\
\text { MMSE 12-24; CDR } \\
1-3 ; \text { able to perform } \\
\text { exercises, qualified } \\
\text { family caregivers }\end{array}$ & $\begin{array}{l}\mathrm{N}=26 \\
\text { Age: } \mathrm{Y} \\
\text { Sex: } \mathrm{Y} \\
\text { \% majority race: } \mathrm{Y} \\
\text { Education: } \mathrm{Y}\end{array}$ & NR & $\begin{array}{l}\mathrm{N}=26 \\
\text { Age: } \mathrm{Y} \\
\text { Sex: } \mathrm{Y} \\
\text { \% majority race: } \mathrm{Y} \\
\text { Education: } \mathrm{Y} \\
\text { Relation to PLWD: } \\
\mathrm{N}\end{array}$ & NR & NR & Training: $Y$ & 6 months & $\begin{array}{l}\text { Cognitive } \\
\text { function; } \\
\text { ADL; BPSD; } \\
\text { caregiver } \\
\text { distress }\end{array}$ & $\begin{array}{l}\text { Caregiver } \\
\text { distress } \\
\text { decreased } \\
\text { from baseline }\end{array}$ \\
\hline
\end{tabular}




\begin{tabular}{|c|c|c|c|c|c|c|c|c|c|c|c|c|c|}
\hline $\begin{array}{l}\text { Bosser 201424 } \\
\text { (248447772) } \\
\text { Netherlands } \\
\text { Pilot }\end{array}$ & $\begin{array}{l}\text { Evaluation of a } \\
\text { combined } \\
\text { aerobic and } \\
\text { strength } \\
\text { training program } \\
\text { for } \\
\text { institutionalized } \\
\text { PLWD; studied } \\
\text { effects on } \\
\text { cognitive and } \\
\text { physical function }\end{array}$ & $\begin{array}{l}\text { Non-randomized } \\
\text { pilot study; } \\
\text { comparison of } \\
\text { training program } \\
\text { and social group } \\
\text { visit program }\end{array}$ & \begin{tabular}{|l} 
PLWD from \\
a single \\
nursing \\
home
\end{tabular} & \begin{tabular}{|l|} 
Diagnosis of \\
dementia; age 70 \\
or older; not \\
wheelchair bound; \\
able to walk
\end{tabular} & $\begin{array}{l}\mathrm{N}=18 \text { PLWD } \\
\text { exercise group } \\
18 \text { PLWD control } \\
\text { group } \\
\text { Age: } Y \\
\text { Sex: } Y \\
\text { Race: } N \\
\text { Education: } N\end{array}$ & NR & NR & NA & NA & NR & 6 weeks & \begin{tabular}{l|} 
Cognitive \\
function tests \\
Physical \\
function tests
\end{tabular} & NA \\
\hline $\begin{array}{l}\text { Suttanon, } \\
2013^{55} \\
(23117349) \\
\text { Australia } \\
\text { Pilot }\end{array}$ & $\begin{array}{l}\text { Individual } \\
\text { tailored home- } \\
\text { based exercise } \\
\text { program; } \\
\text { balance, } \\
\text { strengthening } \\
\text { and walking } \\
\text { exercise }\end{array}$ & $\begin{array}{l}\text { Home-based } \\
\text { education } \\
\text { program }\end{array}$ & \begin{tabular}{|l|} 
Community- \\
based \\
RCT \\
PLWD
\end{tabular} & $\begin{array}{l}\text { PLWD assessed } \\
\text { with mild to } \\
\text { moderate AD }\end{array}$ & $\begin{array}{l}\mathrm{N}=40 \\
\text { Age: yes } \\
\text { Sex: yes } \\
\text { \% majority race: no } \\
\text { Education: no }\end{array}$ & Prior disability: $Y$ & NA & NA & NA & NA & 6 months & & $\begin{array}{l}\text { Exercise arm } \\
\text { PLWD } \\
\text { dropped out } \\
\text { due to inability } \\
\text { of home } \\
\text { caregivers to } \\
\text { maintain the } \\
\text { program }\end{array}$ \\
\hline $\begin{array}{l}\text { Canonici, 201256 } \\
\text { (22994617) } \\
\text { Brazil } \\
\text { Small sample }\end{array}$ & $\begin{array}{l}\text { Evaluation of a } \\
\text { motor } \\
\text { intervention } \\
\text { program for } \\
\text { PLWD wit AD to } \\
\text { promote } \\
\text { functionality and } \\
\text { reduce } \\
\text { caregiver } \\
\text { burden }\end{array}$ & \begin{tabular}{|l} 
Motor \\
intervention \\
compared to \\
control group
\end{tabular} & NR & $\begin{array}{l}\text { PLWD diagnosed } \\
\text { with mild to } \\
\text { moderate AD; CDR } \\
\text { of } 1 \text { to } 2\end{array}$ & $\begin{array}{l}\mathrm{N}=16 \text { PLWD, } 16 \text { FC } \\
\text { exercise arm } \\
11 \text { PLWD, 11 FC } \\
\text { control arm } \\
\text { Age: } \mathrm{Y} \\
\text { Sex: } \mathrm{Y} \\
\text { Race: } \mathrm{N} \\
\text { Education: } \mathrm{Y}\end{array}$ & NR & NA & NA & NR & NR & 6 months & $\begin{array}{l}\text { Measuremen } \\
\text { ts of physical } \\
\text { function, } \\
\text { cognition } \\
\text { and } \\
\text { caregiver } \\
\text { burden }\end{array}$ & NA \\
\hline $\begin{array}{l}\text { Nascimento, } \\
2012^{57} \\
(22499405) \\
\text { Brazil } \\
\text { Small sample }\end{array}$ & $\begin{array}{l}\text { Supervised } \\
\text { exercise } \\
\text { program, } \\
\text { including } \\
\text { aerobic activity, } \\
\text { strength, motor } \\
\text { coordination, } \\
\text { balance } \\
6 \text { months }\end{array}$ & \begin{tabular}{|l|} 
PLWD who did \\
not participate in \\
the exercise \\
program
\end{tabular} & $\begin{array}{l}\text { Community } \\
\text { Non-RCT: } \\
\text { assigned by } \\
\text { willingness } \\
\text { to follow the } \\
\text { exercise } \\
\text { program }\end{array}$ & $\begin{array}{l}\text { Community-based } \\
\text { PLWD diagnosed } \\
\text { with mild to } \\
\text { moderate dementia } \\
\text { and using MMSE; } \\
\text { exclusion criteria } \\
\text { for other health } \\
\text { conditions }\end{array}$ & $\begin{array}{l}\mathrm{N}=20 \text { (10exercise; } \\
10 \text { controls) } \\
\text { Age: } \mathrm{N} \\
\text { Sex: } \mathrm{N} \\
\text { \% majority race: } \mathrm{N} \\
\text { Education: } \mathrm{N}\end{array}$ & NR & NA & NA & NA & NA & 6 months & $\begin{array}{l}\text { Neuropsychi } \\
\text { atric, ADL }\end{array}$ & NA \\
\hline $\begin{array}{l}\text { Vreugdenhil, } \\
2012^{58} \\
(21564154) \\
\text { Australia } \\
\text { Small sample }\end{array}$ & $\begin{array}{l}\text { Home-based } \\
\text { exercise } \\
\text { program }\end{array}$ & Usual care & \begin{tabular}{|l|} 
Community- \\
based \\
RCT
\end{tabular} & $\begin{array}{l}\text { PLWD diagnosed } \\
\text { with AD }\end{array}$ & $\begin{array}{l}\mathrm{N}=80 \\
\text { Age: } \mathrm{Y} \\
\text { Sex: } \mathrm{Y} \\
\% \text { majority race: } \mathrm{N} \\
\text { Education: } \mathrm{N}\end{array}$ & NR & NR & NR & NA & NA & 4 months & $\begin{array}{l}\text { Cognitive } \\
\text { function; } \\
\text { physical } \\
\text { function; } \\
\text { ADL; } \\
\text { depression; } \\
\text { global } \\
\text { function }\end{array}$ & $\begin{array}{l}\text { Caregiver } \\
\text { burden }\end{array}$ \\
\hline $\begin{array}{l}\text { Fan 2011 } \\
(21385519) \\
\text { Taiwan } \\
\text { High RoB }\end{array}$ & $\begin{array}{l}\text { Yoga training } \\
\text { program } \\
3 \times 1 \text { hour/week } \\
12 \text { weeks }\end{array}$ & Usual activities & $\begin{array}{l}\text { Long term } \\
\text { care facilities } \\
\text { Quasi- } \\
\text { experimental } \\
\mathrm{N}=68\end{array}$ & $\begin{array}{l}\text { Age } 60 \text { or older, } \\
\text { living in LTC, mild } \\
\text { dementia per } \\
\text { DSM-IV and MMSE } \\
18-23 \text {, no recent } \\
\text { exercise }\end{array}$ & $\begin{array}{l}\mathrm{N}=68 \text { (33 yoga, } 35 \\
\text { usual activities); } \\
\text { reported } 59 \\
\text { Mean age: } 75 \\
\text { Female: } 59 \% \\
\text { Education: } \leq 9 \mathrm{yrs} \\
\text { Race \& majority: } \mathrm{N}\end{array}$ & $\begin{array}{l}\text { SES: N } \\
\text { Disability: N } \\
\text { Household: NA } \\
\text { Marital status: Y } \\
\text { N comorbidities: Y } \\
\text { Length of } \\
\text { residence: } N \\
\text { Insurance: NA }\end{array}$ & NA & NA & NR & NR & 12 weeks & $\begin{array}{l}\text { Mobility (6 } \\
\text { m. walk), } \\
\text { balance, } \\
\text { strength, } \\
\text { depression } \\
\text { (CSDD), } \\
\text { behavior } \\
\text { (BRS) }\end{array}$ & NR \\
\hline $\begin{array}{l}\text { Roach 2011 } \\
\text { (21937893) } \\
\text { USA } \\
\text { High RoB }\end{array}$ & $\begin{array}{l}\text { 11: activity- } \\
\text { specific exercise } \\
\text { group } \\
\text { 12. Supervised } \\
\text { walking group } \\
\text { 5x/week } \\
16 \text { weeks }\end{array}$ & $\begin{array}{l}\text { Social } \\
\text { conversation } \\
\text { group }\end{array}$ & \begin{tabular}{|l|} 
Long term \\
care sites (7) \\
RCT \\
PLWD \\
$\mathrm{N}=105$
\end{tabular} & $\begin{array}{l}\text { Residence in long } \\
\text { term care, AD per } \\
\text { NINCDS-ADRDA, } \\
\text { able to walk, } \\
\text { dependent in } \geq 1 \\
\text { ADL }\end{array}$ & $\begin{array}{l}\mathrm{N}=82 \text { completed } \\
\text { Age: } \mathrm{Y} \\
\text { Sex: } \mathrm{N} \\
\% \text { majority race: } \mathrm{N} \\
\text { Education: } \mathrm{N}\end{array}$ & $\begin{array}{l}\text { Length of stay: } Y \\
\text { Other: } N\end{array}$ & NA & NA & NR & NR & 16 weeks? & $\begin{array}{l}\text { Walking, } \\
\text { transferring, } \\
\text { bed mobility }\end{array}$ & NR \\
\hline
\end{tabular}




\begin{tabular}{|c|c|c|c|c|c|c|c|c|c|c|c|c|c|}
\hline $\begin{array}{l}\text { Stella, 2011 } \\
\text { (21915483) } \\
\text { Brazil } \\
\text { Small sample }\end{array}$ & $\begin{array}{l}\text { Individual } \\
\text { exercise } \\
\text { (flexibility, } \\
\text { strength, and } \\
\text { agility)\& } \\
\text { functional } \\
\text { balance } \\
\text { exercise) } 60 \\
\text { minutes three } \\
\text { times per week. }\end{array}$ & $\begin{array}{l}\text { Motor } \\
\text { intervention } \\
\text { compared to } \\
\text { PLWD who did } \\
\text { not receive the } \\
\text { intervention }\end{array}$ & $\begin{array}{l}\text { Community- } \\
\text { based } \\
\text { RCT } \\
\text { PLWD }\end{array}$ & \begin{tabular}{|l} 
mild or moderate \\
state of probable \\
AD
\end{tabular} & $\begin{array}{l}\mathrm{N}=16 \text { PLWD } \\
\text { exercise group } \\
16 \text { PLWD control } \\
\text { group } \\
\text { Age: } Y \\
\text { Sex: } Y \\
\text { \% majority race: } N \\
\text { Education: } Y\end{array}$ & NR & $\begin{array}{l}\text { Age: } Y \\
\text { Sex: Y } \\
\text { \% majority race: N } \\
\text { Education: } \mathrm{Y} \\
\text { Relation to PLWD: } \\
\mathrm{N}\end{array}$ & Duration: $Y$ & NA & NA & 6 months & $\begin{array}{l}\text { Neuropsychi } \\
\text { atric } \\
\text { inventory; } \\
\text { Caregiver } \\
\text { burden and } \\
\text { stress }\end{array}$ & $\begin{array}{l}\text { Reduction of } \\
\text { caregiver } \\
\text { stress }\end{array}$ \\
\hline $\begin{array}{l}\text { Venturelli, } \\
2011^{60} \\
(21852281) \\
\text { Italy } \\
\text { Small sample }\end{array}$ & $\begin{array}{l}\text { Group exercise, } \\
30 \text { minutes of } \\
\text { moderate } \\
\text { walking exercise } \\
4 \text { times a week }\end{array}$ & $\begin{array}{l}\text { Daily organized } \\
\text { activities like } \\
\text { bingo, } \\
\text { patchwork } \\
\text { sewing, and } \\
\text { music therapy }\end{array}$ & $\begin{array}{l}\text { Nursing } \\
\text { homes } \\
\text { RCT } \\
\text { PLWD }\end{array}$ & $\begin{array}{l}\text { PLWD diagnosed } \\
\text { with AD, MMSE } \\
<=15 \text { and absence } \\
\text { of mobility } \\
\text { limitations }\end{array}$ & $\begin{array}{l}\mathrm{N}=12 \text { PLWD, } 12 \\
\text { caregivers walking } \\
\text { group } \\
12 \mathrm{PLWD}, 12 \\
\text { caregivers control } \\
\text { group } \\
\text { Age: yes } \\
\text { Sex: no } \\
\% \text { majority race: no } \\
\text { Education: no }\end{array}$ & NR & $\begin{array}{l}\text { Age: Y } \\
\text { Sex: Y } \\
\text { Relation to PLWD: } \\
\text { Y }\end{array}$ & NR & NA & NA & 6 months & $\begin{array}{l}\text { Reduction of } \\
\text { Functional, } \\
\text { cognitive and } \\
\text { physical } \\
\text { decline }\end{array}$ & NR \\
\hline $\begin{array}{l}\text { Steinberg, } \\
2009^{61} \\
\text { (19089875) } \\
\text { US } \\
\text { Pilot }\end{array}$ & $\begin{array}{l}\text { Home-based } \\
\text { exercise } \\
\text { intervention } \\
\text { program: } \\
\text { Aerobic fitness, } \\
\text { strength, } \\
\text { balance \& } \\
\text { flexibility training }\end{array}$ & $\begin{array}{l}\text { Home safety } \\
\text { assessment }\end{array}$ & $\begin{array}{l}\text { Community- } \\
\text { based } \\
\text { RCT } \\
\text { PLWD }\end{array}$ & $\begin{array}{l}\text { PLWD diagnosed } \\
\text { with probable AD, } \\
\text { MMSE score }>=10 ; \\
\text { community- } \\
\text { residing; } \\
\text { ambulatory, with } \\
\text { caregiver }\end{array}$ & $\begin{array}{l}\mathrm{N}=14 \text { exercise } \\
\text { group } \\
13 \text { control group } \\
\text { Age: yes } \\
\text { Sex: yes } \\
\text { \% majority race: yes } \\
\text { Education: no }\end{array}$ & NR & NR & $\mathrm{NR}$ & NA & NA & 12 weeks & $\begin{array}{l}\text { Functional } \\
\text { performance, } \\
\text { connitive } \\
\text { functioning, } \\
\text { neuropsychi } \\
\text { atric } \\
\text { symptoms, } \\
\text { caregiver } \\
\text { burden and } \\
\text { quality of life: }\end{array}$ & NA \\
\hline $\begin{array}{l}\text { Williams, } 2008^{63} \\
(17959874) \\
\text { US } \\
\text { Small sample }\end{array}$ & $\begin{array}{l}\text { I1: Group } \\
\text { exercise } \\
\text { (walking plus } \\
\text { strength } \\
\text { training, } \\
\text { balance, and } \\
\text { flexibility) } \\
\text { 12: Supervised } \\
\text { walking }\end{array}$ & $\begin{array}{l}\text { Social } \\
\text { conversation }\end{array}$ & $\begin{array}{l}\text { Nursing } \\
\text { home } \\
\text { RCT }\end{array}$ & $\begin{array}{l}\text { PLWD diagnosed } \\
\text { with AD and } \\
\text { symptoms of } \\
\text { depression }\end{array}$ & $\begin{array}{l}\text { N=116 } \\
\text { Age: yes } \\
\text { Sex: yes (overall) } \\
\text { \% majority race: yes } \\
\text { (NR by group) } \\
\text { Education: no }\end{array}$ & NR & NA & NA & Education: $Y$ & Training: $Y$ & 16 weeks & $\begin{array}{l}\text { Observed } \\
\text { Affect Scale } \\
\text { (OAS), } \\
\text { Alzheimer } \\
\text { Mood Scale, } \\
\text { Dementia } \\
\text { Mood } \\
\text { Assessment }\end{array}$ & NA \\
\hline $\begin{array}{l}\text { Rolland 200721 } \\
\text { (17302650) } \\
\text { France } \\
\text { High RoB }\end{array}$ & $\begin{array}{l}\text { Group exercise } \\
\text { (walk, strength, } \\
\text { balance, } \\
\text { flexibility) } \\
\text { 2x/week } \\
12 \text { months }\end{array}$ & $\begin{array}{l}\text { Routine medical } \\
\text { care }\end{array}$ & $\begin{array}{l}\text { Nursing } \\
\text { homes (5) } \\
\text { RCT }\end{array}$ & Mild to severe AD & $\begin{array}{l}\mathrm{N}=134 \\
\text { Age: } \mathrm{Y} \\
\text { Sex: } \mathrm{Y} \\
\% \text { majority race: } \mathrm{N} \\
\text { Education: } \mathrm{N}\end{array}$ & $\begin{array}{l}\text { Disability: Hearing } \\
\text { and visual } \\
\text { impairment } \\
\text { Other: NR }\end{array}$ & NA & NA & NR & NR & 12 months & \begin{tabular}{l|} 
Function, \\
Katz ADL, \\
depression, \\
Neuropsychi \\
atric \\
Inventory
\end{tabular} & NA \\
\hline $\begin{array}{l}\text { Stevens, 200664 } \\
\text { (16594879) } \\
\text { Australia } \\
\text { Pilot }\end{array}$ & $\begin{array}{l}\text { Individually } \\
\text { prescribed } \\
\text { exercise } \\
\text { program of } 20 \text { to } \\
30 \text { minutes } 3 x \\
\text { per weeks }\end{array}$ & $\begin{array}{l}\text { Two groups: } \\
\text { 1. no } \\
\text { intervention } \\
\text { 2. social visit of } \\
\text { equal length to } \\
\text { exercise } \\
\text { program }\end{array}$ & \begin{tabular}{|l} 
Nursing \\
home-based \\
RCT
\end{tabular} & \begin{tabular}{|l|} 
PLWD assessed \\
with mild to \\
moderate \\
dementia; MMSE \\
score $<9$ and $<23$; \\
not all PLWD \\
assessed for \\
MMSE \\
\end{tabular} & $\begin{array}{l}\mathrm{N}=24 \text { exercise, } 21 \\
\text { social visit, } \\
30 \text { control } \\
\text { Age: } \mathrm{Y} \\
\text { Sex: } \mathrm{Y} \\
\text { \% majority race: } \mathrm{N} \\
\text { Education: } \mathrm{N}\end{array}$ & NR & NA & NA & NA & NA & 12 weeks & $\begin{array}{l}\text { Assessment } \\
\text { of physical } \\
\text { and } \\
\text { psychiatric } \\
\text { problems, } \\
\text { dependence } \\
\text { on nursing } \\
\text { care }\end{array}$ & NA \\
\hline
\end{tabular}




\begin{tabular}{|c|c|c|c|c|c|c|c|c|c|c|c|c|c|}
\hline $\begin{array}{l}\text { Van de Winkel } \\
2004^{65} \\
\text { (15137556) } \\
\text { Belgium } \\
\text { Small sample }\end{array}$ & $\begin{array}{l}\text { Music-based } \\
\text { exercises }\end{array}$ & $\begin{array}{l}\text { Social } \\
\text { conversation }\end{array}$ & $\begin{array}{l}\text { RCT } \\
\text { Institutionaliz } \\
\text { ed }\end{array}$ & $\begin{array}{l}\text { PLWD with MMSE } \\
\text { score lower than } \\
\text { 24; able to respond } \\
\text { to verbal and visual } \\
\text { commands; } \\
\text { capable of } \\
\text { responding to } \\
\text { music }\end{array}$ & $\begin{array}{l}\mathrm{N}=15 \text { PLWD } \\
\text { exercise group } \\
10 \text { PLWD control } \\
\text { group } \\
\text { Age: } \mathrm{N} \\
\text { Sex: } \mathrm{N} \\
\% \text { majority race: } \mathrm{N} \\
\text { Education: } \mathrm{N}\end{array}$ & NR & NA & NA & NA & NA & 3 months & $\begin{array}{l}\text { Measures of } \\
\text { cognition; } \\
\text { need for } \\
\text { help; } \\
\text { aggressiven } \\
\text { ess; } \\
\text { depression; } \\
\text { level of } \\
\text { activity }\end{array}$ & $\mathrm{NA}$ \\
\hline $\begin{array}{l}\text { Toulotte, } 2003^{66} \\
(12540351) \\
\text { France } \\
\text { Small sample }\end{array}$ & $\begin{array}{l}\text { Exercise: chair- } \\
\text { assisted or } \\
\text { standing } \\
\text { supervised } \\
\text { exercise } \\
1 \text { hour, 2x/week } \\
16 \text { weeks }\end{array}$ & $\begin{array}{l}\text { Usual daily } \\
\text { routine }\end{array}$ & $\begin{array}{l}\text { Home and } \\
\text { institutionaliz } \\
\text { ed PLWD } \\
\text { Crossover } \\
\text { RCT }\end{array}$ & $\begin{array}{l}\text { Demented elderly } \\
\text { people with a } \\
\text { history of falling }\end{array}$ & $\begin{array}{l}\mathrm{N}=20 \\
\text { Age: yes } \\
\text { Sex: yes } \\
\text { \% majority race: no } \\
\text { Education: no }\end{array}$ & $\mathrm{NR}$ & NA & NA & NA & NA & 16 weeks & $\begin{array}{l}\text { Walking, } \\
\text { mobility, } \\
\text { flexibility and } \\
\text { static } \\
\text { balance }\end{array}$ & $\mathrm{NA}$ \\
\hline
\end{tabular}

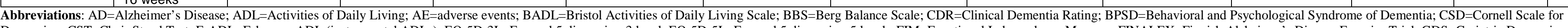

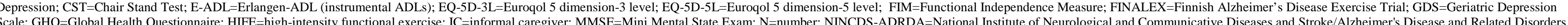

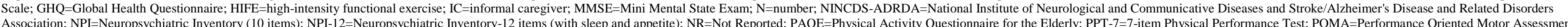
Association; NPI=Neuropsychiatric Inventory (10 items); NPI-12=Neuropsychiatric Inventory-12 items (with sleep and appetite); NR=Not Reported; PAQE=Physical Activity Questionnaire for the Elderly; PPT-7=7-7tem Physical Performance Test; POMA=Performance Oriented Motor Assed
(gait and balance); PMID=PubMed Identification Number; PLWD=Persons with Dementia; QUALID=quality of life in late-stage dementia scale; RCT=Randomized controlled trial; ROB=risk of bias; SAE=serious adverse event; SCL-90=Symptom Checklist-90; SD=standard deviation

SES=socioecono, PMID

\section{Music Intervention}




\begin{tabular}{|c|c|c|c|c|c|c|c|c|c|}
\hline Study (PMID) & $\begin{array}{l}\text { Outcome } \\
\text { Timing }\end{array}$ & $\begin{array}{l}\text { Attrition Bias } \\
\text { Attrition \% }\end{array}$ & $\begin{array}{l}\text { Selection } \\
\text { Bias }\end{array}$ & \begin{tabular}{|l|} 
Detection \\
Bias
\end{tabular} & \begin{tabular}{|l} 
Performance \\
Bias
\end{tabular} & $\begin{array}{l}\text { Reporting } \\
\text { Bias }\end{array}$ & Fidelity Bias & Funder & Overall Rating \\
\hline Ugur, 201967 (30497077) & 3 months & $\begin{array}{l}\text { Medium } \\
14 \%\end{array}$ & Low & Medium & High & Low & High & University & High \\
\hline Cheung, 2018 $8^{68}$ (30235949) & 6 weeks & $\begin{array}{l}\text { Low } \\
6 \text { weeks: } 19.39 \% \\
12 \text { weeks: } 24.84 \%\end{array}$ & Low & Low & Moderate & Low & High & No funding & Medium \\
\hline $\mathrm{Ho}, 2018^{69}(29468887)$ & 8 weeks & $\begin{array}{l}\text { Low } \\
0 \%\end{array}$ & Medium & High & High & Low & Low & The Tung Wah Groups of Hospitals & High \\
\hline Kwak 2018 ${ }^{70}$ (29871544) & 14 weeks & $\begin{array}{l}\text { Low } \\
0 \%\end{array}$ & Low & Medium & Medium & Low & Moderate & Nonprofit, University, Government & Medium \\
\hline Pongan, $2017^{71}((28922159)$ & $\begin{array}{l}12 \text { weeks } \\
\& 20 \text { weeks }\end{array}$ & $\begin{array}{l}\text { Low } \\
20 \%\end{array}$ & Medium & Low & Medium & Low & High & Nonprofit, Government & High \\
\hline Wang, 2017'2 (26443002) & 24 weeks & $\begin{array}{l}\text { Medium } \\
13.37 \%\end{array}$ & High & Medium & High & Low & High & Unclear & High \\
\hline Sarkamo, 2014 & 3 months & $\begin{array}{l}\text { Low } \\
3 \text { months: } 5.61 \% \\
9 \text { months (6months follow up post } \\
\text { intervention): } 16.85 \%\end{array}$ & Low & Low & High & Low & High & Nonprofit, Government & Medium \\
\hline Vink, 2013 $3^{74}(23280604)$ & 4 months & $\begin{array}{l}\text { Low } \\
18 \%\end{array}$ & Medium & Medium & Medium & Low & High & Nonprofit & High \\
\hline Sung, 2012 25 (21823174) & 6 weeks & $\begin{array}{l}\text { Low } \\
8.33 \%\end{array}$ & Low & Medium & High & Low & High & Government & Medium \\
\hline $\begin{array}{l}\text { Lin, 201176 (20672256) } \\
\# 2 \\
\text { Chu, 2014 } \\
\text { (23639952) }\end{array}$ & 6 weeks & $\begin{array}{l}\text { Low } \\
6 \text { weeks: } 3.84 \% \\
10 \text { weeks: } 3.84 \% \\
\text { Low }\end{array}$ & $\begin{array}{l}\text { Low } \\
\text { Low }\end{array}$ & $\begin{array}{l}\text { Medium } \\
\text { Medium }\end{array}$ & $\begin{array}{l}\text { High } \\
\text { Medium }\end{array}$ & $\begin{array}{l}\text { Low } \\
\text { Low }\end{array}$ & $\begin{array}{l}\text { High } \\
\text { Low }\end{array}$ & $\begin{array}{l}\text { NR } \\
\text { The Taipei Medical University Hospital }\end{array}$ & $\begin{array}{l}\text { Medium } \\
\text { Medium }\end{array}$ \\
\hline Raglio, 2008 ${ }^{78}(18525288)$ & $\begin{array}{l}16 \text { weeks } \\
20 \text { weeks post } \\
\text { intervention }\end{array}$ & $\begin{array}{l}\text { Low } \\
3.38 \%\end{array}$ & Medium & Low & High & Low & High & NR & High \\
\hline Ledger, 200779 (17558584) & 1 Year & $\begin{array}{l}\text { High } \\
25 \%\end{array}$ & High & $x$ & $\mathrm{x}$ & $\mathrm{x}$ & $\mathrm{x}$ & No funding & High \\
\hline
\end{tabular}

Abbreviations: NR=Not Reported; PMID=PubMed Identification Number; \#2=Companion 


\begin{tabular}{|c|c|c|c|c|c|c|c|c|c|c|c|c|c|}
\hline $\begin{array}{l}\text { Study (PMID) } \\
\text { Country } \\
\text { RoB* } \\
\text { Type }\end{array}$ & $\begin{array}{l}\text { Intervention } \\
\text { Intervention Focus } \\
\text { Theoretical Model } \\
\text { Delivery Person } \\
\text { Intervention Target } \\
\text { Recipient } \\
\text { Mode } \\
\text { Components } \\
\text { Frequency } \\
\text { Duration }\end{array}$ & \begin{tabular}{|l|} 
Comparison \\
Target \\
Mode \\
Components \\
Frequency \\
Duration
\end{tabular} & \begin{tabular}{|l|} 
Setting \\
and Design \\
\\
Setting \\
Design \\
Cluster N \\
Participants \\
Random- \\
ized N \\
\end{tabular} & $\begin{array}{l}\text { PLWD } \\
\text { Dementia } \\
\text { Characteristics } \\
\text { Dementia Types } \\
\text { Dementia } \\
\text { Severity } \\
\text { Diagnostic } \\
\text { Criteria } \\
\text { Age of Diagnosis }\end{array}$ & $\begin{array}{l}\text { PLWD } \\
\text { Non-Disease } \\
\text { Char } \\
\text { PLWD N } \\
\text { PLWD Age } \\
\text { (mean) } \\
\text { PLWD Sex (\% } \\
\text { female) } \\
\text { PLWD Race (\% } \\
\text { majority) } \\
\text { PLWD } \\
\text { Education } \\
\text { (mean years) }\end{array}$ & $\begin{array}{l}\text { PLWD } \\
\text { Non-Disease } \\
\text { Char Reporting } \\
\text { Status (RS) } \\
\text { PLWD SES } \\
\text { PLWD Prior } \\
\text { Disability } \\
\text { PLWD } \\
\text { Household } \\
\text { Characteristics } \\
\text { PLWD Health } \\
\text { Insurance } \\
\text { PLWD Detailed } \\
\text { Race } \\
\text { Information }\end{array}$ & \begin{tabular}{|l} 
Informal Caregiver \\
(IC) \\
Characteristics \\
II N \\
IC Age (mean) \\
IC Sex (\% female) \\
IC Race (\% \\
majority) \\
IC Education \\
(mean years) \\
IC Relation to \\
PLWD (\% \\
majority)
\end{tabular} & \begin{tabular}{|l} 
Informal Caregiver \\
(IC) Char. RS \\
IC Duration \\
IC Living With \\
PLWD \\
IC Payment \\
IC Health Status \\
IC Dementia \\
Family History \\
IC Employment \\
Status \\
IC Training
\end{tabular} & $\begin{array}{l}\text { Formal Caregiver } \\
\text { (FC) } \\
\text { Characteristics } \\
\text { FC N } \\
\text { FC Age (mean) } \\
\text { FC Sex (\% } \\
\text { female) } \\
\text { FC Race (\% } \\
\text { majority) } \\
\text { FC Education } \\
\text { (mean years) }\end{array}$ & $\begin{array}{l}\text { Formal } \\
\text { Caregiver (FC) } \\
\text { Char. RS } \\
\text { FC Health } \\
\text { Status } \\
\text { FC Training } \\
\text { FC Education } \\
\text { FC Position } \\
\text { FC Length of } \\
\text { Service }\end{array}$ & \begin{tabular}{|l|} 
Outcome \\
Timing(s)
\end{tabular} & PLWD Outcomes & $\begin{array}{l}\text { Caregiver } \\
\text { Outcomes } \\
\text { IC: } \\
\text { FC: }\end{array}$ \\
\hline $\begin{array}{l}\text { Cheung 2018 } \\
(30235949) \\
\text { Hong Kong } \\
\text { Medium } \\
\text { Explanatory }\end{array}$ & $\begin{array}{l}\text { PLWD met } 30 \text { mins, twice a } \\
\text { week for six weeks to } \\
\text { receive music intervention } \\
\text { that involved listening to } \\
\text { their preferred music and } \\
\text { moving their limbs and } \\
\text { trunk. The first author } \\
\text { facilitated the intervention. }\end{array}$ & \begin{tabular}{|l|} 
(1) Music \\
Listening: \\
PLWD in this \\
group listened \\
to their \\
preferred \\
music \\
(2) Social \\
activity: \\
chatted \\
casually, \\
twice a week \\
for six weeks.
\end{tabular} & $\begin{array}{l}\text { Residential } \\
\text { care facilities } \\
\text { RCT } \\
\text { multisite } \\
165 \text { PLWD }\end{array}$ & $\begin{array}{l}\text { Any type of } \\
\text { dementia with } \\
\text { moderate severity } \\
\text { according to } \\
\text { Global } \\
\text { Deterioration } \\
\text { Scale (stage } 5 \text { or } \\
6 \text { ) }\end{array}$ & \begin{tabular}{|l|}
$\mathrm{N}=165$ \\
82.27 years \\
$75.8 \%$ Female \\
$54.5 \%$ had no \\
formal \\
education
\end{tabular} & NR & NA & NA & NA & NA & \begin{tabular}{|l|}
6 weeks \\
12 weeks
\end{tabular} & $\begin{array}{l}\text { Agitation: CMIA- } \\
\text { Nursing home } \\
\text { version (Chinese } \\
\text { version) }\end{array}$ & NA \\
\hline $\begin{array}{l}\text { Cheung 2018 } \\
\text { (278194838) } \\
\text { Taiwan } \\
\text { Medium } \\
\text { Explanatory }\end{array}$ & $\begin{array}{l}\text { PLWD met } 30 \text { mins, twice a } \\
\text { week for six weeks to } \\
\text { receive music intervention } \\
\text { that involved listening to } \\
\text { their preferred music and } \\
\text { moving their limbs and } \\
\text { trunk. The first author } \\
\text { facilitated the intervention. }\end{array}$ & \begin{tabular}{|l|} 
(1) Music \\
Listening: \\
PLWD in this \\
group listened \\
to their \\
preferred \\
music \\
(2) Social \\
activity: \\
chatted \\
casually, \\
twice a week \\
for six weeks
\end{tabular} & $\begin{array}{l}\text { Residential } \\
\text { care facilities } \\
\text { RCT } \\
\text { multisite } \\
165 \text { PLWD }\end{array}$ & $\begin{array}{l}\text { Any type of } \\
\text { dementia with } \\
\text { moderate severity } \\
\text { according to } \\
\text { Global } \\
\text { Deterioration } \\
\text { Scale (stage } 5 \text { or } \\
6 \text { ) }\end{array}$ & \begin{tabular}{|l|}
$\mathrm{N}=165$ \\
82.27 years \\
$75.8 \%$ Female \\
$54.5 \%$ had no \\
formal \\
education
\end{tabular} & NR & NA & NA & NA & NA & \begin{tabular}{|l|}
6 weeks \\
12 weeks
\end{tabular} & $\begin{array}{l}\text { Anxiety: The } \\
\text { Chinese RAID } \\
\text { Depressive } \\
\text { symptoms: GDS }\end{array}$ & NA \\
\hline $\begin{array}{l}\text { Kwak 2018 } \\
\text { (29871544) } \\
\text { USA } \\
\text { Low } \\
\text { Explanatory }\end{array}$ & $\begin{array}{l}\text { Music \& memory: In } \\
\text { addition to treatment as } \\
\text { usual, personalized music } \\
\text { playlists delivered on digital } \\
\text { music players over a 6- } 6 \text { - } \\
\text { week period followed by 2- } \\
\text { week washout period \& } 6 \\
\text { weeks of treatment as } \\
\text { usual only }\end{array}$ & \begin{tabular}{|l|} 
Condition 2: 6 \\
weeks of \\
treatment as \\
usual only \\
followed by 2- \\
week \\
washout \\
period and \\
then music \\
and memory \\
for 6 weeks \\
\end{tabular} & $\begin{array}{l}\text { Nursing } \\
\text { homes } \\
\text { RCT } \\
\text { (Crossover) } \\
59 \text { PLWD }\end{array}$ & $\begin{array}{l}\text { Alzheimer's } \\
\text { disease or other } \\
\text { dementia }\end{array}$ & \begin{tabular}{|l|}
$\mathrm{N}=59$ \\
86.9 years \\
$77.96 \%$ Female \\
$93 \%$ White \\
Education: No
\end{tabular} & $\mathrm{NR}$ & NA & NA & NA & NA & 14 weeks & $\begin{array}{l}\text { Agitation: CMAI } \\
\text { Dementia-related } \\
\text { cognitive- } \\
\text { behavioral issues: } \\
\text { NPI-NH } \\
\text { Medication use: } \\
\text { Standardize } \\
\text { record form }\end{array}$ & NA \\
\hline
\end{tabular}




\begin{tabular}{|c|c|c|c|c|c|c|c|c|c|c|c|c|c|}
\hline \begin{tabular}{|l} 
Sarkamo, \\
$2014^{73}$ \\
$(24009169)$ \\
Finland \\
Medium \\
Explanatory \\
$\# 2$ \\
Sarkamo, \\
$2016^{81}$ \\
$(26519435)$
\end{tabular} & $\begin{array}{l}\text { Group Music: } \\
\text { (1) Singing coaching group: } \\
\text { In a group of 10, PLWD } \\
\text { participated in primarily } \\
\text { singing familiar songs } \\
\text { coupled occasionally with } \\
\text { vocal exercises and } \\
\text { rhythmic movements led by } \\
\text { a trained music teacher } \\
\text { once a week for } 10 \text { weeks } \\
\text { (2) Listening coaching } \\
\text { group: In a group of } 10, \text { led } \\
\text { by a therapist, PLWD } \\
\text { primarily listened to songs } \\
\text { from CD and discussed } \\
\text { about the emotions, } \\
\text { thoughts, and memories, } \\
\text { once a week for } 10 \text { weeks }\end{array}$ & \begin{tabular}{|l|} 
Usual care: \\
PLWD \\
continued \\
with their \\
normal \\
everyday \\
activities and \\
hobbies \\
without \& \\
were not \\
given any \\
additional \\
activities \\
throughout \\
the follow-up.
\end{tabular} & \begin{tabular}{|l} 
Day activity \\
centers \& \\
inpatient \\
centers \\
RCT \\
89 Dyads \\
(59 family \\
members \& \\
30 nurses) \\
\end{tabular} & \begin{tabular}{|l|} 
All types of \\
dementia \\
(diagnosis made \\
by a geriatrician or \\
a primary care \\
Physician) Mild to \\
moderate (CDR)
\end{tabular} & \begin{tabular}{|l}
$\mathrm{N}=84$ \\
Age: 89.6 years \\
$71.42 \%$ Female \\
$54.5 \%$ had no \\
formal \\
education
\end{tabular} & NR & \begin{tabular}{|l|}
59 IC (family \\
members) \\
randomized; \\
No details provided \\
on how many \\
completed or their \\
demographic \\
details
\end{tabular} & NR & \begin{tabular}{|l|}
30 nurses \\
randomized \\
No details \\
provided on how \\
many completed \\
and their \\
demographic \\
details
\end{tabular} & NR & \begin{tabular}{|l|}
10 weeks \\
9 months
\end{tabular} & $\begin{array}{l}\text { PLWD Mood \& } \\
\text { QoL: CBS \& QOL- } \\
\text { AD } \\
\text { Psychological } \\
\text { well-being of CGs: } \\
\text { GHQ and ZBI }\end{array}$ & NA \\
\hline $\begin{array}{l}\text { Sung 2012 } \\
(21823174) \\
\text { Taiwan } \\
\text { Medium } \\
\text { Explanatory }\end{array}$ & $\begin{array}{l}\text { Group music: } \\
\text { PLWD received } \\
\text { 30-min music intervention } \\
\text { using percussion } \\
\text { instruments with } \\
\text { familiar music in a group } \\
\text { setting, delivered by trained } \\
\text { research assistants in } \\
\text { midafternoon twice weekly } \\
\text { for 6weeks, }\end{array}$ & $\begin{array}{l}\text { PLWD in the } \\
\text { control group } \\
\text { received } \\
\text { usual care } \\
\text { and did not } \\
\text { attend the } \\
\text { group music } \\
\text { interventions. }\end{array}$ & $\begin{array}{l}\text { Residential } \\
\text { care facility } \\
\text { RCT } \\
60 \text { PLWD }\end{array}$ & \begin{tabular}{|l} 
Any type of \\
dementia with mild \\
to severe severity \\
(the Short \\
Portable Mental \\
Status \\
Questionnaire)
\end{tabular} & \begin{tabular}{|l}
$\mathrm{N}=55$ \\
Average age \\
81.37 years for \\
the control \\
group and 97.5 \\
years for the \\
control group \\
$65.8 \%$ Female \\
$76.2 \%$ had no \\
formal \\
education
\end{tabular} & NR & NA & NA & NA & NA & 6 weeks & $\begin{array}{l}\text { Anxiety: RAID } \\
\text { Agitation: CMAI }\end{array}$ & NA \\
\hline
\end{tabular}

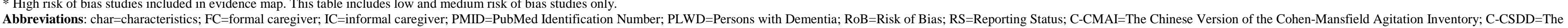

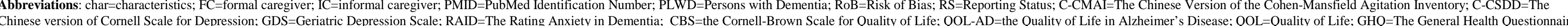
ZBI=Zarit Burden Interview; RoB=Risk of Bias; NPI-NH=the Neuropsychiatric Inventory-Nursing Home Version; CDR=Clinical Dementia Rating; CG=Caregiver; RCT=Randomized controlled trial; MMSE=Mini-Mental State Exam 
Table D-8. PLWD outcomes summary low and medium risk of bias studies: music intervention

\begin{tabular}{|c|c|c|c|c|c|}
\hline $\begin{array}{l}\text { Study (PMID) } \\
\text { Comparison } \\
\text { RoB } \\
\text { Category* }\end{array}$ & $\begin{array}{l}\text { Outcome } \\
\text { Timing }\end{array}$ & Summary Finding & Intervention & Comparator & p-Value \\
\hline $\begin{array}{l}\text { Cheung, } 2018^{68} \text { (27819483) } \\
\text { Music with movement vs. music listening } \\
\text { and social activity } \\
\text { Medium } \\
\text { Explanatory }\end{array}$ & $\begin{array}{l}\text { Adjusted Mean Difference }(95 \% \mathrm{Cl}) \\
\text { Anxiety (RAID- Chinese version) } \\
6 \text { weeks } \\
\text { Depressive symptoms (GDS) } \\
6 \text { weeks } \\
\end{array}$ & $\begin{array}{l}\text { No useable outcome reported } \\
\text { No useable outcome reported }\end{array}$ & & & \\
\hline $\begin{array}{l}\text { Cheung, } 2018^{80}(30235949) \\
\text { Music with movement vs. music listening } \\
\text { and social activity } \\
\text { Medium } \\
\text { Explanatory }\end{array}$ & $\begin{array}{l}\text { Adjusted Mean Difference }(95 \% \mathrm{Cl}) \\
\text { Agitation (CMAI-NH) } \\
6 \text { weeks }\end{array}$ & $\begin{array}{l}\text { Group } x \text { time interaction do not favor intervention } \\
F(d f)=1.22(4,324)\end{array}$ & & & 0.303 \\
\hline $\begin{array}{l}\text { Sarkamo, 201473 (24009169) } \\
\text { Music vs. usual care } \\
\text { Medium } \\
\text { Explanatory }\end{array}$ & $\begin{array}{l}\text { Quality of life ((QOL-AD) - administered in an interview format } \\
3 \text { months } \\
\text { Adjusted Mean Difference }(95 \% \text { Cl) } \\
\text { Mood (CBS) - administered in an interview format }\end{array}$ & $\begin{array}{l}\text { Group } x \text { time interaction favors intervention } \\
F(d f)=12.9(1,81) \\
\text { Group x time interaction favors intervention } \\
F(d f)=4.6(1,62)\end{array}$ & & & $\begin{array}{l}\mathrm{p}=.001 \\
\mathrm{p}=.036\end{array}$ \\
\hline $\begin{array}{l}\text { Sung, } 2012^{75}(21823174) \\
\text { Group music vs. usual care } \\
\text { Medium } \\
\text { Explanatory }\end{array}$ & $\begin{array}{l}\text { Adjusted Mean Difference }(95 \% \mathrm{Cl}) \\
\text { Agitation (CMAI) } \\
6 \text { weeks } \\
\text { Adjusted Mean Difference }(95 \% \mathrm{Cl}) \\
\text { Anxiety (RAID) } \\
6 \text { weeks } \\
\end{array}$ & $\begin{array}{l}\text { Group } x \text { time interaction do not favor intervention } \\
F(d f)=0.33(1,51) \\
\text { The outcome favors intervention: } \\
\text { Mean difference }(95 \% \text { Cl): } 3.77 \text { (1.25 to } 6.3)\end{array}$ & & & $p=0.004$ \\
\hline $\begin{array}{l}\text { Lin, 201176 (20672256) } \\
\text { Group music vs. usual care } \\
\text { Medium } \\
\text { Explanatory } \\
\text { \#2 } \\
\text { Chu, 201477 (23639952) }\end{array}$ & $\begin{array}{l}\text { Adjusted Mean Difference }(95 \% \mathrm{Cl}) \\
\text { Agitated behavior (C-CMAI). } \\
6 \text { weeks } \\
\text { Adjusted Mean Difference }(95 \% \mathrm{Cl}) \\
\text { Depression (C-CSDD) } \\
6 \text { weeks } \\
\end{array}$ & $\begin{array}{l}\text { The outcome favors music intervention } \\
\text { Adjusted Mean Difference }(95 \% \mathrm{Cl}) \text { : } \\
-0.47(-0.74 \text { to }-0.19)\end{array}$ & & & $<0.001$ \\
\hline
\end{tabular}

Abbreviations: CBS=Caregiver Burden Scale,
RAID=the Rating Anxiety in Dementia Scale 
Table D-9. Summary of strength of evidence for PLWD outcomes: music intervention

\begin{tabular}{|c|c|c|c|c|c|c|c|c|}
\hline $\begin{array}{l}\text { Outcome } \\
\text { Comparison }\end{array}$ & Timing & $\begin{array}{l}\text { \# Studies/ Design } \\
\text { (n analyzed) }\end{array}$ & Finding or Summary Statistic & $\begin{array}{l}\text { Study } \\
\text { Limitations }\end{array}$ & Consistency & Directness & Precision & Overall Grade/ Conclusion \\
\hline $\begin{array}{l}\text { Agitation } \\
\text { Group music vs. Usual care }\end{array}$ & 6 weeks & $2 \mathrm{RCT}(\mathrm{n}=155)$ & $\begin{array}{l}\text { Inconsistent finding. One study reported significant reduction of agitation (C- } \\
\text { CMAI) in the music group compared to usual care group, another reported no } \\
\text { significant difference in the reduction of agitation scores (\& CMAI) between two } \\
\text { groups. }\end{array}$ & Moderate & Inconsistent & Direct & Imprecise & Insufficient \\
\hline $\begin{array}{l}\text { Agitation } \\
\text { Music with movement vs. } \\
\text { music listening \& social } \\
\text { activity }\end{array}$ & 6 weeks & $1 \mathrm{RCT}(\mathrm{n}=165)$ & $\begin{array}{l}\text { No sig difference between groups in terms of reducing agitation measured } \\
\text { using CMAI-NH. }\end{array}$ & Moderate & Unknown & Direct & Imprecise & Insufficient \\
\hline $\begin{array}{l}\text { Depression } \\
\text { Group music vs usual care }\end{array}$ & 6 weeks & $\begin{array}{l}1 \text { RCT }(100) \\
\text { Companion study (Lin,2011) }\end{array}$ & $\begin{array}{l}\text { Significant difference between groups in the change in depression (C-CSDD) } \\
\text { level from baseline to posttest }\end{array}$ & Moderate & Unknown & Direct & Imprecise & Insufficient \\
\hline $\begin{array}{l}\text { Anxiety } \\
\text { Music with movement vs. } \\
\text { music listening \& social } \\
\text { activity }\end{array}$ & 6 weeks & $1 \mathrm{RCT}(\mathrm{n}=165)$ & $\begin{array}{l}\text { Results showed that the Music with movement did not differ } \\
\text { from ML and SA in changing the symptoms of anxiety measured using RAID. }\end{array}$ & Moderate & Unknown & Direct & Imprecise & Insufficient \\
\hline $\begin{array}{l}\text { Group music vs. usual care } \\
\text { Mood }\end{array}$ & 3 months & $1 \mathrm{RCT}(\mathrm{n}=84)$ & $\begin{array}{l}\text { A significant effect of music on mood (CBS) was observed in the music group. } \\
\text { Music was effective in temporarily improving mood. }\end{array}$ & Moderate & unknown & Direct & Imprecise & Insufficient \\
\hline $\begin{array}{l}\text { Group music vs. usual care } \\
\text { Quality of Life }\end{array}$ & 3 months & $1 \mathrm{RCT}(\mathrm{n}=84)$ & $\begin{array}{l}\text { Result was inconsistent. There was a long-term specific effect in the self-report } \\
\text { total scores. However, statistical significance could not be established with } \\
\text { caregiver-report QOL-AD scores. Music listening than singing, showed long } \\
\text { term effect }\end{array}$ & Moderate & Inconsistent & Direct & Imprecise & Insufficient \\
\hline
\end{tabular}

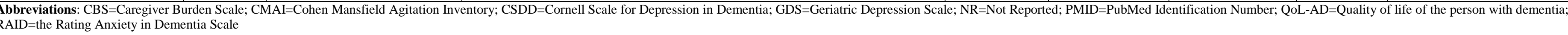

\section{Evidence Map: Music Intervention}

\begin{tabular}{|c|c|c|c|c|c|c|c|c|c|c|c|c|c|}
\hline $\begin{array}{l}\text { Study (PMID) } \\
\text { Country } \\
\text { EM Reason }\end{array}$ & Intervention & Comparison & $\begin{array}{l}\text { Setting } \\
\text { and Design } \\
\text { Setting } \\
\text { Design } \\
\text { Cluster N } \\
\text { Participants } \\
\text { Random- } \\
\text { ized N }\end{array}$ & $\begin{array}{l}\text { PLWD } \\
\text { Dementia } \\
\text { Characteristics }\end{array}$ & $\begin{array}{l}\text { PLWD } \\
\text { Non-Disease Char } \\
\text { PLWD N } \\
\text { PLWD Age (mean) } \\
\text { PLWD Sex (\% } \\
\text { female) } \\
\text { PLWD Race (\% } \\
\text { majority) } \\
\text { PLWD Education } \\
\text { (mean years) }\end{array}$ & $\begin{array}{l}\text { PLWD } \\
\text { Non-Disease } \\
\text { Char Reporting } \\
\text { Status (RS) } \\
\\
\text { PLWD SES } \\
\text { PLWD Prior } \\
\text { Disability } \\
\text { PLWD } \\
\text { Household } \\
\text { Characteristics } \\
\text { PLWD Health } \\
\text { Insurance } \\
\text { PLWD Detailed } \\
\text { Race Information }\end{array}$ & $\begin{array}{l}\text { Informal Caregiver } \\
\text { (IC) } \\
\text { Characteristics } \\
\text { IC N } \\
\text { IC Age (mean) } \\
\text { IC Sex (\% female) } \\
\text { IC Race (\% } \\
\text { majority) } \\
\text { IC Education } \\
\text { (mean years) } \\
\text { IC Relation to } \\
\text { PLWD (\% majority) }\end{array}$ & $\begin{array}{l}\text { Informal Caregiver } \\
\text { (IC) Char. RS } \\
\text { IC Duration } \\
\text { IC Living With } \\
\text { PLWD } \\
\text { IC Payment } \\
\text { IC Health Status } \\
\text { IC Dementia } \\
\text { Family History } \\
\text { IC Employment } \\
\text { Status } \\
\text { IC Training }\end{array}$ & 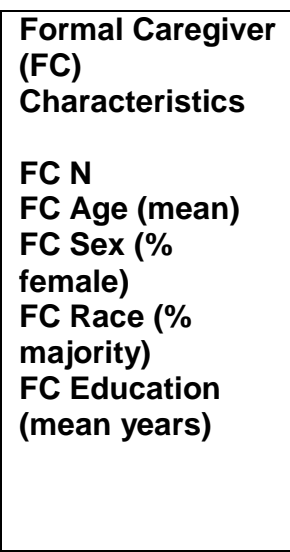 & $\begin{array}{l}\text { Formal } \\
\text { Caregiver (FC) } \\
\text { Char. RS } \\
\text { FC Health } \\
\text { Status } \\
\text { FC Training } \\
\text { FC Education } \\
\text { FC Position } \\
\text { FC Length of } \\
\text { Service }\end{array}$ & $\begin{array}{l}\text { Outcome } \\
\text { Timing(s) }\end{array}$ & \begin{tabular}{|l|} 
PLWD \\
Outcomes
\end{tabular} & $\begin{array}{l}\text { Caregiver } \\
\text { Outcomes } \\
\text { IC: } \\
\text { FC: }\end{array}$ \\
\hline $\begin{array}{l}\text { Weise } 2020^{82} \\
\text { Germany } \\
\text { Pilot }\end{array}$ & $\begin{array}{l}\text { Individualized } \\
\text { music program: } \\
\text { Participants } \\
\text { listened to } \\
\text { personally- } \\
\text { relevant music } \\
\text { playlists for } 30 \\
\text { minutes every } \\
\text { other day for } \\
\text { four weeks }\end{array}$ & Wait-list control & $\begin{array}{l}\mathrm{NH} \\
\text { Residents } \\
\text { RCT } \\
20 \text { PLWD }\end{array}$ & $\begin{array}{l}\text { Clinical diagnosis } \\
\text { of dementia (mild } \\
\text { to severe) and no } \\
\text { severe hearing } \\
\text { problems }\end{array}$ & $\begin{array}{l}\mathrm{N}=20 \\
\text { Mean age: } 85 \text { years } \\
\text { \% Female: } 80 \\
\text { \% majority race: no } \\
\text { Education: no }\end{array}$ & None & NA & None & NA & NA & 4 weeks & CMAI & NA \\
\hline
\end{tabular}




\begin{tabular}{|c|c|c|c|c|c|c|c|c|c|c|c|c|c|}
\hline $\begin{array}{l}\text { Ihara 201983 } \\
\text { (30460747) } \\
\text { US } \\
\text { Quasi- } \\
\text { experimental }\end{array}$ & $\begin{array}{l}\text { MUSIC \& } \\
\text { MEMORY } \\
\text { program: an } \\
\text { individualized } \\
\text { music listening } \\
\text { system where } \\
\text { individuals are } \\
\text { given an iPod } \\
\text { programmed } \\
\text { with their } \\
\text { personalized } \\
\text { playlist }\end{array}$ & $\begin{array}{l}\text { Participated in } \\
\text { daily planned } \\
\text { activities, such } \\
\text { as exercises } \\
\text { and games. }\end{array}$ & \begin{tabular}{|l|} 
Residents of \\
community- \\
based adult \\
day health \\
centers \\
Quasi- \\
experimental \\
51 PLWD
\end{tabular} & $\begin{array}{l}\text { Diagnosis of } \\
\text { dementia and } \\
\text { MMSE score of } \\
\leq 24\end{array}$ & $\begin{array}{l}\mathrm{N}=51 \\
\text { Mean age: } 82 \text { years } \\
\% \text { Female: } 67 \\
\text { \% majority race: no } \\
\text { Education: no }\end{array}$ & None & NA & None & NA & $\mathrm{NA}$ & $\begin{array}{l}\text { During the } \\
\text { intervention } \\
6 \text { weeks }\end{array}$ & $\begin{array}{l}\text { CMAl } \\
\text { CSDD } \\
\text { video } \\
\text { recording of } \\
\text { Behavioral } \\
\text { observations }\end{array}$ & NA \\
\hline $\begin{array}{l}\text { Park, 201984 } \\
\text { (31533443) } \\
\text { US } \\
\text { Pilot }\end{array}$ & $\begin{array}{l}\text { Chair yoga: } \\
\text { Practiced Hatha } \\
\text { yoga (breathing, } \\
\text { physical posture } \\
\text { \& guided } \\
\text { relaxation), in a } \\
\text { chair or } \\
\text { standing and } \\
\text { using a chair as } \\
\text { support twice- } \\
\text { weekly } 45- \\
\text { minute sessions } \\
\text { for } 12 \text { weeks }\end{array}$ & $\begin{array}{l}\text { Music: Attended } \\
\text { music sessions } \\
\text { twice-weekly } \\
\text { 45-minute } \\
\text { sessions for } 12 \\
\text { weeks } \\
\text { facilitated by } \\
\text { music therapy. } \\
\text { Chair-based } \\
\text { exercise: } \\
\text { Attended } 45- \\
\text { min gentle } \\
\text { exercise } \\
\text { session twice } \\
\text { weekly for } 12 \\
\text { weeks }\end{array}$ & \begin{tabular}{|l|} 
Community \\
setting \\
RCT \\
(Cluster) \\
31 PLWD \\
\end{tabular} & \begin{tabular}{|l|} 
All types of \\
dementia \\
Any stage of \\
severity
\end{tabular} & $\begin{array}{l}\mathrm{N}=31 \\
\text { Age: } 84.3 \\
\text { Sex: Yes } \\
\text { \% majority race: } \\
\text { Yes } \\
\text { Education: No }\end{array}$ & $\begin{array}{l}\text { PLWD Household } \\
\text { Characteristics: } \\
\text { Yes } \\
\text { PLWD Detailed } \\
\text { Race Information: } \\
\text { Yes }\end{array}$ & NA & NA & NA & NA & 12 weeks & $\begin{array}{l}\text { Psychological } \\
\text { symptoms: } \\
\text { HADS } \\
\text { Behavioral } \\
\text { problems: } \\
\text { CMAI-SF } \\
\text { QoL; } \\
\text { sleep } \\
\text { problems; } \\
\text { Epworth } \\
\text { Sleepiness } \\
\text { Scale }\end{array}$ & NA \\
\hline $\begin{array}{l}\text { Ugur, 201967 } \\
(30497077) \\
\text { Turkey } \\
\text { High RoB }\end{array}$ & $\begin{array}{l}\text { Music Therapy: } \\
\text { PLWD listened } \\
\text { to instrumental } \\
\text { classical } \\
\text { Turkish music, } 3 \\
\text { sessions per } \\
\text { week in the } \\
\text { mornings in } \\
\text { their own rooms } \\
\text { for } 12 \text { weeks }\end{array}$ & $\begin{array}{l}\text { Control: MT } \\
\text { was not } \\
\text { provided for } \\
\text { the } \\
\text { participants in } \\
\text { the control }\end{array}$ & $\begin{array}{l}\text { Nursing } \\
\text { homes } \\
\text { RCT } \\
\text { 70 PLWD }\end{array}$ & $\begin{array}{l}\text { Non-specified } \\
\text { Mild -severe }\end{array}$ & $\begin{array}{l}\mathrm{N}=60 \\
\text { Age: } 82.91 \text { years } \\
\text { Sex: Yes } \\
\text { \% majority race: } \\
\text { No } \\
\text { Education: Yes }\end{array}$ & NR & $\begin{array}{l}\text { N=60 } \\
\text { Age: Yes } \\
\text { Sex: No } \\
\% \text { majority race: } \\
\text { No } \\
\text { Education: Yes }\end{array}$ & Health status: Yes & NA & NA & 12 weeks & CBS \& ADLS & $\begin{array}{l}\text { Caregiver } \\
\text { burden: } \\
\text { CBS }\end{array}$ \\
\hline $\begin{array}{l}\text { Ho, 2018 } \\
(29468887) \\
\\
\text { Hong Kong } \\
\text { High RoB }\end{array}$ & $\begin{array}{l}\text { Music } \\
\text { Intervention: } 16 \\
\text { half-hour } \\
\text { sessions of } \\
\text { music } \\
\text { intervention with } \\
\text { multi-sensory } \\
\text { components } \\
\text { over eight } \\
\text { weeks }\end{array}$ & $\begin{array}{l}\text { Control: } \\
\text { Received } \\
\text { standard care }\end{array}$ & $\begin{array}{l}\text { Elderly } \\
\text { residential } \\
\text { home } \\
\text { RCT } \\
73\end{array}$ & \begin{tabular}{|l|} 
Moderate dementia \\
(non-specified)
\end{tabular} & $\begin{array}{l}\text { N=73 } \\
\text { Age: } 85.3 \text { years } \\
\text { Sex: Yes } \\
\text { \% majority race: } \\
\text { Yes } \\
\text { Education: Yes }\end{array}$ & NR & NA & NA & NA & NA & 8 weeks & $\begin{array}{l}\text { Behavioral } \\
\text { and } \\
\text { psychological } \\
\text { symptoms: } \\
\text { The NPI } \\
\text { (Chinese } \\
\text { version) } \\
\text { Subjective } \\
\text { mood: VAMS }\end{array}$ & $\mathrm{NA}$ \\
\hline
\end{tabular}




\begin{tabular}{|c|c|c|c|c|c|c|c|c|c|c|c|c|c|}
\hline $\begin{array}{l}\text { Giovagnoli } \\
2017^{85} \\
(29550981) \\
\text { Italy } \\
\text { Small sample }\end{array}$ & $\begin{array}{l}\text { Active music } \\
\text { therapy (AMT) } \\
\text { and neuro- } \\
\text { education (NE): } \\
\text { involving a } \\
\text { regulatory } \\
\text { action of sound } \\
\text { stimulating non- } \\
\text { verbal } \\
\text { communication. } \\
\text { AMT lasted } 245 \\
\text { minutes group } \\
\text { sessions per } \\
\text { week for 12 } \\
\text { weeks. NE also } \\
12 \text { weeks }\end{array}$ & $\begin{array}{l}\text { cognitive } \\
\text { training lasted } 2 \\
45 \text { minutes } \\
\text { group sessions } \\
\text { per week for } 12 \\
\text { weeks }\end{array}$ & $\begin{array}{l}\text { Recruited } \\
\text { from a } \\
\text { center } \\
\text { RCT } \\
50 \text { PLWD }\end{array}$ & \begin{tabular}{|l|} 
Mild to moderate \\
dementia or AD \\
with a MMSE score \\
$>15$
\end{tabular} & $\begin{array}{l}\mathrm{N}=50 \\
\text { Age: } 74 \text { years } \\
\text { Sex: Yes } \\
\text { \% majority race: no } \\
\text { Education: Yes }\end{array}$ & \begin{tabular}{|l|}
$N R$ \\
\end{tabular} & NA & NA & NA & $\mathrm{NA}$ & $\begin{array}{l}12 \text { weeks } \\
24 \text { weeks }\end{array}$ & $\begin{array}{l}\text { Word Fluency } \\
\text { on phonemic } \\
\text { cue } \\
\text { Attentive } \\
\text { Matrices } \\
\text { Trail Making } \\
\text { Test A/B } \\
\text { Weigl Sorting } \\
\text { Test } \\
\text { Short Story } \\
\text { test }\end{array}$ & \\
\hline $\begin{array}{l}\text { Pongan, 2017 } \\
\text { (28922159) } \\
\text { France } \\
\text { High RoB }\end{array}$ & $\begin{array}{l}\text { Choral singing: } \\
\text { participated in } \\
12 \text { weekly two- } \\
\text { hour singing } \\
\text { sessions }\end{array}$ & $\begin{array}{l}\text { Painting: } \\
\text { participated in } \\
12 \text { weekly two- } \\
\text { hour painting } \\
\text { sessions }\end{array}$ & $\begin{array}{l}\text { Community } \\
\text { setting } \\
\text { RCT } \\
65 \text { PLWD }\end{array}$ & $\begin{array}{l}\text { Probable AD with } \\
\text { mild severity }\end{array}$ & $\begin{array}{l}\mathrm{N}=59 \\
\text { Age: Yes } \\
\text { Sex: Yes } \\
\text { \% majority race: No } \\
\text { Education: Yes }\end{array}$ & \begin{tabular}{|l|}
$N R$ \\
\end{tabular} & NA & NA & NA & NA & 12 weeks & $\begin{array}{l}\text { Chronic pain: } \\
\text { NRS, SVS \& } \\
\text { BPI Anxiety: } \\
\text { STAI } \\
\text { Depression: } \\
\text { GDS Quality } \\
\text { of life: EQ-5D }\end{array}$ & NA \\
\hline $\begin{array}{l}\text { Wang, 2017'2 } \\
\text { (26443002) } \\
\text { Taiwan } \\
\text { High RoB }\end{array}$ & $\begin{array}{l}\text { Music: received } \\
\text { Kagayashiki } \\
\text { music care } \\
\text { (KMC) twice per } \\
\text { week for } 24 \\
\text { weeks. }\end{array}$ & $\begin{array}{l}\text { Usual care: } \\
\text { provided with } \\
\text { activities as } \\
\text { usual }\end{array}$ & $\begin{array}{l}\text { Long-term } \\
\text { care facility } \\
\text { Quasi } \\
172 \text { PLWD }\end{array}$ & \begin{tabular}{|l|} 
Any type of \\
dementia \\
Mild - severe
\end{tabular} & $\begin{array}{l}\mathrm{N}=147 \\
\text { Age: Yes } \\
\text { Sex: Yes } \\
\text { \% majority race: No } \\
\text { Education: Yes }\end{array}$ & \begin{tabular}{|l|}
$N R$ \\
\end{tabular} & NA & NA & NA & NA & 24 weeks & $\begin{array}{l}\text { Behavior } \\
\text { problem: } \\
\text { CAPE-BRS } \\
\text { Depression: } \\
\text { CSD }\end{array}$ & NA \\
\hline 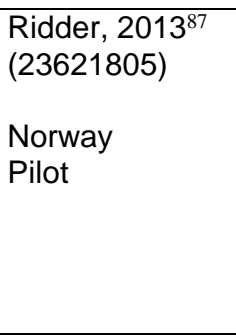 & $\begin{array}{l}\text { Individual music } \\
\text { therapy: } \\
\text { Individual music } \\
\text { therapy was } \\
\text { given biweekly } \\
\text { over a period of } \\
\text { six weeks, } \\
\text { altogether } 12 \\
\text { sessions }\end{array}$ & $\begin{array}{l}\text { Control: } \\
\text { Received } \\
\text { Standard Care } \\
\text { (for some } \\
\text { includes group } \\
\text { sing-along } \\
\text { sessions as } \\
\text { usual) }\end{array}$ & \begin{tabular}{|l|} 
Nursing \\
home \\
RCT(Crosso \\
ver) \\
42 PLWD \\
\end{tabular} & $\begin{array}{l}\text { Moderate to severe } \\
\text { dementia }\end{array}$ & $\begin{array}{l}\mathrm{N}=42 \\
\text { Age: Yes } \\
\text { Sex: Yes } \\
\text { \% majority race: No } \\
\text { Education: No }\end{array}$ & \begin{tabular}{|l|}
$N R$ \\
\end{tabular} & NA & NA & NA & NA & 6 weeks & $\begin{array}{l}\text { CMAI-NH; the } \\
\text { ADRQL; \& } \\
\text { psychotropic } \\
\text { medication }\end{array}$ & NA \\
\hline
\end{tabular}




\begin{tabular}{|c|c|c|c|c|c|c|c|c|c|c|c|c|c|}
\hline $\begin{array}{l}\text { Sakamoto, } \\
2013^{88} \\
(23298693) \\
\\
\text { Japan } \\
\text { Small sample }\end{array}$ & $\begin{array}{l}\text { Music therapy - } \\
\text { Passive OR } \\
\text { interactive: } \\
\text { Each } \\
\text { intervention was } \\
\text { performed for } \\
30 \text { min once a } \\
\text { week for } 10 \\
\text { weeks (10 } \\
\text { sessions in } \\
\text { total). } \\
\text { Passive: } \\
\text { participants } \\
\text { passively } \\
\text { listened to the } \\
\text { selected music } \\
\text { via a CD player. } \\
\text { Interactive: } \\
\text { participants not } \\
\text { only listened to } \\
\text { the selectedd } \\
\text { music via a CD } \\
\text { player but also } \\
\text { participated in } \\
\text { interactive } \\
\text { activities (e.g., } \\
\text { clapping, } \\
\text { singing, \& } \\
\text { dancing) guided } \\
\text { by a music } \\
\text { facilitator }\end{array}$ & $\begin{array}{l}\text { Control- No } \\
\text { music: } \\
\text { participant } \\
\text { spent time with } \\
\text { one caregiver in } \\
\text { their own room } \\
\text { as usual, } \\
\text { without any } \\
\text { music } \\
\text { intervention }\end{array}$ & \begin{tabular}{|l|} 
Nursing \\
home \\
RCT \\
39 PLWD
\end{tabular} & $\begin{array}{l}\text { Severe Alzheimer's } \\
\text { Type dementia }\end{array}$ & $\begin{array}{l}\mathrm{N}=39 \\
\text { Age: Yes } \\
\text { Sex: Yes } \\
\text { \% majority race: } \\
\text { Yes } \\
\text { Education: No }\end{array}$ & \begin{tabular}{|l|}
$N R$ \\
\end{tabular} & NA & NA & NA & NA & 10 weeks & $\begin{array}{l}\text { BPSD } \\
\text { changes: } \\
\text { BEHAVE-AD }\end{array}$ & NA \\
\hline $\begin{array}{l}\text { Vink, 201374 } \\
(23280604) \\
\\
\text { Netherlands } \\
\text { High RoB }\end{array}$ & $\begin{array}{l}\text { Music therapy: } \\
\text { Each music } \\
\text { therapy } \\
\text { intervention } \\
\text { lasted for } 40 \\
\text { min and was } \\
\text { provided twice } \\
\text { weekly, by a } \\
\text { formally trained } \\
\text { music therapist } \\
\text { with at least } 5 \\
\text { years working } \\
\text { experience. }\end{array}$ & $\begin{array}{l}\text { General } \\
\text { recreational day } \\
\text { activities: } 40 \\
\text { minutes of } \\
\text { general daily } \\
\text { recreational } \\
\text { activities }\end{array}$ & $\begin{array}{l}\text { Nursing } \\
\text { homes } \\
\text { RCT } \\
\text { 94 PLWD }\end{array}$ & $\begin{array}{l}\text { Any type of } \\
\text { dementia with mild } \\
\text { to very severe } \\
\text { severity }\end{array}$ & $\begin{array}{l}\mathrm{N}=77 \\
\text { Age: Yes } \\
\text { Sex: Yes } \\
\text { \% majority race: No } \\
\text { Education: No }\end{array}$ & NR & NA & NA & NA & NA & 4 months & CMAI & NA \\
\hline $\begin{array}{l}\text { Ceccato, } 2012^{89} \\
\text { Italy } \\
\text { Small sample }\end{array}$ & $\begin{array}{l}\text { The Sound } \\
\text { Training for } \\
\text { Attention \& } \\
\text { Memory in } \\
\text { Dementia } \\
\text { (STAM-Dem): } \\
\text { Music employed } \\
\text { as stimulus. } \\
\text { Followed the } \\
\text { STAM-Dem for } \\
2 \text { weekly } \\
\text { sessions of } 45 \\
\text { minutes for } 12 \\
\text { weeks (in } \\
\text { addition to } \\
\text { standard care). }\end{array}$ & $\begin{array}{l}\text { Standard care: } \\
\text { Continued with } \\
\text { the normal } \\
\text { "standard care" } \\
\text { provided }\end{array}$ & $\begin{array}{l}\text { RCT } \\
51 \text { PLWD }\end{array}$ & NR & $\begin{array}{l}\mathrm{N}=50 \\
\text { Age: Yes } \\
\text { Sex: Yes } \\
\text { \% majority race: No } \\
\text { Education: No }\end{array}$ & NR & NA & NA & NA & NA & 12 weeks & $\begin{array}{l}\text { Cognitive } \\
\text { function: MPI, } \\
\text { MPD } \\
\text { Mood: GDS } \\
\text { Aggressive } \\
\text { behavior: } \\
\text { CMAI } \\
\text { Functional } \\
\text { status: ADL }\end{array}$ & NA \\
\hline
\end{tabular}




\begin{tabular}{|c|c|c|c|c|c|c|c|c|c|c|c|c|c|}
\hline $\begin{array}{l}\text { McHugh, 2012 } \\
\text { USA } \\
\text { Pilot study }\end{array}$ & $\begin{array}{l}\text { Music therapy - } \\
\text { vocal re- } \\
\text { creative music } \\
\text { therapy (VMT): } \\
\text { residents } \\
\text { participated in } \\
25 \text { mins of } \\
\text { music session } \\
\text { just before their } \\
\text { mid-day meal, } 4 \\
\text { times a week for } \\
3 \text { weeks. }\end{array}$ & $\begin{array}{l}\text { Control wait-list } \\
\text { group: Unclear }\end{array}$ & $\begin{array}{l}\text { Care facility } \\
\text { RCT } \\
15 \text { PLWD }\end{array}$ & $\begin{array}{l}\text { Moderate to severe } \\
\text { dementia (primarily } \\
\text { AD) }\end{array}$ & $\begin{array}{l}\mathrm{N}=15 \\
\text { Age: Yes } \\
\text { Sex: Yes } \\
\text { \% majority race: No } \\
\text { Education: No }\end{array}$ & NR & NA & NA & NA & NA & 3weeks & $\begin{array}{l}\text { Nutritional } \\
\text { intake: Care- } \\
\text { tracker by } \\
\text { Ingenix }\end{array}$ & NA \\
\hline $\begin{array}{l}\text { Chang et. al, } \\
2010^{91} \\
\text { (20492038) } \\
\text { Small sample } \\
(<25 \text { in each } \\
\text { arm) }\end{array}$ & $\begin{array}{l}\text { Music(backgrou } \\
\text { nd) was } \\
\text { administered } \\
\text { over the } \\
\text { institution's } \\
\text { internal } \\
\text { broadcast } \\
\text { network during } \\
\text { the second } \\
\text { week of the } \\
\text { study over the } \\
\text { lunchtime } \\
\text { (11:00-12:00). }\end{array}$ & $\begin{array}{l}\text { No music (time } \\
\text { series) }\end{array}$ & $\begin{array}{l}\text { Institution } \\
\text { housing } \\
\text { ITS } \\
\text { 47 PLWD }\end{array}$ & $\begin{array}{l}\mathrm{AD} \text {, vascular and } \\
\text { other dementia } \\
\text { Mild }\end{array}$ & $\begin{array}{l}\mathrm{N}=41 \\
\text { Age: } 81.68 \text { years } \\
\text { Sex: Yes } \\
\text { \% majority race: No } \\
\text { Education: Yes }\end{array}$ & NR & NA & NA & NA & NA & 8 weeks & $\begin{array}{l}\text { Dementia- } \\
\text { related } \\
\text { problem } \\
\text { behavior: } \\
\text { CMAl } \\
\text { (Chinese } \\
\text { version) }\end{array}$ & NA \\
\hline $\begin{array}{l}\text { Cooke, 201099 } \\
\text { (20603300) } \\
\text { Australia } \\
\text { Small sample } \\
\text { \#2 } \\
\text { Cooke, 201093 } \\
(20635236)\end{array}$ & $\begin{array}{l}\text { Live music: } \\
\text { Participated in a } \\
30 \text { mins of } \\
\text { musician-led } \\
\text { familiar song } \\
\text { singing (with } \\
\text { guitar } \\
\text { accompaniment } \\
\text { ) and } 10 \text { mins of } \\
\text { pre-recorded } \\
\text { instrumental } \\
\text { music for active } \\
\text { listening, three } \\
\text { times a week for } \\
\text { eight weeks, }\end{array}$ & $\begin{array}{l}\text { Reading: } \\
\text { Engaged in a } \\
\text { range of } \\
\text { reading/ } \\
\text { socialal activities } \\
\text { including } \\
\text { reading local } \\
\text { news stories, } \\
\text { short stories, } \\
\text { telling jokes } \\
\text { \& undertaking } \\
\text { quiz activities. }\end{array}$ & $\begin{array}{l}\text { Aged care } \\
\text { facility } \\
\text { RCT(crosso } \\
\text { ver) } \\
\text { 47 PLWD }\end{array}$ & $\begin{array}{l}\text { Early to mid-stage } \\
\text { dementia or } \\
\text { probable dementia }\end{array}$ & $\begin{array}{l}\mathrm{N}=47 \\
\text { Age: Yes } \\
\text { Sex: Yes } \\
\text { \% majority race: No } \\
\text { Education: Yes }\end{array}$ & NR & NA & NA & NA & NA & 8 Weeks & $\begin{array}{l}\text { DQOL; GDS; } \\
\# 2 \\
\text { CMAI-SF; } \\
\text { RAID; }\end{array}$ & NA \\
\hline $\begin{array}{l}\text { Han, 201094 } \\
(21252549) \\
\text { Singapore } \\
(<25 \text { in each } \\
\text { arm) }\end{array}$ & $\begin{array}{l}\text { Music therapy } \\
\text { and activity } \\
\text { program: } \\
\text { Attended a } \\
\text { weekly group } \\
\text { MAP conducted } \\
\text { by a qualified } \\
\text { music therapist } \\
\text { and } \\
\text { occupational } \\
\text { therapist for } \\
\text { once a week for } \\
8 \text { weeks. }\end{array}$ & Waitlist & $\begin{array}{l}\text { Outpatient } \\
\text { dementia } \\
\text { clinic } \\
\text { Non-RCT } \\
45 \text { PLWD }\end{array}$ & $\begin{array}{l}\text { Alzheimer's } \\
\text { disease or vascular } \\
\text { dementia with } \\
\text { moderate severity }\end{array}$ & $\begin{array}{l}\mathrm{N}=43 \\
\text { Age: Yes } \\
\text { Sex: Yes } \\
\text { \% majority race: No } \\
\text { Education: No }\end{array}$ & NR & NA & NA & NA & NA & 8 weeks & $\begin{array}{l}\text { Mood \& } \\
\text { disruptive } \\
\text { behavioral: } \\
\text { RMPCP \& } \\
\text { AES }\end{array}$ & NA \\
\hline
\end{tabular}




\begin{tabular}{|c|c|c|c|c|c|c|c|c|c|c|c|c|c|}
\hline $\begin{array}{l}\text { Raglio 201078 } \\
\text { (20735342) } \\
\text { Italy } \\
\text { Pilot }\end{array}$ & \begin{tabular}{|l} 
Music therapy: \\
received three \\
cycles of 12 \\
sessions each, \\
three times a \\
week. Each \\
session \\
involved a \\
group of three \\
patients and \\
lasted 30 \\
minutes \\
\end{tabular} & \begin{tabular}{|l|} 
Standard care \\
(i.e. educational \\
and \\
entertainment \\
activities such \\
as reading a \\
newspaper, \\
performing \\
physical \\
activities, etc.). \\
\end{tabular} & \begin{tabular}{|l|} 
Nursing \\
home \\
RCT \\
60 PLWD
\end{tabular} & \begin{tabular}{|l|} 
AD diagnosis \\
based on DSM-IV \\
and MMSE \\
between 18 and 30
\end{tabular} & $\begin{array}{l}\mathrm{N}=60 \\
\text { Age: Yes } \\
\text { Sex: Yes } \\
\text { \% majority race: No } \\
\text { Education: No }\end{array}$ & NR & NA & NA & NA & NA & $\begin{array}{l}4 \text { weeks } \\
8 \text { weeks }\end{array}$ & $\begin{array}{l}\text { MMSE } \\
\text { Barthel index } \\
\text { NPI }\end{array}$ & NA \\
\hline $\begin{array}{l}\text { Sung 201095 } \\
\text { (20492050) } \\
\text { Taiwan } \\
\text { Quasi- } \\
\text { experimental }\end{array}$ & \begin{tabular}{|l} 
30-minute \\
music listening \\
intervention \\
based on \\
personal \\
preferences \\
delivered by \\
trained nursing \\
staff, twice a \\
week for six \\
weeks. \\
\end{tabular} & $\begin{array}{l}\text { Standard care } \\
\text { without music }\end{array}$ & $\begin{array}{l}\text { Long term } \\
\text { care facility } \\
\text { RCT } \\
60 \text { PLWD }\end{array}$ & \begin{tabular}{|l|} 
Clinical diagnosis \\
of dementia and \\
moderate to severe \\
cognitive decline \\
with GDS score \\
between 4 and 6 \\
\end{tabular} & $\begin{array}{l}\text { N=52 } \\
\text { Age: Yes } \\
\text { Sex: Yes } \\
\text { \% majority race: } \\
\text { Yes } \\
\text { Education: Yes }\end{array}$ & NR & NA & NA & NA & NA & 6 weeks & RAID & NA \\
\hline $\begin{array}{l}\text { Guetin, 200996 } \\
\text { (19628939) } \\
\text { France } \\
\text { (<25 in each } \\
\text { arm) }\end{array}$ & $\begin{array}{l}\text { Music therapy: } \\
\text { Participated in } \\
\text { weekly sessions } \\
\text { of individual, } \\
\text { receptive music } \\
\text { therapy } \\
\end{array}$ & \begin{tabular}{|l|} 
Reading: \\
Participated \\
under the same \\
conditions in \\
reading \\
sessions. \\
\end{tabular} & \begin{tabular}{|l|} 
Nursing \\
homes \\
RCT \\
30 PLWD \\
\end{tabular} & \begin{tabular}{|l|} 
AD with no \\
specified severity
\end{tabular} & $\begin{array}{l}\mathrm{N}=30 \\
\text { Age: Yes } \\
\text { Sex: Yes } \\
\text { \% majority race: No } \\
\text { Education: Yes }\end{array}$ & $\begin{array}{l}\text { Household } \\
\text { Characteristics: } \\
\text { Yes } \\
\text { Age of Diagnosis: } \\
\text { Yes } \\
\text { Rest: No }\end{array}$ & NA & NA & $\mathrm{NA}$ & NA & 16 weeks & $\begin{array}{l}\text { Anxiety: the } \\
\text { Hamilton } \\
\text { Scale } \\
\text { Depression: } \\
\text { GDS }\end{array}$ & NA \\
\hline $\begin{array}{l}\text { Choi, } 2008^{97} \\
\text { South Korea } \\
\text { Pilot }\end{array}$ & \begin{tabular}{|l} 
Group music: \\
received 50 \\
minutes of \\
music \\
intervention \\
(singing songs, \\
analysis of \\
libretto, making \\
musical \\
instruments, \\
song writing \& \\
playing \\
instruments) 3 \\
times per week \\
for 5 \\
consecutive \\
weeks.
\end{tabular} & \begin{tabular}{|l|} 
Usual care: \\
received usual \\
care, and did \\
not participate \\
in any \\
structured \\
therapeutic \\
programs during \\
the study \\
period.
\end{tabular} & \begin{tabular}{|l|} 
Special \\
dementia \\
care unit \\
Non-RCT \\
20 PLWD
\end{tabular} & $\begin{array}{l}\text { AD, vascular and } \\
\text { other type }\end{array}$ & $\begin{array}{l}\mathrm{N}=20 \\
\text { Age= }=74.9 \text { years } \\
\text { Sex: No } \\
\text { \% majority race: No } \\
\text { Education: No }\end{array}$ & NR & NA & NA & NA & NA & 5 weeks & $\begin{array}{l}\text { Depression: } \\
\text { GDS } \\
\text { Quality of life: } \\
\text { GQoL } \\
\text { Behavioral } \\
\text { disturbances: } \\
\text { NPI-Q }\end{array}$ & NA \\
\hline $\begin{array}{l}\text { Raglio, 2008 }{ }^{78} \\
\text { (18525288) } \\
\text { Italy } \\
\text { High RoB }\end{array}$ & $\begin{array}{l}\text { Music Therapy: } \\
\text { received } 3 \\
\text { cycles of } 10 \mathrm{MT} \\
\text { sessions }(30 \\
\text { min/session) } \\
\text { over } 16 \text { weeks }\end{array}$ & \begin{tabular}{|l|} 
Control: \\
received \\
educational \\
support or \\
entertainment \\
activities.
\end{tabular} & \begin{tabular}{|l|} 
Nursing \\
home \\
RCT \\
59 PLWD
\end{tabular} & \begin{tabular}{|l|} 
Mild Alzheimer type \\
or vascular or \\
mixed dementia
\end{tabular} & $\begin{array}{l}\mathrm{N}=59 \\
\text { Age: Yes } \\
\text { Sex: Yes } \\
\text { \% majority race: No } \\
\text { Education: Yes }\end{array}$ & NR & NA & NA & NA & NA & 16 weeks & BPSD & NA \\
\hline $\begin{array}{l}\text { Ledger, 200779 } \\
\text { (17558584) } \\
\\
\text { Australia } \\
\text { High RoB }\end{array}$ & \begin{tabular}{|l|} 
Group music \\
therapy: \\
Received \\
weekly music \\
therapy for
\end{tabular} & \begin{tabular}{|l|} 
Standard care: \\
Received \\
standard \\
nursing home \\
care
\end{tabular} & $\begin{array}{l}\text { Nursing } \\
\text { home } \\
\text { Non-RCT } \\
\text { 60 PLWD }\end{array}$ & \begin{tabular}{|l|} 
Mild or moderate or \\
moderately severe \\
Alzheimer's type
\end{tabular} & $\begin{array}{l}\mathrm{N}=45 \\
\text { Age: } 71-100 \text { years } \\
\text { Sex: Yes } \\
\text { \% majority race: No } \\
\text { Education: No }\end{array}$ & NR & $\mathrm{NA}$ & NA & NA & NA & 42 weeks & $\begin{array}{l}\text { Agitation; } \\
\text { CMAI }\end{array}$ & NA \\
\hline
\end{tabular}




\begin{tabular}{|c|c|c|c|c|c|c|c|c|c|c|c|c|c|}
\hline $\begin{array}{l}\text { Holmes, 200698 } \\
(16805928) \\
\text { UK } \\
\text { Pilot }\end{array}$ & $\begin{array}{l}\text { Music Therapy: } \\
\text { Participants } \\
\text { listened to } \\
\text { either live or } \\
\text { prerecorded } \\
\text { music for } 30 \\
\text { mins }\end{array}$ & $\begin{array}{l}\text { Silence: } \\
30 \text { mins of } \\
\text { silence }\end{array}$ & $\begin{array}{l}\text { Residential- } \\
\text { care or } \\
\text { nursing- } \\
\text { home facility } \\
\text { RCT } \\
32 \text { PLWD }\end{array}$ & $\begin{array}{l}\text { Moderate or severe } \\
\text { dementia with } \\
\text { apathy }\end{array}$ & $\begin{array}{l}\mathrm{N}=32 \\
\text { Age: Yes } \\
\text { Sex: No } \\
\text { \% majority race: No } \\
\text { Education=No }\end{array}$ & NR & NA & NA & NA & $\mathrm{NA}$ & Unclear & $\begin{array}{l}\text { Engagement: } \\
\text { DCM }\end{array}$ & $\mathrm{NA}$ \\
\hline $\begin{array}{l}\text { Sung, 200699 } \\
(16765849) \\
\text { Taiwan } \\
\text { Small sample }\end{array}$ & $\begin{array}{l}\text { Group music } \\
\text { with movement } \\
\text { intervention: } \\
\text { received 30-min } \\
\text { group music } \\
\text { with movement } \\
\text { intervention in } \\
\text { the afternoon } \\
\text { twice a week } \\
\text { over a 4-week } \\
\text { period by a } \\
\text { nursing } \\
\text { researcher } \\
\text { and two } \\
\text { research } \\
\text { assistants } \\
\text { trained in music } \\
\text { intervention }\end{array}$ & $\begin{array}{l}\text { Control: } \\
\text { Received usual } \\
\text { care without } \\
\text { intervention }\end{array}$ & $\begin{array}{l}\text { Residential } \\
\text { care facility } \\
\text { RCT } \\
36 \text { PLWD }\end{array}$ & $\begin{array}{l}\text { Moderate to severe } \\
\text { dementia }\end{array}$ & $\begin{array}{l}\mathrm{N}=36 \\
\text { Age: Yes } \\
\text { Sex: Yes } \\
\text { \% majority race: No } \\
\text { Education: Yes }\end{array}$ & NR & NA & $\mathrm{NA}$ & NA & $\mathrm{NA}$ & 4 weeks & CMAl; \& RAID & $\mathrm{NA}$ \\
\hline $\begin{array}{l}\text { Svansdottir, } \\
2006^{100} \\
(16618375) \\
\\
\text { Iceland } \\
\text { Small sample }\end{array}$ & $\begin{array}{l}\text { Music therapy: } \\
\text { received } 18 \\
\text { sessions of } \\
\text { music therapy, } \\
\text { each lasting } 30 \\
\text { minutes, three } \\
\text { times a week for } \\
6 \text { weeks }\end{array}$ & $\begin{array}{l}\text { Usual care: had } \\
\text { no change of } \\
\text { care. }\end{array}$ & $\begin{array}{l}\text { Nursing } \\
\text { homes and } \\
\text { psychogeriat } \\
\text { ric wards. } \\
\text { RCT } \\
46 \text { PLWD }\end{array}$ & $\begin{array}{l}\text { Moderate or severe } \\
\text { AD }\end{array}$ & $\begin{array}{l}\mathrm{N}=47 \\
\text { Age: Yes } \\
\text { Sex: No } \\
\text { \% majority race: No } \\
\text { Education: No }\end{array}$ & NR & NA & NA & NA & NA & 6 weeks & $\begin{array}{l}\text { Behavioral \& } \\
\text { psychological } \\
\text { symptoms: } \\
\text { BEHAVE-AD }\end{array}$ & NA \\
\hline $\begin{array}{l}\text { Gerdner, } \\
20000^{101} \\
(10798453) \\
\text { Australia } \\
(<25 \text { in each } \\
\text { arm })\end{array}$ & $\begin{array}{l}\text { G1- } \\
\text { Individualized } \\
\text { music: } \\
\text { Received } \\
\text { individualized } \\
\text { music for } 6 \\
\text { weeks followed } \\
\text { by a 2- } \\
\text { week="washout" } \\
\text { period and } 6 \\
\text { weeks of } \\
\text { classical"relaxat } \\
\text { ion" music. }\end{array}$ & $\begin{array}{l}\text { G2-Classical } \\
\text { "relaxation" } \\
\text { music: } \\
\text { Received } \\
\text { classical } \\
\text { relaxation music } \\
\text { for } 6 \text { weeks } \\
\text { followed by a 2- } \\
\text { week"washout" } \\
\text { period and } 6 \\
\text { weeks of } \\
\text { individualized } \\
\text { music }\end{array}$ & $\begin{array}{l}\text { Long term } \\
\text { care facility } \\
\text { RCT } \\
\text { (crossover) } \\
45 \text { PLWD }\end{array}$ & $\begin{array}{l}\text { Mild to severe } \\
\text { dementia }\end{array}$ & $\begin{array}{l}\mathrm{N}=39 \\
\text { Age: Yes } \\
\text { Sex: Yes } \\
\text { \% majority race: } \\
\text { Yes } \\
\text { Education: No }\end{array}$ & $\begin{array}{l}\text { PLWD Detailed } \\
\text { Race Information: } \\
\text { Yes }\end{array}$ & NA & NA & NA & NA & 6 weeks & $\begin{array}{l}\text { Agitation: } \\
\text { MCMAl }\end{array}$ & NA \\
\hline $\begin{array}{l}\text { Groene, } 1993^{102} \\
\text { USA } \\
(<25 \text { in each } \\
\text { arm) }\end{array}$ & $\begin{array}{l}\text { Mostly Music } \\
\text { therapp: Each } \\
\text { participant } \\
\text { received one } \\
\text { session per day } \\
\text { for } 7 \text { days (five } \\
\text { sessions of } \\
\text { music followed } \\
\text { by two sessions } \\
\text { of reading) }\end{array}$ & $\begin{array}{l}\text { Mostly reading } \\
\text { session: Each } \\
\text { participant } \\
\text { received one } \\
\text { session per day } \\
\text { for } 7 \text { days (five } \\
\text { sessions of } \\
\text { reading followed } \\
\text { by two sessions } \\
\text { of music) }\end{array}$ & $\begin{array}{l}\text { Health care } \\
\text { facility } \\
\text { RCT } \\
30 \text { PLWD }\end{array}$ & \begin{tabular}{|l|} 
Late moderate to \\
severe stages of \\
Alzheimer's \\
disease
\end{tabular} & $\begin{array}{l}\mathrm{N}=30 \\
\text { Age: Yes } \\
\text { Sex: Yes } \\
\text { \% majority race: No } \\
\text { Education: No }\end{array}$ & $\mathrm{NR}$ & NA & NA & NA & NA & 7 days & $\begin{array}{l}\text { Wandering } \\
\text { Behaviour: } \\
\text { researcher } \\
\text { and nursing } \\
\text { staff recorded } \\
\text { the wandering } \\
\text { behavior of } \\
\text { each } \\
\text { participant }\end{array}$ & $\mathrm{NA}$ \\
\hline
\end{tabular}




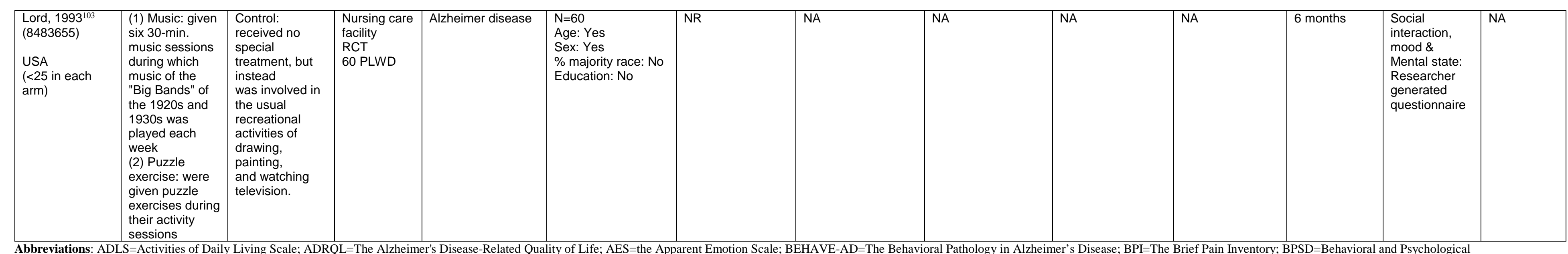

Abbreviations: ADLS=Activities of Daily Living Scale; ADRQL=The Alzheimer's Disease-Related Quality of Life; AES=the Apparent Emotion Scale; BEHAVE-AD=The Behavioral Pathology in Alzheimer's Disease; BPI=The Brief Pain Inventory; BPSD=Behavioral and Psychological

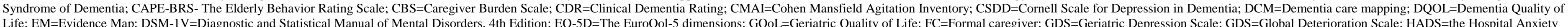

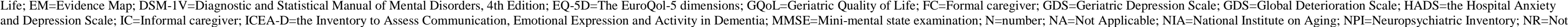

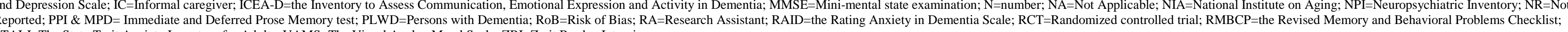

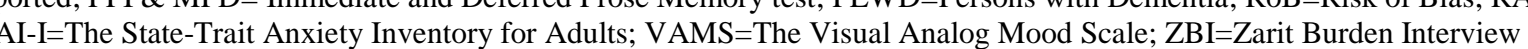

\section{Reminiscence Therapy}




\begin{tabular}{|c|c|c|c|c|c|c|c|c|c|}
\hline Study (PMID) & Outcome Timing & $\begin{array}{l}\text { Attrition Bias } \\
\text { Attrition \% }\end{array}$ & Selection Bias & Detection Bias & \begin{tabular}{|l|} 
Performance Bias \\
\end{tabular} & Reporting Bias & Fidelity Bias & Funder & Overall Rating \\
\hline Li 2019104 (31645180) & $\begin{array}{l}12 \text { weeks } \\
24 \text { weeks }\end{array}$ & $\begin{array}{l}\text { Low } \\
5.6 \%\end{array}$ & Low & Low & Medium & Low & Medium & Government & Medium \\
\hline Lok 2019 ${ }^{105}(30246408)$ & 8 weeks & $\begin{array}{l}\text { Medium } \\
15.5 \%\end{array}$ & Medium & High & High & Medium & $x$ & NR & High \\
\hline Lin $2018^{106}(28881430)$ & $\begin{array}{l}10 \text { week } \\
22 \text { weeks }\end{array}$ & $\begin{array}{l}\text { Low } \\
10 \text { weeks: } 2.6 \% \\
22 \text { weeks: } 9.3 \%\end{array}$ & High & High & Low & Low & $\mathrm{x}$ & Government & High \\
\hline Amieva $2016^{107}(26572551)$ & $\begin{array}{l}3 \text { months } \\
24 \text { months }\end{array}$ & $\begin{array}{l}\text { Medium } \\
3 \text { months: } 10.8 \% \\
\text { High } \\
24 \text { months: } 30.3 \%\end{array}$ & Low & Low & Low & Medium & Medium & Government & $\begin{array}{l}\text { Medium ( } 3 \text { months) } \\
\text { High (24 months) }\end{array}$ \\
\hline Duru Asiret 2016 ${ }^{108}$ (26251112) & 12 weeks & $\begin{array}{l}\text { Low } \\
6 \%\end{array}$ & High & $\mathrm{x}$ & $\mathrm{x}$ & $x$ & $\mathrm{x}$ & NR & High \\
\hline Van Bogaert 2016 $6^{109}(27511740)$ & 9 weeks & \begin{tabular}{|l|} 
Medium \\
$16.7 \%$
\end{tabular} & Medium & Low & High & Low & $\mathrm{x}$ & Foundation, Government & High \\
\hline Wu 2016 & 6 weeks & $\begin{array}{l}\text { Low } \\
2.8 \%\end{array}$ & Low & High & Low & Low & $x$ & Government & High \\
\hline O'Shea 2014 ${ }^{111}(24633858)$ & 2 years & $\begin{array}{l}\text { Medium } \\
17 \%\end{array}$ & Low & Low & High & Low & $\mathrm{x}$ & Government & High \\
\hline Seranni Azcurra $2012^{112}(23429813)$ & $\begin{array}{l}3 \text { months } \\
6 \text { months }\end{array}$ & $\begin{array}{l}\text { Low } \\
3.7 \%\end{array}$ & Medium & Low & Low & High & $x$ & NR & High \\
\hline Woods $2012^{113}(23211271)$ & $\begin{array}{l}3 \text { months } \\
10 \text { months }\end{array}$ & $\begin{array}{l}\text { Medium } \\
3 \text { months: } 19.1 \% \\
\text { High } \\
10 \text { months: } 28.3 \% \\
\end{array}$ & Low & Low & Low & Medium & Medium & Government & $\begin{array}{l}\text { Medium (3 months) } \\
\text { High (10 months) }\end{array}$ \\
\hline Hsieh $2010^{114}(\mathrm{NA})$ & 3 months & $\begin{array}{l}\text { High } \\
33.3 \%\end{array}$ & $x$ & $x$ & $x$ & $x$ & $\mathrm{x}$ & Government & High \\
\hline Wang $2007^{115}$ (17503545) & 8 weeks & \begin{tabular}{|l|} 
Medium \\
NR
\end{tabular} & Medium & Low & Medium & Low & Medium & Government & Medium \\
\hline $\begin{array}{l}\text { Lai 2004 } \\
\text { (116 } \\
\text { (15190995) }\end{array}$ & $\begin{array}{l}6 \text { weeks } \\
12 \text { weeks }\end{array}$ & \begin{tabular}{|l|} 
Medium \\
$15 \%$
\end{tabular} & Medium & Low & Low & High & $x$ & $\mathrm{NR}$ & High \\
\hline Camberg 1999117 (10203120) & 4 weeks & \begin{tabular}{|l} 
Low \\
$0 \%$
\end{tabular} & Medium & High & Low & Low & $x$ & NR & High \\
\hline
\end{tabular}

Abbreviations: NR=Not Reported; PMID=PubMed Identification Number 


\begin{tabular}{|c|c|c|c|c|c|c|c|c|c|c|c|c|c|}
\hline $\begin{array}{l}\text { Study (PMID) } \\
\text { Country } \\
\text { RoB* } \\
\text { Type }\end{array}$ & $\begin{array}{l}\text { Intervention } \\
\text { Intervention } \\
\text { Focus } \\
\text { Theoretical } \\
\text { Model } \\
\text { Delivery } \\
\text { Person } \\
\text { Intervention } \\
\text { Target } \\
\text { Recipient } \\
\text { Mode } \\
\text { Components } \\
\text { Frequency } \\
\text { Duration }\end{array}$ & \begin{tabular}{|l|} 
Comparison \\
\\
Target \\
Mode \\
Components \\
Frequency \\
Duration
\end{tabular} & $\begin{array}{l}\text { Setting } \\
\text { and Design } \\
\text { Setting } \\
\text { Design } \\
\text { cluster N } \\
\text { Participants } \\
\text { Randomized } \\
\text { N }\end{array}$ & $\begin{array}{l}\text { PLWD } \\
\text { Dementia } \\
\text { Characteristics } \\
\text { Dementia Types } \\
\text { Dementia Severity } \\
\text { Diagnostic } \\
\text { Criteria } \\
\text { Age of Diagnosis }\end{array}$ & $\begin{array}{l}\text { PLWD } \\
\text { Non-Disease Char } \\
\text { PLWD N } \\
\text { PLWD Age (mean) } \\
\text { PLWD Sex (\% } \\
\text { female) } \\
\text { PLWD Race (\% } \\
\text { majority) } \\
\text { PLWD Education } \\
\text { (mean years) }\end{array}$ & $\begin{array}{l}\text { PLWD } \\
\text { Non-Disease } \\
\text { Char Reporting } \\
\text { Status (RS) } \\
\text { PLWD SES } \\
\text { PLWD Prior } \\
\text { Disability } \\
\text { PLWD } \\
\text { Household } \\
\text { Characteristics } \\
\text { PLWD Health } \\
\text { Insurance } \\
\text { PLWD Detailed } \\
\text { Race Information }\end{array}$ & $\begin{array}{l}\text { Informal Caregiver } \\
\text { (IC) } \\
\text { Characteristics } \\
\text { IC N } \\
\text { IC Age (mean) } \\
\text { IC Sex (\% female) } \\
\text { IC Race (\% } \\
\text { majority) } \\
\text { IC Education } \\
\text { (mean years) } \\
\text { IC Relation to } \\
\text { PLWD (\% majority) }\end{array}$ & $\begin{array}{l}\text { Informal Caregiver } \\
\text { (IC) Char. RS } \\
\text { IC Duration } \\
\text { IC Living With } \\
\text { PLWD } \\
\text { IC Payment } \\
\text { IC Health Status } \\
\text { IC Dementia } \\
\text { Family History } \\
\text { IC Employment } \\
\text { Status } \\
\text { IC Training }\end{array}$ & 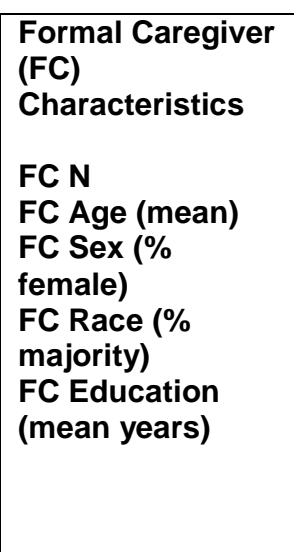 & $\begin{array}{l}\text { Formal } \\
\text { Caregiver (FC) } \\
\text { Char. RS } \\
\text { FC Health } \\
\text { Status } \\
\text { FC Training } \\
\text { FC Education } \\
\text { FC Position } \\
\text { FC Length of } \\
\text { Service }\end{array}$ & \begin{tabular}{|l|} 
Outcome \\
Timing(s)
\end{tabular} & $\begin{array}{l}\text { PLWD } \\
\text { Outcomes }\end{array}$ & $\begin{array}{l}\text { Caregiver } \\
\text { Outcomes } \\
\text { IC: } \\
\text { FC: }\end{array}$ \\
\hline $\begin{array}{l}\text { Li 2019104 } \\
(31645180) \\
\text { China } \\
\text { Medium } \\
\text { Explanatory }\end{array}$ & $\begin{array}{l}\text { Group } \\
\text { reminiscence } \\
\text { therapy in an } \\
\text { antique setting; } \\
30 \text { to } 45-\text {-minute } \\
\text { sessions, } 2 \\
\text { sessions/week } \\
\text { for } 12 \text { weeks }\end{array}$ & \begin{tabular}{|l} 
Usual care \\
\end{tabular} & $\begin{array}{l}\text { Geriatric } \\
\text { hospital } \\
\text { RCT, single } \\
\text { site } \\
90 \text { PLWD }\end{array}$ & $\begin{array}{l}\text { Diagnosis of AD } \\
\text { based on } \\
\text { NINCDS-ADRDA } \\
\text { criteria; } \\
\text { Mild-to-moderate } \\
\text { stage of dementia } \\
\text { based on CDR } \\
\text { score of } 1 \text { or } 2\end{array}$ & $\begin{array}{l}\mathrm{N}=90 \\
83 \text { years } \\
45 \% \text { Female } \\
\text { Race NR } \\
90.5 \% \text { Primary } \\
\text { education and } \\
\text { above }\end{array}$ & NR & NA & NA & NA & NA & \begin{tabular}{|l|}
12 weeks \\
24 weeks
\end{tabular} & $\begin{array}{l}\text { ADAS-Cog } \\
\text { NPI } \\
\text { CSDD } \\
\text { Barthel index }\end{array}$ & $\begin{array}{l}\mathrm{NPI}- \\
\text { Caregiver } \\
\text { Distress }\end{array}$ \\
\hline $\begin{array}{l}\text { Amieva 2016 } \\
(26572551) \\
\text { France } \\
\text { Medium } \\
\text { Explanatory }\end{array}$ & $\begin{array}{l}\text { Group } \\
\text { reminiscence } \\
\text { therapy; } 90- \\
\text { minute weekly } \\
\text { sessions for } 3 \\
\text { months and } \\
\text { monthly } \\
\text { maintenance } \\
\text { sessions for } 21 \\
\text { months } \\
\end{array}$ & \begin{tabular}{|l|} 
Usual care \\
\end{tabular} & \begin{tabular}{|l} 
Memory \\
centers or \\
geriatric day \\
care units \\
RCT; multisite \\
326 PLWD
\end{tabular} & $\begin{array}{l}\text { Mild to moderate } \\
\text { AD diagnosis } \\
\text { based on NINCDS- } \\
\text { ADRDA criteria, } \\
\text { MMSE from } 16 \text { to } \\
26 \text {, and GDS score } \\
\text { of } 2 \text { to } 5\end{array}$ & $\begin{array}{l}\mathrm{N}=326 \\
79 \text { years } \\
60 \% \text { Female } \\
\text { Race NR } \\
\text { 49\% Secondary } \\
\text { education and } \\
\text { above }\end{array}$ & $\mathrm{NR}$ & $\begin{array}{l}\mathrm{N}=326 \\
\text { Age: NR } \\
\text { Sex: NR } \\
\text { Race: NR } \\
\text { Education: NR } \\
\text { Relation to PLWD: } \\
\text { NR }\end{array}$ & NR & NA & NA & \begin{tabular}{|l|}
3 months \\
24 months
\end{tabular} & $\begin{array}{l}\text { ADAS-Cog } \\
\text { NPI } \\
\text { DAD } \\
\text { Apathy } \\
\text { Inventory } \\
\text { MADRS } \\
\text { QOL-AD } \\
\text { RUD Lite }\end{array}$ & ZBI \\
\hline $\begin{array}{l}\text { Woods 2012 } \\
(23211271)^{113} \\
\text { UK } \\
\text { Medium } \\
\text { Explanatory } \\
\text { Woods 2009118 } \\
\text { (19642992) } \\
\text { Woods 2016 } \\
\text { (27093052) }\end{array}$ & $\begin{array}{l}\text { Group } \\
\text { reminiscence } \\
\text { therapy for } \\
\text { PLWD-informal } \\
\text { caregiver } \\
\text { dyads; 2-hour } \\
\text { weekly sessions } \\
\text { for } 12 \text { weeks, } \\
\text { with monthly } \\
\text { maintenance for } \\
7 \text { months } \\
\end{array}$ & \begin{tabular}{|l|} 
Usual care \\
\end{tabular} & $\begin{array}{l}\text { Community- } \\
\text { based setting } \\
\text { RCT; multisite } \\
488 \text { PLWD- } \\
\text { informal } \\
\text { caregiver } \\
\text { dyads }\end{array}$ & $\begin{array}{l}\text { Mild to moderate } \\
\text { dementia (any } \\
\text { type) based on } \\
\text { DSM-IV criteria and } \\
\text { CDR }\end{array}$ & $\begin{array}{l}\mathrm{N}=488 \\
76 \text { years } \\
50 \% \text { Female } \\
95 \% \text { White } \\
\text { Education: NR }\end{array}$ & $\mathrm{NR}$ & $\begin{array}{l}\mathrm{N}=488 \\
70 \text { years } \\
67 \% \text { Female } \\
95 \% \text { White } \\
\text { Education NR } \\
71 \% \text { Spouse }\end{array}$ & NR & NA & NA & \begin{tabular}{|l|}
3 months \\
10 months
\end{tabular} & $\begin{array}{l}\text { QoL-AD } \\
\text { AMIE } \\
\text { CSDD } \\
\text { RAID } \\
\text { Bristol } \\
\text { Activities of } \\
\text { Daily Living } \\
\text { EQ-5-D } \\
\text { Resource } \\
\text { Utilization }\end{array}$ & $\begin{array}{l}\text { GHQ } \\
\text { QCPR } \\
\text { HADS } \\
\text { RSS } \\
\text { EQ5-D }\end{array}$ \\
\hline $\begin{array}{l}\text { Wang 2007115 } \\
(17503545) \\
\text { Medium } \\
\text { Taiwan } \\
\text { Explanatory } \\
\\
\text { Wang 2009'120 } \\
(18930560)\end{array}$ & $\begin{array}{l}\text { Group } \\
\text { reminiscence } \\
\text { therapy; } 60- \\
\text { minute session } \\
\text { one time/week } \\
\text { for } 8 \text { weeks }\end{array}$ & \begin{tabular}{|l|} 
Usual care \\
\end{tabular} & $\begin{array}{l}\text { Elderly care } \\
\text { facilities } \\
\text { RCT; multisite } \\
102 \text { PLWD }\end{array}$ & $\begin{array}{l}\text { Mild to severe } \\
\text { dementia, CDR } \\
\text { score of } 1 \text { to } 3\end{array}$ & \begin{tabular}{|l|}
$\mathrm{N}=102$ \\
79 years \\
$51 \%$ Female \\
Race NR \\
5.2 years education
\end{tabular} & SES: yes & NA & NA & NA & NA & 8 weeks & $\begin{array}{l}\text { MMSE } \\
\text { GDS-SF } \\
\text { CSDD }\end{array}$ & NR \\
\hline
\end{tabular}

(1)

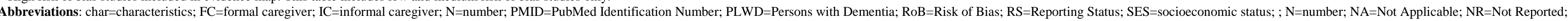

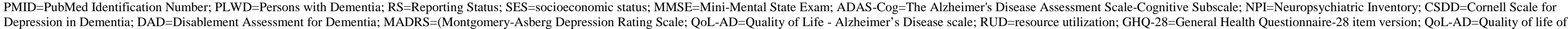

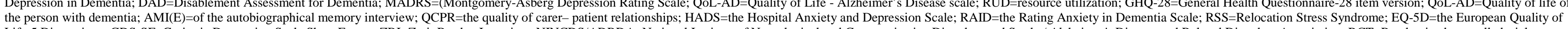

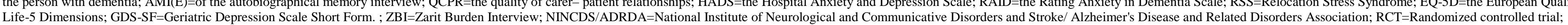
$\mathrm{CDR}=$ Clinical Dementia Rating; $C S D D=$ Cornell Scale for Depression in Dementia; ZBI=Zarit Burden Interview. 
Figure D-1. PLWD outcomes summary low and medium risk of bias studies: activities of daily living, BPSD, depression, and quality of life at 3 months

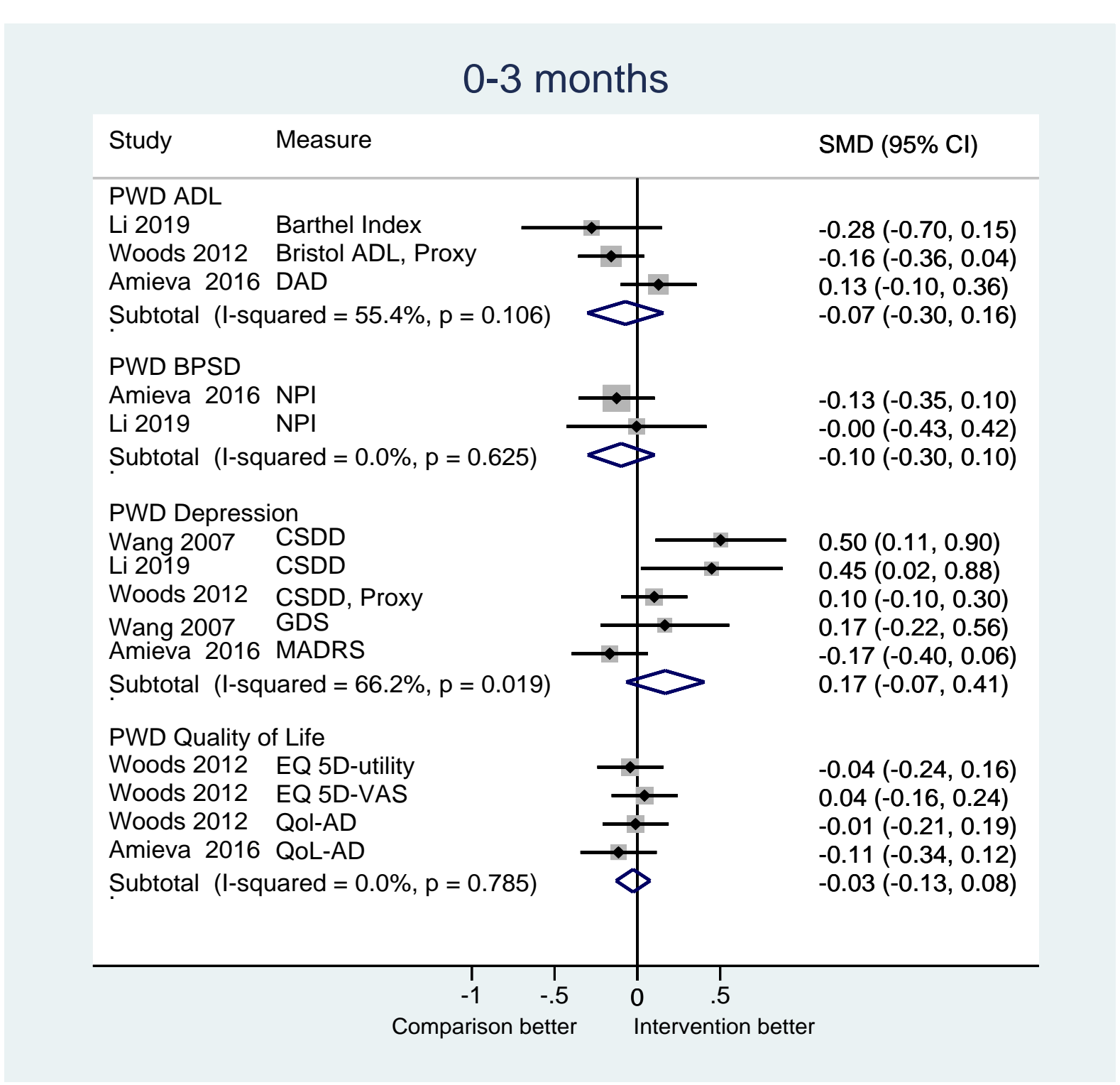


Table D-13. PLWD outcomes summary low and medium risk of bias studies: reminiscence therapy

\begin{tabular}{|c|c|c|c|c|c|}
\hline $\begin{array}{l}\text { Study (PMID) } \\
\text { Comparison } \\
\text { RoB } \\
\text { Category* }\end{array}$ & $\begin{array}{l}\text { Outcome } \\
\text { Timing }\end{array}$ & Summary Finding & Intervention & Comparator & p-Value \\
\hline $\begin{array}{l}\text { Li } 2019^{104}(31645180) \\
\text { Reminiscence Therapy vs. Usual Care } \\
\text { Medium } \\
\text { Explanatory }\end{array}$ & \begin{tabular}{|l} 
Barthel Index \\
Mean (SD) \\
6 months
\end{tabular} & No difference between groups. & $55.47(18.96)$ & $57.07(17.89)$ & NR \\
\hline $\begin{array}{l}\text { Li } 2019^{104}(31645180) \\
\text { Reminiscence Therapy vs. Usual Care } \\
\text { Medium } \\
\text { Explanatory }\end{array}$ & \begin{tabular}{|l}
$\mathrm{NPI}$ \\
Mean (SD) \\
6 months
\end{tabular} & No difference between groups. & $22.21(10.63)$ & $25.57(12.45)$ & NR \\
\hline $\begin{array}{l}\text { Li 2019104 (31645180) } \\
\text { Reminiscence Therapy vs. Usual Care } \\
\text { Medium } \\
\text { Explanatory }\end{array}$ & \begin{tabular}{|l} 
CSDD \\
Mean (SD) \\
6 months
\end{tabular} & Favors intervention. & $0.84(1.41)$ & $2.48(2.41)$ & NR \\
\hline $\begin{array}{l}\text { Amieva } 2016 \text { (26572551) } \\
\text { Reminiscence Therapy vs. Usual Care } \\
\text { Medium } \\
\text { Explanatory }\end{array}$ & \begin{tabular}{|l} 
Apathy Inventory \\
Mean (SD) \\
3 months
\end{tabular} & NA & $11.8(13.1)$ & $10.4(11.8)$ & 0.69 \\
\hline $\begin{array}{l}\text { Amieva } 2016^{107}(26572551) \\
\text { Reminiscence Therapy vs. Usual Care } \\
\text { Medium } \\
\text { Explanatory }\end{array}$ & \begin{tabular}{|l} 
AGGIR \\
Mean (SD) \\
3 months
\end{tabular} & NA & $7.1(8.7)$ & $6.63(7.5)$ & 0.49 \\
\hline $\begin{array}{l}\text { Amieva } 2016^{107}(26572551) \\
\text { Reminiscence Therapy vs. Usual Care } \\
\text { Medium } \\
\text { Explanatory }\end{array}$ & \begin{tabular}{|l} 
RUD-Lite \\
Mean (SD) \\
3 months
\end{tabular} & $\mathrm{NA}$ & $2230.1(3301.8)$ & $2259.3(3078.3)$ & 0.89 \\
\hline $\begin{array}{l}\text { Woods } 2012^{113}(23211271) \\
\text { Reminiscence Therapy vs. Usual Care } \\
\text { Medium } \\
\text { Explanatory }\end{array}$ & \begin{tabular}{|l} 
RAID (Proxy) \\
Mean (SD) \\
3 months
\end{tabular} & $\begin{array}{l}\text { Pooled Mean Difference }(95 \% \mathrm{Cl}) \\
1.2(-0.0364,2.807)\end{array}$ & $8.44(6.92)$ & $7.87(6.45)$ & $\mathrm{NR}$ \\
\hline $\begin{array}{l}\text { Woods } 2012^{113}(23211271) \\
\text { Reminiscence Therapy vs. Usual Care } \\
\text { Medium } \\
\text { Explanatory }\end{array}$ & \begin{tabular}{|l} 
QCPR \\
Mean (SD) \\
3 months
\end{tabular} & $\begin{array}{l}\text { Pooled Mean Difference }(95 \% \mathrm{Cl}) \\
0.99(-1.460,2.424)\end{array}$ & $57.89(6.52)$ & $57.37(6.71)$ & NR \\
\hline
\end{tabular}

Medium
Explanatory

Abbreviations: NA=Not Applicable; NR=Not Reported; PMID=PubMed Identification Number; QCPR=the quality of carer-patient relationships;; RAID=Rating for Anxiety in Dementia; RUD=resource utilization; GAGGIR: Grille d’Autonomie Gérontologique-Groupes Iso-Ressources (standardized dependency scale in France) 
Table D-14. Caregiver outcomes summary low and medium risk of bias studies: reminiscence therapy

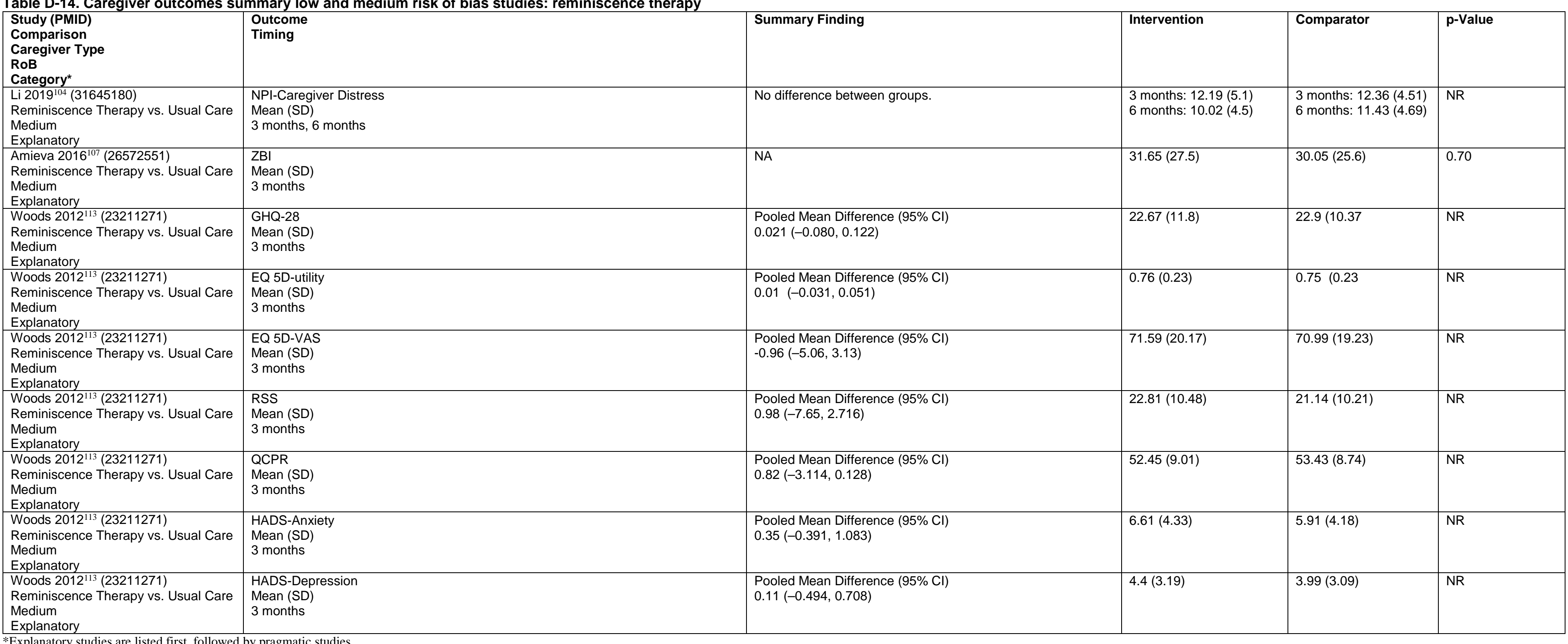

Explanatory studies are listed first, followed by pragmatic studies.

Abbreviations: NA=Not Applicable; NR=Not Reported; PMID=PubMed Identification Number; HADS=Hasegawa dementia scale revised; QCPR=Quality of Caregiver and Patient Relationship; EQ-5D=EuroQoL 5D scale; ZBI=Zarit Burden Interview 
Table D-15. Summary of strength of evidence for PLWD outcomes: reminiscence therapy

\begin{tabular}{|c|c|c|c|c|c|c|c|c|}
\hline \begin{tabular}{|l|} 
Comparison \\
Outcome
\end{tabular} & Timing & $\begin{array}{l}\text { \# Studies/ Design } \\
\text { (n analyzed) }\end{array}$ & Finding or Summary Statistic & Study Limitations & Consistency & Directness & \begin{tabular}{|l} 
Precision \\
\end{tabular} & \begin{tabular}{|l} 
Overall Grade/ Conclusion \\
\end{tabular} \\
\hline $\begin{array}{l}\text { Reminiscence Therapy vs. Usual Care } \\
\text { Activities of Daily Living }\end{array}$ & 3 months & 3 RCTs $(n=802)$ & No difference between groups. & Moderate & Consistent & Direct & Imprecise & Insufficient \\
\hline $\begin{array}{l}\text { Reminiscence Therapy vs. Usual Care } \\
\text { BPSD }\end{array}$ & 3 months & 2 RCTs ( $n=416)$ & No difference between groups. & Moderate & Consistent & Direct & Imprecise & \begin{tabular}{|l} 
Insufficient \\
\end{tabular} \\
\hline \begin{tabular}{|l|} 
Reminiscence Therapy vs. Usual Care \\
Depression
\end{tabular} & 2 to 3 months & 4 RCTs $(n=1,006)$ & No difference between groups. & Moderate & Consistent & Indirect & Imprecise & \begin{tabular}{|l} 
Insufficient \\
\end{tabular} \\
\hline $\begin{array}{l}\text { Reminiscence Therapy vs. Usual Care } \\
\text { Quality of Life }\end{array}$ & 3 months & 2 RCTs ( $n=814)$ & No difference between groups. & Moderate & Consistent & Direct & Imprecise & \begin{tabular}{|l|} 
Insufficient \\
\end{tabular} \\
\hline $\begin{array}{l}\text { Reminiscence Therapy vs. Usual Care } \\
\text { Anxiety }\end{array}$ & 3 months & $1 \mathrm{RCT}(\mathrm{n}=488)$ & No difference between groups. & Moderate & Unclear & Direct & \begin{tabular}{|l} 
Imprecise \\
\end{tabular} & \begin{tabular}{|l} 
Insufficient \\
\end{tabular} \\
\hline \begin{tabular}{|l} 
Alliely \\
Reminiscence Therapy vs. Usual Care \\
PLWD-Caregiver Relationship
\end{tabular} & 3 months & 1 RCT (n=488) & No difference between groups. & Moderate & Unclear & Direct & Imprecise & \begin{tabular}{|l} 
Insufficient \\
\end{tabular} \\
\hline $\begin{array}{l}\text { Reminiscence Therapy vs. Usual Care } \\
\text { Apathy }\end{array}$ & 3 months & $1 \mathrm{RCT}(\mathrm{n}=326)$ & No difference between groups. & Moderate & Unclear & Direct & Imprecise & \begin{tabular}{|l} 
Insufficient \\
\end{tabular} \\
\hline $\begin{array}{l}\text { Reminiscence Therapy vs. Usual Care } \\
\text { Dependency }\end{array}$ & 3 months & $1 \mathrm{RCT}(\mathrm{n}=326)$ & No difference between groups. & Moderate & Unclear & Direct & \begin{tabular}{|l} 
Imprecise \\
\end{tabular} & \begin{tabular}{|l|} 
Insufficient \\
\end{tabular} \\
\hline
\end{tabular}

Abbreviations: RCT=Randomized controlled trial; PLWD=Persons with Dementia

Table D-16. Summary of strength of evidence for caregiver outcomes: reminiscence therapy

\begin{tabular}{|c|c|c|c|c|c|c|c|c|}
\hline $\begin{array}{l}\text { Comparison } \\
\text { Caregiver Type } \\
\text { Outcome }\end{array}$ & Timing & $\begin{array}{l}\text { \# Studies/ Design } \\
\text { ( } \mathrm{n} \text { analyzed) }\end{array}$ & Finding or Summary Statistic & Study Limitations & Consistency & Directness & Precision & Overall Grade/ Conclusion \\
\hline $\begin{array}{l}\text { Reminiscence Therapy vs. Usual Care } \\
\text { Caregiver Burden }\end{array}$ & 3 months & $1 \mathrm{RCT}(\mathrm{n}=326)$ & No difference between groups. & Moderate & Unclear & Direct & Imprecise & Insufficient \\
\hline $\begin{array}{l}\text { Reminiscence Therapy vs. Usual Care } \\
\text { Caregiver Distress }\end{array}$ & \begin{tabular}{|l}
3 months \\
6 months \\
\end{tabular} & $1 \mathrm{RCT}(\mathrm{n}=90)$ & No difference between groups. & Moderate & Unclear & Direct & Imprecise & Insufficient \\
\hline $\begin{array}{l}\text { Reminiscence Therapy vs. Usual Care } \\
\text { Mental Health }\end{array}$ & 3 months & $1 \mathrm{RCT}(\mathrm{n}=488)$ & No difference between groups. & Moderate & Unclear & \begin{tabular}{|l|} 
Direct \\
\end{tabular} & Imprecise & Insufficient \\
\hline $\begin{array}{l}\text { Reminiscence Therapy vs. Usual Care } \\
\text { Quality of Life }\end{array}$ & 3 months & 1 RCT $(n=488)$ & No difference between groups. & Moderate & Unclear & Direct & Imprecise & Insufficient \\
\hline $\begin{array}{l}\text { Reminiscence Therapy vs. Usual Care } \\
\text { Stress }\end{array}$ & 3 months & $1 \mathrm{RCT}(\mathrm{n}=488)$ & No difference between groups. & Moderate & Unclear & Direct & Imprecise & Insufficient \\
\hline $\begin{array}{l}\text { Reminiscence Therapy vs. Usual Care } \\
\text { Anxiety }\end{array}$ & 3 months & $1 \mathrm{RCT}(\mathrm{n}=488)$ & No difference between groups. & Moderate & Unclear & Direct & Imprecise & Insufficient \\
\hline $\begin{array}{l}\text { Reminiscence Therapy vs. Usual Care } \\
\text { Depression }\end{array}$ & 3 months & $1 \mathrm{RCT}(\mathrm{n}=488)$ & No difference between groups. & Moderate & Unclear & Direct & Imprecise & Insufficient \\
\hline $\begin{array}{l}\text { Reminiscence Therapy vs. Usual Care } \\
\text { PLWD-Caregiver Relationship }\end{array}$ & 3 months & $1 \mathrm{RCT}(\mathrm{n}=488)$ & No difference between groups. & Moderate & Unclear & \begin{tabular}{|l|l} 
Direct \\
\end{tabular} & Imprecise & Insufficient \\
\hline
\end{tabular}

\begin{tabular}{|l|l|l}
\hline PLWD-Caregiver Relationship & & \\
\hline
\end{tabular}

\section{Evidence Map: Reminiscence Therapy}




\begin{tabular}{|c|c|c|c|c|c|c|c|c|c|c|c|c|c|}
\hline $\begin{array}{l}\text { Study (PMID) } \\
\text { Country } \\
\text { EM Reason }\end{array}$ & \begin{tabular}{|l|} 
Intervention \\
\end{tabular} & Comparison & \begin{tabular}{|l|} 
Setting \\
and Design \\
Setting \\
Design \\
Cluster N \\
Participants \\
Random- \\
ized N \\
\end{tabular} & $\begin{array}{l}\text { PLWD } \\
\text { Dementia } \\
\text { Characteristics }\end{array}$ & $\begin{array}{l}\text { PLWD } \\
\text { Non-Disease Char } \\
\text { PLWD N } \\
\text { PLWD Age (mean) } \\
\text { PLWD Sex (\% } \\
\text { female) } \\
\text { PLWD Race (\% } \\
\text { majority) } \\
\text { PLWD Education } \\
\text { (mean years) }\end{array}$ & $\begin{array}{l}\text { PLWD } \\
\text { Non-Disease } \\
\text { Char Reporting } \\
\text { Status (RS) } \\
\\
\text { PLWD SES } \\
\text { PLWD Prior } \\
\text { Disability } \\
\text { PLWD } \\
\text { Household } \\
\text { Characteristics } \\
\text { PLWD Health } \\
\text { Insurance } \\
\text { PLWD Detailed } \\
\text { Race Information }\end{array}$ & \begin{tabular}{|l|} 
Informal Caregiver \\
(IC) \\
Characteristics \\
IC N \\
IC Age (mean) \\
IC Sex (\% female) \\
IC Race (\% \\
majority) \\
IC Education \\
(mean years) \\
IC Relation to \\
PLWD (\% majority)
\end{tabular} & $\begin{array}{l}\text { Informal Caregiver } \\
\text { (IC) Char. RS } \\
\text { IC Duration } \\
\text { IC Living With } \\
\text { PLWD } \\
\text { IC Payment } \\
\text { IC Health Status } \\
\text { IC Dementia } \\
\text { Family History } \\
\text { IC Employment } \\
\text { Status } \\
\text { IC Training }\end{array}$ & 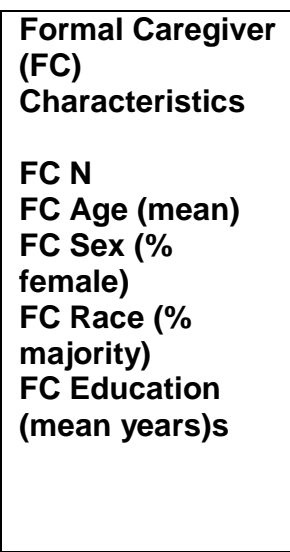 & $\begin{array}{l}\text { Formal } \\
\text { Caregiver (FC) } \\
\text { Char. RS } \\
\text { FC Health } \\
\text { Status } \\
\text { FC Training } \\
\text { FC Education } \\
\text { FC Position } \\
\text { FC Length of } \\
\text { Service }\end{array}$ & $\begin{array}{l}\text { Outcome } \\
\text { Timing(s) }\end{array}$ & \begin{tabular}{|l|} 
PLWD \\
Outcomes
\end{tabular} & $\begin{array}{l}\text { Caregiver } \\
\text { Outcomes } \\
\text { IC: } \\
\text { FC: }\end{array}$ \\
\hline $\begin{array}{l}\text { Ching-Ten, } \\
2020^{121} \\
\text { Taiwan } \\
\text { Small sample }\end{array}$ & \begin{tabular}{|l} 
Group \\
reminiscence \\
therapy 1 hour \\
per week for 8 \\
weeks
\end{tabular} & Usual care & \begin{tabular}{|l|} 
Nursing \\
Home \\
Quasi- \\
experimental \\
24 PLWD \\
\end{tabular} & $\begin{array}{l}\text { Veterans with mild } \\
\text { dementia }\end{array}$ & $\begin{array}{l}\mathrm{N}=24 \\
\text { Age: yes } \\
\text { Sex: yes } \\
\text { \% majority race: no } \\
\text { Education: ves }\end{array}$ & NR & NA & NA & NA & NA & 3 months & \begin{tabular}{|l|} 
GDS \\
Meaning of \\
life scale
\end{tabular} & NA \\
\hline $\begin{array}{l}\text { Lok 2019105 } \\
\text { (30246408) } \\
\text { Turkey } \\
\text { High RoB }\end{array}$ & \begin{tabular}{|l} 
Group \\
reminiscence \\
therapy; 60- \\
minute sessions \\
once/week for 8 \\
weeks
\end{tabular} & Usual care & \begin{tabular}{|l} 
Nursing \\
home \\
RCT; single \\
site \\
60 PLWD
\end{tabular} & \begin{tabular}{|l|} 
AD diagnosis \\
based on IWG-2 \\
criteria, MMSE 13 \\
to 24, CSDD >8
\end{tabular} & $\begin{array}{l}\mathrm{N}=60 \\
\text { Age: no } \\
\text { Sex: yes } \\
\text { \% majority race: no } \\
\text { Education: yes }\end{array}$ & $\mathrm{NR}$ & NA & NA & NA & NA & 8 weeks & \begin{tabular}{|l|} 
MMSE \\
CSDD \\
QOL-AD
\end{tabular} & NA \\
\hline $\begin{array}{l}\text { Manav 2019122 } \\
\text { (30612511) } \\
\text { Turkey } \\
\text { Pilot Study }\end{array}$ & \begin{tabular}{|l|} 
Reminiscence \\
therapy with \\
internet-based \\
videos; 60 \\
minutes a week \\
for 3 months
\end{tabular} & $\begin{array}{l}\text { Unstructured } \\
\text { casual } \\
\text { conversations; } \\
25-30 \text { minutes } \\
\text { once a week for } \\
3 \text { months }\end{array}$ & \begin{tabular}{|l|} 
Elderly care \\
and \\
rehabilitation \\
centers \\
RCT; \\
multisite \\
\end{tabular} & $\begin{array}{l}\text { Mild AD (DSM-V); } \\
\text { MMSE } 18 \text { to } 24 \text {; }\end{array}$ & $\begin{array}{l}\mathrm{N}=32 \\
\text { Age: yes } \\
\text { Sex: yes } \\
\text { \% majority race: no } \\
\text { Education: yes }\end{array}$ & SES: yes & NA & NA & NA & NA & 3 months & \begin{tabular}{|l|} 
MMSE \\
Apathy \\
Rating Scale
\end{tabular} & NA \\
\hline $\begin{array}{l}\text { Martinez } \\
2019^{123} \\
(31453826) \\
\text { US } \\
\text { Pilot Study }\end{array}$ & $\begin{array}{l}\text { Online cognitive } \\
\text { tasks with } \\
\text { positive memory } \\
\text { recall; } 40 \text { tasks } \\
\text { taking } 5 \text { minutes } \\
\text { to complete }\end{array}$ & $\begin{array}{l}\text { Standard } \\
\text { reminders } \\
\text { without positive } \\
\text { reinforcement }\end{array}$ & $\begin{array}{l}\text { Outpatient } \\
\text { memory } \\
\text { clinic } \\
\text { RCT; single } \\
\text { site } \\
22 \text { PLWD }\end{array}$ & \begin{tabular}{|l|}
$\begin{array}{l}\text { Mild AD; MMSE } 18 \\
\text { or higher }\end{array}$ \\
\end{tabular} & $\begin{array}{l}\mathrm{N}=22 \\
\text { Age: yes } \\
\text { Sex: yes } \\
\text { \% majority race: yes } \\
\text { Education: no }\end{array}$ & $\mathrm{NR}$ & NA & NA & NA & NA & 3 months & $\begin{array}{l}\text { Rosenberg } \\
\text { Self-Esteem } \\
\text { Scale } \\
\text { MMSE } \\
\text { GDS }\end{array}$ & NA \\
\hline $\begin{array}{l}\text { Laird 2018 } \\
\text { (30206053) } \\
\text { UK } \\
\text { Pilot Study }\end{array}$ & $\begin{array}{l}\text { Home-based } \\
\text { personalized } \\
\text { reminiscence } \\
\text { using iPad app; } \\
19 \text { weeks }\end{array}$ & NR & \begin{tabular}{|l|} 
Community- \\
based \\
(intervention \\
at home) \\
Quasi \\
experimental \\
60 PLWD- \\
informal \\
caregiver \\
dyads
\end{tabular} & $\begin{array}{l}\text { Early to moderate } \\
\text { dementia }\end{array}$ & $\begin{array}{l}\mathrm{N}=60 \\
\text { Age: yes } \\
\text { Sex: yes } \\
\text { \% majority race: no } \\
\text { Education: no }\end{array}$ & NR & $\begin{array}{l}\mathrm{N}=50 \\
\text { Age: yes } \\
\text { Sex: yes } \\
\text { \% majority race: no } \\
\text { Education: no } \\
\text { Relation to PLWD: } \\
\text { no }\end{array}$ & $\begin{array}{l}\text { Living with PLWD: } \\
\text { yes }\end{array}$ & NA & NA & 19 weeks & \begin{tabular}{|l|} 
Mutuality \\
Scale \\
QCPR \\
WHO-5
\end{tabular} & $\begin{array}{l}\text { Mutuality } \\
\text { Scale } \\
\text { QCPR } \\
\text { WHO-5 }\end{array}$ \\
\hline $\begin{array}{l}\text { Lin 2011106 } \\
\text { (28881430) } \\
\text { Taiwan } \\
\text { High RoB }\end{array}$ & $\begin{array}{l}\text { Reminiscence } \\
\text { therapy; 50- } \\
\text { minute sessions } \\
\text { once/week for } \\
10 \text { weeks }\end{array}$ & Usual care & $\begin{array}{l}\text { Long-term } \\
\text { care } \\
\text { facilities } \\
\text { Quasi } \\
\text { experimental } \\
\text {; multi-site }\end{array}$ & \begin{tabular}{|l|} 
Dementia with \\
agitation \\
MMSE $<17$ for non- \\
high school \\
graduates \\
MMSE<24 for high \\
school graduates
\end{tabular} & $\begin{array}{l}\mathrm{N}=75 \\
\text { Age: yes } \\
\text { Sex: yes } \\
\text { \% majority race: no } \\
\text { Education: yes }\end{array}$ & $\mathrm{NR}$ & NA & NA & NA & NA & $\begin{array}{l}12 \text { weeks } \\
24 \text { weeks }\end{array}$ & \begin{tabular}{|l|} 
MMSE \\
QOL-AD
\end{tabular} & NA \\
\hline
\end{tabular}




\begin{tabular}{|c|c|c|c|c|c|c|c|c|c|c|c|c|c|}
\hline $\begin{array}{l}\text { Duru Asiret } \\
2016^{108} \\
(26251112) \\
\text { Turkey } \\
\text { High RoB }\end{array}$ & $\begin{array}{l}\text { Group } \\
\text { reminiscence } \\
\text { therapy; } 30 \text { to } \\
25 \text {-minute } \\
\text { sessions for } 12 \\
\text { weeks }\end{array}$ & $\begin{array}{l}\text { Group } \\
\text { conversations. } \\
20 \text { to } 25 \\
\text { minutes per } \\
\text { week for } 12 \\
\text { weeks }\end{array}$ & \begin{tabular}{|l|} 
Quasi \\
experimental \\
; single site \\
62 PLWD
\end{tabular} & $\begin{array}{l}\text { Mild to moderate } \\
\text { AD; MMSE from } 10 \\
\text { to } 24\end{array}$ & $\begin{array}{l}\mathrm{N}=62 \\
\text { Age: yes } \\
\text { Sex: yes } \\
\text { \% majority race: no } \\
\text { Education: yes }\end{array}$ & NR & NA & NA & NA & NA & 3 months & $\begin{array}{l}\text { ADL } \\
\text { Observation } \\
\text { Form } \\
\text { MMSE } \\
\text { GDS }\end{array}$ & NA \\
\hline $\begin{array}{l}\text { Van Bogaert } \\
2016^{109} \\
(27511740) \\
\text { Belgium } \\
\text { High RoB }\end{array}$ & $\begin{array}{l}\text { Individual } \\
\text { reminiscence } \\
\text { therapy based } \\
\text { on Solcos } \\
\text { model; } 2 \\
\text { times/iveek for } 8 \\
\text { weeks }\end{array}$ & Usual care & $\begin{array}{l}\text { RCT; } \\
\text { multisite }\end{array}$ & \begin{tabular}{|l} 
Major \\
neurocognitive \\
disorder (DSM-V); \\
MMSE 10 to 24
\end{tabular} & $\begin{array}{l}\mathrm{N}=72 \\
\text { Age: yes } \\
\text { Sex: yes } \\
\text { \% majority race: no } \\
\text { Education: no }\end{array}$ & NR & NA & NA & NA & NA & 8 weeks & $\begin{array}{l}\text { CSDD } \\
\text { MMSE } \\
\text { NPI }\end{array}$ & NA \\
\hline $\begin{array}{l}\text { Wu 2016110 } \\
\text { (25965388) } \\
\text { Taiwan } \\
\text { High RoB }\end{array}$ & $\begin{array}{l}\text { Spiritual } \\
\text { reminiscence } \\
\text { therapy group } \\
\text { therapy; } 6 \\
\text { weeks }\end{array}$ & Usual care & \begin{tabular}{|l|} 
Medical \\
center \\
RCT; single \\
site \\
103 PLWD \\
\end{tabular} & $\begin{array}{l}\text { Mild to moderate } \\
\text { AD } \\
21<\text { MMSE }<24 \text { for } \\
\text { mild } \\
13<\text { MMSE }<20 \text { for } \\
\text { moderate }\end{array}$ & $\begin{array}{l}\mathrm{N}=103 \\
\text { Age: yes } \\
\text { Sex: yes } \\
\text { \% majority race: no } \\
\text { Education: yes }\end{array}$ & NR & NA & NA & NA & NA & 6 weeks & $\begin{array}{l}\text { Heath Hope } \\
\text { Index } \\
\text { Life } \\
\text { Satisfaction } \\
\text { Scale } \\
\text { Spirituality } \\
\text { Index of } \\
\text { Well-being } \\
\text { MMSE }\end{array}$ & NA \\
\hline $\begin{array}{l}\text { Gonzalez } \\
2015^{125} \\
(25765779) \\
\text { Spain } \\
\text { Sample Size }\end{array}$ & \begin{tabular}{l|} 
Group \\
reminiscence \\
therapy; 10 \\
weekly 60- \\
minute sessions
\end{tabular} & Wait-list control & \begin{tabular}{|l|} 
Nursing \\
home \\
Quasi \\
experimental \\
42 PLWD \\
\end{tabular} & $\begin{array}{l}\text { AD (DSM-IV); } \\
\text { MMSE less than } \\
\text { 23; GDS } 3 \text { to } 4\end{array}$ & $\begin{array}{l}\mathrm{N}=42 \\
\text { Age: yes } \\
\text { Sex: yes } \\
\text { \% majority race: no } \\
\text { Education: no }\end{array}$ & NR & NA & NA & NA & NA & 10 weeks & $\begin{array}{l}\text { MMSE } \\
\text { CES-D } \\
\text { RESS } \\
\text { PWB }\end{array}$ & NA \\
\hline $\begin{array}{l}\text { Lalanee 2015126 } \\
(25122521) \\
\text { France } \\
\text { Sample Size }\end{array}$ & $\begin{array}{l}\text { Autobiographica } \\
\text { I conitive } \\
\text { training } \\
\text { reminiscence; } 6 \\
\text { sessions }\end{array}$ & $\begin{array}{l}\text { Cognitive } \\
\text { training program }\end{array}$ & $\begin{array}{l}\text { Health } \\
\text { institutions } \\
\text { RCT; } \\
\text { multisite } \\
\text { 33 PLWD }\end{array}$ & $\begin{array}{l}\text { Mild to moderate } \\
\text { AD (MMSE>18) }\end{array}$ & $\begin{array}{l}\mathrm{N}=33 \\
\text { Age: yes } \\
\text { Sex: no } \\
\text { \% majority race: no } \\
\text { Education: yes }\end{array}$ & NR & NA & NA & NA & NA & $\begin{array}{l}6 \text { weeks } \\
8 \text { weeks }\end{array}$ & $\begin{array}{l}\text { Semi } \\
\text { autographica } \\
\text { I Memory } \\
\text { Episodic } \\
\text { memory } \\
\text { GDS }\end{array}$ & NA \\
\hline $\begin{array}{l}\text { O'Shea 2014111 } \\
\text { (24633858) } \\
\text { High RoB }\end{array}$ & $\begin{array}{l}\text { Staff training to } \\
\text { incorporate } \\
\text { reminiscence } \\
\text { into general } \\
\text { care }\end{array}$ & $\begin{array}{l}\text { Usual care } \\
\text { activities }\end{array}$ & \begin{tabular}{|l|} 
Nursing \\
home \\
Cluster RCT \\
18 units
\end{tabular} & $\begin{array}{l}\text { PLWD with AD, } \\
\text { and no significant } \\
\text { sensory impairment } \\
\text { or acute physical } \\
\text { illness }\end{array}$ & $\begin{array}{l}\mathrm{N}=304 \\
\text { Age: } \mathrm{Y} \\
\text { Sex: } \mathrm{Y} \\
\text { Race: } \mathrm{Y} \\
\text { Education: } \mathrm{N}\end{array}$ & \begin{tabular}{|l|l|} 
Prior disability \\
\end{tabular} & NA & NA & NR & NR & 2 years & $\begin{array}{l}\text { QOL-AD } \\
\text { CMAl-AD } \\
\text { CSDD }\end{array}$ & $\begin{array}{l}\text { MZBI nurse } \\
\text { MZBI heath } \\
\text { care assistant }\end{array}$ \\
\hline $\begin{array}{l}\text { Nakamae } \\
2014^{127} \text { (NA) } \\
\text { Japan } \\
\text { Pilot Study }\end{array}$ & $\begin{array}{l}\text { Six activity } \\
\text { sessions } \\
\text { (making rice } \\
\text { balls) with } \\
\text { reminiscence } \\
\text { therapy; one } \\
\text { session//week }\end{array}$ & Eating rice balls & $\begin{array}{l}\text { Long-term } \\
\text { care } \\
\text { facilities } \\
\text { RCT; } \\
\text { multisite } \\
36 \text { PLWD } \\
\end{array}$ & \begin{tabular}{|l|} 
AD and vascular \\
dementia; MMSE \\
23 or lower
\end{tabular} & $\begin{array}{l}\mathrm{N}=36 \\
\text { Age: yes } \\
\text { Sex: no } \\
\text { \% majority race: no } \\
\text { Education: yes }\end{array}$ & NR & NA & NA & NA & NA & 6 weeks & \begin{tabular}{l|} 
CSDD \\
MOSES \\
MMSE \\
Vitality Index
\end{tabular} & NA \\
\hline $\begin{array}{l}\text { Subramaniam } \\
2014^{128} \\
(24063317) \\
\text { Wales } \\
\text { Pilot Study }\end{array}$ & $\begin{array}{l}\text { Individual life } \\
\text { review sessions } \\
\text { and co-creating } \\
\text { a life story book; } \\
12 \text { sessions }\end{array}$ & $\begin{array}{l}\text { Person life story } \\
\text { book created by } \\
\text { relatives }\end{array}$ & $\begin{array}{l}\text { Care homes } \\
\text { RCT; } \\
\text { multisite } \\
23 \text { PLWD }\end{array}$ & \begin{tabular}{|l|} 
Mild to moderate \\
dementia diagnosis \\
(DSM criteria)
\end{tabular} & $\begin{array}{l}\mathrm{N}=24 \\
\text { Age: yes } \\
\text { Sex: yes } \\
\text { \% majority race: no } \\
\text { Education: no }\end{array}$ & NR & $\begin{array}{l}\mathrm{N}=24 \\
\text { Age: yes } \\
\text { Sex: yes } \\
\text { \% majority race: no } \\
\text { Education: no } \\
\text { Relation to PLWD: } \\
\text { yes }\end{array}$ & NR & $\begin{array}{l}\mathrm{N}=68 \\
\text { Age: yes } \\
\text { Sex: yes } \\
\text { \% majority race: } \\
\text { no } \\
\text { Education: yes }\end{array}$ & $\begin{array}{l}\text { Length of } \\
\text { services: yes }\end{array}$ & $\begin{array}{l}12 \text { weeks } \\
18 \text { weeks }\end{array}$ & \begin{tabular}{l|} 
QOL-AD \\
AMI-E \\
GDS \\
(Residential) \\
QCPR
\end{tabular} & FC: ADQ \\
\hline $\begin{array}{l}\text { Van Bogaret } \\
2013^{129} \\
\text { (23583001) } \\
\text { Belgium } \\
\text { Pilot Study }\end{array}$ & $\begin{array}{l}\text { Individual } \\
\text { thematically- } \\
\text { based } \\
\text { reminiscence } \\
\text { therapy; } 4 \\
\text { weeks with two } \\
45 \text { min } \\
\text { sessions/week }\end{array}$ & No intervention & $\begin{array}{l}\text { Psychiatric } \\
\text { day care, } \\
\text { inpatient, } \\
\text { and long- } \\
\text { term care } \\
\text { facilities } \\
\text { RCT; } \\
\text { 82 PLWD }\end{array}$ & $\begin{array}{l}\text { AD based on } \\
\text { NINCDS-ADRDA }\end{array}$ & $\begin{array}{l}\mathrm{N}=82 \\
\text { Age: yes } \\
\text { Sex: yes } \\
\text { \% majority race: no } \\
\text { Education: no }\end{array}$ & NR & NA & NA & NA & NA & 4 weeks & $\begin{array}{l}\text { MMSE } \\
\text { FAB } \\
\text { NPI } \\
\text { GDS-30 } \\
\text { CSDD }\end{array}$ & NA \\
\hline
\end{tabular}




\begin{tabular}{|c|c|c|c|c|c|c|c|c|c|c|c|c|c|}
\hline $\begin{array}{l}\text { Seranni Azcurra } \\
2012^{12} \\
\text { (23429813) } \\
\text { Argentina } \\
\text { High RoB }\end{array}$ & $\begin{array}{l}\text { Life-story } \\
\text { reminiscence; } \\
\text { biweekly } \\
\text { sessions for } 1 \\
\text { hour over } 12 \\
\text { weeks }\end{array}$ & $\begin{array}{l}\text { Unstructured } \\
\text { social contact; } \\
\text { biweekly } \\
\text { sessions for } 1 \\
\text { hour over } 12 \\
\text { weeks }\end{array}$ & \begin{tabular}{|l|} 
Nursing \\
home \\
RCT; \\
multisite
\end{tabular} & \begin{tabular}{|l|} 
AD (DSM-IV); \\
Folstein Minimental \\
Exam above 10
\end{tabular} & \begin{tabular}{|l|}
$\mathrm{N}=90$ \\
Age: yes \\
Sex: yes \\
\% majority race: no \\
Education: yes
\end{tabular} & NR & NA & NA & $\begin{array}{l}\text { N=NR } \\
\text { Age: no } \\
\text { Sex: no } \\
\% \text { majority race: } \\
\text { no } \\
\text { Education: no } \\
\text { Relation to PLWD: } \\
\text { no }\end{array}$ & NR & $\begin{array}{l}12 \text { weeks } \\
6 \text { months }\end{array}$ & $\begin{array}{l}\text { SRQol } \\
\text { SES } \\
\text { WIB } \\
\text { ADL }\end{array}$ & ZBI \\
\hline $\begin{array}{l}\text { Hsieh } 2010 \\
114(\mathrm{NA}) \\
\text { Taiwan } \\
\text { High RoB }\end{array}$ & $\begin{array}{l}\text { Group } \\
\text { reminiscence } \\
\text { therapy; } 12 \\
\text { sessions } 40 \text { to } \\
50 \text { minutes per } \\
\text { week }\end{array}$ & No intervention & $\begin{array}{l}\text { Nursing } \\
\text { homes } \\
\text { RCT; } \\
\text { multisite } \\
61 \text { PLWD }\end{array}$ & \begin{tabular}{|l|} 
Mild to moderate \\
dementia (DSM-IV)
\end{tabular} & \begin{tabular}{|l|}
$\mathrm{N}=61$ \\
Age: yes \\
Sex: yes \\
\% majority race: no \\
Education: yes
\end{tabular} & NR & NA & NA & NA & NA & 12 weeks & $\begin{array}{l}\text { CDR } \\
\text { GDS } \\
\text { Apathy } \\
\text { Evaluation } \\
\text { Scale } \\
\text { NPI }\end{array}$ & NA \\
\hline 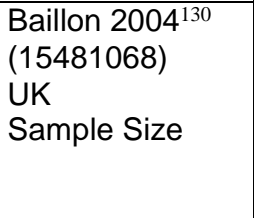 & $\begin{array}{l}\text { Reminiscence } \\
\text { therapy; } 3 \text { one- } \\
\text { on-one } \\
\text { sessions, } 40 \\
\text { minutes/ } \\
\text { session }\end{array}$ & $\begin{array}{l}\text { Snoezelen; } 3 \\
\text { one-on-one } \\
\text { sessions, } 40 \\
\text { minutes/ } \\
\text { session }\end{array}$ & $\begin{array}{l}\text { Crossover } \\
\text { RCT; } \\
20 \text { PLWD }\end{array}$ & \begin{tabular}{|l} 
Dementia \\
diagnosis with \\
BPSD
\end{tabular} & \begin{tabular}{|l|}
$\mathrm{N}=20$ \\
Age: yes \\
Sex: yes \\
\% majority race: no \\
Education: no
\end{tabular} & NR & NA & NA & NA & NA & 4 weeks & $\begin{array}{l}\text { Agitation } \\
\text { Behavior } \\
\text { Mapping } \\
\text { Instrument } \\
\text { Interact } \\
\text { Scale }\end{array}$ & NA \\
\hline $\begin{array}{l}\text { Lai 2004116 } \\
\text { (15190995) } \\
\text { China } \\
\text { High RoB }\end{array}$ & $\begin{array}{l}\text { Life-story } \\
\text { reminiscence; } \\
\text { weekly } 30- \\
\text { minute session } \\
\text { for } 6 \text { weeks }\end{array}$ & $\begin{array}{l}\text { Friendly } \\
\text { discussions; 30- } \\
\text { minute sessions } \\
\text { for } 6 \text { weeks }\end{array}$ & \begin{tabular}{|l|} 
Nursing \\
homes \\
RCT; \\
multisite \\
101 PLWD \\
\end{tabular} & $\begin{array}{l}\text { Dementia } \\
\text { diagnosis (DSM-IV) }\end{array}$ & $\begin{array}{l}\mathrm{N}=101 \\
\text { Age: yes } \\
\text { Sex: yes } \\
\text { \% majority race: no } \\
\text { Education: no } \\
\end{array}$ & NR & NA & NA & NA & NA & $\begin{array}{l}6 \text { weeks } \\
12 \text { weeks }\end{array}$ & $\begin{array}{l}\text { SES } \\
\text { WIB } \\
\text { MMSE }\end{array}$ & NA \\
\hline $\begin{array}{l}\text { Politis 2004'131 } \\
\text { (15481065) } \\
\text { US } \\
\text { Pilot Studies }\end{array}$ & $\begin{array}{l}\text { Kit-based } \\
\text { activity } \\
\text { intervention to } \\
\text { reduce apathy; } \\
\text { 30-minute } \\
\text { sessions for } 4 \\
\text { weeks }\end{array}$ & $\begin{array}{l}\text { One-on-one } \\
\text { meetings with } \\
\text { activity therapist }\end{array}$ & \begin{tabular}{|l|} 
Long-term \\
care facility \\
for PLWD \\
RCT; single \\
site \\
37 PLWD \\
\end{tabular} & \begin{tabular}{|l|} 
Dementia \\
diagnosis (DSM-IV) \\
with apathy and a \\
GDS score of 3 to \\
5
\end{tabular} & $\begin{array}{l}\mathrm{N}=37 \\
\text { Age: yes } \\
\text { Sex: yes } \\
\text { \% majority race: no } \\
\text { Education: yes }\end{array}$ & NR & NA & NA & NA & NA & 4 weeks & $\begin{array}{l}\text { NPI-Apathy } \\
\text { NPI } \\
\text { ADRQRL } \\
\text { CRAI }\end{array}$ & NA \\
\hline $\begin{array}{l}\text { Camberg } \\
1999^{117} \\
(10203120) \\
\text { US } \\
\text { High RoB }\end{array}$ & $\begin{array}{l}\text { Stimulated } \\
\text { presence } \\
\text { continuous play } \\
\text { memory audio } \\
\text { recordings }\end{array}$ & Usual care & $\begin{array}{l}\text { Nursing } \\
\text { homes } \\
\text { RCT; } \\
\text { multisite } \\
\text { 54 PLWD }\end{array}$ & $\begin{array}{l}\text { ARDR with } \\
\text { agitation }\end{array}$ & $\begin{array}{l}\mathrm{N}=54 \\
\text { Age: yes } \\
\text { Sex: yes } \\
\text { \% majority race: yes } \\
\text { Education: no }\end{array}$ & NR & NA & NA & NA & NA & 12 weeks & $\begin{array}{l}\text { Observed } \\
\text { Agitation } \\
\text { Scale } \\
\text { Agitation } \\
\text { VAS } \\
\text { PARS } \\
\text { WVAS } \\
\text { FACE }\end{array}$ & NA \\
\hline
\end{tabular}

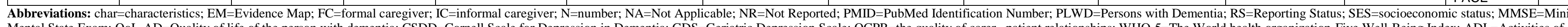

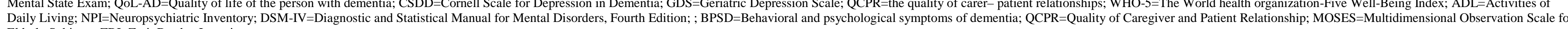
Elderly Subjects; ZBI=Zarit Burden Intervie

\section{Cognitive Rehabilitation}




\begin{tabular}{|c|c|c|c|c|c|c|c|c|c|}
\hline $\begin{array}{l}\text { Author, Year } \\
\text { PMID }\end{array}$ & Outcome Timing & $\begin{array}{l}\text { Attrition Bias } \\
\text { Attrition \% }\end{array}$ & Selection Bias & Detection Bias & Performance Bias & Reporting Bias & Fidelity Bias & Funding & Overall Rating \\
\hline $\begin{array}{l}\text { Chen 2020132 } \\
\text { (No PMID) }\end{array}$ & 1 month & Low (reported no attrition) & High & $\mathrm{x}$ & $\mathrm{x}$ & $\mathrm{x}$ & $\mathrm{x}$ & Not reported & High \\
\hline $\begin{array}{l}\text { Clare 2019193 } \\
30724405\end{array}$ & 9 months & Low $10 \%$ & Low & Low & High & Low & Medium & Government & Medium \\
\hline $\begin{array}{l}\text { Voigt-Radloff } 2017^{134} \\
28335810\end{array}$ & 16 week & Low $13 \%$ & Low & Medium & Medium & Low & Low & $\begin{array}{l}\text { Foundation } \\
\text { (Government-funded) }\end{array}$ & Low \\
\hline $\begin{array}{l}\text { Voigt-Radloff } 2017^{134} \\
28335810\end{array}$ & 26 week & Low $15 \%$ & Low & Medium & Medium & Low & Low & $\begin{array}{l}\text { Foundation } \\
\text { (Government-funded) }\end{array}$ & Low \\
\hline $\begin{array}{l}\text { Amieva 2016 } \\
26572551\end{array}$ & 3 months & Low $10 \%$ & Low & Low & Medium & Low & Medium & Government, Foundation & Low \\
\hline $\begin{array}{l}\text { Amieva 2016 } 207 \\
26572551\end{array}$ & 24 months & Medium 28\% & Low & Low & Medium & Low & Medium & Government, Foundation & Medium \\
\hline $\begin{array}{l}\text { Kumar } 2014^{135} \\
24982692\end{array}$ & 5 weeks & High: attrition NR & Low & High & High & Low & Medium & Government & High \\
\hline $\begin{array}{l}\text { Wu 2014136 } \\
24444172\end{array}$ & 6 months & High: $56 \%$ & $\mathrm{x}$ & $\mathrm{x}$ & $\mathrm{x}$ & $\mathrm{x}$ & $\mathrm{x}$ & Government & High \\
\hline
\end{tabular}

$\mathrm{X}$ indicates that domain was not assessed due to high risk of bias.

Abbreviations: NR=Not Reported; PMID=PubMed Identification Number 


\begin{tabular}{|c|c|c|c|c|c|c|c|c|c|c|c|}
\hline $\begin{array}{l}\text { Author, Year } \\
\text { PMID } \\
\text { Country } \\
\text { RoB* } \\
\text { Type } \\
\text { Funder }\end{array}$ & \begin{tabular}{|l|} 
Target \\
Intervention \\
Group vs Not \\
Components \\
Frequency \\
Duration
\end{tabular} & \begin{tabular}{|l|} 
Target Comparison \\
Group vs Not \\
Components \\
Frequency \\
Duration
\end{tabular} & \begin{tabular}{|l|} 
Setting \\
Design \\
Clusters \\
N Randomized
\end{tabular} & \begin{tabular}{|l} 
PLWD \\
Dementia \\
Characteristics \\
Type(s) \\
Severity \\
Diagnostic Criteria \\
Age Diagnosed
\end{tabular} & \begin{tabular}{|l|} 
PLWD \\
Age (mean) \\
Female \% \\
Race (\% majority) \\
Detailed Race \\
Education (mean \\
years)
\end{tabular} & \begin{tabular}{|l|} 
PLWD \\
SES \\
Prior Disability \\
Household \\
Characteristics \\
Health Insurance
\end{tabular} & $\begin{array}{l}\text { Informal } \\
\text { Caregiver } \\
\mathrm{N} \\
\text { Age (mean) } \\
\text { Female \% } \\
\text { Race (\% } \\
\text { majority) } \\
\text { Education } \\
\text { (mean years) } \\
\text { Relation to } \\
\text { PLWD }\end{array}$ & $\begin{array}{l}\text { Informal Caregiver } \\
\text { Duration } \\
\text { Live With PLWD } \\
\text { IC Paid } \\
\text { Health } \\
\text { Dementia Family } \\
\text { History } \\
\text { Employed } \\
\text { Training }\end{array}$ & \begin{tabular}{|l|} 
Outcome \\
Timing
\end{tabular} & $\begin{array}{l}\text { PLWD Outcomes } \\
\text { Quality of Life } \\
\text { Daily Function } \\
\text { BPSD } \\
\text { Adverse Events } \\
\text { Services } \\
\text { Other }\end{array}$ & \begin{tabular}{|l}
$\mid$ Informal \\
Caregiver \\
Outcome
\end{tabular} \\
\hline $\begin{array}{l}\text { Clare 2019133 } \\
30724405 \\
\text { UK } \\
\text { Medium } \\
\text { Explanatory } \\
\text { Government } \\
\text { Clare 2019 } \\
\text { 137 }\end{array}$ & \begin{tabular}{|l|} 
PLWD \\
Individual goal-oriented \\
cognitive rehabilitition \\
1 hour/week (10 sessions in 3 \\
months) plus 4 maintenance \\
sessions over 6 months. \\
9 months \\
Directed by trained OT or RN
\end{tabular} & \begin{tabular}{|l} 
Usual care \\
\end{tabular} & $\begin{array}{l}\text { Home-based } \\
\text { intervention by } \\
\text { therapist } \\
\mathrm{RCT} \\
\mathrm{N}=475\end{array}$ & \begin{tabular}{|l|} 
Types: 60\% AD, vascular, \\
mixed per ICD-10 code. \\
Early-stage mild- \\
moderate dementia \\
(mean MMSE 24, enrolled \\
MSSE $\geq 18$ ); stable \\
dementia medication \\
acceptable
\end{tabular} & $\begin{array}{l}\mathrm{N}=475 \\
\text { Mean age: } 79 \text { years } \\
\text { 48\% female } \\
96 \% \text { white race } \\
\text { Education: } 13 \text { years }\end{array}$ & Married: $70 \%$ & $\begin{array}{l}\mathrm{N}=474 \\
\text { Mean age: } 69 \text { yrs } \\
\text { Female: } 70 \% \\
\text { 96\% white race } \\
\text { Education: } 14 \\
\text { years } \\
\text { Relation: } 70 \% \\
\text { spouse/partner }\end{array}$ & NR & $\begin{array}{l}3 \text { months and } \\
9 \text { months post } \\
\text { intervention }\end{array}$ & $\begin{array}{l}\text { which are typically } \\
\text { used in cross- } \\
\text { sectional studies } \\
\text { (which are } \\
\text { typically used in } \\
\text { cross-sectional } \\
\text { studies which are } \\
\text { typically used in } \\
\text { cross-sectional } \\
\text { studies }\end{array}$ & $\begin{array}{l}\begin{array}{l}\text { Stress, Quality } \\
\text { of life }\end{array} \\
\text { laty }\end{array}$ \\
\hline $\begin{array}{l}\text { Voigt-Radloff } \\
2017 \\
13428335810 \\
\text { Germany, } \\
\text { Netherlands } \\
\text { Low } \\
\text { Explanatory } \\
\text { Government } \\
\text { Foundation } \\
\\
\text { Voight-Radloff } \\
2011^{138}\end{array}$ & \begin{tabular}{|l} 
PLWD \\
Individual \\
Errorless Learning (feed- \\
forward instructions) at home \\
$9 \times 1$ 1-hour task-training \\
sessions \\
8 weeks
\end{tabular} & $\begin{array}{l}\text { PLWD } \\
\text { Individual } \\
\text { Trial \& Error } \\
\text { learning at home } \\
9 \times 1-\text { hour task- } \\
\text { training sessions } \\
8 \text { weeks }\end{array}$ & $\begin{array}{l}\text { Home } \\
\text { (community) } \\
\text { RCT } \\
\text { Cluster: NA } \\
\mathrm{N}=161\end{array}$ & $\begin{array}{l}\text { Type: AD or mixed } \\
\text { Severity: mild- moderate } \\
\text { (MMSE 14-24), with } \\
\text { available ICG } \\
\text { Diagnosis: NR } \\
\text { Diagnosis age: } 1.9 \text { years } \\
\text { prior }\end{array}$ & \begin{tabular}{|l}
$\mathrm{N}=161$ \\
77 years \\
$57 \%$ female \\
Race: NR \\
More race: NA \\
Education: $98 \% \geq 9$ \\
years
\end{tabular} & $\begin{array}{l}\text { SES: NR } \\
\text { Disability: NR } \\
\text { Household: NA } \\
\text { Insurance: NR } \\
\text { (Germany) }\end{array}$ & $\begin{array}{l}\text { N=NR (161) } \\
\text { Mean age: } 63 \\
\text { Female: 36\% } \\
\text { Race: NR } \\
\text { Education: NR } \\
\text { Relation: NR }\end{array}$ & $\begin{array}{l}\text { Duration: } 25 \text { months } \\
\text { Live together: } 67 \% \\
\text { Paid: NR } \\
\text { Health: NR } \\
\text { Family history: NR } \\
\text { Employed: NR } \\
\text { Training: NR }\end{array}$ & $\begin{array}{l}16 \text { weeks, } 26 \\
\text { weeks }\end{array}$ & \begin{tabular}{|l} 
QoL: QOL-AD \\
Function: IIDD \\
BPSD: NPI-12, \\
AE: NR \\
Services: NR \\
Other: Task \\
performance videos, \\
cognitive, satisfaction
\end{tabular} & NR \\
\hline $\begin{array}{l}\text { Amieva 2016107 } \\
26572551 \\
\text { France } \\
\text { Medium } \\
\text { Explanatory } \\
\text { Government } \\
\text { Foundation } \\
\text { ETNA3 }\end{array}$ & $\begin{array}{l}\text { PLWD (+ ICG support group): } \\
\text { 1. Group cognitive rehabilitation } \\
\text { 2. Group reminiscence } \\
\text { 3. Individually-tailored cognitive } \\
\text { rehabilitation (with psychologist } \\
\text { + weekly call to ICG) } \\
\text { All: } 1.5 \text { hours/week } \times 3 \text { months, } \\
\text { then once every } 6 \text { weeks } \times 21 \\
\text { months }(24 \text { months total) } \\
\text { Supervision: psychologist or } \\
\text { related }\end{array}$ & \begin{tabular}{|l} 
PLWD (+ ICG \\
support group): \\
Individual \\
Usual care \\
(+ ICG support \\
group 14/week $\times 3$ \\
months, then once \\
every 6 weeks $\times 21$ \\
months).
\end{tabular} & \begin{tabular}{|l|} 
Outpatient (small \\
groups or \\
individual). \\
RCT \\
Clusters: NA \\
$\mathrm{N}=653$
\end{tabular} & \begin{tabular}{|l|} 
Type: NR \\
Severity: mild-moderate \\
(mean MMSE=22) \\
Diagnosis: NINCDS- \\
ADRDA \\
Age diagnosed: NR \\
88\% anti-dementia drugs:
\end{tabular} & \begin{tabular}{|l|}
78 years \\
$60 \%$ female \\
Race: NR \\
More race: NR \\
Education: $49 \%$ \\
secondary school or \\
higher
\end{tabular} & $\begin{array}{l}\text { SES: NR } \\
\text { Disability: NR } \\
\text { Household: NR } \\
\text { Insurance: NR } \\
\text { (France) }\end{array}$ & $\begin{array}{l}\mathrm{N}=\mathrm{NR} \\
\text { Mean age: NR } \\
\text { Female: NR } \\
\text { Race: NR } \\
\text { Education: NR } \\
\text { Relation: NR }\end{array}$ & $\begin{array}{l}\text { Duration: NR } \\
\text { Live together: NR } \\
\text { Paid: NR } \\
\text { Health: NR } \\
\text { Family history: NR } \\
\text { Employed: NR } \\
\text { Training: NR }\end{array}$ & 3,24 months & \begin{tabular}{|l|} 
QoL: QOL-AD \\
Function: DAD, \\
GAGGIR \\
BPSD: NPI \\
AE: NR (survival rate \\
reported) \\
Services: RUD Lite, \\
rate of \\
institutionalization \\
Other: ADAS-cog, \\
MMSE, \% without \\
severe dementia
\end{tabular} & Apathy, ZBI \\
\hline
\end{tabular}

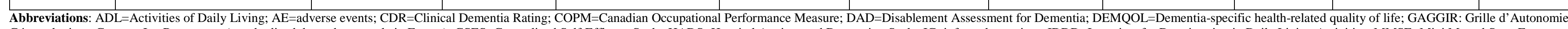

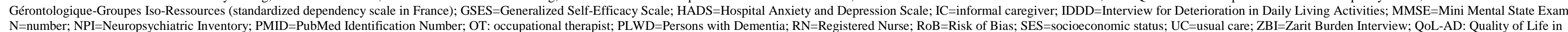
Alzheimer's Disease scale; E=Apathy Evaluation; NR=NR; ADAS-Cog=Alzheimer's Disease Assessment Scale - Cognition RUD=resource utilization; BPSD=Behavioral and psychological symptoms of dementia 
Table D-20. PLWD outcomes summary low and medium risk of bias studies: cognitive rehabilitation

\begin{tabular}{|c|c|c|c|c|c|}
\hline $\begin{array}{l}\text { Study (PMID) } \\
\text { Comparison } \\
\text { RoB } \\
\text { Category* } \\
\text { Type }\end{array}$ & $\begin{array}{l}\text { Outcome } \\
\text { Timing }\end{array}$ & Summary Finding & Intervention & Comparator & $p$-Value \\
\hline $\begin{array}{l}\text { Clare } 2019^{133} \\
\text { (30724405) } \\
\text { Individual cognitive rehabilitation vs } \\
\text { Usual care } \\
\text { Medium } \\
\text { Explanatory }\end{array}$ & $\begin{array}{l}\text { DEMQOL } \\
\text { Mean (SD) } \\
\text { HADS depression } \\
\text { Mean (SD) } \\
\text { HADS anxiety } \\
\text { Mean (SD) } \\
\text { Self-rated goal attainment (COPM, 0-10; } 2 \text { point change=clinically significant) } \\
\text { Mean (SD) } \\
9 \text { months }\end{array}$ & $\begin{array}{l}\text { Mean difference }(95 \% \mathrm{Cl}) 1.08(-0.6,2.8) \\
\text { Mean difference }(95 \% \mathrm{Cl}) 0.12(-0.4,0.6) \\
\text { Mean difference }(95 \% \mathrm{Cl}) 0.26(-0.3,0.8) \\
\text { Mean difference }(95 \% \mathrm{Cl}) 1.7(1.4,2.1)\end{array}$ & $\begin{array}{l}92.36(12.0) \\
4.19(3.2) \\
5.63(3.8) \\
6.05(2.2)\end{array}$ & $\begin{array}{l}92.25(12.8) \\
3.83(2.8) \\
4.88(3.4) \\
4.22(2.0)\end{array}$ & $\begin{array}{l}0.22 \\
0.61 \\
0.33 \\
<0.001\end{array}$ \\
\hline $\begin{array}{l}\text { Amieva 2016107 } \\
\text { (26572551) } \\
\text { Individual cognitive rehabilitation vs } \\
\text { Usual care } \\
\text { Medium } \\
\text { Explanatory }\end{array}$ & $\begin{array}{l}\text { QOL-AD } \\
\text { Mean (SD) } \\
\text { DAD (ADLs) } \\
\text { Mean (SD) } \\
\text { GAGGIR (dependency) } \\
\text { Mean (SD) } \\
\text { NPI } \\
\text { Mean (SD) } \\
24 \text { months }\end{array}$ & $\begin{array}{l}\mathrm{NR} \\
\mathrm{NR} \\
\mathrm{NR} \\
\mathrm{NR}\end{array}$ & $\begin{array}{l}29.05(9.2) \\
27.04(11.9) \\
12.73(11.6) \\
34.44(32.8)\end{array}$ & $\begin{array}{l}28.83(9.5) \\
25.38(13.4) \\
15.21(11.5) \\
39.31(32.3)\end{array}$ & $\begin{array}{l}0.94 \\
0.39 \\
0.025 \\
0.081\end{array}$ \\
\hline $\begin{array}{l}\text { Voigt-Radloff } 2017^{134} \\
\text { (28335810) } \\
\text { Individual: } \\
\text { Errorless Learning (feed-forward } \\
\text { instructions) vs Trial \& Error Learning } \\
\text { Low } \\
\text { Explanatory }\end{array}$ & $\begin{array}{l}\text { IDDD (ADL performance) } \\
\text { Mean (SD) } \\
\text { NPI } \\
\text { Mean (SD) } \\
\text { Task performance rating video (A) } \\
\text { Mean (SD) (1=worst, } 7 \text { =best) } \\
\text { Task performance rating video (B) } \\
\text { Mean (SD) (1=worst, } 7=\text { best) } \\
26 \text { weeks }\end{array}$ & $\begin{array}{l}\text { Difference (CI) } 1.4(-2.1,4.8) \\
\text { Difference (CI) } 0.6(-1.5,2.7) \\
\text { Difference (CI) } 0.3(-0.5,0.8) \\
\text { Difference (CI) }-0.2(-0.9,0.5)\end{array}$ & $\begin{array}{l}22.3(10.2) \\
8.0(5.4) \\
3.8(1.8) \\
4.1(2.0)\end{array}$ & $\begin{array}{l}23.6(10.1) \\
8.6(6.5) \\
4.0(1.7) \\
3.9(1.9)\end{array}$ & $\begin{array}{l}\mathrm{NR} \\
\mathrm{NR} \\
\mathrm{NR} \\
\mathrm{NR}\end{array}$ \\
\hline
\end{tabular}

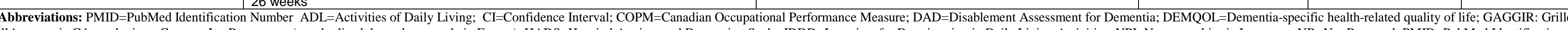

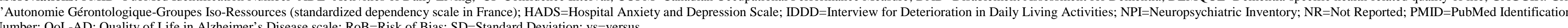


Table D-21. Summary of strength of evidence for PLWD outcomes: cognitive rehabilitation

\begin{tabular}{|c|c|c|c|c|c|c|c|c|}
\hline \begin{tabular}{|l|} 
Comparison \\
Outcome
\end{tabular} & Timing & $\begin{array}{l}\text { \# Studies/ Design } \\
\text { (n analyzed) }\end{array}$ & Finding or Summary Statistic & Study Limitations & Consistency & \begin{tabular}{|l|} 
Directness \\
\end{tabular} & Precision & Overall Grade/ Conclusion \\
\hline $\begin{array}{l}\text { Individual cognitive rehabilitation vs } \\
\text { Usual care } \\
\text { Quality of Life }\end{array}$ & 9 months & $\begin{array}{c}1 \mathrm{RCT}^{133} \\
(\mathrm{n}=475)\end{array}$ & No significant difference between groups & Medium & Unknown & Direct & \begin{tabular}{|l} 
Imprecise \\
\end{tabular} & Insufficient \\
\hline $\begin{array}{l}\text { Individual cognitive rehabilitation vs } \\
\text { Usual care } \\
\text { Quality of Life }\end{array}$ & 24 months & $\begin{array}{l}1 \mathrm{RCT}^{107} \\
(\mathrm{n}=311)\end{array}$ & No significant difference between groups & Medium & Unknown & Direct & Imprecise & Insufficient \\
\hline $\begin{array}{l}\text { Individual cognitive rehabilitation vs usual } \\
\text { care } \\
\text { Daily function }\end{array}$ & 24 months & $\begin{array}{l}\begin{array}{l}\text { RCT107 } \\
(n=311)\end{array}\end{array}$ & No significant difference between groups & Medium & Unknown & Direct & Imprecise & Insufficient \\
\hline $\begin{array}{l}\text { IIdividual cognitive rehabilitation vs usual } \\
\text { care } \\
\text { Self-rated goal attainment }\end{array}$ & 9 months & $\begin{array}{c}1 \mathrm{RCT}^{133} \\
(\mathrm{n}=475)\end{array}$ & $\begin{array}{l}\text { Statistically but not clinically significant } \\
\text { benefit of individual cognitive rehabilitation } \\
\text { compared with usual care on function } \\
\text { assessed as self-rated goal attainment }\end{array}$ & Medium & Unknown & Direct & Imprecise & Insufficient \\
\hline $\begin{array}{l}\text { Errorless Learning (feed-forward } \\
\text { instructions) vs Trial \& Error Learning } \\
\text { Daily function }\end{array}$ & 6 months & $\begin{array}{l}1 \text { RCT134 } \\
\text { (n=161) } \\
6 \text { months }\end{array}$ & \begin{tabular}{|l} 
No difference between groups \\
\end{tabular} & Low & Unknown & Direct & \begin{tabular}{|l|} 
Imprecise \\
\end{tabular} & \begin{tabular}{|l} 
Insufficient \\
\end{tabular} \\
\hline $\begin{array}{l}\text { Individual cognitive rehabilitation vs usual } \\
\text { care } \\
\text { Dependency }\end{array}$ & 24 months & $\begin{array}{l}1 \mathrm{RCT} 107 \\
(\mathrm{n}=311)\end{array}$ & $\begin{array}{l}\text { Less dependency (slower functional } \\
\text { decline) with cognitive rehabilitation over } \\
24 \text { months }\end{array}$ & Medium & Unknown & Direct & Imprecise & Insufficient \\
\hline $\begin{array}{l}\text { Individual cognitive rehabilitation vs usual } \\
\text { care } \\
\text { Neuropsychiatric symptoms }\end{array}$ & 24 months & $\begin{array}{l}\mathrm{RCT}^{107} \\
(\mathrm{n}=311)\end{array}$ & No difference between groups & Medium & Unknown & \begin{tabular}{|l|} 
Direct \\
\end{tabular} & \begin{tabular}{|l} 
Imprecise \\
\end{tabular} & \begin{tabular}{|l} 
Insufficient \\
\end{tabular} \\
\hline $\begin{array}{l}\text { Errorless Learning (feed-forward } \\
\text { instructions) vs Trial \& Error Learning } \\
\text { Neuropsychiatric symptoms }\end{array}$ & 6 months & $\begin{array}{l}1 \mathrm{RCT}^{134} \\
(\mathrm{n}=161)\end{array}$ & No difference between groups & Low & Unknown & \begin{tabular}{|l|} 
Direct \\
\end{tabular} & \begin{tabular}{|l} 
Imprecise \\
\end{tabular} & \begin{tabular}{|l} 
Insufficient \\
\end{tabular} \\
\hline $\begin{array}{l}\text { Individual cognitive rehabilitation vs usual } \\
\text { care }\end{array}$ & 9 months & $\begin{array}{c}1 \mathrm{RCT}^{133} \\
(\mathrm{n}=475)\end{array}$ & No difference between groups & Medium & Unknown & Direct & \begin{tabular}{|l|} 
Imprecise \\
\end{tabular} & Insufficient \\
\hline $\begin{array}{l}\text { Individual cognitive rehabilitation vs usual } \\
\text { care } \\
\text { Anxiety }\end{array}$ & 9 months & $\begin{array}{l}1 \mathrm{RCT}^{133} \\
(\mathrm{n}=475)\end{array}$ & No difference between groups & Medium & Unknown & Direct & Imprecise & Insufficient \\
\hline
\end{tabular}

Abbreviations: PLWD=Persons Living With Dementia; RCT=Randomized Controlled Trial; vs=Versus

\section{Evidence Map: Cognitive Rehabilitation}

\begin{tabular}{|c|c|c|c|c|c|c|c|c|c|c|c|c|c|}
\hline \begin{tabular}{|l} 
Study (PMID) \\
Country \\
EM Reason
\end{tabular} & Intervention & Comparison & \begin{tabular}{|l|} 
Setting \\
and Design \\
\\
Setting \\
Design \\
Cluster N \\
Participants \\
Randomized \\
N
\end{tabular} & $\begin{array}{l}\text { PLWD } \\
\text { Dementia } \\
\text { Characteristics }\end{array}$ & $\begin{array}{l}\text { PLWD } \\
\text { Non-Disease Char } \\
\text { PLWD N } \\
\text { PLWD Age (mean) } \\
\text { PLWD Sex (\% } \\
\text { female) } \\
\text { PLWD Race (\% } \\
\text { majority) } \\
\text { PLWD Education } \\
\text { (mean years) }\end{array}$ & \begin{tabular}{|l|} 
PLWD \\
Non-Disease \\
Char Reporting \\
Status (RS) \\
PLWD SES \\
PLWD Prior \\
Disability \\
PLWD \\
Household \\
Characteristics \\
PLWD Health \\
Insurance \\
PLWD Detailed \\
Race \\
Information
\end{tabular} & $\begin{array}{l}\text { Informal } \\
\text { Caregiver (IC) } \\
\text { Characteristics } \\
\text { IC N } \\
\text { IC Age (mean) } \\
\text { IC Sex (\% } \\
\text { female) } \\
\text { IC Race (\% } \\
\text { majority) } \\
\text { IC Education } \\
\text { (mean years) } \\
\text { IC Relation to } \\
\text { PLWD (\% } \\
\text { majority) }\end{array}$ & \begin{tabular}{|l|} 
Informal \\
Caregiver (IC) \\
Char. RS \\
IC Duration \\
IC Living With \\
PLWD \\
IC Payment \\
IC Health Status \\
IC Dementia \\
Family History \\
IC Employment \\
Status \\
IC Training
\end{tabular} & $\begin{array}{l}\text { Formal } \\
\text { Caregiver (FC) } \\
\text { Characteristics } \\
\mathrm{N} \\
\text { Age (mean) } \\
\text { Sex (\% female) } \\
\text { Race (\% } \\
\text { majority) } \\
\text { Education } \\
\text { (mean years) }\end{array}$ & $\begin{array}{l}\text { Formal } \\
\text { Caregiver (FC) } \\
\text { Char. RS } \\
\text { FC Health } \\
\text { Status } \\
\text { FC Training } \\
\text { FC Education } \\
\text { FC Position } \\
\text { FC Length of } \\
\text { Service }\end{array}$ & $\begin{array}{l}\text { Outcome } \\
\text { Timing(s) }\end{array}$ & $\begin{array}{l}\text { PLWD } \\
\text { Outcomes }\end{array}$ & $\begin{array}{l}\text { Caregiver } \\
\text { Outcomes } \\
\text { IC: } \\
\text { FC: }\end{array}$ \\
\hline
\end{tabular}




\begin{tabular}{|c|c|c|c|c|c|c|c|c|c|c|c|c|c|}
\hline $\begin{array}{l}\text { Mbakile-Mahlanza } \\
2020^{139} \\
(31762434) \\
\text { Australia } \\
\text { Small sample }\end{array}$ & \begin{tabular}{|l|} 
Montessori-based \\
activities for \\
PLWD \\
implemented by \\
family members \\
30 minutes \\
$2 x /$ week \\
2 weeks
\end{tabular} & $\begin{array}{l}\text { Reading a } \\
\text { newspaper } \\
\text { with family } \\
\text { member } \\
30 \text { minutes } \\
2 x / \text { week } \\
2 \text { weeks }\end{array}$ & \begin{tabular}{|l} 
Nine Nursing \\
Homes. \\
Cluster \\
crossover \\
RCT; 1-week \\
between \\
groups (6- \\
week study). \\
N=20 PLWD \\
and 20 ICG \\
\end{tabular} & $\begin{array}{l}\text { Chart diagnosis of } \\
\text { dementia, } \geq 3 \\
\text { months in NH, } \\
\text { family willing to } \\
\text { visit at least } \\
\text { 2x/week \& attend } \\
\text { 3-hr. training. } \\
\text { Dementia severity: } \\
\text { unable to } \\
\text { determine } \\
\end{array}$ & $\begin{array}{l}\mathrm{N}=20 \\
\text { Age: NR } \\
\text { Sex: NR } \\
\text { Other: NR }\end{array}$ & NR & \begin{tabular}{|l|}
$\mathrm{N}=20$ \\
Mean age: 64 yrs. \\
Female: \\
Race: $\mathrm{NR} \%$ \\
Education: \\
secondary or \\
higher=68\% \\
Relation: $50 \%$ \\
offspring
\end{tabular} & $\begin{array}{l}\text { Duration: } 5 \text { yrs. } \\
\text { Not living with } \\
\text { PLWD: } 100 \% \\
\text { (70\% IC lived } \\
\text { with family) } \\
\text { Employed: } 48 \% \\
\text { (45\% retired) }\end{array}$ & NA & NA & $\begin{array}{l}2 \text { weeks X } \\
2 \text { (after } \\
\text { each } \\
\text { group) }\end{array}$ & $\begin{array}{l}\text { Affect, } \\
\text { engagement }\end{array}$ & \begin{tabular}{|l} 
IC: quality of \\
relationship, \\
mutuality, \\
mood, \\
mastery, \\
Carer-QoL
\end{tabular} \\
\hline $\begin{array}{l}\text { Chen 2020132 } \\
\text { (No PMID) } \\
\text { China } \\
\text { High RoB }\end{array}$ & \begin{tabular}{|l} 
Montessori- \\
method of sensory \\
stimulation and \\
feeding training \\
(re-identify food \\
and utensils, use \\
utensils). \\
Details: NR \\
1 month
\end{tabular} & \begin{tabular}{|l|} 
Observation \\
and timely \\
correction by \\
staff, if \\
needed \\
\end{tabular} & \begin{tabular}{|l|} 
Hospital. \\
Retrospective \\
(randomized \\
retrospective \\
data); PLWD \\
with complete \\
records, orally \\
fed, informed \\
consent. \\
Not RCT \\
\end{tabular} & \begin{tabular}{|l|} 
Clinical diagnosis \\
of AD or VD. Mild \\
to severe (54\% \\
moderate, $40 \%$ \\
severe)
\end{tabular} & $\begin{array}{l}\mathrm{N}=85 \\
\text { Mean age: } 71 \text { yrs. } \\
\text { Female: } 46 \% \\
\text { Education: } 43 \% \\
\text { junior high or } \\
\text { above }\end{array}$ & NR & NA & NA & NR & NR & 1 month & $\begin{array}{l}\text { EdFED, EBS, } \\
\text { eating time, } \\
\text { MMSE }\end{array}$ & NR \\
\hline $\begin{array}{l}\text { Yuen 2019190 } \\
\text { (no PMID) } \\
\text { Hong Kong } \\
\text { Small sample }\end{array}$ & \begin{tabular}{|l|} 
DementiAbility: \\
Montessori-based \\
cognitive \\
rehabilitation 6 \\
sessions, 45 \\
minutes each \\
\end{tabular} & \begin{tabular}{|l} 
Structured \\
social \\
activities
\end{tabular} & \begin{tabular}{|l} 
Nursing home \\
RCT \\
N=46 PLWD
\end{tabular} & \begin{tabular}{|l|} 
Dementia \\
diagnosis
\end{tabular} & \begin{tabular}{|l}
$\mathrm{N}=46$ \\
Age: yes \\
Sex: yes \\
Race: no (Hong \\
Kong) \\
Education: no \\
\end{tabular} & NR & NR & NR & NA & NA & & $\begin{array}{l}\text { Agitation; } \\
\text { Global } \\
\text { deterioration } \\
\text { scale }\end{array}$ & NA \\
\hline $\begin{array}{l}\text { Silva 2017 } 141 \\
\text { (28124633) } \\
\text { Portugal } \\
\text { Small sample } \\
\text { Silva } 2017^{142}\end{array}$ & $\begin{array}{l}\text { Use of a sense- } \\
\text { cam as a } \\
\text { cognitive } \\
\text { intervention }\end{array}$ & $\begin{array}{l}\text { Memo- } \\
\text { taking; } \\
\text { Diary }\end{array}$ & \begin{tabular}{|l} 
RCT \\
Community- \\
based or \\
integrated in \\
day-care \\
center
\end{tabular} & $\begin{array}{l}\text { PLWD with a } \\
\text { diagnosis of } \\
\text { probable AD; <6 } \\
\text { months since } \\
\text { diagnosis; MMSE } \\
\text { 15-26; other } \\
\text { inclusion criteria } \\
\text { applied }\end{array}$ & $\begin{array}{l}\mathrm{N}=51 \text { (17 sense- } \\
\text { cam, } 17 \text { memo- } \\
\text { writing, } 17 \text { diary) } \\
\text { Age: } \mathrm{N} \\
\text { Sex: } \mathrm{N} \\
\text { Race \% majority: } \mathrm{N} \\
\text { Education: } \mathrm{Y}\end{array}$ & NR & NA & NA & NR & NR & 6 weeks & $\begin{array}{l}\text { Depression,; } \\
\text { functional } \\
\text { capacity; } \\
\text { quality of life }\end{array}$ & NA \\
\hline $\begin{array}{l}\text { Tanaka 2017143 } \\
(27612310) \\
\text { Japan } \\
\text { Small sample }\end{array}$ & \begin{tabular}{|l|} 
5-dimension \\
cognitive \\
rehabilitation: \\
1. Group (1 hour) \\
2. Individual (20 \\
min.) \\
Both: $2 x /$ week for \\
12 weeks
\end{tabular} & Control & \begin{tabular}{|l} 
One $\mathrm{NH}$ \\
$\mathrm{RCT}$ \\
$\mathrm{N}=60$
\end{tabular} & $\begin{array}{l}\text { Resident of one } \\
\text { geriatric health } \\
\text { facility, MMSE 5- } \\
\text { 23; dementia AD, } \\
\text { VD, mixed, other; } \\
\text { and completed } \\
\text { this rehabilitation } \\
\text { program }\end{array}$ & $\begin{array}{l}\mathrm{N}=60 \text { (reported } 43: \\
13 \text { group, } 20 \\
\text { individual, } 20 \\
\text { control) } \\
21 \text { regular care) } \\
\text { Age: } \mathrm{Y} \\
\text { Sex: } \mathrm{Y} \\
\text { Race: } \mathrm{NR} \\
\text { Education: } \mathrm{N}\end{array}$ & \begin{tabular}{|l|} 
SES: NR \\
Disability: NR \\
Household: NR \\
Insurance: NR \\
(Japan)
\end{tabular} & NR & NR & NR & NR & 12 weeks & $\begin{array}{l}\text { Cognition, } \\
\text { mood, QOL } \\
\text { (Japan), GDS, } \\
\text { communication }\end{array}$ & NR \\
\hline $\begin{array}{l}\text { Tsuchiya 2016 } 6^{144} \\
\text { (27303064) } \\
\text { Japan } \\
\text { Small sample }\end{array}$ & \begin{tabular}{|l} 
Brain-Activating \\
Rehabilitation \\
1 hour 3 times \\
weekly plus \\
standard cognitive \\
rehabilitation \\
\end{tabular} & \begin{tabular}{|l} 
Standard \\
cognitive \\
rehabilitation
\end{tabular} & \begin{tabular}{|l} 
Hospital \\
daycare \\
RCT \\
N=48
\end{tabular} & \begin{tabular}{|l} 
Diagnosed \\
dementia \\
CDR rating 0.5-3
\end{tabular} & $\begin{array}{l}\mathrm{N}=201 \\
\text { Age: } Y \\
\text { Sex: } Y \\
\text { Race: } N \\
\text { Education: } N\end{array}$ & NR & NA & NA & NA & NA & $\begin{array}{l}\text { Post } \\
\text { intervention } \\
\text { (NR) }\end{array}$ & MOSES & NA \\
\hline $\begin{array}{l}\text { Seyun Kim 2015 } 5^{145} \\
\text { (25729212) } \\
\text { Korea } \\
\text { Small sample }\end{array}$ & $\begin{array}{l}\text { Cognitive training } \\
\text { in the } \\
\text { performance of } \\
\text { everyday activities }\end{array}$ & $\begin{array}{l}\text { Unstructured } \\
\text { conversation } \\
\text { and watch } \\
\text { health- } \\
\text { related } \\
\text { videos }\end{array}$ & \begin{tabular}{|l} 
RCT \\
PLWD \\
recruited from \\
a day care \\
center
\end{tabular} & $\begin{array}{l}\text { PLWD diagnosed } \\
\text { with possible or } \\
\text { probable AD; } \\
\text { MMSE } \geq 18\end{array}$ & $\begin{array}{l}\mathrm{N}=43(22 \\
\text { intervention, } 21 \\
\text { control) } \\
\text { Age: } \mathrm{Y} \\
\text { Sex: } \mathrm{Y} \\
\text { \% majority race: } \mathrm{N} \\
\text { Education: } \mathrm{Y}\end{array}$ & NR & NA & NA & NR & NR & 8 weeks & $\begin{array}{l}\text { Task } \\
\text { performance; } \\
\text { satisfaction; } \\
\text { quality of life; } \\
\text { mental status; } \\
\text { memory }\end{array}$ & NA \\
\hline $\begin{array}{l}\text { Kumar 2014135 } \\
\text { (24982692) } \\
\text { India } \\
\text { High RoB }\end{array}$ & \begin{tabular}{|l|} 
Novel \\
occupational \\
therapy program + \\
usual care \\
5 weeks \\
\end{tabular} & Usual care & $\begin{array}{l}\text { Outpatient } \\
\text { Unblinded } \\
\text { RCT }\end{array}$ & \begin{tabular}{|l|} 
Adults with mild \\
(most) to \\
moderate \\
dementia, MMSE \\
$11-23$ \\
\end{tabular} & $\begin{array}{l}\text { n=77 (attrition NR) } \\
\text { Age: } Y \\
\text { Sex: } Y \\
\text { Race: } N \\
\text { Education: } Y \\
\end{array}$ & $\begin{array}{l}\text { SES: NR } \\
\text { Disability: NR } \\
\text { Household: NR } \\
\text { Insurance: NR }\end{array}$ & NA & NA & NA & NA & 5 weeks & $\begin{array}{l}\text { Standard OT } \\
\text { assessment, } \\
\text { WHO-QOL- } \\
\text { BREF }\end{array}$ & NR \\
\hline
\end{tabular}




\begin{tabular}{|c|c|c|c|c|c|c|c|c|c|c|c|c|c|}
\hline $\begin{array}{l}\text { Thivierge 2014 } 24^{146} \\
\text { (23871120) } \\
\text { Canada } \\
\text { Small sample } \\
\text { Brunelle-Hamann } \\
\text { 2015147 }\end{array}$ & \begin{tabular}{|l|} 
Cognitive \\
rehabilitation of \\
instrumental ADL \\
activities
\end{tabular} & \begin{tabular}{|l} 
Delayed \\
training
\end{tabular} & \begin{tabular}{|l|} 
Mix of \\
community \\
and nursing \\
home adults. \\
6 months \\
RCT \\
crossover \\
\end{tabular} & $\begin{array}{l}\text { PLWD diagnosed } \\
\text { with AD; mean } \\
\text { MMSE=22; } \\
\text { Additional criteria } \\
\text { applied }\end{array}$ & $\begin{array}{l}\mathrm{N}=20 \text { (reported } 17) \\
\text { Age: } Y \\
\text { Sex: } Y \\
\text { \% majority race: } N \\
\text { Education: } Y\end{array}$ & NR & NA & NA & NR & NR & 6 months & \begin{tabular}{|l|} 
ADL/ADL, \\
NPI, memory, \\
QOL
\end{tabular} & $\begin{array}{l}\text { caregiver } \\
\text { burden }\end{array}$ \\
\hline $\begin{array}{l}\text { Wu 2014436 } \\
\text { (24444172) } \\
\text { Taiwan } \\
\text { High RoB } \\
\text { Wu 2013 }\end{array}$ & \begin{tabular}{|l} 
Individualized \\
Montessori-based \\
eating activities \\
with spaced \\
retrieval \\
24 sessions over \\
8 weeks; number \\
of sessions \\
adjusted per \\
PLWD's recall \\
\end{tabular} & $\begin{array}{l}1 . \\
\text { Standardized } \\
\text { Montessori- } \\
\text { based eating } \\
\text { activities } \\
24 \text { sessions } \\
\text { over } 8 \text { weeks } \\
\text { 2. No } \\
\text { treatment }\end{array}$ & 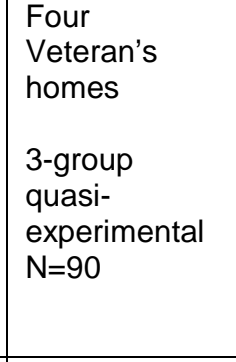 & \begin{tabular}{|l} 
Dementia \\
diagnosis
\end{tabular} & $\begin{array}{l}\text { N=205 (reported } \\
\text { 90) } \\
\text { Age: } Y \\
\text { Sex: } Y \\
\text { Race: } N \\
\text { Education: } Y\end{array}$ & NR & NA & NA & NA & NA & $\begin{array}{l}1,3,6 \\
\text { months }\end{array}$ & \begin{tabular}{|l} 
Edinburgh \\
Feeding \\
Evaluation in \\
Dementia \\
(Chinese)
\end{tabular} & NA \\
\hline $\begin{array}{l}\text { Van der Ploeg } \\
2013^{149} \\
(23237211) \\
\text { Australia } \\
\text { Small sample }\end{array}$ & \begin{tabular}{|l} 
One-to-one \\
Montessori-based \\
activities \\
30 min, 2x/week \\
2 weeks
\end{tabular} & \begin{tabular}{|l|} 
Non- \\
personalized \\
activities
\end{tabular} & \begin{tabular}{|l} 
Nine \\
residential \\
facilities. \\
RCT \\
crossover \\
after 2 weeks \\
\end{tabular} & $\begin{array}{l}\text { Adults diagnosed } \\
\text { with dementia, } \\
\text { agitated behavior } \\
\text { \& resident for > } 3 \\
\text { months }\end{array}$ & $\begin{array}{l}\mathrm{N}=44 \\
\text { Age: } \mathrm{Y} \\
\text { Sex: } \mathrm{Y} \\
\text { \% majority race: } \mathrm{N} \\
\text { Education: } \mathrm{N}\end{array}$ & $\begin{array}{l}\text { Lost English } \\
\text { fluency: } Y\end{array}$ & NA & NA & NR & NR & $\begin{array}{l}\text { Two } \\
\text { periods of } \\
4 \text { weeks }\end{array}$ & $\begin{array}{l}\text { Agitated } \\
\text { behavior; } \\
\text { engagement; } \\
\text { apathy, affect }\end{array}$ & NA \\
\hline $\begin{array}{l}\text { Kurz 2012150 } \\
\text { (21986341) } \\
\text { Germany } \\
\text { Pilot }\end{array}$ & $\begin{array}{l}\text { CORDIAL } 12 \\
\text { individual weekly } \\
\text { sessions. } \\
\text { Combined } 4 \\
\text { established } \\
\text { strategies adopted } \\
\text { from } \\
\text { neurorehabilitation } \\
\text { \& psychotherapy } \\
\end{array}$ & $\begin{array}{l}\text { Standard } \\
\text { care }\end{array}$ & $\begin{array}{l}\text { Home-based } \\
\text { RCT } \\
\text { N=201 PLWD }\end{array}$ & \begin{tabular}{|l} 
Mild AD \\
MMSE >21
\end{tabular} & $\begin{array}{l}\mathrm{N}=201 \\
\text { Age: } Y \\
\text { Sex: } Y \\
\text { Race: } N \\
\text { Education: } Y\end{array}$ & NR & $\begin{array}{l}\text { N=201 } \\
\text { Age: } Y \\
\text { Sex: } Y \\
\text { Race: } N \\
\text { Education: } N \\
\text { Relation to } \\
\text { PLWD: Y }\end{array}$ & NR & NA & NA & $\begin{array}{l}3,9 \\
\text { months }\end{array}$ & \begin{tabular}{|l} 
B-ADL \\
AFIB \\
DEMQOL \\
GDS \\
NPI
\end{tabular} & $\begin{array}{l}\text { ZBI } \\
\text { BDI } \\
\text { WMSRLM } \\
\text { TMT } \\
\text { ZUF-8 }\end{array}$ \\
\hline $\begin{array}{l}\text { Lin 2011 } 151 \\
\text { (20054841) } \\
\text { Taiwan } \\
\text { Small sample }\end{array}$ & \begin{tabular}{|l|} 
Use of a \\
Montessori \\
method to \\
increase eating \\
ability \\
$1 / 2$ hour, 3x/week \\
8 weeks
\end{tabular} & \begin{tabular}{|l|} 
Routine \\
activities
\end{tabular} & \begin{tabular}{|l|} 
RCT \\
crossover \\
with 2-week \\
washout. \\
Randomized \\
by care unit, 2 \\
dementia \\
care facilities \\
\end{tabular} & $\begin{array}{l}\text { PLWD diagnosed } \\
\text { with dementia; } \\
\text { EdFED score of } \\
>=2 \text { and MMSE } \\
10-23\end{array}$ & \begin{tabular}{|l|}
$\mathrm{N}=29$ \\
Age $\mathrm{Y}$ \\
Sex: $\mathrm{Y}$ \\
Race majority: $\mathrm{N}$ \\
Education: $\mathrm{Y}$ (not \\
by group)
\end{tabular} & \begin{tabular}{|l} 
Religion: Y (not \\
by group)
\end{tabular} & NA & NA & NR & NR & $\begin{array}{l}8 \text { week } \\
\text { intervention } \\
\text { with 2 } \\
\text { week } \\
\text { washout } \\
\text { between }\end{array}$ & \begin{tabular}{|l} 
Edinburgh \\
Feeding \\
evaluation, \\
Verbal \\
assistance, \\
Physical \\
assistance, \\
nutrition \\
\end{tabular} & NA \\
\hline $\begin{array}{l}\text { Clare 2010152 } \\
\text { (20808145) } \\
\text { UK } \\
\text { Small sample }\end{array}$ & \begin{tabular}{|l|} 
Individualized \\
cognitive \\
rehabilitation
\end{tabular} & \begin{tabular}{|l} 
1. Relaxation \\
therapy \\
2. No \\
treatment
\end{tabular} & $\begin{array}{l}\text { Community- } \\
\text { based } \\
\text { RCT }\end{array}$ & \begin{tabular}{|l|} 
PLWD with \\
diagnosis of AD, \\
early stage, with \\
MMSE>=18 \\
Screened for \\
exclusion criteria
\end{tabular} & $\begin{array}{l}\mathrm{N}=69 \text { (23 cognitive } \\
\text { rehabilitation, } 24 \\
\text { relaxation, } 22 \text { no } \\
\text { treatment) } \\
\text { Age: } \mathrm{Y} \\
\text { Sex: } \mathrm{Y} \\
\text { Race \% majority: } \mathrm{N} \\
\text { Education: } \mathrm{N} \\
\end{array}$ & Prior disability: $Y$ & NR & $\begin{array}{l}\text { Living with } \\
\text { PLWD: } Y\end{array}$ & NR & NR & $\begin{array}{l}8 \text { week } \\
\text { intervention } \\
\text { period; } \\
6 \text { month } \\
\text { follow-up }\end{array}$ & $\begin{array}{l}\text { Quality of life, } \\
\text { anxiety, } \\
\text { depression, } \\
\text { memory } \\
\text { functioning, } \\
\text { verbal fluency }\end{array}$ & \begin{tabular}{|l|} 
Stress level, \\
anxiety, \\
physical \\
health, social \\
relationships, \\
environment
\end{tabular} \\
\hline $\begin{array}{l}\text { Lam 2010153 } \\
\text { (19582757) } \\
\text { Hong Kong } \\
\text { Pilot }\end{array}$ & $\begin{array}{l}\text { Individualized } \\
\text { functional skills } \\
\text { training for } \\
\text { affective } \\
\text { disturbances and } \\
\text { functional skills } \\
\end{array}$ & \begin{tabular}{|l} 
General \\
occupational \\
therapy \\
program
\end{tabular} & \begin{tabular}{|l} 
Community \\
and \\
residential \\
center based \\
RCT
\end{tabular} & $\begin{array}{l}\text { PLWD diagnosed } \\
\text { with mild or } \\
\text { moderate } \\
\text { dementia; } \\
\text { Screening criteria } \\
\text { applied } \\
\end{array}$ & $\begin{array}{l}\mathrm{N}=74 \text { (37 skills } \\
\text { training, } 37 \text { control) } \\
\text { Age: } \mathrm{Y} \\
\text { Sex: } \mathrm{Y} \\
\text { Race \% majority: } \mathrm{N} \\
\text { Education: } \mathrm{Y}\end{array}$ & NR & NA & NA & NR & NR & $\begin{array}{l}8 \text { week, } 3 \\
\text { months, } 6 \\
\text { months }\end{array}$ & \begin{tabular}{|l} 
MMSE \\
Motor skills \\
Depression \\
Apathy
\end{tabular} & NA \\
\hline $\begin{array}{l}\text { Lin 2010154 } \\
\text { (20054841) } \\
\text { Taiwan } \\
\text { Small sample }\end{array}$ & $\begin{array}{l}\text { 1. Spaced } \\
\text { retrieval activities } \\
3 \times / \text { week, } \\
8 \text { weeks } \\
\text { 2. Montessori- } \\
\text { based activities } \\
3 x / \text { week, } \\
8 \text { weeks } \\
\end{array}$ & \begin{tabular}{|l} 
Routine \\
activity
\end{tabular} & $\begin{array}{l}\text { Nursing } \\
\text { homes (3) } \\
\text { RCT } \\
\text { Clusters: } 3 \\
\text { n=85 }\end{array}$ & \begin{tabular}{|l} 
Adults with chart \\
diagnosis of \\
dementia, lived in \\
1 of 3 NH, scored \\
$\geq 2$ on EdFED, \\
MMSE $10-23$
\end{tabular} & $\begin{array}{l}\mathrm{N}=85 \text { (32 spaced } \\
\text { retrieval, } 29 \\
\text { Montessori, } 24 \\
\text { control) } \\
\text { Age: } Y \\
\text { Sex: } Y \\
\text { Race: } N \\
\text { Education: } N\end{array}$ & $\begin{array}{l}\text { SES: NR } \\
\text { Disability: NR } \\
\text { Household: NA } \\
\text { Insurance: NR } \\
\text { (Taiwan) } \\
\text { More race: NR }\end{array}$ & NA & NA & NR & NR & 8 weeks & \begin{tabular}{|l} 
Eating ability \& \\
feeding \\
assistance \\
required \\
(multiple)
\end{tabular} & NR \\
\hline
\end{tabular}




\begin{tabular}{|c|c|c|c|c|c|c|c|c|c|c|c|c|c|}
\hline $\begin{array}{l}\text { Loewenstein } \\
2004^{155} \\
(15249277) \\
\text { US } \\
\text { Small sample }\end{array}$ & \begin{tabular}{|l|} 
Cognitive \\
rehabilitation \\
program focused \\
on memory and \\
functional \\
performance
\end{tabular} & \begin{tabular}{|l|} 
Mental \\
stimulation \\
program, \\
using \\
computer \\
games
\end{tabular} & \begin{tabular}{|l|} 
Setting NR \\
(assume \\
community \\
due to mean \\
MMSE $=24$ ) \\
RCT
\end{tabular} & $\begin{array}{l}\text { PLWD diagnosed } \\
\text { with probable or } \\
\text { possible AD } \\
\text { (DSM-IV), all on } \\
\text { stable } \\
\text { cholinesterase } \\
\text { inhibitor }\end{array}$ & $\begin{array}{l}\mathrm{N}=44 \text { (25 cognitive } \\
\text { rehabilitation, } 19 \\
\text { mental stimulation) } \\
\text { Age: } \mathrm{Y} \\
\text { Sex: } \mathrm{Y} \\
\text { Race: } \mathrm{NR} \\
\text { Language: } \mathrm{Y} \\
\text { Education: } \mathrm{Y} \\
\end{array}$ & NR & NR & $\mathrm{NR}$ & NA & NA & 12 weeks & \begin{tabular}{|l|} 
Performance \\
on trained \\
skills; \\
memory/recall; \\
ability to \\
perform tasks
\end{tabular} & NA \\
\hline $\begin{array}{l}\text { Beck 2002156 } \\
(12131234) \\
\text { US } \\
\text { Small sample }\end{array}$ & \begin{tabular}{|l|} 
ADL and \\
psychosocial \\
activity \\
combinations (3 \\
groups) \\
1. Combined \\
psychosocial \& \\
ADL intervention \\
2. ADLs \\
3. psycho-social
\end{tabular} & $\begin{array}{l}2 \text { control } \\
\text { groups: } \\
\text { 1. attention } \\
\text { control } \\
\text { 2. usual care }\end{array}$ & \begin{tabular}{|l|} 
Nursing home \\
(7 sites) \\
RCT (5 \\
groups total in \\
each center) \\
Randomized \\
$179 ;$ reported \\
127
\end{tabular} & $\begin{array}{l}\text { PLWD diagnosed } \\
\text { with dementia } \\
\text { MMSE <=20 } \\
\text { Screened for } \\
\text { exclusion criteria }\end{array}$ & $\begin{array}{l}\text { N=179 (reported } \\
129): 28 \text { ADL, } \\
29 \text { psycho-social } \\
\text { activity, } 22 \\
\text { combined, } \\
29 \text { attention } \\
\text { control, } 19 \text { no } \\
\text { intervention. } \\
\text { Age: } Y \\
\text { Sex: } Y \\
\text { Race \% majority: } Y \\
\text { Education: } N\end{array}$ & \% widowed: Y & NA & NA & Education: $Y$ & \begin{tabular}{|l|} 
Training: $Y$ \\
\end{tabular} & $\begin{array}{l}\text { 12-week } \\
\text { intervention } \\
\text { period } \\
\text { One- and } \\
\text { two-month } \\
\text { follow-up } \\
\text { post } \\
\text { intervention }\end{array}$ & $\begin{array}{l}\text { Disruptive } \\
\text { behavior } \\
\text { scores }\end{array}$ & NA \\
\hline $\begin{array}{l}\text { Quayhagen } \\
1995157 \\
7761291 \\
\text { USA } \\
\text { Small sample }\end{array}$ & $\begin{array}{l}\text { Active cognitive } \\
\text { stimulation } \\
\text { training (dyadic } \\
\text { remediation } \\
\text { program) }\end{array}$ & $\begin{array}{l}\text { 1. Passive } \\
\text { activity } \\
\text { 2. Waitlist }\end{array}$ & $\begin{array}{l}\text { Community } \\
\text { (intervention } \\
\text { in home by } \\
\text { ICG) } \\
n=95 \text { eligible }\end{array}$ & $\begin{array}{l}\text { AD, living at home } \\
\text { with caregiver }\end{array}$ & $\begin{array}{l}\mathrm{N}=78 \text { completed } \\
\text { (25 intervention, } 28 \\
\text { passive activity, } 25 \\
\text { control) } \\
\text { Age: } Y \\
\text { Sex: } Y \\
\text { Race: } Y \\
\text { Education: } Y \\
\end{array}$ & NR & \begin{tabular}{|l|}
$\mathrm{N}=78$ \\
Age: $Y$ \\
Sex: $Y$ \\
Race: $N$ \\
Education: $Y$
\end{tabular} & NR & NA & NA & 9 months & \begin{tabular}{|l|} 
Memory \& \\
Behavior \\
Problems \\
Checklist, \\
multiple \\
cognitive
\end{tabular} & NR \\
\hline $\begin{array}{l}\text { Tappen 1994158 } \\
\text { (8184127) } \\
\text { USA } \\
\text { Small sample }\end{array}$ & $\begin{array}{l}\text { 1. Skill training in } \\
\text { performance of } \\
\text { basic daily } \\
\text { activities }\end{array}$ & $\begin{array}{l}\text { 1. Traditional } \\
\text { situational } \\
\text { approach } \\
\text { 2. regular } \\
\text { care }\end{array}$ & \begin{tabular}{|l|} 
RCT \\
Nursing \\
home; \\
random \\
selection from \\
entire NH \\
population
\end{tabular} & \begin{tabular}{|l} 
PLWD diagnosed \\
with dementia; \\
exclusion criteria \\
aplied
\end{tabular} & $\begin{array}{l}\mathrm{N}=63(21 \\
\text { treatment, } 21 \\
\text { situational, } \\
21 \text { regular care) } \\
\text { Age: } \mathrm{Y} \\
\text { Sex: } \mathrm{Y} \\
\text { \% majority race: } \mathrm{N} \\
\text { Education: } \mathrm{N} \\
\end{array}$ & NR & NA & NA & $\mathrm{NR}$ & NR & 20 weeks & \begin{tabular}{|l|} 
Self- \\
maintenance; \\
skills \\
performance; \\
goal \\
attainment
\end{tabular} & NA \\
\hline
\end{tabular}

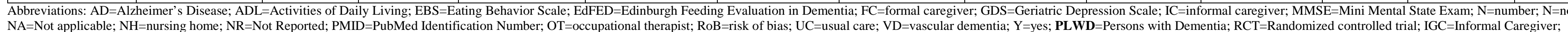
ADL=Instrumental Activities of Daily Living; QoL=Quality of Life; $\mathrm{RCT}=$ Randomized controlled trial

\section{Cognitive Training}




\begin{tabular}{|c|c|c|c|c|c|c|c|c|c|}
\hline Study (PMID) & Outcome Timing & $\begin{array}{l}\text { Attrition Bias } \\
\text { Attrition \% }\end{array}$ & Selection Bias & Detection Bias & Performance Bias & Reporting Bias & Fidelity Bias & Funder & Overall Rating \\
\hline $\begin{array}{l}\text { Tseng 2019159 } \\
(30970666)\end{array}$ & 6 months & Medium & High & $x$ & $\mathrm{x}$ & $x$ & $x$ & NR & High \\
\hline $\begin{array}{l}\text { Kallio 2018 } 8^{160}(29345724) \\
\text { Finland } \\
\text { Medium } \\
\text { Explanatory }\end{array}$ & $\begin{array}{l}3 \text { months } \\
9 \text { months }\end{array}$ & $\begin{array}{l}\text { Low } \\
3 \text { months: } 0 \% \\
\text { High } \\
9 \text { months: } 20.4 \%\end{array}$ & Low & Low & Low & Medium & Medium & Foundation & $\begin{array}{l}\text { Medium (3 months) } \\
\text { High (9 months) }\end{array}$ \\
\hline Amieva 2016 $16^{107}(26572551)$ & $\begin{array}{l}3 \text { months } \\
24 \text { months }\end{array}$ & $\begin{array}{l}\text { Medium } \\
3 \text { months: } 9.9 \% \\
\text { High } \\
24 \text { months: } 28.1 \% \\
\end{array}$ & Low & Low & Low & Medium & Medium & Government & $\begin{array}{l}\text { Medium ( } 3 \text { months) } \\
\text { High (24 months) }\end{array}$ \\
\hline Cavallo 2016 $6^{161}(27600448)$ & $\begin{array}{l}3 \text { months } \\
6 \text { months }\end{array}$ & $\begin{array}{l}\frac{L_{0}}{5 \%} \\
5 \%\end{array}$ & Low & Medium & Low & Medium & Medium & NR & Medium \\
\hline $\begin{array}{l}\text { Giuli 2016162 } \\
\text { (26952713) }\end{array}$ & 10 weeks & $\begin{array}{l}\text { Low } \\
5.9 \%\end{array}$ & Low & High & Medium & Medium & $\mathrm{x}$ & Government & High \\
\hline Hsu 2016 ${ }^{163}(27878873)$ & 6 weeks & $\begin{array}{l}\text { Low } \\
2 \%\end{array}$ & Medium & Low & Medium & Low & Medium & NR & Medium \\
\hline Kao $2016^{164}(27307717)$ & $\begin{array}{l}6 \text { weeks } \\
10 \text { weeks } \\
18 \text { weeks } \\
30 \text { weeks }\end{array}$ & $\begin{array}{l}\text { Low } \\
6 \%\end{array}$ & Medium & Low & Medium & Medium & Medium & Government & Medium \\
\hline
\end{tabular}

X indicates that domain was not assessed due to high risk of bias.

\begin{tabular}{|c|c|c|c|c|c|c|c|c|c|c|c|c|c|}
\hline $\begin{array}{l}\text { Study (PMID) } \\
\text { Country } \\
\text { RoB* } \\
\text { Type }\end{array}$ & $\begin{array}{l}\text { Intervention } \\
\text { Intervention } \\
\text { Focus } \\
\text { Theoretical } \\
\text { Model } \\
\text { Delivery } \\
\text { Person } \\
\text { Intervention } \\
\text { Target } \\
\text { Recipient } \\
\text { Mode } \\
\text { Components } \\
\text { Frequency } \\
\text { Duration }\end{array}$ & $\begin{array}{l}\text { Comparison } \\
\text { Target } \\
\text { Mode } \\
\text { Components } \\
\text { Frequency } \\
\text { Duration }\end{array}$ & $\begin{array}{l}\text { Setting } \\
\text { and Design } \\
\text { Setting } \\
\text { Design } \\
\text { Cluster N } \\
\text { Participants } \\
\text { Randomized } \\
\text { N }\end{array}$ & $\begin{array}{l}\text { PLWD } \\
\text { Dementia } \\
\text { Characteristics } \\
\text { Dementia Types } \\
\text { Dementia Severity } \\
\text { Diagnostic } \\
\text { Criteria } \\
\text { Age of Diagnosis }\end{array}$ & $\begin{array}{l}\text { PLWD } \\
\text { Non-Disease Char } \\
\text { PLWD N } \\
\text { PLWD Age (mean) } \\
\text { PLWD Sex (\% } \\
\text { female) } \\
\text { PLWD Race (\% } \\
\text { majority) } \\
\text { PLWD Education } \\
\text { (mean years) }\end{array}$ & $\begin{array}{l}\text { PLWD } \\
\text { Non-Disease } \\
\text { Char Reporting } \\
\text { Status (RS) } \\
\\
\text { PLWD SES } \\
\text { PLWD Prior } \\
\text { Disability } \\
\text { PLWD } \\
\text { Household } \\
\text { Characteristics } \\
\text { PLWD Health } \\
\text { Insurance } \\
\text { PLWD Detailed } \\
\text { Race Information }\end{array}$ & \begin{tabular}{|l|} 
Informal Caregiver \\
(IC) \\
Characteristics \\
IC N \\
IC Age (mean) \\
IC Sex (\% female) \\
IC Race (\% \\
majority) \\
IC Education \\
(mean years) \\
IC Relation to \\
PLWD (\% majority)
\end{tabular} & $\begin{array}{l}\text { Informal Caregiver } \\
\text { (IC) Char. RS } \\
\text { IC Duration } \\
\text { IC Living With } \\
\text { PLWD } \\
\text { IC Payment } \\
\text { IC Health Status } \\
\text { IC Dementia } \\
\text { Family History } \\
\text { IC Employment } \\
\text { Status } \\
\text { IC Training }\end{array}$ & 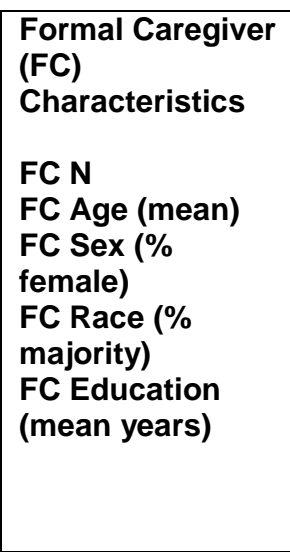 & $\begin{array}{l}\text { Formal } \\
\text { Caregiver (FC) } \\
\text { Char. RS } \\
\text { FC Health } \\
\text { Status } \\
\text { FC Training } \\
\text { FC Education } \\
\text { FC Position } \\
\text { FC Length of } \\
\text { Service }\end{array}$ & $\begin{array}{l}\text { Outcome } \\
\text { Timing(s) }\end{array}$ & \begin{tabular}{|l|} 
PLWD \\
Outcomes
\end{tabular} & $\begin{array}{l}\text { Caregiver } \\
\text { Outcomes } \\
\text { IC: } \\
\text { FC: }\end{array}$ \\
\hline $\begin{array}{l}\text { Kallio 2018 } \\
\text { (29345724) } \\
\text { Finland } \\
\text { Medium } \\
\text { Explanatory } \\
\text { Kallio } \\
2017(N A)^{165}\end{array}$ & \begin{tabular}{|l} 
Group or \\
individual \\
paper-and- \\
pencil cognitive \\
training focused \\
on executive \\
function; 2 \\
times/week, 45 \\
minutes/session \\
for 12 weeks \\
\end{tabular} & Usual care & $\begin{array}{l}\text { Adult day care } \\
\text { RCT; single } \\
\text { site } \\
147 \text { PLWD }\end{array}$ & $\begin{array}{l}\text { AD or other } \\
\text { dementia, very mild } \\
\text { to moderate; } C D R \\
0.5 \text { to } 2\end{array}$ & \begin{tabular}{|l|}
$\mathrm{N}=147$ \\
83 years \\
$72 \%$ Female \\
Race NR \\
$46 \%$ Education $<8$ \\
years
\end{tabular} & $\begin{array}{l}\text { Household } \\
\text { Characteristics: } \\
\text { yes }\end{array}$ & NA & NA & NA & NA & 3 months & \begin{tabular}{|l|} 
ADAS-Cog \\
15-D HRQoL
\end{tabular} & NA \\
\hline
\end{tabular}




\begin{tabular}{|c|c|c|c|c|c|c|c|c|c|c|c|c|c|}
\hline $\begin{array}{l}\text { Amieva 2016 } \\
(26772551) \\
\text { France } \\
\text { Medium } \\
\text { Explanatory }\end{array}$ & $\begin{array}{l}\text { Group cognitive } \\
\text { training program } \\
\text { focused on ADL } \\
\text { and tasks } \\
\text { involving } \\
\text { memory, } \\
\text { attention, } \\
\text { language, or } \\
\text { executive } \\
\text { function; 90- } \\
\text { minute weekly } \\
\text { sessions for } 3 \\
\text { months and } \\
\text { monthly } \\
\text { maintenance } \\
\text { sessions for } 21 \\
\text { months }\end{array}$ & Usual care & \begin{tabular}{|l|} 
Memory \\
centers or \\
geriatric day \\
care units \\
RCT; multisite \\
324 PLWD
\end{tabular} & $\begin{array}{l}\text { Mild to moderate } \\
\text { AD diagnosis } \\
\text { based on NINCDS- } \\
\text { ADRDA criteria, } \\
\text { MMSE from } 16 \text { to } \\
26, \text { and GDS score } \\
\text { of } 2 \text { to } 5\end{array}$ & $\begin{array}{l}\mathrm{N}=324 \\
79 \text { years } \\
58 \% \text { Female } \\
\text { Race NR } \\
52 \% \text { Secondary } \\
\text { education and } \\
\text { above }\end{array}$ & $\mathrm{NR}$ & $\begin{array}{l}\mathrm{N}=324 \\
\text { Age: NR } \\
\text { Sex: NR } \\
\text { Race: NR } \\
\text { Education: NR } \\
\text { Relation to PLWD: } \\
\text { NR }\end{array}$ & $\mathrm{NR}$ & NA & $\mathrm{NA}$ & 3 months & $\begin{array}{l}\text { ADAS-Cog } \\
\text { NPI } \\
\text { DAD } \\
\text { Apathy } \\
\text { Inventory } \\
\text { MADRS } \\
\text { QOL-AD } \\
\text { AGGIR } \\
\text { RUD-Lite }\end{array}$ & ZBI \\
\hline $\begin{array}{l}\text { Cavallo 2016 } \\
(27600448) \\
\text { Italy } \\
\text { Medium } \\
\text { Explanatory }\end{array}$ & $\begin{array}{l}\text { Structured } \\
\text { rehabilitative } \\
\text { cognitive } \\
\text { training } \\
\text { software aimed } \\
\text { at memory, } \\
\text { attention, } \\
\text { executive } \\
\text { function, and } \\
\text { language; three } \\
30 \text { minutes } \\
\text { sessions /week } \\
\text { for } 12 \text { weeks }\end{array}$ & $\begin{array}{l}\text { Using } \\
\text { computer to } \\
\text { read } \\
\text { newspaper or } \\
\text { play games; } \\
\text { three } 30 \\
\text { minutes } \\
\text { sessions } \\
\text { /week for } 12 \\
\text { weeks }\end{array}$ & $\begin{array}{l}\text { Assisted health } \\
\text { residence } \\
\text { RCT; single } \\
\text { site } \\
80 \text { PLWD }\end{array}$ & $\begin{array}{l}\text { Early-stage AD; } \\
\text { NINCDS-ADRDA } \\
\text { criteria- }\end{array}$ & $\begin{array}{l}\mathrm{N}=80 \\
76 \text { years } \\
64 \% \text { Female } \\
\text { Race NR } \\
8 \text { years }\end{array}$ & $\mathrm{NR}$ & $\mathrm{NA}$ & NA & NA & $\mathrm{NA}$ & $\begin{array}{l}3 \text { months } \\
6 \text { months }\end{array}$ & $\begin{array}{l}\text { HADS- } \\
\text { Depression } \\
\text { HADS- } \\
\text { Anxiety } \\
\text { RMBT }\end{array}$ & NA \\
\hline $\begin{array}{l}\text { Hsu 2016 } \\
\text { (27878873) } \\
\text { Taiwan } \\
\text { Medium } \\
\text { Explanatory }\end{array}$ & $\begin{array}{l}\text { Individual } \\
\text { spatial retrieval } \\
\text { training ; } 5 \\
\text { times/week, } 40 \\
\text { min/session, for } \\
6 \text { weeks }\end{array}$ & Usual care & $\begin{array}{l}\text { Nursing } \\
\text { centers and } \\
\text { dementia units } \\
\text { of Veterans } \\
\text { Homes } \\
\text { RCT; multisite } \\
\text { 97 PLWD }\end{array}$ & $\begin{array}{l}\text { Dementia with } \\
\text { hyperphagia } \\
\text { behaviors(all } \\
\text { severities, vascular } \\
\text { and brain injury } \\
\text { excluded) with at } \\
\text { least } 3 \text { points on } \\
\text { the }\end{array}$ & $\begin{array}{l}\mathrm{N}=97 \\
82 \text { years } \\
28 \% \text { Female } \\
\text { Race NR } \\
41 \% \text { Secondary or } \\
\text { higher }\end{array}$ & $\mathrm{NR}$ & NA & NA & NA & NA & 6 weeks & $\begin{array}{l}\text { DHBS } \\
\text { Food Intake }\end{array}$ & NA \\
\hline $\begin{array}{l}\text { Kao 2016 } \\
(27307717) \\
\text { Taiwan } \\
\text { Medium } \\
\text { Explanatory }\end{array}$ & $\begin{array}{l}\text { Spatial retrieval } \\
\text { training with } \\
\text { Montessori } \\
\text { activities during } \\
\text { recall; } 5 \\
\text { times/week, } 40 \\
\text { mins/session, for } \\
6 \text { weeks }\end{array}$ & Usual care & \begin{tabular}{|l} 
Dementia \\
special care \\
units \\
RCT; multisite \\
100 PLWD
\end{tabular} & $\begin{array}{l}\text { Dementia with } \\
\text { hyperphagia } \\
\text { behaviors(all } \\
\text { severities, vascular } \\
\text { and brain injury } \\
\text { excluded) with at } \\
\text { least } 3 \text { points on } \\
\text { hyperphagia scale }\end{array}$ & $\begin{array}{l}\mathrm{N}=100 \\
82 \text { years } \\
28 \% \text { Female } \\
\text { Race NR } \\
44 \% \text { High school or } \\
\text { higher }\end{array}$ & $\mathrm{NR}$ & $\mathrm{NA}$ & NA & $\begin{array}{l}\mathrm{N}=\mathrm{NR} \\
\text { Age NR } \\
\text { Sex NR } \\
\text { Race NR } \\
\text { Education NR }\end{array}$ & NR & $\begin{array}{l}6 \text { weeks } \\
10 \text { weeks } \\
18 \text { weeks } \\
30 \text { weeks }\end{array}$ & $\begin{array}{l}\text { Hyperphagic } \\
\text { behavior } \\
\text { Pica } \\
\text { Behavior } \\
\text { Change in } \\
\text { Eating Habit } \\
\text { Short Meal } \\
\text { Frequency }\end{array}$ & $\begin{array}{l}\text { Caregiver } \\
\text { Distress to } \\
\text { Hyperphagic } \\
\text { Behavior }\end{array}$ \\
\hline
\end{tabular}

*High risk of bias studies included in evidence map. This table includes low and medium risk of bias studies only.

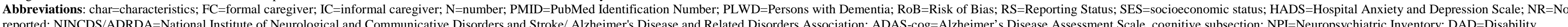

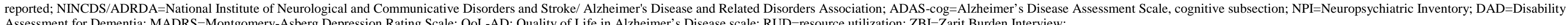

\begin{tabular}{|c|c|c|c|c|c|}
\hline $\begin{array}{l}\text { Study (PMID) } \\
\text { Comparison } \\
\text { RoB } \\
\text { Category* }\end{array}$ & $\begin{array}{l}\text { Outcome } \\
\text { Timing }\end{array}$ & Summary Finding & Intervention & Comparator & p-Value \\
\hline $\begin{array}{l}\text { Kallio } 2018^{160}(29345724) \\
\text { Cognitive Training vs. Usual Care } \\
\text { Medium }\end{array}$ & $\begin{array}{l}\text { 15-D HRQoL } \\
\text { Mean Change from Baseline }(95 \% \mathrm{Cl}) \\
3 \text { months }\end{array}$ & $\mathrm{NR}$ & $-0.04(-0.058,-0.021)$ & $-0.04(-0.056,-0.018)$ & $p=0.82$ \\
\hline
\end{tabular}




\begin{tabular}{|c|c|c|c|c|c|}
\hline $\begin{array}{l}\text { Amieva } 2016^{107}(26572551) \\
\text { Cognitive Training vs. Usual Care } \\
\text { Medium } \\
\text { Explanatory }\end{array}$ & $\begin{array}{l}\text { NPI } \\
\text { Mean (SD) } \\
3 \text { months }\end{array}$ & NR & $25.34(28.8)$ & $23.29(28.4)$ & $p=0.22$ \\
\hline $\begin{array}{l}\text { Amieva 2016107 }(26572551) \\
\text { Cognitive Training vs. Usual Care } \\
\text { Medium } \\
\text { Explanatory }\end{array}$ & $\begin{array}{l}\text { DAD } \\
\text { Mean (SD) } \\
3 \text { months }\end{array}$ & NR & $27.54(9.2)$ & $26.94(9.6)$ & $p=0.62$ \\
\hline $\begin{array}{l}\text { Amieva 2016107 }(26572551) \\
\text { Cognitive Training vs. Usual Care } \\
\text { Medium } \\
\text { Explanatory }\end{array}$ & $\begin{array}{l}\text { Apathy Inventory } \\
\text { Mean (SD) } \\
3 \text { months }\end{array}$ & NR & $10.26(11.7)$ & $10.4(11.8)$ & $p=0.97$ \\
\hline $\begin{array}{l}\text { Amieva 2016107 }(26572551) \\
\text { Cognitive Training vs. Usual Care } \\
\text { Medium } \\
\text { Explanatory }\end{array}$ & $\begin{array}{l}\text { AGGIR } \\
\text { Mean (SD) } \\
3 \text { months }\end{array}$ & NR & $7.0(7.9)$ & $6.63(7.5)$ & $p=0.67$ \\
\hline $\begin{array}{l}\text { Amieva 2016107 }(26572551) \\
\text { Cognitive Training vs. Usual Care } \\
\text { Medium } \\
\text { Explanatory }\end{array}$ & $\begin{array}{l}\text { MADRS } \\
\text { Mean (SD) } \\
3 \text { months }\end{array}$ & NR & $10.65(9.9)$ & $8.82(9.1)$ & $p=0.06$ \\
\hline $\begin{array}{l}\text { Amieva 2016107 }(26572551) \\
\text { Cognitive Training vs. Usual Care } \\
\text { Medium } \\
\text { Explanatory }\end{array}$ & $\begin{array}{l}\text { QOL-AD } \\
\text { Mean (SD) } \\
3 \text { months }\end{array}$ & NR & $31.99(8.0)$ & $33.28(7.7)$ & $p=0.22$ \\
\hline $\begin{array}{l}\text { Amieva 2016 } 107 \text { (26572551) } \\
\text { Cognitive Training vs. Usual Care } \\
\text { Medium } \\
\text { Explanatory }\end{array}$ & $\begin{array}{l}\text { RUD-Lite } \\
\text { Mean (SD) } \\
3 \text { months }\end{array}$ & NR & $2559.3(3078.3)$ & $2199.8(3002.2)$ & $p=0.50$ \\
\hline $\begin{array}{l}\text { Cavallo } 2016^{161}(27600448) \\
\text { Cognitive Training vs. Attention Control } \\
\text { Medium } \\
\text { Explanatory }\end{array}$ & $\begin{array}{l}\text { HADS-Anxiety } \\
\text { Mean (SD) } \\
3 \text { months, } 6 \text { months }\end{array}$ & No difference between groups at either time point & $\begin{array}{l}3 \text { months: } 7.65(2.41) \\
6 \text { months: NR }\end{array}$ & $\begin{array}{l}3 \text { months: } 7.57(1.33) \\
6 \text { months: NR }\end{array}$ & NR \\
\hline $\begin{array}{l}\text { Cavallo } 2016^{161}(27600448) \\
\text { Cognitive Training vs. Attention Control } \\
\text { Medium } \\
\text { Explanatory }\end{array}$ & $\begin{array}{l}\text { HADS-Depression } \\
\text { Mean (SD) } \\
3 \text { months, } 6 \text { months }\end{array}$ & No difference between groups at either time point & $\begin{array}{l}3 \text { months: } 6.42(2.21) \\
6 \text { months: NR }\end{array}$ & $\begin{array}{l}3 \text { months: } 6.35 \text { (2.21) } \\
6 \text { months: NR }\end{array}$ & NR \\
\hline $\begin{array}{l}\text { Hsu 2016 } 26^{163}(27878873) \\
\text { Cognitive Training vs. Usual Care } \\
\text { Medium } \\
\text { Explanatory }\end{array}$ & $\begin{array}{l}\text { DHBS } \\
\text { Mean Change from Baseline (SD) } \\
6 \text { weeks }\end{array}$ & NR & $-0.88(2.48)$ & $1.04(1.98)$ & $p<0.001$ \\
\hline $\begin{array}{l}\text { HSu } 2016^{163} \text { (27878873) } \\
\text { Cognitive Training vs. Usual Care } \\
\text { Medium } \\
\text { Explanatory }\end{array}$ & $\begin{array}{l}\text { Average Food Intake (g) } \\
\text { Mean Change from Baseline (SD) } \\
6 \text { weeks }\end{array}$ & NR & $-38.63(145.51)$ & $18.28(127.34)$ & $\mathrm{p}=0.046$ \\
\hline $\begin{array}{l}\text { Kao } 2016^{164}(27307717) \\
\text { Cognitive Training vs. Usual Care } \\
\text { Medium } \\
\text { Explanatory }\end{array}$ & $\begin{array}{l}\text { Hyperphagic Behavior } \\
\text { Group x Time p-value } \\
6 \text { weeks, } 10 \text { weeks, } 18 \text { weeks, } 30 \text { weeks }\end{array}$ & NR & NR & NR & $\begin{array}{l}\text { All time points } \\
p<0.05\end{array}$ \\
\hline $\begin{array}{l}\text { Kao 2016164 }(27307717) \\
\text { Cognitive Training vs. Usual Care } \\
\text { Medium } \\
\text { Explanatory }\end{array}$ & $\begin{array}{l}\text { Pica Behavior } \\
\text { Group X Time p-value } \\
6 \text { weeks, } 10 \text { weeks, } 18 \text { weeks, } 30 \text { weeks }\end{array}$ & NR & NR & NR & $\begin{array}{l}6 \text { weeks, } 10 \\
\text { weeks, } 18 \text { weeks: } \\
p<0.05 \\
30 \text { weeks: } p=0.092\end{array}$ \\
\hline $\begin{array}{l}\text { Kao } 2016^{164}(27307717) \\
\text { Cognitive Training vs. Usual Care } \\
\text { Medium } \\
\text { Explanatory }\end{array}$ & $\begin{array}{l}\text { Change in Eating Habit } \\
\text { Group X Time p-value } \\
6 \text { weeks, } 10 \text { weeks, } 18 \text { weeks, } 30 \text { weeks }\end{array}$ & NR & NR & NR & $\begin{array}{l}6 \text { weeks, } 10 \\
\text { weeks, } 18 \text { weeks: } \\
\text { p>0.05 } \\
30 \text { weeks: } p=0.037\end{array}$ \\
\hline $\begin{array}{l}\text { Kao } 2016^{164}(27307717) \\
\text { Cognitive Training vs. Usual Care } \\
\text { Medium } \\
\text { Explanatory }\end{array}$ & $\begin{array}{l}\text { Short Meal Frequency } \\
\text { Group X Time } p \text {-value } \\
6 \text { weeks, } 10 \text { weeks, } 18 \text { weeks, } 30 \text { weeks }\end{array}$ & NR & NR & NR & $\begin{array}{l}\text { All time points } \\
p<0.05\end{array}$ \\
\hline
\end{tabular}


Table D-26. Caregiver outcomes summary low and medium risk of bias studies: cognitive training

\begin{tabular}{|c|c|c|c|c|c|}
\hline $\begin{array}{l}\text { Study (PMID) } \\
\text { Comparison } \\
\text { Caregiver Type } \\
\text { RoB } \\
\text { Category* }\end{array}$ & $\begin{array}{l}\text { Outcome } \\
\text { Timing }\end{array}$ & Summary Finding & Intervention & Comparator & $p$-Value \\
\hline $\begin{array}{l}\text { Amieva 2016107 (26572551) } \\
\text { Cognitive Training vs. Usual Care } \\
\text { Medium } \\
\text { Explanatory }\end{array}$ & $\begin{array}{l}\text { ZBI } \\
\text { Mean (SD) } \\
3 \text { months }\end{array}$ & $\mathrm{NR}$ & $30.31(25.9)$ & $30.05(25.6)$ & 0.88 \\
\hline $\begin{array}{l}\text { Kao } 2016^{164}(27307717) \\
\text { Cognitive Training vs. Usual Care } \\
\text { Medium } \\
\text { Explanatory }\end{array}$ & $\begin{array}{l}\text { Caregiver Distress to Hyperphagic Behavior } \\
\text { Group X Time p-value } \\
6 \text { weeks, } 10 \text { weeks, } 18 \text { weeks, } 30 \text { weeks }\end{array}$ & $\mathrm{NR}$ & $\mathrm{NR}$ & $\mathrm{NR}$ & $\begin{array}{l}6 \text { weeks: } p=0.004 \\
10 \text { weeks, } 18 \\
\text { weeks, } 30 \text { weeks: } \\
p>0.05\end{array}$ \\
\hline
\end{tabular}

Abbreviations: NA=Not Applicable; NR=Not Reported; PMID=PubMed Identification Number; ZBI=Zarit Burden Interview

Table D-27. Summary of strength of evidence for PLWD outcomes: cognitive training

\begin{tabular}{|c|c|c|c|c|c|c|c|c|}
\hline $\begin{array}{l}\text { Comparison } \\
\text { Outcome }\end{array}$ & Timing & $\begin{array}{l}\text { \# Studies/ Design } \\
\text { ( } \mathrm{n} \text { analyzed) }\end{array}$ & Finding or Summary Statistic & Study Limitations & Consistency & Directness & Precision & Overall Grade/ Conclusion \\
\hline $\begin{array}{l}\text { Cognitive Training vs. Usual Care } \\
\text { Quality of Life }\end{array}$ & 3 months & 2 RCTS (n=471) & No benefit. & Moderate & Consistent & Direct & Imprecise & Insufficient \\
\hline \begin{tabular}{|l|} 
Cognitive Training vs. Usual Care or Attention \\
Control \\
Depression
\end{tabular} & 3 to 6 months & 2 RCTs $(n=404)$ & No benefit. & Moderate & Consistent & Direct & Imprecise & Insufficient \\
\hline $\begin{array}{l}\text { Cognitive Training vs. Usual Care } \\
\text { Hyperphagia Behavior }\end{array}$ & 6 to 30 weeks & 2 RCTs $(n=197)$ & Benefit favoring intervention. & Moderate & Consistent & Direct & Imprecise & Insufficient \\
\hline $\begin{array}{l}\text { Cognitive Training vs. Usual Care } \\
\text { Food Intake }\end{array}$ & 6 weeks & 1 RCT $(n=97)$ & Benefit favoring intervention. & Moderate & Unknown & Direct & Imprecise & Insufficient \\
\hline $\begin{array}{l}\text { Cognitive Training vs. Usual Care } \\
\text { Short meal frequency }\end{array}$ & 6 to 30 weeks & $1 \mathrm{RCT}(\mathrm{n}=100)$ & Benefit favoring intervention at all time-points. & Moderate & Unknown & Direct & Imprecise & Insufficient \\
\hline \begin{tabular}{|l} 
Cognitive Training vs. Usual Care \\
Pica behavior
\end{tabular} & 6 to 30 weeks & $1 \mathrm{RCT}(\mathrm{n}=100)$ & $\begin{array}{l}\text { Benefit favoring intervention post- } \\
\text { intervention, but not sustained } 6 \text {-months post- } \\
\text { intervention. }\end{array}$ & Moderate & Unknown & Direct & Imprecise & Insufficient \\
\hline $\begin{array}{l}\text { Cognitive Training vs. Usual Care } \\
\text { Change in eating habits }\end{array}$ & 6 to 30 weeks & $1 \mathrm{RCT}(\mathrm{n}=100)$ & No benefit. & Moderate & Unknown & Direct & Imprecise & Insufficient \\
\hline $\begin{array}{l}\text { Cognitive Training vs. Usual Care } \\
\text { BPSD }\end{array}$ & 3 months & $1 \mathrm{RCT}(\mathrm{n}=324)$ & No benefit. & Moderate & Unknown & Direct & Imprecise & Insufficient \\
\hline \begin{tabular}{|l} 
BPSD \\
Cognitive Training vs. Usual Care \\
Function/ADLs
\end{tabular} & 3 months & $1 \mathrm{RCT}(\mathrm{n}=324)$ & No benefit. & Moderate & Unknown & \begin{tabular}{|l|} 
Direct \\
\end{tabular} & Imprecise & Insufficient \\
\hline $\begin{array}{l}\text { Cognitive Training vs. Usual Care } \\
\text { Apathy }\end{array}$ & 3 months & $1 \mathrm{RCT}(\mathrm{n}=324)$ & No benefit. & Moderate & Unknown & Direct & Imprecise & Insufficient \\
\hline $\begin{array}{l}\text { Cognitive Training vs. Usual Care } \\
\text { Dependence }\end{array}$ & 3 months & $1 \mathrm{RCT}(\mathrm{n}=324)$ & No benefit. & Moderate & Unknown & \begin{tabular}{|l|} 
Direct \\
\end{tabular} & Imprecise & Insufficient \\
\hline $\begin{array}{l}\text { Cognitive Training vs. Attention Control } \\
\text { Anxiety }\end{array}$ & 3 and 6 months & 1 RCT $(\mathrm{n}=80)$ & No benefit. & Moderate & Unknown & \begin{tabular}{|l|} 
Direct \\
\end{tabular} & Imprecise & Insufficient \\
\hline
\end{tabular}

Anxiety 
Table D-28. Summary of strength of evidence for caregiver outcomes: cognitive training

\begin{tabular}{|c|c|c|c|c|c|c|c|c|}
\hline $\begin{array}{l}\text { Comparison } \\
\text { Caregiver Type } \\
\text { Outcome }\end{array}$ & Timing & $\begin{array}{l}\text { \# Studies/ Design } \\
\text { (n analyzed) }\end{array}$ & Finding or Summary Statistic & Study Limitations & Consistency & Directness & Precision & $\begin{array}{l}\text { Overall Gradel } \\
\text { Conclusion }\end{array}$ \\
\hline $\begin{array}{l}\text { Cognitive Training vs. Usual Care } \\
\text { Caregiver Distress } \\
\text { Informal caregivers }\end{array}$ & 3 months & $1 \mathrm{RCT}(\mathrm{n}=324)$ & No benefit. & Moderate & Unknown & Direct & Imprecise & Insufficient \\
\hline $\begin{array}{l}\text { Cognitive Training vs. Usual Care } \\
\text { Caregiver Distress, PLWD hyperphagic behavior } \\
\text { Formal caregivers }\end{array}$ & 6 to 30 weeks & $1 \mathrm{RCT}(\mathrm{n}=\mathrm{NR})$ & No benefit. & Moderate & Unknown & Direct & Imprecise & Insufficient \\
\hline
\end{tabular}

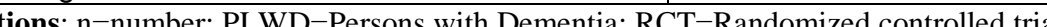

\section{Evidence Map: Cognitive Training}

\begin{tabular}{|c|c|c|c|c|c|c|c|c|c|c|c|c|c|}
\hline $\begin{array}{l}\text { Study (PMID) } \\
\text { Country } \\
\text { EM Reason }\end{array}$ & Intervention & Comparison & $\begin{array}{l}\text { Setting } \\
\text { and Design } \\
\text { Setting } \\
\text { Design } \\
\text { Cluster N } \\
\text { Participants } \\
\text { Random- } \\
\text { ized N }\end{array}$ & $\begin{array}{l}\text { PLWD } \\
\text { Dementia } \\
\text { Characteristics }\end{array}$ & $\begin{array}{l}\text { PLWD } \\
\text { Non-Disease Char } \\
\text { PLWD N } \\
\text { PLWD Age (mean) } \\
\text { PLWD Sex (\% } \\
\text { female) } \\
\text { PLWD Race (\% } \\
\text { majority) } \\
\text { PLWD Education } \\
\text { (mean years) }\end{array}$ & $\begin{array}{l}\text { PLWD } \\
\text { Non-Disease } \\
\text { Char Reporting } \\
\text { Status (RS) } \\
\\
\text { PLWD SES } \\
\text { PLWD Prior } \\
\text { Disability } \\
\text { PLWD } \\
\text { Household } \\
\text { Characteristics } \\
\text { PLWD Health } \\
\text { Insurance } \\
\text { PLWD Detailed } \\
\text { Race Information }\end{array}$ & \begin{tabular}{|l|} 
Informal Caregiver \\
(IC) \\
Characteristics \\
IC N \\
IC Age (mean) \\
IC Sex (\% female) \\
IC Race (\% \\
majority) \\
IC Education \\
(mean years) \\
IC Relation to \\
PLWD (\% majority)
\end{tabular} & $\begin{array}{l}\text { Informal Caregiver } \\
\text { (IC) Char. RS } \\
\text { IC Duration } \\
\text { IC Living With } \\
\text { PLWD } \\
\text { IC Payment } \\
\text { IC Health Status } \\
\text { IC Dementia } \\
\text { Family History } \\
\text { IC Employment } \\
\text { Status } \\
\text { IC Training }\end{array}$ & 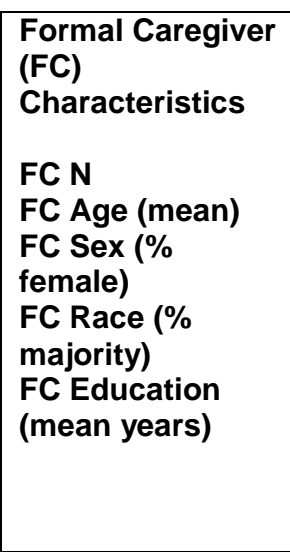 & $\begin{array}{l}\text { Formal } \\
\text { Caregiver (FC) } \\
\text { Char. RS } \\
\text { FC Health } \\
\text { Status } \\
\text { FC Training } \\
\text { FC Education } \\
\text { FC Position } \\
\text { FC Length of } \\
\text { Service }\end{array}$ & $\begin{array}{l}\text { Outcome } \\
\text { Timing(s) }\end{array}$ & \begin{tabular}{|l|} 
PLWD \\
Outcomes
\end{tabular} & $\begin{array}{l}\text { Caregiver } \\
\text { Outcomes } \\
\text { IC: } \\
\text { FC: }\end{array}$ \\
\hline $\begin{array}{l}\text { Tseng 2019159 } \\
\text { (30970666) } \\
\text { Taiwan } \\
\text { High RoB }\end{array}$ & \begin{tabular}{|l} 
Smart Restored \\
Learning \\
exercise
\end{tabular} & No intervention & $\begin{array}{l}\text { Veterans } \\
\text { home } \\
\text { Quasi- } \\
\text { experimental } \\
\text { n=68 }\end{array}$ & $\begin{array}{l}\text { PLWD diagnosed } \\
\text { with dementia, } \\
\text { MMSE <25 and } \\
\text { living in Veterans } \\
\text { Home }\end{array}$ & $\begin{array}{l}\mathrm{N}=68 \text { (35 treatment, } \\
33 \text { control) } \\
\text { Age: } \mathrm{Y} \\
\text { Sex: } \mathrm{N} \\
\text { Race \% majority: } \mathrm{N} \\
\text { Education: } \mathrm{Y}\end{array}$ & NR & NA & NA & NR & NR & 6 months & \begin{tabular}{|l} 
MMSE \\
Neuropsychi \\
atric \\
Inventory \\
Frontal \\
Assessment \\
Battery
\end{tabular} & NA \\
\hline $\begin{array}{l}\text { Giovagnoli } \\
2017^{166} \\
(28577267) \\
\text { Italy } \\
\text { Sample Size }\end{array}$ & \begin{tabular}{|l|} 
Cognitive \\
training
\end{tabular} & $\begin{array}{l}\text { 1. Music } \\
\text { therapy or } \\
\text { 2. neuro- } \\
\text { education }\end{array}$ & $\begin{array}{l}\text { One care } \\
\text { center } \\
\text { RCT single } \\
\text { blind }\end{array}$ & $\begin{array}{l}\text { PLWD with mild- } \\
\text { moderate dementia } \\
\text { or probable AD. } \\
\text { Screened for } \\
\text { additional criteria }\end{array}$ & $\begin{array}{l}\mathrm{N}=50 \text { (17 cognitive } \\
\text { training, } 17 \text { music, } \\
13 \text { neuro-education } \\
\text { Age: } \mathrm{Y} \\
\text { Sex: } \mathrm{Y} \\
\text { Race: } \mathrm{N} \\
\text { Education: } \mathrm{Y}\end{array}$ & $\mathrm{NR}$ & NR & $\mathrm{NR}$ & NR & NR & 3 months & $\begin{array}{l}\text { Word fluency } \\
\text { test } \\
\text { Neuropsych } \\
\text { ological and } \\
\text { behavioral } \\
\text { assessments }\end{array}$ & NA \\
\hline
\end{tabular}




\begin{tabular}{|c|c|c|c|c|c|c|c|c|c|c|c|c|c|}
\hline $\begin{array}{l}\text { Venturelli } \\
2016^{52} \\
(27540967) \\
\text { Italy } \\
\text { Small sample }\end{array}$ & $\begin{array}{l}\text { Cognitive } \\
\text { training based } \\
\text { on reality } \\
\text { orientation } \\
\text { method; 5 } \\
\text { days/week for } 1 \\
\text { hour before } \\
\text { sunset for 3 } \\
\text { months } \\
\end{array}$ & $\begin{array}{l}\text { Walking at } \\
\text { moderate } \\
\text { intensity; } 5 \\
\text { days/week for } 1 \\
\text { hour before } \\
\text { sunset for } 3 \\
\text { months }\end{array}$ & $\begin{array}{l}\text { AD care } \\
\text { units } \\
\text { RCT } \\
40 \text { PLWD }\end{array}$ & \begin{tabular}{|l|} 
AD with MMSE \\
between 10 and 15 \\
and \\
neurobehavioral \\
symptoms of SDS
\end{tabular} & $\begin{array}{l}\mathrm{N}=40 \\
\text { Age: yes } \\
\text { Sex: yes } \\
\text { Race: } n \text { o } \\
\text { Education: no }\end{array}$ & NR & NA & NA & NA & NA & 3 months & $\begin{array}{l}\text { NPI } \\
\text { ABS }\end{array}$ & NA \\
\hline $\begin{array}{l}\text { Chen, 2016167 } \\
\text { (27846102) } \\
\text { Taiwan } \\
\text { Small sample }\end{array}$ & $\begin{array}{l}\text { Individualized } \\
\text { learning therapy } \\
30 \text { minutes } \\
\text { twice weekly for } \\
3 \text { months } \\
\end{array}$ & Usual care & \begin{tabular}{|l} 
Nursing \\
home \\
Quasi- \\
experimental \\
, 2 homes \\
\end{tabular} & \begin{tabular}{|l|} 
Dementia \\
diagnosis, MMSE \\
between 10 and 23
\end{tabular} & $\begin{array}{l}\mathrm{N}=44 \\
\text { Age: yes } \\
\text { Sex: yes } \\
\text { Race: no } \\
\text { Education: no }\end{array}$ & \begin{tabular}{|l|} 
Marital status \\
\end{tabular} & NA & NA & NA & NA & 12 weeks & $\begin{array}{l}\text { Chinese NPI } \\
\text { MMSE }\end{array}$ & NA \\
\hline $\begin{array}{l}\text { De Luca } 2016^{168} \\
\text { Italy } \\
\text { Small sample }\end{array}$ & $\begin{array}{l}\text { Combined } \\
\text { standard } \\
\text { cognitive } \\
\text { training and } \\
\text { web-based } \\
\text { rehabilitation } \\
24 \text { sessions } \times 8 \\
\text { weeks }\end{array}$ & $\begin{array}{l}\text { Standard } \\
\text { treatment }\end{array}$ & $\begin{array}{l}\text { Nursing } \\
\text { home } \\
\text { RCT }\end{array}$ & $\begin{array}{l}\text { Diagnosis of mild to } \\
\text { moderate cognitive } \\
\text { decline MMSE } 25 \\
+ \text { +- } 3.4 \text { ); ability to } \\
\text { perform training }\end{array}$ & $\begin{array}{l}\mathrm{N}=20(10 \\
\text { experimental } \\
10 \text { control) } \\
\text { Age: } Y \\
\text { Sex: } Y \\
\text { Race: } Y \\
\text { Education: } Y\end{array}$ & NR & NA & NA & NR & NR & 8 weeks & $\begin{array}{l}\text { MMSE } \\
\text { Attention } \\
\text { Verbal } \\
\text { fluency } \\
\text { ADL ADL } \\
\text { Behavioral, } \\
\text { Depression }\end{array}$ & NA \\
\hline $\begin{array}{l}\text { Giuli 2016 } \\
(26952713) \\
\text { Italy } \\
\text { High RoB }\end{array}$ & $\begin{array}{l}\text { Comprehensive } \\
\text { cognitive } \\
\text { training }\end{array}$ & No intervention & \begin{tabular}{|l|} 
Community \\
based \\
RCT
\end{tabular} & $\begin{array}{l}\text { Three levels of } \\
\text { cognitive } \\
\text { impairment: healthy } \\
\text { elderly, moderate } \\
\text { cognitive } \\
\text { impairment, mild to } \\
\text { moderate AD; } \\
\text { Aged } 65 \text { or older }\end{array}$ & $\begin{array}{l}\mathrm{N}=321 \text { (47 healthy } \\
\text { experimental, } 53 \\
\text { healthy control, } 48 \\
\text { moderate decline } \\
\text { experimental, } \\
49 \text { moderate decline } \\
\text { control, } \\
48 \mathrm{AD} \text { experimental, } \\
47 \mathrm{AD} \text { control) } \\
\text { Age: } \mathrm{Y} \\
\mathrm{Sex}: \mathrm{Y} \\
\text { Race } \% \text { majority: } \mathrm{N} \\
\text { Education: } \mathrm{Y}\end{array}$ & NR & $\mathrm{NA}$ & NA & $\mathrm{NR}$ & NR & 10 weeks & $\begin{array}{l}\text { MMSE, } \\
\text { Orientation, } \\
\text { Memory, } \\
\text { Attention, } \\
\text { Verbal } \\
\text { fluency, } \\
\text { Depression, } \\
\text { ADL, IADL, } \\
\text { confidence } \\
\text { in own } \\
\text { cognition }\end{array}$ & NA \\
\hline $\begin{array}{l}\text { Kawashima } \\
2015^{169} \\
\text { US } \\
\text { Sample Size }\end{array}$ & $\begin{array}{l}\text { Americanized } \\
\text { SAIDO (working } \\
\text { memory training } \\
\text { program of } \\
\text { basic arithmetic } \\
\text { \& language) }\end{array}$ & Usual care & $\begin{array}{l}\text { Two nursing } \\
\text { homes. } \\
\text { Prospective } \\
\text { non- } \\
\text { randomized, } \\
\text { by NH } \\
\mathrm{n}=47(39 \\
\text { reported) }\end{array}$ & $\begin{array}{l}\text { PLWD diagnosed } \\
\text { with dementia } \\
\text { (DSM-IV) }\end{array}$ & $\begin{array}{l}\mathrm{N}=\mathrm{n}=47(23 \\
\text { intervention, } 24 \\
\text { controls) } \\
\text { Age: } Y \\
\text { Sex: } Y \\
\text { Race \% majority: N } \\
\text { Education: N }\end{array}$ & NR & NA & NA & NR & NR & 6 months & $\begin{array}{l}\text { Cognitive } \\
\text { scores, } \\
\text { mood mental } \\
\text { status }\end{array}$ & NA \\
\hline $\begin{array}{l}\text { Bergamaschi } \\
2013^{170} \\
(23784727) \\
\text { Italy } \\
\text { Sample Size }\end{array}$ & $\begin{array}{l}\text { Repeated } \\
\text { cycles of } \\
\text { cognitive } \\
\text { training } \times 1 \text { year }\end{array}$ & $\begin{array}{l}\text { Non-specific } \\
\text { cognitive } \\
\text { exercises }\end{array}$ & $\begin{array}{l}\text { Day center } \\
\text { RCT }\end{array}$ & $\begin{array}{l}\text { PLWD diagnosed } \\
\text { with mild to } \\
\text { moderate AD; } \\
\text { MMSE 18-24 }\end{array}$ & $\begin{array}{l}\mathrm{N}=32 \text { (16 each } \\
\text { group) } \\
\text { Age: } \mathrm{Y} \\
\text { Sex: } \mathrm{N} \\
\text { Race \% majority: } \mathrm{N} \\
\text { Education: } \mathrm{Y}\end{array}$ & NR & NA & NA & NR & NR & 1 year & $\begin{array}{l}\text { MMSE } \\
\text { Memory and } \\
\text { recall } \\
\text { Verbal } \\
\text { fluency } \\
\text { Depression } \\
\text { ADL }\end{array}$ & NA \\
\hline $\begin{array}{l}\text { Mapelli 2013 } \\
\text { (24052800) } \\
\text { Italy } \\
\text { Sample Size }\end{array}$ & $\begin{array}{l}\text { Cognitive } \\
\text { stimulation } \\
\text { focused on } \\
\text { memory, } \\
\text { language, } \\
\text { spatial and } \\
\text { temporal } \\
\text { orientation, } \\
\text { attention and } \\
\text { logic }\end{array}$ & $\begin{array}{l}\text { Occupational } \\
\text { therapy s } \\
\text { placebo or no } \\
\text { intervention }\end{array}$ & $\begin{array}{l}\text { Nursing } \\
\text { home } \\
\text { RCT } \\
\text { PLWD with } \\
\text { AD, vascular } \\
\text { and mixed } \\
\text { dementia }\end{array}$ & $\begin{array}{l}\text { PLWD diagnosed } \\
\text { with AD, vascular } \\
\text { and mixed } \\
\text { dementia } \\
\text { AD MMSE 19-24; } \\
\text { vascular MMSE14- } \\
18\end{array}$ & $\begin{array}{l}\mathrm{N}=30 \text { (10 each } \\
\text { group) } \\
\text { Age: } \mathrm{Y} \\
\text { Sex: } \mathrm{N} \\
\text { Race \% majority: } \mathrm{N} \\
\text { Education: } \mathrm{Y}\end{array}$ & NR & NA & NA & NR & NR & 8 weeks & $\begin{array}{l}\text { Cognition } \\
\text { Behavior }\end{array}$ & NA \\
\hline
\end{tabular}




\begin{tabular}{|c|c|c|c|c|c|c|c|c|c|c|c|c|c|}
\hline $\begin{array}{l}\text { Tarraga 2006 } 6^{172} \\
\text { (16820420) } \\
\text { Spain } \\
\text { Sample Size }\end{array}$ & $\begin{array}{l}\text { Cognitive } \\
\text { stimulation } \\
\text { using an } \\
\text { interactive multi- } \\
\text { media tool plus } \\
\text { psycho- } \\
\text { stimulation and } \\
\text { medication }\end{array}$ & $\begin{array}{l}\text { Integrated } \\
\text { psycho- } \\
\text { stimulation and } \\
\text { medication, or } \\
\text { medication only }\end{array}$ & \begin{tabular}{|l} 
Community- \\
based \\
RCT \\
PLWD \\
diagnosed \\
as having \\
suspected \\
AD
\end{tabular} & $\begin{array}{l}\text { PLWD diagnosed } \\
\text { with suspected AD } \\
>65 \text { years old; at } \\
\text { least } 3 \text { years } \\
\text { education. MMMSE } \\
\text { 18-24; GDS } 3-4 .\end{array}$ & $\begin{array}{l}\mathrm{N}=43(15 \\
\text { experimental, } \\
16 \text { treatment } \\
\text { control, } 12 \\
\text { medication only } \\
\text { control) } \\
\text { Age: } \mathrm{Y} \\
\text { Sex: } \mathrm{Y} \\
\text { Race \% majority: } \mathrm{N} \\
\text { Education: statistic } \\
\text { only }\end{array}$ & \begin{tabular}{|l|l|}
$N R$ \\
\end{tabular} & NA & NA & NR & NR & 24 weeks & $\begin{array}{l}\text { ADAS-Cog } \\
\text { Additional } \\
\text { tests of } \\
\text { cognition, } \\
\text { verbal } \\
\text { fluency, } \\
\text { recall, level } \\
\text { of disability }\end{array}$ & NA \\
\hline $\begin{array}{l}\text { McGilton } \\
2003^{173} \\
(12959805) \\
\text { Canada } \\
\text { Sample Size }\end{array}$ & $\begin{array}{l}\text { Training in way- } \\
\text { finding in a new } \\
\text { environment }\end{array}$ & No intervention & \begin{tabular}{|l|} 
University \\
geriatric \\
care center \\
RCT
\end{tabular} & \begin{tabular}{|l|} 
PLWD with \\
diagnosis of AD \\
and moderate to \\
severe cognitive \\
decline. Able to \\
ambulate and \\
understand English
\end{tabular} & $\begin{array}{l}\mathrm{N}=32 \text { (17 treatment, } \\
15 \text { control) } \\
\text { Age: } Y \\
\text { Sex: } Y \\
\text { Race \% information: } \\
N \\
\text { Education: } N\end{array}$ & \begin{tabular}{|l|l|}
$N R$ \\
\end{tabular} & NA & NA & NR & Training: $Y$ & $\begin{array}{l}4 \text { weeks } \\
\text { training } \\
\text { Re-evaluation } \\
\text { at } 1 \text { week and } \\
3 \text { months } \\
\text { post-test }\end{array}$ & $\begin{array}{l}\text { Find dining } \\
\text { room } \\
\text { Agitation } \\
\text { Spatial } \\
\text { orientation }\end{array}$ & NA \\
\hline $\begin{array}{l}\text { Davis 2001174 } \\
\text { (11236819) } \\
\text { US } \\
\text { Sample Size }\end{array}$ & $\begin{array}{l}\text { Training in face- } \\
\text { name } \\
\text { association, } \\
\text { spaced retrieval } \\
\text { and cognitive } \\
\text { stimulation } \\
\text { with ICGs }\end{array}$ & $\begin{array}{l}\text { Mock placebo } \\
\text { intervention }\end{array}$ & $\begin{array}{l}\text { AD research } \\
\text { center } \\
\text { RCT } \\
\text { Crossover } \\
\text { study }\end{array}$ & $\begin{array}{l}\text { Diagnosis of } \\
\text { probable AD }\end{array}$ & $\begin{array}{l}\mathrm{N}=37 \text { (19 } \\
\text { intervention, } \\
18 \text { control) } \\
\text { Age: } Y \\
\text { Sex: } Y \\
\text { Race \% majority: N } \\
\text { Education: } Y\end{array}$ & \begin{tabular}{|l|}
$N R$ \\
\end{tabular} & NR & NR & NR & NR & $\begin{array}{l}10 \text { weeks; } \\
\text { Crossover at } \\
5 \text { weeks }\end{array}$ & $\begin{array}{l}\text { MMSE } \\
\text { Verbal and } \\
\text { visual } \\
\text { memory } \\
\text { Attention/con } \\
\text { centration } \\
\text { Word } \\
\text { generation } \\
\text { Motor speed } \\
\text { Depression } \\
\text { Quality of life }\end{array}$ & NR \\
\hline $\begin{array}{l}\text { Zarit 1982175 } \\
\text { (13238424) } \\
\text { US } \\
\text { Sample Size }\end{array}$ & $\begin{array}{l}\text { 1. Group } \\
\text { didactic training } \\
\text { (with imagery) } \\
\text { 2. problem } \\
\text { solving } \\
\text { All groups with } \\
\text { ICG }\end{array}$ & Wait list control & $\begin{array}{l}\text { Community- } \\
\text { based } \\
\text { RCT } \\
n=35 \text { PLWD }\end{array}$ & \begin{tabular}{|l|} 
Community \\
dwelling adults \\
screened for \\
cognitive \\
impairment \\
(Introduction states \\
adults with
\end{tabular} & $\begin{array}{l}\mathrm{N}=35 \text { (14 didactic, } \\
11 \text { problem solving, } \\
10 \text { wait list } \\
\text { Age: } \mathrm{Y} \\
\text { Sex: } \mathrm{N} \\
\text { Race \% majority: N } \\
\text { Education: } \mathrm{Y}\end{array}$ & \begin{tabular}{|l|}
$N R$ \\
\end{tabular} & $\begin{array}{l}\text { Relation to PLWD: } \\
Y\end{array}$ & NR & NA & NA & 3.5 weeks & $\begin{array}{l}\text { Recall trials } \\
\text { Recognition } \\
\text { trials }\end{array}$ & $\begin{array}{l}\text { Burden } \\
\text { Depression } \\
\text { Memory } \\
\text { problems } \\
\text { Behavior } \\
\text { problems } \\
\text { Distress over } \\
\text { problems }\end{array}$ \\
\hline
\end{tabular}

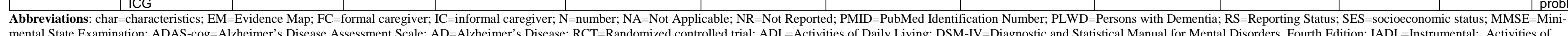

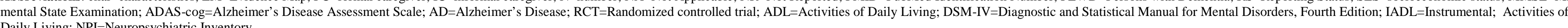

\section{Cognitive Stimulation Therapy}




\begin{tabular}{|c|c|c|c|c|c|c|c|c|c|}
\hline Study (PMID) & Outcome Timing & $\begin{array}{l}\text { Attrition Bias } \\
\text { Attrition \% }\end{array}$ & Selection Bias & Detection Bias & Performance Bias & Reporting Bias & Fidelity Bias & Funder & Overall Rating \\
\hline Lok, $2020^{176}$ & 7 weeks & \begin{tabular}{|l|} 
High \\
$23.08 \%$
\end{tabular} & Low & $x$ & $\mathrm{x}$ & $x$ & $x$ & $x$ & High \\
\hline Lin, 2018 & $\begin{array}{l}12 \text { weeks } \\
24 \text { weeks }\end{array}$ & $\begin{array}{l}\text { Medium } \\
14.91 \%\end{array}$ & Medium & High & High & Low & Low & Taiwanese government & High \\
\hline $\begin{array}{l}\text { Orgeta, 2015'17 }(26292178) \\
\text { Orrell, } 2017^{179}(28350796)\end{array}$ & $\begin{array}{l}13 \text { weeks } \\
26 \text { weeks }\end{array}$ & \begin{tabular}{|l|} 
Medium \\
$23.31 \%$
\end{tabular} & Low & Low & Moderate & Low & High & UK government & High \\
\hline Orrell, 2014 $4^{180}$ (24676963) & $\begin{array}{l}12 \text { weeks } \\
24 \text { weeks }\end{array}$ & \begin{tabular}{|l|} 
Low \\
$15.68 \%$
\end{tabular} & Low & Low & High & Low & Moderate & UK government & Medium \\
\hline $\begin{array}{l}\text { Spector, 20033181 (12948999) } \\
\text { Knapp, 2006-182 (16738349) }\end{array}$ & 8 weeks & $\begin{array}{l}\text { Low } \\
5.47 \%\end{array}$ & Low & Low & Moderate & Low & Low & UK government & Low \\
\hline
\end{tabular}

$X$ indicates that domain was not assessed due to high risk of bias.

Abbreviations: NR=Not Reported; PMID=PubMed Identification Number 


\begin{tabular}{|c|c|c|c|c|c|c|c|c|c|c|c|c|c|}
\hline $\begin{array}{l}\text { Study (PMID) } \\
\text { Country } \\
\text { RoB* } \\
\text { Type }\end{array}$ & $\begin{array}{l}\text { Intervention } \\
\text { Intervention } \\
\text { Focus } \\
\text { Theoretical } \\
\text { Model } \\
\text { Delivery } \\
\text { Person } \\
\text { Intervention } \\
\text { Target } \\
\text { Recipient } \\
\text { Mode } \\
\text { Components } \\
\text { Frequency } \\
\text { Duration }\end{array}$ & $\begin{array}{l}\text { Comparison } \\
\text { Target } \\
\text { Mode } \\
\text { Components } \\
\text { Frequency } \\
\text { Duration }\end{array}$ & $\begin{array}{l}\text { Setting } \\
\text { and Design } \\
\text { Setting } \\
\text { Design } \\
\text { Cluster N } \\
\text { Participants } \\
\text { Randomized } \\
\text { N }\end{array}$ & \begin{tabular}{|l|} 
PLWD \\
Dementia \\
Characteristics \\
Dementia Types \\
Dementia Severity \\
Diagnostic \\
Criteria \\
Age of Diagnosis \\
\end{tabular} & $\begin{array}{l}\text { PLWD } \\
\text { Non-Disease Char } \\
\text { PLWD N } \\
\text { PLWD Age (mean) } \\
\text { PLWD Sex (\% } \\
\text { female) } \\
\text { PLWD Race (\% } \\
\text { majority) } \\
\text { PLWD Education } \\
\text { (mean years) }\end{array}$ & $\begin{array}{l}\text { PLWD } \\
\text { Non-Disease } \\
\text { Char Reporting } \\
\text { Status (RS) } \\
\text { PLWD SES } \\
\text { PLWD Prior } \\
\text { Disability } \\
\text { PLWD } \\
\text { Household } \\
\text { Characteristics } \\
\text { PLWD Health } \\
\text { Insurance } \\
\text { PLWD Detailed } \\
\text { Race Information }\end{array}$ & $\begin{array}{l}\text { Informal Caregiver } \\
\text { (IC) } \\
\text { Characteristics } \\
\text { IC N } \\
\text { IC Age (mean) } \\
\text { IC Sex (\% female) } \\
\text { IC Race (\% } \\
\text { majority) } \\
\text { IC Education } \\
\text { (mean years) } \\
\text { IC Relation to } \\
\text { PLWD (\% majority) }\end{array}$ & $\begin{array}{l}\text { Informal Caregiver } \\
\text { (IC) Char. RS } \\
\text { IC Duration } \\
\text { IC Living With } \\
\text { PLWD } \\
\text { IC Payment } \\
\text { IC Health Status } \\
\text { IC Dementia } \\
\text { Family History } \\
\text { IC Employment } \\
\text { Status } \\
\text { IC Training }\end{array}$ & $\begin{array}{l}\text { Formal Caregiver } \\
\text { (FC) } \\
\text { Characteristics } \\
\text { FC N } \\
\text { FC Age (mean) } \\
\text { FC Sex (\% } \\
\text { female) } \\
\text { FC Race (\% } \\
\text { majority) } \\
\text { FC Education } \\
\text { (mean years) }\end{array}$ & $\begin{array}{l}\text { Formal } \\
\text { Caregiver (FC) } \\
\text { Char. RS } \\
\\
\text { FC Health } \\
\text { Status } \\
\text { FC Training } \\
\text { FC Education } \\
\text { FC Position } \\
\text { FC Length of } \\
\text { Service }\end{array}$ & $\begin{array}{l}\text { Outcome } \\
\text { Timing(s) }\end{array}$ & $\begin{array}{l}\text { PLWD } \\
\text { Outcomes }\end{array}$ & $\begin{array}{l}\text { Caregiver } \\
\text { Outcomes } \\
\text { IC: } \\
\text { FC: }\end{array}$ \\
\hline $\begin{array}{l}\text { Orrell, 2014180 } \\
\text { (24676963) } \\
\text { United Kingdom } \\
\text { Medium } \\
\text { Explanatory }\end{array}$ & $\begin{array}{l}\text { Maintenance of } \\
\text { CST after an } \\
\text { initial 7-week } \\
\text { program of } \\
\text { CST. Program } \\
\text { defined as } \\
\text { grouup sessions } \\
\text { focused on } \\
\text { reality } \\
\text { orientation and } \\
\text { cognitive } \\
\text { stimulation, led } \\
\text { by trained } \\
\text { facilitator; one } \\
\text { 45-minute } \\
\text { session for } 24 \\
\text { weeks }\end{array}$ & $\begin{array}{l}\text { Usual care } \\
\text { after an initial } \\
\text { 7-week } \\
\text { program of } \\
\text { CST }\end{array}$ & $\begin{array}{l}\text { Care homes } \\
\text { and community } \\
\text { centers } \\
\\
\text { RCT } \\
\text { Multisite } \\
\text { 236 PLWD }\end{array}$ & $\begin{array}{l}\text { DSM-IV criteria for } \\
\text { dementia, mild to } \\
\text { moderate dementia } \\
\text { on Clinical } \\
\text { Dementia Rating } \\
\text { scale }\end{array}$ & $\begin{array}{l}\mathrm{N}=236 \\
\text { Age } 83 \text { years } \\
63.6 \% \text { female } \\
\text { Race NR } \\
\text { Education NR }\end{array}$ & NR & $\mathrm{NR}$ & $\mathrm{NR}$ & NR & $\mathrm{NR}$ & $\begin{array}{l}12 \text { weeks } \\
24 \text { weeks }\end{array}$ & \begin{tabular}{l|} 
Quality of life \\
Neuropsychi \\
atric \\
symptoms \\
Function
\end{tabular} & NR \\
\hline $\begin{array}{l}\text { Spector, 2003 } 203^{181} \\
\text { (12948999) } \\
\text { Knapp, 2006 } \\
\text { (16738349) } \\
\text { United Kingdom } \\
\text { Low } \\
\text { Explanatory }\end{array}$ & $\begin{array}{l}\text { Group sessions } \\
\text { focused on } \\
\text { reality } \\
\text { orientation and } \\
\text { cognitive } \\
\text { stimulation, } \\
\text { facilitated by } \\
\text { researcher; two } \\
45 \text {-minute } \\
\text { sessions for } 7 \\
\text { weeks } \\
\end{array}$ & $\begin{array}{l}\text { Usual care, } \\
\text { typically doing } \\
\text { nothing, but } \\
\text { sometimes } \\
\text { including } \\
\text { music, } \\
\text { games, arts } \\
\text { and crafts, } \\
\text { and other } \\
\text { activities }\end{array}$ & $\begin{array}{l}\text { Residential } \\
\text { homes and } \\
\text { day care } \\
\text { centers } \\
\text { RCT } \\
\text { Multisite } \\
201 \text { PLWD }\end{array}$ & $\begin{array}{l}\text { DSM-IV criteria for } \\
\text { dementia, MMSE } \\
\text { between } 10 \text { and } 24\end{array}$ & $\begin{array}{l}\mathrm{N}=201 \\
\text { Age } 85.3 \text { years } \\
78.6 \% \text { female } \\
\text { Race NR } \\
\text { Education NR }\end{array}$ & NR & NR & NR & NR & NR & 8 weeks & $\begin{array}{l}\text { Quality of life } \\
\text { Function } \\
\text { Depression } \\
\text { Anxiety }\end{array}$ & NR \\
\hline
\end{tabular}

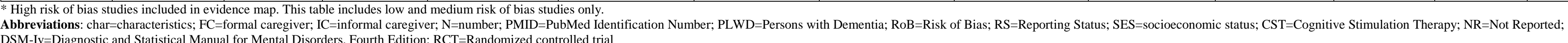

\begin{tabular}{|c|c|c|c|c|c|}
\hline $\begin{array}{l}\text { Study (PMID) } \\
\text { Comparison } \\
\text { RoB } \\
\text { Category* }\end{array}$ & $\begin{array}{l}\text { Outcome } \\
\text { Timing }\end{array}$ & Summary Finding & \begin{tabular}{|l|} 
Intervention \\
\end{tabular} & Comparator & p-Value \\
\hline $\begin{array}{l}\text { Orrell, 2014180 } \\
(24676963) \\
\text { Explanatory }\end{array}$ & $\begin{array}{l}\text { Quality of life, self-rated } \\
\text { QoL-AD, DEMQOL } \\
12 \text { weeks }\end{array}$ & $\begin{array}{l}0.32 \text { points ( } 95 \% \mathrm{Cl}-0.88 \text { to } 1.52 \text {, QoL-AD) } \\
-0.86 \text { points ( }(95 \% \mathrm{Cl}-3.45 \text { to } 1.73 \text {, DEMQOL) }\end{array}$ & $\begin{array}{l}\text { Cognitive stimulation } \\
\text { maintenance therapy } \\
\text { after initial cognitive } \\
\text { stimulation }\end{array}$ & $\begin{array}{l}\text { Usual care after } \\
\text { withdrawal of cognitive } \\
\text { stimulation }\end{array}$ & $\begin{array}{l}P=0.54 \text { (QoL-AD) } \\
P=0.54 \text { (DEMQOL) }\end{array}$ \\
\hline $\begin{array}{l}\text { Orrell, 2014180 } \\
(24676963) \\
\text { Explanatory }\end{array}$ & $\begin{array}{l}\text { Quality of life, caregiver-rated } \\
\text { QoL-AD, DEMQOL } \\
12 \text { weeks }\end{array}$ & $\begin{array}{l}1.53 \text { points ( } 95 \% \text { Cl } 0.37 \text { to } 2.69, \text { QOL-AD) } \\
3.24 \text { points ( } 95 \% \mathrm{Cl} 0.29 \text { to } 6.19, \mathrm{DEMQOL})\end{array}$ & $\begin{array}{l}\text { Cognitive stimulation } \\
\text { maintenance therapy } \\
\text { after initial cognitive } \\
\text { stimulation }\end{array}$ & $\begin{array}{l}\text { Usual care after } \\
\text { withdrawal of cognitive } \\
\text { stimulation }\end{array}$ & $\begin{array}{l}\mathrm{P}=0.01 \text { (QoL-AD) } \\
\mathrm{P}=0.03 \text { (DEMQOL) }\end{array}$ \\
\hline
\end{tabular}




\begin{tabular}{|c|c|c|c|c|c|}
\hline $\begin{array}{l}\text { Orrell, 2014480 } \\
\text { (24676963) } \\
\text { Explanatory }\end{array}$ & $\begin{array}{l}\text { Neuropsychiatric symptoms } \\
\text { NPI } \\
12 \text { weeks }\end{array}$ & 1.47 points (95\% $\mathrm{Cl}-1.59$ to 4.53$)$ & $\begin{array}{l}\text { Cognitive stimulation } \\
\text { maintenance therapy } \\
\text { after initial cognitive } \\
\text { stimulation }\end{array}$ & $\begin{array}{l}\text { Usual care after } \\
\text { withdrawal of cognitive } \\
\text { stimulation }\end{array}$ & $P=0.34$ \\
\hline $\begin{array}{l}\text { Orrell, 2014180 } \\
\text { (24676963) } \\
\text { Explanatory }\end{array}$ & $\begin{array}{l}\text { Function } \\
\text { ADCS-ADL } \\
12 \text { weeks }\end{array}$ & 2.64 points (95\% $\mathrm{Cl} 0.08$ to 5.20 ) & $\begin{array}{l}\text { Cognitive stimulation } \\
\text { maintenance therapy } \\
\text { after initial cognitive } \\
\text { stimulation }\end{array}$ & $\begin{array}{l}\text { Usual care after } \\
\text { withdrawal of cognitive } \\
\text { stimulation }\end{array}$ & $P=0.04$ \\
\hline $\begin{array}{l}\text { Orrell, 2014180 } \\
\text { (24676963) } \\
\text { Explanatory }\end{array}$ & $\begin{array}{l}\text { Quality of life, self-rated } \\
\text { QoL-AD, DEMQOL } \\
24 \text { weeks }\end{array}$ & $\begin{array}{l}1.78 \text { points (95\% Cl }-0.01 \text { to } 3.57 \text {, QoL-AD) } \\
0.30 \text { points ( } 95 \% \mathrm{Cl}-3.45 \text { to } 1.73, \mathrm{DEMQOL})\end{array}$ & $\begin{array}{l}\text { Cognitive stimulation } \\
\text { maintenance therapy } \\
\text { after initial cognitive } \\
\text { stimulation }\end{array}$ & $\begin{array}{l}\text { Usual care after } \\
\text { withdrawal of cognitive } \\
\text { stimulation }\end{array}$ & $\begin{array}{l}\mathrm{P}=0.03 \text { (QoL-AD) } \\
\mathrm{P}=0.87 \text { (DEMQOL) }\end{array}$ \\
\hline $\begin{array}{l}\text { Orrell, 2014180 } \\
\text { (24676963) } \\
\text { Explanatory }\end{array}$ & $\begin{array}{l}\text { Quality of life, caregiver-rated } \\
\text { QoL-AD, DEMQOL } \\
24 \text { weeks }\end{array}$ & $\begin{array}{l}0.07 \text { points (95\% Cl }-1.39 \text { to } 1.53 \text {, QoL-AD) } \\
1.13 \text { points ( } 95 \% \mathrm{Cl}-2.24 \text { to } 4.51 \text {, DEMQOL) }\end{array}$ & $\begin{array}{l}\text { Cognitive stimulation } \\
\text { maintenance therapy } \\
\text { after initial cognitive } \\
\text { stimulation }\end{array}$ & $\begin{array}{l}\text { Usual care after } \\
\text { withdrawal of cognitive } \\
\text { stimulation }\end{array}$ & $\begin{array}{l}\mathrm{P}=0.95 \text { (QOL-AD) } \\
\mathrm{P}=0.50 \text { (DEMQOL) }\end{array}$ \\
\hline $\begin{array}{l}\text { Orrell, 2014180 } \\
\text { (24676963) } \\
\text { Explanatory }\end{array}$ & $\begin{array}{l}\text { Neuropsychiatric symptoms } \\
\text { NPI } \\
24 \text { weeks }\end{array}$ & 1.58 points ( $95 \% \mathrm{Cl}-2.67$ to 5.84$)$ & $\begin{array}{l}\text { Cognitive stimulation } \\
\text { maintenance therapy } \\
\text { after initial cognitive } \\
\text { stimulation }\end{array}$ & $\begin{array}{l}\text { Usual care after } \\
\text { withdrawal of cognitive } \\
\text { stimulation }\end{array}$ & $P=0.53$ \\
\hline $\begin{array}{l}\text { Orrell, 2014180 } \\
\text { (24676963) } \\
\text { Explanatory }\end{array}$ & $\begin{array}{l}\text { Function } \\
\text { ADCS-ADL } \\
24 \text { weeks }\end{array}$ & 0.94 points ( $95 \% \mathrm{Cl}-2.04$ to 3.92$)$ & $\begin{array}{l}\text { Cognitive stimulation } \\
\text { maintenance therapy } \\
\text { after initial cognitive } \\
\text { stimulation }\end{array}$ & $\begin{array}{l}\text { Usual care after } \\
\text { withdrawal of cognitive } \\
\text { stimulation }\end{array}$ & $P=0.54$ \\
\hline $\begin{array}{l}\text { Spector, 2003181 } \\
\text { (12948999) } \\
\text { Knapp, 2006 } \\
\text { (16738349) } \\
\text { Explanatory }\end{array}$ & $\begin{array}{l}\text { Quality of life } \\
\text { QoL-AD } \\
8 \text { weeks }\end{array}$ & 1.64 points (95\% Cl 0.09 to 3.18 ) & Cognitive stimulation & Usual care & $\mathrm{P}=0.028$ \\
\hline $\begin{array}{l}\text { Spector, 2003181 } \\
\text { (12948999) } \\
\text { Knapp, 2006 } \\
\text { (16738349) } \\
\text { Explanatory }\end{array}$ & $\begin{array}{l}\text { Function } \\
\text { HCS } \\
8 \text { weeks }\end{array}$ & 2.3 points $(95 \% \mathrm{Cl}-0.45$ to 4.15$)$ & Cognitive stimulation & Usual care & $P=0.09$ \\
\hline $\begin{array}{l}\text { Spector, 2003181 } \\
\text { (12948999) } \\
\text { Knapp, 2006 } \\
\text { (16738349) } \\
\text { Explanatory }\end{array}$ & $\begin{array}{l}\text { Neuropsychiatric symptoms } \\
\text { CAPE-BRS } \\
8 \text { weeks }\end{array}$ & 0.40 points ( $95 \% \mathrm{Cl}-0.9$ to 1.69 points) & Cognitive stimulation & Usual care & $\mathrm{P}=0.449$ \\
\hline $\begin{array}{l}\text { Spector, 2003181 } \\
\text { (12948999) } \\
\text { Knapp, 2006 } \\
\text { (16738349) } \\
\text { Explanatory }\end{array}$ & $\begin{array}{l}\text { Anxiety } \\
\text { RAID } \\
8 \text { weeks }\end{array}$ & -1.30 points $(95 \% \mathrm{Cl}-3.48$ to 0.87$)$ & Cognitive stimulation & Usual care & $\mathrm{P}=0.200$ \\
\hline $\begin{array}{l}\text { Spector, 2003181 } \\
\text { (12948999) } \\
\text { Knapp, 2006 } \\
\text { (16738349) } \\
\text { Explanatory }\end{array}$ & $\begin{array}{l}\text { Depression } \\
\text { CSDD } \\
8 \text { weeks }\end{array}$ & 0.12 points $(95 \% \mathrm{Cl} 1.56$ to 1.31$)$ & Cognitive stimulation & Usual care & $P=0.648$ \\
\hline
\end{tabular}

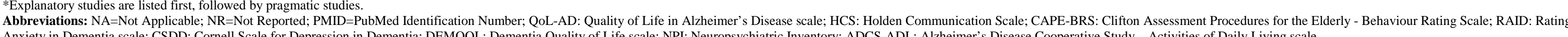
Anxiety in Dementia scale; CSDD: Cornell Scale for Depression in Dementia; DEMQQL: Dementia Quality of Life scale; NPI: Neuropsychiatric Inventory; ADCS-ADL: Alzheimer's Disease Cooperative Study - Activities of Daily Living scale 
Table D-33 Summary of strength of evidence for PLWD outcomes: cognitive stimulation therapy

\begin{tabular}{|c|c|c|c|c|c|c|c|c|}
\hline $\begin{array}{l}\text { Comparison } \\
\text { Outcome }\end{array}$ & Timing & $\begin{array}{l}\text { \# Studies/ Design } \\
\text { (n analyzed) }\end{array}$ & Finding or Summary Statistic & Study Limitations & Consistency & Directness & Precision & Overall Grade/ Conclusion \\
\hline $\begin{array}{l}\text { CS vs. Active control } \\
\text { Quality of life } \\
\end{array}$ & 8 weeks & $1 \mathrm{RCT}(\mathrm{n}=201)$ & Improvement in quality of life & Low & Unknown & Direct & Imprecise & Insufficient \\
\hline $\begin{array}{l}\text { CS vs. Active control } \\
\text { Function } \\
\end{array}$ & 8 weeks & $1 \mathrm{RCT}(\mathrm{n}=201)$ & No change in function & Low & Unknown & Direct & Imprecise & Insufficient \\
\hline $\begin{array}{l}\text { CS vs. Active control } \\
\text { Neuropsychiatric } \\
\text { symptoms }\end{array}$ & 8 weeks & $1 \mathrm{RCT}(\mathrm{n}=201)$ & No change in neuropsychiatric symptoms & Low & Unknown & Direct & Imprecise & Insufficient \\
\hline $\begin{array}{l}\text { CS vs. Active control } \\
\text { Anxiety }\end{array}$ & 8 weeks & $1 \mathrm{RCT}(\mathrm{n}=201)$ & No change in anxiety & Low & Unknown & Direct & Imprecise & Insufficient \\
\hline $\begin{array}{l}\text { CS vs. Active control } \\
\text { Depression }\end{array}$ & 8 weeks & $1 \mathrm{RCT}(\mathrm{n}=201)$ & No change in depression & Low & Unknown & Direct & Imprecise & Insufficient \\
\hline $\begin{array}{l}\text { CS maintenance } \\
\text { therapy vs. Usual care } \\
\text { after initial CS therapy } \\
\text { Quality of life } \\
\end{array}$ & 24 weeks & $1 \mathrm{RCT}(\mathrm{n}=236)$ & Improvement in quality of life & Moderate & Unknown & Direct & Imprecise & Insufficient \\
\hline $\begin{array}{l}\text { CS maintenance } \\
\text { therapy vs. Usual care } \\
\text { after initial CS therapy } \\
\text { Neuropsychiatric } \\
\text { symptoms }\end{array}$ & 24 weeks & $1 \mathrm{RCT}(\mathrm{n}=236)$ & Improvement in quality of life & Moderate & Unknown & Direct & Imprecise & Insufficient \\
\hline $\begin{array}{l}\text { CS maintenance } \\
\text { therapy vs. Usual care } \\
\text { after initial CS therapy } \\
\text { Function } \\
\end{array}$ & 24 weeks & $1 \mathrm{RCT}(\mathrm{n}=236)$ & Improvement in quality of life & Moderate & Unknown & Direct & Imprecise & Insufficient \\
\hline
\end{tabular}

\section{Evidence Map: Cognitive Stimulation Therapy}

\begin{tabular}{|c|c|c|c|c|c|c|c|c|c|c|c|c|c|}
\hline $\begin{array}{l}\text { Study (PMID) } \\
\text { Country } \\
\text { EM Reason }\end{array}$ & Intervention & Comparison & $\begin{array}{l}\text { Setting } \\
\text { and Design } \\
\text { Setting } \\
\text { Design } \\
\text { Cluster N } \\
\text { Participants } \\
\text { Random- } \\
\text { ized N }\end{array}$ & $\begin{array}{l}\text { PLWD } \\
\text { Dementia } \\
\text { Characteristics }\end{array}$ & $\begin{array}{l}\text { PLWD } \\
\text { Non-Disease Char } \\
\text { PLWD N } \\
\text { PLWD Age (mean) } \\
\text { PLWD Sex (\% } \\
\text { female) } \\
\text { PLWD Race (\% } \\
\text { majority) } \\
\text { PLWD Education } \\
\text { (mean years) }\end{array}$ & $\begin{array}{l}\text { PLWD } \\
\text { Non-Disease } \\
\text { Char Reporting } \\
\text { Status (RS) } \\
\\
\text { PLWD SES } \\
\text { PLWD Prior } \\
\text { Disability } \\
\text { PLWD } \\
\text { Household } \\
\text { Characteristics } \\
\text { PLWD Health } \\
\text { Insurance } \\
\text { PLWD Detailed } \\
\text { Race Information }\end{array}$ & \begin{tabular}{|l|} 
Informal Caregiver \\
(IC) \\
Characteristics \\
IC N \\
IC Age (mean) \\
IC Sex (\% female) \\
IC Race (\% \\
majority) \\
IC Education \\
(mean years) \\
IC Relation to \\
PLWD (\% majority)
\end{tabular} & $\begin{array}{l}\text { Informal Caregiver } \\
\text { (IC) Char. RS } \\
\text { IC Duration } \\
\text { IC Living With } \\
\text { PLWD } \\
\text { IC Payment } \\
\text { IC Health Status } \\
\text { IC Dementia } \\
\text { Family History } \\
\text { IC Employment } \\
\text { Status } \\
\text { IC Training }\end{array}$ & $\begin{array}{l}\text { Formal Caregiver } \\
\text { (FC) } \\
\text { Characteristics } \\
\text { FC N } \\
\text { FC Age (mean) } \\
\text { FC Sex (\% } \\
\text { female) } \\
\text { FC Race (\% } \\
\text { majority) } \\
\text { FC Education } \\
\text { (mean years) }\end{array}$ & $\begin{array}{l}\text { Formal } \\
\text { Caregiver (FC) } \\
\text { Char. RS } \\
\text { FC Health } \\
\text { Status } \\
\text { FC Training } \\
\text { FC Education } \\
\text { FC Position } \\
\text { FC Length of } \\
\text { Service }\end{array}$ & $\begin{array}{l}\text { Outcome } \\
\text { Timing(s) }\end{array}$ & \begin{tabular}{|l|} 
PLWD \\
Outcomes
\end{tabular} & $\begin{array}{l}\text { Caregiver } \\
\text { Outcomes } \\
\text { IC: } \\
\text { FC: }\end{array}$ \\
\hline $\begin{array}{l}\text { Lok, } 20200^{176} \\
\text { Turkey } \\
\text { High RoB }\end{array}$ & \begin{tabular}{|l} 
Cognitive \\
stimulation \\
therapy
\end{tabular} & Usual care & $\begin{array}{l}\text { Hospital; } \\
\text { single site } \\
78 \text { PLWDD }\end{array}$ & $\begin{array}{l}\text { MMSE between } 13 \\
\text { and } 24\end{array}$ & $\begin{array}{l}\mathrm{N}=60 \\
\text { Age NR } \\
\text { Sex } \\
\text { Race NR } \\
\text { Education }\end{array}$ & $\begin{array}{l}\text { Household } \\
\text { characteristics }\end{array}$ & NR & NR & NR & NR & 7 weeks & $\begin{array}{l}\text { MMSE } \\
\text { CAPS } \\
\text { QoL-AD }\end{array}$ & NR \\
\hline $\begin{array}{l}\text { Cheung, } 2019 \\
183 \\
\text { Hong Kong } \\
\text { Small sample }\end{array}$ & \begin{tabular}{|l} 
Cognitive \\
stimulating play \\
intervention
\end{tabular} & Social activities & $\begin{array}{l}\text { Community } \\
\text { residents } \\
30 \text { PLWD }\end{array}$ & Dementia type NR & $\begin{array}{l}\text { N=30 } \\
\text { Age: yes } \\
\text { Sex:yes } \\
\text { Race: NR } \\
\text { Education:yes }\end{array}$ & NR & NR & $\mathrm{NR}$ & NR & NR & 8 weeks & $\begin{array}{l}\text { MoCA } \\
\text { FOME }\end{array}$ & \\
\hline
\end{tabular}




\begin{tabular}{|c|c|c|c|c|c|c|c|c|c|c|c|c|c|}
\hline $\begin{array}{l}\text { Lin, 2018 } \\
\text { (28881430) } \\
\text { Taiwan } \\
\text { High RoB }\end{array}$ & $\begin{array}{l}\text { Cognitive } \\
\text { stimulation } \\
\text { therapy }\end{array}$ & $\begin{array}{l}\text { Reminiscence } \\
\text { therapy; usual } \\
\text { care }\end{array}$ & \begin{tabular}{|l|} 
Long-term \\
care \\
institutions; \\
multisite \\
105 PLWD \\
\end{tabular} & \begin{tabular}{|l|} 
MMSE between 17 \\
and 24, with \\
agitation or \\
depressive \\
symptoms present \\
\end{tabular} & $\begin{array}{l}\mathrm{N}=105 \\
\text { Age: no } \\
\text { Sex:yes } \\
\text { Race: NR } \\
\text { Education: NR }\end{array}$ & \begin{tabular}{|l|} 
Marital status \\
\end{tabular} & $\mathrm{NR}$ & NR & NR & NR & $\begin{array}{l}12 \text { weeks } \\
24 \text { weeks }\end{array}$ & $\begin{array}{l}\text { MMSE } \\
\text { QoL-AD }\end{array}$ & NR \\
\hline $\begin{array}{l}\text { Capotosto, } \\
2017^{184} \\
(27272538) \\
\text { Italy } \\
\text { Small sample }\end{array}$ & $\begin{array}{l}\text { Cognitive } \\
\text { stimulation } \\
\text { therapy }\end{array}$ & $\begin{array}{l}\text { General } \\
\text { activities }\end{array}$ & \begin{tabular}{|l} 
Residential \\
homes; \\
multisite \\
39 PLWD
\end{tabular} & \begin{tabular}{|l} 
Mild to moderate \\
dementia
\end{tabular} & $\begin{array}{l}\mathrm{N}=39 \\
\text { Mean age } \\
\text { Mean education }\end{array}$ & NR & NR & NR & NR & NR & 7 weeks & $\begin{array}{l}\text { MMSE } \\
\text { ADAS-Cog } \\
\text { QoL-AD } \\
\text { CSDD } \\
\text { DAD } \\
\text { NPI }\end{array}$ & NR \\
\hline $\begin{array}{l}\text { Wong, 2016185 } \\
\text { (29717527) } \\
\text { Hong Kong } \\
\text { Pilot }\end{array}$ & $\begin{array}{l}\text { Cognitive } \\
\text { stimulation } \\
\text { therapy }\end{array}$ & $\begin{array}{l}\text { No control } \\
\text { group }\end{array}$ & $\begin{array}{l}\text { Community } \\
\text { and } \\
\text { residential } \\
\text { care units of } \\
\text { major } \\
\text { NGOs; } \\
\text { multisite } \\
30 \text { PLWD }\end{array}$ & $\begin{array}{l}\text { Clinical diagnosis } \\
\text { of dementia, } \\
\text { criteria unspecified }\end{array}$ & $\begin{array}{l}\mathrm{N}=30 \\
\text { Mean age } \\
\text { Race NR } \\
\text { Sex } \\
\text { Education }\end{array}$ & \begin{tabular}{|l|} 
Living situation \\
\end{tabular} & Sex & NR & NR & NR & 7 weeks & $\begin{array}{l}\text { QoL-AD } \\
\text { ADAS-Cog }\end{array}$ & NR \\
\hline $\begin{array}{l}\text { Orgeta, 2015'178 } \\
(26292178) \\
\text { Orrell, 2017179 } \\
\text { (28350796) } \\
\text { United Kingdom } \\
\text { High RoB }\end{array}$ & $\begin{array}{l}\text { Carer-delivered } \\
\text { individual } \\
\text { cognitive } \\
\text { stimulation } \\
\text { therapy }\end{array}$ & Usual care & $\begin{array}{l}\text { Unspecified } \\
\text { community } \\
\text { settings; } \\
\text { multisite } \\
356 \text { PLWD }\end{array}$ & \begin{tabular}{|l|} 
Dementia \\
unspecified
\end{tabular} & $\begin{array}{l}\mathrm{N}=356 \\
\text { Age NR } \\
\text { Race } \\
\text { Sex } \\
\text { Education }\end{array}$ & $\begin{array}{l}\text { Marital status } \\
\text { Living situation }\end{array}$ & $\begin{array}{l}356 \text { informal } \\
\text { caregivers } \\
\text { Sex } \\
\text { Race }\end{array}$ & $\begin{array}{l}\text { Marital status } \\
\text { Living situation }\end{array}$ & NR & $\mathrm{NR}$ & 26 weeks & $\begin{array}{l}\text { ADAS-Cog } \\
\text { QoL-AD } \\
\text { DEMQOL } \\
\text { NPI } \\
\text { BADLs } \\
\text { GDS-15 } \\
\text { QCPR }\end{array}$ & $\begin{array}{l}\text { QCPR } \\
\text { EQ-5D } \\
\text { HADS }\end{array}$ \\
\hline $\begin{array}{l}\text { Cove, 2014186 } \\
(25525349) \\
\text { United Kingdom } \\
\text { Small sample }\end{array}$ & $\begin{array}{l}\text { Cognitive } \\
\text { stimulation } \\
\text { therapy plus } \\
\text { carer training; } \\
\text { cognitive } \\
\text { stimulation } \\
\text { therapy alone }\end{array}$ & Waitlist control & $\begin{array}{l}\text { Unspecified } \\
\text { community } \\
\text { settings; } \\
\text { multisite } \\
72 \text { PLWD }\end{array}$ & $\begin{array}{l}\text { DSM-IV diagnosis } \\
\text { of mild to moderate } \\
\text { dementia }\end{array}$ & $\begin{array}{l}\mathrm{N}=72 \\
\text { Mean age } \\
\text { Race/ethnicity } \\
\text { Sex } \\
\text { Education NR }\end{array}$ & \begin{tabular}{|l|} 
Detailed \\
race/ethnicity
\end{tabular} & $\mathrm{NR}$ & NR & NR & & 15 weeks & $\begin{array}{l}\text { MMSE } \\
\text { ADAS-Cog } \\
\text { QoL-AD } \\
\text { QCPR }\end{array}$ & QCPR \\
\hline $\begin{array}{l}\text { Yamanaka, } \\
2013^{187} \\
(23550665) \\
\text { Japan } \\
\text { Pilot }\end{array}$ & $\begin{array}{l}\text { Cognitive } \\
\text { stimulation } \\
\text { therapy }\end{array}$ & Usual care & $\begin{array}{l}\text { Japanese } \\
\text { residential } \\
\text { and nursing } \\
\text { homes; } \\
\text { multisite } \\
56 \text { PLWD }\end{array}$ & $\begin{array}{l}\text { Mild to moderate } \\
\text { AD }\end{array}$ & $\begin{array}{l}\mathrm{N}=56 \\
\text { Mean age } \\
\text { Sex } \\
\text { Education NR } \\
\text { Race NR }\end{array}$ & NR & $\mathrm{NR}$ & NR & NR & NR & 7 weeks & $\begin{array}{l}\text { MMSE } \\
\text { COGNISTAT } \\
\text { QoL-AD } \\
\text { EQ-5D } \\
\text { Face scale } \\
\text { for mood }\end{array}$ & $\mathrm{NR}$ \\
\hline $\begin{array}{l}\text { Niu, 2010188 } \\
\text { (20713437) } \\
\text { China } \\
\text { Small sample }\end{array}$ & $\begin{array}{l}\text { Cognitive } \\
\text { stimulation } \\
\text { therapy }\end{array}$ & $\begin{array}{l}\text { Communication } \\
\text { exercise }\end{array}$ & $\begin{array}{l}\text { Military } \\
\text { sanatorium; } \\
\text { single site } \\
\text { 32 PLWD }\end{array}$ & \begin{tabular}{|l|} 
Mild to moderate \\
AD with marked \\
neuropsychiatric \\
symptoms
\end{tabular} & $\begin{array}{l}\mathrm{N}=32 \\
\text { Mean age } \\
\text { Sex } \\
\text { Education }\end{array}$ & NR & $\mathrm{NR}$ & NR & NR & NR & 10 weeks & $\begin{array}{l}\text { MMSE } \\
\text { NPI }\end{array}$ & NR \\
\hline $\begin{array}{l}\text { Matsuda, } \\
2007^{189} \\
(17005066) \\
\text { Japan } \\
\text { Small sample }\end{array}$ & $\begin{array}{l}\text { Cognitive } \\
\text { stimulation } \\
\text { therapy plus } \\
\text { donepezil }\end{array}$ & Donepezil alone & $\begin{array}{l}\text { Outpatient } \\
\text { neuropsychi } \\
\text { atric clinic; } \\
\text { single site } \\
30 \text { PLWD }\end{array}$ & $\begin{array}{l}\text { DSM-IV diagnosis } \\
\text { of dementia }\end{array}$ & $\begin{array}{l}\mathrm{N}=30 \\
\mathrm{NR}\end{array}$ & NR & NR & NR & NR & $\mathrm{NR}$ & 1 year & MMSE & $\mathrm{NR}$ \\
\hline
\end{tabular}

Abbreviations: char=characteristics; EM=Evidence Map; FC=formal caregiver; IC=informal caregiver; N=number; NA=Not Applicable; NR=Not Reported; PMID=PubMed Identification Number; PLWD=Persons with Dementia; RS=Reporting Status; SES=socioeconomic status; MMS
Mental State Examination; CAPS=Coping and Adaptation Processing Scale; MoCA=Montreal Cognitive Assessment; FOME=Fuld Object Memory Evaluation; NPI=Neuropsychiatric Inventory; COGNISTAT=Neurobehavioral Cognitive Status Examination; QoL-AD: Quality of Life in

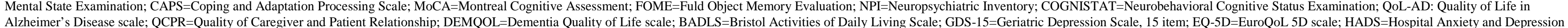
Scale; ADAS-Cog=Alzheimer's Disease Assessment Scale - Cognition; CSDD=Cornell Scale for Depression in Dementia· DAD=Disability Assessment for Dementia

\section{Recreation Therapy}




\begin{tabular}{|c|c|c|c|c|c|c|c|c|c|}
\hline Study (PMID) & Outcome Timing & $\begin{array}{l}\text { Attrition Bias } \\
\text { Attrition \% }\end{array}$ & Selection Bias & \begin{tabular}{|l} 
Detection Bias \\
\end{tabular} & Performance Bias & Reporting Bias & Fidelity Bias & Funder & Overall Rating \\
\hline $\begin{array}{l}\text { Pedrinolla, 2019190 } \\
\text { (31424399) }\end{array}$ & 24 weeks & $\begin{array}{l}\text { Low } \\
6.75 \% \\
\end{array}$ & Low & Low & Moderate & Low & High & \begin{tabular}{|l|} 
Foundations \\
\end{tabular} & Medium \\
\hline $\begin{array}{l}\text { Tse, 2018191 } \\
\text { (30255637) }\end{array}$ & \begin{tabular}{|l}
8 weeks \\
12 weeks
\end{tabular} & $\begin{array}{l}\text { High } \\
\text { NR }\end{array}$ & $\mathrm{x}$ & $x$ & $\mathrm{x}$ & $x$ & $x$ & Unfunded & High \\
\hline $\begin{array}{l}\text { Lin, 2015192 } \\
(607203463)\end{array}$ & 24 weeks & $\begin{array}{l}\text { High } \\
\text { NR }\end{array}$ & $\mathrm{x}$ & $x$ & $x$ & $x$ & $\mathrm{x}$ & NR & High \\
\hline $\begin{array}{l}\text { Van Haitsma, 2015 } 293 \\
\text { (24304555) }\end{array}$ & 0 weeks & $\begin{array}{l}\text { Low } \\
7.69 \% \\
\end{array}$ & Moderate & Moderate & High & Low & Moderate & $\begin{array}{l}\text { US state government, } \\
\text { foundation }\end{array}$ & High \\
\hline $\begin{array}{l}\text { Low, 2013 } \\
(23315520)\end{array}$ & \begin{tabular}{|l}
13 weeks \\
26 weeks \\
\end{tabular} & \begin{tabular}{|l|} 
Medium \\
$13.82 \%$ \\
\end{tabular} & Low & Low & Moderate & Low & Low & Australian government & Medium \\
\hline $\begin{array}{l}\text { Kolanowski, } 2011^{195} \\
\text { (21649633) }\end{array}$ & Up to 3 weeks & $\begin{array}{l}\text { Low } \\
4.69 \%\end{array}$ & Low & Medium & Low & Low & Low & $\begin{array}{l}\text { National Institutes of } \\
\text { Health }\end{array}$ & Low \\
\hline $\begin{array}{l}\text { Buettner, 2002 } \\
\text { (119546 }\end{array}$ & \begin{tabular}{|l|}
2 weeks \\
10 weeks
\end{tabular} & $\begin{array}{l}\text { High } \\
\text { NR }\end{array}$ & $\mathrm{x}$ & $x$ & $\mathrm{x}$ & $x$ & $\mathrm{x}$ & US state government & High \\
\hline $\begin{array}{l}\text { Hopman-Rock, 1999197 } \\
\text { (29403282) }\end{array}$ & 24 weeks & $\begin{array}{l}\text { High } \\
31.3 \%\end{array}$ & $x$ & $x$ & $\mathrm{x}$ & $x$ & $x$ & Foundations, health insurer & High \\
\hline
\end{tabular}

(29403282)

X indicates that domain was not assessed due to high risk of bias.
Abbreviations: NR=Not Reported; PMID=PubMed Identification Number 


\begin{tabular}{|c|c|c|c|c|c|c|c|c|c|c|c|c|c|}
\hline $\begin{array}{l}\text { Study (PMID) } \\
\text { Country } \\
\text { RoB* } \\
\text { Type }\end{array}$ & $\begin{array}{l}\text { Intervention } \\
\text { Intervention } \\
\text { Focus } \\
\text { Theoretical } \\
\text { Model } \\
\text { Delivery } \\
\text { Person } \\
\text { Intervention } \\
\text { Target } \\
\text { Recipient } \\
\text { Mode } \\
\text { Components } \\
\text { Frequency } \\
\text { Duration }\end{array}$ & $\begin{array}{l}\text { Comparison } \\
\text { Target } \\
\text { Mode } \\
\text { Components } \\
\text { Frequency } \\
\text { Duration }\end{array}$ & $\begin{array}{l}\text { Setting } \\
\text { and Design } \\
\text { Setting } \\
\text { Design } \\
\text { Cluster N } \\
\text { Participants } \\
\text { Randomized } \\
\text { N }\end{array}$ & \begin{tabular}{|l|} 
PLWD \\
Dementia \\
Characteristics \\
\\
Dementia Types \\
Dementia Severity \\
Diagnostic \\
Criteria \\
Age of Diagnosis
\end{tabular} & $\begin{array}{l}\text { PLWD } \\
\text { Non-Disease Char } \\
\text { PLWD N } \\
\text { PLWD Age (mean) } \\
\text { PLWD Sex (\% } \\
\text { female) } \\
\text { PLWD Race (\% } \\
\text { majority) } \\
\text { PLWD Education } \\
\text { (mean years) }\end{array}$ & $\begin{array}{l}\text { PLWD } \\
\text { Non-Disease } \\
\text { Char Reporting } \\
\text { Status (RS) } \\
\text { PLWD SES } \\
\text { PLWD Prior } \\
\text { Disability } \\
\text { PLWD } \\
\text { Household } \\
\text { Characteristics } \\
\text { PLWD Health } \\
\text { Insurance } \\
\text { PLWD Detailed } \\
\text { Race Information }\end{array}$ & $\begin{array}{l}\text { Informal Caregiver } \\
\text { (IC) } \\
\text { Characteristics } \\
\text { IC N } \\
\text { IC Age (mean) } \\
\text { IC Sex (\% female) } \\
\text { IC Race (\% } \\
\text { majority) } \\
\text { IC Education } \\
\text { (mean years) } \\
\text { IC Relation to } \\
\text { PLWD (\% majority) }\end{array}$ & $\begin{array}{l}\text { Informal Caregiver } \\
\text { (IC) Char. RS } \\
\text { IC Duration } \\
\text { IC Living With } \\
\text { PLWD } \\
\text { IC Payment } \\
\text { IC Health Status } \\
\text { IC Dementia } \\
\text { Family History } \\
\text { IC Employment } \\
\text { Status } \\
\text { IC Training }\end{array}$ & $\begin{array}{l}\text { Formal Caregiver } \\
\text { (FC) } \\
\text { Characteristics } \\
\text { FC N } \\
\text { FC Age (mean) } \\
\text { FC Sex (\% } \\
\text { female) } \\
\text { FC Race (\% } \\
\text { majority) } \\
\text { FC Education } \\
\text { (mean years) }\end{array}$ & $\begin{array}{l}\text { Formal } \\
\text { Caregiver (FC) } \\
\text { Char. RS } \\
\text { FC Health } \\
\text { Status } \\
\text { FC Training } \\
\text { FC Education } \\
\text { FC Position } \\
\text { FC Length of } \\
\text { Service }\end{array}$ & $\begin{array}{l}\text { Outcome } \\
\text { Timing(s) }\end{array}$ & $\begin{array}{l}\text { PLWD } \\
\text { Outcomes }\end{array}$ & $\begin{array}{l}\text { Caregiver } \\
\text { Outcomes } \\
\text { IC: } \\
\text { FC: }\end{array}$ \\
\hline $\begin{array}{l}\text { Pedrinolla, } \\
2019^{190} \\
(31424399) \\
\text { Italy }\end{array}$ & $\begin{array}{l}\text { Unstructured } \\
\text { group } \\
\text { interaction with } \\
\text { enclosed } \\
\text { natural } \\
\text { environment } \\
\text { including plants } \\
\text { and flowers }\end{array}$ & $\begin{array}{l}\text { Usual care in } \\
\text { AD unit of a } \\
\text { nursing } \\
\text { home, } \\
\text { including long } \\
\text { corridor for } \\
\text { walking plus } \\
\text { recreation } \\
\text { rooms }\end{array}$ & $\begin{array}{l}\text { Nursing home } \\
\text { Single site } \\
163 \text { PLWD }\end{array}$ & \begin{tabular}{|l|}
$\mathrm{N}=163$ \\
Probable AD \\
diagnosis \\
MMSE $\leq 15$
\end{tabular} & $\begin{array}{l}\mathrm{N}=163 \\
\text { Age } 77 \text { years } \\
74.2 \% \text { female } \\
\text { Race NR } \\
\text { Education NR }\end{array}$ & $\mathrm{NR}$ & $\mathrm{NR}$ & NR & NR & $\mathrm{NR}$ & 24 weeks & $\begin{array}{l}\text { Neuropsychi } \\
\text { atric } \\
\text { symptoms } \\
\text { Function } \\
\text { Antipsychoti } \\
\text { c dosage }\end{array}$ & NR \\
\hline $\begin{array}{l}\text { Low, 2013194 } \\
(23315520) \\
\text { Australia } \\
\text { Medium } \\
\text { Explanatory }\end{array}$ & $\begin{array}{l}\text { Individual, } \\
\text { tailored humor } \\
\text { therapy } \\
\text { sessions for } \\
\text { residents } \\
\text { focused on } \\
\text { humorous } \\
\text { interactions; } \\
\text { delivered by a } \\
\text { nominated staff } \\
\text { member who } \\
\text { received one } \\
\text { day of training; } \\
\text { weekly therapy } \\
\text { sessions for } 9 \text { to } \\
12 \text { weeks }\end{array}$ & $\begin{array}{l}\text { Usual care, } \\
\text { not otherwise } \\
\text { specified }\end{array}$ & $\begin{array}{l}\text { Nursing homes } \\
\text { Cluster RCT } \\
\text { Multisite } \\
36 \text { nursing } \\
\text { homes } \\
389 \text { PLWD }\end{array}$ & $\begin{array}{l}\mathrm{N}=389 \\
\text { Dementia type NR } \\
\text { Mean Global } \\
\text { Deterioration Scale } \\
5.0\end{array}$ & $\begin{array}{l}\mathrm{N}=389 \\
\text { Age } 84.5 \text { years } \\
64.1 \% \text { female } \\
\text { Race NR } \\
\text { Education NR }\end{array}$ & NR & NR & NR & NR & NR & $\begin{array}{l}13 \text { weeks } \\
26 \text { weeks }\end{array}$ & $\begin{array}{l}\text { Depression } \\
\text { Neuropsychi } \\
\text { atric } \\
\text { symptoms } \\
\text { Social } \\
\text { engagement } \\
\text { Quality of life }\end{array}$ & NR \\
\hline $\begin{array}{l}\text { Kolanowski, } \\
2011^{195} \\
\text { (21649633) } \\
\text { United States } \\
\text { Low } \\
\text { Explanatory }\end{array}$ & $\begin{array}{l}\text { Individual } \\
\text { recreational } \\
\text { activities based } \\
\text { on Need-driven } \\
\text { Dementia- } \\
\text { compromised } \\
\text { Behavior model, } \\
\text { tailored to } \\
\text { residents based } \\
\text { on either } \\
\text { physical/cognitiv } \\
\text { e function, } \\
\text { personality as } \\
\text { assessed } \\
\text { through the Five } \\
\text { Factor Model, or } \\
\text { both: }\end{array}$ & $\begin{array}{l}\text { Active control: } \\
\text { same } \\
\text { individual } \\
\text { recreational } \\
\text { activities } \\
\text { targeted } \\
\text { opposite to } \\
\text { both } \\
\text { physical/cogni } \\
\text { tive function } \\
\text { and } \\
\text { personality }\end{array}$ & $\begin{array}{l}\text { Nursing homes } \\
\text { Multisite } \\
9 \text { nursing } \\
\text { homes } \\
128 \text { PLWD }\end{array}$ & $\begin{array}{l}\mathrm{N}=128 \\
\text { Dementia by DSM- } \\
\text { IV criteria } \\
\text { Mean MMSE } 12.68 \\
\text { to } 15.78\end{array}$ & $\begin{array}{l}\mathrm{N}=128 \\
\text { Mean age } 85.87 \text { to } \\
87.21 \text { years } \\
\text { Percent White } \\
81.25 \% \text { to } 93.55 \% \\
\text { Mean years of } \\
\text { education } 11.64 \text { to } \\
13.87\end{array}$ & NR & NR & NR & NR & NR & Up to 7 weeks & $\begin{array}{l}\text { Agitation } \\
\text { Affect } \\
\text { Social } \\
\text { engagement }\end{array}$ & NR \\
\hline
\end{tabular}

High risk of bias studies included in evidence map. This table includes low and medium risk of bias studies only.

Abbreviations: char=characteristics; FC=formal caregiver; IC=informal caregiver; N=number; PMID=PubMed Identification Number; PLWD=Persons with Dementia; RoB=Risk of Bias; RS=Reporting Status; SES=socioeconomic status; NR=NR; RCT=Randomized controlled trial 
Table D-37. PLWD outcomes summary low and medium risk of bias studies: recreation therapy

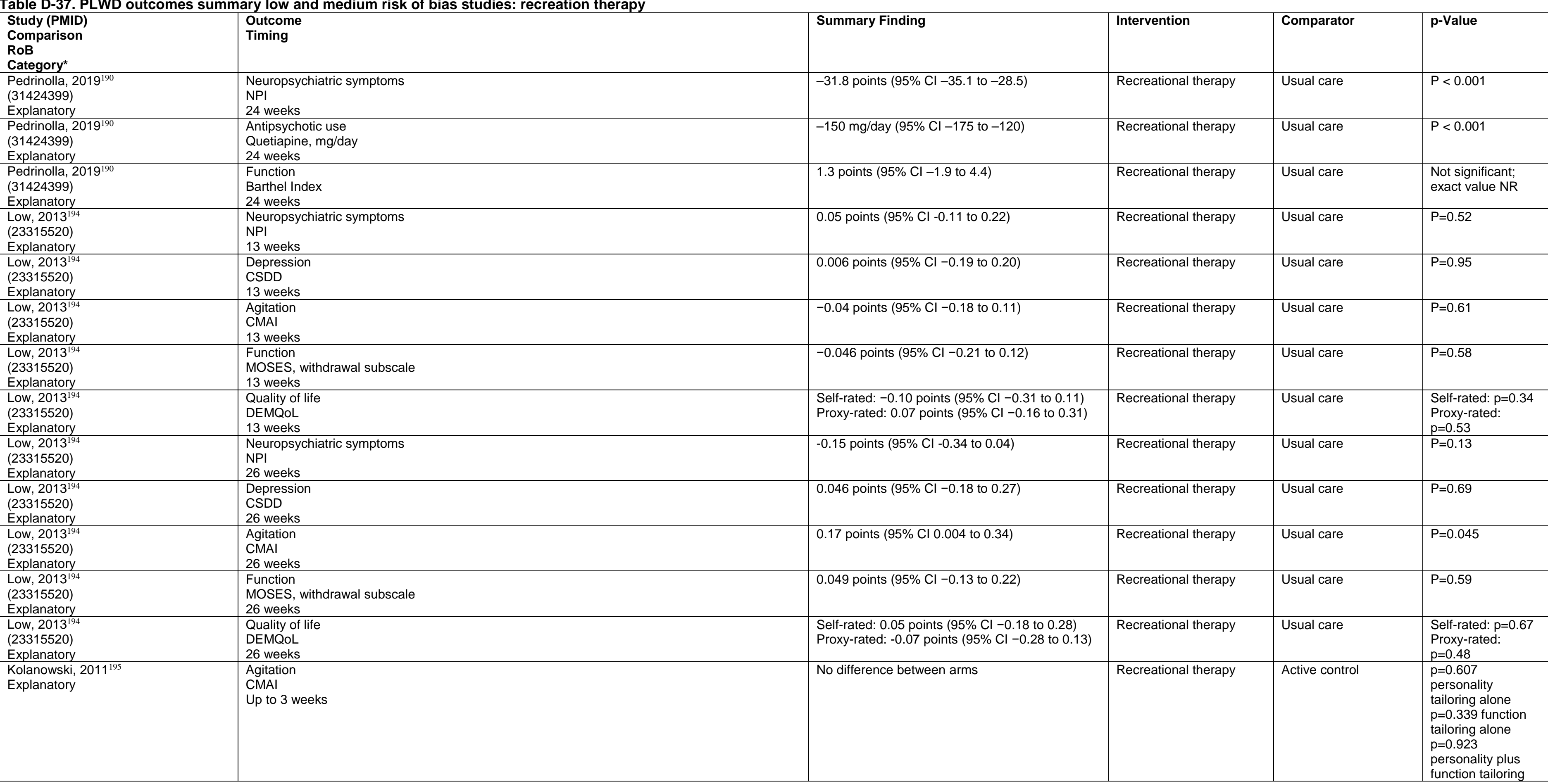

Explanatory studies are listed first, followed by pragmatic studies.

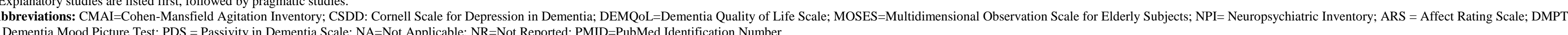


Table D-38. Summary of strength of evidence for PLWD outcomes: recreation therapy

\begin{tabular}{|c|c|c|c|c|c|c|c|c|}
\hline $\begin{array}{l}\text { Comparison } \\
\text { Outcome }\end{array}$ & Timing & $\begin{array}{l}\text { \# Studies/ Design } \\
\text { ( } \mathrm{n} \text { analyzed) }\end{array}$ & Finding or Summary Statistic & Study Limitations & Consistency & Directness & Precision & Overall Grade/ Conclusion \\
\hline $\begin{array}{l}\text { RT vs. Usual care } \\
\text { Neuropsychological } \\
\text { symptoms }\end{array}$ & 24-26 weeks & 2 RCTs (n=552) & Reduction in neuropsychological symptoms & Medium & Inconsistent & Direct & Imprecise & Insufficient \\
\hline \begin{tabular}{|l|} 
RT vs. Usual care \\
Depression \\
\end{tabular} & 26 weeks & $1 \mathrm{RCT}(\mathrm{n}=389)$ & Increase in depression symptoms & Medium & $\begin{array}{l}\text { Unknown (single } \\
\text { study) }\end{array}$ & Direct & Imprecise & Insufficient \\
\hline \begin{tabular}{|l} 
RT vs. Usual care \\
Agitation
\end{tabular} & $\begin{array}{l}\text { Up to } 13 \\
\text { weeks }\end{array}$ & $2 \mathrm{RCT}(\mathrm{n}=517)$ & No change in agitation symptoms & Medium & Inconsistent & Direct & Imprecise & Insufficient \\
\hline $\begin{array}{l}\text { RT vs. Usual care } \\
\text { Function, social } \\
\end{array}$ & 26 weeks & $1 \mathrm{RCT}(\mathrm{n}=389)$ & No change in social function & Medium & $\begin{array}{l}\text { Unknown (single } \\
\text { study) }\end{array}$ & Direct & Imprecise & Insufficient \\
\hline $\begin{array}{l}\text { RT vs. Usual care } \\
\text { Function, physical }\end{array}$ & 24 weeks & $1 \mathrm{RCT}(\mathrm{n}=163)$ & Improvement in physical function & Medium & $\begin{array}{l}\text { Unknown (single } \\
\text { study) }\end{array}$ & Direct & Imprecise & Insufficient \\
\hline $\begin{array}{l}\text { RT vs. Usual care } \\
\text { Quality of life, self-rated } \\
\end{array}$ & 26 weeks & $1 \mathrm{RCT}(\mathrm{n}=389)$ & Improvement in self-rated quality of life & Medium & $\begin{array}{l}\text { Unknown (single } \\
\text { study) }\end{array}$ & Direct & Imprecise & Insufficient \\
\hline $\begin{array}{l}\text { RT vs. Usual care } \\
\text { Quality of life, proxy- } \\
\text { rated }\end{array}$ & 26 weeks & $1 \mathrm{RCT}(\mathrm{n}=389)$ & Decline in proxy-rated quality of life & Medium & $\begin{array}{l}\text { Unknown (single } \\
\text { study) }\end{array}$ & Indirect & Imprecise & Insufficient \\
\hline \begin{tabular}{|l|} 
RT vs. Usual care \\
Antipsychotic use \\
\end{tabular} & 24 weeks & $1 \mathrm{RCT}(\mathrm{n}=163)$ & Decrease in antipsychotic dosage & Medium & $\begin{array}{l}\text { Unknown (single } \\
\text { study) }\end{array}$ & Direct & Imprecise & Insufficient \\
\hline
\end{tabular}

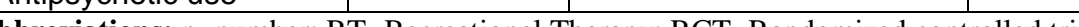

\section{Evidence Map: Recreation Therapy}

\begin{tabular}{|c|c|c|c|c|c|c|c|c|c|c|c|c|c|}
\hline $\begin{array}{l}\text { Study (PMID) } \\
\text { Country } \\
\text { EM Reason }\end{array}$ & Intervention & Comparison & $\begin{array}{l}\text { Setting } \\
\text { and Design } \\
\\
\text { Setting } \\
\text { Design } \\
\text { Cluster N } \\
\text { Participants } \\
\text { Random- } \\
\text { ized N }\end{array}$ & $\begin{array}{l}\text { PLWD } \\
\text { Dementia } \\
\text { Characteristics }\end{array}$ & $\begin{array}{l}\text { PLWD } \\
\text { Non-Disease Char } \\
\text { PLWD N } \\
\text { PLWD Age (mean) } \\
\text { PLWD Sex (\% } \\
\text { female) } \\
\text { PLWD Race (\% } \\
\text { majority) } \\
\text { PLWD Education } \\
\text { (mean years) }\end{array}$ & $\begin{array}{l}\text { PLWD } \\
\text { Non-Disease } \\
\text { Char Reporting } \\
\text { Status (RS) } \\
\\
\text { PLWD SES } \\
\text { PLWD Prior } \\
\text { Disability } \\
\text { PLWD } \\
\text { Household } \\
\text { Characteristics } \\
\text { PLWD Health } \\
\text { Insurance } \\
\text { PLWD Detailed } \\
\text { Race Information }\end{array}$ & $\begin{array}{l}\text { Informal Caregiver } \\
\text { (IC) } \\
\text { Characteristics } \\
\text { IC N } \\
\text { IC Age (mean) } \\
\text { IC Sex (\% female) } \\
\text { IC Race (\% } \\
\text { majority) } \\
\text { IC Education } \\
\text { (mean years) } \\
\text { IC Relation to } \\
\text { PLWD (\% majority) }\end{array}$ & $\begin{array}{l}\text { Informal Caregiver } \\
\text { (IC) Char. RS } \\
\text { IC Duration } \\
\text { IC Living With } \\
\text { PLWD } \\
\text { IC Payment } \\
\text { IC Health Status } \\
\text { IC Dementia } \\
\text { Family History } \\
\text { IC Employment } \\
\text { Status } \\
\text { IC Training }\end{array}$ & \begin{tabular}{|l} 
Formal Caregiver \\
(FC) \\
Characteristics \\
FC N \\
FC Age (mean) \\
FC Sex (\% \\
female) \\
FC Race (\% \\
majority) \\
FC Education \\
(mean years)
\end{tabular} & $\begin{array}{l}\text { Formal } \\
\text { Caregiver (FC) } \\
\text { Char. RS } \\
\text { FC Health } \\
\text { Status } \\
\text { FC Training } \\
\text { FC Education } \\
\text { FC Position } \\
\text { FC Length of } \\
\text { Service }\end{array}$ & $\begin{array}{l}\text { Outcome } \\
\text { Timing(s) }\end{array}$ & \begin{tabular}{|l|} 
PLWD \\
Outcomes
\end{tabular} & $\begin{array}{l}\text { Caregiver } \\
\text { Outcomes } \\
\text { IC: } \\
\text { FC: }\end{array}$ \\
\hline $\begin{array}{l}\text { Laksono, } 2019 \\
198 \\
\text { Indonesia } \\
\text { Small sample }\end{array}$ & Chess game & NR & $\begin{array}{l}\text { Community } \\
\text { residents } \\
\text { Single site } \\
20 \text { PLWD }\end{array}$ & NR & NR & NR & NR & $\mathrm{NR}$ & NR & NR & 2 weeks & MoCA & $\mathrm{NR}$ \\
\hline $\begin{array}{l}\text { Lathifah, } 2019 \\
199 \\
\text { India } \\
\text { Small sample }\end{array}$ & $\begin{array}{l}\text { Dhakonan } \\
\text { game }\end{array}$ & NR & $\begin{array}{l}\text { Community } \\
\text { residents } \\
\text { Single site } \\
20 \text { PLWD }\end{array}$ & NR & NR & NR & NR & NR & NR & NR & 0 weeks & GDS & NR \\
\hline
\end{tabular}




\begin{tabular}{|c|c|c|c|c|c|c|c|c|c|c|c|c|c|}
\hline $\begin{array}{l}\text { Tse, 2018191 } \\
\text { (30255637) } \\
\text { Hong Kong } \\
\text { High RoB }\end{array}$ & $\begin{array}{l}\text { Play activity } \\
\text { program }\end{array}$ & \begin{tabular}{|l|} 
Reading \\
sessions, \\
unstructured \\
group
\end{tabular} & $\begin{array}{l}\text { Nursing } \\
\text { homes } \\
\text { Multisite } \\
4 \text { clusters } \\
\text { 53 PLWD }\end{array}$ & $\begin{array}{l}\mathrm{N}=53 \\
\text { MMSE between } 10 \\
\text { and } 25\end{array}$ & \begin{tabular}{|l}
$\mathrm{N}=53$ \\
Age \\
Percent female \\
Education \\
Race NR
\end{tabular} & $\mathrm{NR}$ & NR & NR & NR & $\mathrm{NR}$ & $\begin{array}{l}8 \text { weeks } \\
12 \text { weeks }\end{array}$ & $\begin{array}{l}\text { Visual } \\
\text { analog scale } \\
\text { for } \\
\text { happiness } \\
\text { GDS } \\
\text { Katz ADL } \\
\text { scale } \\
\text { Index of } \\
\text { Social } \\
\text { Engagement } \\
\text { MDS } \\
\text { Behavior } \\
\text { Rating Scale } \\
\text { Non- } \\
\text { Pharmacolo } \\
\text { gical } \\
\text { Therapy } \\
\text { Experience } \\
\text { Scale }\end{array}$ & $\mathrm{NR}$ \\
\hline $\begin{array}{l}\text { Li, 2017200 } \\
(27345629) \\
\text { China } \\
\text { Small sample }\end{array}$ & $\begin{array}{l}\text { Folk recreation } \\
\text { intervention } \\
\text { involving art, } \\
\text { music, and } \\
\text { games }\end{array}$ & Usual care & $\begin{array}{l}\text { Long-term } \\
\text { care facility } \\
\text { Single site } \\
48 \text { PLWD }\end{array}$ & $\begin{array}{l}\mathrm{N}=48 \\
\text { Physician } \\
\text { diagnosis of } \\
\text { dementia, CDR > }\end{array}$ & $\begin{array}{l}\mathrm{N}=48 \\
\text { Age } \\
\text { Percent female } \\
\text { Education } \\
\text { Race NR }\end{array}$ & NR & NR & NR & NR & NR & 16 weeks & $\begin{array}{l}\text { Barthel } \\
\text { Index } \\
\text { NPI }\end{array}$ & NR1 \\
\hline $\begin{array}{l}\text { Pongan, 2017 } \\
\text { (31288544) } \\
\text { France } \\
\text { Pilot }\end{array}$ & $\begin{array}{l}\text { Directed singing } \\
\text { led by a choir } \\
\text { conductor }\end{array}$ & $\begin{array}{l}\text { Painting } \\
\text { sessions }\end{array}$ & $\begin{array}{l}\text { Memory } \\
\text { clinics } \\
\text { Multisite } \\
59 \text { PLWD }\end{array}$ & $\begin{array}{l}\text { N=59 } \\
\text { Mild AD, DSM-V } \\
\text { criteria }\end{array}$ & $\begin{array}{l}\mathrm{N}=59 \\
\text { Age } \\
\text { Percent female } \\
\text { Education } \\
\text { Race } \\
\end{array}$ & NR & NR & NR & NR & NR & 16 weeks & $\begin{array}{l}\text { STAI } \\
\text { GDS } \\
\text { EQ-5D } \\
\text { RSES }\end{array}$ & NR \\
\hline $\begin{array}{l}\text { Schall, 2017201 } \\
(28914089) \\
\text { Germany } \\
\text { Small sample }\end{array}$ & $\begin{array}{l}\text { Directed visits } \\
\text { to art museums } \\
\text { plus art-making } \\
\text { sessions }\end{array}$ & \begin{tabular}{|l} 
Unstructured \\
visits to art \\
museums
\end{tabular} & $\begin{array}{l}\text { Community } \\
44 \text { PLWD }\end{array}$ & $\begin{array}{l}\mathrm{N}=44 \\
\text { Diagnosis of mild to } \\
\text { moderate } \\
\text { dementia, criteria } \\
\mathrm{NR}\end{array}$ & $\begin{array}{l}\mathrm{N}=44 \\
\text { Age } \\
\text { Percent female } \\
\text { Education NR } \\
\text { Race NR } \\
\end{array}$ & $\mathrm{NR}$ & $\begin{array}{l}\mathrm{N}=44 \\
\mathrm{NR}\end{array}$ & $\mathrm{NR}$ & $\mathrm{NR}$ & $\mathrm{NR}$ & 12 weeks & $\begin{array}{l}\text { GDS } \\
\text { QoL-AD } \\
\text { NPI } \\
\text { FAHW } \\
\text { Smiley Scale }\end{array}$ & $\mathrm{NR}$ \\
\hline $\begin{array}{l}\text { Lin, 2015192 } \\
(607203463) \\
\text { China } \\
\text { High RoB }\end{array}$ & $\begin{array}{l}\text { Strategy board } \\
\text { game sessions, } \\
1-2 \text { hours daily }\end{array}$ & Not stated & $\begin{array}{l}\text { Hospital } \\
\text { Single site } \\
147 \text { PLWD }\end{array}$ & $\begin{array}{l}\mathrm{N}=147 \\
\text { NINCDS-ADRDA } \\
\text { diagnosis of } \\
\text { dementia }\end{array}$ & $\begin{array}{l}\mathrm{N}=147 \\
\mathrm{NR}\end{array}$ & NR & NR & NR & NR & NR & 24 weeks & $\begin{array}{l}\text { MADRS } \\
\text { HADS } \\
\text { RAND-36 }\end{array}$ & NR \\
\hline $\begin{array}{l}\text { Van Haitsma, } \\
2015^{193} \\
\text { (24304555) } \\
\text { US } \\
\text { High RoB }\end{array}$ & $\begin{array}{l}\text { Activity } \\
\text { sessions } \\
\text { tailored to } \\
\text { individual } \\
\text { preferences }\end{array}$ & \begin{tabular}{|l} 
Standard \\
activity \\
sessions, \\
involving \\
magazine \\
reading and \\
conversation
\end{tabular} & $\begin{array}{l}\text { Nursing } \\
\text { home } \\
\text { Single site } \\
180 \text { PLWD }\end{array}$ & $\mathrm{N}=180$ & $\begin{array}{l}\mathrm{N}=180 \text { Age } \\
\text { Percent female } \\
\text { Education } \\
\text { Race NR }\end{array}$ & NR & $\mathrm{NR}$ & NR & NR & NR & 3 weeks & $\begin{array}{l}\text { Direct } \\
\text { observation } \\
\text { of resident } \\
\text { behavior } \\
\text { MOSES } \\
\text { MDS ADL } \\
\text { scale }\end{array}$ & $\mathrm{NR}$ \\
\hline $\begin{array}{l}\text { Hattori, 2011202 } \\
(21518170) \\
\text { Japan } \\
\text { Small sample }\end{array}$ & $\begin{array}{l}\text { Art therapy in } \\
\text { groups }\end{array}$ & $\begin{array}{l}\text { Basic } \\
\text { mathematical } \\
\text { exercises }\end{array}$ & $\begin{array}{l}\text { Outpatient } \\
\text { clinic } \\
\text { Single site } \\
39 \text { PLWD }\end{array}$ & $\begin{array}{l}\mathrm{N}=39 \\
\text { NINCDS-ADRDA } \\
\text { diagnosis of } \\
\text { dementia } \\
\text { MMSE } \geq 20\end{array}$ & $\begin{array}{l}\mathrm{N}=39 \\
\text { Age } \\
\text { Percent female } \\
\text { Race NR } \\
\text { Education NR } \\
\end{array}$ & NR & NR & $\mathrm{NR}$ & NR & NR & 12 weeks & $\begin{array}{l}\text { GDS } \\
\text { Apathy } \\
\text { Scale } \\
\text { SF-8 } \\
\text { DBD }\end{array}$ & $\mathrm{NR}$ \\
\hline $\begin{array}{l}\text { Buettner, } \\
2002^{196} \\
(11954670) \\
\text { US } \\
\text { High RoB }\end{array}$ & $\begin{array}{l}\text { 15-minute } \\
\text { wheelchair } \\
\text { tandem bicycle } \\
\text { ride with a staff } \\
\text { member } \\
\text { pedaling the } \\
\text { bicycle }\end{array}$ & Not stated & $\begin{array}{l}\text { Nursing } \\
\text { home and } \\
\text { assisted } \\
\text { living facility } \\
\text { Multisite } \\
70 \text { PLWD }\end{array}$ & $\begin{array}{l}\mathrm{N}=70 \\
\mathrm{MMSE} \leq 24\end{array}$ & $\begin{array}{l}\mathrm{N}=70 \\
\mathrm{NR}\end{array}$ & $\mathrm{NR}$ & NR & $\mathrm{NR}$ & NR & $\mathrm{NR}$ & $\begin{array}{l}2 \text { weeks } \\
12 \text { weeks }\end{array}$ & $\begin{array}{l}\text { GDS } \\
\text { CMAI }\end{array}$ & $\mathrm{NR}$ \\
\hline
\end{tabular}




\begin{tabular}{|c|c|c|c|c|c|c|c|c|c|c|c|c|c|}
\hline $\begin{array}{l}\text { Hopman-Rock, } \\
\text { 1999197 } \\
\text { Netherlands } \\
\text { (29403282) } \\
\text { High RoB }\end{array}$ & $\begin{array}{l}\text { Biweekly } \\
\text { sporting activity } \\
\text { and game } \\
\text { sessions, } \\
\text { preferably in a } \\
\text { separate room, } \\
\text { targeting } \\
\text { communication, } \\
\text { reactivation, } \\
\text { resocialization, } \\
\text { and function }\end{array}$ & $\begin{array}{l}\text { Usual activities, } \\
\text { including light } \\
\text { housenhold } \\
\text { activities, bible } \\
\text { reading, singing }\end{array}$ & $\begin{array}{l}\text { Nursing } \\
\text { homes } \\
\text { Multisite } \\
12 \text { clusters } \\
134 \text { PLWD }\end{array}$ & $\begin{array}{l}N=134 \\
N R\end{array}$ & $\begin{array}{l}\mathrm{N}=134 \\
\text { Age } \\
\text { Percent female } \\
\text { Race NR } \\
\text { Education NR }\end{array}$ & $\overline{N R}$ & NR & NR & $\begin{array}{l}\mathrm{NR} \\
\end{array}$ & $\begin{array}{l}\mathrm{NR} \\
\end{array}$ & 24 weeks & $\begin{array}{l}\mathrm{BIP} \\
\text { SIPO }\end{array}$ & NR \\
\hline
\end{tabular}

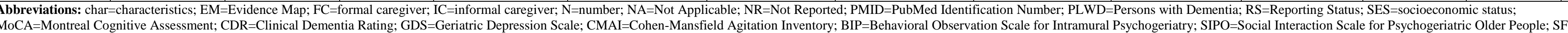

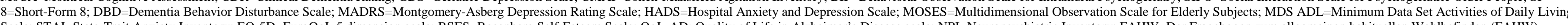

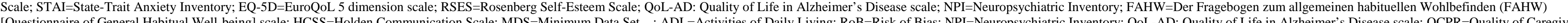
and Patient Relationship; DEMQOL=Dementia Quality of Life scale; CSDD=Cornell Scale for Depression in Dementia

\section{Psychosocial Interventions}

Table D-40. Risk of bias assessment: psychosocial interventions

\begin{tabular}{|c|c|c|c|c|c|c|c|c|c|}
\hline Study (PMID) & Outcome Timing & $\begin{array}{l}\text { Attrition Bias } \\
\text { Attrition \% }\end{array}$ & Selection Bias & Detection Bias & Performance Bias & Reporting Bias & Fidelity Bias & Funder & Overall Rating \\
\hline Onder 2005³ (16260821) & 25 weeks & $\begin{array}{l}\text { Medium } \\
12 \%\end{array}$ & Low & High & Medium & Low & High & Italian government & High \\
\hline
\end{tabular}

\section{Evidence Map: Psychosocial Interventions}

\begin{tabular}{|c|c|c|c|c|c|c|c|c|c|c|c|c|c|}
\hline $\begin{array}{l}\text { Study (PMID) } \\
\text { Country } \\
\text { EM Reason }\end{array}$ & Intervention & $\begin{array}{l}\text { Compari- } \\
\text { son }\end{array}$ & $\begin{array}{l}\text { Setting } \\
\text { and Design } \\
\text { Setting } \\
\text { Design } \\
\text { Cluster N } \\
\text { Participants } \\
\text { Randomized } \\
\text { N }\end{array}$ & $\begin{array}{l}\text { PLWD } \\
\text { Dementia } \\
\text { Characteristics }\end{array}$ & \begin{tabular}{|l} 
PLWD \\
Non-Disease Char \\
PLWD N \\
PLWD Age (mean) \\
PLWD Sex (\% \\
female) \\
PLWD Race (\% \\
majority) \\
PLWD Education \\
(mean years)
\end{tabular} & \begin{tabular}{|l} 
PLWD \\
Non-Disease \\
Char Reporting \\
Status (RS) \\
\\
PLWD SES \\
PLWD Prior \\
Disability \\
PLWD \\
Household \\
Characteristics \\
PLWD Health \\
Insurance \\
PLWD Detailed \\
Race Information \\
\end{tabular} & $\begin{array}{l}\text { Informal Caregiver } \\
\text { (IC) } \\
\text { Characteristics } \\
\text { IC N } \\
\text { IC Age (mean) } \\
\text { IC Sex (\% female) } \\
\text { IC Race (\% } \\
\text { majority) } \\
\text { IC Education } \\
\text { (mean years) } \\
\text { IC Relation to } \\
\text { PLWD (\% majority) }\end{array}$ & $\begin{array}{l}\text { Informal Caregiver } \\
\text { (IC) Char. RS } \\
\text { IC Duration } \\
\text { IC Living With } \\
\text { PLWD } \\
\text { IC Payment } \\
\text { IC Health Status } \\
\text { IC Dementia } \\
\text { Family History } \\
\text { IC Employment } \\
\text { Status } \\
\text { IC Training }\end{array}$ & $\begin{array}{l}\text { Formal Caregiver } \\
\text { (FC) } \\
\text { Characteristics } \\
\text { FC N } \\
\text { FC Age (mean) } \\
\text { FC Sex (\% } \\
\text { female) } \\
\text { FC Race (\% } \\
\text { majority) } \\
\text { FC Education } \\
\text { (mean years) }\end{array}$ & $\begin{array}{l}\text { Formal } \\
\text { Caregiver (FC) } \\
\text { Char. RS } \\
\text { FC Health } \\
\text { Status } \\
\text { FC Training } \\
\text { FC Education } \\
\text { FC Position } \\
\text { FC Length of } \\
\text { Service }\end{array}$ & $\begin{array}{l}\text { Outcome } \\
\text { Timing(s) }\end{array}$ & \begin{tabular}{|l|} 
PLWD \\
Outcomes
\end{tabular} & $\begin{array}{l}\text { Caregiver } \\
\text { Outcomes } \\
\text { IC: } \\
\text { FC: }\end{array}$ \\
\hline
\end{tabular}




\begin{tabular}{|c|c|c|c|c|c|c|c|c|c|c|c|c|c|}
\hline $\begin{array}{l}\text { Quinn 2016204 } \\
(26674087) \\
\text { UK } \\
\text { Pilot } \\
\\
\text { Quinn 2014205 } \\
\text { (24606601) } \\
\text { UK } \\
\text { Pilot }\end{array}$ & $\begin{array}{l}\text { Self-management } \\
\text { group intervention } \\
\text { for } 8 \text { weeks: } \\
\text { Weekly 90-minute } \\
\text { sessions covering } \\
\text { information } \\
\text { sharing on various } \\
\text { aspects such as } \\
\text { enjoying hobbies, } \\
\text { activities staying } \\
\text { well, practical } \\
\text { ways to manage } \\
\text { memory } \\
\text { difficulties, } \\
\text { maintaining } \\
\text { relationships and } \\
\text { social networks, } \\
\text { planning for the } \\
\text { future, coping } \\
\text { skills and } \\
\text { accessing local } \\
\text { resources. }\end{array}$ & TAU $(n=11)$ & \begin{tabular}{|l|} 
A memory \\
clinic in a \\
semi-rural area \\
of North Wales \\
RCT \\
24 PLWD
\end{tabular} & $\begin{array}{l}\text { ICD-10 diagnosis } \\
\text { of AD, vascular } \\
\text { dementia, or mixed } \\
\text { AD and vascular } \\
\text { dementia with } \\
\text { MMSE score } \geq 20\end{array}$ & $\begin{array}{l}\mathrm{N}=24 \\
\text { Mean age: } 76 \text { years } \\
\text { Female: } 25 \% \\
\% \text { majority race: no } \\
\text { Education: } 63 \% \\
\text { college/university }\end{array}$ & NR & $\begin{array}{l}\mathrm{N}=24 \\
\text { Mean age: } 67 \text { years } \\
\text { Female: } 79 \% \\
\text { \% majority race: no } \\
\text { Education: : } 42 \% \\
\text { college/university } \\
\text { Relationship with } \\
\text { PLWD: } 79 \% \\
\text { spouse/partner }\end{array}$ & $\begin{array}{l}\text { Living with PLWD: } \\
92 \%\end{array}$ & $\mathrm{NA}$ & NA & $\begin{array}{l}3 \text { months } \\
6 \text { months }\end{array}$ & $\begin{array}{l}\text { ACE-III } \\
\text { GSES } \\
\text { HADS } \\
\text { CORE-OM } \\
\text { EQ-5D-3L } \\
\text { ICECAP-O }\end{array}$ & NR \\
\hline $\begin{array}{l}\text { Young 2014206 } \\
\text { (25587218) } \\
\text { Hong Kong } \\
\text { Small sample }\end{array}$ & $\begin{array}{l}10 \text { weekly } \\
\text { sessions of } \\
\text { support group } \\
\text { (structured, time } \\
\text { limited, and } \\
\text { allowed } \\
\text { participants to } \\
\text { share on different } \\
\text { topics) for 90 } \\
\text { minutes }\end{array}$ & $\begin{array}{l}\text { standardized } \\
\text { educational } \\
\text { written } \\
\text { material on } \\
\text { dementia } \\
\text { that provided } \\
\text { basic } \\
\text { information } \\
\text { on dementia } \\
(n=19)\end{array}$ & $\begin{array}{l}\text { Non- } \\
\text { governmental } \\
\text { geriatric } \\
\text { centers } \\
\text { RCT } \\
\text { 39 PLWD }\end{array}$ & $\begin{array}{l}\text { Diagnosis of } \\
\text { dementia, MMSE } \\
\text { score } \geq 18\end{array}$ & $\begin{array}{l}\mathrm{N}=39 \\
\text { Mean age: } 80 \text { years } \\
\text { Female: } 44 \% \\
\text { \% majority race: no } \\
\text { Education: } 33 \% \\
\text { illiterate }\end{array}$ & NR & NA & NA & NA & NA & 10 weeks & $\begin{array}{l}\text { GDS-C } \\
\text { RSES-C } \\
\text { GSE-C } \\
\text { IMMEL }\end{array}$ & NA \\
\hline $\begin{array}{l}\text { Tappen } \\
2009^{207} \\
\text { (20077983) } \\
\text { US } \\
\text { Small sample }\end{array}$ & $\begin{array}{l}30 \text { minutes } \\
\text { modified } \\
\text { counseling } \\
\text { sessions, } 3 \text { times } \\
\text { per week for } 16 \\
\text { weeks }\end{array}$ & $\begin{array}{l}\text { Usual care } \\
(n=15)\end{array}$ & $\begin{array}{l}\text { Long-term care } \\
\text { facility } \\
\text { RCT } \\
36 \text { PLWD }\end{array}$ & $\begin{array}{l}\text { Clinical diagnosis } \\
\text { of probable AD } \\
\text { using NINCDS- } \\
\text { ADRDA; an MMSE } \\
\text { score } \leq 25\end{array}$ & $\begin{array}{l}\mathrm{N}=30 \\
\text { Mean age }=87 \text { years } \\
\text { Female: no } \\
\text { \% majority race: no } \\
\text { Education: } 39 \% \\
\text { illiterate }\end{array}$ & NR & NA & NA & NA & NA & $\begin{array}{l}6 \text { months } \\
12 \text { months }\end{array}$ & $\begin{array}{l}\text { DMAS } \\
\text { ADRD } \\
\text { MADRS }\end{array}$ & NA \\
\hline $\begin{array}{l}\text { Burns 2005208 } \\
(16055825) \\
\text { UK } \\
\text { Small sample }\end{array}$ & $\begin{array}{l}\text { Six, } 50 \text { minutes } \\
\text { sessions of } \\
\text { psychodynamic } \\
\text { interpersonal } \\
\text { therapy }\end{array}$ & TAU $(n=20)$ & $\begin{array}{l}\text { Referrals to } \\
\text { memory clinic } \\
\text { in South } \\
\text { Manchester, } \\
\text { UK } \\
\text { RCT } \\
40 \text { PLWD }\end{array}$ & $\begin{array}{l}\text { Diagnosis of AD } \\
\text { according to } \\
\text { NINCDS-ADRDA } \\
\text { criteria with a } \\
\text { clinical dementia } \\
\text { rating of } 1 \text { and a } \\
\text { score of } 15 \text { or } \\
\text { above on MMSE }\end{array}$ & $\begin{array}{l}\mathrm{N}=40 \\
\text { Mean age: } 76 \text { years } \\
\text { Female: } 48 \% \\
\text { \% majority race: no } \\
\text { Education: no }\end{array}$ & NR & NA & NR & NA & NA & $\begin{array}{l}6 \text { weeks } \\
3 \text { months }\end{array}$ & $\begin{array}{l}\text { CSDD } \\
\text { BADLS } \\
\text { RMPBC } \\
\text { MMSE } \\
\text { CIB-GIC }\end{array}$ & $\begin{array}{l}\text { GHQ } \\
\text { BDI } \\
\text { WCC }\end{array}$ \\
\hline
\end{tabular}




\begin{tabular}{|c|c|c|c|c|c|c|c|c|c|c|c|c|c|}
\hline $\begin{array}{l}\text { Onder 2005203 } \\
(16260821) \\
\text { Italy } \\
\text { High RoB }\end{array}$ & $\begin{array}{l}\text { PLWD treated } \\
\text { with donepezil got } \\
\text { a reality } \\
\text { orientation } \\
\text { program. CG then } \\
\text { trained to offer the } \\
\text { group were } \\
\text { trained to offer the } \\
\text { program at home } \\
3 \text { days a week, } 30 \\
\text { minutes/day, for } \\
25 \text { consecutive } \\
\text { weeks, and were } \\
\text { invited to stimulate } \\
\text { and involve PLWD } \\
\text { in reality-based } \\
\text { communication. }\end{array}$ & No treatment & \begin{tabular}{|l|} 
Community- \\
dwelling PLWD \\
RCT \\
156 PLWD
\end{tabular} & \begin{tabular}{|l|} 
Diagnosis of AD \\
according to \\
NINCDS-ADRA \\
with MMSE \\
between 14 and \\
27, did not present \\
with major aphasia \\
or blindness and \\
got donepezil \\
treatment for at \\
least 3 months
\end{tabular} & $\begin{array}{l}\mathrm{N}=156 \\
\text { Mean age: } 75.8 \\
\text { years } \\
\text { Female: } 72 \% \\
\text { \% majority race: no } \\
\text { Education: yes }\end{array}$ & NR & $\begin{array}{l}\mathrm{N}=156 \\
\text { Mean age: } 57 \text { years } \\
\text { Female: } 63 \% \\
\text { \% majority race: no } \\
\text { Education: no }\end{array}$ & NA & NA & NA & 25 weeks & $\begin{array}{l}\text { MMSE } \\
\text { ADAS-cog } \\
\text { IADL } \\
\text { NPI } \\
\text { medication } \\
\text { use }\end{array}$ & $\begin{array}{l}\text { HRSD } \\
\text { HRSA } \\
\text { SF-36 } \\
\text { Burden of } \\
\text { care }\end{array}$ \\
\hline $\begin{array}{l}\text { Cohen 1999209 } \\
(10495580) \\
\text { US } \\
\text { Quasi- } \\
\text { experimental }\end{array}$ & $\begin{array}{l}\text { The Buddy } \\
\text { Program: } \\
\text { Nondemented } \\
\text { residents were } \\
\text { trained via } 8 \text { hours } \\
\text { educational } \\
\text { sessions and } \\
\text { acted as "buddies" } \\
\text { to their demented } \\
\text { partner and } \\
\text { encouraged to } \\
\text { spend at least } 30 \\
\text { minutes of social } \\
\text { interaction } \\
\text { (game/music/book } \\
\text { /communication) }\end{array}$ & $\begin{array}{l}\text { No training } \\
\text { or partners } \\
(\mathrm{n}=31)\end{array}$ & $\begin{array}{l}\text { Nursing homes } \\
\text { Quasiexperime } \\
\text { ntal } \\
\text { 73 PLWD } \\
\text { (assigned; not } \\
\text { randomized) }\end{array}$ & $\begin{array}{l}\text { MMSE score } \leq 24 \\
\text { were considered as } \\
\text { potential dementia } \\
\text { participants but did } \\
\text { not show memory } \\
\text { or verbal } \\
\text { impairment }\end{array}$ & $\begin{array}{l}\mathrm{N}=73 \\
\text { Mean age: } 80 \text { years } \\
\text { Female: } 70 \% \\
\text { \% majority race: } \\
50 \% \text { African } \\
\text { American } \\
\text { Education: yes }\end{array}$ & \begin{tabular}{|l|}
$N R$ \\
\end{tabular} & NA & $\mathrm{NR}$ & NA & NA & $\begin{array}{l}3 \text { months } \\
6 \text { months }\end{array}$ & $\begin{array}{l}\text { MMSE } \\
\text { GDS } \\
\text { CSDD } \\
\text { CMAI } \\
\text { BEHAVE-AD } \\
\text { FAST } \\
\text { PGCMS } \\
\text { NPD } \\
\text { ADL } \\
\text { ESS } \\
\text { NSS }\end{array}$ & $\mathrm{NA}$ \\
\hline $\begin{array}{l}\text { Wallis } \\
1983^{210}(63542 \\
48) \\
\text { UK } \\
\text { Small Sample }\end{array}$ & $\begin{array}{l}\text { Reality Orientation } \\
\text { therapy for } 30 \\
\text { minutes, } 5 \\
\text { days/week for } 3 \\
\text { months }\end{array}$ & \begin{tabular}{|l} 
A variety of \\
group and \\
individual \\
activities \\
was offered \\
to them each \\
day \\
(attention \\
control)
\end{tabular} & \begin{tabular}{|l|} 
High Royds \\
Hospital \\
Quasiexperime \\
ntal \\
Unclear \\
\end{tabular} & \begin{tabular}{|l|} 
No diagnosis \\
criteria mentioned
\end{tabular} & $\begin{array}{l}\mathrm{N}=38 \\
\text { Mean age: } 70 \text { years } \\
\text { Female: } 34 \% \\
\text { \% majority race: no } \\
\text { Education: no }\end{array}$ & NR & NA & $\mathrm{NR}$ & NA & NA & $\begin{array}{l}2 \text { weeks } \\
3 \text { months } \\
4 \text { months }\end{array}$ & $\begin{array}{l}\text { Crichton scale } \\
\text { for behavioral } \\
\text { functioning } \\
\text { Cognitive } \\
\text { abilities }\end{array}$ & NA \\
\hline
\end{tabular}

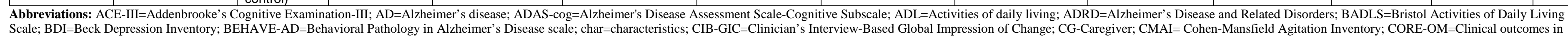

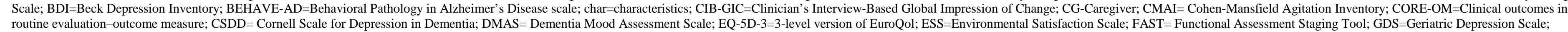

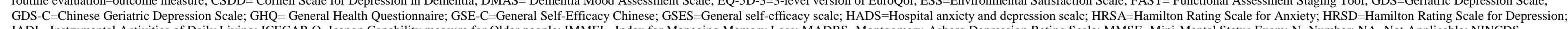

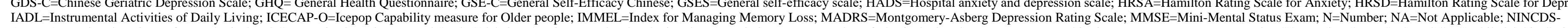

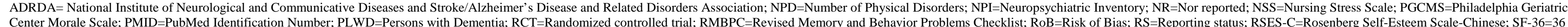
Item Shorte

\section{Creative Expression Therapy}

Table D-42. Risk of bias assessment: creative-expression therapy

\begin{tabular}{|c|c|c|c|c|c|c|c|c|c|}
\hline Study (PMID) & Outcome Timing & $\begin{array}{l}\text { Attrition Bias } \\
\text { Attrition } \%\end{array}$ & Selection Bias & Detection Bias & \begin{tabular}{|l} 
Performance Bias \\
\end{tabular} & Reporting Bias & Fidelity Bias & Funder & Overall Rating \\
\hline Fritsch $2009^{211}(19363009)$ & & High & $x$ & $\mathrm{x}$ & $x$ & $\mathrm{x}$ & $x$ & Nonprofit & High \\
\hline
\end{tabular}

Abbreviations: PMID=PubMed Identification Number 


\section{Evidence Map: Creative-Expression Therapy}

\begin{tabular}{|c|c|c|c|c|c|c|c|c|c|c|c|c|c|}
\hline $\begin{array}{l}\text { Study (PMID) } \\
\text { Country } \\
\text { EM Reason }\end{array}$ & Intervention & Comparison & $\begin{array}{l}\text { Setting } \\
\text { and Design } \\
\\
\text { Setting } \\
\text { Design } \\
\text { Cluster N } \\
\text { Participants } \\
\text { Randomized N }\end{array}$ & $\begin{array}{l}\text { PLWD } \\
\text { Dementia } \\
\text { Characteristics }\end{array}$ & $\begin{array}{l}\text { PLWD } \\
\text { Non-Disease } \\
\text { Char } \\
\\
\text { PLWD N } \\
\text { PLWD Age } \\
\text { (mean) } \\
\text { PLWD Sex (\% } \\
\text { female) } \\
\text { PLWD Race (\% } \\
\text { majority) } \\
\text { PLWD } \\
\text { Education } \\
\text { (mean years) }\end{array}$ & $\begin{array}{l}\text { PLWD } \\
\text { Non-Disease } \\
\text { Char Reporting } \\
\text { Status (RS) } \\
\text { PLWD SES } \\
\text { PLWD Prior } \\
\text { Disability } \\
\text { PLWD } \\
\text { Household } \\
\text { Characteristics } \\
\text { PLWD Health } \\
\text { Insurance } \\
\text { PLWD Detailed } \\
\text { Race } \\
\text { Information }\end{array}$ & $\begin{array}{l}\text { Informal } \\
\text { Caregiver (IC) } \\
\text { Characteristics } \\
\text { IC N } \\
\text { IC Age (mean) } \\
\text { IC Sex (\% } \\
\text { female) } \\
\text { IC Race (\% } \\
\text { majority) } \\
\text { IC Education } \\
\text { (mean years) } \\
\text { IC Relation to } \\
\text { PLWD (\% } \\
\text { majority) }\end{array}$ & \begin{tabular}{|l} 
Informal \\
Caregiver (IC) \\
Char. RS \\
IC Duration \\
IC Living With \\
PLWD \\
IC Payment \\
IC Health \\
Status \\
IC Dementia \\
Family History \\
IC Employment \\
Status \\
IC Training
\end{tabular} & $\begin{array}{l}\text { Formal } \\
\text { Caregiver (FC) } \\
\text { Characteristics } \\
\text { FC N } \\
\text { FC Age (mean) } \\
\text { FC Sex (\% } \\
\text { female) } \\
\text { FC Race (\% } \\
\text { majority) } \\
\text { FC Education } \\
\text { (mean years) }\end{array}$ & $\begin{array}{l}\text { Formal } \\
\text { Caregiver (FC) } \\
\text { Char. RS } \\
\text { FC Health } \\
\text { Status } \\
\text { FC Training } \\
\text { FC Education } \\
\text { FC Position } \\
\text { FC Length of } \\
\text { Service }\end{array}$ & $\begin{array}{l}\text { Outcome } \\
\text { Timing(s) }\end{array}$ & \begin{tabular}{|l|} 
PLWD \\
Outcomes
\end{tabular} & $\begin{array}{l}\text { Caregiver } \\
\text { Outcomes } \\
\text { IC: } \\
\text { FC: }\end{array}$ \\
\hline $\begin{array}{l}\text { Lin 2019212 } \\
\text { (31440055) } \\
\text { China } \\
\text { Pilot }\end{array}$ & $\begin{array}{l}\text { Creative } \\
\text { Expression } \\
\text { Therapy } \\
\text { (Timeslip) twice } \\
\text { a week for } 6 \\
\text { weeks }\end{array}$ & $\begin{array}{l}\text { Standard } \\
\text { Cognitive } \\
\text { Training }\end{array}$ & $\begin{array}{l}\text { Long-Term Care } \\
\text { Facilities, } \\
\text { Prospective } \\
\text { non-blinded } \\
\text { RCT, } \\
100 \text { PLWD }\end{array}$ & Not specified & $\begin{array}{l}\mathrm{N}=91 \text { (43 } \\
\text { treatment vs } 48 \\
\text { control) } \\
\text { Age: yes } \\
\text { Sex: yes } \\
\text { Race: yes } \\
\text { Education: yes }\end{array}$ & NR & NA & NA & NA & NA & 6 weeks & $\begin{array}{l}\text { Cognitive } \\
\text { Function, } \\
\text { Quality of Life, } \\
\text { Depression, } \\
\text { Communication } \\
\text { ability, } \\
\text { Emotion status }\end{array}$ & NA \\
\hline $\begin{array}{l}\text { Houser 2014 } \\
(23602304) \\
\text { US } \\
\text { Small sample }\end{array}$ & $\begin{array}{l}\text { Two 1-hour } \\
\text { Timeslip } \\
\text { sessions per } \\
\text { week for } 6 \\
\text { weeks }\end{array}$ & $\begin{array}{l}\text { Facilities } \\
\text { standard of care }\end{array}$ & $\begin{array}{l}\text { Nursing homes } \\
\text { Cluster RCT; } 1 \\
\text { site } \\
20 \text { PLWD }\end{array}$ & Not specified & $\begin{array}{l}\mathrm{N}=20(10 \\
\text { treatment vs } 10 \\
\text { control) } \\
\text { Age: yes } \\
\text { Sex: yes } \\
\% \text { majority race: } \\
\text { no } \\
\text { Education: no }\end{array}$ & NR & NA & NA & NA & NA & 4 months & $\begin{array}{l}\text { Mood score } \\
\text { Behavior score }\end{array}$ & NA \\
\hline
\end{tabular}




\begin{tabular}{|c|c|c|c|c|c|c|c|c|c|c|c|c|c|}
\hline $\begin{array}{l}\text { Van Dijk 2012214 } \\
\text { (15802911) } \\
\text { Netherlands } \\
\text { Pilot }\end{array}$ & $\begin{array}{l}\text { Group 1: Veder } \\
\text { method living } \\
\text { room theater } \\
\text { group with } \\
\text { trained care } \\
\text { professionals } \\
\text { Group 2: Veder } \\
\text { method living } \\
\text { room group with } \\
\text { professional } \\
\text { actors }\end{array}$ & $\begin{array}{l}\text { Standard } \\
\text { Reminiscence } \\
\text { group activity }\end{array}$ & $\begin{array}{l}\text { Psychiatric } \\
\text { Nursing Home, } \\
\text { Quasi- } \\
\text { experimental } \\
\text { non-equivalent } \\
\text { three group, } \\
\text { Multisite, } \\
155 \text { PLWD, } \\
20 \text { Trained } \\
\text { professional } \\
\text { caregivers, } \\
10 \text { professional } \\
\text { actors, } \\
11 \text { therapists } \\
\text { and nurses }\end{array}$ & $\begin{array}{l}\text { Alzheimer, } \\
\text { Vascular } \\
\text { dementia, } \\
\text { Dementia NOS }\end{array}$ & $\begin{array}{l}\mathrm{N}=155 \text { (69 } \\
\text { treatment 1, 31 } \\
\text { treatment 2 vs } \\
55 \text { control) } \\
\text { Age: yes } \\
\text { Sex: yes } \\
\% \text { majority race: } \\
\text { no } \\
\text { Education: yes }\end{array}$ & NR & NR & NR & NR & NR & 1 week & $\begin{array}{l}\text { Behavior } \\
\text { Mood } \\
\text { Quality of Life } \\
\text { Alertness } \\
\text { Memory recall } \\
\text { Social isolation } \\
\text { behaviors }\end{array}$ & NA \\
\hline $\begin{array}{l}\text { Phillips 2010215 } \\
(21048483) \\
\text { US } \\
\text { Pilot }\end{array}$ & $\begin{array}{l}\text { Timeslip } \\
\text { sessions twice a } \\
\text { week for } 6 \\
\text { weeks }\end{array}$ & $\begin{array}{l}\text { Facilities } \\
\text { standard of care }\end{array}$ & $\begin{array}{l}\text { Nursing homes } \\
\text { and Assisted } \\
\text { Living Facilities, } \\
\text { Quasi- } \\
\text { experimental } \\
\text { repeated } \\
\text { measures, } \\
\text { Multisite } \\
56 \text { PLWD }\end{array}$ & Not specified & $\begin{array}{l}\mathrm{N}=56(28 \\
\text { treatment vs } 28 \\
\text { control) } \\
\text { Age } \\
\text { Sex } \\
\text { Race } \\
\text { Education }\end{array}$ & $\begin{array}{l}\text { Household } \\
\text { characteristics }\end{array}$ & NA & NA & NA & NA & 6 weeks & $\begin{array}{l}\text { Cornell Scale for } \\
\text { Depression in } \\
\text { Dementia, } \\
\text { Neuropsychiatric } \\
\text { Inventory- } \\
\text { Nursing Home } \\
\text { Version, } \\
\text { Functional } \\
\text { Assessment of } \\
\text { Communication } \\
\text { Skills, } \\
\text { Quality of Life- } \\
\text { AD, } \\
\text { Observed } \\
\text { Emotion Rating } \\
\text { Scale }\end{array}$ & NA \\
\hline $\begin{array}{l}\text { Fritsch 2009211 } \\
\text { (19363009) } \\
\text { US } \\
\text { High RoB }\end{array}$ & $\begin{array}{l}\text { Staff members } \\
\text { trained in } \\
\text { Timeslip; } \\
\text { Timeslips group } \\
\text { once a week for } \\
1 \text { hour for } 10 \\
\text { weeks }\end{array}$ & $\begin{array}{l}\text { Facilities' } \\
\text { standard of care }\end{array}$ & $\begin{array}{l}\text { Nursing homes, } \\
\text { randomized } \\
\text { matched pair, } \\
20 \text { sites, } \\
\text { Undefined N } \\
\text { PLWD, } \\
\text { Undefined N } \\
\text { Daytime staff }\end{array}$ & Not specified & NR & NR & $\mathrm{NA}$ & NA & $\begin{array}{l}\mathrm{N}=\text { not given } \\
\text { Age: yes } \\
\text { Sex: yes } \\
\text { Race: yes } \\
\text { Education: no }\end{array}$ & $\begin{array}{l}\text { Health status: } \\
\text { no } \\
\text { Training: yes } \\
\text { Education: no } \\
\text { Position: yes } \\
\text { Service: yes }\end{array}$ & Two weeks & $\begin{array}{l}\text { Resident } \\
\text { Engagement, } \\
\text { Resident Affect }\end{array}$ & $\begin{array}{l}\text { FC: Staff- } \\
\text { Initiated } \\
\text { interactions with } \\
\text { Residents, } \\
\text { Attitudes } \\
\text { towards PLWD, } \\
\text { Job } \\
\text { Satisfaction, } \\
\text { Burnout }\end{array}$ \\
\hline
\end{tabular}

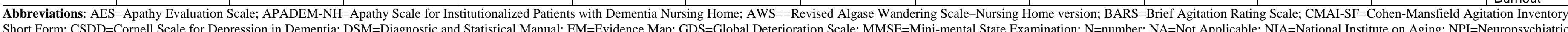

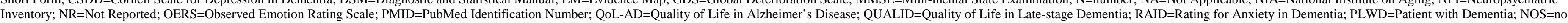
otherwise specified; FC=Formal Caregiver 


\section{Multicomponent for PLWD Well-Being}

\begin{tabular}{|c|c|c|c|c|c|c|c|c|}
\hline Study (PMID) & Outcome Timing & $\begin{array}{l}\text { Attrition Bias } \\
\text { Attrition \% }\end{array}$ & Selection Bias & Detection Bias & Performance Bias & Reporting Bias & Fidelity Bias & Overall Rating \\
\hline \begin{tabular}{|l|} 
Okamura $2018^{216}(30036871)$ \\
\end{tabular} & 3 months & $\begin{array}{l}\text { Low } \\
3 \text { months: } 1 \%\end{array}$ & Medium & High & High & Low & Low & High \\
\hline Rokstad 2018 217 (30445937) & 24 months & NA & High & $x$ & $x$ & $x$ & $x$ & High \\
\hline \begin{tabular}{|l} 
Masayuki Satoh $2017^{218}(28222531)$ \\
\end{tabular} & 6 months & $\begin{array}{l}\text { High } \\
6 \text { months:27\% }\end{array}$ & Medium & $x$ & $\mathrm{x}$ & $x$ & $x$ & High \\
\hline \begin{tabular}{|l|} 
Middelstadt 2016 \\
219 \\
(27497474)
\end{tabular} & 14 weeks & $\begin{array}{l}\text { Medium } \\
14 \text { weeks:20\% }\end{array}$ & Low & Low & Medium & Low & High & High \\
\hline \begin{tabular}{|l|} 
Fernandez-Calvo 2015²0 (25121567) \\
\end{tabular} & 16 weeks & $\begin{array}{l}\text { Low } \\
16 \text { weeks: } 10 \%\end{array}$ & Medium & Low & High & Low & High & High \\
\hline \begin{tabular}{|l} 
Luttenberger 2012221 \\
(22468985)
\end{tabular} & 6 months & $\begin{array}{l}\text { Medium } \\
6 \text { months: } 16 \%\end{array}$ & Low & High & Medium & Low & Medium & Medium \\
\hline \begin{tabular}{|l} 
Yamagami 2012222 (23300492) \\
\end{tabular} & 12 weeks & $\begin{array}{l}\text { Low } \\
12 \text { weeks: } 1 \%\end{array}$ & Medium & High & High & Low & Medium & High \\
\hline Brooker 2011223 (21702705) & 18 months & \begin{tabular}{|l|} 
High \\
18 months: $32 \%$
\end{tabular} & Low & $\mathrm{x}$ & $\mathrm{x}$ & $x$ & $\mathrm{x}$ & High \\
\hline \begin{tabular}{|l} 
Ferrero-Arias 2011224 (21346517) \\
\end{tabular} & $\begin{array}{l}4 \text { weeks } \\
8 \text { weeks }\end{array}$ & $\begin{array}{l}\text { High } \\
\text { Unclear }\end{array}$ & Medium & $x$ & $\mathrm{x}$ & $\mathrm{x}$ & $x$ & High \\
\hline \begin{tabular}{|l} 
McCurry $2011^{225}$ (21797835) \\
\end{tabular} & 2 months & $\begin{array}{l}\text { Low } \\
2 \text { months: } 13 \%\end{array}$ & Low & Medium & High & Low & Low & Medium \\
\hline McCurry $2011^{225}(21797835$ & 6 months & $\begin{array}{l}\text { Low } \\
6 \text { months: } 16 \%\end{array}$ & Low & Medium & High & Low & Low & Medium \\
\hline Femia $2007^{226}(18192631)$ & 2 months & $x$ & High & $\mathrm{x}$ & $x$ & $x$ & $x$ & High \\
\hline \begin{tabular}{|l} 
Chapman 200427 (15603468) \\
\end{tabular} & 4 months & $\begin{array}{l}\text { High } \\
4 \text { months: } 24 \%\end{array}$ & Low & $\mathrm{x}$ & $\mathrm{x}$ & $x$ & $x$ & High \\
\hline Tadaka $2004^{228}$ (No PMID) & 3 months & $\begin{array}{l}\text { Low } \\
3 \text { months: } 8 \%\end{array}$ & Low & Medium & Medium & Low & High & Medium \\
\hline \begin{tabular}{|l} 
Tadaka $2004^{228}$ (No PMID) \\
\end{tabular} & 6 months & $\begin{array}{l}\text { Medium } \\
6 \text { months: } 17 \%\end{array}$ & Low & Medium & Medium & Low & High & Medium \\
\hline Wimo $1993^{229}$ (8356361) & 12 months & $x$ & High & $\mathrm{x}$ & $\mathrm{x}$ & $x$ & $x$ & High \\
\hline
\end{tabular}

Abbreviations: PMID=PubMed Identification Number; PLWD=persons with dementia 
Table D-45. Characteristics of included studies: multicomponent for PLWD well-being

\begin{tabular}{|c|c|c|c|c|c|c|c|c|c|c|c|c|c|}
\hline $\begin{array}{l}\text { Study (PMID) } \\
\text { Country } \\
\text { RoB* } \\
\text { Type }\end{array}$ & $\begin{array}{l}\text { Intervention } \\
\text { Intervention } \\
\text { Focus } \\
\text { Theoretical } \\
\text { Model } \\
\text { Delivery } \\
\text { Person } \\
\text { Intervention } \\
\text { Target } \\
\text { Recipient } \\
\text { Mode } \\
\text { Components } \\
\text { Frequency } \\
\text { Duration }\end{array}$ & $\begin{array}{l}\text { Comparison } \\
\text { Target } \\
\text { Mode } \\
\text { Components } \\
\text { Frequency } \\
\text { Duration }\end{array}$ & $\begin{array}{l}\text { Setting } \\
\text { and Design } \\
\text { Setting } \\
\text { Design } \\
\text { Cluster N } \\
\text { Participants } \\
\text { Random- } \\
\text { ized N }\end{array}$ & $\begin{array}{l}\text { LWD } \\
\text { Dementia } \\
\text { Characteristics } \\
\text { Dementia Types } \\
\text { Dementia Severity } \\
\text { Diagnostic } \\
\text { Criteria } \\
\text { Age of Diagnosis }\end{array}$ & $\begin{array}{l}\text { PLWD } \\
\text { Non-Disease Char } \\
\text { PLWD N } \\
\text { PLWD Age (mean) } \\
\text { PLWD Sex (\% } \\
\text { female) } \\
\text { PLWD Race (\% } \\
\text { majority) } \\
\text { PLWD Education } \\
\text { (mean years) }\end{array}$ & $\begin{array}{l}\text { PLWD } \\
\text { Non-Disease } \\
\text { Char Reporting } \\
\text { Status (RS) } \\
\text { PLWD SES } \\
\text { PLWD Prior } \\
\text { Disability } \\
\text { PLWD } \\
\text { Household } \\
\text { Characteristics } \\
\text { PLWD Health } \\
\text { Insurance } \\
\text { PLWD Detailed } \\
\text { Race Information } \\
\end{array}$ & $\begin{array}{l}\text { Informal Caregiver } \\
\text { (IC) } \\
\text { Characteristics } \\
\text { IC N } \\
\text { IC Age (mean) } \\
\text { IC Sex (\% female) } \\
\text { IC Race (\% } \\
\text { majority) } \\
\text { IC Education } \\
\text { (mean years) } \\
\text { IC Relation to } \\
\text { PLWD (\% majority) }\end{array}$ & $\begin{array}{l}\text { Informal Caregiver } \\
\text { (IC) Char. RS } \\
\text { IC Duration } \\
\text { IC Living With } \\
\text { PLWD } \\
\text { IC Payment } \\
\text { IC Health Status } \\
\text { IC Dementia } \\
\text { Family History } \\
\text { IC Employment } \\
\text { Status } \\
\text { IC Training }\end{array}$ & 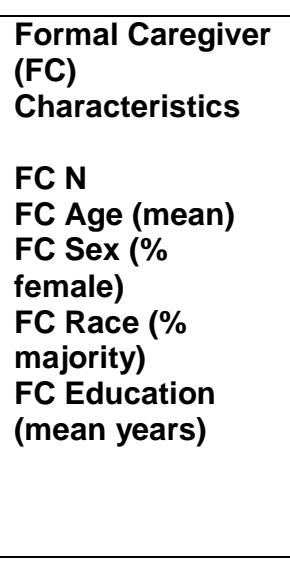 & $\begin{array}{l}\text { Formal } \\
\text { Caregiver (FC) } \\
\text { Char. RS } \\
\text { FC Health } \\
\text { Status } \\
\text { FC Training } \\
\text { FC Education } \\
\text { FC Position } \\
\text { FC Length of } \\
\text { Service }\end{array}$ & $\begin{array}{l}\text { Outcome } \\
\text { Timing(s) }\end{array}$ & $\begin{array}{l}\text { PLWD } \\
\text { Outcomes }\end{array}$ & $\begin{array}{l}\text { Caregiver } \\
\text { Outcomes } \\
\text { IC: } \\
\text { FC: }\end{array}$ \\
\hline $\begin{array}{l}\text { Luttenberger } \\
2012^{221} \\
\text { (22468985) } \\
\text { Germany } \\
\text { Medium } \\
\text { Explanatory }\end{array}$ & $\begin{array}{l}\text { MAKS } \\
\text { intervention } \\
\text { consisting of } \\
\text { motor } \\
\text { stimulation, ADL } \\
\text { activities and } \\
\text { cognitive } \\
\text { stimulation }\end{array}$ & Standard care & $\begin{array}{l}\text { Nursing } \\
\text { homes } \\
\text { RCT }\end{array}$ & $\begin{array}{l}\text { PLWD diagnosed } \\
\text { with primary } \\
\text { degenerative } \\
\text { dementia } \\
\text { MMSE <24 } \\
\text { Exclusion criteria }\end{array}$ & $\begin{array}{l}\mathrm{N}=30 \text { Intervention } \\
\text { group } \\
22 \text { Control group } \\
\text { Age: } \mathrm{Y} \\
\text { Sex: } \mathrm{Y} \\
\text { Education: } \mathrm{Y} \\
\text { Race \% majority: } \mathrm{N}\end{array}$ & Marital Status & NA & NA & Education: $Y$ & Training: $Y$ & $\begin{array}{l}12 \text { months } \\
\text { Additional } \\
\text { follow-up after } \\
10 \text { additional } \\
\text { months }\end{array}$ & ADL & NA \\
\hline $\begin{array}{l}\text { McCurry 2011225 } \\
(21797835) \\
\text { US } \\
\text { Medium } \\
\text { Explanatory }\end{array}$ & $\begin{array}{l}\text { Increased } \\
\text { walking and/or } \\
\text { bright light } \\
\text { exposure to } \\
\text { improve sleep }\end{array}$ & Contact only & $\begin{array}{l}\text { Community- } \\
\text { based } \\
\text { RCT }\end{array}$ & $\begin{array}{l}\text { PLWD diagnosed } \\
\text { with probable or } \\
\text { possible AD } \\
\text { Two or more sleep } \\
\text { problems multiple } \\
\text { times a week } \\
\text { Average wake time } \\
\text { of } 1 \text { hour per night } \\
\text { or greater }\end{array}$ & $\begin{array}{l}\mathrm{N}=27 \text { Walking group } \\
29 \text { Light group } \\
\text { 33Insomnia } \\
\text { Treatment and } \\
\text { Education group } \\
\text { Age: } Y \\
\text { Sex } Y \\
\text { Education: } Y \\
\text { Race \% majority: } Y\end{array}$ & NR & $\begin{array}{l}\text { Age: } Y \\
\text { Sex: } Y \\
\text { Education: } Y \\
\text { Race \% majority: } Y \\
\text { Spouse or other: } Y\end{array}$ & $\mathrm{NR}$ & $\begin{array}{l}\text { Education: } \mathrm{Y} \\
\text { Experience: } \mathrm{Y}\end{array}$ & Training: $Y$ & 6 months & $\begin{array}{l}\text { Sleep } \\
\text { Disorders } \\
\text { Inventory } \\
\text { Secondary } \\
\text { sleep } \\
\text { outcomes } \\
\text { measuremen } \\
\text { ts }\end{array}$ & $\begin{array}{l}\text { Satisfaction } \\
\text { survey }\end{array}$ \\
\hline \begin{tabular}{|l} 
Tadaka 2000228 \\
(PMID NR) \\
Japan \\
Medium \\
Explanatory
\end{tabular} & $\begin{array}{l}\text { Group care } \\
\text { program of } \\
\text { reminiscence } \\
\text { and reality } \\
\text { orientation care }\end{array}$ & Routine care & $\begin{array}{l}\text { Community- } \\
\text { based } \\
\text { RCT }\end{array}$ & $\begin{array}{l}\text { PLWD diagnosed } \\
\text { with AD or vascular } \\
\text { dementia } \\
\text { CDR score of } 1 \text { or } 2 \\
\text { Exclusion criteria }\end{array}$ & $\begin{array}{l}\mathrm{N}=2 \text { Intervention } \\
\text { group26 Control } \\
\text { group } \\
\text { Age: } \mathrm{Y} \\
\text { Sex: } \mathrm{Y} \\
\text { Education: } \mathrm{N} \\
\text { Race \% majority: } \mathrm{N}\end{array}$ & NR & $\begin{array}{l}\text { Relation to PLWD: } \\
\mathrm{Y}\end{array}$ & NR & $\begin{array}{l}\text { Education: } \mathrm{Y} \\
\text { Experience: } \mathrm{Y}\end{array}$ & Training: $Y$ & $\begin{array}{l}10 \text { weeks with } \\
\text { follow-up at } 6 \\
\text { months }\end{array}$ & $\begin{array}{l}\text { MMSE } \\
\text { MOSES } \\
\text { Self-care } \\
\text { Disorientatio } \\
n \\
\text { Depression } \\
\text { Irritability } \\
\text { Withdrawal } \\
\end{array}$ & NR \\
\hline
\end{tabular}

* High risk of bias studies included in evidence map. This table includes low and medium risk of bias studies only

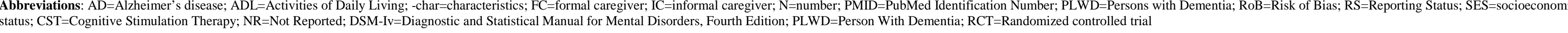

\begin{tabular}{|c|c|c|c|c|c|}
\hline $\begin{array}{l}\text { Study (PMID) } \\
\text { Comparison } \\
\text { RoB } \\
\text { Category* }\end{array}$ & $\begin{array}{l}\text { Outcome } \\
\text { Timing }\end{array}$ & Summary Finding & Intervention & Comparator & $\begin{array}{l}\text { p-Value } \\
\end{array}$ \\
\hline $\begin{array}{l}\text { Luttenberger } 2012230(22468985) \\
\text { MAKS therapy vs. Usual care } \\
\text { Medium } \\
\text { Explanatory }\end{array}$ & $\begin{array}{l}\text { E-ADL test (Range } 0 \text { to } 30 \text {, higher better) } \\
\text { Cohen's d } \\
12 \text { months }\end{array}$ & $\begin{array}{l}\text { No difference between groups for ITT analysis (Cohen's } d \\
0.23 \text { ) } \\
\text { Significant difference in PP analysis }(d=0.50)\end{array}$ & NR & NR & $\mathrm{NR}$ \\
\hline $\begin{array}{l}\text { McCurry 2011225 (21797835) } \\
\text { NITE-AD (Sleep education) vs. contact } \\
\text { control } \\
\text { Medium } \\
\text { Explanatory }\end{array}$ & $\begin{array}{l}\text { Sleep Disorders Inventory } \\
\text { Daily mean (SE) } \\
6 \text { months }\end{array}$ & No difference between groups & $0.8(0.2)$ & $0.5(0.1)$ & 0.12 \\
\hline
\end{tabular}




\begin{tabular}{|c|c|c|c|c|c|}
\hline $\begin{array}{l}\text { Study (PMID) } \\
\text { Comparison } \\
\text { RoB } \\
\text { Category* }\end{array}$ & \begin{tabular}{l|} 
Outcome \\
Timing
\end{tabular} & Summary Finding & Intervention & Comparator & p-Value \\
\hline $\begin{array}{l}\text { Tadaka } 2004^{228} \text { (PMID NR) } \\
\text { Group care program consisting of } \\
\text { reminiscence and reality orientation } \\
\text { care methods in addition to a routine } \\
\text { day-care service vs. Routine day-care } \\
\text { service } \\
\text { Medium } \\
\text { Explanatory }\end{array}$ & $\begin{array}{l}\text { Self-care category of MOSES } \\
\text { LS Mean (SE) adjusted for baseline and clinical dementia rating } \\
6 \text { months }\end{array}$ & No difference between groups & $16.1(1.0)$ & $18.5(1.0)$ & 0.05 \\
\hline $\begin{array}{l}\text { Tadaka } 2000^{228}(\text { PMID NR) } \\
\text { Group care program consisting of } \\
\text { reminiscence and reality orientation } \\
\text { care methods in addition to a routine } \\
\text { day-care service vs. Routine day-care } \\
\text { service } \\
\text { Medium } \\
\text { Explanatory }\end{array}$ & $\begin{array}{l}\text { Disorientation category of MOSES } \\
\text { LS Mean (SE) adjusted for baseline and clinical dementia rating } \\
6 \text { months }\end{array}$ & Favors intervention (lower score better) & $16.0(1.3)$ & $18.3(1.3)$ & 0.01 \\
\hline $\begin{array}{l}\text { Tadaka } 2004^{228} \text { (PMID NR) } \\
\text { Group care program consisting of } \\
\text { reminiscence and reality orientation } \\
\text { care methods in addition to a routine } \\
\text { day-care service vs. Routine day-care } \\
\text { service } \\
\text { Medium } \\
\text { Explanatory }\end{array}$ & $\begin{array}{l}\text { Depression category of MOSES } \\
\text { LS Mean (SE) adjusted for baseline and clinical dementia rating } \\
6 \text { months }\end{array}$ & No difference between groups & $17.1(1.2)$ & $15.9(1.2)$ & 0.97 \\
\hline $\begin{array}{l}\text { Tadaka } 2004^{228} \text { (PMID NR) } \\
\text { Group care program consisting of } \\
\text { reminiscence and reality orientation } \\
\text { care methods in addition to a routine } \\
\text { day-care service vs. Routine day-care } \\
\text { service } \\
\text { Medium } \\
\text { Explanatory }\end{array}$ & $\begin{array}{l}\text { Irritability category of MOSES } \\
\text { LS Mean (SE) adjusted for baseline and clinical dementia rating } \\
6 \text { months }\end{array}$ & No difference between groups & $14.9(1.0)$ & $15.0(1.0)$ & 0.34 \\
\hline $\begin{array}{l}\text { Tadaka } 2004^{228} \text { (PMID NR) } \\
\text { Group care program consisting of } \\
\text { reminiscence and reality orientation } \\
\text { care methods in addition to a routine } \\
\text { day-care service vs. Routine day-care } \\
\text { service } \\
\text { Medium } \\
\text { Explanatory }\end{array}$ & $\begin{array}{l}\text { Withdrawal category of MOSES } \\
\text { LS Mean (SE) adjusted for baseline and clinical dementia rating } \\
6 \text { months }\end{array}$ & Favors intervention (lower score better) & $16.6(1.1)$ & $19.5(1.1)$ & 0.02 \\
\hline
\end{tabular}

Explanatory 
Table D-47. Summary of strength of evidence for PLWD outcomes: multicomponent for PLWD well-being

\begin{tabular}{|c|c|c|c|c|c|c|c|c|}
\hline $\begin{array}{l}\text { Comparison } \\
\text { Outcome }\end{array}$ & Timing & $\begin{array}{l}\text { \# Studies/ Design } \\
\text { (n analyzed) }\end{array}$ & Finding or Summary Statistic & Study Limitations & Consistency & Directness & Precision & Overall Grade/ Conclusion \\
\hline \begin{tabular}{|l|} 
Instrumental Activities \\
of Daily Living \\
Multicomponent: motor \\
stimulation, activities of \\
daily living, and \\
cognitive stimulation \\
components contact \\
control \\
\end{tabular} & 6 months & $1 \mathrm{RCT}(\mathrm{n}=98)$ & No difference in instrumental activities of daily living & Medium & Unknown & Direct & Imprecise & Insufficient \\
\hline \begin{tabular}{|l|} 
Sleep \\
Walking, light, \\
combination treatment \\
versus contact control
\end{tabular} & 6 months & $1 \mathrm{RCT}(\mathrm{n}=66)$ & No difference in sleep disorders inventory & Medium & Unknown & Direct & Imprecise & Insufficient \\
\hline $\begin{array}{l}\text { Cognitive and Social } \\
\text { Function Reminiscence } \\
\text { and reality orientation } \\
\text { care methods, routine } \\
\text { day-care service versus } \\
\text { usual care }\end{array}$ & 6 months & $1 \mathrm{RCT}(\mathrm{n}=60)$ & $\begin{array}{l}\text { No difference in self-care, depression, or irritability } \\
\text { Favors intervention for withdrawal and disorientation }\end{array}$ & Medium & Unknown & Direct & Imprecise & Insufficient \\
\hline
\end{tabular}

\section{Evidence Map: Multicomponent for PLWD Well-Being}

\begin{tabular}{|c|c|c|c|c|c|c|c|c|c|c|c|c|c|}
\hline $\begin{array}{l}\text { Study (PMID) } \\
\text { Country } \\
\text { EM Reason }\end{array}$ & Intervention & Comparison & $\begin{array}{l}\text { Setting } \\
\text { and Design } \\
\text { Setting } \\
\text { Design } \\
\text { Cluster N } \\
\text { Participants } \\
\text { Random- } \\
\text { ized N }\end{array}$ & $\begin{array}{l}\text { PLWD } \\
\text { Dementia } \\
\text { Characteristics }\end{array}$ & $\begin{array}{l}\text { PLWD } \\
\text { Non-Disease Char } \\
\text { PLWD N } \\
\text { PLWD Age (mean) } \\
\text { PLWD Sex (\% } \\
\text { female) } \\
\text { PLWD Race (\% } \\
\text { majority) } \\
\text { PLWD Education } \\
\text { (mean years) }\end{array}$ & $\begin{array}{l}\text { PLWD } \\
\text { Non-Disease } \\
\text { Char Reporting } \\
\text { Status (RS) } \\
\\
\text { PLWD SES } \\
\text { PLWD Prior } \\
\text { Disability } \\
\text { PLWD } \\
\text { Household } \\
\text { Characteristics } \\
\text { PLWD Health } \\
\text { Insurance } \\
\text { PLWD Detailed } \\
\text { Race Information }\end{array}$ & $\begin{array}{l}\text { Informal Caregiver } \\
\text { (IC) } \\
\text { Characteristics } \\
\text { IC N } \\
\text { IC Age (mean) } \\
\text { IC Sex (\% female) } \\
\text { IC Race (\% } \\
\text { majority) } \\
\text { IC Education } \\
\text { (mean years) } \\
\text { IC Relation to } \\
\text { PLWD (\% majority) }\end{array}$ & $\begin{array}{l}\text { Informal Caregiver } \\
\text { (IC) Char. RS } \\
\text { IC Duration } \\
\text { IC Living With } \\
\text { PLWD } \\
\text { IC Payment } \\
\text { IC Health Status } \\
\text { IC Dementia } \\
\text { Family History } \\
\text { IC Employment } \\
\text { Status } \\
\text { IC Training }\end{array}$ & $\begin{array}{l}\text { Formal Caregiver } \\
\text { (FC) } \\
\text { Characteristics } \\
\text { FC N } \\
\text { FC Age (mean) } \\
\text { FC Sex (\% } \\
\text { female) } \\
\text { FC Race (\% } \\
\text { majority) } \\
\text { FC Education } \\
\text { (mean years) }\end{array}$ & $\begin{array}{l}\text { Formal } \\
\text { Caregiver (FC) } \\
\text { Char. RS } \\
\text { FC Health } \\
\text { Status } \\
\text { FC Training } \\
\text { FC Education } \\
\text { FC Position } \\
\text { FC Length of } \\
\text { Service }\end{array}$ & \begin{tabular}{|l|} 
Outcome \\
Timing(s)
\end{tabular} & $\begin{array}{l}\text { PLWD } \\
\text { Outcomes }\end{array}$ & $\begin{array}{l}\text { Caregiver } \\
\text { Outcomes } \\
\text { IC: } \\
\text { FC: }\end{array}$ \\
\hline $\begin{array}{l}\text { Sado 2020231 } \\
\text { (32116248) } \\
\text { Japan } \\
\text { Pilot }\end{array}$ & $\begin{array}{l}\text { Combination of } \\
\text { cognitive } \\
\text { training and } \\
\text { stimulation }\end{array}$ & $\begin{array}{l}\text { Unspecified } \\
\text { control group } \\
\text { volunteers from } \\
\text { within nursing } \\
\text { home }\end{array}$ & $\begin{array}{l}\text { Nursing } \\
\text { homes } \\
\text { Controlled } \\
\text { Trial (CT) } \\
\text { Cluster } \mathrm{N}=23 \\
\mathrm{~N}=57 \text { (non- } \\
\text { randomized) }\end{array}$ & $\begin{array}{l}\text { Aged } 6 \text { years or } \\
\text { older } \\
\text { Diagnosis of } \\
\text { dementia } \\
\text { Long-term- } \\
\text { care insurance } \\
\text { MMSE between } 10 \\
\text { and } 26 \\
\text { Living in nursing } \\
\text { homes; } \\
\text { No previous } \\
\text { experience of the } \\
\text { intervention }\end{array}$ & $\begin{array}{l}\mathrm{N}=30 \\
\text { Age: Yes } \\
\text { Sex: Yes } \\
\text { Race: No } \\
\text { Education: No }\end{array}$ & NR & NA & NA & NR & NR & 12 months & ADL & NR \\
\hline
\end{tabular}




\begin{tabular}{|c|c|c|c|c|c|c|c|c|c|c|c|c|c|}
\hline $\begin{array}{l}\text { Young 2019232 } \\
\text { (29781725) } \\
\text { Hong Kong } \\
\text { Pilot }\end{array}$ & $\begin{array}{l}\text { Structured } \\
\text { cognitive } \\
\text { stimulation } \\
\text { therapy and tai } \\
\text { chi }\end{array}$ & $\begin{array}{l}\text { Treatment as } \\
\text { usual; waitlist }\end{array}$ & \begin{tabular}{|l} 
Community- \\
based \\
RCT with \\
waitlist for \\
control \\
group \\
101 PLWD
\end{tabular} & $\begin{array}{l}\text { PLWD diagnosed } \\
\text { with mild stage } \\
\text { dementia } \\
\text { MMSE }>=18 \\
\text { Aged } 60 \text { or older }\end{array}$ & $\begin{array}{l}\mathrm{N}=101 \\
\text { Age: Yes } \\
\text { Sex: Yes } \\
\text { Race \% majority: No } \\
\text { Education: Yes }\end{array}$ & $\begin{array}{l}\text { Household } \\
\text { characteristics: } \\
\text { Yes }\end{array}$ & $\mathrm{NR}$ & NR & NA & NA & 7 weeks & $\begin{array}{l}\text { DSR } \\
\text { dementia } \\
\text { rating tests } \\
\text { MMSE }\end{array}$ & NA \\
\hline $\begin{array}{l}\text { Chen 2018 } \\
\text { (29881235) } \\
\text { Taiwan } \\
\text { Small sample }\end{array}$ & $\begin{array}{l}\text { Music-based } \\
\text { dual task } \\
\text { training }\end{array}$ & $\begin{array}{l}\text { Non-music- } \\
\text { based walking }\end{array}$ & $\begin{array}{l}\text { Community- } \\
\text { based } \\
\text { RCT } \\
28 \text { PLWD }\end{array}$ & $\begin{array}{l}\text { PLWD diagnosed } \\
\text { with mild to } \\
\text { moderate dementia } \\
\text { CDR score } 0.5,1 \\
\text { or 2; aged } 55 \text { or } \\
\text { older, ambulatory } \\
\text { Exclusion criteria } \\
\text { applied }\end{array}$ & $\begin{array}{l}\mathrm{N}=28 \\
\text { Age: Yes } \\
\text { Sex: Yes } \\
\text { \% majority race: No } \\
\text { Education: No }\end{array}$ & $\mathrm{NR}$ & $\mathrm{NR}$ & NR & NR & $\mathrm{NR}$ & $\begin{array}{l}2 \text { months; up } \\
\text { to } 3 \text { make-up } \\
\text { sessions } \\
\text { allowed }\end{array}$ & $\begin{array}{l}\text { Evaluation of } \\
\text { walking and } \\
\text { stride } \\
\text { Ability to } \\
\text { stand } \\
\text { Fall efficacy } \\
\text { measuremen } \\
t \\
\text { Agitation } \\
\text { Memory } \\
\text { inventory }\end{array}$ & NA \\
\hline $\begin{array}{l}\text { Okamura } \\
2018^{216} \\
(30036871) \\
\text { Japan } \\
\text { High RoB }\end{array}$ & $\begin{array}{l}\text { Combined } \\
\text { exercise and } \\
\text { cognitive } \\
\text { training }\end{array}$ & $\begin{array}{l}\text { Pedaling with } \\
\text { arm ergometer }\end{array}$ & $\begin{array}{l}\text { Combined } \\
\text { day care } \\
\text { and } \\
\text { residential } \\
\text { care } \\
\text { RCT } \\
100 \text { PLWD }\end{array}$ & $\begin{array}{l}\text { PLWD with } \\
\text { diagnosis of } \\
\text { dementia o } \\
\text { No restriction on } \\
\text { type or severity } \\
\text { Aged } 65 \text { or older } \\
\text { and requiring } \\
\text { assistance } \\
\text { Exclusion criteria } \\
\text { applied }\end{array}$ & $\begin{array}{l}\mathrm{N}=100 \\
\text { Age: Yes } \\
\text { Sex: Yes } \\
\text { Race \% majority: No } \\
\text { Education: No }\end{array}$ & NR & NA & NA & NR & NR & $\begin{array}{l}6 \text { weeks; } \\
1 \text { month } \\
3 \text { months }\end{array}$ & $\begin{array}{l}\text { MMSE } \\
\text { Trail making } \\
\text { test } \\
\text { ADL }\end{array}$ & NA \\
\hline $\begin{array}{l}\text { Reisberg } \\
2017^{234} \\
\text { (28122366) } \\
\text { US } \\
\text { Small sample }\end{array}$ & $\begin{array}{l}\text { Comprehensive, } \\
\text { Individualized, } \\
\text { Person- } \\
\text { Centered } \\
\text { Management } \\
\text { with following } \\
\text { components: } \\
\text { CG training, } \\
\text { management } \\
\text { assessment, } \\
\text { therapeutic } \\
\text { home visits and } \\
\text { CG support } \\
\text { groups }\end{array}$ & $\begin{array}{l}\text { Usual } \\
\text { community care }\end{array}$ & $\begin{array}{l}\text { Community } \\
\text { dwelling } \\
\text { PLWD and } \\
\text { CG } \\
\text { RCT } \\
20 \text { PLWD }\end{array}$ & $\begin{array}{l}\text { Diagnosis based } \\
\text { on DSM-IV-TR } \\
\text { criteria fulfilled } \\
\text { NINCDS-ADRDA } \\
\text { criteria for probable } \\
\text { AD; moderate-to- } \\
\text { severe AD with } \\
\text { GDS } 5 \text { or } 6 ; \\
\text { FAST } 6 \text {; MMSE } \\
\text { between } 3 \text { and } 14\end{array}$ & $\begin{array}{l}\mathrm{N}=20 \\
\text { Age: yes } \\
\text { Sex: yes } \\
\text { Race \% majority: no } \\
\text { Education: yes }\end{array}$ & NR & NR & NA & NA & NA & $\begin{array}{l}4 \text { weeks } \\
12 \text { weeks } \\
28 \text { weeks }\end{array}$ & $\begin{array}{l}\text { NYU-CIBIC- } \\
\text { Plus } \\
\text { ADCS- } \\
\text { ADLsev-abv } \\
\text { MMSE } \\
\text { FAST-DS } \\
\text { BEHAVE- } \\
\text { AD-FW } \\
\text { RMBPC }\end{array}$ & NA \\
\hline $\begin{array}{l}\text { Rokstad 2018217 } \\
(30445937) \\
\text { Norway } \\
\text { High RoB }\end{array}$ & $\begin{array}{l}\text { Attending } \\
\text { daycare-based } \\
\text { dementia } \\
\text { programs at } \\
\text { least } 2 \text { days per } \\
\text { week }\end{array}$ & Care as usual & $\begin{array}{l}\text { Community } \\
\text { dwelling } \\
\text { PLWD using } \\
\text { daycare } \\
\text { services and } \\
\text { their CG } \\
\text { Quasi- } \\
\text { experimental } \\
\text { 257 PLWD } \\
\text { recruited } \\
\end{array}$ & $\begin{array}{l}\text { Dementia } \\
\text { diagnosis } \\
\text { according to ICD- } \\
10 ; \text { MMSE } \geq 15 \text { and } \\
\text { consent of CG }\end{array}$ & $\begin{array}{l}\mathrm{N}=257 \\
\text { Age: yes } \\
\text { Sex: yes } \\
\text { Race \% majority: no } \\
\text { Education: yes }\end{array}$ & $\mathrm{NR}$ & $\begin{array}{l}\mathrm{N}=257 \\
\text { Age: yes } \\
\text { Sex: yes } \\
\text { Race \% majority: no } \\
\text { Education: no } \\
\text { Relation to } \\
\text { PLWD=yes }\end{array}$ & NR & NA & NA & 24 months & $\begin{array}{l}\text { PSMS } \\
\text { IADL } \\
\text { MADRS } \\
\text { NPI-Q } \\
\text { REED }\end{array}$ & $\begin{array}{l}\text { RSS } \\
\text { MADRS }\end{array}$ \\
\hline $\begin{array}{l}\text { Kampragkou } \\
2017^{235} \\
\text { (ID\# not } \\
\text { available) } \\
\text { Greece } \\
\text { Small sample } \\
\end{array}$ & $\begin{array}{l}\text { Aerobic } \\
\text { exercise, } \\
\text { memory games } \\
\text { and music } \\
\text { therapy }\end{array}$ & No intervention & $\begin{array}{l}\text { Institutional } \\
\text { setting } \\
\text { RCT } \\
36 \text { PLWD }\end{array}$ & $\begin{array}{l}\text { PLWD diagnosed } \\
\text { with moderate AD, } \\
\text { ambulatory, aged } \\
65 \text { or older } \\
\text { Exclusion criteria } \\
\text { applied }\end{array}$ & $\begin{array}{l}\mathrm{N}=30 \\
\text { Age: No } \\
\text { Sex: Yes } \\
\text { Race \% majority: No } \\
\text { Education: No }\end{array}$ & $\mathrm{NR}$ & NA & NA & NA & NA & 12 weeks & $\begin{array}{l}\text { MMSE } \\
\text { ADAS } \\
\text { ADL } \\
\text { Ability to } \\
\text { stand } \\
\text { Balance } \\
\end{array}$ & NA \\
\hline
\end{tabular}




\begin{tabular}{|c|c|c|c|c|c|c|c|c|c|c|c|c|c|}
\hline $\begin{array}{l}\text { Masayuki Satoh } \\
2017^{236} \\
\text { (28222531) } \\
\text { Japan } \\
\text { High RoB }\end{array}$ & $\begin{array}{l}\text { Physical } \\
\text { exercise with } \\
\text { music (muscle } \\
\text { training for the } \\
\text { upper and lower } \\
\text { extremities, } \\
\text { hand clapping } \\
\text { to music, breath } \\
\text { and voice } \\
\text { training, and } \\
\text { singing) for } 40 \\
\text { minutes } 1 x \\
\text { week for } 6 \\
\text { months }\end{array}$ & $\begin{array}{l}\text { Cognitive } \\
\text { stimulation } \\
\text { using a portable } \\
\text { game console } \\
\text { (Nintendo DS } \\
\text { LL), and drills } \\
\text { consisting of } \\
\text { easy } \\
\text { calculations, } \\
\text { mazes, and } \\
\text { mistake- } \\
\text { searching in } \\
\text { pictures for } 40 \\
\text { minutes } 1 x \\
\text { week for } 6 \\
\text { months }\end{array}$ & \begin{tabular}{|l|} 
PLWD using \\
geriatric \\
nursing \\
services \\
RCT \\
85 PLWD \\
\end{tabular} & $\begin{array}{l}\text { Neurological } \\
\text { specialist } \\
\text { diagnosed } \\
\text { dementia according } \\
\text { to ICD-10; MMSE } \\
\text { score between } 16 \\
\text { and 26; }\end{array}$ & $\begin{array}{l}\mathrm{N}=85 \\
\text { Age: yes } \\
\text { Sex: no } \\
\text { Race \% majority: no } \\
\text { Education: yes }\end{array}$ & NR & NA & $\mathrm{NR}$ & NA & NA & 6 months & $\begin{array}{l}\text { RCPM } \\
\text { RBMT } \\
\text { WF } \\
\text { TMT-A } \\
\text { FIM } \\
\text { BEHAVE-AD } \\
\text { VSRAD }\end{array}$ & NA \\
\hline $\begin{array}{l}\text { Kim } 2016^{237} \\
\text { (ID\# not } \\
\text { available) } \\
\text { South Korea } \\
\text { Small sample }\end{array}$ & $\begin{array}{l}\text { Cognitive } \\
\text { stimulation, } \\
\text { including art, } \\
\text { music, } \\
\text { recollection ad } \\
\text { horticultural } \\
\text { therapy }\end{array}$ & $\begin{array}{l}\text { Pharmacologica } \\
\text { I treatment only }\end{array}$ & $\begin{array}{l}\text { Institutional } \\
\text { setting } \\
\text { RCT } \\
64 \text { PLWD }\end{array}$ & $\begin{array}{l}\text { PLWD diagnosed } \\
\text { with probable AD } \\
\text { Exclusion criteria } \\
\text { applied }\end{array}$ & $\begin{array}{l}\mathrm{N}=53 \\
\text { Age: Yes } \\
\text { Sex: Yes } \\
\text { Race \% majority: No } \\
\text { Education: Yes }\end{array}$ & NR & NR & NR & NR & NR & 6 months & $\begin{array}{l}\text { MMSE } \\
\text { Verbal } \\
\text { fluency and } \\
\text { word recall } \\
\text { Depression } \\
\text { Judgement } \\
\text { and problem } \\
\text { solving } \\
\text { QoL }\end{array}$ & QoL \\
\hline $\begin{array}{l}\text { Middelstadt } \\
2016^{219} \\
(27497474) \\
\text { Germany } \\
\text { High RoB }\end{array}$ & $\begin{array}{l}\text { Cognitive } \\
\text { stimulation } \\
\text { program twice } \\
\text { weekly for eight } \\
\text { weeks }\end{array}$ & Usual care & $\begin{array}{l}\text { PLWD } \\
\text { recruited } \\
\text { from long } \\
\text { term care } \\
\text { facilities } \\
\text { RCT } \\
71 \text { PLWD }\end{array}$ & $\begin{array}{l}\text { Mild to moderate } \\
\text { PLWD diagnosed } \\
\text { according to ICD- } \\
10 \text { and MMSE } \\
\text { score } 10-25\end{array}$ & $\begin{array}{l}\mathrm{N}=71 \\
\text { Age: Yes } \\
\text { Sex: Yes } \\
\text { Race \% majority: No } \\
\text { Education: No }\end{array}$ & NR & NA & NA & NA & NA & $\begin{array}{l}8 \text { weeks } \\
14 \text { weeks }\end{array}$ & $\begin{array}{l}\text { ADAS-cog } \\
\text { QoL-AD } \\
\text { NPI-NH } \\
\text { ADCS-ADL }\end{array}$ & NA \\
\hline $\begin{array}{l}\text { Fernandez- } \\
\text { Calvo 2015 } 220 \\
\text { (25121567) } \\
\text { Spain } \\
\text { High RoB }\end{array}$ & $\begin{array}{l}\text { Multicomponent } \\
\text { intervention } \\
\text { including new } \\
\text { recreational and } \\
\text { cognitive tasks, } \\
\text { a module of } \\
\text { functional } \\
\text { activities based } \\
\text { on real-life } \\
\text { situations, } \\
\text { cognitive } \\
\text { training } \\
\text { exercises to } \\
\text { carry out at } \\
\text { home with the } \\
\text { caregiver; and } \\
\text { psychoeducatio } \\
\text { nal activities; } 48 \\
\text { sessions (90 } \\
\text { minutes } \\
\text { duration, and } 3 \\
\text { sessions per } \\
\text { week) for } 16 \\
\text { weeks } \\
\text { wats }\end{array}$ & Waitlist group & $\begin{array}{l}\text { Community } \\
\text { dwelling } \\
\text { PLWD } \\
\text { RCT } \\
\text { 61 PLWD }\end{array}$ & $\begin{array}{l}\text { Diagnosis of } \\
\text { probable AD using } \\
\text { NINCDS/ADRDA; } \\
\text { mild dementia; } \\
\text { MMSE } \geq 18 \text { and } \\
\text { having a CG for at } \\
\text { least } 6 \text { months }\end{array}$ & $\begin{array}{l}\mathrm{N}=61 \\
\text { Age: yes } \\
\text { Sex: yes } \\
\text { Race \% majority: no } \\
\text { Education: yes }\end{array}$ & NR & NA & NR & NA & $\mathrm{NA}$ & 16 weeks & $\begin{array}{l}\text { ADAS-Cog } \\
\text { NPI-Q } \\
\text { CSDD } \\
\text { RDRS-2 }\end{array}$ & NA \\
\hline
\end{tabular}




\begin{tabular}{|c|c|c|c|c|c|c|c|c|c|c|c|c|c|}
\hline $\begin{array}{l}\text { Wesson 2013238 } \\
\text { (24004682) } \\
\text { Australia } \\
\text { Pilot }\end{array}$ & $\begin{array}{l}\text { Home hazard } \\
\text { reduction } \\
\text { program to } \\
\text { reduce falls and } \\
\text { Increase } \\
\text { balance and } \\
\text { strength }\end{array}$ & Usual care & $\begin{array}{l}\text { Community- } \\
\text { based } \\
\text { RCT } \\
\text { 22 PLWD } \\
\text { and CG }\end{array}$ & \begin{tabular}{|l|} 
PLWD diagnosed \\
with dementia or \\
ACE-R score $<=82$ \\
Age 65 or older \\
with non-paid \\
caregiver \\
MMSE.82 \\
Exclusion criteria \\
applied
\end{tabular} & \begin{tabular}{|l|}
$\mathrm{N}=22$ \\
Age: Yes \\
Sex: Yes \\
Race \% majority: No \\
Education: Yes
\end{tabular} & $\begin{array}{l}\text { Household } \\
\text { characteristics: } \\
\text { Yes } \\
\text { Prior disability: } \\
\text { Yes }\end{array}$ & $\begin{array}{l}\text { Sex: Yes } \\
\text { Relation to PLWD: } \\
\text { Yes }\end{array}$ & Living with PLWD & NA & $\mathrm{NA}$ & 12 weeks & $\begin{array}{l}\text { Fall risk tests } \\
\text { Step and } \\
\text { balance } \\
\text { tests } \\
\text { ADL } \\
\text { Depression } \\
\text { Agitation }\end{array}$ & $\begin{array}{l}\text { Caregiver } \\
\text { burden } \\
\text { Task strategy } \\
\text { management } \\
\text { index }\end{array}$ \\
\hline $\begin{array}{l}\text { Yamagami } \\
2012^{222} \\
(23300492) \\
\text { Japan } \\
\text { High RoB }\end{array}$ & $\begin{array}{l}24 \text { sessions for } \\
1 \text {-hour } 2 \times \text { week, } \\
\text { for 12-weeks of } \\
\text { intervention } \\
\text { based on brain- } \\
\text { activating } \\
\text { rehabilitation } \\
\text { combined reality } \\
\text { orientation and } \\
\text { various } \\
\text { activities (e.g. } \\
\text { cooking, } \\
\text { singing, and } \\
\text { sewing) along } \\
\text { with } \\
\text { reminiscence } \\
\text { therapy. }\end{array}$ & No treatment & \begin{tabular}{|l} 
Recruited \\
from \\
residential \\
care homes \\
(group living \\
homes) \\
RCT \\
54 PLWD
\end{tabular} & \begin{tabular}{|l|} 
Dementia \\
diagnosis without \\
severe visual or \\
auditory \\
impairments
\end{tabular} & \begin{tabular}{|l|}
$\mathrm{N}=54$ \\
Age: Yes \\
Sex: Yes \\
Race \% majority: No \\
Education: No
\end{tabular} & NR & NA & NA & NA & NA & 12 weeks & $\begin{array}{l}\text { CDR } \\
\text { MOSES } \\
\text { HDS-R } \\
\text { TMT-A } \\
\text { Staff } \\
\text { interview }\end{array}$ & NA \\
\hline $\begin{array}{l}\text { Brooker 2011223 } \\
\text { (21702705) } \\
\text { UK } \\
\text { High RoB }\end{array}$ & $\begin{array}{l}\text { Occupational } \\
\text { activities to } \\
\text { foster well-being } \\
\text { and goal } \\
\text { achievement }\end{array}$ & $\begin{array}{l}\text { Trained staff } \\
\text { employed to try } \\
\text { to increase } \\
\text { PLWD activities }\end{array}$ & \begin{tabular}{|l} 
Assisted \\
living \\
Cluster RCT \\
293 PLWD
\end{tabular} & \begin{tabular}{|l|} 
PLWD exhibiting \\
confused behavior, \\
communication, \\
difficulties, \\
challenging \\
behavior or low \\
mood \\
Exclusion criteria \\
applied
\end{tabular} & \begin{tabular}{|l|}
$\mathrm{N}=293$ \\
Age: Yes \\
Sex: Yes \\
Race \% majority: No \\
Education: No
\end{tabular} & $\begin{array}{l}\text { Household } \\
\text { characteristics: } \\
\text { Yes }\end{array}$ & NA & NA & NR & $\begin{array}{l}\text { Training: Yes } \\
\text { Position: Yes }\end{array}$ & 18 months & $\begin{array}{l}\text { QoL } \\
\text { Level of } \\
\text { social } \\
\text { support } \\
\text { Quality of } \\
\text { relationships } \\
\text { Dementia } \\
\text { Care } \\
\text { Mapping } \\
\text { index }\end{array}$ & NA \\
\hline $\begin{array}{l}\text { Burgener } \\
2011^{239} \\
\text { (20509596) } \\
\text { US } \\
\text { Small sample }\end{array}$ & $\begin{array}{l}\text { Taiji exercises, } \\
\text { cognitive- } \\
\text { behavioral } \\
\text { therapies and } \\
\text { cognitive } \\
\text { training } \\
\text { exercises, as } \\
\text { well as support } \\
\text { group } \\
\text { participation }\end{array}$ & $\begin{array}{l}\text { Education } \\
\text { program for } \\
\text { caregivers }\end{array}$ & $\begin{array}{l}\text { Community- } \\
\text { based } \\
\text { RCT } \\
\text { Self- } \\
\text { referred, } \\
\text { caregiver } \\
\text { referred, or } \\
\text { provider } \\
\text { referred } \\
\text { Unclear } \\
\end{array}$ & $\begin{array}{l}\text { PLWD diagnosed } \\
\text { with dementia at } \\
\text { early to middle } \\
\text { disease stage }\end{array}$ & $\begin{array}{l}\mathrm{N}=32 \\
\text { Age: No } \\
\text { Sex: No } \\
\text { Race\% majority: No } \\
\text { Education: No }\end{array}$ & NR & $\begin{array}{l}\text { N=32 } \\
\text { Age: Yes } \\
\text { Sex: Yes } \\
\text { Race \% majority: } \\
\text { No } \\
\text { Education: Yes }\end{array}$ & NR & NA & NA & 20 weeks & $\begin{array}{l}\text { MMSE } \\
\text { ECFR } \\
\text { RSS }\end{array}$ & $\begin{array}{l}\text { Stress } \\
\text { Quality of } \\
\text { family } \\
\text { relationship }\end{array}$ \\
\hline $\begin{array}{l}\text { Ferrero-Arias } \\
2011^{224} \\
(21346517) \\
\text { Spain } \\
\text { High RoB }\end{array}$ & $\begin{array}{l}\text { Occupational } \\
\text { therapy } \\
\text { sessions (music } \\
\text { and art therapy } \\
\text { and } \\
\text { psychomotor } \\
\text { activity) }\end{array}$ & $\begin{array}{l}\text { Control } \\
\text { participants } \\
\text { remained in the } \\
\text { day rooms } \\
\text { performing } \\
\text { activities of their } \\
\text { own choosing }\end{array}$ & \begin{tabular}{|l} 
Recruited \\
from centers \\
of the \\
Association \\
of Alzheimer \\
PLWD' \\
Families of \\
Burgos \\
Crossover \\
RCT \\
146 PLWD \\
\end{tabular} & $\begin{array}{l}\text { Dementia criteria of } \\
\text { CDR } 1 \text { or } 2 \text {, } \\
\text { undergoing } \\
\text { occupational } \\
\text { therapy }\end{array}$ & $\begin{array}{l}\mathrm{N}=146 \\
\text { Age: } \text { yes } \\
\text { Sex: yes } \\
\text { Race\% majority: No } \\
\text { Education: yes }\end{array}$ & NR & NA & NA & NA & NA & $\begin{array}{l}4 \text { weeks } \\
8 \text { weeks }\end{array}$ & $\begin{array}{l}\text { NPI-Q } \\
\text { DAIR }\end{array}$ & NA \\
\hline
\end{tabular}




\begin{tabular}{|c|c|c|c|c|c|c|c|c|c|c|c|c|c|}
\hline $\begin{array}{l}\text { Burgener } \\
2008^{240} \\
(18453642) \\
\text { US } \\
\text { Small sample }\end{array}$ & $\begin{array}{l}\text { Taiji exercises, } \\
\text { cognitive } \\
\text { behavioral } \\
\text { therapies and } \\
\text { support group }\end{array}$ & $\begin{array}{l}\text { Attention control } \\
\text { education } \\
\text { program }\end{array}$ & \begin{tabular}{|l|} 
Community- \\
based \\
RCT \\
Self- \\
referred, \\
caregiver \\
referred, or \\
provider \\
referred \\
43 PLWD \\
\end{tabular} & \begin{tabular}{|l|} 
PLWD diagnosed \\
with dementia at \\
early to middle \\
disease stage
\end{tabular} & \begin{tabular}{l|}
$\mathrm{N}=43$ \\
Age: Yes \\
Sex: Yes \\
Race \% majority: No \\
Education: Yes
\end{tabular} & NR & NR & NR & NA & NA & $\begin{array}{l}20 \text { weeks; } \\
40 \text { weeks }\end{array}$ & $\begin{array}{l}\text { MMSE } \\
\text { Physical } \\
\text { functioning } \\
\text { Depression } \\
\text { Self esteem }\end{array}$ & NR \\
\hline $\begin{array}{l}\text { Femia 2007226 } \\
(18192631) \\
\text { US } \\
\text { High RoB }\end{array}$ & $\begin{array}{l}\text { Adult day } \\
\text { service } 3 \text { days } \\
\text { per week for } 6 \\
\text { hours for } 2 \\
\text { months: } 5 \text { to } 6 \\
\text { different } \\
\text { activities per } \\
\text { day in addition } \\
\text { to daily routines } \\
\text { (i.e., Iunch), } \\
\text { which included } \\
30 \text { minutes of } \\
\text { physical } \\
\text { activities on } \\
\text { average, } 1 \text { to } 2 \\
\text { hours of social } \\
\text { activities, and } \\
\text { about } 1 \text { hour of } \\
\text { cognitively } \\
\text { stimulating } \\
\text { activities }\end{array}$ & $\begin{array}{l}\text { Not using adult } \\
\text { day services }\end{array}$ & \begin{tabular}{|l|} 
Community \\
dwelling \\
PLWD and \\
their CG \\
participating \\
in the \\
Dementia \\
Day Care \\
Program of \\
the NJ \\
Statewide \\
Respite \\
Care \\
Program \\
Quasi- \\
experimental \\
234 PLWD \\
recruited
\end{tabular} & \begin{tabular}{|l|} 
Dementia \\
diagnosis
\end{tabular} & $\begin{array}{l}\mathrm{N}=201 \\
\text { Age: no } \\
\text { Sex: yes } \\
\text { Race \% majority: no } \\
\text { Education: yes }\end{array}$ & Income=yes & $\begin{array}{l}\mathrm{N}=201 \\
\text { Age: yes } \\
\text { Sex: yes } \\
\text { Race \% majority: } \\
\text { yes } \\
\text { Education: yes } \\
\text { Relation to } \\
\text { PLWD:yes }\end{array}$ & Employment=yes & NA & NA & 2 months & $\begin{array}{l}\text { BPSD } \\
\text { Domains } \\
\text { IADL } \\
\text { PADL } \\
\text { MMSE } \\
\text { DRB }\end{array}$ & NA \\
\hline $\begin{array}{l}\text { Tappen 2000 } \\
\text { (11186596) } \\
\text { US }\end{array}$ & $\begin{array}{l}\text { Skill training } \\
\text { program }\end{array}$ & $\begin{array}{l}\text { Traditional } \\
\text { stimulation or } \\
\text { regular care }\end{array}$ & \begin{tabular}{|l|} 
Nursing \\
home \\
RCT \\
3 groups
\end{tabular} & $\begin{array}{l}\text { PLWD diagnosed } \\
\text { with dementia } \\
\text { Able to stand with } \\
\text { assistance } \\
\text { Exclusion criteria } \\
\text { applied }\end{array}$ & $\begin{array}{l}\mathrm{N}=21 \text { skill group } \\
21 \text { stimulation group } \\
21 \text { regular care } \\
\text { group } \\
\text { Age: NR by group } \\
\text { Sex: NR by group } \\
\text { Race \% majority: } N \\
\text { Education: } N\end{array}$ & NR & NA & NA & Education: Y & NR & 20 weeks & $\begin{array}{l}\text { Self- } \\
\text { maintenance } \\
\text { ADL } \\
\text { Goal } \\
\text { attainment }\end{array}$ & NA \\
\hline $\begin{array}{l}\text { Wimo 1993229 } \\
\text { (8356361) } \\
\text { Sweden } \\
\text { High RoB }\end{array}$ & $\begin{array}{l}6 \text { hours of } \\
\text { daycare } \\
\text { activities } \\
\text { including } \\
\text { everyday } \\
\text { routines such as } \\
\text { meals, coffee, } \\
\text { short walks, and } \\
\text { excursions with } \\
\text { stimulating } \\
\text { elements, e.g. } \\
\text { newspaper } \\
\text { reading and } \\
\text { videos }\end{array}$ & $\begin{array}{l}\text { Not attending } \\
\text { daycare }\end{array}$ & $\begin{array}{l}\text { Non- } \\
\text { institutionaliz } \\
\text { ed PLWD } \\
\text { attending } \\
\text { daycare in } \\
\text { Sundsvall } \\
\text { Quasi- } \\
\text { experimental } \\
\text { 99 PLWD }\end{array}$ & $\begin{array}{l}\text { Dementia } \\
\text { diagnosis } \\
\text { according to DSM- } \\
\text { IIIR criteria }\end{array}$ & $\begin{array}{l}\text { N=99 } \\
\text { Age: yes } \\
\text { Sex: yes } \\
\text { Race \% majority: no } \\
\text { Education: no }\end{array}$ & $\begin{array}{l}\text { Home } \\
\text { support=yes }\end{array}$ & NA & NA & NA & NA & 12 months & $\begin{array}{l}\text { MMSE } \\
\text { MDDAS } \\
\text { institutionaliz } \\
\text { ation }\end{array}$ & NA \\
\hline
\end{tabular}




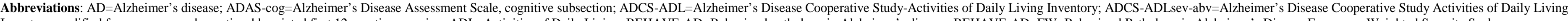
Inventory modified for more severe dementia, abbreviated first 12 questions version; $\mathrm{ADL}=\mathrm{Activities} \mathrm{of} \mathrm{Daily} \mathrm{Living;} \mathrm{BEHAVE-AD=Behavioral} \mathrm{pathology} \mathrm{in} \mathrm{Alzheimer's} \mathrm{disease;} \mathrm{BEHAVE-AD=FW=Behavioral} \mathrm{Pathology} \mathrm{in} \mathrm{Alzheimer's} \mathrm{Disease} \mathrm{Frequency-Weighted} \mathrm{Severity} \mathrm{Scale;}$

BPSD=Behavioral and psychological symptoms of dementia; CDR=Clinical Dementia Rating; $C G=$ Caregiver; char=characteristics; CIBIC=Clinician Interview-Based Impression of change; CSDD=Cornell Scale for Depression in Dementia; DAIR=Dementia Apathy Interview and Rating;

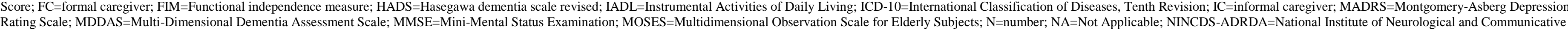

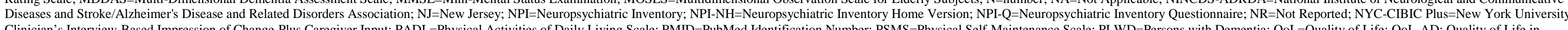

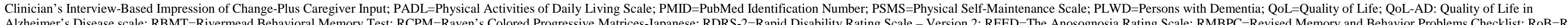
作

\section{Assistive Technology}




\section{Evidence Map: Assistive Technology}

\begin{tabular}{|c|c|c|c|c|c|c|c|c|c|c|c|c|c|}
\hline $\begin{array}{l}\text { Study (PMID) } \\
\text { Country } \\
\text { EM Reason }\end{array}$ & Intervention & Comparison & $\begin{array}{l}\text { Setting } \\
\text { and Design } \\
\\
\text { Setting } \\
\text { Design } \\
\text { Cluster N } \\
\text { Participants } \\
\text { Random- } \\
\text { ized N }\end{array}$ & $\begin{array}{l}\text { PLWD } \\
\text { Dementia } \\
\text { Characteristics }\end{array}$ & $\begin{array}{l}\text { PLWD } \\
\text { Non-Disease Char } \\
\text { PLWD N } \\
\text { PLWD Age (mean) } \\
\text { PLWD Sex (\% } \\
\text { female) } \\
\text { PLWD Race (\% } \\
\text { majority) } \\
\text { PLWD Education } \\
\text { (mean years) }\end{array}$ & $\begin{array}{l}\text { PLWD } \\
\text { Non-Disease } \\
\text { Char Reporting } \\
\text { Status (RS) } \\
\\
\text { PLWD SES } \\
\text { PLWD Prior } \\
\text { Disability } \\
\text { PLWD } \\
\text { Household } \\
\text { Characteristics } \\
\text { PLWD Health } \\
\text { Insurance } \\
\text { PLWD Detailed } \\
\text { Race Information }\end{array}$ & $\begin{array}{l}\text { Informal Caregiver } \\
\text { (IC) } \\
\text { Characteristics } \\
\text { IC N } \\
\text { IC Age (mean) } \\
\text { IC Sex (\% female) } \\
\text { IC Race (\% } \\
\text { majority) } \\
\text { IC Education } \\
\text { (mean years) } \\
\text { IC Relation to } \\
\text { PLWD (\% majority) }\end{array}$ & $\begin{array}{l}\text { Informal Caregiver } \\
\text { (IC) Char. RS } \\
\text { IC Duration } \\
\text { IC Living With } \\
\text { PLWD } \\
\text { IC Payment } \\
\text { IC Health Status } \\
\text { IC Dementia } \\
\text { Family History } \\
\text { IC Employment } \\
\text { Status } \\
\text { IC Training }\end{array}$ & \begin{tabular}{|l} 
Formal Caregiver \\
(FC) \\
Characteristics \\
FC N \\
FC Age (mean) \\
FC Sex (\% \\
female) \\
FC Race (\% \\
majority) \\
FC Education \\
(mean years)
\end{tabular} & $\begin{array}{l}\text { Formal } \\
\text { Caregiver (FC) } \\
\text { Char. RS } \\
\text { FC Health } \\
\text { Status } \\
\text { FC Training } \\
\text { FC Education } \\
\text { FC Position } \\
\text { FC Length of } \\
\text { Service }\end{array}$ & $\begin{array}{l}\text { Outcome } \\
\text { Timing(s) }\end{array}$ & \begin{tabular}{|l|} 
PLWD \\
Outcomes
\end{tabular} & $\begin{array}{l}\text { Caregiver } \\
\text { Outcomes } \\
\text { IC: } \\
\text { FC: }\end{array}$ \\
\hline $\begin{array}{l}\text { Lauriks, } 2018 \\
241 \\
\text { Amsterdam } \\
\text { Pilot }\end{array}$ & $\begin{array}{l}\text { Application of } \\
\text { Assistive Home } \\
\text { Technology } \\
\text { (AHT); life } \\
\text { circles, pathway } \\
\text { lighting, } \\
\text { automated } \\
\text { lighting, } \\
\text { automated } \\
\text { alerts, } \\
\text { automated sun } \\
\text { blinds }\end{array}$ & $\begin{array}{l}\text { Lived in same } \\
\text { type of group } \\
\text { home, but w/out } \\
\text { AHT }\end{array}$ & $\begin{array}{l}\text { Group } \\
\text { homes in } \\
\text { residential } \\
\text { care facility } \\
(\mathrm{N}=9) \\
\mathrm{RCT} \\
\mathrm{PLWD} \\
(\mathrm{N}=54) \\
\text { Caregivers } \\
(\mathrm{N}=25)\end{array}$ & $\begin{array}{l}\text { Diagnosis of } \\
\text { dementia } \\
\text { confirmed by a } \\
\text { physician }\end{array}$ & $\begin{array}{l}\mathrm{N}=54 \text { ( } 30 \text { treatment } \\
\text { vs } 24 \text { control) } \\
\text { Age: yes } \\
\text { Sex: yes } \\
\text { Race: NR }\end{array}$ & NR & NA & NA & $\begin{array}{l}\mathrm{N}=25 \text { (14 } \\
\text { treatment vs } 11 \\
\text { control) } \\
\text { Sex }\end{array}$ & Training & $\begin{array}{l}\text { On average } \\
\text { four months } \\
\text { after baseline } \\
\text { measurement } \\
\text { completed } \\
\text { and AHT } \\
\text { switched on. }\end{array}$ & \begin{tabular}{|l} 
Quality of life \\
(Qaullidem \\
instrument)
\end{tabular} & $\begin{array}{l}\text { FC: Job } \\
\text { satisfaction } \\
\text { and general } \\
\text { health }\end{array}$ \\
\hline $\begin{array}{l}\text { Tchalla, } 2013 \\
242 \\
\text { France } \\
\text { Pilot }\end{array}$ & \begin{tabular}{|l|} 
Home-based \\
technologies \\
coupled with \\
teleassistance \\
service (HBTec- \\
TS); nightlight \\
path and \\
electronic \\
bracelet \\
coupled with a \\
teleassistance \\
service
\end{tabular} & $\begin{array}{l}\text { No HBTec-TS } \\
\text { system was } \\
\text { implemented }\end{array}$ & $\begin{array}{l}\text { Community } \\
\text { Experimenta } \\
\text { I prospective } \\
\text { study }\end{array}$ & $\begin{array}{l}\text { Suffering from } \\
\text { Alzheimer's } \\
\text { disease, living at } \\
\text { home and } \\
\text { rogistered on the } \\
\text { frail elderly people } \\
\text { register } \\
\text { Considered high } \\
\text { risk of falling and } \\
\text { were receiving a } \\
\text { county allocation }\end{array}$ & $\begin{array}{l}\mathrm{N}=96 \text { ( } 49 \text { treatment } \\
\text { vs } 47 \text { control) } \\
\text { Age: yes } \\
\text { Sex: yes } \\
\text { Race: NR }\end{array}$ & Comorbidity & NA & NA & NA & NA & 12 months & \begin{tabular}{|l|}
$\begin{array}{l}\text { Incidence of } \\
\text { falls }\end{array}$ \\
\end{tabular} & NA \\
\hline $\begin{array}{l}\text { Rowe, } 2010^{243} \\
\text { United States } \\
\text { Pilot }\end{array}$ & \begin{tabular}{|l} 
Home \\
monitoring \\
system \\
designed to \\
reliably awaken \\
a caregiver \\
when the PLWD \\
left the bed at \\
night
\end{tabular} & $\begin{array}{l}\text { Paid } \$ 15 \text { at } \\
\text { each data } \\
\text { collection point } \\
\text { and provided } \\
\text { with some } \\
\text { eduction } \\
\text { material } \\
\text { unrelated to any } \\
\text { study goals }\end{array}$ & $\begin{array}{l}\text { Community } \\
\text { Pretest- } \\
\text { posttest } \\
\text { control } \\
\text { group } \\
\text { design } \\
\text { Caregivers } \\
(\mathrm{N}=49)\end{array}$ & $\begin{array}{l}\text { Medical diagnosis } \\
\text { of Alzheimer's } \\
\text { disease or other } \\
\text { dementia }\end{array}$ & NR & NR & \begin{tabular}{|l|}
$\mathrm{N}=49$ \\
Age \\
Sex \\
Race \\
Education \\
Relation to PLWD
\end{tabular} & $\begin{array}{l}\text { Living with PLWD } \\
\text { Health status } \\
\text { Employment status }\end{array}$ & NA & NA & $\begin{array}{l}\text { Post-test } \\
\text { months; } 2,3, \\
4,5,6,8,10 \\
\text { and } 12(7 \\
\text { consecutive } \\
\text { nights at each } \\
\text { data collection } \\
\text { month) }\end{array}$ & NA & $\begin{array}{l}\text { IC: Subjective } \\
\text { sleep, } \\
\text { objective } \\
\text { sleep and } \\
\text { distress }\end{array}$ \\
\hline $\begin{array}{l}\text { Gaugler } 2019 \\
(29982413)^{244} \\
\text { United States } \\
\text { Pilot }\end{array}$ & $\begin{array}{l}\text { Wearable / on- } \\
\text { body monitoring } \\
\text { system alerts } \\
\text { caregiver to } \\
\text { activities, } \\
\text { provides reports } \\
\text { behavioral } \\
\text { patterns } \\
\text { associated } \\
\text { potential health } \\
\text { problems }\end{array}$ & Usual care & $\begin{array}{l}\text { Community } \\
\text { RCT } \\
\text { PLWD } \\
(\mathrm{N}=132) \\
\text { Caregivers } \\
(\mathrm{N}=132)\end{array}$ & $\begin{array}{l}\text { English speaking } \\
\text { Diagnosis of } \\
\text { NINCDS/ADRDA } \\
\text { Not receiving } \\
\text { similar services } \\
\text { Age } \geq 55 \text { years }\end{array}$ & $\begin{array}{l}\mathrm{N}=132 \\
\text { Age yes } \\
\text { Sex yes } \\
\text { Race yes } \\
\text { Education yes }\end{array}$ & $\begin{array}{l}\text { SES } \\
\text { Household } \\
\text { Characteristics } \\
\text { Health Insurance } \\
\text { Race Information }\end{array}$ & \begin{tabular}{|l|}
$\mathrm{N}=132$ \\
Age yes \\
Sex yes \\
Race yes \\
Education yes \\
Relation to PLWD: \\
yes
\end{tabular} & $\begin{array}{l}\text { Living with PLWD } \\
\text { Employment Status }\end{array}$ & NA & NA & 6 months & NA & $\begin{array}{l}\text { Sense of } \\
\text { competence } \\
\text { (SSCQ), } \\
\text { Caregiver } \\
\text { Distress } \\
\text { (ZBI), } \\
\text { Depression } \\
\text { (CES-D) }\end{array}$ \\
\hline
\end{tabular}

Abbreviations: CES-D=Center for Epidemiologic Studies Depression Scale; NA=Not Applicable; NINCDS/ADRDA= National Institute of
Identification Number; $P$ PLWD=Patients living with Dementia; SSCQ=Short Sense of Competence Questionnaire; ZBI=Zarit Burden Index

\section{Electrostimulation}

\section{Evidence Map: Electrostimulation}




\begin{tabular}{|c|c|c|c|c|c|c|c|c|c|c|c|c|c|}
\hline $\begin{array}{l}\text { Study (PMID) } \\
\text { Country } \\
\text { EM Reason }\end{array}$ & Intervention & Comparison & $\begin{array}{l}\text { Setting } \\
\text { and Design } \\
\text { Setting } \\
\text { Design } \\
\text { Cluster N } \\
\text { Participants } \\
\text { Randomized N }\end{array}$ & $\begin{array}{l}\text { PWD } \\
\text { Dementia } \\
\text { Characteristics }\end{array}$ & $\begin{array}{l}\text { PWD } \\
\text { Non-Disease } \\
\text { Char } \\
\text { PWD N } \\
\text { PWD Age (mean) } \\
\text { PWD Sex (\% } \\
\text { female) } \\
\text { PWD Race (\% } \\
\text { majority) } \\
\text { PWD Education } \\
\text { (mean years) }\end{array}$ & $\begin{array}{l}\text { PWD } \\
\text { Non-Disease } \\
\text { Char Reporting } \\
\text { Status (RS) } \\
\text { PWD SES } \\
\text { PWD Prior } \\
\text { Disability } \\
\text { PWD Household } \\
\text { Characteristics } \\
\text { PWD Health } \\
\text { Insurance } \\
\text { PWD Detailed } \\
\text { Race } \\
\text { Information } \\
\end{array}$ & $\begin{array}{l}\text { Informal } \\
\text { Caregiver (IC) } \\
\text { Characteristics } \\
\text { IC N } \\
\text { IC Age (mean) } \\
\text { IC Sex (\% } \\
\text { female) } \\
\text { IC Race (\% } \\
\text { majority) } \\
\text { IC Education } \\
\text { (mean years) } \\
\text { IC Relation to } \\
\text { PWD (\% } \\
\text { majority) }\end{array}$ & $\begin{array}{l}\text { Informal } \\
\text { Caregiver (IC) } \\
\text { Char. RS } \\
\text { IC Duration } \\
\text { IC Living With } \\
\text { PWD } \\
\text { IC Payment } \\
\text { IC Health } \\
\text { Status } \\
\text { IC Dementia } \\
\text { Family History } \\
\text { IC Employment } \\
\text { Status } \\
\text { IC Training } \\
\end{array}$ & $\begin{array}{l}\text { Formal } \\
\text { Caregiver (FC) } \\
\text { Characteristics } \\
\text { FC N } \\
\text { FC Age (mean) } \\
\text { FC Sex (\% } \\
\text { female) } \\
\text { FC Race (\% } \\
\text { majority) } \\
\text { FC Education } \\
\text { (mean years) }\end{array}$ & $\begin{array}{l}\text { Formal } \\
\text { Caregiver (FC) } \\
\text { Char. RS } \\
\\
\text { FC Health } \\
\text { Status } \\
\text { FC Training } \\
\text { FC Education } \\
\text { FC Position } \\
\text { FC Length of } \\
\text { Service }\end{array}$ & $\begin{array}{l}\text { Outcome } \\
\text { Timing(s) }\end{array}$ & PWD Outcomes & $\begin{array}{l}\text { Caregiver } \\
\text { Outcomes } \\
\text { IC: } \\
\text { FC: }\end{array}$ \\
\hline $\begin{array}{l}\text { Elder 2019 } \\
\text { (30658705) } \\
\text { UK } \\
\text { Small sample }\end{array}$ & $\begin{array}{l}2 \text { c consecutive } \\
20-m i n \text { sessions } \\
\text { of active } \\
\left(0.048 \mathrm{~mA} / \mathrm{cm}^{2}\right) \\
\text { tDCS separated } \\
\text { by a } 30 \text {-min } \\
\text { break, over } 5 \\
\text { consecutive } \\
\text { days. The } \\
\text { anodal } \\
\text { electrode was } \\
\text { applied to the } \\
\text { right parietal } \\
\text { cortex }(\mathrm{P} 4) \text { and } \\
\text { the cathodal } \\
\text { electrode was } \\
\text { applied to the } \\
\text { occipital cortex. }\end{array}$ & Sham tDCS & $\begin{array}{l}\text { Community } \\
\text { dwelling PLWDs } \\
\text { RCT } \\
\text { 40 PLWD }\end{array}$ & $\begin{array}{l}\text { Clinical diagnosis } \\
\text { of Lewy body } \\
\text { dementia; } \\
\text { experiencing visual } \\
\text { hallucinations of a } \\
\text { moderate to severe } \\
\text { nature and MMSE } \\
\text { score e } \geq 12\end{array}$ & $\begin{array}{l}\mathrm{N}=40 \\
\text { Mean age: } 76 \\
\text { years } \\
\% \text { Female: } \\
\text { \% Fojojority race: } \\
\text { no } \\
\text { Education: no }\end{array}$ & None & NA & None & $\mathrm{NA}$ & NA & $\begin{array}{l}\text { Day 5 } \\
1 \text { month } \\
3 \text { months }\end{array}$ & $\begin{array}{l}\text { MMSE } \\
\text { CAMCOG } \\
\text { GDS-15 } \\
\text { TMT A/B } \\
\text { ODFAAS } \\
\text { computerized } \\
\text { attentional and } \\
\text { visuoperceptual tasks }\end{array}$ & $\begin{array}{l}\text { NPI } \\
\text { CAF } \\
\text { ODFAS } \\
\text { CGI }\end{array}$ \\
\hline $\begin{array}{l}\text { Khedr 2019245 } \\
\text { (30940012) } \\
\text { Egypt } \\
\text { Small sample }\end{array}$ & $\begin{array}{l}\text { tDCS, } 20 \\
\text { minutes on } \\
\text { each side. } 2 \\
\text { weeks, } 10 \\
\text { sessions }\end{array}$ & $\begin{array}{l}\text { 21 PLWD got } \\
\text { tDCS }\end{array}$ & $\begin{array}{l}1 \text { clinical center } \\
R C T \\
N=44\end{array}$ & $\begin{array}{l}\text { Diagnosis of } \\
\text { probable } A D\end{array}$ & $\begin{array}{l}\mathrm{N}=23 \\
\text { Age: yes } \\
\text { Sex: yes } \\
\text { Education: yes } \\
\text { Race: no }\end{array}$ & NR & NA & NA & NA & NA & 2 weeks & $\begin{array}{l}\text { MMSE } \\
\text { MoCA } \\
\text { Clock drawing test } \\
\text { Cornell Depression } \\
\text { Scale }\end{array}$ & NA \\
\hline $\begin{array}{l}\text { Sabbagh } 2019 \\
\text { (31879235) } \\
\text { US and Israel } \\
\text { Safety study }\end{array}$ & $\begin{array}{l}\text { Combines short } \\
\text { bursts of rTMS } \\
\text { with } \\
\text { computerized } \\
\text { cognitive } \\
\text { training. Daily } \\
\text { sessions } \\
\text { applied across } \\
\text { three targeted } \\
\text { regions, with a } \\
\text { total of } 1300 \\
\text { rTMS pulses at } \\
10 \mathrm{~Hz} \text { in short } \\
\text { burts of } 20 \\
\text { pulses. }\end{array}$ & Sham rTMS. & $\begin{array}{l}\text { Community } \\
\text { dwelling PLWDs } \\
\text { RCT } \\
129 \text { PLWD }\end{array}$ & $\begin{array}{l}\text { Diagnosis of mild to } \\
\text { moderate AD using } \\
\text { DSM-IV and MMSE } \\
\text { score of between } \\
18 \text { to } 26 \text {; ADAS- } \\
\text { Cog }>17 \text {; }\end{array}$ & $\begin{array}{l}\mathrm{N}=129 \\
\text { Mean age: } 77 \\
\text { years } \\
\text { \% Female: } 46 \\
\% \text { majority race: } \\
\text { no } \\
\text { Education: yes }\end{array}$ & None & NA & None & NA & NA & $\begin{array}{l}\text { Week } 7 \\
\text { week } 12\end{array}$ & $\begin{array}{l}\text { ADAS-Cog } \\
\text { ADCS-CGI-C }\end{array}$ & NA \\
\hline
\end{tabular}




\begin{tabular}{|c|c|c|c|c|c|c|c|c|c|c|c|c|c|}
\hline $\begin{array}{l}\text { Zhang } 2019 \\
(\mathrm{xxx}) \\
\text { China } \\
\text { Small sample }\end{array}$ & $\begin{array}{l}\text { Repetitive } \\
\text { transcranial } \\
\text { magnetic } \\
\text { stimulation } \\
\text { combined with } \\
\text { cognitive } \\
\text { training: rTMS } \\
\text { operation lasted } \\
\text { approximately } \\
10 \text { minutes at } \\
\text { one targeted } \\
\text { encephalic } \\
\text { region. Every } \\
\text { day, the } \\
\text { magnetic coil } \\
\text { was first placed } \\
\text { over the left } \\
\text { DLPFC and } \\
\text { then over the } \\
\text { left temporal } \\
\text { lobe. The } \\
\text { treatment } \\
\text { protocol was } \\
\text { applied for } 4 \\
\text { weeks (5 } \\
\text { times/week) and } \\
\text { with no } \\
\text { maintenance } \\
\text { sessions }\end{array}$ & Sham rTMS & \begin{tabular}{|l} 
PLWDS were \\
recruited from \\
Department of \\
Neurology in \\
Tongji Hospital \\
at Huazhong \\
University of \\
Science and \\
Technology \\
(Wuhan, China) \\
RCT \\
30 PLWD \\
\end{tabular} & $\begin{array}{l}\text { Diagnosis of AD } \\
\text { (mild to moderate) } \\
\text { using NINCDS- } \\
\text { ADRDA }\end{array}$ & $\begin{array}{l}\mathrm{N}=28 \\
\text { Mean age: } 69 \\
\text { years } \\
\text { \% Female: } 79 \\
\text { \% majority race: } \\
\text { no } \\
\text { Education: no }\end{array}$ & None & $\mathrm{NA}$ & None & NA & NA & $\begin{array}{l}\text { Week } 4 \\
\text { week } 8\end{array}$ & $\begin{array}{l}\text { ADAS-Cog } \\
\text { MMSE } \\
\text { ACE-III } \\
\text { ADL } \\
\text { NPI }\end{array}$ & \begin{tabular}{|l|}
$N A$ \\
\end{tabular} \\
\hline $\begin{array}{l}\text { Lozano 2016 } \\
(27567810) \\
\text { Canada, US } \\
\text { Small sample } \\
\text { Leoutsakos } \\
2018^{247} \\
\text { (29914028) } \\
\text { Canada, US }\end{array}$ & $\begin{array}{l}12 \text { months } \\
\text { continuous } \\
\text { Fornix deep } \\
\text { brain stimulation } \\
\text { post-surgery }\end{array}$ & $\begin{array}{l}21 \text { control } \\
\text { PLWD got } \\
\text { sham pocedure } \\
\text { proced }\end{array}$ & $\begin{array}{l}7 \text { centers in } \\
\text { Canada and the } \\
\text { US } \\
\text { RCT- Phase II } \\
\mathrm{N}=42\end{array}$ & $\begin{array}{l}\text { Mild dementia with } \\
\text { CDR-SB of } 0.5 \text { or } 1 \\
\text { or ADAD-Cog } 13 \text { of } \\
12 \text { to } 24\end{array}$ & $\begin{array}{l}\mathrm{N}=21 \\
\text { Age: yes } \\
\text { Sex: yes } \\
\text { Race: no } \\
\text { Education: no }\end{array}$ & NR & NA & NA & NA & NA & 12 months & $\begin{array}{l}\text { Adverse events } \\
\text { Cerebral glucose } \\
\text { metabolism } \\
\text { Effects of age on } \\
\text { metabolism }\end{array}$ & NA \\
\hline $\begin{array}{l}\text { Wu 2015248 } \\
\text { (26977125) } \\
\text { China } \\
\text { Pilot }\end{array}$ & $\begin{array}{l}\text { Risperidone } 1 \\
\text { mg per day plus } \\
\text { rTMS } \\
\text { treatments for a } \\
\text { total of } 20 \\
\text { sessions, } 5 \\
\text { days a week for } \\
4 \text { consecutive } \\
\text { weeks }\end{array}$ & $\begin{array}{l}\text { Risperidone } 1 \\
\text { mg per day } \\
\text { plus sham } \\
\text { rTMS } \\
\text { treatments for } \\
\text { a total of } 20 \\
\text { sessions, } 5 \\
\text { days a week } \\
\text { for } 4 \\
\text { consecutive } \\
\text { weeks }\end{array}$ & $\begin{array}{l}1 \text { clinical center } \\
\text { (The Wuxi } \\
\text { Mental Health } \\
\text { Center) } \\
\text { RCT } \\
\mathrm{N}=54\end{array}$ & $\begin{array}{l}\text { Diagnosis of } \\
\text { probable AD } \\
\text { according to } \\
\text { NINCDS/ ADRDA } \\
\text { criteria with MMSE } \\
\text { score less than } 24 \\
\text { and BEHAVE-AD } \\
\text { score greater than } \\
8\end{array}$ & $\begin{array}{l}\mathrm{N}=27 \\
\text { Age: yes } \\
\text { Sex: yes } \\
\text { Education: yes } \\
\text { Race: no }\end{array}$ & NR & NA & NA & NA & NA & 4 weeks & $\begin{array}{l}\text { BEHAVE-AD } \\
\text { ADAS-Cog } \\
\text { Vital signs } \\
\text { TESS } \\
\text { Routine blood tests, } \\
\text { urine tests, } \\
\text { electrocardiogram, } \\
\text { blood biochemistry } \\
\text { adverse events }\end{array}$ & NA \\
\hline $\begin{array}{l}\text { Cotelli 2014年9 } \\
(24678298) \\
\text { Italy } \\
\text { Small sample }\end{array}$ & $\begin{array}{l}\text { AtDCS (25 } \\
\text { minutes) plus IC } \\
\text { Memory training } \\
\text { (5 days per } \\
\text { week f for 2 } \\
\text { weeks }\end{array}$ & $\begin{array}{l}12 \text { PLWD got } \\
\text { PtDCS ( } 25 \\
\text { minutes) plus } \\
\text { IC Memory } \\
\text { training (5 } \\
\text { days per week) } \\
12 \text { PLWD got } \\
\text { AtDCS (25 } \\
\text { minutes) plus } \\
\text { motor training } \\
\text { ( } 5 \text { days per } \\
\text { week) }\end{array}$ & $\begin{array}{l}1 \text { clinical center } \\
3 \text { arm } R C T \\
N=36\end{array}$ & $\begin{array}{l}\text { Diagnosis of mild to } \\
\text { moderate AD, } \\
\text { according to the } \\
\text { NINCDS-ADRDA } \\
\text { criteria, }\end{array}$ & $\begin{array}{l}\mathrm{N}=12 \\
\text { Age: yes } \\
\text { Sex: yes } \\
\text { Race: } n \\
\text { Education: yes }\end{array}$ & NR & $\mathrm{NA}$ & NA & NA & NA & $\begin{array}{l}3 \text { months } \\
6 \text { months }\end{array}$ & $\begin{array}{l}\text { MMSE } \\
\text { ADL } \\
\text { IADL } \\
\text { Balance and gait } \\
\text { NPI } \\
\text { Language } \\
\text { Complex figure copy } \\
\text { Attention and executive } \\
\text { function }\end{array}$ & \begin{tabular}{|l|} 
NA \\
\end{tabular} \\
\hline $\begin{array}{l}\text { Olazaran } \\
2014^{250} \\
(24898637) \\
\text { Spain } \\
\text { Pilot study }\end{array}$ & $\begin{array}{l}2 \text { hours of } \\
\text { REAC NPO per } \\
\text { day for } 2 \text { weeks }\end{array}$ & $\begin{array}{l}\text { 60 PLWD were } \\
\text { treated with } \\
\text { sham } \\
\text { procedure }\end{array}$ & $\begin{array}{l}\text { Residential care } \\
\text { facility } \\
\text { Crossover RCT } \\
\text { Single site } \\
\mathrm{N}=60\end{array}$ & $\begin{array}{l}\text { Diagnosis of AD or } \\
\text { probable AD } \\
\text { Nursing home } \\
\text { PLWD capable of } \\
\text { standing alone; day } \\
\text { care PLWD GDS } \\
\text { level } 6 \text { or } 7\end{array}$ & $\begin{array}{l}\mathrm{N}=60 \\
\text { Age: yes } \\
\text { Sex: yes } \\
\text { Race: no } \\
\text { Education: no }\end{array}$ & NR & NA & NA & NA & NA & $\begin{array}{l}2 \text { weeks each } \\
\text { arm }\end{array}$ & $\begin{array}{l}\text { GDS } \\
\text { Study to assess safety } \\
\text { of stimulation in AD } \\
\text { PLWD }\end{array}$ & \begin{tabular}{|l|} 
NA \\
\end{tabular} \\
\hline $\begin{array}{l}\text { Olazaran } \\
2013^{251} \\
(23603397) \\
\text { Spain } \\
\text { Pilot, }\end{array}$ & $\begin{array}{l}1 \text { treatment of } \\
\text { REAC NPO for } \\
2 \text { hours with } \\
\text { follow-up at } 2 \text { to } \\
3 \text { weeks }\end{array}$ & $\begin{array}{l}14 \text { control } \\
\text { PLWD got } \\
\text { sham } \\
\text { treatment }\end{array}$ & $\begin{array}{l}\text { Nursing home } \\
\text { PLWD } \\
\text { RCT } \\
\text { N=31 }\end{array}$ & $\begin{array}{l}\text { Diagnosis of } \\
\text { probable AD, not } \\
\text { bedridden, some } \\
\text { gait dysfunction }\end{array}$ & $\begin{array}{l}\mathrm{N}=17 \\
\text { Age: yes } \\
\text { Sex: yes } \\
\text { Race: no } \\
\text { Education: no } \\
\mathrm{N} \text { of medications: } \\
\text { yes }\end{array}$ & NR & NA & NA & NA & NA & 2 to 3 weeks & $\begin{array}{l}\text { Axial movement test } \\
\text { RSGE-CD } \\
\text { Walking test } \\
\text { SMMSE } \\
\text { Barthel Index } \\
\text { NPI }\end{array}$ & \begin{tabular}{|l|} 
NA \\
\end{tabular} \\
\hline
\end{tabular}




\begin{tabular}{|c|c|c|c|c|c|c|c|c|c|c|c|c|c|}
\hline $\begin{array}{l}\text { Suemoto } \\
2013^{252} \\
(24262299) \\
\text { Brazil } \\
\text { Small sample }\end{array}$ & $\begin{array}{l}\text { Anodal tDCS for } \\
20 \text { minutes; } 2 \\
\text { weeks; } 6 \\
\text { sessions }\end{array}$ & $\begin{array}{l}20 \text { PLWD got } \\
\text { sham tDCS }\end{array}$ & $\begin{array}{l}1 \text { clinical center } \\
\text { RCT-Phase II } \\
\mathrm{N}=40\end{array}$ & $\begin{array}{l}\text { Diagnosis of } \\
\text { possible or } \\
\text { probable AD and } \\
\text { score of } 14 \text { or more } \\
\text { on Starkstein } \\
\text { Apathy Scale }\end{array}$ & $\begin{array}{l}\mathrm{N}=20 \\
\text { Age: yes } \\
\text { Sex: yes } \\
\text { Education: yes } \\
\text { Race: yes }\end{array}$ & \begin{tabular}{|l|}
$N R$ \\
\end{tabular} & NA & NA & NA & NA & $\begin{array}{l}1 \text { week } \\
2 \text { weeks } \\
3 \text { week }\end{array}$ & $\begin{array}{l}\text { Starkstein Apathy Scale } \\
\text { Cornell Depression } \\
\text { Scale } \\
\text { NPI Caregiver Distress } \\
\text { ADAS-Cog } \\
\text { Digit Cancellation Task } \\
\text { Word list learning task } \\
\text { Word recognition task } \\
\end{array}$ & NA \\
\hline $\begin{array}{l}\text { Ahmed 2012253 } \\
\text { (21671144) } \\
\text { Egypt } \\
\text { Small sample }\end{array}$ & $\begin{array}{l}1 \text { daily } \\
\text { treatment of } \\
\text { rTMS on } \\
\text { DLPFC }(20 \mathrm{~Hz}) \\
\text { with follow-Hu at } \\
1 \text { month and } 3 \\
\text { months }\end{array}$ & $\begin{array}{l}15 \text { PLWD got } \\
\text { rTMS on } \\
\text { DLPFC (1 Hz) } \\
15 \text { PLWD } \\
\text { sham } \\
\text { procedure }\end{array}$ & $\begin{array}{l}1 \text { clinical center } \\
3 \text { arm } \mathrm{RCT} \\
\mathrm{N}=45\end{array}$ & $\begin{array}{l}\text { Diagnosis of } \\
\text { probable AD; } \\
\text { Dementia assessed } \\
\text { using MMSE, IADL, } \\
\text { GDS }\end{array}$ & $\begin{array}{l}\text { N=15 } \\
\text { Age: yes } \\
\text { Sex: yes } \\
\text { Education: yes } \\
\text { Residence: } y \text { es } \\
\text { Duration of } \\
\text { illness: yes }\end{array}$ & NR & NA & NA & NA & NA & 3 months & $\begin{array}{l}\text { MMSE } \\
\text { IADL } \\
\text { GDS }\end{array}$ & NA \\
\hline $\begin{array}{l}\text { Scherder } \\
2006^{254} \\
(16788393) \\
\text { The } \\
\text { Netherlands } \\
\text { Small sample }\end{array}$ & $\begin{array}{l}\text { High frequency } \\
\text { CES } 30 \text { min per } \\
\text { day, } 5 \text { days per } \\
\text { week for } 6 \\
\text { weeks }\end{array}$ & $\begin{array}{l}10 \text { control } \\
\text { PLWD treated } \\
\text { same way } \\
\text { without any } \\
\text { current }\end{array}$ & $\begin{array}{l}\text { Institutionalized } \\
\text { PLWD; } \\
\text { treatment and } \\
\text { control groups } \\
\text { blindly selected } \\
\mathrm{N}=21 \\
\end{array}$ & $\begin{array}{l}\text { Diagnosis based on } \\
\text { NINCDS-ADRDA } \\
\text { for probable AD } \\
\text { and stage } 5 \text { of GDS }\end{array}$ & $\begin{array}{l}\text { N=11 } \\
\text { Age: } \text { no } \\
\text { Sex: yes } \\
\text { Race: no } \\
\text { Education: yes }\end{array}$ & NR & NA & NA & NA & NA & 6 weeks & $\begin{array}{l}\text { BOP } \\
\text { BDI }\end{array}$ & NA \\
\hline $\begin{array}{l}\text { Van Dijk 2005255 } \\
\text { (15802911) } \\
\text { The } \\
\text { Netherlands } \\
\text { Pilot }\end{array}$ & $\begin{array}{l}\text { Electrical } \\
\text { stimulation was } \\
\text { given } 30 \\
\text { minutes a day } \\
\text { for } 7 \text { days a } \\
\text { week for } 6 \\
\text { weeks }\end{array}$ & $\begin{array}{l}\text { Placebo } \\
\text { stimulation }\end{array}$ & $\begin{array}{l}1 \text { clinical center } \\
\text { and } 1 \text { home } \\
\text { care center } \\
\\
\mathrm{RCT} \\
\mathrm{N}=68\end{array}$ & $\begin{array}{l}\text { Diagnosis of AD } \\
\text { according to } \\
\text { NINCDS/ ADRDA } \\
\text { criteria with MMSEE } \\
\text { of } 26 \text { or lower along } \\
\text { with sufficient } \\
\text { hearing and vision }\end{array}$ & $\begin{array}{l}\mathrm{N}=34 \\
\text { Age: } y e s \\
\text { Sex: yes } \\
\text { Education: yes } \\
\text { Race: no }\end{array}$ & NR & NA & NA & NA & NA & $\begin{array}{l}6 \text { weeks of } \\
\text { treatment for } \\
30 \text { min per day } \\
\text { Additional } \\
\text { follow-up for } 6 \\
\text { weeks }\end{array}$ & $\begin{array}{l}\text { Cognitive measures } \\
\text { Behavioral measures }\end{array}$ & NA \\
\hline $\begin{array}{l}\text { Hozumi 1996 } \\
\text { (9003961) } \\
\text { Japan } \\
\text { Small sample }\end{array}$ & $\begin{array}{l}\text { Transcranial } \\
\text { electro- } \\
\text { stimulation was } \\
\text { given for } 20 \\
\text { minutes for } 2 \\
\text { weeks }\end{array}$ & $\begin{array}{l}\text { 13 PLWD got } \\
\text { placebo } \\
\text { transcranial } \\
\text { electro- } \\
\text { stimulation }\end{array}$ & $\begin{array}{l}1 \text { clinical center } \\
\mathrm{RCT} \\
\mathrm{N}=27\end{array}$ & $\begin{array}{l}\text { Elderly PLWD with } \\
\text { sleep-wake } \\
\text { disorder and } \\
\text { dementia }\end{array}$ & $\begin{array}{l}\mathrm{N}=14 \\
\text { Age: yes } \\
\text { Sex" yes } \\
\text { Education: no } \\
\text { Race: no } \\
\text { Severity: yes }\end{array}$ & NR & NA & NA & NA & NA & 2 weeks & $\begin{array}{l}\text { Motivation } \\
\text { Behavior disorder } \\
\text { Sleep disorder } \\
\text { Intelligence } \\
\text { Emotion } \\
\text { Language } \\
\text { Neurological signs } \\
\text { Subjective complaint } \\
\text { Activity level }\end{array}$ & NA \\
\hline
\end{tabular}

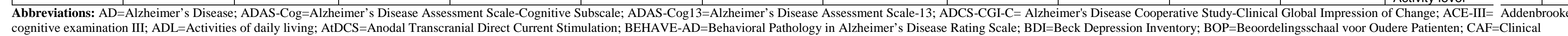

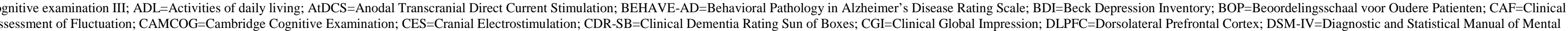

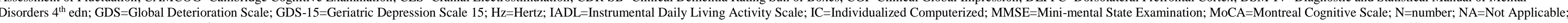

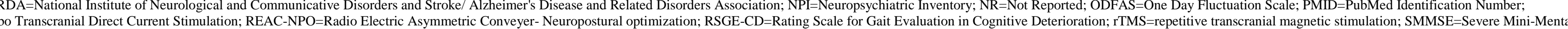
State Examination; tDCS=Transcranial Direct Current Stimulation; TESS=Treatment Emergent Symptom Scale; TMT A/B= Trail Making Test A and B

\section{Other Interventions for PLWD Well-Being}

\section{Evidence Map: Other Interventions for PLWD Well-Being}

\begin{tabular}{|c|c|c|c|c|c|c|c|c|c|c|c|c|c|}
\hline $\begin{array}{l}\text { Study (PMID) } \\
\text { Country } \\
\text { EM Reason }\end{array}$ & Intervention & $\begin{array}{l}\text { Compari- } \\
\text { son }\end{array}$ & $\begin{array}{l}\text { Setting } \\
\text { and Design } \\
\\
\text { Setting } \\
\text { Design } \\
\text { Cluster N } \\
\text { Participants } \\
\text { Randomized N }\end{array}$ & $\begin{array}{l}\text { PLWD } \\
\text { Dementia } \\
\text { Characteristics }\end{array}$ & \begin{tabular}{|l} 
PLWD \\
Non-Disease Char \\
PLWD N \\
PLWD Age (mean) \\
PLWD Sex (\% \\
female) \\
PLWD Race (\% \\
majority) \\
PLWD Education \\
(mean years)
\end{tabular} & \begin{tabular}{|l|} 
PLWD \\
Non-Disease \\
Char Reporting \\
Status (RS) \\
PLWD SES \\
PLWD Prior \\
Disability \\
PLWD \\
Household \\
Characteristics \\
PLWD Health \\
Insurance \\
PLWD Detailed \\
Race Information
\end{tabular} & $\begin{array}{l}\text { Informal Caregiver } \\
\text { (IC) } \\
\text { Characteristics } \\
\text { IC N } \\
\text { IC Age (mean) } \\
\text { IC Sex (\% female) } \\
\text { IC Race (\% } \\
\text { majority) } \\
\text { IC Education } \\
\text { (mean years) } \\
\text { IC Relation to } \\
\text { PLWD (\% majority) }\end{array}$ & $\begin{array}{l}\text { Informal Caregiver } \\
\text { (IC) Char. RS } \\
\text { IC Duration } \\
\text { IC Living With } \\
\text { PLWD } \\
\text { IC Payment } \\
\text { IC Health Status } \\
\text { IC Dementia } \\
\text { Family History } \\
\text { IC Employment } \\
\text { Status } \\
\text { IC Training }\end{array}$ & $\begin{array}{l}\text { Formal Caregiver } \\
\text { (FC) } \\
\text { Characteristics } \\
\text { FC N } \\
\text { FC Age (mean) } \\
\text { FC Sex (\% } \\
\text { female) } \\
\text { FC Race (\% } \\
\text { majority) } \\
\text { FC Education } \\
\text { (mean years) }\end{array}$ & $\begin{array}{l}\text { Formal } \\
\text { Caregiver (FC) } \\
\text { Char. RS } \\
\text { FC Health } \\
\text { Status } \\
\text { FC Training } \\
\text { FC Education } \\
\text { FC Position } \\
\text { FC Length of } \\
\text { Service }\end{array}$ & $\begin{array}{l}\text { Outcome } \\
\text { Timing(s) }\end{array}$ & \begin{tabular}{|l|} 
PLWD \\
Outcomes
\end{tabular} & $\begin{array}{l}\text { Caregiver } \\
\text { Outcomes } \\
\text { IC: } \\
\text { FC: }\end{array}$ \\
\hline $\begin{array}{l}\text { Liu 2018257 } \\
\text { (30464426) } \\
\text { China } \\
\text { Small sample }\end{array}$ & $\begin{array}{l}\text { Passive finger } \\
\text { movement } \\
\text { exercise } \\
\text { program based } \\
\text { on active finger } \\
\text { exercise along } \\
\text { with routine } \\
\text { care }\end{array}$ & Routine care & $\begin{array}{l}\text { Residents from } \\
\text { Hangzhou Love } \\
\text { Heart Older's } \\
\text { home in China } \\
\text { RCT } \\
\text { 54 PLWD }\end{array}$ & $\begin{array}{l}\text { Diagnosis of } \\
\text { dementia by } \\
\text { medical doctors } \\
\text { with PLWD's } \\
\text { fingers free from } \\
\text { disease restricting } \\
\text { hand movement } \\
\text { along with visual } \\
\text { and oral } \\
\text { communication } \\
\text { function and no } \\
\text { history of taking } \\
\text { anti-dementia } \\
\text { drugs }\end{array}$ & $\begin{array}{l}\mathrm{N}=18 \\
\text { Mean age }=80 \text { years } \\
\text { Female: } 67 \% \\
\text { majority race: } \mathrm{no} \\
\text { Education: } 39 \% \\
\text { illiterate }\end{array}$ & $\begin{array}{l}N R \\
\end{array}$ & NA & NA & NA & NA & 12 weeks & $\begin{array}{l}\text { Grip strength } \\
\text { (electrical } \\
\text { hand muscle } \\
\text { dynamometer) } \\
\text { Barthel index }\end{array}$ & NA \\
\hline
\end{tabular}




\begin{tabular}{|c|c|c|c|c|c|c|c|c|c|c|c|c|c|}
\hline $\begin{array}{l}\text { Rostad 2018258 } \\
\text { (29763832) } \\
\text { Noway } \\
\text { Pilot }\end{array}$ & $\begin{array}{l}\text { Pain } \\
\text { assessment } \\
\text { twice a week for } \\
12 \text { weeks using } \\
\text { Doloplus-2 pain } \\
\text { scale }\end{array}$ & Usual care & $\begin{array}{l}\text { Nursing home } \\
\text { Cluster RCT } \\
121 \text { PLWD }\end{array}$ & \begin{tabular}{|l} 
Dementia \\
diagnosis in \\
medical record with \\
being non-verbal or \\
inability to self- \\
report pain
\end{tabular} & $\begin{array}{l}\mathrm{N}=58 \\
\text { Mean age: } 84 \text { years } \\
\text { Female: } 78 \% \\
\text { \% majority race: no } \\
\text { Education: no }\end{array}$ & \begin{tabular}{|l|}
$N R$ \\
\end{tabular} & $\mathrm{NA}$ & $\mathrm{NR}$ & NA & $\mathrm{NA}$ & $\begin{array}{l}\text { Twice a week } \\
\text { for } 12 \text { weeks }\end{array}$ & $\begin{array}{l}\text { Doloplus-2 } \\
\text { pain scale } \\
\text { ATC NO2A } \\
\text { ATC No2B } \\
\text { ATC M01A } \\
\text { OMEQ }\end{array}$ & NA \\
\hline $\begin{array}{l}\text { Batchelor- } \\
\text { Murphy 2017259 } \\
\text { (28165618) } \\
\text { US } \\
\text { Pilot }\end{array}$ & $\begin{array}{l}\text { 11: Direct Hand } \\
\text { (DH): CG holds } \\
\text { the utensil or } \\
\text { cup for the } \\
\text { resident without } \\
\text { PLWD active } \\
\text { involvement } \\
\text { I2: Over Hand } \\
\text { (OH): CG puts } \\
\text { his/her hand } \\
\text { over the } \\
\text { resident's hand } \\
\text { to assist the } \\
\text { PLWDD. } \\
\text { I3: Under Hand } \\
\text { (UH): CG holds } \\
\text { the utensil or } \\
\text { cup and places } \\
\text { his/her hand } \\
\text { under the } \\
\text { resident's hand. } \\
\text { This technique } \\
\text { theoretically } \\
\text { allows the } \\
\text { resident to feel } \\
\text { as though } \\
\text { he/she initiated } \\
\text { the movement, } \\
\text { and is in control }\end{array}$ & NR & $\begin{array}{l}\text { Nursing Homes } \\
3 \text { arm RCT } \\
53 \text { PLWWD }\end{array}$ & $\begin{array}{l}\text { Diagnosis of AD or } \\
\text { related dementia, } \\
\text { BIMS score } \\
\text { between 0-12 }\end{array}$ & $\begin{array}{l}\mathrm{N}=23 \\
\text { Mean age: } 84 \text { years } \\
\text { Female: } 59 \% \\
\text { \% majority race: yes } \\
\text { Education: no }\end{array}$ & NR & NA & NA & NA & NA & 2 years & EdFED & NA \\
\hline $\begin{array}{l}\text { Kim 2017260 } \\
(27594544) \\
\text { Korea } \\
\text { Quasi- } \\
\text { experimental }\end{array}$ & $\begin{array}{l}\text { Participated in a } \\
\text { suicie } \\
\text { prevention } \\
\text { program twice a } \\
\text { week for } 5 \\
\text { weeks with one } \\
\text { pretest and } 2 \\
\text { posttests. }\end{array}$ & $\mathrm{NR}$ & $\begin{array}{l}\text { Recruited from } \\
\text { geriatric } \\
\text { daycare centers } \\
\text { Randomized } \\
\text { Pretest Posttest } \\
\text { design } \\
66 \text { PLWD }\end{array}$ & \begin{tabular}{|l|} 
Early-stage and \\
questionable \\
dementia selected \\
based on MMSE- \\
KC (score between \\
16 to 19 ; ; score > 5 \\
on both SIS and \\
GDSSF-K scale
\end{tabular} & $\begin{array}{l}\mathrm{N}=32 \\
\text { Mean age: } 82 \text { years } \\
\text { Female: } 77 \% \\
\text { \% majority race: no } \\
\text { Education: } 55 \% \\
\text { illiterate }\end{array}$ & NR & NA & $\mathrm{NR}$ & NA & NA & $\begin{array}{l}5 \text { weeks } \\
7 \text { weeks }\end{array}$ & $\begin{array}{l}\text { SIS } \\
\text { Perceived } \\
\text { health status } \\
\text { Barthel index } \\
\text { SS-A scale } \\
\text { GDSSF-K }\end{array}$ & NA \\
\hline $\begin{array}{l}\text { Hobbelen } \\
2012^{261} \\
(22185768) \\
\text { Netherlands } \\
\text { Pilot } \\
\text { Hobbelen } \\
2007^{261} \\
\text { (18093298) } \\
\text { Netherlands } \\
\text { Study Protocol }\end{array}$ & $\begin{array}{l}\text { PLWD, 20 } \\
\text { minutes session } \\
\text { of PMT by } \\
\text { trained physical } \\
\text { therapists, } 3 \\
\text { times a week for } \\
4 \text { weeks }\end{array}$ & $\begin{array}{l}\text { No PMT } \\
\text { (attention } \\
\text { control) }\end{array}$ & $\begin{array}{l}\text { Nursing home } \\
\text { residents } \\
\text { RCT } \\
102 \text { PLWD }\end{array}$ & $\begin{array}{l}\text { Dementia } \\
\text { diagnosis based on } \\
\text { DSM-IV-TR and } \\
\text { have paratonia with } \\
\text { a MAS score of at } \\
\text { least } 2 \text { in at least } 1 \\
\text { limb; }\end{array}$ & $\begin{array}{l}\mathrm{N}=35 \\
41 \% \text { Female } \\
82 \text { years } \\
\text { Race NR } \\
\text { Education NR } \\
\text { Use of psychotropic } \\
\text { meds: } 51 \%\end{array}$ & NR & NA & NA & NA & NA & $\begin{array}{l}2 \text { weeks } \\
4 \text { weeks }\end{array}$ & $\begin{array}{l}\text { MAS } \\
\text { CGI } \\
\text { PCS } \\
\text { PACSLAC-D }\end{array}$ & NA \\
\hline $\begin{array}{l}\text { Coyne 19977262 } \\
\text { (9281930 2) } \\
\text { US } \\
\text { Pilot }\end{array}$ & $\begin{array}{l}\text { Using directed } \\
\text { verbal prompts } \\
\text { (6 sets) and } \\
\text { positive } \\
\text { reinforcements } \\
\text { to complete } \\
\text { eating tasks }\end{array}$ & Unclear & $\begin{array}{l}\text { Dementia unit of } \\
\text { SNF } \\
3 \text { arm RCT } \\
24 \text { PLWD }\end{array}$ & $\begin{array}{l}\text { Diagnosis of } \\
\text { dementia; eat more } \\
\text { than half of the } \\
\text { meal without } \\
\text { assistance in the } \\
\text { communal dining } \\
\text { hall of the facility; }\end{array}$ & $\begin{array}{l}\mathrm{N}=12 \\
\text { Mean age: } 82 \text { years } \\
\text { Female: } 100 \% \\
\% \text { majority race: no } \\
\text { Education: no }\end{array}$ & NR & NA & NA & NA & NA & $\begin{array}{l}\text { Day } 6 \\
\text { Day } 7 \\
\text { Day } 12 \\
\text { Day } 13\end{array}$ & LEI & NA \\
\hline
\end{tabular}

=Anatomical Therapeutic Chemical; ATC-M01A=Anti-inflammatory and anti-rheumatic products; ATC-N02A=opioids; ATC-N02B=Other analgesics and antipyretics; BIMS=Brief Interview for Mental Status; Char=Characteristics; CG=Caregiver

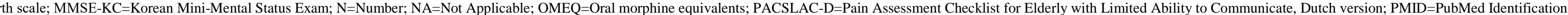
Number; PMT=Passive Movement Therapy; PSC=Patient specific complaints-modified; PLWD=Persons with Dementia; RCT=Randomized controlled trial; RoB=Risk of Bias; SIS=Suicidal Ideation Scale; SNF=Skilled Nursing Facility; SS-A=Social support appraisal 


\section{References for Appendix D}

1. Fleiner T, Dauth H, Zijlstra W, et al. A Structured Physical Exercise Program Reduces Professional Caregiver's Burden Caused by

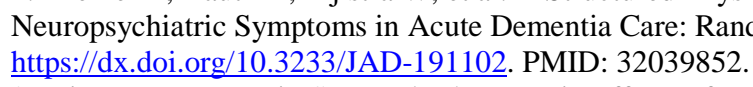

(a)d Controlled Trial Results. J Alzheimers Dis. 2020;74(2):429-33. doi:

. Liu IT, Lee WJ, Lin SY, et al. Therapeutic Effects of Exercise Training on Elderly Patients With Dementia: A Randomized Controlled Trial. arch Phys Med Rehabil. $2020 \mathrm{Feb}$ 19;19:19. doi: https:///dx.doi.org/10.1016/j.apmr.2020.01.012. PMID: 32084347.

4. Huang N, Li W, Rong X, et al. Effects of a Modified Tai Chi Program on Older People with Mild Dementia: A Randomized Controlled Trial. J Alzheimers Dis. 2019;72(3):947-56. doi: https://dx.doi.org/10.3233/JAD-190487. PMID: 31743998.

Karssemeijer EGA, Bossers WJR, Aaronson JA, et al. Exergaming as a Physical Exercise Strategy Reduces Frailty in People With Dementia:

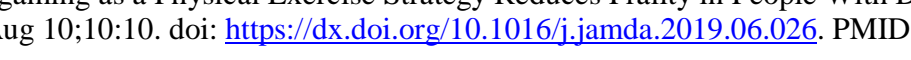

Henskens M, Nauta IM, Drost KT, et al. The effects of movement stimulation on activities of daily living performance and quality of life in nursing home residents with dementia: a randomized controlled trial. Clin Interv Aging. 2018;13:805-17. doi:

https://dx.doi.org/10.2147/CIA.S160031. PMID: 29750023.

. Ho RTH, Fong TCT, Chan WC, et al. Psychophysiological effects of Dance Movement Therapy and physical exercise on older adults with doi: https://dx doi. org/10 1093/geronb/gbyl45. PMID: 30496547.

8. Lamb SE, Sheehan B, Atherton N, et al. Dementia And Physical Activity (DAPA) trial of moderate to high intensity exercise training for

people with dementia: randomised controlled trial. Bmj. 2018 05 16;361:k1675. doi: https://dx.doi.org/10.1136/bmj.k1675. PMID: 29769247 9. Chen KM, Kuo CC, Chang YH, et al. Resistance Band Exercises Reduce Depression and Behavioral Problems of Wheelchair-Bound Older

Ittps://dx.doi.org/10.1111/igs.14526. PMID: 27879982. Individuals with Dementia: A 9-Week Randomized, Controlled Trial. J Am Geriatr Soc. 2016 Jun;64(6):1258-66. doi

hitps://dx.doi.org/10.1111/jgs.14160. PMID: 27321604.

11. Cancela JM, Ayan C, Varela S, et al. Effects of a long-term aerobic exercise intervention on institutionalized patients with dementia. J Sci ed Sport. 2016 Apr; 19(4):293-8. doi: https:///dx.doi.org/10.1016/j.jsams.2015.05.007. PMID: 26087884.

12. Hoffmann K, Sobol NA, Frederiksen KS, et al. Moderate-to-High Intensity Physical Exercise in Patients with Alzheimer's Disease: A
Randomized Controlled Trial. J Alzheimers Dis. 2016;50(2):443-53. doi: 10.3233/JAD-150817. PMID: 112439259. Language: English. Entry Date: 20161122. Revision Date: 20180627. Publication Type: journal article.

13. Toots A, Littbrand H, Lindelof N, et al. Effects of a High-Intensity Functional Exercise Program on Dependence in Activities of Daily Living and Balance in Older Adults with Dementia. J Am Geriatr Soc. 2016 Jan;64(1):55-64. doi: https://dx.doi.org/10.1111//gs.13880. PMID:

14. Telenius EW, Engedal K, Bergland A. Long-term effects of a 12 weeks high-intensity functional exercise program on physical function and https://dx.doi.org/10.1186/s12877-015-0151-8. PMID: 26630910.

15. Yang SY, Shan CL, Qing H, et al. The Effects of Aerobic Exercise on Cognitive Function of Alzheimer's Disease Patients. CNS Neurol isord Drug Targets. 2015;14(10):1292-7. PMID: 26556080.

16. Pitkala KH, Poysti MM, Laakkonen ML, et al. Effects of the Finnish Alzheimer disease exercise trial (FINALEX): a randomized controlled

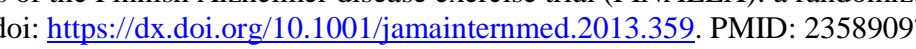
7. Hauer K, Schwenk M, Zieschang T, et al. Physical training improves motor performance in people with dementia: a randomized controlled trial. J Am Geriatr Soc. 2012 Jan;60(1):8-15. doi: https://dx.doi.org/10.1111/j.1532-5415.2011.03778.x. PMID: 22211512. 18. Fan J, Chen K. Using silver yoga exercises to promote physical and mental health of elders with dementia in long-term care facilities. In International psychogeriatrics / IPA

Sirk-Sanchez $\mathrm{N}$, et al. A randomized controlled trial of an activity specific exercise program for individuals with Alzheimer disease in long-term care settings. J Geriatr Phys Ther. 2011 Apr-Jun;34(2):50-6. doi:

Ittps://dx.doi.org/10.1519/JPT.0b013e31820aab9c. PMID: 21937893

20. Eggermont LH, Knol DL, Hol EM, et al. Hand motor activity, cognition, mood, and the rest-activity rhythm in dementia: a clustered RCT.

21. Rolland Y, Pillard F, Klapouszczak A, et al. Exercise program for nursing home residents with Alzheimer's disease: a 1-year randomized,

, Khan K, Mistry D, et al. Does Structured Exercise Improve Cognitive Impairment in People with Mild to Moderate Dementia? A Cost-Effectiveness Analysis from a Confirmatory Randomised Controlled Trial: The Dementia and Physical Activity (DAPA) Trial.
PharmacoEconomics - Open. 201901 Jun; $3(2): 215-27$. doi: http://dx.doi. org $10.1007 / 541669-018-0097-9$. PMID: 627968385.

23. Bossers WJ, van der Woude LH, Boersma F, et al. A 9-Week Aerobic and Strength Training Program Improves Cognitive and Motor Function in Patients with Dementia: A Randomized, Controlled Trial. Am J Geriatr Psychiatry. 2015 Nov;23(11):1106-16. doi:

24. Bossers WJ Scherder EJ, Boersma F, et al. Feasibility of a conb.

physical function in institutionalized dementia patients. A pilot study. PLoS ONE. 2014;9(5):e97577. doi:

hitps://dx.doi.org/10.1371/journal.pone.0097577. PMID: 24844772.

25. Sobol NA, Hoffmann K, Frederiksen KS, et al. Effect of aerobic exercise on physical performance in patients with Alzheimer's disease

Alzheimer's dement. 2016 12;12(12):1207-15. doi: https:///dx.doi.org/10.1016/j.jalz.2016.05.004. PMID: 27344641.

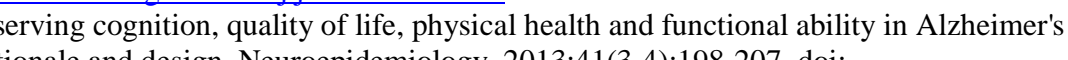
https://dx.doi.org/10.1159/000354632. PMID: 24135720.

27. Sondell A, Rosendahl E, Gustafson Y, et al. The Applicability of a High-Intensity Functional Exercise Program Among Older People With Dementia Living in Nursing Homes. J Geriatr Phys Ther. 2018 May 25;25:25. doi: https://dx.doi.org/10.1519/JPT.0000000000000199. PMID

28. Toots A, Wiklund R, Littbrand H, et al. The Effects of Exercise on Falls in Older People With Dementia Living in Nursing Homes: A
Randomized Controlled Trial J Am Med Dir Assoc. 2018 Nov 28:28:28. doi: https://dx.doi.org/10.1016/jijamda.2018.10.009. PMID:

30503589 .

29. Toots A, Littbrand H, Bostrom G, et al. Effects of Exercise on Cognitive Function in Older People with Dementia: A Randomized

Controlled Trial. J Alzheimers Dis. 2017;60(1):323-32. doi: https:///dx.doi.org/10.3233/JAD-170014. PMID: 28800328.

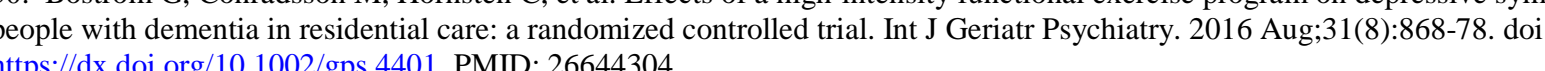

31. Roitto HM, Kautiainen H, Ohman H, et al. Relationship of Neuropsychiatric Symptoms with Falls in Alzheimer's Disease - Does Exercise Modify the Risk? J Am Geriatr Soc. 2018 Dec;66(12):2377-81. doi: https://dx.doi.org/10.1111/jgs.15614. PMID: 30320427 32. Ohman H, Savikko N, Strandeberg Ge, et al. Effects of Exercise on Cognition: The Finnish Alzheimer Disease Exercise Trial: A Randomized, Controlled Trial.J Am Geriatr Soc. 2016 Apr;64(4):731-8. doil. hitps://dx.doi.org/0.1111/gs.14059. PMID: 27037872.

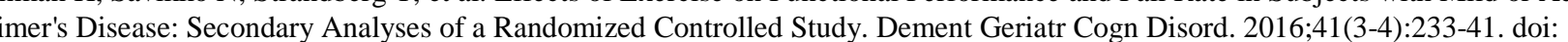
34. Perttila N Ohmman H, Strandberg T, et al. Severity of frailty and the outcome of exercise intervention among participants with Alzheimer disease: a sub-group analysis of a randonized controlled trial. hn European geriatric medich

35. Pitkala KH, Raivio MM, Laakkonen ML, et al. Exercise rehabilitation on home-dwelling patients with Alzheimer disease: A randomized, controlled trial. Baseline findings and feasibility. European Geriatric Medicine. 2011 December;2(6):338-43. doi:

36. Pomiersky R, Abel B, Werner C, et al. Increasing Physical Activity in Persons With Dementia: A Randomized Controlled Trial. J Agin Phys Activity. 2020 Jan 01:1-10. doi: httpp://dx.doi.org/10.1123/japa. 2019-0183. PMID: 31896078.

37. Enette L, Vogel T, Merle S, et al. Effect of 9 weeks continuous vs. interval aerobic training on plasma BDNF levels, aerobic fitness, cognitive capacity and quality of life among seniors with mild to moderate Alzheimer's disease: a randomized controlled trial. Eur. 2020;17:2.

作

39. Sanders LMJ, Hortobagyi T, Karssemeijer EGA, et al. Effects of low- and high-intensity physical exercise on physical and cognitive

function in older persons with dementia: a randomized controlled trial. Alzheimers Res Ther. 2020 Mar 19;12(1):28. do

https://dx.doi.org/10.1186/s13195-020-00597-3. PMID: 32192537.

thysical performance and fall incidents of individuals living with dementia in nursing homes: a randomized controlled trial. Physiother. 2019
https://dx.doi.org/10.1080/09593985.2019.1594470. PMID: 30912690.

41. Nyman SR, Ingram W, Sanders J, et al. Randomised Controlled Trial Of The Effect Of Tai Chi On Postural Balance Of People With Dementia. Clin Interv Aging. 2019;14:2017-29. doi: https://dx.doi.org/10.2147/CIA.S228931. PMID: 31819385.

42. Todri J, Lena O, Martinez Gil JL. A single blind randomized controlled trial of globl plo PM. disease patients. European Journal of Psychiatry. 2019 April - June;:33(2):83-90.
43. Liu JYW, Kwan RYC, Lai CK, et al. A simplified 10-step Tai-chi programme 44. Dawson N, Judge KS, Gerhart H. Improved Functional Performance in Individuals With Dementia After a Moderate-Intensity Home-Based Exercise Program: A Randomized Controlled Trial. J Geriatr Phys Ther. 2017 Mar 01;01:01. doi:

https://dx.doi.org/10.1519/JPT.0000000000000128. PMID: 28252473.

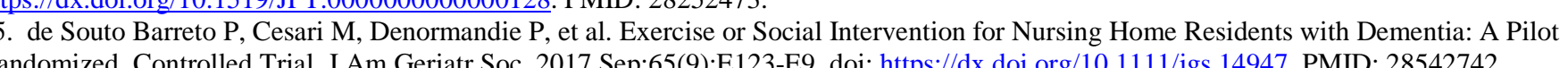
46. Hamilton G, Gallagher M, Pierson K, et al. Palliative care for dementia: a randomized controlled study of a replicable and financially viable model for organizations providing caregiver support. In Journal of pain and symptom management
47. Henwood $T$, Neville $C$, Baguley $C$, et al. Aquatic exercise for residential gaed care ed

47. Henwood T, Nevile C, Baguley C, et al. Aquatic exercise for residential aged care adults with dementia: benefits and barriers to

48. Lam F, Liao L, Kwok $\mathrm{T}$, et al. Effects of adding whole-body vibration to routine day activity program on physical functioning in elderly 49. Morris JK Viterate dementia: a randomized controlled trial. In Int J Geriatr Psychiatry

49. Morris JK, Vidoni ED, Johnson DK, et al. Aerobic exercise for Alzheimer's disease: A rand.
2017; 12(2): :01770547. doi: https://dx.doi.org/10.1371/journal.pone.0170547. PMID: 28187125.

50. Padala KP, Padala PR, Lensing SY, et al. Home-Based Exercise Program Improves Balance and Fear of Falling in Community-Dwelling Older Adults with Mild Al

51. Burge E, Berchtold A, Maupetit C, et al. Does physical exercise improve adl capacities in people over 65 years with moderate or severe
dementia hospitalized in an acute psychiatric setting? A multisite randomized clinical trial Int Psychogeriatr. 2016. PMID: CN-01308751. dementia hospitalized in an acute psychiatric setting? A multisite randomized clinical trial. Int Psychogeriatr. 2016. PMID: CN-0130875 52. Venturelli M, Sollima A, Ce E, et al. Effectiveness of Exercise- and Cognitive-Based Treatments on Salivary Colt
Sundowning Syndrome Symptoms in Patients with Alzheimer's Disease. J Alzheimers Dis. 2016;53(4):1631-40. doi:

http://dx.doi.org/10.3233/IAD-160392 PMID: 611692625.

53. Holthoff VA, Marschner K, Scharf M, et al. Effects of physical activity training in patients with Alzheimer's dementia: results of a pilot RCT study. PLoS ONE. 2015;10(4):e0121478. doi: https://dx.doi.org/10.1371/journal.pone.0121478. PMID: 25884637. 54. Yu F, Thomas W, Nelson NW, et al. Impact of 6-month aerobic exercise
500. doi: https://dx doi org 10.111770733464813512895 . PMID: 24652914.

55. Suttanon P, Hill KD, Said CM, et al. Feasibility, safety and preliminary evidence of the effectiveness of a home-based exercise programme for older people with Alzheimer's disease: a pilot randomized controll

56. Canonici P, Andrade LP, Gobbi S, et al. Functional dependence and caregiver burden in Alzheimer's disease: a controlled trial on the benefits of motor intervention. Psychogeriatrics. 2012;12(3):186-92. doi: 10.1111/j.1479-8301.2012.00407.x. PMID: CN-00863385

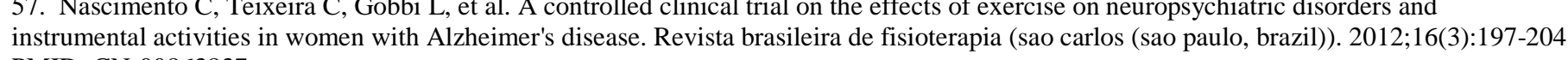
58. Vreugdenhil A, Cannell J, Davies A, et al. A community-based exercise programme to improve functional ability in people with
Alzheimer's disease: a randomized controlled trial. Scand J Caring Sci. 2012 Mar;26(1):12-9. doi: https://dx.doi.org/10.1111/j.1471-

6712.2011.00895.x. PMID: 21564154.
51. Stella F. Canonici A, Gobbi s, et al. Attenuation of neuropsychiatric symptoms and caregiver burden in Alzheimer's disease by motor
intervention. a controlled trial. Clinics (sao paulo, brazil). 2011:66(8):1353-60. PMID: CN-00860745. thervention: a controlled trial. Clinics (sa pa

60. Venturelli M, Scarsini R, Schena F. Six-month walking program changes cognitive and ADL performance in patients with Alzheimer. Am Alzheimers Dis Other Demen. 2011 Aug;26(5):381-8. doi: https://dx.doi.org/10.117/1533317511418956. PMID: 21852281.

61. Steinberg M, Leoutsakos JM, Podewils LJ, et al. Evaluation of a home-based exercise program in the treatment of Alzheimer's disease: the
Maximizing Independence in Dementia (MIND) study. Int J Geriatr Psychiatry. 2009 Jul;24(7):680-5. doi: https://dx.doi.org/10.1002/gps.2175. 62. Kwak YS, Um SY, Son TG, et al. Effect of regular exercise on senile dementia patients. Int J Sports Med. 2008 Jun;29(6):471-4. PMID: 18050054 . 

63. Williams CL, Tappen RM. Exercise training for depressed older adults with Alzheimer's disease. Aging Ment Health. 2008 Jan;12(1):72-80.
doi: https://dx.doi.org/10.1080/13607860701529932. PMID: 18297481.
64. Stevens J, Killeen M. A randomised controlled trial testing the impact of exercise on cognitive symptoms and disability of residents with

64. Stevens J, Killeen M. A randomised controlled trial testing the impact of exercise on cognitive symptoms and disability of residents with
dementia. Contemp Nurse. 2006 Feb-Mar;21(1):32-40. PMID: 16594879 .

65. Van de Winckel A, Feys H, De Weerdt W, et al. Cognitive and behavioural effects of music-based exercises in patients with dementia. Cli Rehabil. 2004 May;18(3):253-60. PMID: 15137556. 66. Toulotte C, Fabre C, Dangremont B, et al. Effects of physical training on the physical
falling: a randomised controlled trial. Age Ageing. 2003 Jan;32(1):67-73. PMID: 12540351 .

67. Ugur HG, Orak OS, Aktas YY, et al. Effects of music therapy on the care burden of in-home caregivers and physiological parameters of
their in-home dementia patients: a randomized controlled trial Complementary medicine research 2019-26(1):22-30. PMID . CN-10191571 68. Cheung DSK, Lai CKY, Wong FKY, et al. The effects of the music-with-movement intervention on the cognitive functions of people with moderate dementia: a randomized controlled trial. Aging Ment Health. 2018 Mar;22(3):306-15. doi:

https://dx.doi.org/10.1080/13607863.2016.1251571. PMID: 27819483.

69. Ho RT, Fong TC, Sing CY, et al. Managing behavioral and psychological symptoms in Chinese elderly with dementia via group-based

https:///dx.doi.org/10.11777/1471301218760023. PMID: 29468887.

作

PMID: 29871544.
71. Pongan E, Tillmann B, Leveque Y, et al. Can Musical or Painting Interventions Improve Chronic Pain, Mood, Quality of Life, and Cognition in Patients with Mild Alzheimer's Disease? Evide
https://dx.doi.org/10.3233/JAD-170410. PMID: 28922159.

quasi-experimental, longitudinal study. Aging Ment Health. 2017 02;21(2):156-62. doi: https://dx.doi.org/10.1080/13607863.2015.1093602. PMID: 26443002.

73. Sarkamo T, Tervaniemi M, Laitinen S, et al. Cognitive, emotional, and social benefits of regular musical activities in early dementia
randomized controlled study. Gerontologist. 2014 Aug:54(4):634-50, doi: https://dx. doi org/10 1093/geront/gnt100 PMID: 24009169 . randomized controlled study. Gerontologist. 2014 Aug;54(4):634-50. doi: https:///dx.doi.org/10.1093/geront/gnt100. PMID: 24009169.
74. Vink AC, Zuidersma M, Boersma F, et al. The effect of music therapy compared with general recreational activities in reducing agitation people with dementia: a randomised controlled trial. Int J Geriatr Psychiatry. 2013 Oct;28(10):1031-8. doi: https://dx.doi.org/10.1002/gps.3924. PMID: 23280604.
75. Sung HC, Lee WL, Li TL, et al. A group music intervention using percussion instruments with familiar music to reduce anxiety and 75. Sung HC, Lee WL, Li TL, et al. A group music intervention using percussion instruments with familiar
agitation of institutionalized older adults with dementia. Int J Geriatr Psychiatry. 2012 Jun;27(6):621-7. doi:

https://dx.doi.org/10.1002/gps.2761. PMID: 21823174.

music intervention against agitated behavior in elderly persons with dementia. Int $J$ 77. Chu H, Yang CY, Lin Y, et al. The impact of $/$ goup.doi.org/10.1002/gps.2580. PMID: 20672256.

randomized controlled study. Biol Res Nurs. 2014 Apr;16(2):209-17. doi: https://dx.doi.org/10.1177/1099800413485410. PMID: 23639952 78. Raglio A, Bellelli G, Traficante D, et al. Efficacy of music therapy in the treatment of behavioral and psychiatric symptoms of dementia. 79. Ledger A Baker F. An inestigtion of long Aging Ment Health. 2007;11(3):330-8. PMID: CN-00738375.

80. Cheung DSK, Lai CKY, Wong FKY, et al. Is music-with-movement intervention better than music listening and social activities in alleviating agitation of people with moderate dementia? A randomized controlled trial. Dementia. 2018 Sep 20:1471301218800195. do https:///dx.doi.org/10.1177//1471301218800195. PMID: 30235949.

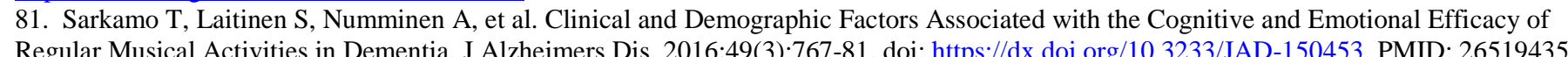
82. Weise L, Töpfer NF, Deux J, et al. Feasibility and effects of individualized recorded music for people with dementia: A pilot RCT study. Nordic Journal of Music Therapy. 2020;29(1):39-56. doi: 10.1080/08098131.2019.1661507. PMID: 141134381. Language: English. Entry Date: 20200117. Revision Date: 20200117. Publication Type: Article.

83. Thara ES, Tompkins CJ, Inoue M, et al. Results from a person-centered music intervention for individuals living with dementia. Geriatr
Gerontol Int 2019 Jan: 19(1):30-4, doi hthps:

Gerontol Int. 2019 Jan;19(1):30-4. doi: https://dx.doi.org/10.1111/ggi.13563. PMID: 30460747.

84. Park J, Tolea MI, Sherman D, et al. Feasibility of Conducting Nonpharmacological Interventions to Manage Dementia Symptoms in 18:1533317519872635. doi: https://dx.doi.org/10.1177/15333175198872635. PMID: 31533443.

85. Giovagnoli AR, Manfredi V, Schifano L, et al. Combining drug and music therapy in patients with moderate Alzheimer's disease: a

86. Narme P, Clement $\mathrm{S}$, Ehrle N, et al. Efficacy of musical interventions in dementia: evidence from a randomized controlled trial. $J$

87. Ridder HM, Stige B, Qvale LG, et al. Individual music therapy for agitation in dementia: an exploratory randomized controlled trial. Aging

Ment Health. 2013;17(6):667-78. doi: htttps://dx.doi.org/10.1080/13607863.2013.790926. PMID: 23621805.
88. Sakamoto M, Ando H, Tsutuou A. Comparing the effects of different individualized music interventions for elderly individuals with severe dementia. Int Psychogeriatr. 2013 May;25(5):775-84. doi: https://dx.doi.org/10.1017/S1041610212002256. PMID: 23298693 .

Alzheimers Dis Other Demen 2012 Aug.27(5):301-10. doi: https.//dx doi murg/ticenter, single-blind, randomized, and controlled trial. Am J 90. McHugh L, Gardstrom S, Hiller J, et al. The effect of pre-meal, vocal re-creative music therapy on nutritional intake of residents with Alzheimer's disease and related dementias: a pilot study. Music ther perspect. 2012;30(1):32-42. PMID: CN-01140174. 91. Chang F, Huang H, Lin K, et al. The effect of a music programme during lunchtime on the problem behaviour of the older residents with
dementia at an institution in Taiwan. J Clin Nurs. 2010;19(7-8):939-48. doi: 10.1111/j.1365-2702.2009.02801.x. PMID: CN-00752730 dementia at an institution in Taiwan. J Clin Nurs. 2010;19(7-8):939-48. doi: 10.1111/j.1365-2702.2009.02801.x. PMID: CN-00752730. people with dementia. J Health Psychol. 2010 Jul;15(5):765-76. doi: https://dx.doi.org/10.1177/1359105310368188. PMID: 20603300. 93. Cooke ML, Moyle W, Shum DH, et al. A randomized controlled trial exploring the effect of music on agitated behaviours and anxiety older people with dementia. Aging Ment Health. 2010 Nov;14(8):905-16. doi: https://dx.doi.org/10.1080/13607861003713190. PMID: 20635236.
94. Han P. Kwan M, Chen D, et al. A controlled naturalistic study on a weekly music therapy and activity program on disruptive and depressive 作

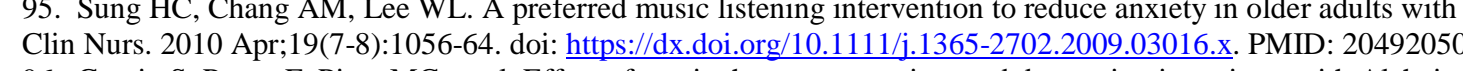

96. Guetin S, Portet F, Picot MC, et al. Effect of music therapy on anxiety and depression in patients with Alzheimer's type dementia:

randomised, controlled study. Dement Geriatr Cogn Disord. 2009;28(1):36-46. doi: hthps:///dx.doi.org/10.1159/000229024. PMID: 19628939 .
97. Choi A, Lee M. Cheong K, et al. Effects of group music intervention on behavioral and psychological symptoms in patients with dementions 97. Choi A, Lee M, Cheong K, et al. Effects of group music intervention on behavioral and psychological symptoms in patients with demential
a pilot-controlled trial. Int J Neurosci. 2009;119(4):471-81. doi: 10.1080/00207450802328136. PMID: CN-00688678. 98. Holmes C, Knights A, Dean C, et al. Keep music live: music and the alleviation of apathy in dementia subjects. Int Psychogeriatr. 2006
Dec;18(4):623-30. PMID: 16805928.
99. Sung HC, Chang SM, Lee WL, et al. The effects of group music with movement intervention on agitated behaviours of institutionalized

100. Svansdottir HB, Snaedal J. Music therapy in moderate and severe dementia of Alzheimer's type: a case-control study. Int Psychogeriatr.

101. Gerdner LA. Effects of individualized versus classical "relaxation" music on the frequency of agitation in elderly persons with Alzheimer's 101. Gerdner LA.
disease and relats of individidualized versus classical "relaxation" Int music on the frequegeriatr. 2000 Mar;12(1):49-65. PMID: 10798453.

102. Groene RW. Effectiveness of music therapy 1:1 intervention with individuals having Senile Dementia of the Alzheimer's Type. J Music Ther. 1993 Fal;30(3):138-57. doi: http://dx.doi.org/10.1093//mt/30.3.138. PMID: 1994-10861-00

103. Lord TR, Garner JE. Effects of music on Alzheimer patients. Percept Mot Skills. 1993 Apr;76(2):451-5. PMID: 8483655.

Activities of Daily Living for Patients With Alzheimer Disease I Geriatr Psychiatry Neurol. 2019 Oct 23:8919887198082099. doims, and https://dx.doi.org/10.1177/0891988719882099. PMID: 31645180.

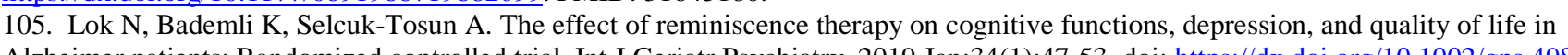
Alzheimer patients: Randomized controlled trial. Int J Geriatr Psychiatry. 2019 Jan;34(1):47-53. doi: https://dx.doi.org/10.1002/gps.4980.

106. Lin H-C, Yang Y-P, Cheng W-Y, et al. Distinctive effects between cognitive stimulation and reminiscence therapy on cognitive function

107. Amieva H, Robert PH, Grandoulier AS, et al. Group and individual cognitive therapies in Alzheimer's disease: the ETNA3 randomize Alzheimer Disease. J Geriatr Psychiatry Neurol. 2016 Jan;29(1):31-7. doi: https:///Xx.doi.org/10.1177/0891988 s of Daily Liviving for Patients Witt Alzheimer Disease. J Geriatr Psychiatry Neurol. 2016 Jan;29(1):31-7. doi: https://dx.doi.org/10.1177/0891988715598233. PMID: 26251112.
109. Van Bogaert P, Tolson D, Eerlingen R, et al. SolCos model-based individual reminiscence for older adults with mild to moderate dementia in nursing homes: a randomized controlled intervention study. J Psychiatr Ment Health Nurs. 2016 Nov;23(9-10):568-75. doi:

https://dx.doi.org $/ 10.1111 / j p m .12336$. PMID: 27511740 . being in elderly with mild and moderate dementia. Int J Geriatr Psychiatry. 2016 Feb;31(2):120-7. doi: https:///dx.doi.org/10.1002/gps.4300.
PMID: 25965388.

111. O'Shea E, Devane D, Cooney A, et al. The impact of reminiscence on the quality of life of residents with dementia in long-stay care. Int $J$

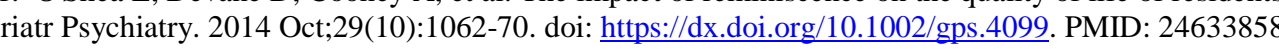

112. Serrani Azcurra DJ. A reminiscence program intervention to improve the quality of life of long-term care residents with Alzheimer's

effectiveness and cost-effectiveness pragmatic multicentre remdoniscence groups for people with dementita and their family caregiver

https://dx.doi.org/10.3310/hta16480. PMID: 23211271.

114. Hsieh C-J, Chang C, Su S-F, et al. Reminiscence group therapy on depression and apathy in nursing home residents with mild-to-moderate dementia. In Journal of experimental and clinical medicine

(15) 116. Alzheimer's disease. J Am Geriatr Soc. 1999 Apr;47(4):446-52. PMID: 10203120.

118. Woods RT, Bruce E, Edwards RT, et al. Reminiscence groups for people with dementia and their family carers: pragmatic eight-centre randomised trial of joint reminiscence and maintenance versus usual treatment: a protocol. Trials. 2009 Jul 30;10:64. doi:

https://dx.doi.org/10.1186/1745-6215-10-64. PMID: 19642992. Dementia and their Family Carers: Effectiveness and Economic Analysis. PLoS ONE. 2016;11(4):e0152843. doi

https://dx.doi.org/10.1371/journal.pone.0152843. PMID: 27093052.

120. Wang J, Yen M, OuYang W. Group reminiscence intervention in Taiwanese elders with dementia. In Archives of gerontology and

geriatrics
121 . Ching-Teng Y, Ya-Ping Y, Chia-Ju L, et al. Effect of group reminiscence therapy on depression and perceived meaning of life of veterans
diagnoed with dementia 141877576. Language: English. Entry Date: 20200227. Revision Date: 20200319. Publication Type: Article.

122. Manav AI, Simsek N. The effect of reminiscence therapy with internet-based videos on cognitive status and apathy of older people with mild dementia. Journal of geriatric psychiatry and neurology. 2019;32(2):104-13. PMID: CN-01707450.
123. Martinez N, Stutzman SE, Olson DM. Flectronic interventions aimed at increasing self-worth in 123. Martinez N, Stutzman SE, Olson DM. Electronic interventions aimed at increasing self-worth in mild dementia may not be feasible. J Am
Assoc Nurse Pract. 2019 Aug 22;22:22. doi: https://dx.doi.org/10.1097/JXX.00000000000000280. PMID: 31453826 . ApraicCauley C, et al. Using Mobile Technology to Provide Personalized Reminiscence for People Living With Dementia https://dx.doi.org/10.2196/mental.9684. PMID: 30206053 .

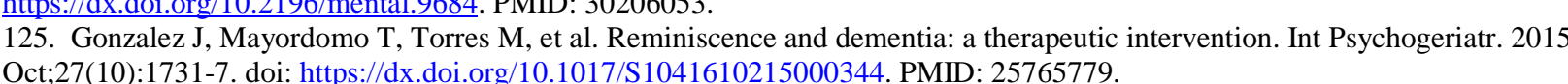
Oct;27(10):1731-7. doi: https://dx.doi.org/10.1017/S1041610215000344. PMID: 25765779.
126. Lalanne J Gallarda T, Piolino P. "The Castle of Remembrance": New insights from a co

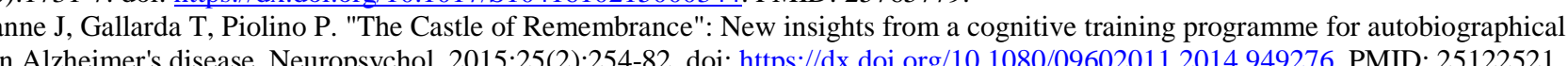
memory in Alzheimer's disease. Neuropsychol. 2015,25(2):254-82. doi: htps://dX.doi.org/10.1080/09602011.2014.949276. PMID: 25122521 dementia: A pilot randomized controlled study. Hong Kong Jour

http://dx.doi.org/10.1016/jihkjot.2014.01.003. PMID: 53104158.

128. Subramaniam P, Woods B, Whitaker C. Life review and life story books for people with mild to moderate dementia: a randomised controlled trial. Aging Ment Health. 2014;18(3):363-75. doi: https://dx.doi.org/10.1080/13607863.2013.837144. PMID: 24063317 .
129. Van Bogaert P, Van Grinsven R, Tolson D, et al. Effects of SolCos model-based individual reminiscence on older adults with mild to moderate dementia due to Alzheimer disease: a pilot study. J Am Med Dir Assoc. 2013 Jul;14(7):528.e9-13. doi:

https://dx.doi.org/10.1016/j.jamda.2013.01.020. PMID: 23583001. 130. Baillon S, Van Diepen E, Prettyman R, et al. A comparison of the effects of Snoezelen and reminiscence therapy on the agitated behaviour
of patients with dementia. Int J Geriatr Psychiatry. 2004 Nov;19(11):1047-52. PMID: 15481068.

(n) 132. Chen Z, Yu H. Effect of intervention guided by montessori method on improving feeding capacity of patients with dementia. Int J Clin

Exp Med. 2020;13(2):1148-55.
133. Clare L, Kudlicka A, Oyebode JR, et al. Individual goal-oriented cognitive rehabilitation to improve everyday functioning for people with
early-stage dementia: A multicentre randomised controlled trial (the GREAT trial). Int J Geriatr Psychiatry. 2019 May:34(5):709-21. doi: early-stage dementia: A multicentre randomised controlled
http://dx.doi.org/10.1002/gps.5076. PMID: 626609676. 
134. Voigt-Radloff S, de Werd MM, Leonhart R, et al. Structured relearning of activities of daily living in dementia: the randomized controlled REDALI-DEM trial on errorless learning. Alzheimers Res Ther. 2017 Mar 23;9(1):22. doi: https://dx.doi.org/10.1186/s13195-017-0247-9. PMID: 28335810.

政

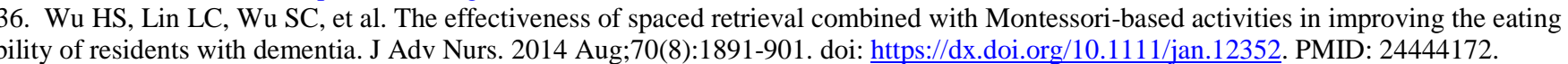
37. Clare L, Kudlicka A, Oyebode JR, et al. Goal-oriented cognitive rehabilitation for early-stage Alzheimer's and related dementias: the GREAT RCT. Health technology assessment. 2019;23(10):1-+. PMID: CN-01911576.

Vigt-Radloff S, Leonhart R, Rikkert MO, et al. Study protocol of the multi-site randomised controlled REDALI-DEM trial--the effects of dementia. BMC geriatr. 2011 Aug 18;11:44. doi:

139. Mbakile-Mahlanza L, van der Ploeg ES, Busija L, et al. A cluster-randomized crossover trial of Montessori activities delivered by family carers to nursing home residents with behavioral and psychological symptoms of dementia. Int Psychogeriatr. 2020 Mar;32(3):347-58. doi ittps://dx.doi.ong

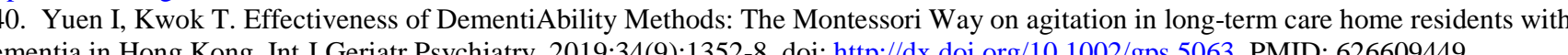
141. Silva AR, Pinho MS, Macedo L, et al. It is not only memory: effects of sensecam on improving well-being in patients with mild alzheim disease. Int Psychogeriatr. 2017 May;29(5):741-54. doi: https://dx.doi.org/10.1017/S104161021600243X. PMID: 28124633.

42. Silva AR, Pinho MS, Macedo L, et al. The cognitive effects of wearable cameras in mild Alzheimer disease-An experimental study. Curr

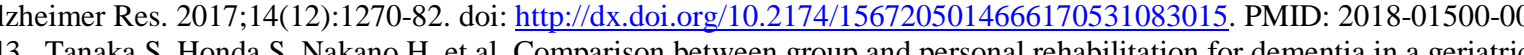
143. Tanaka S, Honda S, Nakano H, et al. Comparison between group and personal rehabilitation for dementia in a geriatric health service
facility: single-blinded randomized controlled study. Psychogeriatrics. 2017 May;17(3):177-85. doi: https://dx.doi.org/10.1111/psyg. 12212 PMID: 27612310

144. Tsuchiya K, Yamaguchi T, Fujita T, et al. A Quasi-Randomized Controlled Trial of Brain-Activating Rehabilitation in an Acute Hospital. m J Alzheimers Dis Other Demen. 2016 Dec;31(8):612-7. PMID: 27303064.

45. Kim S. Cognitive rehabilitation for elderly people with early-stage Alzheimer's disease. J Phys Ther Sci. 2015 Feb;27(2):543-6. doi: 146. Thivierge S, Jean L, Simard M. A randomized cross-or

living in Alzheimer disease. Am J Geriatr Psychiatry. 2014 Nov;22(11):1188-99. doi: https://dx.doi.org/10.1016/j.jagp.2013.03.008. PMID:

23871120.
147. Brunelle-Hamann L, Thivierge S, Simard M. Impact of a cognitive rehabilitation intervention on neuropsychiatric symptoms in mild to
moderate Alzheimer's disease. Neuropsychol. 2015;25(5):677-707. doi: https://dx.doi.org/10.1080/09602011.2014.964731. PMID: 25312605 . moderate Alzheimer's disease. Neuropsychol. 2015;25(5):677-707. doi: https://dx.doi.org/10.1080/09602011.2014.964731. PMID: 25312605. combined with Montessori-based activities. J Adv Nurs. 2013 Oct; 69 (10):2229-41. doi: https://dx.doi.org/10.1111/jann.12097. PMID: 23444942 149. van der Ploeg ES, Eppingstall B, Camp CJ, et al. A randomized crossover trial to study the effect of personalized, one-to-one interaction using Montessori-based activities on agitation, affect, and engagement in nursing home res
Apr;25(4):565-75. doi: https://dx.doi.org/10.1017/S1041610212002128. PMID: 23237211 .

Apr:25(4):565-75. doi: https://dX.doi.org/10.1017/S1041610212002128. PMID: 23237211.
150. Kurz A, Thone-OOtto A, Cramer B, et al. CORDIAL: cognitive rehabilitation and cognitive-behavioral treatment for early dementia in Alzheimer disease: a multicenter, randomized, controlled trial. Alzheimer Dis Assoc Disord. 2012 Jul-Sep;26(3):246-53. PMID: 21986341 151. Lin LC, Huang YJ, Watson R, et al. Using a Montessori method to increase eating ability for institutionalised residents with dementia: a
crossover design. J Clin Nurs. 2011 Nov:20(21-22):3092-101. doi: https://dx.doi.org/10.1111/j.1365-2702.2011. 03858.x. PMID: 21981704 .

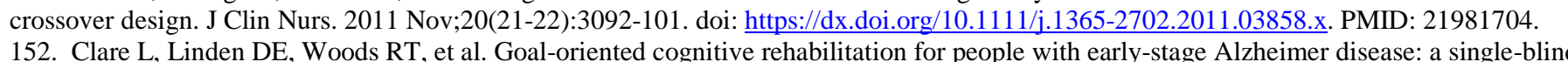
152. Clare L, Linden DE, Woods RT, et al. Goal-oriented cognitive rehabilitation for people with early
randomized controlled trial of clinical efficacy. Am J Geriatr Psychiatry. 2010 Oct; $18(10): 928-39$. doi:

hantps:///dx.doi.org/10.1097/JGP.06013e3181d5792a. PMID: 20808145.

153. Lam LC, Lui VW, Luk DN, et al. Effectiveness of an individualized functional training program on affective disturbances and functional skills in mild and moderate dementia--a randomized control trial. Int J Geriatr Psychiatry. 2010 Feb;25(2):133-41. do

https://dx.doi..0rg/10.1002/gps.2309. PMID: 19582757.

154. Lin LC, Huang YJ, Su SG, et al. Using spaced retrieval and Montessori-based activities in improving eating ability for residents with dementia. Int J Geriatr Psychiatry. 2010 Oct;25(10):953-9. doi: https://dx.doi.org/10.1002/gps.2433. PMID: 2005484 PMID: 1524927 on disruptive behavior and affect in demented nursing home 156. Beck CK, Vogelpohl TS, Rasin JH, et al. Effects of behaviord

157. Quayhagen MP, Quayhagen M, Corbeil RR, et al. A dyadic remediation program for care recipients with dementia. Nurs Res. 1995 May-
15;44(3):153-9. PMID: 7761291

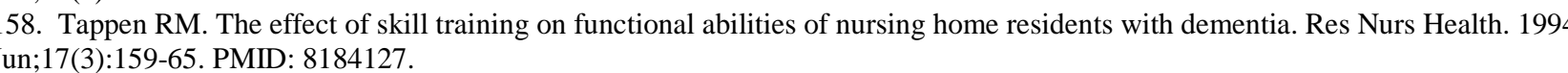
159. Tseng CF, Lee SH, Hsieh TC, et al. Smart Restored by Learning Exercise Alleviates the Deterioration of Cognitive Function in Older Adults with Dementia-A Quasi-Experimental Research. Int $J$ Environ Res Public Health. 2019 04 09;16(7):09. do

https://dx.doi.org/10.3390/ijerph16071270. PMID: 30970666.

160. Kallio EL, Ohman H, Hietanen M, et al. Effects of Cognitive Training on Cognition and Quality of Life of Older Persons with Dementia. J Am Geriatr Soc. 2018 Apr:66(4):664-70. doi: https://dx.doi.org/10.1111/3is.19

Alzheimer's Disease is Feasible and Effective: A Randomized Controlled Study. Arch Clin Neuropsychol. 2016 Sep 06;06:06. PMID: 27600448.

162. Giuli C, Papa R, Lattanzio F, et al. The Effects of Cognitive Training for Elderly: Results from My Mind Project. Rejuvenation Res. 2016 Dec;19(6):485-94. PMID: 26952713.

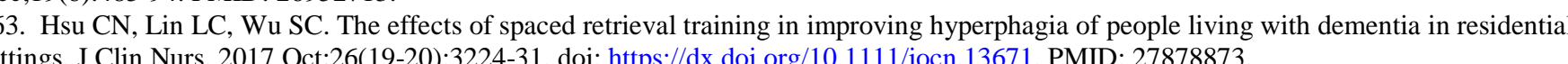
20).3224-31. doi: https:///dx.doi.org/10.1111/jocn.13671. PMID: 27878873.

164. Kao CC, Lin LC, Wu SC, et al. Effectiveness of different memory training programs on improving hyperphagic behaviors of residents
with dementia: a longitudinal single-blind study. Clin Interv Aging. 2016;11:707-20. doi: https://dx.doi.org/10.2147/CIA.S102027. PMID:

27307717.

作 http:///dx.doi.org/10.1016/j.eurger.2017.04.008. PMID: 616108233.

166. Giovagnoli AR, Manfredi V, Parente A, et al. Cognitive training in Alzheimer's disease: a controlled randomized study. Neurol Sci. 2017 Aug;38(8): 1485-93. doi: https://dx.doi.org/10.1007/s10072-017-3003-9. PMID: 28577267.

167. Chen HM, Tsai LJ, Chao SY, et al. Study on the Effects of Individualized Learning Therapy on Cognitive Function and Behavioral and
Psychological Symptoms of Dementia in the Institutionalized Older Adults. J Nurs Res. 2016 Dec;24(4):300-10. PMID: 27846102.

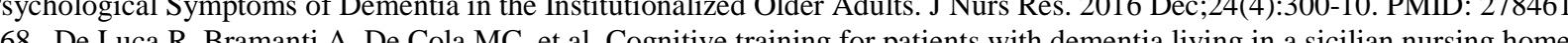

168. De Luca R, Bramanti A, De Cola MC, et al. Cognitive training for patients with dementia living in a sicilian nursing home:
based approach. Neurol Sci. 201601 Oct;37(10):1685-91. doi: http://dx.doi.org/10.1007/s10072-016-2659--. PMID: 611200073.
169. Kawashima R, Hiller D, Sereda S, et al. SAIDO Learning as a Cognitive Intervention for Dementia Care: aPreliminary Study. In J Am

170. Bergamaschi S, Arcara G, Calza A, et al. One-year repeated cycles of cognitive training (CT) for Alzheimer's disease. Aging Clin Exp
Res. 2013 Aug;25(4):421-6. doi: https://dx.doi.org-10.1007/s40520-013-0065-2. PMID: 23784727.

171. Mapelli D, Di Roca E N Nocita R, et al. Cognitive stimulation in patients with dementia: randomized controlled trial. Dement Geriatr Cog Dis Extra. 2013;3(1):263-71. doi: https:///xx.doi.org/10.1159/000353457. PMID: 24052800.

17. Tarraga L, Boada M, Modinos G, et al. A randomised pilot study to assess the efficacy of an interactive, multimedia tool of cognitive stimulation in Alzheimer's disease. J Neurol Neurosurg Psychiatry. 2006 Oct; 77(10):1116-21. PMID: 16820420.

173. McGilton KS, Rivera TM, Dawson P. Can we help persons with dementia find their way in a new environment?. [Erratum appears in
Aging Ment Health. 2003 Nov; 7(6):following author index]. Aging Ment Health. 2003 Sep; 7(5):363-71. PMID: 22959805

174. Davis RN, Massman PJ, Doody RS. Cognitive intervention in Alzheimer disease: a randomized placebo-controlled study. Alzheimer Dis Assoc Disord. 2001 Jan-Mar;15(1):1-9. PMID: 11236819.

175. Zarit SH, Zarit JM, Reever KE. Memory training for severe memory loss: Effects on senile dementia patients and their families. Gerontologist. 1982;22(4):373-7. PMID: 13238424. cognitive functions, coping-adaptation skills, and quality of life: A randomized controlled trial. Perspect Psychiatr Care. 2020 Jan 12;12:12. do
https://dx.doi.org/10.1111/ppc.12472. PMID: 31930518 . 177. Lin HC, Yang YP, Cheng WY, et al. Distinctive effects between cognitive stimulation and reminiscence therapy on
quality of life for different types of behavioural problems in dementia. Scand J Caring Sci. 2018 Jun;32(2):594-602. doi:

https://dx.doi.org/10.1111/scs.12484. PMID: 28881430.

ind ct. Individual cognitive stimulation therapy for dementia: a clinical effectiveness and cost-effectiveness PMID: 26292178. 179. Orrell M, Yates L, Leung P, et al. The impact of individual Cognitive Stimulation Therapy (iCST) on cognition, quil
health, and family relationships in dementia: A randomised controlled trial. PLoS Med. 2017 Mar;14(3):e1002269. doi: https://dx.doi.org/10.1371/journal.pmed.1002269. PMID: 28350796.

180. Orrell M, Aguirre E, Spector A, et al. Maintenance cognitive stimulation therapy for dementia: single-blind, multicentre, pragmatic randomised controlled trial. Br J Psychiatry. 2014 Jun; 204(6):454-61. doi: https://dx.doi.org/10.1192/bjp.bp.113.137414. PMID: 24676963. dementia: randomised controlled trial. Br J Psychiatry. 2003 Sep; $183: 248-54$. PMID: 12948999.

the Psychiatry. 2006 Jun;188:574-80. PMID: 16738349.

183. Cheung DSK, Li B, Lai DWL, et al. Cognitive Stimulating Play Intervention for Dementia: A Feasibility Randomized Controlled Trial.
Am J Alzheimers Dis Other Demen. 2019 02;34(1):63-71. doi: https://dx.doi.org/10.1177/1533317518808036. PMID: 30370782 . 184. Capotosto E, Belacchi C, Gardini S, et al. Cognitive stimulation therapy in the Italian context: Its efficacy in cognitive and non-cognitive
masurus in older adults with dementia. Int J Geriatr Psychiatry. 2017 Mar;32(3):331-40. doi: http://dx.doi.org/10.1002/gps.4521. PMID: 201705902-0 in. 185. Wong G, Yek O, Zhang A, et al. Cultural adaptation of cognitive stimulation therapy (CST) for Chinese people with dementia: multicentre pilot study. In Int $J$ Geriatr Psychiatry

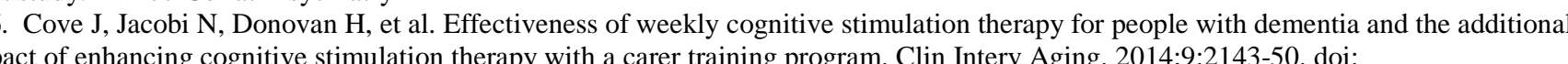
https://dx.doi.org/10.2147/CIA.S66232. PMID: 25525349

187. Yamanaka K, Kawano Y, Noguchi D, et al. Effects of cognitive stimulation therapy Japanese version (CST-J) for people with dementia: single-blind, controlled clinical trial.[Erratum appears in Aging Ment Health. 2013;17(5):654]. Aging Ment Health. 2013;17(5):579-86. doi
https://dx.doi.org/10.1080/13607863.2013.777395. PMID: 23550665.

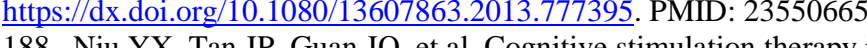

randomized controlled trial. Clin Rehabil. 2010 Dec;24(12):1102-11. doi: https:///dx.doi.org/10.1177/0269215510376004. PMID: 20713437. 189. Matsuda $\mathrm{O}$. Cognitive stimulation therapy for Alzheimer's disease: the effect of cognitive stimulation therapy on the progression of mild Alzheimer's disease in patients treated with donepezil. Int Psychogeriatr. 2007;19(2):241-52. doi: 10.1017/S1041610206004194. PMID: CN-

190. Pedrinolla A, Tamburin S, Brasioli A, et al. An Indoor Therapeutic Garden for Behavioral Symptoms in Alzheimer's Disease: A Randomized Controlled Trial. J Alzheimers Dis. 2019;71(3):813-23. doi: https:///dx.doi.org/10.3233/JAD-190394. PMID: 31424399 .
191. Tse MMY, Lau JL, Kwan R, et al. Effects of play activities program for nursing home residents with dementia on pain and psychological well-being: Cluster randomized controlled trial. Geriatr Gerontol Int. 2018 Oct;18(10):1485-90. doi: https://dx.doi.org/10.1111/ggi.13509. PMID: 30255637
192. Lin Q Cao Y,

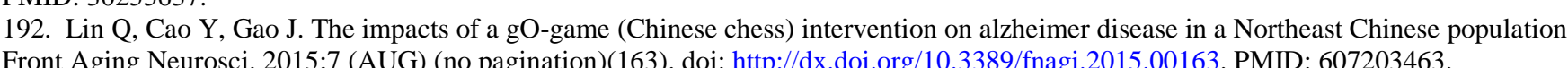
Front Aging Neurosci. 2015;7 (AUG) (no pagination)(163). doi: http:///dx.doi.org/10.3389/fnagi.2015.00163. PMID: 607203463 .
193. Van Haitsma KS, Curyto K, Abbott KM, et al. A randomized controlled trial for an individualized positive psychosocial inter the affective and behavioral symptoms of dementia in nursing home residents. J Gerontol B Psychol Sci Soc Sci. 2015 Jan;70(1):35-45. doi:

https://dx.doi.org/10.1093/geronb/gbt102. PMID: 24304555. randomised trial of humour therapy in nursing homes. BMJ Open. 2013 Jan 11:3(1):11, doi: https://dx doi org/10 1136/hmiopen-2012-002072. 195. Kolanowski A, Litaker M, Buettner L, et al. A randomized clinical trial of theory-based activities for the behavioral symptoms of dementia in nursing home residents. J Am Geriatr Soc. 2011 Jun;59(6):1032-41. doi: https://dx.doi.org/10.1111/j.1532-5415.2011.03449.x. PMID: 21649633.

196. Buettner LL, Fitzsimmons S. AD-venture program: therapeutic biking for the treatment of depression in long-term care residents with 197. Ha. Am J Alzheimers Dis Other Demen. 2002 Mar-Apr,1 (2).121 7 . Phr h. 1195460 of

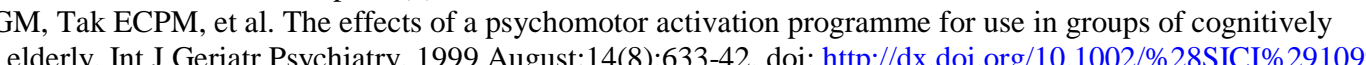
1166\%28199908\%2914:8\%3C633::AID-GPS996\%3E3.0.CO;2-W. PMID: 29403282.

198. Laksono WAW, Haryanto J, Bahiyah K. The impact of chess games towards comfortableness of cognitive mind on elderly. Indian Journal of Public Health Research and Development. 2019 September;10(9):873-7.

199. Lathifah MN, Haryanto J, Fauziningtyas R. The influence of dhakonan games to impede dementia in elders. Indian Journal of Public
Health Resarch and Development 2019 September: 10(l):1440-4

200. Li DM, Li XX. The effect of folk recreation program in improving symptoms: a study of Chinese elder dementia patients. Int J Geriatr Psychiatry. 2017 08;32(8):901-8. doi: https://dx.doi.org/10.1002/gps.4543. PMID: 27345629 .
201. Schall A, Tesky VA, Adams AK, et al. Art museum-based intervention to promote emotional well-being and improve quality of life in people with dementia: The ARTEMIS project. Dementia. 2017 Jan 01:1471301217730451. doi: https:///dx.doi.org $10.1177 / 14771301217730451$
PMID: 28914089

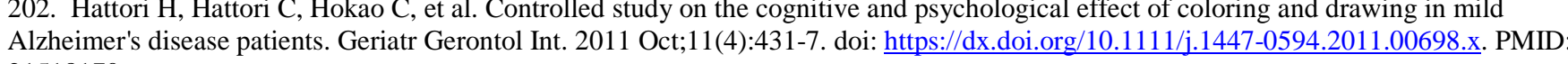
21518170. 
203. Onder G, Zanetti O, Giacobini E, et al. Reality orientation therapy combined with cholinesterase inhibitors in Alzheimer's disease: randomised controlled trial. Br J Psychiatry. 2005 Nov;187:450-5. PMID: 16260821.

204. Quinn C, Toms G, Jones C, et al. A pilot randomized controlled trial of a self-management group intervention for people with early-stage
dementia (The SMART study). Int Psychogeriatr. 2016 May-28(5):787-800 doi:-thes dementia $($ C.

205. Quinn C, Anderson D, Toms G, et al. Self-management in early-stage dementia: a pilot randomised controlled trial of the efficacy and cost-effectiveness of a self-management group intervention (the SMART study). Trials. 2014 Mar 08;15:74. doi

https:///dx.doi.org/10.1186/1745-6215-15-74. PMID: 24606601.
206. Young DK, Kwok TC, Ng PY. A single blind randomized control trial on support groups for Chinese persons with mild dementia. Clin
Interv Aging. 2014;9:2105-12. doi: https://dx.doi.org/10.2147/CIA.S68687. PMID: 25587218. 207. Tappen RM, Williams CL. Therapeutic conversation to improve mood in nursing home
Oct;22(4):267-75. doi: https://dx.doi.org/10.3928/19404921-20090428-02. PMID: 20077983.

208. Burns A, Guthrie E, Marino-Francis F, et al. Brief psychotherapy in Alzheimer's disease: randomised controlled trial. Br J Psychiatry.
2005 2005 Aug;:187:143-7. PMID: 16055825. 209. Cohen CI, Hyland K, Devlin M. An evaluation of the use of the natural helping network model to enhance the well-being of nursing home
residents. Gerontologist. 1999;39(4):426-33. PMID: 107227653. Language: English. Entry Date: 19991201. Revision Date: 20150711.
Publication Type: Journal Article.

210. Wallis GG, Baldwin M, Higginbotham P. Reality orientation therapy--a controlled trial. Br J Med Psychol. 1983 Sep;56(Pt 3):271-7.

PMID: 6354248.
211. Fritsch T, Kwak J, Grant S, et al. Impact of TimeSlips, a Creative Expression Intervention Program, on Nursing Home Residents with Dementia and heir

212. Lin LC, Yang MH, Kao CC, et al. Using acupressure and Montessori-based activities to decrease agitation for residents with dementia: a 213. Houser WS, George DR, Chinchilli VM. Impact of TimeSlips creative expression program on behavioral symptoms and psychotropic medication use in persons with dementiti in long-tertm care: a cluster-randonized pilot study. Am J Geriatr Psychiatry. 2014 Apry,22(4):337-40.
doi: https://dx.doi.org/10.1016/j.jagp.2012.12.005. PMID: 23602304.

214. van Dijk AM, van Weert JC, Droes RM. Does theatre improve the quality of life of people with dementia? Int Psychogeriatr. 2012
Mar;24(3):367-81. doi: https://dx.doi.org/10.1017/S1041610211001992 PMID 2 22040605

with dementia. Nurs Res. 2010;59(6):417-25. doi: 10.1097/NNR.0b013e3181faff52. PMID: CN-00770848.

Coll

217. Rokstad AMM, Engedal K, Kogn Disord. 2018;45(5-6):318-25. doi: https://dx.doi.org/10.1159/000490613. PMID: 30036871.

nursing home admission: a 24-month controlled study. BMC Health Serv Res. 2018 Nov 16;18(1):864. doi: https://dx.doi.org/10.1186/s12913

218. Satoh M, Ogawa J, Tokita T, et al. Physical exercise with music maintains activities of daily living in patients with dementia: mihama-kiho project part 2. In Journal of alzheimers disease

219. Middelstadt J, Folkerts AK, Blawath S, et al. Cognitive Stimulation for People with Dementia in Long-Term Care Facilities: Baseline Cognitive Level Predicts Cognitive Gains, Moderated by Depression. J Alzheimers Dis. 2016 08 04;54(1):253-68. doi:

220. Fernandez-Calvo B, Contador I, Ramos F, et al. Effect of unawareness on rehabilitation outcome in a randomised controlled trial of multicomponent intervention for patients with mild Alzheimer's disease. Neuropsychol. 2015;25(3):448-77. do

https://dx.doi.org/10.1080/09602011.2014.948461. PMID: 25121567.

221. Luttenberger K, Donath C, Uter W, et al. Effects of multimodal nondrug therapy on dementia symptoms and need for care in nursing home residents with degenerative dementia: a randomized-controlled study wit 6 -

hitps://dx.doi.org/10.1111/1.1532-5415.2012.03938.X. PMID: 22468985. dementia in residential care homes. Dement Geriatr Cogn Dis Extra. 2012 Jan;2(1):372-80. doi: https://dx.doi.org/10.1159/000342614. PMID

223. Brooker DJ, Argyle E, Scally AJ, et al. The enriched opportunities programme for people with dementia: a cluster-randomised controlled trial in 10 extra care housing schemes. Aging Ment Health. 2011 Nov;15(8):1008-17. doi: https://dx.doi.org/10.1080/13607863.2011.583628. PMID: 21702705.

224. Ferrero-Arias J, Goni-Imizcoz M, Gonzalez-Bernal J, et al. The efficacy of nonpharmacological treatment for dementia-related apathy. Alzheimer Dis Assoc Disord. 2011 Jul-Sep;25(3):213-9. doi: https:///dx.doi.org/10.1097/WAD.0b013e3182087dbc. PMID: 21346517. 225. McCurry SM, Pike KC, Vitiello MV, et al. Increasing walking and bright light exposure to improve sleep in community-dwelling person with Alzheimer's disease: results of a randomized, controlled trial. J Am G

hitps://dx.doi.org/10.1111/j.1532-5415.2011.03519.x. PMID: 21797835 . Gerontologist. 2007 Dec;47(6):775-88. PMID: 18192631. Gerontologist. 2007 Dec;47(6):775-88. PMID: 18192631.
227. Chapman SB, Weiner MF, Rackley A, et al. Effects of cognitive-communication stimulation for Alzheimer's disease patients treated with
donepezil. J Speech Lang Hear Res. 2004 Oct; 47 (5):1149-63. PMID: 15603468.

228. Tadaka E, Kanagawa K. Randomized controlled trial of a group care program for community-dwelling elderly people with dementia.
Japan Journal of Nursing Science. 2004 Aug; $1(1): 19-25$. doi: http://dx.doi.org/10.1111/i.1742-7924.2004.00006.x. PMID: 2007-06056-002.

229. Wimo A, Mattsson B, Adolfsson R, et al. Dementia day care and its effects on symptoms and institutionalization--a controlled Swedish

study. Scand J Prim Health Care. 1993 Jun;11(2):117-23. PMID: 8356361.

230. Luttenberger K, Hofner B, Graessel E. Are the effects of a non-drug multimodal activation therapy of dementia sustainable? Follow-up study 10 months after completion of a randomised controlled trial. BMC Neurol. 2012 Dec 05;12:151. doi: https:///dx.doi.org/10.1186/14712377-12-151. PMID: 23217188.

Save the Long-Term Care Cost? A Pilot Study of Effectiveness and Cost Caving Analysis of "Learning Therapy" for People with Diving and Save the Long-Term Care Cost? A Pilot Study of Effectiveness and Cost Saving Analysis of "Learning Therapy" for People with Dementia.
Alzheimers Dis. 2020 Feb 27;27:27. doi: https://dx.doi.org/10.3233/JAD-190886. PMID: 32116248 .
232. Young DK, Ng PY, Kwok T, et al. The effects of an expanded cognitive stimulation therapy model on the improvement of cognitive 232. Young DK, Ng PY, Kwok T, et al. The effects of an expanded cognitive stimulation therapy model on the improvement of cognitive
ability of elderly with mild stage Dementia living in a community - a randomized waitlist controlled trial. Aging Ment Health. 2019 ability of elderly with mild stage Dementia living in a community - a randomized waitlist conter
Jul;23(7):855-62. doi: https://dx.doi.org/10.1080/13607863.2018.1471586. PMID: 29781725.
233. Chen YL, Pei YC. Musical dual-task training in patients with mild-to-moderate dementia: a randomized controlled trial. Neuropsychiatr. 2018;14:1381-93. doi: https://dx.doi.org/10.2147/NDT.S159174. PMID: 29881275.

234. Reisberg B, Shao Y, Golomb J, et al. Comprehensive, Individualized, Person-Centered Management of Community-Residing Persons with https://dx.doi.org/10.1159/000455397. PMID: 28122366

235. Kampragkou C, Iakovidis P, Kampragkou E, et al. Effects of a 12-week aerobic exercise program combined with music therapy and

memory exercises on cognitive and functional ability in people with middle type of alzheimer's disease. 2017;4(5):262-8. doi:
10.15621/ijphy/2017/v4is/159420. PMID: CN-01425336.

236. Masayuki S, Jun-ichi O, Tomoko T, et al. Physical Exercise with Music Maintains Activities of Daily Living in Patients with Dementia Date: 20180302. Revision Date: 20180619. Publication Type: journal articl.

237. Kim H-J, Yang Y, Oh J-G, et al. Effectiveness of a community-based multidomain cognitive intervention program in patients with Alzheimer's disease. Geriatr Gerontol Int. 2016;16(2):191-9. doi: 10.1111/ggi.12453. PMID: CN-01132849.

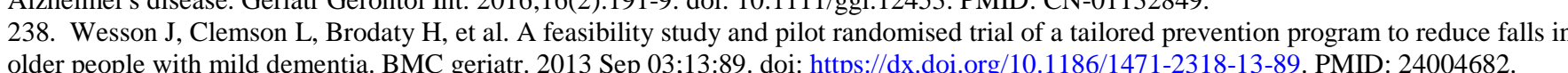
239. Burgener SC, Marsh-Yant S, Nega KK. A Combined, Multimodal Intervention for Individuals with Dementia. Res. 2011:411:64. 239. Burgener SC, Marsh-Yant S, Nega KK. A Combined, Multimodal Intervention for Individuals with Dementia. Res. 2011;4(1):64-75. doi:
10.3928/19404921-20100504-01. PMID: 104897556. Language: English. Entry Date: 20110622. Revision Date: 20150711. Publication Type: Journal Article.
240. Burgener SC, Yang Y, Gilbert R, et al. The effects of a multimodal intervention on outcomes of persons with early-stage dementia. Am J
Alzheimers Dis Other Demen. 2008 Aug-Sep;23(4):382-94. doi: https://dx.doi.org/10.1177/1533317508317527. PMID: 18453642 . Alzheimers Dis Other Demen. 2008 Aug-Sep;23(4):382-94. doi: https://dx.doi.org/10.1177/1533317508317527. PMID: 18453642.
241. Lauriks SM, Meiland FJMP, Oste JPM, et al. Effects of Assistive Home Technology on quality of life and falls of people with dementia 241. Lauriks SM, Meiland pjers; Oste JPults from a a pilot randomized controlled trial. Assist Technol. 2018 Dec 28:1-8. doi:
and job satisfaction of caregivers; and job satisfaction of caregivers; results from a pilot randomized conttold
https://dx.doi.org/10.1080/10400435.2018.1531952. PMID: 30592439.

242. Tchalla AE, Lachal F, Cardinaud N, et al. Preventing and managing indoor falls with home-based technologies in mild and moderate Alzheimer's disease patients: Pilot study in a community dwelling. Dementia and Geriatric Cognitive Disorders. 2013 September;36(3-4):251-
61. doi: http.//lx do 61. doi: hittp://dx.doi.org/10.1159/000351863. PMID: 36990027 243. Rowe MA, Kairalla JA, McCrae CS. Sleep in dementia caregivers and the effect of a nighttime monitoring system. J Nurs Scholarsh. 2010
Sep 01;42(3):338-47. doi: https://dx.doi.org/10.1111/ji.1547-5069.2010.01337.x. PMID: 20738745.
244. Gaugler IJ, Zmora R. Mitchell LL et al al. Six-Month Effectiveness of Remote Activity Monitoring for Persons Living With Dementia and

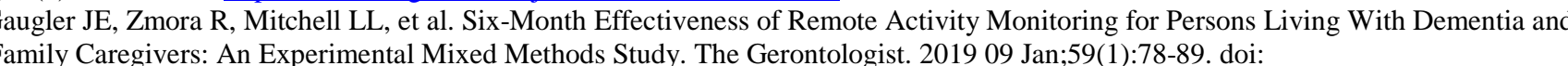
http://dx.doi.org/10.1093/geront/gny078. PMID: 625875732.

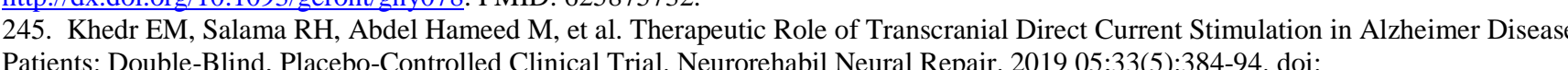
Patients: Double-Blind, Placebo-Controlled Clinical Trial. Neuror
https://dx.doi. org $10.1177 / 1545968319840285$. PMID: 30940012.

246. Lozano AM, Fosdick L, Chakravarty MM, et al. A Phase II Study of Fornix Deep Brain Stimulation in Mild Alzheimer's Disease. J Alzheimers Dis. 2016 09 06;54(2):777-87. doi: https://dx.doi.org/10.3233/JAD-160017. PMID: 27567810.

Dementia (the ADvance Trial): A Two Year Follow-up Including Results of Delayed Activation. J Alzheimers Dis. 2018;64(2):597-606. doi:

24tps. Wu Y X Xu W, Liu X, et al Adjunctive treatment with

a requency repetitive transcranial magnetic stimulation for the behavioral and 2015 Oct;27(5):280-8. doi: https:///dx.doi.org/10.11919/j/.issn. 1002-0829.215107. PMID: 26977125. Aging Neurosci. 2014;6:38. doi: https://dx.doi.org/10.3389/fnagi.2014.00038. PMID: 24678298.

250. Olazaran J, Gonzalez B, Osa-Ruiz E, et al. Motor effects of radio electric asymmetric conveyer in Alzheimer's disease: results from a

251. Olazaran J, Gonzalez B, Lopez-Alvarez J, et al. Motor effects of REAC in advanced Alzheimer's disease: results from a pilot trial. $J$

252. Suemoto CK Apolin:297-302. doi: https://dx.doi.org/10.3233/JAD-130077. PMID: 23603397. 252. Suemoto CK, Apolinario D, Nakamura-Palacios EM, et al. Effects of a non-focal plasticity protocol
disease: a randomized, double-blind, sham--controlled trial. Brain Stimul. 2014 Mar-Apr; $7(2): 308-13$. doi:

hitps://dx.doi.org/10.1016/i.brs.2013.10.003. PMID: 24262299 ,

cognitive function and cortical excitability in Alzheimer's dementia. J Neurol. 2012 Jan:259(1):83-92. doi: httres://dx.doi.org/10.1007/s00415-

011-6128-4. PMID: 21671144.

254. Scherder EJ, van Tol MJ, Swaab DF. High-frequency cranial electrostimulation (CES) in patients with probable Alzheimer's disease. Am

255. van Dijk KR, Scheltens P, Luijpen MW, et al. Peripheral electrical stimulation in Alzheimer's disease. Dement Geriatr Cogn Disord

256. Hozumi S, Hori H, Okawa M, et al. Favorable effect of transcranial electrostimulation on behavior disorders in elderly patients with 257. Liu B, Chen X, Li Y et al. Ef J Neurosci. 1996 Nov;88(1-2):1-10. PMID: 9003961.

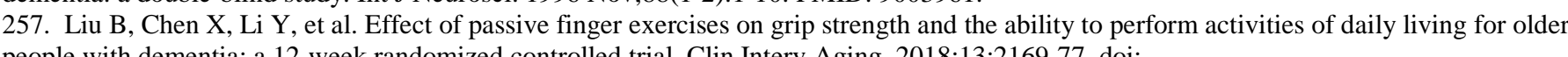
https://dx.doi.org/10.2147/CIA.S174756. PMID: 30464426 .

EK, et al. The impact of a pain assessment intervention on pain score and analgesic use in older nursing home .

https://dx.doi.org/10.1016/ji.ijnurstu.2018.04.017. PMID: 29763832.

259. Batchelor-Murphy M, McConnell E, Amella E, et al. Experimental Comparison of Efficacy for Three Handfeeding Techniques in

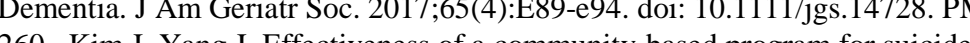

位 observational study. In Geriatric nursing (new york, NY)

261. Hobbelen JH, Tan FE, Verhey FR, et al. Passive movement therapy in severe paratonia: a multicenter randomized clinical trial. In
Psychogeriatr. 2012 May;24(5):834-44. doi: https://dx.doi.org/10.1017/S1041610211002468. PMID: 22185768.

262. Coyne ML, Hoskins L. Improving eating behaviors in dementia using behavioral strategies. Clin Nurs Res. 1997 Aug;6(3):275-90. PMID 9281930 


\section{Appendix E. Psychosocial Interventions for Caregiver Well-Being}

\section{Psychosocial Interventions for Caregiver Well-Being}

Table E-1. Risk of bias assessment: psychosocial interventions for caregiver well-being

\begin{tabular}{|c|c|c|c|c|c|c|c|c|c|}
\hline Study (PMID) & Outcome Timing & $\begin{array}{l}\text { Attrition Bias } \\
\text { Attrition \% }\end{array}$ & Selection Bias & Detection Bias & Performance Bias & Reporting Bias & Fidelity Bias & Funder & Overall Rating \\
\hline \begin{tabular}{|l} 
Au $2020^{1}$ (no PMID) \\
\end{tabular} & 8 weeks & $\begin{array}{l}\text { Low } \\
8 \%\end{array}$ & Low & Low & Low & Low & Low & University & Low \\
\hline Au 2019² (31279613) & 5 months & $\begin{array}{l}\text { Low } \\
15 \%\end{array}$ & Low & Medium & Medium & Low & Low & Government & Low \\
\hline \begin{tabular}{|l|l|} 
Kunik $2020^{3}(32115311)$ \\
\end{tabular} & $\begin{array}{l}3 \text { months } \\
6 \text { months } \\
12 \text { months }\end{array}$ & $\begin{array}{l}\text { Low } \\
18 \%\end{array}$ & Low & Medium & High & Low & Low & National Institute of Nursing & Low \\
\hline \begin{tabular}{|l|l|} 
Bjorge $2019^{4}(31651321)$ \\
\end{tabular} & 3 months & $\begin{array}{l}\text { Low } \\
15 \%\end{array}$ & Low & Medium & Medium & Low & Medium & None & Medium \\
\hline \begin{tabular}{|l} 
Ghaffari $2019^{5}$ (no PMID) \\
\end{tabular} & 8 weeks & $\begin{array}{l}\text { Low } \\
7 \%\end{array}$ & Low & Medium & Medium & Medium & Medium & University & Medium \\
\hline Meng $2019^{6}(30884961)$ & 3 months & $\begin{array}{l}\text { Low } \\
0 \%\end{array}$ & Medium & Medium & Medium & Low & Medium & Government & Medium \\
\hline \begin{tabular}{|l} 
Moskowitz 20197 \\
(31045422)
\end{tabular} & 6 weeks & $\begin{array}{l}\text { Low } \\
9 \%\end{array}$ & Low & Medium & Medium & Low & Medium & Government & Medium \\
\hline $\begin{array}{l}\text { Nordheim 20198 } \\
\text { (30775982) }\end{array}$ & 6 months & \begin{tabular}{|l|} 
Medium \\
$19 \%$
\end{tabular} & Low & Medium & Medium & Low & Medium & Government & Medium \\
\hline \begin{tabular}{|l|} 
Terraccianono 2019 \\
(31866419)
\end{tabular} & 6 weeks & $\begin{array}{l}\text { High } \\
27 \%\end{array}$ & $x$ & $x$ & $x$ & $x$ & $x$ & $\begin{array}{l}\text { Government } \\
\text { Nonprofit }\end{array}$ & High \\
\hline Uyar $2019^{10}$ (no PMID) & 16 weeks & \begin{tabular}{|l|} 
Medium \\
$12 \%$
\end{tabular} & Medium & Medium & Medium & Low & Medium & NR & $\begin{array}{l}\text { High } \\
\text { Very unclear methods } \\
\text { and intervention } \\
\text { description }\end{array}$ \\
\hline \begin{tabular}{|l} 
Wawrziczny 201911 \\
(29665714)
\end{tabular} & 10 weeks & $x$ & High & $x$ & $x$ & $\mathrm{x}$ & $x$ & Government & High \\
\hline Boots 2018 ${ }^{12}(30006327)$ & 8 weeks & \begin{tabular}{|l|} 
Medium \\
$16 \%$
\end{tabular} & Low & Low & Medium & Low & Low & $\begin{array}{l}\text { Alzheimer Nederland and the } \\
\text { Alzheimer Research Fund } \\
\text { Limburg. }\end{array}$ & High \\
\hline \begin{tabular}{|l} 
Gitlin 2018 \\
(29192967)
\end{tabular} & 4 months & $\begin{array}{l}\text { Medium } \\
31 \%\end{array}$ & Low & Low & Medium & Low & Low & Government & Medium \\
\hline \begin{tabular}{|l} 
Spalding-Wilson $2018^{14}$ \\
(30258974)
\end{tabular} & $1,3,6$ months & $\begin{array}{l}\text { Low } \\
9 \%\end{array}$ & Medium & Medium & High & Low & Low & $\begin{array}{l}\text { National Science Foundation, } \\
\text { private foundations }\end{array}$ & High \\
\hline \begin{tabular}{|l|} 
Wilz 2018 \\
15 \\
(29190357)
\end{tabular} & $\begin{array}{l}6 \text { months } \\
\text { Follow up at } 12 \text { month }\end{array}$ & $\begin{array}{l}\text { Medium } \\
17 \%\end{array}$ & Low & High & Medium & Low & Low & $\begin{array}{l}\text { The German Federal Ministry } \\
\text { of Health }\end{array}$ & High \\
\hline \begin{tabular}{|l|} 
Callahan 201716 \\
$(27893087)$
\end{tabular} & 2 years & $\begin{array}{l}\text { Medium } \\
35 \%\end{array}$ & Low & Low & High & Low & Medium & NIA & High \\
\hline \begin{tabular}{|l} 
Whitlatch 2017 \\
(29171296)
\end{tabular} & 6 months & \begin{tabular}{|l|} 
Medium \\
$15 \%$
\end{tabular} & Medium & Medium & High & Low & High & Government & High \\
\hline \begin{tabular}{|l} 
Charlesworth 2016 \\
(27521377)
\end{tabular} & $\begin{array}{l}5 \text { months } \\
1 \text { year }\end{array}$ & $\begin{array}{l}\text { Low } \\
5 \text { months: } 7 \% \\
12 \text { months: } 13 \%\end{array}$ & Low & Low & Medium & Low & Medium & Government & Low \\
\hline \begin{tabular}{|l|l|} 
Cheng $2016^{19}(27401052)$ \\
\end{tabular} & 2 months & NR & Medium & Medium & Medium & Low & Medium & Government & Medium \\
\hline \begin{tabular}{|l} 
Gonyea $2016^{20}(24855313)$ \\
\end{tabular} & $\begin{array}{l}3 \text { months } \\
6 \text { months }\end{array}$ & $\begin{array}{l}\text { Low } \\
15 \%\end{array}$ & Medium & Medium & Medium & Low & Medium & Nonprofit & Medium \\
\hline $\begin{array}{l}\text { Laakkonen 2016 } 6^{21} \\
\text { (27060101) }\end{array}$ & $\begin{array}{l}3 \text { months } \\
9 \text { months }\end{array}$ & $\begin{array}{l}\text { Low } \\
4 \%\end{array}$ & Low & Medium & Medium & Low & Medium & $\begin{array}{l}\text { Government } \\
\text { Nonprofit } \\
\text { University }\end{array}$ & Medium \\
\hline \begin{tabular}{|l} 
Taati $2016^{22}$ \\
(no PMID)
\end{tabular} & 8 weeks & $\begin{array}{l}\text { High } \\
23 \%\end{array}$ & Low & $\mathrm{x}$ & $\mathrm{x}$ & $\mathrm{x}$ & $\mathrm{x}$ & No funding & High \\
\hline
\end{tabular}




\begin{tabular}{|c|c|c|c|c|c|c|c|c|c|}
\hline $\begin{array}{l}\text { Blom 2015'3 } \\
\text { (no PMID) }\end{array}$ & 5-6 months & $\begin{array}{l}\text { Medium } \\
28 \%\end{array}$ & Low & Medium & Low & Low & High & $\begin{array}{l}\text { the Alzheimer's Society in the } \\
\text { Netherlands, the health care } \\
\text { provider Geriant and the VU } \\
\text { University }\end{array}$ & High \\
\hline Chiu $2015^{24}(25615434)$ & 4 weeks & $\begin{array}{l}\text { Medium } \\
16 \%\end{array}$ & High & High & Low & Low & Low & $\begin{array}{l}\text { The UHN AMO Innovation } \\
\text { Fund }\end{array}$ & High \\
\hline $\begin{array}{l}\text { Gallagher-Thompson } \\
2015^{25} \text { (25590939) }\end{array}$ & 10 months & $\begin{array}{l}\text { High } \\
25 \%\end{array}$ & Medium & $\mathrm{x}$ & $\mathrm{x}$ & $\mathrm{x}$ & $\mathrm{x}$ & $\begin{array}{l}\text { The National Office of the } \\
\text { Alzheimer's Association \& } \\
\text { the Alzheimer's Disease } \\
\text { Center at University of } \\
\text { California, Davis }\end{array}$ & High \\
\hline Losada $2015^{26}(26075381)$ & $2 \% 6$ months & $\begin{array}{l}\text { High } \\
30 \%\end{array}$ & $x$ & $x$ & $x$ & $x$ & $x$ & NR & High \\
\hline Otero $2015^{27}$ (25331992) & $1,3,6,12$ months & $\begin{array}{l}\text { Low } \\
4.62 \%\end{array}$ & Low & Low & Medium & Low & Low & Spanish government & Low \\
\hline Tremont $2015^{28}(25074341)$ & 6 months & $\begin{array}{l}\text { Medium } \\
15 \%\end{array}$ & Low & Medium & Medium & Low & Medium & Government & Medium \\
\hline $\begin{array}{l}\text { Arango-Lasprilla } 2014^{29} \\
\text { (24550547) }\end{array}$ & 3 months & $\begin{array}{l}\text { High } \\
\text { Unclear }\end{array}$ & $\mathrm{x}$ & $x$ & $\mathrm{x}$ & $\mathrm{x}$ & $\mathrm{x}$ & No funding & High \\
\hline $\begin{array}{l}\text { Livingston 2014 } \\
\text { (25300037) }\end{array}$ & \begin{tabular}{|l}
4 months \\
8 months
\end{tabular} & $\begin{array}{l}\text { Low } \\
4 \text { months: } 9 \% \\
8 \text { months: } 13 \%\end{array}$ & Low & Medium & Medium & Low & Low & Government & Low \\
\hline $\begin{array}{l}\text { Martin-Carrasco 201431 } \\
\text { (24113563) }\end{array}$ & 4 months & $\begin{array}{l}\text { Medium } \\
4 \text { months: } 26 \% \\
8 \text { months: } 39 \%\end{array}$ & Low & Low & Medium & Low & Medium & Government & Medium \\
\hline Passoni 201432 (24614271) & 6 months & $x$ & High & $x$ & $\mathrm{x}$ & $x$ & $x$ & Not reported & High \\
\hline \begin{tabular}{|l|} 
Bruvik $2013^{33}(24348500)$ \\
\end{tabular} & 12 months & $\begin{array}{l}\text { Medium } \\
13 \%\end{array}$ & Low & Low & Medium & Low & High & Government, foundations & High \\
\hline Huang $2013^{34}(23933422)$ & 2 weeks, $3 \& 6$ months & $\begin{array}{l}\text { Medium } \\
10 \%\end{array}$ & Medium & Medium & Low & Low & High & Government & High \\
\hline Judge $2013^{35}$ (22899427) & 15 weeks & $\begin{array}{l}\text { Medium } \\
13 \%\end{array}$ & Medium & Medium & High & Low & Low & NR & High \\
\hline $\begin{array}{l}\text { Kajiyama 201336 } \\
\text { (23461355) }\end{array}$ & 3 months & $\begin{array}{l}\text { High } \\
31 \%\end{array}$ & \begin{tabular}{|l|} 
Medium \\
\end{tabular} & $x$ & $x$ & $x$ & $x$ & $\begin{array}{l}\text { The National Institute on } \\
\text { Aging, part of the National } \\
\text { Institutes of Health. }\end{array}$ & High \\
\hline Kuo $2013^{37}(22778053)$ & 2 weeks, $3 \& 6$ months & $\begin{array}{l}\text { Low } \\
16 \%\end{array}$ & Medium & Medium & Low & Low & High & Government & High \\
\hline Moore $2013^{38}(23916631)$ & 6 weeks & $\begin{array}{l}\text { Low } \\
14 \%\end{array}$ & Low & Medium & Medium & Low & Low & National Institute on Aging & Low \\
\hline Joling 2012 $2^{39}$ (22303473) & 12 months & $\begin{array}{l}\text { Low } \\
13 \%\end{array}$ & Low & Medium & Medium & Low & Low & Government & Low \\
\hline 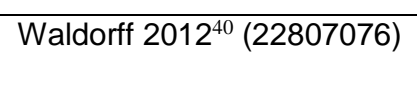 & 1 year & $\begin{array}{l}\text { Medium } \\
16 \%\end{array}$ & Low & Low & Medium & Low & Medium & Government & Medium \\
\hline Chu 2011 & 1 month & $\begin{array}{l}\text { High } \\
29 \%\end{array}$ & $\mathrm{x}$ & $x$ & $\mathrm{x}$ & $x$ & $\mathrm{x}$ & University & High \\
\hline $\begin{array}{l}\text { de Rotrou 201142 } \\
\text { (20922772) }\end{array}$ & $\begin{array}{l}3 \text { months } \\
6 \text { months }\end{array}$ & $\begin{array}{l}\text { Medium } \\
31 \%\end{array}$ & Low & Medium & Medium & Low & Medium & Government & Medium \\
\hline Guerra 201143 (20602013) & 6 months & $\begin{array}{l}\text { Low } \\
3 \%\end{array}$ & Low & Low & High & Low & High & Nonprofit & Medium \\
\hline Losada $2011^{14}(21061414)$ & 3 months & $\begin{array}{l}\text { High } \\
29 \%\end{array}$ & $x$ & $x$ & $\mathrm{x}$ & $\mathrm{x}$ & $x$ & NR & High \\
\hline Spijker 201145 (21358385) & 12 months & $\begin{array}{l}\text { Medium } \\
36 \%\end{array}$ & Low & Medium & Low & Low & High & $\begin{array}{l}\text { The Dutch Organization of } \\
\text { Health Research and } \\
\text { Development (ZonMw). }\end{array}$ & High \\
\hline $\begin{array}{l}\text { Voight-Radloff } 2011^{46} \\
\text { (22021760) }\end{array}$ & \begin{tabular}{|l|}
6 weeks \\
16 weeks \\
26 weeks \\
52 weeks
\end{tabular} & $\begin{array}{l}\text { Low } 6-16 \text { weeks } \\
\text { Medium } 26-52 \text { weeks } \\
6 \text { weeks: } 9 \% \\
16 \text { weeks: } 19 \% \\
\text { 26 weeks: } 25 \% \\
52 \text { weeks: } 26 \%\end{array}$ & Low & Medium & Medium & Low & Medium & Government & $\begin{array}{l}\text { Low 6-16 weeks } \\
\text { Medium 26-52 weeks }\end{array}$ \\
\hline
\end{tabular}




\begin{tabular}{|c|c|c|c|c|c|c|c|c|c|}
\hline Wang $2011^{47}$ (21752121) & 6 months & $\begin{array}{l}\text { Low } \\
2 \%\end{array}$ & Medium & Medium & Medium & Low & Medium & University & Medium \\
\hline Wilz 2011 & 3 months & $\begin{array}{l}\text { Medium } \\
25 \%\end{array}$ & Medium & Medium & Medium & Low & Low & Government & Medium \\
\hline Gitlin 2010 & $\begin{array}{l}4 \text { months } \\
9 \text { months }\end{array}$ & $\begin{array}{l}\text { High } \\
12 \%\end{array}$ & Low & Medium & Medium & Low & Low & $\begin{array}{l}\text { The National Institute on } \\
\text { Aging and National Institute }\end{array}$ & High \\
\hline 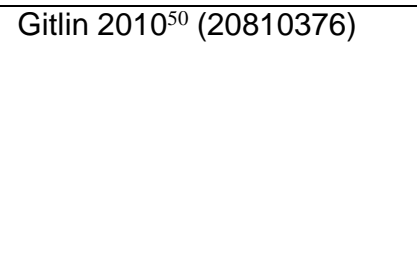 & $\begin{array}{l}4 \text { months } \\
9 \text { months }\end{array}$ & \begin{tabular}{|l|} 
Medium \\
$12 \%$ \\
$27 \%$ at 9 month
\end{tabular} & Low & Medium & High & Low & High & $\begin{array}{l}\text { In part by funds from the } \\
\text { National Institute on Aging \& } \\
\text { the National Institute on } \\
\text { Nursing Research \& the } \\
\text { Pennsylvania } \\
\text { Department of Health, } \\
\text { Tobacco Settlement }\end{array}$ & High \\
\hline Kurz 2010 (19946869) & 15 months & $\begin{array}{l}\text { High } \\
\text { Unclear }\end{array}$ & $\mathrm{x}$ & $\mathrm{x}$ & $x$ & $x$ & $x$ & $\begin{array}{l}\text { German } \\
\text { Federal Ministry of Education } \\
\text { and Research }\end{array}$ & High \\
\hline Williams 201052 (20978227) & \begin{tabular}{|l}
3,6 months \\
\end{tabular} & $\mathrm{x}$ & High & $x$ & $x$ & $x$ & $x$ & National Institutes on Aging & High \\
\hline $\begin{array}{l}\text { Gavrilova 200953 } \\
(18814197)\end{array}$ & & \begin{tabular}{|l|} 
Medium \\
$12 \%$
\end{tabular} & Low & Low & Medium & Low & High & $\begin{array}{l}\text { The World Health } \\
\text { Organization (WHO) }\end{array}$ & High \\
\hline $\begin{array}{l}\text { Martin-Carrasco } 2009^{54} \\
(18949763)\end{array}$ & \begin{tabular}{|l|}
4 months \\
10 months \\
\end{tabular} & $\begin{array}{l}\text { Medium } \\
10 \%\end{array}$ & Low & Medium & Medium & Low & Medium & Industry & Medium \\
\hline Andrén 20085 (18269429) & $6 \& 12$ months & $x$ & High & $\mathrm{x}$ & $x$ & $x$ & $x$ & $\begin{array}{l}\text { Swedish Council for Social } \\
\text { Research, foundations, } \\
\text { university, and municipality }\end{array}$ & High \\
\hline $\begin{array}{l}\text { Gallagher- Thompson } \\
2008^{56}(25067886)\end{array}$ & 6 months & $\begin{array}{l}\text { Medium } \\
15 \%\end{array}$ & Medium & Medium & Medium & Medium & Medium & Government & Medium \\
\hline $\begin{array}{l}\text { Marquez-Gonzalez 200757 } \\
(18074249)\end{array}$ & 2 months & $\begin{array}{l}\text { High } \\
47 \%\end{array}$ & $\mathrm{x}$ & $\mathrm{x}$ & $\mathrm{x}$ & $x$ & $\mathrm{x}$ & Not reported & High \\
\hline Ulstein $2007^{58}(17986818)$ & 1 year & \begin{tabular}{|l|} 
Medium \\
$14 \%$
\end{tabular} & Low & Medium & Medium & Low & High & Government, foundation & High \\
\hline Gonyea 2006 ${ }^{59}(17169938)$ & 6 weeks & $\begin{array}{l}\text { Medium } \\
12 \%\end{array}$ & Low & Medium & High & Low & High & Foundations & High \\
\hline Graff 2006 $6^{60}(17114212)$ & $\begin{array}{l}6 \text { weeks } \\
3 \text { months }\end{array}$ & $\begin{array}{l}\text { Medium } 6 \text { weeks } \\
\text { High } 12 \text { weeks } \\
6 \text { weeks: } 16 \% \\
12 \text { weeks: } 23 \%\end{array}$ & Low & Medium & Medium & Low & Medium & $\begin{array}{l}\text { Nonprofit } \\
\text { University }\end{array}$ & $\begin{array}{l}\text { Medium } 6 \text { weeks } \\
\text { High } 12 \text { weeks }\end{array}$ \\
\hline $\begin{array}{l}\text { Beauchamp } 2005^{61} \text { (no } \\
\text { PMID) }\end{array}$ & 1 month & $\begin{array}{l}\text { Low } \\
3 \%\end{array}$ & Medium & High & High & Low & Low & Not reported & High \\
\hline Farran 200462 (41552352) & 18 months & $\begin{array}{l}\text { Medium } \\
17 \%\end{array}$ & Low & Medium & Medium & Low & Medium & Government & Medium \\
\hline Burgio $2003^{63}(12937335)$ & 6 months & $\begin{array}{l}\text { Medium } \\
16 \%\end{array}$ & Medium & High & Medium & Low & Low & $\begin{array}{l}\text { National Institute for Nursing } \\
\text { Research }\end{array}$ & High \\
\hline Burns $2003^{64}(12937333)$ & 2 years & $\begin{array}{l}\text { High } \\
55 \%\end{array}$ & $\mathrm{x}$ & $\mathrm{x}$ & $\mathrm{x}$ & $x$ & $x$ & $\begin{array}{l}\text { National Institute on Aging, } \\
\text { National Institute of Nursing } \\
\text { Research, Department of } \\
\text { Veterans Affairs }\end{array}$ & High \\
\hline Coon 200365 (14570964) & 4,7 months & $\begin{array}{l}\text { High } \\
23 \%\end{array}$ & $\mathrm{x}$ & $x$ & $x$ & $x$ & $x$ & $\begin{array}{l}\text { National Institute of Mental } \\
\text { Health }\end{array}$ & High \\
\hline $\begin{array}{l}\text { Gallagher Thompson } \\
2003^{66}(12937336)\end{array}$ & 3 months & $\begin{array}{l}\text { Low } \\
0 \%\end{array}$ & Medium & Medium & Medium & Medium & Medium & Government & Medium \\
\hline Gitlin 2003 ${ }^{67}$ (12937332) & 3 months & $\begin{array}{l}\text { Medium } \\
15 \%\end{array}$ & Medium & High & Medium & Low & Low & Government & Medium \\
\hline Hebert $2003^{68}(12496309)$ & 4 months & $\begin{array}{l}\text { Medium } \\
18 \%\end{array}$ & Low & Medium & High & Low & Low & $\begin{array}{l}\text { The Quebec Health } \\
\text { Research Fund, the } \\
\text { Alzheimer Society of } \\
\text { Canada, the FRSQ Network } \\
\text { for Geronto-Geriatric } \\
\text { Research, and the Quebec } \\
\text { Council for Social Research } \\
\end{array}$ & High \\
\hline
\end{tabular}




\begin{tabular}{|c|c|c|c|c|c|c|c|c|c|}
\hline Fung 2002 ${ }^{69}(12037799)$ & 3 months & $\begin{array}{l}\text { Medium } \\
13 \%\end{array}$ & Medium & High & High & Low & Low & Not reported & High \\
\hline Stolley 200270 (11954669) & 3, 6 \&12 months & $\begin{array}{l}\text { High } \\
27 \%\end{array}$ & Medium & $\mathrm{x}$ & $x$ & $\mathrm{x}$ & $x$ & NR & High \\
\hline Wright 2001 11 (11885210) & $\begin{array}{l}2 \& 6 \text { weeks, } 3,6 \& 12 \\
\text { months }\end{array}$ & $\begin{array}{l}\text { High } \\
\text { Unclear }\end{array}$ & $\mathrm{X}$ & $x$ & $x$ & $x$ & $x$ & Foundation & High \\
\hline $\begin{array}{l}\text { Buckwalter 199972 } \\
\text { (10222636) }\end{array}$ & $6 \& 12$ months & $\begin{array}{l}\text { High } \\
29 \%\end{array}$ & $x$ & $x$ & $\mathrm{x}$ & $x$ & $x$ & National Institutes of Health & High \\
\hline Chang $1999^{73}(10337848)$ & 4,8 \& 12 weeks & $\begin{array}{l}\text { Medium } \\
25 \%\end{array}$ & Medium & Medium & Medium & Low & High & NR & High \\
\hline Ostwald 199974 (10396888) & 5 months & $\begin{array}{l}\text { Low } \\
19.7 \%\end{array}$ & Low & Medium & High & Low & Low & \begin{tabular}{|l|} 
National Institute of Nursing \\
Research
\end{tabular} & Medium \\
\hline
\end{tabular}

X indicates that domain was not assessed due to high risk of bias.
Abbreviations: NR=Not Reported; PMID=PubMed Identification Number

Table E-2. Characteristics of included studies: psychosocial interventions for caregiver well-being

\begin{tabular}{|c|c|c|c|c|c|c|c|c|c|c|c|}
\hline $\begin{array}{l}\text { Study (PMID) } \\
\text { Country } \\
\text { RoB* } \\
\text { Type }\end{array}$ & $\begin{array}{l}\text { Intervention } \\
\text { Intervention } \\
\text { Focus } \\
\text { Theoretical } \\
\text { Model } \\
\text { Delivery Person } \\
\text { Intervention } \\
\text { Target } \\
\text { Recipient } \\
\text { Mode } \\
\text { Components } \\
\text { Frequency } \\
\text { Duration }\end{array}$ & $\begin{array}{l}\text { Comparison } \\
\text { Target } \\
\text { Mode } \\
\text { Components } \\
\text { Frequency } \\
\text { Duration }\end{array}$ & $\begin{array}{l}\text { Setting } \\
\text { and Design } \\
\\
\text { Setting } \\
\text { Design } \\
\text { Cluster N } \\
\text { Participants } \\
\text { Randomized N }\end{array}$ & $\begin{array}{l}\text { PLWD } \\
\text { Dementia } \\
\text { Characteristics } \\
\text { Dementia Types } \\
\text { Dementia Severity } \\
\text { Diagnostic Criteria } \\
\text { Age of Diagnosis }\end{array}$ & $\begin{array}{l}\text { PLWD } \\
\text { Non-Disease Char } \\
\text { PLWD N } \\
\text { PLWD Age (mean) } \\
\text { PLWD Sex (\% } \\
\text { female) } \\
\text { PLWD Race (\% } \\
\text { majority) } \\
\text { PLWD Education } \\
\text { (mean years) }\end{array}$ & $\begin{array}{l}\text { PLWD } \\
\text { Non-Disease Char } \\
\text { Reporting Status } \\
\text { (RS) } \\
\text { PLWD SES } \\
\text { PLWD Prior } \\
\text { Disability } \\
\text { PLWD Household } \\
\text { Characteristics } \\
\text { PLWD Health } \\
\text { Insurance } \\
\text { PLWD Detailed } \\
\text { Race Information }\end{array}$ & $\begin{array}{l}\text { Informal Caregiver } \\
\text { (IC) Characteristics } \\
\text { IC N } \\
\text { IC Age (mean) } \\
\text { IC Sex (\% female) } \\
\text { IC Race (\% majority) } \\
\text { IC Education (mean } \\
\text { years) } \\
\text { IC Relation to PLWD } \\
\text { (\% majority) }\end{array}$ & $\begin{array}{l}\text { Informal Caregiver } \\
\text { (IC) Char. RS } \\
\text { IC Duration } \\
\text { IC Living With PLWD } \\
\text { IC Payment } \\
\text { IC Health Status } \\
\text { IC Dementia Family } \\
\text { History } \\
\text { IC Employment } \\
\text { Status } \\
\text { IC Training }\end{array}$ & $\begin{array}{l}\text { Outcome } \\
\text { Timing(s) }\end{array}$ & PLWD Outcomes & $\begin{array}{l}\text { Caregiver } \\
\text { Outcomes } \\
\text { IC: } \\
\text { FC: }\end{array}$ \\
\hline $\begin{array}{l}\text { Au 20201 (no } \\
\text { PMID) } \\
\text { Hong Kong } \\
\text { Low } \\
\text { Explanatory }\end{array}$ & $\begin{array}{l}\text { Connecting } \\
\text { through caregiving } \\
\text { Intergenerational } \\
\text { perspective-taking } \\
\text { reappraisals } \\
\text { Aim to promote } \\
\text { balance between } \\
\text { self-care and } \\
\text { caring of others: } \\
\text { (1) enhance self- } \\
\text { awareness (2) } \\
\text { connect with } \\
\text { PLWD } \\
\text { through } \\
\text { empathetic } \\
\text { understanding (3) } \\
\text { connect with help } \\
\text { Initial home visit, } \\
\text { then } 7 \text { weekly } \\
\text { telephone } \\
\text { sessions } \\
8 \text { weeks total }\end{array}$ & $\begin{array}{l}\text { Basic skill building } \\
\text { intervention } \\
\text { Initial home visit, } \\
\text { then } 7 \text { weekly } \\
\text { telephone } \\
\text { sessions } \\
8 \text { weeks total }\end{array}$ & $\begin{array}{l}\text { Home-based } \\
\text { RCT } \\
\mathrm{N}=72 \text { dyads }\end{array}$ & $\begin{array}{l}\text { Alzheimer's disease } \\
\text { NPI } 20 \\
\text { Physician-diagnosed }\end{array}$ & $\begin{array}{l}\mathrm{N}=72 \\
79 \text { years } \\
72 \% \text { Female } \\
\text { Race NR } \\
\text { Education NR }\end{array}$ & NR & $\begin{array}{l}\mathrm{N}=72 \\
52 \text { years } \\
81 \% \text { Female } \\
\text { Race NR } \\
\text { Education } \\
11 \text { Years formal } \\
\text { education (mean) } \\
100 \% \text { Child }\end{array}$ & $\begin{array}{l}3 \text { years caregiving } \\
\text { (mean) } \\
57 \% \text { Living with } \\
\text { PLWD } \\
53 \% \text { Employed }\end{array}$ & 8 weeks & NR & $\begin{array}{l}\text { CESD } \\
\text { ZBI } \\
\text { Satisfaction with } \\
\text { Life Survey } \\
\text { (all Chinese } \\
\text { versions) }\end{array}$ \\
\hline
\end{tabular}




\begin{tabular}{|c|c|c|c|c|c|c|c|c|c|c|c|}
\hline $\begin{array}{l}\text { Au 2019² } \\
\text { (31279613) } \\
\text { Hong Kong } \\
\text { Low } \\
\text { Explanatory }\end{array}$ & \begin{tabular}{|l} 
Telephone- \\
administered \\
psycho-education \\
with \\
behavioral \\
activation \\
intervention. 8 \\
biweekly sessions.
\end{tabular} & \begin{tabular}{|l|} 
Telephone based \\
psycho-education \\
with general \\
monitoring. 8 \\
biweekly \\
monitoring \\
sessions.
\end{tabular} & $\begin{array}{l}\text { Home-based } \\
\text { RCT } \\
\mathrm{N}=111\end{array}$ & $\begin{array}{l}\text { Alzheimer's disease } \\
\text { NINCDS-ADRDA }\end{array}$ & $\mathrm{NR}$ & NR & \begin{tabular}{|l|}
$\mathrm{N}=111$ \\
57 years \\
$81 \%$ Female \\
Race NR \\
Education \\
$32 \%$ Primary \\
$50 \%$ Secondary \\
$18 \%$ Tertiary \\
$29 \%$ Spouse or \\
partner \\
$65 \%$ Child \\
\end{tabular} & $\begin{array}{l}4+\text { years caregiving } \\
\text { (mean) } \\
11 \text { hours daily } \\
\text { caregiving }\end{array}$ & 5 months & NR & $\begin{array}{l}\text { CESD } \\
\text { ZBI } \\
\text { RAS } \\
\text { Caregiver: self- } \\
\text { efficacy for } \\
\text { controlling } \\
\text { upsetting } \\
\text { thoughts } 5 \text { item }\end{array}$ \\
\hline $\begin{array}{l}\text { Kunik 202033 } \\
(32115311) \\
\text { United States } \\
\text { Low } \\
\text { Pragmatic }\end{array}$ & \begin{tabular}{|l} 
Psychosocial \\
approach to \\
understanding \\
behavioral \\
problems / Unmet \\
Needs Model \\
including \\
education, 6 to 8 \\
weekly skills \\
sessions, and \\
telephone wrap-up \\
session for PLWD \\
and caregivers for \\
3 months
\end{tabular} & $\begin{array}{l}\text { booklet on } \\
\text { memory } \\
\text { problems, } \\
\text { community } \\
\text { resources and } \\
\text { caregivers, eight } \\
\text { brief, weekly calls, } \\
\text { and encouraged to } \\
\text { bring concerns to } \\
\text { primary care } \\
\text { physician for } 3 \\
\text { months }\end{array}$ & $\begin{array}{l}\text { Community } \\
\mathrm{N}=228\end{array}$ & $\begin{array}{l}\text { Documented } \\
\text { diagnosed dementia }\end{array}$ & \begin{tabular}{|l|}
$\mathrm{N}=228$ \\
77 years \\
$42 \%$ Female \\
$67 \%$ Non-Hispanic \\
white \\
$69 \% 14$ years
\end{tabular} & $\begin{array}{l}\text { 48\% \$20k-49,999/yr } \\
21 \% \text { Black } \\
9 \% \text { Hispanic } \\
\text { 2\% Other }\end{array}$ & \begin{tabular}{|l|}
$\mathrm{N}=228$ \\
68 years \\
$77 \%$ Female \\
$66 \%$ Non-Hispanic \\
white \\
$78 \% 14$ years \\
$69 \%$ Spouse
\end{tabular} & $\begin{array}{l}\text { 88\% Living with } \\
\text { PLWD }\end{array}$ & \begin{tabular}{|l|}
3 months \\
6 months \\
12 months
\end{tabular} & \begin{tabular}{|l} 
Aggression \\
(CMAI) \\
Pain (PGPIS)
\end{tabular} & $\begin{array}{l}\text { Depression } \\
\text { (GDS) } \\
\text { Pain (PGPIS) } \\
\text { Burden (ZBS) }\end{array}$ \\
\hline $\begin{array}{l}\text { Bjorge 20194 } \\
\text { (31651321) } \\
\text { Norway } \\
\text { Medium } \\
\text { Explanatory }\end{array}$ & $\begin{array}{l}\text { Psychosocial } \\
\text { intervention } \\
\text { (education about } \\
\text { dementia, } \\
\text { counselling and } \\
\text { group sessions) } \\
\text { over } 12 \text { months }\end{array}$ & Usual care & $\begin{array}{l}\text { Community- } \\
\text { based } \\
\text { RCT } \\
\text { N=208 dyads }\end{array}$ & $\begin{array}{l}\text { Non-specified } \\
\text { dementia } \\
\text { ICD } 10\end{array}$ & \begin{tabular}{|l|}
$\mathrm{N}=208$ \\
79 years \\
$53 \%$ Female \\
Race NR \\
9 Years of education
\end{tabular} & NR & \begin{tabular}{|l}
$\mathrm{N}=208$ \\
64 years \\
$76 \%$ Female \\
Race NR \\
Education NR \\
$57 \%$ Spouse or \\
partner \\
$44 \%$ Child
\end{tabular} & 58\% Live with PLWD & 3 months & \begin{tabular}{|l|} 
MMSE \\
NPI-Q \\
Lawton \& Brody's \\
IADL \\
CSDD \\
PLWD nursing \\
home placement
\end{tabular} & $\begin{array}{l}\text { RSS } \\
\text { GDS } \\
\text { FEERS }\end{array}$ \\
\hline $\begin{array}{l}\text { Ghaffari } 2019^{5} \text { (no } \\
\text { PMID) } \\
\text { Iran } \\
\text { Medium } \\
\text { Explanatory }\end{array}$ & \begin{tabular}{|l} 
Resilience \\
education \\
8 weekly 45- \\
minute group \\
sessions \\
Training and \\
education program \\
aimed to promote \\
resilience \\
PowerPoint \\
presentations and \\
educational \\
pamphlets
\end{tabular} & $\begin{array}{l}\text { No treatment } \\
\text { Received } \\
\text { resilience } \\
\text { development } \\
\text { education after the } \\
\text { study finished }\end{array}$ & $\begin{array}{l}\text { Community- } \\
\text { based } \\
\text { RCT } \\
\mathrm{N}=54 \text { caregivers }\end{array}$ & $\begin{array}{l}\text { Alzheimer's disease } \\
\text { Physician-diagnosed }\end{array}$ & NR & NR & \begin{tabular}{|l|}
$\mathrm{N}=54$ \\
43 years \\
$74 \%$ Female \\
Race NR \\
Education \\
$22 \%$ Primary \\
education \\
$35 \%$ High school \\
diploma \\
$35 \%$ University \\
education \\
$77 \%$ Spouse or \\
partner \\
$23 \%$ Child
\end{tabular} & $\begin{array}{l}\text { Months caregiving } \\
4 \%<6 \text { months } \\
54 \% 6-24 \text { months } \\
42 \%>24 \text { months } \\
\text { Employment } \\
18 \% \text { Retired } \\
50 \% \text { Working } \\
32 \% \text { Homemaker }\end{array}$ & 8 weeks & NR & GHQ \\
\hline $\begin{array}{l}\text { Meng 20196 } \\
\text { (30884961) } \\
\text { USA } \\
\text { Medium } \\
\text { Explanatory }\end{array}$ & \begin{tabular}{|l|} 
Telephone based \\
cognitive \\
behavioral therapy
\end{tabular} & \begin{tabular}{|l|} 
In-person \\
cognitive \\
behavioral therapy
\end{tabular} & $\begin{array}{l}\text { Home and } \\
\text { Community- } \\
\text { based } \\
\mathrm{RCT} \\
\mathrm{N}=109 \text { dyads }\end{array}$ & $\begin{array}{l}\text { Alzheimer's disease } \\
\text { or progressive } \\
\text { dementia } \\
\text { Physician-diagnosed }\end{array}$ & \begin{tabular}{|l|}
$\mathrm{N}=109$ \\
81 years \\
$66 \%$ Female \\
$100 \%$ African- \\
American \\
12 Years education
\end{tabular} & NR & \begin{tabular}{|l|}
$\mathrm{N}=109$ \\
59 years \\
$50 \%$ Female \\
$100 \%$ African- \\
American \\
15 Years education \\
$23 \%$ Spouse or \\
partner \\
$66 \%$ Child
\end{tabular} & $\begin{array}{l}4 \text { years caregiving } \\
\text { (mean) } \\
66 \% \text { Live with PLWD }\end{array}$ & 3 months & NR & $\begin{array}{l}\text { Caregiver health } \\
\text { (physician office } \\
\text { visits, } \\
\text { mental health } \\
\text { visits, } \\
\text { prescription } \\
\text { medication, ER } \\
\text { visits, } \\
\text { hospital days, } \\
\text { out of pocket } \\
\text { expenditures in } \\
\text { dollars, } \\
\text { total monthly } \\
\text { expenditures in } \\
\text { dollars) }\end{array}$ \\
\hline
\end{tabular}




\begin{tabular}{|c|c|c|c|c|c|c|c|c|c|c|c|}
\hline $\begin{array}{l}\text { Moskowitz 20197 } \\
\text { (31045422) } \\
\text { USA } \\
\text { Medium } \\
\text { Explanatory }\end{array}$ & \begin{tabular}{|l|} 
Life Enhancing \\
Activities for \\
Family Caregivers \\
(LEAF). Taught \\
eight emotion- \\
regulating skills \\
over 6 sessions.
\end{tabular} & $\begin{array}{l}\text { Waitlist control, } \\
\text { emotion-reporting }\end{array}$ & \begin{tabular}{|l|} 
Home-based \\
RCT \\
$\mathrm{N}=170$
\end{tabular} & \begin{tabular}{|l|} 
Alzheimer's disease \\
Frontotemporal \\
Lewy body \\
Parkinson's dementia \\
Nonspecified \\
dementia
\end{tabular} & NR & NR & \begin{tabular}{|l|}
$\mathrm{N}=170$ \\
63 years \\
$84 \%$ Female \\
$88 \%$ White \\
Education \\
$6 \%$ High school \\
education or less \\
$40 \%$ College degree \\
or some college \\
$40 \%$ Postgraduate \\
education \\
$67 \%$ Spouse or \\
partner \\
$27 \%$ Child \\
\end{tabular} & $\begin{array}{l}\begin{array}{l}4+\text { years caregiving } \\
\text { (mean) }\end{array} \\
\end{array}$ & 6 weeks & \begin{tabular}{|l|} 
Dementia Severity \\
Rating Scale
\end{tabular} & $\begin{array}{l}\text { PROMIS } \\
\text { (depressive } \\
\text { symptoms, } \\
\text { mental health, } \\
\text { physical health) } \\
\text { NeuroQOL } \\
\text { ZBI } \\
\text { Caregiver Strain } \\
\text { Index (CSI) } \\
\text { Differential } \\
\text { Emotions Scale } \\
\text { (DES) } \\
\text { the Positive } \\
\text { Aspects of } \\
\text { Caregiving } \\
\text { Scale } \\
\text { The Perceived } \\
\text { Stress Scale }\end{array}$ \\
\hline $\begin{array}{l}\text { Nordheim 20198 } \\
(30775982) \\
\text { Germany } \\
\text { Medium } \\
\text { Explanatory }\end{array}$ & \begin{tabular}{|l} 
Couple-based \\
interdisciplinary \\
psychosocial \\
intervention \\
9 sessions over \\
10-12 weeks
\end{tabular} & Usual care & $\begin{array}{l}\text { Community- } \\
\text { based } \\
\text { RCT } \\
\mathrm{N}=108 \text { dyads }\end{array}$ & $\begin{array}{l}\text { Non-specified } \\
\text { dementia } \\
\text { NIA criteria }\end{array}$ & \begin{tabular}{|l}
$\mathrm{N}=108$ \\
81 years \\
$39 \%$ Female \\
Race NR \\
Education NR
\end{tabular} & NR & \begin{tabular}{|l|}
$\mathrm{N}=108$ \\
78 years \\
$61 \%$ Female \\
Race NR \\
Education NR
\end{tabular} & NR & 6 months & \begin{tabular}{|l|} 
QOL in \\
Alzheimer's \\
Disease (QOLAD) \\
ADL \\
IADL \\
Older Adults \\
Overprotection \\
Scale (OPSA) \\
Geriatric \\
depression scale \\
(GDS) \\
PLWD social \\
support: (F-SOZU) \\
MMSE
\end{tabular} & $\begin{array}{l}\text { WHOQOL- } \\
\text { BREF short form } \\
\text { Global QOL } \\
\text { subscale } \\
\text { Sense of } \\
\text { Competence } \\
\text { Questionnaire } \\
\text { (SCQ) (personal } \\
\text { life, performance } \\
\text { as a caregiver, } \\
\text { satisfaction with } \\
\text { PLWD) } \\
\text { Caregiver social } \\
\text { supportt (F- } \\
\text { SOZU) } \\
\text { Perceived stress } \\
\text { GDS }\end{array}$ \\
\hline $\begin{array}{l}\text { Gitlin 2018 } \\
(29192967) \\
\text { Gittlin 201375 } \\
(29192967) \\
\\
\text { USA } \\
\text { Medium } \\
\text { Explanatory }\end{array}$ & \begin{tabular}{|l} 
Home-based \\
Tailored Activity \\
Program. 8 \\
sessions \\
with occupational \\
therapists to \\
customize \\
activities \\
to the interests \\
and abilities of the \\
veterans and \\
educate \\
their caregivers \\
about dementia \\
and use of \\
customized \\
activity. \\
Delivery by \\
occupational \\
therapist. 4 \\
months.
\end{tabular} & $\begin{array}{l}\text { Attention control } \\
\text { using } 8 \text { telephone } \\
\text { visits. }\end{array}$ & $\begin{array}{l}\text { Home-based } \\
\mathrm{RCT} \\
\mathrm{N}=160 \text { dyads }\end{array}$ & \begin{tabular}{|l|} 
Veterans with \\
dementia and their \\
carers. MMSE $\leq 23$ \\
and documented \\
dementia diagnosis.
\end{tabular} & \begin{tabular}{|l|}
$\mathrm{N}=160$ \\
80 years \\
$3 \%$ Female \\
$79 \%$ White \\
$<41 \%$ High school \\
diploma or less
\end{tabular} & NR & \begin{tabular}{|l|}
$\mathrm{N}=160$ \\
72 years \\
$98 \%$ Female \\
$82 \%$ White \\
$<38 \%$ High school \\
diploma or less \\
$87 \%$ Spouse or \\
partner
\end{tabular} & $\begin{array}{l}\text { 100\% Living with } \\
\text { PLWD }\end{array}$ & 4 months & \begin{tabular}{|l|} 
Neuropsychiatric \\
Inventory- \\
Clinician (NPI-C) - \\
number of \\
behavioral \\
symptoms \\
Caregiver \\
Assessment of \\
Function and \\
Upset Scale \\
(CAFU) (number \\
of ADL/IADL \\
dependencies, \\
level of \\
dependence) \\
PLWD QOL \\
(caregiver \\
perception of \\
affect)
\end{tabular} & $\begin{array}{l}\text { CES-D } \\
\text { ZBS Short Form } \\
\text { (12 item) } \\
\text { Neuropsychiatric } \\
\text { Inventory- } \\
\text { Clinician (NPI-C) } \\
\text { distress } \\
\text { Time spent } \\
\text { caregiving (time } \\
\text { on ADLs and } \\
\text { IADLs, hours on } \\
\text { duty, hours on } \\
\text { doing things) }\end{array}$ \\
\hline
\end{tabular}




\begin{tabular}{|c|c|c|c|c|c|c|c|c|c|c|c|}
\hline $\begin{array}{l}\text { Charlesworth } \\
2016^{18} \\
(27521377) \\
\text { Melunsky 201576 } \\
(24381218) \\
\text { Charlesworth } \\
2011^{77} \\
(21917187) \\
\text { UK } \\
\text { Low } \\
\text { Explanatory }\end{array}$ & \begin{tabular}{|l|} 
1) One-to-one \\
peer support to \\
family carers from \\
experienced \\
carers (Carer \\
Supporter \\
Programme (CSP) \\
2) Group \\
reminiscence \\
therapy \\
Remembering \\
Yesterday, Caring \\
Today (RYCT) \\
Once a week for \\
the first we weeks \\
followed by \\
monthly meetings \\
for the next 7 \\
months (12 \\
months total)
\end{tabular} & 3) Usual care & $\begin{array}{l}\text { Community- } \\
\text { based } \\
\text { RCT } \\
\text { N=291 dyads }\end{array}$ & \begin{tabular}{|l|} 
Alzheimer's disease \\
Vascular dementia \\
Non-specified \\
dementia \\
DSM-IV
\end{tabular} & \begin{tabular}{|l|}
$\mathrm{N}=291$ \\
80 years \\
$53 \%$ Female \\
$88 \%$ White \\
$75 \%$ High school \\
education or less
\end{tabular} & NR & \begin{tabular}{|l|}
$\mathrm{N}=291$ \\
67 years \\
$67 \%$ Female \\
$89 \%$ White \\
$62 \%$ High school \\
education or less \\
$64 \%$ Spouse or \\
partner
\end{tabular} & \begin{tabular}{|l|}
$\begin{array}{l}\text { 4+ years caregiving } \\
\text { (mean) }\end{array}$ \\
\end{tabular} & $\begin{array}{l}5 \text { months } \\
1 \text { year }\end{array}$ & \begin{tabular}{|l|} 
QOL-AD (self and \\
proxy reported) \\
Alzheimer's \\
Disease \\
Cooperative \\
Study-ADL \\
NPI
\end{tabular} & $\begin{array}{l}\text { SF-12 (mental, } \\
\text { physical } \\
\text { component) } \\
\text { Hospital Anxiety } \\
\text { and Depression } \\
\text { Scale (HADS) } \\
\text { (anxiety, } \\
\text { Depression) } \\
\text { EQ-5D global } \\
\text { health visual } \\
\text { analog scale } \\
\text { Carers of Older } \\
\text { People in } \\
\text { Europe Index } \\
\text { (COPE index) } \\
\text { positive aspects } \\
\text { Caregiver } \\
\text { Quality of } \\
\text { Caregiver- } \\
\text { PLWD } \\
\text { Relationship } \\
\text { (QCPR) }\end{array}$ \\
\hline $\begin{array}{l}\text { Cheng 2016 } 6^{19} \\
(27401052) \\
\text { Cheng 2019a } \\
(31076215) \\
\text { Cheng 2019b79 } \\
(3155647) \\
\text { Cheng 2016 } \\
(28287803) \\
\text { Cheng 2014 } \\
(24688081) \\
\text { Hong Kong } \\
\text { Medium } \\
\text { Explanatory }\end{array}$ & $\begin{array}{l}\text { 1) Benefit finding } \\
\text { intervention (BF) } \\
\text { 2) Simplified } \\
\text { psychoeducation } \\
\text { (SIM-PE) }\end{array}$ & $\begin{array}{l}\text { 3. Standard } \\
\text { psychoeducation } \\
\text { (STD-PE) }\end{array}$ & $\begin{array}{l}\text { Community- } \\
\text { based } \\
\text { Cluster- } \\
\text { randomized RCT } \\
\mathrm{N}=129 \text { dyads }\end{array}$ & $\begin{array}{l}\text { Alzheimer's disease } \\
\text { NINCDS-ADRDA }\end{array}$ & $\begin{array}{l}\mathrm{N}=129 \\
\mathrm{NR}\end{array}$ & NR & \begin{tabular}{|l}
$\mathrm{N}=129$ \\
55 years \\
$85 \%$ Female \\
Race NR \\
Education \\
$28 \%$ Primary or less \\
$57 \%$ Secondary \\
$15 \%$ Tertiary \\
$27 \%$ Spouse or \\
partner \\
$73 \%$ Child
\end{tabular} & $\begin{array}{l}2 \text { years caregiving } \\
\text { (mean) } \\
13 \text { hours per day } \\
31 \% \text { Employed }\end{array}$ & 2 months & NR & $\begin{array}{l}\text { Hamilton } \\
\text { Depression } \\
\text { Rating Scale } \\
\text { ZBI } \\
\text { Caregiver role } \\
\text { overload } \\
\text { Ryff's } \\
\text { Psychological } \\
\text { Well-being } \\
\text { Scale }\end{array}$ \\
\hline $\begin{array}{l}\text { Gonyea 201620 } \\
(24855313) \\
\text { USA } \\
\text { Medium } \\
\text { Explanatory }\end{array}$ & \begin{tabular}{|l|} 
Circulo de \\
Cuidado, a \\
culturally- \\
sensitive, cognitive \\
behavioral (CBT) \\
group intervention. \\
(1) 5 weekly $90-$ \\
minute group \\
sessions \\
(2) Telephone \\
coaching at week \\
$3,6,9$ and 12 \\
\end{tabular} & $\begin{array}{l}\text { Psychoeducational } \\
\text { (PED) control } \\
\text { condition }\end{array}$ & $\begin{array}{l}\text { Community- } \\
\text { based } \\
\mathrm{RCT} \\
\mathrm{N}=67 \text { dyads }\end{array}$ & $\begin{array}{l}\text { Possible or probable } \\
\text { Alzheimer's } \\
\text { Disease } \\
\text { Criteria NR }\end{array}$ & \begin{tabular}{|l}
$\mathrm{N}=291$ \\
75 years \\
$63 \%$ Female \\
Race NR \\
Education NR
\end{tabular} & NR & \begin{tabular}{|l|}
$\mathrm{N}=291$ \\
54 years \\
$51 \%$ Female \\
$100 \%$ Latino \\
$28 \%$ Less than high \\
school education \\
$48 \%$ High school \\
certificate or GED \\
$24 \%$ Spouse or \\
partner \\
$55 \%$ Child
\end{tabular} & \begin{tabular}{|l|} 
Employment \\
$37 \%$ Fulltime \\
$24 \%$ Part time \\
$63 \%$ Living together \\
12 hours daily \\
caregiving
\end{tabular} & \begin{tabular}{|l|}
3 months \\
6 months
\end{tabular} & \begin{tabular}{|l|} 
Neuropsychiatric \\
Inventory-Severity \\
scale (NPI-S) \\
(Spanish)
\end{tabular} & $\begin{array}{l}\text { NPI distress } \\
\text { Revised Scale } \\
\text { for Caregiving } \\
\text { Self-Efficacy } \\
\text { (RSCSE) } \\
\text { CES-D } \\
\text { State Anxiety } \\
\text { Inventory-State } \\
\text { (STAl-S) } \\
\text { (all Spanish) }\end{array}$ \\
\hline
\end{tabular}




\begin{tabular}{|c|c|c|c|c|c|c|c|c|c|c|c|}
\hline $\begin{array}{l}\text { Laakkonen 2016 } \\
(27060101) \\
\text { Laakkonen 201381 } \\
\text { (52813196) } \\
\text { Laakkonen 2012 } \\
\text { (22871 } \\
\text { Finland } \\
\text { Medium } \\
\text { Explanatory }\end{array}$ & $\begin{array}{l}\text { Self-management } \\
\text { groups. 4-hour } \\
\text { group sessions in } \\
\text { a day center once } \\
\text { a week for } \\
\text { an 8-week period. }\end{array}$ & Usual care & $\begin{array}{l}\text { Clinic-based } \\
\text { RCT } \\
\mathrm{N}=136 \text { dyads }\end{array}$ & $\begin{array}{l}\text { Non-specified } \\
\text { dementia }\end{array}$ & $\begin{array}{l}\mathrm{N}=136 \\
77 \text { years } \\
27 \% \text { Female } \\
\text { Race NR } \\
45 \%<8 \text { years } \\
\text { education }\end{array}$ & $\mathrm{NR}$ & $\begin{array}{l}\mathrm{N}=136 \\
74 \text { years } \\
63 \% \text { Female } \\
\text { Race NR } \\
40 \%<8 \text { years } \\
\text { education } \\
100 \% \text { Spouse or } \\
\text { partner }\end{array}$ & $\mathrm{NR}$ & $\begin{array}{l}3 \text { months } \\
9 \text { months }\end{array}$ & $\begin{array}{l}\text { PLWD quality of } \\
\text { life: } 15 D \text { D } \\
\text { Verbal Fluency } \\
\text { (VF) } \\
\text { Clock Drawing } \\
\text { Test (CDT) } \\
\text { PLWD Health \& } \\
\text { social services } \\
\text { cost }\end{array}$ & $\begin{array}{l}\text { SF-36 } \\
\text { Caregiver } \\
\text { quality of life } \\
\text { (physical, } \\
\text { mental) } \\
\text { Spousal Sense } \\
\text { of Competence } \\
\text { Questionnaire } \\
\text { (SCQ) } \\
\text { Caregiver } \\
\text { mastery Pearlin } \\
\text { Mastery Scale } \\
\text { (PMS) } \\
\text { Caregiver } \\
\text { Health \& Social } \\
\text { services cost }\end{array}$ \\
\hline $\begin{array}{l}\text { Otero 2015.33 } \\
(25331992) \\
\text { Spain } \\
\text { High ROB }\end{array}$ & $\begin{array}{l}\text { Group-based } \\
\text { cognitive } \\
\text { behavioral } \\
\text { intervention for } \\
\text { problem solving } \\
5 \text { g0-minute } \\
\text { weekly sessions }\end{array}$ & Usual care & $\begin{array}{l}\text { Community- } \\
\text { based } \\
\mathrm{RCT} \\
\mathrm{N}=173 \text { informal } \\
\text { caregivers }\end{array}$ & $\begin{array}{l}\text { Dementia diagnosis } \\
(47 \%)\end{array}$ & NR & NR & $\begin{array}{l}\mathrm{N}=173 \\
54 \text { years } \\
\text { Sex NR } \\
\text { Race NR } \\
75 \% \text { primary/higher } \\
\text { education } \\
51 \% \text { Daughter or son }\end{array}$ & $\begin{array}{l}\text { Mean of } 10 \text { years } \\
\text { caring for PLWDD } \\
84 \% \text { unemployed }\end{array}$ & $\begin{array}{l}1,3,6,12 \\
\text { months }\end{array}$ & NA & $\begin{array}{l}\text { CES-D } \\
\text { SCID-CV }\end{array}$ \\
\hline $\begin{array}{l}\text { Tremont 201528 } \\
(25074341) \\
\text { Tremont 201784 } \\
(28008609) \\
\text { Tremont 2013 } \\
\text { (23916916) } \\
\text { USA } \\
\text { Medium } \\
\text { Explanatory }\end{array}$ & $\begin{array}{l}\text { Telephone } \\
\text { Tracking-- } \\
\text { Caregiver (FITT- } \\
\text { C) } \\
\text { received } 1615- \\
60 \text { min telephone } \\
\text { contacts over } 6 \\
\text { months. Provided } \\
\text { education, } \\
\text { emotional support, } \\
\text { directing to } \\
\text { resources, } \\
\text { encouraging } \\
\text { caregivers to } \\
\text { attend to their } \\
\text { physical, } \\
\text { emotional, and } \\
\text { social needs, and } \\
\text { teaching } \\
\text { caregivers } \\
\text { strategies to cope } \\
\text { with ongoing } \\
\text { problems }\end{array}$ & $\begin{array}{l}\text { Telephone } \\
\text { Support } \\
\text { Control: Received } \\
\text { non-directive } \\
\text { support through } \\
\text { empathic and } \\
\text { reflective listening } \\
\text { and open-ended } \\
\text { questioning } \\
\text { without directive } \\
\text { strategies, such as } \\
\text { education, } \\
\text { problem-solving, } \\
\text { advice-giving, or } \\
\text { task directives. }\end{array}$ & $\begin{array}{l}\text { Academic } \\
\text { medical center } \\
\text { RCT } \\
\mathrm{N}=250\end{array}$ & NR & $\mathrm{NR}$ & NR & $\begin{array}{l}\mathrm{N}=250 \\
63 \text { years } \\
78 \% \text { Female } \\
96 \% \text { White } \\
15 . \text { years } \\
51 \% \text { Spouse or } \\
\text { partner } \\
42 \% \text { Child }\end{array}$ & $\begin{array}{l}80 \% \text { Living together } \\
3.8 \text { years caregiving }\end{array}$ & 6 months & NR & $\begin{array}{l}\text { CESD } \\
\text { ZBI } \\
\text { RMBPC-RT } \\
\text { Reaction Score } \\
\text { Family } \\
\text { Assessment } \\
\text { Device (FAD) } \\
\text { Self-Efficacy } \\
\text { Questionnaire } \\
\text { (symptom } \\
\text { management, } \\
\text { support } \\
\text { services) } \\
\text { Positive Aspects } \\
\text { of Caregiving } \\
\text { (PAC) } \\
\text { EuroQoL Visual } \\
\text { Analog scale }\end{array}$ \\
\hline $\begin{array}{l}\text { Martinn-Carrasco } \\
2014^{31} \\
(24113563) \\
\text { Spain } \\
\text { Medium } \\
\text { Explanatory }\end{array}$ & $\begin{array}{l}\text { Psychoeducational } \\
\text { Intervention } \\
\text { Program to teach } \\
\text { strategies for } \\
\text { confronting } \\
\text { problems of PLWD } \\
\text { care. } 7 \text { biweekly } \\
90-120 \text { min } \\
\text { sessions over } 4 \\
\text { months. }\end{array}$ & Usual care & $\begin{array}{l}\text { Clinic-based } \\
R C T \\
N=238 \text { dyads }\end{array}$ & $\begin{array}{l}\text { Alzheimer's disease } \\
\text { Vascular dementia } \\
\text { Non-specified } \\
\text { dementia } \\
\text { DSM-IV-TR }\end{array}$ & $\begin{array}{l}\mathrm{N}=238 \\
78 \text { years } \\
62 \% \text { Female } \\
\text { Race NR } \\
\text { Education } \\
61 \% \text { Primary school } \\
10 \% \text { Secondary school } \\
7 \% \text { College }\end{array}$ & NR & $\begin{array}{l}\mathrm{N}=238 \\
62 \text { years } \\
77 \% \text { Female } \\
\text { Race NR } \\
\text { Education } \\
48 \% \text { Primary school } \\
30 \% \text { Secondary } \\
\text { school } \\
13 \% \text { College } \\
59 \% \text { Spouse or } \\
\text { partner } \\
45 \% \text { Child }\end{array}$ & $\begin{array}{l}5 \text { years providing care } \\
12 \text { hours per day } \\
25 \% \text { Working } \\
39 \% \text { Homemaker } \\
27 \% \text { Retired }\end{array}$ & 4 months & NR & $\begin{array}{l}\text { ZBI } \\
\text { General Health } \\
\text { Questionnaire } \\
\text { 28 item (GHQ- } \\
\text { 28) } \\
\text { SF-12 } \\
\text { (all Spanish) }\end{array}$ \\
\hline
\end{tabular}




\begin{tabular}{|c|c|c|c|c|c|c|c|c|c|c|c|}
\hline $\begin{array}{l}\text { Waldorff 201240 } \\
(22807076) \\
\text { Phung 2013 } \\
(370414872) \\
\text { Denmark } \\
\text { Medium } \\
\text { Explanatory }\end{array}$ & \begin{tabular}{|l|} 
DAISY plus \\
support \\
(multificaceded and \\
semi-tailored \\
counselling, \\
education, and \\
support). Up to 7 \\
sessions over 12 \\
months.
\end{tabular} & $\begin{array}{l}\text { Support control } \\
\text { group }\end{array}$ & $\begin{array}{l}\text { Home and clinic- } \\
\text { based } \\
\mathrm{RCT} \\
\mathrm{N}=330 \text { dyads }\end{array}$ & $\begin{array}{l}\text { Alzheimer's disease } \\
\text { Vascular dementia } \\
\text { Non-specified } \\
\text { dementia } \\
\text { Mixed-type } \\
\text { DSM-IV } \\
\text { NINCDS-ADRDA }\end{array}$ & \begin{tabular}{|l|}
$\mathrm{N}=330$ \\
76 years \\
$54 \%$ Female \\
Race NR \\
Education \\
$36 \%$ NR \\
$26 \%<3$ years \\
$38 \% \geq 3$ years
\end{tabular} & NR & \begin{tabular}{|l}
$\mathrm{N}=330$ \\
66 years \\
$67 \%$ Female \\
Race NR \\
Education \\
$24 \%$ NR \\
$33 \%<3$ years \\
$43 \% \geq 3$ years \\
$65 \%$ Spouse or \\
partner \\
$27 \%$ Child \\
\end{tabular} & 65\% Live with PLWD & 1 year & \begin{tabular}{|l} 
MMSE \\
CSDD \\
EQ-VAS (PLWD \\
and proxy rated) \\
QoL-AD (PLWD \\
and proxy rated) \\
NPI \\
ADSC-ADL
\end{tabular} & \begin{tabular}{|l|} 
Geriatric \\
depression \\
scale
\end{tabular} \\
\hline $\begin{array}{l}\text { de Rotrou 201142 } \\
\text { (20922772) } \\
\text { France } \\
\text { Medium } \\
\text { Explanatory }\end{array}$ & $\begin{array}{l}\text { Psycho Education } \\
\text { Program. } 12 \\
\text { weekly 2-hour } \\
\text { sessions. Content } \\
\text { included } \\
\text { education, } \\
\text { problem-solving } \\
\text { techniques and } \\
\text { emotion-centered } \\
\text { coping strategies, } \\
\text { management of } \\
\text { PLWD behavior, } \\
\text { communication } \\
\text { skills, crisis } \\
\text { management, } \\
\text { resource } \\
\text { information and } \\
\text { practical advice. } \\
\text { Both groups also } \\
\text { received } \\
\text { antidementia } \\
\text { drugs. }\end{array}$ & Usual care & $\begin{array}{l}\text { Memory clinic } \\
\text { RCT } \\
\mathrm{N}=167 \text { dyads }\end{array}$ & $\begin{array}{l}\text { Alzheimer's disease } \\
\text { DSM-IV }\end{array}$ & \begin{tabular}{|l|}
$\mathrm{N}=167$ \\
79 years \\
$60 \%$ Female \\
Race NR \\
9 Years education
\end{tabular} & NR & \begin{tabular}{|l|}
$\mathrm{N}=167$ \\
65 years \\
$68 \%$ Female \\
Race NR \\
11 Years education \\
$57 \%$ Spouse or \\
partner \\
$29 \%$ Daughter
\end{tabular} & NR & $\begin{array}{l}3 \text { months } \\
6 \text { months }\end{array}$ & NR & \begin{tabular}{|l|} 
MADRS \\
ZBI \\
Caregiver \\
perception of \\
disease \\
understanding \\
Caregiver \\
coping
\end{tabular} \\
\hline $\begin{array}{l}\text { Guerra 2011433 } \\
\text { (20602013) } \\
\text { Peru } \\
\text { Medium } \\
\text { Explanatory }\end{array}$ & $\begin{array}{l}\text { 10/66 Caregiver } \\
\text { Intervention. } \\
\text { Provide education } \\
\text { and } \\
\text { training on } \\
\text { managing problem } \\
\text { behaviors. } \\
\text { Delivered in } 3 \\
\text { modules over } 5 \\
\text { 30-min weekly } \\
\text { sessions. }\end{array}$ & Waitlist & $\begin{array}{l}\text { Home-based } \\
\mathrm{RCT} \\
\mathrm{N}=58\end{array}$ & $\begin{array}{l}\text { Nonspecified } \\
\text { dementia } \\
\text { DSM-IV } \\
\text { NPI-Q 4.6 }\end{array}$ & \begin{tabular}{|l|}
$\mathrm{N}=58$ \\
Age 82 \\
$72 \%$ Female \\
Race NR \\
Education NR
\end{tabular} & NR & \begin{tabular}{|l|}
$\mathrm{N}=58$ \\
51 years \\
$88 \%$ Female \\
Race NR \\
Education NR \\
$12 \%$ Spouse \\
$43 \%$ Child \\
\end{tabular} & NR & 6 months & \begin{tabular}{|l|} 
NPI-Q \\
DEMQOL
\end{tabular} & $\begin{array}{l}\text { ZBI } \\
\text { Self-Reporting } \\
\text { Questionnaire } \\
20 \\
\text { NPI-Q } \\
\text { WHOQOL- } \\
\text { BREF }\end{array}$ \\
\hline
\end{tabular}




\begin{tabular}{|c|c|c|c|c|c|c|c|c|c|c|c|}
\hline $\begin{array}{l}\text { Voight-Radloff } \\
2011^{46} \\
\text { (2201760) } \\
\text { Voight-Radloff } \\
2011^{86} \text { (no PMID) } \\
\text { Voight-Radloff } \\
2009^{87} \\
\text { (355485226) } \\
\\
\text { Germany } \\
\text { Low 6-16 weeks } \\
\text { Medium 26-52 } \\
\text { weeks } \\
\text { Explanatory }\end{array}$ & $\begin{array}{l}\text { Community } \\
\text { Occupational } \\
\text { Therapy in } \\
\text { Dementia } \\
\text { Program. } 10 \text { home } \\
\text { visits over } 5 \text { weeks } \\
\text { by an occupational } \\
\text { therapist. }\end{array}$ & $\begin{array}{l}\text { Usual care (one } \\
\text { session home } \\
\text { consultation) }\end{array}$ & \begin{tabular}{|l} 
Home-based \\
RCT \\
$\mathrm{N}=141$ dyads
\end{tabular} & $\begin{array}{l}\text { Alzheimer's disease } \\
\text { or mixed-type } \\
\text { dementia } \\
\text { ICD-10 }\end{array}$ & \begin{tabular}{|l|}
$\mathrm{N}=141$ \\
78 years \\
$58 \%$ Female \\
Race NR \\
$22 \%$ High school \\
diploma \\
$77 \%$ Less than high \\
school education
\end{tabular} & NR & \begin{tabular}{|l|}
$\mathrm{N}=141$ \\
65 years \\
$70 \%$ Female \\
Race NR \\
Education NR \\
$57 \%$ Spouse or \\
partner \\
$37 \%$ Child \\
\end{tabular} & NR & \begin{tabular}{|l|}
6 weeks \\
16 weeks \\
26 weeks \\
52 weeks
\end{tabular} & $\begin{array}{l}\text { Interview for } \\
\text { Deterioration in } \\
\text { Daili Living } \\
\text { Activities in } \\
\text { Dementia (IDDD) } \\
\text { (performance, } \\
\text { initiative) } \\
\text { Cornell Scale for } \\
\text { Depression in } \\
\text { Dementia } \\
\text { Perceive, Recall, } \\
\text { Plan and Perform } \\
\text { System of Task } \\
\text { Analysis (PRPP) } \\
\text { (independence) } \\
\text { Dementia Quality } \\
\text { of Life Instrument - } \\
\text { overall } \\
\text { SF-12 (physical, } \\
\text { mental) } \\
\text { Number of } \\
\text { adverse events } \\
\text { Resource } \\
\text { Utilization in } \\
\text { Dementia (nights } \\
\text { in nursing home, } \\
\text { night in hospital) }\end{array}$ & $\begin{array}{l}\text { Sense of } \\
\text { Competence } \\
\text { Questionnaire } \\
\text { Center for } \\
\text { Epidemiologic } \\
\text { Depression } \\
\text { Scale } \\
\text { Dementia QOL } \\
\text { Instrument } \\
\text { SF-12 (2hysical, } \\
\text { mental) } \\
\text { Resource } \\
\text { utilization in } \\
\text { Dementia - } \\
\text { Basic ADL Care } \\
\text { by primary carer } \\
\text { (hours per day) }\end{array}$ \\
\hline $\begin{array}{l}\text { Wang 201147 } \\
(21752121) \\
\text { USA } \\
\text { Medium } \\
\text { Explanatory }\end{array}$ & \begin{tabular}{|l} 
Family Mutual \\
Support Program \\
in Dementia Care \\
(FMSP-DC). 8 2- \\
hour biweekly \\
sessions over 6 \\
months.
\end{tabular} & Usual care & $\begin{array}{l}\text { Home-based } \\
\mathrm{N}=80 \text { dyads }\end{array}$ & $\begin{array}{l}\text { Alzheimer's disease } \\
\text { DSM-IV }\end{array}$ & \begin{tabular}{|l}
$\mathrm{N}=80$ \\
68 years \\
$81 \%$ Female \\
Race NR \\
Education NR
\end{tabular} & NR & \begin{tabular}{|l|}
$\mathrm{N}=80$ \\
41 years \\
$65 \% \%$ Female \\
Race NR \\
$73 \%$ Secondary \\
education \\
40\% Spouse or \\
partner \\
38\% Child
\end{tabular} & NR & 6 months & $\begin{array}{l}\text { MMSE (Chinese) } \\
\text { PLWD } \\
\text { institutionalizations } \\
\text { (number, duration) }\end{array}$ & $\begin{array}{l}\text { Family } \\
\text { Caregiving } \\
\text { Burden } \\
\text { Inventory (FCBI) } \\
\text { WHOQOL- } \\
\text { BREF } \\
\text { Six-item Social } \\
\text { Support } \\
\text { Questionnaire } \\
\text { (SSO6) }\end{array}$ \\
\hline $\begin{array}{l}\text { Gitlin 201049 } \\
\text { (20662955) } \\
\text { USA } \\
\text { Medium } \\
\text { Explanatory }\end{array}$ & \begin{tabular}{|l|} 
Advancing \\
Caregiving \\
Training (ACT) \\
Health \\
professionals \\
helped target \\
behavioral \\
symptoms of \\
dementia, created \\
plan to help \\
caregivers \\
manage \\
11 home and \\
phone contacts \\
over 4 months
\end{tabular} & $\begin{array}{l}\text { No treatment or } \\
\text { contact }\end{array}$ & $\begin{array}{l}\text { Home-based } \\
\mathrm{RCT} \text {-b dyads } \\
\mathrm{N}=272 \text { dy }\end{array}$ & MMSE $<24$ & \begin{tabular}{|l}
$\mathrm{N}=272$ \\
82 years \\
$53 \%$ Female \\
$70 \%$ White \\
$30 \%$ Nonwhite \\
Education NR
\end{tabular} & NR & \begin{tabular}{|l|}
$\mathrm{N}=272$ \\
66 years \\
$82 \%$ Female \\
$70 \%$ White \\
$30 \% \%$ Nonwhite \\
Education \\
$7 \%<$ <igh school \\
$26 \%$ High school \\
degree \\
$67 \%$ Higher education \\
$51 \%$ Spouse
\end{tabular} & 4 years caregiving & 4,6 months & $\begin{array}{l}\text { Frequency of } \\
\text { problem behavior } \\
\text { being targeted }\end{array}$ & $\begin{array}{l}\text { ZBI } \\
\text { CES-D } \\
\text { Perceived } \\
\text { Change Index } \\
\text { Skill } \\
\text { enhancement }\end{array}$ \\
\hline $\begin{array}{l}\text { Martin-Carrasco } \\
\text { 200954 } \\
(18949763) \\
\text { Spain } \\
\text { Medium } \\
\text { Explanatory }\end{array}$ & $\begin{array}{l}\text { Psychoeducational } \\
\text { Intervention } \\
\text { Program to teach } \\
\text { strategies for } \\
\text { confronting } \\
\text { problems of } \\
\text { PLWD care. } 8 \\
\text { sessions over } 4 \\
\text { months. }\end{array}$ & Usual care & $\begin{array}{l}\text { Hospital and } \\
\text { outpatient clinics } \\
\mathrm{N}=115 \text { dyads } \\
\text { RCT }\end{array}$ & $\begin{array}{l}\text { Alzheimer's disease } \\
\text { DSM-IV }\end{array}$ & \begin{tabular}{|l|}
$\mathrm{N}=115$ \\
77 years \\
$63 \%$ Female \\
Race NR \\
Education NR
\end{tabular} & NR & \begin{tabular}{|l}
$\mathrm{N}=115$ \\
77 years \\
$63 \%$ Female \\
Race NR \\
Education NR \\
55\% Spouse or \\
partner \\
$37 \%$ Child
\end{tabular} & $\begin{array}{l}12 \text { Hours per day } \\
\text { caregiving } \\
3 \text { years caregiving } \\
39 \% \text { Retired } \\
28 \% \text { Homemaker } \\
25 \% \text { Working }\end{array}$ & \begin{tabular}{|l|}
4 months \\
10 months
\end{tabular} & $\begin{array}{l}\text { PLWD Caregiver } \\
\text { healthcare and } \\
\text { social resources } \\
\text { use (number of } \\
\text { visits, } \\
\text { time spent on } \\
\text { medical care) }\end{array}$ & $\begin{array}{l}\text { ZBI } \\
\text { Spanish SF-36 } \\
\text { GHQ-28 } \\
\text { Caregiver } \\
\text { healthcare and } \\
\text { social resources } \\
\text { use (number of } \\
\text { visits, time spent } \\
\text { on medical care) }\end{array}$ \\
\hline
\end{tabular}




\begin{tabular}{|c|c|c|c|c|c|c|c|c|c|c|c|}
\hline $\begin{array}{l}\text { Gallagher- } \\
\text { Thompson 200856 } \\
\text { (25067886) } \\
\text { USA } \\
\text { Medium } \\
\text { Explanatory }\end{array}$ & $\begin{array}{l}\text { Coping with } \\
\text { Caregiving (CWC). } \\
\text { Instruction and } \\
\text { practice in small } \\
\text { groups to learn } \\
\text { specific cognitive } \\
\text { and behavioral } \\
\text { skills. Weekly 2- } \\
\text { hour sessions over } \\
4 \text { months. } \\
\end{array}$ & \begin{tabular}{|l} 
Minimal \\
telephone-based \\
control condition \\
(TSC). Empathetic \\
support via 15-20 \\
min telephone \\
calls every 2 \\
weeks.
\end{tabular} & \begin{tabular}{|l} 
Home-based \\
RCT \\
$\mathrm{N}=184$
\end{tabular} & NR & NR & NR & \begin{tabular}{|l|}
$\mathrm{N}=184$ \\
58 years \\
$100 \%$ Female \\
$52 \%$ White \\
48\% Latina \\
13 Years education \\
$33 \%$ Spouse or \\
partner
\end{tabular} & $\begin{array}{l}10 \text { Hours per day } \\
\text { caregiving } \\
65 \% \text { Live with PLWD }\end{array}$ & 6 months & NR & $\begin{array}{l}\text { CES-D } \\
\text { Perceived } \\
\text { Stress Scale } \\
\text { (PSS-10) } \\
\text { RMBPC-CB } \\
\text { (both) }\end{array}$ \\
\hline $\begin{array}{l}\text { Graff 2006 } \\
(17114212) \\
\text { Graff 200888 } \\
(18171718) \\
\text { Graff 200789 } \\
(17895439) \\
\\
\text { Netherlands } \\
\text { Medium } \\
\text { Explanatory }\end{array}$ & $\begin{array}{l}\text { Community based } \\
\text { occupational } \\
\text { therapy program. } \\
10 \text { sessions over } 5 \\
\text { weeks. }\end{array}$ & $\begin{array}{l}\text { Waitlist for } \\
\text { occupational } \\
\text { therapy }\end{array}$ & $\begin{array}{l}\text { Home-based } \\
\text { RCT } \\
\mathrm{N}=135 \text { dyads }\end{array}$ & $\begin{array}{l}\text { Mild to moderate } \\
\text { dementia } \\
\text { Diagnosed with } \\
\text { DSM-IV }\end{array}$ & \begin{tabular}{|l}
$\mathrm{N}=135$ \\
78 years \\
$56 \%$ Female \\
Race NR \\
Education NR
\end{tabular} & NR & $\begin{array}{l}\mathrm{N}=135 \\
63 \text { years } \\
70 \% \text { Female } \\
\text { Race NR } \\
\text { Education NR } \\
59 \% \text { Spouse or } \\
\text { partner } \\
32 \% \text { Child }\end{array}$ & NR & $\begin{array}{l}6 \text { weeks } \\
3 \text { months }\end{array}$ & \begin{tabular}{|l|} 
Assessment of \\
Motor and Process \\
Skills (AMPS) \\
(process) \\
Interview of \\
Deterioration in \\
Daily Activities in \\
Dementia (IDDDD) \\
(performance)
\end{tabular} & $\begin{array}{l}\text { Sense of } \\
\text { Competence } \\
\text { Questionnaire } \\
\text { (SCQ) }\end{array}$ \\
\hline $\begin{array}{l}\text { Farran 200462 } \\
(41552352) \\
\text { Farran 200790 } \\
(175) \\
\text { Farran 2004 } \\
\text { USA } \\
\text { Medium } \\
\text { Explanatory }\end{array}$ & \begin{tabular}{|l|} 
Caregiver skill \\
building (CSB) \\
12 weekly \\
sessions (Five \\
group sessions \& \\
7 individual \\
telephone \\
contacts), 2 group \\
booster sessions \\
$\&$ as-needed \\
phone contacts \\
\end{tabular} & $\begin{array}{l}\text { Information and } \\
\text { support group } \\
\text { (ISO) Information } \\
\text { \& support oriented } \\
\text { (ISO) group: } \\
\text { Routine care- } \\
\text { related issues } \\
\text { were addressed, } \\
\text { but did not include } \\
\text { individualized skill } \\
\text { enhancement }\end{array}$ & $\begin{array}{l}\text { Home-based } \\
\text { RCT } \\
\mathrm{N}=295\end{array}$ & NR & NR & NR & \begin{tabular}{|l|}
$\mathrm{N}=295$ \\
65 years \\
$83 \%$ Female \\
$88 \%$ White \\
$62 \%$ High school \\
education or less \\
$58 \%$ Spouse or \\
partner
\end{tabular} & NR & 18 months & \begin{tabular}{|l|} 
RMPBC \\
Time to \\
institutionalization \\
\end{tabular} & $\begin{array}{l}\text { CES-D } \\
\text { Behavior } \\
\text { Management } \\
\text { Skill-Revised } \\
\text { (BMS-R) }\end{array}$ \\
\hline $\begin{array}{l}\text { Gallagher- } \\
\text { Thompson 200366 } \\
\text { (12937336) } \\
\text { Rabinowitz 200692 } \\
\text { (16861368) } \\
\text { USA } \\
\text { Medium } \\
\text { Explanatory }\end{array}$ & $\begin{array}{l}\text { Coping With } \\
\text { Caregiving } \\
\text { psycheoeducational } \\
\text { program } \\
\text { (instruction and } \\
\text { practice in small } \\
\text { groups to learn } \\
\text { specific cognitive } \\
\text { and behavioral } \\
\text { skills) } \\
\text { a) Once a week } \\
\text { for the first } 10 \\
\text { weeks } \\
\text { b) once a month } \\
\text { during the } \\
\text { "booster } \\
\text { phase" for the } \\
\text { next } 8 \text { months. }\end{array}$ & $\begin{array}{l}\text { Enhanced Support } \\
\text { Group condition } \\
\text { (guided discussion } \\
\text { and empathic } \\
\text { listening to } \\
\text { develop reciprocal } \\
\text { support within the } \\
\text { group) }\end{array}$ & $\begin{array}{l}\text { Community- } \\
\text { based } \\
\text { RCT } \\
\text { N=213 }\end{array}$ & NR & NR & NR & $\begin{array}{l}\mathrm{N}=213 \\
57 \text { years } \\
100 \% \text { Female } \\
57 \% \text { White } \\
43 \% \text { Latino } \\
12 \text { years } \\
38 \% \text { Spouse or } \\
\text { partner } \\
62 \% \text { Child }\end{array}$ & 5 years (mean) & 3 months & NR & $\begin{array}{l}\text { CES-D } \\
\text { Revised Ways } \\
\text { of Coping } \\
\text { Checklist } \\
\text { (RWCCL) } \\
\text { (positive, } \\
\text { negative) } \\
\text { Inventory of } \\
\text { Socially } \\
\text { Supportive } \\
\text { Behaviors } \\
\text { (ISSP) } \\
\text { (satisfaction, } \\
\text { negative } \\
\text { interaction) } \\
\text { RMBPC }\end{array}$ \\
\hline $\begin{array}{l}\text { Gitlin 200193 } \\
\text { (11220813) } \\
\text { USA } \\
\text { Medium } \\
\text { Explanatory }\end{array}$ & \begin{tabular}{|l} 
Home \\
Environmental \\
Intervention \\
Five 90-min home \\
visits by \\
occupational \\
therapists who \\
provided \\
education and \\
physical and social \\
environmental \\
modifications. \\
\end{tabular} & Usual care & $\begin{array}{l}\text { Home-based } \\
\text { RCT } \\
\mathrm{N}=202 \text { dyads }\end{array}$ & NR & \begin{tabular}{|l}
$\mathrm{N}=202$ \\
78 years \\
$66 \%$ Female \\
Race NR \\
Education NR
\end{tabular} & NR & \begin{tabular}{|l}
$\mathrm{N}=202$ \\
61 years \\
$73 \%$ Female \\
$74 \%$ White \\
14 years \\
25\% Spouse or \\
partner
\end{tabular} & $\begin{array}{l}\text { 100\% Living with } \\
\text { PLWD }\end{array}$ & 3 months & \begin{tabular}{|l} 
Functional \\
Independence \\
Measure \\
(modified) (ADL, \\
IADL) \\
MBPC
\end{tabular} & $\begin{array}{l}\text { Caregiver self- } \\
\text { efficacy and } \\
\text { upset (managing } \\
\text { ADL, IADL, } \\
\text { behaviors, } \\
\text { dependency) }\end{array}$ \\
\hline
\end{tabular}




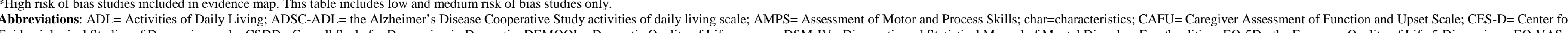

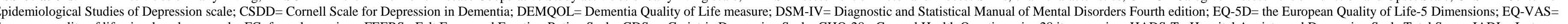

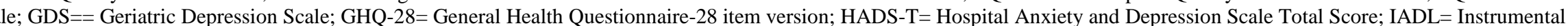

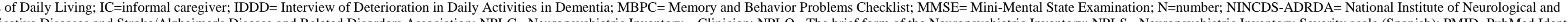

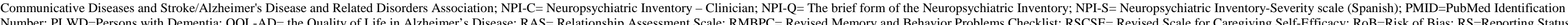

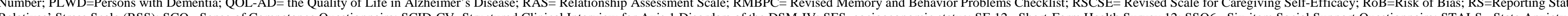

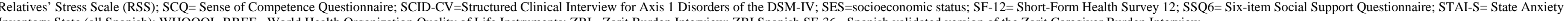


Figure E-1. SMD calculated from difference between baseline and FU in intervention minus difference between baseline and FU in comparison group

Caregiver Depression

\begin{tabular}{llrr} 
Study & $\begin{array}{c}\text { SMD } \\
\text { with 95\% Cl }\end{array}$ & $\begin{array}{c}\text { Weight } \\
(\%)\end{array}$ \\
\hline Au 2019 & $0.233(-0.141,0.606)$ & 18.62 \\
Cheng 2016 & $0.979(0.532,1.426)$ & 16.96 \\
Gitlin 2018 & $0.590(-0.555,1.735)$ & 6.43 \\
Gonyea 2016 & $0.068(-0.411,0.547)$ & 16.24 \\
Tremont 2015 & $0.210(-0.039,0.459)$ & 21.29 \\
Gallagher-Thompson 2008 & $-0.178(-0.468,0.112)$ & 20.45 \\
Overall & & $0.267(-0.164,0.698)$
\end{tabular}

Heterogeneity: $\mathrm{T}^{2}=0.12, \mathrm{I}^{2}=76.67 \%, \mathrm{H}^{2}=4.29$ Favors comparison Favors intervention

Test of $\theta=0: t(5)=1.59, p=0.17$

\begin{tabular}{lllll}
\hline-2 & -1 & 0 & 1 & 2
\end{tabular}

Table E-3. Intervention components of psychosocial interventions for informal caregivers

\begin{tabular}{|c|c|c|c|c|}
\hline \begin{tabular}{|l|} 
Study (PMID) \\
Intervention \& Comparison \\
Country
\end{tabular} & Duration of & Interventionist & Dose/Format & Components \\
\hline $\begin{array}{l}\text { Au } 2020^{1} \text { (no PMID) } \\
\text { Intergenerational perspective-taking in-home } \\
\text { intervention vs. basic skill building in-home } \\
\text { intervention } \\
\text { Hong Kong }\end{array}$ & 8 weeks & Therapists & $\begin{array}{l}140 \text {-minute in-home session followed by } 7 \text { weekly } 35- \\
\text { minute telephone sessions } \\
\text { Total hours: } 5\end{array}$ & $\begin{array}{l}\text { Connecting through Caregiving an Intergenerational Perspective-Taking Intervention: } \\
\text { discussed the following topics through the lens of intergenerational perspective-taking: self- } \\
\text { monitoring mood, scheduling pleasant events, monitoring behavioral problems of PLWD, } \\
\text { communicating with PLWD, identifying help, creating implementation plans } \\
\text { Basic Skill Building Intervention: discussed the same topics more generally }\end{array}$ \\
\hline $\begin{array}{l}\text { Au } 2019^{2}(31279613) \\
\text { Telephone-administered psycho-education with } \\
\text { behavioral activation intervention vs. telephone- } \\
\text { based psycho-education with general monitoring } \\
\text { Hong Kong }\end{array}$ & 20 weeks & Social workers, paraprofessionals & $\begin{array}{l}4 \text { weekly individual telephone sessions with social worker } \\
\text { followed by } 8 \text { biweekly sessions with a paraprofessional, } \\
\text { each session lasted approximately } 20 \text { minutes } \\
\text { Total hours: } 4\end{array}$ & $\begin{array}{l}\text { Behavioral Activation Intervention: Updating recent caregiving situation; Reporting health } \\
\text { and needs of CG and CR; Reporting daily routines; Reporting family communication; } \\
\text { Activity planning; Review to improve on scheduling; Develop new help-seeking skills; } \\
\text { Reviewing to improve communication } \\
\text { General Monitoring Intervention: Update caregiving situation: Discuss caregiver's health; } \\
\text { Update care giving situation; Discuss care-recipient's needs; Update caregiving situation; } \\
\text { Discuss daily/weekly routines; Review support from family/ friends/ agencies; }\end{array}$ \\
\hline \begin{tabular}{|l|} 
Bjorge $2019^{4}(31651321)$ \\
Psychosocial intervention vs. usual care \\
Norway
\end{tabular} & 1 year & Nurses, occupational therapists & $\begin{array}{l}\text { 51-hour individual counseling sessions followed by } 62- \\
\text { hour group meetings } \\
\text { Total hours: } 17\end{array}$ & $\begin{array}{l}\text { Psychosocial support intervention: Education about dementia; counselling on problem } \\
\text { solving and setting limits; and group sessions } \\
\text { Usual care intervention: information on available support }\end{array}$ \\
\hline $\begin{array}{l}\text { Ghafftari } 2019^{5} \text { (no PMID) } \\
\text { Resilience education group classes vs. usual care } \\
\text { Iran }\end{array}$ & 8 weeks & Study researchers & $\begin{array}{l}8 \text { weekly } 45 \text { minute sessions } \\
\text { Total hours: } 6\end{array}$ & $\begin{array}{l}\text { Resilience Education Program: education on Alzheimer's disease, internal supportive } \\
\text { factors (self-esteem, optimism, etc), external supportive factors and responsibility, resilience } \\
\text { promotion solutions (commitment, control, challenging, coping), problem solving } \\
\text { Usual care: received resilience development education after the study finished }\end{array}$ \\
\hline $\begin{array}{l}\text { Meng } 2019^{6}(30884961) \\
\text { Telephone based cognitive behavioral therapy vs. } \\
\text { in-person cognitive behavioral therapy } \\
\text { United States }\end{array}$ & 12 weeks & NR & $\begin{array}{l}\text { Telephone based cognitive therapy } \\
\text { Dose not reported }\end{array}$ & Cognitive Behavioral Therapy \\
\hline $\begin{array}{l}\text { Moskowitz } 2019^{7}(31045422) \\
\text { Life Enhancing Activities for Family Caregivers } \\
\text { (LEAF) vs. waitlist control } \\
\text { United States }\end{array}$ & 6 weeks & Facilitator discipline not specified & 6 weekly live online sessions using a computer tablet & $\begin{array}{l}\text { Life Enhancing Activities for Family Caregivers (LEAF): positive emotion regulation skills: } \\
\text { noticing positive events; capitalizing on positive events; gratitude; mindfulness, positive } \\
\text { reappraisal; personal strengths; setting antainable goals; acts of kindness } \\
\text { Waitlist control: completed daily emotion survey for } 6 \text { weeks then crossed over to LEAF } \\
\text { program. }\end{array}$ \\
\hline
\end{tabular}




\begin{tabular}{|c|c|c|c|c|}
\hline $\begin{array}{l}\text { Nordheim } 2019^{8} \text { (30775982) } \\
\text { Couple-based Interdisciplinary Psychosocial } \\
\text { Intervention vs. 1-2 hours of memory care } \\
\text { consultation } \\
\text { Germany }\end{array}$ & $10-12$ weeks & Psychotherapist, social worker & $\begin{array}{l}7 \text { home-based couples' sessions with two additional } \\
\text { telephone sessions }\end{array}$ & $\begin{array}{l}\text { Sessions included information about dementia, couple communication training, coping and } \\
\text { problem-solving strategies, network and activity analysis, counseling for living space } \\
\text { adaptions, and relaxation techniques } \\
\text { Manualized program }\end{array}$ \\
\hline $\begin{array}{l}\text { Gitlin 2018 }{ }^{13}(29192967) \\
\text { Home-based Tailored Activity Program vs. } \\
\text { attention control using telephone visits } \\
\text { United States Veterans }\end{array}$ & NR & Occupational therapists & 8 in-home sessions & $\begin{array}{l}\text { Customize activities to the interests and abilities of the veterans and educate their } \\
\text { caregivers about dementia and use of customized activity. }\end{array}$ \\
\hline $\begin{array}{l}\text { Voigt-Radloff } 2017^{94}(22021760) \\
\text { Community Occupational Therapy in Dementia } \\
\text { Program vs. usual care (one session home } \\
\text { consultation) } \\
\text { Germany }\end{array}$ & 5 weeks & Occupational therapists & $\begin{array}{l}\text { 10 1-hour in-home sessions } \\
\text { Total hours: } 10\end{array}$ & $\begin{array}{l}\text { Evidence-based manual } \\
\text { Educating PLWD in the performance of simplified daily activities and in the use of aids to } \\
\text { compensate for cognitive decline; and educating carers in coping with behavior of the } \\
\text { PLWDD and in giving supervision to the PLWD. } \\
\text { Content included: (1) } \\
\text { The PLWD preferences and history of daily activities; (2) their ability to perform activities } \\
\text { and to use compensatory strategies within the familiar environment: (3) the possibilities of } \\
\text { modifying the PLWD home; (4) the carer's activity preferences, problems in care giving, } \\
\text { coping strategies and abilities to supervise; and (5) the interaction between carer and } \\
\text { PLWD. } \\
\text { Carer received practical and emotional support and was coached in effective supervision, } \\
\text { problem-solving and coping strategies by means of cognitive-behavioral interventions. }\end{array}$ \\
\hline $\begin{array}{l}\text { Charlesworth } 2016^{18}(27521377) \\
\text { Carer Supporter Programme (CSP) program vs. } \\
\text { Remembering Yesterday Caring Today (RYCT) } \\
\text { program } \\
\text { England }\end{array}$ & $\begin{array}{l}8 \text { months } \\
10 \text { months }\end{array}$ & $\begin{array}{l}\text { CSP: Volunteer carer supporters } \\
\text { RYCT: Unspecified }\end{array}$ & $\begin{array}{l}\text { CSP: } 12 \text { one-to-one peer support 1-hour sessions } \\
\text { occuring weekly, followed by twice monthly meetings for } \\
\text { the next } 5 \text { months - } \\
\text { Total hours: } 22 \\
\text { RYCT: } 12 \text { weekly 2-hour sessions followed by } 7 \text { months } \\
\text { of monthly sessions - } 19 \text { sessions over } 10 \text { months } \\
\text { Total hours: } 38\end{array}$ & $\begin{array}{l}\text { CSP: One-to-One Peer support. Volunteer carer supporters asked to listen, encourage and } \\
\text { give moral support. Though they could also signpost to resources and services, we } \\
\text { instructed them not to offer tangible support, respite or direct advice. } \\
\text { RYCT: Group Reminiscence }\end{array}$ \\
\hline $\begin{array}{l}\text { Cheng } 2016^{19}(27401052) \\
\text { Benefit finding intervention vs. simplified lecture } \\
\text { only psychoeducation vs. standard } \\
\text { psychoeducation } \\
\text { Hong Kong }\end{array}$ & 8 weeks & $\begin{array}{l}\text { Research Assistants with } \\
\text { undergraduate degree in psychology } \\
\text { or related field. }\end{array}$ & $\begin{array}{l}8 \text { weekly 2-hour group sessions of 7-11 caregivers } \\
\text { Total hours: } 16\end{array}$ & $\begin{array}{l}\text { Benefit finding intervention: Benefit finding, positive reappraisal cognitive techniques; } \\
\text { Information on dementia, communication skills, and stress management; causes and coping } \\
\text { strategies for BPSD; homebased activities; Skills for helping } \\
\text { with ADLs, creating an appropriate home environment } \\
\text { for the care recipient, and community resources; goal setting } \\
\text { Simplified lecture only psychoeducation Intervention: Lectures and discussions, no practical } \\
\text { elements } \\
\text { Standard psychoeducation intervention: same benefit finding intervention components } \\
\text { excluding dysfunctional thoughts, maintenance cycle, and positive reappraisal }\end{array}$ \\
\hline $\begin{array}{l}\text { Gonyea } 2016^{20}(24855313) \\
\text { Circulo de Cuidado, a culturally-sensitive, } \\
\text { cognitive behavioral therapy group intervention vs. } \\
\text { psychoeducational control condition } \\
\text { United States }\end{array}$ & 17 weeks & $\begin{array}{l}\text { MSW Social workers bilingual in } \\
\text { Spanish }\end{array}$ & $\begin{array}{l}5 \text { weekly 90-minute small group sessions, followed by } 10- \\
15 \text {-minute individual telephone } \\
\text { coaching sessions at } 3,6,9 \text { - and 12-weeks post-group } \\
\text { Total hours: } 5.5\end{array}$ & $\begin{array}{l}\text { Culturally-sensitive Cognitive Behavioral Group Intervention; antecedents-behaviors- } \\
\text { consequences (A-B-C) problem solving } \\
\text { approach to behavior change; relaxation techniques or exercises; increasing engagement in } \\
\text { pleasant activities for caregiver and PLWD; improving communication with PLWD; stress } \\
\text { management; relaxation techniques; weekly at-home assignments } \\
\text { Psychoeducational group control intervention: dementia education; finding community } \\
\text { resources; working with physicians; home safety; communication in context of dementia }\end{array}$ \\
\hline $\begin{array}{l}\text { Laakkonen } 2016^{21}(27060101) \\
\text { Self-management group rehabilitation } \\
\text { Finland }\end{array}$ & 8 weeks & $\begin{array}{l}\text { Nurses, Occupational therapists, } \\
\text { Physiotherapists }\end{array}$ & $\begin{array}{l}8 \text { weekly 4-hour group sessions } \\
\text { Total hours: } 32\end{array}$ & $\begin{array}{l}\text { Group sessions aim to enhance self-efficacy and problem-solving skills and to provide peer } \\
\text { support. } \\
\text { Self-management capabilities such as problem-solving skills, self-efficacy, and mastery are } \\
\text { built gradually during the intervention } \\
\text { Usual preferred topics were dementia, medication, nutrition, active lifestyle, marital } \\
\text { relationship, caregiving, available social and health services, and advance care planning. }\end{array}$ \\
\hline
\end{tabular}




\begin{tabular}{|c|c|c|c|c|}
\hline $\begin{array}{l}\text { Otero } 2015^{83}(25331992) \\
\text { Cognitive behavioral problem solving vs. usual } \\
\text { care } \\
\text { Spain }\end{array}$ & 5 weeks & Psychologists & $\begin{array}{l}\text { 51.5-hour group sessions occurring weekly } \\
\text { Total hours: } 7.5\end{array}$ & $\begin{array}{l}\text { Cognitive behavioral problem solving group-based program including: problem solving } \\
\text { moduel, goal setting, decision making and planning, and relapse prevention. }\end{array}$ \\
\hline $\begin{array}{l}\text { Tremont } 20155^{28}(25074341) \\
\text { Family Intervention Telephone Tracking- } \\
\text { Caregiver (FITT-C) vs. Telephone Support (TS) } \\
\text { attention control. } \\
\text { United States }\end{array}$ & 6 months & $\begin{array}{l}\text { Master's degree prepared therapists } \\
\text { with training in dementia and } \\
\text { caregiving }\end{array}$ & $\begin{array}{l}16 \text { telephone contacts averaging } 36 \text { minutes each } \\
\text { Total hours: } 9.6\end{array}$ & $\begin{array}{l}\text { Providing dementia education, emotional support, directing caregivers to appropriate } \\
\text { resources, encouraging caregivers to attend to their physical, emotional, and social needs, } \\
\text { and teaching caregivers' strategies to cope with ongoing problems. }\end{array}$ \\
\hline $\begin{array}{l}\text { Livingston } 2014^{30}(25300037) \\
\text { START (STrAtegies for RelaTives vs. usual care } \\
\text { United Kingdom }\end{array}$ & unspecified & $\begin{array}{l}\text { Supervised psychology } \\
\text { graduates }\end{array}$ & 8 home-based individual sessions & $\begin{array}{l}\text { REACH Palo Alto Coping with Caregiving program modified with fewer sessions and home- } \\
\text { based sessions. Coping with Caregiving Manual } \\
\text { Individual sessions } \\
\text { Topics: Psychoeducation about dementia, carer stress and understanding behaviors, } \\
\text { discussion of behaviors or situations the carer finds difficult, incorporating behavioral } \\
\text { management, identifying and changing unhelpful thoughts, assertive communication, } \\
\text { increasing communication, acceptance, accessing emotional support and positive } \\
\text { reframing. future planning, pleasant activities, maintaining skills over time, \& stress } \\
\text { reduction techniques }\end{array}$ \\
\hline $\begin{array}{l}\text { Martin-Carrasco } 2014^{31}(24113563) \\
\text { Group psychoeducational intervention (PIP) vs. } \\
\text { usual care } \\
\text { Spain }\end{array}$ & 14 weeks & Therapist (unspecified training) & $\begin{array}{l}\text { 790-120-minute group sessions administered biweekly } \\
\text { Total hours: } 10.5-14 \text { hours }\end{array}$ & $\begin{array}{l}\text { REACH Palo Alto Coping with Caregiving program modified for Latinos living in Spain and } \\
\text { group sessions. Modified Martin-Carrasco } 2014 \text { intervention from individual to group } \\
\text { sessions. } \\
\text { Caregivers received standardized information about the clinical course of dementia and } \\
\text { were trained on cognitive and behavioral skills and relaxation techniques to increase their } \\
\text { care abilities, communicative skills, and emotional control. } \\
7 \text { modules related with strain and well-being, changing maladaptive behaviors, negative } \\
\text { thoughts, ways to communicate, planning the future, and planning enjoyable activities. }\end{array}$ \\
\hline $\begin{array}{l}\text { Waldorff } 2012^{40}(22807076) \\
\text { Danish Alzheimer's Study (DAISY) vs. control } \\
\text { support } \\
\text { Denmark }\end{array}$ & 4 months & Nurse trained in counselling approach & $\begin{array}{l}\text { Up to } 7 \text { counselling sessions including: } 2 \text { sessions with } \\
\text { the PLWD and care giver; } 2 \text { sessions with the PLWD } \\
\text { alone; } 2 \text { sessions with the care giver alone; and an } \\
\text { optional network session with the PLWD, caregiver, and } \\
\text { family network. } \\
52 \text {-hour courses for caregiver } \\
5 \text { 2-hour courses for PLWD } \\
\text { 5-8 telephone contacts with study coordinator to discuss } \\
\text { counselling session and course information. }\end{array}$ & $\begin{array}{l}\text { The counselling was based on a philosophical approach in which each PLWD or care giver } \\
\text { was given the possibility of expressing his or her life story and what is of personal } \\
\text { importance and of great value. The counsellor offered the participants guidance with } \\
\text { common decision making, advice, and activities that help the participants construct a } \\
\text { meaningful life. } \\
\text { Caregiver courses: formalized education program on Alzheimer's disease, also supported } \\
\text { by printed handouts with information on specific topics. } \\
\text { PLWD courses: information on key issues of the disease and its consequences supported } \\
\text { by printed handouts with information on specific topics. }\end{array}$ \\
\hline $\begin{array}{l}\text { de Rotrou 201142 }(20922772) \\
\text { Psych-Educational Program vs. waitlist control } \\
\text { group } \\
\text { France }\end{array}$ & 12 weeks & $\begin{array}{l}\text { Psychologists were group leaders } \\
\text { Disease education provided by health } \\
\text { professional (geriatrician, psychiatrist, } \\
\text { psychologist, social worker, speech } \\
\text { therapist, occupational therapist) }\end{array}$ & $\begin{array}{l}12 \text { weekly 2-hour group sessions of } 6-10 \text { caregivers } \\
\text { Total hours: } 24\end{array}$ & $\begin{array}{l}\text { Education on dementia, problem-solving techniques and emotion-centered coping } \\
\text { strategies, management of PLWD behavior, communication skills, crisis management, } \\
\text { resource information and practical advice, ecological stimulation - stimulate their relative in } \\
\text { daily activities and social situations } \\
\text { Waitlist control: } \\
\text { PLWD in both groups received cholinesterase-inhibitor pharmacotherapy }\end{array}$ \\
\hline $\begin{array}{l}\text { Guerra } 2011^{43} \\
10 / 66 \text { Helping Carers to Care vs waitlist control } \\
\text { Peru }\end{array}$ & 5 weeks & Junior Psychologists, social workers & $\begin{array}{l}5 \text { weekly 30-minute sessions over } 5 \text { weeks } \\
\text { Total hours: } 2.5\end{array}$ & Helping carers to care: dementia education, training on managing problem behaviors \\
\hline $\begin{array}{l}\text { Wang } 2011^{47}(21752121) \\
\text { Family Mutual Support Programme in Dementia } \\
\text { Care (FMSP-DC) vs. usual care } \\
\text { China }\end{array}$ & 6 months & Psychiatric nurse & $\begin{array}{l}8 \text { biweekly 2-hour group sessions } \\
\text { Family led } \\
\text { Total hours: } 16\end{array}$ & $\begin{array}{l}\text { Community based program addressing (1) information about client's illness, prognosis and } \\
\text { current treatment and care; (2) development of social relationships with close relatives } \\
\text { and friends and thus a satisfactory extended social support network; (3) sharing and } \\
\text { adaptation of emotional impacts of caregiving; (4) learning about self-care and motivation; } \\
\text { (5) improvement of interpersonal relationships with family members and the client; } \\
\text { (6)establishing support trom the community groups and health-care resources; and (7) } \\
\text { improvement of problem solving skills in family care. }\end{array}$ \\
\hline
\end{tabular}




\begin{tabular}{|c|c|c|c|}
\hline $\begin{array}{l}\text { Gitlin 201049 (20662955) } \\
\text { Advancing Caregiving Training (ACT) vs. no } \\
\text { treatment } \\
\text { United States }\end{array}$ & $\begin{array}{l}\text { 16-week active } \\
\text { phase followed } \\
\text { by } \\
\text { 8-week } \\
\text { maintenance } \\
\text { phase }\end{array}$ & $\begin{array}{l}\text { Occupational therapists } \\
\text { Advanced Practice Nurses }\end{array}$ & $\begin{array}{l}\text { During active phase, up to } 9 \text { OT sessions } \& 2 \text { nursing } \\
\text { sessions (one home visit, one telephone visitt) } \\
\text { During maintenance phase: } 3 \text { telephone calls from } \\
\text { occupational therapist } \\
\text { Total hours: } 16\end{array}$ \\
\hline $\begin{array}{l}\text { Martin-Carrasco } 2009^{54}(18949763) \\
\text { Psychoeducational Intervention Program (PIP) vs. } \\
\text { usual care } \\
\text { Spain }\end{array}$ & 4 months & $\begin{array}{l}\text { Clinical psychologist, } \\
\text { nurse or social worker }\end{array}$ & $\begin{array}{l}8 \text { individual } 90 \text {-minute sessions at 1-2-week intervals over } \\
4 \text { months } \\
\text { Total hours: } 12\end{array}$ \\
\hline $\begin{array}{l}\text { Gallagher-Thompson } 2008^{56}(25067886) \\
\text { Coping with Caregiving vs. Minimal telephone- } \\
\text { based control condition } \\
\text { United States }\end{array}$ & 13-16 weeks & $\begin{array}{l}\text { Post-doctoral fellows or advanced } \\
\text { graduate students in psychology or } \\
\text { related fields who had relevant } \\
\text { bilingual/bicultural backgrounds. } \\
2 \text { Interventionists led each session }\end{array}$ & $\begin{array}{l}\text { Weekly 2-hour group sessions of 4-8 female caregivers. } \\
\text { Total hours: } 26-32\end{array}$ \\
\hline $\begin{array}{l}\text { Graff } 2006^{60}(17114212) \\
\text { Community based occupational therapy program } \\
\text { vs. Waitlist for occupational therapy } \\
\text { The Netherlands }\end{array}$ & 5 weeks & Occupational therapists & $\begin{array}{l}10 \text { 1-hour sessions with caregiver and PLWD } \\
\text { Total hours: } 10\end{array}$ \\
\hline 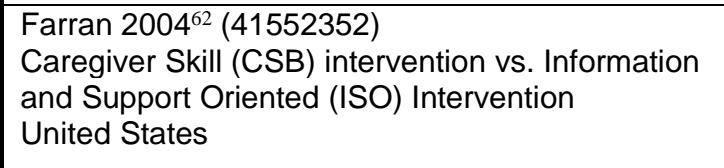 & 1 year & Nurses, social workers & $\begin{array}{l}12 \text { weekly sessions consisting of } 5 \text { group sessions and } 7 \\
\text { individual telephone contact sessions; Followed with } 2 \\
\text { booster sessions at } 6 \text { and } 12 \text { months and as needed } \\
\text { telephone sessions. }\end{array}$ \\
\hline $\begin{array}{l}\text { Gallagher-Thompson } 2003^{66}(12937336) \\
\text { Coping with Caregiving vs. Enhanced support } \\
\text { group condition } \\
\text { United States }\end{array}$ & \begin{tabular}{|l|}
120 weeks \\
(10 months)
\end{tabular} & $\begin{array}{l}\text { Psychologists, social workers or other } \\
\text { helping professionals, and predoctoral } \\
\text { or postdoctoral psychology and social } \\
\text { work graduate students }\end{array}$ & $\begin{array}{l}\text { First } 10 \text { weeks used } 2 \text {-hour group sessions of } 7-9 \text { female } \\
\text { caregivers followed by } 8 \text { monthly booster sessions. } \\
\text { Total hours: } 28\end{array}$ \\
\hline $\begin{array}{l}\text { Gitlin } 2001^{93}(11220813) \\
\text { Home Environmental Intervention vs. usual care } \\
\text { United States }\end{array}$ & 3 months & Occupational therapists & $\begin{array}{l}5 \text { 90-minute home visits occurring every other month } \\
\text { Total hours: } 7.5\end{array}$ \\
\hline
\end{tabular}

Abbreviations: PLWD=person with dementia
Advanced Caregiver Training (ACT): identification of PLWD unmet needs, discomfort, \& medical problems, caregiver skill building, problem solving, and communication building, fications \& provision

No treatment control group

REACH Palo Alto Coping with Caregiving program modified for Latinos IIving in Spain. Structured psycho-educational-type psychosocial intervention program

Skills training: (a) to help the caregiver control tension and stress deriving from caregiving (b) to teach the caregiver different strategies for handling their relative's behavioral REACH Palo Alto Coping with Caregiving Program updated (Gallagher-Thompson 2003 (12937336))

Coping with Caregiving: cognitive behavioral therapy to develop cognitive and behaviora managing care recipient's problem behaviors; skills to take better care of the caregiver, including changing unhelpful thoughts, increasing assertive communication, and identifying everyday pleasant activities (both for self and shared with care recipient); planning for the care-recipient's future needs; develop action plan for future

Minimal telephone-based control intervention: Individual sessions of empathic support (7 biweekly calls lasting 15-20 minutes)

Cognitive and behavioral interventions, to train PLWD in the use of aids to compensate for cognitive decline and care givers in coping behaviors and supervision

Diagnostics and goal defining. PLWD and primary care givers learnt to choose and prioritize meaningful activities they wanted to improve

optimize these compensatory and environmental strategies to improve their performance of

daily activities symptoms, prevention of behavior during cares, management of restless behaviors,

management of hallucinations and delusions.

ISO: Standardized implementation of psychoeducational methods, generalized information and support, no individualized behavioral symptom management. 5 topics: understanding and finding/using help. Both programs were tailored to be sensitive to the cultural concerns of Anglo and Latino caregivers, and they were delivered in either English or Spanish by trained interventionists. REACH Palo Alto Coping with Caregiving Group: dementia education, cognitive-behavioral mood management skills

Enhanced Support Group: dementia education, guided discussion, empathic listening

\section{Eduction and physical and social environmental modifications.}

Dementia education;

Education on daily care needs specified by caregiver;

Enclay, den colve; Problem solving; 
Table E-4. PLWD outcomes summary low and medium risk of bias studies: psychosocial interventions for caregiver well-being

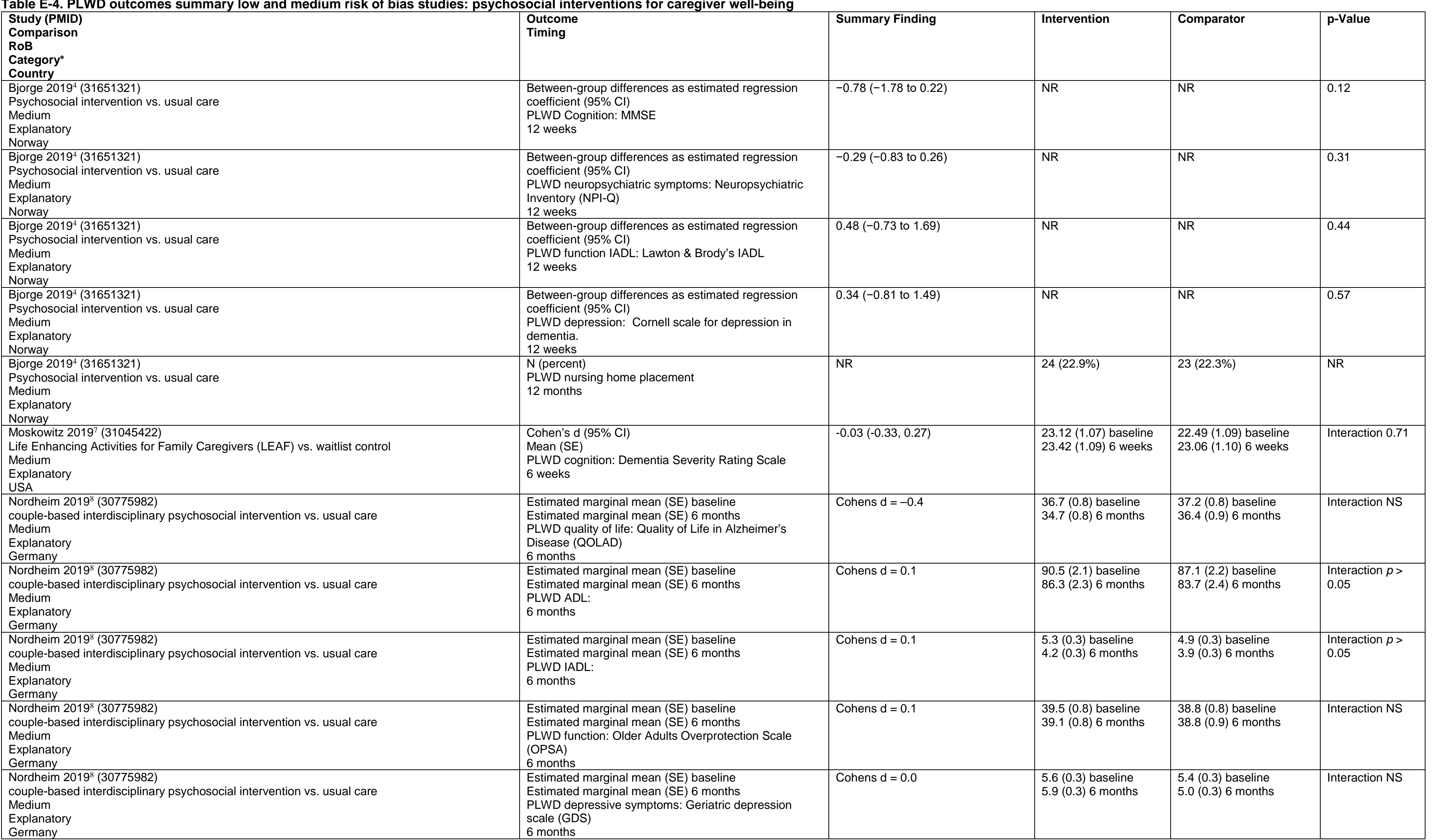




\begin{tabular}{|c|c|c|c|c|c|}
\hline $\begin{array}{l}\text { Nordheim } 2019^{8}(30775982) \\
\text { couple--based interdisciplinary psychosocial intervention vs. usual care } \\
\text { Medium } \\
\text { Explanatory } \\
\text { Germany }\end{array}$ & $\begin{array}{l}\text { Estimated marginal mean (SE) baseline } \\
\text { Estimated marginal mean (SE) } 6 \text { months } \\
\text { PLWD cognition: MMSE } \\
6 \text { months }\end{array}$ & Cohens $\mathrm{d}=0.4$ & $\begin{array}{l}23.0(0.6) \text { baseline } \\
21.6(0.7) 6 \text { months }\end{array}$ & $\begin{array}{l}22.7(0.7) \text { baseline } \\
19.6(0.8) 6 \text { months }\end{array}$ & $\begin{array}{l}\text { Interaction } p< \\
0.05\end{array}$ \\
\hline $\begin{array}{l}\text { Nordheim } 2019^{8}(30775982) \\
\text { couple-based interdisciplinary psychosocial intervention vs. usual care } \\
\text { Medium } \\
\text { Explanatory } \\
\text { Germany }\end{array}$ & $\begin{array}{l}\text { Estimated marginal mean (SE) baseline } \\
\text { Estimated marginal mean (SE) } 6 \text { months } \\
\text { PLWD social support: (F-SOZU) } \\
6 \text { months }\end{array}$ & Cohens $d=-0.1$ & $\begin{array}{l}54.8 \text { (1.4) baseline } \\
54.0 \text { (1.3) } 6 \text { months }\end{array}$ & $\begin{array}{l}54.1 \text { (1.4) baseline } \\
55.1 \text { (1.4) } 6 \text { months }\end{array}$ & Interaction NS \\
\hline $\begin{array}{l}\text { Gitlin } 2018^{13}(29192967) \\
\text { Mome-based Tailored Activity Program vs. attention control using telephone visits } \\
\text { Medium } \\
\text { Explanatory } \\
\text { USA }\end{array}$ & $\begin{array}{l}\text { Mean difference }(95 \% \mathrm{Cl}) \\
\text { PLWD neuropsychiatric symptoms: Neuropsychiatric } \\
\text { Inventory - Clinician (NPI-C) -number of behavioral } \\
\text { symptoms } \\
4 \text { months }\end{array}$ & $-0.68(-1.23 ;-0.13)$ & $\mathrm{NR}$ & NR & 0.02 \\
\hline $\begin{array}{l}\text { Gitlin } 2018^{13}(29192967) \\
\text { Home-based Tailored Activity Program vs. attention control using telephone visits } \\
\text { Medium Explanatory } \\
\text { ESA }\end{array}$ & $\begin{array}{l}\text { Mean difference }(95 \% \mathrm{Cl}) \\
\text { PLWD neuropsychiatric symptoms: Neuropsychiatric } \\
\text { Inventory - Clinician (NPI-C) } \\
4 \text { months }\end{array}$ & $-24.3(-45.6 ;-3.1)$ & NR & NR & 0.02 \\
\hline $\begin{array}{l}\text { Gitlin } 2018^{13}(29192967) \\
\text { Home-based Tailored Activity Program vs. attention control using telephone visits } \\
\text { Medium Explanatory } \\
\text { USA }\end{array}$ & $\begin{array}{l}\text { Mean difference }(95 \% \mathrm{Cl}) \\
\text { PLWD function ADL//ADL: Caregiver Assessment of } \\
\text { Function and Upset Scale (CAFU) - number of } \\
\text { ADL/IADL dependencies subscale } \\
4 \text { months }\end{array}$ & $-0.80(-1.41 ;-0.20)$ & NR & NR & 0.009 \\
\hline $\begin{array}{l}\text { Gitlin } 2018^{13}(29192967) \\
\text { Home-based Tailored Activity Program vs. attention control using telephone visits } \\
\text { Medium Explanatory } \\
\text { USA }\end{array}$ & $\begin{array}{l}\text { Mean difference }(95 \% \text { CI) } \\
\text { PLWD function ADL/IADL: Caregiver Assessment of } \\
\text { Function and Upset Scale (CAFU) - level of ADL/IADL } \\
\text { dependence (total) } \\
4 \text { months }\end{array}$ & $4.09(1.06 ; 7.13)$ & NR & NR & 0.009 \\
\hline $\begin{array}{l}\text { Gitlin } 2018^{13}(29192967) \\
\text { Home-based Tailored Activity Program vs. attention control using telephone visits } \\
\text { Medium Explanatory } \\
\text { USA }\end{array}$ & $\begin{array}{l}\text { Mean difference }(95 \% \mathrm{Cl}) \\
\text { PLWD QOL: affect (caregiver perception) } \\
4 \text { months }\end{array}$ & $0.47(-1.37 ; 0.43)$ & NR & NR & 0.30 \\
\hline $\begin{array}{l}\text { Gitlin } 2018^{13}(29192967) \\
\text { Home-based Tailored Activity Program vs. attention control using telephone visits } \\
\text { Medium Explanatory } \\
\text { USA }\end{array}$ & $\begin{array}{l}\text { Mean difference }(95 \% \text { Cl) } \\
\text { PLWD function ADL: Caregiver Assessment of Function } \\
\text { and Upset Scale (CAFU) - number of ADL } \\
\text { dependencies subscale } \\
4 \text { months }\end{array}$ & \begin{tabular}{|l}
$-0.61(-1.08 ;-0.14)$ \\
\end{tabular} & NR & $\mathrm{NR}$ & 0.01 \\
\hline $\begin{array}{l}\text { Gitlin } 2018^{13} \text { (29192967) } \\
\text { Home-based Tailored Activity Program vs. attention control using telephone visits } \\
\text { Medium Explanatory } \\
\text { ESA }\end{array}$ & $\begin{array}{l}\text { Mean difference }(95 \% \mathrm{Cl}) \\
\text { PLWD function IADL: Caregiver Assessment of } \\
\text { Function and Upset Scale (CAFU) - number of IADL } \\
\text { dependencies subscale } \\
4 \text { months }\end{array}$ & $-0.25(-0.54 ; 0.04)$ & NR & NR & 0.09 \\
\hline $\begin{array}{l}\text { Gitlin } 2018^{13}(29192967) \\
\text { Home-based Tailored Activity Program vs. attention control using telephone visits } \\
\text { Medium Explanatory } \\
\text { ESA }\end{array}$ & $\begin{array}{l}\text { Mean difference }(95 \% \text { Cl) } \\
\text { PLWD function ADL: Caregiver Assessment of Function } \\
\text { and Upset Scale (CAFU) - level of ADL dependence } \\
\text { subscale } \\
4 \text { months }\end{array}$ & $2.37(0.32 ; 4.42)$ & NR & NR & 0.02 \\
\hline $\begin{array}{l}\text { Gitlin } 2018^{13}(29192967) \\
\text { Home-based Tailored Activity Program vs. attention control using telephone visits } \\
\text { Medium } \\
\text { Explanatory } \\
\text { USA }\end{array}$ & $\begin{array}{l}\text { Mean difference (95\% CI)PLWD function IADL: } \\
\text { Caregiver Assessment of Function and Upset Scale } \\
\text { (CAFU) - level of IADL dependence subscale } \\
4 \text { months }\end{array}$ & $1.57(0.05 ; 3.08)$ & NR & NR & 0.04 \\
\hline $\begin{array}{l}\text { Tremont } 2017^{84}(28008609) \text { companion to Tremont } 2015(25074341) \\
\text { Telephone Tracking-Caregiver (FITT-C) vs. Telephone Support (TS) } \\
\text { Medium } \\
\text { Explanatory }\end{array}$ & $\begin{array}{l}\text { Mean (SD) } \\
\text { PLWD health care use: Number of monthly emergency } \\
\text { department visits during intervention } \\
6 \text { months }\end{array}$ & NR & $0.08(0.16)$ & $0.08(0.17)$ & 0.83 \\
\hline
\end{tabular}




\begin{tabular}{|c|c|c|c|c|c|}
\hline $\begin{array}{l}\text { Tremont } 2017^{84} \text { (28008609) companion to Tremont } 2015 \text { (25074341) } \\
\text { Telephone Tracking-Caregiver (FITT-C) vs. Telephone Support (TS) } \\
\text { Medium } \\
\text { Explanatory } \\
\text { USA }\end{array}$ & $\begin{array}{l}\text { Mean (SD) } \\
\text { PLWD health care use: Number of monthly doctor visits } \\
\text { during intervention } \\
6 \text { months }\end{array}$ & NR & $1.32(1.35)$ & $1.30(1.05)$ & 0.88 \\
\hline $\begin{array}{l}\text { Tremont } 2017^{84} \text { (28008609) companion to Tremont } 2015 \text { (25074341) } \\
\text { Telephone Tracking-Caregiver (FITT-C) vs. Telephone Support (TS) } \\
\text { Medium } \\
\text { Uxplanatory } \\
\text { USA }\end{array}$ & $\begin{array}{l}\text { Mean (SD) } \\
\text { PLWD community support use: Number of community } \\
\text { support services used in final month of intervention } \\
6 \text { months }\end{array}$ & NR & $6.79(13.72)$ & $5.11(10.68)$ & 0.34 \\
\hline $\begin{array}{l}\text { Tremont } 2017^{84} \text { (28008609) companion to Tremont } 2015 \text { (25074341) } \\
\text { Telephone Tracking-Caregiver (FITT-C) vs. Telephone Support (TS) } \\
\text { Medium } \\
\text { Explanatory } \\
\text { USA }\end{array}$ & $\begin{array}{l}\text { Frequency (\%) } \\
\text { PLWDD nursing home admission: number of PLWD } \\
\text { admitted to nursing home during the intervention }\end{array}$ & $12(6 \%)$ & NR & NR & $\begin{array}{l}0.70 \text { (no group } \\
\text { difference) }\end{array}$ \\
\hline $\begin{array}{l}\text { Charlesworth } 2016^{18}(27521377) \\
3 \text { groups: } 1 \text { ) One-to-one peer support to family carers from experienced carers (Carer } \\
\text { Supporter Programme (CSP), 2) group reminiscence therapy Remembering Yesterday, Caring } \\
\text { Today (RYCT), \& 3) usual care } \\
\text { Low } \\
\text { Explanatory } \\
\text { UK }\end{array}$ & $\begin{array}{l}\text { Adjusted mean difference }(95 \% \mathrm{Cl}) \text { for CSP vs other } 2 \\
\text { groups } \\
\text { PLWD Health related quality of life: QOL-AD self- } \\
\text { reported } \\
12 \text { months }\end{array}$ & $-0.162(-1.87$ to 1.54$)$ & Means reported & Means reported & 0.85 \\
\hline $\begin{array}{l}\text { Charlesworth } 2016^{18}(27521377) \\
3 \text { groups: } 1) \text { One-to-one peer support to family carers from experienced carers (Carer } \\
\text { Supporter Programme (CSP), 2) group reminiscence therapy Remembering Yesterday, Caring } \\
\text { Today (RYCT), \& 3) usual care } \\
\text { Low } \\
\text { Explanatory } \\
\text { UK }\end{array}$ & $\begin{array}{l}\text { Adjusted mean difference }(95 \% \mathrm{Cl}) \text { for RYCT vs other } 2 \\
\text { groups } \\
\text { PLWD Health related quality of life: QOL-AD self- } \\
\text { reported } \\
12 \text { months }\end{array}$ & $0.702(-1.05$ to 2.45$)$ & & & 0.43 \\
\hline $\begin{array}{l}\text { Charlesworth } 2016^{18}(27521377) \\
3 \text { groups: } 1 \text { ) One-to-one peer support to family carers from experienced carers (Carer } \\
\text { Supporter Programme (CSP), 2) group reminiscence therapy Remembering Yesterday, Caring } \\
\text { Today (RYCT), \& 3) usual care } \\
\text { Low } \\
\text { Explanatory } \\
\text { UK }\end{array}$ & $\begin{array}{l}\text { Adjusted mean difference }(95 \% \mathrm{Cl}) \text { for CSP vs other } 2 \\
\text { groups } \\
\text { PLWD Health related quality of life: QOL-AD proxy } \\
\text { reported } \\
12 \text { months }\end{array}$ & $0.050(-1.21$ to 1.31$)$ & & & 0.94 \\
\hline $\begin{array}{l}\text { Charlesworth } 2016^{18}(27521377) \\
3 \text { groups: } 1 \text { ) One-to-one peer support to family carers from experienced carers (Carer } \\
\text { Supporter Programme (CSP), 2) group reminiscence therapy Remembering Yesterday, Caring } \\
\text { Today (RYCT), \& 3) usual care } \\
\text { Low } \\
\text { Explanatory } \\
\text { UK }\end{array}$ & $\begin{array}{l}\text { Adjusted mean difference }(95 \% \mathrm{Cl}) \text { for RYCT vs other } 2 \\
\text { groups } \\
\text { PLWD Health related quality of life: QOL-AD proxy } \\
\text { reported } \\
12 \text { months }\end{array}$ & $0.0660(-1.17$ to 1.30$)$ & & & 0.92 \\
\hline $\begin{array}{l}\text { Charlesworth } 2016^{18}(27521377) \\
3 \text { groups: } 1 \text { ) One-to-one peer support to family carers from experienced carers (Carer } \\
\text { Supporter Programme (CSP), 2) group reminiscence therapy Remembering Yesterday, Caring } \\
\text { Today (RYCT), \& 3) usual care } \\
\text { Low } \\
\text { Explanatory } \\
\text { UK }\end{array}$ & $\begin{array}{l}\text { Adjusted mean difference }(95 \% \mathrm{Cl}) \text { for CSP vs other } 2 \\
\text { groups } \\
\text { PLWD function: Alzheimer's Disease Cooperative } \\
\text { Study - Activities of Daily Living } \\
12 \text { months }\end{array}$ & $-2.18(-6.07$ to 1.71$)$ & & & 0.27 \\
\hline $\begin{array}{l}\text { Charlesworth } 2016^{18}(27521377) \\
3 \text { groups: } 1) \text { One-to-one peer support to family carers from experienced carers (Carer } \\
\text { Supporter Programme (CSP), 2) group reminiscence therapy Remembering Yesterday, Caring } \\
\text { Today (RYCT), \& 3) usual care } \\
\text { Low } \\
\text { Explanatory } \\
\text { UK }\end{array}$ & $\begin{array}{l}\text { Adjusted mean difference }(95 \% \mathrm{Cl}) \text { for RYCT vs other } 2 \\
\text { groups } \\
\text { PLWD function: Alzheimer's Disease Cooperative } \\
\text { Study - Activities of Daily Living } \\
12 \text { months }\end{array}$ & $-2.45(-5.95$ to 1.06$)$ & & & 0.17 \\
\hline
\end{tabular}


Charlesworth 2016 ${ }^{18}(27521377)$

3 groups: 1) One-to-one peer support to family carers from experienced carers (Carer

Supporter Programme (CSP), 2) group reminiscence therapy Remembering Yesterday, Caring

Explanatory

UK
Charlesworth 2016 18 (27521377)

3 groups: 1) One-to-one peer support to family carers from experienced carers (Carer

Supporter Programme (CSP), 2) group reminiscence therapy Remembering Yesterday, Caring

ual care

Explanatory

UK

Circulo de Cuidado, a culturally-sensitive, cognitive behavioral (CBT) group intervention vs.

psychoeducational (PED) control condition

Laakkonen 2016 21 (27060101)

Self-management groups vs. usual care

Explanatory

Finland

Laakkonen 2016 21 (27060101)

Self-management groups vs. usual care

Explanatory

Explanatory
Finland

Laakkonen 2016 21 (27060101)

Self-management groups vs. usual care

Medium

Explanatory

Laakkonen $2016^{21}$ (27060101)

Self-management groups vs, usual care

Medium

Explanatory

Finland

Self-management groups vs. usual care

Medium

Explanatory

Finland

Self-managenent (27060101)

Medium

Explanatory

Finland

Livingston 201430 (25300037)

START (STrAtegies for RelaTives) manual-based coping strategy programme in promoting the

Explanatory
Adjusted mean difference $(95 \% \mathrm{CI})$ for CSP vs other 2

groups

12 months

Adjusted mean difference $(95 \% \mathrm{Cl})$ for RYCT vs other 2

groups

ensy

inventory (NPI)

Mean (SD)

PLWD neuropsychiatric symptoms: Spanish version

the Neuropsychiatric Inventory-Severity scale (NPI-S)

24 week

Adjusted Mean change $(95 \% \mathrm{Cl})$

QLWD quality of life: $15 \mathrm{D}$

Adjusted Mean change $(95 \% \mathrm{Cl})$

PLWD cognition: Verbal Fluency (VF)

Adjusted Mean change $(95 \% \mathrm{Cl})$

PLWD cognition: Verbal Fluency (VF)

Adjusted Mean change $(95 \% \mathrm{Cl})$

PLWD cognition: Clock Drawing Test (CDT)

3 months

\section{Adjusted Mean change $(95 \% \mathrm{Cl})$}

Adjusted Mean change (95\% CI)
PLWD cognition: Clock Drawing Test (CDT)

9 months

Adjusted Mean cost in $€$ per year $(95 \% \mathrm{Cl})$

24 months

Adjusted mean difference $(95 \% \mathrm{Cl})$

PLWD QOL: (QOL-AD)

years

\begin{tabular}{|c|c|c|c|}
\hline$-1.20(-6.64$ to 4.23$)$ & & & 0.66 \\
\hline $0.236(-4.83$ to 5.30$)$ & & & 0.93 \\
\hline NR & $\begin{array}{l}21.70(6.90) \text { baseline } \\
20.67(6.94) 12 \text { weeks } \\
20.52(6.98) 24 \text { weeks }\end{array}$ & $\begin{array}{l}21.76(7.98) \text { baseline } \\
22.03(9.10) 12 \text { weeks } \\
22.09(8.46) 24 \text { weeks }\end{array}$ & $\begin{array}{l}\text { Repeated } \\
\text { measures } \\
\text { ANCOVA } \\
<0.001\end{array}$ \\
\hline $\mathrm{d}=\mathrm{NR}$ & $-0.03(-0.0$ to -0.01$)$ & $-0.04(-0.05$ to -0.02$)$ & 0.55 \\
\hline $\mathrm{d}=\mathrm{NR}$ & 0.26 (-0.38 to 0.91$)$ & $-0.60(-1.26$ to 0.07$)$ & 0.07 \\
\hline $\mathrm{d}=\mathrm{NR}$ & $-0.38(-1.03$ to 0.27$)$ & $-1.60(-2.26$ to -0.94$)$ & 0.01 \\
\hline $\mathrm{d}=\mathrm{NR}$ & $0.22(-0.13$ to 0.58$)$ & $-0.18(-0.55$ to 0.18$)$ & 0.12 \\
\hline $\mathrm{d}=0.28$ & $-0.11(-0.46$ to 0.25$)$ & $-0.65(-1.02$ to -0.30$)$ & 0.03 \\
\hline$-436(-4,986 ; 4,115)$ & $8,947(6,455-12,415)$ & $9,383(6,398-13,374)$ & .035 \\
\hline $0.17(-1.37$ to 1.70$)$ & NR & NR & NR \\
\hline
\end{tabular}




\begin{tabular}{|c|c|c|c|c|c|}
\hline $\begin{array}{l}\text { Waldorff } 2012^{40}(22807076) \\
\text { DAISY plus support (multifaceted and semi-tailored counselling, } \\
\text { education, and support) vs. support control group } \\
\text { Medium } \\
\text { Explanatory } \\
\text { Denmark }\end{array}$ & $\begin{array}{l}\text { Mean change score }(95 \% \text { Cl) } \\
\text { PLWD QOL: European quality of life visual analogue } \\
\text { scale (EQ-VAS) score } \\
12 \text { months }\end{array}$ & $\mathrm{NR}$ & -0.31 (-3.41 to 2.79$)$ & $0.20(-2.31$ to 2.71$)$ & 0.7799 \\
\hline $\begin{array}{l}\text { Waldorff } 2012^{40}(22807076) \\
\text { DAISY plus support (multifaceted and semi-tailored counselling, } \\
\text { education, and support) vs. support control group } \\
\text { Medium } \\
\text { Explanatory } \\
\text { Denmark }\end{array}$ & $\begin{array}{l}\text { Mean change score }(95 \% \mathrm{Cl}) \\
\text { PLWD cognition: MMSE } \\
12 \text { months }\end{array}$ & NR & $-1.55(-2.19$ to -0.91$)$ & $-1.80(-2.56$ to -1.04$)$ & 0.6502 \\
\hline $\begin{array}{l}\text { Waldorff } 2012^{40}(22807076) \\
\text { DAISY plus support (multifaceted and semi-tailored counselling, } \\
\text { education, and support) vs. support control group } \\
\text { Medium } \\
\text { Explanatory } \\
\text { Denmark }\end{array}$ & $\begin{array}{l}\text { Mean change score }(95 \% \mathrm{Cl}) \\
\text { PLWD depression: Cornell depression scale } \\
12 \text { months }\end{array}$ & $\mathrm{NR}$ & $-0.20(-1.04$ to 0.64$)$ & 1.38 (0.49 to 2.27 ) & 0.0103 \\
\hline $\begin{array}{l}\text { Waldorff } 2012^{40}(22807076) \\
\text { DAISY plus support (multifaceted and semi-tailored counselling, } \\
\text { education, and support) vs. support control group } \\
\text { Medium, } \\
\text { Explanatory } \\
\text { Denmark }\end{array}$ & $\begin{array}{l}\text { Mean change score }(95 \% \text { Cl) } \\
\text { PLWD QOL: proxy rated European quality of life visual } \\
\text { analogue scale (EQ-VAS) (proxy rated) score } \\
12 \text { months }\end{array}$ & NR & $-3.88(-7.38$ to -0.37$)$ & $-6.83(-10.10$ to -3.56$)$ & 0.2308 \\
\hline $\begin{array}{l}\text { Waldorff } 2012^{40}(22807076) \\
\text { DAISY plus support (multifaceted and semi-tailored counselling, } \\
\text { education, and support) vs. support control group } \\
\text { Medium } \\
\text { Explanatory } \\
\text { Denmark }\end{array}$ & $\begin{array}{l}\text { Mean change score }(95 \% \text { Cl) } \\
\text { PLWD QOL: European quality of life visual analogue } \\
\text { scale EQ VAS (PLWD rated) } \\
12 \text { months }\end{array}$ & NR & 0.73 (-2.41 to 3.88$)$ & $-4.20(-8.29$ to -0.11$)$ & 0.0667 \\
\hline $\begin{array}{l}\text { Waldorff } 2012^{40}(22807076) \\
\text { DAISY plus support (multifaceted and semi-tailored counselling, } \\
\text { education, and support) vs. support control group } \\
\text { Medium } \\
\text { Explanatory } \\
\text { Denmark }\end{array}$ & $\begin{array}{l}\text { Mean change score }(95 \% \mathrm{Cl}) \\
\text { PLWD WOL: Quality of life scale for Alzheimer's } \\
\text { disease QoL-AD (PLWD rated) } \\
12 \text { months }\end{array}$ & NR & -0.82 (-1.77 to 0.12$)$ & $-1.04(-2.04$ to -0.04$)$ & 0.7213 \\
\hline $\begin{array}{l}\text { Waldorff } 2012^{40}(22807076) \\
\text { DAISY plus support (multifaceted and semi-tailored counselling, } \\
\text { education, and support) vs. support control group } \\
\text { Medium } \\
\text { Explanatory } \\
\text { Denmark }\end{array}$ & $\begin{array}{l}\text { Mean change score }(95 \% \mathrm{Cl}) \\
\text { PLWD QOL: quality of life scale for Alzheimer's disease } \\
\text { QoL-AD (proxy rated) } \\
12 \text { months }\end{array}$ & NR & -0.82 (-1.77 to 0.12$)$ & $-2.61(-3.58$ to -1.64$)$ & 0.0013 \\
\hline $\begin{array}{l}\text { Waldorff } 2012^{40}(22807076) \\
\text { DAISY plus support (multifaceted and semi-tailored counselling, } \\
\text { education, and support) vs. support control group } \\
\text { Medium } \\
\text { Explanatory } \\
\text { Denmark }\end{array}$ & $\begin{array}{l}\text { Mean change score }(95 \% \mathrm{CI}) \\
\text { PLWD neuropsychiatric symptoms: neuropsychiatric } \\
\text { inventory questionnaire NPI-Q } \\
12 \text { months }\end{array}$ & NR & 0.85 (0.16 to 1.53$)$ & $0.43(-0.26$ to 1.13$)$ & 0.4045 \\
\hline $\begin{array}{l}\text { Waldorff } 2012^{40}(22807076) \\
\text { DAISY plus support (multifaceted and semi-tailored counselling, } \\
\text { education, and support) vs. support control group } \\
\text { Medium } \\
\text { Explanatory } \\
\text { Denmark }\end{array}$ & $\begin{array}{l}\text { Mean change score }(95 \% \text { CI) } \\
\text { PLWD function: Allzheimer's Disease Cooperative Study } \\
\text { activities of daily living scale. ADSC-ADL } \\
12 \text { months }\end{array}$ & NR & $-6.39(-8.51$ to -4.27$)$ & $-4.63(-6.92$ to -2.53$)$ & 0.2689 \\
\hline $\begin{array}{l}\text { Guerra } 2011^{43} \\
10 / 66 \text { Helping Carers to Care vs waitlist control } \\
\text { Medium } \\
\text { Explanatory } \\
\text { Peru }\end{array}$ & $\begin{array}{l}\text { Standardized Mean Difference }(95 \% \mathrm{Cl}) \\
\text { PLWD neuropsychiatric symptoms: Neuropsychiatric } \\
\text { Inventory brief form (NPI-Q) } \\
6 \text { months }\end{array}$ & $-0.05(-0.59$ to .52$)$ & NR & $\mathrm{NR}$ & $\mathrm{NR}$ \\
\hline
\end{tabular}




\begin{tabular}{|c|c|c|c|c|c|}
\hline $\begin{array}{l}\text { Guerra } 2011^{43} \\
10 / 66 \text { Helping Carers to Care vs waitlist control } \\
\text { Medium } \\
\text { Explanatory } \\
\text { Peru }\end{array}$ & $\begin{array}{l}\text { Mean change (SD) } \\
\text { PLWD neuropsychiatric symptoms: Neuropsychiatric } \\
\text { Inventory brief form (NPI-Q) } \\
6 \text { months }\end{array}$ & $\mathrm{NR}$ & $-1.7(3.3)$ & $-1.6(206)$ & 0.87 \\
\hline $\begin{array}{l}\text { Voight-Radloff } 2011^{46}(22021760) \\
\text { Community Occupational Therapy in Dementia Program vs. usual care (one session home } \\
\text { consultation) } \\
\text { Medium } 26-52 \text { weeks } \\
\text { Explanatory } \\
\text { Germany }\end{array}$ & $\begin{array}{l}\text { Mean difference }(95 \% \mathrm{Cl}) \\
\text { PLWD function: Interview for Deterioration in Daily } \\
\text { Living Activities in Dementia (IDDD) - performance } \\
\text { subscale } \\
52 \text { weeks }\end{array}$ & $-2.4(-7.1 ; 2.3)$ & NR & NR & NS \\
\hline $\begin{array}{l}\text { Voight-Radloff } 2011^{46}(22021760) \\
\text { Community Occupational Therapy in Dementia Program vs. usual care (one session home } \\
\text { consultation) } \\
\text { Medium } 26-52 \text { weeks } \\
\text { Explanatory } \\
\text { Germany }\end{array}$ & $\begin{array}{l}\text { Mean difference }(95 \% \mathrm{Cl}) \\
\text { PLWD function: Interview for Deterioration in Daily } \\
\text { Living Activities in Dementia (IDDD) - initiative subscale } \\
52 \text { weeks }\end{array}$ & $-1.0(-5.0 ; 3.0)$ & NR & $\mathrm{NR}$ & NS \\
\hline $\begin{array}{l}\text { Voight-Radloff } 2011^{46}(22021760) \\
\text { Community Occupational Therapy in Dementia Program vs. usual care (one session home } \\
\text { consultation) } \\
\text { Medium } 26-52 \text { weeks } \\
\text { Explanatory } \\
\text { Germany }\end{array}$ & $\begin{array}{l}\text { Mean difference }(95 \% \mathrm{Cl}) \\
\text { PLWD depression: Cornell Scale for Depression in } \\
\text { Dementia } \\
52 \text { weeks }\end{array}$ & $-2.0(-5.1 ; 1.0)$ & $\mathrm{NR}$ & $\mathrm{NR}$ & NS \\
\hline $\begin{array}{l}\text { Voight-Radloff } 2011^{46}(22021760) \\
\text { Community Occupational Therapy in Dementia Program vs. usual care (one session home } \\
\text { consultation) } \\
\text { Medium } 26-52 \text { weeks } \\
\text { Explanatory } \\
\text { Germany }\end{array}$ & $\begin{array}{l}\text { Mean difference }(95 \% \text { Cl) } \\
\text { PLWD function: Perceive, Recall, Plan and Perform } \\
\text { System of Task Analysis (PRPP)- independence } \\
\text { subscale } \\
26 \text { weeks }\end{array}$ & $3.3(-8.3 ; 14.9)$ & NR & $\mathrm{NR}$ & NS \\
\hline $\begin{array}{l}\text { Voight-Radloff } 2011^{46}(22021760) \\
\text { Community Occupational Therapy in Dementia Program vs. usual care (one session home } \\
\text { consultation) } \\
\text { Medium } 26-52 \text { weeks } \\
\text { Explanatory } \\
\text { Germany }\end{array}$ & $\begin{array}{l}\text { Mean difference }(95 \% \mathrm{Cl}) \\
\text { PLWD QOL: Dementia Quality of Life Instrument - } \\
\text { overall } \\
26 \text { weeks }\end{array}$ & $0.2(-0.1 ; 0.5)$ & NR & NR & NS \\
\hline $\begin{array}{l}\text { Voight-Radloff } 2011^{46}(22021760) \\
\text { Community Occupational Therapy in Dementia Program vs. usual care (one session home } \\
\text { consultation) } \\
\text { Medium } 26-52 \text { weeks } \\
\text { Explanatory } \\
\text { Germany }\end{array}$ & $\begin{array}{l}\text { Mean difference }(95 \% \mathrm{Cl}) \\
\text { PLWDD QOL: Short-Form } 12 \text { Health Survey } \\
\text { Questionnaire -Physical component } \\
26 \text { weeks }\end{array}$ & $3.0(-1.6 ; 7.6)$ & NR & $\mathrm{NR}$ & NS \\
\hline $\begin{array}{l}\text { Voight-Radloff } 2011^{46}(22021760) \\
\text { Community Occupational Therapy in Dementia Program vs. usual care (one session home } \\
\text { consultation) } \\
\text { Medium } 26-52 \text { weeks } \\
\text { Explanatory } \\
\text { Germany }\end{array}$ & $\begin{array}{l}\text { Mean difference }(95 \% \mathrm{Cl}) \\
\text { PLWDD QOL: Short-Form } 12 \text { Health Survey } \\
\text { Questionnaire - mental component } \\
26 \text { weeks }\end{array}$ & $-0.8(-4.9 ; 3.3)$ & NR & NR & NS \\
\hline $\begin{array}{l}\text { Voight-Radloff } 2011^{46}(22021760) \\
\text { Community Occupational Therapy in Dementia Program vs. usual care (one session home } \\
\text { consultation) } \\
\text { Medium } 26-52 \text { weeks } \\
\text { Explanatory } \\
\text { Germany }\end{array}$ & $\begin{array}{l}\text { N } \\
\text { PLWD harms: number of adverse events } \\
52 \text { weeks }\end{array}$ & $\mathrm{NR}$ & 0 & 0 & NS \\
\hline $\begin{array}{l}\text { Voight-Radloff } 2011^{46}(22021760) \\
\text { Community Occupational Therapy in Dementia Program vs. usual care (one session home } \\
\text { consultation) } \\
\text { Medium } 26-52 \text { weeks } \\
\text { Explanatory } \\
\text { Germany }\end{array}$ & $\begin{array}{l}\text { N } \\
\text { PLWD nursing home: Resource Utilisation in Dementia, } \\
\text { nights in nursing home (except respite care) } \\
52 \text { weeks }\end{array}$ & NR & 2 & 1 & NS \\
\hline
\end{tabular}




\begin{tabular}{|c|c|c|c|c|c|}
\hline $\begin{array}{l}\text { Voight-Radloff } 2011^{46}(22021760) \\
\text { Community Occupational Therapy in Dementia Program vs. usual care (one session home } \\
\text { consultation) } \\
\text { Medium } 26-52 \text { weeks } \\
\text { Explanatory } \\
\text { Germany }\end{array}$ & $\begin{array}{l}\text { N } \\
\text { PLWD hospitalization: Resource Utilisation in Dementia, } \\
\text { nights in hospital } \\
52 \text { weeks }\end{array}$ & NR & \begin{tabular}{l|}
14 PLWD \\
were admitted to \\
hospital for an average \\
of 15 nights
\end{tabular} & $\begin{array}{l}10 \text { PLWD were admitted } \\
\text { to hospital for an average } \\
\text { of } \\
18 \text { nights. }\end{array}$ & 0.1 \\
\hline $\begin{array}{l}\text { Wang } 2011^{47}(21752121) \\
\text { Family Mutual Support Program in Dementia Care (FMSP-DC) vs. usual care } \\
\text { Medium } \\
\text { Explanatory } \\
\text { USA }\end{array}$ & $\begin{array}{l}\text { Mean (SD) baseline } \\
\text { Mean (SD) } 6 \text { months } \\
\text { PLWD cognition: MMSE (Chinese) } \\
6 \text { months }\end{array}$ & NR & $\begin{array}{l}17.5 \text { (4.7) baseline } \\
18.1 \text { (4.0) } 6 \text { months }\end{array}$ & $\begin{array}{l}17.3 \text { (3.9) baseline } \\
17.2(4.1) 6 \text { months }\end{array}$ & NS \\
\hline $\begin{array}{l}\text { Wang 201147 }(21752121) \\
\text { Family Mutual Support Program in Dementia Care (FMSP-DC) vs. usual care } \\
\text { Medium } \\
\text { Explanatory } \\
\text { USA }\end{array}$ & $\begin{array}{l}\text { Mean (SD) baseline } \\
\text { Mean (SD) } 6 \text { months } \\
\text { PLWD institutionalization: Number } \\
6 \text { months }\end{array}$ & NR & $\begin{array}{l}5.1 \text { (0.9) baseline } \\
3.8 \text { (1.0) } 6 \text { months }\end{array}$ & $\begin{array}{l}5.5(1.2) \text { baseline } \\
5.2(1.3) 6 \text { months }\end{array}$ & $<0.01$ \\
\hline $\begin{array}{l}\text { Wang } 2011^{47}(21752121) \\
\text { Family Mutual Support Program in Dementia Care (FMSP-DC) vs. usual care } \\
\text { Medium } \\
\text { Explanatory } \\
\text { USA }\end{array}$ & $\begin{array}{l}\text { Mean (SD) baseline } \\
\text { Mean (SD) } 6 \text { months } \\
\text { PLWD institutionalization: duration } \\
6 \text { months }\end{array}$ & $\mathrm{NR}$ & $\begin{array}{l}13.8(4.0) \text { baseline } \\
10.1 \text { (5.1) } 6 \text { months }\end{array}$ & $\begin{array}{l}14.2 \text { (3.8) baseline } \\
17.9(7.1) 6 \text { months }\end{array}$ & $<0.001$ \\
\hline $\begin{array}{l}\text { Gitlin } 2010^{49}(20662955) \\
\text { Advancing Caregiver Training (ACT) vs. no treatment control } \\
\text { Medium } \\
\text { Explanatory } \\
\text { USA }\end{array}$ & $\begin{array}{l}\text { Percentage reporting improvement in problem behavior } \\
\text { PLWD neuropsychiatric symptoms: number of problem } \\
\text { behaviors } \\
4 \text { months }\end{array}$ & NR & 67.5 & 45.8 & 0.002 \\
\hline $\begin{array}{l}\text { Martin-Carrasco } 2009^{54}(18949763) \\
\text { Psychoedcuational Intervention Program to teach strategies for confronting problems of PLWD } \\
\text { care vs. usual care } \\
\text { Medium } \\
\text { Explanatory } \\
\text { Spain }\end{array}$ & $\begin{array}{l}\text { Frequency (percent) } \\
\text { PLWD Caregiver healthcare and social resources use: } \\
\text { number of visits } \\
4 \text { months }\end{array}$ & NR & $\begin{array}{l}0 \text { visits: } 29 / 44(66 \%) \\
1-2 \text { visits: } 13 / 44(36 \%)\end{array}$ & $\begin{array}{l}0 \text { visits: } 25 / 38(66 \%) \\
1-2 \text { visits: } 13 / 38(36 \%)\end{array}$ & NR (NS) \\
\hline $\begin{array}{l}\text { Martin-Carrasco } 2009^{54}(18949763) \\
\text { Psychoedcuational Intervention Program to teach strategies for confronting problems of PLWD } \\
\text { care vs. usual care } \\
\text { Medium } \\
\text { Explanatory } \\
\text { Spain }\end{array}$ & $\begin{array}{l}\text { Mean (SD) } \\
\text { PLWD Caregiver healthcare and social resources use: } \\
\text { time spent on medical care } \\
4 \text { months }\end{array}$ & NR & $28.5(25.5)$ & $21.8(11.0)$ & NR \\
\hline $\begin{array}{l}\text { Graff } 2006^{60}(17114212) \\
\text { Community based occupational therapy program vs. Waitlist for occupational therapy } \\
\text { Medium } \\
\text { Explanatory }\end{array}$ & $\begin{array}{l}\text { Adjusted Mean difference }(95 \% \mathrm{Cl}) \\
\text { Effect size } \\
\text { Number needed to treat } \\
\text { PLWD function: Assessment of Motor and Process } \\
\text { Skills (AMPS) - process subscale } \\
6 \text { weeks }\end{array}$ & $\begin{array}{l}1.5(1.3 ; 1.7) \\
2.5 \\
1.3(1.2 \text { to } 1.4) \text { NNT }\end{array}$ & NR & NR & $<0.0001$ \\
\hline $\begin{array}{l}\text { Graff } 2000^{60}(17114212) \\
\text { Community based occupational therapy program vs. Waitlist for occupational therapy } \\
\text { Medium } \\
\text { Explanatory }\end{array}$ & $\begin{array}{l}\text { Adjusted Mean difference }(95 \% \text { Cl) } \\
\text { PLWD function: Interview of Deterioration in Daily } \\
\text { Activities in Dementia (IDDD) - performance subscale } \\
6 \text { weeks }\end{array}$ & \begin{tabular}{|l|}
$-11.7(-13.6 ;-9.7)$ \\
2.3 \\
$1.5(1.4$ to 1.6$) \mathrm{NNT}$
\end{tabular} & NR & NR & $<0.0001$ \\
\hline $\begin{array}{l}\text { Graff } 2006^{60}(17114212) \text { Graff } 2007^{89}(17895439) \\
\text { Community based occupational therapy program vs. Waitlist for occupational therapy } \\
\text { Medium } \\
\text { Explanatory }\end{array}$ & $\begin{array}{l}\text { Adjusted treatment difference }(95 \% \mathrm{Cl}) \\
\text { Effect size } \\
\text { Mean (SD) } \\
\text { PLWD quality of life: Dementia Quality of Life } \\
\text { Instrument } \\
6 \text { weeks }\end{array}$ & $\begin{array}{l}0.8 \text { (0.6 to } 1.1) \\
1.3 \text { (effect size) }\end{array}$ & $4.0(0.6)$ & $3.1(0.8)$ & $<0.0001$ \\
\hline $\begin{array}{l}\text { Graff } 2006^{60}(17114212) \\
\text { Community based occupational therapy program vs. Waitlist for occupational therapy } \\
\text { Medium } \\
\text { Explanatory }\end{array}$ & $\begin{array}{l}\text { Adjusted treatment difference }(95 \% \mathrm{Cl}) \\
\text { Effect size } \\
\text { Mean (SD) } \\
\text { PLWD depression: Cornell scale for depression } \\
6 \text { weeks }\end{array}$ & $\begin{array}{l}-2.8(-4.3 \text { to }-1.3) \\
0.7 \text { effect size }\end{array}$ & $6.5(5.3)$ & $9.2(6.4)$ & $<0.0001$ \\
\hline
\end{tabular}




\begin{tabular}{|c|c|c|c|c|c|}
\hline $\begin{array}{l}\text { Farran } 2004^{62}(41552352) \\
\text { Caregiver skill building (CSB) vs. Information and support group (ISO) } \\
\text { Medium } \\
\text { Explanatory } \\
\text { USA }\end{array}$ & $\begin{array}{l}\text { Regression coefficient (SE) } \\
\text { PLWD neuropsychiatric symptoms: Revised Memory } \\
\text { and Problem Behavior Checklist (RMPBC) } \\
18 \text { months }\end{array}$ & $-0.046(0.035)$ & NR & $\mathrm{NR}$ & 0.193 \\
\hline $\begin{array}{l}\text { Farran } 2004^{62}(41552352) \\
\text { Caregiver skill building (CSB) vs. Information and support group (ISO) } \\
\text { Medium } \\
\text { Explanatory } \\
\text { USA }\end{array}$ & $\begin{array}{l}\text { Log rank from lifetable } \\
\text { PLWD Time to institutionalization } \\
18 \text { months }\end{array}$ & $0.11, \mathrm{df}=1$ & NR & NR & $p>0.75$ \\
\hline $\begin{array}{l}\text { Gitlin } 2001^{93}(11220813) \\
\text { Home Environmental Intervention vs. usual care } \\
\text { Medium } \\
\text { Explanatory } \\
\text { USA } \\
\end{array}$ & $\begin{array}{l}\text { Adjusted mean difference }(95 \% \mathrm{Cl}) \\
\text { Mean (SD) baseline } \\
\text { Mean (SD) } 3 \text { month } \\
\text { PLWD function ADL: Functional Independence Measure } \\
\text { (modified) ADL subscale } \\
3 \text { months }\end{array}$ & $-0.06(-0.30$ to 0.18$)$ & $\begin{array}{l}2.93(1.49) \text { baseline } \\
3.24(1.59) 3 \text { months }\end{array}$ & $\begin{array}{l}3.23 \text { (1.36) baseline } \\
3.57 \text { (1.38) } 3 \text { months }\end{array}$ & 0.599 \\
\hline $\begin{array}{l}\text { Gitlin } 20011^{93}(11220813) \\
\text { Home Environmental Intervention vs. usual care } \\
\text { Medium } \\
\text { Explanatory } \\
\text { USA }\end{array}$ & $\begin{array}{l}\text { Adjusted mean difference }(95 \% \mathrm{Cl}) \\
\text { Mean (SD) baseline } \\
\text { Mean (SD) } 3 \text { month } \\
\text { PLWD function IADL: Functional Independence } \\
\text { Measure (modified) IADL subscale } \\
3 \text { months }\end{array}$ & $-0.13(-0.24$ to -0.01$)$ & $\begin{array}{l}5.43 \text { (.62) baseline } \\
5.54(.60) 3 \text { months }\end{array}$ & $\begin{array}{l}5.56 \text { (.50) baseline } \\
5.75 \text { (.36) } 3 \text { months }\end{array}$ & 0.030 \\
\hline $\begin{array}{l}\text { Gitlin } 2001^{93}(11220813) \\
\text { Home Environmental Intervention vs. usual care } \\
\text { Medium } \\
\text { Explanatory } \\
\text { USA }\end{array}$ & $\begin{array}{l}\text { Adjusted mean difference }(95 \% \mathrm{Cl}) \\
\text { Mean (SD) baseline } \\
\text { Mean (SD) } 3 \text { month } \\
\text { PLWD neuropsychiatric symptoms: Memory and } \\
\text { Behavior Problems Checklist (MBPC) } \\
3 \text { months }\end{array}$ & $1.85(-0.42$ to 4.13$)$ & $\begin{array}{l}20.25 \text { (5.39) baseline } \\
17.20(7.73) 3 \text { months }\end{array}$ & $\begin{array}{l}18.74(6.31) \\
\text { baseline14.43 (9.82) } 3 \\
\text { months }\end{array}$ & 0.110 \\
\hline
\end{tabular}

Abbreviations: ADL= Activities of Daily Living; CI= Confidence Interval; IADL= Instrumental Activities of Daily Living; MMSE= Mini-Mental State Examination; NR=Not Reported; PMID=PubMed Identification Number; QOL-AD= the Quality of Life in Alzheimer's; SD= Standard Deviation; $\mathrm{SE}=$ Standard Error

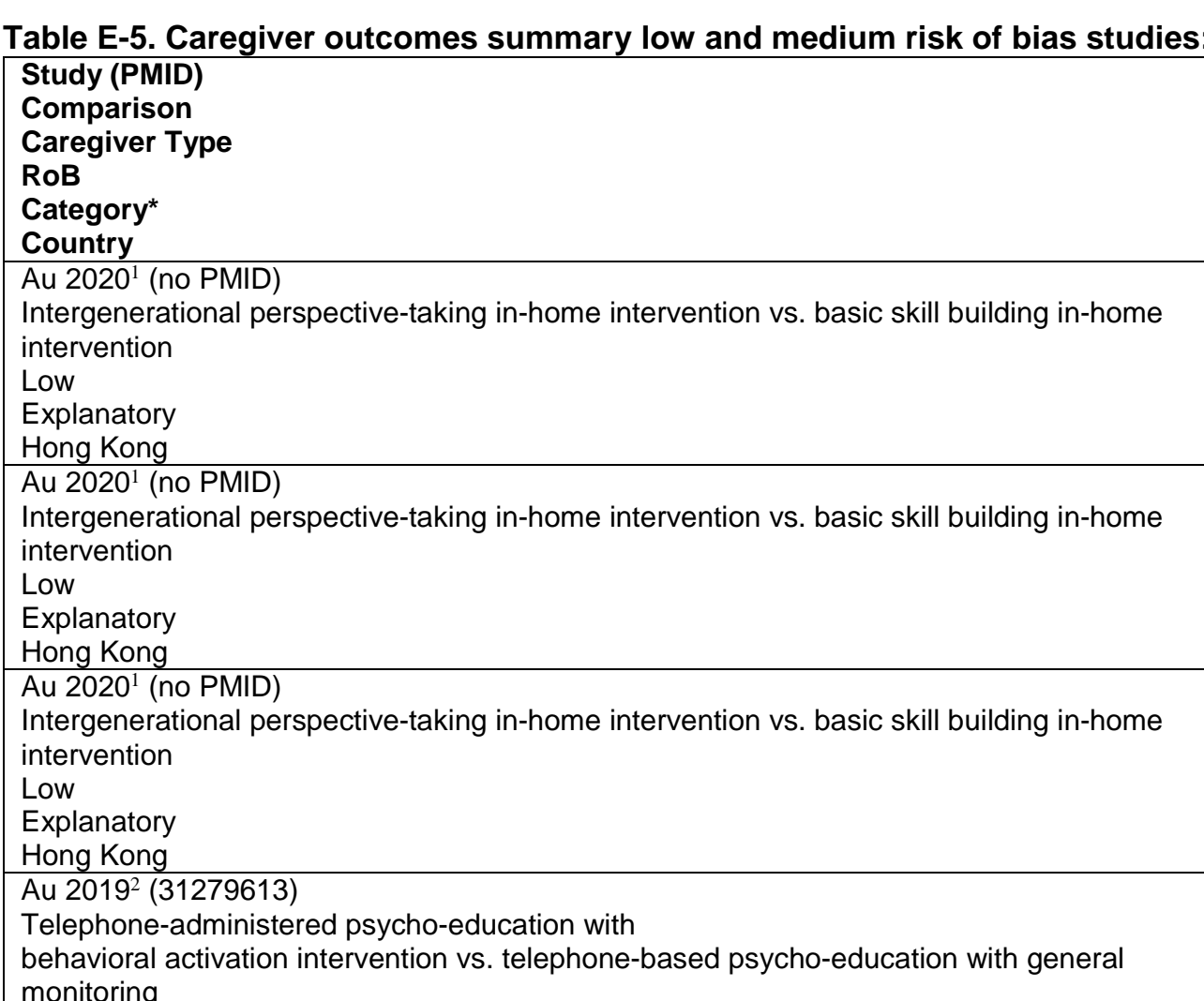

\begin{tabular}{l} 
psychosocial interventions for caregiver well-being \\
Outcome \\
Timing \\
$\begin{array}{l}\text { Mean (SD) } \\
\text { Caregiver life satisfaction: Satisfaction With Life Scale } \\
8 \text { weeks }\end{array}$ \\
$\begin{array}{l}\text { Mean (SD) } \\
\text { Caregiver depression: Center for Epidemiological Studies- } \\
\text { Depression (CESD) } \\
8 \text { weeks }\end{array}$ \\
$\begin{array}{l}\text { Mean (SD) } \\
\text { Caregiver burden: Zarit Burden Interview } \\
8 \text { weeks }\end{array}$ \\
\hline $\begin{array}{l}\text { Cohen's d (95\% Cl) } \\
\text { Mean (SD) } \\
\text { Caregiver depression: Center for Epidemiological Studies- }\end{array}$ \\
\hline
\end{tabular}

\begin{tabular}{|l|l|}
\hline Summary Finding & Intervention \\
\hline NR & \\
& $\begin{array}{l}17.20(3.79) \text { baseline } \\
23.46(5.90) 8 \text { weeks }\end{array}$ \\
\hline NR & \\
& $\begin{array}{l}15.97(4.02) \text { baseline } \\
10.76(4.34) 8 \text { weeks }\end{array}$ \\
\hline NR & \\
& $23.32(7.49)$ baseline \\
& $16.30(7.14) 8$ weeks \\
& \\
\hline$-0.66(-1.04,-0.74)$ & $\begin{array}{l}13.29(7.63) \text { baseline } \\
8.62(8.53) 20 \\
\text { months }\end{array}$ \\
&
\end{tabular}

\begin{tabular}{|l|l|}
\hline Comparator & $p$-Value \\
\hline $\begin{array}{l}18.09(4.98) \text { baseline } \\
19.66(5.62) 8 \text { weeks }\end{array}$ & $p<0.001$ \\
\hline $15.02(3.53)$ baseline & $p<0.001$ \\
$13.34(3.76) 8$ weeks & \\
\hline $22.83(6.66)$ baseline & $p<0.001$ \\
$21.14(6.14) 8$ weeks & \\
\hline $13.92(9.05)$ baseline & $p<0.001$ \\
$15.55(11.35) 20$ months & \\
&
\end{tabular}




\begin{tabular}{|c|c|c|c|c|c|}
\hline $\begin{array}{l}\text { Study (PMID) } \\
\text { Comparison } \\
\text { Caregiver Type } \\
\text { RoB } \\
\text { Category* } \\
\text { Country }\end{array}$ & \begin{tabular}{|l} 
Outcome \\
Timing
\end{tabular} & Summary Finding & Intervention & Comparator & $p$-Value \\
\hline $\begin{array}{l}\text { Low } \\
\text { Explanatory } \\
\text { Hong Kong }\end{array}$ & $\begin{array}{l}\text { Depression (CESD) } \\
20 \text { weeks }\end{array}$ & & & & \\
\hline $\begin{array}{l}\text { Au } 2019^{2}(31279613) \\
\text { Telephone-administered psycho-education with } \\
\text { behavioral activation intervention vs. telephone-based psycho-education with general } \\
\text { monitoring } \\
\text { Low } \\
\text { Explanatory } \\
\text { Hong Kong }\end{array}$ & $\begin{array}{l}\text { Cohen's d }(95 \% \mathrm{Cl}) \\
\text { Mean (SD) } \\
\text { Caregiver burden: Zarit Burden Interview } \\
20 \text { weeks }\end{array}$ & $-1.14(-1.54 \cdot-0.75)$ & $\begin{array}{l}32.26(17.43) \\
\text { baseline } \\
21.82(14.06) 20 \\
\text { months }\end{array}$ & $\begin{array}{l}29.47 \text { (17.06) baseline } \\
37.47 \text { (17.28) } 20 \text { months }\end{array}$ & $p<0.001$ \\
\hline $\begin{array}{l}\text { Au } 2019^{2}(31279613) \\
\text { Telephone-administered psycho-education with } \\
\text { behavioral activation intervention vs. telephone-based psycho-education with general } \\
\text { monitoring } \\
\text { Low } \\
\text { Explanatory } \\
\text { Hong Kong }\end{array}$ & $\begin{array}{l}\text { Cohen's d }(95 \% \mathrm{Cl}) \\
\text { Mean (SD) } \\
\text { Caregiver satisfaction with relationship with care recipient: } \\
\text { Relationship Assessment Scale (RAS) } \\
20 \text { weeks }\end{array}$ & $0.44(0.82,0.07)$ & $\begin{array}{l}27.78(5.90) \text { baseline } \\
30.05(4.79) 20 \\
\text { months }\end{array}$ & \begin{tabular}{|l|}
$27.41(5.86)$ baseline \\
$27.60(6.08) 20$ months
\end{tabular} & $p=0.01$ \\
\hline $\begin{array}{l}\text { Au } 2019^{2}(31279613) \\
\text { Telephone-administered psycho-education with } \\
\text { behavioral activation intervention vs. telephone-based psycho-education with general } \\
\text { monitoring } \\
\text { Low } \\
\text { Explanatory } \\
\text { Hong Kong }\end{array}$ & $\begin{array}{l}\text { Cohen's d }(95 \% \mathrm{Cl}) \\
\text { Mean (SD) } \\
\text { Caregiver : self-efficacy for controlling upsetting thoughts } 5 \\
\text { item } \\
20 \text { weeks }\end{array}$ & $0.86(1.24,0.46)$ & $\begin{array}{l}36.08(8.03) \text { baseline } \\
40.15(9.02) 20 \\
\text { months }\end{array}$ & \begin{tabular}{|l|}
$33.58(10.03)$ baseline \\
$34.05(10.07) 20$ months
\end{tabular} & $p<0.01$ \\
\hline $\begin{array}{l}\text { Kunik } 2020^{3}(32115311) \\
\text { Unmet Needs Psychosocial vs education and information support } \\
\text { Low } \\
\text { Explanatory } \\
\text { United States }\end{array}$ & $\begin{array}{l}\text { Cohen's d (95\% Cl) } \\
\text { Mean (SD) } \\
3 \text { months }\end{array}$ & $-6.9(2.32,0.78)$ & NR & $\mathrm{NR}$ & $p=0.27$ \\
\hline $\begin{array}{l}\text { Bjorge } 2019^{4} \text { (31651321) } \\
\text { Psychosocial intervention vs. usual care } \\
\text { Medium } \\
\text { Explanatory } \\
\text { Norway }\end{array}$ & $\begin{array}{l}\text { Between-group differences as estimated regression } \\
\text { coefficient (95\% CI) } \\
\text { Caregiver stress: Relatives' Stress Scale (RSS) } \\
12 \text { months }\end{array}$ & $1.12(-1.23$ to 3.48$)$ & NR & NR & 0.35 \\
\hline $\begin{array}{l}\text { Bjorge } 2019^{4}(31651321) \\
\text { Psychosocial intervention vs. usual care } \\
\text { Medium } \\
\text { Explanatory } \\
\text { Norway }\end{array}$ & $\begin{array}{l}\text { Between-group differences as estimated regression } \\
\text { coefficient }(95 \% \mathrm{Cl}) \\
\text { Caregiver depression: Geriatric Depression Scale (GDS) } \\
12 \text { months }\end{array}$ & 1.70 (0.41 to 3.0$)$ & NR & $\mathrm{NR}$ & 0.01 \\
\hline $\begin{array}{l}\text { Bjorge } 2019^{4}(31651321) \\
\text { Psychosocial intervention vs. usual care } \\
\text { Medium } \\
\text { Explanatory } \\
\text { Norway }\end{array}$ & $\begin{array}{l}\text { Between-group differences as estimated regression } \\
\text { coefficient (95\% Cl) } \\
\text { Caregiver emotions: Felt Expressed Emotion Rating Scale } \\
\text { (FEERS) } \\
12 \text { months }\end{array}$ & $0.36(-0.62$ to 1.34$)$ & NR & NR & 0.47 \\
\hline $\begin{array}{l}\text { Ghaffari } 2019^{5} \text { (no PMID) } \\
\text { Resilience education group classes vs. usual care } \\
\text { Medium } \\
\text { Explanatory } \\
\text { Iran }\end{array}$ & $\begin{array}{l}\text { Mean difference (SD) } \\
\text { Quality of life: General Health Questionnaire } 28 \text { item (GHQ- } \\
\text { 28) } \\
\text { 8 weeks }\end{array}$ & & $\begin{array}{l}39.9(8.4) \text { baseline } \\
16.08 \text { (3.7) } 8 \text { weeks }\end{array}$ & $\begin{array}{l}32.0(7.0) \text { baseline } \\
32.9(7.2) 8 \text { weeks }\end{array}$ & $p<0.001$ \\
\hline $\begin{array}{l}\text { Meng } 2019^{6}(30884961) \\
\text { Telephone based cognitive behavioral therapy vs. in-person cognitive behavioral therapy } \\
\text { Medium } \\
\text { Explanatory } \\
\text { USA }\end{array}$ & $\begin{array}{l}\text { Mean (SD) } \\
\text { Caregiver health service use: physician office visits } \\
3 \text { months }\end{array}$ & NR & \begin{tabular}{|l|}
3.6 (2.9) baseline \\
2.9 (2.6) 3 months
\end{tabular} & \begin{tabular}{|l|}
2.4 (3.1) baseline \\
$3.6(4.3) 3$ months
\end{tabular} & NS \\
\hline
\end{tabular}




\begin{tabular}{|c|c|c|c|c|c|}
\hline $\begin{array}{l}\text { Study (PMID) } \\
\text { Comparison } \\
\text { Caregiver Type } \\
\text { RoB } \\
\text { Category* } \\
\text { Country }\end{array}$ & \begin{tabular}{|l|} 
Outcome \\
Timing
\end{tabular} & Summary Finding & Intervention & Comparator & p-Value \\
\hline $\begin{array}{l}\text { Meng } 2019^{6}(30884961) \\
\text { Telephone based cognitive behavioral therapy vs. in-person cognitive behavioral therapy } \\
\text { Medium } \\
\text { Explanatory } \\
\text { USA }\end{array}$ & $\begin{array}{l}\text { Mean (SD) } \\
\text { Caregiver health service use: mental health visits } \\
3 \text { months }\end{array}$ & NR & $\begin{array}{l}1.7 \text { (0.6) baseline } \\
2(0) 3 \text { months }\end{array}$ & $\begin{array}{l}0.1 \text { (0.3) baseline } \\
1.5 \text { (.7) } 3 \text { months }\end{array}$ & NS \\
\hline $\begin{array}{l}\text { Meng } 2019^{6}(30884961) \\
\text { Telephone based cognitive behavioral therapy vs. in-person cognitive behavioral therapy } \\
\text { Medium } \\
\text { Explanatory } \\
\text { USA }\end{array}$ & $\begin{array}{l}\text { Mean (SD) } \\
\text { Caregiver health service use: prescription medication } \\
3 \text { months }\end{array}$ & NR & $\begin{array}{l}3.5(2.2) \text { baseline } \\
3.5(2.5) 3 \text { months }\end{array}$ & $\begin{array}{l}3.5 \text { (3.1) baseline } \\
4.1(2.6) 3 \text { months }\end{array}$ & NS \\
\hline $\begin{array}{l}\text { Meng } 2019^{6}(30884961) \\
\text { Telephone based cognitive behavioral therapy vs. in-person cognitive behavioral therapy } \\
\text { Medium } \\
\text { Explanatory } \\
\text { USA }\end{array}$ & \begin{tabular}{|l|} 
Mean (SD) \\
Caregiver health service use: ER visits \\
3 months
\end{tabular} & NR & $\begin{array}{l}1.8(1.2) \text { baseline } \\
1.6(0.9) 3 \text { months }\end{array}$ & $\begin{array}{l}0.1 \text { (0.5) baseline } \\
1.2(0.4) 3 \text { months }\end{array}$ & NS \\
\hline $\begin{array}{l}\text { Meng } 2019^{6}(30884961) \\
\text { Telephone based cognitive behavioral therapy vs. in-person cognitive behavioral therapy } \\
\text { Medium } \\
\text { Explanatory } \\
\text { USA }\end{array}$ & $\begin{array}{l}\text { Mean (SD) } \\
\text { Caregiver health service use: hospital days } \\
3 \text { months }\end{array}$ & NR & $\begin{array}{l}1.4 \text { (0.9) baseline } \\
3 \text { (1.4) } 3 \text { months }\end{array}$ & $\begin{array}{l}0.1 \text { (0.8) baseline } \\
3.3 \text { (1.5) } 3 \text { months }\end{array}$ & NS \\
\hline $\begin{array}{l}\text { Meng } 2019^{6}(30884961) \\
\text { Telephone based cognitive behavioral therapy vs. in-person cognitive behavioral therapy } \\
\text { Medium } \\
\text { Explanatory } \\
\text { USA }\end{array}$ & \begin{tabular}{|l|} 
Mean (SD) \\
Caregiver health service use: out of pocket expenditures in \\
dollars \\
3 months
\end{tabular} & NR & $\begin{array}{l}\text { 104.9 (94.4) baseline } \\
216.3(504.3) 3 \\
\text { months }\end{array}$ & $\begin{array}{l}41 \text { (72.7) baseline } \\
191.7 \text { (228.8) } 3 \text { months }\end{array}$ & NS \\
\hline $\begin{array}{l}\text { Meng } 2019^{6}(30884961) \\
\text { Telephone based cognitive behavioral therapy vs. in-person cognitive behavioral therapy } \\
\text { Medium } \\
\text { Explanatory } \\
\text { USA }\end{array}$ & $\begin{array}{l}\text { Mean (SD) } \\
\text { Caregiver health service use: total monthly expenditures in } \\
\text { dollars } \\
3 \text { months }\end{array}$ & NR & $\begin{array}{l}1019.9(1412.1) \\
\text { baseline } \\
883.2(749.5) 3 \\
\text { months }\end{array}$ & $\begin{array}{l}844 \text { (1851) baseline } \\
768.9 \text { (661.2) } 3 \text { months }\end{array}$ & NS \\
\hline $\begin{array}{l}\text { Moskowitz } 2019^{7}(31045422) \\
\text { Life Enhancing Activities for Family Caregivers (LEAF) vs. waitlist control } \\
\text { Medium } \\
\text { Explanatory } \\
\text { USA }\end{array}$ & $\begin{array}{l}\text { Cohen's d }(95 \% \mathrm{Cl}) \\
\text { Mean (SE) } \\
\text { Caregiver depression: PROMIS depressive symptoms scale } \\
6 \text { weeks }\end{array}$ & $-.25(-.55, .06)$ & $\begin{array}{l}65.85(2.02) \text { baseline } \\
55.18(2.08) 6 \text { weeks }\end{array}$ & $\begin{array}{l}65.62(2.04) \text { baseline } \\
59.65(2.15) 6 \text { weeks }\end{array}$ & Interaction 0.02 \\
\hline $\begin{array}{l}\text { Moskowitz } 2019^{7}(31045422) \\
\text { Life Enhancing Activities for Family Caregivers (LEAF) vs. waitlist control } \\
\text { Medium } \\
\text { Explanatory } \\
\text { USA }\end{array}$ & $\begin{array}{l}\text { Cohen's d }(95 \% \mathrm{Cl}) \\
\text { Mean (SE) } \\
\text { Caregiver mental health: PROMIS mental health scale } \\
6 \text { weeks }\end{array}$ & $0.21[-.09, .52]$ & $\begin{array}{l}37.4(4.10) \text { baseline } \\
39.8(4.10) 6 \text { weeks }\end{array}$ & $\begin{array}{l}39.8(4.10) \text { baseline } \\
39.8(4.10) 6 \text { weeks }\end{array}$ & Interaction 0.49 \\
\hline $\begin{array}{l}\text { Moskowitz } 2019^{7}(31045422) \\
\text { Life Enhancing Activities for Family Caregivers (LEAF) vs. waitlist control } \\
\text { Medium } \\
\text { Explanatory } \\
\text { USA }\end{array}$ & $\begin{array}{l}\text { Cohen's d }(95 \% \mathrm{Cl}) \\
\text { Mean (SE) } \\
\text { Caregiver physical health: PROMIS physical health scale } \\
6 \text { weeks }\end{array}$ & $0.24[-.07, .54]$ & $\begin{array}{l}\text { 42.3 (4.20) baseline } \\
44.9(4.30) 6 \text { weeks }\end{array}$ & $\begin{array}{l}44.9(4.30) \text { baseline } \\
44.9(4.30) 6 \text { weeks }\end{array}$ & Interaction 0.03 \\
\hline $\begin{array}{l}\text { Moskowitz } 2019^{7}(31045422) \\
\text { Life Enhancing Activities for Family Caregivers (LEAF) vs. waitlist control } \\
\text { Medium } \\
\text { Explanatory } \\
\text { USA }\end{array}$ & $\begin{array}{l}\text { Cohen's } d(95 \% \mathrm{Cl}) \\
\text { Mean ( } \mathrm{SE}) \\
\text { Caregiver anxiety: Quality of Life in Neurological Disorders } \\
\text { (NeuroQOL) anxiety measure } \\
6 \text { weeks } \\
\end{array}$ & $-0.32[-.63,-.02]$ & $\begin{array}{l}52.15(1.63) \text { baseline } \\
44.64(1.68) 6 \text { weeks }\end{array}$ & $\begin{array}{l}51.38(1.65) \text { baseline } \\
48.91(1.76) 6 \text { weeks }\end{array}$ & Interaction .009 \\
\hline $\begin{array}{l}\text { Moskowitz } 2019^{7}(31045422) \\
\text { Life Enhancing Activities for Family Caregivers (LEAF) vs. waitlist control } \\
\text { Medium } \\
\text { Explanatory } \\
\text { USA }\end{array}$ & $\begin{array}{l}\text { Cohen's d }(95 \% \mathrm{Cl}) \\
\text { Mean (SE) } \\
\text { Caregiver burden: Zarit Burden Interview } \\
6 \text { weeks }\end{array}$ & $-0.16[-.46, .14]$ & $\begin{array}{l}42.19(1.58) \text { baseline } \\
38.18(1.60) 6 \text { weeks }\end{array}$ & $\begin{array}{l}42.18(1.59) \text { baseline } \\
41.13(1.65) 6 \text { weeks }\end{array}$ & Interaction .066 \\
\hline
\end{tabular}




\begin{tabular}{|c|c|c|c|c|c|}
\hline $\begin{array}{l}\text { Study (PMID) } \\
\text { Comparison } \\
\text { Caregiver Type } \\
\text { RoB } \\
\text { Category* } \\
\text { Country }\end{array}$ & $\begin{array}{l}\text { Outcome } \\
\text { Timing }\end{array}$ & \begin{tabular}{|l|} 
Summary Finding \\
\end{tabular} & Intervention & Comparator & $p$-Value \\
\hline $\begin{array}{l}\text { Moskowitz } 2019^{7}(31045422) \\
\text { Life Enhancing Activities for Family Caregivers (LEAF) vs. waitlist control } \\
\text { Medium } \\
\text { Explanatory } \\
\text { USA }\end{array}$ & $\begin{array}{l}\text { Cohen's d }(95 \% \mathrm{Cl}) \\
\text { Mean (SE) } \\
\text { Caregiver strain: Caregiver Strain Index (CSI) } \\
6 \text { weeks }\end{array}$ & $.01[-.30, .30]$ & $\begin{array}{l}8.40(.33) \text { baseline } \\
8.46(.33) 6 \text { weeks }\end{array}$ & $\begin{array}{l}8.061(.35) \text { baseline } \\
8.55(.33) 6 \text { weeks }\end{array}$ & Interaction 0.74 \\
\hline $\begin{array}{l}\text { Moskowitz } 2019^{7}(31045422) \\
\text { Life Enhancing Activities for Family Caregivers (LEAF) vs. waitlist control } \\
\text { Medium } \\
\text { Explanatory } \\
\text { USA }\end{array}$ & $\begin{array}{l}\text { Cohen's d }(95 \% \mathrm{Cl}) \\
\text { Mean (SE) } \\
\text { Caregiver positive emotion: Differential Emotions Scale (DES) } \\
6 \text { weeks }\end{array}$ & $0.58[.27, .88]$ & $\begin{array}{l}4.64(.16) \text { baseline } \\
4.86(.16) 6 \text { weeks }\end{array}$ & $\begin{array}{l}4.92(.17) \text { baseline } \\
4.31(.17) 6 \text { weeks }\end{array}$ & Interaction $<.001$ \\
\hline $\begin{array}{l}\text { Moskowitz } 2019^{7} \text { (31045422) } \\
\text { Life Enhancing Activities for Family Caregivers (LEAF) vs. waitlist control } \\
\text { Medium } \\
\text { Explanatory } \\
\text { USA }\end{array}$ & $\begin{array}{l}\text { Cohen's d }(95 \% \mathrm{Cl}) \\
\text { Mean (SE) } \\
\text { Caregiver negative emotion: Differential Emotions Scale } \\
\text { (DES) } \\
6 \text { weeks }\end{array}$ & $-0.22[-.52, .08]$ & $\begin{array}{l}3.66(.13) \text { baseline } \\
2.6(.13) 6 \text { weeks }\end{array}$ & $\begin{array}{l}3.70(.13) \text { baseline } \\
2.91(.14) 6 \text { weeks }\end{array}$ & Interaction 0.13 \\
\hline $\begin{array}{l}\text { Moskowitz } 2019^{7}(31045422) \\
\text { Life Enhancing Activities for Family Caregivers (LEAF) vs. waitlist control } \\
\text { Medium } \\
\text { Explanatory } \\
\text { USA }\end{array}$ & $\begin{array}{l}\text { Cohen's d ( } 95 \% \mathrm{Cl}) \\
\text { Mean (SE) } \\
\text { Caregiver positive aspects of caregiving: the Positive Aspects } \\
\text { of Caregiving Scale } \\
6 \text { weeks }\end{array}$ & $0.35[.05, .66]$ & $\begin{array}{l}35.92(1.09) \text { baseline } \\
39.04(1.12) 6 \text { weeks }\end{array}$ & $\begin{array}{l}26.88 \text { (1.11) baseline } \\
36.41(1.16) 6 \text { weeks }\end{array}$ & Interaction 0.001 \\
\hline $\begin{array}{l}\text { Moskowitz } 2019^{7}(31045422) \\
\text { Life Enhancing Activities for Family Caregivers (LEAF) vs. waitlist control } \\
\text { Medium } \\
\text { Explanatory } \\
\text { USA }\end{array}$ & $\begin{array}{l}\text { Cohen's d }(95 \% \mathrm{Cl}) \\
\text { Mean (SE) } \\
\text { Caregiver perceived stress: The Perceived Stress Scale } \\
6 \text { weeks }\end{array}$ & $-0.20[-.50, .11]$ & $\begin{array}{l}30.64(.71) \text { baseline } \\
27.48(.72) 6 \text { weeks }\end{array}$ & $\begin{array}{l}31.08(.73) \text { baseline } \\
29.24(.76) 6 \text { weeks }\end{array}$ & Interaction 0.10 \\
\hline $\begin{array}{l}\text { Nordheim } 2019^{8}(30775982) \\
\text { couple-based interdisciplinary psychosocial intervention vs. usual care } \\
\text { Medium } \\
\text { Explanatory } \\
\text { Germany }\end{array}$ & $\begin{array}{l}\text { Estimated marginal mean (SE) baseline } \\
\text { Estimated marginal mean ( (E) } 6 \text { months } \\
\text { Caregiver quality of life: World Health Organization Quality of } \\
\text { Life questionnaire (WHOQOL-BREF) short form Global QOL } \\
\text { subscale } \\
6 \text { month }\end{array}$ & Cohens $d=-0.2$ & $\begin{array}{l}60.9 \text { (2.4) baseline } \\
53.2(2.4) 6 \text { months }\end{array}$ & $\begin{array}{l}58.5 \text { (2.4) baseline } \\
54.5 \text { (2.6) } 6 \text { months }\end{array}$ & $\begin{array}{l}\text { NS } \\
(p \text {-value NR) }\end{array}$ \\
\hline $\begin{array}{l}\text { Nordheim } 2019^{8}(30775982) \\
\text { couple-based interdisciplinary psychosocial intervention vs. usual care. } \\
\text { Medium } \\
\text { Explanatory } \\
\text { Germany }\end{array}$ & $\begin{array}{l}\text { Estimated marginal mean (SE) baseline } \\
\text { Estimated marginal mean (SE) } 6 \text { months } \\
\text { Caregiver confidence: Sense of Competence Questionnaire } \\
\text { (SCQ) personal life subscale } \\
6 \text { months }\end{array}$ & Cohens $d=-0.5$ & $\begin{array}{l}16.9 \text { (1.1) baseline } \\
19.9(1.0) 6 \text { months }\end{array}$ & $\begin{array}{l}17.4 \text { (1.1) baseline } \\
16.5 \text { (1.1) } 6 \text { months }\end{array}$ & $\begin{array}{l}\text { NS } \\
(p \text {-value NR) }\end{array}$ \\
\hline $\begin{array}{l}\text { Nordheim } 2019^{8}(30775982) \\
\text { couple-based interdisciplinary psychosocial intervention vs. usual care } \\
\text { Medium } \\
\text { Explanatory } \\
\text { Germany }\end{array}$ & $\begin{array}{l}\text { Estimated marginal mean (SE) baseline } \\
\text { Estimated marginal mean (SE) } 6 \text { months } \\
\text { Caregiver confidence: Sense of Competence Questionnaire } \\
\text { (SCQ) performance as a caregiver subscale } \\
6 \text { months }\end{array}$ & Cohens $d=-0.4$ & $\begin{array}{l}17.2(1.0) \text { baseline } \\
20.9(0.9) 6 \text { months }\end{array}$ & $\begin{array}{l}18.0 \text { (1.0) baseline } \\
20.0(1.0) 6 \text { months }\end{array}$ & $\begin{array}{l}\text { NS } \\
\text { (p-value NR) }\end{array}$ \\
\hline $\begin{array}{l}\text { Nordheim } 2019^{8} \text { (30775982) } \\
\text { couple-based interdisciplinary psychosocial intervention vs. usual care } \\
\text { Medium } \\
\text { Explanatory } \\
\text { Germany }\end{array}$ & $\begin{array}{l}\text { Estimated marginal mean (SE) baseline } \\
\text { Estimated marginal mean (SE) } 6 \text { months } \\
\text { Caregiver confidence: Sense of Competence Questionnaire } \\
\text { (SCQ) satisfaction with PLWD subscale } \\
6 \text { months }\end{array}$ & Cohens $d=-0.5$ & $\begin{array}{l}8.9(0.7) \text { baseline } \\
10.4(0.7) 6 \text { months }\end{array}$ & \begin{tabular}{|l|}
$10.1(0.7)$ baseline \\
$9.7(0.7) 6$ months
\end{tabular} & $\begin{array}{l}\text { NS } \\
\text { (p-value NR) }\end{array}$ \\
\hline $\begin{array}{l}\text { Nordheim } 2019^{8}(30775982) \\
\text { couple-based interdisciplinary psychosocial intervention vs. usual care } \\
\text { Medium } \\
\text { Explanatory } \\
\text { Germany }\end{array}$ & $\begin{array}{l}\text { Estimated marginal mean (SE) baseline } \\
\text { Estimated marginal mean (SE) } 6 \text { months } \\
\text { Caregiver social support: (F-SOZU) } \\
6 \text { months }\end{array}$ & Cohens $\mathrm{d}=-0.1$ & $\begin{array}{l}53.3 \text { (1.1) baseline } \\
50.5 \text { (1.3) } 6 \text { months }\end{array}$ & \begin{tabular}{|l|}
$53.2(1.4)$ baseline \\
53.5 (1.4) 6 months
\end{tabular} & $\begin{array}{l}\text { NS } \\
\text { (p-value NR) }\end{array}$ \\
\hline $\begin{array}{l}\text { Nordheim } 2019^{8}(30775982) \\
\text { couple-based interdisciplinary psychosocial intervention vs. usual care Medium } \\
\text { Explanatory }\end{array}$ & $\begin{array}{l}\text { Estimated marginal mean (SE) baseline } \\
\text { Estimated marginal mean (SE) } 6 \text { months } \\
\text { Caregiver perceived stress }\end{array}$ & Cohens $\mathrm{d}=0.1$ & $\begin{array}{l}25.5(1.0) \text { baseline } \\
27.3(1.2) 6 \text { months }\end{array}$ & $\begin{array}{l}27.2 \text { (1.) baseline } \\
26.9 \text { (1.2) } 6 \text { months }\end{array}$ & $\begin{array}{l}\text { NS } \\
\text { (p-value NR) }\end{array}$ \\
\hline
\end{tabular}




\begin{tabular}{|c|c|c|c|c|c|}
\hline $\begin{array}{l}\text { Study (PMID) } \\
\text { Comparison } \\
\text { Caregiver Type } \\
\text { RoB } \\
\text { Category* } \\
\text { Country }\end{array}$ & $\begin{array}{l}\text { Outcome } \\
\text { Timing }\end{array}$ & Summary Finding & Intervention & Comparator & $p$-Value \\
\hline $\begin{array}{l}\text { Nordheim } 2019^{8}(30775982) \\
\text { couple-based interdisciplinary psychosocial intervention vs. usual care } \\
\text { Medium } \\
\text { Explanatory } \\
\text { Germany }\end{array}$ & $\begin{array}{l}\text { Estimated marginal mean (SE) baseline } \\
\text { Estimated marginal mean (SE) } 6 \text { months } \\
\text { Caregiver depressive symptoms: Geriatric depression scale } \\
\text { (GDS) } 6 \text { months }\end{array}$ & Cohens $d=0.0$ & $\begin{array}{l}4.9(0.3) \text { baseline } \\
4.8(0.3) 6 \text { months }\end{array}$ & $\begin{array}{l}4.5(0.3) \text { baseline } \\
5.0(0.3) 6 \text { months }\end{array}$ & $\begin{array}{l}N S \\
\text { (p-value NR) }\end{array}$ \\
\hline $\begin{array}{l}\text { Gittin } 2018^{13}(29192967) \\
\text { Home-based Tailored Activity Program vs. attention control using telephone visits } \\
\text { Medium } \\
\text { Explanatory } \\
\text { USA }\end{array}$ & $\begin{array}{l}\text { Mean difference }(95 \% \mathrm{Cl}) \\
\text { Caregiver depressive symptoms: Centers for Epidemiologic } \\
\text { Study Depression Scale (CES-D) } \\
4 \text { months }\end{array}$ & $-0.59(-1.74$ to 0.55$)$ & NR & NR & 0.31 \\
\hline $\begin{array}{l}\text { Gitlin } 2018^{13}(29192967) \\
\text { Home-based Tailored Activity Program vs. attention control using telephone visits } \\
\text { Medium } \\
\text { Explanatory } \\
\text { USA }\end{array}$ & $\begin{array}{l}\text { Mean difference }(95 \% \mathrm{Cl}) \\
\text { Caregiver burden: Zarit Burden Short Form (12 item) } \\
4 \text { months }\end{array}$ & $-0.39(-2.06$ to 1.29$) .65$ & $\mathrm{NR}$ & NR & 0.65 \\
\hline $\begin{array}{l}\text { Gitlin } 2018^{13}(29192967) \\
\text { Home-based Tailored Activity Program vs. attention control using telephone visits } \\
\text { Medium } \\
\text { Explanatory } \\
\text { USA }\end{array}$ & $\begin{array}{l}\text { Mean difference }(95 \% \mathrm{Cl}) \\
\text { Caregiver distress: Neuropsychiatric Inventory - Clinician } \\
\text { (NPI-C) distress subscale } \\
4 \text { months }\end{array}$ & $-0.07(-0.14$ to -0.01$)$ & NR & NR & 0.03 \\
\hline $\begin{array}{l}\text { Gitlin } 2018^{13}(29192967) \\
\text { Home-based Tailored Activity Program vs. attention control using telephone visits } \\
\text { Medium } \\
\text { Explanatory } \\
\text { USA }\end{array}$ & $\begin{array}{l}\text { Mean difference }(95 \% \mathrm{Cl}) \\
\text { Caregiver time spent caregiving: hours on ADLS } \\
4 \text { months }\end{array}$ & $-0.53(-1.20$ to 0.14$)$ & $\mathrm{NR}$ & $\mathrm{NR}$ & 0.12 \\
\hline $\begin{array}{l}\text { Gitlin } 2018^{13}(29192967) \\
\text { Home-based Tailored Activity Program vs. attention control using telephone visits } \\
\text { Medium } \\
\text { Explanatory } \\
\text { USA }\end{array}$ & $\begin{array}{l}\text { Mean difference }(95 \% \mathrm{Cl}) \\
\text { Caregiver time spent caregiving: hours on IADLs } \\
4 \text { months }\end{array}$ & $-1.09(-2.34$ to 0.15$)$ & NR & $\mathrm{NR}$ & 0.08 \\
\hline $\begin{array}{l}\text { Gitlin } 2018^{13}(29192967) \\
\text { Home-based Tailored Activity Program vs. attention control using telephone visits } \\
\text { Medium } \\
\text { Explanatory } \\
\text { USA }\end{array}$ & $\begin{array}{l}\text { Mean difference }(95 \% \mathrm{Cl}) \\
\text { Caregiver time spent caregiving: hours on duty } \\
4 \text { months }\end{array}$ & $-0.28(-2.25$ to 1.68$)$ & NR & NR & 0.78 \\
\hline $\begin{array}{l}\text { Gitlin } 2018^{13,20}(29192967) \\
\text { Mome-based Tailored Activity Program vs. attention control using telephone visits } \\
\text { Medium } \\
\text { Explanatory } \\
\text { USA }\end{array}$ & $\begin{array}{l}\text { Mean difference }(95 \% \mathrm{Cl}) \\
\text { Caregiver time spent caregiving: hours on doing things } \\
4 \text { months }\end{array}$ & $-0.99(-2.28$ to 0.30$)$ & NR & $\mathrm{NR}$ & 0.13 \\
\hline $\begin{array}{l}\text { Tremont } 2017^{84}(28008609) \text { companion to Tremont } 2015^{28}(25074341) \\
\text { Telephone Tracking-Caregiver (FITT-C) vs. Telephone Support (TS) } \\
\text { Medium Explanatory } \\
\text { ExA }\end{array}$ & $\begin{array}{l}\text { Mean (SD) } \\
\text { Caregiver health service use: Number of monthly emergency } \\
\text { department visits during intervention } \\
6 \text { months }\end{array}$ & NR & $0.02(0.06)$ & $0.05(0.13)$ & 0.02 \\
\hline $\begin{array}{l}\text { Tremont } 2017^{84}(28008609) \text { companion to Tremont } 2015^{28}(25074341) \\
\text { Telephone Tracking-Caregiver (FITT-C) vs. Telephone Support (TS) } \\
\text { Medium Explanatory } \\
\text { USA }\end{array}$ & $\begin{array}{l}\text { Mean (SD) } \\
\text { Caregiver health service use: Number of monthly hospital } \\
\text { visits during intervention } \\
6 \text { months }\end{array}$ & NR & $0.01(0.04)$ & $0.04(0.10)$ & 0.006 \\
\hline $\begin{array}{l}\text { Tremont } 2017^{84}(28008609) \text { companion to Tremont } 2015^{28}(25074341) \\
\text { Telephone Tracking-Caregiver (FITT-C) vs. Telephone Support (TS) } \\
\text { Medium Explanatory } \\
\text { USA }\end{array}$ & $\begin{array}{l}\text { Mean (SD) } \\
\text { Caregiver health service use: number of monthly doctor visits } \\
\text { during intervention } \\
6 \text { months }\end{array}$ & NR & $0.85(0.89)$ & $0.71(0.61)$ & 0.88 \\
\hline
\end{tabular}




\begin{tabular}{|c|c|c|c|c|c|}
\hline $\begin{array}{l}\text { Study (PMID) } \\
\text { Comparison } \\
\text { Caregiver Type } \\
\text { RoB } \\
\text { Category* } \\
\text { Country }\end{array}$ & \begin{tabular}{|l|} 
Outcome \\
Timing
\end{tabular} & Summary Finding & Intervention & Comparator & $p$-Value \\
\hline $\begin{array}{l}\text { Tremont } 2017^{84}(28008609) \text { companion to Tremont } 2015^{28}(25074341) \\
\text { Telephone Tracking-Caregiver (FITT-C) vs. Telephone Support (TS) } \\
\text { Medium Explanatory } \\
\text { ExpA }\end{array}$ & $\begin{array}{l}\text { Absolute risk reduction }(95 \% \mathrm{Cl}) \\
\text { Caregiver hospital admissions } \\
6 \text { months }\end{array}$ & $11.4 \%(0.023$ to 0.25$)$ & $\mathrm{NR}$ & NR & 0.01 \\
\hline $\begin{array}{l}\text { Tremont } 2017^{84}(28008609) \text { companion to Tremont } 2015^{28}(25074341) \\
\text { Telephone Tracking-Caregiver (FITT-C) vs. Telephone Support (TS) } \\
\text { Medium } \\
\text { Explanatory } \\
\text { USA }\end{array}$ & $\begin{array}{l}\text { Absolute risk reduction }(95 \% \mathrm{Cl}) \\
\text { Caregiver emergency department visits } \\
6 \text { months }\end{array}$ & $9.5 \%$ (0.01 to 0.19$)$ & $\mathrm{NR}$ & NR & 0.048 \\
\hline 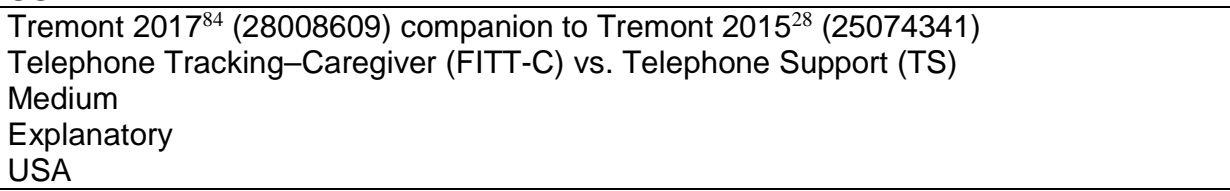 & \begin{tabular}{|l|} 
Mean (SD) \\
Caregiver community support use: Number of community \\
support services used in final month of intervention \\
6 months
\end{tabular} & $\mathrm{NR}$ & $8.47(5.14)$ & \begin{tabular}{|l|}
$5.14(7.05)$ \\
\end{tabular} & 0.02 \\
\hline 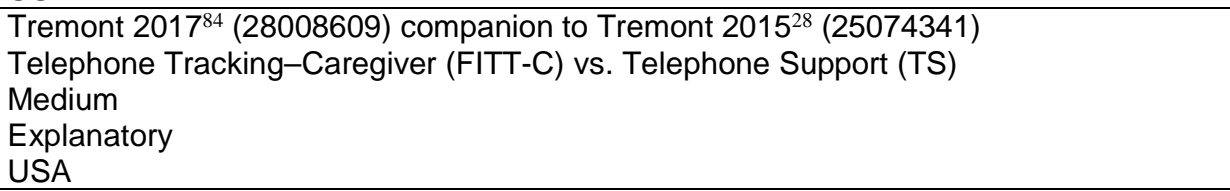 & $\begin{array}{l}\text { Mean (SD) } \\
\text { Caregiver out of pocket cost on formal care for PLWD for final } \\
\text { month of intervention in dollars } \\
6 \text { months }\end{array}$ & $\$ 274.43(901.68)$ & $\mathrm{NR}$ & NR & $\begin{array}{l}0.73 \text { (no difference } \\
\text { between groups) }\end{array}$ \\
\hline $\begin{array}{l}\text { Charlesworth } 2016^{18}(27521377) \\
3 \text { groups: 1) One--o-one peer support to family carers from experienced carers (Carer } \\
\text { Supporter Programme (CSP), 2) group reminiscence therapy } \\
\text { Remembering Yesterday, Caring Today (RYCT), \& 3) usual care } \\
\text { Low } \\
\text { Explanatory } \\
\text { UK }\end{array}$ & $\begin{array}{l}\text { Adjusted mean difference (95\% CI) for CSP vs other } 2 \text { groups } \\
\text { Caregiver Health related Quality of life: SF-12 mental } \\
\text { component } \\
12 \text { months }\end{array}$ & 0.52 (-1.28 to 2.32$)$ & NR & NR & 0.57 \\
\hline $\begin{array}{l}\text { Charlesworth } 2016^{18}(27521377) \\
3 \text { groups: 1) One-to-one peer support to family carers from experienced carers (Carer } \\
\text { Supporter Programme (CSP), 2) group reminiscence therapy Remembering Yesterday, } \\
\text { Caring Today (RYCT), \& 3) usual care } \\
\text { Low } \\
\text { Explanatory } \\
\text { UK }\end{array}$ & $\begin{array}{l}\text { Adjusted mean difference }(95 \% \text { Cl) for RYCT vs other } 2 \\
\text { groups } \\
\text { Caregiver Health related Quality of life: SF-12 mental } \\
\text { component } \\
12 \text { months }\end{array}$ & $0.10(-1.72$ to 1.93$)$ & $\mathrm{NR}$ & NR & 0.91 \\
\hline $\begin{array}{l}\text { Charlesworth } 2016^{18}(27521377) \\
3 \text { groups: 1) One-to-one peer support to family carers from experienced carers (Carer } \\
\text { Supporter Programme (CSP), 2) group reminiscence therapy Remembering Yesterday, } \\
\text { Caring Today (RYCT), \& 3) usual care } \\
\text { Low } \\
\text { Explanatory } \\
\text { UK }\end{array}$ & $\begin{array}{l}\text { Adjusted mean difference }(95 \% \text { CI) for CSP vs other } 2 \text { groups } \\
\text { Caregiver Health related Quality of life: SF-12 physical } \\
\text { component } \\
12 \text { months }\end{array}$ & $0.613(-1.23$ to 2.46$)$ & NR & NR & 0.52 \\
\hline $\begin{array}{l}\text { Charlesworth } 2016^{18}(27521377) \\
3 \text { groups: 1) One-to-one peer support to family carers from experienced carers (Carer } \\
\text { Supporter Programme ( (SP), 2) group reminiscence therapy Remembering Yesterday, } \\
\text { Caring Today (RYCT), \& 3) usual care } \\
\text { Low } \\
\text { Explanatory } \\
\text { UK }\end{array}$ & $\begin{array}{l}\text { Adjusted mean difference }(95 \% \mathrm{Cl}) \text { for RYCT vs other } 2 \\
\text { groups } \\
\text { Caregiver Health related Quality of life: } \mathrm{SF}-12 \text { physical } \\
\text { component } \\
12 \text { months }\end{array}$ & $-0.428(-2.29$ to 1.43$)$ & $\mathrm{NR}$ & NR & 0.65 \\
\hline $\begin{array}{l}\text { Charlesworth } 2016^{18}(27521377) \\
3 \text { groups: 1) One-to-one peer support to family carers from experienced carers (Carer } \\
\text { Supporter Programme (CSP), 2) group reminiscence therapy Remembering Yesterday, } \\
\text { Caring Today (RYCT), \& 3) usual care } \\
\text { Low } \\
\text { Explanatory } \\
\text { UK }\end{array}$ & $\begin{array}{l}\text { Adjusted mean difference }(95 \% \text { CI) for CSP vs other } 2 \text { groups } \\
\text { Caregiver anxiety: Hospital Anxiety and Depression Scale } \\
\text { (HADS) anxiety subscale } \\
12 \text { months }\end{array}$ & $-0.33(-1.31$ to 0.60$)$ & $\mathrm{NR}$ & NR & 0.47 \\
\hline
\end{tabular}




\begin{tabular}{|c|c|c|c|c|c|}
\hline $\begin{array}{l}\text { Study (PMID) } \\
\text { Comparison } \\
\text { Caregiver Type } \\
\text { RoB } \\
\text { Category* } \\
\text { Country }\end{array}$ & \begin{tabular}{|l|} 
Outcome \\
Timing
\end{tabular} & \begin{tabular}{|l|} 
Summary Finding \\
\end{tabular} & Intervention & Comparator & p-Value \\
\hline $\begin{array}{l}\text { Charlesworth 201618 (27521377) } \\
3 \text { groups: 1) One-to-one peer support to family carers from experienced carers (Carer } \\
\text { Supporter Programme (CSP), 2) group reminiscence therapy Remembering Yesterday, } \\
\text { Caring Today (RYCT), \& 3) usual care } \\
\text { Low } \\
\text { Explanatory } \\
\text { UK }\end{array}$ & $\begin{array}{l}\text { Adjusted mean difference }(95 \% \mathrm{Cl}) \text { for RYCT vs other } 2 \\
\text { groups } \\
\text { Caregiver depression: Hospital Anxiety and Depression Scale } \\
\text { (HADS) anxiety subscale } \\
12 \text { months }\end{array}$ & $0.298(-0.65$ to 1.25$)$ & NR & NR & 0.54 \\
\hline $\begin{array}{l}\text { Charlesworth } 2016^{18}(27521377) \\
3 \text { groups: } 1 \text { ) One-to-one peer support to family carers from experienced carers (Carer } \\
\text { Supporter Programme (CSP), 2) group reminiscence therapy Remembering Yesterday, } \\
\text { Caring Today (RYCT), \& 3) usual care } \\
\text { Low } \\
\text { Explanatory } \\
\text { UK }\end{array}$ & $\begin{array}{l}\text { Adjusted mean difference }(95 \% \text { Cl) for CSP vs other } 2 \text { groups } \\
\text { Caregiver depression: Hospital Anxiety and Depression } \\
\text { Scale (HADS) depression subscale } \\
12 \text { months }\end{array}$ & 0.0144 (-0.85 to 0.88$)$ & NR & NR & 0.97 \\
\hline $\begin{array}{l}\text { Charlesworth } 2016^{18}(27521377) \\
3 \text { groups: 1) One-to-one peer support to family carers from experienced carers (Carer } \\
\text { Supporter Programme (CSP), 2) group reminiscence therapy Remembering Yesterday, } \\
\text { Caring Today (RYCT), \& 3) usual care } \\
\text { Low } \\
\text { Explanatory } \\
\text { UK }\end{array}$ & $\begin{array}{l}\text { Adjusted mean difference }(95 \% \mathrm{Cl}) \text { for RYCT vs other } 2 \\
\text { groups } \\
\text { Caregiver depression: Hospital Anxiety and Depression Scale } \\
\text { (HADS) depression subscale } \\
12 \text { months }\end{array}$ & $-0.0700(-0.95$ to 0.81$)$ & NR & NR & 0.88 \\
\hline $\begin{array}{l}\text { Charlesworth } 2016^{18}(27521377) \\
3 \text { groups: 1) One-to-one peer support to family carers from experienced carers (Carer } \\
\text { Supporter Programme (CSP), 2) group reminiscence therapy Remembering Yesterday, } \\
\text { Caring Today (RYCT), \& 3) usual care } \\
\text { Low } \\
\text { Explanatory } \\
\text { UK }\end{array}$ & $\begin{array}{l}\text { Adjusted mean difference }(95 \% \text { CI) for CSP vs other } 2 \text { groups } \\
\text { Caregiver Health-related quality of life: EQ-5D global health } \\
\text { visual analog scale } \\
12 \text { months }\end{array}$ & $0.384(-4.20$ to 4.96$)$ & NR & NR & 0.87 \\
\hline $\begin{array}{l}\text { Charlesworth } 2016^{18}(27521377) \\
3 \text { groups: 1) One-to-one peer support to family carers from experienced carers (Carer } \\
\text { Supporter Programme (CSP), 2) group reminiscence therapy Remembering Yesterday, } \\
\text { Caring Today (RYCT), \& 3) usual care } \\
\text { Low } \\
\text { Explanatory } \\
\text { UK }\end{array}$ & $\begin{array}{l}\text { Adjusted mean difference }(95 \% \mathrm{Cl}) \text { for RYCT vs other } 2 \\
\text { groups } \\
\text { Caregiver Health-related quality of life: } \mathrm{EQ}-5 \mathrm{D} \text { global health } \\
\text { visual analog scale } \\
12 \text { months }\end{array}$ & $0.915(-3.65$ to 5.48$)$ & NR & NR & 0.69 \\
\hline $\begin{array}{l}\text { Charlesworth } 2016^{18}(27521377) \\
3 \text { groups: 1) One-to-one peer support to family carers from experienced carers (Carer } \\
\text { Supporter Programme (CSP), 2) group reminiscence therapy Remembering Yesterday, } \\
\text { Caring Today (RYCT), \& 3) usual care } \\
\text { Low } \\
\text { Explanatory } \\
\text { UK }\end{array}$ & $\begin{array}{l}\text { Adjusted mean difference }(95 \% \text { CI) for CSP vs other } 2 \text { groups } \\
\text { Caregiver Positive Aspects of Caring: Carers of Older People } \\
\text { in Europe Index (COPE index) positive aspects subscale } \\
12 \text { months }\end{array}$ & $0.0125(-0.54$ to 0.56$)$ & NR & NR & 0.96 \\
\hline $\begin{array}{l}\text { Charlesworth } 2016^{18}(27521377) \\
3 \text { groups: 1) One-to-one peer support to family carers from experienced carers (Carer } \\
\text { Supporter Programme (CSP), 2) group reminiscence therapy Remembering Yesterday, } \\
\text { Caring Today (RYCT), \& 3) usual care } \\
\text { Low } \\
\text { Explanatory } \\
\text { UK }\end{array}$ & $\begin{array}{l}\text { Adjusted mean difference }(95 \% \mathrm{Cl}) \text { for RYCT vs other } 2 \\
\text { groups } \\
\text { Caregiver Positive Aspects of Caring: Carers of Older People } \\
\text { in Europe Index (COPE index) positive aspects subscale } \\
12 \text { months }\end{array}$ & $0.0784(-0.45$ to 0.61$)$ & NR & NR & 0.77 \\
\hline $\begin{array}{l}\text { Charlesworth } 2016^{18}(27521377) \\
3 \text { groups: } 1 \text { ) One-to-one peer support to family carers from experienced carers (Carer } \\
\text { Supporter Programme (CSP), 2) group remiliscence therapy Remembering Yesterday, } \\
\text { Caring Today (RYCT), \& 3) usual care } \\
\text { Low } \\
\text { Explanatory } \\
\text { UK }\end{array}$ & $\begin{array}{l}\text { Adjusted mean difference }(95 \% \text { Cl) for CSP over other } 2 \\
\text { groups } \\
\text { Caregiver Quality of Caregiver-PLWD Relationship (QCPR) } \\
12 \text { months }\end{array}$ & $1.416(-0.47$ to 3.30$)$ & NR & NR & 0.14 \\
\hline
\end{tabular}




\begin{tabular}{|c|c|c|c|c|c|}
\hline $\begin{array}{l}\text { Study (PMID) } \\
\text { Comparison } \\
\text { Caregiver Type } \\
\text { RoB } \\
\text { Category* } \\
\text { Country }\end{array}$ & $\begin{array}{l}\text { Outcome } \\
\text { Timing }\end{array}$ & Summary Finding & Intervention & Comparator & p-Value \\
\hline $\begin{array}{l}\text { Charlesworth 201618 }(27521377) \\
3 \text { groups: } 1 \text { ) One-to-one peer support to family carers from experienced carers (Carer } \\
\text { Supporter Programme (CSP), 2) group reminiscence therapy Remembering Yesterday, } \\
\text { Caring Today (RYCT), \& 3) usual care } \\
\text { Low } \\
\text { Explanatory } \\
\text { UK }\end{array}$ & $\begin{array}{l}\text { Adjusted mean difference }(95 \% \mathrm{Cl}) \text { for RYCT over other } 2 \\
\text { groups } \\
\text { Caregiver Quality of Caregiver-PLWD Relationship (QCPR) } \\
12 \text { months }\end{array}$ & $1.869(-0.02$ to 3.75$)$ & NR & NR & 0.05 \\
\hline $\begin{array}{l}\text { Cheng } 2016^{19}(27401052) \\
\text { Benefit finding intervention (BF) vs. simplified psychoeducation (SIM-PE) vs. standard } \\
\text { psychoeducation (STD-PE) } \\
\text { Medium } \\
\text { Explanatory } \\
\text { Hong Kong }\end{array}$ & $\begin{array}{l}\text { d effect size } \\
\text { Adjusted mean (SD) } \\
\text { Caregiver depressive symptoms: Hamilton Depression Rating } \\
\text { Scale } \\
8 \text { weeks }\end{array}$ & $d=-0.77$ & $\begin{array}{l}2.441(3.499) \\
B F\end{array}$ & $\begin{array}{l}5.137(3.476) \\
\text { SIM-PE }\end{array}$ & $\begin{array}{l}\text { Regression } \\
\mathrm{p}<0.001\end{array}$ \\
\hline $\begin{array}{l}\text { Cheng } 2016^{19}(27401052) \\
\text { Benefit finding intervention (BF) vs. simplified psychoeducation (SIM-PE) vs. standard } \\
\text { psychoeducation (STD-PE) } \\
\text { Medium } \\
\text { Explanatory } \\
\text { Hong Kong }\end{array}$ & $\begin{array}{l}\text { d effect size } \\
\text { Adjusted mean (SD) } \\
\text { Caregiver burden: Zarit Caregiver Burden Interview (ZBI) } \\
8 \text { weeks }\end{array}$ & $d=-0.47$ & $\begin{array}{l}27.133(11.704) \\
\mathrm{BF}\end{array}$ & $\begin{array}{l}32.544 \text { (11.431) } \\
\text { SIM-PE }\end{array}$ & $\begin{array}{l}\text { Regression } \\
p=0.028\end{array}$ \\
\hline $\begin{array}{l}\text { Cheng } 2016{ }^{19}(27401052) \\
\text { Benefit finding intervention (BF) vs. simplified psychoeducation (SIM-PE) vs. standard } \\
\text { psychoeducation (STD-PE) } \\
\text { Medium } \\
\text { Explanatory } \\
\text { Hong Kong }\end{array}$ & $\begin{array}{l}\text { d effect size } \\
\text { Adjusted mean (SD) } \\
\text { Caregiver role overload: } \\
8 \text { weeks }\end{array}$ & $d=-0.43$ & $\begin{array}{l}9.650(2.424) \\
\text { BF }\end{array}$ & $\begin{array}{l}10.705(2.478) \\
\text { SIM-PE }\end{array}$ & $\begin{array}{l}\text { Regression } \\
p=0.046\end{array}$ \\
\hline $\begin{array}{l}\text { Cheng } 2016^{19}(27401052) \\
\text { Benefit finding intervention (BF) vs. simplified psychoeducation (SIM-PE) vs. standard } \\
\text { psychoeducation (STD-PE) } \\
\text { Medium } \\
\text { Explanatory } \\
\text { Hong Kong }\end{array}$ & $\begin{array}{l}\text { e effect size } \\
\text { Adjusted mean (SD) } \\
\text { Caregiver psychological wellbeing: Ryff's Psychological Well- } \\
\text { being Scale } \\
8 \text { weeks }\end{array}$ & $d=0.42$ & $\begin{array}{l}66.652(6.753) \\
\mathrm{BF}\end{array}$ & $\begin{array}{l}63.867(6.559) \\
\text { SIM-PE }\end{array}$ & $\begin{array}{l}\text { Regression } \\
p=0.048\end{array}$ \\
\hline $\begin{array}{l}\text { Cheng } 2016^{19}(27401052) \\
\text { Benefit finding intervention (BF) vs. simplified psychoeducation (SIM-PE) vs. standard } \\
\text { psychoeducation (STD-PE) } \\
\text { Medium } \\
\text { Explanatory } \\
\text { Hong Kong }\end{array}$ & $\begin{array}{l}\text { effect size } \\
\text { Adjusted mean (SD) } \\
\text { Caregiver depressive symptoms: Hamilton Depression Rating } \\
\text { Scale } \\
8 \text { weeks }\end{array}$ & $d=-0.96$ & $\begin{array}{l}2.441(3.499) \\
B F\end{array}$ & $\begin{array}{l}5.833(3.432) \\
\text { STD-PE }\end{array}$ & $\begin{array}{l}\text { Regression } \\
p<0.001\end{array}$ \\
\hline $\begin{array}{l}\text { Cheng } 2016^{19}(27401052) \\
\text { Benefit finding intervention (BF) vs. simplified psychoeducation (SIM-PE) vs. standard } \\
\text { psychoeducation (STD-PE) } \\
\text { Medium } \\
\text { Explanatory } \\
\text { Hong Kong }\end{array}$ & $\begin{array}{l}\text { d effect size } \\
\text { Adjusted mean (SD) } \\
\text { Caregiver burden: Zarit Caregiver Burden Interview (ZBI) } \\
8 \text { weeks }\end{array}$ & $d=-0.65$ & $\begin{array}{l}27.133(11.704) \\
\mathrm{BF}\end{array}$ & $\begin{array}{l}34.749(11.886) \\
\text { STD-PE }\end{array}$ & $\begin{array}{l}\text { Regression } \\
p=0.003\end{array}$ \\
\hline $\begin{array}{l}\text { Cheng } 2016^{19}(27401052) \\
\text { Benefit finding intervention (BF) vs. simplified psychoeducation (SIM-PE) vs. standard } \\
\text { psychoeducation (STD-PE) } \\
\text { Medium } \\
\text { Explanatory } \\
\text { Hong Kong }\end{array}$ & $\begin{array}{l}\text { d effect size } \\
\text { Adjusted mean (SD) } \\
\text { Caregiver role overload: } \\
8 \text { weeks }\end{array}$ & $d=N R$ & $\begin{array}{l}9.650(2.424) \\
\text { BF }\end{array}$ & $\begin{array}{l}10.243(2.387) \\
\text { STD-PE }\end{array}$ & $\begin{array}{l}\text { Regression } \\
\mathrm{p}=0.234\end{array}$ \\
\hline $\begin{array}{l}\text { Cheng } 2016^{19}(27401052) \\
\text { Benefit finding intervention (BF) vs. simplified psychoeducation (SIM-PE) vs. standard } \\
\text { psychoeducation (STD-PE) } \\
\text { Medium } \\
\text { Explanatory } \\
\text { Hong Kong }\end{array}$ & $\begin{array}{l}\text { d effect size } \\
\text { Adjusted mean (SD) } \\
\text { Caregiver psychological wellbeing: Ryff's Psychological Well- } \\
\text { being Scale } \\
8 \text { weeks }\end{array}$ & $d=N R$ & $\begin{array}{l}66.652(6.753) \\
\mathrm{BF}\end{array}$ & $\begin{array}{l}\text { 65.027 (6.894) } \\
\text { STD-PE }\end{array}$ & $\begin{array}{l}\text { Regression } \\
p=0.264\end{array}$ \\
\hline
\end{tabular}




\begin{tabular}{|c|c|c|c|c|c|}
\hline $\begin{array}{l}\text { Study (PMID) } \\
\text { Comparison } \\
\text { Caregiver Type } \\
\text { RoB } \\
\text { Category* } \\
\text { Country }\end{array}$ & $\begin{array}{l}\text { Outcome } \\
\text { Timing }\end{array}$ & \begin{tabular}{|l|} 
Summary Finding \\
\end{tabular} & Intervention & Comparator & p-Value \\
\hline $\begin{array}{l}\text { Gonyea } 2016^{20}(24855313) \\
\text { Circulo de Cuidado, a culturally-sensitive, cognitive behavioral (CBT) group intervention vs. } \\
\text { psychoeducational (PED) control condition } \\
\text { Medium } \\
\text { Explanatory } \\
\text { USA }\end{array}$ & $\begin{array}{l}\text { Mean (SD) } \\
\text { Caregiver distress of PLWD neuropsychiatric symptoms: } \\
\text { Spanish version of the Neuropsychiatric Inventory-Distress } \\
\text { (NPI-D) } \\
12 \text { week } \\
24 \text { week }\end{array}$ & NR & $\begin{array}{l}17.94(6.84) \text { baseline } \\
16.64(7.02) 12 \\
\text { weeks } 12 \\
16.39(7.02) 24 \\
\text { weeks }\end{array}$ & \begin{tabular}{|l}
$17.91(7.30)$ baseline \\
$17.85(7.26) 12$ weeks \\
$18.26(7.37) 24$ weeks
\end{tabular} & $\begin{array}{l}\text { Repeated } \\
\text { measures } \\
\text { ANCOVA } \\
<0.001\end{array}$ \\
\hline $\begin{array}{l}\text { Gonyea } 2016^{20}(24855313) \\
\text { Circulo de Cuidado, a culturally-sensitive, cognitive behavioral (CBT) group intervention vs. } \\
\text { psychoeducational (PED) control condition } \\
\text { Medium } \\
\text { Explanatory } \\
\text { USA }\end{array}$ & $\begin{array}{l}\text { Mean (SD) } \\
\text { Caregiver self-efficacy: Spanish version of the Revised Scale } \\
\text { for Caregiving Self-Efficacy (RSCSE) } \\
12 \text { week } \\
24 \text { week }\end{array}$ & NR & $\begin{array}{l}69.11(12.75) \\
\text { baseline } \\
70.36(12.44) 12 \\
\text { weeks } \\
70.27(12.43) 24 \\
\text { weeks }\end{array}$ & \begin{tabular}{|l}
$69.19(9.01)$ baseline \\
$69.29(8.80) 12$ weeks \\
$69.15(8.88) 24$ weeks
\end{tabular} & $\begin{array}{l}\text { Repeated } \\
\text { measures } \\
\text { ANCOVA } \\
<0.001\end{array}$ \\
\hline $\begin{array}{l}\text { Gonyea } 2016^{20}(24855313) \\
\text { Circulo de Cuidado, a culturally-sensitive, cognitive behavioral (CBT) group intervention vs. } \\
\text { psychoeducational (PED) control condition } \\
\text { Medium } \\
\text { Explanatory } \\
\text { USA }\end{array}$ & $\begin{array}{l}\text { Mean (SD) } \\
\text { Caregiver depressive symptoms: Spanish version of the } \\
\text { Center for Epidemiological Studies- } \\
\text { Depression Scale (CES-D) } \\
12 \text { week } \\
24 \text { week }\end{array}$ & NR & $\begin{array}{l}14.39(6.14) \text { baseline } \\
13.79(5.85) 12 \\
\text { weeks } 12 \\
13.82(5.88) 24 \\
\text { weeks }\end{array}$ & \begin{tabular}{|l}
$14.41(6.31)$ baseline \\
$14.38(6.10) 12$ weeks \\
$14.44(6.07) 24$ weeks
\end{tabular} & $\begin{array}{l}\text { Repeated } \\
\text { measures } \\
\text { ANCOVA } \\
<0.01\end{array}$ \\
\hline $\begin{array}{l}\text { Gonyea } 2016^{20}(24855313) \\
\text { Circulo de Cuidado, a culturally-sensitive, cognitive behavioral (CBT) group intervention vs. } \\
\text { psychoeducational (PED) control condition } \\
\text { Medium } \\
\text { Explanatory } \\
\text { USA }\end{array}$ & $\begin{array}{l}\text { Mean (SD) } \\
\text { Caregiver anxiety: Spanish version of the State Anxiety } \\
\text { Inventory-State (STAl-S) } \\
12 \text { week } \\
24 \text { week }\end{array}$ & NR & $\begin{array}{l}37.09(10.25) \\
\text { baseline } \\
36.85(9.88) 12 \\
\text { weeks } 12 \\
36.96(10.00) 24 \\
\text { weeks }\end{array}$ & $\begin{array}{l}37.12(9.69) \text { baseline } \\
37.09(9.61) 12 \text { weeks } \\
37.12(9.68) 24 \text { weeks }\end{array}$ & $\begin{array}{l}\text { Repeated } \\
\text { measures } \\
\text { ANCOVA } \\
p>0.05 \text { NS }\end{array}$ \\
\hline $\begin{array}{l}\text { Laakkonen } 2016^{21}(27060101) \\
\text { Self-management groups vs. usual care Medium } \\
\text { Explanatory } \\
\text { Finland }\end{array}$ & $\begin{array}{l}\text { Adjusted Mean change }(95 \% \mathrm{Cl}) \\
\text { Caregiver quality of life: physical component SF-36 } \\
3 \text { months }\end{array}$ & $d=0.38$ & $1.0(-0.5$ to 2.5$)$ & $-2.0(-3.5$ to -0.5$)$ & .006 \\
\hline $\begin{array}{l}\text { Laakkonen 2016621 (27060101) } \\
\text { Self-management groups vs. usual care } \\
\text { Medium } \\
\text { Explanatory } \\
\text { Finland }\end{array}$ & $\begin{array}{l}\text { Adjusted Mean change }(95 \% \mathrm{Cl}) \\
\text { Caregiver quality of life: physical component SF-36 } \\
9 \text { months }\end{array}$ & $d=N R$ & $-0.0(-1.5$ to 1.4$)$ & $-1.7(-3.2$ to -0.2$)$ & 0.13 \\
\hline $\begin{array}{l}\text { Laakkonen } 2016^{21}(27060101) \\
\text { Self-management groups vs. usual care } \\
\text { Medium } \\
\text { Explanatory } \\
\text { Finland }\end{array}$ & $\begin{array}{l}\text { Adjusted Mean change }(95 \% \mathrm{Cl}) \\
\text { Caregiver quality of life: mental component SF-36 } \\
3 \text { months }\end{array}$ & $d=N R$ & NR & NR & 0.99 \\
\hline $\begin{array}{l}\text { Laakkonen } 2016^{21}(27060101) \\
\text { Self-management groups vs. usual care } \\
\text { Medium } \\
\text { Explanatory } \\
\text { Finland }\end{array}$ & $\begin{array}{l}\text { Adjusted Mean change }(95 \% \mathrm{Cl}) \\
\text { Caregiver quality of life: mental component SF-36 } \\
9 \text { months }\end{array}$ & $d=N R$ & NR & NR & 0.58 \\
\hline $\begin{array}{l}\text { Laakkonen } 2016^{21}(27060101) \\
\text { Self-management groups vs. usual care } \\
\text { Medium } \\
\text { Explanatory } \\
\text { Finland }\end{array}$ & $\begin{array}{l}\text { Adjusted Mean change }(95 \% \mathrm{Cl}) \\
\text { Caregiver competence: spousal Sense of Competence } \\
\text { Questionnaire (SCQ) } \\
3 \text { months }\end{array}$ & $d=N R$ & NR & NR & 0.76 \\
\hline $\begin{array}{l}\text { Laakkonen 201621 (27060101) } \\
\text { Self-management groups vs. usual care } \\
\text { Medium } \\
\text { Explanatory } \\
\text { Finland }\end{array}$ & $\begin{array}{l}\text { Adjusted Mean change }(95 \% \mathrm{Cl}) \\
\text { Caregiver competence: spousal Sense of Competence } \\
\text { Questionnaire (SCQ) } \\
9 \text { months }\end{array}$ & $d=N R$ & $\mathrm{NR}$ & NR & 0.38 \\
\hline
\end{tabular}




\begin{tabular}{|c|c|c|c|c|c|}
\hline $\begin{array}{l}\text { Study (PMID) } \\
\text { Comparison } \\
\text { Caregiver Type } \\
\text { RoB } \\
\text { Category* } \\
\text { Country }\end{array}$ & \begin{tabular}{|l|} 
Outcome \\
Timing
\end{tabular} & Summary Finding & Intervention & Comparator & $p$-Value \\
\hline $\begin{array}{l}\text { Laakkonen } 2016^{21}(27060101) \\
\text { Self-management groups vs. usual care } \\
\text { Medium } \\
\text { Explanatory } \\
\text { Finland }\end{array}$ & $\begin{array}{l}\text { Adjusted Mean change }(95 \% \mathrm{Cl}) \\
\text { Caregiver mastery Pearlin Mastery Scale (PMS) } \\
3 \text { months }\end{array}$ & $d=N R$ & NR & NR & 0.06 \\
\hline $\begin{array}{l}\text { Laakkonen } 2016^{21}(27060101) \\
\text { Self-management groups vs. usual care } \\
\text { Medium } \\
\text { Explanatory } \\
\text { Finland }\end{array}$ & $\begin{array}{l}\text { Adjusted Mean change }(95 \% \mathrm{Cl}) \\
\text { Caregiver mastery Pearlin Mastery Scale (PMS) } \\
9 \text { months }\end{array}$ & $d=N R$ & NR & NR & 0.11 \\
\hline $\begin{array}{l}\text { Laakkonen } 2016^{21}(27060101) \\
\text { Self-management groups vs. usual care Medium } \\
\text { Explanatory } \\
\text { Finland }\end{array}$ & $\begin{array}{l}\text { Adjusted Mean cost in } € \text { per year }(95 \% \mathrm{Cl}) \\
\text { Caregiver Health \& Social services cost: } \\
24 \text { months }\end{array}$ & $-896(-3,657 ; 1,864)$ & $2,658(1,473-4,176)$ & $3,555(1,674-6,718)$ & 0.51 \\
\hline $\begin{array}{l}\text { Otero } 2015^{83}(25331992) \\
\text { Cognitive behavioral problem solving vs. usual care } \\
\text { Low } \\
\text { Explanatory } \\
\text { Spain }\end{array}$ & $\begin{array}{l}\text { Relative risk (RR) } \\
\text { Number needed to treat (NNT) } \\
\text { Caregiver depression: Structured Clinical Interview for Axis } 1 \\
\text { Disorders of the DSM-IV (SCID-CV) } \\
12 \text { months } \\
\end{array}$ & $\begin{array}{l}0.40 \mathrm{RR} \\
6.7 \mathrm{NNT}\end{array}$ & & & 0.010 \\
\hline $\begin{array}{l}\text { Otero } 2015^{83}(25331992) \\
\text { Cognitive behavioral problem solving vs. usual care } \\
\text { Low } \\
\text { Explanatory } \\
\text { Spain }\end{array}$ & $\begin{array}{l}\text { Standardized mean difference } \\
\text { Center for Epidemiologic Depression Scale (CESD) Spanish } \\
\text { version } \\
12 \text { months }\end{array}$ & 1.14 & & & $\mathrm{NR}$ \\
\hline $\begin{array}{l}\text { Tremont } 2015^{28}(25074341) \\
\text { Telephone Tracking-Caregiver (FITT-C) vs. Telephone Support (TS) } \\
\text { Medium } \\
\text { Uxplanatory } \\
\text { USA }\end{array}$ & $\begin{array}{l}\text { Mean (SD) baseline } \\
\text { Mean (SD) } 6 \text { months } \\
\text { Caregiver depressive symptoms: Center for Epidemiology } \\
\text { Studies Depression Scale (CESD) } \\
6 \text { months } \\
\end{array}$ & NR & $\begin{array}{l}17.04(10.22) \\
\text { baseline } \\
14.15(10.00) 6 \\
\text { months }\end{array}$ & $\begin{array}{l}15.19 \text { (9.98) baseline } \\
15.62(10.18) 6 \text { months }\end{array}$ & 0.003 \\
\hline $\begin{array}{l}\text { Tremont } 2015^{28}(25074341) \\
\text { Telephone Tracking-Caregiver (FITT-C) vs. Telephone Support (TS) } \\
\text { Medium } \\
\text { Explanatory } \\
\text { USA }\end{array}$ & $\begin{array}{l}\text { Mean (SD) baseline } \\
\text { Mean (SD) } 6 \text { months } \\
\text { Caregiver burden: Zarit Burden Interview (ZBI) } \\
6 \text { months }\end{array}$ & NR & $\begin{array}{l}38.61(13.98) \\
\text { baseline } \\
35.95(14.34) 6 \\
\text { months }\end{array}$ & \begin{tabular}{|l|}
$38.82(14.63)$ baseline \\
37.17 (13.93) 6 months
\end{tabular} & 0.485 \\
\hline $\begin{array}{l}\text { Tremont } 2015^{28}(25074341) \\
\text { Telephone Tracking-Caregiver (FITT-C) vs. Telephone Support (TS) } \\
\text { Medium } \\
\text { Explanatory } \\
\text { USA }\end{array}$ & $\begin{array}{l}\text { Mean (SD) baseline } \\
\text { Mean (SD) } 6 \text { months } \\
\text { Caregiver reactions to care: Revised Memory and Behavior } \\
\text { Problem Checklist (RMBPC-RT) Reaction Score } \\
6 \text { months } \\
\end{array}$ & NR & $\begin{array}{l}22.98(12.89) \\
\text { baseline } \\
20.04(13.68) 6 \\
\text { months }\end{array}$ & $\begin{array}{l}22.59 \text { (13.94) baseline } \\
22.00 \text { (12.82) } 6 \text { months }\end{array}$ & 0.160 \\
\hline $\begin{array}{l}\text { Tremont } 2015^{28}(25074341) \\
\text { Telephone Tracking-Caregiver (FITT-C) vs. Telephone Support (TS) } \\
\text { Medium } \\
\text { Explanatory } \\
\text { USA }\end{array}$ & $\begin{array}{l}\text { Mean (SD) baseline } \\
\text { Mean (SD) } 6 \text { months } \\
\text { Caregiver : Family Assessment Device (FAD) } \\
6 \text { months }\end{array}$ & NR & $\begin{array}{l}2.11(0.61) \text { baseline } \\
2.09(0.63) 6 \text { months }\end{array}$ & $\begin{array}{l}2.05(0.55) \text { baseline } \\
2.00(0.50) 6 \text { months }\end{array}$ & 0.798 \\
\hline $\begin{array}{l}\text { Tremont } 2015^{28}(25074341) \\
\text { Telephone Tracking-Caregiver (FITT-C) vs. Telephone Support (TS) } \\
\text { Medium } \\
\text { Explanatory } \\
\text { USA }\end{array}$ & $\begin{array}{l}\text { Mean (SD) baseline } \\
\text { Mean (SD) } 6 \text { months } \\
\text { Caregiver confidence: Self-Efficacy Questionnaire (SEQ-SM) } \\
\text { Symptom Management subscale } \\
6 \text { months }\end{array}$ & NR & $\begin{array}{l}29.35(10.78) \\
\text { baseline } \\
34.17(9.55) 6 \\
\text { months }\end{array}$ & $\begin{array}{l}29.74 \text { (11.86) baseline } \\
32.27 \text { (10.04)6 months }\end{array}$ & 0.108 \\
\hline $\begin{array}{l}\text { Tremont } 2015^{28}(25074341) \\
\text { Telephone Tracking-Caregiver (FITT-C) vs. Telephone Support (TS) } \\
\text { Medium Explanatory } \\
\text { Expl }\end{array}$ & $\begin{array}{l}\text { Mean (SD) baseline } \\
\text { Mean (SD) } 6 \text { months } \\
\text { Caregiver confidence: Self-Efficacy Questionnaire (SEQ-SS) } \\
\text { Support Services subscale } \\
6 \text { months }\end{array}$ & NR & $\begin{array}{l}23.39(10.89) \\
\text { baseline } \\
31.06(7.77) 6 \\
\text { months }\end{array}$ & $\begin{array}{l}24.00(9.93) \text { baseline } \\
30.64(7.28) 6 \text { months }\end{array}$ & 0.467 \\
\hline
\end{tabular}




\begin{tabular}{|c|c|c|c|c|c|}
\hline \begin{tabular}{|l|} 
Study (PMID) \\
Comparison \\
Caregiver Type \\
RoB \\
Category* \\
Country \\
\end{tabular} & $\begin{array}{l}\text { Outcome } \\
\text { Timing }\end{array}$ & Summary Finding & Intervention & Comparator & p-Value \\
\hline $\begin{array}{l}\text { Tremont } 2015^{28}(25074341) \\
\text { Telephone Tracking-Caregiver (FITT-C) vs. Telephone Support (TS) } \\
\text { Medium } \\
\text { Explanatory } \\
\text { USA }\end{array}$ & $\begin{array}{l}\text { Mean (SD) baseline } \\
\text { Mean (SD) } 6 \text { months } \\
\text { Caregiver : Positive Aspects of Caregiving (PAC) } \\
6 \text { months }\end{array}$ & NR & $\begin{array}{l}31.51(7.58) \text { baseline } \\
34.13(8.31) 6 \\
\text { months }\end{array}$ & $\begin{array}{l}30.35 \text { (9.65) baseline } \\
35.24(7.55) 6 \text { months }\end{array}$ & 0.041 \\
\hline $\begin{array}{l}\text { Tremont } 2015^{28}(25074341) \\
\text { Telephone Tracking-Caregiver (FITT-C) vs. Telephone Support (TS) } \\
\text { Medium } \\
\text { Explanatory } \\
\text { USA }\end{array}$ & $\begin{array}{l}\text { Mean (SD) baseline } \\
\text { Mean (SD) } 6 \text { months } \\
\text { Caregiver health related quality of life; EuroQoL Visual Analog } \\
\text { scale } \\
6 \text { months }\end{array}$ & $\mathrm{NR}$ & $\begin{array}{l}80.08(16.07) \\
\text { baseline } \\
79.87(15.00) 6 \\
\text { months }\end{array}$ & $\begin{array}{l}77.14 \text { (17.61) baseline } \\
77.59 \text { (15.69) } 6 \text { months }\end{array}$ & 0.748 \\
\hline $\begin{array}{l}\text { Livingston } 2014^{30}(25300037) \\
\text { START ( STrAtegies for RelaTives) manual-based coping strategy programme in promoting } \\
\text { the mental health of carers of people with dementia vs. usual care } \\
\text { Low } \\
\text { Explanatory } \\
\text { UK }\end{array}$ & $\begin{array}{l}\text { Adjusted mean difference }(95 \% \mathrm{Cl}) \\
\text { Caregiver Affective symptoms [Hospital Anxiety and } \\
\text { Depression Scale-total (HADS-T)] } \\
2 \text { years }\end{array}$ & $-2.58(-4.26$ to -0.90$)$ & NR & NR & NR \\
\hline $\begin{array}{l}\text { Livingston } 2014^{30}(25300037) \\
\text { START (STrAtegies for RelaTives) manual-based coping strategy programme in promoting } \\
\text { the mental health of carers of people with dementia vs. usual care } \\
\text { Low } \\
\text { Explanatory } \\
\text { UK }\end{array}$ & $\begin{array}{l}\text { Adjusted mean difference }(95 \% \mathrm{Cl}) \\
\text { Caregiver Anxiety: Hospital Anxiety and Depression Scale- } \\
\text { anxiety (HADS-A) } \\
2 \text { years }\end{array}$ & $-1.16(-2.15$ to -0.18$)$ & NR & $\mathrm{NR}$ & NR \\
\hline $\begin{array}{l}\text { Livingston } 20144^{30}(25300037) \\
\text { START (STrAtegies for Relatives) manual-based coping strategy programme in promoting } \\
\text { the mental health of carers of people with dementia vs. usual care } \\
\text { Low } \\
\text { Explanatory } \\
\text { UK }\end{array}$ & $\begin{array}{l}\text { OR }(95 \% \text { Cl) } \\
\text { Caregiver Anxiety: Hospital Anxiety and Depression Scale- } \\
\text { anxiety (HADS-A) (score } \geq 9 \text { ) } \\
2 \text { years }\end{array}$ & 0.57 (0.26 to 1.24$)$ & $57(43.2) \mathrm{N}(\%)$ & $32(50.0) \mathrm{N}(\%)$ & NR \\
\hline $\begin{array}{l}\text { Livingston } 2014^{30}(25300037) \\
\text { START ( STrAtegies for RelaTives) manual-based coping strategy programme in promoting } \\
\text { the mental health of carers of people with dementia vs. usual care } \\
\text { Low } \\
\text { Explanatory } \\
\text { UK }\end{array}$ & $\begin{array}{l}\text { Adjusted mean difference }(95 \% \mathrm{Cl}) \\
\text { Caregiver Depression: Hospital Anxiety and Depression } \\
\text { Scale-depression (HADS-D) } \\
2 \text { years }\end{array}$ & $-1.45(-2.32$ to -0.57$)$ & NR & $\mathrm{NR}$ & $\mathrm{NR}$ \\
\hline $\begin{array}{l}\text { Livingston } 2014^{30}(25300037) \\
\text { START ( STrAtegies for RelaTives) manual-based coping strategy programme in promoting } \\
\text { the mental health of carers of people with dementia vs. usual care } \\
\text { Low } \\
\text { Explanatory } \\
\text { UK }\end{array}$ & $\begin{array}{l}\text { OR ( }(95 \% \text { Cl) } \\
\text { Caregiver Depression: Hospital Anxiety and Depression } \\
\text { Scale-depression (HADS-D) (score } \geq 9 \text { ) } \\
2 \text { years }\end{array}$ & 0.14 (0.04 to 0.53$)$ & $30(22.7) \mathrm{N}(\%)$ & $19(29.7) \mathrm{N}(\%)$ & NR \\
\hline $\begin{array}{l}\text { Livingston } 2014^{30}(25300037) \\
\text { START ( STrAtegies for RelaTives) manual-based coping strategy programme in promoting } \\
\text { the mental health of carers of people with dementia vs. usual care } \\
\text { Low } \\
\text { Explanatory } \\
\text { UK }\end{array}$ & $\begin{array}{l}\text { Adjusted mean difference }(95 \% \text { Cl) } \\
\text { Caregiver QOL: Health Status Questionnaire (HSQ) - mental } \\
\text { health } \\
2 \text { years }\end{array}$ & 7.47 (2.87 to 12.08$)$ & NR & NR & $\mathrm{NR}$ \\
\hline $\begin{array}{l}\text { Livingston } 2014^{30}(25300037) \\
\text { START (STrAtegies for Relatives) manual-based coping strategy programme in promoting } \\
\text { the mental health of carers of people with dementia vs. usual care } \\
\text { Low } \\
\text { Explanatory } \\
\text { UK }\end{array}$ & $\begin{array}{l}\text { OR }(95 \% \text { Cl) } \\
\text { Caregiver Abusive behaviors: Modified Conflict Tactic Score } \\
\text { (MCTS) (at least one item with score of } \geq 2 \text { ) } \\
2 \text { years }\end{array}$ & 0.83 (0.36 to 1.93$)$ & $28(29.5) \mathrm{N}(\%)$ & $11(23.4) \mathrm{N}(\%)$ & $\mathrm{NR}$ \\
\hline \begin{tabular}{l|} 
Livingston $2014^{30}(25300037)$ \\
START (STrAtegies for RelaTives) manual-based coping strategy programme in promoting \\
the mental health of carers of people with dementia vs. usual care
\end{tabular} & $\begin{array}{l}\text { Mean difference }(95 \% \mathrm{Cl}) \\
\text { Caregiver health care cost: Quality-Adjusted Life-Years }\end{array}$ & $£ 336(95 \% \mathrm{Cl}-£ 223$ to $£ 895)$ & NR & $\mathrm{NR}$ & $\mathrm{NR}$ \\
\hline
\end{tabular}




\begin{tabular}{|c|c|c|c|c|c|}
\hline $\begin{array}{l}\text { Study (PMID) } \\
\text { Comparison } \\
\text { Caregiver Type } \\
\text { RoB } \\
\text { Category* } \\
\text { Country }\end{array}$ & $\begin{array}{l}\text { Outcome } \\
\text { Timing }\end{array}$ & Summary Finding & Intervention & Comparator & p-Value \\
\hline $\begin{array}{l}\text { Low } \\
\text { Explanatory } \\
\text { UK }\end{array}$ & $\begin{array}{l}\text { (QALYs) from the EQ-5D } \\
2 \text { years }\end{array}$ & & & & \\
\hline $\begin{array}{l}\text { Livingston } 2014^{30}(25300037) \\
\text { START (STRAtegies for Relaives) manual-based coping strategy programme in promoting } \\
\text { the mental health of carers of people with dementia vs. usual care } \\
\text { Low } \\
\text { Explanatory } \\
\text { UK }\end{array}$ & $\begin{array}{l}\text { Mean difference }(95 \% \mathrm{Cl}) \\
\text { Caregiver health care cost: Quality-Adjusted Life-Years } \\
\text { (QALYS) from the Hospital Anxiety and Depression Scale- } \\
\text { total (HADS-T) } \\
2 \text { years }\end{array}$ & $£ 303(95 \% \mathrm{Cl}-£ 206$ to $£ 812)$ & NR & NR & NR \\
\hline $\begin{array}{l}\text { Martin-Carrasco } 2014^{31}(24113563) \\
\text { Psychoedcuational Intervention Program to teach strategies for confronting problems of } \\
\text { PLWD care vs. usual care } \\
\text { Medium } \\
\text { Explanatory } \\
\text { Spain }\end{array}$ & $\begin{array}{l}\text { Mean difference }(95 \% \mathrm{Cl}) \\
\text { SMD }(95 \% \mathrm{CI}) \\
\text { Caregiver burden: Spanish Zarit caregiver burden interview } \\
4 \text { months }\end{array}$ & $\begin{array}{l}-0.55(-3.64 ; 2.55) \text { - Mean } \\
\text { difference } \\
-0.04(-0.30 ; 0.21) \text { - SDM }\end{array}$ & $-1.17(12.3)$ & $-0.63(12.0)$ & 0.73 \\
\hline $\begin{array}{l}\text { Martin-Carrasco } 2014^{31}(24113563) \\
\text { Psychoedcuational Intervention Program to teach strategies for confronting problems of } \\
\text { PLWD care vs. usual care } \\
\text { Medium } \\
\text { Explanatory } \\
\text { Spain }\end{array}$ & $\begin{array}{l}\text { Mean difference }(95 \% \mathrm{Cl}) \\
\text { SMD }(95 \% \mathrm{Cl}) \\
\text { Caregiver psychological distress: Spanish General Health } \\
\text { Questionnaire } 28 \text { item (GHQ-28) } \\
4 \text { months }\end{array}$ & $\begin{array}{l}-2.34(-5.27 ; 0.59) \text { mean diff } \\
-0.20(-0.46 ; 0.05) \text { SDM }\end{array}$ & $-4.76(12.6)$ & $-2.42(10.3)$ & $>.05$ \\
\hline $\begin{array}{l}\text { Martin-Carrasco } 2014^{31}(24113563) \\
\text { Psychoedcuational Intervention Program to teach strategies for confronting problems of } \\
\text { PLWD care vs. usual care } \\
\text { Medium Explanatory } \\
\text { Expan }\end{array}$ & $\begin{array}{l}\text { Mean difference }(95 \% \mathrm{Cl}) \\
\text { SMD }(95 \% \mathrm{Cl}) \\
\text { Caregiver quality of life: Spanish SF-12 physical function } \\
4 \text { months }\end{array}$ & $\begin{array}{l}-1.02(-11.10 \text { to } 9.06) \\
-0.03(-0.30 \text { to } 0.25)\end{array}$ & $-1.02(30.0)$ & $0.0(41.3)$ & $>.05$ \\
\hline $\begin{array}{l}\text { Martin-Carrasco } 2014^{31}(24113563) \\
\text { Psychoedcuational Intervention Program to teach strategies for confronting problems of } \\
\text { PLWD care vs. usual care } \\
\text { Medium Explanatory } \\
\text { Expan }\end{array}$ & $\begin{array}{l}\text { Mean difference }(95 \% \mathrm{Cl}) \\
\text { SMD }(95 \% \mathrm{Cl}) \\
\text { Caregiver quality of life: Spanish SF-12 role physical } \\
4 \text { months }\end{array}$ & $\begin{array}{l}1.80(-5.63 \text { to } 9.22) \\
0.07(-0.21 \text { to } 0.34)\end{array}$ & $3.09(26.9)$ & $1.30(26.7)$ & $>.05$ \\
\hline $\begin{array}{l}\text { Martin-Carrasco } 2014^{31}(24113563) \\
\text { Psychoedcuational Intervention Program to teach strategies for confronting problems of } \\
\text { PLWD care vs. usual care } \\
\text { Medium } \\
\text { Explanatory } \\
\text { Spain }\end{array}$ & $\begin{array}{l}\text { Mean difference }(95 \% \mathrm{Cl}) \\
\text { SMD }(95 \% \mathrm{Cl}) \\
\text { Caregiver quality of life: Spanish SF-12 bodily pain } \\
4 \text { months }\end{array}$ & $\begin{array}{l}6.85(-1.58 \text { to } 15.28) \\
0.22(-0.05 \text { to } 0.50)\end{array}$ & $6.38(25.7)$ & $-0.47(34.3)$ & 0.11 \\
\hline $\begin{array}{l}\text { Martin-Carrasco } 2014^{31}(24113563) \\
\text { Psychoedcuational Intervention Program to teach strategies for confronting problems of } \\
\text { PLWD care vs. usual care } \\
\text { Medium } \\
\text { Explanatory } \\
\text { Spain }\end{array}$ & $\begin{array}{l}\text { Mean difference }(95 \% \mathrm{Cl}) \\
\text { SMD }(95 \% \mathrm{Cl}) \\
\text { Caregiver quality of life: Spanish SF-12 general health } \\
4 \text { months }\end{array}$ & $\begin{array}{l}-5.01(-10.48 \text { to } 0.45) \\
-0.25(-0.53 \text { to } 0.02)\end{array}$ & $-3.12(19.3)$ & $1.89(20.0)$ & 0.07 \\
\hline $\begin{array}{l}\text { Martin-Carrasco } 2014^{31}(24113563) \\
\text { Psychoedcuational Intervention Program to teach strategies for confronting problems of } \\
\text { PLWD care vs. usual care } \\
\text { Medium Explanatory } \\
\text { Spain }\end{array}$ & $\begin{array}{l}\text { Mean difference }(95 \% \mathrm{Cl}) \\
\text { SMD (95\% CI) } \\
\text { Caregiver quality of life: Spanish SF-12 vitality } \\
4 \text { months }\end{array}$ & \begin{tabular}{|l|}
$3.71(-4.34$ to 11.76$)$ \\
$0.13(-0.15$ to 0.40$)$
\end{tabular} & $2.04(28.6)$ & $-1.67(29.5)$ & $>.05$ \\
\hline $\begin{array}{l}\text { Martin-Carrasco } 2014^{31}(24113563) \\
\text { Psychoedcuational Intervention Program to teach strategies for confronting problems of } \\
\text { PLWD care vs. usual care } \\
\text { Medium }\end{array}$ & $\begin{array}{l}\text { Mean difference }(95 \% \mathrm{Cl}) \\
\text { SMD }(95 \% \mathrm{Cl}) \\
\text { Caregiver quality of life: Spanish SF-12 social functioning } \\
4 \text { months }\end{array}$ & $\begin{array}{l}-0.78(-9.33 \text { to } 7.77) \\
-0.02(-0.30 \text { to } 0.25)\end{array}$ & $-4.08(31.6)$ & $-3.30(30.3)$ & $>.05$ \\
\hline
\end{tabular}




\begin{tabular}{|c|c|c|c|c|c|}
\hline $\begin{array}{l}\text { Study (PMID) } \\
\text { Comparison } \\
\text { Caregiver Type } \\
\text { RoB } \\
\text { Category* } \\
\text { Country }\end{array}$ & $\begin{array}{l}\text { Outcome } \\
\text { Timing }\end{array}$ & Summary Finding & Intervention & Comparator & $p$-Value \\
\hline \multicolumn{6}{|l|}{$\begin{array}{l}\text { Explanatory } \\
\text { Spain }\end{array}$} \\
\hline $\begin{array}{l}\text { Martin-Carrasco } 2014^{31}(24113563) \\
\text { Psychoedcuational Intervention Program to teach strategies for confronting problems of } \\
\text { PLWD care vs. usual care } \\
\text { Medium } \\
\text { Explanatory } \\
\text { Spain }\end{array}$ & $\begin{array}{l}\text { Mean difference }(95 \% \mathrm{Cl}) \\
\text { SMD (95\% CI) } \\
\text { Caregiver quality of life: Spanish SF-12 role emotional } \\
4 \text { months }\end{array}$ & \begin{tabular}{|l}
$-4.25(-11.48$ to 2.98$)$ \\
$-0.16(-0.44$ to 0.11$)$
\end{tabular} & $-3.06(27.7)$ & 1.19 (24.5) & $>.05$ \\
\hline $\begin{array}{l}\text { Martin-Carrasco } 2014^{31}(24113563) \\
\text { Psychoedcuational Intervention Program to teach strategies for confronting problems of } \\
\text { PLWD care vs. usual care } \\
\text { Medium } \\
\text { Explanatory } \\
\text { Spain }\end{array}$ & $\begin{array}{l}\text { Mean difference }(95 \% \mathrm{Cl}) \\
\text { SMD }(95 \% \mathrm{Cl}) \\
\text { Caregiver quality of life: Spanish SF-12 mental health } \\
4 \text { months }\end{array}$ & $\begin{array}{l}-1.23(-7.22 \text { to } 4.75) \\
-0.06(-0.33 \text { to } 0.22)\end{array}$ & $1.53(23.8)$ & \begin{tabular}{|l|}
$2.76(19.2)$ \\
\end{tabular} & $>.05$ \\
\hline $\begin{array}{l}\text { Waldorff } 2012^{40}(22807076) \\
\text { DAISY plus support (multifaceted and semi-tailored counselling, } \\
\text { education, and support) vs. support control group } \\
\text { Medium } \\
\text { Explanatory } \\
\text { Denmark }\end{array}$ & $\begin{array}{l}\text { Mean change score }(95 \% \mathrm{Cl}) \\
\text { Caregiver depressive symptom: Geriatric depression scale } \\
12 \text { months }\end{array}$ & $\mathrm{NR}$ & 0.96 (0.05 to 1.86$)$ & \begin{tabular}{|l|}
$0.26(-0.51$ to 1.03$)$ \\
\end{tabular} & 0.2500 \\
\hline $\begin{array}{l}\text { de Rotrou } 2011^{42}(20922772) \\
\text { Psycho Education Program vs. usual care } \\
\text { Medium } \\
\text { Explanatory } \\
\text { France }\end{array}$ & $\begin{array}{l}\text { Imputed mean (SD) } \\
\text { Caregiver depressive symptoms: Montgomery and Asberg } \\
\text { Depression Rating Scale (MADRS) } \\
\text { Baseline } \\
3 \text { months }\end{array}$ & $\mathrm{NR}$ & $\begin{array}{l}9.03(0.79) \text { baseline } \\
8.61(0.94) 3 \text { months }\end{array}$ & \begin{tabular}{|l|}
$10.16(1.22)$ baseline \\
$10.11(1.18) 3$ months
\end{tabular} & $\begin{array}{l}\text { NS } \\
\text { Value not reported }\end{array}$ \\
\hline $\begin{array}{l}\text { de Rotrou } 2011^{42}(20922772) \\
\text { Psycho Education Program vs. usual care } \\
\text { Medium } \\
\text { Explanatory } \\
\text { France }\end{array}$ & $\begin{array}{l}\text { Imputed mean (SD) } \\
\text { Caregiver burden: Zarit Caregiver Burden Interview (ZBI) } \\
\text { Baseline } \\
3 \text { months }\end{array}$ & $\mathrm{NR}$ & $\begin{array}{l}22.97(2.79) \text { baseline } \\
22.23(1.59) 3 \\
\text { months } 3\end{array}$ & \begin{tabular}{|l|}
$24.30(4.15)$ baseline \\
$23.53(2.05) 3$ months
\end{tabular} & $\begin{array}{l}\text { NS } \\
\text { Value not reported }\end{array}$ \\
\hline $\begin{array}{l}\text { de Rotrou } 2011^{42}(20922772) \\
\text { Psycho Education Program vs. usual care } \\
\text { Medium } \\
\text { Explanatory } \\
\text { France }\end{array}$ & $\begin{array}{l}\text { Imputed mean (SD) } \\
\text { Caregiver perception of disease understanding: Visual } \\
\text { Analogue Scales (VAS) } \\
\text { Baseline } \\
3 \text { months }\end{array}$ & NR & $\begin{array}{l}12.52(0.27) \text { baseline } \\
14.44(0.47) 3 \\
\text { months }\end{array}$ & \begin{tabular}{|l}
$12.05(0.27)$ baseline \\
$12.54(0.53) 3$ months
\end{tabular} & NR \\
\hline $\begin{array}{l}\text { de Rotrou } 2011^{42}(20922772) \\
\text { Psycho Education Program vs. usual care } \\
\text { Medium } \\
\text { Explanatory } \\
\text { France }\end{array}$ & $\begin{array}{l}\text { Imputed mean (SD) } \\
\text { Caregiver coping: Visual Analogue Scales (VAS) } \\
\text { Baseline } \\
3 \text { months }\end{array}$ & NR & $\begin{array}{l}12.53(0.24) \text { baseline } \\
13.47(0.44) 3 \\
\text { months } 3\end{array}$ & \begin{tabular}{|l|}
$12.72(0.29)$ baseline \\
$12.63(0.52) 3$ months
\end{tabular} & NR \\
\hline $\begin{array}{l}\text { de Rotrou } 2011^{42}(20922772) \\
\text { Psycho Education Program vs. usual care } \\
\text { Medium } \\
\text { Explanatory } \\
\text { France }\end{array}$ & $\begin{array}{l}\text { Imputed mean (SD) } \\
\text { Caregiver depressive symptoms: Montgomery and Asberg } \\
\text { Depression Rating Scale (MADRS) } \\
\text { Baseline } \\
6 \text { months }\end{array}$ & NR & $\begin{array}{l}9.03(0.79) \text { baseline } \\
9.28(0.99) 3 \text { months }\end{array}$ & $\begin{array}{l}\text { 10.16 (1.22) baseline } \\
11.43(1.24) 3 \text { months }\end{array}$ & Interaction 0.373 \\
\hline $\begin{array}{l}\text { de Rotrou 201142 (20922772) } \\
\text { Psycho Education Program vs. usual care } \\
\text { Medium } \\
\text { Explanatory } \\
\text { France }\end{array}$ & $\begin{array}{l}\text { Imputed mean (SD) } \\
\text { Caregiver burden: Zarit Caregiver Burden Interview (ZBI) } \\
\text { Baseline } \\
6 \text { months }\end{array}$ & NR & $\begin{array}{l}22.97(2.79) \text { baseline } \\
23.90(1.86) 3 \\
\text { months } 3\end{array}$ & \begin{tabular}{|l|}
$24.30(4.15)$ baseline \\
$25.95(2.09) 3$ months
\end{tabular} & Interaction 0.657 \\
\hline $\begin{array}{l}\text { de Rotrou } 2011^{42}(20922772) \\
\text { Psycho Education Program vs. usual care } \\
\text { Medium } \\
\text { Explanatory } \\
\text { France }\end{array}$ & $\begin{array}{l}\text { Imputed mean (SD) } \\
\text { Caregiver perception of disease understanding: Visual } \\
\text { Analogue Scales (VAS) } \\
\text { Baseline } \\
6 \text { months }\end{array}$ & NR & $\begin{array}{l}12.52(0.27) \text { baseline } \\
15.03(0.38) 3 \\
\text { months } 3\end{array}$ & \begin{tabular}{|l|l}
$12.05(0.27)$ baseline \\
$12.52(0.50) 3$ months
\end{tabular} & Interaction 0.003 \\
\hline
\end{tabular}




\begin{tabular}{|c|c|c|c|c|c|}
\hline $\begin{array}{l}\text { Study (PMID) } \\
\text { Comparison } \\
\text { Caregiver Type } \\
\text { RoB } \\
\text { Category* } \\
\text { Country }\end{array}$ & \begin{tabular}{|l|} 
Outcome \\
Timing
\end{tabular} & Summary Finding & Intervention & Comparator & $p$-Value \\
\hline $\begin{array}{l}\text { de Rotrou 201142 (20922772) } \\
\text { Psycho Education Program vs. usual care } \\
\text { Medium } \\
\text { Explanatory } \\
\text { France }\end{array}$ & $\begin{array}{l}\text { Imputed mean (SD) } \\
\text { Caregiver coping: Visual Analogue Scales (VAS) } \\
\text { Baseline } \\
6 \text { months }\end{array}$ & $\mathrm{NR}$ & $\begin{array}{l}12.53(0.24) \text { baseline } \\
13.63(0.44) 3 \\
\text { months } 3\end{array}$ & $\begin{array}{l}12.72(0.29) \text { baseline } \\
12.24(0.57) 3 \text { months }\end{array}$ & Interaction 0.025 \\
\hline $\begin{array}{l}\text { Guerra } 2011^{43} \\
10 / 66 \text { Helping Carers to Care vs waitlist control } \\
\text { Medium } \\
\text { Explanatory } \\
\text { Peru }\end{array}$ & $\begin{array}{l}\text { Standardized mean difference }(95 \% \mathrm{Cl}) \text { summary finding } \\
\text { Mean change score (SD) } \\
\text { Caregiver burden: Zarit Caregiver Burden Interview (ZBI) } \\
6 \text { months }\end{array}$ & $-1.05(-1.60$ to -0.48$)$ & $-3.6(4.6)$ & $0.3(2.9)$ & $<0.001$ \\
\hline $\begin{array}{l}\text { Guerra } 2011^{43} \\
10 / 66 \text { Helping Carers to Care vs waitlist control } \\
\text { Medium } \\
\text { Explanatory } \\
\text { Peru }\end{array}$ & $\begin{array}{l}\text { Standardized mean difference }(95 \% \mathrm{Cl}) \text { summary finding } \\
\text { Mean change score (SD) } \\
\text { Caregiver mental health: Self reporting questionnaire } 20 \\
\text { (SRQ-20) psychological morbidity } \\
\text { 6 months } \\
\end{array}$ & $-0.01(-0.55$ to 0.55$)$ & $-3.1(4.0)$ & $-3.0(3.1)$ & 0.97 \\
\hline $\begin{array}{l}\text { Guerra } 2011^{43} \\
10 / 66 \text { Helping Carers to Care vs waitlist control } \\
\text { Medium } \\
\text { Explanatory } \\
\text { Peru }\end{array}$ & $\begin{array}{l}\text { Standardized mean difference }(95 \% \mathrm{Cl}) \text { summary finding } \\
\text { Mean change score (SD) } \\
\text { Caregiver distress: Neuropsychiatric Inventory (NPI-Q) carer } \\
\text { distress score } \\
6 \text { months } \\
\end{array}$ & $0.01(-0.55$ to 0.57$)$ & $-2.3(4.7)$ & $-2.4(4.6)$ & 0.96 \\
\hline $\begin{array}{l}\text { Guerra } 2011^{43} \\
10 / 66 \text { Helping Carers to Care vs waitlist control } \\
\text { Medium } \\
\text { Explanatory } \\
\text { Peru }\end{array}$ & $\begin{array}{l}\text { Standardized mean difference }(95 \% \mathrm{Cl}) \text { summary finding } \\
\text { Mean change score (SD) } \\
\text { Caregiver QOL: WHOQOL-BREF physical subscale } \\
6 \text { months }\end{array}$ & $0.36(-0.19$ to 0.92$)$ & $-9.7(18.7)$ & $-15.5(13.9)$ & 0.19 \\
\hline $\begin{array}{l}\text { Guerra } 2011^{43} \\
10 / 66 \text { Helping Carers to Care vs waitlist control } \\
\text { Medium } \\
\text { Explanatory } \\
\text { Peru }\end{array}$ & $\begin{array}{l}\text { Standardized mean difference }(95 \% \mathrm{Cl}) \text { summary finding } \\
\text { Mean change score (SD) } \\
\text { Caregiver QOL: WHOQOL-BREF psychological subscale } \\
6 \text { months }\end{array}$ & $0.10(-0.44$ to 0.65$)$ & $10.0(11.5)$ & $8.9(11.1)$ & 0.71 \\
\hline $\begin{array}{l}\text { Guerra } 2011^{43} \\
10 / 66 \text { Helping Carers to Care vs waitlist control } \\
\text { Medium } \\
\text { Explanatory } \\
\text { Peru }\end{array}$ & $\begin{array}{l}\text { Standardized mean difference }(95 \% \mathrm{Cl}) \text { summary finding } \\
\text { Mean change score (SD) } \\
\text { Caregiver QOL: WHOQOL-BREF social subscale } \\
6 \text { months }\end{array}$ & $0.39(-0.15$ to 0.94$)$ & $7.1(12.6)$ & $1.7(15.2)$ & 0.16 \\
\hline $\begin{array}{l}\text { Guerra } 2011^{43} \\
10 / 66 \text { Helping Carers to Care vs waitlist control } \\
\text { Medium } \\
\text { Explanatory } \\
\text { Peru }\end{array}$ & $\begin{array}{l}\text { Standardized mean difference }(95 \% \mathrm{Cl}) \text { summary finding } \\
\text { Mean change score (SD) } \\
\text { Caregiver QOL: WHOQOL-BREF environmental subscale } \\
6 \text { months }\end{array}$ & $-0.15(-0.70$ to 0.39$)$ & $7.6(11.4)$ & $9.5(13.0)$ & 0.58 \\
\hline $\begin{array}{l}\text { Voight-Radloff } 2011^{46}(22021760) \\
\text { Community Occupational Therapy in Dementia Program vs. usual care (one session home } \\
\text { consultation) } \\
\text { Medium } 26-52 \text { weeks } \\
\text { Explanatory } \\
\text { Germany }\end{array}$ & $\begin{array}{l}\text { Mean difference }(95 \% \mathrm{Cl}) \\
\text { Caregiver interaction with PLWD: Sense of Competence } \\
\text { Questionnaire } \\
52 \text { weeks }\end{array}$ & 3.8 (-3.5 to 11.2$)$ & NR & NR & NS \\
\hline $\begin{array}{l}\text { Voight-Radloff } 2011^{46}(22021760) \\
\text { Community Occupational Therapy in Dementia Program vs. usual care (one session home } \\
\text { consultation) } \\
\text { Medium } 26-52 \text { weeks } \\
\text { Explanatory } \\
\text { Germany }\end{array}$ & $\begin{array}{l}\text { Mean difference }(95 \% \mathrm{Cl}) \\
\text { Caregiver depression: Center for Epidemiologic Depression } \\
\text { Scale } \\
52 \text { weeks }\end{array}$ & $-1.4(-5.1$ to 2.3$)$ & $\mathrm{NR}$ & NR & NS \\
\hline $\begin{array}{l}\text { Voight-Radloff } 2011^{46}(22021760) \\
\text { Community Occupational Therapy in Dementia Program vs. usual care (one session home } \\
\text { consultation) }\end{array}$ & $\begin{array}{l}\text { Mean difference }(95 \% \mathrm{Cl}) \\
\text { Caregiver QOL: Dementia Quality of Life Instrument overall } \\
52 \text { weeks }\end{array}$ & $0.2(-0.1$ to 0.5$)$ & NR & NR & NS \\
\hline
\end{tabular}




\begin{tabular}{|c|c|c|c|c|c|}
\hline $\begin{array}{l}\text { Study (PMID) } \\
\text { Comparison } \\
\text { Caregiver Type } \\
\text { RoB } \\
\text { Category* } \\
\text { Country }\end{array}$ & $\begin{array}{l}\text { Outcome } \\
\text { Timing }\end{array}$ & Summary Finding & Intervention & Comparator & $p$-Value \\
\hline \multicolumn{6}{|l|}{$\begin{array}{l}\text { Medium } 26-52 \text { weeks } \\
\text { Explanatory } \\
\text { Germany }\end{array}$} \\
\hline $\begin{array}{l}\text { Voight-Radloff } 2011^{46}(22021760) \\
\text { Community Occupational Therapy in Dementia Program vs. usual care (one session home } \\
\text { consultation) } \\
\text { Medium } 26-52 \text { weeks } \\
\text { Explanatory } \\
\text { Germany }\end{array}$ & $\begin{array}{l}\text { Mean difference }(95 \% \mathrm{Cl}) \\
\text { Caregiver QOL: Short-Form } 12 \text { Health Survey Questionnaire } \\
\text { - physical component } \\
52 \text { weeks }\end{array}$ & $-1.0(-6.1$ to 4.0$)$ & $\mathrm{NR}$ & NR & NS \\
\hline $\begin{array}{l}\text { Voight-Radloff } 2011^{46}(22021760) \\
\text { Community Occupational Therapy in Dementia Program vs. usual care (one session home } \\
\text { consultation) } \\
\text { Medium } 26-52 \text { weeks } \\
\text { Explanatory } \\
\text { Germany }\end{array}$ & $\begin{array}{l}\text { Mean difference }(95 \% \mathrm{Cl}) \\
\text { Caregiver QOL: Short-Form } 12 \text { Health Survey Questionnaire } \\
\text { - mental component } \\
52 \text { weeks }\end{array}$ & $-1.7(-6.9$ to 3.4$)$ & NR & NR & NS \\
\hline $\begin{array}{l}\text { Voight-Radloff } 2011^{46}(22021760) \\
\text { Community Occupational Therapy in Dementia Program vs. usual care (one session home } \\
\text { consultation) } \\
\text { Medium } 26-52 \text { weeks } \\
\text { Explanatory } \\
\text { Germany }\end{array}$ & $\begin{array}{l}\text { Mean difference }(95 \% \mathrm{Cl}) \\
\text { Caregiver work: Resource utilization in Dementia - Basic } \\
\text { Activities of Daily Living care by primary carer (hours per day) } \\
\text { subscale } \\
52 \text { weeks }\end{array}$ & $0.1(-0.8$ to 1.0$)$ & NR & NR & NS \\
\hline $\begin{array}{l}\text { Voight-Radloff } 2011^{46}(22021760) \\
\text { Community Occupational Therapy in Dementia Program vs. usual care (one session home } \\
\text { consultation) } \\
\text { Medium } 26-52 \text { weeks } \\
\text { Explanatory } \\
\text { Germany }\end{array}$ & $\begin{array}{l}\text { Mean difference }(95 \% \mathrm{Cl}) \\
\text { Caregiver work: Resource utilization in Dementia - } \\
\text { Instrumental Activities of Daily Living -care by primary carer } \\
\text { (hours per day) subscale } \\
52 \text { weeks }\end{array}$ & $0.5(-0.6$ to 1.6$)$ & $\mathrm{NR}$ & NR & NS \\
\hline $\begin{array}{l}\text { Wang } 2011^{47}(21752121) \\
\text { Family Mutual Support Program in Dementia Care (FMSP-DC) vs. usual care } \\
\text { Medium } \\
\text { Explanatory } \\
\text { USA }\end{array}$ & $\begin{array}{l}\text { Mean (SD) baseline } \\
\text { Mean (SD) } 6 \text { months } \\
\text { Caregiver burden: Family Caregiving Burden Inventory (FCBI) } \\
6 \text { months }\end{array}$ & NR & $\begin{array}{l}68.2(11.9) \text { baseline } \\
55.2(15.0) 6 \text { months }\end{array}$ & $\begin{array}{l}68.8(16.7) \text { baseline } \\
65.0(18.1) 6 \text { months }\end{array}$ & $<0.001$ \\
\hline $\begin{array}{l}\text { Wang } 2011^{47}(21752121) \\
\text { Family Mutual Support Program in Dementia Care (FMSP-DC) vs. usual care } \\
\text { Medium } \\
\text { Explanatory } \\
\text { USA }\end{array}$ & $\begin{array}{l}\text { Mean (SD) baseline } \\
\text { Mean (SD) } 6 \text { months } \\
\text { Caregiver quality of life: World Health Organization Quality of } \\
\text { Life Scale (Brief H.K. version) (WHOQOL-BREF) } \\
6 \text { months }\end{array}$ & NR & $\begin{array}{l}65.9 \text { (13.0) baseline } \\
78.8 \text { (19.0) } 6 \text { months }\end{array}$ & $\begin{array}{l}67.0 \text { (13.5) baseline } \\
68.9 \text { (15.7) } 6 \text { months }\end{array}$ & $<0.001$ \\
\hline $\begin{array}{l}\text { Wang } 2011^{47}(21752121) \\
\text { Family Mutual Support Program in Dementia Care (FMSP-DC) vs. usual care } \\
\text { Medium } \\
\text { Explanatory } \\
\text { USA }\end{array}$ & $\begin{array}{l}\text { Mean (SD) baseline } \\
\text { Mean (SD) } 6 \text { months } \\
\text { Caregiver social support: Six-item Social Support } \\
\text { Questionnaire (SSQ6) - number of support persons subscale } \\
6 \text { months }\end{array}$ & NR & $\begin{array}{l}3.1(1.0) \text { baseline } \\
4.4(2.0) 6 \text { months }\end{array}$ & $\begin{array}{l}2.9(1.1) \text { baseline } \\
2.9(1.3) 6 \text { months }\end{array}$ & NS \\
\hline $\begin{array}{l}\text { Wang } 2011^{47}(21752121) \\
\text { Family Mutual Support Program in Dementia Care (FMSP-DC) vs. usual care } \\
\text { Medium Explanatory } \\
\text { USA }\end{array}$ & $\begin{array}{l}\text { Mean (SD) baseline } \\
\text { Mean (SD) } 6 \text { months } \\
\text { Caregiver social support: Six-item Social Support } \\
\text { Questionnaire (SSQ6) - satisfaction subscale } \\
6 \text { months }\end{array}$ & NR & $\begin{array}{l}5.3(1.8) \text { baseline } \\
6.2(3.1) 6 \text { months }\end{array}$ & $\begin{array}{l}5.5(1.1) \text { baseline } \\
5.6(2.1) 6 \text { months }\end{array}$ & NS \\
\hline $\begin{array}{l}\text { Gitlin } 2010^{49}(20662955) \\
\text { Advancing Caregiver Training (ACT) vs. no treatment control } \\
\text { Medium } \\
\text { Explanatory } \\
\text { USA }\end{array}$ & $\begin{array}{l}\text { Adjusted mean difference }(95 \% \mathrm{Cl}) \\
\text { Cohen's D } \\
\text { Caregiver upset with PLWD problem behaviors } \\
4 \text { months }\end{array}$ & $\begin{array}{l}-0.93(-1.76 \text { to }-0.10) \\
0.30\end{array}$ & $\mathrm{NR}$ & NR & 0.03 \\
\hline $\begin{array}{l}\text { Gittin } 2010^{49}(20662955) \\
\text { Advancing Caregiver Training (ACT) vs. no treatment control } \\
\text { Medium }\end{array}$ & $\begin{array}{l}\text { Adjusted mean difference }(95 \% \mathrm{Cl}) \\
\text { Cohen's D }\end{array}$ & $\begin{array}{l}0.33(0.08 \text { to } 0.58) \\
0.30\end{array}$ & NR & NR & 0.01 \\
\hline
\end{tabular}




\begin{tabular}{|c|c|c|c|c|c|}
\hline $\begin{array}{l}\text { Study (PMID) } \\
\text { Comparison } \\
\text { Caregiver Type } \\
\text { RoB } \\
\text { Category* } \\
\text { Country }\end{array}$ & $\begin{array}{l}\text { Outcome } \\
\text { Timing }\end{array}$ & Summary Finding & Intervention & Comparator & p-Value \\
\hline $\begin{array}{l}\text { Explanatory } \\
\text { USA }\end{array}$ & $\begin{array}{l}\text { Caregiver confidence managing PLWD problem behaviors } \\
4 \text { months }\end{array}$ & & & & \\
\hline $\begin{array}{l}\text { Gitlin } 2010^{49}(20662955) \\
\text { Advancing Caregiver Training (ACT) vs. no treatment control } \\
\text { Medium } \\
\text { Explanatory } \\
\text { USA }\end{array}$ & $\begin{array}{l}\text { Adjusted mean difference }(95 \% \mathrm{Cl}) \\
\text { Caregiver skills: task simplification strategies } \\
4 \text { months }\end{array}$ & $0.06(-0.03$ to 0.15$)$ & $\mathrm{NR}$ & NR & 0.21 \\
\hline $\begin{array}{l}\text { Gitlin } 2010^{49}(20662955) \\
\text { Advancing Caregiver Training (ACT) vs. no treatment control } \\
\text { Medium } \\
\text { Explanatory } \\
\text { USA }\end{array}$ & $\begin{array}{l}\text { Adjusted mean difference }(95 \% \mathrm{CI}) \\
\text { Caregiver burden: Zarit Caregiver Burden Interview (ZBI) } \\
4 \text { months }\end{array}$ & $-1.37(-2.75$ to 0.01$)$ & $\mathrm{NR}$ & $\mathrm{NR}$ & 0.05 \\
\hline $\begin{array}{l}\text { Gitlin } 2010^{49}(20662955) \\
\text { Advancing Caregiver Training (ACT) vs. no treatment control } \\
\text { Medium } \\
\text { Explanatory } \\
\text { USA }\end{array}$ & $\begin{array}{l}\text { Percentage with CES-D score > } \\
\text { Caregiver depressive symptoms: Centers for Epidemiologic } \\
\text { Study Depression Scale (CES-D) } \\
4 \text { months }\end{array}$ & NR & 53.0 & 67.8 & $\begin{array}{l}0.02 \text { for chi-square } \\
\text { test }\end{array}$ \\
\hline $\begin{array}{l}\text { Gitlin } 2010^{49}(20662955) \\
\text { Advancing Caregiver Training (ACT) vs. no treatment control } \\
\text { Medium } \\
\text { Explanatory } \\
\text { USA }\end{array}$ & $\begin{array}{l}\text { Adjusted mean difference }(95 \% \mathrm{Cl}) \\
\text { Cohen's D } \\
\text { Caregiver affect: } \\
4 \text { months }\end{array}$ & $\begin{array}{l}0.46(0.27-0.65) \\
0.58\end{array}$ & NR & NR & 0.004 \\
\hline $\begin{array}{l}\text { Martin-Carrasco } 2009^{54}(18949763) \\
\text { Psychoedcuational Intervention Program to teach strategies for confronting problems of } \\
\text { PLWD care vs. usual care } \\
\text { Medium } \\
\text { Explanatory } \\
\text { Spain }\end{array}$ & $\begin{array}{l}\text { Mean change score (SD) } \\
\text { Caregiver burden: Zarit caregiver burden interview } \\
10 \text { months }\end{array}$ & NR & $-8.1(17.3)$ & $2.1(16.5)$ & .0083 \\
\hline $\begin{array}{l}\text { Martin-Carrasco } 2009^{54}(18949763) \\
\text { Psychoedcuational Intervention Program to teach strategies for confronting problems of } \\
\text { PLWD care vs. usual care } \\
\text { Medium } \\
\text { Explanatory } \\
\text { Spain }\end{array}$ & $\begin{array}{l}\text { Mean (SD) } \\
\text { Caregiver quality of life: Spanish SF-36 physical functioning } \\
\text { subscale } \\
10 \text { months }\end{array}$ & NR & $80.1(20.7)$ & $68.8(25.6)$ & 0.0310 \\
\hline $\begin{array}{l}\text { Martin-Carrasco } 2009^{54}(18949763) \\
\text { Psychoedcuational Intervention Program to teach strategies for confronting problems of } \\
\text { PLWD care vs. usual care } \\
\text { Medium } \\
\text { Explanatory } \\
\text { Spain }\end{array}$ & $\begin{array}{l}\text { Mean (SD) } \\
\text { Caregiver quality of life: Spanish SF-36 physical role subscale } \\
10 \text { months }\end{array}$ & NR & $84.7(34.6)$ & $56.8(47.0)$ & 0.0074 \\
\hline $\begin{array}{l}\text { Martin-Carrasco } 2009^{54}(18949763) \\
\text { Psychoedcuational Intervention Program to teach strategies for confronting problems of } \\
\text { PLWD care vs. usual care } \\
\text { Medium } \\
\text { Explanatory } \\
\text { Spain }\end{array}$ & $\begin{array}{l}\text { Mean (SD) } \\
\text { Caregiver quality of life: Spanish SF-36 bodily pain subscale } \\
10 \text { months }\end{array}$ & NR & $74.0(18.7)$ & $61.7(26.9)$ & 0.0157 \\
\hline $\begin{array}{l}\text { Martin-Carrasco } 2009^{54}(18949763) \\
\text { Psychoedcuational Intervention Program to teach strategies for confronting problems of } \\
\text { PLWD care vs. usual care } \\
\text { Medium } \\
\text { Explanatory } \\
\text { Spain }\end{array}$ & $\begin{array}{l}\text { Mean (SD) } \\
\text { Caregiver quality of life: Spanish SF-36 General Health } \\
\text { subscale } \\
10 \text { months }\end{array}$ & NR & $53.4(18.0)$ & $40.1(15.7)$ & 0.0011 \\
\hline $\begin{array}{l}\text { Martin-Carrasco } 2009^{54}(18949763) \\
\text { Psychoedcuational Intervention Program to teach strategies for confronting problems of } \\
\text { PLWD care vs. usual care }\end{array}$ & $\begin{array}{l}\text { Mean (SD) } \\
\text { Caregiver quality of life: Spanish SF-36 Vitality } \\
10 \text { months }\end{array}$ & NR & $53.8(15.9)$ & $38.9(17.9)$ & 0.0002 \\
\hline
\end{tabular}




\begin{tabular}{|c|c|c|c|c|c|}
\hline $\begin{array}{l}\text { Study (PMID) } \\
\text { Comparison } \\
\text { Caregiver Type } \\
\text { RoB } \\
\text { Category* } \\
\text { Country }\end{array}$ & \begin{tabular}{|l} 
Outcome \\
Timing
\end{tabular} & \begin{tabular}{|l|} 
Summary Finding \\
\end{tabular} & Intervention & Comparator & p-Value \\
\hline \multicolumn{6}{|l|}{$\begin{array}{l}\text { Medium } \\
\text { Explanatory } \\
\text { Spain }\end{array}$} \\
\hline $\begin{array}{l}\text { Martin-Carrasco } 2009^{54}(18949763) \\
\text { Psychoedcuational Intervention Program to teach strategies for confronting problems of } \\
\text { PLWD care vs. usual care } \\
\text { Medium Explanatory } \\
\text { Expain }\end{array}$ & $\begin{array}{l}\text { Mean (SD) } \\
\text { Caregiver quality of life: Spanish SF-36 Social Functioning } \\
10 \text { months }\end{array}$ & NR & $71.0(23.4)$ & \begin{tabular}{|l|}
$58.9(27.7)$ \\
\end{tabular} & 0.0488 \\
\hline $\begin{array}{l}\text { Martin-Carrasco } 2009^{54}(18949763) \\
\text { Psychoedcuational Intervention Program to teach strategies for confronting problems of } \\
\text { PLWD care vs. usual care } \\
\text { Medium } \\
\text { Explanatory } \\
\text { Spain }\end{array}$ & \begin{tabular}{|l|} 
Mean (SD) \\
Caregiver quality of life: Spanish SF-36 Emotional Role \\
10 months
\end{tabular} & NR & 73.5 (41.0) & $47.4(48.2)$ & 0.0160 \\
\hline $\begin{array}{l}\text { Martin-Carrasco } 2009^{54}(18949763) \\
\text { Psychoedcuational Intervention Program to teach strategies for confronting problems of } \\
\text { PLWD care vs. usual care } \\
\text { Medium } \\
\text { Explanatory } \\
\text { Spain }\end{array}$ & $\begin{array}{l}\text { Mean (SD) } \\
\text { Caregiver quality of life: Spanish SF-36 Mental Health } \\
10 \text { months }\end{array}$ & NR & $63.0(9.2)$ & $60.9(8.3)$ & 0.3197 \\
\hline $\begin{array}{l}\text { Martin-Carrasco } 2009^{54}(18949763) \\
\text { Psychoedcuational Intervention Program to teach strategies for confronting problems of } \\
\text { PLWD care vs. usual care } \\
\text { Medium } \\
\text { Explanatory } \\
\text { Spain }\end{array}$ & $\begin{array}{l}\text { Mean (SD) } \\
\text { Caregiver mental health status: General Health Questionnaire } \\
\text { (GHQ-28) } \\
4 \text { months }\end{array}$ & NR & $4.7(7.2)$ & $6.3(6.6)$ & 0.0340 \\
\hline $\begin{array}{l}\text { Martin-Carrasco } 2009^{54}(18949763) \\
\text { Psychoedcuational Intervention Program to teach strategies for confronting problems of } \\
\text { PLWD care vs. usual care } \\
\text { Medium } \\
\text { Explanatory } \\
\text { Spain }\end{array}$ & $\begin{array}{l}\text { Mean (SD) } \\
\text { Caregiver mental health status: General Health Questionnaire } \\
\text { (GHQ-28) } \\
10 \text { months }\end{array}$ & NR & $2.2(4.0)$ & $7.8(7.6)$ & 0.0004 \\
\hline $\begin{array}{l}\text { Martin-Carrasco } 2009^{54}(18949763) \\
\text { Psychoedcuational Intervention Program to teach strategies for confronting problems of } \\
\text { PLWD care vs. usual care } \\
\text { Medium } \\
\text { Explanatory } \\
\text { Spain }\end{array}$ & $\begin{array}{l}\text { Frequency (percent) } \\
\text { Caregiver healthcare and social resources use: number of } \\
\text { visits } \\
4 \text { months }\end{array}$ & NR & $\begin{array}{l}\text { O visits: } 34 / 44(77 \%) \\
\text { 1-2 visits: } 10 / 44 \\
\text { (23\%) }\end{array}$ & $\begin{array}{l}0 \text { visits: } 29 / 38(77 \%) \\
1-2 \text { visits: } 9 / 38(23 \%)\end{array}$ & \begin{tabular}{|l}
$N R(N S)$ \\
\end{tabular} \\
\hline $\begin{array}{l}\text { Martin-Carrasco } 2009^{54}(18949763) \\
\text { Psychoedcuational Intervention Program to teach strategies for confronting problems of } \\
\text { PLWD care vs. usual care } \\
\text { Medium } \\
\text { Explanatory }\end{array}$ & $\begin{array}{l}\text { Mean (SD) } \\
\text { Caregiver healthcare and social resources use: time spent on } \\
\text { medical care } \\
4 \text { months }\end{array}$ & NR & 24.6 (19.6) & \begin{tabular}{|l|}
$14.5(10.1)$ \\
\end{tabular} & NR \\
\hline $\begin{array}{l}\text { Gallagher-Thompson } 2008^{56}(25067886) \\
\text { Coping with Caregiving (CWC) vs. minimal telephone-based control condition (TSC) } \\
\text { Medium } \\
\text { Explanatory }\end{array}$ & $\begin{array}{l}\text { Beta (SE) for linear regression model } \\
\text { Caregiver depressive symptoms: CESD Center for } \\
\text { Epidemiological Studies Depression scale (CESD) } \\
6 \text { months }\end{array}$ & \begin{tabular}{|l}
$-2.135(1.073)$ \\
\end{tabular} & NR & NR & ANOVA $p=.048$ \\
\hline $\begin{array}{l}\text { Gallagher-Thompson } 2008^{56}(25067886) \\
\text { Coping with Caregiving (CWC) vs. minimal telephone-based control condition (TSC) } \\
\text { Medium } \\
\text { Explanatory }\end{array}$ & $\begin{array}{l}\text { Beta (SE) for linear regression model } \\
\text { Caregiver perceived psychological stress: Perceived Stress } \\
\text { Scale (PSS-10) } \\
6 \text { months }\end{array}$ & $-1.530(0.760)$ & NR & NR & ANOVA $p=.046$ \\
\hline 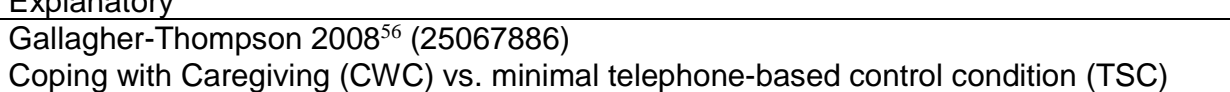 & $\begin{array}{l}\text { Beta (SE) for linear regression model } \\
\text { Caregiver bother of PLWD neuropsychiatric symptoms: }\end{array}$ & \begin{tabular}{|l|}
$-0.250(0.092)$ \\
\end{tabular} & NR & NR & ANOVA $p=.007$ \\
\hline
\end{tabular}




\begin{tabular}{|c|c|c|c|c|c|}
\hline $\begin{array}{l}\text { Study (PMID) } \\
\text { Comparison } \\
\text { Caregiver Type } \\
\text { RoB } \\
\text { Category* } \\
\text { Country }\end{array}$ & \begin{tabular}{|l|} 
Outcome \\
Timing
\end{tabular} & \begin{tabular}{|l} 
Summary Finding \\
\end{tabular} & Intervention & Comparator & p-Value \\
\hline \begin{tabular}{|l} 
Medium \\
Explanatory
\end{tabular} & $\begin{array}{l}\text { Subscale of Revised Memory and Behavior Problem } \\
\text { Checklist (RMBPC-CB) } \\
6 \text { months } \\
\end{array}$ & & & & \\
\hline $\begin{array}{l}\text { Graff } 2006^{60}(17114212) \\
\text { Community based occupational therapy program vs. Waitlist for occupational therapy } \\
\text { Medium } \\
\text { Explanatory }\end{array}$ & $\begin{array}{l}\text { Adjusted Mean difference }(95 \% \mathrm{Cl}) \\
\text { Caregiver competence: Sense of Competence Questionnaire } \\
\text { (SCQ) } \\
6 \text { weeks }\end{array}$ & \begin{tabular}{|l|}
$11.0(9.2 ; 12.8)$ \\
1.2 \\
$2.5(2.3$ to 2.7$) \mathrm{NNT}$
\end{tabular} & NR & NR & $<0.0001$ \\
\hline $\begin{array}{l}\text { Graff } 2006^{60}(17114212) \text { Graff } 2007^{89}(17895439) \\
\text { Community based occupational therapy program vs. Waitlist for occupational therapy } \\
\text { Medium } \\
\text { Explanatory }\end{array}$ & $\begin{array}{l}\text { Adjusted treatment difference }(95 \% \mathrm{Cl}) \\
\text { Effect size } \\
\text { Mean (SD) } \\
\text { Caregiver quality of life: Dementia Quality of Life Instrument } \\
6 \text { weeks }\end{array}$ & \begin{tabular}{|l}
0.7 (0.5 to 0.9$)$ \\
1.2 (effect size)
\end{tabular} & $4.0(0.6)$ & $3.4(0.7)$ & $<0.0001$ \\
\hline $\begin{array}{l}\text { Graff } 2006^{60}(17114212) \text { Graff } 2000^{89}(17895439) \\
\text { Community based occupational therapy program vs. Waitlist for occupational therapy } \\
\text { Medium } \\
\text { Explanatory }\end{array}$ & $\begin{array}{l}\text { Adjusted treatment difference }(95 \% \mathrm{Cl}) \\
\text { Effect size } \\
\text { Mean (SD) } \\
\text { Caregiver depression: Center for Epidemiologic Studies } \\
\text { Depression Scale CES-D } \\
6 \text { weeks } \\
\end{array}$ & \begin{tabular}{|l|}
$7.6(5.3$ to 9.7$)$ \\
1.3 effect size
\end{tabular} & $5.8(4.8)$ & $12.6(8.5)$ & $<0.0001$ \\
\hline $\begin{array}{l}\text { Farran } 2004^{62}(41552352) \\
\text { Caregiver skill building (CSB) vs. Information and support group (ISO) } \\
\text { Medium } \\
\text { Explanatory } \\
\text { USA }\end{array}$ & $\begin{array}{l}\text { Regression coefficient (SE) } \\
\text { Caregiver depression: Center for Epidemiological Studies of } \\
\text { Depression scale (CES-D) } \\
18 \text { months }\end{array}$ & \begin{tabular}{|l}
$0.034(0.032)$ \\
\end{tabular} & NR & NR & 0.707 \\
\hline $\begin{array}{l}\text { Farran } 2004^{62}(41552352) \\
\text { Caregiver skill building (CSB) vs. Information and support group (ISO) } \\
\text { Medium } \\
\text { Explanatory } \\
\text { USA }\end{array}$ & $\begin{array}{l}\text { Regression coefficient (SE) } \\
\text { Caregiver skills: Behavior Management Skill-Revised (BMS- } \\
\text { R) } \\
18 \text { months }\end{array}$ & $\mid-0.006(0.010)$ & NR & NR & 0.538 \\
\hline $\begin{array}{l}\text { Gitlin } 2001^{93}(11220813) \\
\text { Home Environmental Intervention vs. usual care } \\
\text { Medium } \\
\text { Explanatory } \\
\text { USA }\end{array}$ & $\begin{array}{l}\text { Adjusted mean difference }(95 \% \mathrm{Cl}) \\
\text { Mean (SD) baseline } \\
\text { Mean (SD) } 3 \text { month } \\
\text { Caregiver self-efficacy: managing ADL dependency } \\
3 \text { months }\end{array}$ & .03 (-.03; .08) & $\begin{array}{l}.81(.33) \text { baseline } \\
.93(.18) 3 \text { months }\end{array}$ & \begin{tabular}{|l|}
$.80(.34)$ baseline \\
$.90(.21) 3$ months
\end{tabular} & 0.375 \\
\hline $\begin{array}{l}\text { Gitlin } 2001^{93}(11220813) \\
\text { Home Environmental Intervention vs. usual care } \\
\text { Medium } \\
\text { Explanatory } \\
\text { USA }\end{array}$ & $\begin{array}{l}\text { Adjusted mean difference }(95 \% \mathrm{Cl}) \\
\text { Mean (SD) baseline } \\
\text { Mean (SD) } 3 \text { month } \\
\text { Caregiver self-efficacy: managing IADL dependency } \\
3 \text { months }\end{array}$ & .01 (-.03; .05) & $\begin{array}{l}.87(.30) \text { baseline } \\
.96(.15) 3 \text { months }\end{array}$ & \begin{tabular}{|l|}
$.87(.26)$ baseline \\
$.95(.14) 3$ months
\end{tabular} & 0.704 \\
\hline $\begin{array}{l}\text { Gitlin } 2001^{93}(11220813) \\
\text { Home Environmental Intervention vs. usual care } \\
\text { Medium } \\
\text { Explanatory } \\
\text { USA }\end{array}$ & $\begin{array}{l}\text { Adjusted mean difference }(95 \% \mathrm{Cl}) \\
\text { Mean (SD) baseline } \\
\text { Mean (SD) } 3 \text { month } \\
\text { Caregiver self-efficacy: managing behaviors } \\
3 \text { months }\end{array}$ & $.03(-.03 ; .10)$ & $\begin{array}{l}.77(.27) \text { baseline } \\
.84(.24) 3 \text { months }\end{array}$ & $\begin{array}{l}.74(.32) \text { baseline } \\
.80(.27) 3 \text { months }\end{array}$ & 0.314 \\
\hline $\begin{array}{l}\text { Gitlin } 2001^{93}(11220813) \\
\text { Home Environmental Intervention vs. usual care } \\
\text { Medium } \\
\text { Explanatory } \\
\text { USA }\end{array}$ & $\begin{array}{l}\text { Adjusted mean difference }(95 \% \mathrm{Cl}) \\
\text { Mean (SD) baseline } \\
\text { Mean (SD) } 3 \text { month } \\
\text { Caregiver upset: managing ADL dependency } \\
3 \text { months }\end{array}$ & $-.06(-.16 ; .03)$ & $\begin{array}{l}.26(.35) \text { baseline } \\
.25(.34) 3 \text { months }\end{array}$ & \begin{tabular}{|l}
$.29(.36)$ baseline \\
$.34(.37) 3$ months
\end{tabular} & 0.156 \\
\hline $\begin{array}{l}\text { Gitlin } 2001^{93}(11220813) \\
\text { Home Environmental Intervention vs. usual care } \\
\text { Medium } \\
\text { Explanatory } \\
\text { USA }\end{array}$ & $\begin{array}{l}\text { Adjusted mean difference }(95 \% \mathrm{Cl}) \\
\text { Mean (SD) baseline } \\
\text { Mean (SD) 3 month } \\
\text { Caregiver upset: managing IADL dependency } \\
3 \text { months }\end{array}$ &.$- .02(-.10 ; .07)$ & $\begin{array}{l}.17(.30) \text { baseline } \\
.17(.29) 3 \text { months }\end{array}$ & $\begin{array}{l}.22(.33) \text { baseline } \\
.22(.32) 3 \text { months }\end{array}$ & 0.663 \\
\hline $\begin{array}{l}\text { Gitlin } 2001^{93}(11220813) \\
\text { Home Environmental Intervention vs. usual care }\end{array}$ & $\begin{array}{l}\text { Adjusted mean difference }(95 \% \mathrm{Cl}) \\
\text { Mean (SD) baseline }\end{array}$ & $\mid-0.02(-0.09 ; .05)$ & $\begin{array}{l}.48(.27) \\
.43(.31)\end{array}$ & $\begin{array}{l}.47(.30) \\
.45(.29)\end{array}$ & 0.501 \\
\hline
\end{tabular}




\begin{tabular}{|c|c|c|c|c|c|}
\hline $\begin{array}{l}\text { Study (PMID) } \\
\text { Comparison } \\
\text { Caregiver Type } \\
\text { RoB } \\
\text { Category* } \\
\text { Country }\end{array}$ & \begin{tabular}{|l} 
Outcome \\
Timing
\end{tabular} & Summary Finding & Intervention & Comparator & $p$-value \\
\hline $\begin{array}{l}\text { Medium } \\
\text { Explanatory } \\
\text { USA }\end{array}$ & $\begin{array}{l}\text { Mean (SD) } 3 \text { month } \\
\text { Caregiver upset: managing behaviors } \\
3 \text { months }\end{array}$ & & & & \\
\hline
\end{tabular}

Abbreviations: ADL= Activities of Daily Living. CI= Confidence Interval; $\mathrm{IADL}=$ Instrumental Activities of Daily Living; MMSE= Mini-Mental State Examination; NR=Not Reported; PMID=PubMed Identification Number; QOL-AD= the Quality of Life in Alzheimer's; SD= Standard

Deviation: SE= Standard Error

Table E-6. Summary of strength of evidence for PLWD outcomes: psychosocial interventions for caregiver well-being \begin{tabular}{|l|l|l|l|l}
\hline $\begin{array}{l}\text { Outcome } \\
\text { Comparison }\end{array}$ & Timing & $\begin{array}{l}\text { \# Studies/ Design } \\
\text { (n analyzed) }\end{array}$ & Finding or Summary Statistic & Study Limitations \\
\hline
\end{tabular}

\begin{tabular}{|l|l|l|l|}
\hline Comparison & & (n analyzed) & \\
\hline $\begin{array}{l}\text { Depression } \\
\text { Usual care }\end{array}$ & $6-52$ weeks & $\begin{array}{l}4 \text { RCTs } \\
(n=592)\end{array}$ & $\begin{array}{l}1 \text { found benefit } \\
3 \text { found no difference }\end{array}$ \\
\hline
\end{tabular}

\begin{tabular}{|l|l|l|l|}
$\begin{array}{l}\text { Depression } \\
\text { Usual care }\end{array}$ & $6-52$ weeks & $\begin{array}{l}4 \mathrm{RCTs} \\
(\mathrm{n}=592)\end{array}$ & $\begin{array}{l}1 \text { found benefit } \\
3 \text { found no difference }\end{array}$ \\
\hline $\begin{array}{l}\text { Depression } \\
\text { Atention control }\end{array}$ & 52 weeks & $\begin{array}{l}1 \mathrm{RCT} \\
\mathrm{n}=330\end{array}$ & $\begin{array}{l}1 \text { found benefit } \\
0 \text { found no difference }\end{array}$ \\
\hline
\end{tabular}

Attention control

Function
Usual care
Functon

Usual care

Function
Usual care

0 found no difference

Function

6-12 weeks

3 RCTs

Attention control

Neuropsychiatric

symptoms

\begin{tabular}{l|l} 
Usual care & \\
\hline Neuropsychiatric & $4-6$ months
\end{tabular}

Neuropsychiatic
symptoms

symptoms
Attention control

Neuropsychiatric

symptoms
Attention contro

Quality of life

Usual care

Usual care

Quality of life

Attention control

Social suppor
Usual care
Social support

Social support
Attention control

Health care usage

Usual care

Health care usage

Attention control

events
Usual care

Institutionalization

\begin{tabular}{l} 
Usual care \\
\hline Institutionalization
\end{tabular}

6-12 months

\begin{tabular}{ll}
\hline $\mathrm{R} C \mathrm{~s}$ & 2 found benefit \\
$(\mathrm{n}=545)$ & 1 found no difference \\
\hline $\mathrm{RCTs}$ & 0 found benefit \\
\hline
\end{tabular}

0 found benefit

1 found benefit

\begin{tabular}{l|l}
\hline$(n=490)$ & 1 found no difference \\
\hline $4 R C T s$ & 1 found benefit \\
$(n=740)$ & 3 found no difference \\
\hline
\end{tabular}

Institutionalization
Attention control

2 found benefit

\begin{tabular}{l|l}
$\begin{array}{l}2 \mathrm{RCTS} \\
(\mathrm{n}=227)\end{array}$ & $\begin{array}{l}2 \text { found benefit } \\
0 \text { found no difference }\end{array}$ \\
\hline
\end{tabular}

\begin{tabular}{l|l|l}
\hline $12-18$ & 3 RCTs
\end{tabular}

$3 \mathrm{RCTS}$
$(\mathrm{n}=916)$

6 weeks

$1 \mathrm{RCT}$

found no difference

\begin{tabular}{l|l|l|l} 
alization & $6-12$ months & $\begin{array}{l}2 \mathrm{RCTs} \\
(\mathrm{n}=288)\end{array}$ & $\begin{array}{l}1 \text { found benefit } \\
1 \text { found no difference }\end{array}$ \\
\hline lization & $6-18$ months & $\begin{array}{l}2 \mathrm{RCT} \\
(\mathrm{n}=545)\end{array}$ & $\begin{array}{l}0 \text { found benefit } \\
2 \text { found no difference }\end{array}$ \\
\hline
\end{tabular}

0 found benefit
3 found no difference

1 found benefit

0 found benefit

4-12 months

\begin{tabular}{l|l}
2 RCTs \\
$(n=490)$
\end{tabular}

\begin{tabular}{l|l} 
& $\begin{array}{l}1 \mathrm{RCT} \\
(\mathrm{n}=108)\end{array}$ \\
\hline 6 months & $1 \mathrm{RCT}$ \\
\hline
\end{tabular}

\begin{tabular}{l|l}
2 RCTs & 1 found benefit \\
$(\mathrm{n}=490)$ & 1 found no difference \\
\hline RCT & 0 found benefit
\end{tabular}

1 found no difference

4-24 months

$\mathrm{RCT}$
$(\mathrm{n}=250)$

$(n=250)$
RCTs
$(n=652)$

6 months

\begin{tabular}{l|l}
1 found benefit \\
3 found no differe
\end{tabular}

3 found no differen

1 found no difference

1 found no difference

2 found no difference

\begin{tabular}{|l|l|}
\hline Study Limitations \\
\hline Medium \\
\hline Medium \\
\hline Medium \\
\hline Medium \\
\hline Medium \\
\hline Medium \\
\hline Medium \\
\hline Medium \\
\hline Medium \\
\hline Medium \\
\hline Medium \\
\hline Medium \\
\hline Medium \\
\hline Medium \\
\hline Medium \\
\hline Medium \\
\hline
\end{tabular}

\begin{tabular}{|l|l|l|l|}
\hline Consistency & Directness & Precision & Overall Gradel Conclusion \\
\hline Inconsistent & Indirect & Imprecise & Insufficient \\
\hline Unknown & Indirect & Imprecise & Insufficient \\
\hline Inconsistent & Direct & Imprecise & Insufficient \\
\hline Inconsistent & Direct & Imprecise & Insufficient \\
\hline Consistent & Direct & Imprecise & Insufficient \\
\hline Inconsistent & Direct & Imprecise & Insufficient \\
\hline Consistent & Direct & Imprecise & Insufficient \\
\hline Inconsistent & Direct & Imprecise & Insufficient \\
\hline Unknown & Indirect & Imprecise & Insufficient \\
\hline Inconsistent & Indirect & Imprecise & Insufficient \\
\hline Consistent & Indirect & Imprecise & Insufficient \\
\hline Unknown & Direct & Imprecise & Insufficient \\
\hline Unknown & Direct & Imprecise & Insufficient \\
\hline Inconsistent & Direct & Imprecise & Insufficient \\
\hline Unknown & Direct & Imprecise & Insufficient \\
\hline Unknown & Direct & Imprecise & Insufficient \\
\hline Inconsistent & Direct & Imprecise & Insufficient \\
\hline Unknown & Direct & Imprecise & Insufficient \\
\hline
\end{tabular}


Table E-7. Summary of strength of evidence for caregiver outcomes: psychosocial interventions

\begin{tabular}{|c|c|c|c|c|c|c|c|c|}
\hline $\begin{array}{l}\text { Outcome } \\
\text { Comparison }\end{array}$ & Timing & $\begin{array}{l}\text { \# Studies/ Design } \\
\text { (n analyzed) }\end{array}$ & Finding or Summary Statistic & Study Limitations & Consistency & Directness & Precision & Overall Grade/ Conclusion \\
\hline $\begin{array}{l}\text { Anxiety } \\
\text { Attention control }\end{array}$ & 24-52 weeks & \begin{tabular}{|l}
$2 \mathrm{RCT}$ \\
$\mathrm{N}=358$
\end{tabular} & \begin{tabular}{|l|}
0 found benefit \\
2 found no difference
\end{tabular} & Medium & Consistent & Direct & Imprecise & Insufficient \\
\hline $\begin{array}{l}\text { Anxiety } \\
\text { Usual care }\end{array}$ & 6 weeks & \begin{tabular}{|l|}
$1 \mathrm{RCT}$ \\
$\mathrm{n}=170$ \\
\end{tabular} & \begin{tabular}{|l|}
1 found benefit \\
0 found no difference
\end{tabular} & Medium & Unknown & Direct & Imprecise & Insufficient \\
\hline $\begin{array}{l}\text { Anxiety } \\
\text { Usual care }\end{array}$ & 2 years & \begin{tabular}{|l|}
1 RCT \\
$\mathrm{n}=260$ \\
\end{tabular} & $\begin{array}{l}0 \text { found benefit } \\
1 \text { found no difference } \\
\end{array}$ & Low & Unknown & Direct & Imprecise & Insufficient \\
\hline $\begin{array}{l}\text { Burden of care } \\
\text { Buttention control }\end{array}$ & 8-24 weeks & \begin{tabular}{|l|l|}
$5 \mathrm{RCT}$ \\
$\mathrm{N}=725$ \\
\end{tabular} & \begin{tabular}{|l}
3 found benefit \\
2 found no difference \\
\end{tabular} & Medium & Consistent & Direct & Imprecise & Insufficient \\
\hline $\begin{array}{l}\text { Burden of care } \\
\text { Usual care }\end{array}$ & 6-16 weeks & \begin{tabular}{|l|}
$3 \mathrm{RCT}$ \\
$\mathrm{N}=680$ \\
\end{tabular} & \begin{tabular}{|l|}
0 found benefit \\
3 found no difference
\end{tabular} & Medium & Consistent & Direct & Imprecise & Insufficient \\
\hline $\begin{array}{l}\text { Burden of care } \\
\text { Usual care }\end{array}$ & 24-52 weeks & \begin{tabular}{|l}
$5 \mathrm{RCT}$ \\
$\mathrm{N}=561$
\end{tabular} & \begin{tabular}{|l|}
2 found benefit \\
3 found no difference
\end{tabular} & Medium & Inconsistent & Direct & Imprecise & Insufficient \\
\hline $\begin{array}{l}\text { Depressive symptoms } \\
\text { Attention control }\end{array}$ & 8-24 weeks & $\begin{array}{l}\text { 7 RCT } \\
n=976 \\
\end{array}$ & \begin{tabular}{|l|}
6 \\
6 found benefit \\
1 found no difference
\end{tabular} & Medium & Consistent & Direct & Imprecise & Insufficient \\
\hline $\begin{array}{l}\text { Depressive symptoms } \\
\text { Attention control }\end{array}$ & $52-72$ weeks & $\begin{array}{l}3 R C T \\
n=916\end{array}$ & \begin{tabular}{|l|}
0 found benefit \\
3 found no difference
\end{tabular} & Medium & Inconsistent & Direct & Imprecise & Insufficient \\
\hline $\begin{array}{l}\text { Depressive symptoms } \\
\text { Usual care }\end{array}$ & 6-24 weeks & \begin{tabular}{|l|}
$5 \mathrm{RCT}$ \\
$\mathrm{N}=852$ \\
\end{tabular} & \begin{tabular}{|l|}
2 found benefit \\
3 found no difference \\
\end{tabular} & Medium & Consistent & Direct & Imprecise & Insufficient \\
\hline $\begin{array}{l}\text { Depressive symptoms } \\
\text { Usual care }\end{array}$ & \begin{tabular}{|l}
$52-104$ \\
weeks
\end{tabular} & $\begin{array}{l}\mathrm{RCT} \\
\mathrm{N}=1073\end{array}$ & $\begin{array}{l}2 \text { found benefit } \\
3 \text { found no difference }\end{array}$ & Medium & Inconsistent & Indirect & Imprecise & Insufficient \\
\hline $\begin{array}{l}\text { Caregiving } \\
\text { bother/distress/affect } \\
\text { Attention control }\end{array}$ & 4-12 months & $\begin{array}{l}5 \mathrm{RCT} \\
\mathrm{N}=952\end{array}$ & \begin{tabular}{|l}
3 found benefit \\
2 found no difference \\
Have means SD \\
\end{tabular} & Medium & Inconsistent & Direct & Imprecise & Insufficient \\
\hline $\begin{array}{l}\text { Caregiving } \\
\text { bother/distress/affect } \\
\text { Usual care }\end{array}$ & 6-24 weeks & $\begin{array}{l}4 \mathrm{RCT} \\
\mathrm{N}=702\end{array}$ & $\begin{array}{l}1 \text { found benefit } \\
3 \text { found no difference }\end{array}$ & Medium & Consistent & Direct & Imprecise & Insufficient \\
\hline $\begin{array}{l}\text { Health care usage } \\
\text { Attention control }\end{array}$ & 3-6 months & $\begin{array}{l}2 R C T \\
n=359\end{array}$ & \begin{tabular}{|l|}
1 found benefit \\
1 found no difference \\
\end{tabular} & Medium & Inconsistent & Direct & Imprecise & Insufficient \\
\hline $\begin{array}{l}\text { Health care usage } \\
\text { Usual care }\end{array}$ & 4-24 months & $\begin{array}{l}2 \mathrm{RCT} \\
\mathrm{n}=251\end{array}$ & \begin{tabular}{|l|}
0 found benefit \\
2 found no difference
\end{tabular} & Medium & Inconsistent & Direct & Imprecise & Insufficient \\
\hline $\begin{array}{l}\text { Caregiving knowledge } \\
\text { Usual care }\end{array}$ & 24 weeks & $\begin{array}{l}\text { RCT } \\
n=167\end{array}$ & \begin{tabular}{|l}
1 found benefit \\
0 found no difference \\
\end{tabular} & Medium & Unknown & Direct & Imprecise & Insufficient \\
\hline $\begin{array}{l}\text { Quality of life } \\
\text { Attention control }\end{array}$ & 8 weeks & $\begin{array}{l}\text { RCT } \\
\mathrm{n}=132 \\
\end{array}$ & \begin{tabular}{|l}
1 found benefit \\
0 found no difference
\end{tabular} & Medium & Unknown & Direct & Imprecise & Insufficient \\
\hline $\begin{array}{l}\text { Quality of life } \\
\text { Attention control }\end{array}$ & 24-52 weeks & $\begin{array}{l}1-102 \\
2 R C T \\
n=541 \\
\end{array}$ & $\begin{array}{l}0 \text { found benefit } \\
2 \text { found no difference } \\
\end{array}$ & Medium & Inconsistent & Direct & Imprecise & Insufficient \\
\hline $\begin{array}{l}\text { Quality of life } \\
\text { Usual care }\end{array}$ & 6-24 weeks & $\begin{array}{l}7 \mathrm{RCT} \\
\mathrm{n}=843\end{array}$ & $\begin{array}{l}4 \text { found benefit } \\
3 \text { found no difference }\end{array}$ & Medium & Inconsistent & Direct & Imprecise & Insufficient \\
\hline $\begin{array}{l}\text { Quality of life } \\
\text { Usual care }\end{array}$ & \begin{tabular}{|l|}
9 months to \\
2 years \\
\end{tabular} & $\begin{array}{l}4 \mathrm{RCT} \\
\mathrm{N}=652 \\
\end{array}$ & \begin{tabular}{|l|}
1 found benefit \\
3 found no difference \\
\end{tabular} & Medium & Inconsistent & Direct & Imprecise & Insufficient \\
\hline $\begin{array}{l}\text { Relationship with PLWD } \\
\text { Attention control }\end{array}$ & 20-52 weeks & $\begin{array}{l}3 R C T \\
n=652\end{array}$ & \begin{tabular}{|l|}
1 found benefit \\
2 found no difference
\end{tabular} & Medium & Inconsistent & Direct & Imprecise & Insufficient \\
\hline $\begin{array}{l}\text { Caregiving confidence } \\
\text { Attention control }\end{array}$ & 20-24 weeks & $\begin{array}{l}3 \mathrm{RCT} \\
\mathrm{N}=428\end{array}$ & \begin{tabular}{|l|}
2 found benefit \\
1 found no difference
\end{tabular} & Medium & Consistent & Direct & Imprecise & Insufficient \\
\hline $\begin{array}{l}\text { Caregiving confidence } \\
\text { Usual care }\end{array}$ & $12-24$ weeks & \begin{tabular}{|l|} 
3 RCT \\
$\mathrm{N}=582$ \\
\end{tabular} & \begin{tabular}{|l|l|}
1 found benefit \\
2 found no difference
\end{tabular} & Medium & Consistent & Direct & Imprecise & Insufficient \\
\hline $\begin{array}{l}\text { Caregiving skill } \\
\text { Attention control }\end{array}$ & 6-18 months & $\begin{array}{l}2 \mathrm{RCT} \\
\mathrm{N}=545\end{array}$ & \begin{tabular}{|l}
0 found benefit \\
2 found no difference
\end{tabular} & Medium & Inconsistent & Direct & Imprecise & Insufficient \\
\hline
\end{tabular}




\begin{tabular}{|c|c|c|c|c|c|c|c|c|}
\hline $\begin{array}{l}\text { Caregiving skill } \\
\text { Usual care }\end{array}$ & 6-16 weeks & $\begin{array}{l}2 \mathrm{RCT} \\
\mathrm{N}=407\end{array}$ & $\begin{array}{l}1 \text { found benefit } \\
1 \text { found no difference }\end{array}$ & Medium & Consistent & Direct & Imprecise & Insufficient \\
\hline $\begin{array}{l}\text { Caregiving skill } \\
\text { Usual care }\end{array}$ & 24-52 weeks & $\begin{array}{l}3 \mathrm{RCT} \\
\mathrm{N}=444\end{array}$ & $\begin{array}{l}0 \text { found benefit } \\
3 \text { found no difference }\end{array}$ & Medium & Inconsistent & Direct & Imprecise & Insufficient \\
\hline \begin{tabular}{|l|} 
Stress \\
Attention
\end{tabular} & 6 months & $\begin{array}{l}\mathrm{RCT} \\
\mathrm{N}=184 \\
\end{array}$ & $\begin{array}{l}1 \text { found benefit } \\
0 \text { found no difference }\end{array}$ & Medium & Unknown & Direct & Imprecise & Insufficient \\
\hline \begin{tabular}{|l|} 
Stress \\
Usual care
\end{tabular} & 6-52 weeks & $\begin{array}{l}\mathrm{RCT} \\
\mathrm{n}=486\end{array}$ & $\begin{array}{l}0 \text { found benefit } \\
3 \text { found no difference }\end{array}$ & Medium & Inconsistent & Direct & Imprecise & Insufficient \\
\hline $\begin{array}{l}\text { Social support } \\
\text { Attention control }\end{array}$ & 6 months & $\begin{array}{l}1 \mathrm{RCT} \\
\mathrm{N}=250 \\
\end{array}$ & $\begin{array}{l}1 \text { found benefit } \\
0 \text { found no difference }\end{array}$ & Medium & Unknown & Direct & Imprecise & Insufficient \\
\hline $\begin{array}{l}\text { Social support } \\
\text { Usual care }\end{array}$ & $12-24$ weeks & $\begin{array}{l}2 \mathrm{RCT} \\
\mathrm{n}=188\end{array}$ & $\begin{array}{l}0 \text { found benefit } \\
2 \text { found no difference }\end{array}$ & Medium & Inconsistent & Direct & Imprecise & Insufficient \\
\hline $\begin{array}{l}\text { Caregiving coping } \\
\text { Usual care }\end{array}$ & 24 weeks & \begin{tabular}{|l} 
1 RCT \\
$n=167$
\end{tabular} & $\begin{array}{l}1 \text { found benefit } \\
0 \text { found no difference }\end{array}$ & Medium & Unknown & Direct & Imprecise & Insufficient \\
\hline
\end{tabular}

\section{Evidence Map: Psychosocial Interventions for Caregiver Well-Being}

\begin{tabular}{|c|c|c|c|c|c|c|c|c|c|c|c|c|c|}
\hline $\begin{array}{l}\text { Study (PMID) } \\
\text { Country } \\
\text { EM Reason }\end{array}$ & Intervention & Comparison & $\begin{array}{l}\text { Setting } \\
\text { and Design } \\
\text { Setting } \\
\text { Design } \\
\text { Cluster N } \\
\text { Participants } \\
\text { Random- } \\
\text { ized N }\end{array}$ & $\begin{array}{l}\text { PLWD } \\
\text { Dementia } \\
\text { Characteristics }\end{array}$ & $\begin{array}{l}\text { PLWD } \\
\text { Non-Disease Char } \\
\text { PLWD N } \\
\text { PLWD Age (mean) } \\
\text { PLWD Sex (\% } \\
\text { female) } \\
\text { PLWD Race (\% } \\
\text { majority) } \\
\text { PLWD Education } \\
\text { (mean years) }\end{array}$ & $\begin{array}{l}\text { PLWD } \\
\text { Non-Disease } \\
\text { Char Reporting } \\
\text { Status (RS) } \\
\text { PLWD SES } \\
\text { PLWD Prior } \\
\text { Disability } \\
\text { PLWD } \\
\text { Household } \\
\text { Characteristics } \\
\text { PLWD Health } \\
\text { Insurance } \\
\text { PLWD Detailed } \\
\text { Race Information }\end{array}$ & $\begin{array}{l}\text { Informal Caregiver } \\
\text { (IC) } \\
\text { Characteristics } \\
\text { IC N } \\
\text { IC Age (mean) } \\
\text { IC Sex (\% female) } \\
\text { IC Race (\% } \\
\text { majority) } \\
\text { IC Education } \\
\text { (mean years) } \\
\text { IC Relation to } \\
\text { PLWD (\% majority) }\end{array}$ & $\begin{array}{l}\text { Informal Caregiver } \\
\text { (IC) Char. RS } \\
\text { IC Duration } \\
\text { IC Living With } \\
\text { PLWD } \\
\text { IC Payment } \\
\text { IC Health Status } \\
\text { IC Dementia } \\
\text { Family History } \\
\text { IC Employment } \\
\text { Status } \\
\text { IC Training }\end{array}$ & $\begin{array}{l}\text { Formal Caregiver } \\
\text { (FC) } \\
\text { Characteristics } \\
\text { FC N } \\
\text { FC Age (mean) } \\
\text { FC Sex (\% } \\
\text { female) } \\
\text { FC Race (\% } \\
\text { majority) } \\
\text { FC Education } \\
\text { (mean years) }\end{array}$ & $\begin{array}{l}\text { Formal } \\
\text { Caregiver (FC) } \\
\text { Char. RS } \\
\\
\text { FC Health } \\
\text { Status } \\
\text { FC Training } \\
\text { FC Education } \\
\text { FC Position } \\
\text { FC Length of } \\
\text { Service }\end{array}$ & $\begin{array}{l}\text { Outcome } \\
\text { Timing(s) }\end{array}$ & \begin{tabular}{|l|} 
PLWD \\
Outcomes
\end{tabular} & $\begin{array}{l}\text { Caregiver } \\
\text { Outcomes } \\
\text { IC: } \\
\text { FC: }\end{array}$ \\
\hline $\begin{array}{l}\text { Meichsner, } \\
2019^{95} \\
(31384663) \\
\\
\text { Germany } \\
\text { Small Sample }\end{array}$ & \begin{tabular}{|l|} 
CBT \\
intervention \\
delivered via \\
internet, weekly \\
message \\
exchanges with \\
therapist, 8 \\
weeks
\end{tabular} & No treatment & \begin{tabular}{|l} 
Community \\
$2 \times 3$ RCT \\
Caregivers
\end{tabular} & $\begin{array}{l}\text { Dementia, } \\
\text { unspecified }\end{array}$ & $\begin{array}{l}\mathrm{N}=37 \\
\text { Age: yes } \\
\text { Sex: yes } \\
\text { Race: no } \\
\text { Education: no }\end{array}$ & NR & \begin{tabular}{|l|}
$\mathrm{N}=37$ \\
Age: $y$ yes \\
Sex: yes \\
Race: $n$ \\
Education: yes \\
Relation to PLWD: \\
yes
\end{tabular} & $\begin{array}{l}\text { Duration: yes } \\
\text { Living with PLWD: } \\
\text { yes }\end{array}$ & NA & NA & $\begin{array}{l}\text { Post } \\
\text { intervention, } 3 \\
\text { months post } \\
\text { intervention }\end{array}$ & NA & $\begin{array}{l}\text { CES-D } \\
\text { CGS } \\
\text { Burden of } \\
\text { care } \\
\text { Emotional } \\
\text { wellbeing }\end{array}$ \\
\hline $\begin{array}{l}\text { Orgeta, 201996 } \\
\text { (31609689) } \\
\\
\text { England } \\
\text { Pilot }\end{array}$ & \begin{tabular}{|l|} 
Weekly \\
individual \\
behavioral \\
activation \\
intervention \\
delivered at \\
home, 8 weeks, \\
1-hour sessions \\
\end{tabular} & $\begin{array}{l}\text { Treatment as } \\
\text { usual }\end{array}$ & $\begin{array}{l}\text { Community } \\
\text { RCT } \\
\text { Caregiver- } \\
\text { PLWD } \\
\text { Dyads }\end{array}$ & $\begin{array}{l}\text { Diagnosis of } \\
\text { dementia of any } \\
\text { type, defined by an } \\
\text { MMSE score of } \\
\geq 18\end{array}$ & $\begin{array}{l}\mathrm{N}=63 \\
\text { Age: } \text { yes } \\
\text { Sex: yes } \\
\text { Race: yes } \\
\text { Education: yes }\end{array}$ & Household status & \begin{tabular}{|l} 
N=63 \\
Age: yes \\
Sex: yes \\
Race: yes \\
Education: yes \\
Relation to PLWD: \\
yes
\end{tabular} & Health Status: yes & NA & NA & $\begin{array}{l}3 \text { months after } \\
\text { start of } \\
\text { treatment, } 6 \\
\text { months after } \\
\text { start of } \\
\text { treatment }\end{array}$ & \begin{tabular}{|l|} 
CSDD \\
BADLS \\
DEMQOL \\
(self and \\
proxy) \\
EQ-5D (self \\
and proxy) \\
NPI \\
\end{tabular} & $\begin{array}{l}\text { SF-12 } \\
\text { HADS } \\
\text { EQ-5D }\end{array}$ \\
\hline
\end{tabular}




\begin{tabular}{|c|c|c|c|c|c|c|c|c|c|c|c|c|c|}
\hline $\begin{array}{l}\text { Ta Park, 201997 } \\
(31609689) \\
(\text { Embase } \\
629157769)^{98} \\
\text { United States } \\
\text { Pilot }\end{array}$ & $\begin{array}{l}\text { Weekly group } \\
\text { sessions of } \\
\text { Vietnamese- } \\
\text { language } \\
\text { cognitive- } \\
\text { behavioral skills } \\
\text { evidence-based } \\
\text { program, } 4 \\
\text { weeks, 120- } \\
\text { minute sessions }\end{array}$ & $\begin{array}{l}\text { Received } \\
\text { educational } \\
\text { materials on } \\
\text { dementia }\end{array}$ & \begin{tabular}{|l|} 
Community \\
RCT \\
Caregivers
\end{tabular} & \begin{tabular}{|l|} 
Dementia or \\
neurocognitive \\
disorder, memory \\
loss confirmed by \\
Montreal Cognitive \\
Assessment if in \\
doubt
\end{tabular} & $\mathrm{NR}$ & $\mathrm{NR}$ & $\begin{array}{l}\mathrm{N}=60 \\
\text { Sex: yes } \\
\text { Race: yes } \\
\text { Education: yes } \\
\text { Relation to PLWD: } \\
\text { Yes }\end{array}$ & $\begin{array}{l}\text { Living with PLWD: } \\
\text { yes } \\
\text { Employment Status: } \\
\text { yes }\end{array}$ & NA & NA & $\begin{array}{l}3 \text { months post } \\
\text { enrollment in } \\
\text { study }\end{array}$ & $\mathrm{NR}$ & $\begin{array}{l}\text { CES-D } \\
\text { RMBPC } \\
\text { (Conditional } \\
\text { Bother } \\
\text { Subscale) }\end{array}$ \\
\hline $\begin{array}{l}\text { Terracciano } \\
2019^{9} \\
(31866419) \\
\\
\text { United States } \\
\text { High ROB }\end{array}$ & $\begin{array}{l}\text { Powerful Tools } \\
\text { for Caregivers } \\
\text { educational } \\
\text { progeram } \\
\text { Weekly 2-hours } \\
\text { classes for 6 } \\
\text { weeks }\end{array}$ & Waitlist control & $\begin{array}{l}\text { Community- } \\
\text { based } \\
\mathrm{RCT} \\
\mathrm{N}=60 \\
\text { informal } \\
\text { caregivers }\end{array}$ & $\begin{array}{l}\text { Dementia } \\
\text { diagnosis } \\
\text { BPSD symptoms } \\
\text { (assessed with } \\
\text { RMBPC) }\end{array}$ & $\begin{array}{l}\text { N=60 } \\
\text { Age: yes } \\
\text { Sex: yes } \\
\text { Race: no } \\
\text { Education: no }\end{array}$ & NR & $\begin{array}{l}\text { N=60 } \\
\text { Age: yes } \\
\text { Sex: yes } \\
\text { Race: yes } \\
\text { Education: no } \\
\text { Relation to PLWD: } \\
\text { yes }\end{array}$ & $\begin{array}{l}\text { Duration: yes } \\
\text { Living with PLWD: } \\
\text { no } \\
\text { Employment Status: } \\
\text { yes }\end{array}$ & NA & NA & 6 weeks & $\begin{array}{l}\text { RMBPC } \\
\text { CMAI }\end{array}$ & $\begin{array}{l}\text { ZBI } \\
\text { CES-D } \\
\text { NPI } \\
\text { Caregiver } \\
\text { satisfaction }\end{array}$ \\
\hline $\begin{array}{l}\text { Uyar 201910 (no } \\
\text { PMID) } \\
\text { Turkey } \\
\text { High ROB }\end{array}$ & $\begin{array}{l}\text { Dementia Care } \\
\text { and Support } \\
\text { Program } \\
\text { Multicomponent } \\
\text { intervention } \\
\text { 8 sessions over } \\
16 \text { weeks } \\
\end{array}$ & Waitlist control & \begin{tabular}{|l|} 
Community- \\
based \\
$\mathrm{RCT}$ \\
$\mathrm{N}=61$ \\
informal \\
caregivers \\
\end{tabular} & \begin{tabular}{|l|} 
Dementia \\
diagnosis \\
Physician- \\
diagnosed
\end{tabular} & $\mathrm{NR}$ & $\mathrm{NR}$ & $\begin{array}{l}\mathrm{N}=61 \\
\text { Age: } \text { yes } \\
\text { Sex: yes } \\
\text { Race: no } \\
\text { Education: yes } \\
\text { Relation to PLWD: } \\
\text { yes }\end{array}$ & $\begin{array}{l}\text { Duration: no } \\
\text { Living with PLWD: } \\
\text { no } \\
\text { Employment Status: } \\
\text { yes }\end{array}$ & NA & NA & 16 weeks & $\begin{array}{l}\text { QOL-AD } \\
\text { NPI-S }\end{array}$ & $\begin{array}{l}\text { ZBI } \\
\text { SF-36 } \\
\text { BDI } \\
\text { Beck Anxiety } \\
\text { Inventory }\end{array}$ \\
\hline $\begin{array}{l}\text { Wawrziczny } \\
2019^{11} \\
(29665714) \\
\\
\text { France } \\
\text { High ROB }\end{array}$ & $\begin{array}{l}\text { Individualized } \\
\text { psychoeducatio } \\
\text { n for } 7 \text { weeks, } \\
90 \text { in sessions }\end{array}$ & Usual support & \begin{tabular}{|l} 
Community \\
Quasi- \\
experimental \\
Caregivers
\end{tabular} & $\begin{array}{l}\text { Physician } \\
\text { diagnosed } \\
\text { Alzheimer's, mixed, } \\
\text { Lewy body, or } \\
\text { frontotemporal } \\
\text { dementias }\end{array}$ & $\begin{array}{l}\mathrm{N}=102 \\
\text { Age: yes } \\
\text { Sex: no } \\
\text { Race: no } \\
\text { Education: no }\end{array}$ & $\begin{array}{l}\text { Household } \\
\text { characteristics } \\
\text { SES }\end{array}$ & $\begin{array}{l}\text { N=102 } \\
\text { Age: yes } \\
\text { Sex: yes } \\
\text { Race: no } \\
\text { Education: yes } \\
\text { Relation to PLWD: } \\
\text { yes }\end{array}$ & $\begin{array}{l}\text { Duration: yes } \\
\text { Employment: yes }\end{array}$ & NA & NA & 7 weeks & NR & $\begin{array}{l}\text { Caregiving } \\
\text { selffesteem, } \\
\text { distress } \\
\text { Self-efficacy } \\
\text { Depression } \\
\text { Impact on } \\
\text { finances } \\
\text { Self-rated } \\
\text { health }\end{array}$ \\
\hline $\begin{array}{l}\text { Whitlatch } \\
2019^{99} \\
\text { (29171296) } \\
\text { US } \\
\text { High ROB }\end{array}$ & $\begin{array}{l}\text { Psychoeducatio } \\
\text { n } \\
\text { Support, Health, } \\
\text { Activities, } \\
\text { Resources, and } \\
\text { Education } \\
\text { (SHARE) } \\
\text { Program } \\
\text { Dyadic } \\
\text { counseling for } \\
\text { early stage } \\
\text { dementia } \\
6 \text { sessions }\end{array}$ & $\begin{array}{l}\text { Education } \\
\text { One 90-minute } \\
\text { session }\end{array}$ & $\begin{array}{l}\text { Home-based } \\
\text { setting } \\
\mathrm{RCT} \\
\mathrm{N}=128 \\
\text { dyads }\end{array}$ & \begin{tabular}{|l} 
Dementia \\
diagnosis
\end{tabular} & $\begin{array}{l}\mathrm{N}=128 \\
\text { Age: yes } \\
\text { Sex: yes } \\
\text { Race: no } \\
\text { Education: yes }\end{array}$ & NR & $\begin{array}{l}\mathrm{N}=128 \\
\text { Age: yes } \\
\text { Sex: yes } \\
\text { Race: no } \\
\text { Education: yes } \\
\text { Relation to PLWD: } \\
\text { yes }\end{array}$ & $\begin{array}{l}\text { Duration: yes } \\
\text { Living with PLWD: } \\
\text { yes } \\
\text { Employment Status: } \\
\text { yes } \\
\text { Training: no }\end{array}$ & NA & NA & 2 months & NR & $\begin{array}{l}\text { Preferences } \\
\text { for Care } \\
\text { Tasks Scale } \\
\text { Service } \\
\text { Availability } \\
\text { Measure } \\
\text { Dyadic } \\
\text { Relationship } \\
\text { Scale } \\
\text { Care-related } \\
\text { Agreement } \\
\text { Scale } \\
\text { Emotional- } \\
\text { Intimacy } \\
\text { Disruptive } \\
\text { Behavior } \\
\text { Scale } \\
\text { Dementia } \\
\text { Quality of Life }\end{array}$ \\
\hline
\end{tabular}




\begin{tabular}{|c|c|c|c|c|c|c|c|c|c|c|c|c|c|}
\hline $\begin{array}{l}\text { Yoo, 2019100 } \\
(30938110) \\
\text { Korea } \\
\text { Small Sample }\end{array}$ & $\begin{array}{l}\text { Psycho- } \\
\text { educational } \\
\text { intervention, } 4 \\
\text { in-person } \\
\text { sessions } \\
\text { delivered over } \\
8-10 \text { weeks, } 60 \\
\text { minutes } \\
\text { sessions, } 1^{\text {st }} \\
\text { session } \\
\text { delivered to } \\
\text { group, } \\
\text { subsequent } \\
\text { sessions } \\
\text { individual }\end{array}$ & No treatment & \begin{tabular}{|l|} 
Community \\
Multicenter \\
RCT \\
Caregivers
\end{tabular} & \begin{tabular}{|l|} 
Possible and \\
probable \\
Alzheimer's \\
disease per \\
NINCDS-ADRDA \\
and DSM-V criteria, \\
a CDR score of \\
$0.5-2.0$ and an \\
MMSE score of 10- \\
26 , with BPSD \\
within last 6 \\
months to one year
\end{tabular} & $\mathrm{NR}$ & $\mathrm{NR}$ & $\begin{array}{l}\mathrm{N}=76 \\
\text { Age: yes } \\
\text { Sex: yes } \\
\text { Race: no } \\
\text { Education: yes } \\
\text { Relation to PLWD: } \\
\text { yes }\end{array}$ & NR & NA & $\mathrm{NA}$ & $\begin{array}{l}2 \text { weeks post } \\
\text { intervention }\end{array}$ & NA & $\begin{array}{l}\text { ZBI (Korean), } \\
\text { GDS } \\
\text { Philadelphia } \\
\text { Geriatric } \\
\text { Center } \\
\text { Morale Scale } \\
\text { (PGCMS) } \\
\text { CGA-NPI } \\
\text { (burden) } \\
\text { Positive } \\
\text { Affect and } \\
\text { Negative } \\
\text { Affect } \\
\text { Schedule } \\
\text { (PANAS), } \\
\text { Self- } \\
\text { Compassion } \\
\text { Scale (SCS) }\end{array}$ \\
\hline $\begin{array}{l}\text { Boots 2018 } \\
(30006327) \\
\\
(27142676)^{101} \\
(29258980)^{102} \\
\text { Netherlands } \\
\text { High ROB }\end{array}$ & $\begin{array}{l}\text { 8-week, } \\
\text { blended care } \\
\text { self- } \\
\text { management } \\
\text { Partner in } \\
\text { Balance } \\
\text { program } \\
\text { Group modified } \\
\text { mindfulness- } \\
\text { based cognitive } \\
\text { therapy, } 72- \\
\text { hour sessions } \\
\text { over } 10 \text { weeks }\end{array}$ & Waitlist control & $\begin{array}{l}\text { Community- } \\
\text { based } \\
\mathrm{RCT} \\
\mathrm{N}=81 \\
\text { informal } \\
\text { caregivers }\end{array}$ & \begin{tabular}{|l|} 
Dementia \\
diagnosis
\end{tabular} & NR & NR & $\begin{array}{l}\mathrm{N}=81 \\
\text { Age: yes } \\
\text { Sex: yes } \\
\text { Race: no } \\
\text { Education: yes } \\
\text { Relation to PLWD: } \\
\text { yes }\end{array}$ & $\begin{array}{l}\text { Living with PLWD: } \\
\text { yes }\end{array}$ & NA & NA & 8 weeks & NR & $\begin{array}{l}\text { Caregiver } \\
\text { Self-Efficacy } \\
\text { Scale } \\
\text { CESD } \\
\text { Pearlin } \\
\text { Mastery } \\
\text { Scale } \\
\text { Investigation } \\
\text { Choice } \\
\text { Experiments } \\
\text { for the } \\
\text { Preferences } \\
\text { of Older } \\
\text { People } \\
\text { HADS }\end{array}$ \\
\hline $\begin{array}{l}\text { De Oliveira, } \\
2018^{103} \\
(30035341) \\
\text { Brazil } \\
\text { Small Sample }\end{array}$ & $\begin{array}{l}\text { Activity-based } \\
\text { intervention } \\
\text { (TAP) delivered } \\
\text { face-to-face to } \\
\text { dementia PLWD } \\
\text { and their } \\
\text { caregivers, } 8 \\
\text { one-hour } \\
\text { sessions over a } \\
\text { 3-month period }\end{array}$ & $\begin{array}{l}\text { Psychoeducatio } \\
\text { n intervention } \\
\text { delivered face- } \\
\text { to-face to } \\
\text { dementia PLWD } \\
\text { and their } \\
\text { caregivers, } 8 \\
\text { sessions over a } \\
\text { 3-month period }\end{array}$ & $\begin{array}{l}\text { Community } \\
\text { RCT } \\
\text { Caregiver- } \\
\text { PLWD } \\
\text { dyads }\end{array}$ & $\begin{array}{l}\text { Diagnosis of } \\
\text { dementia by } \\
\text { physician, MMSE } \\
\text { score }<24 \text {, and } \\
\text { presence of at least } \\
\text { three types of } \\
\text { neuropsychiatric } \\
\text { symptoms }\end{array}$ & $\begin{array}{l}\mathrm{N}=21 \\
\text { Age: } \text { yes } \\
\text { Sex: yes } \\
\text { Race: } n \text { o } \\
\text { Education: yes }\end{array}$ & NR & $\begin{array}{l}\mathrm{N}=21 \\
\text { Age: yes } \\
\text { Sex: yes } \\
\text { Race: no } \\
\text { Education: no } \\
\text { Relation to PLWD: } \\
\text { yes }\end{array}$ & $\mathrm{NR}$ & NA & $\mathrm{NA}$ & $\begin{array}{l}\text { Post } \\
\text { intervention }\end{array}$ & NPI-C & $\mathrm{ZBI}$ \\
\hline $\begin{array}{l}\text { Kales, 2018 } \\
(29747583) \\
\text { United States } \\
\text { Pilot }\end{array}$ & $\begin{array}{l}\text { Use of } \\
\text { WeCareAdvisor } \\
\text { tool (for } \\
\text { assessment, } \\
\text { management, } \\
\text { and tracking of } \\
\text { BPSD) for one } \\
\text { month, with } \\
\text { weekly check-in } \\
\text { calls of } 20 \\
\text { minutes max }\end{array}$ & No Treatment & $\begin{array}{l}\text { Community } \\
\text { RCT } \\
\text { Caregivers }\end{array}$ & $\begin{array}{l}\text { Clinical diagnosis } \\
\text { of dementia or } \\
\text { MMSE }<24\end{array}$ & $\begin{array}{l}\text { N=57 } \\
\text { Age: yes } \\
\text { Sex: yes } \\
\text { Race: yes } \\
\text { Education: no }\end{array}$ & $\mathrm{NR}$ & $\begin{array}{l}\mathrm{N}=57 \\
\text { Age: } \text { yes } \\
\text { Sex: yes } \\
\text { Race: yes } \\
\text { Education: yes } \\
\text { Relationship: yes }\end{array}$ & $\mathrm{NR}$ & NA & NA & $\begin{array}{l}\text { Post } \\
\text { intervention }\end{array}$ & $\begin{array}{l}\text { Functional } \\
\text { Dependence }\end{array}$ & $\begin{array}{l}\text { Confidence in } \\
\text { Using } \\
\text { Activities, } \\
\text { Caregiver } \\
\text { Distress (NPI- } \\
\text { Q) } \\
\text { Caregiver } \\
\text { Stress } \\
\text { CES-D } \\
\text { ZBI } \\
\text { Negative } \\
\text { Communicati } \\
\text { on Scale }\end{array}$ \\
\hline
\end{tabular}




\begin{tabular}{|c|c|c|c|c|c|c|c|c|c|c|c|c|c|}
\hline $\begin{array}{l}\text { Meichsner, } \\
2018^{105} \\
(30597537) \\
\text { Germany } \\
\text { Pilot }\end{array}$ & $\begin{array}{l}\text { Telephone- } \\
\text { based CBT } \\
\text { intervention } \\
\text { including grief } \\
\text { module } \\
\text { delivered via } 12 \\
\text { 50-minute } \\
\text { individual } \\
\text { therapy } \\
\text { sessions over } \\
\text { six months }\end{array}$ & $\begin{array}{l}\text { Written } \\
\text { information on } \\
\text { dementia and } \\
\text { caregiving }\end{array}$ & \begin{tabular}{|l|} 
Community \\
RCT \\
Caregivers
\end{tabular} & $\begin{array}{l}\text { Diagnosis of } \\
\text { dementia }\end{array}$ & $\begin{array}{l}\mathrm{N}=273 \\
\text { Age: yes } \\
\text { Sex: yes } \\
\text { Race: no } \\
\text { Education: no }\end{array}$ & NR & $\begin{array}{l}\mathrm{N}=273 \\
\text { Age: yes } \\
\text { Sex: yes } \\
\text { Race: no } \\
\text { Education: no } \\
\text { Relation to PLWD: } \\
\text { yes }\end{array}$ & $\begin{array}{l}\text { Duration: yes } \\
\text { Living with PLWD: } \\
\text { yes }\end{array}$ & NA & $\mathrm{NA}$ & $\begin{array}{l}\text { Post } \\
\text { intervention, } \\
\text { six months } \\
\text { post } \\
\text { intervention }\end{array}$ & NR & $\begin{array}{l}\text { Caregiver } \\
\text { Grief Scale } \\
\text { (coping) }\end{array}$ \\
\hline $\begin{array}{l}\text { Novelli, 2018 } \\
\text { Brazil } \\
\text { Pilot }\end{array}$ & $\begin{array}{l}\text { Activity-based } \\
\text { intervention } \\
\text { (TAP), 8 } \\
\text { sessions in the } \\
\text { home over 4- } \\
\text { month period }\end{array}$ & Usual care & $\begin{array}{l}\text { Community } \\
\text { RCT } \\
\text { Caregiver- } \\
\text { PLWD } \\
\text { dyads }\end{array}$ & $\begin{array}{l}\text { Previous diagnosis } \\
\text { of dementia per } \\
\text { NIAAA criteria and } \\
\text { able to perform at } \\
\text { least } 2 \text { basic ADLs, } \\
\text { with presence of } \geq \\
2 \text { BPSD in last } 30 \\
\text { days }\end{array}$ & $\begin{array}{l}\mathrm{N}=30 \\
\text { Age: yes } \\
\text { Sex: yes } \\
\text { Race: no } \\
\text { Education: yes }\end{array}$ & NR & $\begin{array}{l}\mathrm{N}=30 \\
\text { Age: } \text { yes } \\
\text { Sex: yes } \\
\text { Race: no } \\
\text { Education: yes } \\
\text { Relation to PLWD: } \\
\text { yes }\end{array}$ & NR & NA & NA & $\begin{array}{l}\text { Post } \\
\text { Intervention }\end{array}$ & $\begin{array}{l}\mathrm{NPI} \\
\text { (frequency, } \\
\text { severity, and } \\
\text { carer } \\
\text { distress } \\
\text { related to } \\
\text { behaviors), } \\
\text { Quality of } \\
\text { Life Scale }\end{array}$ & $\begin{array}{l}\text { NPI Distress } \\
\text { ZBI } \\
\text { Quality of Life } \\
\text { Scale }\end{array}$ \\
\hline $\begin{array}{l}\text { Spalding-Wilson } \\
2018^{14} \\
\text { (30258974) } \\
\text { US } \\
\text { High ROB }\end{array}$ & $\begin{array}{l}\text { Two day- } \\
\text { intervention } \\
\text { using } \\
\text { techniques } \\
\text { aimed at } \\
\text { fostering self- } \\
\text { care for } \\
\text { caregivers and } \\
\text { improving } \\
\text { communication } \\
\text { between } \\
\text { caregivers }\end{array}$ & Waitlist & $\begin{array}{l}\text { Community- } \\
\text { based } \\
\mathrm{RCT} \\
\mathrm{N}=104 \\
\text { informal } \\
\text { caregivers }\end{array}$ & \begin{tabular}{|l} 
Dementia \\
diagnosis
\end{tabular} & $\begin{array}{l}\text { N=104 } \\
\text { Age: no } \\
\text { Sex: no } \\
\text { Race: no } \\
\text { Education: no }\end{array}$ & NR & $\begin{array}{l}\mathrm{N}=104 \\
\text { Age: yes } \\
\text { Sex: yes } \\
\text { Race: no } \\
\text { Education: yes } \\
\text { Relation to PLWD: } \\
\text { no }\end{array}$ & $\begin{array}{l}\text { Duration: yes } \\
\text { Living with PLWD: } \\
\text { yes }\end{array}$ & NA & NA & $1,3,6$ months & $\mathrm{NR}$ & $\begin{array}{l}\text { PSS } \\
\text { CBI } \\
\text { BDI-II } \\
\text { Beck Anxiety } \\
\text { Inventory }\end{array}$ \\
\hline $\begin{array}{l}\text { Van } \\
\text { Knippenberg, } \\
2011^{107} \\
\text { (30126766) } \\
\text { Netherlands } \\
\text { Pilot }\end{array}$ & $\begin{array}{l}\text { Experience } \\
\text { sampling } \\
\text { method (ESM) } \\
\text { self-monitoring } \\
3 \text { days//week for } \\
6 \text { weeks, with } \\
\text { personalized } \\
\text { face-to-face } \\
\text { feedback } \\
\text { received every } \\
2 \text { weeks OR } \\
\text { ESM self- } \\
\text { monitoring } 3 \\
\text { days/week for } 6 \\
\text { weeks with no } \\
\text { ESM feedback } \\
\text { (semi-structured } \\
\text { well-being } \\
\text { interview } \\
\text { instead) }\end{array}$ & Usual Care & \begin{tabular}{|l|} 
Community \\
RCT \\
Caregivers
\end{tabular} & $\begin{array}{l}\text { Dementia of any } \\
\text { subtype or stage }\end{array}$ & NR & NR & $\begin{array}{l}\text { N=76 } \\
\text { Age: yes } \\
\text { Sex: yes } \\
\text { Race: no } \\
\text { Education: yes } \\
\text { Relation to PLWD }\end{array}$ & $\begin{array}{l}\text { Living with PLWD: } \\
\text { yes }\end{array}$ & NA & NA & $\begin{array}{l}\text { Post } \\
\text { intervention, } 2 \\
\text { months post } \\
\text { intervention }\end{array}$ & $\begin{array}{l}\text { NEO Five } \\
\text { Factor } \\
\text { Inventory } \\
\text { (neuroticism) } \\
\text { Utrecht } \\
\text { Coping List } \\
\text { NPI }\end{array}$ & $\begin{array}{l}\text { Short Sense } \\
\text { of } \\
\text { Competence } \\
\text { Questionnaire } \\
\text { Pearlin } \\
\text { Mastery } \\
\text { Scale } \\
\text { CES-D PSS } \\
\text { HADS } \\
\text { Affect } \\
\text { (positive, } \\
\text { negative) }\end{array}$ \\
\hline $\begin{array}{l}\text { Wilz 2018 } \\
(29190357) \\
\\
(\text { Embase } \\
626130265)^{108} \\
(\text { Embase } \\
626123635)^{109} \\
\\
\text { Germany } \\
\text { High ROB }\end{array}$ & $\begin{array}{l}\text { Tele.TAnDem } \\
\text { Intervention } \\
\text { Telephone- } \\
\text { based CBT for } \\
\text { family } \\
\text { caregivers } \\
1250 \text {-minute } \\
\text { sessions }\end{array}$ & Usual care & $\begin{array}{l}\text { Community- } \\
\text { based } \\
\mathrm{RCT} \\
\mathrm{N}=273 \\
\text { informal } \\
\text { caregivers }\end{array}$ & \begin{tabular}{|l|} 
Dementia \\
diagnosis
\end{tabular} & $\begin{array}{l}\mathrm{N}=273 \\
\text { Age: } \text { yes } \\
\text { Sex: yes } \\
\text { Race: no } \\
\text { Education: no }\end{array}$ & NR & $\begin{array}{l}\mathrm{N}=273 \\
\text { Age: yes } \\
\text { Sex: yes } \\
\text { Race: no } \\
\text { Education: yes } \\
\text { Relation to PLWD: } \\
\text { yes }\end{array}$ & $\begin{array}{l}\text { Duration: yes } \\
\text { Living with PLWD: } \\
\text { yes } \\
\text { Employment Status: } \\
\text { no } \\
\text { Training: no }\end{array}$ & NA & $\mathrm{NA}$ & 6 months & NR & $\begin{array}{l}\text { CESD } \\
\text { BDI } \\
\text { Emotional } \\
\text { wellbeing } \\
\text { Gießen } \\
\text { Body } \\
\text { Complaints } \\
\text { List } \\
\text { BEHAVE-AD } \\
\text { coping item }\end{array}$ \\
\hline
\end{tabular}




\begin{tabular}{|c|c|c|c|c|c|c|c|c|c|c|c|c|c|}
\hline $\begin{array}{l}\text { Callahan 201716 } \\
\text { (27893087) } \\
\text { US } \\
\text { High ROB }\end{array}$ & $\begin{array}{l}\text { Collaborative } \\
\text { care and } \\
\text { occupational } \\
\text { therapy } \\
24 \text { sessions } \\
\text { over } 2 \text { years }\end{array}$ & Usual care & \begin{tabular}{|l|} 
Home-based \\
setting \\
$\mathrm{RCT}$ \\
$\mathrm{N}=180$ \\
dyads \\
\end{tabular} & \begin{tabular}{|l|} 
Dementia \\
diagnosis
\end{tabular} & $\begin{array}{l}\text { N=180 } \\
\text { Age: } \text { yes } \\
\text { Sex: yes } \\
\text { Race: yes } \\
\text { Education: yes }\end{array}$ & $\mathrm{NR}$ & $\begin{array}{l}\mathrm{N}=180 \\
\text { Age: yes } \\
\text { Sex: no } \\
\text { Race: no } \\
\text { Education: no } \\
\text { Relation to PLWD: } \\
\text { yes }\end{array}$ & NR & NA & NA & 24 months & $\mathrm{NR}$ & $\begin{array}{l}\text { ADCS ADL } \\
\text { SPPB }\end{array}$ \\
\hline $\begin{array}{l}\text { Lok, 2017 } \\
\text { Turkey } \\
\text { Pilot }\end{array}$ & $\begin{array}{l}\text { "First You } \\
\text { Should Get } \\
\text { Stronger" } \\
\text { program } \\
\text { delivered to } \\
\text { individuals } \\
\text { weekly, } 7 \\
\text { weeks, 45- } \\
\text { minute sessions }\end{array}$ & No Treatment & $\begin{array}{l}\text { Community } \\
\text { RCT } \\
\text { Caregivers }\end{array}$ & \begin{tabular}{|l|} 
Diagnosis of \\
dementia per DSM \\
V criteria
\end{tabular} & $\begin{array}{l}\mathrm{N}=40 \\
\text { Age: } \text { yes } \\
\text { Gender: yes } \\
\text { Race: } \mathrm{no} \\
\text { Education: No }\end{array}$ & NR & $\begin{array}{l}\text { N=40 } \\
\text { Age: yes } \\
\text { Sex: yes } \\
\text { Race: no } \\
\text { Education: yes } \\
\text { Relationship: no }\end{array}$ & Duration & NA & NA & $\begin{array}{l}\text { Post } \\
\text { intervention }\end{array}$ & $\mathrm{NR}$ & $\begin{array}{l}\text { IC: Zarit } \\
\text { Burden } \\
\text { Interview, } \\
\text { Health Life } \\
\text { Style } \\
\text { Behavior } \\
\text { Scale II }\end{array}$ \\
\hline $\begin{array}{l}\text { O'Connor, } \\
2017^{111} \\
\text { Australia } \\
\text { Pilot }\end{array}$ & $\begin{array}{l}\text { Activity-based } \\
\text { intervention } \\
\text { (TAP), } 8 \text { home } \\
\text { visits over 4- } \\
\text { month period }\end{array}$ & $\begin{array}{l}\text { Three phone } \\
\text { calls to } \\
\text { caregivers over } \\
\text { 4-month period } \\
\text { on dementia } \\
\text { education }\end{array}$ & $\begin{array}{l}\text { Community } \\
\text { RCT } \\
\text { Caregiver- } \\
\text { PLWD } \\
\text { dyads }\end{array}$ & \begin{tabular}{|l|} 
Diagnosis of \\
frontotemporal \\
dementia according \\
to current \\
diagnostic criteria, \\
presence of \\
behavioral \\
disturbances over \\
the past month as \\
rated by the carer, \\
score of $>3.31$ on \\
Informant \\
Questionnaire on \\
Cognitive Decline \\
for the Elderly, and \\
able to perform at \\
least 2 basic ADLs \\
\end{tabular} & $\begin{array}{l}\mathrm{N}=20 \\
\text { Age: } \text { yes } \\
\text { Sex: yes } \\
\text { Race: } \text { no } \\
\text { Education: yes }\end{array}$ & no & $\begin{array}{l}\mathrm{N}=-20 \\
\text { Age: yes } \\
\text { Sex: yes } \\
\text { Race: no } \\
\text { Education: yes } \\
\text { Relation to PLWD: } \\
\text { yes }\end{array}$ & $\begin{array}{l}\text { Living with PLWD: } \\
\text { yes }\end{array}$ & NA & NA & $\begin{array}{l}\text { Post } \\
\text { Intervention }\end{array}$ & $\begin{array}{l}\text { NPI-C } \\
\text { (frequency, } \\
\text { severity, and } \\
\text { carer } \\
\text { distress } \\
\text { related to } \\
\text { behaviors), } \\
\text { DAD (ADLs } \\
\text { and IADLs), } \\
\text { EruoQol5-D }\end{array}$ & $\begin{array}{l}\text { Vigilance } \\
\text { Items (carer } \\
\text { time feeling } \\
\text { "on duty" and } \\
\text { "doing things" } \\
\text { for PLWD) }\end{array}$ \\
\hline $\begin{array}{l}\text { Supiano, } \\
2017^{112} \\
\\
\text { United States } \\
\text { Small Sample }\end{array}$ & $\begin{array}{l}\text { Weekly } \\
\text { Complicated } \\
\text { Grief Group } \\
\text { Therapy } \\
\text { (CGGT), 120- } \\
\text { minute } \\
\text { sessions, 16 } \\
\text { weeks, with } \\
\text { weekly } \\
\text { homework on } \\
\text { grief status and } \\
\text { emotions }\end{array}$ & $\begin{array}{l}\text { Weekly phone } \\
\text { calls, 30 } \\
\text { minutes } \\
\text { duration, } \\
\text { discussing } \\
\text { caregiver's grief } \\
\text { status and } \\
\text { emotions }\end{array}$ & \begin{tabular}{|l|} 
Community \\
Randomized \\
attention \\
control \\
Caregivers \\
\end{tabular} & \begin{tabular}{|l} 
Dementia, \\
unspecified
\end{tabular} & NR & NR & $\begin{array}{l}\text { N=35 } \\
\text { Age: yes } \\
\text { Sex: yes } \\
\text { Race: no } \\
\text { Education: no } \\
\text { Relation to PLWD: } \\
\text { yes }\end{array}$ & NR & NA & NA & $\begin{array}{l}\text { Weekly } \\
\text { throughout } \\
\text { trial, post } \\
\text { intervention }\end{array}$ & NR & $\begin{array}{l}\text { Brief Grief } \\
\text { Questionnaire } \\
\text { (BGQ) } \\
\text { Inventory of } \\
\text { Complicated } \\
\text { Grief-revised } \\
\text { (ICG-r) } \\
\text { Clinical } \\
\text { Global } \\
\text { Impressions - } \\
\text { Severity } \\
\text { Scale (CGI-S) } \\
\text { Clinical } \\
\text { Global } \\
\text { Impressions- } \\
\text { Improvement } \\
\text { Scale (CGI-I) }\end{array}$ \\
\hline
\end{tabular}




\begin{tabular}{|c|c|c|c|c|c|c|c|c|c|c|c|c|c|}
\hline $\begin{array}{l}\text { Whitlatch } \\
2017^{17} \\
(29171296) \\
\text { US } \\
\text { High ROB }\end{array}$ & $\begin{array}{l}\text { Psychoeducatio } \\
\mathrm{n} \\
\text { Support, Health, } \\
\text { Activities, } \\
\text { Resources, and } \\
\text { Education } \\
\text { (SHARE) } \\
\text { Program } \\
\text { Dyadic } \\
\text { counseling for } \\
\text { early stage } \\
\text { dementia } \\
6 \text { sessions }\end{array}$ & $\begin{array}{l}\text { Education } \\
\text { One 90-minute } \\
\text { session }\end{array}$ & \begin{tabular}{|l|} 
Home-based \\
setting \\
$\mathrm{RCT}$ \\
$\mathrm{N}=128$ \\
dyads \\
\end{tabular} & \begin{tabular}{|l|} 
Dementia \\
diagnosis
\end{tabular} & $\begin{array}{l}\mathrm{N}=128 \\
\text { Age: } \text { yes } \\
\text { Sex: yes } \\
\text { Race: yes } \\
\text { Education: yes }\end{array}$ & NR & $\begin{array}{l}\mathrm{N}=128 \\
\text { Age: yes } \\
\text { Sex: yes } \\
\text { Race: yes } \\
\text { Education: yes } \\
\text { Relation to PLWD: } \\
\text { yes }\end{array}$ & $\begin{array}{l}\text { Duration: yes } \\
\text { Living with PLWD: } \\
\text { yes } \\
\text { Employment Status: } \\
\text { yes } \\
\text { Training: no }\end{array}$ & NA & $\mathrm{NA}$ & 2 months & NR & $\begin{array}{l}\text { Preferences } \\
\text { for Care } \\
\text { Tasks Scale } \\
\text { Service } \\
\text { Availability } \\
\text { Measure } \\
\text { Dyadic } \\
\text { Relationship } \\
\text { Scale } \\
\text { Care-related } \\
\text { Agreement } \\
\text { Scale } \\
\text { Emotional- } \\
\text { Intimacy } \\
\text { Disruptive } \\
\text { Behavior } \\
\text { Scale } \\
\text { Dementia } \\
\text { Quality of Life }\end{array}$ \\
\hline $\begin{array}{l}\text { Nunez-Naveira, } \\
2016^{113} \\
\text { Denmark, } \\
\text { Poland, Spain } \\
\text { Pilot }\end{array}$ & $\begin{array}{l}\text { UnderstAID } \\
\text { application--with } \\
\text { information, } \\
\text { skills training, } \\
\text { social network } \\
\text { forum, and daily } \\
\text { reminders-- } \\
\text { made available } \\
\text { via mobile } \\
\text { device and/or } \\
\text { PC for three } \\
\text { months with } \\
\text { periodic check- } \\
\text { ins by research } \\
\text { staff }\end{array}$ & No Treatment & $\begin{array}{l}\text { Community, } \\
\text { RCT } \\
\text { Caregivers }\end{array}$ & \begin{tabular}{|l|} 
Diagnosis of \\
dementia by a \\
specialist or \\
neurologist per \\
criteria of CMBD- \\
10, DSM-IV, \\
NINDSADRDA \\
\end{tabular} & NR & $\mathrm{NR}$ & $\begin{array}{l}\text { N=77 } \\
\text { Age: yes } \\
\text { Sex: yes } \\
\text { Race: no } \\
\text { Education: no } \\
\text { Relationship: yes }\end{array}$ & $\begin{array}{l}\text { Duration: yes } \\
\text { Payment: yes } \\
\text { Health Status: yes } \\
\text { Employment Status: } \\
\text { yes }\end{array}$ & NA & NA & $\begin{array}{l}\text { Post } \\
\text { intervention }\end{array}$ & NR & $\begin{array}{l}\text { CES-D } \\
\text { Revised } \\
\text { Caregiving } \\
\text { Satisfaction } \\
\text { Scale }\end{array}$ \\
\hline $\begin{array}{l}\text { Sepe-Monti, } \\
2016^{114} \\
\text { Italy } \\
\text { Pilot }\end{array}$ & $\begin{array}{l}\text { Six weekly } \\
\text { group sessions } \\
\text { of psycho- } \\
\text { education } \\
\text { program } \\
\text { delivered by a } \\
\text { psychologist, 2- } \\
\text { hour sessions }\end{array}$ & $\begin{array}{l}\text { Two group } \\
\text { sessions } \\
\text { providing } \\
\text { medical } \\
\text { information and } \\
\text { discussing } \\
\text { management of } \\
\text { care recipient } \\
\text { behaviors, 2- } \\
\text { hour sessions, } \\
\text { one session in } \\
\text { week two of } \\
\text { trial, one } \\
\text { session in week } \\
\text { four; } \\
\text { informational } \\
\text { leaflet provided }\end{array}$ & $\begin{array}{l}\text { Community } \\
\text { Multicenter } \\
\text { RCT } \\
\text { Caregivers }\end{array}$ & \begin{tabular}{|l|} 
Probable or \\
possible AD \\
according to \\
NINCDS-ADRDA \\
criteria
\end{tabular} & $\begin{array}{l}\mathrm{N}=381 \\
\text { Age: } \text { yes } \\
\text { Sex: yes } \\
\text { Race: no } \\
\text { Education: no }\end{array}$ & NR & $\begin{array}{l}\mathrm{N}=164 \\
\text { Age: yes } \\
\text { Sex: yes } \\
\text { Race: no } \\
\text { Education: yes } \\
\text { Relation to PLWD: } \\
\text { yes }\end{array}$ & $\begin{array}{l}\text { Living with PLWD: } \\
\text { yes }\end{array}$ & NA & NA & $\begin{array}{l}\text { Two weeks } \\
\text { post } \\
\text { intervention, } \\
\text { six months } \\
\text { post } \\
\text { intervention }\end{array}$ & NR & $\begin{array}{l}\text { Coping } \\
\text { Orientations } \\
\text { to Problems } \\
\text { Experienced- } \\
\text { New Italian } \\
\text { Version } \\
\text { (COPE-NIV) } \\
\text { Caregiver } \\
\text { Burden } \\
\text { Inventory } \\
\text { (CBI) } \\
\text { NPI } \\
\text { NPI-Distress } \\
\text { Scale } \\
\text { SF-12 } \\
\text { (physical and } \\
\text { mental } \\
\text { health) } \\
\text { CES-D } \\
\text { STAl-Y }\end{array}$ \\
\hline $\begin{array}{l}\text { Taati 201622 } \\
\text { (no PMID) } \\
\text { Iran } \\
\text { High ROB }\end{array}$ & $\begin{array}{l}\text { Support group } \\
\text { for family } \\
\text { caregivers } \\
8 \sim 2 \text {-hour } \\
\text { sessions }\end{array}$ & $\begin{array}{l}\text { Nonspecified } \\
\text { control }\end{array}$ & $\begin{array}{l}\text { Community- } \\
\text { based } \\
\text { setting } \\
R C T \\
\mathrm{~N}=80 \\
\text { informal } \\
\text { caregivers }\end{array}$ & \begin{tabular}{|l} 
Dementia \\
diagnosis
\end{tabular} & NR & NR & $\begin{array}{l}\mathrm{N}=80 \\
\text { Age: } n 0 \\
\text { Sex: yes } \\
\text { Race: no } \\
\text { Education: yes } \\
\text { Relation to PLWD: } \\
\text { yes }\end{array}$ & $\begin{array}{l}\text { Duration: no } \\
\text { Employment Status: } \\
\text { yes } \\
\text { Training: no }\end{array}$ & NA & NA & 8 weeks & $\mathrm{NR}$ & $\begin{array}{l}\text { DASS-21 } \\
\text { (depression, } \\
\text { anxiety, } \\
\text { stress level) }\end{array}$ \\
\hline
\end{tabular}




\begin{tabular}{|c|c|c|c|c|c|c|c|c|c|c|c|c|c|}
\hline $\begin{array}{l}\text { Zauszniewski, } \\
2016^{115} \\
(27500286) \\
(26735507)^{116} \\
\text { United States } \\
\text { Pilot }\end{array}$ & $\begin{array}{l}\text { 40-minute } \\
\text { individual } \\
\text { resourcefulness } \\
\text { training with } \\
\text { daily journaling } \\
\text { or recording as } \\
\text { practice/reinforc } \\
\text { ement methods } \\
\text { for four weeks, } \\
\text { with random } \\
\text { assignment to } \\
\text { choice of } \\
\text { method vs. } \\
\text { assignment of } \\
\text { method }\end{array}$ & $\begin{array}{l}\text { Journaling or } \\
\text { recording } \\
\text { without learning } \\
\text { training skills }\end{array}$ & \begin{tabular}{|l|} 
Community \\
Modified \\
partially \\
randomized \\
preference \\
trial \\
Caregivers \\
\end{tabular} & Dementia, any form & $\begin{array}{l}\mathrm{N}=138 \\
\text { Age: yes } \\
\text { Sex: yes } \\
\text { Race: no } \\
\text { Education: no }\end{array}$ & NR & $\begin{array}{l}\mathrm{N}=138 \\
\text { Age: yes } \\
\text { Sex: yes } \\
\text { Race: yes } \\
\text { Education: yes } \\
\text { Relationship: no }\end{array}$ & $\begin{array}{l}\text { Duration: yes } \\
\text { Living with PLWD: } \\
\text { yes } \\
\text { Health Status: yes }\end{array}$ & NA & NA & \begin{tabular}{l|} 
Post \\
intervention, 6 \\
weeks post \\
intervention
\end{tabular} & NR & $\begin{array}{l}\text { PSS } \\
\text { DCS } \\
\text { ESC }\end{array}$ \\
\hline $\begin{array}{l}\text { Au, 2015117 } \\
(25848237) \\
\text { China } \\
\text { Small Sample }\end{array}$ & $\begin{array}{l}\text { Individual } \\
\text { psychoeducatio } \\
\text { n by telephone } \\
\text { for } 4 \text { weeks, } 30 \\
\text { minutes } \\
\text { sessions, } \\
\text { followed by } 8 \\
\text { biweekly } \\
\text { telephone } \\
\text { sessions, } 15-20 \\
\text { minutes each, } \\
\text { of behavioral } \\
\text { activation }\end{array}$ & $\begin{array}{l}\text { Individual } \\
\text { psychoeducatio } \\
\text { n by telephone } \\
\text { for } 4 \text { weeks, } 30- \\
\text { minute } \\
\text { sessions, } \\
\text { followed by } 8 \\
\text { biweekly } \\
\text { telephone } \\
\text { sessions, } 15-20 \\
\text { minutes each, } \\
\text { of general } \\
\text { discussion of } \\
\text { psychoeducatio } \\
\text { n and related } \\
\text { information }\end{array}$ & $\begin{array}{l}\text { Community } \\
\text { RCT } \\
\text { Caregivers }\end{array}$ & \begin{tabular}{|l|} 
Alzheimer's \\
diagnosis for at \\
least 3 months
\end{tabular} & $\begin{array}{l}\text { N=59 } \\
\text { Age: yes } \\
\text { Sex: yes } \\
\text { Race: no } \\
\text { Education: no }\end{array}$ & NR & $\begin{array}{l}\mathrm{N}=62 \\
\text { Age: yes } \\
\text { Sex: yes } \\
\text { Race: no } \\
\text { Education: no } \\
\text { Relationship: yes }\end{array}$ & $\begin{array}{l}\text { Duration: yes } \\
\text { Living with PLWD: } \\
\text { yes }\end{array}$ & NA & NA & $\begin{array}{l}4 \text { weeks (i.e., } \\
\text { after first } \\
\text { arm), } 6 \\
\text { months (i.e., } \\
\text { after second } \\
\text { arm) }\end{array}$ & NR & $\begin{array}{l}\text { CES-D } \\
\text { RAS }\end{array}$ \\
\hline $\begin{array}{l}\text { Au 2015 } \\
\text { PMID) } \\
\text { Hong Kong } \\
\text { High ROB }\end{array}$ & $\begin{array}{l}\text { Psychoeducatio } \\
\text { n with } \\
\text { telephone- } \\
\text { assisted } \\
\text { behavioral } \\
\text { activation } \\
\text { intervention } \\
8 \text { biweekly } \\
\text { sessions }\end{array}$ & $\begin{array}{l}\text { Psychoeducatio } \\
\text { n only }\end{array}$ & \begin{tabular}{|l|} 
Community- \\
based \\
setting \\
$\mathrm{RCT}$ \\
$\mathrm{N}=96$ \\
informal \\
caregivers
\end{tabular} & \begin{tabular}{|l|} 
Dementia \\
diagnosis
\end{tabular} & NR & NR & $\begin{array}{l}\mathrm{N}=96 \\
\text { Age: } \text { yes } \\
\text { Sex: yes } \\
\text { Race: no } \\
\text { Education: no } \\
\text { Relation to PLWD: } \\
\text { yes }\end{array}$ & $\begin{array}{l}\text { Duration: yes } \\
\text { Employment Status: } \\
\text { no } \\
\text { Training: no }\end{array}$ & NA & NA & 4 months & NR & CES-D \\
\hline $\begin{array}{l}\text { Blom 2015'23 } \\
\text { (no PMID) } \\
\\
(23305463)^{119} \\
(25648589)^{120} \\
\text { Netherlands } \\
\text { High ROB }\end{array}$ & $\begin{array}{l}\text { Mastery of } \\
\text { Dementia } \\
\text { Intervention } \\
\text { Problem } \\
\text { solving, } \\
\text { relaxation, } \\
\text { cognitive } \\
\text { restructuring, } \\
\text { communication } \\
8 \text { lessons + } \\
\text { booster session }\end{array}$ & $\begin{array}{l}\text { Education via e- } \\
\text { bulletins }\end{array}$ & \begin{tabular}{|l|} 
Home-based \\
setting \\
RCT \\
$N=251$ \\
dyads \\
\end{tabular} & \begin{tabular}{|l|} 
Dementia \\
diagnosis
\end{tabular} & NR & $\mathrm{NR}$ & $\begin{array}{l}\mathrm{N}=251 \\
\text { Age: yes } \\
\text { Sex: yes } \\
\text { Race: no } \\
\text { Education: yes } \\
\text { Relation to PLWD: } \\
\text { yes }\end{array}$ & $\begin{array}{l}\text { Duration: no } \\
\text { Living with PLWD: } \\
\text { yes } \\
\text { Employment Status: } \\
\text { no } \\
\text { Training: no }\end{array}$ & NA & NA & 3,6 months & NR & $\begin{array}{l}\text { CES-D } \\
\text { HADS-A }\end{array}$ \\
\hline
\end{tabular}




\begin{tabular}{|c|c|c|c|c|c|c|c|c|c|c|c|c|c|}
\hline $\begin{array}{l}\text { Chen, 2015'121 } \\
(25515800) \\
\text { Taiwan } \\
\text { Small Sample }\end{array}$ & $\begin{array}{l}\text { Biweekly in- } \\
\text { person } \\
\text { education } \\
\text { sessions about } \\
\text { problem-solving } \\
\text { skills, dementia, } \\
\text { social } \\
\text { resources, and } \\
\text { emotional } \\
\text { support, six } \\
\text { sessions over } 3 \\
\text { months (session } \\
\text { length not } \\
\text { specified) } \\
\end{array}$ & \begin{tabular}{|l} 
Biweekly phone \\
calls for usual \\
clinical \\
management \\
(phone call \\
length not \\
specified
\end{tabular} & \begin{tabular}{|l} 
Community \\
or Clinic \\
RCT \\
Caregivers
\end{tabular} & $\begin{array}{l}\text { Dementia, } \\
\text { unspecified }\end{array}$ & \begin{tabular}{|l}
$\mathrm{N}=$ unclear \\
Age: yes \\
Sex: yes \\
Race: no \\
Education: no
\end{tabular} & NR & $\begin{array}{l}\mathrm{N}=46 \\
\text { Age: yes } \\
\text { Sex: yes } \\
\text { Race: no } \\
\text { Education: yes } \\
\text { Relationship: yes }\end{array}$ & $\begin{array}{l}\text { Living with PLWD: } \\
\text { yes }\end{array}$ & NA & NA & \begin{tabular}{|l|}
2 weeks post \\
intervention
\end{tabular} & NR & \begin{tabular}{|l|} 
WCCL-R \\
Caregiver \\
Burden \\
Inventory, \\
RMBPC
\end{tabular} \\
\hline $\begin{array}{l}\text { Chiu 201524 } \\
\text { (25615434) } \\
\text { Canada } \\
\text { High ROB }\end{array}$ & $\begin{array}{l}\text { Problem-solving } \\
\text { technique- } \\
\text { based } \\
\text { intervention } \\
31 \text {-hour } \\
\text { sessions over 3- } \\
4 \text { weeks }\end{array}$ & $\begin{array}{l}\text { Nonspecified } \\
\text { control }\end{array}$ & $\begin{array}{l}\text { Home-based } \\
\text { setting } \\
\mathrm{RCT} \\
\mathrm{N}=56 \\
\text { informal } \\
\text { caregivers }\end{array}$ & \begin{tabular}{|l|} 
Dementia \\
diagnosis
\end{tabular} & NR & NR & $\begin{array}{l}\mathrm{N}=56 \\
\text { Age: } \text { yes } \\
\text { Sex: yes } \\
\text { Race: no } \\
\text { Education: no } \\
\text { Relation to PLWD: } \\
\text { yes }\end{array}$ & $\begin{array}{l}\text { Duration: no } \\
\text { Employment Status: } \\
\text { yes } \\
\text { Training: no }\end{array}$ & NA & NA & 4 weeks & NR & \begin{tabular}{|l|} 
ZBI \\
Pearlin Self- \\
Mastery and \\
Competency \\
Scales \\
PSS
\end{tabular} \\
\hline 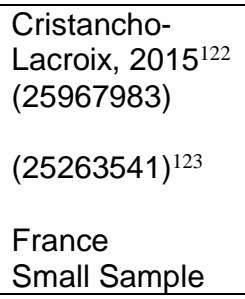 & $\begin{array}{l}\text { Web-based } \\
\text { psycho- } \\
\text { educational } \\
\text { program } \\
\text { delivered in 15- } \\
\text { 30-minute } \\
\text { weekly sessions } \\
\text { for } 12 \text { weeks } \\
\end{array}$ & Usual care only & \begin{tabular}{|l|} 
Day care \\
center \\
geriatric unit \\
RCT \\
Caregivers
\end{tabular} & \begin{tabular}{|l|} 
Alzheimer's \\
diagnosis per \\
DSM-IV criteria
\end{tabular} & $\begin{array}{l}\mathrm{N}=49 \\
\text { Age: no } \\
\text { Sex: no } \\
\text { Race: no } \\
\text { Education: no }\end{array}$ & NR & $\begin{array}{l}\text { N=49 } \\
\text { Age: yes } \\
\text { Sex: yes } \\
\text { Race: no } \\
\text { Education: yes } \\
\text { Relationship: yes }\end{array}$ & $\begin{array}{l}\text { Living with PLWD: } \\
\text { yes } \\
\text { Health status: yes }\end{array}$ & NA & NA & \begin{tabular}{|l} 
Post \\
intervention, 3 \\
months post \\
intervention
\end{tabular} & NR & \begin{tabular}{|l|} 
RSCS \\
RMBPC \\
ZBI \\
NHP \\
\end{tabular} \\
\hline $\begin{array}{l}\text { Gallagher- } \\
\text { Thompson } \\
2015^{25} \\
(25590939) \\
\text { US } \\
\text { High ROB }\end{array}$ & $\begin{array}{l}\text { Psychoeducatio } \\
\text { n Fotonovela for } \\
\text { reducing stress } \\
\text { and depression } \\
\text { in Latino } \\
\text { caregivers }\end{array}$ & $\begin{array}{l}\text { Usual care with } \\
\text { basic } \\
\text { information }\end{array}$ & $\begin{array}{l}\text { Home-based } \\
\text { setting } \\
\mathrm{RCT} \\
\mathrm{N}=147 \\
\text { dyads }\end{array}$ & \begin{tabular}{|l} 
Dementia \\
diagnosis
\end{tabular} & \begin{tabular}{|l}
$\mathrm{N}=147$ \\
Age: $y$ es \\
Sex: no \\
Race: yes \\
Education: yes
\end{tabular} & NR & $\begin{array}{l}\mathrm{N}=147 \\
\text { Age: yes } \\
\text { Sex: yes } \\
\text { Race: yes } \\
\text { Education: yes } \\
\text { Relation to PLWD: } \\
\text { yes }\end{array}$ & $\begin{array}{l}\text { Duration: yes } \\
\text { Living with PLWD: } \\
\text { yes } \\
\text { Employment Status: } \\
\text { yes } \\
\text { Training: no }\end{array}$ & NA & NA & 4,6 months & NR & \begin{tabular}{|l|} 
CES-D \\
RMBPC \\
Reaction
\end{tabular} \\
\hline $\begin{array}{l}\text { Gaugler, } \\
2015^{124} \\
(25751083) \\
\text { United States } \\
\text { Pilot }\end{array}$ & $\begin{array}{l}\text { Individual } \\
\text { psychosocial } \\
\text { intervention } \\
\text { (other family } \\
\text { members or } \\
\text { care decision } \\
\text { makers present } \\
\text { per participant } \\
\text { discretion), six } \\
\text { sessions, } \\
\text { weekly for three } \\
\text { weeks, then } \\
\text { monthly for } 3 \\
\text { months, 60-120- } \\
\text { minute sessions }\end{array}$ & No Treatment & \begin{tabular}{|l|} 
Community \\
RCT \\
Caregivers
\end{tabular} & Dementia & $\begin{array}{l}\mathrm{N}=36 \\
\text { Age: } \text { yes } \\
\text { Sex: yes } \\
\text { Race: yes } \\
\text { Education: yes }\end{array}$ & NR & $\begin{array}{l}\mathrm{N}=36 \\
\text { Age: yes } \\
\text { Sex: yes } \\
\text { Race: yes } \\
\text { Education: yes } \\
\text { Relation to PLWD: } \\
\text { yes }\end{array}$ & $\begin{array}{l}\begin{array}{l}\text { Employment Status: } \\
\text { yes }\end{array} \\
\text { : }\end{array}$ & NA & NA & \begin{tabular}{|l} 
Post \\
intervention, 4 \\
months post \\
intervention
\end{tabular} & $\mathrm{NR}$ & \begin{tabular}{|l|} 
ZBI \\
CES-D \\
GDS \\
NPI-Q \\
Role overload \\
PSS
\end{tabular} \\
\hline $\begin{array}{l}\text { Kamkhagi, } \\
2015^{125} \\
\text { (no PMID) } \\
\\
\text { Brazil } \\
\text { Small Sample }\end{array}$ & $\begin{array}{l}\text { Psychological } \\
\text { intervention } \\
\text { delivered via } \\
\text { weekly group } \\
\text { sessions, 90- } \\
\text { minute } \\
\text { sessions, } 14 \\
\text { weeks }\end{array}$ & $\begin{array}{l}\text { Non- } \\
\text { psychologically- } \\
\text { oriented } \\
\text { intervention } \\
\text { delivered via } \\
\text { weekly group } \\
\text { sessions, 90- } \\
\text { minute } \\
\text { sessions, } 14 \\
\text { weeks } \\
\end{array}$ & \begin{tabular}{|l|} 
Community \\
RCT \\
Caregivers
\end{tabular} & $\begin{array}{l}\text { Mild or moderate } \\
\text { Alzheimer's } \\
\text { disease }\end{array}$ & NR & NR & $\begin{array}{l}\mathrm{N}=37 \\
\text { Age: } \text { yes } \\
\text { Sex: yes } \\
\text { Race: no } \\
\text { Education: yes } \\
\text { Relation to PLWD: } \\
\text { yes }\end{array}$ & $\begin{array}{l}\text { Living with PLWD: } \\
\text { yes }\end{array}$ & NA & NA & \begin{tabular}{|l|} 
Post \\
intervention
\end{tabular} & NR & \begin{tabular}{|l} 
ZBI \\
BDI \\
WHO-QoL \\
Scale
\end{tabular} \\
\hline
\end{tabular}




\begin{tabular}{|c|c|c|c|c|c|c|c|c|c|c|c|c|c|}
\hline $\begin{array}{l}\text { Losada 201526 } \\
(21061414) \\
\text { Spain } \\
\text { High ROB }\end{array}$ & $\begin{array}{l}\text { 1. CBT } \\
\text { 2. ACT } \\
\text { (Acceptance } \\
\text { and } \\
\text { Commitment } \\
\text { Therapy) to } \\
\text { reduce } \\
\text { depression in } \\
\text { caregivers } \\
8 \text { weekly 90- } \\
\text { minute sessions }\end{array}$ & $\begin{array}{l}\text { 3. Minimal } \\
\text { support control }\end{array}$ & \begin{tabular}{|l} 
Community- \\
based \\
setting \\
$\mathrm{RCT}$ \\
$\mathrm{N}=135$ \\
informal \\
caregivers
\end{tabular} & \begin{tabular}{|l|} 
Dementia \\
diagnosis
\end{tabular} & $\mathrm{NR}$ & NR & $\begin{array}{l}\mathrm{N}=135 \\
\text { Age: yes } \\
\text { Sex: yes } \\
\text { Race: no } \\
\text { Education: yes } \\
\text { Relation to PLWD: } \\
\text { yes }\end{array}$ & $\begin{array}{l}\text { Duration: yes } \\
\text { Employment Status: } \\
\text { yes } \\
\text { Training: yes }\end{array}$ & NA & $\mathrm{NA}$ & 2 months & $\begin{array}{l}\text { Barthel } \\
\text { Index } \\
\text { RMBPC }\end{array}$ & $\begin{array}{l}\text { CES-D } \\
\text { Profile of } \\
\text { Mood States }\end{array}$ \\
\hline $\begin{array}{l}\text { Paun, 2015 } \\
(24510968) \\
\text { United States } \\
\text { Pilot }\end{array}$ & $\begin{array}{l}12 \text { weekly group } \\
\text { sessions of } \\
\text { chronic grief } \\
\text { management } \\
\text { intervention, } 60- \\
90-\text { minute } \\
\text { sessions }\end{array}$ & $\begin{array}{l}\text { No treatment } \\
\text { (Two check-in } \\
\text { calls at } 1.5 \\
\text { months and } 4.5 \\
\text { months) }\end{array}$ & $\begin{array}{l}\text { Long-term } \\
\text { care } \\
\text { facilities } \\
\text { Pre- } \\
\text { test/multiple } \\
\text { post-test } \\
\text { quasi- } \\
\text { experimental } \\
\text { design } \\
\text { Careaiver }\end{array}$ & $\begin{array}{l}\text { Diagnosis of AD or } \\
\text { a related dementia }\end{array}$ & NR & NR & $\begin{array}{l}\mathrm{N}=93 \\
\text { Age: yes } \\
\text { Sex: yes } \\
\text { Race: yes } \\
\text { Education: yes } \\
\text { Relation to PLWD: } \\
\text { yes }\end{array}$ & NR & NA & NA & $\begin{array}{l}\text { Post } \\
\text { intervention, } 3 \\
\text { months post } \\
\text { intervention }\end{array}$ & NR & $\begin{array}{l}\text { FPCR (Family } \\
\text { Perception of } \\
\text { Caregiver } \\
\text { Role - role } \\
\text { stress) } \\
\text { MMCGI (grief } \\
\text { in caregivers } \\
\text { of persons } \\
\text { with AD) } \\
\text { CES-D }\end{array}$ \\
\hline $\begin{array}{l}\text { Aboulafia- } \\
\text { Brakha, 2014 } \\
\text { (no PMID) } \\
\text { Brazil } \\
\text { Small Sample }\end{array}$ & $\begin{array}{l}\text { Weekly 90- } \\
\text { minute group } \\
\text { sessions of } \\
\text { psychoeducatio } \\
\text { n and cognitive } \\
\text { behavioral } \\
\text { therapy, or } \\
\text { weekly 60- } \\
\text { minute group } \\
\text { sessions of } \\
\text { psychoeducatio } \\
\text { n alone, } 8 \\
\text { weeks }\end{array}$ & $\begin{array}{l}\text { No Control } \\
\text { Group }\end{array}$ & $\begin{array}{l}\text { Community } \\
\text { Semi- } \\
\text { Random } \\
\text { Comparative } \\
\text { Trial } \\
\text { Caregivers }\end{array}$ & $\begin{array}{l}\text { Clinical diagnosis } \\
\text { of possible or } \\
\text { probable } \\
\text { Alzheimer's } \\
\text { disease in the } \\
\text { moderate or severe } \\
\text { stage, with } \\
\text { diagnosis and } \\
\text { staging based on } \\
\text { the DSM-IV, the } \\
\text { FAST, and the } \\
\text { MMSE (Portuguese } \\
\text { version) }\end{array}$ & NR & NR & $\begin{array}{l}\mathrm{N}=35 \\
\text { Age: yes } \\
\text { Sex: yes } \\
\text { Race: no } \\
\text { Education: no } \\
\text { Relation to PLWD: } \\
\text { yes }\end{array}$ & $\begin{array}{l}\text { Duration: yes } \\
\text { Living with PLWD: } \\
\text { yes } \\
\text { Employment Status: } \\
\text { yes }\end{array}$ & NA & $\mathrm{NA}$ & $\begin{array}{l}\text { Post } \\
\text { intervention }\end{array}$ & NR & $\begin{array}{l}\text { PSS } \\
\text { ZBI } \\
\text { BDI } \\
\text { STAI-S } \\
\text { STAI-T } \\
\text { Portuguese } \\
\text { version }\end{array}$ \\
\hline $\begin{array}{l}\text { Arango- } \\
\text { Lasprilla 2014 } \\
(24550547) \\
\text { Spain } \\
\text { High ROB }\end{array}$ & $\begin{array}{l}\text { Group-based } \\
\text { cognitive } \\
\text { behavioral } \\
\text { intervention for } \\
\text { caregiver } \\
\text { mental health }\end{array}$ & $\begin{array}{l}\text { Educational } \\
\text { control }\end{array}$ & $\begin{array}{l}\text { Community- } \\
\text { based } \\
\text { setting } \\
\mathrm{RCT} \\
\mathrm{N}=69 \\
\text { informal } \\
\text { caregivers }\end{array}$ & $\begin{array}{l}\text { Dementia } \\
\text { diagnosis }\end{array}$ & NR & NR & $\begin{array}{l}\text { N=69 } \\
\text { Age: yes } \\
\text { Sex: yes } \\
\text { Race: yes } \\
\text { Education: no } \\
\text { Relation to PLWD: } \\
\text { yes }\end{array}$ & $\begin{array}{l}\text { Duration: yes } \\
\text { Employment Status: } \\
\text { no } \\
\text { Training: no }\end{array}$ & NA & NA & $\begin{array}{l}8 \text { weeks } \\
3 \text { months }\end{array}$ & NA & $\begin{array}{l}\text { PHQ-9 } \\
\text { ZBI } \\
\text { Satisfaction } \\
\text { with Life } \\
\text { Scale } \\
\text { PSS }\end{array}$ \\
\hline $\begin{array}{l}\text { Dowling, } \\
2014^{128} \\
(24113564) \\
\\
\text { United States } \\
\text { Small Sample }\end{array}$ & $\begin{array}{l}\text { Weekly, one-on- } \\
\text { one, positive } \\
\text { affect skill- } \\
\text { building } \\
\text { intervention } \\
\text { sessions } \\
\text { delivered in } \\
\text { person or via } \\
\text { video- } \\
\text { conference, } 5 \\
\text { weeks, 1-hour } \\
\text { cecsions }\end{array}$ & $\begin{array}{l}\text { Weekly, one- } \\
\text { one one, } \\
\text { sessions } \\
\text { delivered in } \\
\text { person or via } \\
\text { video- } \\
\text { conference } \\
\text { consisting of a } \\
\text { thematic } \\
\text { interview, } 5 \\
\text { weeks, } 1-\text { hour }\end{array}$ & $\begin{array}{l}\text { Community } \\
\text { Randomized } \\
\text { attention } \\
\text { control } \\
\text { Caregivers }\end{array}$ & $\begin{array}{l}\text { Frontotemporal } \\
\text { dementia (FTD) }\end{array}$ & $\mathrm{NR}$ & NR & $\begin{array}{l}\mathrm{N}=26 \\
\text { Age: yes } \\
\text { Sex: yes } \\
\text { Race: yes } \\
\text { Education: yes } \\
\text { Relation to PLWD: } \\
\text { yes }\end{array}$ & $\begin{array}{l}\text { Duration: yes } \\
\text { Living with PLWD: } \\
\text { yes }\end{array}$ & NA & NA & $\begin{array}{l}\text { One-week } \\
\text { post } \\
\text { intervention, } \\
\text { One-month } \\
\text { post } \\
\text { intervention }\end{array}$ & NR & $\begin{array}{l}\text { DES } \\
\text { CES-D } \\
\text { PSS } \\
\text { NPI } \\
\text { ZBI }\end{array}$ \\
\hline $\begin{array}{l}\text { Gonzalez, } \\
2014^{129} \\
(25328754) \\
\\
\text { United States } \\
\text { Pilot }\end{array}$ & $\begin{array}{l}\text { Weekly group } \\
\text { resourcefulness } \\
\text { trainings } \\
\text { delivered for } 6 \\
\text { weeks, } 2 \text { hours } \\
\text { per training }\end{array}$ & Usual Care & $\begin{array}{l}\text { Community } \\
\text { RCT } \\
\text { Caregivers }\end{array}$ & $\begin{array}{l}\text { Probably diagnosis } \\
\text { of AC using the } \\
\text { NINCDS/ADRD } \\
\text { criteria for probable } \\
\text { AC }\end{array}$ & $\begin{array}{l}\mathrm{N}=102 \\
\text { Age: yes } \\
\text { Sex: yes } \\
\text { Race: yes } \\
\text { Education: no }\end{array}$ & NR & $\begin{array}{l}\mathrm{N}=102 \\
\text { Age: yes } \\
\text { Sex yes } \\
\text { Race: yes } \\
\text { Education: yes } \\
\text { Relationship: yes }\end{array}$ & Health status: yes & NA & $\mathrm{NA}$ & $\begin{array}{l}\text { Within one- } \\
\text { week post } \\
\text { intervention, } \\
12 \text { weeks post } \\
\text { intervention }\end{array}$ & RMBPC & $\begin{array}{l}\text { STAI } \\
\text { CESD } \\
\text { CRS }\end{array}$ \\
\hline
\end{tabular}




\begin{tabular}{|c|c|c|c|c|c|c|c|c|c|c|c|c|c|}
\hline $\begin{array}{l}\text { Passoni 2014 } \\
(24614271) \\
\text { Italy } \\
\text { High ROB }\end{array}$ & $\begin{array}{l}\text { 1. Group-based } \\
\text { cognitive } \\
\text { behavioral } \\
\text { intervention for } \\
\text { caregiver } \\
\text { mental health } \\
\text { 2. Manual only }\end{array}$ & Control & \begin{tabular}{|l} 
Community- \\
based \\
setting \\
$\mathrm{RCT}$ \\
$\mathrm{N}=100$ \\
informal \\
caregivers
\end{tabular} & \begin{tabular}{|l} 
Dementia \\
diagnosis
\end{tabular} & $\begin{array}{l}\text { N=100 } \\
\text { Age: yes } \\
\text { Sex: yes } \\
\text { Race: no } \\
\text { Education: no }\end{array}$ & NR & $\begin{array}{l}\mathrm{N}=100 \\
\text { Age: yes } \\
\text { Sex: yes } \\
\text { Race: no } \\
\text { Education: no } \\
\text { Relation to PLWD: } \\
\text { no }\end{array}$ & $\begin{array}{l}\text { Duration: no } \\
\text { Employment Status: } \\
\text { no } \\
\text { Training: no }\end{array}$ & NA & $\mathrm{NA}$ & 6 months & NA & $\begin{array}{l}\text { Anxiety and } \\
\text { Depression } \\
\text { Scale- } \\
\text { Reduced } \\
\text { Form } \\
\text { Caregiver } \\
\text { Need } \\
\text { Assessment } \\
\text { Family Strain } \\
\text { Questionnaire }\end{array}$ \\
\hline $\begin{array}{l}\text { Bruvik 2013 } \\
(24348500) \\
\text { Norway } \\
\text { High ROB }\end{array}$ & $\begin{array}{l}\text { Tailored } \\
\text { psychosocial } \\
\text { support } \\
\text { intervention for } \\
\text { depression }\end{array}$ & Usual care & \begin{tabular}{|l|} 
Home-based \\
setting \\
$R C T$ \\
$N=230$ \\
dyads \\
\end{tabular} & $\begin{array}{l}\text { Dementia } \\
\text { diagnosis }\end{array}$ & $\begin{array}{l}\mathrm{N}=230 \\
\text { Age: yes } \\
\text { Sex: yes } \\
\text { Race: } \mathrm{no} \\
\text { Education: yes }\end{array}$ & NR & $\begin{array}{l}\mathrm{N}=230 \\
\text { Age: yes } \\
\text { Sex: yes } \\
\text { Race: no } \\
\text { Education: yes } \\
\text { Relation to PLWD: } \\
\text { yes }\end{array}$ & $\begin{array}{l}\text { Duration: yes } \\
\text { Living with PLWD: } \\
\text { yes } \\
\text { Employment Status: } \\
\text { yes } \\
\text { Training: no }\end{array}$ & NA & NA & 12 months & CSDD & GDS \\
\hline $\begin{array}{l}\text { Huang 201334 } \\
\text { (23933422) } \\
\text { Taiwan } \\
\text { High ROB }\end{array}$ & $\begin{array}{l}\text { Training } \\
\text { program for } \\
\text { caregivers for } \\
\text { skills managing } \\
\text { behavioral } \\
\text { problems }\end{array}$ & $\begin{array}{l}\text { Written } \\
\text { instructions and } \\
\text { telephone } \\
\text { followup }\end{array}$ & \begin{tabular}{|l|} 
Home-based \\
setting \\
$\mathrm{RCT}$ \\
$\mathrm{N}=129$ \\
dyads \\
\end{tabular} & $\begin{array}{l}\text { Dementia } \\
\text { diagnosis }\end{array}$ & $\begin{array}{l}\text { N=129 } \\
\text { Age: yes } \\
\text { Sex: yes } \\
\text { Race: no } \\
\text { Education: no }\end{array}$ & NR & $\begin{array}{l}\mathrm{N}=129 \\
\text { Age: yes } \\
\text { Sex: yes } \\
\text { Race: no } \\
\text { Education: yes } \\
\text { Relation to PLWD: } \\
\text { yes }\end{array}$ & $\begin{array}{l}\text { Duration: yes } \\
\text { Employment Status: } \\
\text { no } \\
\text { Training: no }\end{array}$ & NA & NA & 3,6 months & CMAI & $\begin{array}{l}\text { Agitation } \\
\text { Management } \\
\text { Self-efficacy } \\
\text { Scale } \\
\text { Preparedness } \\
\text { and } \\
\text { Competence } \\
\text { Scales }\end{array}$ \\
\hline $\begin{array}{l}\text { Judge 201335 } \\
(22899427 \text { ) } \\
\\
\text { Judge 2011 } \\
\text { (19808841) }^{130} \\
\text { Judge 2011 (no } \\
\text { PMID) })^{131} \\
\text { US } \\
\text { High ROB }\end{array}$ & $\begin{array}{l}\text { ANSWERS } \\
\text { Intervention } \\
\text { (Acquiring New } \\
\text { Skills While } \\
\text { Enhancing } \\
\text { Remaining } \\
\text { Strengths) } \\
\text { Education and } \\
\text { cognitive } \\
\text { rehabilitation } \\
\text { skills }\end{array}$ & $\begin{array}{l}\text { Educational } \\
\text { pamphlets }\end{array}$ & \begin{tabular}{|l|} 
Home-based \\
setting \\
$R C T$ \\
$N=128$ \\
dyads
\end{tabular} & $\begin{array}{l}\text { Dementia } \\
\text { diagnosis }\end{array}$ & $\begin{array}{l}\mathrm{N}=128 \\
\text { Age: yes } \\
\text { Sex: yes } \\
\text { Race: yes } \\
\text { Education: yes }\end{array}$ & NR & $\begin{array}{l}\mathrm{N}=128 \\
\text { Age: yes } \\
\text { Sex: yes } \\
\text { Race: yes } \\
\text { Education: yes } \\
\text { Relation to PLWD: } \\
\text { yes }\end{array}$ & $\begin{array}{l}\text { Duration: no } \\
\text { Employment Status: } \\
\text { yes } \\
\text { Training: no }\end{array}$ & NA & NA & 11,19 weeks & NA & $\begin{array}{l}\text { Pearlin } \\
\text { Caregiver } \\
\text { Mastery } \\
\text { scales } \\
\text { Depression } \\
\text { Anxiety } \\
\text { QOL } \\
\text { Self-Esteem }\end{array}$ \\
\hline $\begin{array}{l}\text { Kajiyama } \\
2013^{36} \\
(23461355) \\
\text { US } \\
\text { High ROB }\end{array}$ & $\begin{array}{l}\text { iCare Stress } \\
\text { Management e- } \\
\text { Training } \\
\text { Program }\end{array}$ & Information only & \begin{tabular}{|l|} 
Home-based \\
setting \\
$R C T$ \\
$N=150$ \\
dyads \\
\end{tabular} & $\begin{array}{l}\text { Dementia } \\
\text { diagnosis }\end{array}$ & $\begin{array}{l}\mathrm{N}=150 \\
\text { Age: yes } \\
\text { Sex: no } \\
\text { Race: } \text { no } \\
\text { Education: no }\end{array}$ & NR & $\begin{array}{l}\mathrm{N}=150 \\
\text { Age: yes } \\
\text { Sex: yes } \\
\text { Race: no } \\
\text { Education: yes } \\
\text { Relation to PLWD: } \\
\text { yes }\end{array}$ & $\begin{array}{l}\text { Duration: yes } \\
\text { Employment Status: } \\
\text { no } \\
\text { Training: no }\end{array}$ & NA & NA & 3 months & NA & $\begin{array}{l}\text { CES-D } \\
\text { PSS } \\
\text { RMBPC } \\
\text { Bother } \\
\text { PQOL }\end{array}$ \\
\hline $\begin{array}{l}\text { Kuo 201337 } \\
(22778053) \\
(27653753)^{132} \\
\text { Kuo 2014 (no } \\
\text { PMID) } \\
\text { Taiwan } \\
\text { High ROB }\end{array}$ & $\begin{array}{l}\text { Training } \\
\text { program to } \\
\text { improve QOL } \\
\text { and decrease } \\
\text { depression } \\
2 \text { 2-3-hour } \\
\text { sessions 1 } \\
\text { week apart }\end{array}$ & Attention control & $\begin{array}{l}\text { Home-based } \\
\text { setting } \\
\mathrm{RCT} \\
\mathrm{N}=129 \\
\text { informal } \\
\text { caregivers }\end{array}$ & \begin{tabular}{|l} 
Dementia \\
diagnosis
\end{tabular} & NR & NR & $\begin{array}{l}\mathrm{N}=129 \\
\text { Age: yes } \\
\text { Sex: yes } \\
\text { Race: no } \\
\text { Education: yes } \\
\text { Relation to PLWD: } \\
\text { yes }\end{array}$ & $\begin{array}{l}\text { Duration: yes } \\
\text { Employment Status: } \\
\text { no } \\
\text { Training: no }\end{array}$ & NA & NA & $\begin{array}{l}2 \text { weeks } \\
3,6 \text { months }\end{array}$ & NA & $\begin{array}{l}\text { HRQOL } \\
\text { CES-D }\end{array}$ \\
\hline
\end{tabular}




\begin{tabular}{|c|c|c|c|c|c|c|c|c|c|c|c|c|c|}
\hline $\begin{array}{l}\text { Kwok, 2013 } \\
\text { (24072965) } \\
\text { China } \\
\text { Small Sample }\end{array}$ & \begin{tabular}{|l} 
Psychoeducatio \\
$\mathrm{n}$ for caregivers \\
by telephone, \\
twelve $30-$ \\
minute weekly \\
sessions, plus \\
DVD containing \\
eductional \\
information \\
about dementia \\
caregivina
\end{tabular} & $\begin{array}{l}\text { DVD containing } \\
\text { educational } \\
\text { information } \\
\text { about dementia } \\
\text { caregiving }\end{array}$ & \begin{tabular}{|l} 
Community \\
RCT \\
Caregivers
\end{tabular} & $\begin{array}{l}\text { Clinical diagnosis } \\
\text { of dementia of any } \\
\text { stage }\end{array}$ & $\begin{array}{l}\text { N: no } \\
\text { Age: no } \\
\text { Sex: yes } \\
\text { Race: no } \\
\text { Education: no }\end{array}$ & NR & $\begin{array}{l}\mathrm{N}=42 \\
\text { Age: yes } \\
\text { Sex: yes } \\
\text { Race: no } \\
\text { Education: yes } \\
\text { Relation to PLWD: } \\
\text { yes }\end{array}$ & NR & NA & $\mathrm{NA}$ & $\begin{array}{l}\text { Post } \\
\text { intervention }\end{array}$ & NR & $\begin{array}{l}\text { ZBI } \\
\text { Revised } \\
\text { Scale for } \\
\text { Caregiving } \\
\text { Self-efficacy }\end{array}$ \\
\hline $\begin{array}{l}\text { Moore 201338 } \\
(23916631) \\
\text { US } \\
\text { High ROB }\end{array}$ & $\begin{array}{l}\text { Pleasant Events } \\
\text { Program } \\
\text { Behavioral } \\
\text { Activation } \\
\text { therapy for } \\
\text { improving } \\
\text { physical and } \\
\text { mental health in } \\
\text { caregivers } \\
6 \text { weeks }\end{array}$ & $\begin{array}{l}\text { Educational } \\
\text { manual }\end{array}$ & $\begin{array}{l}\text { Community- } \\
\text { based } \\
\text { setting } \\
\text { RCT } \\
\mathrm{N}=100 \\
\text { dyads }\end{array}$ & \begin{tabular}{|l|} 
Dementia \\
diagnosis
\end{tabular} & $\begin{array}{l}\mathrm{N}=100 \\
\text { Age: no } \\
\text { Sex: no } \\
\text { Race: no } \\
\text { Education: no }\end{array}$ & NR & $\begin{array}{l}\mathrm{N}=100 \\
\text { Age: yes } \\
\text { Sex: yes } \\
\text { Race: yes } \\
\text { Education: yes } \\
\text { Relation to PLWD: } \\
\text { no }\end{array}$ & $\begin{array}{l}\text { Duration: yes } \\
\text { Employment Status: } \\
\text { no } \\
\text { Training: no }\end{array}$ & NA & NA & $\begin{array}{l}6 \text { weeks } \\
12 \text { months }\end{array}$ & NA & $\begin{array}{l}\text { CES-D } \\
\text { Health status } \\
\text { Social } \\
\text { Support Scale } \\
\text { PANAS }\end{array}$ \\
\hline $\begin{array}{l}\text { Joling 201239 } \\
(22303473) \\
\\
(22876304)^{135} \\
(24053631)^{136} \\
(18208607)^{137} \\
\text { Netherlands } \\
\text { High ROB }\end{array}$ & $\begin{array}{l}\text { Family Meeting } \\
\text { Intervention for } \\
\text { depression and } \\
\text { anxiety in } \\
\text { caregivers } \\
\text { Four sessions } \\
\text { every 2-3 } \\
\text { months }\end{array}$ & Usual care & \begin{tabular}{|l|} 
Home-based \\
setting \\
$\mathrm{RCT}$ \\
$\mathrm{N}=192$ \\
caregivers \\
\end{tabular} & $\begin{array}{l}\text { Dementia } \\
\text { diagnosis }\end{array}$ & $\begin{array}{l}\mathrm{N}=192 \\
\text { Age: yes } \\
\text { Sex: yes } \\
\text { Race: } n \text { o } \\
\text { Education: no }\end{array}$ & \begin{tabular}{|l|}
$N R$ \\
\end{tabular} & $\begin{array}{l}\text { N=192 } \\
\text { Age: yes } \\
\text { Sex: yes } \\
\text { Race: no } \\
\text { Education: yes } \\
\text { Relation to PLWD: } \\
\text { yes }\end{array}$ & $\begin{array}{l}\text { Duration: no } \\
\text { Living with PLWD: } \\
\text { yes } \\
\text { Employment Status: } \\
\text { no } \\
\text { Training: no }\end{array}$ & NA & NA & $\begin{array}{l}3,6,9,12 \\
\text { months }\end{array}$ & NA & $\begin{array}{l}\text { CES-D } \\
\text { HADS-A } \\
\text { Caregiver } \\
\text { Reaction } \\
\text { Assessment } \\
\text { SF-12 }\end{array}$ \\
\hline $\begin{array}{l}\text { Chu 201141 } \\
(20847363) \\
\text { Taiwan } \\
\text { High ROB }\end{array}$ & $\begin{array}{l}\text { Support group } \\
\text { for caregiver } \\
\text { burden and } \\
\text { depression } \\
12 \text { weeks } \\
\end{array}$ & Usual care & $\begin{array}{l}\text { Home-based } \\
\text { setting } \\
R C T \\
N=85 \text { dyads }\end{array}$ & \begin{tabular}{|l|} 
Dementia \\
diagnosis
\end{tabular} & NR & NR & $\begin{array}{l}\mathrm{N}=85 \\
\text { Age: no } \\
\text { Sex: yes } \\
\text { Race: no } \\
\text { Education: no } \\
\text { Relation to PLWD: } \\
\text { yes }\end{array}$ & $\begin{array}{l}\text { Duration: no } \\
\text { Living with PLWD: } \\
\text { yes } \\
\text { Employment Status: } \\
\text { no } \\
\text { Training: no }\end{array}$ & NA & NA & 3,4 months & NA & $\begin{array}{l}\mathrm{BDI}-\mathrm{II} \\
\mathrm{CBI}\end{array}$ \\
\hline $\begin{array}{l}\text { Davis, 2011 } \\
\text { (20842759) } \\
\text { United States } \\
\text { Small Sample }\end{array}$ & \begin{tabular}{|l} 
Psychosocial \\
intervention \\
delivered by \\
telephone, 10 \\
contacts over 3 \\
months: initial \\
call and 7 \\
weekly follow- \\
up calls $(60$ \\
minutes each), \\
and 2 bimonthly \\
termination calls \\
(35-45 minutes \\
each)
\end{tabular} & No Treatment & \begin{tabular}{|l} 
Community \\
RCT \\
Caregivers
\end{tabular} & \begin{tabular}{|l|} 
Diagnosis of \\
dementia
\end{tabular} & $\begin{array}{l}\text { N=53 } \\
\text { Age: yes } \\
\text { Sex: no } \\
\text { Race: no } \\
\text { Education: no }\end{array}$ & NR & $\begin{array}{l}\mathrm{N}=53 \\
\text { Age: } \text { yes } \\
\text { Sex: yes } \\
\text { Race: no } \\
\text { Education: yes } \\
\text { Relation to PLWD: } \\
\text { yes }\end{array}$ & NR & NA & NA & $\begin{array}{l}\text { Post } \\
\text { intervention }\end{array}$ & NR & $\begin{array}{l}\text { CES-D } \\
\text { ZBI } \\
\text { SF-36 }\end{array}$ \\
\hline $\begin{array}{l}\text { Losada 201114 } \\
(21061414) \\
\text { Spain } \\
\text { High ROB }\end{array}$ & \begin{tabular}{|l|} 
CBT for \\
caregiver \\
behavioral \\
activation and \\
modifying \\
dysfunctional \\
thoughts \\
12 sessions \\
\end{tabular} & Usual care & $\begin{array}{l}\text { Community- } \\
\text { based } \\
\text { setting } \\
\mathrm{RCT} \\
\mathrm{N}=170 \\
\text { dyads }\end{array}$ & \begin{tabular}{|l|} 
Dementia \\
diagnosis
\end{tabular} & $\begin{array}{l}\mathrm{N}=170 \\
\text { Age: yes } \\
\text { Sex: no } \\
\text { Race: no } \\
\text { Education: no }\end{array}$ & NR & $\begin{array}{l}\mathrm{N}=170 \\
\text { Age: yes } \\
\text { Sex: yes } \\
\text { Race: no } \\
\text { Education: no } \\
\text { Relation to PLWD: } \\
\text { yes }\end{array}$ & $\begin{array}{l}\text { Duration: yes } \\
\text { Employment Status: } \\
\text { no } \\
\text { Training: no }\end{array}$ & NA & $\mathrm{NA}$ & 3 months & RMBPC & $\begin{array}{l}\text { CES-D } \\
\text { Dysfunctional } \\
\text { Thoughts } \\
\text { about } \\
\text { Caregiving } \\
\text { Questionnaire } \\
\text { Leisure Time } \\
\text { Satisfaction }\end{array}$ \\
\hline
\end{tabular}




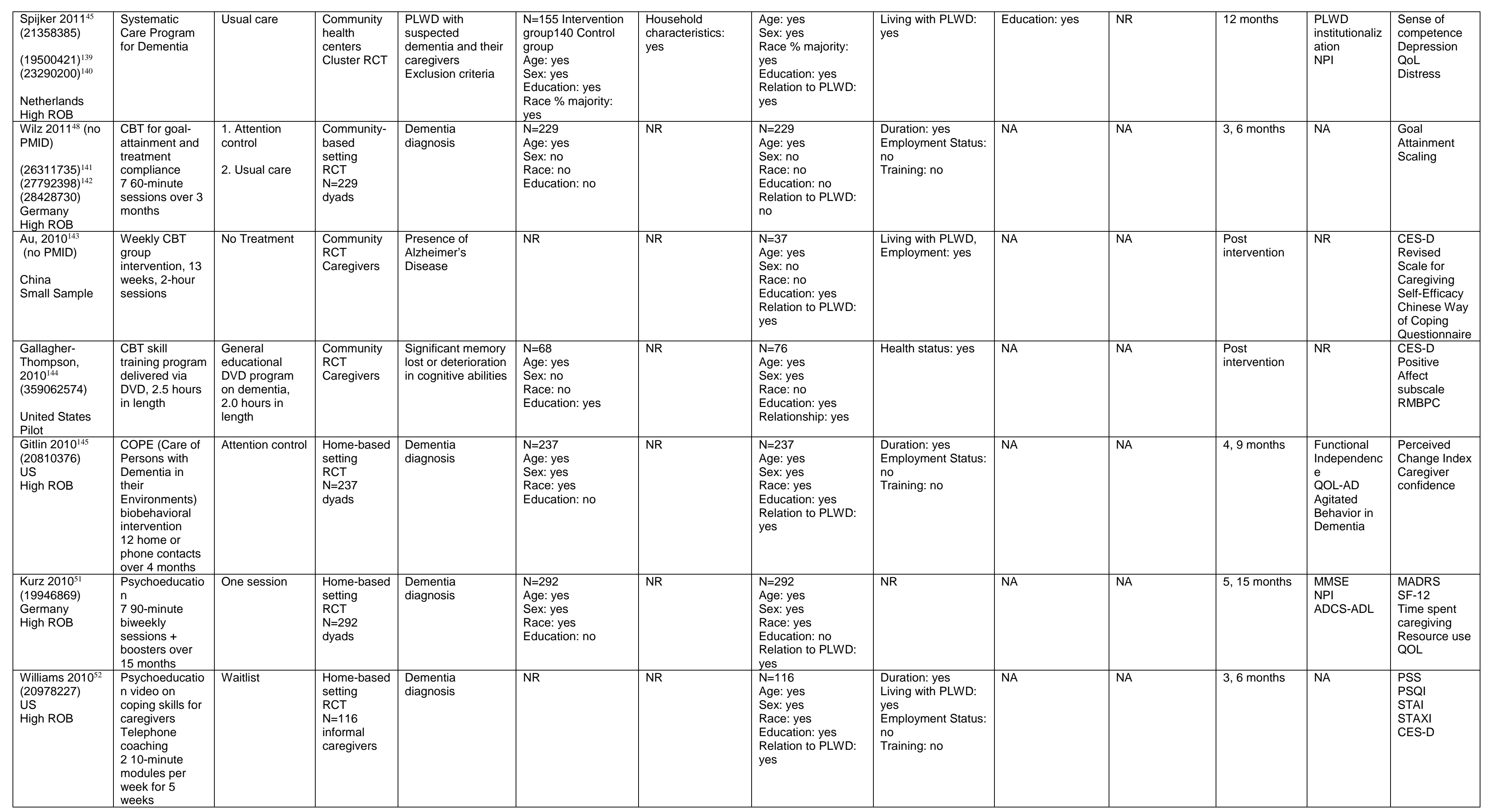




\begin{tabular}{|c|c|c|c|c|c|c|c|c|c|c|c|c|c|}
\hline $\begin{array}{l}\text { Gavrilova } \\
2009^{53} \\
(18814197) \\
\text { Russia } \\
\text { High ROB }\end{array}$ & $\begin{array}{l}\text { Psychoeducatio } \\
\text { n } \\
\text { i0/66 } \\
\text { intervention } \\
\text { basic education } \\
\text { and training for } \\
\text { managing } \\
\text { problem } \\
\text { behaviors } \\
5 \text { weekly } 30- \\
\text { minute sessions } \\
\end{array}$ & Usual care & $\begin{array}{l}\text { Community- } \\
\text { based } \\
\text { setting } \\
\text { RCT } \\
\mathrm{N}=60 \text { dyads }\end{array}$ & \begin{tabular}{|l|} 
Dementia \\
diagnosis
\end{tabular} & $\begin{array}{l}\mathrm{N}=60 \\
\text { Age: yes } \\
\text { Sex: yes } \\
\text { Race: } n \text { o } \\
\text { Education: no }\end{array}$ & NR & $\begin{array}{l}\mathrm{N}=60 \\
\text { Age: yes } \\
\text { Sex: yes } \\
\text { Race: no } \\
\text { Education: no } \\
\text { Relation to PLWD: } \\
\text { yes }\end{array}$ & $\begin{array}{l}\text { Duration: yes } \\
\text { Employment Status: } \\
\text { no } \\
\text { Training: no }\end{array}$ & NA & NA & 6 months & NA & $\begin{array}{l}\text { ZBI } \\
\text { SRQ 20 } \\
\text { WHOQOL- } \\
\text { BREF } \\
\text { NPI-Q } \\
\text { DEMQOL }\end{array}$ \\
\hline $\begin{array}{l}\text { Dias 2008146 } \\
(18523642) \\
\\
\text { India } \\
\text { Pilot }\end{array}$ & $\begin{array}{l}\text { Support and } \\
\text { guidance from } \\
\text { Home Care } \\
\text { Advisors team }\end{array}$ & $\begin{array}{l}\text { Caregivers } \\
\text { received only } \\
\text { education and } \\
\text { information on } \\
\text { dementia; } \\
\text { Intervention } \\
\text { delayed for } 6 \\
\text { months } \\
\end{array}$ & $\begin{array}{l}\text { Home-based } \\
\text { RTC }\end{array}$ & \begin{tabular}{|l|} 
PLWD diagnosed \\
with mild to \\
moderate dementia \\
and their home \\
caregivers \\
Exclusion criteria
\end{tabular} & $\begin{array}{l}\mathrm{N}=33 \text { intervention } \\
\text { group } \\
26 \text { control group } \\
\text { Age: yes } \\
\text { Sex: yes } \\
\text { Education: yes } \\
\text { Race \% majority: N }\end{array}$ & \begin{tabular}{|l|} 
Household \\
characteristics: \\
yes \\
Availability of paid \\
help: yes \\
Income: yes
\end{tabular} & $\begin{array}{l}\text { Age: yes } \\
\text { Sex: yes } \\
\text { Rae \% majority: no } \\
\text { Education: yes } \\
\text { Relation to PLWD: } \\
\text { yes }\end{array}$ & NR & NR & Training: yes & 6 months & \begin{tabular}{|l} 
Activities of \\
daily living \\
NPI
\end{tabular} & $\begin{array}{l}\text { Caregiver } \\
\text { mental health } \\
\text { Caregiver } \\
\text { burden }\end{array}$ \\
\hline 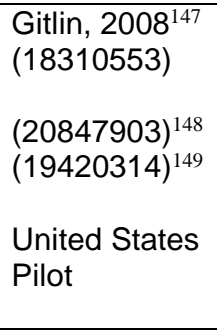 & $\begin{array}{l}\text { Activity-based } \\
\text { intervention } \\
\text { (TAP), } 6 \text { home } \\
\text { visits and two } \\
15 \text {-minute } \\
\text { phone calls over } \\
4 \text { months }\end{array}$ & $\begin{array}{l}\text { Tailored activity } \\
\text { intervention } \\
\text { administered } \\
\text { upon conclusion } \\
\text { of main trial (6 } \\
\text { home visits and } \\
\text { two } 15 \text {-minutes } \\
\text { phone calls over } \\
4 \text { months) } \\
\end{array}$ & $\begin{array}{l}\text { Community } \\
\text { RCT } \\
\text { Caregiver- } \\
\text { PLWD } \\
\text { dyads }\end{array}$ & \begin{tabular}{|l|} 
Diagnosis of \\
dementia by \\
physician or MMSE \\
score $<24$
\end{tabular} & $\begin{array}{l}\mathrm{N}=60 \\
\text { Age: } \text { yes } \\
\text { Sex: yes } \\
\text { Race: yes } \\
\text { Education: yes }\end{array}$ & NR & $\begin{array}{l}\mathrm{N}=60 \\
\text { Age: yes } \\
\text { Sex: yes } \\
\text { Race: yes } \\
\text { Education: yes } \\
\text { Relation to PLWD: } \\
\text { yes }\end{array}$ & Health status: yes & NA & NA & $\begin{array}{l}\text { Post } \\
\text { intervention }\end{array}$ & \begin{tabular}{|l} 
CSDD \\
Quality of \\
Life-AD \\
Behavioral \\
occurrences
\end{tabular} & $\begin{array}{l}\text { ZBB } \\
\text { Subjective } \\
\text { burden } \\
\text { Objective } \\
\text { burden } \\
\text { CES-D }\end{array}$ \\
\hline $\begin{array}{l}\text { Tremont, } \\
2008^{150} \\
(20228893) \\
(18838742)^{151} \\
\text { Pilot }\end{array}$ & $\begin{array}{l}\text { Psychosocial } \\
\text { telephone } \\
\text { intervention } \\
\text { delivered on set } \\
\text { schedule, } \\
\text { completing } 23 \\
\text { calls over one } \\
\text { year, calls } \\
\text { ranged from 15- } \\
60 \text { minutes, } \\
\text { totaling } \\
\text { approximately } \\
12 \text { hours }\end{array}$ & Standard Care & \begin{tabular}{|l} 
Community \\
RCT \\
Caregivers
\end{tabular} & $\begin{array}{l}\text { Formal dementia } \\
\text { diagnosis per } \\
\text { DSM-IV criteria and } \\
\text { CDR score of } 1 \text { or } \\
2\end{array}$ & $\begin{array}{l}\mathrm{N}=60 \\
\text { Age: } \text { yes } \\
\text { Sex: no } \\
\text { Race: no } \\
\text { Education: no }\end{array}$ & NR & $\begin{array}{l}\text { N=60 } \\
\text { Age: yes } \\
\text { Sex: no } \\
\text { Race: no } \\
\text { Education: yes } \\
\text { Relation to PLWD: } \\
\text { no }\end{array}$ & $\begin{array}{l}\text { Duration: yes } \\
\text { Living with PLWD: } \\
\text { yes } \\
\text { Health Status: yes }\end{array}$ & NA & $\mathrm{NA}$ & $\begin{array}{l}\text { Post } \\
\text { intervention }\end{array}$ & NR & $\begin{array}{l}\text { ZBI } \\
\text { RMBPC } \\
\text { GDS } \\
\text { Self-Efficacy } \\
\text { Scale } \\
\text { SF-36 }\end{array}$ \\
\hline $\begin{array}{l}\text { Gant, 2007152 } \\
(17545139) \\
\\
\text { United States } \\
\text { Pilot }\end{array}$ & $\begin{array}{l}\text { Psychosocial } \\
\text { intervention } \\
\text { delivered via } \\
\text { vo-session } \\
\text { video series } \\
\text { with workbook } \\
\text { and } 12 \text { weekly } \\
\text { telephone } \\
\text { coaching } \\
\text { sessions (phone } \\
\text { call length not } \\
\text { specified) }\end{array}$ & $\begin{array}{l}\text { Education } \\
\text { intervention } \\
\text { delivered via } \\
\text { booklet and } 7 \\
\text { bi-weekly } \\
\text { check-in calls } \\
\text { (phone call } \\
\text { length not } \\
\text { specified) }\end{array}$ & \begin{tabular}{|l} 
Community \\
RCT \\
Caregivers
\end{tabular} & \begin{tabular}{|l} 
Diagnosis of \\
dementia \\
secondary to: \\
Alzheimer's \\
disease, \\
Parkinson's \\
disease, \\
cerebrovascular \\
disease, \\
Huntington's \\
disease, \\
Korsakoff's \\
disease, multiple \\
sclerosis, or other \\
\end{tabular} & $\begin{array}{l}\text { N=32 } \\
\text { Age: yes } \\
\text { Sex: no } \\
\text { Race: no } \\
\text { Education: no }\end{array}$ & NR & $\begin{array}{l}\mathrm{N}=32 \\
\text { Age: yes } \\
\text { Sex: yes } \\
\text { Race: } \mathrm{no} \\
\text { Education: yes } \\
\text { Relationship: yes }\end{array}$ & $\begin{array}{l}\text { Living with PLWD: } \\
\text { yes } \\
\text { Employment status: } \\
\text { yes }\end{array}$ & NA & NA & $\begin{array}{l}\text { Post } \\
\text { intervention }\end{array}$ & NR & $\begin{array}{l}\text { RMBPC } \\
\text { RSCSE } \\
\text { PNAS } \\
\text { Target } \\
\text { complaints } \\
\text { (upset, sad, } \\
\text { frustrated, } \\
\text { and irritated) }\end{array}$ \\
\hline
\end{tabular}




\begin{tabular}{|c|c|c|c|c|c|c|c|c|c|c|c|c|c|}
\hline $\begin{array}{l}\text { Glueckauf, } \\
2007^{153} \\
\text { (no PMID) } \\
\text { United States } \\
\text { Small Sample }\end{array}$ & $\begin{array}{l}\text { Weekly } \\
\text { cognitive- } \\
\text { behavioral } \\
\text { intervention } \\
\text { delivered via at- } \\
\text { home video with } \\
\text { telephone } \\
\text { follow-up: } 7 \\
\text { weeks of } 45- \\
\text { minute group } \\
\text { phone sessions, } \\
\text { followed by } 5 \\
\text { weeks of one- } \\
\text { hour individual } \\
\text { phone sessions, } \\
12 \text { weeks }\end{array}$ & No treatment & \begin{tabular}{|l|} 
Community \\
RCT \\
Caregivers
\end{tabular} & $\begin{array}{l}\text { Medical diagnosis } \\
\text { of Alzheimer's } \\
\text { disease or another } \\
\text { type of progressive } \\
\text { dementia }\end{array}$ & \begin{tabular}{|l|}
$\mathrm{N}=24$ \\
Age: $y e s$ \\
Sex: yes \\
Race: yes \\
Education: no
\end{tabular} & NR & $\begin{array}{l}\mathrm{N}=24 \\
\text { Age: yes } \\
\text { Sex: yes } \\
\text { Race: yes } \\
\text { Education: yes } \\
\text { Relation to PLWD: } \\
\text { yes }\end{array}$ & Duration: yes & NA & NA & $\begin{array}{l}\text { One-week } \\
\text { post } \\
\text { intervention }\end{array}$ & $\mathrm{NR}$ & $\begin{array}{l}\text { Caregiver } \\
\text { Appraisal } \\
\text { Inventory CAI } \\
\text { Caregiver } \\
\text { Self-Efficacy } \\
\text { CES-D }\end{array}$ \\
\hline $\begin{array}{l}\text { Marquez- } \\
\text { Gonzalez } \\
2007^{57} \\
(18074249) \\
(22899425)^{154} \\
\text { Spain } \\
\text { High ROB }\end{array}$ & $\begin{array}{l}\text { Cognitive } \\
\text { behavioral } \\
\text { intervention for } \\
\text { caregiver } \\
\text { coping } \\
8 \text { weekly 2-hour } \\
\text { sessions }\end{array}$ & Waitlist & $\begin{array}{l}\text { Community- } \\
\text { based } \\
\text { setting } \\
\mathrm{RCT} \\
\mathrm{N}=74 \\
\text { informal } \\
\text { caregivers }\end{array}$ & $\begin{array}{l}\text { Dementia } \\
\text { diagnosis }\end{array}$ & NR & NR & $\begin{array}{l}\mathrm{N}=74 \\
\text { Age: yes } \\
\text { Sex: yes } \\
\text { Race: no } \\
\text { Education: yes } \\
\text { Relation to PLWD: } \\
\text { yes }\end{array}$ & $\begin{array}{l}\text { Duration: yes } \\
\text { Employment Status: } \\
\text { no } \\
\text { Training: no }\end{array}$ & NA & NA & 8 weeks & $\begin{array}{l}\text { MBPC } \\
\text { (proxy) }\end{array}$ & $\begin{array}{l}\text { CES-D } \\
\text { Dysfunctional } \\
\text { Thoughts } \\
\text { about } \\
\text { Caregiving } \\
\text { Questionnaire }\end{array}$ \\
\hline $\begin{array}{l}\text { Ulstein 200758 } \\
(17986818) \\
\text { Norway } \\
\text { High ROB }\end{array}$ & $\begin{array}{l}\text { Psychosocial } \\
\text { intervention for } \\
\text { education about } \\
\text { dementia and } \\
\text { structured } \\
\text { problem-solving } \\
\text { 3-hour } \\
\text { education } \\
\text { program + } 6 \text { 2- } \\
\text { hour group } \\
\text { meetings over } 1 \\
\text { year }\end{array}$ & Usual care & $\begin{array}{l}\text { Community- } \\
\text { based } \\
\text { setting } \\
\mathrm{RCT} \\
\mathrm{N}=180 \\
\text { dyads }\end{array}$ & $\begin{array}{l}\text { Dementia } \\
\text { diagnosis }\end{array}$ & $\begin{array}{l}\text { N=180 } \\
\text { Age: no } \\
\text { Sex: yes } \\
\text { Race: no } \\
\text { Education: no }\end{array}$ & NR & $\begin{array}{l}\mathrm{N}=180 \\
\text { Age: } n \mathrm{n} \\
\text { Sex: yes } \\
\text { Race: no } \\
\text { Education: no } \\
\text { Relation to PLWD: } \\
\text { yes }\end{array}$ & NR & NA & NA & 1 year & NPI & $\begin{array}{l}\text { Relative } \\
\text { Stress Scale }\end{array}$ \\
\hline $\begin{array}{l}\text { Gonyea 200659 }(17169938) \\
\text { US } \\
\text { High ROB }\end{array}$ & $\begin{array}{l}\text { Behavioral } \\
\text { intervention to } \\
\text { reduce } \\
\text { caregiver } \\
\text { distress, } \\
\text { burden, and } \\
\text { PLWD symptom } \\
\text { severity } \\
5 \text { g9-minute } \\
\text { weekly } \\
\text { sessions, } 16-20 \\
\text { hours total } \\
\text { training }\end{array}$ & Education only & $\begin{array}{l}\text { Community- } \\
\text { based } \\
\text { setting } \\
\mathrm{RCT} \\
\mathrm{N}=80 \\
\text { informal } \\
\text { caregivers }\end{array}$ & $\begin{array}{l}\text { Dementia } \\
\text { diagnosis }\end{array}$ & $\mathrm{NR}$ & $\mathrm{NR}$ & $\begin{array}{l}\mathrm{N}=80 \\
\text { Age: yes } \\
\text { Sex: yes } \\
\text { Race: yes } \\
\text { Education: no } \\
\text { Relation to PLWD: } \\
\text { yes }\end{array}$ & $\begin{array}{l}\text { Duration: yes } \\
\text { Employment Status: } \\
\text { no } \\
\text { Training: no }\end{array}$ & NA & NA & 6 weeks & NPI & $\mathrm{ZBI}$ \\
\hline $\begin{array}{l}\text { Beauchamp } \\
2005^{61} \\
\text { (no PMID) } \\
\text { US } \\
\text { High ROB }\end{array}$ & $\begin{array}{l}\text { Multimedia } \\
\text { support } \\
\text { program } \\
\text { delivered via } \\
\text { internet }\end{array}$ & Waitlist & $\begin{array}{l}\text { Community- } \\
\text { based } \\
\text { setting } \\
R C T \\
N=299 \\
\text { informal } \\
\text { caregivers } \\
\end{array}$ & $\begin{array}{l}\text { Dementia } \\
\text { diagnosis }\end{array}$ & $\mathrm{NR}$ & $\mathrm{NR}$ & $\begin{array}{l}\mathrm{N}=299 \\
\text { Age: yes } \\
\text { Sex: yes } \\
\text { Race: yes } \\
\text { Education: yes } \\
\text { Relation to PLWD: } \\
\text { yes }\end{array}$ & $\begin{array}{l}\text { Duration: yes } \\
\text { Employment Status: } \\
\text { no } \\
\text { Training: no }\end{array}$ & NA & NA & 1 month & NA & $\begin{array}{l}\text { CES-D } \\
\text { STAI } \\
\text { CSS } \\
\text { Positive } \\
\text { Aspects of } \\
\text { Caregiving }\end{array}$ \\
\hline
\end{tabular}




\begin{tabular}{|c|c|c|c|c|c|c|c|c|c|c|c|c|c|}
\hline $\begin{array}{l}\text { Akkerman, } \\
2004^{155} \\
(15106393) \\
\text { United States } \\
\text { Small Sample }\end{array}$ & $\begin{array}{l}\text { Weekly } \\
\text { cognitive- } \\
\text { behavioral } \\
\text { group } \\
\text { intervention, } 9 \\
\text { weeks, 2-hour } \\
\text { meetings }\end{array}$ & No Treatment & $\begin{array}{l}\text { Community } \\
\text { RCT } \\
\text { Caregivers }\end{array}$ & $\begin{array}{l}\text { Diagnosis of } \\
\text { Alzheimer's }\end{array}$ & $\mathrm{NR}$ & $\mathrm{NR}$ & $\begin{array}{l}\mathrm{N}=38 \\
\text { Age: yes } \\
\text { Sex: yes } \\
\text { Race: yes } \\
\text { Education: yes } \\
\text { Relation to PLWD: } \\
\text { yes }\end{array}$ & Duration: yes & NA & $\mathrm{NA}$ & $\begin{array}{l}\text { Post } \\
\text { intervention, } 6 \\
\text { weeks post } \\
\text { intervention }\end{array}$ & $\mathrm{NR}$ & $\begin{array}{l}\text { HAMA } \\
\text { BAI }\end{array}$ \\
\hline $\begin{array}{l}\text { Nobili, 2004156 } \\
\text { (15249851) } \\
\text { Italy } \\
\text { Pilot }\end{array}$ & $\begin{array}{l}\text { 60-minute home } \\
\text { visit from a } \\
\text { psychologist, } \\
\text { separate 90- } \\
\text { minute home } \\
\text { visit from } \\
\text { occupational } \\
\text { therabist }\end{array}$ & $\begin{array}{l}\text { Standard } \\
\text { counseling via } \\
\text { help line, } \\
\text { information } \\
\text { about services } \\
\text { and supports }\end{array}$ & $\begin{array}{l}\text { Community } \\
\text { RCT } \\
\text { Caregiver- } \\
\text { PLWD } \\
\text { Dyads }\end{array}$ & $\begin{array}{l}\text { Clinical diagnosis } \\
\text { of dementia made } \\
\text { by a neurologist, } \\
\text { geriatrician, or } \\
\text { psychiatrist }\end{array}$ & $\begin{array}{l}\text { N=69 } \\
\text { Age: } \text { yes } \\
\text { Sex: yes } \\
\text { Race: no } \\
\text { Education: yes }\end{array}$ & NR & $\begin{array}{l}\mathrm{N}=69 \\
\text { Age: yes } \\
\text { Sex: yes } \\
\text { Race: no } \\
\text { Education: yes } \\
\text { Relation to PLWD: } \\
\text { yes }\end{array}$ & $\begin{array}{l}\text { Duration: yes } \\
\text { Living with PLWD: } \\
\text { yes } \\
\text { Employment Status: } \\
\text { yes }\end{array}$ & NA & NA & $\begin{array}{l}6 \text { months post } \\
\text { intervention, } \\
12 \text { months } \\
\text { post } \\
\text { intervention }\end{array}$ & $\begin{array}{l}\text { ADLS } \\
\text { SBI-C } \\
\text { (problem } \\
\text { behaviors) }\end{array}$ & RSS \\
\hline $\begin{array}{l}\text { Burgio 200363 } \\
(12937335) \\
(16625937)^{157} \\
\text { US } \\
\text { High ROB }\end{array}$ & $\begin{array}{l}\text { Skills training } \\
\text { group workshop } \\
+16 \text { home- } \\
\text { based treatment } \\
\text { sessions over } \\
12 \text { months }\end{array}$ & $\begin{array}{l}\text { Educational } \\
\text { materials and } \\
\text { telephone } \\
\text { support }\end{array}$ & $\begin{array}{l}\text { Home-based } \\
\text { setting } \\
\mathrm{RCT} \\
\mathrm{N}=118 \\
\text { dyads }\end{array}$ & $\begin{array}{l}\text { Dementia } \\
\text { diagnosis }\end{array}$ & $\begin{array}{l}\mathrm{N}=118 \\
\text { Age: yes } \\
\text { Sex: yes } \\
\text { Race: yes } \\
\text { Education: yes }\end{array}$ & NR & $\begin{array}{l}\mathrm{N}=118 \\
\text { Age: yes } \\
\text { Sex: yes } \\
\text { Race: yes } \\
\text { Education: yes } \\
\text { Relation to PLWD: } \\
\text { yes }\end{array}$ & $\begin{array}{l}\text { Duration: yes } \\
\text { Employment Status: } \\
\text { yes } \\
\text { Training: no }\end{array}$ & NA & NA & 6 months & $\begin{array}{l}\text { MMSE } \\
\text { RMBPC } \\
\text { (proxy) }\end{array}$ & $\begin{array}{l}\text { Positive } \\
\text { Aspects of } \\
\text { Caregiving } \\
\text { RMBPC } \\
\text { bother } \\
\text { Lubben } \\
\text { Social } \\
\text { Network } \\
\text { Index } \\
\text { Leisure Time } \\
\text { Satisfaction } \\
\text { CES-D } \\
\text { Desire to } \\
\text { Institutionaliz } \\
\text { e }\end{array}$ \\
\hline $\begin{array}{l}\text { Burns 200364 } \\
(12937333) \\
(19290751)^{158} \\
\text { US } \\
\text { High ROB }\end{array}$ & $\begin{array}{l}\text { REACH } \\
\text { PLWD behavior } \\
\text { management } \\
\text { and caregiver } \\
\text { stress } \\
\text { management }\end{array}$ & $\begin{array}{l}\text { Targeted } \\
\text { educational } \\
\text { materials }\end{array}$ & $\begin{array}{l}\text { Home-based } \\
\text { setting } \\
\text { RCT } \\
N=167 \\
\text { dyads }\end{array}$ & $\begin{array}{l}\text { Dementia } \\
\text { diagnosis }\end{array}$ & $\begin{array}{l}\text { N=167 } \\
\text { Age: yes } \\
\text { Sex: yes } \\
\text { Race: yes } \\
\text { Education: yes }\end{array}$ & $\mathrm{NR}$ & $\begin{array}{l}\mathrm{N}=167 \\
\text { Age: yes } \\
\text { Sex: yes } \\
\text { Race: yes } \\
\text { Education: yes } \\
\text { Relation to PLWD: } \\
\text { yes }\end{array}$ & $\begin{array}{l}\text { Duration: yes } \\
\text { Employment Status: } \\
\text { yes } \\
\text { Training: no }\end{array}$ & NA & $\mathrm{NA}$ & 2 years & RMBPC & $\begin{array}{l}\text { General } \\
\text { wellbeing } \\
\text { scale } \\
\text { CES-D }\end{array}$ \\
\hline $\begin{array}{l}\text { Coon 200365 } \\
(14570964) \\
\text { US } \\
\text { High ROB }\end{array}$ & $\begin{array}{l}\text { Psychoeducatio } \\
\text { n skill training } \\
\text { for } \\
\text { 1. anger or } \\
\text { 2. depression } \\
\text { management } \\
\text { over 3-4 months }\end{array}$ & Waitlist & $\begin{array}{l}\text { Home-based } \\
\text { setting } \\
\mathrm{RCT} \\
\mathrm{N}=169 \\
\text { dyads }\end{array}$ & $\begin{array}{l}\text { Dementia } \\
\text { diagnosis }\end{array}$ & $\begin{array}{l}\text { N=169 } \\
\text { Age: no } \\
\text { Sex: no } \\
\text { Race: no } \\
\text { Education: no }\end{array}$ & NR & $\begin{array}{l}\mathrm{N}=169 \\
\text { Age: yes } \\
\text { Sex: yes } \\
\text { Race: no } \\
\text { Education: no } \\
\text { Relation to PLWD: } \\
\text { yes }\end{array}$ & $\begin{array}{l}\text { Duration: yes } \\
\text { Employment Status: } \\
\text { no } \\
\text { Training: no }\end{array}$ & NA & NA & 4,7 months & NA & $\begin{array}{l}\text { STAXI } \\
\text { Multiple Affect } \\
\text { Adjective } \\
\text { Checklist } \\
\text { Hostility and } \\
\text { Depression } \\
\text { subscales } \\
\text { Ways of } \\
\text { Coping } \\
\text { Checklist } \\
\text { Positive } \\
\text { Coping } \\
\text { subscales } \\
\text { BDI } \\
\text { Caregiving } \\
\text { Self Efficacy }\end{array}$ \\
\hline
\end{tabular}




\begin{tabular}{|c|c|c|c|c|c|c|c|c|c|c|c|c|c|}
\hline $\begin{array}{l}\text { Gitlin 200367 } \\
(12937332) \\
\\
(15860476)^{159} \\
(17595426)^{160} \\
\text { (17563191) }^{161} \\
\text { US } \\
\text { High ROB }\end{array}$ & $\begin{array}{l}\text { REACH Home } \\
\text { Environmental } \\
\text { Skill-Building } \\
\text { Program } \\
5 \text { home-based } \\
\text { sessions + } 1 \\
\text { telephone } \\
\text { session }\end{array}$ & Usual care & $\begin{array}{l}\text { Home-based } \\
\text { setting } \\
\text { RCT } \\
\mathrm{N}=255 \\
\text { dyads }\end{array}$ & $\begin{array}{l}\text { Dementia } \\
\text { diagnosis }\end{array}$ & $\begin{array}{l}\mathrm{N}=255 \\
\text { Age: yes } \\
\text { Sex: yes } \\
\text { Race: } \mathrm{no} \\
\text { Education: no }\end{array}$ & NR & $\begin{array}{l}\mathrm{N}=255 \\
\text { Age: yes } \\
\text { Sex: yes } \\
\text { Race: yes } \\
\text { Education: yes } \\
\text { Relation to PLWD: } \\
\text { yes }\end{array}$ & $\begin{array}{l}\text { Duration: yes } \\
\text { Employment Status: } \\
\text { no } \\
\text { Training: no }\end{array}$ & NA & NA & 6 months & NA & $\begin{array}{l}\text { RMBPC } \\
\text { Upset } \\
\text { Caregiving } \\
\text { Mastery Index } \\
\text { Task } \\
\text { Management } \\
\text { Strategy } \\
\text { Index }\end{array}$ \\
\hline $\begin{array}{l}\text { Hebert 2003 }^{68} \\
(12496309) \\
\\
(15841829)^{162} \\
\text { Canada } \\
\text { High ROB }\end{array}$ & $\begin{array}{l}\text { Psychoeducatio } \\
\text { n Group } \\
\text { Program on } \\
\text { stress appraisal } \\
\text { and coping } \\
15 \text { 2-hour } \\
\text { weekly sessions }\end{array}$ & Usual care & $\begin{array}{l}\text { Home-based } \\
\text { setting } \\
\text { RCT } \\
N=158 \\
\text { dyads }\end{array}$ & $\begin{array}{l}\text { Dementia } \\
\text { diagnosis }\end{array}$ & NR & NR & $\begin{array}{l}\mathrm{N}=158 \\
\text { Age: yes } \\
\text { Sex: yes } \\
\text { Race: no } \\
\text { Education: yes } \\
\text { Relation to PLWD: } \\
\text { yes }\end{array}$ & $\begin{array}{l}\text { Duration: yes } \\
\text { Living with PLWD: } \\
\text { yes } \\
\text { Employment Status: } \\
\text { yes } \\
\text { Training: no }\end{array}$ & NA & NA & 4 months & RMBPC & $\begin{array}{l}\text { RMBPC } \\
\text { reaction } \\
\text { ZBI } \\
\text { STAI } \\
\text { Bradburn } \\
\text { Revised } \\
\text { Affective } \\
\text { Scale } \\
\text { Inventory of } \\
\text { Socially } \\
\text { Supportive } \\
\text { Behavior } \\
\text { Ilfeld } \\
\text { Psychiatric } \\
\text { Symptoms } \\
\text { Index }\end{array}$ \\
\hline $\begin{array}{l}\text { Huang, 2003163 } \\
\text { Taiwan } \\
\text { Pilot }\end{array}$ & $\begin{array}{l}\text { Two-session in- } \\
\text { home caregiver } \\
\text { training, } \\
\text { sessions } \\
\text { separated by } \\
\text { one week, 2-3- } \\
\text { hour sessions; } \\
\text { training } \\
\text { accompanied } \\
\text { by follow-up } \\
\text { phone calls } \\
\text { once a week for } \\
\text { two weeks; } \\
\text { accompanied by } \\
\text { further weekly } \\
\text { follow-wu } \\
\text { consultation } \\
\text { calls beginning } \\
\text { three weeks } \\
\text { after completion } \\
\text { of two-session } \\
\text { program }\end{array}$ & $\begin{array}{l}\text { Written } \\
\text { educational } \\
\text { materials with } \\
\text { social telephone } \\
\text { phone calls } \\
\text { once a week for } \\
\text { two weeks; } \\
\text { accompanied by } \\
\text { weekly follow- } \\
\text { up social phone } \\
\text { calls beginning } \\
\text { three weeks } \\
\text { after } \\
\text { experimental } \\
\text { group } \\
\text { completed two- } \\
\text { session } \\
\text { program }\end{array}$ & $\begin{array}{l}\text { Community } \\
\text { RCT } \\
\text { Caregivers }\end{array}$ & $\begin{array}{l}\text { Diagnosis of } \\
\text { dementia by } \\
\text { psychiatrist or } \\
\text { neurologist }\end{array}$ & $\begin{array}{l}\text { N=59 } \\
\text { Age: yes } \\
\text { Sex: yes } \\
\text { Race: no } \\
\text { Education: yes }\end{array}$ & $\mathrm{NR}$ & $\begin{array}{l}\mathrm{N}=59 \\
\text { Age: } \text { yes } \\
\text { Sex: yes } \\
\text { Race: no } \\
\text { Education: yes } \\
\text { Relation to PLWD: } \\
\text { yes }\end{array}$ & $\mathrm{NR}$ & NA & NA & $\begin{array}{l}\text { Three weeks } \\
\text { after } \\
\text { experimental } \\
\text { group } \\
\text { completed } \\
\text { two-session } \\
\text { training } \\
\text { program; } \\
\text { again three } \\
\text { months after } \\
\text { training } \\
\text { completion }\end{array}$ & $\begin{array}{l}\text { Cohen- } \\
\text { Manstield } \\
\text { Agitation } \\
\text { Inventory } \\
\text { (CMAl; } \\
\text { problematic } \\
\text { behaviors) }\end{array}$ & $\begin{array}{l}\text { Agitation } \\
\text { Management } \\
\text { Self-Efficacy } \\
\text { Scale (AMSS) }\end{array}$ \\
\hline $\begin{array}{l}\text { Martin-Cook, } \\
2003^{164} \\
(14682086) \\
\text { United States } \\
\text { Pilot }\end{array}$ & $\begin{array}{l}\text { Weekly group } \\
\text { psychoeducatio } \\
\text { nal intervention, } \\
4 \text { weeks, 2-hour } \\
\text { sessions }\end{array}$ & Standard Care & $\begin{array}{l}\text { Community } \\
\text { RCT } \\
\text { Caregivers }\end{array}$ & $\begin{array}{l}\text { Diagnosis of } \\
\text { dementing illness } \\
\text { with behavioral } \\
\text { disturbance }\end{array}$ & NR & NR & $\begin{array}{l}\text { N=37 } \\
\text { Age: no } \\
\text { Sex: no } \\
\text { Race: no } \\
\text { Education: no } \\
\text { Relationship: no }\end{array}$ & NR & NA & NA & $\begin{array}{l}\text { Two weeks } \\
\text { post } \\
\text { intervention, } \\
10 \text { weeks post } \\
\text { intervention }\end{array}$ & NR & $\begin{array}{l}\text { Caregiver } \\
\text { Resentment } \\
\text { Scale } \\
\text { CES-D } \\
\text { NPI }\end{array}$ \\
\hline $\begin{array}{l}\text { Fung 200269 } \\
(12037799) \\
\\
\text { Hong Kong } \\
\text { High ROB }\end{array}$ & $\begin{array}{l}\text { Support group } \\
\text { for family } \\
\text { caregivers } \\
12 \text { 1-hour } \\
\text { sessions }\end{array}$ & Usual care & $\begin{array}{l}\text { Community- } \\
\text { based } \\
\text { setting } \\
\text { RCT } \\
N=52 \text { dyads }\end{array}$ & $\begin{array}{l}\text { Dementia } \\
\text { diagnosis }\end{array}$ & $\mathrm{NR}$ & $\mathrm{NR}$ & $\begin{array}{l}\mathrm{N}=52 \\
\text { Age: yes } \\
\text { Sex: yes } \\
\text { Race: no } \\
\text { Education: yes } \\
\text { Relation to PLWD: } \\
\text { yes }\end{array}$ & $\begin{array}{l}\text { Duration: yes } \\
\text { Employment Status: } \\
\text { yes } \\
\text { Training: no }\end{array}$ & NA & NA & 3 months & NA & $\begin{array}{l}\text { NPI-D } \\
\text { WHOQOL- } \\
\text { BREF } \\
\text { Mental health } \\
\text { service use }\end{array}$ \\
\hline
\end{tabular}




\begin{tabular}{|c|c|c|c|c|c|c|c|c|c|c|c|c|c|}
\hline $\begin{array}{l}\text { Garand, 2002165 } \\
(12143075) \\
\text { United States } \\
\text { Pilot }\end{array}$ & $\begin{array}{l}\text { Psychoeducatio } \\
\text { nal nursing } \\
\text { intervention } \\
\text { delivered } \\
\text { through two in- } \\
\text { home visits, } 3 \\
\text { hours each, } \\
\text { followed by } \\
\text { twice weekly } \\
\text { phone calls for } \\
6 \text { months } \\
\text { (phone call } \\
\text { length not } \\
\text { specified) }\end{array}$ & $\begin{array}{l}\text { Psychological } \\
\text { support, } \\
\text { traditional } \\
\text { dementia } \\
\text { information, and } \\
\text { referral to } \\
\text { community- } \\
\text { based services, } \\
\text { delivered } \\
\text { through two in- } \\
\text { home visits, } \\
\text { hours each, } \\
\text { followed by } \\
\text { twice weekkly } \\
\text { phone calls for } \\
6 \text { months } \\
\text { (phone call } \\
\text { length not } \\
\text { specified) }\end{array}$ & $\begin{array}{l}\text { Community } \\
\text { RCT } \\
\text { Caregivers }\end{array}$ & $\begin{array}{l}\text { Dementia, } \\
\text { diagnosed or } \\
\text { undiagnosed }\end{array}$ & $\begin{array}{l}\mathrm{N}=39 \\
\text { Age: yes } \\
\text { Sex: yes } \\
\text { Race: yes } \\
\text { Education: no }\end{array}$ & NR & $\begin{array}{l}\mathrm{N}=39 \\
\text { Age: yes } \\
\text { Sex: yes } \\
\text { Race: yes } \\
\text { Education: yes } \\
\text { IC Relation to } \\
\text { PLWD: yes }\end{array}$ & $\begin{array}{l}\text { Health Status: yes } \\
\text { Employment Status, } \\
\text { Training: yes }\end{array}$ & $\mathrm{NA}$ & NA & $\begin{array}{l}\text { One week } \\
\text { after in-home } \\
\text { phase, again } \\
\text { after } 6 \text { months } \\
\text { of biweekly } \\
\text { phone calls }\end{array}$ & $\mathrm{NR}$ & $\begin{array}{l}\text { Total mood } \\
\text { disturbance }\end{array}$ \\
\hline $\begin{array}{l}\text { Stolley, 2002 } \\
(11954669) \\
\text { US } \\
\text { High ROB }\end{array}$ & $\begin{array}{l}\text { Psychoeducatio } \\
\mathrm{n} \text { based on } \\
\text { progressively } \\
\text { lowered stress } \\
\text { theory }\end{array}$ & $\begin{array}{l}\text { Basic dementia } \\
\text { education }\end{array}$ & $\begin{array}{l}\text { Community } \\
\text { RCT } \\
\text { Caregivers }\end{array}$ & $\begin{array}{l}\text { Physician- } \\
\text { diagnosed } \\
\text { dementia; } 66 \% A D\end{array}$ & $\mathrm{NR}$ & NR & $\begin{array}{l}\mathrm{N}=241 \\
\text { Age: yes } \\
\text { Sex: yes } \\
\text { Race: yes } \\
\text { Education: yes } \\
\text { Relation to PLWD: } \\
\text { yes }\end{array}$ & Duration: yes & NA & NA & 12 months & NA & $\begin{array}{l}\text { ZBI } \\
\text { Mastery }\end{array}$ \\
\hline $\begin{array}{l}\text { Wright 2001 } \\
(11885210) \\
\text { US } \\
\text { High ROB }\end{array}$ & $\begin{array}{l}\text { Nurse education } \\
\text { and counseling } \\
\text { program } \\
\text { Home visits and } \\
\text { phone calls }\end{array}$ & Attention control & $\begin{array}{l}\text { Community- } \\
\text { based } \\
\text { setting } \\
\mathrm{RCT} \\
\mathrm{N}=93 \\
\text { informal } \\
\text { caregivers }\end{array}$ & $\begin{array}{l}\text { Dementia } \\
\text { diagnosis }\end{array}$ & $\begin{array}{l}\mathrm{N}=93 \\
\text { Age: yes } \\
\text { Sex: yes } \\
\text { Race: no } \\
\text { Education: no }\end{array}$ & NR & $\begin{array}{l}\mathrm{N}=93 \\
\text { Age: yes } \\
\text { Sex: yes } \\
\text { Race: yes } \\
\text { Education: yes } \\
\text { Relation to PLWD: } \\
\text { yes }\end{array}$ & $\begin{array}{l}\text { Duration: yes } \\
\text { Living with PLWD: } \\
\text { yes } \\
\text { Employment Status: } \\
\text { no } \\
\text { Training: no }\end{array}$ & NA & NA & $\begin{array}{l}2,6,12 \text { weeks } \\
6,12 \text { months }\end{array}$ & \begin{tabular}{|l|} 
Blessed \\
Dementia \\
Rating Scale \\
CMAl
\end{tabular} & $\begin{array}{l}\text { Caregiving } \\
\text { Hassle Scale } \\
\text { CES-D } \\
\text { Multilevel } \\
\text { Assessment } \\
\text { Inventory }\end{array}$ \\
\hline $\begin{array}{l}\text { Marriott, 2000166 } \\
(10974962) \\
\text { United Kingdom } \\
\text { Small Sample }\end{array}$ & $\begin{array}{l}\text { Cognitive- } \\
\text { behavioral } \\
\text { family } \\
\text { intervention, } \\
\text { individual } \\
\text { sessions every } \\
\text { two weeks, 14 } \\
\text { sessions (length } \\
\text { of sessions } \\
\text { unspecified) }\end{array}$ & $\begin{array}{l}\text { In-depth } \\
\text { interview of } \\
\text { caregiver OR no } \\
\text { interview }\end{array}$ & $\begin{array}{l}\text { Community } \\
\text { RCT } \\
\text { Caregivers }\end{array}$ & $\begin{array}{l}\text { Satisfaction of } \\
\text { DSM-III-R criteria } \\
\text { for primary } \\
\text { degenerative } \\
\text { dementia of the } \\
\text { Alzheimer type }\end{array}$ & $\begin{array}{l}\mathrm{N}=42 \\
\text { Age: yes } \\
\text { Sex: yes } \\
\text { Race: no } \\
\text { Education: no }\end{array}$ & NR & $\begin{array}{l}\mathrm{N}=42 \\
\text { Age: yes } \\
\text { Sex: yes } \\
\text { Race: no } \\
\text { Education: no } \\
\text { Relation to PLWD: } \\
\text { yes }\end{array}$ & $\begin{array}{l}\text { Duration: yes } \\
\text { Living with PLWD: } \\
\text { yes }\end{array}$ & NA & NA & $\begin{array}{l}\text { Post } \\
\text { intervention, } \\
\text { three months } \\
\text { post } \\
\text { intervention }\end{array}$ & $\begin{array}{l}\text { ADLs } \\
\text { CSDD }\end{array}$ & $\begin{array}{l}\text { GHQ } \\
\text { (caseness/ps } \\
\text { ychiatric } \\
\text { morbidity) } \\
\text { BDI }\end{array}$ \\
\hline $\begin{array}{l}\text { Steffen, 2000167 } \\
\text { (no PMID) } \\
\text { United States } \\
\text { Pilot }\end{array}$ & $\begin{array}{l}\text { Weekly 30- } \\
\text { minute } \\
\text { psychoeducatio } \\
\text { nal videos-- } \\
\text { viewed either at } \\
\text { home with 20- } \\
\text { minute weekly } \\
\text { telephone } \\
\text { sessions or in a } \\
\text { class setting } \\
\text { with a trained } \\
\text { facilitator--with } \\
\text { accompanying } \\
\text { workbook, } \\
\text { weeks, } \\
\text { minute- } \\
\text { classroom } \\
\text { sessions }\end{array}$ & No Treatment & $\begin{array}{l}\text { Community } \\
\text { RCT } \\
\text { Caregivers }\end{array}$ & $\begin{array}{l}\text { Diagnosis of } \\
\text { Alzheimer's } \\
\text { disease, multi- } \\
\text { infarct dementia or } \\
\text { some other } \\
\text { dementing illness }\end{array}$ & $\mathrm{NR}$ & NR & $\begin{array}{l}\mathrm{N}=33 \\
\text { Age: yes } \\
\text { Sex: yes } \\
\text { Race: yes } \\
\text { Education: yes } \\
\text { Relationship: yes }\end{array}$ & $\mathrm{NR}$ & NA & NA & $\begin{array}{l}\text { Post } \\
\text { intervention }\end{array}$ & NR & $\begin{array}{l}\text { CgAl } \\
\text { BDI } \\
\text { Revised } \\
\text { Care-Giving } \\
\text { Self-Efficacy } \\
\text { Scale }\end{array}$ \\
\hline
\end{tabular}




\begin{tabular}{|c|c|c|c|c|c|c|c|c|c|c|c|c|c|}
\hline $\begin{array}{l}\text { Buckwalter } \\
1999^{72} \\
(10222636) \\
(12464756)^{168} \\
\text { US } \\
\text { High ROB }\end{array}$ & $\begin{array}{l}\text { Psychoeducatio } \\
\text { n for managing } \\
\text { problem } \\
\text { behaviors } \\
\text { Information, } \\
\text { case } \\
\text { management } \\
\text { referrals, } \\
\text { service } \\
\text { provision, } \\
\text { support groups }\end{array}$ & NR & \begin{tabular}{|l} 
Community- \\
based \\
setting \\
$\mathrm{RCT}$ \\
$\mathrm{N}=245$ \\
dyads
\end{tabular} & \begin{tabular}{|l|} 
Dementia \\
diagnosis
\end{tabular} & \begin{tabular}{|l|}
$\mathrm{N}=245$ \\
Age: no \\
Sex: yes \\
Race: $\mathrm{no}$ \\
Education: no
\end{tabular} & NR & $\begin{array}{l}\mathrm{N}=245 \\
\text { Age: yes } \\
\text { Sex: yes } \\
\text { Race: yes } \\
\text { Education: yes } \\
\text { Relation to PLWD: } \\
\text { yes }\end{array}$ & $\begin{array}{l}\text { Duration: yes } \\
\text { Employment Status: } \\
\text { yes } \\
\text { Training: yes }\end{array}$ & NA & NA & 6,12 months & NA & $\begin{array}{l}\text { POMS } \\
\text { GDRS }\end{array}$ \\
\hline $\begin{array}{l}\text { Chang 199973 } \\
(10337848) \\
(15471059)^{169} \\
\text { USA } \\
\text { High ROB }\end{array}$ & $\begin{array}{l}\text { Cognitive- } \\
\text { behavioral } \\
\text { intervention for } \\
\text { PLWD and } \\
\text { care-giver }\end{array}$ & $\begin{array}{l}\text { Attention only } \\
\text { placebo } \\
\text { telephone calls }\end{array}$ & $\begin{array}{l}\text { Homebased } \\
\text { RCT }\end{array}$ & $\begin{array}{l}\text { PLWD diagnosed } \\
\text { with dementia } \\
\text { MMSE<21 } \\
\text { Significant eating } \\
\text { and dressing } \\
\text { problems } \\
\text { Home caregivers }\end{array}$ & $\begin{array}{l}\mathrm{N}=34 \text { Intervention } \\
\text { group } \\
\text { 31 Control group } \\
\text { Age: yes } \\
\text { Sex: no } \\
\text { Education: yes } \\
\text { Race \% majority: } \\
\text { yes }\end{array}$ & NR & $\begin{array}{l}\text { Age: yes } \\
\text { Sex: yes } \\
\text { Race \% majority: } \\
\text { yes } \\
\text { Education: yes } \\
\text { Relation to PLWD: } \\
\text { yes }\end{array}$ & $\begin{array}{l}\text { Duration: yes } \\
\text { Living with PLWD: } \\
\text { yes } \\
\text { Training: yes }\end{array}$ & NR & NR & 12 weeks & MMSE & $\begin{array}{l}\text { Depression } \\
\text { Burden } \\
\text { Satisfaction } \\
\text { Anxiety }\end{array}$ \\
\hline $\begin{array}{l}\text { Ostwald 19997 } \\
\text { (10396888) } \\
\text { US } \\
\text { High ROB }\end{array}$ & $\begin{array}{l}\text { Psychoeducatio } \\
\text { n group } \\
\text { intervention for } \\
\text { caregiver } \\
\text { burden and } \\
\text { depression } \\
7 \text { weekly 2-hour } \\
\text { sessions }\end{array}$ & Waitlist & $\begin{array}{l}\text { Community- } \\
\text { based } \\
\text { setting } \\
\mathrm{RCT} \\
\mathrm{N}=117 \\
\text { dyads }\end{array}$ & \begin{tabular}{|l|} 
Dementia \\
diagnosis
\end{tabular} & $\begin{array}{l}\mathrm{N}=117 \\
\text { Age: yes } \\
\text { Sex: yes } \\
\text { Race: } \text { no } \\
\text { Education: yes }\end{array}$ & PLWD SES: yes & $\begin{array}{l}\mathrm{N}=117 \\
\text { Age: yes } \\
\text { Sex: yes } \\
\text { Race: no } \\
\text { Education: yes } \\
\text { Relation to PLWD: } \\
\text { no }\end{array}$ & $\begin{array}{l}\text { Duration: no } \\
\text { Employment Status: } \\
\text { no } \\
\text { Training: no }\end{array}$ & NA & NA & 5 months & RMBPC & $\begin{array}{l}\text { ZBI } \\
\text { CES-D }\end{array}$ \\
\hline $\begin{array}{l}\text { McCurry, } \\
1998^{170} \\
(9520929) \\
\\
\text { United States } \\
\text { Pilot }\end{array}$ & $\begin{array}{l}\text { Weekly small } \\
\text { group } \\
\text { behavioral } \\
\text { treatment } \\
\text { sessions, } 6 \\
\text { weeks (session } \\
\text { length not } \\
\text { specified) OR } \\
\text { weekly } \\
\text { individidual } \\
\text { behavioral } \\
\text { treatment, 4 } \\
\text { weeks (session } \\
\text { length not } \\
\text { specified) }\end{array}$ & No Treatment & \begin{tabular}{|l|} 
Community \\
RCT \\
Caregivers
\end{tabular} & $\begin{array}{l}\text { Diagnosis of senile } \\
\text { dementia }\end{array}$ & $\begin{array}{l}\text { N=36 } \\
\text { Age: yes } \\
\text { Sex: yes } \\
\text { Race: no } \\
\text { Education: no }\end{array}$ & NR & $\begin{array}{l}\mathrm{N}=36 \\
\text { Age: yes } \\
\text { Sex: yes } \\
\text { Race: yes } \\
\text { Education: yes } \\
\text { Relationship: yes }\end{array}$ & $\begin{array}{l}\text { Living with PLWD: } \\
\text { yes }\end{array}$ & NA & NA & $\begin{array}{l}\text { Post } \\
\text { intervention, } 3 \\
\text { months post } \\
\text { intervention }\end{array}$ & NR & $\begin{array}{l}\text { CES-D } \\
\text { SCB } \\
\text { RMBPC }\end{array}$ \\
\hline $\begin{array}{l}\text { Moniz-Cook, } \\
\text { 1998 }^{171} \text { (no } \\
\text { PMID) } \\
\text { Pilot }\end{array}$ & $\begin{array}{l}\text { Advice } \\
\text { pamphlets plus } \\
\text { home-based } \\
\text { individualized } \\
\text { intervention } \\
\text { delivered after } \\
\text { dementia } \\
\text { diagnosis, six to } \\
12 \text { hours in } \\
\text { duration, four to } \\
\text { fourteen weeks } \\
\end{array}$ & $\begin{array}{l}\text { Advice } \\
\text { pamphlets plus } \\
\text { usual care (i.e.,. } \\
\text { referred to local } \\
\text { psychogeriatric } \\
\text { community } \\
\text { team upon } \\
\text { diagnosis) }\end{array}$ & \begin{tabular}{|l|} 
Community \\
RCT \\
Caregiver- \\
PLWD \\
Dyads
\end{tabular} & \begin{tabular}{|l|} 
Probable diagnosis \\
of Alzheimer's \\
disease, multi- \\
infarct dementia, or \\
frontal lobe \\
dementia per \\
neuropsychological \\
profiles and ICD-10 \\
operational criteria \\
for diagnosing \\
dementia \\
\end{tabular} & $\begin{array}{l}\mathrm{N}=30 \\
\text { Age: yes } \\
\text { Sex: yes } \\
\text { Race: no } \\
\text { Education: no }\end{array}$ & $\begin{array}{l}\text { Household } \\
\text { characteristics }\end{array}$ & $\begin{array}{l}\mathrm{N}=30 \\
\text { Age: } n \text { no } \\
\text { Sex: no } \\
\text { Race: no } \\
\text { Education: no } \\
\text { Relation to PLWD: } \\
\text { no }\end{array}$ & $\mathrm{NR}$ & NA & NA & $\begin{array}{l}6 \text { months after } \\
\text { diagnosis } \\
\text { (caregivers), } \\
18 \text { months } \\
\text { after } \\
\text { diagnosis } \\
\text { (PLWD and } \\
\text { caregivers) }\end{array}$ & $\begin{array}{l}\text { Memory } \\
\text { impairment } \\
\text { RBMT }\end{array}$ & $\begin{array}{l}\text { GHQ } \\
\text { BDI } \\
\text { HADS }\end{array}$ \\
\hline
\end{tabular}




\begin{tabular}{|c|c|c|c|c|c|c|c|c|c|c|c|c|c|}
\hline $\begin{array}{l}\text { Brodaty, 1997 } \\
(9520929) \\
\text { Australia } \\
\text { Pilot }\end{array}$ & $\begin{array}{l}\text { Caregiver } \\
\text { training program } \\
\text { delivered daily } \\
\text { for } 10 \text { days in } \\
\text { residential } \\
\text { setting } \\
\text { immediately } \\
\text { unpon enrollment } \\
\text { in study, } \\
\text { followed by } \\
\text { telephone } \\
\text { check-ins at } \\
\text { decreasing time } \\
\text { intervals over } \\
\text { the next } 12 \\
\text { months OR } \\
\text { same treatment } \\
\text { and follow-up } \\
\text { delivered } 6 \\
\text { months after } \\
\text { studdy } \\
\text { enrollment }\end{array}$ & $\begin{array}{l}\text { No Treatment } \\
\text { (PLWD memory } \\
\text { retraining only, } \\
\text { delivered to all } \\
\text { PLWD in study) }\end{array}$ & \begin{tabular}{|l} 
Community \\
RCT \\
Caregivers
\end{tabular} & \begin{tabular}{|l} 
Diagnosis of \\
Dementia
\end{tabular} & $\begin{array}{l}\mathrm{N}=96 \\
\text { Age: } y e s \\
\text { Sex: yes } \\
\text { Race: } n \text { o } \\
\text { Education: no }\end{array}$ & \begin{tabular}{|l|}
$N R$ \\
\end{tabular} & $\begin{array}{l}\mathrm{N}=96 \\
\text { Age: yes } \\
\text { Sex: yes } \\
\text { Race: no } \\
\text { Education: no } \\
\text { Relation to PLWD: } \\
\text { yes }\end{array}$ & $\begin{array}{l}\begin{array}{l}\text { Living with PLWD: } \\
\text { yes }\end{array} \\
\end{array}$ & \begin{tabular}{|l|}
$N A$ \\
\end{tabular} & NA & \begin{tabular}{|l|}
3 months \\
post- \\
intervention, 6 \\
months post- \\
intervention, \\
12 months \\
post- \\
intervention, \\
and annually \\
thereafter until \\
conclusion of \\
8-year study
\end{tabular} & \begin{tabular}{|l} 
Nursing \\
home \\
admission, \\
time until \\
death
\end{tabular} & NR \\
\hline $\begin{array}{l}\text { Teri, 1997173 } \\
(9224439) \\
\\
\text { United States } \\
\text { Small Sample }\end{array}$ & $\begin{array}{l}\text { Behavior } \\
\text { therapy focused } \\
\text { on increasing } \\
\text { pleasant events } \\
\text { OR behavior } \\
\text { therapy focused } \\
\text { on problem } \\
\text { solving } \\
\text { istuations of } \\
\text { concern, both } \\
\text { treatments } \\
\text { administered } \\
\text { weekly for } 9 \\
\text { weeks, } 60- \\
\text { minute sessions }\end{array}$ & $\begin{array}{l}\text { Typical } \\
\text { therapeutic care } \\
\text { weekly for } 9 \\
\text { weeks, 60- } \\
\text { minute sessions } \\
\text { OR no } \\
\text { treatment } \\
\text { (waitlist) }\end{array}$ & $\begin{array}{l}\text { Community } \\
\text { RCT } \\
\text { Caregiver- } \\
\text { PLWD } \\
\text { dyads }\end{array}$ & \begin{tabular}{|l|} 
Probable \\
Alzheimer's \\
disease per \\
NINCDS-ADRDA \\
criteria
\end{tabular} & $\begin{array}{l}\mathrm{N}=88 \\
\text { Age: } \text { yes } \\
\text { Sex: yes } \\
\text { Race: } \text { no } \\
\text { Education: yes }\end{array}$ & NR & $\begin{array}{l}\mathrm{N}=88 \\
\text { Age: } \text { yes } \\
\text { Sex: yes } \\
\text { Race: } \mathrm{no} \\
\text { Education: yes } \\
\text { Relation to PLWD: } \\
\text { yes }\end{array}$ & NR & NA & NA & \begin{tabular}{|l} 
Post \\
intervention, \\
six months \\
post \\
intervention
\end{tabular} & \begin{tabular}{|l} 
HDRS \\
CSDD \\
BDI \\
Record of \\
Independent \\
Living (RIL) \\
\end{tabular} & $\begin{array}{l}\text { HDRS } \\
\text { ZBI }\end{array}$ \\
\hline $\begin{array}{l}\text { Gendron, } \\
1996^{174} \\
(26250550) \\
\text { Pilot }\end{array}$ & $\begin{array}{l}\text { Weekly } \\
\text { cognitive- } \\
\text { behavioral skills } \\
\text { training group } \\
\text { intervention, } 8 \\
\text { weeks, 90- } \\
\text { minute sessions }\end{array}$ & $\begin{array}{l}\text { Weekly support } \\
\text { group } \\
\text { emphasizing } \\
\text { information and } \\
\text { social exchange } \\
8 \text { weeks, 90- } \\
\text { minute sessions }\end{array}$ & \begin{tabular}{|l} 
Community \\
RCT \\
Caregivers
\end{tabular} & $\begin{array}{l}\text { Diagnosis of } \\
\text { dementia }\end{array}$ & NR & NR & $\begin{array}{l}\mathrm{N}=35 \\
\text { Age: yes } \\
\text { Sex: yes } \\
\text { Race: no } \\
\text { Education: yes } \\
\text { Relation to PLWD: } \\
\text { yes }\end{array}$ & $\begin{array}{l}\text { Duration: yes } \\
\text { Living with PLWD: } \\
\text { yes } \\
\text { Health Status: yes }\end{array}$ & NA & NA & $\begin{array}{l}\text { Post } \\
\text { intervention, } \\
\text { 3-month } \\
\text { follow-up, } 6 \\
\text { month follow } \\
\text { up }\end{array}$ & NR & \begin{tabular}{|l} 
Hopkins \\
Symptom \\
Checklist \\
(distress) \\
Automatic \\
Thoughts \\
Questionnaire \\
(depressive \\
thinking) \\
ZBI \\
RMBPC \\
\end{tabular} \\
\hline $\begin{array}{l}\text { Hebert, 1994 } \\
(24087060) \\
(25107702)^{176} \\
\text { Canada } \\
\text { Small Sample }\end{array}$ & $\begin{array}{l}\text { Weekly } \\
\text { structured } \\
\text { support group } \\
\text { sessions for } 8 \\
\text { weeks, 2-hour } \\
\text { sessions }\end{array}$ & $\begin{array}{l}\text { Referral to } \\
\text { informal } \\
\text { monthly } \\
\text { meetings of the } \\
\text { Alzheimer's } \\
\text { Society }\end{array}$ & \begin{tabular}{|l} 
Community \\
RCT \\
Caregivers
\end{tabular} & $\begin{array}{l}\text { Dementia } \\
\text { confirmed by DSM- } \\
\text { IIIR criteria }\end{array}$ & NR & NR & $\begin{array}{l}\text { N= } 45 \\
\text { Age: yes } \\
\text { Sex: yes } \\
\text { Race: no } \\
\text { Education: yes } \\
\text { Relationship: yes }\end{array}$ & $\begin{array}{l}\text { Living with PLWD: } \\
\text { yes } \\
\text { Employment status: } \\
\text { yes }\end{array}$ & NA & NA & \begin{tabular}{|l} 
Post \\
intervention, 6 \\
months post \\
intervention
\end{tabular} & SMAF & \begin{tabular}{|l} 
ZBI \\
BSI \\
RMBPC
\end{tabular} \\
\hline
\end{tabular}




\begin{tabular}{|c|c|c|c|c|c|c|c|c|c|c|c|c|c|}
\hline $\begin{array}{l}\text { Tappen, } 1994^{177} \\
\text { United States } \\
\text { Small Sample }\end{array}$ & \begin{tabular}{|l|} 
Group \\
interventions of \\
(a) skill training \\
or (b) mental \\
stimulation \\
delivered 5 days \\
a week, 20 \\
weeks, 2.5 \\
hours per \\
session \\
\end{tabular} & No treatment & \begin{tabular}{|l} 
Nursing \\
home \\
RCT \\
PLWD
\end{tabular} & \begin{tabular}{|l|} 
Diagnosis of \\
dementia with six \\
or more errors out \\
of 10 items on the \\
Short Portable \\
Mental Status \\
Questionnaire
\end{tabular} & $\begin{array}{l}\mathrm{N}=63 \\
\text { Age: yes } \\
\text { Gender: yes } \\
\text { Race: no } \\
\text { Education: no }\end{array}$ & NR & $\mathrm{NA}$ & NA & $\mathrm{NA}$ & NA & $\begin{array}{l}\text { Post } \\
\text { intervention }\end{array}$ & $\begin{array}{l}\text { Physical } \\
\text { Self- } \\
\text { Maintenance } \\
\text { Scale } \\
\text { Performance } \\
\text { Test of ADLs }\end{array}$ & NA \\
\hline
\end{tabular}

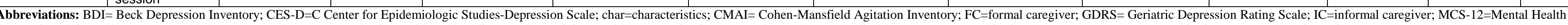

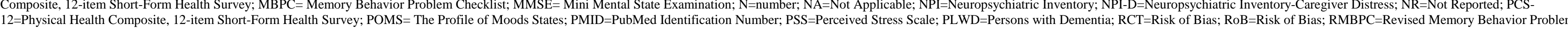
Checklist; RS=Reporting Status; SES=socioeconomic status; STAI= Spielberger State-Trait Anxiety Inventory; STAXI=Spielberger State-Trait Anger Inventory; WHOQOL-BREF= World Health Organization Quality of Life Instruments; ZBI=Zarit Burden Interview

\section{Social Support}

Table E-9. Risk of bias assessment: social support

\begin{tabular}{|c|c|c|c|c|c|c|c|c|c|}
\hline Study (PMID) & Outcome Timing & $\begin{array}{l}\text { Attrition Bias } \\
\text { Attrition \% }\end{array}$ & Selection Bias & Detection Bias & Performance Bias & Reporting Bias & Fidelity Bias & Funder & Overall Rating \\
\hline \begin{tabular}{|l} 
Huis $2019^{178}$ (32130142) \\
\end{tabular} & $\begin{array}{l}6 \text { weeks } \\
12 \text { weeks }\end{array}$ & $\begin{array}{l}\text { Medium } \\
14 \%\end{array}$ & Low & Medium & High & Low & Medium & \begin{tabular}{|l} 
Netherlands Organization \\
for Health Research and \\
Development program \\
Tussen Weten en Doen \\
\end{tabular} & High \\
\hline \begin{tabular}{|l|l} 
Van Mierlo $2015^{179}(25872457)$ \\
\end{tabular} & $\begin{array}{l}6 \text { months } \\
12 \text { months }\end{array}$ & $\begin{array}{l}\text { Medium } \\
6 \text { months: } 12 \% \\
\text { High } \\
12 \text { months: } 33 \%\end{array}$ & Medium & High & Medium & Medium & $x$ & Government & High \\
\hline \begin{tabular}{|l} 
Wang $2012^{180}(22554214)$ \\
\end{tabular} & 28 weeks & $\begin{array}{l}\text { Low } \\
7.6 \%\end{array}$ & Medium & Low & Low & Low & Medium & NR & Medium \\
\hline Logsdon 2010 ${ }^{181}$ (20693265) & 2 months & $\begin{array}{l}\text { Low } \\
4 \%\end{array}$ & Medium & Low & Medium & High & $x$ & Government & High \\
\hline $\begin{array}{l}\text { Charlesworth 2008 } \\
\text { Charlesworth } 2008^{183}(18284895) \\
\text { Wilson } 2009^{184}(19101921)\end{array}$ & $\begin{array}{l}6 \text { months } \\
15 \text { months } \\
24 \text { months }\end{array}$ & $\begin{array}{l}\text { Medium } \\
6 \text { months: } 10 \% \\
15 \text { months: } 16 \% \\
\text { High } \\
24 \text { months: } 21 \%\end{array}$ & Low & Medium & High & Low & $x$ & Government & High \\
\hline Winter $2006^{185}(17267370)$ & 6 months & $\begin{array}{l}\text { Low } \\
8.7 \%\end{array}$ & Medium & High & High & Medium & $x$ & Non-profit & High \\
\hline \begin{tabular}{|l|} 
Mahoney $2003^{186}(12937334)$ \\
Mahoney $2001^{187}(11346473)$
\end{tabular} & $\begin{array}{l}6 \text { months } \\
12 \text { months } \\
18 \text { months }\end{array}$ & $\begin{array}{l}\text { Low } \\
6 \text { months: } 8 \% \\
\text { Medium } \\
12 \text { months: } 14 \% \\
18 \text { months:18\% }\end{array}$ & Low & Low & $\begin{array}{l}\text { Medium (6 months) } \\
\text { High (12 and } 18 \\
\text { months) }\end{array}$ & Medium & Medium & Government & $\begin{array}{l}\text { Medium (6 months) } \\
\text { High (12 and } 18 \text { months) }\end{array}$ \\
\hline \begin{tabular}{|l} 
Pillemer $2002^{188}$ (NA) \\
\end{tabular} & 6 months & $\begin{array}{l}\text { High } \\
22 \%\end{array}$ & $x$ & $\mathrm{x}$ & $\mathrm{x}$ & $x$ & $\mathrm{x}$ & Government & High \\
\hline $\begin{array}{l}\text { Goodman 1990189 (2354800) } \\
\text { Goodman 1990189 (2284602) }\end{array}$ & NR & $\begin{array}{l}\text { High } \\
34 \%\end{array}$ & $x$ & $x$ & $x$ & $x$ & $x$ & NR & High \\
\hline
\end{tabular}

indicates that domain was not assessed due to high risk of bias.

s: NR=Not Reported; PMID=PubMed Identification Number 
Table E-10. Characteristics of included studies: social support

\begin{tabular}{|c|c|c|c|c|c|c|c|c|c|c|c|c|c|}
\hline \begin{tabular}{|l|} 
Study (PMID) \\
Country \\
RoB* \\
Type \\
\end{tabular} & \begin{tabular}{|l|} 
Intervention \\
Intervention \\
Focus \\
Theoretical \\
Model \\
Delivery \\
Person \\
Intervention \\
Target \\
Recipient \\
Mode \\
Components \\
Frequency \\
Duration \\
\end{tabular} & \begin{tabular}{|l|} 
Comparison \\
Target \\
Mode \\
Components \\
Frequency \\
Duration
\end{tabular} & \begin{tabular}{|l} 
Setting \\
and Design \\
\\
Setting \\
Design \\
Cluster N \\
Participants \\
Randomized \\
N
\end{tabular} & \begin{tabular}{|l|} 
PLWD \\
Dementia \\
Characteristics \\
Dementia Types \\
Dementia Severity \\
Diagnostic \\
Criteria \\
Age of Diagnosis
\end{tabular} & \begin{tabular}{|l} 
PLWD \\
Non-Disease Char \\
PLWD N \\
PLWD Age (mean) \\
PLWD Sex (\% \\
female) \\
PLWD Race (\% \\
majority) \\
PLWD Education \\
(mean years)
\end{tabular} & \begin{tabular}{|l|} 
PLWD \\
Non-Disease \\
Char Reporting \\
Status (RS) \\
\\
PLWD SES \\
PLWD Prior \\
Disability \\
PLWD \\
Household \\
Characteristics \\
PLWD Health \\
Insurance \\
PLWD Detailed \\
Race Information \\
\end{tabular} & \begin{tabular}{|l} 
Informal Caregiver \\
(IC) \\
Characteristics \\
IC N \\
IC Age (mean) \\
IC Sex (\% female) \\
IC Race (\% \\
majority) \\
IC Education \\
(mean years) \\
IC Relation to \\
PLWD (\% majority)
\end{tabular} & \begin{tabular}{|l} 
Informal Caregiver \\
(IC) Char. RS \\
IC Duration \\
IC Living With \\
PLWD \\
IC Payment \\
IC Health Status \\
IC Dementia \\
Family History \\
IC Employment \\
Status \\
IC Training
\end{tabular} & $\begin{array}{l}\text { Formal Caregiver } \\
\text { (FC) } \\
\text { Characteristics } \\
\text { FC N } \\
\text { FC Age (mean) } \\
\text { FC Sex (\% } \\
\text { female) } \\
\text { FC Race (\% } \\
\text { majority) } \\
\text { FC Education } \\
\text { (mean years) }\end{array}$ & $\begin{array}{l}\text { Formal } \\
\text { Caregiver (FC) } \\
\text { Char. RS } \\
\text { FC Health } \\
\text { Status } \\
\text { FC Training } \\
\text { FC Education } \\
\text { FC Position } \\
\text { FC Length of } \\
\text { Service }\end{array}$ & $\begin{array}{l}\text { Outcome } \\
\text { Timing(s) }\end{array}$ & \begin{tabular}{|l|} 
PLWD \\
Outcomes
\end{tabular} & $\begin{array}{l}\text { Caregiver } \\
\text { Outcomes } \\
\text { IC: } \\
\text { FC: }\end{array}$ \\
\hline $\begin{array}{l}\text { Wang 2012180 } \\
(22554214) \\
\text { China } \\
\text { Medium } \\
\text { Explanatory }\end{array}$ & $\begin{array}{l}\text { In person, peer- } \\
\text { led mutual } \\
\text { support group } \\
\text { for caregivers; } \\
\text { bi-weekly 90- } \\
\text { minute sessions } \\
\text { for } 24 \text { weeks } \\
\text { (12 total } \\
\text { sessions) }\end{array}$ & $\begin{array}{l}\text { Usual care } \\
\text { (conventional } \\
\text { family } \\
\text { services) }\end{array}$ & $\begin{array}{l}\text { Dementia } \\
\text { resource and } \\
\text { respite centers } \\
\text { RCT } \\
\text { Multisite } \\
78 \text { Informal } \\
\text { Caregivers }\end{array}$ & $\begin{array}{l}\text { All dementiat types } \\
\text { and severities } \\
\text { (primarily AD and } \\
\text { cerebrovascular). } \\
\text { Dementia } \\
\text { diagnosis based on } \\
\text { DSM-IV criteria }\end{array}$ & $\begin{array}{l}\mathrm{N}=78 \\
81 \% \text { Age } 61+ \\
53 \% \text { Female } \\
\text { Race NR } \\
\text { Education NR }\end{array}$ & NR & $\begin{array}{l}\mathrm{N}=78 \\
66 \% \text { Age } 31-70 \\
62 \% \text { Female } \\
73 \% \text { Secondary } \\
\text { School or Higher } \\
\text { Race NR } \\
\text { 37\% Spouse }\end{array}$ & $\begin{array}{l}\text { Duration: Yes } \\
\text { Employment Status: } \\
\text { Yes }\end{array}$ & NA & NA & 28 weeks & NA & $\begin{array}{l}\text { NPI-D } \\
\text { WHOQOL- } \\
\text { BREF(HK } \\
\text { Family } \\
\text { Supports } \\
\text { Services } \\
\text { Index }\end{array}$ \\
\hline \begin{tabular}{|l|} 
Mahoney \\
$2003^{186}$ \\
$(12937334)$ \\
US \\
Medium \\
Explanatory \\
\\
Mahoney \\
$2001^{187}$ \\
$(11346473)$
\end{tabular} & $\begin{array}{l}\text { Automated } \\
\text { telephone } \\
\text { support system } \\
\text { for caregivers } \\
\text { which included } \\
\text { a weekly } \\
\text { automated } \\
\text { conversation, a } \\
\text { personal voice } \\
\text { mailibox, } \\
\text { telephone } \\
\text { support group, } \\
\text { and an activity- } \\
\text { respite module; } \\
\text { available } 22 \\
\text { hours a day for } \\
12 \text { months } \\
\end{array}$ & Usual care & $\begin{array}{l}\text { Community- } \\
\text { based } \\
\text { recruitment } \\
\text { from health } \\
\text { and social } \\
\text { agencies. } \\
\text { RCT } \\
\text { Single site } \\
100 \text { Informal } \\
\text { Caregivers }\end{array}$ & \begin{tabular}{|l} 
Diagnosis of \\
probable AD or \\
MMSE $\leq 23$, with \\
two IADL \\
impairments and \\
one AD-related \\
disturbing behavior
\end{tabular} & \begin{tabular}{|l|}
$\mathrm{N}=100$ \\
77 years \\
$48 \%$ Female \\
Race NR \\
$70 \%$ High School \\
Diploma or Higher
\end{tabular} & NR & \begin{tabular}{|l|}
$N=100$ \\
63 years \\
$78 \%$ Female \\
$79 \%$ White \\
$82 \%$ High School \\
Diploma or Higher \\
$54 \%$ Spouse
\end{tabular} & \begin{tabular}{|l} 
Employment Status: \\
Yes
\end{tabular} & NA & NA & 6 months & NA & $\begin{array}{l}\text { CES-D } \\
\text { STAl }\end{array}$ \\
\hline
\end{tabular}

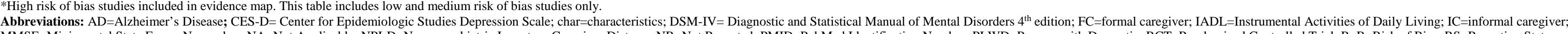

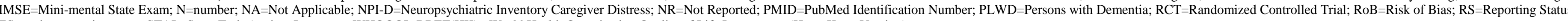


Table E-11. Caregiver outcomes summary low and medium risk of bias studies: social support

\begin{tabular}{|c|c|c|c|c|c|}
\hline $\begin{array}{l}\text { Study (PMID) } \\
\text { Comparison } \\
\text { Caregiver Type } \\
\text { RoB } \\
\text { Category* }\end{array}$ & $\begin{array}{l}\text { Outcome } \\
\text { Timing }\end{array}$ & Summary Finding & Intervention & Comparator & p-Value \\
\hline $\begin{array}{l}\text { Wang } 2012^{180} \text { (22554214) } \\
\text { In-person support group vs. Usual } \\
\text { care } \\
\text { Informal } \\
\text { Medium } \\
\text { Explanatory }\end{array}$ & $\begin{array}{l}\text { Mean (SD) } \\
\text { NPI-D Total Score } \\
28 \text { weeks }\end{array}$ & NR & $37.47(9.68)$ & $43.88(13.56)$ & $\mathrm{p}=0.005$ \\
\hline $\begin{array}{l}\text { Wang } 2012^{180}(22554214) \\
\text { In-person support group vs. Usual } \\
\text { care } \\
\text { Informal } \\
\text { Medium } \\
\text { Explanatory }\end{array}$ & $\begin{array}{l}\text { Mean (SD) } \\
\text { WHOQOL-BREF(HK) Total Score } \\
28 \text { weeks }\end{array}$ & NR & $114.02(13.98)$ & $88.19(7.56)$ & $p=0.001$ \\
\hline $\begin{array}{l}\text { Wang } 2012^{180}(22554214) \\
\text { In-person support group vs. Usual } \\
\text { care } \\
\text { Informal } \\
\text { Medium } \\
\text { Explanatory }\end{array}$ & $\begin{array}{l}\text { Mean (SD) } \\
\text { Mental Health Services Utilization, Family Supports Services } \\
\text { Index } \\
28 \text { weeks }\end{array}$ & $\begin{array}{l}\text { No difference between groups in utilization of } \\
\text { mental health services. }\end{array}$ & NR & NR & NR \\
\hline $\begin{array}{l}\text { Mahoney } 2003^{186}(12937334) \\
\text { Automated support vs. Usual care } \\
\text { Informal } \\
\text { Medium } \\
\text { Explanatory } \\
\text { Mahoney } 2001^{187}(11346473)\end{array}$ & $\begin{array}{l}\text { Mean (SD) } \\
\text { CES-D } \\
6 \text { months }\end{array}$ & No difference between groups. & $12.3(9.1)$ & $14.9(11.7)$ & NR \\
\hline $\begin{array}{l}\text { Mahoney } 2003^{186}(12937334) \\
\text { Automated support vs. Usual care } \\
\text { Informal } \\
\text { Medium } \\
\text { Explanatory } \\
\text { Mahoney 2001 }\end{array}$ & \begin{tabular}{|l} 
Mean (SD) \\
STAI \\
6 months
\end{tabular} & No difference between groups. & $19.8(5.7)$ & $20.6(7.7)$ & $\mathrm{NR}$ \\
\hline
\end{tabular}

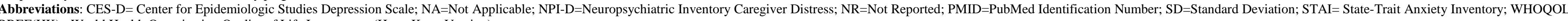
BREF(HK) = World Health Organization Quality of Life Instruments (Hong Kong Version)

Table E-12. Summary of strength of evidence for caregiver outcomes: social support

\begin{tabular}{|c|c|c|c|c|c|c|c|c|}
\hline $\begin{array}{l}\text { Comparison } \\
\text { Caregiver Type } \\
\text { Outcome }\end{array}$ & Timing & $\begin{array}{l}\text { \# Studies/ Design } \\
\text { (n analyzed) }\end{array}$ & Finding or Summary Statistic & Study Limitations & Consistency & Directness & Precision & Overall Grade/ Conclusion \\
\hline \begin{tabular}{|l} 
In-person support vs. \\
Usual care \\
Informal \\
All Outcomes
\end{tabular} & 28 weeks & $1 \mathrm{RCT}(\mathrm{n}=78)$ & Insufficient evidence to draw conclusions about all outcomes. & Moderate & Unknown & Direct & Imprecise & Insufficient \\
\hline \begin{tabular}{|l} 
Automated support vs. \\
Usual care \\
Informal \\
All Outcomes
\end{tabular} & 6 months & $1 \mathrm{RCT}(\mathrm{n}=100)$ & Insufficient evidence to draw conclusions about all outcomes. & Moderate & Unknown & Direct & Imprecise & Insufficient \\
\hline
\end{tabular}

Abbreviations: n=number: RCT=Randomized Controlled Tri 


\section{Evidence Map: Social Support for Caregivers}

\begin{tabular}{|c|c|c|c|c|c|c|c|c|c|c|c|c|c|}
\hline $\begin{array}{l}\text { Study (PMID) } \\
\text { Country } \\
\text { EM Reason }\end{array}$ & Intervention & Comparison & $\begin{array}{l}\text { Setting } \\
\text { and Design } \\
\text { Setting } \\
\text { Design } \\
\text { Cluster N } \\
\text { Participants } \\
\text { Random- } \\
\text { ized N }\end{array}$ & $\begin{array}{l}\text { PLWD } \\
\text { Dementia } \\
\text { Characteristics }\end{array}$ & $\begin{array}{l}\text { PLWD } \\
\text { Non-Disease Char } \\
\text { PLWD N } \\
\text { PLWD Age (mean) } \\
\text { PLWD Sex (\% } \\
\text { female) } \\
\text { PLWD Race (\% } \\
\text { majority) } \\
\text { PLWD Education } \\
\text { (mean years) }\end{array}$ & $\begin{array}{l}\text { PLWD } \\
\text { Non-Disease } \\
\text { Char Reporting } \\
\text { Status (RS) } \\
\text { PLWD SES } \\
\text { PLWD Prior } \\
\text { Disability } \\
\text { PLWD } \\
\text { Household } \\
\text { Characteristics } \\
\text { PLWD Health } \\
\text { Insurance } \\
\text { PLWD Detailed } \\
\text { Race Information }\end{array}$ & $\begin{array}{l}\text { Informal Caregiver } \\
\text { (IC) } \\
\text { Characteristics } \\
\text { IC N } \\
\text { IC Age (mean) } \\
\text { IC Sex (\% female) } \\
\text { IC Race (\% } \\
\text { majority) } \\
\text { IC Education } \\
\text { (mean years) } \\
\text { IC Relation to } \\
\text { PLWD (\% majority) }\end{array}$ & $\begin{array}{l}\text { Informal Caregiver } \\
\text { (IC) Char. RS } \\
\text { IC Duration } \\
\text { IC Living With } \\
\text { PLWD } \\
\text { IC Payment } \\
\text { IC Health Status } \\
\text { IC Dementia } \\
\text { Family History } \\
\text { IC Employment } \\
\text { Status } \\
\text { IC Training }\end{array}$ & 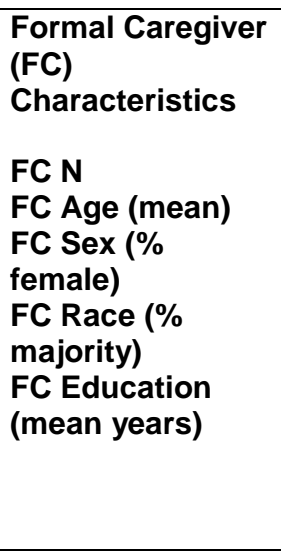 & $\begin{array}{l}\text { Formal } \\
\text { Caregiver (FC) } \\
\text { Char. RS } \\
\text { FC Health } \\
\text { Status } \\
\text { FC Training } \\
\text { FC Education } \\
\text { FC Position } \\
\text { FC Length of } \\
\text { Service }\end{array}$ & $\begin{array}{l}\text { Outcome } \\
\text { Timing(s) }\end{array}$ & $\begin{array}{l}\text { PLWD } \\
\text { Outcomes }\end{array}$ & $\begin{array}{l}\text { Caregiver } \\
\text { Outcomes } \\
\text { IC: } \\
\text { FC: }\end{array}$ \\
\hline $\begin{array}{l}\text { Huis 2020178 } \\
\text { (32130142) } \\
\text { Netherlands } \\
\text { High ROB }\end{array}$ & $\begin{array}{l}\text { Email contacts } \\
\text { with a specialist } \\
\text { dementia nurse } \\
\text { and } \\
\text { informational } \\
\text { videos and e- } \\
\text { bullletins vs. } \\
\text { informational } \\
\text { videos and e- } \\
\text { bullletins vs. e- } \\
\text { bulletins }\end{array}$ & & $\begin{array}{l}\text { Community } \\
3 \text {-arm RCT } \\
\mathrm{N}=81 \\
\text { informal } \\
\text { caregivers }\end{array}$ & $\begin{array}{l}\text { AD } \\
\text { Vascular dementia } \\
\text { Frontotemporal } \\
\text { dementia } \\
\text { Dementia with } \\
\text { Lewy bodies } \\
\text { Mixed dementia } \\
\text { Dementia type not } \\
\text { known } \\
\text { PLWD lives at } \\
\text { home and has } \\
\text { minimum weekly } \\
\text { contact with } \\
\text { informal caregiver } \\
\text { who is parner or } \\
\text { family member }\end{array}$ & $\begin{array}{l}\mathrm{N}=81 \\
\text { Age: } \text { yes } \\
\text { Sex: yes } \\
\text { Race: no } \\
\text { Education: no }\end{array}$ & $\begin{array}{l}\text { Household } \\
\text { Characteristics: } \\
\text { yes }\end{array}$ & $\begin{array}{l}\mathrm{N}=81 \\
\text { Age: yes } \\
\text { Sex: yes } \\
\text { Education: yes } \\
\text { Relation to PLWD: } \\
\text { yes }\end{array}$ & $\begin{array}{l}\text { Living with PLWD: } \\
\text { yes }\end{array}$ & NA & NA & $\begin{array}{l}6 \text { weeks, } 12 \\
\text { weeks }\end{array}$ & $\begin{array}{l}\text { Disruptive } \\
\text { behavior } \\
\text { (RMBPC) }\end{array}$ & $\begin{array}{l}\text { Self-efficacy } \\
\text { (TRUST) } \\
\text { Dyadic } \\
\text { relationship } \\
\text { quality (DRS) }\end{array}$ \\
\hline $\begin{array}{l}\text { Gustafson } \\
2019^{190} \\
(31256126) \\
\text { US } \\
\text { Pilot study }\end{array}$ & $\begin{array}{l}\text { Dementia- } \\
\text { Comprehensive } \\
\text { Health } \\
\text { Enhancement } \\
\text { Support System } \\
\text { (D-CHESS) } \\
\text { website access } \\
\text { for } 6 \text { months }\end{array}$ & Caregiving book & $\begin{array}{l}\text { Community- } \\
\text { based } \\
\text { (intervention } \\
\text { at home) } \\
\text { RCT; single } \\
\text { site } \\
31 \text { informal } \\
\text { caregivers }\end{array}$ & AD, all severities & $\begin{array}{l}\mathrm{N}=31 \\
\text { Age: } \text { yes } \\
\text { Sex: no } \\
\text { Race: no } \\
\text { Education: no }\end{array}$ & NR & $\begin{array}{l}\mathrm{N}=31 \\
\text { Age: } \text { yes } \\
\text { Sex: yes } \\
\text { Race: } n \mathrm{no} \\
\text { Education: yes } \\
\text { Relation to PLWD: } \\
\text { yes }\end{array}$ & Payment: yes & NA & NA & 6 months & NA & $\begin{array}{l}\text { Caregiver } \\
\text { Burden } \\
\text { (composite) } \\
\text { Family } \\
\text { Conflict } \\
\text { (Aneshensel } \\
\text { Scale) } \\
\text { Satisfaction } \\
\text { with Decision } \\
\text { Scale } \\
\text { MOS Social } \\
\text { Support } \\
\text { Survey } \\
\text { UCLA } \\
\text { Loneliness } \\
\text { Scale } \\
\text { Generalized } \\
\text { Anniety } \\
\text { Disorder } \\
\text { Scale } \\
\text { PHQ } \\
\text { Coping } \\
\text { Competence } \\
\text { (Lauton } \\
\text { Scale) }\end{array}$ \\
\hline
\end{tabular}




\begin{tabular}{|c|c|c|c|c|c|c|c|c|c|c|c|c|c|}
\hline $\begin{array}{l}\text { Metcalfe } \\
2019^{191} \\
\text { (31111516) } \\
\text { Multinational } \\
\text { Pilot study }\end{array}$ & $\begin{array}{l}\text { Online } \\
\text { information and } \\
\text { support } \\
\text { program for } \\
\text { caregivers of } \\
\text { individuals } \\
\text { with young } \\
\text { onset dementia; } \\
\text { access for } 12 \\
\text { weeks }\end{array}$ & Waitlist control & $\begin{array}{l}\text { Community- } \\
\text { based } \\
\text { memory } \\
\text { clinics } \\
\text { (intervention } \\
\text { at home) } \\
\text { RCT; } \\
\text { multisite } \\
61 \text { informal } \\
\text { caregivers } \\
\end{array}$ & $\begin{array}{l}\text { AD or behavioral } \\
\text { variant } \\
\text { frontotemporal } \\
\text { degeneration onset } \\
\text { before the age of } \\
65\end{array}$ & $\begin{array}{l}\mathrm{N}=61 \\
\text { Age: yes } \\
\text { Sex: yes } \\
\text { Race: no } \\
\text { Education: no }\end{array}$ & NR & $\begin{array}{l}\mathrm{N}=61 \\
\text { Age: yes } \\
\text { Sex: yes } \\
\text { Race: no } \\
\text { Education: yes } \\
\text { Relation to PLWD: } \\
\text { yes }\end{array}$ & $\begin{array}{l}\text { Duration: yes } \\
\text { Employment Status: } \\
\text { yes }\end{array}$ & NA & NA & 12 weeks & NA & $\begin{array}{l}\text { RSCE } \\
\text { PSS } \\
\text { Burden Scale } \\
\text { for Family } \\
\text { Caregivers } \\
\text { EQ-5D-5L }\end{array}$ \\
\hline $\begin{array}{l}\text { Van Mierlo } \\
2015^{179} \\
(25872457) \\
\text { Netherlands } \\
\text { High ROB }\end{array}$ & $\begin{array}{l}\text { DEMentia- } \\
\text { specific } \\
\text { dynamic } \\
\text { interactive } \\
\text { social chart } \\
\text { (DEM-DISC) } \\
\text { providing } \\
\text { information on } \\
\text { support, coping, } \\
\text { dementia, and } \\
\text { other topics; } \\
\text { accessible to } \\
\text { both carers and } \\
\text { case managers }\end{array}$ & No intervention & $\begin{array}{l}\text { Community- } \\
\text { based } \\
\text { setting } \\
\text { (intervention } \\
\text { at home) } \\
\text { Cluster } \\
\text { RCT } \\
\text { multisite } \\
27 \text { clusters } \\
73 \text { informal } \\
\text { caregivers }\end{array}$ & $\begin{array}{l}\text { Dementia (not } \\
\text { specified) }\end{array}$ & $\begin{array}{l}\text { N=73 } \\
\text { Age: yes } \\
\text { Sex: yes } \\
\text { Race: no } \\
\text { Education: no }\end{array}$ & NR & $\begin{array}{l}\mathrm{N}=73 \\
\text { Age: yes } \\
\text { Sex: yes } \\
\text { Race: no } \\
\text { Education: yes } \\
\text { Relation to PLWD: } \\
\text { yes }\end{array}$ & $\begin{array}{l}\text { Living with PLWD: } \\
\text { yes } \\
\text { Payment: yes } \\
\text { Health Status: yes }\end{array}$ & NA & NA & $\begin{array}{l}6 \text { months } \\
12 \text { months }\end{array}$ & $\begin{array}{l}\text { QOL-AD } \\
\text { EQ-5D }\end{array}$ & $\begin{array}{l}\text { Camberwell } \\
\text { Assessment } \\
\text { of Needs for } \\
\text { the } \\
\text { Elderly } \\
\text { NPI-Distress } \\
\text { Short Sense } \\
\text { of } \\
\text { Competence } \\
\text { Questionnaire }\end{array}$ \\
\hline $\begin{array}{l}\text { Torkamani } \\
2014^{192} \\
(24643137) \\
\text { UK } \\
\text { Pilot Study }\end{array}$ & $\begin{array}{l}\text { Computerized } \\
\text { social support } \\
\text { platform } \\
\text { provided } \\
\text { information, } \\
\text { social } \\
\text { networking, and } \\
\text { educational } \\
\text { materials }\end{array}$ & No intervention & $\begin{array}{l}\text { Hospital } \\
\text { outpatients } \\
\text { RCT, } \\
\text { multisite } \\
60 \text { PLWD- } \\
\text { informal } \\
\text { caregiver } \\
\text { dyads }\end{array}$ & $\begin{array}{l}\text { Dementia with BI } \\
\text { score } 35 \text { or greater } \\
\text { and MMSE score } \\
\text { from } 9 \text { to } 21\end{array}$ & $\begin{array}{l}\text { N=60 } \\
\text { Age: } y \text { es } \\
\text { Sex: no } \\
\text { Race: no } \\
\text { Education: no }\end{array}$ & NR & $\begin{array}{l}\text { N=60 } \\
\text { Age: yes } \\
\text { Sex: no } \\
\text { Race: no } \\
\text { Education: no } \\
\text { Relation to PLWD: } \\
\text { no }\end{array}$ & NR & NA & NA & $\begin{array}{l}3 \text { months } \\
6 \text { months }\end{array}$ & $\begin{array}{l}\text { NPI } \\
\text { MMSE } \\
\text { MDRS } \\
\text { BDRS } \\
\text { CDR } \\
\text { RMBPC } \\
\text { GDS } \\
\text { BI } \\
\text { Lawton ADL }\end{array}$ & $\begin{array}{l}\text { ZBI } \\
\text { BDI } \\
\text { Zung } \\
\text { Depression } \\
\text { Self-rating } \\
\text { Scale } \\
\text { EQ-5D } \\
\text { Quality of Life } \\
\text { Scale }\end{array}$ \\
\hline $\begin{array}{l}\text { Logsdon } \\
2010^{181} \\
\text { (20693265) } \\
\text { US } \\
\text { High ROB }\end{array}$ & $\begin{array}{l}\text { Early-stage } \\
\text { memory loss } \\
\text { support group } \\
\text { for PLWD- } \\
\text { caregiver dyad; } \\
\text { 90-minute } \\
\text { sessions weekly } \\
\text { for } 9 \text { weeks }\end{array}$ & Waitlist control & $\begin{array}{l}\text { Community- } \\
\text { based } \\
\text { setting } \\
\text { RCT; single } \\
\text { site } \\
142 \text { PLWD- } \\
\text { informal } \\
\text { caregiver } \\
\text { dyads }\end{array}$ & $\begin{array}{l}\text { Dementia } \\
\text { diagnosis with } \\
\text { MMSE score } 18 \text { or } \\
\text { higher }\end{array}$ & $\begin{array}{l}\mathrm{N}=142 \\
\text { Age: } \text { yes } \\
\text { Sex: yes } \\
\text { Race: yes } \\
\text { Education: yes }\end{array}$ & NR & $\begin{array}{l}\text { N=142 } \\
\text { Age: yes } \\
\text { Sex: yes } \\
\text { Race: no } \\
\text { Education: yes } \\
\text { Relation to PLWD: } \\
\text { yes }\end{array}$ & NR & NA & NA & 9 weeks & $\begin{array}{l}\text { QOL-AD } \\
\text { SF-36 } \\
\text { GDS } \\
\text { RMBPC }\end{array}$ & $\begin{array}{l}\text { GDS } \\
\text { Family } \\
\text { Assessment } \\
\text { Measure, } \\
\text { Communicati } \\
\text { on } \\
\text { PSS }\end{array}$ \\
\hline $\begin{array}{l}\text { van der Roest } \\
2010^{193} \\
(20455122) \\
\text { Netherlands } \\
\text { Pilot Study }\end{array}$ & $\begin{array}{l}\text { DEMentia- } \\
\text { specific } \\
\text { dynamic } \\
\text { interactive } \\
\text { social chart } \\
\text { (DEM-DISC) } \\
\text { providing } \\
\text { information on } \\
\text { support, coping, } \\
\text { dementia, and } \\
\text { other topics }\end{array}$ & No intervention & $\begin{array}{l}\text { Community- } \\
\text { based } \\
\text { setting } \\
\text { (intervention } \\
\text { at home) } \\
\text { CCT; single } \\
\text { site } \\
29 \text { informal } \\
\text { caregivers }\end{array}$ & $\begin{array}{l}\text { Dementia (not } \\
\text { specified) }\end{array}$ & $\begin{array}{l}\text { N=29 } \\
\text { Age: yes } \\
\text { Sex: yes } \\
\text { Race: no } \\
\text { Education: no }\end{array}$ & NR & $\begin{array}{l}\mathrm{N}=29 \\
\text { Age: yes } \\
\text { Sex: no } \\
\text { Race: no } \\
\text { Education: yes } \\
\text { Relation to PLWD: } \\
\text { yes }\end{array}$ & Health status: yes & NA & NA & 2 months & $\begin{array}{l}\text { Camberwell } \\
\text { Assessment } \\
\text { of Need for } \\
\text { the Elderly } \\
\text { QOL-AD }\end{array}$ & $\begin{array}{l}\text { Short Sense } \\
\text { of } \\
\text { Competence } \\
\text { Questionnaire } \\
\text { Pearlin } \\
\text { Mastery } \\
\text { Scale }\end{array}$ \\
\hline
\end{tabular}




\begin{tabular}{|c|c|c|c|c|c|c|c|c|c|c|c|c|c|}
\hline $\begin{array}{l}\text { Charlesworth } \\
2008^{182} \\
(18284895) \\
\text { High ROB } \\
\text { UK } \\
\text { Charlesworth } \\
2008^{183} \\
(18505757) \\
\text { Wilson 2009 } \\
(19101921)\end{array}$ & $\begin{array}{l}\text { Befriending } \\
\text { volunteers to } \\
\text { provide } \\
\text { emotional } \\
\text { support to } \\
\text { informal } \\
\text { caregivers; } \\
\text { weekly contact } \\
\text { for } 6 \text { months }\end{array}$ & Usual care & $\begin{array}{l}\text { Community- } \\
\text { based } \\
\text { setting } \\
\text { RCT; single } \\
\text { site } \\
236 \text { informal } \\
\text { caregivers }\end{array}$ & \begin{tabular}{|l|} 
Primary \\
progressive \\
dementia
\end{tabular} & $\begin{array}{l}\mathrm{N}=236 \\
\text { Age: yes } \\
\text { Sex: no } \\
\text { Race: no } \\
\text { Education: no }\end{array}$ & \begin{tabular}{|l|}
$N R$ \\
\end{tabular} & $\begin{array}{l}\mathrm{N}=236 \\
\text { Age: yes } \\
\text { Sex: yes } \\
\text { Race: yes } \\
\text { Education: yes } \\
\text { Relation to PLWD: } \\
\text { yes }\end{array}$ & Employment: yes & NA & NA & $\begin{array}{l}6 \text { months } \\
15 \text { months } \\
24 \text { months }\end{array}$ & NA & $\begin{array}{l}\text { HADS- } \\
\text { Depression } \\
\text { EuroQoL } \\
\text { Positive and } \\
\text { negative } \\
\text { affectivity } \\
\text { scale } \\
\text { HADS- } \\
\text { Anxiety } \\
\text { MSPSS }\end{array}$ \\
\hline $\begin{array}{l}\text { Winter 2006 } \\
\text { (17265 } \\
\text { US } \\
\text { High ROB }\end{array}$ & $\begin{array}{l}\text { Telephone- } \\
\text { based support } \\
\text { groups; } 1 \text { hour } \\
\text { weekly for } 6 \\
\text { months }\end{array}$ & No intervention & $\begin{array}{l}\text { Community- } \\
\text { based } \\
\text { setting } \\
\text { (intervention } \\
\text { at home) } \\
\text { RCT; single } \\
\text { site } \\
106 \text { female } \\
\text { caregivers }\end{array}$ & $\begin{array}{l}\text { AD and related } \\
\text { dementias }\end{array}$ & $\begin{array}{l}\mathrm{N}=106 \\
\text { Age: yes } \\
\text { Sex: yes } \\
\text { Race: } n \text { o } \\
\text { Education: no }\end{array}$ & \begin{tabular}{|l|}
$N R$ \\
\end{tabular} & $\begin{array}{l}\mathrm{N}=106 \\
\text { Age: yes } \\
\text { Sex: yes } \\
\text { Race: yes } \\
\text { Education: yes } \\
\text { Relation to PLWD: } \\
\text { yes }\end{array}$ & Duration: yes & NA & NA & 6 months & NA & $\begin{array}{l}\text { CES-D } \\
\text { ZBI } \\
\text { Gain Through } \\
\text { Group } \\
\text { Involvement } \\
\text { Scale }\end{array}$ \\
\hline $\begin{array}{l}\text { Pillemer } 2002^{188} \\
\text { (NA) } \\
\text { US } \\
\text { High ROB }\end{array}$ & $\begin{array}{l}\text { One-on-one in } \\
\text { person peer } \\
\text { support for new } \\
\text { informal } \\
\text { caregivers; } \\
\text { weekly 2-hour } \\
\text { sessions for } 8 \\
\text { weeks }\end{array}$ & No intervention & $\begin{array}{l}\text { Community- } \\
\text { based } \\
\text { setting } \\
\text { (intervention } \\
\text { at home) } \\
\text { RCT; single } \\
\text { site } \\
147 \text { informal } \\
\text { caregivers }\end{array}$ & $A D$ & $\begin{array}{l}\mathrm{N}=147 \\
\text { Age: yes } \\
\text { Sex: yes } \\
\text { Race: no } \\
\text { Education: no }\end{array}$ & \begin{tabular}{|l|}
$N R$ \\
\end{tabular} & $\begin{array}{l}\mathrm{N}=147 \\
\text { Age: yes } \\
\text { Sex: yes } \\
\text { Race: no } \\
\text { Education: yes } \\
\text { Relation to PLWD: } \\
\text { yes }\end{array}$ & $\begin{array}{l}\text { Living with PLWD: } \\
\text { yes }\end{array}$ & NA & NA & 6 months & NA & $\begin{array}{l}\text { CES-D } \\
\text { Index of } \\
\text { Disruptive } \\
\text { Behaviors } \\
\text { (Caregiving } \\
\text { Stress) }\end{array}$ \\
\hline $\begin{array}{l}\text { Goodman } \\
1990^{189} \\
(2354800) \\
\text { US } \\
\text { High ROB } \\
\text { Goodman } \\
1990^{189} \\
(2284602)\end{array}$ & $\begin{array}{l}\text { Telephone } \\
\text { support } \\
\text { network; } 2 \text { calls } \\
\text { a week for } 12 \\
\text { weeks }\end{array}$ & $\begin{array}{l}\text { Telephone- } \\
\text { accessed taped } \\
\text { lectures on AD } \\
\text { and care } \\
\text { management } \\
\text { accessed } \\
\text { anytime over } 12 \\
\text { weeks }\end{array}$ & $\begin{array}{l}81 \text { informal } \\
\text { caregivers }\end{array}$ & $\begin{array}{l}\text { AD and other } \\
\text { dementias }\end{array}$ & $\begin{array}{l}\mathrm{N}=81 \\
\text { No } \\
\text { Sex: no } \\
\text { Race: no } \\
\text { Education: no }\end{array}$ & \begin{tabular}{|l|}
$N R$ \\
\end{tabular} & $\begin{array}{l}\mathrm{N}=81 \\
\text { Age: yes } \\
\text { Sex: yes } \\
\text { Race: yes } \\
\text { Education: yes } \\
\text { Relation to PLWD: } \\
\text { yes }\end{array}$ & $\begin{array}{l}\text { Health Status: yes } \\
\text { Employment status: } \\
\text { yes }\end{array}$ & NA & NA & 12 weeks & MPBC & $\begin{array}{l}\text { ZBI } \\
\text { Perceived } \\
\text { Social } \\
\text { Support and } \\
\text { Social } \\
\text { Conflict } \\
\text { Mental Health } \\
\text { Index }\end{array}$ \\
\hline
\end{tabular}

Abbreviations: AD=Alzheimer's disease; ADL=Activities of Daily Living; BDI=Beck Depression Inventory; BDRS= Blessed Dementia Rating Scale; BI=Barthel Index; CDR=Clinical Dementia Rating; CES-D=Center for Epidemiologic Studies Depression Scale; char=characteristics; EQ-

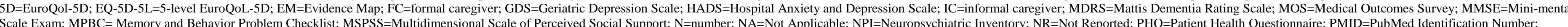

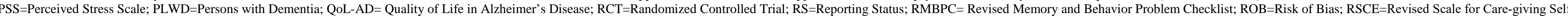
Efficacy; SES=socioeconomic status; SF-36; 36-Item Short Form Survey; ZBI=Zarit Burden Interview 


\section{Lifestyle Interventions}

Table E-14. Risk of bias assessment: lifestyle interventions

\begin{tabular}{|c|c|c|c|c|c|c|c|c|c|}
\hline \begin{tabular}{|l|l|} 
Study (PMID) \\
\end{tabular} & Outcome Timing & $\begin{array}{l}\text { Attrition Bias } \\
\text { Attrition \% }\end{array}$ & Selection Bias & Detection Bias & Performance Bias & Reporting Bias & Fidelity Bias & Funder & Overall Rating \\
\hline \begin{tabular}{|l|l} 
Salamizadeh $2017^{194}(28670585)$ \\
\end{tabular} & 3 weeks & $\begin{array}{l}\text { Medium } \\
10 \%\end{array}$ & Medium & High & High & Low & $x$ & NR & High \\
\hline \begin{tabular}{|l|} 
Farran 2016 $195(28752016)$ \\
Faran $2016^{196}(29147683)$ \\
Cothran 2017'97 28956706$)$
\end{tabular} & 12 months & $\begin{array}{l}\text { High } \\
27 \%\end{array}$ & $\mathrm{x}$ & $\mathrm{x}$ & $x$ & $\mathrm{x}$ & $x$ & Government & High \\
\hline Mahdavi $2016^{198}(28499566)$ & 5 weeks & $\begin{array}{l}\text { Medium } \\
11 \%\end{array}$ & Medium & Medium & High & Medium & $x$ & NR & High \\
\hline \begin{tabular}{|l|} 
Lowery 2014199 (24338799) \\
D'Amico 2016 $200(26489776)$
\end{tabular} & 12 weeks & $\begin{array}{l}\text { Medium } \\
11 \%\end{array}$ & Medium & Low & High & High & $x$ & Government & High \\
\hline \begin{tabular}{|l|} 
Whitebird $2013^{201}$ \\
\end{tabular} & \begin{tabular}{|l|}
2 months \\
6 months
\end{tabular} & $\begin{array}{l}\text { Low } \\
2 \text { months: } 8 \% \\
\text { Medium } \\
6 \text { months:10\% }\end{array}$ & Medium & Low & \begin{tabular}{|l|} 
Low \\
\end{tabular} & Medium & Medium & \begin{tabular}{|l} 
Government \\
\end{tabular} & Medium \\
\hline Connell 2009'202 (21709757) & $\begin{array}{l}6 \text { months } \\
12 \text { months }\end{array}$ & $\begin{array}{l}\text { Medium } \\
17 \%\end{array}$ & Medium & High & High & Low & $x$ & Government & High \\
\hline $\begin{array}{l}\text { King } 2002(11773209) \\
\text { Castro } 2002^{203}(12021419)\end{array}$ & \begin{tabular}{|l}
12 months \\
\end{tabular} & $\begin{array}{l}\text { Medium } \\
15 \%\end{array}$ & Low & Low & High & Low & $x$ & Government & High \\
\hline
\end{tabular}

indicates that domain was not assessed due to high risk of bias.

Abbreviations: NR=Not Reported; PMID=PubMed Identification Number 
Table E-15. Characteristics of included studies: lifestyle interventions

\begin{tabular}{|c|c|c|c|c|c|c|c|c|c|c|c|c|c|}
\hline \begin{tabular}{|l|} 
Study (PMID) \\
Country \\
RoB* \\
Type \\
\end{tabular} & \begin{tabular}{|l|} 
Intervention \\
Intervention \\
Focus \\
Theoretical \\
Model \\
Delivery \\
Person \\
Intervention \\
Target \\
Recipient \\
Mode \\
Components \\
Frequency \\
Duration
\end{tabular} & \begin{tabular}{l|} 
Comparison \\
Target \\
Mode \\
Components \\
Frequency \\
Duration
\end{tabular} & \begin{tabular}{|l|} 
Setting \\
and Design \\
Setting \\
Design \\
Cluster N \\
Participants \\
Randomized \\
N
\end{tabular} & $\begin{array}{l}\text { PLWD } \\
\text { Dementia } \\
\text { Characteristics } \\
\text { Dementia Types } \\
\text { Dementia Severity } \\
\text { Diagnostic } \\
\text { Criteria } \\
\text { Age of Diagnosis }\end{array}$ & $\begin{array}{l}\text { PLWD } \\
\text { Non-Disease Char } \\
\text { PLWD N } \\
\text { PLWD Age (mean) } \\
\text { PLWD Sex (\% } \\
\text { female) } \\
\text { PLWD Race (\% } \\
\text { majority) } \\
\text { PLWD Education } \\
\text { (mean years) }\end{array}$ & $\begin{array}{l}\text { PLWD } \\
\text { Non-Disease } \\
\text { Char Reporting } \\
\text { Status (RS) } \\
\\
\text { PLWD SES } \\
\text { PLWD Prior } \\
\text { Disability } \\
\text { PLWD } \\
\text { Household } \\
\text { Characteristics } \\
\text { PLWD Health } \\
\text { Insurance } \\
\text { PLWD Detailed } \\
\text { Race Information } \\
\end{array}$ & \begin{tabular}{|l|} 
Informal Caregiver \\
(IC) \\
Characteristics \\
IC N \\
IC Age (mean) \\
IC Sex (\% female) \\
IC Race (\% \\
majority) \\
IC Education \\
(mean years) \\
IC Relation to \\
PLWD (\% majority)
\end{tabular} & $\begin{array}{l}\text { Informal Caregiver } \\
\text { (IC) Char. RS } \\
\text { IC Duration } \\
\text { IC Living With } \\
\text { PLWD } \\
\text { IC Payment } \\
\text { IC Health Status } \\
\text { IC Dementia } \\
\text { Family History } \\
\text { IC Employment } \\
\text { Status } \\
\text { IC Training }\end{array}$ & 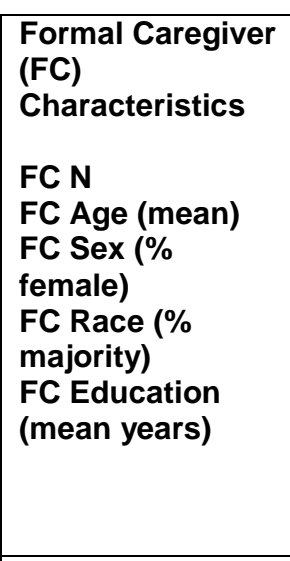 & $\begin{array}{l}\text { Formal } \\
\text { Caregiver (FC) } \\
\text { Char. RS } \\
\text { FC Health } \\
\text { Status } \\
\text { FC Training } \\
\text { FC Education } \\
\text { FC Position } \\
\text { FC Length of } \\
\text { Service }\end{array}$ & $\begin{array}{l}\text { Outcome } \\
\text { Timing(s) }\end{array}$ & \begin{tabular}{|l|} 
PLWD \\
Outcomes
\end{tabular} & $\begin{array}{l}\text { Caregiver } \\
\text { Outcomes } \\
\text { IC: } \\
\text { FC: }\end{array}$ \\
\hline $\begin{array}{l}\text { Whitebird } \\
2013^{201} \\
(23070934) \\
\text { US } \\
\text { Medium } \\
\text { Explanatory }\end{array}$ & \begin{tabular}{|l|} 
Group \\
mindfulness- \\
based stress \\
reduction for \\
informal \\
caregivers; one \\
2.5-hour \\
session/week \\
for 8 weeks and \\
a 5-hour retreat \\
with CDs and \\
written materials \\
\end{tabular} & \begin{tabular}{l|} 
Group \\
community \\
caregiver \\
education and \\
support for \\
informal \\
caregivers; \\
one 2.5-hour \\
session//week \\
for 8 weeks, a \\
5-hour \\
retreat, and \\
telephone \\
support calls
\end{tabular} & $\begin{array}{l}\text { Community- } \\
\text { based setting } \\
\text { RCT, single } \\
\text { site } \\
78 \text { family } \\
\text { caregivers }\end{array}$ & $\begin{array}{l}\text { Memory loss } \\
\text { consistent with } \\
\text { dementia }\end{array}$ & $\begin{array}{l}\mathrm{N}=78 \\
\text { Age NR } \\
\text { Sex NR } \\
\text { Race NR } \\
\text { Education NR }\end{array}$ & NR & \begin{tabular}{|l}
$\mathrm{N}=78$ \\
57 years \\
$89 \%$ Female \\
$99 \%$ White \\
$56 \%$ College or \\
Graduation \\
Education \\
$74 \%$ Adult child
\end{tabular} & $\begin{array}{l}\text { Duration: yes } \\
\text { Employment status: } \\
\text { yes }\end{array}$ & NA & NA & $\begin{array}{l}2 \text { months } \\
6 \text { months }\end{array}$ & NA & $\begin{array}{l}\text { PSS } \\
\text { CES-D } \\
\text { STAl } \\
\text { Montgomery } \\
\text { Borgatta } \\
\text { Caregiver } \\
\text { Burden Scale } \\
\text { Medical } \\
\text { Outcomes } \\
\text { Study Social } \\
\text { Support } \\
\text { MCS-12 } \\
\text { PCS-12 }\end{array}$ \\
\hline
\end{tabular}

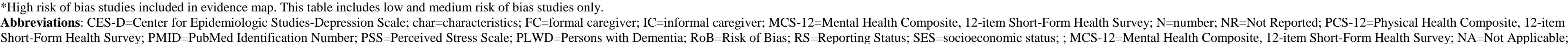

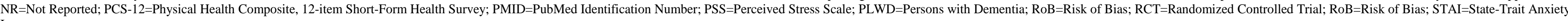
Inventory 
Table E-16. Caregiver outcomes summary low and medium risk of bias studies: lifestyle interventions

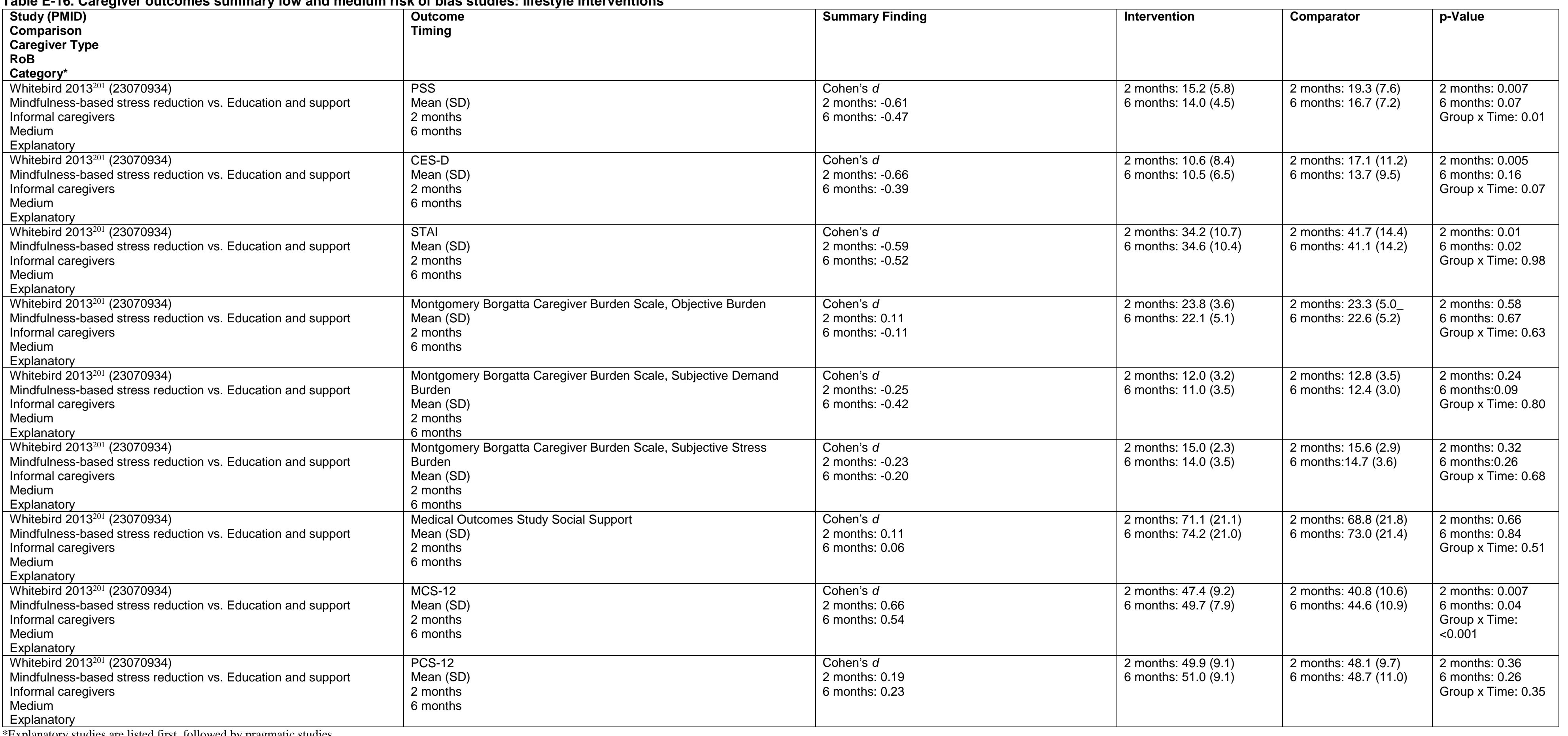

Explanatory studies are listed first, followed by pragmatic studies.

Depression Scale; NA=Not Applicable; MCS-12=Mental Health Composite, 12-item Short-Form Health Survey; NR=Not Reported; PCS-12=Physical Health Composite, 12-item Short-Form Health Survey; PMID=PubMed Identification

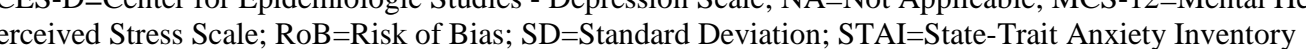


Table E-17. Summary of strength of evidence for caregiver outcomes: lifestyle interventions

\begin{tabular}{|c|c|c|c|c|c|c|c|c|}
\hline $\begin{array}{l}\text { Comparison } \\
\text { Caregiver Type } \\
\text { Outcome }\end{array}$ & Timing & $\begin{array}{l}\text { \# Studies/ Design } \\
\text { (n analyzed) }\end{array}$ & Finding or Summary Statistic & Study Limitations & Consistency & Directness & Precision & Overall Grade/ Conclusion \\
\hline $\begin{array}{l}\text { Mindfulness-based } \\
\text { stress reduction vs. } \\
\text { Education and support } \\
\text { Informal caregivers } \\
\text { All Outcomes }\end{array}$ & $\begin{array}{l}2 \text { months } \\
6 \text { months }\end{array}$ & $1 \mathrm{RCT}(\mathrm{n}=78)$ & $\begin{array}{l}\text { Insufficient evidence to draw conclusions about the effect of } \\
\text { mindfulness-based stress reduction on informal caregivers. }\end{array}$ & Moderate & Unknown & Direct & Imprecise & Insufficient \\
\hline
\end{tabular}

$\begin{array}{lll}\text { All Outcomes } & \\ \text { Abbreviations: n=number; RCT=randomized controlled trial }\end{array}$ 


\section{Evidence Map: Lifestyle Interventions}

\begin{tabular}{|c|c|c|c|c|c|c|c|c|c|c|c|c|c|}
\hline $\begin{array}{l}\text { Study (PMID) } \\
\text { Country } \\
\text { EM Reason }\end{array}$ & Intervention & Comparison & \begin{tabular}{|l|} 
Setting \\
and Design \\
\\
Setting \\
Design \\
Cluster N \\
Participants \\
Random- \\
ized N \\
\end{tabular} & \begin{tabular}{|l} 
PLWD \\
Dementia \\
Characteristics
\end{tabular} & $\begin{array}{l}\text { PLWD } \\
\text { Non-Disease Char } \\
\text { PLWD N } \\
\text { PLWD Age (mean) } \\
\text { PLWD Sex (\% } \\
\text { female) } \\
\text { PLWD Race (\% } \\
\text { majority) } \\
\text { PLWD Education } \\
\text { (mean years) }\end{array}$ & $\begin{array}{l}\text { PLWD } \\
\text { Non-Disease } \\
\text { Char Reporting } \\
\text { Status (RS) } \\
\\
\text { PLWD SES } \\
\text { PLWD Prior } \\
\text { Disability } \\
\text { PLWD } \\
\text { Household } \\
\text { Characteristics } \\
\text { PLWD Health } \\
\text { Insurance } \\
\text { PLWD Detailed } \\
\text { Race Information } \\
\end{array}$ & $\begin{array}{l}\text { Informal Caregiver } \\
\text { (IC) } \\
\text { Characteristics } \\
\text { IC N } \\
\text { IC Age (mean) } \\
\text { IC Sex (\% female) } \\
\text { IC Race (\% } \\
\text { majority) } \\
\text { IC Education } \\
\text { (mean years) } \\
\text { IC Relation to } \\
\text { PLWD (\% majority) }\end{array}$ & $\begin{array}{l}\text { Informal Caregiver } \\
\text { (IC) Char. RS } \\
\text { IC Duration } \\
\text { IC Living With } \\
\text { PLWD } \\
\text { IC Payment } \\
\text { IC Health Status } \\
\text { IC Dementia } \\
\text { Family History } \\
\text { IC Employment } \\
\text { Status } \\
\text { IC Training }\end{array}$ & $\begin{array}{l}\text { Formal Caregiver } \\
\text { (FC) } \\
\text { Characteristics } \\
\text { FC N } \\
\text { FC Age (mean) } \\
\text { FC Sex (\% } \\
\text { female) } \\
\text { FC Race (\% } \\
\text { majority) } \\
\text { FC Education } \\
\text { (mean years) }\end{array}$ & $\begin{array}{l}\text { Formal } \\
\text { Caregiver (FC) } \\
\text { Char. RS } \\
\text { FC Health } \\
\text { Status } \\
\text { FC Training } \\
\text { FC Education } \\
\text { FC Position } \\
\text { FC Length of } \\
\text { Service }\end{array}$ & $\begin{array}{l}\text { Outcome } \\
\text { Timing(s) }\end{array}$ & $\begin{array}{l}\text { PLWD } \\
\text { Outcomes }\end{array}$ & $\begin{array}{l}\text { Caregiver } \\
\text { Outcomes } \\
\text { IC: } \\
\text { FC: }\end{array}$ \\
\hline $\begin{array}{l}\text { Kor 2019204 } \\
\text { (30922609) } \\
\text { China } \\
\text { Pilot Study }\end{array}$ & $\begin{array}{l}\text { Group modified } \\
\text { mindfulness- } \\
\text { based cognitive } \\
\text { therapy, } 72- \\
\text { hour sessions } \\
\text { over } 10 \text { weeks }\end{array}$ & $\begin{array}{l}\text { Usual care with } \\
\text { brief education } \\
\text { on dementia } \\
\text { care } 7 \text { group } \\
\text { sessions) }\end{array}$ & \begin{tabular}{|l|} 
Community- \\
based \\
setting \\
RCT; single \\
site \\
36 informal \\
caregivers \\
\end{tabular} & $\begin{array}{l}\text { Dementia } \\
\text { diagnosis }\end{array}$ & $\begin{array}{l}\mathrm{N}=36 \\
\text { Age: no } \\
\text { Sex: no } \\
\text { Race: } n \\
\text { Education: no }\end{array}$ & NR & $\begin{array}{l}\mathrm{N}=36 \\
\text { Age: yes } \\
\text { Sex: yes } \\
\text { Race: no } \\
\text { Education: yes } \\
\text { Relation to PLWD: } \\
\text { yes }\end{array}$ & $\begin{array}{l}\text { Duration: yes } \\
\text { Employment Status: } \\
\text { yes } \\
\text { Training: yes }\end{array}$ & NA & NA & $\begin{array}{l}10 \text { weeks } \\
3 \text { months }\end{array}$ & NA & $\begin{array}{l}\text { PSS } \\
\text { ZBI } \\
\text { CED-S } \\
\text { HADS-A } \\
\text { Brief } \\
\text { Resilience } \\
\text { Scale } \\
\text { SF-12 } \\
\text { Five Facets } \\
\text { Mindfulness } \\
\text { Questionnaire } \\
\text { Short Form }\end{array}$ \\
\hline $\begin{array}{l}\text { Richards } \\
2018^{205} \\
\text { (29653492) } \\
\text { US } \\
\text { Sample Size }\end{array}$ & $\begin{array}{l}\text { Visual arts } \\
\text { education group } \\
\text { (producing } \\
\text { different artistic } \\
\text { work each week } \\
\text { with increasing } \\
\text { difficulty) for } \\
\text { PLWDD-informal } \\
\text { caregiver } \\
\text { dyads; 1 } \\
\text { session/week } \\
\text { for } 8 \text { weeks }\end{array}$ & $\begin{array}{l}\text { Discussion and } \\
\text { slide shows } \\
\text { about art and } \\
\text { painting for } \\
\text { PLWD-informal } \\
\text { caregiver } \\
\text { dyads; } 1 \\
\text { session/week } \\
\text { for } 8 \text { weeks }\end{array}$ & \begin{tabular}{|l|} 
Community- \\
based \\
setting \\
RCT; single \\
site \\
35 PLWD- \\
informal \\
caregiver \\
pairs
\end{tabular} & \begin{tabular}{|l|} 
Mild to moderate \\
AD and related \\
dementias, MMSE \\
12 to 26
\end{tabular} & $\begin{array}{l}\mathrm{N}=35 \\
\text { Age: yes } \\
\text { Sex: yes } \\
\text { Race: } n \text { o } \\
\text { Education: no }\end{array}$ & NR & $\begin{array}{l}\mathrm{N}=35 \\
\text { Age: } y e s \\
\text { Sex: yes } \\
\text { Race: no } \\
\text { Education: no } \\
\text { Relation to PLWD: } \\
\text { no }\end{array}$ & NR & NA & NA & $\begin{array}{l}8 \text { weeks } \\
6 \text { months }\end{array}$ & $\begin{array}{l}\text { Rosenberg } \\
\text { Self-Esteem } \\
\text { Scale } \\
\text { QOL-AD } \\
\text { ADL (FAQ) }\end{array}$ & $\begin{array}{l}\text { ZBI } \\
\text { Rosenberg } \\
\text { Self-Esteem } \\
\text { Scale } \\
\text { QOL-AD }\end{array}$ \\
\hline $\begin{array}{l}\text { Salamizadeh } \\
2017^{194} \\
(28670585) \\
\text { Iran } \\
\text { High ROB }\end{array}$ & $\begin{array}{l}\text { Spiritual care } \\
\text { education; } 5 \\
\text { educational } \\
\text { sessions, } 45 \text { to } \\
60 \text { minutes }\end{array}$ & Usual care & \begin{tabular}{|l|} 
Community- \\
based \\
setting \\
Quasi- \\
experimental \\
60 informal \\
caregivers \\
\end{tabular} & AD diagnosis & $\begin{array}{l}\mathrm{N}=60 \\
\text { Age: no } \\
\text { Sex: no } \\
\text { Race: no } \\
\text { Education: no }\end{array}$ & $\mathrm{NR}$ & $\begin{array}{l}\mathrm{N}=60 \\
\text { Age: yes } \\
\text { Sex: yes } \\
\text { Race: yes } \\
\text { Education: yes } \\
\text { Relation to PLWD: } \\
\text { yes }\end{array}$ & Duration: yes & NA & NA & 3 weeks & $\mathrm{NA}$ & GSE-10 \\
\hline $\begin{array}{l}\text { Waelde 2017206 } \\
\text { (28263398) } \\
\text { US } \\
\text { Sample Size }\end{array}$ & $\begin{array}{l}\text { Group stress } \\
\text { mindfulness and } \\
\text { mantra training } \\
\text { for female } \\
\text { informal } \\
\text { caregivers; } 10 \\
\text { sessions over } \\
12 \text { weeks }\end{array}$ & $\begin{array}{l}\text { Telephone } \\
\text { psychoeducatio } \\
\text { n and telephone } \\
\text { supports, } \\
\text { biweekly 10-15- } \\
\text { minute calls } \\
\text { over } 12 \text { weeks }\end{array}$ & \begin{tabular}{|l|} 
Community- \\
based \\
setting \\
RCT; single \\
site \\
31 informal \\
caregivers \\
\end{tabular} & $\begin{array}{l}\text { AD or other } \\
\text { dementia }\end{array}$ & $\begin{array}{l}\text { N=31 } \\
\text { Age: no } \\
\text { Sex: no } \\
\text { Race: no } \\
\text { Education: no }\end{array}$ & $\mathrm{NR}$ & $\begin{array}{l}\mathrm{N}=31 \\
\text { Age: yes } \\
\text { Sex: yes } \\
\text { Race: yes } \\
\text { Relation to PLWD: } \\
\text { no }\end{array}$ & $\begin{array}{l}\text { Living with PLWD: } \\
\text { yes } \\
\text { Employment: yes }\end{array}$ & NA & NA & 12 weeks & MMSE & $\begin{array}{l}\text { Satisfaction } \\
\text { with Life } \\
\text { Scale } \\
\text { CES-D } \\
\text { Self-Efficacy } \\
\text { for Controlling } \\
\text { Upsetting } \\
\text { Thoughts, } \\
\text { Revised } \\
\text { Scale for } \\
\text { Caregiving } \\
\text { Self-Efficacy }\end{array}$ \\
\hline
\end{tabular}




\begin{tabular}{|c|c|c|c|c|c|c|c|c|c|c|c|c|c|}
\hline $\begin{array}{l}\text { Brown 2016207 } \\
\text { (26211415) } \\
\text { US } \\
\text { Sample Size }\end{array}$ & $\begin{array}{l}\text { Group } \\
\text { mindfulness- } \\
\text { based stress } \\
\text { reduction for } \\
\text { informal } \\
\text { caregivers; } 1.5 \\
\text { to } 2 \text { hours/week } \\
\text { for } 8 \text { weeks }\end{array}$ & $\begin{array}{l}\text { Group social } \\
\text { support for } \\
\text { informal } \\
\text { caregivers; } 1.5 \\
\text { to } 2 \text { hours/week } \\
\text { for } 8 \text { weeks }\end{array}$ & \begin{tabular}{|l} 
Community- \\
based \\
setting \\
RCT, single \\
site \\
38 informal \\
caregivers
\end{tabular} & $\begin{array}{l}\text { Early stage AD or } \\
\text { other dementia, } \\
\text { FAST stage } 5 \text { or } \\
\text { lower }\end{array}$ & $\begin{array}{l}\text { N=38 } \\
\text { Age: no } \\
\text { Sex: no } \\
\text { Race: no } \\
\text { Education: no }\end{array}$ & $\mathrm{NR}$ & $\begin{array}{l}\mathrm{N}=211 \\
\text { Age: yes } \\
\text { Sex: yes } \\
\text { Race: yes } \\
\text { Education: yes } \\
\text { Relation to PLWD: } \\
\text { yes }\end{array}$ & Duration: yes & NA & NA & 3 months & NA & $\begin{array}{l}\text { PSS } \\
\text { Acceptance } \\
\text { and Action } \\
\text { Questionnaire } \\
\text { II } \\
\text { Profile of } \\
\text { Mood States } \\
\text { SF-36 } \\
\text { ZBI } \\
\text { Mortality } \\
\text { Scale, Family } \\
\text { Care } \\
\text { Inventory }\end{array}$ \\
\hline $\begin{array}{l}\text { Farran 2016 } \\
\text { (28752016) } \\
\text { US } \\
\text { High ROB } \\
\text { Faran 2016196 } \\
\text { (29147683) } \\
\text { Cothran 2017197 } \\
\text { (28956706) }\end{array}$ & $\begin{array}{l}\text { Telephone- } \\
\text { based } \\
\text { intervention to } \\
\text { support and } \\
\text { encourage } \\
\text { informal } \\
\text { caregivers to } \\
\text { participate in } \\
\text { moderate } \\
\text { vigorous } \\
\text { physical activity, } \\
\text { including goal- } \\
\text { setting; regular } \\
\text { phone calls over } \\
12 \text { months }\end{array}$ & $\begin{array}{l}\text { Caregiver skill } \\
\text { building to } \\
\text { provide } \\
\text { information, } \\
\text { support, and } \\
\text { problem-solving } \\
\text { to caregivers; } \\
\text { regular phone } \\
\text { calls over } 12 \\
\text { months }\end{array}$ & $\begin{array}{l}\text { Community- } \\
\text { based } \\
\text { setting with } \\
\text { intervention } \\
\text { received at } \\
\text { home } \\
\text { RCT; single } \\
\text { site } \\
\text { 211 Informal } \\
\text { Caregivers }\end{array}$ & $\begin{array}{l}\text { AD or related } \\
\text { dementia }\end{array}$ & $\begin{array}{l}\mathrm{N}=211 \\
\text { Age: yes } \\
\text { Sex: yes } \\
\text { Race: no } \\
\text { Education: no }\end{array}$ & NR & $\begin{array}{l}\mathrm{N}=211 \\
\text { Age: yes } \\
\text { Sex: yes } \\
\text { Race: yes } \\
\text { Education: yes } \\
\text { Relation to PLWD: } \\
\text { yes }\end{array}$ & $\begin{array}{l}\text { Living with PLWD: } \\
\text { yes } \\
\text { Health status: yes } \\
\text { Employment Status: } \\
\text { yes }\end{array}$ & NA & NA & 12 months & NA & $\begin{array}{l}\text { 2-minute Step } \\
\text { Test } \\
\text { 30s Chair } \\
\text { Stand Test } \\
\text { Caregiver } \\
\text { Burden } \\
\text { (PADL/IADL) } \\
\text { PSS }\end{array}$ \\
\hline $\begin{array}{l}\text { Hirano 2016208 } \\
\text { (26289496) } \\
\text { Japan } \\
\text { Pilot Study }\end{array}$ & $\begin{array}{l}\text { Informal } \\
\text { caregiver } \\
\text { selected leisure } \\
\text { activity, } 3 \\
\text { times/week for } \\
30 \text { minutes for } \\
24 \text { weeks }\end{array}$ & Usual care & $\begin{array}{l}\text { Community- } \\
\text { based } \\
\text { setting } \\
\text { RCT; single } \\
\text { site } \\
54 \text { informal } \\
\text { caregivers }\end{array}$ & AD, DSM-IV criteria & $\begin{array}{l}\text { N=54 } \\
\text { Age: yes } \\
\text { Sex: yes } \\
\text { Race: no } \\
\text { Education: no }\end{array}$ & NR & $\begin{array}{l}\mathrm{N}=54 \\
\text { Age: yes } \\
\text { Sex: yes } \\
\text { Race: no } \\
\text { Education: no } \\
\text { Relation to PLWD: } \\
\text { no }\end{array}$ & $\begin{array}{l}\text { Living with PLWD: } \\
\text { yes } \\
\text { Health status: yes }\end{array}$ & NA & NA & 12 months & $\begin{array}{l}\text { MMSE } \\
\text { NPI }\end{array}$ & $\mathrm{ZBI}$ \\
\hline $\begin{array}{l}\text { Mahdavi } \\
2016^{198} \\
(28499566) \\
\text { Iran } \\
\text { High ROB }\end{array}$ & $\begin{array}{l}\text { Spiritual group } \\
\text { therapy for } \\
\text { informal } \\
\text { caregivers } \\
\text { based on } \\
\text { Iranian } \\
\text { culture/lslam; } \\
\text { weekkly } 45 \text { to } 60- \\
\text { minute sessions } \\
\text { for } 5 \text { weeks }\end{array}$ & $\begin{array}{l}\text { 1. Caregiver } \\
\text { discussion } \\
\text { group; weekly } \\
45 \text { to } 60 \text {-minute } \\
\text { sessions for } 5 \\
\text { weeks } \\
\text { 2. No } \\
\text { intervention }\end{array}$ & $\begin{array}{l}\text { Community- } \\
\text { based } \\
\text { setting } \\
\text { RCT; single } \\
\text { site } \\
100 \text { Informal } \\
\text { Caregivers }\end{array}$ & Diagnosis of AD & $\begin{array}{l}\mathrm{N}=100 \\
\text { Age: yes } \\
\text { Sex: no } \\
\text { Race: yes } \\
\text { Education: no }\end{array}$ & $\mathrm{NR}$ & $\begin{array}{l}\mathrm{N}=100 \\
\text { Age: Yes } \\
\text { Sex: no } \\
\text { Race: yes } \\
\text { Education: no } \\
\text { Relation to PLWD: } \\
\text { yes }\end{array}$ & $\mathrm{NR}$ & NA & NA & 5 weeks & NA & $\begin{array}{l}\text { Caregiver } \\
\text { Strain Index }\end{array}$ \\
\hline $\begin{array}{l}\text { Danucalov } 2015 \\
\text { (26685923) } \\
\text { Danucalov 2013 } \\
\text { (368865412) } \\
\text { Brazil } \\
\text { Sample Size }\end{array}$ & $\begin{array}{l}\text { Yoga and } \\
\text { compassion } \\
\text { meditation; i in- } \\
\text { person and at } \\
\text { home; three } 75 \\
\text { min } \\
\text { sessions/week } \\
\text { for } 8 \text { weeks }\end{array}$ & Waitlist control & $\begin{array}{l}\text { Community- } \\
\text { based } \\
\text { setting } \\
\text { RCT; single } \\
\text { site } \\
53 \text { informal } \\
\text { caregivers }\end{array}$ & Diagnosis of AD & $\begin{array}{l}\text { N=53 } \\
\text { Age: no } \\
\text { Sex: no } \\
\text { Race: yes } \\
\text { Education: no }\end{array}$ & NR & $\begin{array}{l}\mathrm{N}=53 \\
\text { Sex: yes } \\
\text { Age: yes } \\
\text { Race: no } \\
\text { Education: no } \\
\text { Relation to PLWD: } \\
\text { no }\end{array}$ & $\mathrm{NR}$ & NA & NA & 8 weeks & $\mathrm{NA}$ & $\begin{array}{l}\text { WHOQOL- } \\
\text { Bref } \\
\text { Subjective } \\
\text { Vitality Scales } \\
\text { Mindfulness } \\
\text { Attention } \\
\text { Awareness } \\
\text { Scale } \\
\text { Self- } \\
\text { Compassion } \\
\text { Sale }\end{array}$ \\
\hline
\end{tabular}




\begin{tabular}{|c|c|c|c|c|c|c|c|c|c|c|c|c|c|}
\hline $\begin{array}{l}\text { Leach 2015209 } \\
\text { (25952550) } \\
\text { Australia } \\
\text { Pilot Study } \\
\text { Leach 2014 } \\
\text { (24044373) } \\
\text { Leach 201611 } \\
\text { (27227995) }\end{array}$ & $\begin{array}{l}\text { Transcendental } \\
\text { meditation; 14- } \\
\text { hour program } \\
\text { over } 12 \text { weeks } \\
\text { with face to face } \\
12 \text {-week follow- } \\
\text { up }\end{array}$ & Waitlist control & $\begin{array}{l}\text { Community- } \\
\text { based } \\
\text { setting } \\
\text { RCT; single } \\
\text { site } \\
17 \text { informal } \\
\text { caregivers }\end{array}$ & $\begin{array}{l}\text { Diagnosis of } \\
\text { dementia }\end{array}$ & $\begin{array}{l}\mathrm{N}=17 \\
\text { Age: no } \\
\text { Sex: no } \\
\text { Race: yes } \\
\text { Education: no }\end{array}$ & \begin{tabular}{|l|}
$N R$ \\
\end{tabular} & $\begin{array}{l}\text { N-17 } \\
\text { Sex: yes } \\
\text { Age: yes } \\
\text { Race: no } \\
\text { Education: no } \\
\text { Relation to PLWD: } \\
\text { yes }\end{array}$ & $\begin{array}{l}\text { Duration: yes } \\
\text { Health Status: yes }\end{array}$ & NA & NA & $\begin{array}{l}12 \text { weeks } \\
24 \text { weeks }\end{array}$ & NA & $\begin{array}{l}\text { AQoL-8D } \\
\text { WebNeuro } \\
\text { Test Battery }\end{array}$ \\
\hline $\begin{array}{l}\text { Lowery 2014 } \\
\text { (24338799) } \\
\text { UK } \\
\text { High ROB } \\
\text { D'Amico 2016200 } \\
\text { (26489776) }\end{array}$ & $\begin{array}{l}\text { Individually } \\
\text { tailored, } \\
\text { progressively } \\
\text { intensive } \\
\text { walking regimen } \\
\text { for PLWD and } \\
\text { informal } \\
\text { caregiver dyad, } \\
20-30 \text { min at } \\
\text { least } 5 \\
\text { times/week for } \\
12 \text { weeks }\end{array}$ & Usual care & $\begin{array}{l}\text { Community- } \\
\text { based } \\
\text { setting with } \\
\text { intervention } \\
\text { at home } \\
\text { RCT; single } \\
\text { site } \\
\text { 131 PLWD- } \\
\text { informal } \\
\text { caregiver } \\
\text { dyads }\end{array}$ & $\begin{array}{l}\text { Clinical diagnosis } \\
\text { of dementia with } \\
\text { ICD-10 Diagnostic } \\
\text { Criteria for } \\
\text { Research and NPI } \\
\text { minimum score of } 2 \\
\text { and frequency of } 2 \\
\text { (hallucinations and } \\
\text { delusions } \\
\text { excluded) }\end{array}$ & $\begin{array}{l}\mathrm{N}=131 \\
\text { Age: } \text { yes } \\
\text { Sex: yes } \\
\text { Race: yes } \\
\text { Education: yes }\end{array}$ & NR & $\begin{array}{l}\mathrm{N}=131 \\
\text { Age: yes } \\
\text { Sex: yes } \\
\text { Race: no } \\
\text { Education: no } \\
\text { Relation to PLWD: } \\
\text { yes }\end{array}$ & NR & NA & NA & 12 weeks & $\begin{array}{l}\text { NPI } \\
\text { DemQOL- } \\
\text { Proxy } \\
\text { Change of } \\
\text { Domiciliary } \\
\text { Status } \\
\text { Mortality }\end{array}$ & $\begin{array}{l}\text { ZBI } \\
\text { NPI Caregiver } \\
\text { Distress } \\
\text { GHQ }\end{array}$ \\
\hline $\begin{array}{l}\text { Lavretsky } \\
2013^{212} \\
(22407663) \\
\text { US } \\
\text { Pilot Study }\end{array}$ & $\begin{array}{l}\text { Kirtan Kriya } \\
\text { yogic } \\
\text { meditation, } 12 \\
\text { min/day for } 8 \\
\text { weeks }\end{array}$ & $\begin{array}{l}\text { Relation, } 12 \\
\text { min/day for } 8 \\
\text { weeks }\end{array}$ & $\begin{array}{l}\text { RCT, single } \\
\text { site }\end{array}$ & $\begin{array}{l}\text { Dementia based on } \\
\text { DSM-IV-R without } \\
\text { major depression } \\
\text { (screened by HAM- } \\
\text { D-24) }\end{array}$ & $\begin{array}{l}\text { N=39 } \\
\text { Age: no } \\
\text { Sex: no } \\
\text { Race: no } \\
\text { Education: no }\end{array}$ & \begin{tabular}{|l|}
$N R$ \\
\end{tabular} & $\begin{array}{l}\mathrm{N}=39 \\
\text { Age: } \text { yes } \\
\text { Sex: yes } \\
\text { Race: no } \\
\text { Education: yes } \\
\text { Relation to PLWD: } \\
\text { yes }\end{array}$ & $\begin{array}{l}\text { Duration: yes } \\
\text { Health Status: yes }\end{array}$ & NA & NA & 8 weeks & NA & $\begin{array}{l}\text { SF-36 } \\
\text { HAM-D } \\
\text { MMSE } \\
\text { Cumulative } \\
\text { Illness Rating } \\
\text { Scale } \\
\text { CVLT II } \\
\text { TMT A } \\
\text { TMT B }\end{array}$ \\
\hline $\begin{array}{l}\text { Hirano 2010 } \\
\text { (20850878) } \\
\text { Japan } \\
\text { Sample Size }\end{array}$ & $\begin{array}{l}\text { Regular } \\
\text { exercise for } \\
\text { informal } \\
\text { caregivers with } \\
\text { moderate } \\
\text { intensity, } 3 \\
\text { times a week for } \\
12 \text { weeks }\end{array}$ & No exercise & $\begin{array}{l}\text { Community- } \\
\text { based } \\
\text { setting } \\
\text { RCT; single } \\
\text { site } \\
36 \text { informal } \\
\text { caregivers }\end{array}$ & $\begin{array}{l}\text { AD according to } \\
\text { DSM-IV criteria }\end{array}$ & $\begin{array}{l}\mathrm{N}=36 \\
\text { Age: no } \\
\text { Sex: no } \\
\text { Race: no } \\
\text { Education: no }\end{array}$ & \begin{tabular}{|l|l}
$N R$ \\
\end{tabular} & $\begin{array}{l}\mathrm{N}=36 \\
\text { Age: yes } \\
\text { Sex: yes } \\
\text { Race: no } \\
\text { Education: no } \\
\text { Relation to PLWD: } \\
\text { no }\end{array}$ & $\begin{array}{l}\text { Health status: yes } \\
\text { Living with PLWD: } \\
\text { yes }\end{array}$ & NA & NA & 12 weeks & $\begin{array}{l}\text { NPI } \\
\text { MMSE }\end{array}$ & $\begin{array}{l}\text { ZBI } \\
\text { VAS }\end{array}$ \\
\hline $\begin{array}{l}\text { Carbpnneau } \\
2011^{213} \\
(20598758) \\
\text { Canada } \\
\text { Sample Size }\end{array}$ & $\begin{array}{l}\text { Adapted leisure } \\
\text { education } \\
\text { program for } \\
\text { informal } \\
\text { caregiver- } \\
\text { PLWD dyads; } 4 \\
\text { to } 6 \text { in person } \\
\text { sessions with } \\
\text { telephone } \\
\text { follow-ups } \\
\end{array}$ & Usual care & $\begin{array}{l}\text { Community } \\
\text { and nursing } \\
\text { home } \\
\text { RCT; single } \\
\text { site } \\
\text { 49 PLWD- } \\
\text { informal } \\
\text { caregiver } \\
\text { dyads }\end{array}$ & Not specified & $\begin{array}{l}\text { N=49 } \\
\text { Age: no } \\
\text { Sex: no } \\
\text { Race: no } \\
\text { Education: no }\end{array}$ & NR & $\begin{array}{l}\mathrm{N}=49 \\
\text { Age: yes } \\
\text { Sex: yes } \\
\text { Race: no } \\
\text { Education: yes } \\
\text { Relation to PLWD: } \\
\text { yes }\end{array}$ & Health status: yes & NA & NA & $\begin{array}{l}8 \text { weeks } \\
12 \text { weeks }\end{array}$ & NA & $\begin{array}{l}\text { General Well- } \\
\text { being Scale } \\
\text { Elder Care } \\
\text { Scale } \\
\text { Self-Efficacy }\end{array}$ \\
\hline
\end{tabular}




\begin{tabular}{|c|c|c|c|c|c|c|c|c|c|c|c|c|c|}
\hline $\begin{array}{l}\text { Oken 2010214 } \\
\text { (20929380) } \\
\text { US } \\
\text { Pilot Study }\end{array}$ & $\begin{array}{l}\text { Mindfulness- } \\
\text { based cognitive } \\
\text { therapy, one 90- } \\
\text { minute session } \\
\text { in-person/week } \\
\text { with at home } \\
\text { implementation } \\
\text { for } 7 \text { weeks }\end{array}$ & $\begin{array}{l}\text { 1. Educational } \\
\text { class based on } \\
\text { Powerful Tools } \\
\text { for caregivers } \\
\text { with weekly } \\
\text { assignments, } \\
\text { one 90-minute } \\
\text { session/week } \\
\text { for } 7 \text { weeks } \\
\text { 2. Respite only, } \\
3 \text { hours of } \\
\text { respite/week for } \\
7 \text { weeks }\end{array}$ & $\begin{array}{l}\text { Community- } \\
\text { based } \\
\text { RCT; single } \\
\text { site } \\
31 \text { informal } \\
\text { caregivers }\end{array}$ & $\begin{array}{l}\text { Progressive } \\
\text { dementia }\end{array}$ & $\begin{array}{l}\mathrm{N}=31 \\
\text { Age: no } \\
\text { Sex: no } \\
\text { Race: no } \\
\text { Education: no }\end{array}$ & $\mathrm{NR}$ & $\begin{array}{l}\mathrm{N}=31 \\
\text { Age: yes } \\
\text { Sex: yes } \\
\text { Race: no } \\
\text { Education: No } \\
\text { Relation to PLWD: } \\
\text { yes }\end{array}$ & NR & NA & NA & 8 weeks & NPI & $\begin{array}{l}\text { RMBPC } \\
\text { Caregiver } \\
\text { Appraisal } \\
\text { Tool } \\
\text { PSS } \\
\text { CESD } \\
\text { General } \\
\text { Perceived } \\
\text { Self-Efficacy } \\
\text { Scale } \\
\text { PSQI } \\
\text { Epworth } \\
\text { Sleep } \\
\text { Questionnaire } \\
\text { Global } \\
\text { Impression of } \\
\text { Change }\end{array}$ \\
\hline $\begin{array}{l}\text { Connell 2009202 } \\
(21709757) \\
\text { US } \\
\text { High ROB }\end{array}$ & $\begin{array}{l}\text { Telephone- } \\
\text { based exercise } \\
\text { counseling and } \\
\text { goal-setting for } \\
\text { female spouse } \\
\text { caregivers with } \\
\text { exercise } \\
\text { workbooks and } \\
\text { videos; } 14 \text { calls } \\
\text { over } 6 \text { months }\end{array}$ & No intervention & $\begin{array}{l}\text { Community- } \\
\text { based } \\
\text { setting with } \\
\text { intervention } \\
\text { at home } \\
\text { RCT; single } \\
\text { site } \\
157 \text { female } \\
\text { spouse } \\
\text { caregivers }\end{array}$ & No specified & $\begin{array}{l}\text { N=157 } \\
\text { Age: no } \\
\text { Sex: no } \\
\text { Race: no } \\
\text { Education: no }\end{array}$ & NR & $\begin{array}{l}\mathrm{N}=157 \\
\text { Age: yes } \\
\text { Sex: yes } \\
\text { Race: yes } \\
\text { Education: yes } \\
\text { Relation to PLWD: } \\
\text { yes }\end{array}$ & $\begin{array}{l}\text { Health status: yes } \\
\text { Employment status: } \\
\text { yes }\end{array}$ & NA & NA & $\begin{array}{l}6 \text { months } \\
12 \text { months }\end{array}$ & NA & $\begin{array}{l}\text { MOS Short- } \\
\text { Form General } \\
\text { Health Survey } \\
\text { RMBPC } \\
\text { (Caregiver } \\
\text { Burden) } \\
\text { CES-D }\end{array}$ \\
\hline $\begin{array}{l}\text { Wilz 2008215 } \\
\text { (18381838) } \\
\text { Germany } \\
\text { Pilot study }\end{array}$ & $\begin{array}{l}\text { Assisted } \\
\text { vacations } \\
\text { (participating in } \\
\text { positive } \\
\text { activities, } \\
\text { improving } \\
\text { utilization of } \\
\text { support, } \\
\text { psychoeducatio } \\
\text { n) based on the } \\
\text { needs of the } \\
\text { caregiver and } \\
\text { PLWWDi w week } \\
\text { in a hotel or } \\
\text { rehabilitation } \\
\text { clinic }\end{array}$ & Waitlist control & $\begin{array}{l}\text { Hotel or } \\
\text { rehabilitation } \\
\text { centers } \\
\text { Quasi } \\
\text { experimental } \\
29 \\
\text { caregiving } \\
\text { wives }\end{array}$ & $\begin{array}{l}\text { Medical diagnosis } \\
\text { of dementia }\end{array}$ & $\begin{array}{l}\mathrm{N}=29 \\
\text { Age: yes } \\
\text { Sex: yes } \\
\text { Race: no } \\
\text { Education: no }\end{array}$ & NR & $\begin{array}{l}\mathrm{N}=29 \\
\text { Age: yes } \\
\text { Sex: yes } \\
\text { Race: no } \\
\text { Education: no } \\
\text { Relation to PLWD: } \\
\text { yes }\end{array}$ & $\begin{array}{l}\text { Living with PLWD: } \\
\text { yes }\end{array}$ & NA & NA & 3 months & NA & $\begin{array}{l}\text { Giessen } \\
\text { Subjective } \\
\text { Complaints } \\
\text { List } \\
\text { BDI }\end{array}$ \\
\hline $\begin{array}{l}\text { King 2002 } \\
\text { (11773209) } \\
\text { Castro 2002203 } \\
\text { (12021419) } \\
\text { US } \\
\text { High ROB }\end{array}$ & $\begin{array}{l}\text { Home-based, } \\
\text { telephone } \\
\text { supervised } \\
\text { moderate } \\
\text { intensity } \\
\text { exercise training } \\
\text { for female } \\
\text { caregivers, 30- } \\
40 \text {-minute } \\
\text { sessions } 4 \\
\text { times/week for } \\
12 \text { months, } 15 \\
\text { telephone } \\
\text { contacts over } \\
12 \text { months }\end{array}$ & $\begin{array}{l}\text { Telephone- } \\
\text { based nutrition } \\
\text { education, } 15 \\
\text { telephone } \\
\text { contacts over } \\
12 \text { months }\end{array}$ & $\begin{array}{l}\text { Community- } \\
\text { based } \\
\text { setting with } \\
\text { intervention } \\
\text { at home } \\
\text { RCT; single } \\
\text { site } \\
100 \text { female } \\
\text { caregivers }\end{array}$ & $\begin{array}{l}\text { AD or another form } \\
\text { of dementia }\end{array}$ & $\begin{array}{l}\text { N=100 } \\
\text { Age: yes } \\
\text { Sex: no } \\
\text { Race: no } \\
\text { Education: no }\end{array}$ & NR & $\begin{array}{l}\mathrm{N}=100 \\
\text { Age: yes } \\
\text { Sex: yes } \\
\text { Race: yes } \\
\text { Education: yes } \\
\text { Relation to PLWD: } \\
\text { yes }\end{array}$ & $\begin{array}{l}\text { Duration: yes } \\
\text { Employment Status: } \\
\text { yes }\end{array}$ & NA & NA & 12 months & NA & $\begin{array}{l}\text { Screen for } \\
\text { Caregiver } \\
\text { Burden } \\
\text { TMAS } \\
\text { BDI } \\
\text { PSS } \\
\text { Interpersonal } \\
\text { Social } \\
\text { Evaluation } \\
\text { List } \\
\text { RMBPC } \\
\text { (Caregiver } \\
\text { Burden) } \\
\text { PSQI }\end{array}$ \\
\hline
\end{tabular}

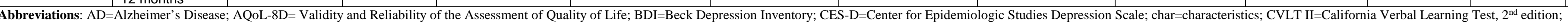

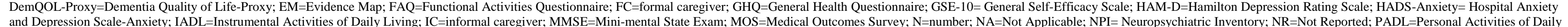

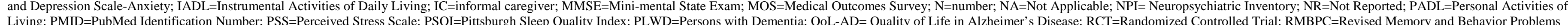




\section{Respite Care}

Table E-19. Risk of bias assessment: respite care vs. usual care

\begin{tabular}{|l|l|l|}
\hline Study (PMID) & Outcome Timing & $\begin{array}{l}\text { Attrition Bias } \\
\text { Attrition \% }\end{array}$ \\
\hline
\end{tabular}

Vandepitte 2019216 (31243801)

Vandepitte $2016^{217}(27912740)$

Kim 2012'19 (21322030)

Lawton $1989^{220}$ (NA)

6 months

\begin{tabular}{l} 
High \\
$24.7 \%$ \\
\hline
\end{tabular}

\begin{tabular}{|l|l|}
\hline 6 months & $\begin{array}{l}\text { High } \\
24.7\end{array}$ \\
\hline 3 months & High \\
\hline
\end{tabular}

High

\begin{tabular}{|l|l}
\hline 12 months & 3 months: $42.7 \%$ \\
& 12 months: $65.9 \%$ \\
\hline 12 months & High \\
\hline
\end{tabular}

\begin{tabular}{|l|l|}
\hline Selection Bias & Detection Bias \\
\hline
\end{tabular}

Performance Bias

Reporting Bias

Fidelity Bias

Funder

\begin{tabular}{|l|l|}
\hline & Overall Rating \\
\hline Hent & High \\
\hline High \\
\hline
\end{tabular}

Abbreviations: NR=Not Reported; PMID=PubMed Identification Number; NA=Not Applicable 


\section{Evidence Map: Respite Care}

\begin{tabular}{|c|c|c|c|c|c|c|c|c|c|c|c|c|c|}
\hline $\begin{array}{l}\text { Study (PMID) } \\
\text { Country } \\
\text { EM Reason }\end{array}$ & Intervention & Comparison & $\begin{array}{l}\text { Setting } \\
\text { and Design } \\
\text { Setting } \\
\text { Design } \\
\text { Cluster N } \\
\text { Participants } \\
\text { Random- } \\
\text { ized N }\end{array}$ & $\begin{array}{l}\text { PLWD } \\
\text { Dementia } \\
\text { Characteristics }\end{array}$ & \begin{tabular}{|l|} 
PLWD \\
Non-Disease Char \\
PLWD N \\
PLWD Age (mean) \\
PLWD Sex (\% \\
female) \\
PLWD Race (\% \\
majority) \\
PLWD Education \\
(mean years)
\end{tabular} & $\begin{array}{l}\text { PLWD } \\
\text { Non-Disease } \\
\text { Char Reporting } \\
\text { Status (RS) } \\
\text { PLWD SES } \\
\text { PLWD Prior } \\
\text { Disability } \\
\text { PLWD } \\
\text { Household } \\
\text { Characteristics } \\
\text { PLWD Health } \\
\text { Insurance } \\
\text { PLWD Detailed } \\
\text { Race Information }\end{array}$ & $\begin{array}{l}\text { Informal Caregiver } \\
\text { (IC) } \\
\text { Characteristics } \\
\text { IC N } \\
\text { IC Age (mean) } \\
\text { IC Sex (\% female) } \\
\text { IC Race (\% } \\
\text { majority) } \\
\text { IC Education } \\
\text { (mean years) } \\
\text { IC Relation to } \\
\text { PLWD (\% majority) }\end{array}$ & $\begin{array}{l}\text { Informal Caregiver } \\
\text { (IC) Char. RS } \\
\text { IC Duration } \\
\text { IC Living With } \\
\text { PLWD } \\
\text { IC Payment } \\
\text { IC Health Status } \\
\text { IC Dementia } \\
\text { Family History } \\
\text { IC Employment } \\
\text { Status } \\
\text { IC Training }\end{array}$ & 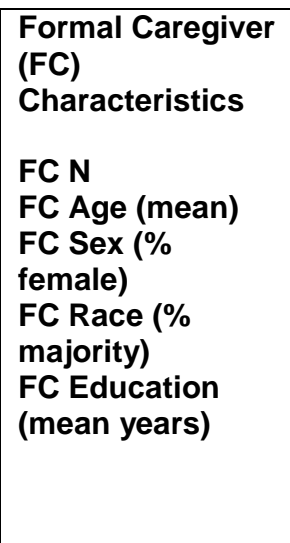 & $\begin{array}{l}\text { Formal } \\
\text { Caregiver (FC) } \\
\text { Char. RS } \\
\text { FC Health } \\
\text { Status } \\
\text { FC Training } \\
\text { FC Education } \\
\text { FC Position } \\
\text { FC Length of } \\
\text { Service }\end{array}$ & \begin{tabular}{|l} 
Outcome \\
Timing(s)
\end{tabular} & $\begin{array}{l}\text { PLWD } \\
\text { Outcomes }\end{array}$ & $\begin{array}{l}\text { Caregiver } \\
\text { Outcomes } \\
\text { IC: } \\
\text { FC: }\end{array}$ \\
\hline $\begin{array}{l}\text { Vandepitte } \\
2019^{216} \\
(31243801) \\
\text { Belgium } \\
\text { High ROB } \\
\text { Vandepitte } \\
2016^{217} \\
(27912740)\end{array}$ & $\begin{array}{l}\text { In-home respite } \\
\text { care for informal } \\
\text { caregivers with } \\
\text { support diary for } \\
\text { additional } \\
\text { caregiver } \\
\text { support; } 5 \text { to } 14 \\
\text { days of } 24 \text {-hour } \\
\text { care for PLWD } \\
\text { from trained } \\
\text { employee }\end{array}$ & \begin{tabular}{|l|} 
Usual care \\
\end{tabular} & $\begin{array}{l}\text { Community- } \\
\text { setting with } \\
\text { in home } \\
\text { care } \\
\text { Quasi } \\
\text { experimental } \\
198 \text { Informal } \\
\text { Caregiver- } \\
\text { PLWD } \\
\text { Dyads }\end{array}$ & Not specified & $\begin{array}{l}\mathrm{N}=198 \\
\text { Age: yes } \\
\text { Sex: yes } \\
\text { Race: } \text { no } \\
\text { Education: yes }\end{array}$ & NR & $\begin{array}{l}\mathrm{N}=198 \\
\text { Sex: yes } \\
\text { Race: no } \\
\text { Education: yes } \\
\text { Relation to PLWD: } \\
\text { yes }\end{array}$ & $\begin{array}{l}\text { Living with PLWD: } \\
\text { yes } \\
\text { Employment Status: } \\
\text { yes }\end{array}$ & NA & NA & 6 months & RMBPC & \begin{tabular}{|l} 
ZBI \\
EQ-5D-5 L \\
Desire-to- \\
Institutionaliz \\
e scale
\end{tabular} \\
\hline $\begin{array}{l}\text { Zarit 1998218 } \\
\text { (9750575) } \\
\text { US } \\
\text { High ROB } \\
\text { Kim 201219 } \\
(21322030)\end{array}$ & $\begin{array}{l}\text { Adult day care; } \\
\text { at least } 2 \\
\text { times/week for } \\
12 \text { months }\end{array}$ & $\begin{array}{l}\text { Usual care (no } \\
\text { intervention) }\end{array}$ & $\begin{array}{l}\text { Community- } \\
\text { based adult } \\
\text { day care } \\
\text { Quasi } \\
\text { experimental } \\
566 \text { informal } \\
\text { caregivers }\end{array}$ & Not specified & $\begin{array}{l}\mathrm{N}=566 \\
\text { Age: yes } \\
\text { Sex: yes } \\
\text { Race: no } \\
\text { Education: no }\end{array}$ & $\mathrm{NR}$ & $\begin{array}{l}\mathrm{N}=566 \\
\text { Age: yes } \\
\text { Sex: yes } \\
\text { Race: yes } \\
\text { Education: yes } \\
\text { Relation to PLWD: } \\
\text { yes }\end{array}$ & NR & NA & NA & $\begin{array}{l}3 \text { months } \\
12 \text { months }\end{array}$ & $\begin{array}{l}\text { PADL } \\
\text { IADL }\end{array}$ & $\begin{array}{l}\text { Involvement } \\
\text { in Caregiving } \\
\text { CES-D } \\
\text { Positive } \\
\text { and Negative } \\
\text { Affect } \\
\text { Schedule } \\
\text { Role Captivity }\end{array}$ \\
\hline $\begin{array}{l}\text { Lawton } 1989^{220} \\
\text { (NA) } \\
\text { US } \\
\text { High ROB }\end{array}$ & $\begin{array}{l}\text { Respite } \\
\text { program with } \\
\text { options for in- } \\
\text { home respite } \\
\text { services, adult } \\
\text { day care, and } \\
\text { institutional } \\
\text { respite care } \\
\text { (limited to } 21 \\
\text { days); any } \\
\text { combination of } \\
\text { services } \\
\text { (caregiver } \\
\text { discretion) over } \\
12 \text { months }\end{array}$ & $\begin{array}{l}\text { Usual care with } \\
\text { list of local } \\
\text { services }\end{array}$ & $\begin{array}{l}\text { In-home, } \\
\text { adult care, } \\
\text { and/or } \\
\text { nursing } \\
\text { home } \\
\text { RCT; Single } \\
\text { site } \\
632 \text { Informal } \\
\text { caregivers }\end{array}$ & Not specified & $\begin{array}{l}\mathrm{N}=632 \\
\text { Age: yes } \\
\text { Sex: yes } \\
\text { Race: } \text { no } \\
\text { Education: yes }\end{array}$ & $\begin{array}{l}\text { Household } \\
\text { Characteristics: } \\
\text { Yes }\end{array}$ & $\begin{array}{l}\mathrm{N}=632 \\
\text { Age: yes } \\
\text { Sex: yes } \\
\text { Race: no } \\
\text { Education: yes } \\
\text { Relation to PLWD: } \\
\text { yes }\end{array}$ & $\begin{array}{l}\begin{array}{l}\text { Employment Status: } \\
\text { yes }\end{array} \\
\end{array}$ & NA & NA & 12 months & NA & $\begin{array}{l}\text { Multilevel } \\
\text { Assessment } \\
\text { Instrument } \\
\text { CES-D } \\
\text { The Affect } \\
\text { Balance } \\
\text { Scale }\end{array}$ \\
\hline
\end{tabular}

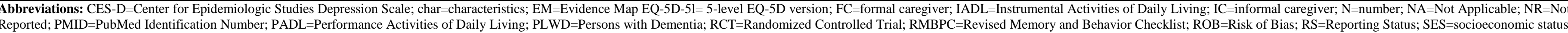
RBI=Zarit Burden Interview 


\section{Multicomponent Interventions}

Table E-21. Risk of bias assessment: multicomponent interventions for informal caregivers

\begin{tabular}{|c|c|c|c|c|c|c|c|c|}
\hline Study (PMID) & Outcome Timing & $\begin{array}{l}\text { Attrition Bias } \\
\text { Attrition \% }\end{array}$ & Selection Bias & Detection Bias & Performance Bias & Reporting Bias & Fidelity Bias & Overall Rating \\
\hline $\begin{array}{l}\text { Luchsinger 2018 } \\
(30084133)\end{array}$ & 6 months & $\begin{array}{l}\text { Low } \\
6 \text { months: } 5 \%\end{array}$ & Medium & Medium & Medium & Low & Medium & Medium \\
\hline Berwig 2017222 (29233097) & 6 months & $\begin{array}{l}\text { Low } \\
6 \text { months: } 12 \%\end{array}$ & Low & Medium & Medium & Low & Low & Medium \\
\hline Berwig 2017222 (29233097) & 9 months & $\begin{array}{l}\text { High } \\
9 \text { months: } 33 \%\end{array}$ & Low & Medium & Medium & Low & Low & High \\
\hline Brijoux 2016 & 16 weeks & $\begin{array}{l}\text { Medium } \\
16 \text { weeks: } 17 \%\end{array}$ & Low & High & Medium & Low & Low & High \\
\hline Koivisto 201624 (26177825) & 36 months & $\begin{array}{l}\text { High } \\
36 \text { months: } 45 \%\end{array}$ & Low & NA & NA & NA & NA & High \\
\hline Baglio $2015^{225}(24788581)$ & 32 weeks & $\begin{array}{l}\text { Medium } \\
32 \text { weeks: 17\% }\end{array}$ & Low & Medium & High & Low & Low & High \\
\hline \begin{tabular}{|l} 
Prick $2015^{226}(26004290)$ \\
\end{tabular} & 3 months & $\begin{array}{l}\text { Low } \\
3 \text { months: } 11 \%\end{array}$ & Low & Low & Medium & Medium & Low & Low \\
\hline \begin{tabular}{|l|} 
Prick $2015^{226}(26004290)$ \\
\end{tabular} & 6 months & $\begin{array}{l}\text { Medium } \\
6 \text { months: } 21 \%\end{array}$ & Low & Low & Medium & Medium & Low & Medium \\
\hline \begin{tabular}{|l} 
Gaugler 2013227 (23339050) \\
\end{tabular} & 12 months & $\begin{array}{l}\text { High } \\
\text { Unclear }\end{array}$ & Low & NA & NA & NA & NA & High \\
\hline Martindale-Adams $2013^{228}$ (24617278) & 12 months & $\begin{array}{l}\text { Medium } \\
\text { months: } 11 \%\end{array}$ & Medium & High & Medium & Low & Low & High \\
\hline Mittleman $2008^{229}(18978250)$ & 24 months & $\begin{array}{l}\text { Low } \\
24 \text { months: } 2 \%\end{array}$ & Low & Low & High & Medium & Medium & Medium \\
\hline Signe 200855 (18269429) & $\begin{array}{l}6 \text { months } \\
12 \text { months }\end{array}$ & NA & High & NA & NA & NA & NA & High \\
\hline Belle $2006^{230}$ (17116917) & 6 months & $\begin{array}{l}\text { Low } \\
6 \text { months: } 9 \%\end{array}$ & Low & Medium & High & Low & Low & Medium \\
\hline \begin{tabular}{|l|l|} 
Ducharme $2005^{231}(16024406)$ \\
\end{tabular} & 6 months & $\begin{array}{l}\text { High } \\
6 \text { months: } 34 \%\end{array}$ & Medium & NA & NA & NA & NA & High \\
\hline \begin{tabular}{|l} 
Mittleman 2004'32 (14722336) \\
\end{tabular} & 4 months & $\begin{array}{l}\text { Low } \\
4 \text { months:9\% }\end{array}$ & Low & Low & High & Low & Medium & Medium \\
\hline \begin{tabular}{|l|} 
Mittleman 2004'32 (14722336) \\
\end{tabular} & 12 months & $\begin{array}{l}\text { High } \\
12 \text { months: } 35 \%\end{array}$ & Low & NA & NA & NA & NA & High \\
\hline
\end{tabular}




\begin{tabular}{|c|c|c|c|c|c|c|c|c|}
\hline \begin{tabular}{|l|} 
Mittleman 2004'32 (14722336) \\
\end{tabular} & 24 months & \begin{tabular}{|l|} 
High \\
24 months: 53\%
\end{tabular} & Low & NA & NA & NA & NA & High \\
\hline Mittleman 2004'32 (14722336) & 36 months & $\begin{array}{l}\text { High } \\
36 \text { months: } 67 \%\end{array}$ & Low & NA & NA & NA & NA & High \\
\hline \begin{tabular}{|l|l|} 
Mittleman 2004'32 (14722336) \\
\end{tabular} & 48 months & $\begin{array}{l}\text { High } \\
46 \text { months: } 74 \%\end{array}$ & Low & NA & NA & NA & NA & High \\
\hline Eisdorfer 2003 ${ }^{233}(12937331)$ & 18 months & \begin{tabular}{|l|} 
High \\
18 months: $32 \%$
\end{tabular} & Low & NA & NA & NA & NA & High \\
\hline Teri 2003 234 (14559955) & 3 months & \begin{tabular}{|l|} 
Low \\
3 months: $9 \%$
\end{tabular} & Low & Medium & Medium & Low & Low & Medium \\
\hline \begin{tabular}{|l|} 
Teri $2003^{234}$ (14559955) \\
\end{tabular} & 6 months & \begin{tabular}{|l|} 
Low \\
6 months: $18 \%$
\end{tabular} & Low & Medium & Medium & Low & Low & Medium \\
\hline \begin{tabular}{|l|} 
Teri $2003^{234}$ (14559955) \\
\end{tabular} & 12 months & \begin{tabular}{|l|} 
Medium \\
12 months: $25 \%$
\end{tabular} & Low & Medium & Medium & Low & Low & Medium \\
\hline \begin{tabular}{|l|} 
Teri $2003^{234}$ (14559955) \\
\end{tabular} & 18 months & $\begin{array}{l}\text { High } \\
18 \text { months: } 37 \%\end{array}$ & Low & NA & NA & NA & NA & High \\
\hline \begin{tabular}{|l} 
Teri 2003 \\
234 \\
(14559955)
\end{tabular} & 24 months & \begin{tabular}{|l} 
High \\
24 months: $42 \%$
\end{tabular} & Low & NA & NA & NA & NA & High \\
\hline Mittelman 1993235 (8314099) & 12 months & $\begin{array}{l}\text { Low } \\
12 \text { months: } 2 \%\end{array}$ & Medium & High & High & Low & Medium & High \\
\hline Mohide $1990^{236}$ (2184186) & 6 months & \begin{tabular}{|l|} 
Medium \\
6 months:30\%
\end{tabular} & Low & High & Medium & Low & Low & High \\
\hline
\end{tabular}

* High risk of bias studies included in evidence map. This table includes low and medium risk of bias studies only.

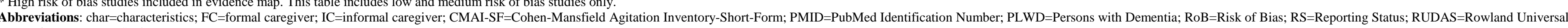
ia Assessment Scale

Table E-22. Characteristics of included studies: multicomponent interventions for informal caregivers

\begin{tabular}{|c|c|c|c|c|c|c|c|c|c|c|c|c|c|}
\hline $\begin{array}{l}\text { Study (PMID) } \\
\text { Country } \\
\text { RoB* } \\
\text { Type }\end{array}$ & \begin{tabular}{|l|} 
Intervention \\
Intervention \\
Focus \\
Theoretical \\
Model \\
Delivery \\
Person \\
Intervention \\
Target \\
Recipient \\
Mode \\
Components \\
Frequency \\
Duration \\
\end{tabular} & \begin{tabular}{|l|} 
Comparison \\
Target \\
Mode \\
Components \\
Frequency \\
Duration
\end{tabular} & $\begin{array}{l}\text { Setting } \\
\text { and Design } \\
\text { Setting } \\
\text { Design } \\
\text { Cluster N } \\
\text { Participants } \\
\text { Randomized } \\
\text { N }\end{array}$ & \begin{tabular}{|l} 
PLWD \\
Dementia \\
Characteristics \\
Dementia Types \\
Dementia Severity \\
Diagnostic \\
Criteria \\
Age of Diagnosis
\end{tabular} & $\begin{array}{l}\text { PLWD } \\
\text { Non-Disease Char } \\
\text { PLWD N } \\
\text { PLWD Age (mean) } \\
\text { PLWD Sex (\% } \\
\text { female) } \\
\text { PLWD Race (\% } \\
\text { majority) } \\
\text { PLWD Education } \\
\text { (mean years) }\end{array}$ & $\begin{array}{l}\text { PLWD } \\
\text { Non-Disease } \\
\text { Char Reporting } \\
\text { Status (RS) } \\
\text { PLWD SES } \\
\text { PLWD Prior } \\
\text { Disability } \\
\text { PLWD } \\
\text { Household } \\
\text { Characteristics } \\
\text { PLWD Health } \\
\text { Insurance } \\
\text { PLWD Detailed } \\
\text { Race Information }\end{array}$ & $\begin{array}{l}\text { Informal Caregiver } \\
\text { (IC) } \\
\text { Characteristics } \\
\text { IC N } \\
\text { IC Age (mean) } \\
\text { IC Sex (\% female) } \\
\text { IC Race (\% } \\
\text { majority) } \\
\text { IC Education } \\
\text { (mean years) } \\
\text { IC Relation to } \\
\text { PLWD (\% majority) }\end{array}$ & $\begin{array}{l}\text { Informal Caregiver } \\
\text { (IC) Char. RS } \\
\text { IC Duration } \\
\text { IC Living With } \\
\text { PLWD } \\
\text { IC Payment } \\
\text { IC Health Status } \\
\text { IC Dementia } \\
\text { Family History } \\
\text { IC Employment } \\
\text { Status } \\
\text { IC Training }\end{array}$ & $\begin{array}{l}\text { Formal Caregiver } \\
\text { (FC) } \\
\text { Characteristics } \\
\text { FC N } \\
\text { FC Age (mean) } \\
\text { FC Sex (\% } \\
\text { female) } \\
\text { FC Race (\% } \\
\text { majority) } \\
\text { FC Education } \\
\text { (mean years) }\end{array}$ & $\begin{array}{l}\text { Formal } \\
\text { Caregiver (FC) } \\
\text { Char. RS } \\
\text { FC Health } \\
\text { Status } \\
\text { FC Training } \\
\text { FC Education } \\
\text { FC Position } \\
\text { FC Length of } \\
\text { Service }\end{array}$ & \begin{tabular}{|l|} 
Outcome \\
Timing(s)
\end{tabular} & \begin{tabular}{|l|l|} 
PLWD \\
Outcomes
\end{tabular} & $\begin{array}{l}\text { Caregiver } \\
\text { Outcomes } \\
\text { IC: } \\
\text { FC: }\end{array}$ \\
\hline
\end{tabular}




\begin{tabular}{|c|c|c|c|c|c|c|c|c|c|c|c|c|c|}
\hline $\begin{array}{l}\text { Teri 2003234 } \\
\text { (14559955) } \\
\text { US } \\
\text { Low } \\
\text { Explanatory }\end{array}$ & $\begin{array}{l}\text { Dyad, trained } \\
\text { home health } \\
\text { care facilitated } \\
12 \text {-hour training } \\
\text { sessions with } \\
\text { PLWD, PLWD } \\
\text { caregiver } \\
\text { exercise and } \\
\text { behavioral } \\
\text { management } \\
\text { techniques } \\
\text { (RDAD) (2 } \\
\text { sessions per } \\
\text { week for } 3 \\
\text { weeks, } 1 \\
\text { session per } \\
\text { week for } 4 \\
\text { weeks, followed } \\
\text { by biweekly } \\
\text { sessions for } 4 \\
\text { weeks.) } \\
12 \text { weeks }\end{array}$ & $\begin{array}{l}\text { Routine } \\
\text { medical care } \\
\text { in community. }\end{array}$ & $\begin{array}{l}\text { In-person, in- } \\
\text { home visits at } \\
\text { homes of } \\
\text { community } \\
\text { dwelling PLWD } \\
\text { and PLWD } \\
\text { caregivers. } \\
\text { RCT } \\
153 \text { PLWD }\end{array}$ & \begin{tabular}{l|} 
National Institute of \\
Neurological and \\
Communicative \\
Diseases and \\
Stroke/Alzheimer \\
Disease and \\
Related Disorders \\
Association criteria \\
for Alzheimer \\
disease. Dementia \\
diagnosis for an \\
average of 4.3 \\
years.
\end{tabular} & $\begin{array}{l}\mathrm{N}=153 \\
78 \text { years } \\
41 \% \text { female } \\
89 \% \text { white } \\
13 \text { years education }\end{array}$ & $\begin{array}{l}\text { Detailed Race } \\
\text { Information: yes }\end{array}$ & $\begin{array}{l}\mathrm{N}=153 \\
70 \text { years } \\
70 \% \text { female } \\
87 \% \text { white } \\
13 \text { years education } \\
80 \% \text { spouse }\end{array}$ & Living Status: yes & NA & NA & 12 weeks & $\begin{array}{l}\text { Physical role } \\
\text { functioning } \\
\text { scores (SF- } \\
\text { 36) } \\
\text { SIP Mobility } \\
\text { Cornell } \\
\text { Depression } \\
\text { in Dementia } \\
\text { Restricted } \\
\text { Activity } \\
\text { (number of } \\
\text { days } \\
\text { reported) }\end{array}$ & NA \\
\hline $\begin{array}{l}\text { Prick 2015 } \\
(26004290) \\
\\
(28120631)^{237} \\
(27099480)^{238} \\
(25336121)^{239} \\
\\
\text { Netherlands } \\
\text { Medium } \\
\text { Explanatory }\end{array}$ & $\begin{array}{l}\text { During three } \\
\text { months, a } \\
\text { personal coach } \\
\text { visited } \\
\text { PLWD/PLWD } \\
\text { caregiver dyads } \\
\text { for eight one- } \\
\text { hour-long home } \\
\text { visits. } \\
\text { The first month } \\
\text { the dyads were } \\
\text { visited weekly, } \\
\text { followed by } \\
\text { biweekly home } \\
\text { visists over the } \\
\text { next eight } \\
\text { weeks. } \\
12 \text { weeks }\end{array}$ & $\begin{array}{l}\text { Monthly } \\
\text { mailed } \\
\text { bulletins and } \\
\text { phone calls. }\end{array}$ & $\begin{array}{l}\text { Community- } \\
\text { dwelling PLWD } \\
\text { living with their } \\
\text { caregivers. } \\
\text { RCT } \\
111 \text { PLWD }\end{array}$ & $\begin{array}{l}\text { Diagnosis of } \\
\text { dementia, age } 55+ \\
\text { years, living at } \\
\text { home with } \\
\text { a caregiver, no use } \\
\text { of antidepressants, } \\
\text { no psychotic } \\
\text { symptoms, } \\
\text { MMSE score }<14 \text {, } \\
\text { receiving } \\
\text { more than two days } \\
\text { respite care in a } \\
\text { day care facility. }\end{array}$ & $\begin{array}{l}\mathrm{N}=111 \\
77 \text { years } \\
41 \% \text { Female } \\
\text { Race NR } \\
\text { Education } 4.01(0-6 \\
\text { scale) }\end{array}$ & None & $\begin{array}{l}\mathrm{N}=111 \\
72 \text { years } \\
72 \% \text { female } \\
\text { Education } 4.08 \text { (0-6 } \\
\text { scale) } \\
90.1 \% \text { spouse }\end{array}$ & $\begin{array}{l}\text { Living with PLWD: } \\
\text { yes }\end{array}$ & NA & NA & 12 weeks & NA & $\begin{array}{l}\text { Family } \\
\text { caregiver } \\
\text { mood } \\
\text { Self- } \\
\text { Perceived } \\
\text { Pressure from } \\
\text { Family Care } \\
\text { (Dutch } \\
\text { version) } \\
\text { RMBPC } \\
\text { (Dutch } \\
\text { version) }\end{array}$ \\
\hline $\begin{array}{l}\text { Belle 2006 } \\
(17116917) \\
(20122038)^{230} \\
(28295134)^{241} \\
(21357811)^{242} \\
243 \\
(24652899)^{244} \\
(20056684)^{245} \\
(27294833)^{246} \\
(23983230)^{247} \\
(16920998)^{248} \\
(14518801)^{249} \\
\text { US } \\
\text { Medium } \\
\text { Explanatory }\end{array}$ & $\begin{array}{l}12 \text { in-home and } \\
\text { telephone } \\
\text { Structured } \\
\text { sessions } \\
\text { involving } \\
\text { preliminary talk, } \\
\text { information } \\
\text { booklet, } \\
\text { structured } \\
\text { telephone- } \\
\text { based support } \\
\text { groups, and } \\
\text { structured } \\
\text { evaluation of } \\
\text { each support } \\
\text { group session. } \\
6 \text { months }\end{array}$ & $\begin{array}{l}\text { Mailed packet } \\
\text { of educational } \\
\text { materials and } \\
\text { provided 2 } \\
\text { brief (<15 } \\
\text { minute) } \\
\text { telephone } \\
\text { "check-in" } \\
\text { calls at } 3 \\
\text { months and } 5 \\
\text { months atter } \\
\text { randomization } \\
6 \text { months }\end{array}$ & $\begin{array}{l}\text { PLWD } \\
\text { caregiver living } \\
\text { with } \\
\text { or sharing } \\
\text { cooking } \\
\text { facilities with } \\
\text { PLWD relative } \\
\text { with diagnosed } \\
\text { Alzheimer } \\
\text { disease or } \\
\text { related } \\
\text { disorders } \\
\text { providing care } \\
\text { least } 4 \text { hours } \\
\text { per day for at } \\
\text { least the past } 6 \\
\text { months. } \\
\text { RCT } \\
642 \text { PLWD }\end{array}$ & $\begin{array}{l}\text { Alzheimer disease } \\
\text { or related } \\
\text { disorders. } \\
\text { MMSE }>0\end{array}$ & $\begin{array}{l}\mathrm{N}=642 \\
77 \text { years } \\
60 \% \text { female } \\
\text { Education }<12 \\
\text { years }\end{array}$ & $\begin{array}{l}\text { SES: yes } \\
\text { Prior disability: yes } \\
\text { Household } \\
\text { characteristics: } \\
\text { yes } \\
\text { Detailed race: yes }\end{array}$ & $\begin{array}{l}\mathrm{N}=642 \\
60 \text { years } \\
83 \% \text { female } \\
35 \% \text { white } \\
\text { Education } 57 \%>12 \\
\text { years } \\
43 \% \text { spouse }\end{array}$ & $\begin{array}{l}\text { Duration: yes } \\
\text { Living with PLWD: } \\
\text { yes } \\
\text { Employment status: } \\
\text { yes }\end{array}$ & NA & NA & 6 months & & $\begin{array}{l}\text { Prevalence of } \\
\text { clinical } \\
\text { depression } \\
\text { for caregivers } \\
\text { (CES-D score } \\
\geq 15 \text { ) }\end{array}$ \\
\hline
\end{tabular}




\begin{tabular}{|c|c|c|c|c|c|c|c|c|c|c|c|c|c|}
\hline $\begin{array}{l}\text { Berwig 2017222 } \\
\text { (NCT01690117) } \\
\text { Germany } \\
\text { Medium } \\
\text { Explanatory }\end{array}$ & $\begin{array}{l}12 \text { individual } \\
\text { two-weekly } \\
\text { sessions } 9 \text { at } \\
\text { home with the } \\
\text { informal } \\
\text { caregiver and } 3 \\
\text { via telephone) } \\
\text { and combined } \\
\text { five modules. } \\
6 \text { months. }\end{array}$ & $\begin{array}{l}\text { Mailed packet } \\
\text { of educational } \\
\text { materials and } \\
\text { provided 2 } \\
\text { brief }(<15 \\
\text { minute) } \\
\text { telephone } \\
\text { "check-in" } \\
\text { calls at } 3 \\
\text { months and } 5 \\
\text { months after } \\
\text { randomization } \\
6 \text { months }\end{array}$ & $\begin{array}{l}\text { Community/in- } \\
\text { home dwelling } \\
\text { PLWD and } \\
\text { caregivers. } \\
\text { RCT } \\
\text { 92 PLWD }\end{array}$ & $\begin{array}{l}\text { AD or related } \\
\text { disorders. } \\
\text { MMSE }>0\end{array}$ & $\begin{array}{l}\mathrm{N}=92 \\
73 \text { years } \\
72 \% \text { female } \\
\text { Race NR } \\
\text { Education NR }\end{array}$ & $\mathrm{NR}$ & $\begin{array}{l}\text { Education } 13 \text { years } \\
89 \% \text { spouse }\end{array}$ & 92 living with PLWD & NA & NA & 6 months & NA & $\begin{array}{l}\text { Benefit } \\
\text { caregiver } \\
\text { depression } \\
\text { (PHQ4) } \\
\text { Benefit quality } \\
\text { of life (SF-12) }\end{array}$ \\
\hline $\begin{array}{l}\text { Mittelman } \\
2004^{232} \\
(14722336)^{2} \\
\left(151216500^{250}\right. \\
(17101889)^{251} \\
\left(178048311^{252}\right. \\
(166166466)^{253} \\
(191392477)^{254} \\
(16420138)^{255} \\
(181794955)^{256} \\
(21543959)^{257} \\
(15197285)^{258} \\
\text { US } \\
\text { Medium } \\
\text { Explanatory }\end{array}$ & $\begin{array}{l}\text { Counseling } \\
\text { sessions (in- } \\
\text { person, family, } \\
\text { and telephone) } \\
\text { for caregivers } \\
\text { vs. usual care } \\
\text { with home- } \\
\text { based phone } \\
\text { interviews; } \\
\text { Pearlin stress } \\
\text { process model }\end{array}$ & $\begin{array}{l}\text { Usual care for } \\
4 \text { months }\end{array}$ & $\begin{array}{l}\text { Spouse } \\
\text { caregivers } \\
\text { living at home } \\
\text { with patient } \\
\text { RCT } \\
371 \text { PLWD }\end{array}$ & $\begin{array}{l}\text { AD or related } \\
\text { disorders. }\end{array}$ & $\begin{array}{l}\text { N=371 } \\
\text { Age: NR } \\
90 \% \text { White } \\
\text { Sex NR } \\
\text { Education :NR }\end{array}$ & $\begin{array}{l}\text { Detailed race } \\
\text { information: yes }\end{array}$ & $\begin{array}{l}\mathrm{N}=371 \\
71 \text { years } \\
60 \% \text { female } \\
90 \% \text { white }\end{array}$ & $\begin{array}{l}371 \text { spouse } \\
\text { caregivers living at } \\
\text { home with patient. }\end{array}$ & NA & NA & 4 months & NA & $\begin{array}{l}\text { Benefit } \\
\text { caregiver } \\
\text { stress } \\
\text { perceptions } \\
\text { Benefit } \\
\text { caregiver } \\
\text { depression }\end{array}$ \\
\hline $\begin{array}{l}\text { Mittelman } \\
2008^{229} \\
(18978250) \\
(21071943)^{259} \\
(19705599)^{260} \\
\\
\text { Multinational } \\
\text { Medium } \\
\text { Explanatory }\end{array}$ & $\begin{array}{l}\text { Counseling } \\
\text { sessions (in- } \\
\text { person, family, } \\
\text { and telephone) } \\
\text { for caregivers } \\
\text { vs. usual care; } \\
\text { five sessions } \\
\text { individual and } \\
\text { family } \\
\text { counselling w/in } \\
\text { first } 3 \text { months, } \\
\text { continuous ad } \\
\text { hoc telephone } \\
\text { counselling } \\
\text { Donepezil for all } \\
\text { patients } \\
\text { Pearlin stress } \\
\text { process model }\end{array}$ & No control. & $\begin{array}{l}\text { Community- } \\
\text { based spouse } \\
\text { caregivers } \\
\text { living at home } \\
\text { with patient } \\
\text { RCT } \\
158 \text { PLWD }\end{array}$ & $\begin{array}{l}\text { AD or related } \\
\text { disorders. mild to } \\
\text { moderate dementia } \\
\text { at enrollment }\end{array}$ & $\begin{array}{l}\mathrm{N}=158 \\
\text { Age: NR } \\
\text { Sex NR } \\
\text { Race NR } \\
\text { Education NR }\end{array}$ & None & $\begin{array}{l}\mathrm{N}=158 \\
\text { Age: NR } \\
\text { Sex NR } \\
\text { Race NR } \\
\text { Education NR }\end{array}$ & None & NA & NA & 6 months & NA & $\begin{array}{l}\text { Depressive } \\
\text { symptoms } \\
\text { Beck } \\
\text { Depression } \\
\text { Inventory } \\
\text { (revised). }\end{array}$ \\
\hline $\begin{array}{l}\text { Luchsinger } \\
2018^{221} \\
\text { (14722336) } \\
\text { US } \\
\text { Medium } \\
\text { Explanatory }\end{array}$ & $\begin{array}{l}\text { Counseling } \\
\text { sessions (in- } \\
\text { person, family, } \\
\text { and telephone) } \\
\text { for caregivers } \\
\text { vs. usual care } \\
6 \text { planned visits } \\
\text { of similar } \\
\text { duration in } 6 \\
\text { months and ad } \\
\text { hoc contacts. } \\
\text { Pearlin stress } \\
\text { process model }\end{array}$ & No control. & $\begin{array}{l}\text { Community- } \\
\text { based spouse } \\
\text { caregivers } \\
\text { living at home } \\
\text { with patient } \\
\text { RCT } \\
221 \text { PLWD }\end{array}$ & $\begin{array}{l}\text { AD or related } \\
\text { disorders. }\end{array}$ & $\begin{array}{l}\mathrm{N}=221 \\
58 \text { years } \\
82 \% \text { female } \\
\text { Race NR } \\
\text { Education NR }\end{array}$ & None & $\begin{array}{l}\mathrm{N}=221 \\
58 \text { years } \\
32 \% \text { spouses. } \\
\text { Race NR } \\
\text { Education } 12 \text { years }\end{array}$ & $\begin{array}{l}\text { Employment Status: } \\
\text { yes }\end{array}$ & NA & NA & 4 months & NA & $\begin{array}{l}\text { Depressive } \\
\text { symptoms, } \\
\text { GDS } \\
\text { Caregiver } \\
\text { burden } \\
\text { ZBI }\end{array}$ \\
\hline
\end{tabular}


* High risk of bias studies included in evidence map. This table includes low and medium risk of bias studies only.

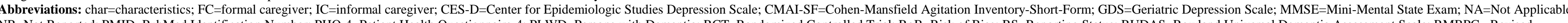
isk of Bias; RS=Reporting Status; RUDAS=Rowland Universal Dementia Assessment Scale; RMBPC= Revised 作

Table E-23. Caregiver outcomes summary low and medium risk of bias studies: multicomponent interventions for informal caregivers

\begin{tabular}{|c|c|c|c|c|c|}
\hline $\begin{array}{l}\text { Study (PMID) } \\
\text { Comparison } \\
\text { RoB } \\
\text { Category* }\end{array}$ & $\begin{array}{l}\text { Outcome } \\
\text { Timing }\end{array}$ & Summary Finding & Intervention & Comparator & p-Value \\
\hline $\begin{array}{l}\text { Belle 2006 } 230 \\
\text { (17116917) } \\
12 \text { in-home and telephone } \\
\text { Structured sessions } \\
\text { involving preliminary talk, information } \\
\text { booklet, structured telephone-based } \\
\text { support groups, and structured evaluation } \\
\text { of each support group session vs Mailed } \\
\text { packet of educational materials and } \\
\text { provided } 2 \text { brief (<15 minute) telephone } \\
\text { "check-in" calls at } 3 \text { months and } 5 \text { months } \\
\text { atter randomization } \\
\text { Medium } \\
\text { Explanatory }\end{array}$ & $\begin{array}{l}\text { Prevalence of clinical depression for caregivers } \\
\text { (CES-D score } \geq 15) \\
\mathrm{n} / \mathrm{N}(\%) \\
6 \text { months }\end{array}$ & $\begin{array}{l}\text { Overall prevalence of clinical depression at } 6 \text { month follow-up was significantly greater } \\
\text { among caregivers in the control group than among those in the intervention group }\end{array}$ & $12.6 \%$ & $22.7 \%$ & 0.001 \\
\hline $\begin{array}{l}\text { Belle } 2006224 \\
(17116917) \\
12 \text { in-home and telephone } \\
\text { Structured sessions } \\
\text { involving preliminary talk, information } \\
\text { booklet, structured telephone-based } \\
\text { support groups, and structured evaluation } \\
\text { of each support group session vs Mailed } \\
\text { packet of educational materials and } \\
\text { provided } 2 \text { brief (<15 minute) telephone } \\
\text { "check-in" calls at } 3 \text { months and } 5 \text { months } \\
\text { after randomization } \\
\text { Medium } \\
\text { Explanatory }\end{array}$ & $\begin{array}{l}\text { Burden (modified Zarit Burden) } \\
\text { Mean (Cl) } \\
6 \text { months }\end{array}$ & No difference & $3(-15,21)$ & $4.2(-16.9,25.7)$ & NR \\
\hline $\begin{array}{l}\text { Berwig } 2017 \text { 222 } \\
\text { (NCTO1690117) } \\
\text { homdividual two-weekly sessions ( } 9 \text { at } \\
\text { home with the informal caregiver and } 3 \\
\text { via telephonon) and combined five } \\
\text { modules vs. Mailed packet of educational } \\
\text { materials and provided } 2 \text { brief (<15 } \\
\text { minute) telephone "check-in" calls at } 3 \\
\text { months and } 5 \text { months after randomization } \\
\text { Medium } \\
\text { Explanatory }\end{array}$ & $\begin{array}{l}\text { ZBI } \\
\text { Mean change from baseline (SD) } \\
9 \text { months }\end{array}$ & $\begin{array}{l}\text { Cohen's } \mathrm{d}=0.623 \text {. Informal caregivers' burden increased in both groups, but much more } \\
\text { strongly in the control group }\end{array}$ & $2.67(8.86)$ & $8.10(8.58)$ & 0.017 \\
\hline $\begin{array}{l}\text { Berwig } 2017 \text { 222 } \\
\text { (NCTO1690117) } \\
\text { home wividual two-weekly sessions ( } 9 \text { at } \\
\text { homformal caregiver and } 3 \\
\text { vo telephonoe) and combined five } \\
\text { modules vs. Mailed packet of educational } \\
\text { materials and provided } 2 \text { brief (<15 } \\
\text { minute) telephone "check-in" calls at } 3 \\
\text { months and } 5 \text { months after randomization } \\
\text { Medium } \\
\text { Explanatory }\end{array}$ & $\begin{array}{l}\text { PHQ-15 } \\
\text { Mean change from baseline (SD) } \\
9 \text { months }\end{array}$ & Cohen's $d=0.502$ & $-0.601(3.999)$ & $1.098(2.773)$ & 0.057 \\
\hline
\end{tabular}




\begin{tabular}{|c|c|c|c|c|c|}
\hline $\begin{array}{l}\text { Berwig } 2017222 \\
\text { (NCTO160117) } \\
12 \text { individual two-weekly sessions ( } 9 \text { at } \\
\text { home with the informal caregiver and } 3 \\
\text { via telephone) and combined five } \\
\text { modules vs. Mailed packet of educational } \\
\text { materials and provided } 2 \text { brief ( (<15 } \\
\text { minute) telephone "check-in" calls at } 3 \\
\text { months and } 5 \text { months after randomization } \\
\text { Medium } \\
\text { Explanatory }\end{array}$ & $\begin{array}{l}\text { SF-12 Psychological } \\
\text { Mean change from baseline (SD) } \\
9 \text { months }\end{array}$ & Cohen's d=0.902 & $3.868(10.662)$ & $-4.618(8.157)$ & 0.001 \\
\hline $\begin{array}{l}\text { Luchsinger 2018221 (14722336) } \\
\text { NYUCl vs. REACH-OUT } \\
\text { Medium } \\
\text { Explanatory }\end{array}$ & $\begin{array}{l}\text { GDS } \\
\text { Mean (SD) } \\
6 \text { months }\end{array}$ & $\begin{array}{l}\text { There was no difference between groups } \\
\text { There was no significant change from baseline in either group }\end{array}$ & $\begin{array}{l}\text { REACH-OUT } \\
9.8(7.2)\end{array}$ & $\begin{array}{l}\mathrm{NYUCl} \\
9.6(7.1)\end{array}$ & NR \\
\hline $\begin{array}{l}\text { Luchsinger 2018221(14722336) } \\
\text { NYUCI vs. REACH-OUT } \\
\text { Medium } \\
\text { Explanatory }\end{array}$ & $\begin{array}{l}\text { ZCBS } \\
\text { Mean (SD) } \\
6 \text { months }\end{array}$ & $\begin{array}{l}\text { There was no difference between groups } \\
\text { There was a decrease in the ZCBS for } \\
\text { REACH-OUT ( } 5.2 \text { points }(2.2-8.1), p<.001) \text { and the NYUCI (4.6 points, }(1.7-7.5 \text {, } \\
\text { p=.002). }\end{array}$ & $\begin{array}{l}\text { REACH-OUT } \\
35.5(18.0)\end{array}$ & $\begin{array}{l}\mathrm{NYUCl} \\
36.2(15.9)\end{array}$ & NR \\
\hline $\begin{array}{l}\text { Reri } 2003234 \text { (14559955) } \\
\text { Reducing Disability in Alzheimer Disease } \\
\text { (RDAD) vs. Routine Medical Care (RMC) } \\
\text { Low } \\
\text { Pragmatic }\end{array}$ & $\begin{array}{l}\text { SF-36 } \\
\text { Mean (SD) } \\
12 \text { weeks }\end{array}$ & Significant difference between groups & $72.1(33.0)$ & $50.7(39.1)$ & $<0.001$ \\
\hline $\begin{array}{l}\text { Teri } 2003234 \text { (14559955) } \\
\text { Reducing Disability in Alzheimer Disease } \\
\text { (RDAD) vs. Routine Medical Care (RMC) } \\
\text { Low } \\
\text { Explanatory }\end{array}$ & $\begin{array}{l}\text { SIP Mobility } \\
\text { Mean (SD) } \\
12 \text { weeks }\end{array}$ & No significant difference between groups & $16.0(17.1)$ & $15.2(17.1)$ & 0.17 \\
\hline $\begin{array}{l}\text { Teri } 2003234 \text { (14559955) } \\
\text { Reducing Disability in Alzheimer Disease } \\
\text { (RDAD) vs. Routine Medical Care (RMC) } \\
\text { Low } \\
\text { Explanatory }\end{array}$ & $\begin{array}{l}\text { Cornell Depression in Dementia Scale } \\
\text { Mean (SD) } \\
12 \text { weeks }\end{array}$ & Significant difference between groups & $5.2(3.6)$ & $6.2(3.8)$ & 0.02 \\
\hline $\begin{array}{l}\text { Teri } 20033^{234}(14559955) \\
\text { Reducing Disability in Alzheimer Disease } \\
\text { (RDAD) vs. Routine Medical Care (RMC) } \\
\text { Low } \\
\text { Explanatory }\end{array}$ & $\begin{array}{l}\text { Restricted Activity (number of days reported) } \\
\text { Mean (SD) } \\
12 \text { weeks }\end{array}$ & Significant difference between groups & $0.1(0.4)$ & $0.6(2.5)$ & $<0.001$ \\
\hline $\begin{array}{l}\text { Prick } 2015226 \text { (26004290) } \\
\text { During three months, a personal coach } \\
\text { visited PLWD/PLWD caregiver dyads for } \\
\text { eight one-hourlong home visits. } \\
\text { The first month the dyads were visited } \\
\text { weekly, followed by biweekly home visits } \\
\text { over the next eight weeks vs. Monthlyly } \\
\text { mailed bulletins and phone calls } \\
\text { Medium } \\
\text { Explanatory }\end{array}$ & $\begin{array}{l}\text { Family caregiver mood (Dutch version CES-D) } \\
\text { Mean (SD) } \\
12 \text { weeks }\end{array}$ & All analyses showed no benefits of the intervention over time on any of the outcomes & $13.71(8.18)$ & $10.94(8.42)$ & 0.08 \\
\hline $\begin{array}{l}\text { Prick } 2015^{226}(26004290) \\
\text { During three months, a personal coach } \\
\text { visited PLWD/PLWD caregiver dyads for } \\
\text { eight one-hour-long home visits. } \\
\text { The first month the dyads were visited } \\
\text { weekly, followed by biweekly home visits } \\
\text { over the next eight weeks vs. Monthly } \\
\text { mailed bulletins and phone calls } \\
\text { Medium } \\
\text { Explanatory }\end{array}$ & $\begin{array}{l}\text { Dutch Self-Perceived Pressure from Family } \\
\text { Care (SPICC) } \\
12 \text { weeks }\end{array}$ & All analyses showed no benefits of the intervention over time on any of the outcomes & $5.67(2.36)$ & $5.85(2.13)$ & 0.45 \\
\hline
\end{tabular}




\begin{tabular}{|c|c|c|c|c|c|}
\hline $\begin{array}{l}\text { Prick } 20155^{226} \text { (26004290) } \\
\text { During three months, a personal coach } \\
\text { visited PLWDDPLWD caregiver dyads for } \\
\text { eight one-hour-long home visits. } \\
\text { The first month the dyads were visited } \\
\text { weekly, followed by biweekly home visits } \\
\text { over the next eight weeks vs. Monthly } \\
\text { mailed bulletins and phone calls } \\
\text { Medium } \\
\text { Explanatory }\end{array}$ & $\begin{array}{l}\text { Dutch version of Revised Memory and Behavior } \\
\text { Problem Checklist (RMBPC) } \\
12 \text { weeks }\end{array}$ & All analyses showed no benefits of the intervention over time on any of the outcomes & $13.06(10.38)$ & $12.13(8.55)$ & 0.82 \\
\hline $\begin{array}{l}\text { Mittelman } 20088^{229} \\
\text { (18978250) } \\
\text { Five sessions of individual and family } \\
\text { counselling within } 3 \text { months of enrollment } \\
\text { and continuous availability of ad hoc } \\
\text { telephone counselling and drug treatment } \\
\text { vs. drug alone. } \\
\text { Medium } \\
\text { Explanatory }\end{array}$ & $\begin{array}{l}\text { Beck Depression Inventory (BDI) (revised) } \\
\text { Regression coefficients } \\
6 \text { months }\end{array}$ & $\begin{array}{l}\text { The predicted BDI scores decreased for treatment caregivers and increased for control } \\
\text { caregivers. }\end{array}$ & NR & NR & NR \\
\hline $\begin{array}{l}\text { Mittelman } 2004232 \text { (14722336) } \\
\text { Counseling sessions (in-person, family, } \\
\text { and telephone) for caregivers vs. usual } \\
\text { care } \\
\text { Medium } \\
\text { Explanatory }\end{array}$ & $\begin{array}{l}\text { MBPC } \\
\text { Regression coefficients } \\
4 \text { months }\end{array}$ & $\begin{array}{l}\text { Intervention did not affect the frequency of patient behavioral problems, it did } \\
\text { significantly reduce caregivers' reaction ratings }\end{array}$ & NR & NR & NR \\
\hline
\end{tabular}

"Explanatory studies are listed first, followed by pragmatic studies.

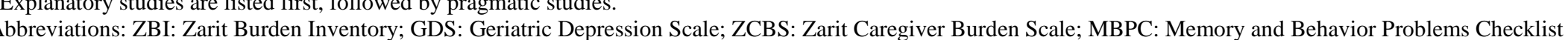

Table E-24. Summary of strength of evidence for caregiver outcomes: multicomponent interventions for informal caregivers

\begin{tabular}{|c|c|c|c|c|c|c|c|c|}
\hline $\begin{array}{l}\text { Comparison } \\
\text { Outcome }\end{array}$ & Timing & $\begin{array}{l}\text { \# Studies/ Design } \\
\text { ( } \mathrm{n} \text { analyzed) }\end{array}$ & $\begin{array}{l}\text { Finding or Summary } \\
\text { Statistic }\end{array}$ & Study Limitations & Consistency & Directness & Precision & Overall Grade/ Conclusion \\
\hline $\begin{array}{l}\text { Health in PLWD and informal caregiver } \\
\text { Combined exercise \& support vs. monthly phone calls \& } \\
\text { mailed bulletins }\end{array}$ & 12 weeks & $\begin{array}{l}1 \mathrm{RCT} \\
(\mathrm{n}=153)\end{array}$ & 1 showed benefit & Medium & Inconsistent & Direct & Imprecise & Insufficient \\
\hline $\begin{array}{l}\text { Depression } \\
\text { Counseling sessions (in-person, family, and phone) for } \\
\text { caregivers vs. usual care }\end{array}$ & 4-6 months & $\begin{array}{l}1 \text { RCT } \\
(n=371\end{array}$ & 1 showed benefit & Medium & Unknown & Direct & Imprecise & Insufficient \\
\hline $\begin{array}{l}\text { Caregiver stress perception } \\
\text { Counseling sessions (in-person, family, and phone) for } \\
\text { caregivers vs. usual care }\end{array}$ & 4-6 months & $\begin{array}{l}1 \mathrm{RCT} \\
(\mathrm{n}=158)\end{array}$ & 1 showed benefit & Medium & Unknown & Direct & Imprecise & Insufficient \\
\hline $\begin{array}{l}\text { Depression } \\
\text { Education, group discussion, in-home and phone } \\
\text { support sessions, and caregiver feedback vs usual } \\
\text { care }\end{array}$ & 6 months & $\begin{array}{l}3 \mathrm{RCT} \\
(\mathrm{n}=895)\end{array}$ & $\begin{array}{l}2 \text { showed benefit } \\
1 \text { showed no difference }\end{array}$ & Medium & Consistent & Direct & Imprecise & $\begin{array}{l}\text { Low } \\
\text { REACH II }\end{array}$ \\
\hline $\begin{array}{l}\text { Caregiver stress, burden } \\
\text { Education, group discussion, in-home and phone } \\
\text { support sessions, and caregiver feedback vs usual } \\
\text { care }\end{array}$ & 6 months & $\begin{array}{l}\text { 3 RCT } \\
(\mathrm{n}=895)\end{array}$ & $\begin{array}{l}1 \text { showed benefit } \\
2 \text { showed no difference }\end{array}$ & Medium & Inconsistent & Direct & Imprecise & Insufficient \\
\hline
\end{tabular}




\section{Evidence Map: Multicomponent Interventions for Informal Caregivers}

\begin{tabular}{|c|c|c|c|c|c|c|c|c|c|c|c|c|c|}
\hline $\begin{array}{l}\text { Study (PMID) } \\
\text { Country } \\
\text { EM Reason }\end{array}$ & Intervention & Comparison & $\begin{array}{l}\text { Setting } \\
\text { and Design } \\
\text { Setting } \\
\text { Design } \\
\text { Cluster N } \\
\text { Participants } \\
\text { Random- } \\
\text { ized N }\end{array}$ & $\begin{array}{l}\text { PLWD } \\
\text { Dementia } \\
\text { Characteristics }\end{array}$ & $\begin{array}{l}\text { PLWD } \\
\text { Non-Disease Char } \\
\text { PLWD N } \\
\text { PLWD Age (mean) } \\
\text { PLWD Sex (\% } \\
\text { female) } \\
\text { PLWD Race (\% } \\
\text { majority) } \\
\text { PLWD Education } \\
\text { (mean years) }\end{array}$ & $\begin{array}{l}\text { PLWD } \\
\text { Non-Disease } \\
\text { Char Reporting } \\
\text { Status (RS) } \\
\text { PLWD SES } \\
\text { PLWD Prior } \\
\text { Disability } \\
\text { PLWD } \\
\text { Household } \\
\text { Characteristics } \\
\text { PLWD Health } \\
\text { Insurance } \\
\text { PLWD Detailed } \\
\text { Race Information }\end{array}$ & $\begin{array}{l}\text { Informal Caregiver } \\
\text { (IC) } \\
\text { Characteristics } \\
\text { IC N } \\
\text { IC Age (mean) } \\
\text { IC Sex (\% female) } \\
\text { IC Race (\% } \\
\text { majority) } \\
\text { IC Education } \\
\text { (mean years) } \\
\text { IC Relation to } \\
\text { PLWD (\% majority) }\end{array}$ & $\begin{array}{l}\text { Informal Caregiver } \\
\text { (IC) Char. RS } \\
\text { IC Duration } \\
\text { IC Living With } \\
\text { PLWD } \\
\text { IC Payment } \\
\text { IC Health Status } \\
\text { IC Dementia } \\
\text { Family History } \\
\text { IC Employment } \\
\text { Status } \\
\text { IC Training }\end{array}$ & $\begin{array}{l}\text { Formal Caregiver } \\
\text { (FC) } \\
\text { Characteristics } \\
\text { FC N } \\
\text { FC Age (mean) } \\
\text { FC Sex (\% } \\
\text { female) } \\
\text { FC Race (\% } \\
\text { majority) } \\
\text { FC Education } \\
\text { (mean years) }\end{array}$ & $\begin{array}{l}\text { Formal } \\
\text { Caregiver (FC) } \\
\text { Char. RS } \\
\text { FC Health } \\
\text { Status } \\
\text { FC Training } \\
\text { FC Education } \\
\text { FC Position } \\
\text { FC Length of } \\
\text { Service }\end{array}$ & $\begin{array}{l}\text { Outcome } \\
\text { Timing(s) }\end{array}$ & $\begin{array}{l}\text { PLWD } \\
\text { Outcomes }\end{array}$ & $\begin{array}{l}\text { Caregiver } \\
\text { Outcomes } \\
\text { IC: } \\
\text { FC: }\end{array}$ \\
\hline $\begin{array}{l}\text { Mazurek, } \\
2019^{261} \\
\text { (30666097) } \\
\text { Poland } \\
\text { Small sample }\end{array}$ & $\begin{array}{l}\text { Meeting } \\
\text { Centres Support } \\
\text { Programme } \\
\text { (MCSP) } \\
\text { Integrated } \\
\text { group support } \\
\text { activities } \\
\text { (cognitive } \\
\text { stimulation, } \\
\text { activity groups, } \\
\text { music therapy, } \\
\text { psychomotor } \\
\text { therapy, family } \\
\text { support groups, } \\
\text { psychoeducatio } \\
\text { n, counseling) }\end{array}$ & Usual care & $\begin{array}{l}\text { Persons with } \\
\text { mild-to-- } \\
\text { moderately } \\
\text { severe } \\
\text { dementia, } \\
\text { living at } \\
\text { home, and a } \\
\text { with a } \\
\text { caregiver } \\
\text { prepared to } \\
\text { participate }\end{array}$ & $\begin{array}{l}\text { Mild-to-moderate } \\
\text { dementia (Global } \\
\text { Deterioration Scale } \\
\text { / GDS) }\end{array}$ & $\begin{array}{l}\mathrm{N}=42 \\
\text { Age: yes } \\
\text { Sex: yes } \\
\text { Race: no } \\
\text { Education: yes }\end{array}$ & $\begin{array}{l}\text { Household } \\
\text { characteristics }\end{array}$ & $\begin{array}{l}\mathrm{N}=42 \\
\text { Age: yes } \\
\text { Sex: yes } \\
\text { Race: yes } \\
\text { Education: yes }\end{array}$ & NR & NA & NA & 6 months & $\mathrm{NR}$ & $\begin{array}{l}\text { caregiver } \\
\text { unmet } \\
\text { needs }\end{array}$ \\
\hline $\begin{array}{l}\text { Zhang, 20199262 } \\
\text { (31466807) } \\
\text { China } \\
\text { Pilot }\end{array}$ & $\begin{array}{l}6 \text { bi-weekly self- } \\
\text { management } \\
\text { support } \\
\text { sessions; } \\
\text { education, }\end{array}$ & $\begin{array}{l}\text { Education } \\
\text { material }\end{array}$ & $\begin{array}{l}\text { Community- } \\
\text { based } \\
\text { Quasi- } \\
\text { experimental } \\
\text { Caregiver }\end{array}$ & $\begin{array}{l}85 \% \text { AD, } 15 \% \\
\text { other subtypes }\end{array}$ & $\begin{array}{l}\mathrm{N}=41 \\
\text { Age: yes } \\
\text { Sex: yes } \\
\text { Race: no } \\
\text { Education: yes }\end{array}$ & None & $\begin{array}{l}\mathrm{N}=41 \\
\text { Age: yes } \\
\text { Sex: yes } \\
\text { Race: no } \\
\text { Education: yes } \\
\text { Relationship:yes }\end{array}$ & $\begin{array}{l}\text { Health status } \\
\text { Employment }\end{array}$ & NA & NA & 36 weeks & $\begin{array}{l}\mathrm{NPI} \\
\text { IADL } \\
\text { DAD }\end{array}$ & $\begin{array}{l}\text { SF-36 } \\
\text { Self-efficacy }\end{array}$ \\
\hline $\begin{array}{l}\text { Brijoux 2016.23 } \\
(27839534) \\
\text { Germany } \\
\text { High RoB }\end{array}$ & $\begin{array}{l}\text { Qualified family } \\
\text { companions } \\
\text { provided } \\
\text { support to the } \\
\text { caregiver }\end{array}$ & $\begin{array}{l}\text { Caregivers were } \\
\text { supported by } \\
\text { conventional } \\
\text { care } \\
\text { companions }\end{array}$ & $\begin{array}{l}\text { Community- } \\
\text { dwelling } \\
\text { RCT } \\
76 \text { families }\end{array}$ & $\begin{array}{l}\text { Clinical diagnosis } \\
\text { of dementia (all } \\
\text { severity) }\end{array}$ & $\begin{array}{l}\mathrm{N}=73 \\
\text { Age: yes } \\
\text { Sex: yes } \\
\text { Race: no } \\
\text { Education: no }\end{array}$ & None & $\begin{array}{l}\text { N=73 } \\
\text { Age: yes } \\
\text { Sex: yes } \\
\text { Race: no } \\
\text { Education: no } \\
\text { Relationship: yes }\end{array}$ & NR & NA & NA & 16 weeks & NR & $\begin{array}{l}\mathrm{HRQOL} \\
\text { Burden } \\
\text { reduction } \\
\text { Better } \\
\text { networking } \\
\text { to get } \\
\text { support }\end{array}$ \\
\hline $\begin{array}{l}\text { Koivisto 2016 } \\
\text { (26177825) } \\
\text { Finland }\end{array}$ & $\begin{array}{l}\text { Psychosocial } \\
\text { interventions, } \\
\text { including } \\
\text { education, } \\
\text { counseling and } \\
\text { social support }\end{array}$ & $\begin{array}{l}\text { Annual follow- } \\
\text { up }\end{array}$ & $\begin{array}{l}\text { Community- } \\
\text { based }-3 \\
\text { clinics } \\
\text { RCT }\end{array}$ & $\begin{array}{l}\text { Patients diagnosed } \\
\text { with mild AD } \\
\text { CDR of } 0.5 \text { or } 1.0 \\
\text { Free of interfering } \\
\text { comorbidities } \\
\text { Family caregiver } \\
\text { present }\end{array}$ & $\begin{array}{l}\mathrm{N}=54 \text { Intervention } \\
\text { group } \\
76 \text { Control group } \\
\text { Age: yes } \\
\text { Sex: yes } \\
\text { Education: yes } \\
\text { Race \% majority: no }\end{array}$ & Comorbidities: Y & $\begin{array}{l}\text { N=54 Intervention } \\
\text { group } \\
76 \text { Control group } \\
\text { Age: yes } \\
\text { Sex: yes } \\
\text { Education: yes } \\
\text { Race \% majority: no } \\
\text { Relation to PLWD: } \\
\text { yes }\end{array}$ & Employment: & NA & NA & $\begin{array}{l}2 \text { years } \\
36 \text { months of } \\
\text { follow-up }\end{array}$ & $\begin{array}{l}\text { Admission to } \\
\text { nursing home } \\
\text { CDR-SOB } \\
\text { CERAD-NB } \\
\text { MMSE } \\
\text { NPI } \\
\text { BDI } \\
\text { QoL }\end{array}$ & $\begin{array}{l}\mathrm{QOL} \\
\text { GHQ } \\
\text { Sense of } \\
\text { coherence } \\
\text { BDI }\end{array}$ \\
\hline
\end{tabular}




\begin{tabular}{|c|c|c|c|c|c|c|c|c|c|c|c|c|c|}
\hline $\begin{array}{l}\text { Baglio, 2015225 } \\
(24788581) \\
\text { Italy } \\
\text { High RoB }\end{array}$ & $\begin{array}{l}\text { Multidimensiona } \\
\text { I Stimulation } \\
\text { group Therapy } \\
\text { (MST) to } \\
\text { improve } \\
\text { cognition, } \\
\text { behavior, and } \\
\text { motor } \\
\text { functioning in } \\
\text { PWA with } \\
\text { informal } \\
\text { caregivers. }\end{array}$ & No comparison & $\begin{array}{l}\text { Memory } \\
\text { Clinic } \\
\text { Outpatients } \\
\text { RCT } \\
\text { PLWD }\end{array}$ & $\begin{array}{l}\text { Right-handed } \\
\text { persons diagnosed } \\
\text { with AD with MRI } \\
\text { evidence of AD } \\
\text { pathophysiological } \\
\text { processes and } \\
\text { MMSE score 15-24 } \\
\text { and } \\
\text { Clinical Dementia } \\
\text { Rating scale score } \\
\text { of 1-2, age 65-85 } \\
\text { years, school } \\
\text { attendance } \\
\text { range 5-17 years }\end{array}$ & $\mathrm{NR}$ & $\mathrm{NR}$ & $\mathrm{NR}$ & $\mathrm{NR}$ & NA & NA & $\begin{array}{l}10 \text { weeks } \\
\text { (pathophysiol } \\
\text { ogical } \\
\text { outcomes } \\
\text { only) }\end{array}$ & $\begin{array}{l}\text { BPSD, } \\
\text { language, } \\
\text { physiological } \\
\text { brain } \\
\text { activation }\end{array}$ & $\mathrm{NR}$ \\
\hline $\begin{array}{l}\text { Marshall, } \\
20155^{263} \\
\text { (no PMID) } \\
\text { UK } \\
\text { Pilot }\end{array}$ & $\begin{array}{l}\text { Living Well with } \\
\text { Dementia group } \\
\text { intervention } \\
\text { lasting for } 10 \\
\text { weekly sessions } \\
\text { delivered by } \\
\text { nurses from a } \\
\text { memory clinic } \\
\end{array}$ & Waitlist-control & \begin{tabular}{l|} 
NHS \\
hospital or \\
community \\
settings or \\
PLWD home \\
RCT
\end{tabular} & $\begin{array}{l}\text { AD, vascular, or } \\
\text { Lewy body } \\
\text { diagnosed within } \\
\text { previous } 18 \text { months } \\
\text { MMSE 18+ }\end{array}$ & $\begin{array}{l}\text { N=58 } \\
\text { Age: yes } \\
\text { Sex: yes } \\
\text { Race: yes } \\
\text { Education: yes }\end{array}$ & $\begin{array}{l}\text { Household } \\
\text { characteristics }\end{array}$ & $\begin{array}{l}\text { N=58 } \\
\text { Age: yes } \\
\text { Sex: yes } \\
\text { Race: yes } \\
\text { Education: yes } \\
\text { Relationship: no }\end{array}$ & $\mathrm{NR}$ & NA & NA & 10 weeks & $\begin{array}{l}\text { QOL-AD } \\
\text { CSDD }\end{array}$ & None \\
\hline $\begin{array}{l}\text { Czaja 2013264 } \\
(23831174) \\
\text { US } \\
\text { Pilot }\end{array}$ & $\begin{array}{l}\text { Technology- } \\
\text { based multi- } \\
\text { component } \\
\text { psychosocial } \\
\text { intervention was } \\
\text { delivered in- } \\
\text { home and via } \\
\text { videophone } \\
\text { technology over } \\
5 \text { months. }\end{array}$ & $\begin{array}{l}\text { Attention control } \\
\text { caregiver group } \\
\\
\text { Caregiver } \\
\text { received } \\
\text { information only }\end{array}$ & $\begin{array}{l}\text { Community- } \\
\text { dwelling } \\
\text { 3-arm RCT } \\
110 \text { CG }\end{array}$ & $\begin{array}{l}\text { Physician } \\
\text { diagnosis of AD or } \\
\text { other type of } \\
\text { dementia with } \\
\text { MMSE score }<24 \\
\text { and at least one } \\
\text { limitation in ADL or } \\
\text { two in IADL }\end{array}$ & $\begin{array}{l}\mathrm{N}=93 \\
\text { Age: yes } \\
\text { Sex: no } \\
\text { Race: no } \\
\text { Education: yes }\end{array}$ & None & $\begin{array}{l}\mathrm{N}=93 \\
\text { Age: yes } \\
\text { Sex: yes } \\
\text { Race: yes } \\
\text { Education: no } \\
\text { Relationship: yes }\end{array}$ & Duration: yes & NA & NA & 5 months & $\mathrm{NR}$ & $\begin{array}{l}\text { CESD } \\
\text { RMBPC } \\
\text { Positive } \\
\text { aspects of } \\
\text { caregiving. } \\
\text { Social } \\
\text { Support }\end{array}$ \\
\hline $\begin{array}{l}\text { Gaugler, } \\
2013^{227} \\
(23339050) \\
(262382266)^{265} \\
(25628299)^{266} \\
(29562359)^{267} \\
(30009268)^{268} \\
\text { US } \\
\text { High RoB }\end{array}$ & $\begin{array}{l}\text { Indivividual and } \\
\text { family } \\
\text { counseling, } \\
\text { support } \\
\text { group referral, } \\
\text { and ad hoc } \\
\text { consultation for } \\
\text { adult children of } \\
\text { PLWD }\end{array}$ & Contact-control & $\begin{array}{l}\text { Community/ } \\
\text { independent } \\
\text { living to } \\
\text { residential } \\
\text { setting } \\
\text { Adult } \\
\text { children of } \\
\text { PLWD } \\
N=107\end{array}$ & NR & $\mathrm{NR}$ & $\mathrm{NR}$ & $\begin{array}{l}\mathrm{N}=107 \\
\text { Age: yes } \\
\text { Sex: yes } \\
\text { Race: yes } \\
\text { Education: yes } \\
\text { Relationship: no }\end{array}$ & Employment & NA & NA & 2 years & $\begin{array}{l}\text { Residential } \\
\text { care } \\
\text { placement }\end{array}$ & $\begin{array}{l}\text { caregiver } \\
\text { subjective } \\
\text { health } \\
\text { social } \\
\text { support }\end{array}$ \\
\hline $\begin{array}{l}\text { Martindale- } \\
\text { Adams 2013228 } \\
\text { (24617278) } \\
\text { US }\end{array}$ & $\begin{array}{l}\text { Telephone } \\
\text { support and } \\
\text { periodic group } \\
\text { meetings for } \\
\text { caregivers }\end{array}$ & $\begin{array}{l}\text { Printed } \\
\text { materials for } \\
\text { support }\end{array}$ & $\begin{array}{l}\text { Community- } \\
\text { based } \\
\text { RCT }\end{array}$ & $\begin{array}{l}\text { Veteran patients } \\
\text { diagnosed with } \\
\text { dementia or } \\
\text { dementia patients } \\
\text { with veteran } \\
\text { caregivers } \\
\text { MMSE of } 23 \text { or less } \\
\text { At least one ADL or } \\
2 \text { IADL limitations } \\
\text { Exclusion criteria }\end{array}$ & $\begin{array}{l}\mathrm{N}=77 \text { intervention } \\
\text { group } \\
77 \text { control group } \\
\text { Age: yes } \\
\text { Sex: yes } \\
\text { Education: yes } \\
\text { Race } \% \text { majority: no }\end{array}$ & Not reported & $\begin{array}{l}\text { Age: yes } \\
\text { Sex: yes } \\
\text { Education: yes } \\
\text { Race \% majority: } \\
\text { yes } \\
\text { Relation to PLWD: } \\
\text { yes }\end{array}$ & $\begin{array}{l}\text { Duration } \\
\text { Income } \\
\text { Employment }\end{array}$ & NA & NA & 1 year & No reported & $\begin{array}{l}\text { Bother } \\
\text { Burden } \\
\text { Depression } \\
\text { General } \\
\text { well-being }\end{array}$ \\
\hline $\begin{array}{l}\text { Signe, 200855 } \\
(18269429) \\
\text { Sweden } \\
\text { High ROB }\end{array}$ & $\begin{array}{l}\text { Psychosocial } \\
\text { intervention \& } \\
\text { conversation } \\
\text { group, } 5 \text { weeks }\end{array}$ & $\begin{array}{l}\text { Control group } \\
\text { (comparison } \\
\text { NR) }\end{array}$ & $\begin{array}{l}\text { In-home } \\
\text { Quasi- } \\
\text { experimental } \\
\text { Caregiver }\end{array}$ & $\begin{array}{l}\text { Dementia } \\
\text { unspecified }\end{array}$ & $\begin{array}{l}\text { N=150 } \\
\text { Age: yes } \\
\text { Sex: yes } \\
\text { Race: no } \\
\text { Education: no }\end{array}$ & $\begin{array}{l}\text { Household } \\
\text { characteristics }\end{array}$ & $\begin{array}{l}\mathrm{N}=155 \\
\text { Age: yes } \\
\text { Sex: yes } \\
\text { Race: no } \\
\text { Education: no } \\
\text { Relationship: yes }\end{array}$ & Employment & NA & NA & $\begin{array}{l}6 \text { month and } \\
12 \text { month } \\
\text { measures }\end{array}$ & $\mathrm{NR}$ & $\begin{array}{l}\text { Caregiver } \\
\text { Burden } \\
\text { Scale } \\
\text { CASI } \\
\text { Nottingham } \\
\text { Health } \\
\text { Profile }\end{array}$ \\
\hline
\end{tabular}




\begin{tabular}{|c|c|c|c|c|c|c|c|c|c|c|c|c|c|}
\hline $\begin{array}{l}\text { Finkel, 2007269 } \\
\text { (17463195) } \\
\text { US } \\
\text { Pilot }\end{array}$ & $\begin{array}{l}\text { Technology- } \\
\text { based } \\
\text { psychoeducatio } \\
\text { nal intervention } \\
\text { for } \\
\text { family } \\
\text { caregivers of } \\
\text { dementia } \\
\text { patients }\end{array}$ & Information-only & \begin{tabular}{|l|}
$\mathrm{NR}$ \\
$\mathrm{RCT}$ \\
Caregivers
\end{tabular} & \begin{tabular}{|l} 
Diagnosis AD or \\
other type of \\
dementia \\
and MMSE score \\
23 (excluding \\
scores of zero).
\end{tabular} & $\begin{array}{l}\mathrm{N}=46 \\
\text { Age: } \text { yes } \\
\text { Sex: yes } \\
\text { Race: yes } \\
\text { Education: yes }\end{array}$ & None & $\begin{array}{l}\text { N=46 } \\
\text { Age: yes } \\
\text { Sex: yes } \\
\text { Race: yes } \\
\text { Education: yes } \\
\text { Relationship: yes }\end{array}$ & None & NA & NA & 6 months & $\mathrm{NR}$ & $\begin{array}{l}\text { CED-S } \\
\text { RMBPC }\end{array}$ \\
\hline $\begin{array}{l}\text { Ducharme } \\
2005^{231} \\
(16024406) \\
\text { Canada } \\
\text { High RoB }\end{array}$ & $\begin{array}{l}90 \text { minutes of } \\
\text { weekly sessions } \\
\text { (10 weeks) for } \\
\text { women CG } \\
\text { comprising of a } \\
\text { psychoeducatio } \\
\text { nal program } \\
\text { focusing on } \\
\text { cognitive } \\
\text { appraisal of } \\
\text { stress, } \\
\text { perception of } \\
\text { control over a } \\
\text { situation and } \\
\text { problem solving, } \\
\text { reframing, and } \\
\text { stress } \\
\text { symptoms } \\
\text { management. }\end{array}$ & $\begin{array}{l}\text { Comparison } \\
\text { program offered } \\
\text { by an Alzheimer } \\
\text { Society } \\
\text { Control: No } \\
\text { program }\end{array}$ & $\begin{array}{l}\begin{array}{l}\text { Long term } \\
\text { care and } \\
\text { residential } \\
\text { centers }\end{array} \\
\text { 3-arm RCT } \\
137 \\
\text { caregivers }\end{array}$ & $\begin{array}{l}\text { Diagnosed with } \\
\text { irreversible } \\
\text { dementia }\end{array}$ & $\begin{array}{l}\text { N=unclear } \\
\text { Age: yes } \\
\text { Sex: yes } \\
\text { Race: no } \\
\text { Education: no }\end{array}$ & None & $\begin{array}{l}\mathrm{N}=\text { unclear } \\
\text { Age: yes } \\
\text { Sex: yes } \\
\text { Race: no } \\
\text { Education: no } \\
\text { Relationship: yes }\end{array}$ & NR & $\mathrm{NA}$ & $\mathrm{NA}$ & $\begin{array}{l}10 \text { weeks } \\
3 \text { months }\end{array}$ & NR & $\begin{array}{l}\text { PDI } \\
\text { Caregiver } \\
\text { Overload } \\
\text { SAM } \\
\text { CAMI } \\
\text { Competenc } \\
\text { e in dealing } \\
\text { with } \\
\text { healthcare }\end{array}$ \\
\hline
\end{tabular}




\begin{tabular}{|c|c|c|c|c|c|c|c|c|c|c|c|c|c|}
\hline $\begin{array}{l}\text { Eisdorfer } \\
2003^{233} \\
(12937331) \\
\text { US } \\
\text { High RoB }\end{array}$ & $\begin{array}{l}\text { SET: 60-90 } \\
\text { minutes } \\
\text { structural family } \\
\text { therapy } \\
\text { sessions for } 12 \\
\text { months, with } \\
\text { weekly sessions } \\
\text { for the firss } 4 \\
\text { months } \\
\text { biveekly } \\
\text { sessions for the } \\
\text { subsequent } 2 \\
\text { months, and } \\
\text { monthly } \\
\text { sessions for the } \\
\text { final } 6 \text { months } \\
\text { of treatment. } \\
\text { SETTCTIS: } \\
\text { CTIS is an } \\
\text { information } \\
\text { network that } \\
\text { utilizes } \\
\text { computer- } \\
\text { telephone } \\
\text { technology } \\
\text { failitating } \\
\text { linkages of the } \\
\text { caregivers with } \\
\text { both their family } \\
\text { and with } \\
\text { supportive } \\
\text { resources } \\
\text { outside of the } \\
\text { home. }\end{array}$ & $\begin{array}{l}\text { Telephone- } \\
\text { based, minimal } \\
\text { support } \\
\text { condition; } \\
\text { biweekly yalls } \\
\text { for the first } 6 \\
\text { months and } \\
\text { monthly calls } \\
\text { during the next } \\
7-12 \text { months. } \\
\text { The duration of } \\
\text { the calls ranged } \\
\text { from } 5 \text { min to } 15 \\
\text { min and } \\
\text { consisted of } \\
\text { active listening } \\
\text { and empathic } \\
\text { comments when } \\
\text { appropriate. }\end{array}$ & $\begin{array}{l}\text { Community- } \\
\text { dwelling } \\
\text { 3-arm RCT } \\
225 \text { CG }\end{array}$ & $\begin{array}{l}\text { Medical diagnosis } \\
\text { of AD or other type } \\
\text { of dementia with } \\
\text { MMSE score }<24 \\
\text { and at least one } \\
\text { limitation in ADL or } \\
\text { two in IADL }\end{array}$ & $\begin{array}{l}\mathrm{N}=148 \\
\text { Age: yes } \\
\text { Sex: yes } \\
\text { Race: yes } \\
\text { Education: no }\end{array}$ & None & $\begin{array}{l}N=148 \\
\text { Age: yes } \\
\text { Sex: yes } \\
\text { Race: yes } \\
\text { Education: yes } \\
\text { Relationship: yes }\end{array}$ & Duration: yes & NA & NA & $\begin{array}{l}6 \text { months } \\
18 \text { months }\end{array}$ & NR & $\begin{array}{l}\text { CESD } \\
\text { RMBPC } \\
\text { Satisfaction } \\
\text { with social } \\
\text { support } \\
\text { MMSE } \\
\text { ADL } \\
\text { IADL }\end{array}$ \\
\hline $\begin{array}{l}\text { Mittelman } \\
1993^{235} \\
\text { (8314099) } \\
\text { US }\end{array}$ & $\begin{array}{l}\text { Individual and } \\
\text { family } \\
\text { counseling, } \\
\text { support group } \\
\text { and ad hoc } \\
\text { consultation }\end{array}$ & Routine support & $\begin{array}{l}\text { Community- } \\
\text { based } \\
\text { RCT }\end{array}$ & $\begin{array}{l}\text { Patients with a } \\
\text { clinical diagnosis of } \\
\text { AD } \\
\text { Spouse is primary } \\
\text { caregiver } \\
\text { Additional family } \\
\text { support locally } \\
\text { Exclusion criteria }\end{array}$ & $\begin{array}{l}\mathrm{N}=103 \text { Intervention } \\
\text { group } \\
103 \text { control group } \\
\text { Age: yes } \\
\text { Sex: yes (inferred) } \\
\text { Education: no } \\
\text { Race } \% \text { majority: no }\end{array}$ & Patient income & $\begin{array}{l}\mathrm{N}=103 \text { Intervention } \\
\text { group } \\
103 \text { control group } \\
\text { Age: yes } \\
\text { Sex: yes } \\
\text { Education: yes } \\
\text { Race \% majority: } \\
\text { yes } \\
\text { Relation to PLWD: } \\
\text { yes }\end{array}$ & $\begin{array}{l}\text { Duration } \\
\text { Employment status } \\
\text { Religion }\end{array}$ & NA & NA & 12 months & $\begin{array}{l}\text { Admission to } \\
\text { nursing home } \\
\text { Predictors of } \\
\text { nursing home } \\
\text { placement } \\
\text { regression } \\
\text { analysis }\end{array}$ & Not reported \\
\hline $\begin{array}{l}\text { Mohide, 1990236 } \\
\text { (2184186) } \\
\text { Canada } \\
\text { High ROB }\end{array}$ & $\begin{array}{l}\text { Caregiver } \\
\text { Support } \\
\text { Program (CSP): } \\
\text { caregiver- } \\
\text { focused health } \\
\text { care, education } \\
\text { about dementia } \\
\text { and caregiving, } \\
\text { assistance with } \\
\text { problem solving, } \\
\text { regularly } \\
\text { scheduled in- } \\
\text { home respite, } \\
\text { self-help family } \\
\text { caregiver } \\
\text { support group }\end{array}$ & $\begin{array}{l}\text { Conventional } \\
\text { community } \\
\text { nursing care }\end{array}$ & $\begin{array}{l}\text { In-home } \\
\text { RCT } \\
\text { Caregiver }\end{array}$ & $\begin{array}{l}\text { Moderate to severe } \\
\text { dementia }\end{array}$ & $\begin{array}{l}\text { N=30 } \\
\text { Age: yes } \\
\text { Sex: yes } \\
\text { Race: no } \\
\text { Education: no }\end{array}$ & SES & $\begin{array}{l}\mathrm{N}=30 \\
\text { Age: yes } \\
\text { Sex: yes } \\
\text { Race: no } \\
\text { Education: no }\end{array}$ & Duration & NA & NA & 6 months & $\mathrm{NR}$ & $\begin{array}{l}\text { CES-D } \\
\text { Self- } \\
\text { Anchoring } \\
\text { Striving } \\
\text { Scale } \\
\text { Caregiver } \\
\text { Quality of } \\
\text { Life } \\
\text { Inventory }\end{array}$ \\
\hline
\end{tabular}




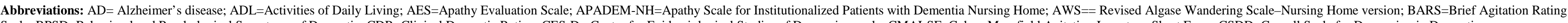
Scale; BPSD=Behavioral and Psychological Symptoms of Dementia; CDR=Clinical Dementia Rating; CES-D= Center for Epidemiological Studies of Depression scale; CMAI-SF=Cohen-Mansfield Agitation Inventory-Short Form; CSDD=Cornell Scale for Depression in Dementia

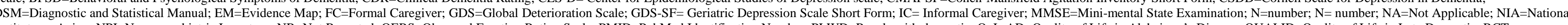

\section{Other Interventions for Informal Caregiver Well-Being}

\begin{tabular}{|c|c|c|c|c|c|c|c|c|c|}
\hline Study (PMID) & Outcome Timing & $\begin{array}{l}\text { Atrition Bias } \\
\text { Attrition \% }\end{array}$ & Selection Bias & Detection Bias & Performance Bias & Reporting Bias & Fidelity Bias & Funder & Overall Rating \\
\hline $\begin{array}{l}\text { Jutten, 2018270 }(30631337) \\
\text { Jutten 2017271 }(28827242)\end{array}$ & $\begin{array}{l}2.5 \text { months } \\
15 \text { months }\end{array}$ & $\begin{array}{l}\text { High } \\
2.5 \text { months: } 16 \% \\
12 \text { months: } 57 \%\end{array}$ & High & $x$ & $x$ & $\mathrm{x}$ & $x$ & Government and Nonprofit & High \\
\hline Stern $2008^{272}$ (19064472) & 8 weeks & $\begin{array}{l}\text { Medium } \\
\text { NR }\end{array}$ & Medium & High & Medium & High & $x$ & Government & High \\
\hline Simpson $2006^{273}(16734920)$ & $\begin{array}{l}6 \text { months } \\
12 \text { months }\end{array}$ & $\begin{array}{l}\text { High } \\
12 \text { months: } 39 \%\end{array}$ & $x$ & $x$ & $x$ & $x$ & $x$ & Government & High \\
\hline
\end{tabular}

Abbreviations: NR=Not Reported; PMID=PubMed Identification Number 


\section{Evidence Map: Other Interventions for Informal Caregiver Well-Being}

\begin{tabular}{|c|c|c|c|c|c|c|c|c|c|c|c|c|c|}
\hline $\begin{array}{l}\text { Study (PMID) } \\
\text { Country } \\
\text { EM Reason }\end{array}$ & Intervention & Comparison & $\begin{array}{l}\text { Setting } \\
\text { and Design } \\
\\
\text { Setting } \\
\text { Design } \\
\text { Cluster N } \\
\text { Participants } \\
\text { Random- } \\
\text { ized N }\end{array}$ & $\begin{array}{l}\text { PLWD } \\
\text { Dementia } \\
\text { Characteristics }\end{array}$ & $\begin{array}{l}\text { PLWD } \\
\text { Non-Disease Char } \\
\text { PLWD N } \\
\text { PLWD Age (mean) } \\
\text { PLWD Sex (\% } \\
\text { female) } \\
\text { PLWD Race (\% } \\
\text { majority) } \\
\text { PLWD Education } \\
\text { (mean years) }\end{array}$ & $\begin{array}{l}\text { PLWD } \\
\text { Non-Disease } \\
\text { Char Reporting } \\
\text { Status (RS) } \\
\\
\text { PLWD SES } \\
\text { PLWD Prior } \\
\text { Disability } \\
\text { PLWD } \\
\text { Household } \\
\text { Characteristics } \\
\text { PLWD Health } \\
\text { Insurance } \\
\text { PLWD Detailed } \\
\text { Race Information }\end{array}$ & $\begin{array}{l}\text { Informal Caregiver } \\
\text { (IC) } \\
\text { Characteristics } \\
\text { IC N } \\
\text { IC Age (mean) } \\
\text { IC Sex (\% female) } \\
\text { IC Race (\% } \\
\text { majority) } \\
\text { IC Education } \\
\text { (mean years) } \\
\text { IC Relation to } \\
\text { PLWD (\% majority) }\end{array}$ & $\begin{array}{l}\text { Informal Caregiver } \\
\text { (IC) Char. RS } \\
\text { IC Duration } \\
\text { IC Living With } \\
\text { PLWD } \\
\text { IC Payment } \\
\text { IC Health Status } \\
\text { IC Dementia } \\
\text { Family History } \\
\text { IC Employment } \\
\text { Status } \\
\text { IC Training }\end{array}$ & 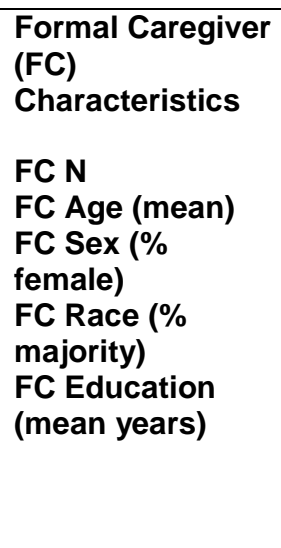 & $\begin{array}{l}\text { Formal } \\
\text { Caregiver (FC) } \\
\text { Char. RS } \\
\text { FC Health } \\
\text { Status } \\
\text { FC Training } \\
\text { FC Education } \\
\text { FC Position } \\
\text { FC Length of } \\
\text { Service }\end{array}$ & $\begin{array}{l}\text { Outcome } \\
\text { Timing(s) }\end{array}$ & \begin{tabular}{|l|} 
PLWD \\
Outcomes
\end{tabular} & $\begin{array}{l}\text { Caregiver } \\
\text { Outcomes } \\
\text { IC: } \\
\text { FC: }\end{array}$ \\
\hline $\begin{array}{l}\text { Jutten, 2018 } \\
\text { (30631337) } \\
\text { Netherlands } \\
\text { High ROB } \\
\text { Jutten 2017271 } \\
\text { (28827242) }\end{array}$ & \begin{tabular}{|l} 
Mixed virtual \\
reality simulator \\
(simulate \\
experience of \\
dementia)
\end{tabular} & No virtual reality & $\begin{array}{l}\text { Community- } \\
\text { based } \\
\text { Quasi- } \\
\text { experimental } \\
\text { caregivers }\end{array}$ & $\begin{array}{l}\text { Dementia } \\
\text { unspecified }\end{array}$ & $\begin{array}{l}\mathrm{N}=201 \\
\text { Age: yes } \\
\text { Sex: yes } \\
\text { Race: no } \\
\text { Education: no }\end{array}$ & Duration & $\begin{array}{l}\mathrm{N}=201 \\
\text { Age: } \text { yes } \\
\text { Sex: yes } \\
\text { Race: yes } \\
\text { Education: no } \\
\text { Relation to PLWD: } \\
\text { yes }\end{array}$ & $\begin{array}{l}\text { Living with PLWD: } \\
\text { yes }\end{array}$ & NA & NA & $\begin{array}{l}2.5 \text { and } 15 \\
\text { months }\end{array}$ & NA & $\begin{array}{l}\text { Empathy } \\
\text { Hospital } \\
\text { Anxiety and } \\
\text { Depression } \\
\text { Scale } \\
\text { Caregiver } \\
\text { Reaction } \\
\text { Assessment } \\
\text { Relationship } \\
\text { Quality Index }\end{array}$ \\
\hline $\begin{array}{l}\text { Lord 2017274 } \\
\text { (28243460) } \\
\text { UK } \\
\text { Pilot Study }\end{array}$ & $\begin{array}{l}\text { DECIDE } \\
\text { decision aid for } \\
\text { informal } \\
\text { caregivers } \\
\text { about place of } \\
\text { care for PLWD; } \\
\text { one 45-minute } \\
\text { session with a } \\
\text { decision coach }\end{array}$ & Usual care & $\begin{array}{l}\text { Community- } \\
\text { based } \\
\text { memory } \\
\text { clinics } \\
\text { RCT, single } \\
\text { site } \\
41 \text { informal } \\
\text { caregivers }\end{array}$ & $\begin{array}{l}\text { Moderate or severe } \\
\text { dementia } \\
\text { MMSE }<20\end{array}$ & $\begin{array}{l}\mathrm{N}=41 \\
\text { Age: no } \\
\text { Sex: no } \\
\text { Race: no } \\
\text { Education: no }\end{array}$ & NR & $\begin{array}{l}\mathrm{N}=41 \\
\text { Age: yes } \\
\text { Sex: yes } \\
\text { Race: yes } \\
\text { Education: no } \\
\text { Relation to PLWD: } \\
\text { yes }\end{array}$ & $\begin{array}{l}\text { Living with PLWD: } \\
\text { yes }\end{array}$ & NA & NA & 10 weeks & NA & $\begin{array}{l}\text { Decisional } \\
\text { Conflict Scale } \\
\text { Hospital } \\
\text { Anxiety and } \\
\text { Depression } \\
\text { Scale }\end{array}$ \\
\hline $\begin{array}{l}\text { Stirling 2012275 } \\
\text { (22429384) } \\
\text { Australia } \\
\text { Pilot Study }\end{array}$ & \begin{tabular}{|l|} 
Decision aid \\
about \\
community \\
services, \\
including respite \\
care, for \\
informal \\
caregivers; \\
mailed directly \\
to caregivers \\
and completed \\
over a week \\
\end{tabular} & Waitlist control & $\begin{array}{l}\text { Community- } \\
\text { based } \\
\text { setting with } \\
\text { in-home } \\
\text { decision aid } \\
\text { RCT; single } \\
\text { site } \\
31 \text { informal } \\
\text { caregivers }\end{array}$ & Not specified & $\begin{array}{l}\mathrm{N}=31 \\
\text { Age: no } \\
\text { Sex: no } \\
\text { Race: no } \\
\text { Education: no }\end{array}$ & NR & $\begin{array}{l}\mathrm{N}=31 \\
\text { Age: yes } \\
\text { Sex: yes } \\
\text { Race: yes } \\
\text { Education: yes } \\
\text { Relation to PLWD: } \\
\text { yes }\end{array}$ & Duration: Yes & NA & NA & $\begin{array}{l}2 \text { weeks } \\
3 \text { months }\end{array}$ & NA & $\begin{array}{l}\text { Modified } \\
\text { Caregiver } \\
\text { Strain Index } \\
\text { Decisional } \\
\text { Conflict Scale } \\
\text { Control } \\
\text { Preference } \\
\text { Scale }\end{array}$ \\
\hline
\end{tabular}




\begin{tabular}{|c|c|c|c|c|c|c|c|c|c|c|c|c|c|}
\hline $\begin{array}{l}\text { Rose 2009276 } \\
\text { (19427574) } \\
\text { US } \\
\text { Pilot Study }\end{array}$ & $\begin{array}{l}\text { At home } \\
\text { AlphaStim }{ }^{-} \\
\text {cranial electrical } \\
\text { stimulation } \\
\text { Alzheimer's } \\
\text { disease or } \\
\text { multi-infarct } \\
\text { dementia for } \\
\text { spouse } \\
\text { caregivers; } 60 \\
\text { minutes per day } \\
\text { for } 4 \text { weeks }\end{array}$ & Sham & \begin{tabular}{|l|} 
Rural \\
community \\
setting, \\
intervention \\
at home \\
RCT; single \\
site \\
39 spouse \\
caregivers
\end{tabular} & $\begin{array}{l}\text { AD or multi-infarct } \\
\text { dementia }\end{array}$ & $\begin{array}{l}\text { N=39 } \\
\text { Age: yes } \\
\text { Sex: no } \\
\text { Race: no } \\
\text { Education: no }\end{array}$ & \begin{tabular}{|l|}
$N R$ \\
\end{tabular} & $\begin{array}{l}\mathrm{N}=39 \\
\text { Age: yes } \\
\text { Sex: yes } \\
\text { Race: no } \\
\text { Education: yes } \\
\text { Relation to PLWD: } \\
\text { yes }\end{array}$ & Duration: Yes & NA & $\mathrm{NA}$ & $\begin{array}{l}2 \text { weeks } \\
4 \text { weeks }\end{array}$ & NA & $\begin{array}{l}\text { PSQI } \\
\text { General } \\
\text { Sleep } \\
\text { Disturbance } \\
\text { Scale } \\
\text { Geriatric } \\
\text { Depression } \\
\text { Scale } \\
\text { Philadelphia } \\
\text { Geriatric } \\
\text { Center } \\
\text { Caregiving } \\
\text { Appraisal } \\
\text { Scales } \\
\end{array}$ \\
\hline $\begin{array}{l}\text { Stern 2000272 } \\
\text { (19064472) } \\
\text { US } \\
\text { High ROB }\end{array}$ & $\begin{array}{l}\text { Family } \\
\text { caregiver } \\
\text { psychoeducatio } \\
\text { nal support } \\
\text { meetings for } \\
\text { PLWD driving } \\
\text { cessation; 4 } \\
\text { weekly 2-hour } \\
\text { meetings }\end{array}$ & $\begin{array}{l}\text { 1. Written } \\
\text { educational } \\
\text { information and } \\
\text { resource list for } \\
\text { PLWD driving } \\
\text { cessation. } \\
\text { 2. No } \\
\text { intervention } \\
\text { (waitlist control) }\end{array}$ & \begin{tabular}{|l|} 
Community- \\
based \\
setting \\
Cluster RCT \\
14 sites \\
66 informal \\
caregivers \\
\end{tabular} & \begin{tabular}{|l|} 
Caregiver's report \\
of MCl, AD, or \\
other dementia
\end{tabular} & $\begin{array}{l}\mathrm{N}=66 \\
\text { Age: no } \\
\text { Sex: no } \\
\text { Race: no } \\
\text { Education: no }\end{array}$ & NR & $\begin{array}{l}\mathrm{N}=66 \\
\text { Age: yes } \\
\text { Sex: yes } \\
\text { Race: no } \\
\text { Education: no } \\
\text { Relation to PLWD: } \\
\text { yes }\end{array}$ & NR & NA & NA & 8 weeks & NA & $\begin{array}{l}\text { Brief COPE } \\
\text { Scale } \\
\text { Self-Efficacy } \\
\text { Questionnaire }\end{array}$ \\
\hline $\begin{array}{l}\text { Simpson } \\
2006^{273} \\
(16734920) \\
\text { UK } \\
\text { High ROB }\end{array}$ & $\begin{array}{l}\text { Carer held } \\
\text { record folder } \\
\text { with sections for } \\
\text { carerffamily, } \\
\text { professional } \\
\text { feedback, and a } \\
\text { carer diary; held } \\
\text { at home and } \\
\text { maintained for } \\
12 \text { months }\end{array}$ & Usual Care & $\begin{array}{l}\text { Community- } \\
\text { based } \\
\text { mental } \\
\text { health care } \\
\text { and at home } \\
\text { CCT } \\
84 \text { informal } \\
\text { caregiver- } \\
\text { PLWD } \\
\text { dyads }\end{array}$ & Not specified & $\begin{array}{l}\mathrm{N}=84 \\
\text { Age: no } \\
\text { Sex: no } \\
\text { Race: no } \\
\text { Education: no }\end{array}$ & NR & $\begin{array}{l}\mathrm{N}=84 \\
\text { Age: no } \\
\text { Sex: no } \\
\text { Race: no } \\
\text { Education: no } \\
\text { Relation to PLWD: } \\
\text { no }\end{array}$ & NR & NA & NA & $\begin{array}{l}6 \text { months } \\
12 \text { months }\end{array}$ & $\begin{array}{l}\text { Crichton } \\
\text { Behavioral } \\
\text { Scale } \\
\text { Global } \\
\text { Deterioration } \\
\text { Scale }\end{array}$ & $\begin{array}{l}\text { Dementia } \\
\text { Knowledge } \\
\text { Questionnaire } \\
\text { Caregiver } \\
\text { Strain Index } \\
\text { GHQ } \\
\text { SF-36 } \\
\text { Locus of } \\
\text { Control Scale }\end{array}$ \\
\hline
\end{tabular}

MMSE=Mini Mental State Exam; N=number; NA=Not Applicable; NR=Not Reported; PMID=PubMed Identification Number; PSQI= Pittsburgh Sleep Quality Index; PLWD=Persons with Dementia; RCT=Randomized Controlled Trial; ROB=Risk of Bias; RS=Reporting Status; SES=socioeconomic status; SF-36=36-item Short Form Survey 


\section{Care Interventions for Formal Caregiver Staff Well-Being}

\section{Evidence Map: Formal Caregiver Staff}

\begin{tabular}{|c|c|c|c|c|c|c|c|c|c|c|c|c|c|}
\hline \begin{tabular}{|l|} 
Study (PMID) \\
Country \\
EM Reason
\end{tabular} & Intervention & Comparison & \begin{tabular}{|l|} 
Setting \\
and Design \\
\\
Setting \\
Design \\
Cluster N \\
Participants \\
Random- \\
ized N
\end{tabular} & \begin{tabular}{|l} 
PLWD \\
Dementia \\
Characteristics
\end{tabular} & $\begin{array}{l}\text { PLWD } \\
\text { Non-Disease Char } \\
\text { PLWD N } \\
\text { PLWD Age (mean) } \\
\text { PLWD Sex (\% } \\
\text { female) } \\
\text { PLWD Race (\% } \\
\text { majority) } \\
\text { PLWD Education } \\
\text { (mean years) }\end{array}$ & \begin{tabular}{|l|} 
PLWD \\
Non-Disease \\
Char Reporting \\
Status (RS) \\
PLWD SES \\
PLWDD Prior \\
Disability \\
PLWD \\
Household \\
Characteristics \\
PLWD Health \\
Insurance \\
PLWD Detailed \\
Race Information
\end{tabular} & $\begin{array}{l}\text { Informal Caregiver } \\
\text { (IC) } \\
\text { Characteristics } \\
\text { IC N } \\
\text { IC Age (mean) } \\
\text { IC Sex (\% female) } \\
\text { IC Race (\% } \\
\text { majority) } \\
\text { IC Education } \\
\text { (mean years) } \\
\text { IC Relation to } \\
\text { PLWD (\% majority) }\end{array}$ & $\begin{array}{l}\text { Informal Caregiver } \\
\text { (IC) Char. RS } \\
\text { IC Duration } \\
\text { IC Living With } \\
\text { PLWD } \\
\text { IC Payment } \\
\text { IC Health Status } \\
\text { IC Dementia } \\
\text { Family History } \\
\text { IC Employment } \\
\text { Status } \\
\text { IC Training }\end{array}$ & \begin{tabular}{|l} 
Formal Caregiver \\
(FC) \\
Characteristics \\
FC N \\
FC Age (mean) \\
FC Sex (\% \\
female) \\
FC Race (\% \\
majority) \\
FC Education
\end{tabular} & $\begin{array}{l}\text { Formal } \\
\text { Caregiver (FC) } \\
\text { Char. RS } \\
\text { FC Health } \\
\text { Status } \\
\text { FC Training } \\
\text { FC Education } \\
\text { FC Position } \\
\text { FC Length of } \\
\text { Service }\end{array}$ & \begin{tabular}{|l} 
Outcome \\
Timing(s)
\end{tabular} & $\begin{array}{l}\text { PLWD } \\
\text { Outcomes }\end{array}$ & $\begin{array}{l}\text { Caregiver } \\
\text { Outcomes } \\
\text { IC: } \\
\text { FC: }\end{array}$ \\
\hline \begin{tabular}{|l} 
Barbosa, \\
2015277 \\
$(25237132)$ \\
$(263994850)^{278}$ \\
Portugal \\
Pilot
\end{tabular} & $\begin{array}{l}8 \text { weekly } \\
\text { sessions of } 60 \\
\text { minutes } \\
\text { psychoeducatio } \\
\text { n plus } 30 \\
\text { minutes } \\
\text { relaxation and } \\
\text { stress } \\
\text { management } \\
\text { strategies }\end{array}$ & Education only & \begin{tabular}{|l|} 
Aged-care \\
residential \\
facilities \\
Cluster RCT \\
4 facilities
\end{tabular} & $\begin{array}{l}\text { Dementia } \\
\text { unspecified } \\
\text { (private, non-profit } \\
\text { institutions with } \\
\text { staff to resident } \\
\text { ratio 1:2 or 1:3) }\end{array}$ & NR & NR & NA & NA & \begin{tabular}{|l}
$\mathrm{N}=58$ \\
Age: yes \\
Sex: yes \\
Race: no \\
Education: yes
\end{tabular} & Length of service & 10 weeks & NR & $\begin{array}{l}\text { Perceived } \\
\text { stress scale } \\
\text { MBI } \\
\text { Intrinsic } \\
\text { satisfaction } \\
\text { with job }\end{array}$ \\
\hline 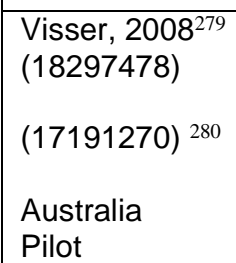 & $\begin{array}{l}\text { 8-week staff } \\
\text { education plus } \\
\text { peer support } \\
\text { group }\end{array}$ & $\begin{array}{l}\text { Waitlist } \\
\text { Education only }\end{array}$ & \begin{tabular}{|l|} 
Aged-care \\
facility \\
Cluster RCT \\
3 facilities
\end{tabular} & \begin{tabular}{|l|} 
Residents selected \\
by staff for \\
displaying \\
agitation; dementia \\
unspecified
\end{tabular} & $\begin{array}{l}\mathrm{N}=76 \\
\text { Age: yes } \\
\text { Sex: yes } \\
\text { Race: no } \\
\text { Education: no }\end{array}$ & NR & NA & NA & $\begin{array}{l}\mathrm{N}=52 \\
\text { Age: yes } \\
\text { Sex: yes } \\
\text { Race: no } \\
\text { Education: no }\end{array}$ & NR & 6 months & $\begin{array}{l}\text { ADRQL } \\
\text { CMAI }\end{array}$ & \begin{tabular}{|l|} 
The Staff \\
Attitudes \\
Questionnaire \\
MBI, third \\
edition
\end{tabular} \\
\hline \begin{tabular}{|l|} 
Mackenzie, \\
$2003^{281}$ \\
$(14569646)$ \\
Canada \\
Pilot
\end{tabular} & $\begin{array}{l}4 \text { module } \\
\text { training and } \\
\text { education to } \\
\text { decrease formal } \\
\text { caregiver stress } \\
\text { and burn-out }\end{array}$ & $\begin{array}{l}\text { Usual care } \\
\text { activities }\end{array}$ & \begin{tabular}{|l} 
Dementia \\
care units \\
Quasi- \\
experimental \\
2 care units
\end{tabular} & NR & NR & NR & NA & NA & $\begin{array}{l}\mathrm{N}=41 \\
\text { Age: } y \text { yes } \\
\text { Sex: yes } \\
\text { Race: no } \\
\text { Education: yes }\end{array}$ & Position & \begin{tabular}{|l} 
3-month post- \\
intervention
\end{tabular} & NR & \begin{tabular}{|l|} 
Inventory of \\
Geriatric \\
Nursing Self- \\
Efficacy \\
MBI \\
Satisfaction \\
with \\
teamwork
\end{tabular} \\
\hline
\end{tabular}




\section{References for Appendix E}

1. Au A, Lai DWL, Biggs S, et al. Perspective-taking interventions for intergenerational caregivers of alzheimer's diseases: a randomized controlled trial Research on social work practice. 2020;30(3):306-19. PMID: CN-02084449.

2. Au A, Yip HM, Lai S, et al. Telephone-based behavioral activation intervention for dementia family caregivers: Outcomes and mediation effect of a randomized controlled trial. Patient Educ Couns. 2019 Nov;102(11):2049-59. doi: https://dx.doi.org/10.1016/j.pec.2019.06.009. PMID: 31279613.

Kunik ME, Stanley MA, Shrestha S, et al. Aggression Prevention Training for Individuals With Dementia and Their Caregivers: A Randomize

4. Bjorge H, Kvaal K, Ulstein I. The effect of psychosocial support on caregivers' perceived criticism and emotional over-involvement of persons with dementia: an assessor-blinded randomized controlled trial. BMC Health Serv Res. 2019 Oct 24;19(1):744. doi:

https://dx.doi.org/10.1186/s12913-019-4551-x. PMID: 31651321.

. with Alate expenditures among African-American dementia caregivers with depressive symptoms. Ethn Health. 2019 Mar 19:1-14. doi:

https://dx.doi.org/10.1080/13557858.2019.1590536. PMID: 3088496

Moskowitz JT, Cheung EO, Snowberg KE, et al. Randomized controlled trial of a facilitated online positive emotion regulation interventio for dementia caregivers. Health Psychol. 2019 May;38(5):391-402. doi: https://dx.doi.org/10.1037/hea0000680. PMID: 31045422 . Worker: The DY ADEM Trias. J Alzheimers Dis. 2019;68(2):745-55. doi: https://dx.doi. org D/10.3233/JAD-180812. PMID: 30775982 . 9. Terracciano A, Artese A, Yeh J, et al. Effectiveness of Powerful Tools for Caregivers on Caregiver Burden and on Care Recipient Behavioral and Psychological Symptoms of Dementia: A Randomized Controlled Trial. J Am Med Dir Assoc. 2019 Dec 19;19:19. do: https:///dx.doi.org/10.1016/.jamda.2019.11.011. PMID.31866419.

0. Uyar F, Ozmen D, Mavioglu $\mathrm{H}$, et al. Assessment of the impact of dementia care and support program in both patient and caregiver

11. Wawrziczny E, Larochette C, Papo D, et al. A Customized Intervention for Dementia Caregivers: A Quasi-Experimental Design. J Aging Health. 2019 Aug;31(7):1172-95. doi: https:///dx.doi.org/10.1177/0898264318770056. PMID: 29665714.

2. Boots LM, de Vugt ME, Kempen GI, et al. Effectiveness of a Blended Care Self-Management Program for Caregivers of People With

Early-Stage Dementia (Partner in Balance): Randomized Controlled Trial. J Med Internet Res. 2018 Jul 13;20(7):e10017. doi:

ittp: //dx.doi.org/10.2196/10017. PMID: 30006327.

Am Geriatr Soc. 2018 Feb;66(2):339-45. doi: https:///dx.doi.org/10.1111/jigs.15194. PMID: 29192967. dementia. Alzheimer's and Dementia: Translational Research and
http://dx.doi.org/10.1016/j.trci.2018.08.004. PMID: 2001115918.

Ittp://dx.doi.org/10.1016/.trci.2018.08.004. PMID: 2001115918.

作

16. Callahan CM, Boustani MA, Schmid AA, et al. Targeting Functional Decline in Alzheimer Disease: A Randomized Trial. Ann Intern Med.

2017 Feb 07;166(3):164-71. doi: https://dx.doi.org/10.7326/M16-0830. PMID: 27893087.

17. Whitlatch CJ, Heid AR, Femia EE, et al. The Support, Health, Activities, Resources, and Education program for early stage dementia: Results from a rais: 29171296

18. Charlesworth G, Burnell K, Crellin N, et al. Peer support and reminiscence therapy for people with dementia and their family carers: a factorial pragmatic randomised trial. J Neurol Neurosurg Psychiatry. 2016 Nov;87(11):1218-28. doi: https://dx.doi.org/10.1136/jinnp-2016-

313736. PMID: 27521377

19. Cheng ST, Fung HH, Chan WC, et al. Short-Term Effects of a Gain-Focused Reappraisal Intervention for Dementia Caregivers: A Doublelind Cluster-Randomized Controlled Trial. Am J Geriatr Psychiatry. 2016 Sep;24(9):740-50. do:

https://dx.doi.org/10.1016/.j.jagp.2016.04.012. PMID: 27401052.

作 1. Laakkonen ML, Kautiainen H, Holtta E et al. Fffects of Self-Man hags://dx.doi.org/10.1093/geront/gnu045. PMID: 24855313. Randomized Controlled Trial. J Am Geriatr Soc. 2016 Apr;64(4):752-60. doi: Gttps:///x.doi.org/10.1111/jgs.14055. PMID: 27060101. 22. Taati $\mathrm{F}$, Bahramnezhad $\mathrm{F}$, Seyedfatemi $\mathrm{N}$, et al. The effect of participation in support groups on depression, anxiety and stress in family PMID: CN-01308282

23. Blom MM, Zarit SH, Groot Zwaaftink RB, et al. Effectiveness of an Internet intervention for family caregivers of people with dementia: results of a randomized controlled trial. PLoS ONE. 2015;10(2):e0116622. doi: https://dx.doi.org/10.1371/journal.pone.0116622. PMID
24. Chiu M, Pauley T, Wesson V, et al. Evaluation of a problem-solving (PS) techniques-based intervention for informal carers of patients with
dementia receiving in-home care. Int Pyychogeriatr. 2015-27(6):-937-48, doi: 10.1017/S1041610214002798 PMID: CN-01102329. 24. Cla family caregivers. Alzheimer Dis Assoc Disord. 2015 Apr-Jun;29(2):146-53. doi: https://dx.doi.org/10.1097/WAD.00000000000000077. PMID 25590939.

26. Losada A, Marquez-Gonzalez M, Romero-Moreno R, et al. Cognitive-behavioral therapy (CBT) versus acceptance and commitment therapy (ACT) for dementia family caregivers with significant depressive symptoms: Results of a
Psychol. 2015 Aug;83(4):760-72. doi: $\frac{\text { https://dx.doi.org/10.1037/ccpo000028. PMID: 26075381. }}{\text {. }}$. 27. Otero P, Smit F, Cuijpers P, et al. Long-term efficacy of indicated prevention of depression in non-profes
controlled trial. Psychol Med. 2015;45(7):1401-12. doi: 10.1017/S0033291714002505. PMID: 25331992.

28. Tremont G, Davis JD, Papandonatos GD, et al. Psychosocial telephone intervention for dementia caregivers: A randomized, controlled trial Alzheimer's dement. 2015 May;11(5):541-8. doi: https://dx.doi.org/10.1016/j.jalz.2014.05.1752. PMID: 25074341.

29. Arango-Lasprilla JC, Panyavin I, Merchan EJH, et al. Evaluation of a group cognitive-behavioral dementia caregiver intervention in Latin America. American Journal of Alzheimer's Disease and Other Dementis.
http://dx.doi.org/10.1177/1533317514523668. PMID: 2014-38564-010.

30. Livingston G, Barber J, Rapaport P, et al. START (STrAtegies for RelaTives) study: a pragmatic randomised controlled trial to determine

the clinical effectiveness and cost-effectiveness of a manual-based coping strategy programme in promoting the mental health of carers
people with dementia. Health Technol Assess. 2014 Oct;1816161:1-242. doi: https://ddx.doi.org/10.3310/tha18610. PMID: 25300037.

31. Martin-Carrasco M, Dominguez-Panchon AI, Gonzzalez-Fraile E, et al. Effectiveness of a psychoeducational intervention group program in
the reduction of the burden experienced by caregivers of patients with dem entia: the EDUCA-II randomized trial. Alzheimer Dis A ssoc Disord the reduction of the burden experienced by caregivers of patients with dementia: the EDUCA-II randomiz 32. Passoni S, Moroni L, Toraldo A, et al. Cognitite behavioral group intervention for Alzheimer caregivers. Alzheimer Dis Assoc Disord.
2014;28(3):275-82. di: 10.1097/WAD.000000000000033. PMID: 107799151. Language: English. Entry Date: 20150508. Revision Date: 20150712. Publication Type: Journal Article.

33. family caregivers: an assessor-blinded randomized controlled trial. Dement Geriatr Cogn Dis Extra. 2013;3(1):386-97. doi:

34ttps://dx.doi.org/10.1159/000355912. PMID: 24348500. behaviors: a randomized controlled trial. Am J Geriatr Psychiatry. 2013 Nov;21(11):1060-70. doi: https://dx.doi.org/10.1016/j.jagp.2012.09.009

35. Judge KS, Yarry SJ, Looman WJ, et al. Improved Strain and Psychosocial Outcomes for Caregivers of Individuals with Dementia: Finding 36. Kajivama B, Thompson LW, Eto-Iwase T, et al. Exploring the effectiveness of an internet-based program for reducing caregiver distress using the iCare Stress Management e-Training Program.[Erratum appears in Aging Ment Health. 2013;17(5):c1]. Aging Ment Health.

2013;17(5):544-54. doi: https:///dx.doi.org/10.1080/13607863.2013.775641. PMID: 23461355.

37. Kuo LM, Huang HL, Huang HL, et al. A home-based training program improves Taiwanese family caregivers' quality of life and decreases https://dx.doi.org/10.1002/gps.3853. PMID: 22778053

38. Moore RC, Chattillion EA, Ceglowski J, et al. A randomized clinical trial of Behavioral Activation (BA) therapy for improving psychological and physical health in dementia caregivers: results of the Pleasant Events Program (PEP). Behav Res Ther. 2013 Oct;51(10):62332. doi: https://dx.doi.org/10.1016/i.brat.2013.07.005. PMID: 23916631.

39. Joling KJ, van Marwijk HW, Smit F, et al. Does a family meetings intervention prevent depression and anxiety in family caregivers of dementia patients? A randomized trial. PLoS ONE. 2012;7(1):e30936. doi: https:///dx.doi.org/10.1371/journal.pone.0030936. PMII: 2230
40. Waldorff FB, Buss DV, Eckermann A, et al. Efficacy of psychosocial intervention in patients with mild Alzheimer's disease: the multicentre, rater blinded, randomised Danish Alzheimer Intervention Study (DAISY). Bmj. 2012 Jul 17;345:e4693. do :

https://dx.doi.org/10.1136/bmj.e4693. PMID: 22807076.

41. Chu H, Yang CY, Liao YH, et al. The effects of a support group on dementia caregivers' burden and depression. Journal of aging an

42. de Rotrou J, Cantegreil I, Faucounau V, et al. Do patients diagnosed with Alzheimer's disease benefit from a psycho-educational https://dx.doi.org/10.1002/gps.2611. PMID: 20922772.

43. Guerra M, Ferri CP, Fonseca M, et al. Helping carers to care: the 10/66 dementia research group's randomized control trial of a caregive

intervention in Peru. Rev Bras Psiquiatr. 2011 Mar;33(1):47-54. PMID: 20602013.
44. Losada A, Marquez-Gonzalez M, Romero-Moreno R. Mechanisms of action of a psychological intervention for dementia caregivers: effects of behavioral activation and modification of dysfunctional thoughts. Int J Geriatr Psychiatry. 2011 Nov;26(11):1119-27. doi

45. Spijker A, Wollersheim H, Teerenstra S, et al. Systematic care for caregivers of patients with dementia: a multicenter, cluster-randomized, controlled trial. Am J Geriatr Psychiatry. 2011 Jun;19(6):521-31. doi: https://dx.doi.org/10.1097/JGP.0b013e3182110599. PMID: 21358385. 46. Voigt-Radloft S, Graff M, Leonhart R, et al. A multicentre RCT on comminity occupatg7h 
47. Wang LQ, Chien WT. Randomised controlled trial of a family-led mutual support programme for people with dementia. J Clin Nurs. 2011 Aug;20(15-16):2362-6. doi: https:///dx.doi.org/10.1111/j.1365-2702.2011.03746.x. PMID: 21752121,

(3) family Sep;24(3):115-25. doi 49. Gitlin LN, Winter L, Dennis MP, et al. Targeting and managing behavioral symptoms in individuals with dementia: a randomized trial of a nonpharmacological intervention. J Am Geriatr Soc. 2010 Aug;58(8):1465-74. doi: https://dx.doi.org/10.1111/1.1532-5415.2010.02971.x. PMID: 20662955.

0. Gitlin LN, Winter L, Dennis MP, et al. A biobehavioral home-based intervention and the well-being of patients with dementia and their

0.1001/jama.2010.1253. PMID: 105093294. Language: English. Entry Date: 20100917. Revision Date: 20161116. Publication Type: journal article. Pychiz A, Wagenpfeil S, Hallauer J, et al. Evaluation of a brief educational program for denentia carers: the AENEAS study. Int $J$ Geria Psychiatry. 2010 Aug;25(8):861-9. doi: https://dx.doi.org/10.1002/gps.2428. PMID: 19946869.

Pisk and Improve Psychological and Physica Well-Being in Alzheimer's Disease Family Caregivers. Psychosom Med. 2010;72(9):897-904. doi: 10.1097/PSY.0b013e3181fc2d09. PMID:

caregiver intervention in Russia. Int J Geriatr Psychiatry. 2009 Apr;24(4):347-54. doi: https://dx.doi.org/10.1002/gps.2126. PMID: 18814197. 54. Martin-Carrasco M, Martin MF, Valero CP, et al. Effectiveness of a psychoeducational intervention program in the reduction of caregiver PMID: 18949763.
55 .

effect after 6 and 12 months. Scand J Caring Sci. 2008;22(1):98-109. doi: 10.1111/j.1471-6712.2007.00498.x. PMID: CN-00630033. 56. Gallagher-Thompson D, Gray HL, Dupart T, et al. Effectiveness of Cognitive/Behavioral Small Group Intervention for Reduction of Depression and Stress in Non-Hispanic White and Hispanic/Latino Women Dementia Family Caregivers: Outcomes and Mediators of Change
Journal of Rational-Emotive \& Cognitive Behavior Therapy. 2008 Dec 01;26(4):286-303. PMID: 25067886.

(a) 58. Ustein ID, Sandvik L, Wyller TB, et al. A one-year randomized controlled psychosocial intervention study among family carers of 59. Gonyea JG, O'Connor MK, Boyle PA. Project CARE: a randomized controlled trial of a behavioral intervention group for Alzheimer's disease caregivers. Gerontologist. 2006 De, 4 (1) 827-32. PMID: 17169938.

2006 Dec 09:333(7580):1196. PMID: 17114212 .

61. Beauchamp N, Irvine AB, Seeley J, et al. Worksite-based

62. Farran CJ, Gilley DW, McCann JJ, et al. Psychosocial interventions to reduce depressive symptoms of dementia caregivers: A randomized

63. Burgio L, Stevens A, Guy D, et al. Impact of two psychosocial interventions on white and African American family caregivers of

individuals with dementia. Gerontologist. 2003 Aug; ;3(4):568-79. PMID: 12937335.

study. Gerontologist. 2003 Aug;;3(4):547-55. PMID: 12937333.

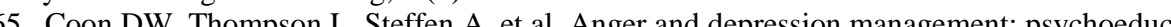

caregivers of a relative with dementia. Gerontologist. 2003 Oct; $43(5)$ : 678-89. PMID: 14570964.

作 relatives wit.

67. Gitlin LN, Winter L, Corcoran M, et al. Effects of the home environmental skill-building program on the caregiver-care recipient dyad: 6-

68. Hebert R, Levesque L, Vezina J, et al. Efficacy of a psychoeducative group program for caregivers of demented persons living at home: a Fized controlled trial. J Gerontol B Psychol Sci Soc Sci. 2003 Jan;58(1):S58-67. PMID: 12496309.

2002;16(3):134-44. PMID: 106975399 . of a mutual support group for family caregivers of a relative with dementia. Arch Psychiatr Nurs. 70. Stolley JM, Reed D, Buckwalter KC. Caregiving appraisal and interventions based on the progressively lowered stress threshold model. Am

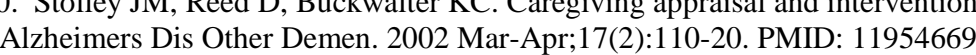
71. Wright LK, Litaker M, Laraia MT, et al. Continumum of care for Alzheimer's disease: a nurse education and counseling program. Issues
Ment Health Nurs. 2001 Apr-May;22(3):231-52. PMID: 11885210.

72. Buckwalter KC, Gerdner L, Kohout F, et al. A nursing inte
Arch Psychiatr Nurs. 1999 Apr;13(2):80-8. PMID: 10222636.

3. Chang BL. Cognitive-behavioral intervention for homebound caregivers of persons with dementia. Nurs Res. 1999 May-Jun;48(3):173-82 MID: 10337848.
74. Ostwald SK, Hepburn KW, Caron W, et al. Reducing caregiver burden: a randomized psychoeducational intervention for caregivers of persons with der, He

(2) Gep 23;13:96. doi: https://dx.doi.org/10.1186/1471-231873.96. PMID: 24060106 . A thematic analysis. Dementia. 2015 Nov;14(6):842-59. doi: https://dx.doi.org/10.1177/1471301213516332. PMID: 24381218 77. Charlesworth G, Burnell K, Beecham J, et al. Peer support for family carers of people with dementia, alone or in combination with grou

78. Cheng ST, Chan WC, Lam LCW. Long-Term Outcomes of the Benefit-Finding Group Intervention for Alzheimer Family Caregivers: A Cluster-Randomized Double-Blind Controlled Trial. Am J Geriatr Psychiatry. 2019 Sep;27(9):984-94. doi:

https://dx.doi.org/10.1016/1/.jagp.2019.03.013. PMID: 31076215. Outcomes of a Randomized Double-Blind Controlled Trial. Journals of Gerontology Series B Psychological Sciences \& Social Sciences. 2019
Sep 26;26:26. doi: https://dx.doi.org/10.1093/geronb/gbz118. PMID: 31556447.
80. Cheng ST, Lau RW, Mak EP, et al. Benefit-finding intervention for Alzheimer caregivers: conceptual framework, implementation issues, and preliminary efficacc. Gerontologist. 2014 Dec; $54(6): 1049-58$. doi: htpps://dx.doi.org/10.1093/geront/gnu018. PMID: 24688081.
81. Laakkonen ML, Savikko N, Holtta E, et al. Self-management groups for people with dementia and their spousal caregivers. A randomized, controlled trial. Baseline findings and feasibility. European Geriatric Medicine. 2013 December;4(6):389-93. do

http://dx.doi.org/10.1016/.eurger.2013.09.006. PMID: 52813196.

. 13-133. PMID: 22871107.

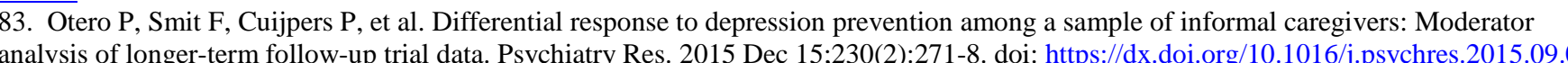
PMID: 26456895. 84. Tremont G, Davis JD, Ott BR, et al. Randomized Trial of the Family Intervention: Telephone Tracking-Caregiver for Dementia Caregivers Use of Com 28008609 .

85. Tremont G, Davis J, Papandonatos GD, et al. A telephone intervention for dementia caregivers: background, design, and baseline characteristics. Contemp Clin Trials. 2013 Nov;36(2):338-47. doi: https://dx.doi.org/10.1016/i.cct.2013.07.011. PMID: 23916916. 86. Voigt-Radloff S, Graff M, Leonhart R, et al. Why did an effective Dutch complex psycho-social intervention for people with dementia not
work in the German healthcare context? Lessons learnt from a process evaluation alongside a multicentre RCT BMJ Open. 2011;1 (1) (10 pagination)(e000094). doi: http://dx.doi.org/10.1136//mjopen-2011-000094. PMID: 611053874.

87. Voigt-Radloff S, Graff M, Leonhart R, et al. WHEDA study: Effectiveness of occupational therapy at home for older people with dementia and their caregivers - The design of a pragmatic randomised controlled trial evaluating a Dutch programme in seven German centres. BMC geriatr. 2009;9 (1) (no pagination)(44). doi: http:///dx.doi.org/10.1186/1471-2318-9-44. PMID: 355485226.

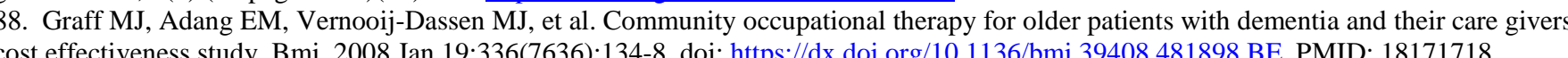
cost effectiveness study. Bmj. 2008 Jan 19;336(7636):134-8. doi: https://dx.doi.org/10.1136/bmj.39408.481898. BE. PMID: 18171718.
89. Graff MJ, Vernooij-Dassen MJ, Thiijsen M, et al. Effects of community occupational therapy on quality of life, mood, and health status dementia patients and their caregivers: a randomized controlled trial. J Gerontol A Biol Sci Med Sci. 2007 Sep;62(9):1002-9. PMID: 17895439 90. Farran CJ, Gilley DW, McCann JJ, et al. Efficacy of behavioral interventions for dementia caregivers. West J Nurs Res. 200

91. Farran CJ, Loukissa D, Perraud S, et al. Alzheimer's disease caregiving information and skills. Part II: family caregiver issues and concerns

Rabinowitz YG, Mausbach BT, Coon DW, et al. The moderating effect of self-efficacy on intervention response in women family caregivers of older adults with dementia. Am J Geriatr Psychiatry. 2006 Aug;14(8):642-9. PMID: 16861368.

caregivers and on daily function of persons with dementia. Gerontologist. $2001 \mathrm{Feb} ; 41(1): 4-14$. PMID: 11220813 effect on efficacy and upset 94. Voigt-Radloff S, de Werd MM, Leonhart R, et al. Structured relearning of activities of daily living in dementia: the randomized controlled

PMID: 28335810 .
95. Meichsner F, Theurer C, Wilz G. Acceptance and treatment effects of an internet-delivered cognitive-behavioral intervention for family caregivers of people with dementia: A randomized-controlled trial. J Clin Psychol. 2019 Apr;75(4):594-613. do:

https://dx.doi.org/10.1002/jiclp.22739. PMID: 30597537

作 97. Ta Park VM, Ton V, Tiet QQ, et al. Promising results from a pilot study to reduce distress in Vietnamese American dementia and memory loss caregivers. Alzheimers Dement (N Y). 2019;5:319-27. doi: htttps://dx.doi.org/10.1016/j.trci.2019.05.006. PMID: 31384663 

98. Ta Park VM, Ton V, Yeo G, et al. Vietnamese American Dementia Caregivers' Perceptions and Experiences of a Culturally Tailored,
Evidence-Based Program to Reduce Stress and Depression. J Gerontol Nurs. 201901 Sep;45(9):39-50. doi: http://dx.doi.org/10.3928/0098913420190813-05. PMID: 629157769.

Femia EE, et al. The Support, Health, Activities, Resources, and Education program for early stage dementia 29171296. 100. Yoo R, Yeom J, Kim GH, et al. A Multicenter, Randomized Clinical Trial to Assess the Efficacy of a Therapeutic Intervention Program for Caregivers of People with Dementia. J Clin Neurol. 2019 Apr;15(2):235-42. doi: https://dx.doi.org/10.3988/jcn.2019.15.2.235. PMID:

101. Boots LM, de Vugt ME, Kempen GI, et al. Effectiveness of the blended care self-management program "Partner in Balance" for earlystage dementia caregivers: study protocol for a randomized controlled trial. Trials. 2016 May 04;17(1):231. doi

102. Boots LM, de Vugt ME, Smeets CM, et al. Implementation of the Blended Care Self-Management Program for Caregivers of People Wi Early-Stage Dementia (Partner in Balance): Process Evaluation of a Randomized Controlled Trial. J Med Internet Res. 2017 De

9;19(12):e423. doi: https://dx.doi.org/10.2196/jmir.7666. PMID: 29258980.

103. de Oliveira AM, Radanovic M, Homem de Mello PC, et al. An intervention to reduce neuropsychiatric symptoms and caregiver burden in 23;23:23. doi: https://dx.doi.org/10.1002/gps.4958. PMID: 30035341

andomized controlled trial. BMC geriatr. 201805 10;18(1):113. doi: https://dx.doi.org/10.1186/s12877-018-0801-8 P. PMDia: a pilot

Meichsner F, Theurer C, Wilz G. Acceptance and treatment effects of an internet-delivered cognitive-behavioral intervention for family caregivers of people with dementia: A randomized-controll
https://dx.doi.org/10.1002/iclp.22739. PMID: 30597537.

106. Novelli M, Machado SCB, Lima GB, et al. Effects of the Tailored Activity Program in Brazil (TAP-BR) for Persons With Dementia: A Randomized Pilot Trial. Alzheimer Dis Assoc Disord. 2018 Apr 25;25:25. doi: https://dx.doi.org/10.1097/WAD.0000000000000256. PMID 29698251

107. van Knippenberg RJM, de Vugt ME, Ponds RW, et al. An Experience Sampling Method Intervention for Dementia Caregivers: Results of

108. Meichsner F, Topfer NF, Reder M, et al. Telephone-Based Cognitive Behavioral Intervention Improves Dementia Caregivers' Quality of Life. American Journal of Alzheimer's Disease and other Dementias. 2019. doi: http://dx.doi.org/10.1177/1533317518822100. PMID: 626130265.

109. Topfer NF, Wilz G. Tele.Tandem increases the psychosocial resource utilization of dementia caregivers. GeroPsych: The Journal of 110. Lok N, Bademli K. Pilot testing of the "First You Should Get Stronger" program among caregivers of older adults with dementia. Arch Gerontol Geriatr. 2017 Jan - Feb;68:84-9. doi: https://dx.doi.org/10.1016/j.archger.2016.09.006. PMID: 27689315, 111. O'Connor CM, Clemson L, Brodaty H, et al. The tailored activity program (TAP) to address behavioral disturbances in frontotempor dementia: a feasibility and pilot study. Disabil Rehabil. 2017 Oct 15:1-12. doi: https://dx.doi.org/10.1080/09638288.2017.1387614. PMID: Evaluation Using the Meaning of Loss Codebook. J Gerontol Soc Work. 2017 Feb-Mar;60(2):155-69. dor https:///dx.doi.org/10.1080/01634372.2016.1274930. PMID: 28051926.

13. Nunez-Naveira L, Alonso-Bua B, de Labra C, et al. UnderstAID, an ICT Platform to Help Informal Caregivers of People with Dementis A Pilot Randomized Controlled Study. Biomed Res Int. 2016;2016:5726465. doi: https://dx.doi.org/10.1155/2016/5726465. PMID: 28116300. 114. Sepe-Monti M, Vanacore N, Bartorelli L, et al. The Savvy Caregiver Program: A Probe Multicenter Randomized Controlled Pilot Trial in
Caregivers of Patients Affected by Alzheimer's Disease. J Alzheimers Dis. 2016 10 04;54(3):1235-46. PMID: 2757824. 115. Zauszniewski $J$, Lekhak N, Burant $C J$, et al. Preliminary Evidence for Effectiveness of Resourcefulness Training in

Caregivers. J Fam Med. 2016;3(5). PMID: 27500286.

116. Zauszniewski JA, Lekhak N, Yolpant W, et al. Need for Resourcefulness Training for Women Caregivers of Elders with Dementia. Issues

117. Au A, Gallagher-Thompson D, Wong MK, et al. Behavioral activation for dementia caregivers: scheduling pleasant events and enhancin

118. Au A. Developing Volunteer-Assisted Behavioral Activation Teleprograms to Meet the Needs of Chinese Dementia Caregivers. Clin
Gerontol. 2015;38(3):190-202. PMID: CN-01071414. 作 MID: 23305463.

20. Pot AM, Blom MM, Willemse BM. Acceptability of a guided self-help Internet intervention for family caregivers: mastery over dementia. Int Psychogeriatr. 2015 Aug;27(8):1343-54. doi: https://dx.doi.org/10.1017/S1041610215000034. PMID: 25648589.

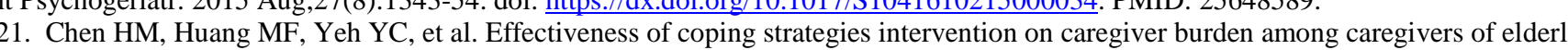

patients with dementio. Psychogeriatrics. 2015 Mar:15(1):20-5. doi: https://dx.doi.org/10.1111/psyg.12071. PMID: 25515800.
122. Cristancho-Lacroix V, Wrobel J, Cantegreil-Kallen I, et al. A web-based psychoeducational program for informal caregivers of patients Med Internet Res. 2015 May 12;17(5).e117. doi: https://dx.doi.org/10.2196//mir.3717. PMID: 25967983,

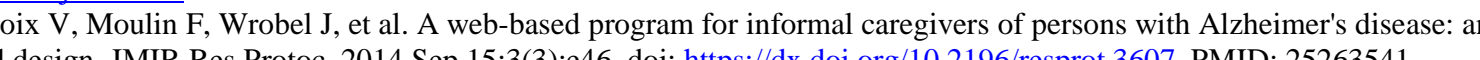
iterative user-cented d24. Gaugler JE, Reese M, Sauld J. A Pilot Evaluation of Psychosocial Support for Family Caregivers of Relatives with Dementia in L Term Care: The Residential Care Transition Module. Res. 2015 Jul-Aug;8(4):161-72. doi: https://dx.doi.org/10.3928/19404921-20150304-01 PMID: 25751083.

125. Kamkhagi D, Costa A, Kusminsky S, et al. Benefits of psychodynamic group therapy on depression, burden and quality of life of family 01162210 .

126. Paun O, Farran CJ, Fogg L, et al. A chronic grief intervention for dementia family caregivers in long-term care. West J Nurs Res. 2015 Jan;37(1):6-27. doi: https://dx.doi.org/10.1177/0193945914521040. PMID: 24510968.

127. Aboulafia-Brakha T, Suchecki D, Gouveia-Paulino F, et al. Cognitive-behavioural group therapy improves a psychophysiological marker of stress in caregivers of patients with Alzheimer's disease. Aging $M$

128. Dowling GA, Merrilees J, Mastick J, et al. Life enhancing activities for family caregivers of people with frontotemporal dementia. Alzheimer Dis Assoc Disord. 2014 Apr-Jun;28(2):175-81. doi: https://dx.doi.org/10.1097/WAD.0b013e3182a6b905. PMID: 24113564. 129. Gonzalez EW, Polansky M, Lippa CF, et al. Enhancing resourcefulness to improve outcomes in family caregivers and persons with
Alzheimer's disease: a pilot randomized trial. Int J Alzheimer Dis. 2014;2014:323478. doi: https://dx.doi.org/10.1155/2014/323478. PMID:

130. Judge KS, Yarry SJ, Orsulic-Jeras S. Acceptability and feasibility results of a strength-based skills training program for dementia caregiving dyads. Gerontologist. 2010 Jun;50(3):408-17. doi: https://dx.doi.org/10.1093/geront/gnp138. PMID: 19808841.

caregivers. In Gerontologis

132. Kuo LM, Huang HL, Liang J et a A andomized con caregivers of persons with dementia J J Adv Nurs. 2017 Mar-73(3):585-98. doi: https://dx.doi.org/10.1111/jan.13157. PMID: 27653753 L of vascular dementia and alzheimer's disease patients. Den.
http://dx.doi.org/10.1159/000360414. PMID: 603852835.

134. Kwok T, Wong B, Ip I, et al. Telephone-delivered psychoeducational intervention for Hong Kong Chinese dementia caregivers: a singleblinded randomized controlled trial. Clin Interv Aging. 2013;8:1191-7. doi: https://dx.doi.org/10.2147/CIA.S48264. PMID: 24072965,

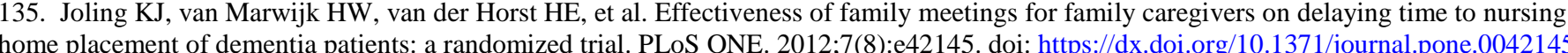
PMID: 22876304.

136. Joling KJ, Bosmans JE, in family caregivers of patients with dementia: a randomized trial. Trials. 2013 Sep 22;14:305. doi: https://dx.doi.org/10.1186/1745-6215-14305. PMID: 2405363

symptoms and disordeut HP, Scheltens P, et al. (Cost)-effectiveness of family meetings on indicated prevention of anxiety and depressive symptoms and disorders of primary family caregivers of patients with dem.

138. Davis JD, Tremont G, Bishop DS, et al. A telephone-delivered psychosocial intervention improves dementia caregiver adjustment following nursing home placement. Int J Geriatr Psychiatry. 2011 Apr;26(4):380-7. doi: https://dx.doi.org/10.1002/gps.2537. PMID: 20842759. 139. PMID: 19500421.

140. Spijker A, Teerenstra S, Wollersheim H, et al. Influence of adherence to a systematic care program for caregivers of Am J Geriatr Psychiatry. 2013 Jan;21(1):26-36. doi: https://dx.doi.org/10.1016jijagp.2012.10.003. PMID: 23290200

141. Meichsner F, Schinkothe D, Wilz G. Managing Loss and Change: Grief Interventions for Dementia Caregivers in a CBT-Based Trial. An

142. Meichsner F, Wilz G. Dementia caregivers' coping with pre-death grief: effects of a CBT-based intervention. Aging Ment Health. 2018 (2):218-25. doi: https://dx.doi.org/10.1080/13607863.2016.1247428. PMID: 27792398.

Kong. In Patient education and counseli
Kong

144. Gallagher-Th Chinese American dementia caregivers: results of a preliminary study. Aging Ment Health. 2010 Apr;14(3):263-73. doi

MID: 359062574

caregivers: the COPE randomized trial. Jama. 2010 Sep 01;304(9):983-91. doi: https:///dx doi org 110 10 1001/jama.2010.1253 PMID: 2081037 146. Dias A, Dewey ME, D'Souza J, et al. The effectiveness of a home care program for supporting caregivers of persons with dementia in https://dx.doi.org/10.1371/journal.pone.0002333. PMID: 18523642. 
147. Gitlin LN, Winter L, Burke J, et al. Tailored activities to manage neuropsychiatric behaviors in persons with dementia and reduce caregiver burden: a randomized pilot study. Am J Geriatr Psychiatry. 2008 Mar;16(3):229-39. do

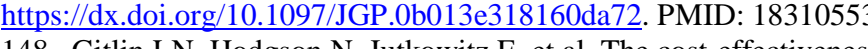

of a nonpharmacologic intervention for individuals with dementia and family caregivers: the tailored activity program. Am J Geriatr Psychiatry. 2010 Jun;18(6):510-9. doi:

https:///dx.doi.org/10.1097/JGP.0b013e3181c37d13. PMID: 20847903. feasibility, acceptability, and replication potential. Gerontologist. 2009 Jun;49(3):428-39. doi: https://dx.doi.org/10.1093/geront/gnp087. PMID

150. Tremont G, Davis JD, Bishop DS, et al. Telephone-delivered psychosocial intervention reduces burden in dementia caregivers. Dementia: The International

151. Epstein-Lubow G, Davis JD, Miller IW, et al. Persisting burden predicts depressive symptoms in dementia caregivers. J Geriatr Psychiatry Neurol. 2008 Sep;21(3): 1988-203. doi: https://dx.doi.org/10.11777/0891988708320972. PMID: 18838742.

152. Gant JR, Steffen AM, Lauderdale SA. Comparative outcomes of two distance-based interventions for male caregivers of family members

with dementia. Am J Alzheimers Dis Other Demen. 153 . Glueckauf R, Sharma D, Davis W, et al. Telephone-based cognitive-behavioral intervention for distressed rural dementia caregivers: initial

findings. In Clin Gerontol

154. Rodriguez-Sanchez E, Patino-Alonso MC, Mora-Simon S, et al. Effects of a psychological intervention in a primary health care center for caregivers of dependent relatives: a randomized trial. Gerontologist. 2013 Jun;53(3):397-406. doi: https://dx.doi.org/10.1093/geront/gns086.
PMID 22899425 PMID: 22899425.

155. Akkerman RL, Ostwald SK. Reducing anxiety in Alzheimer's disease family caregivers: the effectivenes
behavioral intervention. Am J Alzheimers Dis Other Demen. 2004 Mar-Apr;19(2):117-23. PMID: 15106393.

156. Nobili A, Riva E, Tettamanti M, et al. The effect of a structured intervention on caregivers of patients with dementia and problen

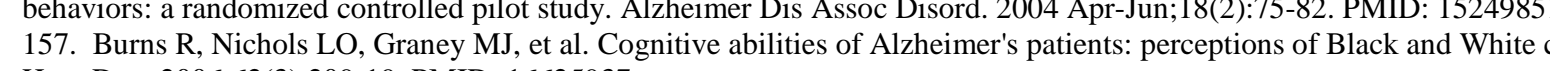

Hum Dev. 2006.62(3):209-19. PMID: 16625937.

158. Holland JM, Currier JM, Galloger-To37.

mes from the Resources for Enhancing Alzheimer's Caregiver Health (REACH) 159. Gitlin LN, Hauck WW, Dennis MP, et al. Maintenarce of effects of the home environiment 10.1037/a0014303. PMID: 19290751. and individuals with Alzheimer's disease and related disorders. J Gerontol A Biol Sci Med Sci. 2005 Mar;60(3):368-74. PMID: 15860476. 160. Chee YK, Gitlin LN, Dennis MP, et al. Predictors of adherence to a skill-building intervention in dementia caregivers. J Gerontol A Bio
Sci Med Sci. 2007 Jun;62(6):673-8. PMID: 17595426 . 161. Hilgeman MM, Allen RS, Decoster J et al. Positive Aging. 2007;22(2):361-71. PMID: 105991138. Language: English. Entry Date: 20080222 Revision Date: 20161222. Publication Type: journal article. 162. Lavoie JP, Ducharme F, Levesque L, et al. Understanding the outcomes of a psycho-educational group intervention for caregivers of
persons with dementia living

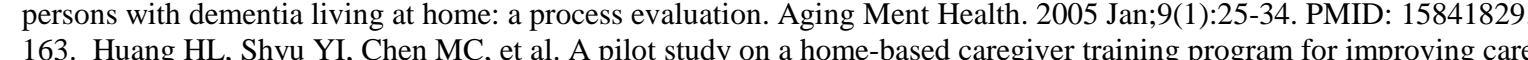

163. Huang HL, Shyu YI, Chen MC, et al. A pilot study on a home-based caregiver training program for improving caregiver self-efficacy and decreasing the behavioral problems of elders with dementia in Taiwan. Int J Geriatr Psychiatry. 2003 Apr; 18(4):337-45. PMID: 12673611.
164. Martin-Cook K, Remakel-Davis B, Svetlik D, et al. Caregiver attribution and resentment in dementia care. Am J Alzheimers Dis Othe Demen. 2003 Nov-Dec;18(6):366-74. PMID: 14682086.

165. Garand L, Buckwalter KC, Lubaroff D, et al. A pilot study of immune and mood outcomes of a community-based intervention for dementia caregivers: the PLST intervention. Arch Psychiatr Nurs. 2002 Aug;16(4):156-67. PMID: 12143075.

166. Marriott A, Donaldson $\mathrm{C}$, Tarrier $\mathrm{N}$, et al. Effectiveness of cognitive-behavioural family intervention in reducing the burden of care in

167. Steffen AM. Anger management for dementia caregivers: A preliminary study using video and telephone interventions. Behavior Therapy.

2000 Spr;31(2):281-99. doi: http:///dx.doi.org/10.1016/S0005-7894\%2800\%2980016-7. PMID: 2000-16043-005.

168. Gerdner LA, Buckwalter KC, Reed D. Impact of a psychoeducational intervention on caregiver response to behavioral problems. Nurs

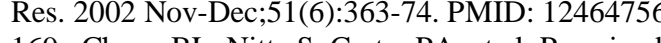

169. Chang BL, Nitta S, Carter PA, et al. Perceived helpfulness of tel
dementia. J Gerontol Nurs. 2004 Sep;30(9):14-21. PMID: 15471059.

170. McCurry SM, Logsdon RG, Vitiello MV, et al. Successful behavioral treatment for reported sleep problems in elderly caregivers of

dementia patients: a controlled study. J Gerontol B Psychol Sci Soc Sci. 1998 Mar;53(2):P122-9. PMID: 9520929.
171. Moniz-Cook E, Agar S, Gibson G, et al. A preliminary study of the effects of early intervention with people with dementia and their

Date: 20150711. Publication Type: Journal Article.

172. Brodaty H, Gresham M, Luscombe G. The Pr

Feb;12(2):183-92. PMID: 9097211.

173. Teri $\mathrm{L}$, Logsdon RG, Uomoto J, et al. Behavioral treatment of depression in dementia patients: a controlled clinical trial. J Gerontol

Psychol Sci Soc Sci. 1997 Jul;52(4):P159-66. PMID: 9224439.
174. Gendron C, Poitras L, Dastoor DP, et al. Cognitive-behavioral group intervention for spousal caregivers: Findings and clinical :i//dx.doi.org/10.1300/J018v17n01 02. PMID: 26250550. 175. Hebert R, Leclerc G, Bravo G, et al. Efficacy of a support group programme for care-givers of demented patients in the community: A 4943\%2894\%2990043-4. PMID: 24087060.

176. Hebert R, Girouard D, Leclerc G, et al. The impact of a support group programme for care-givers on the institutionalisation of demented patients. Archives of Gerontology and Geriatrics. 1995;20(2):129-34. doi: http://dx.doi.org/10.1016/0167-4943\%2894\%2900590-4. PMID: 25107702.

177. Tappen RM. The effect of skill training on functional abilities of nursing home residents with dementia. Res Nurs Health. 1994

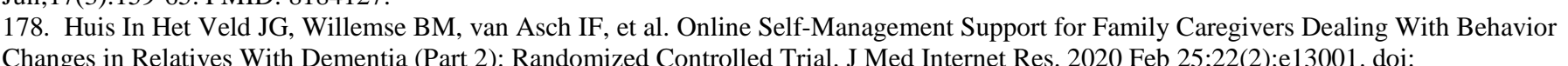
https://dx.doi.org/10.2196/13001. PMID: 32130142 .

179. Van Mierlo LD, Meiland FJ, Van de Ven PM, et al. Evaluation of DEM-DISC, customized e-advice on health and social support services for informal carers and case managers of people with dementia; a c
httrs://dx. doi.org/10 1017/S1041610215000423. PMID: 25872457.

https://dx.doi.org/10.1017//10416I02 An experimental study on the effectiveness of a mutual support group for family caregivers of a relative with dementia in mainland China. Contemp Nurse. 2012 Feb;40(2):210-24. doi: https://dx.doi.org/10.5172/conu.2012.40.2.210. PMID: 22554214.

181. Logsdon RG, Pike KC, McCurry SM, et al. Early-stage memory loss support groups: outcomes from a randomized controlled clinical trial Journals of Gerontology Series B: Psychological Sciences \& Social Sciences. 2010;65B(6):691-7. doi: gerorobl/b054. PMID; 104963362 .

182. Charlesworth $G$, Shepstone $L$, Wilson $E$, et al. Does befriending by trained lay workers improve psychological well-being and quality of life for carers of people with dementia, and at what cost? A randomised controlled trial. Health Technol Assess. 2008 Mar; 12(4):iii, v-ix, 1-78. PMID: 18284895 .

183. Charlesworth G, Shepstone L, Wilson E, et al. Befriending carers of people with dementia: randomised controlled trial. Bmj. 2008 Jun 07:336(7656):1295-7. doi: https://dx.doi.org/10.1136//mi.39549.548831.AE. PMID: 18505757.
184. Wilson E Thalanany M, Shepstone L, et al. Befriending carers of people with dementia:

列 $J$ Geriatr Psychiatry.

185. Winter L, Gitlin LN. Evaluation of a telephone-based support group intervention for female caregivers of community-dwelling individuals with dementia. Am J Alzheimers Dis Other Demen. 2006 Dec-2007 Jan;21(6):391-7. PMID: 17267370.

186. Mahoney DF, Tarlow BJ, Jones RN. Effects of an automated telephone support system on caregiver burden and anxiety: findings from the

vention for caregivers of people with

Alzheimer's disease. J Telemed Telecare. 2001;7(3):139-48. PMID: 11346473.
18illemer K, Suitor JJ. Peer support for Alzheimer's caregivers: Is it enough to make a difference? Research on Aging. 2002;24(2):171-92.

PMID: 34163761 . 189 . Goodman C. Evaluation of a model self-help telephone program: impact on natural networks. Soc Work. 1990 Nov;35(6):556-62. PMID:

190. Gustafson DH, Cody OJ, Chih MY, et al. Pilot Test of a Computer-Based System to Help Family Caregivers of Dementia Patients. Alzheimers Dis. 2019;70(2):539-50. doi: http://dx.doi.org/10.3233/JAD-190052. PMID: 628746285.

191. Metcalfe A, Jones B, Mayer J, et al. Online information and support for carers of people with young-onset dementia: A multi-site randomised co

192. Torkamani M McDonald L Saez Aguayo I et al. A randomized controlled pilot study to evaluate a technology platform for the assisted 192. Torkamani $\mathrm{M}$, M 24643137.

193. van der Roest HG, Meiland FJ, Jonker C, et al. User evaluation of the DEMentia-specific Digital Interactive Social Chart (DEM-DISC). A pilot study among informal carers on its impact, user friendliness and, usefulness. Aging Ment Health. 2010 May;14(4):461-70. doi: https:///dx.doi.org/10.1080/13607860903311741. PMID: 20455122.
194. Salamizadeh AMS, Mirzaei TP, Ravari AP. The Impact of Spiritual Care Education on the Self-Efficacy of the Family Caregivers of Elderly P

195. Farran CJ, Etkin CD, Eisenstein A, et al. Effect of Moderate to Vigorous Physical Activity Intervention on Improving Dementia Family Caregiver Physical Function: A Randomized Controlled Trial. J Alzheimers Dis Parkinsonism. 2016 Aug;6(4). doi:

https://dx.doi.org/10.4172/2161-0460.1000253. PMID: 28752016.

Family Carregsers of Persons with, et al. Impact of an Individualized Physical Activity Intervention on Improving Mental Health Outcomes in https://dx.doi.org/10.3934/medsci.2016.1.15. PMID: 29147683.

197. Cothran FA, Paun O, Barnes LL, et al. Comparing the Effect of a Moderate Physical Activity Intervention on the Mental Health Outcomes of African American and Caucasian Dementia Family Caregivers: A Secondary Data Analysis. Issues Ment Health Nurs. 2017 Dec;38(12):996-
1004. doi: https://dx.doi.org/10.1080/01612840.2017.1364807. PMID: 28956706. 
198. Mahdavi B, Fallahi-Khoshknab M, Mohammadi F, et al. Effects of spiritual group therapy on caregiver strain in home caregivers of the elderly with Alzheimer's disease. Arch Psychiatr Nurs. 2016. PMID: CN-01308784.

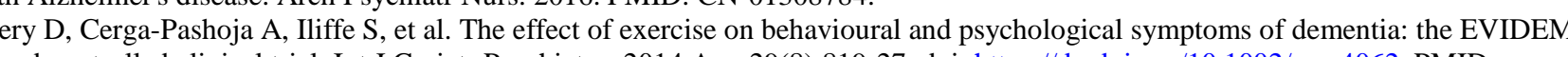
29(8):819-27. doi: https://dx.doi.org/10.1002/gps.4062. PMID:

200. D'Amico F, Rehill A, Knapp M, et al. Cost-effectiveness of exercise as a therapy for behavioural and psychological symptoms of dementia within the EVIDEM-E randomised controlled trial. Int J Geriatr Psychiatry. 2016 Jun;31(6):656-65. doi: https://dx.doi.org/10.1002/gps.4376. PMID: 26489776.

201. Whitebird RR, Kreitzer M, Crain AL, et al. Mindfulness-based stress reduction for family caregivers: a randomized controlled trial. Gerontologist. 2013 Aug;53(4):676-86. doi: https://dx.doi.org/10.1093/geront/gns126. PMID: 23070934.

Trial. J Appl Gerontol. 2009 Apr:28(2):171-94. PMID: 21709757. 2002 May-Jun;64(3):458-68. PMID: 12021419.

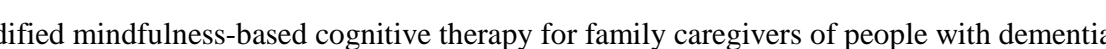
A pilot randomized controlled trial. Int J Nurs Stud. 2019 Oct;98:107-17. doi: https://dx.doi.org/10.1016/ji.ijnurstu.2019.02.020. PMID: 205. Richards AG, Tietyen AC, Jicha GA, et al. Visual Arts Education improves self-esteem for persons with dementia and reduces caregiver
burden: A r andomized controlled trial. Dementia. 2018 Jan 01:1471301218769071. doi: https:///dx.doi.org $10.11777 / 1471301218769071$. PMID

206. Waelde LC, Meyer H, Thompson JM, et al. Randomized Controlled Trial of Inner Resources Meditation for Family Dementia Caregivers. .

208. Hirano A, Umegaki H, Suzuki Y, et al. Effects of leisure activities at home on perceived care burden and the endocrine system of

caregivers of dementia patients: a randomized controlled study. Int Psychogeriatr. 2016 Feb;28(2):261-8. do:

https:///d.doi.org/10.1017/S1041610215001295. PMID: 26289496.

itation for the improvement of health and wellbeing in community-dwelling dementia

https://dx.doi.org/10.1186/s12906-015-0666-8. PMID: 25952550.

210. Leach MJ, Francis A, Ziaian T. Improving the health and well-being of community-dwelling caregivers of dementia sufferers: study protocol of a randomized controlled trial of structured meditation

ittps:///dx.doi.org/10.1089/acm.2013.0170. PMID: 24044373.

TRANSCENDENT Trial. Alzheimer Dis Assoc Disord. 2016 Oct-Dec;30(4):338-44. PMID: 27227995.

212. Lavretsky H, Epel ES, Siddarth P, et al. A pilot study of yogic meditation for family dementia caregivers with depressive symptoms: effects on mental health, cognition, and telomerase activity. Int J Geriatr Psychiatry. 2013 Jan;28(1):57-65. doi;

https://dx.doi.org/10.1002/gps.3790. PMID: 22407663

213. Carbonneau H, Caron CD, Desrosiers J. Effects of an adapted leisure education program as a means of support for caregivers of people
with dementia. Arch Gerontol Geriatr. 2011 Jul-Aug:53(1):31-9. doi: https://dx.doi.org/10 1016/jarchger 2010 06 009. PMID: 20598758 with dementia. Arch Gerontol Geriatr. 2011 Jul-Aug;53(1):31-9. doi: https://dx.doi.org/10.1016/.jarchger.2010.06.009. PMID: 20598758 . Complement Med. 2010 Oct;16(10):1031-8. doi: https://dx.doi.org/10.1089/acm.2009.0733. PMID: 20929380.

215. Wilz G, Fink-Heitz M. Assisted vacations for men with dementia and their caregiving spouses: evaluation of health-related effects. Gerontologist. 2008 Feb;48(1):115-20. PMID: 18381838.

216. Vandepitte S, Putman K, Van Den Noortgate N, et al. Effectiveness of an in-home respite care program to support informal dementia
caregivers: A comparative study. Int J Geriatr Psychiatry. 2019 Oct;34(10):1534-44. doi: https://dx.doi.org/10.1002/gps.5164. PMID: 31243801.20 217. Vandepitte S, Van Den Noortgate N, Putman K, et al. Effectiveness and cost-effectiveness of an in-home respite care program in
2. supporting informal caregivers of people with dementia: design of a comparative study. BMC geriatr. 2016 12 02;16(1):207. PMID: 27912740 218. Zarit SH, Stephens MA, Townsend A, et al. Stress reduction for family caregivers: effects of adult day care use. J Gerontol B Psychol S

219. Kim K, Zarit SH, Femia EE, et al. Kin relationship of caregivers and people with dementia: stress and response to intervention. Int $J$ (1).59-66. doi: https://dx.doi.org/10.1002/gps.2689. PMID: 21322030.

220. Lawton MP, Brody EM, Saperstein A, et al. Respite services for caregivers: research findings for service planning. Home Health Care

Serv Q. 1989;10(1-2):5-32. PMID: 10318337.

221. Luchsinger JA, Burgio L, Mittelman M, et al. Comparative Effectiveness of 2 Interventions for Hispanic Caregivers of Persons with

Dementia. J Am Geriatr Soc. 2018 Sep;66(9):1708-15. doi: https://dx.doi.org/10.1111//gs.15450. PMID: 30084133.

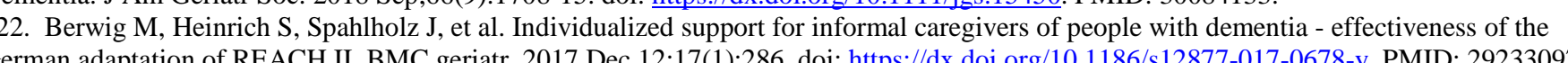

223. Brijoux T, Kricheldorff C, Hull M, et al. Supporting families living with dementia in rural areas: a randomized controlled trial of quality of

life improvement using qualified volunteers. In Dtsch
224. Koivisto AM, Hallikainen I, Valimaki T, et al. Early psychosocial intervention does not delay institutionalization in persons with mild Alzheimer disease and has impact on neither disease progression nor caregivers' well-being: ALSOVA 3-year follow-up. Int J Geriat Psychiatry. 2016 Mar; 31(3):273-83. doi: https:///dx.doi.org/10.1002/gps.4321. PMID: 26177825.

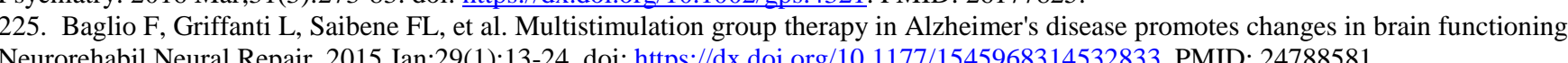
226. Prick AE, de Lange J, Twisk J, et al. The effects of a multi-component dyadic intervention on the psychological distress of family caregivers providing care to people with dementia: a randomized controlled trial. Int Psychogeriatr. 2015 Dec;27(12):2031-44. doi: https://dx.doi.org/10.1017/S104161021500071X. PMID: 26004290.

227. Gaugler JE, Reese M, Mittelman MS. Effects of the NYU caregiver intervention-adult child on residential care placement. Gerontologist.

228. Martindale-Adams J, Nichols LO, Burns R, et al. A trial of dementia caregiver telephone support. Can J Nurs Res. 2013 Dec;45(4):30-48 229. Mittelman MS, Brodaty H, Wallen AS, et al. A three-country randomized controlled trial of a psychosocial intervention for caregivers combined with pharmacological treatment for patients with Alzheimer disease: effects on caregiver depression. Am J Geriatr Psychiatry. 20161207. Publication Type: journal article. 230. Belle SH, Burgio L, Burns R, et al. Enhancing the quality of life of dementia caregivers from different ethnic or racial groups: a
and randomized, controlled trial.[Summary for

Nov 21;145(10):727-38. PMID: 17116917 .

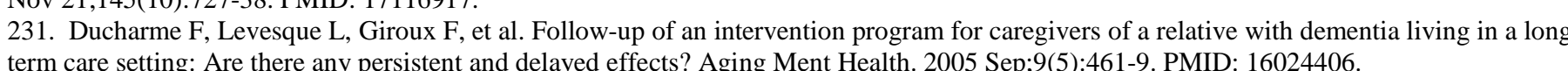
term care setting: Are the any persister.

232. Mittelman MS, Roth DL, Haley WE, et al. Effects of a caregiver intervention on negative caregiver appraisals of behavior problems in
patients with Alzheimer's disease: results of a randomized trial. J Gerontol B Psychol Sci Soc Sci. 2004 Jan;59(1):P27-34. PMID: 14722336 . 233. Eisdorfer C, Czaja SJ, Loewenstein DA, et al. The effect of a family therapy and technology-based intervention on caregiver depression. Gerontologist. 2003 Aug; $43(4)$ :521-31. PMID: 12937331

234. Teri L, Gibbons LE, McCurry SM, et al. Exercise plus behavioral management in patients with Alzheimer disease: a randomized

controlled trial. Jama. 2003 Oct 15;290(15):2015-22. PMID: 14559955.

hat delays institutionalization of Alzheimer's disease patients: treatment of 236. Mohide EA, Pringle DM, Streiner DL, et al. A randomized trial of family caregiver support in the home management of dementia. J Am Geriatr Soc. 1990 Apr;38(4):446-54. PMID: 2184186,

237. Prick AE, de Lange J, Scherder E, et al. The Effects of a Multicomponent Dyadic Intervention With Physical Exercise on the Cognitive ing of People With Dementia: A Randomized Controlled Trial. J Aging Phys Activity. 2017 Oct 01:25(4):539-52, do:

https://dx.doi.org/10.1123/japa.2016-0038. PMID: 28120631. people with dementia: a randomized controlled trial. Clin Interv Aging. 2016;11:383-95. doi: https://dx.doi.org/10.2147/CIA.S95789. PMID: 27099480.

239. Prick AE, de Lange J, van 't Leven N, et al. Process evaluation of a multicomponent dyadic intervention study with exercise and support

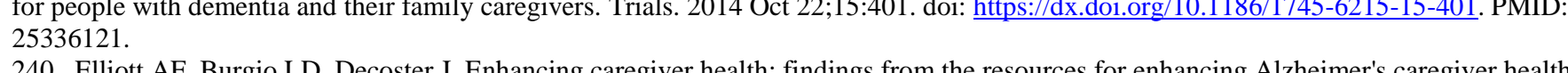

240. Elliott AF, Burgio LD, Decoster J. Enhancing caregiver health: findings from the resources for enhancing Alzheimer's caregiver health
intervention. J Am Geriatr Soc. 2010 Jan:58(1):30-7. doi: https://dx. doi.org/10.1111/i 1532-5415.2009.02631 x. PMID: 20122038. intervention. J Am Geriatr Soc. 2010 Jan;58(1):30-7. doi: https://dx.doi.org/10.1111/i.1532-5415.2009.02631.x. PMID: 20122038.
241. Nichols LO, Martindale-Adams J, Zhu CW, et al. Impact of the REACH II and REACH VA Dementia Caregiver Interventions on Healthcare Costs. J Am Geriatr Soc. 2017 May;65(5)::931-6. doi: https:///dx.doi.org/10.1111/jgs.14716. PMID: 28295134.

242. Nichols LO, Martindale-Adams J, Burns R, et al. Translation of a dementia caregiver support program in a health care system--REACH 48. PMID: 21357811.

243. Nichols LO, Chang C, Lummus A, et al. The cost-effectiveness of a behavior intervention with caregivers of patients with Alzheimer's disease. J Am Geriatr Soc. 2008:56(3):413-20. doi: 10.1111/j.1532-5415.2007.01569.x. PMID: 105900573. Corporate Author: Resources fo
Enhancing Alzheimer's Caregivers Health II Investigators. Language: English. Entry Date: 20080425. Revision Date: 20150711. Publication

244. Basu R, Hochhalter AK, Stevens AB. The Impact of the REACH II Intervention on Caregivers' Perceived Health. J Appl Gerontol. 2015 Lee CC, C08. doi: https://dx.doi.org/10.1177/0733464813499640. PMID: 24652899.

24f. Lee CC, Czaja SJ, Schulz R. The moderating influence of demographic characteristics, social support, and religious coping on the Psychological Sciences \& Social Sciences. 2010;65B(2):185-94. doi: geronb/gbp131. PMID: 105127911. Language: English. Entry Date: 20100430. Revision Date: 20150711. Publication Type: Journal Article.

Caregiver Health lI II Intervention. J Am Geriatr Soc. Racial and Ethnic Differences in the Delivery of the Resources for Enhancing Alzheimer's 247. Hatch DJ, DeHart WB, Norton MC. Subjective stressors moderate effectiveness of a multi-component, multi-site intervention on caregiver depression and burden. Int J Geriatr Psychiatry. 2014 Apr;29(4):406-13. doi: https://dx.doi.org/10.1002/gps.4019. PMID: 23983230 .
248. McGinnis KA, Schulz R, Stone RA, et al. Concordance of race or ethnicity of interventionists and caregivers of dementia patients: 248. MeGinhis KA, Schulz $\mathrm{R}$, Stone RA, et al. Concordance of race or elhicity of interventionists and caregivers of dementia patient: 
249. Wisniewski SR, Belle SH, Coon DW, et al. The Resources for Enhancing Alzheimer's Caregiver Health (REACH): project design and baseline characteristics. Psychol Aging. 2003 Sep;18(3):375-84. PMID: 14518801.

250. Mittelman MS, Roth DL, Coon DW, et al. Sustained benefit of supportive intervention for depressive symproms in caregivers of patients 251. Mittelman MS, Haley WE, Clay OJ, et al. Improving caregive
disease. Neurology. 2006 Nov 14;67(9):1592-9. PMID: 17101889. Geriatr Psychiatry. 2007 Sep;15(9): $780-9$-9. PMID: 17804831.

53. Drentea P, Clay OJ, Roth DL, et al. Predictors of improvement in social support: Five-year effects of a structured intervention for

symptoms. Gerontologist. 2008 Dec:48(6):732-40. PMID: 19139247 .

aregivers of persons with Alzheimer's disease. Psychol in social support as mediators of the impact of a psychosocial intervention for spouse (16420138.

(C) Geriatr Soc, 2008 Mar:56(3):421-8 doi: https//dx doiorg/10.1111/j1532-

25\%. Gaugler JE, Roth DL, Haley WE, et al. Modeling trajectories and transitions: results from the New York University caregiver intervention 258. Res. 2011 May-Jun;60(3 Suppl):S28-37. doi: hittps://dx.doi.org/10.1097/NNR.0b013e318216007d. PMID. 21543959.

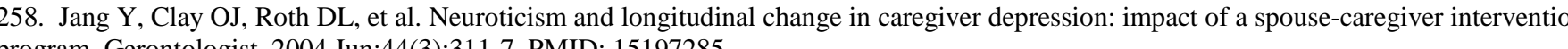
250. B. A, Mitelmat

in dementia care: observations from a psychosocial intervention study.

nd of country of residence on rason L, et al. The effects of counseling spouse caregivers of people with Alzheimer disease taking donepezil

261. Mazurek J, Szczesniak D, Lion KM, et al. Does the Meeting Centres Support Programme reduce unmet care needs of community-dwelling older people with dementia? A controlled, 6-month follow-up Polish study. Clin Interv Aging. 2019;14:113-22. doi:

(14)

262. Zhang SY, Wu F, Tang DL, et al. Pilot testing the caregiver self-management intervention for caregivers of relatives with dementia.

263. Marshall A, Spreadbury $\mathrm{J}$, Cheston R, et al. A pilot randomised controlled trial to compare changes in quality of life for participants with 2015:19(6):526-35. PMID: CN-01071410.

264. Czaja SJ, Loewenstein D, Schulz R, et al. A videophone psychosocial intervention for dementia caregivers. Am J Geriatr Psychiatry. 2013 Nov;21(11):1071-81. doi: https:///dx.doi.org/10.1016/j.jagp.2013.02.019. PMID: 23831174.

265. Gaugler JE, Reese M, Mittelman MS. Effects of the Minnesota Adaptation of the NYU Caregiver Intervention on Depressive Symptom

.
266. Gaugler JE, Reese M, Mittelman MS. Effects of the Minnesota Adaptation of the NYU Caregiver Intervention on Primary Subjective Stress of Adult Child Caregivers of Persons With Dementia. Gerontologist. 2016 Jun;56(3):461-74. doi:

265.//Ax.doi.org/10.1093/geront/gnu125. PMID: 25628299.

intelman MS. Process Evaluation of the NYU Caregiver Intervention-Adult Child. Gerontologist. 201803

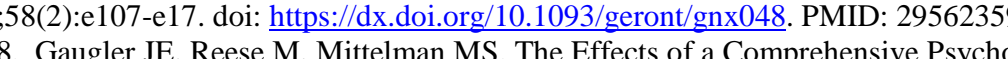

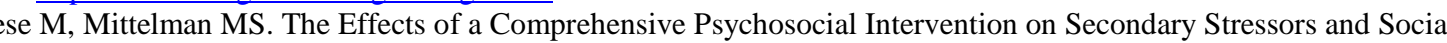
(n)

https://dx.doi.org/10.1093/geroni/igy015. PMID: 30009268. Jutten LH, Mark RE, Sitskoorn MM. Can the Mixed V.

s.ase Burden in Informal Dementia Caregivers? Dement Geriatr Cogn Dis Extra. 2018 Sep-Dec;8(3):453-66. do:

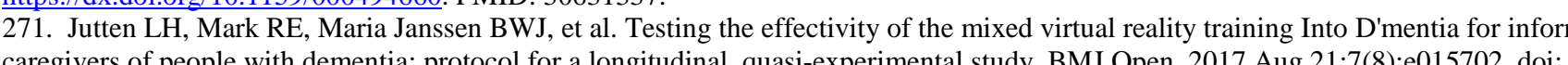
https://dx.doi.org/10.1136/bmjopen-2016-015702. PMID: 28827242.

RA, D'Ambrosio LA, Mohyde M, et al. At the crossroads: development and evaluation of a dementia caregiver group intervention to assist in driving cessation. Gerontol Geriatr Educ. 2008;29(4):363-82. doi: https://dx.doi.org/10.1080/02701960802497936. PMID: 19064472. 273. Simpson R, Wakefield P, Spiers N, et al. Carer-held records for dementia: a controlled trial. Int Psychogeriatr. 2006 Jun;18(2):259-68. PMID: 16734920.

274. Lord K, Livingston G, Cooper C. A feasibility randomised controlled trial of the DECIDE intervention: dementia carers making informed 275. Stirling $C$, Legoett $S$, Lloyd $B$, et al. Decision aids for res

RCT. BMC Med Inf Decis Mak. 2012 Mar 19;12:21. doi: htttps:///dx.doi.org/10.1186/1472-6947-12-21. PMID: 22429384. 276. Rose KM, Taylor AG, Bourguignon C. Effects of cranial electrical sin caregiving appraisal in spousal caregivers of persons with Alzheimer's disease. Appl Nurs Res. 2009 May;22(2):119-25. doi:

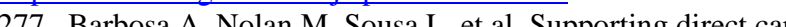

workers in dementia care: effects of a psychoeducational intervention. Am $J$ Mar;30(2):130-8. doi: https://dx.doi.org/10.1177/1533317514550331. PMID: 25237132. 278. Barbosa A, Nolan M, Sousa L, et al. Effects of a Psychoeducational Intervention for Direct Care Workers Caring for People With https://dx.doi.org/10.1177/15333317515603500. PMID: 26399485.

Visser SM, McCabe MP, Hudgson C, et al. Managing behavioural symptoms of dementia: effectiveness of staff education and peer (12) 2008 Jan;12(1):47-55. doi: https://dx.doi.org/10.1080/13607860701366012. PMID; 18297478

280. Davison T, McCabe M, Visser S, et al. Controlled trial of dementia training with a peer support group for aged care staff. Int J Geriatr Psychiatry. 2007;22(9):868-73. doi: 10.1002/gps.1754. PMID: CN-00627010. 281. Mackenzie CS, Peragine G. Measuring and enhancing self-efficacy among professional caregivers of individuals with dementia. Am 


\section{Consultation Service}

Appendix F. Care Delivery Interventions

Table F-1. Risk of bias assessment: consultation services

\begin{tabular}{|c|c|c|c|c|c|c|c|c|}
\hline Study (PMID) & Outcome Timing & $\begin{array}{l}\text { Attrition Bias } \\
\text { Attrition \% }\end{array}$ & Selection Bias & Detection Bias & Performance Bias & Reporting Bias & Fidelity Bias & Overall Rating \\
\hline \begin{tabular}{|l|l} 
Fortinsky $2009^{1}(19347683)$ \\
\end{tabular} & 12 months & $\begin{array}{l}\text { Medium } \\
12 \text { months: } 10.7 \%\end{array}$ & Medium & Medium & Low & Medium & Medium & Medium \\
\hline \begin{tabular}{|l|} 
Borbasi $2011^{2}(21692400)$ \\
\end{tabular} & 12 months & $\mathrm{x}$ & High & $\mathrm{x}$ & $\mathrm{x}$ & $\mathrm{x}$ & $\mathrm{x}$ & High \\
\hline \begin{tabular}{|l} 
McSweeney $2012^{3}$ (22344753) \\
\end{tabular} & 15 weeks & $\begin{array}{l}\text { Medium } \\
11 \%\end{array}$ & Low & Low & High & Low & High & High \\
\hline Opie $2002^{4}(11802224)$ & 4 weeks & Low & $\begin{array}{l}\text { Medium } \\
3 \%\end{array}$ & High & High & Low & Medium & High \\
\hline \begin{tabular}{|l} 
Orrell $2007^{5}$ (17394129) \\
\end{tabular} & 20 weeks & \begin{tabular}{|l} 
Medium \\
$19 \%$
\end{tabular} & Medium & Low & Medium & Low & High & High \\
\hline
\end{tabular}

Table F-2. Characteristics of included studies: consultation services

\begin{tabular}{|c|c|c|c|c|c|c|c|c|c|c|c|c|c|}
\hline $\begin{array}{l}\text { Study (PMID) } \\
\text { Country } \\
\text { RoB* } \\
\text { Type }\end{array}$ & $\begin{array}{l}\text { Intervention } \\
\text { Intervention } \\
\text { Focus } \\
\text { Theoretical } \\
\text { Model } \\
\text { Delivery } \\
\text { Person } \\
\text { Intervention } \\
\text { Target } \\
\text { Recipient } \\
\text { Mode } \\
\text { Components } \\
\text { Frequency } \\
\text { Duration }\end{array}$ & $\begin{array}{l}\text { Comparison } \\
\text { Target } \\
\text { Mode } \\
\text { Components } \\
\text { Frequency } \\
\text { Duration }\end{array}$ & $\begin{array}{l}\text { Setting } \\
\text { and Design } \\
\text { Setting } \\
\text { Design } \\
\text { Cluster N } \\
\text { Participants } \\
\text { Randomized } \\
\text { N }\end{array}$ & \begin{tabular}{|l|} 
PLWD \\
Dementia \\
Characteristics \\
\\
Dementia Types \\
Dementia Severity \\
Diagnostic \\
Criteria \\
Age of Diagnosis
\end{tabular} & $\begin{array}{l}\text { PLWD } \\
\text { Non-Disease Char } \\
\text { PLWD N } \\
\text { PLWD Age (mean) } \\
\text { PLWD Sex (\% } \\
\text { female) } \\
\text { PLWD Race (\% } \\
\text { majority) } \\
\text { PLWD Education } \\
\text { (mean years) }\end{array}$ & \begin{tabular}{|l} 
PLWD \\
Non-Disease \\
Char Reporting \\
Status (RS) \\
\\
PLWD SES \\
PLWD Prior \\
Disability \\
PLWD \\
Household \\
Characteristics \\
PLWD Health \\
Insurance \\
PLWD Detailed \\
Race Information
\end{tabular} & $\begin{array}{l}\text { Informal Caregiver } \\
\text { (IC) } \\
\text { Characteristics } \\
\text { IC N } \\
\text { IC Age (mean) } \\
\text { IC Sex (\% female) } \\
\text { IC Race (\% } \\
\text { majority) } \\
\text { IC Education } \\
\text { (mean years) } \\
\text { IC Relation to } \\
\text { PLWD (\% majority) }\end{array}$ & $\begin{array}{l}\text { Informal Caregiver } \\
\text { (IC) Char. RS } \\
\text { IC Duration } \\
\text { IC Living With } \\
\text { PLWD } \\
\text { IC Payment } \\
\text { IC Health Status } \\
\text { IC Dementia } \\
\text { Family History } \\
\text { IC Employment } \\
\text { Status } \\
\text { IC Training }\end{array}$ & $\begin{array}{l}\text { Formal Caregiver } \\
\text { (FC) } \\
\text { Characteristics } \\
\text { FC N } \\
\text { FC Age (mean) } \\
\text { FC Sex (\% } \\
\text { female) } \\
\text { FC Race (\% } \\
\text { majority) } \\
\text { FC Education } \\
\text { (mean years) }\end{array}$ & $\begin{array}{l}\text { Formal } \\
\text { Caregiver (FC) } \\
\text { Char. RS } \\
\text { FC Health } \\
\text { Status } \\
\text { FC Training } \\
\text { FC Education } \\
\text { FC Position } \\
\text { FC Length of } \\
\text { Service }\end{array}$ & \begin{tabular}{|l|} 
Outcome \\
Timing(s)
\end{tabular} & $\begin{array}{l}\text { PLWD } \\
\text { Outcomes }\end{array}$ & $\begin{array}{l}\text { Caregiver } \\
\text { Outcomes } \\
\text { IC: } \\
\text { FC: }\end{array}$ \\
\hline \begin{tabular}{|l} 
Fortinsky 2009 \\
(19347683) \\
US \\
Medium \\
Explanatory
\end{tabular} & \begin{tabular}{|l} 
Intervention \\
group family \\
caregivers \\
received the \\
dementia care \\
consultation \\
intervention per \\
protocol as well \\
as educational \\
materials about \\
dementia and \\
community \\
resources over \\
12 months \\
period
\end{tabular} & $\begin{array}{l}\text { Educational } \\
\text { materials }\end{array}$ & $\begin{array}{l}\text { Community- } \\
\text { based in-home } \\
\\
\text { Cluster RCT } \\
26 \text { practice } \\
\text { sites }\end{array}$ & \begin{tabular}{|l} 
Diagnosed with AD \\
o other dementia. \\
With following ICD- \\
9 codes: \\
arteriosclerotic \\
dementia \\
$(290.40,290.41,290$ \\
$.42,290.43) ;$ senile \\
dementia \\
$(290.00,290.20,290$ \\
$.21,290.30) ;$ pre- \\
senile dementia \\
$(290.10,290.11,290$ \\
$.12,290.13) ;$ \\
memory loss, mild \\
(310.10); or AD \\
(331.00).
\end{tabular} & \begin{tabular}{|l|}
$\mathrm{N}=84$ \\
$66 \%$ Female \\
82 years \\
Race NR \\
Education NR
\end{tabular} & None & NA & \begin{tabular}{|l|}
$\mathrm{N}=84$ \\
$72 \%$ Female \\
61 years \\
Race NR \\
Education NR
\end{tabular} & NA & NA & 12 months & $\begin{array}{l}\text { Nursing } \\
\text { home } \\
\text { admission; }\end{array}$ & $\begin{array}{l}\text { IC: } 2 \\
\text { measures of } \\
\text { self-efficacy } \\
\text { Caregiver } \\
\text { Burden } \\
\text { CES-D } \\
\text { HSC } \\
\text { measures of } \\
\text { symptom } \\
\text { severity } \\
\text { CSC } \\
\text { PBS }\end{array}$ \\
\hline
\end{tabular}

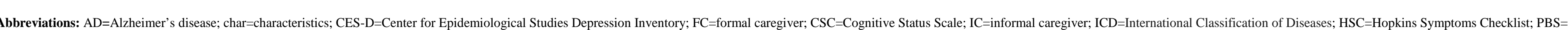

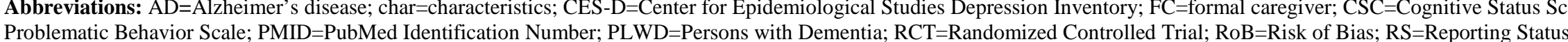

Table F-3. PLWD outcomes summary low and medium risk of bias studies: consultation services

\begin{tabular}{|c|c|c|c|c|c|c|}
\hline \multirow{2}{*}{$\begin{array}{l}\text { Study (PMID) } \\
\text { Comparison } \\
\text { RoB } \\
\text { Category }\end{array}$} & \multirow{2}{*}{$\begin{array}{l}\text { Outcome } \\
\text { Timing }\end{array}$} & \multicolumn{4}{|c|}{ 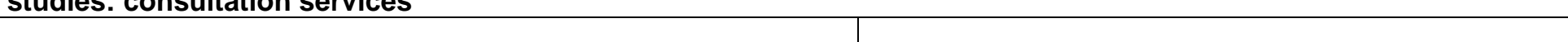 } & \multirow[t]{2}{*}{$\mathrm{p}$-Value } \\
\hline & & Intervention: Before & Intervention: After 12 months & Comparator: Before & $\begin{array}{l}\text { Comparator: After } 12 \\
\text { months }\end{array}$ & \\
\hline $\begin{array}{l}\text { Fortinsky 20091 } \\
\text { (19347663) } \\
\text { Consultation vs Control } \\
\text { Medium } \\
\text { Exploratory }\end{array}$ & $\begin{array}{l}\text { Adjusted Odds Ratio }(95 \% \mathrm{Cl}) \\
\text { Nursing home admission } \\
12 \text { months }\end{array}$ & NR & $0.4(0.14,1.18)$ & NR & 1.0 & $p=0.10$ \\
\hline
\end{tabular}


Table F-4. Informal caregiver outcomes summary low and medium risk of bias studies: consultation services

\begin{tabular}{|c|c|c|c|c|c|c|}
\hline \multirow{2}{*}{$\begin{array}{l}\text { Study (PMID) } \\
\text { Comparison } \\
\text { RoB } \\
\text { Category }\end{array}$} & \multirow{2}{*}{$\begin{array}{l}\text { Outcome } \\
\text { Timing }\end{array}$} & & \multirow[t]{2}{*}{ p-Value } \\
\hline & & Intervention: Before & Intervention: After 12 months & Comparator: Before & $\begin{array}{l}\text { Comparator: After } 12 \\
\text { months }\end{array}$ & \\
\hline $\begin{array}{l}\text { Fortinsky 20091 } \\
\text { (19347683) } \\
\text { Consultation vs Control } \\
\text { Medium } \\
\text { Exploratory }\end{array}$ & $\begin{array}{l}\text { Adjusted Mean }(95 \% \mathrm{Cl}) \\
\text { Symptom management self-efficacy score } \\
12 \text { months }\end{array}$ & $33.52(27.77-39.26)$ & 33.88 (28.04-39-73) & 33.51 (27.31-39.71) & $34.21(27.92-40.49)$ & $p=0.89$ \\
\hline $\begin{array}{l}\text { Fortinsky 20091 } \\
\text { (19347663) } \\
\text { Consultation vs Control } \\
\text { Medium } \\
\text { Exploratory }\end{array}$ & \begin{tabular}{|l} 
Adjusted Mean $(95 \% \mathrm{Cl})$ \\
Support service self-efficacy \\
12 months
\end{tabular} & $27.96(25.17-30.75)$ & $31.95(28.90-35.00)$ & $24.63(20.95-28.31)$ & $27.92(24.17-31.66)$ & $P=0.80$ \\
\hline $\begin{array}{l}\text { Fortinsky 20091 } \\
\text { (19347683) } \\
\text { Consultation vs Control } \\
\text { Medium } \\
\text { Exploratory }\end{array}$ & \begin{tabular}{|l} 
Adjusted Mean $(95 \% \mathrm{Cl})$ \\
CES-D score \\
12 months
\end{tabular} & $12.13(8.90-15.35)$ & \begin{tabular}{|l}
$9.80(6.24-13.36)$ \\
\end{tabular} & \begin{tabular}{|l}
$15.10(10.77-19.43)$ \\
\end{tabular} & $15.00(10.51-19.49)$ & $p=0.41$ \\
\hline $\begin{array}{l}\text { Fortinsky 20091 } \\
\text { (19347683) } \\
\text { Consultation vs Control } \\
\text { Medium } \\
\text { Exploratory }\end{array}$ & $\begin{array}{l}\text { Adjusted Mean }(95 \% \mathrm{Cl}) \\
\text { Zarit burden score } \\
12 \text { months }\end{array}$ & $30.42(26.30-34.53)$ & $26.18(21.81-30.55)$ & 36.02 (30.71-41.33) & $30.57(25.03-36.10)$ & $p=0.73$ \\
\hline $\begin{array}{l}\text { Lapuraury } \\
\text { Fortinsky 20091 } \\
\text { (19347663) } \\
\text { Consultation vs Control } \\
\text { Medium } \\
\text { Exploratory }\end{array}$ & $\begin{array}{l}\text { Adjusted Mean }(95 \% \mathrm{Cl}) \\
\text { Hopkins symptom checklist score } \\
12 \text { months }\end{array}$ & $2.33(1.57-3.09)$ & $2.92(2.10-3.74)$ & \begin{tabular}{|l|}
$3.23(2.31-4.17)$ \\
\end{tabular} & $3.74(2.78-4.70)$ & $p=0.87$ \\
\hline
\end{tabular}

Exploratory

(I)

Table F-5. Summary of strength of evidence for PLWD outcomes: consultation services

\begin{tabular}{|l|l|l|l|l|l|l|l|}
\hline $\begin{array}{l}\text { Comparison } \\
\text { Outcome }\end{array}$ & Timing & $\begin{array}{l}\text { \# Studies/ Design } \\
(\mathbf{n} \text { analyzed) }\end{array}$ & Finding or Summary Statistic & Study Limitations & Consistency & Directness & Precision \\
\hline $\begin{array}{l}\text { Consultation vS Control } \\
\text { Nursing home admission }\end{array}$ & 12 months & 1 RCT (n=84) & $\begin{array}{l}\text { Nursing home admissions showed no difference between the } \\
\text { comparison groups. }\end{array}$ & Moderate & Unknown & Direct \\
\hline Abbreviations: CES-D=Center for Epidemiological Studies Depression Inventory $n=$ number; RCT=Randomized Controlled Trial & Imprecise & Insufficient \\
\hline
\end{tabular}

Consel

Table F-6. Summary of strength of evidence for caregiver outcomes: consultation services

\begin{tabular}{|c|c|c|c|c|c|c|c|c|}
\hline $\begin{array}{l}\text { Comparison } \\
\text { Outcome }\end{array}$ & Timing & $\begin{array}{l}\text { \# Studies/ Design } \\
\text { ( } \mathrm{n} \text { analyzed) }\end{array}$ & Finding or Summary Statistic & Study Limitations & Consistency & Directness & Precision & $\begin{array}{l}\text { Overall Gradel } \\
\text { Conclusion }\end{array}$ \\
\hline $\begin{array}{l}\text { Consultation vs Control } \\
\text { Symptom management } \\
\text { self-efficacy score }\end{array}$ & 12 months & $1 \mathrm{RCT}(\mathrm{n}=84)$ & $\begin{array}{l}\text { Symptom management self-efficacy scores were not different } \\
\text { between the comparison groups }\end{array}$ & Moderate & Unknown & Direct & Imprecise & Insufficient \\
\hline $\begin{array}{l}\text { Consultation vs Control } \\
\text { Support service self- } \\
\text { efficacy }\end{array}$ & 12 months & $1 \mathrm{RCT}(\mathrm{n}=84)$ & $\begin{array}{l}\text { Support service self-efficacy was not different between the } \\
\text { comparison groups }\end{array}$ & Moderate & Unknown & Direct & Imprecise & Insufficient \\
\hline $\begin{array}{l}\text { Consultation vs Control } \\
\text { CES-D score }\end{array}$ & 12 months & $1 \mathrm{RCT}(\mathrm{n}=84)$ & $\begin{array}{l}\text { CES-D scores were not different between the comparison } \\
\text { groups }\end{array}$ & Moderate & Unknown & Direct & Imprecise & Insufficient \\
\hline $\begin{array}{l}\text { Consultation vs Control } \\
\text { Zarit burden score }\end{array}$ & 12 months & $1 \mathrm{RCT}(\mathrm{n}=84)$ & $\begin{array}{l}\text { Zarit burden scores were not different between the comparison } \\
\text { groups }\end{array}$ & Moderate & Unknown & Direct & Imprecise & Insufficient \\
\hline $\begin{array}{l}\text { Consultation vs Control } \\
\text { Hopkins symptom } \\
\text { checklist score }\end{array}$ & 12 months & $1 \mathrm{RCT}(\mathrm{n}=84)$ & $\begin{array}{l}\text { Hopkins symptom checklist scores were not different between } \\
\text { the comparison groups }\end{array}$ & Moderate & Unknown & Direct & Imprecise & Insufficient \\
\hline
\end{tabular}

Abbreviations: CES-D=Center for Epidemiological Studies Depression Inventory; $\mathrm{n}=$ Number; RCT=Randomized Controlled Tria 


\section{Evidence Map: Consultation Services}

\begin{tabular}{|c|c|c|c|c|c|c|c|c|c|c|c|c|c|}
\hline $\begin{array}{l}\text { Study (PMID) } \\
\text { Country } \\
\text { EM Reason }\end{array}$ & Intervention & Comparison & \begin{tabular}{l|} 
Setting \\
and Design \\
Setting \\
Design \\
Cluster N \\
Participants \\
Random- \\
ized N
\end{tabular} & $\begin{array}{l}\text { PLWD } \\
\text { Dementia } \\
\text { Characteristics }\end{array}$ & $\begin{array}{l}\text { PLWD } \\
\text { Non-Disease Char } \\
\text { PLWD N } \\
\text { PLWD Age (mean) } \\
\text { PLWD Sex (\% } \\
\text { female) } \\
\text { PLWD Race (\% } \\
\text { majority) } \\
\text { PLWD Education } \\
\text { (mean years) }\end{array}$ & $\begin{array}{l}\text { PLWD } \\
\text { Non-Disease } \\
\text { Char Reporting } \\
\text { Status (RS) } \\
\text { PLWD SES } \\
\text { PLWD Prior } \\
\text { Disability } \\
\text { PLWD } \\
\text { Household } \\
\text { Characteristics } \\
\text { PLWD Health } \\
\text { Insurance } \\
\text { PLWD Detailed } \\
\text { Race Information }\end{array}$ & $\begin{array}{l}\text { Informal Caregiver } \\
\text { (IC) } \\
\text { Characteristics } \\
\text { IC N } \\
\text { IC Age (mean) } \\
\text { IC Sex (\% female) } \\
\text { IC Race (\% } \\
\text { majority) } \\
\text { IC Education } \\
\text { (mean years) } \\
\text { IC Relation to } \\
\text { PLWD (\% majority) }\end{array}$ & $\begin{array}{l}\text { Informal Caregiver } \\
\text { (IC) Char. RS } \\
\text { IC Duration } \\
\text { IC Living With } \\
\text { PLWD } \\
\text { IC Payment } \\
\text { IC Health Status } \\
\text { IC Dementia } \\
\text { Family History } \\
\text { IC Employment } \\
\text { Status } \\
\text { IC Training }\end{array}$ & $\begin{array}{l}\text { Formal Caregiver } \\
\text { (FC) } \\
\text { Characteristics } \\
\text { FC N } \\
\text { FC Age (mean) } \\
\text { FC Sex (\% } \\
\text { female) } \\
\text { FC Race (\% } \\
\text { majority) } \\
\text { FC Education } \\
\text { (mean years) }\end{array}$ & $\begin{array}{l}\text { Formal } \\
\text { Caregiver (FC) } \\
\text { Char. RS } \\
\text { FC Health } \\
\text { Status } \\
\text { FC Training } \\
\text { FC Education } \\
\text { FC Position } \\
\text { FC Length of } \\
\text { Service }\end{array}$ & $\begin{array}{l}\text { Outcome } \\
\text { Timing(s) }\end{array}$ & $\begin{array}{l}\text { PLWD } \\
\text { Outcomes }\end{array}$ & $\begin{array}{l}\text { Caregiver } \\
\text { Outcomes } \\
\text { IC: } \\
\text { FC: }\end{array}$ \\
\hline $\begin{array}{l}\text { Borbasi 20112 } \\
(21692400) \\
\text { Australia } \\
\text { High RoB }\end{array}$ & $\begin{array}{l}\text { Dementia } \\
\text { Outreach } \\
\text { Service } \\
\text { (DEMOS): } \\
\text { comprised a } \\
\text { multidisciplinary } \\
\text { healthcare team } \\
\text { to assess and } \\
\text { manage } \\
\text { residents } \\
\text { experiencing } \\
\text { cognitive } \\
\text { deterioration as } \\
\text { a result of } \\
\text { dementia. } \\
\text { Conducted for } \\
12 \text { months in } 3 \\
\text { stages }\end{array}$ & $\mathrm{NR}$ & \begin{tabular}{l|} 
Residential \\
aged care \\
facilities \\
Quasi- \\
experimental
\end{tabular} & Not specified & $\mathrm{NR}$ & None & $\mathrm{NR}$ & None & $\begin{array}{l}\mathrm{N}=320 \\
\text { Age: no } \\
\text { Sex: yes } \\
\text { Race: yes } \\
\text { Education: no }\end{array}$ & NA & 12 months & $\mathrm{NR}$ & $\begin{array}{l}\text { Improved } \\
\text { capacity } \\
\text { and clinical } \\
\text { skills of staff } \\
\text { (stress, } \\
\text { knowledge } \\
\text { and self- } \\
\text { confidence) } \\
\text { Improvemen } \\
\text { t in } \\
\text { timeliness } \\
\text { and } \\
\text { appropriate } \\
\text { ness of } \\
\text { referrals } \\
\text { Satisfaction } \\
\text { Barriers to } \\
\text { success }\end{array}$ \\
\hline $\begin{array}{l}\text { McSweeney } \\
2012^{3} \\
(22344753) \\
\text { Australia } \\
\text { High RoB }\end{array}$ & $\begin{array}{l}\text { Psychiatrist and } \\
\text { psychologist's } \\
\text { consultation } \\
\text { regarding best- } \\
\text { practice } \\
\text { management of } \\
\text { depression via } \\
\text { psychosocial } \\
\text { (individually } \\
\text { tailored, } \\
\text { psychosocial } \\
\text { care plan) and } \\
\text { medical care } \\
\text { plan }\end{array}$ & Care as usual & $\begin{array}{l}\text { Aged care } \\
\text { facilities } \\
\text { RCT; } \\
\text { 44 PLWD }\end{array}$ & $\begin{array}{l}\text { MMSE cut point } \\
\text { score of } 23 \text { and } \\
\text { PAS Informant } \\
\text { History } 24 \\
\text { PAS Cognitive } \\
\text { Decline Informant } \\
\text { History and CSDD }\end{array}$ & $\begin{array}{l}\mathrm{N}=44 \\
\text { Age: yes } \\
\text { Sex: yes } \\
\text { Race: no } \\
\text { Education: no }\end{array}$ & None & NA & NA & NA & NA & 15 weeks & $\begin{array}{l}\text { CSDD } \\
\text { RAID } \\
\text { MMSE } \\
\text { BEHAVE-AD } \\
\text { CANE }\end{array}$ & NA \\
\hline $\begin{array}{l}\text { Opie 20024 } \\
\text { (11802224) } \\
\text { Australia } \\
\text { High RoB }\end{array}$ & $\begin{array}{l}\text { Multidisciplinary } \\
\text { interventions } \\
\text { (early and late } \\
\text { groups) } \\
\text { encompassing } \\
\text { psychosocial } \\
\text { strategies, } \\
\text { nursing } \\
\text { approaches, } \\
\text { psychotropic } \\
\text { approaches and } \\
\text { management of } \\
\text { pains }\end{array}$ & NA & \begin{tabular}{l|} 
Nursing \\
homes \\
Quasi- \\
experimental
\end{tabular} & $\begin{array}{l}\text { Based on CMAl, } \\
\text { BAGS and MMSE } \\
\text { scores }\end{array}$ & $\begin{array}{l}\text { N=99 } \\
\text { Age: yes } \\
\text { Sex: no } \\
\text { Race: no } \\
\text { Education: no }\end{array}$ & None & NA & NA & NA & NA & $\begin{array}{l}\text { Weekly up to } \\
4 \text { weeks }\end{array}$ & $\begin{array}{l}\text { Restlessness } \\
\text { Physical } \\
\text { aggression } \\
\text { Verbal } \\
\text { disruption } \\
\text { Inappropriate } \\
\text { behavior }\end{array}$ & NA \\
\hline $\begin{array}{l}\text { Orrell 20075 } \\
(17394129) \\
\text { UK } \\
\text { High RoB }\end{array}$ & $\begin{array}{l}1 \text { hour per week } \\
\text { liaison mediated } \\
\text { personalized } \\
\text { intervention } \\
\text { package over } \\
\text { 20 weeks to } \\
\text { address the } \\
\text { unmet needs }\end{array}$ & Care as usual & $\begin{array}{l}\text { residential } \\
\text { care homes } \\
\text { Cluster } \\
\text { RCT; } \\
238 \text { PLWD }\end{array}$ & $\begin{array}{l}\text { Diagnosis based } \\
\text { on DSM-IV criteria } \\
\text { for dementia }\end{array}$ & $\begin{array}{l}\mathrm{N}=238 \\
\text { Age: yes } \\
\text { Sex: yes } \\
\text { Race: no } \\
\text { Education: no }\end{array}$ & None & NA & NA & NA & NA & 20 weeks & $\begin{array}{l}\text { CANE } \\
\text { QoL-AD } \\
\text { MMSE } \\
\text { CDR } \\
\text { CAPE-BRS } \\
\text { BI } \\
\text { CBS } \\
\text { CSDD } \\
\text { RAID }\end{array}$ & $\begin{array}{l}\text { CANE } \\
\text { QoL-AD }\end{array}$ \\
\hline
\end{tabular}

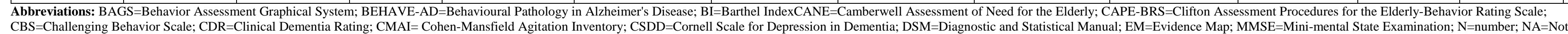
CBS=Challenging Behavior Scale; CDR=Clinical Dementia Rating; CMAI= Cohen-Mansfield Agitation Inventory; CSDD=Cornell Scale for Depression in Dementia; DSM=Diagnostic and Statistical Man
Applicable; NR=Not Reported; PAS=Psychogeriatric Assessment Scale; PMID=PubMed Identification Number; QoL-AD=Quality of Life in Alzheimer's Disease; RAID=Rating for Anxiety in Dementia 


\section{Case Management}

Table F-8. Risk of bias assessment: case management

\begin{tabular}{|c|c|c|c|c|c|c|c|c|c|}
\hline Study (PMID) & Outcome Timing & \begin{tabular}{|l} 
Attrition Bias \\
Attrition \%
\end{tabular} & Selection Bias & Detection Bias & \begin{tabular}{|l|} 
Performance Bias \\
\end{tabular} & Reporting Bias & Fidelity Bias & Funder & Overall Rating \\
\hline \begin{tabular}{|l|} 
Xiao, $2016^{6}$ (25805891) \\
\end{tabular} & 12 months & \begin{tabular}{|l|} 
Medium \\
$15 \%$
\end{tabular} & Low & Medium & High & Low & Low & Government & High \\
\hline \begin{tabular}{|l|l} 
MacNeil Vroomen, 2015 (26170035) \\
\end{tabular} & 24 months & $\begin{array}{l}\text { High } \\
40 \%\end{array}$ & Medium & $x$ & $\mathrm{x}$ & $\mathrm{x}$ & $\mathrm{x}$ & & High \\
\hline \begin{tabular}{|l} 
Chien, $2011^{8}$ (21198803) \\
\end{tabular} & 12,18 months & \begin{tabular}{|l} 
Low \\
$2 \%$ \\
\end{tabular} & Low & Low & Medium & Low & High & $\begin{array}{l}\text { Government } \\
\text { University }\end{array}$ & Medium \\
\hline \begin{tabular}{|l} 
Lam, $2010^{9}(19606455)$ \\
\end{tabular} & 12 months & \begin{tabular}{|l} 
Low \\
$3 \%$
\end{tabular} & Low & Medium & Medium & Low & High & Government & Medium \\
\hline \begin{tabular}{|l} 
Brodaty, 2003 \\
$(12590626)$
\end{tabular} & 12 weeks & \begin{tabular}{|l|} 
Medium \\
$16 \%$
\end{tabular} & Low & Low & High & Low & High & & High \\
\hline Challis, 2002 ${ }^{11}(11994884)$ & 6 months & \begin{tabular}{|l|} 
Low \\
$0 \%$
\end{tabular} & High & High & $x$ & $\mathrm{x}$ & $x$ & & High \\
\hline Weinberger, $1993^{13}(8426038)$ & 6 months & $\begin{array}{l}\text { Medium } \\
14 \%\end{array}$ & Medium & High & High & Low & High & & High \\
\hline
\end{tabular}

Abbreviations: NR=Not Reported; PMID=PubMed Identification Number

\begin{tabular}{|c|c|c|c|c|c|c|c|c|c|c|c|c|c|}
\hline \begin{tabular}{|l|} 
Study (PMID) \\
Country \\
RoB* \\
Type
\end{tabular} & $\begin{array}{l}\text { Intervention } \\
\text { Intervention } \\
\text { Focus } \\
\text { Theoretical } \\
\text { Model } \\
\text { Delivery } \\
\text { Person } \\
\text { Intervention } \\
\text { Target } \\
\text { Recipient } \\
\text { Mode } \\
\text { Components } \\
\text { Frequency } \\
\text { Duration }\end{array}$ & $\begin{array}{l}\text { Comparison } \\
\text { Target } \\
\text { Mode } \\
\text { Components } \\
\text { Frequency } \\
\text { Duration }\end{array}$ & $\begin{array}{l}\text { Setting } \\
\text { and Design } \\
\\
\text { Setting } \\
\text { Design } \\
\text { Cluster N } \\
\text { Participants } \\
\text { Randomized } \\
\text { N }\end{array}$ & $\begin{array}{l}\text { PLWD } \\
\text { Dementia } \\
\text { Characteristics } \\
\text { Dementia Types } \\
\text { Dementia Severity } \\
\text { Diagnostic } \\
\text { Criteria } \\
\text { Age of Diagnosis }\end{array}$ & $\begin{array}{l}\text { PLWD } \\
\text { Non-Disease Char } \\
\text { PLWD N } \\
\text { PLWD Age (mean) } \\
\text { PLWD Sex (\% } \\
\text { female) } \\
\text { PLWD Race (\% } \\
\text { majority) } \\
\text { PLWD Education } \\
\text { (mean years) }\end{array}$ & \begin{tabular}{|l|} 
PLWD \\
Non-Disease \\
Char Reporting \\
Status (RS) \\
PLWD SES \\
PLWD Prior \\
Disability \\
PLWD \\
Household \\
Characteristics \\
PLWD Health \\
Insurance \\
PLWD Detailed \\
Race Information
\end{tabular} & $\begin{array}{l}\text { Informal Caregiver } \\
\text { (IC) } \\
\text { Characteristics } \\
\text { IC N } \\
\text { IC Age (mean) } \\
\text { IC Sex (\% female) } \\
\text { IC Race (\% } \\
\text { majority) } \\
\text { IC Education } \\
\text { (mean years) } \\
\text { IC Relation to } \\
\text { PLWD (\% majority) }\end{array}$ & \begin{tabular}{|l|} 
Informal Caregiver \\
(IC) Char. RS \\
IC Duration \\
IC Living With \\
PLWD \\
IC Payment \\
IC Health Status \\
IC Dementia \\
Family History \\
IC Employment \\
Status \\
IC Training
\end{tabular} & $\begin{array}{l}\text { Formal Caregiver } \\
\text { (FC) } \\
\text { Characteristics } \\
\text { FC N } \\
\text { FC Age (mean) } \\
\text { FC Sex (\% } \\
\text { female) } \\
\text { FC Race (\% } \\
\text { majority) } \\
\text { FC Education } \\
\text { (mean years) }\end{array}$ & $\begin{array}{l}\text { Formal } \\
\text { Caregiver (FC) } \\
\text { Char. RS } \\
\text { FC Health } \\
\text { Status } \\
\text { FC Training } \\
\text { FC Education } \\
\text { FC Position } \\
\text { FC Length of } \\
\text { Service }\end{array}$ & \begin{tabular}{|l|} 
Outcome \\
Timing(s)
\end{tabular} & \begin{tabular}{|l|} 
PLWD \\
Outcomes
\end{tabular} & $\begin{array}{l}\text { Caregiver } \\
\text { Outcomes } \\
\text { IC: } \\
\text { FC: }\end{array}$ \\
\hline \begin{tabular}{|l|} 
Chien 20118 \\
$(21198803)$ \\
\\
Hong Kong \\
Medium \\
Explanatory
\end{tabular} & $\begin{array}{l}\text { Orientation } \\
\text { to dementia } \\
\text { care, } \\
\text { educational } \\
\text { workshop, } \\
\text { family role and } \\
\text { strength } \\
\text { rebuilding, } \\
\text { community } \\
\text { support } \\
\text { resources, } \\
\text { program, } \\
\text { evaluation } \\
\text { For } 6 \text { months }\end{array}$ & $\begin{array}{l}\text { Routine care } \\
\text { Usual } \\
\text { services } \\
\text { provided by } \\
\text { the dementia } \\
\text { resources } \\
\text { center }\end{array}$ & $\begin{array}{l}\text { Community } \\
\text { RCT } \\
N=92 \text { dyads }\end{array}$ & \begin{tabular}{|l|} 
Nonspecified \\
dementia \\
DSM-IV
\end{tabular} & $\begin{array}{l}\mathrm{N}=92 \\
\text { Age } 68 \\
44 \% \text { Female } \\
\text { Race NR } \\
\text { Education NR }\end{array}$ & $\begin{array}{l}\text { Mean monthly } \\
\text { household income } \\
14,000 \text { Hong Kong } \\
\text { dollars }\end{array}$ & $\begin{array}{l}\text { NR } \\
\text { Age 45 } \\
66 \% \text { Female } \\
\text { Race NR } \\
\text { Education } \\
27 \% \text { Primary or less } \\
64 \% \text { Secondary } \\
8 \% \text { Tertiary }\end{array}$ & NR & NA & NA & 12,18 months & $\begin{array}{l}\text { NPI } \\
\text { Institutionaliz } \\
\text { ation }\end{array}$ & \begin{tabular}{|l} 
Family \\
Caregiving \\
Burden \\
Inventory \\
WHOQOL- \\
BREF \\
Social \\
Support \\
Questionnaire \\
Family \\
Support \\
Services \\
Index
\end{tabular} \\
\hline \begin{tabular}{|l} 
Lam 20109 \\
(19606455) \\
\\
Hong Kong \\
Medium \\
Explanatory
\end{tabular} & $\begin{array}{l}\text { Case } \\
\text { Management } \\
\text { model: } \\
\text { Assessment } \\
\text { and advice. } \\
\text { Home-based } \\
\text { advice and } \\
\text { training on } \\
\text { cognitive } \\
\text { stimulation } \\
\text { strategies, } \\
\text { support sites \& } \\
\text { telephone calls } \\
\text { For } 4 \text { months }\end{array}$ & $\begin{array}{l}\text { One home } \\
\text { visit for home } \\
\text { safety at the } \\
\text { beginning of } \\
\text { the trial, no } \\
\text { case } \\
\text { management }\end{array}$ & $\begin{array}{l}\text { Community- } \\
\text { based } \\
\text { RCT } \\
\mathrm{N}=102\end{array}$ & \begin{tabular}{|l} 
Nonspecified \\
dementia \\
Chinese Mini- \\
Mental State \\
Examination \\
(CMMSE) 15+ \\
Clinical \\
Dementia Rating \\
Scale 1
\end{tabular} & $\begin{array}{l}\mathrm{N}=102 \\
\text { Age 78 } \\
58 \% \text { Female } \\
\text { Race NR } \\
\text { Education NR }\end{array}$ & NR & $\begin{array}{l}\mathrm{N}=102 \\
\text { Age NR } \\
74 \% \text { Female } \\
\text { Race NR } \\
\text { Education NR } \\
29 \% \text { Spouse or } \\
\text { partner } \\
51 \% \text { Child }\end{array}$ & NR & NA & NA & 12 months & \begin{tabular}{|l|} 
CSDD \\
NPI \\
Personal \\
Wellbeing \\
Index- \\
Intellectual \\
Disability
\end{tabular} & \begin{tabular}{|l} 
ZBI \\
General \\
Health \\
Questionnaire \\
Personal \\
Well-Being \\
Index \\
Social \\
support use
\end{tabular} \\
\hline \begin{tabular}{|l} 
Eloniemi- \\
Sulkava 200114 \\
$(11890485)$ \\
Finland \\
Medium \\
Explanatory
\end{tabular} & $\begin{array}{l}\text { Care } \\
\text { coordination } \\
\text { support } \\
\text { program } \\
2 \text { years }\end{array}$ & $\begin{array}{l}\text { Usual care } \\
\text { activities }\end{array}$ & $\begin{array}{l}\text { Home-based } \\
\text { RCT } \\
\mathrm{N}=100 \text { dyads }\end{array}$ & \begin{tabular}{|l|} 
Alzheimer's \\
disease \\
Vascular dementia \\
DSM-III
\end{tabular} & $\begin{array}{l}\mathrm{N}=100 \\
\text { Age } 79 \\
53 \% \text { Female } \\
\text { Race NR } \\
\text { Education NR }\end{array}$ & NR & $\begin{array}{l}\mathrm{N}=100 \\
\text { Age } 64 \\
69 \% \text { Female } \\
\text { Race NR } \\
\text { Education NR } \\
56 \% \% \text { Spouse } \\
35 \% \text { Child }\end{array}$ & NR & NA & NA & 2 years & $\begin{array}{l}\text { Rate of } \\
\text { institutionaliz } \\
\text { ation }\end{array}$ & \begin{tabular}{|l|} 
General \\
Health \\
Questionnaire
\end{tabular} \\
\hline
\end{tabular}

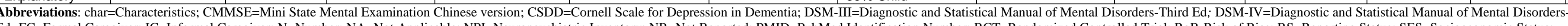
Ed; FC=Formal Caregiver; IC=Informal Caregiver; $N=$ Number; NA=Not Applicable; NPI=Neuropsychiatric Inventory;
WHOQOL-BREF= World Health Organization Quality of Life Measure-Brief Version; ZBI=Zarit Burden Interview 
Table F-10. PLWD outcomes summary for low and medium risk of bias studies: case management

\begin{tabular}{|c|c|c|c|c|c|}
\hline \begin{tabular}{|l|} 
Study (PMID) \\
Comparison \\
RoB \\
Category* \\
\end{tabular} & $\begin{array}{l}\text { Outcome } \\
\text { Timing }\end{array}$ & Summary Finding & Intervention & Comparator & p-Value \\
\hline $\begin{array}{l}\text { Chien, } 2011^{8}(21198803) \\
\text { Case management vs usual care } \\
\text { Medium } \\
\text { Explanatory }\end{array}$ & \begin{tabular}{|l|}
$\mathrm{NPI}$ \\
18 months
\end{tabular} & $\begin{array}{l}\text { Favors intervention } \\
\mathrm{F}(1,90)=3.70\end{array}$ & $\begin{array}{l}\text { Baseline 81.8 (SD 9.1) } \\
18 \text { month 76.5 (SD 8.9) }\end{array}$ & \begin{tabular}{|l|} 
Baseline 80.9 (SD 9.5) \\
18 month 82.4 (SD 11.0)
\end{tabular} & $<0.01$ \\
\hline $\begin{array}{l}\text { Chien, } 2011^{8}(21198803) \\
\text { Case management vs usual care } \\
\text { Medium } \\
\text { Explanatory }\end{array}$ & $\begin{array}{l}\text { Average number institutionalizations over previous } 6 \text { months } \\
18 \text { months }\end{array}$ & $\begin{array}{l}\text { Favors intervention } \\
\mathrm{F}(1,90)=4.32\end{array}$ & $\begin{array}{l}\text { Baseline 5.1 (SD 0.9) } \\
18 \text { month 2.6 (SD 1.9) }\end{array}$ & $\begin{array}{l}\text { Baseline } 5.5 \text { (SD 1.2) } \\
18 \text { month 6.0 (SD 2.9) }\end{array}$ & $<0.01$ \\
\hline $\begin{array}{l}\text { Lam, 20109 }(19606455) \\
\text { Medium } \\
\text { Explanatory }\end{array}$ & $\begin{array}{l}\text { Personal well-being index for intellectually disabled (PWI-ID) } \\
\text { difference in difference } \\
12 \text { month }\end{array}$ & No statistical difference & $\begin{array}{l}\text { (reported data must be } \\
\text { incorrect for NS finding) }\end{array}$ & $\begin{array}{l}\text { Change from baseline } 1.4 \\
(-7.1,10.7)\end{array}$ & NS \\
\hline $\begin{array}{l}\text { Lam, } 2010^{9}(19606455) \\
\text { Medium } \\
\text { Explanatory }\end{array}$ & $\begin{array}{l}\text { NPI } \\
\text { difference in difference } \\
12 \text { month }\end{array}$ & No statistical difference & $\begin{array}{l}\text { Change from baseline }-2.0 \\
(-18.5,6.0)\end{array}$ & $\begin{array}{l}\text { Change from baseline }-7.0 \\
(-19.0,0.0)\end{array}$ & NS \\
\hline $\begin{array}{l}\text { Eloniemi-Sulkava, 2001'12 (11890485) } \\
\text { Medium } \\
\text { Explanatory }\end{array}$ & \begin{tabular}{|l|} 
Rate of institutionalization \\
2 years
\end{tabular} & No statistical difference & $32 \%$ placement & $30 \%$ placement & NS \\
\hline
\end{tabular}

"Explanatory studies are listed first, followed by pragmatic studies.

Abbreviations: NA=Not Applicable; NR=Not Reported; PMID=PubMed Identification Number

\begin{tabular}{|c|c|c|c|c|c|}
\hline $\begin{array}{l}\text { Study (PMID) } \\
\text { Comparison } \\
\text { Caregiver Type } \\
\text { RoB } \\
\text { Category* }\end{array}$ & $\begin{array}{l}\text { Outcome } \\
\text { Timing }\end{array}$ & Summary Finding & Intervention & Comparator & $p$-Value \\
\hline $\begin{array}{l}\text { Chien, } 2011^{8}(21198803) \\
\text { Case management vs usual care } \\
\text { Medium } \\
\text { Explanatory }\end{array}$ & $\begin{array}{l}\text { Family caregiving burden inventory } \\
18 \text { months }\end{array}$ & $F(1,90)=7.09$ & $\begin{array}{l}\text { Baseline } 68.0 \text { (SD 14.8) } \\
18 \text { month } 45.5 \text { (SD 10.0) }\end{array}$ & $\begin{array}{l}\text { Baseline 66.9.0 (SD 14.8) } \\
18 \text { month 64.1 (SD 11.4) }\end{array}$ & $<0.001$ \\
\hline $\begin{array}{l}\text { Chien, } 2011^{8}(21198803) \\
\text { Case management vs usual care } \\
\text { Medium } \\
\text { Explanatory }\end{array}$ & $\begin{array}{l}\text { WHOQOL } \\
18 \text { months }\end{array}$ & $F(1,90)=6.81$ & $\begin{array}{l}\text { Baseline } 64.8 \text { (SD 13.0) } \\
18 \text { month } 82.7 \text { (SD 13.5) }\end{array}$ & $\begin{array}{l}\text { Baseline 64.8 (SD 13.0) } \\
18 \text { month } 64.5 \text { (SD 13.1) }\end{array}$ & $<0.001$ \\
\hline $\begin{array}{l}\text { Lam, } 2010^{9}(19606455) \\
\text { Medium } \\
\text { Explanatory }\end{array}$ & $\begin{array}{l}\text { Personal well-being Index for Adult } \\
12 \text { month }\end{array}$ & No statistical difference & $\begin{array}{l}\text { Change from baseline } 2.9 \\
(-15.0,7.1)\end{array}$ & $\begin{array}{l}\text { Change from baseline } 0.0 \\
(-6.1,5.0)\end{array}$ & NS \\
\hline $\begin{array}{l}\text { Lam, 20109 }(19606455) \\
\text { Medium } \\
\text { Explanatory }\end{array}$ & $\begin{array}{l}Z \mathrm{ZBI} \\
12 \text { month }\end{array}$ & No statistical difference & $\begin{array}{l}\text { Change from baseline } 5.0 \\
(-10.5,12.0)\end{array}$ & $\begin{array}{l}\text { Change from baseline } 3.5 \\
(-9.3,12.3)\end{array}$ & NS \\
\hline $\begin{array}{l}\text { Lam, } 2010^{9}(19606455) \\
\text { Medium } \\
\text { Explanatory }\end{array}$ & $\begin{array}{l}\text { General Health Questionnaire } \\
12 \text { month }\end{array}$ & No statistical difference & $\begin{array}{l}\text { Change from baseline } 1.0(-2.0 \text {, } \\
5.5)\end{array}$ & $\begin{array}{l}\text { Change from baseline } 0.0 \\
(-2.0,3.0)\end{array}$ & NS \\
\hline
\end{tabular}

abbreviations: NS=Not Significant; PMID=PubMed Identification Number; RoB-Risk of Bias; SD=Standard deviation; WHOQOL=World Health Organization Quality of Life Measure; ZBI=Zarit Burden Interview

Table F-12. Summary of strength of evidence for PLWD outcomes: consultation services

\begin{tabular}{|c|c|c|c|c|c|c|c|c|}
\hline $\begin{array}{l}\text { Comparison } \\
\text { Outcome }\end{array}$ & Timing & $\begin{array}{l}\text { \# Studies/ Design } \\
\text { ( } \mathrm{n} \text { analyzed) }\end{array}$ & Finding or Summary Statistic & Study Limitations & Consistency & Directness & Precision & $\begin{array}{l}\text { Overall Grade/ } \\
\text { Conclusion }\end{array}$ \\
\hline $\begin{array}{l}\text { Institutionalization } \\
\text { Case management vs } \\
\text { usual care }\end{array}$ & 18-24 months & 2 RCTs (n=192) & Mixed findings for institutionalization & Moderate & Unknown & Direct & Imprecise & Insufficient \\
\hline $\begin{array}{l}\mathrm{NPI} \\
\text { Case management vs } \\
\text { usual care }\end{array}$ & $12-18$ months & 2 RCT (n=194) & Mixed findings for NPI & Moderate & Unknown & Direct & Imprecise & Insufficient \\
\hline $\begin{array}{l}\text { Quality of Life } \\
\text { Case management vs } \\
\text { usual care } \\
\end{array}$ & 12 months & $1 \mathrm{RCT}(\mathrm{n}=102)$ & No significant difference between groups & Moderate & Unknown & Direct & Imprecise & Insufficient \\
\hline \begin{tabular}{|l} 
Depression \\
Case management vs \\
usual care
\end{tabular} & 12 months & $1 \mathrm{RCT}(\mathrm{n}=102)$ & No significant difference between groups & Moderate & Unknown & Direct & Imprecise & Insufficient \\
\hline
\end{tabular}

Abbreviations: N=Number; NPI=Neuropsychiatric Inventory; RCT=Randomized Controlled TriaL 
Table F-13. Summary of strength of evidence for caregiver outcomes: consultation services

\begin{tabular}{|c|c|c|c|c|c|c|c|c|}
\hline $\begin{array}{l}\text { Comparison } \\
\text { Outcome }\end{array}$ & Timing & $\begin{array}{l}\text { \# Studies/ Design } \\
\text { (n analyzed) }\end{array}$ & Finding or Summary Statistic & Study Limitations & Consistency & Directness & Precision & $\begin{array}{l}\text { Overall Gradel } \\
\text { Conclusion }\end{array}$ \\
\hline $\begin{array}{l}\text { Quality of Life } \\
\text { Case management vs } \\
\text { usual care }\end{array}$ & $12-18$ months & 2 RCTs (n=194) & Mixed findings for NPI & Moderate & Unknown & Direct & Imprecise & Insufficient \\
\hline $\begin{array}{l}\text { Caregiver Burden } \\
\text { Case management vs } \\
\text { usual care }\end{array}$ & $12-18$ months & 2 RCT (n=194) & Mixed findings for NPI & Moderate & Unknown & Direct & Imprecise & Insufficient \\
\hline $\begin{array}{l}\text { Caregiver health status } \\
\text { Case management vs } \\
\text { usual care }\end{array}$ & 12 months & $1 \mathrm{RCT}(\mathrm{n}=102)$ & No significant difference between groups & Moderate & Unknown & Direct & Imprecise & Insufficient \\
\hline
\end{tabular}

Abbreviations: N=Number; NPI=Neuropsychiatric Inventory; RCT=Randomized Controlled TriaL 


\section{Evidence Map: Case Management}

\begin{tabular}{|c|c|c|c|c|c|c|c|c|c|c|c|c|c|}
\hline $\begin{array}{l}\text { Study (PMID) } \\
\text { Country } \\
\text { EM Reason }\end{array}$ & Intervention & Comparison & $\begin{array}{l}\text { Setting } \\
\text { and Design } \\
\text { Setting } \\
\text { Design } \\
\text { Cluster N } \\
\text { Participants } \\
\text { Randomized } \\
\text { N }\end{array}$ & \begin{tabular}{|l|} 
PLWD \\
Dementia \\
Characteristics
\end{tabular} & \begin{tabular}{|l|} 
PLWD \\
Non-Disease Char \\
PLWD N \\
PLWD Age (mean) \\
PLWD Sex (\% \\
female) \\
PLWD Race (\% \\
majority) \\
PLWD Education \\
(mean years)
\end{tabular} & $\begin{array}{l}\text { PLWD } \\
\text { Non-Disease } \\
\text { Char Reporting } \\
\text { Status (RS) } \\
\text { PLWD SES } \\
\text { PLWD Prior } \\
\text { Disability } \\
\text { PLWD } \\
\text { Household } \\
\text { Characteristics } \\
\text { PLWD Health } \\
\text { Insurance } \\
\text { PLWD Detailed } \\
\text { Race Information }\end{array}$ & $\begin{array}{l}\text { Informal Caregiver } \\
\text { (IC) } \\
\text { Characteristics } \\
\text { IC N } \\
\text { IC Age (mean) } \\
\text { IC Sex (\% female) } \\
\text { IC Race (\% } \\
\text { majority) } \\
\text { IC Education } \\
\text { (mean years) } \\
\text { IC Relation to } \\
\text { PLWD (\% majority) }\end{array}$ & $\begin{array}{l}\text { Informal Caregiver } \\
\text { (IC) Char. RS } \\
\text { IC Duration } \\
\text { IC Living With } \\
\text { PLWD } \\
\text { IC Payment } \\
\text { IC Health Status } \\
\text { IC Dementia } \\
\text { Family History } \\
\text { IC Employment } \\
\text { Status } \\
\text { IC Training }\end{array}$ & 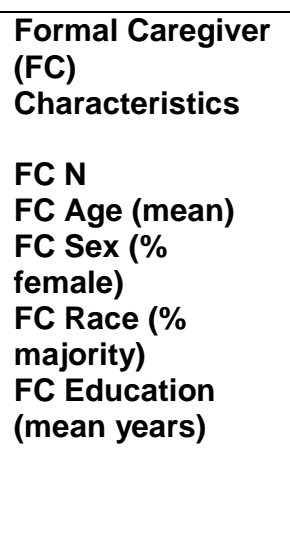 & $\begin{array}{l}\text { Formal } \\
\text { Caregiver } \\
\text { (FC) Char. } \\
\text { RS } \\
\text { FC Health } \\
\text { Status } \\
\text { FC Training } \\
\text { FC } \\
\text { Education } \\
\text { FC Position } \\
\text { FC Length of } \\
\text { Service }\end{array}$ & $\begin{array}{l}\text { Outcome } \\
\text { Timing(s) }\end{array}$ & $\begin{array}{l}\text { PLWD } \\
\text { Outcomes }\end{array}$ & $\begin{array}{l}\text { Caregiver } \\
\text { Outcomes } \\
\text { IC: } \\
\text { FC: }\end{array}$ \\
\hline $\begin{array}{l}\text { Xiao, 2016 } \\
(25805891) \\
\\
\text { Australia } \\
\text { High ROB }\end{array}$ & $\begin{array}{l}\text { Case manager, } \\
\text { home visit for } \\
\text { initial } \\
\text { assessment, } \\
\text { monthly } \\
\text { telephone } \\
\text { contact }\end{array}$ & $\begin{array}{l}\text { Usual care } \\
\text { activities }\end{array}$ & $\begin{array}{l}\text { Community } \\
\text { RCT } \\
\text { Caregivers }\end{array}$ & \begin{tabular}{|l|} 
Dementia \\
unspecified, from \\
minority groups
\end{tabular} & $\begin{array}{l}\mathrm{N}=61 \\
\text { Age: Yes } \\
\text { Sex: Yes } \\
\text { Race: No } \\
\text { Education: no }\end{array}$ & $\begin{array}{l}\text { (minority groups } \\
\text { not specified) }\end{array}$ & $\begin{array}{l}\mathrm{N}=61 \\
\text { Age: Yes } \\
\text { Sex: Yes } \\
\text { \% majority race: No } \\
\text { Education: no } \\
\text { Relationship: Yes }\end{array}$ & \begin{tabular}{|l|} 
Duration \\
Living with PLWD \\
Health status
\end{tabular} & NA & NA & $\begin{array}{l}6,12 \\
\text { months }\end{array}$ & $\begin{array}{l}\text { Blessed } \\
\text { dementia } \\
\text { score } \\
\text { NPI } \\
\text { QUORE }\end{array}$ & $\begin{array}{l}\text { SSCQ } \\
\text { SF-36v2 }\end{array}$ \\
\hline $\begin{array}{l}\text { MacNeil } \\
\text { Vroomen, 20157 } \\
\text { (26170035) } \\
\text { Netherlands } \\
\text { High RoB }\end{array}$ & $\begin{array}{l}\text { Case } \\
\text { management: } \\
\text { (1) provided } \\
\text { within one care } \\
\text { organization } \\
\text { (ICMM), (2)case } \\
\text { management } \\
\text { where } \\
\text { multiple case } \\
\text { management } \\
\text { organizations } \\
\text { are present } \\
\text { within one } \\
\text { region (LM) }\end{array}$ & $\begin{array}{l}\text { Control: group } \\
\text { with no access } \\
\text { to case } \\
\text { management }\end{array}$ & $\begin{array}{l}\text { Home setting } \\
\text { Non-RCT } \\
521 \text { dyads }\end{array}$ & & $\begin{array}{l}\text { N=521 } \\
\text { Age: Yes } \\
\text { Sex: Yes } \\
\text { Race: No } \\
\text { Education: Yes }\end{array}$ & $\begin{array}{l}\text { Marital status } \\
\text { Living condition }\end{array}$ & $\begin{array}{l}\text { N=521 } \\
\text { Age: Yes } \\
\text { Sex: Yes } \\
\text { Race: No } \\
\text { Education: Yes } \\
\text { Relationship: Yes }\end{array}$ & $\begin{array}{l}\text { Living condition: } \\
\text { Yes }\end{array}$ & NA & NA & 2 years & $\begin{array}{l}\text { Neuropsychi } \\
\text { atric } \\
\text { problems: } \\
\text { NPI }\end{array}$ & $\begin{array}{l}\text { Psychological } \\
\text { health: (GHQ- } \\
\text { 12) }\end{array}$ \\
\hline $\begin{array}{l}\text { Brodaty, 200310 } \\
(12590626) \\
\text { Australia } \\
\text { High RoB }\end{array}$ & $\begin{array}{l}\text { Different models } \\
\text { of care: } \\
\text { (1) } \\
\text { Psychogeriatric } \\
\text { case } \\
\text { management } \\
\text { (2) } \\
\text { Psychogeriatric } \\
\text { consultation }\end{array}$ & $\begin{array}{l}\text { Control: } \\
\text { continued to } \\
\text { receive } \\
\text { whatever } \\
\text { treatment they } \\
\text { would have had } \\
\text { were the survey } \\
\text { not to have } \\
\text { taken place. }\end{array}$ & $\begin{array}{l}\text { Nursing home } \\
\text { RCT } \\
102 \text { PLWD }\end{array}$ & Not specified & $\begin{array}{l}\mathrm{N}=102 \\
\text { Age: Yes } \\
\text { Sex: Yes } \\
\text { Race: No } \\
\text { Education: No }\end{array}$ & None & NA & NA & NA & NA & 12 weeks & $\begin{array}{l}\text { Depression: } \\
\text { HAM-D; } \\
\text { CSD; GDS \& } \\
\text { EBAS-DEP } \\
\text { Psychopatho } \\
\text { logy: (NPII) } \\
\text { Behavior } \\
\text { pathology: } \\
\text { BEHAVE-AD }\end{array}$ & NA \\
\hline $\begin{array}{l}\text { Challis, 2002 } \\
(11994884) \\
\text { UK } \\
\text { Hiah ROB }\end{array}$ & $\begin{array}{l}\text { Intensive case } \\
\text { management, } \\
\text { protected small } \\
\text { caseloads per } \\
\text { worker }\end{array}$ & $\begin{array}{l}\text { Usual care } \\
\text { activities }\end{array}$ & $\begin{array}{l}\text { Community } \\
\text { Matched case } \\
\text { control } \\
43 \text { matched } \\
\text { pairs }\end{array}$ & Not specified & $\begin{array}{l}\mathrm{N}=86 \\
\text { Age: Yes } \\
\text { Sex: Yes } \\
\text { Race: } \text { No } \\
\text { Education: No }\end{array}$ & SES & NR & NR & NA & NA & 2 years & $\begin{array}{l}\text { Unmet } \\
\text { needs } \\
\text { Service } \\
\text { patterns and } \\
\text { costs }\end{array}$ & $\begin{array}{l}\text { IC: Caregiver } \\
\text { burden }\end{array}$ \\
\hline $\begin{array}{l}\text { Newcomer, } \\
1999^{15} \\
(10445896) \\
\text { US } \\
\text { Pilot } \\
\text { demonstration }\end{array}$ & $\begin{array}{l}\text { Medicare } \\
\text { demonstration } \\
\text { of case } \\
\text { management } \\
\text { (high attrition } \\
\text { over 3 years) }\end{array}$ & $\begin{array}{l}\text { Higher client to } \\
\text { case manager } \\
\text { ratio }\end{array}$ & $\begin{array}{l}\text { Community } \\
\text { RCT } \\
\text { Caregivers }\end{array}$ & $\begin{array}{l}\text { Alzheimer's } \\
\text { disease }\end{array}$ & $\begin{array}{l}\text { N=2731 } \\
\text { Age: Yes } \\
\text { Sex: Yes } \\
\text { Race: yes } \\
\text { Education: No }\end{array}$ & None & $\begin{array}{l}\mathrm{N}=2576 \\
\text { Age: Yes } \\
\text { Sex: Yes } \\
\text { Race: No } \\
\text { Education: no } \\
\text { Relationship: Yes }\end{array}$ & None & $\mathrm{NA}$ & NA & $\begin{array}{l}3 \text { years } \\
\text { (attrition } \\
64 \%)\end{array}$ & NR & $\begin{array}{l}\text { Caregiver } \\
\text { burden and } \\
\text { depression }\end{array}$ \\
\hline $\begin{array}{l}\text { Weinberger, } \\
1993^{13} \\
(8426038) \\
\text { Us } \\
\text { High RoB }\end{array}$ & $\begin{array}{l}\text { Social work } \\
\text { case } \\
\text { management }\end{array}$ & $\begin{array}{l}\text { Usual care } \\
\text { activities }\end{array}$ & $\begin{array}{l}\text { Community } \\
\text { RCT } \\
\text { Caregivers }\end{array}$ & $\begin{array}{l}\text { Progressive } \\
\text { dementia } \\
\text { unspecified }\end{array}$ & $\begin{array}{l}\text { N=264 } \\
\text { Age: Yes } \\
\text { Sex: Yes } \\
\text { Race: Yes } \\
\text { Education: No }\end{array}$ & $\begin{array}{l}\text { SES } \\
\text { Household } \\
\text { characteristics }\end{array}$ & $\begin{array}{l}\mathrm{N}=264 \\
\text { Age: Yes } \\
\text { Sex: Yes } \\
\text { Race: Yes } \\
\text { Education: Yes } \\
\text { Relationship: Yes }\end{array}$ & $\begin{array}{l}\text { Duration } \\
\text { Health status } \\
\text { Employment }\end{array}$ & NA & NA & 6 months & $\begin{array}{l}\text { Health } \\
\text { utilization }\end{array}$ & NR \\
\hline
\end{tabular}

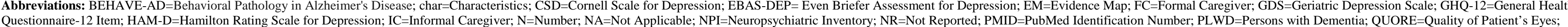
Questionnaire-12 Item; HAM-D=Hamilton Rating Scale for Depression; IC=Informal Caregiver; N=Number; NA=Not Applicable; NPI=Neuropsychiatric Inventory; NR=Not Reported; PMID=PubM
RCT=Randomized Controlled Trial; RoB=Risk of Bias; RS=Reporting Status; SES=Socioeconomic Status; SF-36V2=Quality of Life Questionnaire; SSCQ=Sense of Competence Questionnaire 


\section{Care Protocols for PLWD}

Table F-15. Risk of bias assessment: care protocols for PLWD

\begin{tabular}{|c|c|c|c|c|c|c|c|c|c|}
\hline Study (PMID) & Outcome Timing & $\begin{array}{l}\text { Attrition Bias } \\
\text { Attrition } \%\end{array}$ & Selection Bias & Detection Bias & Performance Bias & Reporting Bias & Fidelity Bias & Funder & Overall Rating \\
\hline \begin{tabular}{|l|l|} 
Appelhof, 201916 (30799167) \\
\end{tabular} & \begin{tabular}{|l}
18 months stepped \\
wedge
\end{tabular} & Stepped wedge & $x$ & $x$ & $x$ & $x$ & High & & High \\
\hline 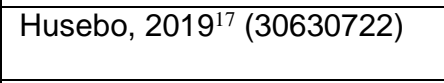 & 9 months & $\begin{array}{l}\text { Medium } \\
27 \%\end{array}$ & Low & $\mathrm{X}$ & $\mathrm{x}$ & $\mathrm{X}$ & High & $\mathrm{x}$ & High \\
\hline \begin{tabular}{|l} 
Livingston $2019^{18}(30872010)$ \\
\end{tabular} & 8 months & $\begin{array}{l}\text { Medium } \\
21 \%\end{array}$ & Medium & Medium & Medium & Low & High & Government & High \\
\hline \begin{tabular}{|l|l} 
Nakanishi $2017^{19}(28857263)$ \\
\end{tabular} & 6 months & $\begin{array}{l}\text { Medium } \\
15 \%\end{array}$ & Low & High & Medium & Low & High & Government & High \\
\hline \begin{tabular}{|l|l|} 
Pieper $2016^{20}(26804064)$ \\
\end{tabular} & & $\begin{array}{l}\text { Medium } \\
11 \%\end{array}$ & Low & Low & Low & Low & High & Nonprofit & High \\
\hline \begin{tabular}{|l|l|} 
McCabe, $2015^{21}$ (25319535) \\
\end{tabular} & 3 months & $\begin{array}{l}\text { Medium } \\
13 \% \\
\end{array}$ & Low & Medium & Medium & Low & High & & High \\
\hline Zwijsen, 201442 (24878214) & Stepped wedge & & $\mathrm{X}$ & $\mathrm{x}$ & $\mathrm{x}$ & $\mathrm{X}$ & High & & High \\
\hline 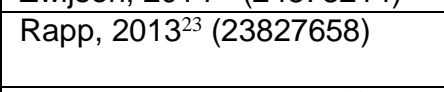 & 10 months & $\begin{array}{l}\text { Medium } \\
15 \% \\
\end{array}$ & Medium & Low & High & Low & High & & High \\
\hline $\begin{array}{l}\begin{array}{l}\text { Cohen-Mansfield, } 2012^{24} \\
(23059151)\end{array} \\
\end{array}$ & 2 weeks & $\begin{array}{l}\text { High } \\
46 \%\end{array}$ & $\mathrm{x}$ & $\mathrm{x}$ & $x$ & $\mathrm{x}$ & $x$ & & High \\
\hline Kovach, $2012^{25}(22998656)$ & & Low & $\begin{array}{l}\text { High } \\
30 \%\end{array}$ & $\mathrm{x}$ & $x$ & $\mathrm{X}$ & $x$ & & High \\
\hline Salva, 2011'26 (22159768) & & Low & $\begin{array}{l}\text { High } \\
31 \%\end{array}$ & $\mathrm{X}$ & $x$ & $\mathrm{x}$ & $\mathrm{x}$ & & High \\
\hline 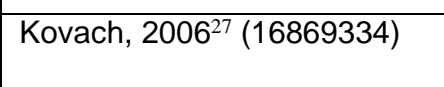 & 8 weeks & $\begin{array}{l}\text { Medium } \\
11 \%\end{array}$ & Low & Low & High & Low & Low & Government & High \\
\hline
\end{tabular}

$\mathrm{X}$ indicates that domain was not assessed due to high risk of bias.

Abbreviations: PMID=PubMed Identification Number

\section{Evidence Map: Care Protocols for PLWD}

\begin{tabular}{|c|c|c|c|c|c|c|c|c|c|c|c|c|c|}
\hline \begin{tabular}{|l|} 
Study (PMID) \\
Country \\
EM Reason
\end{tabular} & Intervention & Comparison & \begin{tabular}{|l|} 
Setting \\
and Design \\
Setting \\
Design \\
cluster N \\
Participants \\
Randomized \\
N
\end{tabular} & $\begin{array}{l}\text { PLWD } \\
\text { Dementia } \\
\text { Characteristics }\end{array}$ & $\begin{array}{l}\text { PLWD } \\
\text { Non-Disease Char } \\
\text { PLWD N } \\
\text { PLWD Age (mean) } \\
\text { PLWD Sex (\% } \\
\text { female) } \\
\text { PLWD Race (\% } \\
\text { majority) } \\
\text { PLWD Education } \\
\text { (mean years) }\end{array}$ & $\begin{array}{l}\text { PLWD } \\
\text { Non-Disease } \\
\text { Char Reporting } \\
\text { Status (RS) } \\
\text { PLWD SES } \\
\text { PLWD Prior } \\
\text { Disability } \\
\text { PLWD } \\
\text { Household } \\
\text { Characteristics } \\
\text { PLWD Health } \\
\text { Insurance } \\
\text { PLWD Detailed } \\
\text { Race Information }\end{array}$ & \begin{tabular}{|l|} 
Informal Caregiver \\
(IC) \\
Characteristics \\
IC N \\
IC Age (mean) \\
IC Sex (\% female) \\
IC Race (\% \\
majority) \\
IC Education \\
(mean years) \\
IC Relation to \\
PLWD (\% majority)
\end{tabular} & $\begin{array}{l}\text { Informal Caregiver } \\
\text { (IC) Char. RS } \\
\text { IC Duration } \\
\text { IC Living With } \\
\text { PLWD } \\
\text { IC Payment } \\
\text { IC Health Status } \\
\text { IC Dementia } \\
\text { Family History } \\
\text { IC Employment } \\
\text { Status } \\
\text { IC Training }\end{array}$ & \begin{tabular}{|l} 
Formal Caregiver \\
(FC) \\
Characteristics \\
FC N \\
FC Age (mean) \\
FC Sex (\% \\
female) \\
FC Race (\% \\
majority) \\
FC Education \\
(mean years)
\end{tabular} & $\begin{array}{l}\text { Formal } \\
\text { Caregiver (FC) } \\
\text { Char. RS } \\
\text { FC Health } \\
\text { Status } \\
\text { FC Training } \\
\text { FC Education } \\
\text { FC Position } \\
\text { FC Length of } \\
\text { Service }\end{array}$ & \begin{tabular}{|l|} 
Outcome \\
Timing(s)
\end{tabular} & $\begin{array}{l}\text { PLWD } \\
\text { Outcomes }\end{array}$ & $\begin{array}{l}\text { Caregiver } \\
\text { Outcomes } \\
\text { IC: } \\
\text { FC: }\end{array}$ \\
\hline \begin{tabular}{|l|} 
Appelhof, \\
201916 \\
(30799167) \\
Netherlands \\
\\
High RoB
\end{tabular} & \begin{tabular}{|l} 
The care \\
program: \\
Involves five \\
steps: \\
evaluation of \\
psychotropic \\
drug \\
prescription, \\
detection, \\
analysis, \\
treatment, \& \\
evaluation of \\
treatment of \\
NPS
\end{tabular} & $\begin{array}{l}\text { Control: } \\
\text { consisted of } \\
\text { care } \\
\text { as usual, } \\
\text { without the } \\
\text { educational } \\
\text { program and } \\
\text { use of } \\
\text { the care } \\
\text { program }\end{array}$ & \begin{tabular}{|l|} 
Young-onset \\
dementia \\
Special care \\
unit \\
RCT (stepped \\
wedge) \\
274 PLWD \\
\end{tabular} & $\begin{array}{l}\text { Mild-severe } \\
\text { dementia (All } \\
\text { types) }\end{array}$ & $\begin{array}{l}\mathrm{N}=274 \\
\text { Age: Yes } \\
\text { Sex: yes } \\
\text { Race: No } \\
\text { Education: No }\end{array}$ & None & NA & NA & NA & NA & 18 months & $\begin{array}{l}\text { Agitation \& } \\
\text { aggression: } \\
\text { CMAI (Dutch) } \\
\text { NPS: NPI-NH } \\
\text { (Dutch) }\end{array}$ & NA \\
\hline
\end{tabular}




\begin{tabular}{|c|c|c|c|c|c|c|c|c|c|c|c|c|c|}
\hline $\begin{array}{l}\text { Livingston } \\
2019^{18} \\
(30872010) \\
\text { UK } \\
\text { High RoB }\end{array}$ & $\begin{array}{l}\text { MARQUE:6 } \\
\text { sessions of } \\
\text { manual-based } \\
\text { intervention, } \\
\text { followed by an } \\
\text { implementation } \\
\text { and supervision } \\
\text { period (panel) }\end{array}$ & $\begin{array}{l}\text { Treatment as } \\
\text { usual }\end{array}$ & \begin{tabular}{|l|} 
Care homes \\
in Uk \\
Cluster RCT \\
20 Care \\
homes \\
404 PLWD \\
492 CG
\end{tabular} & $\begin{array}{l}\text { Clinical diagnosis } \\
\text { of dementia }\end{array}$ & $\begin{array}{l}\text { N=404 } \\
\text { Age: Yes } \\
\text { Sex: yes } \\
\text { Race: yes } \\
\text { Education: yes }\end{array}$ & None & NA & NA & $\begin{array}{l}\mathrm{N}=492 \\
\text { Age: Yes } \\
\text { Sex: yes } \\
\text { Race: yes } \\
\text { Education: yes }\end{array}$ & $\begin{array}{l}\text { Length of service } \\
\text { Training }\end{array}$ & 8 months & $\begin{array}{l}\text { CMAl } \\
\text { NPI } \\
\text { DEMQOL } \\
\text { EQ-5D 5L } \\
\text { CDR }\end{array}$ & $\begin{array}{l}\text { MBI } \\
\text { SCID } \\
\text { STS }\end{array}$ \\
\hline $\begin{array}{l}\text { Nakanishi } \\
2017^{19} \\
(28857263) \\
\text { Japan } \\
\text { High RoB }\end{array}$ & $\begin{array}{l}\text { BASE: } \\
\text { consisted of } \\
\text { training course; } \\
\text { a web-based } \\
\text { tool for ongoing } \\
\text { monitoring and } \\
\text { assessment for } \\
\text { challenging } \\
\text { behavior, and a } \\
\text { multiagency } \\
\text { discussion } \\
\text { meeting. }\end{array}$ & $\begin{array}{l}\text { Same as the } \\
\text { intervention } \\
\text { group, the care } \\
\text { professionals' } \\
\text { input of } \\
\text { measures of } \\
\text { their persons } \\
\text { with dementia } \\
\text { using the web- } \\
\text { based tool at } \\
\text { base-line. } \\
\text { However, the } \\
\text { web-based tool } \\
\text { for the control } \\
\text { group had no } \\
\text { assessment of } \\
\text { unmet needs } \\
\text { nor an action } \\
\text { plan }\end{array}$ & \begin{tabular}{|l|} 
Home care \\
service \\
providers \\
Cluster RCT \\
283 PLWD \\
45 Home \\
care \\
providers \\
98 case \\
managers \\
and CG
\end{tabular} & $\begin{array}{l}\text { Dementia } \\
\text { diagnosis }\end{array}$ & $\begin{array}{l}\text { N=283 } \\
\text { Age: Yes } \\
\text { Sex: yes } \\
\text { Race: No } \\
\text { Education: No }\end{array}$ & None & NA & NA & $\mathrm{NR}$ & $\mathrm{NR}$ & 6 months & $\begin{array}{l}\text { NPI-NH } \\
\text { Abbey Pain } \\
\text { Scale } \\
\text { BI } \\
\text { SMQ }\end{array}$ & NR \\
\hline $\begin{array}{l}\text { Chen, 2016 } \\
\text { (26584896) } \\
\text { Taiwan } \\
\text { Training }\end{array}$ & $\begin{array}{l}\text { PRT: Basic pain } \\
\text { education + } \\
\text { PRT }\end{array}$ & $\begin{array}{l}\text { Basic pain } \\
\text { education alone }\end{array}$ & \begin{tabular}{|l|} 
Dementia \\
special care \\
unit \\
RCT (Cluster) \\
37 NRs +195 \\
PLWDs \\
\end{tabular} & NR & $\mathrm{NA}$ & NA & $\begin{array}{l}\mathrm{N}=195 \\
\text { Age: Yes } \\
\text { Sex: Yes } \\
\text { Race: No } \\
\text { Education: Yes }\end{array}$ & None & $\begin{array}{l}\text { N=37 } \\
\text { Age: Yes } \\
\text { Sex: No } \\
\text { Race: No } \\
\text { Education: No }\end{array}$ & $\begin{array}{l}\text { Years of } \\
\text { experience: Yes }\end{array}$ & $\begin{array}{l}4 \text { months? } \\
\text { (Unclear) } \\
\text { 3-month } \\
\text { follow up }\end{array}$ & Pain reduction: & $\begin{array}{l}\text { Improving the } \\
\text { pain } \\
\text { management } \\
\text { performance } \\
\text { of RNs }\end{array}$ \\
\hline $\begin{array}{l}\text { Pieper 201629 } \\
\text { Netherlands } \\
\text { High ROB }\end{array}$ & $\begin{array}{l}\text { STA OP! } \\
\text { Stepwise } \\
\text { multicomponent } \\
\text { intervention for } \\
\text { NH healthcare } \\
\text { professionals } \\
\text { Training on } \\
\text { general nursing } \\
\text { skills, dementia } \\
\text { management } \\
\text { and pain }\end{array}$ & $\begin{array}{l}\text { Treatment as } \\
\text { usual }\end{array}$ & \begin{tabular}{l|} 
Nursing \\
home-based \\
Cluster RCT \\
21 clusters in \\
12 nursing \\
homes \\
$\mathrm{N}=288$ PLWD
\end{tabular} & $\begin{array}{l}\text { Nonspecified } \\
\text { advanced } \\
\text { dementia } \\
\text { Reisberg Global } \\
\text { Deterioration } \\
\text { Scale 5-7 }\end{array}$ & $\begin{array}{l}\mathrm{N}=288 \\
\text { Age yes } \\
\text { Sex: yes } \\
\text { Race: } n \text { no } \\
\text { Education no }\end{array}$ & NR & $\mathrm{NA}$ & NA & $\mathrm{NR}$ & $\mathrm{NR}$ & $\begin{array}{l}3,6 \\
\text { months }\end{array}$ & $\begin{array}{l}\text { CMAI } \\
\text { NPI-NH } \\
\text { CSDD } \\
\text { Minimum } \\
\text { Dataset } \\
\text { Depression in } \\
\text { Dementia } \\
\text { Psychotropic } \\
\text { medication use }\end{array}$ & NR \\
\hline $\begin{array}{l}\text { Kovach, 201530 } \\
(26250849) \\
\text { USA } \\
\text { Feasibility study }\end{array}$ & T3 Protocol & Usual care & $\begin{array}{l}\text { Long term } \\
\text { care facility } \\
\text { RCT } \\
78 \text { PLWD }\end{array}$ & NR & $\begin{array}{l}\text { N=127 } \\
\text { Age: Yes } \\
\text { Sex: Yes } \\
\text { Race: No } \\
\text { Education: No }\end{array}$ & None & NA & NA & NA & NA & 8 weeks & $\begin{array}{l}\text { Feasibility, } \\
\text { assessments, } \\
\text { treatment } \\
\text { changes, nurse } \\
\text { time, and drug } \\
\text { costs }\end{array}$ & NA \\
\hline $\begin{array}{l}\text { McCabe, 2015 } \\
(25319535) \\
\text { Australia } \\
\text { High RoB }\end{array}$ & $\begin{array}{l}\text { The BPSD } \\
\text { protocol } \\
\text { training: } \\
\text { (1) Training \& } \\
\text { support } \\
\text { condition: } \\
\text { Training in the } \\
\text { use of a BPSD - } \\
\text { structured } \\
\text { clinical protocol, } \\
\text { plus external } \\
\text { clinical support } \\
\text { (2)Support } \\
\text { condition: a } \\
\text { workshop on } \\
\text { BPSD, plus } \\
\text { external clinical } \\
\text { support } \\
\text { (3) Training } \\
\text { condition: } \\
\text { training in the } \\
\text { use of the } \\
\text { structured } \\
\text { clinical protocol } \\
\text { alone }\end{array}$ & Care as usual & $\begin{array}{l}\text { Residential } \\
\text { care facility } \\
\text { RCT } \\
475 \\
\text { (staff=261 + } \\
\text { PLWD=214) }\end{array}$ & & $\begin{array}{l}\text { N=187 } \\
\text { Age: Yes } \\
\text { Sex: Yes } \\
\text { Race: No } \\
\text { Education: No }\end{array}$ & None & NA & NA & $\begin{array}{l}\mathrm{N}=204 \\
\text { Age: Yes } \\
\text { Sex: No } \\
\text { Race: No } \\
\text { Education: no }\end{array}$ & $\begin{array}{l}\text { Job position: Yes } \\
\text { Service length: } \\
\text { Yes } \\
\text { Dementia } \\
\text { training: Yes }\end{array}$ & 3 months & CMAI & $\begin{array}{l}\text { Staff stress: } \\
\text { Carer stress } \\
\text { scale } \\
\text { General strain: } \\
\text { SDCS } \\
\text { Self-efficacy: } \\
\text { Staff attitudde } \\
\text { toward PLWD: } \\
\text { (ADQ) }\end{array}$ \\
\hline
\end{tabular}




\begin{tabular}{|c|c|c|c|c|c|c|c|c|c|c|c|c|c|}
\hline $\begin{array}{l}\text { Zwijsen, 201531 } \\
\text { (25458804) } \\
\text { Netherlands } \\
\text { High RoB }\end{array}$ & $\begin{array}{l}\text { A new care } \\
\text { programme for } \\
\text { challenging } \\
\text { behaviour: care } \\
\text { staff was } \\
\text { educated on } \\
\text { how to detect } \\
\text { and reflect on } \\
\text { signs of } \\
\text { challenging } \\
\text { behavior, } \\
\text { consisting of } \\
\text { four steps; } \\
\text { detection, } \\
\text { analysis, } \\
\text { treatment \& } \\
\text { evaluation }\end{array}$ & $\begin{array}{l}\text { Control } \\
\text { (Stepped } \\
\text { wedged Cluster) }\end{array}$ & \begin{tabular}{|l|} 
Dementia \\
special care \\
Unit \\
RCT \\
(Stepped \\
wedge \\
cluster) \\
380?
\end{tabular} & NA & $\mathrm{NA}$ & $\mathrm{NA}$ & $\mathrm{NA}$ & NA & $\begin{array}{l}\mathrm{N}=380 \\
\text { Age: Yes } \\
\text { Sex: No } \\
\text { Race: No } \\
\text { Education: no }\end{array}$ & $\begin{array}{l}\text { Education: Yes } \\
\text { Job position: Yes } \\
\text { Service length: } \\
\text { Yes } \\
\text { Dementia } \\
\text { training: Yes }\end{array}$ & 4 months & NA & $\begin{array}{l}\text { Burnout: MBI } \\
\text { Job satisfaction } \\
\text { \& job demands: } \\
\text { Stress: Saliva } \\
\text { samples } \\
\text { Comorbid } \\
\text { burden: }\end{array}$ \\
\hline $\begin{array}{l}\text { Rapp, 201323 } \\
\text { 23827658) } \\
\\
\text { German } \\
\text { High ROB } \\
\text { (Protocol in } \\
\text { German) }\end{array}$ & $\begin{array}{l}\text { Guideline-based } \\
\text { intervention } \\
\text { includes clinical } \\
\text { assessments, } \\
\text { nondrug } \\
\text { interventions, } \\
\text { medication } \\
\text { review to } \\
\text { reduce agitation }\end{array}$ & Usual care & $\begin{array}{l}\text { Nursing } \\
\text { homes } \\
\text { Cluster RCT } \\
18 \text { nursing } \\
\text { homes } \\
\text { PIWD }\end{array}$ & $\begin{array}{l}\text { Dementia not } \\
\text { specified }\end{array}$ & $\begin{array}{l}\text { N=304 } \\
\text { Age: Yes } \\
\text { Sex: Yes } \\
\text { Race: No } \\
\text { Education: no } \\
\text { Marital status: no }\end{array}$ & None & NA & NA & $\mathrm{NR}$ & NR & 10 month & $\begin{array}{l}\text { CMAI } \\
\text { Number of } \\
\text { neuroleptics }\end{array}$ & NR \\
\hline $\begin{array}{l}\text { Cohen- } \\
\text { Mansfield, } \\
2012^{22} \\
(23059151) \\
\text { US } \\
\text { High ROB }\end{array}$ & $\begin{array}{l}\text { TREA decision } \\
\text { tree protocol to } \\
\text { assess unmet } \\
\text { needs for } \\
\text { agitation }\end{array}$ & Usual care & $\begin{array}{l}\text { Nursing } \\
\text { homes } \\
\text { Cluster RCT } \\
11 \text { nursing } \\
\text { homes } \\
\text { PLWD }\end{array}$ & $\begin{array}{l}\text { Dementia not } \\
\text { specified }\end{array}$ & $\begin{array}{l}\text { N=125 } \\
\text { Age: Yes } \\
\text { Sex: Yes } \\
\text { Race: yes } \\
\text { Education: yes } \\
\text { Marital status: yes }\end{array}$ & None & $\mathrm{NA}$ & NA & NR & NR & 2 weeks & $\begin{array}{l}\text { ABMI } \\
\text { LMBS }\end{array}$ & NR \\
\hline $\begin{array}{l}\text { Kovach, 2012 } \\
\text { (22998656) } \\
\text { USA } \\
\text { Hiah RoB }\end{array}$ & $\begin{array}{l}\text { Serial Trial } \\
\text { Intervention } \\
\text { (STI): A } \\
\text { decision support } \\
\text { tool-9-Step STI }\end{array}$ & 5-Step STI & \begin{tabular}{|l|} 
Nursing home \\
Quasi \\
125 PLWD
\end{tabular} & $\begin{array}{l}\text { Moderate to } \\
\text { severe dementia }\end{array}$ & $\begin{array}{l}\mathrm{N}=125 \\
\text { Age: Yes } \\
\text { Sex: Yes } \\
\text { Race: Yes } \\
\text { Education: No }\end{array}$ & None & $\mathrm{NA}$ & NA & NA & $\mathrm{NA}$ & 6 weeks & $\begin{array}{l}\text { Discomfort \& } \\
\text { agitation }\end{array}$ & NA \\
\hline $\begin{array}{l}\text { Montgomery, } \\
2011^{34} \\
(21840840) \\
\text { US } \\
\text { Pilot }\end{array}$ & $\begin{array}{l}\text { TCARE protocol } \\
\text { decision } \\
\text { algorithm } \\
\text { assists care } \\
\text { managers } \\
\text { create care } \\
\text { plans }\end{array}$ & $\begin{array}{l}\text { Usual care } \\
\text { activities }\end{array}$ & \begin{tabular}{|l|} 
Community \\
RCT \\
266 \\
caregivers
\end{tabular} & $\begin{array}{l}\text { Dementia } \\
\text { unspecified; } \\
\text { caregivers with } \\
\text { high burden such } \\
\text { that considering } \\
\text { nursing home } \\
\text { placement }\end{array}$ & NR & NR & $\begin{array}{l}\text { N=266 } \\
\text { Age: Yes } \\
\text { Sex: Yes } \\
\text { Race: No } \\
\text { Education: Yes } \\
\text { Relationship: yes }\end{array}$ & Health status & NA & NA & 9 months & NR & $\begin{array}{l}\text { Modified } \\
\text { Montgomery } \\
\text { Borgatta } \\
\text { Caregiver } \\
\text { Burden Scale; } \\
\text { CESD; } \\
\text { Intention to } \\
\text { place }\end{array}$ \\
\hline $\begin{array}{l}\text { Salva, 201126 } \\
(22159768) \\
\text { Spain } \\
\text { High ROB }\end{array}$ & $\begin{array}{l}\text { Health and } \\
\text { nutrition } \\
\text { promotion, } \\
\text { includes } \\
\text { nutritional } \\
\text { counseling }\end{array}$ & $\begin{array}{l}\text { Usual care } \\
\text { activities }\end{array}$ & $\begin{array}{l}\text { Community } \\
\text { Cluster RCT } \\
11 \text { Medical } \\
\text { centers } \\
\text { dyad }\end{array}$ & $\begin{array}{l}\text { Mild to moderate } \\
\text { dementia }\end{array}$ & $\begin{array}{l}\text { N=946 } \\
\text { Age: Yes } \\
\text { Sex: Yes } \\
\text { Race: no } \\
\text { Education: yes }\end{array}$ & & $\begin{array}{l}\text { N=946 } \\
\text { Age: no } \\
\text { Sex: no } \\
\text { Race :No } \\
\text { Education: no } \\
\text { Relationship: yes }\end{array}$ & $\begin{array}{l}\text { Duration } \\
\text { Received payment }\end{array}$ & NA & NA & 1 year & $\begin{array}{l}\text { CDR } \\
\text { NPI } \\
\text { MMSE } \\
\text { Eating behavior } \\
\text { scale }\end{array}$ & Zarit burden \\
\hline $\begin{array}{l}\text { Verkaik, 201135 } \\
\text { (21495077) } \\
\text { Netherlands } \\
\text { Training }\end{array}$ & $\begin{array}{l}\text { Introduction of a } \\
\text { nursing } \\
\text { guideleline: } \\
\text { Nursing team } \\
\text { introduced } \\
\text { nursing } \\
\text { guideline that } \\
\text { entails ting } \\
\text { increasing } \\
\text { individualized } \\
\text { pleasant } \\
\text { activities and } \\
\text { decreasing } \\
\text { unpleasant } \\
\text { events. }\end{array}$ & Usual care & \begin{tabular}{|l|} 
Nursing home \\
RCT \\
100 PLWD \\
\end{tabular} & $\begin{array}{l}\text { moderately severe } \\
\text { dementia (All } \\
\text { types) }\end{array}$ & $\begin{array}{l}\text { N=195 } \\
\text { Age: Yes } \\
\text { Sex: Yes } \\
\text { Race: No } \\
\text { Education: Yes } \\
\text { Marital status: Yes }\end{array}$ & None & NA & NA & NA & NA & 11 months & $\begin{array}{l}\text { Depession; } \\
\text { CSDD (Dutch } \\
\text { version } \\
\text { Mood }\end{array}$ & NA \\
\hline $\begin{array}{l}\text { Cohen- } \\
\text { Mansfield, } \\
2007^{36} \\
(17702884) \\
\text { US } \\
\text { Pilot }\end{array}$ & $\begin{array}{l}\text { TREA decision } \\
\text { tree protocol to } \\
\text { assess unmet } \\
\text { needs for } \\
\text { agitation }\end{array}$ & Usual care & \begin{tabular}{|l|} 
Nursing \\
homes \\
Quasi- \\
experimental \\
PLWD
\end{tabular} & $\begin{array}{l}\text { Dementia not } \\
\text { specified }\end{array}$ & $\begin{array}{l}\mathrm{N}=167 \\
\text { Age: Yes } \\
\text { Sex: Yes } \\
\text { Race: yes } \\
\text { Education: yes } \\
\text { Marital status: yes }\end{array}$ & None & $\mathrm{NA}$ & NA & $\mathrm{NR}$ & $\mathrm{NR}$ & 10 days & $\begin{array}{l}\text { ABMI } \\
\text { LMBS }\end{array}$ & NR \\
\hline
\end{tabular}




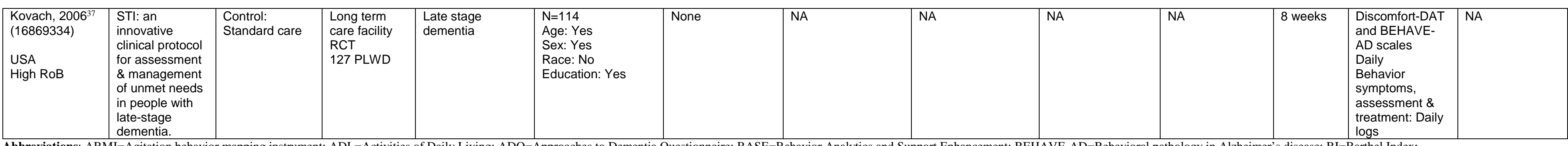

Abbreviations: ABMI=Agitation behavior mapping instrument; ADL=Activities of Daily Living; ADQ=Approaches to Dementia Questionnaire; BASE=Behavior Analytics and Support Enhancement; BEHAVE-AD=Behavioral pathology in Alzheimer's disease; BI=Barthel Index;

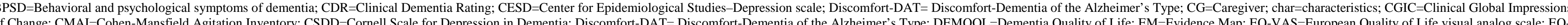
5D-5L=European quality of life five dimensions questionnaire; FC=formal caregiver; IC=informal caregiver; LMBS=Lawtons modified behavior stream; MBI=Maslach Burnout Inventory; MARQUE=Managing Agitation and Raising Quality of Life; MBI=Maslach Burnout Inventory;
MMSE=Mini-Mental Status Examination; N=number; NA=Not Applicable; NPI=Neuropsychiatric Inventory: NPI-NH=Neuropsychiatric Inventory Home Version; NPI-Q=Neuropsychiatric Inventory Questionnaire; NR=Not Reported; NPS=Neuropsychiatric symptoms; PMID=PubMed

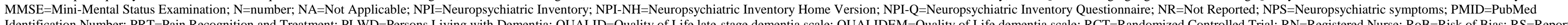

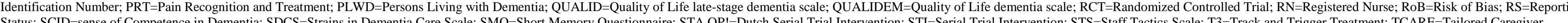
Assessment and Referral; TREA=Treatment Routes for Exploring Agitation; YOD=Young-onset Dementia

\section{Advance Care Planning}

Table F-17. Risk of bias assessment: advance care planning

\begin{tabular}{|c|c|c|c|c|c|c|c|c|c|}
\hline Study (PMID) & Outcome Timing & $\begin{array}{l}\text { Attrition Bias } \\
\text { Attrition \% }\end{array}$ & Selection Bias & Detection Bias & Performance Bias & Reporting Bias & Fidelity Bias & Funder & Overall Rating \\
\hline Brazil 201838 (28786323) & 6 weeks & $\begin{array}{l}\text { Medium } \\
19.3 \%\end{array}$ & Low & Low & High & Low & $\mathrm{x}$ & Government, Foundation & High \\
\hline \begin{tabular}{|l|} 
Mitchell 201839 29868778$)$ \\
Cohen 2019 $20(30273717)$
\end{tabular} & $\begin{array}{l}6 \text { months } \\
12 \text { months }\end{array}$ & $\begin{array}{l}\text { Low } \\
12 \text { months: } 2 \%\end{array}$ & Low & Low & Low & Medium & Low & Government & Medium \\
\hline $\begin{array}{l}\text { Hanson 201741 }(27893884) \\
\text { Hanson 2016 }\end{array}$ & \begin{tabular}{|l}
3 months \\
6 months \\
9 months
\end{tabular} & $\begin{array}{l}\text { Low } \\
3 \text { months: } 1 \% \\
6 \text { months: } 8.2 \% \\
\text { Medium } \\
9 \text { months: } 15.6 \&\end{array}$ & Low & Low & $\begin{array}{l}3 \text { months, } 9 \text { months: } \\
\text { Low } \\
6 \text { months: High }\end{array}$ & High & $\mathrm{x}$ & Government & High \\
\hline \begin{tabular}{|l|l} 
Reinhardt 201443 (24835382) \\
\end{tabular} & $\begin{array}{l}3 \text { months } \\
6 \text { months }\end{array}$ & $\begin{array}{l}\text { Medium } \\
3 \text { months: } 12.7 \% \\
6 \text { months: } 18.1 \% \\
\end{array}$ & Medium & Low & High & Low & $\mathrm{x}$ & Foundation & High \\
\hline
\end{tabular}

indicates that domain was not assessed due to bigh risk of bis.

Abbreviations: NR=Not Reported; PMID=PubMed Identification Number

\begin{tabular}{|c|c|c|c|c|c|c|c|c|c|c|c|c|c|}
\hline $\begin{array}{l}\text { Study (PMID) } \\
\text { Country } \\
\text { RoB* } \\
\text { Type }\end{array}$ & \begin{tabular}{|l|} 
Intervention \\
Intervention \\
Focus \\
Theoretical \\
Model \\
Delivery \\
Person \\
Intervention \\
Target \\
Recipient \\
Mode \\
Components \\
Frequency \\
Duration
\end{tabular} & $\begin{array}{l}\text { Comparison } \\
\text { Target } \\
\text { Mode } \\
\text { Components } \\
\text { Frequency } \\
\text { Duration }\end{array}$ & $\begin{array}{l}\text { Setting } \\
\text { and Design } \\
\\
\text { Setting } \\
\text { Design } \\
\text { Cluster N } \\
\text { Participants } \\
\text { Randomized } \\
\text { N }\end{array}$ & \begin{tabular}{|l|} 
PLWD \\
Dementia \\
Characteristics \\
Dementia Types \\
Dementia Severity \\
Diagnostic \\
Criteria \\
Age of Diagnosis \\
\end{tabular} & $\begin{array}{l}\text { PLWD } \\
\text { Non-Disease Char } \\
\text { PLWD N } \\
\text { PLWD Age (mean) } \\
\text { PLWD Sex (\% } \\
\text { female) } \\
\text { PLWD Race (\% } \\
\text { majority) } \\
\text { PLWD Education } \\
\text { (mean years) }\end{array}$ & \begin{tabular}{|l|} 
PLWD \\
Non-Disease \\
Char Reporting \\
Status (RS) \\
\\
PLWD SES \\
PLWD Prior \\
Disability \\
PLWD \\
Household \\
Characteristics \\
PLWD Health \\
Insurance \\
PLWD Detailed \\
Race Information \\
\end{tabular} & $\begin{array}{l}\text { Informal Caregiver } \\
\text { (IC) } \\
\text { Characteristics } \\
\text { IC N } \\
\text { IC Age (mean) } \\
\text { IC Sex (\% female) } \\
\text { IC Race (\% } \\
\text { majority) } \\
\text { IC Education } \\
\text { (mean years) } \\
\text { IC Relation to } \\
\text { PLWD (\% majority) }\end{array}$ & \begin{tabular}{|l} 
Informal Caregiver \\
(IC) Char. RS \\
IC Duration \\
IC Living With \\
PLWD \\
IC Payment \\
IC Health Status \\
IC Dementia \\
Family History \\
IC Employment \\
Status \\
IC Training
\end{tabular} & \begin{tabular}{|l} 
Formal Caregiver \\
(FC) \\
Characteristics \\
FC N \\
FC Age (mean) \\
FC Sex (\% \\
female) \\
FC Race (\% \\
majority) \\
FC Education \\
(mean years)
\end{tabular} & $\begin{array}{l}\text { Formal } \\
\text { Caregiver (FC) } \\
\text { Char. RS } \\
\text { FC Health } \\
\text { Status } \\
\text { FC Training } \\
\text { FC Education } \\
\text { FC Position } \\
\text { FC Length of } \\
\text { Service }\end{array}$ & \begin{tabular}{|l|} 
Outcome \\
Timing(s)
\end{tabular} & \begin{tabular}{|l|} 
PLWD \\
Outcomes
\end{tabular} & $\begin{array}{l}\text { Caregiver } \\
\text { Outcomes } \\
\text { IC: } \\
\text { FC: }\end{array}$ \\
\hline \begin{tabular}{|l} 
Mitchell 2018 \\
(29868778) \\
US \\
Medium \\
Explanatory \\
Cohen 201940 \\
$(30273717)$
\end{tabular} & $\begin{array}{l}\text { Advanced Care } \\
\text { Planning } \\
12 \text {-minute video } \\
\text { for goal-oriented } \\
\text { care (advanced } \\
\text { care directives, } \\
\text { care } \\
\text { preferences, } \\
\text { etc) }\end{array}$ & Usual care & $\begin{array}{l}\text { Nursing home- } \\
\text { based } \\
\text { Cluster RCT } \\
64 \text { nursing } \\
\text { homes } \\
402 \text { PLWD }\end{array}$ & $\begin{array}{l}\text { Nonspecified } \\
\text { advanced dementia } \\
\text { Primarily AD } \\
\text { Global } \\
\text { Deterioration Scale } \\
7\end{array}$ & \begin{tabular}{|l|}
$\mathrm{N}=402$ \\
Age 87 \\
$80 \%$ Female \\
$87 \%$ White \\
Education NR
\end{tabular} & None & $\begin{array}{l}\mathrm{N}=402 \\
\text { Age } 62 \\
666 \% \text { Female } \\
87 \% \text { White } \\
99 \% \text { High school } \\
\text { diploma or more } \\
63 \% \text { Child } \\
13 \% \text { Spouse }\end{array}$ & None & NA & NA & \begin{tabular}{|l|}
6 months \\
12 months
\end{tabular} & $\begin{array}{l}\text { Hospitalizati } \\
\text { ons }\end{array}$ & \begin{tabular}{|l} 
Documented \\
directives \\
Goal of care \\
discussions \\
Comfort Care \\
Preference \\
Prevalence
\end{tabular} \\
\hline
\end{tabular}

Abbreviations: char=characteristics; FC=formal caregiver; IC=informal caregiver; N=number; PMID=PubMed Identification Number; PLWD=Persons with Dementia; RoB=Risk of Bias; RS=Reporting Status; SES=socioeconomic status 
Table F-19. PLWD outcomes summary low and medium risk of bias studies: advance care planning

\begin{tabular}{|c|c|c|c|c|c|}
\hline $\begin{array}{l}\text { Study (PMID) } \\
\text { Comparison } \\
\text { RoB } \\
\text { Category* }\end{array}$ & $\begin{array}{l}\text { Outcome } \\
\text { Timing }\end{array}$ & Summary Finding & \begin{tabular}{|l} 
Intervention \\
\end{tabular} & Comparator & $p$-Value \\
\hline $\begin{array}{l}\text { Mitchell } 2018^{39}(29868778) \\
\text { Decision Support Tool vs. Usual Care } \\
\text { Medium } \\
\text { Explanatory }\end{array}$ & $\begin{array}{l}\text { Hospitalizations } \\
N(\%) \\
12 \text { months }\end{array}$ & NR & $20(9.5 \%)$ & $21(11.1 \%)$ & NR \\
\hline $\begin{array}{l}\text { Hanson } 2011^{44}(22091750) \\
\text { Decision Support Tool vs. Usual Care } \\
\text { Medium } \\
\text { Explanatory }\end{array}$ & $\begin{array}{l}\text { Assisted Feeding Intervention-Specialized Dysphagia Diet } \\
(\%) \\
3 \text { months }\end{array}$ & NR & $89 \%$ & $76 \%$ & 0.04 \\
\hline
\end{tabular}

Explanatory studies are listed first, followed by pragmatic studies.

Abbreviations: NA=Not Applicable; NR=Not Reported; PMID=PubMed Identification Number

\begin{tabular}{|c|c|c|c|c|c|}
\hline \begin{tabular}{|l|} 
Study (PMID) \\
Comparison \\
Caregiver Type \\
RoB \\
Category*
\end{tabular} & $\begin{array}{l}\text { Outcome } \\
\text { Timing }\end{array}$ & Summary Finding & Intervention & Comparator & p-Value \\
\hline $\begin{array}{l}\text { Mitchell } 2018^{39}(29868778) \\
\text { Decision Support Tool vs. Usual Care } \\
\text { Medium } \\
\text { Explanatory }\end{array}$ & $\begin{array}{l}\text { Documented Do Not Hospitalize Directive } \\
\mathrm{N}(\%) \\
6 \text { months, } 12 \text { months }\end{array}$ & $\begin{array}{l}\text { Adjusted Odds Ratio }(95 \% \mathrm{Cl}) \\
6 \text { months: } 1.08(0.69,1.69) \\
\text { 12: months: } 1.07(0.66,1.72)\end{array}$ & $\begin{array}{l}6 \text { months: } 133(63.0 \%) \\
12 \text { months: } 144(68.2 \%)\end{array}$ & $\begin{array}{l}6 \text { months: } 119(63.0 \%) \\
12 \text { months: } 126(66.7 \%)\end{array}$ & NR \\
\hline $\begin{array}{l}\text { Mitchell } 2018^{39} \text { (29868778) } \\
\text { Decision Support Tool vs. Usual Care } \\
\text { Medium } \\
\text { Explanatory }\end{array}$ & $\begin{array}{l}\text { Goal of care discussions } \\
\mathrm{N}(\%) \\
6 \text { months, } 12 \text { months }\end{array}$ & $\begin{array}{l}\text { Adjusted Odds Ratio (95\% Cl) } \\
6 \text { months: } 1.70(0.94,3.07) \\
12 \text { months: } 1.46(0.86,2.70)\end{array}$ & $\begin{array}{l}6 \text { months: } 49(23.2 \%) \\
12 \text { months: } 72(34.1 \%)\end{array}$ & $\begin{array}{l}6 \text { months: } 29(15.3 \%) \\
12 \text { months: } 48(25.4 \%)\end{array}$ & NR \\
\hline $\begin{array}{l}\text { Mitchell } 2018^{39} \text { (29868778) } \\
\text { Decision Support Tool vs. Usual Care } \\
\text { Medium } \\
\text { Explanatory }\end{array}$ & $\begin{array}{l}\text { Proxies Preferring Comfort Care } \\
\mathrm{N}(\%) \\
6 \text { months, } 12 \text { months }\end{array}$ & $\begin{array}{l}\text { Adjusted Odds Ratio }(95 \% \text { Cl) } \\
6 \text { months: } 1.28(0.85,1.94) \\
12 \text { months: } 0.72(0.38,1.38)\end{array}$ & $\begin{array}{l}6 \text { months: } 153(73.2 \%) \\
12 \text { months: } 159(76.1 \%)\end{array}$ & $\begin{array}{l}6 \text { months: } 140(76.9 \%) \\
12 \text { months: } 151(82.1 \%)\end{array}$ & NR \\
\hline
\end{tabular}

Explanatory studies are listed first, followed by pragmatic studies.

Abbreviations: NA=Not Applicable; NR=Not Reported; PMID=PubMed Identification Number

Table F-21. Summary of strength of evidence for PLWD outcomes: advance care planning

\begin{tabular}{|c|c|c|c|c|c|c|c|c|}
\hline $\begin{array}{l}\text { Comparison } \\
\text { Outcome }\end{array}$ & Timing & $\begin{array}{l}\text { \# Studies/ Design } \\
\text { (n analyzed) }\end{array}$ & Finding or Summary Statistic & Study Limitations & Consistency & Directness & Precision & Overall Grade/ Conclusion \\
\hline $\begin{array}{l}\text { Decision Support Tool vs. Usual Care } \\
\text { Hospitalizations }\end{array}$ & 12 months & 1 cluster RCT $(n=402)$ & $\begin{array}{l}\text { Similar rates of hospitalizations between intervention and } \\
\text { control groups. }\end{array}$ & Moderate & Unclear & Direct & Imprecise & Insufficient \\
\hline
\end{tabular}

Table F-22. Summary of strength of evidence for caregiver outcomes: advance care planning

\begin{tabular}{|c|c|c|c|c|c|c|c|c|}
\hline $\begin{array}{l}\text { Comparison } \\
\text { Caregiver Type } \\
\text { Outcome }\end{array}$ & Timing & $\begin{array}{l}\text { \# Studies/ Design } \\
\text { (n analyzed) }\end{array}$ & Finding or Summary Statistic & Study Limitations & Consistency & Directness & Precision & Overall Grade/ Conclusion \\
\hline $\begin{array}{l}\text { Decision Support Tool vs. Usual Care } \\
\text { Documented Do Not Hospitalize } \\
\text { Directive }\end{array}$ & $\begin{array}{l}6 \text { months } \\
12 \text { months }\end{array}$ & 1 cluster RCT (n=402) & No difference between groups. & Moderate & Unclear & Direct & Imprecise & Insufficient \\
\hline \begin{tabular}{|l|} 
Decision Support Tool vs. Usual Care \\
Proxies Preferring Comfort Care
\end{tabular} & \begin{tabular}{|l|}
6 months \\
12 months
\end{tabular} & 1 cluster RCT (n=402) & No difference between groups. & Moderate & Unclear & Direct & Imprecise & Insufficient \\
\hline
\end{tabular}

\begin{tabular}{l|l}
6 month \\
Dextes Prefering Comfort Care 12 months
\end{tabular} 


\section{Evidence Map: Advance Care Planning}

\begin{tabular}{|c|c|c|c|c|c|c|c|c|c|c|c|c|c|}
\hline $\begin{array}{l}\text { Study (PMID) } \\
\text { Country } \\
\text { EM Reason }\end{array}$ & \begin{tabular}{|l|} 
Intervention \\
\end{tabular} & Comparison & $\begin{array}{l}\text { Setting } \\
\text { and Design } \\
\\
\text { Setting } \\
\text { Design } \\
\text { Cluster N } \\
\text { Participants } \\
\text { Random- } \\
\text { ized N }\end{array}$ & $\begin{array}{l}\text { PLWD } \\
\text { Dementia } \\
\text { Characteristics }\end{array}$ & $\begin{array}{l}\text { PLWD } \\
\text { Non-Disease Char } \\
\text { PLWD N } \\
\text { PLWD Age (mean) } \\
\text { PLWD Sex (\% } \\
\text { female) } \\
\text { PLWD Race (\% } \\
\text { majority) } \\
\text { PLWD Education } \\
\text { (mean years) }\end{array}$ & $\begin{array}{l}\text { PLWD } \\
\text { Non-Disease } \\
\text { Char Reporting } \\
\text { Status (RS) } \\
\\
\text { PLWD SES } \\
\text { PLWD Prior } \\
\text { Disability } \\
\text { PLWD } \\
\text { Household } \\
\text { Characteristics } \\
\text { PLWD Health } \\
\text { Insurance } \\
\text { PLWD Detailed } \\
\text { Race Information }\end{array}$ & $\begin{array}{l}\text { Informal Caregiver } \\
\text { (IC) } \\
\text { Characteristics } \\
\text { IC N } \\
\text { IC Age (mean) } \\
\text { IC Sex (\% female) } \\
\text { IC Race (\% } \\
\text { majority) } \\
\text { IC Education } \\
\text { (mean years) } \\
\text { IC Relation to } \\
\text { PLWD (\% majority) }\end{array}$ & $\begin{array}{l}\text { Informal Caregiver } \\
\text { (IC) Char. RS } \\
\text { IC Duration } \\
\text { IC Living With } \\
\text { PLWD } \\
\text { IC Payment } \\
\text { IC Health Status } \\
\text { IC Dementia } \\
\text { Family History } \\
\text { IC Employment } \\
\text { Status } \\
\text { IC Training }\end{array}$ & 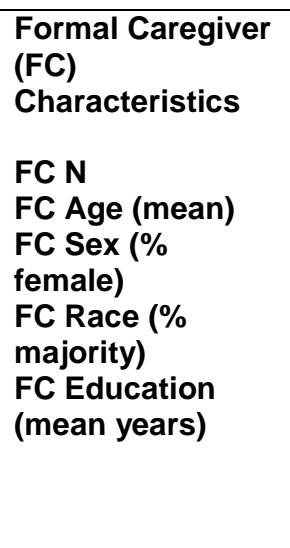 & $\begin{array}{l}\text { Formal } \\
\text { Caregiver (FC) } \\
\text { Char. RS } \\
\text { FC Health } \\
\text { Status } \\
\text { FC Training } \\
\text { FC Education } \\
\text { FC Position } \\
\text { FC Length of } \\
\text { Service }\end{array}$ & $\begin{array}{l}\text { Outcome } \\
\text { Timing(s) }\end{array}$ & $\begin{array}{l}\text { PLWD } \\
\text { Outcomes }\end{array}$ & $\begin{array}{l}\text { Caregiver } \\
\text { Outcomes } \\
\text { IC: } \\
\text { FC: }\end{array}$ \\
\hline $\begin{array}{l}\text { Goossens } \\
2019^{45} \\
(31818522) \\
\\
\text { Goossens } \\
2019^{46} \\
\text { Belgium } \\
\text { High RoB }\end{array}$ & \begin{tabular}{|l|} 
Knowledge- \\
sharing on \\
shared decision \\
making and \\
internal policies \\
role-play \\
exercises on \\
advance care \\
planning \\
\end{tabular} & Waitlist control & $\begin{array}{l}\text { Nursing } \\
\text { Home } \\
\text { Cluster RCT } \\
65 \text { Nursing } \\
\text { homes } \\
\mathrm{N}=311 \\
\text { formal } \\
\text { caregivers }\end{array}$ & None & None & NA & NA & NA & $\begin{array}{l}\mathrm{N}=311 \\
\text { Age: yes } \\
\text { Sex: yes } \\
\text { Race: no } \\
\text { Education: yes }\end{array}$ & $\begin{array}{l}\text { Training: yes } \\
\text { Education: yes } \\
\text { Position: yes } \\
\text { Length of } \\
\text { Service: yes }\end{array}$ & NR & None & Training \\
\hline $\begin{array}{l}\text { Song, 201947 } \\
\text { (31373868) } \\
\text { US } \\
\text { Small sample }\end{array}$ & \begin{tabular}{|l|} 
Adapt SPIRIT \\
(Sharing \\
Patient's IIIness \\
Representation \\
to Increase \\
Trust) for PLWD \\
and surrogates; \\
60 psycho- \\
education
\end{tabular} & $\begin{array}{l}\text { In-person vs } \\
\text { videoconferenc } \\
\mathrm{e}\end{array}$ & $\begin{array}{l}\text { Community } \\
\text { Mixed- } \\
\text { methods } \\
\text { Dyad }\end{array}$ & $\begin{array}{l}\text { Early stage } \\
\text { dementia }\end{array}$ & $\begin{array}{l}\mathrm{N}=23 \\
\text { Age: yes } \\
\text { Sex: yes } \\
\text { Race: yes } \\
\text { Education: yes }\end{array}$ & None & $\begin{array}{l}\mathrm{N}=23 \\
\text { Age: yes } \\
\text { Sex: yes } \\
\text { Race: yes } \\
\text { Education: yes } \\
\text { Relation to PLWD: } \\
\text { yes }\end{array}$ & Duration & NA & NA & 3 days & \begin{tabular}{l|} 
Dyad \\
congruence \\
Patient \\
decisional \\
conflict scale
\end{tabular} & $\begin{array}{l}\text { Surrogate } \\
\text { decision- } \\
\text { making } \\
\text { confidence } \\
\text { scale }\end{array}$ \\
\hline $\begin{array}{l}\text { Brazil 201838 } \\
(28786323) \\
\text { Northern Ireland } \\
\text { High RoB }\end{array}$ & \begin{tabular}{|l|} 
Advance care \\
planning \\
intervention \\
(education, \\
meetings \\
documentation); \\
2 family \\
meetings
\end{tabular} & Usual care & $\begin{array}{l}\text { Nursing } \\
\text { homes } \\
\text { Cluster RCT } \\
24 \text { nursing } \\
\text { homes } \\
197 \text { Informal } \\
\text { caregivers }\end{array}$ & $\begin{array}{l}\text { Dementia without } \\
\text { capacity to } \\
\text { complete ACP }\end{array}$ & $\begin{array}{l}\text { N=197 } \\
\text { Age: no } \\
\text { Sex: no } \\
\text { Race: no } \\
\text { Education: no }\end{array}$ & None & $\begin{array}{l}\mathrm{N}=197 \\
\text { Age: yes } \\
\text { Sex: yes } \\
\text { Race: no } \\
\text { Education: no } \\
\text { Relation to PLWD: } \\
\text { yes }\end{array}$ & None & NA & NA & 6 weeks & None & $\begin{array}{l}\text { Decisional } \\
\text { Conflict Scale } \\
\text { FPCS } \\
\text { GHQ } \\
\text { QOD-LTC }\end{array}$ \\
\hline $\begin{array}{l}\text { Hanson 201741 } \\
(27893884) \\
\text { US } \\
\text { High RoB } \\
\text { Hanson 2016 } \\
\text { (27271683) }\end{array}$ & \begin{tabular}{|l} 
Goals of care \\
video decision \\
aid and \\
structured \\
discussion with \\
health care \\
providers
\end{tabular} & $\begin{array}{l}\text { Informational } \\
\text { video }\end{array}$ & $\begin{array}{l}\text { Nursing } \\
\text { homes } \\
\text { Cluster RCT } \\
22 \text { Nursing } \\
\text { homes } \\
\text { 302 PLWD } \\
\text { and informal } \\
\text { caregivers }\end{array}$ & $\begin{array}{l}\text { Severe to } \\
\text { advanced } \\
\text { dementia; GDS } 5 \\
\text { to } 7\end{array}$ & $\begin{array}{l}\mathrm{N}=302 \\
\text { Age: yes } \\
\text { Sex: yes } \\
\text { Race: yes } \\
\text { Education no }\end{array}$ & None & $\begin{array}{l}\mathrm{N}=302 \\
\text { Age: } \text { yes } \\
\text { Sex: yes } \\
\text { Race: yes } \\
\text { Education: yes } \\
\text { Relation to PLWD: } \\
\text { yes }\end{array}$ & None & NA & NA & $\begin{array}{l}3 \text { months } \\
9 \text { months }\end{array}$ & None & $\begin{array}{l}\text { Quality of } \\
\text { Communicati } \\
\text { on } \\
\text { Concordance } \\
\text { of GOC } \\
\text { ACP problem } \\
\text { score } \\
\text { SWC-EOLD } \\
\text { SM-EOLD }\end{array}$ \\
\hline $\begin{array}{l}\text { Bonner, 201448 } \\
\text { (24381040) } \\
\text { US } \\
\text { Pilot Study }\end{array}$ & $\begin{array}{l}\text { Advance Care } \\
\text { Treatment Plan } \\
\text { (ACT-Plan); a } \\
\text { group-based } \\
\text { education } \\
\text { intervention, } \\
\text { with AA } \\
\text { dementia } \\
\text { caregivers. }\end{array}$ & $\begin{array}{l}\text { Attention-control } \\
\text { health } \\
\text { promotion } \\
\text { conditions. }\end{array}$ & $\begin{array}{l}\text { Adult } \\
\text { Daycare } \\
\text { centers } \\
\text { Non-RCT } \\
68 \\
\text { caregivers }\end{array}$ & NA & NA & NA & $\begin{array}{l}\mathrm{N}=32 \\
\text { Age: Yes } \\
\text { Sex: Yes } \\
\text { Race: No } \\
\text { Education: No }\end{array}$ & $\begin{array}{l}\text { Income: Yes } \\
\text { Living with PLWD: } \\
\text { Yes }\end{array}$ & NA & NA & 4 weeks & Feasibility: & $\begin{array}{l}\text { Knowledge of } \\
\text { dementia, } \\
\text { CPR, MV \& } \\
\text { TF: } \\
\text { Knowledge of } \\
\text { dementia } \\
\text { scale; } \\
\text { Self-efficacy: } \\
\text { the } \\
\text { Confidence in } \\
\text { Treatment } \\
\text { Decisions } \\
\text { Made } \\
\text { guestionnaire }\end{array}$ \\
\hline $\begin{array}{l}\text { Einterz, 201449 } \\
(24508326) \\
\text { US } \\
\text { Pilot study }\end{array}$ & \begin{tabular}{|l|} 
GOC decision \\
aid video \\
viewed by the \\
SDM and \\
structured care \\
plan meeting \\
between the \\
SDM and \\
interdisciplinary \\
NH team. \\
\end{tabular} & Usual care & $\begin{array}{l}\text { Nursing } \\
\text { home } \\
\text { RCT } \\
18 \text { Dyads }\end{array}$ & $\begin{array}{l}\text { All types of } \\
\text { dementia with } \\
\text { moderate to severe } \\
\text { (GDS) }\end{array}$ & $\begin{array}{l}\mathrm{N}=18 \\
\text { Age: yes } \\
\text { Sex: yes } \\
\text { Race: yes } \\
\text { Education: no }\end{array}$ & None & $\begin{array}{l}\mathrm{N}=18 \\
\text { Age: yes } \\
\text { Sex: yes } \\
\text { Race: yes } \\
\text { Education: yes } \\
\text { Relationship with } \\
\text { PLWD: yes }\end{array}$ & None & NA & NA & 3 months & None & $\begin{array}{l}\text { Quality of } \\
\text { communicatio } \\
\mathrm{n} \text { and } \\
\text { decision- } \\
\text { making }\end{array}$ \\
\hline
\end{tabular}




\begin{tabular}{|c|c|c|c|c|c|c|c|c|c|c|c|c|c|}
\hline $\begin{array}{l}\text { Reinhardt } \\
2014^{43} \\
(24835382) \\
\text { US } \\
\text { High RoB }\end{array}$ & $\begin{array}{l}\text { Structured } \\
\text { conversations } \\
\text { about end of life } \\
\text { care with } \\
\text { palliative care } \\
\text { team }\end{array}$ & $\begin{array}{l}\text { Social contact } \\
\text { via telephone }\end{array}$ & \begin{tabular}{|l|} 
Skilled \\
nursing \\
facility \\
110 informal \\
caregivers \\
\end{tabular} & $\begin{array}{l}\text { Advance dementia, } \\
\text { CPS score of } 4 \text { to } 6\end{array}$ & $\begin{array}{l}\mathrm{N}=110 \\
\text { Age: yes } \\
\text { Sex: yes } \\
\text { Race: yes } \\
\text { Education: no }\end{array}$ & None & $\begin{array}{l}\text { N=101 } \\
\text { Age: yes } \\
\text { Sex: yes } \\
\text { Race: yes } \\
\text { Education: yes } \\
\text { Relationship with } \\
\text { PLWD: yes }\end{array}$ & $\begin{array}{l}\text { Employment status: } \\
\text { yes }\end{array}$ & NA & NA & $\begin{array}{l}3 \text { months } \\
6 \text { months }\end{array}$ & None & $\begin{array}{l}\text { Satisfaction of } \\
\text { care at end- } \\
\text { of-life in } \\
\text { dementia } \\
\text { scale } \\
\text { PHQ-9 } \\
\text { Satisfaction } \\
\text { with Life } \\
\text { Scale }\end{array}$ \\
\hline $\begin{array}{l}\text { Sampson, } \\
2011^{50} \\
(21228087) \\
\text { UK } \\
\text { Pilot }\end{array}$ & $\begin{array}{l}\text { A palliative care } \\
\text { and advance } \\
\text { care plan (ACP) } \\
\text { intervention }\end{array}$ & Usual care & \begin{tabular}{|l|} 
Hospital \\
RCT \\
32 dyads
\end{tabular} & Severe dementia & $\begin{array}{l}\mathrm{N}=32 \\
\text { Age: yes } \\
\text { Sex: yes } \\
\text { Race: yes } \\
\text { Education: no }\end{array}$ & None & $\begin{array}{l}\mathrm{N}=32 \\
\text { Age: yes } \\
\text { Sex: yes } \\
\text { Race: yes } \\
\text { Education: no } \\
\text { Relationship: yes }\end{array}$ & None & NA & NA & $\begin{array}{l}6 \text { 6 weeks } \\
6 \text { months }\end{array}$ & $\begin{array}{l}\text { Number of } \\
\text { caregivers } \\
\text { making an } \\
\text { ACP: } \\
\text { Feasibility: } \\
\text { Pain: Visual } \\
\text { analogue } \\
\text { scale } \\
\text { distress: } \\
\text { KD10 } \\
\text { DSI: DCS; } \\
\text { STAXI; LSQ } \\
\end{array}$ & $\begin{array}{l}\text { distress: } \\
\text { KD10 } \\
\text { DSI: DCS; } \\
\text { STAXI; LSQ }\end{array}$ \\
\hline
\end{tabular}

A

\section{Palliative Care}

Table F-24. Risk of bias assessment: palliative

\begin{tabular}{|c|c|c|c|c|c|c|c|c|c|}
\hline Study (PMID) & Outcome Timing & $\begin{array}{l}\text { Attrition Bias } \\
\text { Attrition \% }\end{array}$ & Selection Bias & Detection Bias & Performance Bias & Reporting Bias & Fidelity Bias & Funder & Overall Rating \\
\hline \begin{tabular}{|l|} 
Verrault , 201851 (28731379) \\
\end{tabular} & 1 year & $\begin{array}{l}\text { Medium (response } \\
\text { rates for caregivers) } \\
57 \% \text { control, } 73 \% \\
\text { intervention }\end{array}$ & Medium & Medium & High & Low & $x$ & Government & High \\
\hline \begin{tabular}{|l} 
Boogaard, $2018^{52}$ (29343173) \\
\end{tabular} & 10 months & $\begin{array}{l}\text { Medium } \\
11 \% \text { nursing homes } \\
\text { dropped }\end{array}$ & Medium & $\mathrm{x}$ & $\mathrm{x}$ & $\mathrm{x}$ & High & Gov't & High \\
\hline \begin{tabular}{|l|} 
Agar, $2017^{53}$ (28786995) \\
\end{tabular} & & Cluster RCT & $\begin{array}{l}\text { Medium } \\
64 \% \text { decline rate }\end{array}$ & $\mathrm{x}$ & $x$ & $x$ & $\begin{array}{l}\text { High } \\
\text { (could not measure } \\
\text { as planned) }\end{array}$ & & High \\
\hline 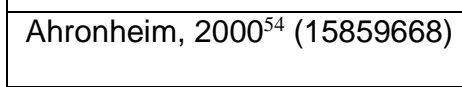 & Over 3 years & $\begin{array}{l}\text { Low } \\
0 \%\end{array}$ & Medium & Low & Medium & Low & High & & High \\
\hline
\end{tabular}

Xindicates that domain was not assessed due to high risk of bias.

Abbreviations: PMID=PubMed Identification Number 


\section{Evidence Map: Palliative Care}

\begin{tabular}{|c|c|c|c|c|c|c|c|c|c|c|c|c|c|}
\hline $\begin{array}{l}\text { Study (PMID) } \\
\text { Country } \\
\text { EM Reason }\end{array}$ & Intervention & Comparison & $\begin{array}{l}\text { Setting } \\
\text { and Design } \\
\\
\text { Setting } \\
\text { Design } \\
\text { Cluster N } \\
\text { Participants } \\
\text { Random- } \\
\text { ized N }\end{array}$ & $\begin{array}{l}\text { PLWD } \\
\text { Dementia } \\
\text { Characteristics }\end{array}$ & $\begin{array}{l}\text { PLWD } \\
\text { Non-Disease Char } \\
\text { PLWD N } \\
\text { PLWD Age (mean) } \\
\text { PLWD Sex (\% } \\
\text { female) } \\
\text { PLWD Race (\% } \\
\text { majority) } \\
\text { PLWD Education } \\
\text { (mean years) }\end{array}$ & $\begin{array}{l}\text { PLWD } \\
\text { Non-Disease } \\
\text { Char Reporting } \\
\text { Status (RS) } \\
\text { PLWD SES } \\
\text { PLWD Prior } \\
\text { Disability } \\
\text { PLWD } \\
\text { Household } \\
\text { Characteristics } \\
\text { PLWD Health } \\
\text { Insurance } \\
\text { PLWD Detailed } \\
\text { Race Information }\end{array}$ & $\begin{array}{l}\text { Informal Caregiver } \\
\text { (IC) } \\
\text { Characteristics } \\
\text { IC N } \\
\text { IC Age (mean) } \\
\text { IC Sex (\% female) } \\
\text { IC Race (\% } \\
\text { majority) } \\
\text { IC Education } \\
\text { (mean years) } \\
\text { IC Relation to } \\
\text { PLWD (\% majority) }\end{array}$ & $\begin{array}{l}\text { Informal Caregiver } \\
\text { (IC) Char. RS } \\
\text { IC Duration } \\
\text { IC Living With } \\
\text { PLWD } \\
\text { IC Payment } \\
\text { IC Health Status } \\
\text { IC Dementia } \\
\text { Family History } \\
\text { IC Employment } \\
\text { Status } \\
\text { IC Training }\end{array}$ & $\begin{array}{l}\text { Formal Caregiver } \\
\text { (FC) } \\
\text { Characteristics } \\
\text { FC N } \\
\text { FC Age (mean) } \\
\text { FC Sex (\% } \\
\text { female) } \\
\text { FC Race (\% } \\
\text { majority) } \\
\text { FC Education } \\
\text { (mean years) }\end{array}$ & $\begin{array}{l}\text { Formal } \\
\text { Caregiver (FC) } \\
\text { Char. RS } \\
\text { FC Health } \\
\text { Status } \\
\text { FC Training } \\
\text { FC Education } \\
\text { FC Position } \\
\text { FC Length of } \\
\text { Service }\end{array}$ & $\begin{array}{l}\text { Outcome } \\
\text { Timing(s) }\end{array}$ & $\begin{array}{l}\text { PLWD } \\
\text { Outcomes }\end{array}$ & $\begin{array}{l}\text { Caregiver } \\
\text { Outcomes } \\
\text { IC: } \\
\text { FC: }\end{array}$ \\
\hline $\begin{array}{l}\text { Hanson, 201955 } \\
\text { (30342242) } \\
\text { USA } \\
\text { Pilot }\end{array}$ & $\begin{array}{l}\text { Triggered } \\
\text { Palliative Care: } \\
\text { Received } \\
\text { denetia-specific } \\
\text { specialty } \\
\text { palliative care } \\
\text { consultation } \\
\text { plus postacute } \\
\text { transitional } \\
\text { care. }\end{array}$ & $\begin{array}{l}\text { Control: } \\
\text { Received usual } \\
\text { care and } \\
\text { educational } \\
\text { information }\end{array}$ & $\begin{array}{l}\text { Hospital } \\
\text { RCT } \\
62 \text { dyads }\end{array}$ & $\begin{array}{l}\text { Late-stage (5-7) } \\
\text { dementia }\end{array}$ & $\begin{array}{l}\text { N=62 } \\
\text { Age: Yes } \\
\text { Sex: Yes } \\
\text { Race: Yes } \\
\text { Education: No }\end{array}$ & None & $\begin{array}{l}\mathrm{N}=62 \\
\text { Age: yes } \\
\text { Sex: Yes } \\
\text { Race: No } \\
\text { Education: no } \\
\text { Relation: yes }\end{array}$ & None & NA & NA & 60 days & $\begin{array}{l}\text { 60-day } \\
\text { hospital or } \\
\text { emergency } \\
\text { department } \\
\text { visits } \\
\text { Patients } \\
\text { comfort, } \\
\text { family } \\
\text { distress etc. }\end{array}$ & $\mathrm{NR}$ \\
\hline $\begin{array}{l}\text { Boogaard, } \\
2018^{52} \\
(29343173) \\
\\
\text { Netherlands } \\
\text { High ROB }\end{array}$ & $\begin{array}{l}\text { Generic or } \\
\text { personal } \\
\text { feedback to } \\
\text { staff from } \\
\text { PLWD about } \\
\text { end of life care }\end{array}$ & No feedback & $\begin{array}{l}\text { Nursing } \\
\text { homes } \\
\text { Cluster RCT } \\
18 \text { nursing } \\
\text { homes }\end{array}$ & $\begin{array}{l}\text { Late stage } \\
\text { dementia }\end{array}$ & $\begin{array}{l}\text { N=668 } \\
\text { Age: Yes } \\
\text { Sex: Yes } \\
\text { Race: no } \\
\text { Education: No }\end{array}$ & $\begin{array}{l}\text { None } \\
\text { (length of stay) }\end{array}$ & $\begin{array}{l}\mathrm{N}=193 \\
\text { Age: yes } \\
\text { Sex: Yes } \\
\text { Race: No } \\
\text { Education: yes } \\
\text { Relation: yes }\end{array}$ & None & NA & NA & 10 months & NR & $\begin{array}{l}\text { End-of Life in } \\
\text { Dementia } \\
\text { scales }\end{array}$ \\
\hline $\begin{array}{l}\text { Verrault, 201851 } \\
\text { (28731379) } \\
\text { Canada } \\
\text { High ROB }\end{array}$ & $\begin{array}{l}\text { End of Life care: } \\
\text { training for } \\
\text { physicians and } \\
\text { staff; pain } \\
\text { monitoring; } \\
\text { regular mouth } \\
\text { care; family } \\
\text { communication; } \\
\text { nurse facilitator }\end{array}$ & $\begin{array}{l}\text { Usual care } \\
\text { activities }\end{array}$ & $\begin{array}{l}\text { Long-term } \\
\text { care } \\
\text { facilities } \\
\text { Quasi- } \\
\text { experimental } \\
193 \text { dyads }\end{array}$ & $\begin{array}{l}\text { Late stage } \\
\text { dementia }\end{array}$ & $\begin{array}{l}\text { N=193 } \\
\text { Age: Yes } \\
\text { Sex: Yes } \\
\text { Race: no } \\
\text { Education: No }\end{array}$ & $\begin{array}{l}\text { None } \\
\text { (length of stay) } \\
\text { (duration of } \\
\text { terminal phase) }\end{array}$ & $\begin{array}{l}\text { N=193 } \\
\text { Age: no } \\
\text { Sex: Yes } \\
\text { Race: No } \\
\text { Education: no } \\
\text { Relation: yes }\end{array}$ & None & NA & NA & 1 year & NR & $\begin{array}{l}\text { Family } \\
\text { perception of } \\
\text { care scale; } \\
\text { Symptom } \\
\text { management } \\
\text { for EoL in } \\
\text { dementia } \\
\text { Comfort } \\
\text { assessment } \\
\text { in dying scale }\end{array}$ \\
\hline $\begin{array}{l}\text { Agar, 2017 } \\
(28786995) \\
\\
\text { Australia } \\
\text { High ROB }\end{array}$ & $\begin{array}{l}\text { Facilitated } \\
\text { family case } \\
\text { conference for } \\
\text { end of life care, } \\
\text { registered nurse }\end{array}$ & $\begin{array}{l}\text { Usual care } \\
\text { activities }\end{array}$ & $\begin{array}{l}\text { Nursing } \\
\text { homes } \\
\text { Cluster RCT } \\
20 \text { nursing } \\
\text { homes }\end{array}$ & $\begin{array}{l}\text { Late stage } \\
\text { dementia }\end{array}$ & $\begin{array}{l}\text { N=131 } \\
\text { Age: Yes } \\
\text { Sex: Yes } \\
\text { Race: } n \text { o } \\
\text { Education: No }\end{array}$ & $\begin{array}{l}\text { None } \\
\text { (length of stay) } \\
\text { (time to death) } \\
\text { (visitor frequency) }\end{array}$ & None & None & NA & NA & $\begin{array}{l}\text { 4-6 weeks } \\
\text { post-death }\end{array}$ & $\begin{array}{l}\text { Nurse-rated } \\
\text { QUALID }\end{array}$ & $\begin{array}{l}\text { Family-rated } \\
\text { End of Life } \\
\text { Care scales }\end{array}$ \\
\hline $\begin{array}{l}\text { Ahronheim, } \\
2000^{54} \\
(15859668) \\
\text { US } \\
\text { High ROB }\end{array}$ & $\begin{array}{l}\text { Palliative care } \\
\text { team } \\
\text { recommendatio } \\
\text { ns }\end{array}$ & $\begin{array}{l}\text { Usual care } \\
\text { activities }\end{array}$ & Hospital & $\begin{array}{l}\text { Late stage } \\
\text { dementia }\end{array}$ & $\begin{array}{l}\mathrm{N}=99 \\
\text { Age: Yes } \\
\text { Sex: Yes } \\
\text { Race: yes } \\
\text { Education: no }\end{array}$ & $\begin{array}{l}\text { None } \\
\text { (length of stay) }\end{array}$ & None & None & NA & NA & $\begin{array}{l}\text { Time until } \\
\text { death }\end{array}$ & $\begin{array}{l}\text { Number of } \\
\text { medical } \\
\text { interventions } \\
\text {, procedures, } \\
\text { and } \\
\text { readmission } \\
\mathrm{s}\end{array}$ & NR \\
\hline
\end{tabular}

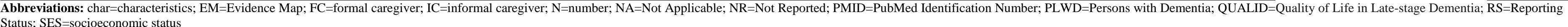

\section{Other Service Provision Interventions}

Table F-26. Risk of bias assessment: other service provision interventions

\begin{tabular}{|c|c|c|c|c|c|c|c|c|c|}
\hline \begin{tabular}{|l|} 
Study (PMID) \\
\end{tabular} & Outcome Timing & $\begin{array}{l}\text { Attrition Bias } \\
\text { Attrition \% }\end{array}$ & Selection Bias & Detection Bias & Performance Bias & Reporting Bias & Fidelity Bias & Funder & Overall Rating \\
\hline $\begin{array}{l}\begin{array}{l}\text { Horvath 2013 } \\
(24195007)\end{array} \\
\end{array}$ & & $\begin{array}{l}\text { Medium } \\
15 \%\end{array}$ & Low & Medium & High & Low & High & & High \\
\hline $\begin{array}{l}\text { Hanson 201144 } 22091750) \\
\text { Hanson } 2010^{57}(20729251) \\
\text { Snyder } 2013^{58}(23273855)\end{array}$ & $\begin{array}{l}3 \text { months } \\
9 \text { months }\end{array}$ & $\begin{array}{l}\text { Low } \\
3 \text { months: } 1 \% \\
\text { High } \\
9 \text { months: }\end{array}$ & Low & Medium & Low & Low & \begin{tabular}{|l} 
Medium \\
\end{tabular} & Government & Medium \\
\hline
\end{tabular}

indicates that domain was not assessed due to high risk of bias.

Abbreviations: PMID=PubMed Identification Number 
Table F-27. Characteristics of included studies: other service provision interventions

\begin{tabular}{|c|c|c|c|c|c|c|c|c|c|c|c|c|c|}
\hline \begin{tabular}{|l|} 
Study (PMID) \\
Country \\
RoB* \\
Type
\end{tabular} & \begin{tabular}{|l|} 
Intervention \\
Intervention \\
Focus \\
Theoretical \\
Model \\
Delivery \\
Person \\
Intervention \\
Target \\
Recipient \\
Mode \\
Components \\
Frequency \\
Duration
\end{tabular} & $\begin{array}{l}\text { Comparison } \\
\text { Target } \\
\text { Mode } \\
\text { Components } \\
\text { Frequency } \\
\text { Duration }\end{array}$ & $\begin{array}{l}\text { Setting } \\
\text { and Design } \\
\\
\text { Setting } \\
\text { Design } \\
\text { Cluster N } \\
\text { Participants } \\
\text { Randomized } \\
\text { N }\end{array}$ & $\begin{array}{l}\text { PLWD } \\
\text { Dementia } \\
\text { Characteristics } \\
\text { Dementia Types } \\
\text { Dementia Severity } \\
\text { Diagnostic } \\
\text { Criteria } \\
\text { Age of Diagnosis }\end{array}$ & \begin{tabular}{|l|} 
PLWD \\
Non-Disease Char \\
PLWD N \\
PLWD Age (mean) \\
PLWD Sex (\% \\
female) \\
PLWD Race (\% \\
majority) \\
PLWD Education \\
(mean years)
\end{tabular} & \begin{tabular}{|l|} 
PLWD \\
Non-Disease \\
Char Reporting \\
Status (RS) \\
\\
PLWD SES \\
PLWD Prior \\
Disability \\
PLWD \\
Household \\
Characteristics \\
PLWD Health \\
Insurance \\
PLWD Detailed \\
Race Information \\
\end{tabular} & $\begin{array}{l}\text { Informal Caregiver } \\
\text { (IC) } \\
\text { Characteristics } \\
\text { IC N } \\
\text { IC Age (mean) } \\
\text { IC Sex (\% female) } \\
\text { IC Race (\% } \\
\text { majority) } \\
\text { IC Education } \\
\text { (mean years) } \\
\text { IC Relation to } \\
\text { PLWD (\% majority) }\end{array}$ & $\begin{array}{l}\text { Informal Caregiver } \\
\text { (IC) Char. RS } \\
\text { IC Duration } \\
\text { IC Living With } \\
\text { PLWD } \\
\text { IC Payment } \\
\text { IC Health Status } \\
\text { IC Dementia } \\
\text { Family History } \\
\text { IC Employment } \\
\text { Status } \\
\text { IC Training }\end{array}$ & $\begin{array}{l}\text { Formal Caregiver } \\
\text { (FC) } \\
\text { Characteristics } \\
\text { FC N } \\
\text { FC Age (mean) } \\
\text { FC Sex (\% } \\
\text { female) } \\
\text { FC Race (\% } \\
\text { majority) } \\
\text { FC Education } \\
\text { (mean years) }\end{array}$ & $\begin{array}{l}\text { Formal } \\
\text { Caregiver (FC) } \\
\text { Char. RS } \\
\text { FC Health } \\
\text { Status } \\
\text { FC Training } \\
\text { FC Education } \\
\text { FC Position } \\
\text { FC Length of } \\
\text { Service }\end{array}$ & $\begin{array}{l}\text { Outcome } \\
\text { Timing(s) }\end{array}$ & \begin{tabular}{|l|} 
PLWD \\
Outcomes
\end{tabular} & $\begin{array}{l}\text { Caregiver } \\
\text { Outcomes } \\
\text { IC: } \\
\text { FC: }\end{array}$ \\
\hline \begin{tabular}{|l} 
Hanson 2011 \\
$(22091750)$ \\
US \\
Medium \\
Explanatory \\
\\
Hanson 201057 \\
$(20729251)$ \\
Snyder 2013 \\
${ }^{58}(23273855)$
\end{tabular} & $\begin{array}{l}\text { Decision aid } \\
\text { about dementia, } \\
\text { feeding options } \\
\text { (feeding tubes, } \\
\text { assisted oral } \\
\text { feeding); print } \\
\text { format reviewed } \\
\text { for } ~ \\
20 \text { minutes }\end{array}$ & Usual care & \begin{tabular}{|l|} 
Nursing homes \\
Cluster RCT \\
24 Nursing \\
homes \\
256 PLWD- \\
surrogate \\
dyads
\end{tabular} & $\begin{array}{l}\text { Advanced } \\
\text { dementia with } \\
\text { feeding problems; } \\
\text { score of } 5 \text { to } 6 \text { on } \\
\text { Cognitive } \\
\text { Performance Scale } \\
\text { in the MDS and } \\
\text { severity stage } 6 \text { to } \\
7 \text { on GDS }\end{array}$ & \begin{tabular}{|l|}
$\mathrm{N}=256$ \\
85 years \\
$78 \%$ Female \\
$70 \%$ White \\
Education NR
\end{tabular} & $\begin{array}{l}\text { Detailed Race } \\
\text { Information: yes }\end{array}$ & $\begin{array}{l}\mathrm{N}=256 \\
59 \text { years } \\
63 \% \text { Female } \\
70 \% \text { White } \\
\text { Education NR } \\
\text { 45\% Daughter }\end{array}$ & None & NA & NA & 3 months & \begin{tabular}{|l|} 
Assisted \\
Feeding \\
Intervention, \\
Specialized \\
dysphagia \\
diet
\end{tabular} & \begin{tabular}{|l} 
Decisional \\
Conflict Scale \\
Frequency of \\
Discussion \\
about \\
Feeding \\
Satisfaction \\
with Decision \\
Scale \\
Decisional \\
Regret Index
\end{tabular} \\
\hline
\end{tabular}

Abbreviations: char=characteristics; FC=formal caregiver; IC=informal caregiver; N=number; PMID=PubMed Identification Number; PLWD=Persons with Dementia; RoB=Risk of Bias; RS=Reporting Status; SES=socioeconomic status

Table F-28. PLWD outcomes summary low and medium risk of bias studies: other service provision interventions

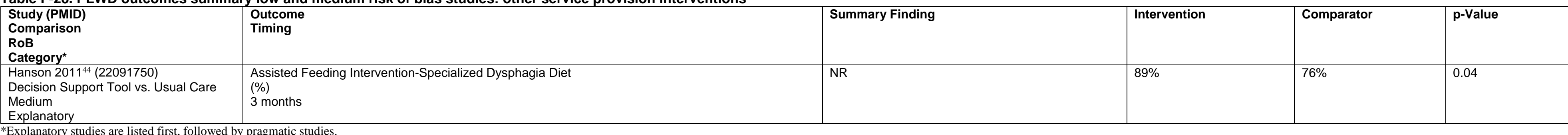

*Explanatory studies are listed first, followed by pragmatic studies.

Abbreviations: NR=Not Reported; PMID=PubMed Identification Number; RoB=Risk of Bias

\begin{tabular}{|c|c|c|c|c|c|}
\hline $\begin{array}{l}\text { Study (PMID) } \\
\text { Comparison } \\
\text { Caregiver Type } \\
\text { RoB } \\
\text { Category* }\end{array}$ & $\begin{array}{l}\text { Outcome } \\
\text { Timing }\end{array}$ & Summary Finding & Intervention & Comparator & p-Value \\
\hline $\begin{array}{l}\text { Hanson } 2011^{44}(22091750) \\
\text { Decision Support Tool vs. Usual Care } \\
\text { Medium } \\
\text { Explanatory }\end{array}$ & $\begin{array}{l}\text { Decisional Conflict Scale, Overall } \\
\text { Mean } \\
\text { Mean Change from Baseline } \\
3 \text { months }\end{array}$ & NR & $\begin{array}{l}1.65 \\
-0.60\end{array}$ & \begin{tabular}{|l}
1.97 \\
-0.13
\end{tabular} & $<0.001$ \\
\hline $\begin{array}{l}\text { Hanson } 2011^{44}(22091750) \\
\text { Decision Support Tool vs. Usual Care } \\
\text { Medium } \\
\text { Explanatory }\end{array}$ & $\begin{array}{l}\text { Satisfaction with Decision Scale } \\
\text { Mean } \\
3 \text { months }\end{array}$ & NR & 1.61 & \begin{tabular}{|l|l|}
1.66 \\
\end{tabular} & 0.5 \\
\hline $\begin{array}{l}\text { Hanson } 2011^{44}(22091750) \\
\text { Decision Support Tool vs. Usual Care } \\
\text { Medium } \\
\text { Explanatory }\end{array}$ & $\begin{array}{l}\text { Decisional Regret Index } \\
\text { Mean } \\
3 \text { months }\end{array}$ & NR & 11.9 & 14.3 & 0.14 \\
\hline $\begin{array}{l}\text { Hanson } 2011^{44}(22091750) \\
\text { Decision Support Tool vs. Usual Care } \\
\text { Medium } \\
\text { Explanatory }\end{array}$ & $\begin{array}{l}\text { Feeding Discussions, With Physician, NP, or PA } \\
\text { Feeding Discussions, Other Nursing Home Staff } \\
\% \\
3 \text { months }\end{array}$ & NR & $\begin{array}{l}45 \% \\
\text { Other staff: } 64 \%\end{array}$ & $\begin{array}{l}33 \% \\
\text { Other staff: } 71 \%\end{array}$ & $\begin{array}{l}0.04 \\
\text { Other staff: } 0.42\end{array}$ \\
\hline
\end{tabular}

Explanatory studies are listed first, followed by pragmatic studies.

Abbreviations: NA=Not Applicable; NR=Not Reported; PMID=PubMed Identification Number

Table F-30. Summary of strength of evidence for PLWD outcomes: other service provision interventions

\begin{tabular}{|c|c|c|c|c|c|c|c|c|}
\hline $\begin{array}{l}\text { Comparison } \\
\text { Outcome }\end{array}$ & Timing & $\begin{array}{l}\text { \# Studies/ Design } \\
\text { (n analyzed) }\end{array}$ & Finding or Summary Statistic & Study Limitations & Consistency & Directness & Precision & Overall Grade/ Conclusion \\
\hline $\begin{array}{l}\text { Decision Support Tool vs. Usual Care } \\
\text { Assisted Feeding Intervention- } \\
\text { Specialized Dysphagia Diet }\end{array}$ & 3 months & 1 cluster RCT $(n=256)$ & $\begin{array}{l}\text { At } 3 \text { months, more PLWD in intervention group had a } \\
\text { specialized dysphagia diet. }\end{array}$ & Moderate & Unclear & Direct & Imprecise & Insufficient \\
\hline
\end{tabular}


Table F-31. Summary of strength of evidence for caregiver outcomes: other service provision interventions

\begin{tabular}{|c|c|c|c|c|c|c|c|c|}
\hline $\begin{array}{l}\text { Comparison } \\
\text { Caregiver Type } \\
\text { Outcome }\end{array}$ & Timing & $\begin{array}{l}\text { \# Studies/ Design } \\
\text { (n analyzed) }\end{array}$ & Finding or Summary Statistic & Study Limitations & Consistency & Directness & Precision & Overall Grade/ Conclusion \\
\hline $\begin{array}{l}\text { Decision Support Tool vs. Usual Care } \\
\text { Decisional conflict }\end{array}$ & 3 months & 1 cluster RCT $(n=256)$ & $\begin{array}{l}\text { At } 3 \text { months, intervention group had less decisional conflict } \\
\text { than control group. }\end{array}$ & Moderate & Unclear & Direct & Imprecise & Insufficient \\
\hline \begin{tabular}{|l} 
Decision Support Tool vs. Usual Care \\
Satisfaction with Decisions
\end{tabular} & 3 months & 1 cluster RCT ( $(=256)$ & No difference between groups. & Moderate & Unclear & Direct & Imprecise & Insufficient \\
\hline \begin{tabular}{|l|} 
Decision Support Tool vs. Usual Care \\
Decisional Regret
\end{tabular} & 3 months & 1 cluster RCT $(n=256)$ & No difference between groups. & Moderate & Unclear & Direct & Imprecise & Insufficient \\
\hline $\begin{array}{l}\text { Decision Support Tool vs. Usual Care } \\
\text { Feeding Discussions }\end{array}$ & 3 months & 1 cluster RCT $(n=256)$ & $\begin{array}{l}\text { At } 3 \text { months, more feeding discussions with doctor, PA, or } \\
\text { NP in intervention group versus control. No difference } \\
\text { between groups in feeding discussions with other nursing } \\
\text { home staff. }\end{array}$ & Moderate & Unclear & Direct & Imprecise & Insufficient \\
\hline
\end{tabular}

\section{Evidence Map: Other Service Provision Interventions}

\begin{tabular}{|c|c|c|c|c|c|c|c|c|c|c|c|c|c|}
\hline $\begin{array}{l}\text { Study (PMID) } \\
\text { Country } \\
\text { EM Reason }\end{array}$ & Intervention & Comparison & \begin{tabular}{l|} 
Setting \\
and Design \\
Setting \\
Design \\
Cluster N \\
Participants \\
Random- \\
ized N
\end{tabular} & $\begin{array}{l}\text { PLWD } \\
\text { Dementia } \\
\text { Characteristics }\end{array}$ & \begin{tabular}{|l|} 
PLWD \\
Non-Disease Char \\
PLWD N \\
PLWD Age (mean) \\
PLWD Sex (\% \\
female) \\
PLWD Race (\% \\
majority) \\
PLWD Education \\
(mean years)
\end{tabular} & $\begin{array}{l}\text { PLWD } \\
\text { Non-Disease } \\
\text { Char Reporting } \\
\text { Status (RS) } \\
\\
\text { PLWD SES } \\
\text { PLWD Prior } \\
\text { Disability } \\
\text { PLWD } \\
\text { Household } \\
\text { Characteristics } \\
\text { PLWD Health } \\
\text { Insurance } \\
\text { PLWD Detailed } \\
\text { Race Information }\end{array}$ & $\begin{array}{l}\text { Informal Caregiver } \\
\text { (IC) } \\
\text { Characteristics } \\
\text { IC N } \\
\text { IC Age (mean) } \\
\text { IC Sex (\% female) } \\
\text { IC Race (\% } \\
\text { majority) } \\
\text { IC Education } \\
\text { (mean years) } \\
\text { IC Relation to } \\
\text { PLWD (\% majority) }\end{array}$ & $\begin{array}{l}\text { Informal Caregiver } \\
\text { (IC) Char. RS } \\
\text { IC Duration } \\
\text { IC Living With } \\
\text { PLWD } \\
\text { IC Payment } \\
\text { IC Health Status } \\
\text { IC Dementia } \\
\text { Family History } \\
\text { IC Employment } \\
\text { Status } \\
\text { IC Training }\end{array}$ & 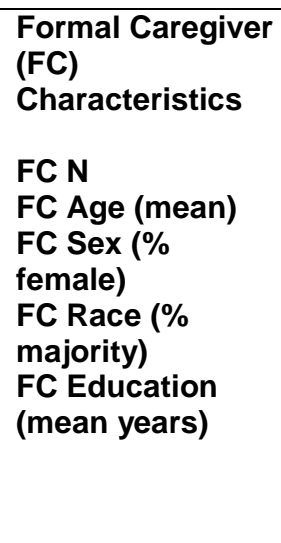 & $\begin{array}{l}\text { Formal } \\
\text { Caregiver (FC) } \\
\text { Char. RS } \\
\text { FC Health } \\
\text { Status } \\
\text { FC Training } \\
\text { FC Education } \\
\text { FC Position } \\
\text { FC Length of } \\
\text { Service }\end{array}$ & $\begin{array}{l}\text { Outcome } \\
\text { Timing(s) }\end{array}$ & $\begin{array}{l}\text { PLWD } \\
\text { Outcomes }\end{array}$ & $\begin{array}{l}\text { Caregiver } \\
\text { Outcomes } \\
\text { IC: } \\
\text { FC: }\end{array}$ \\
\hline $\begin{array}{l}\text { Laver } 2020^{59} \\
32234275 \\
\\
\text { Australia } \\
\text { Pilot }\end{array}$ & Telehealth & Usual care & $\mathrm{N}=63$ & $\begin{array}{l}\text { Diagnosed } \\
\text { dementia or } \\
\text { probable dementia } \\
\text { or MMSE }<24 / 30\end{array}$ & \begin{tabular}{|l} 
N-63 \\
Age: yes \\
Ses: yes
\end{tabular} & $\begin{array}{l}\text { Age: yes } \\
\text { Sex: yes' } \\
\text { Household } \\
\text { characteristics: } \\
\text { yes }\end{array}$ & NA & $\begin{array}{l}\text { Age: yes } \\
\text { Sex: yes' } \\
\text { Household } \\
\text { characteristics: yes }\end{array}$ & NA & NA & 16 weeks & & $\begin{array}{l}\text { CMI } \\
\text { PCS } \\
\text { CAPU }\end{array}$ \\
\hline $\begin{array}{l}\text { Villar 201960 } \\
(29149789) \\
\text { Spain } \\
\text { Pilot }\end{array}$ & $\begin{array}{l}\text { Including PLWD } \\
\text { with } \\
\text { dementia in } \\
\text { care planning } \\
\text { meetings }\end{array}$ & Usual care & \begin{tabular}{l|} 
Nursing \\
homes \\
Cluster RCT \\
Clusters $=4$ \\
$\mathrm{~N}=52$
\end{tabular} & $\begin{array}{l}\text { PLWD living in a } \\
\text { residential facility } \\
\text { for at least six } \\
\text { months, diagnosed } \\
\text { with moderate-to- } \\
\text { severe dementia }\end{array}$ & \begin{tabular}{|l|}
$\mathrm{N}=52$ \\
Age: $y$ yes \\
Sex: yes
\end{tabular} & NR & NA & NA & NR & NR & 20-30 minutes & $\begin{array}{l}\text { the GDS and } \\
\text { GENCAT }\end{array}$ & NR \\
\hline $\begin{array}{l}\text { Williams 201961 } \\
\text { (31660443) } \\
\text { US } \\
\text { Pilot }\end{array}$ & $\begin{array}{l}\text { FamTechCare } \\
\text { video recording } \\
\text { support }\end{array}$ & $\begin{array}{l}\text { Telephone } \\
\text { support/ } \\
\text { attention control }\end{array}$ & $\begin{array}{l}\text { Community- } \\
\text { based } \\
\text { RCT }\end{array}$ & $\begin{array}{l}\text { Patients diagnosed } \\
\text { with dementia } \\
\text { Living at home } \\
\text { Exclusion criteria }\end{array}$ & \begin{tabular}{|l|}
$\mathrm{N}=39$ Intervention \\
group with 42 \\
caregivers \\
32 Control group \\
with 41 caregivers \\
Age: $Y$ \\
Sex: $Y$ \\
Education: $Y$ \\
Race $\%$ majority: $Y$
\end{tabular} & $\begin{array}{l}\text { N caregivers: } Y \\
\text { Ethnicity: } Y\end{array}$ & $\begin{array}{l}\text { Age: } Y \\
\text { Sex: Y } \\
\text { Race \% majority: Y } \\
\text { Education: } Y \\
\text { Relation to PLWD: } \\
\text { Y }\end{array}$ & $\begin{array}{l}\text { Ethnicity: } Y \\
\text { Marital status: } Y \\
\text { Care provided: } Y\end{array}$ & NR & NR & 3 months & NR & $\begin{array}{l}\text { Burden } \\
\text { Depression } \\
\text { Sleep } \\
\text { disturbance } \\
\text { Competence } \\
\text { Desire to } \\
\text { institutionalize } \\
\text { PLWD } \\
\text { Reaction to } \\
\text { behavioral } \\
\text { symptoms }\end{array}$ \\
\hline $\begin{array}{l}\text { Horvath 2013 } \\
\text { (24195007) } \\
\text { US } \\
\text { High ROB }\end{array}$ & $\begin{array}{l}\text { Self-directed } \\
\text { educational } \\
\text { program for } \\
\text { caregiver } \\
\text { competence } \\
\text { and patient } \\
\text { safety; home- } \\
\text { based safety } \\
\text { tool kit }\end{array}$ & Customary care & $\begin{array}{l}\text { Community- } \\
\text { based } \\
\text { Recruited } \\
\text { from } 2 \text { care } \\
\text { centers } \\
\text { Block } \\
\text { randomizatio } \\
\text { n RCT }\end{array}$ & $\begin{array}{l}\text { Patients diagnosed } \\
\text { with progressive } \\
\text { AD } \\
\text { PLWD reads and } \\
\text { speaks English } \\
\text { MMSE <=24 } \\
\text { Primary informal } \\
\text { caregiver at least } 4 \\
\text { hours per day } \\
\text { Exclusion criteria }\end{array}$ & $\begin{array}{l}\mathrm{N}=60 \text { Experimental } \\
\text { group with } 60 \\
\text { caregivers } \\
48 \text { control group } \\
\text { with } 48 \text { caregivers } \\
\text { Age: } Y \\
\text { Sex: } Y \\
\text { Education: } N \\
\text { Race } \% \text { majority: } Y\end{array}$ & NR & $\begin{array}{l}\text { Age: Y } \\
\text { Sex: Y } \\
\text { Race \% majority } \\
\text { Education: N } \\
\text { Relation to PLWD: } \\
\text { Y }\end{array}$ & NR & NR & Training: Y & 12 weeks & $\begin{array}{l}\text { Home safety } \\
\text { evaluation } \\
\text { Risky } \\
\text { behaviors } \\
\text { and } \\
\text { accidents }\end{array}$ & $\begin{array}{l}\text { Self-efficacy } \\
\text { Strain }\end{array}$ \\
\hline
\end{tabular}




\section{Collaborative/Coordinated Care Models}

Table F-33. Risk of bias assessment: multidisciplinary integrated team collaborative care

\begin{tabular}{|c|c|c|c|c|c|c|c|c|c|}
\hline Study (PMID) & Outcome Timing & $\begin{array}{l}\text { Attrition Bias } \\
\text { Attrition \% }\end{array}$ & Selection Bias & Detection Bias & Performance Bias & Reporting Bias & Fidelity Bias & Funder & Overall Rating \\
\hline Halek 202062 (32062052) & 15 days/unclear & $\begin{array}{l}\text { Medium } \\
24 \%\end{array}$ & Low & Low & High & Low & Low & Foundation & High \\
\hline $\begin{array}{l}\text { Possin, 201963 } \\
31566651\end{array}$ & 12 month & Not high & Not high & $x$ & $x$ & $x$ & Not high & Gov't & Move to PRECIS-2 \\
\hline Laporte Uribe $2017^{64}$ (28249632) & 12 months & $\mathrm{x}$ & High & $\mathrm{x}$ & $\mathrm{x}$ & $\mathrm{x}$ & $\mathrm{x}$ & $\mathrm{X}$ & High \\
\hline $\begin{array}{l}\text { Thyrian, } 2017^{65} \\
28746708\end{array}$ & 12 months & $\begin{array}{l}\text { Medium } \\
21 \%\end{array}$ & $\begin{array}{l}\text { Medium } \\
\text { (selection concern } \\
\text { at GP level) }\end{array}$ & $\mathrm{x}$ & $x$ & $x$ & Medium & Gov't & Move to PRECIS-2 \\
\hline $\begin{array}{l}\text { Chodosh, 201566 } \\
25656074\end{array}$ & 6,12 month & $\begin{array}{l}\text { Medium } \\
36 \% 6 \text { month }\end{array}$ & Low & Low & Medium & Low & Low & Gov't & $\begin{array}{l}\text { Medium } \\
\text { (12 month High) }\end{array}$ \\
\hline $\begin{array}{l}\text { Leontjevas, } 2014^{67} \\
23643110\end{array}$ & 4 months & $\begin{array}{l}\text { Low } \\
\text { (stepped wedge) }\end{array}$ & Low & High & Medium & Low & $\begin{array}{l}\text { High (43\% } \\
\text { adherence to } \\
\text { treatment) }\end{array}$ & Gov't & $\begin{array}{l}\text { High (for pragmatic) } \\
\text { High (for traditional) }\end{array}$ \\
\hline $\begin{array}{l}\text { Bass, } 2013^{68} \\
23869899\end{array}$ & 6 months & $\begin{array}{l}\text { Medium } \\
18 \% \\
\text { (12 month almost 40\%) }\end{array}$ & Medium & $\mathrm{x}$ & $x$ & $\mathrm{x}$ & Low & Gov't & Move to PRECIS-2 \\
\hline $\begin{array}{l}\text { Chapman, } 2007^{69} \\
\text { 8537594 }\end{array}$ & 8 weeks & $\begin{array}{l}\text { Low } \\
2 \%\end{array}$ & Medium & High & Medium & Low & Medium & Gov't & High \\
\hline $\begin{array}{l}\text { Callahan, 2006 } \\
16684985\end{array}$ & 12 month & $\begin{array}{l}\text { Medium } \\
18 \%\end{array}$ & Low & Low & Medium & Low & Medium & Gov't & $\begin{array}{l}\text { Medium } \\
\text { (18 month High ROB) }\end{array}$ \\
\hline $\begin{array}{l}\text { Vickodeyos, } 2006^{71} \\
17116916\end{array}$ & 22 months (mean) & Not high & \begin{tabular}{|l} 
Not high \\
\end{tabular} & $\mathrm{x}$ & $x$ & $\mathrm{x}$ & \begin{tabular}{|l} 
Not high \\
\end{tabular} & Gov't & Move to PRECIS-2 \\
\hline $\begin{array}{l}\text { Surr, 201972 } \\
31056923\end{array}$ & 16 months & OK & OK & $\mathrm{x}$ & $\mathrm{x}$ & $\mathrm{x}$ & $\begin{array}{l}\text { High } \\
(26 \%)\end{array}$ & & High ROB for Pragmatic \\
\hline $\begin{array}{l}\text { Ballard, 2018 } 2013 \\
29408901\end{array}$ & 9 months & Medium & Low & Medium & Medium & Low & Medium & Gov't & Medium \\
\hline Lichtwarck, $2018^{74}$ & 12 weeks & $\begin{array}{l}\text { Medium } \\
12 \%\end{array}$ & Low & Low & Med & Low & Low & Gov't & Medium \\
\hline $\begin{array}{l}\text { Dichter, } 2015^{75} \\
26138674\end{array}$ & & Rolling enrollment & Medium & $\mathrm{x}$ & $\mathrm{x}$ & $\mathrm{x}$ & \begin{tabular}{|l|} 
Medium \\
\end{tabular} & Gov't & High \\
\hline $\begin{array}{l}\text { Chenoweth, 2014 } \\
24666667\end{array}$ & 8 months & $\begin{array}{l}\text { High } \\
31 \%\end{array}$ & $x$ & $\mathrm{x}$ & $x$ & $x$ & $x$ & Gov't & High \\
\hline van de Ven, $2013^{77}$ & 8 months & Rolling stable & Low & $\mathrm{x}$ & $x$ & $\mathrm{x}$ & Medium & Gov't & Move to PRECIS-2 \\
\hline $\begin{array}{l}\text { Rokstad, 201378 } \\
24022375\end{array}$ & & $\begin{array}{l}\text { Medium } \\
25 \%\end{array}$ & Low & $x$ & $\mathrm{x}$ & $x$ & High & Gov't & High \\
\hline $\begin{array}{l}\text { Chenoweth, } 2009^{79} \\
19282246\end{array}$ & 8 months & $\begin{array}{l}\text { Low } \\
18 \%\end{array}$ & Low & Low & High & Low & High & Gov't & Medium \\
\hline $\begin{array}{l}\text { Eloniemi-Sulkava, } 2009^{80} \\
\text { (20121986) }\end{array}$ & 1.6 years & $\begin{array}{l}\text { Low } \\
0 \% \text { (use of census data) }\end{array}$ & Low & Low & Medium & Low & \begin{tabular}{|l|} 
Medium \\
\end{tabular} & Gov't & Low \\
\hline $\begin{array}{l}\text { Fossey, 200681 } \\
16543297\end{array}$ & 12 months & Unclear/High & Low & Medium & Medium & Low & High & Gov't & High \\
\hline $\begin{array}{l}\text { Rovner, } 1996^{82} \\
8537594\end{array}$ & 6 months & $\begin{array}{l}\text { Low } \\
9 \%\end{array}$ & Low & Medium & High & Low & High & Gov't & Medium \\
\hline
\end{tabular}

Abbreviations: NR=Not Reported; PMID=PubMed Identification Number

\begin{tabular}{|c|c|c|c|c|c|c|c|c|c|c|c|c|c|}
\hline $\begin{array}{l}\text { Study (PMID) } \\
\text { Country } \\
\text { RoB } \\
\text { Type }\end{array}$ & \begin{tabular}{|l|} 
Intervention \\
Intervention \\
Focus \\
Theoretical \\
Model \\
Delivery \\
Person \\
Intervention \\
Target \\
Recipient \\
Mode \\
Components \\
Frequency \\
Duration
\end{tabular} & $\begin{array}{l}\text { Comparison } \\
\text { Target } \\
\text { Mode } \\
\text { Components } \\
\text { Frequency } \\
\text { Duration }\end{array}$ & $\begin{array}{l}\text { Setting } \\
\text { and Design } \\
\text { Setting } \\
\text { Design } \\
\text { Cluster N } \\
\text { Participants } \\
\text { Randomized } \\
\text { N }\end{array}$ & \begin{tabular}{|l} 
PLWD \\
Dementia \\
Characteristics \\
\\
Dementia Types \\
Dementia Severity \\
Diagnostic \\
Criteria \\
Age of Diagnosis
\end{tabular} & $\begin{array}{l}\text { PLWD } \\
\text { Non-Disease Char } \\
\text { PLWD N } \\
\text { PLWD Age (mean) } \\
\text { PLWD Sex (\% } \\
\text { female) } \\
\text { PLWD Race (\% } \\
\text { majority) } \\
\text { PLWD Education } \\
\text { (mean years) }\end{array}$ & \begin{tabular}{|l|l|} 
PLWD \\
Non-Disease \\
Char Reporting \\
Status (RS) \\
PLWD SES \\
PLWD Prior \\
Disability \\
PLWD \\
Household \\
Characteristics \\
PLWD Health \\
Insurance \\
PLWD Detailed \\
Race Information
\end{tabular} & \begin{tabular}{|l} 
Informal Caregiver \\
(IC) \\
Characteristics \\
IC N \\
IC Age (mean) \\
IC Sex (\% female) \\
IC Race (\% \\
majority) \\
IC Education \\
(mean years) \\
IC Relation to \\
PLWD (\% majority)
\end{tabular} & \begin{tabular}{|l} 
Informal Caregiver \\
(IC) Char. RS \\
IC Duration \\
IC Living With \\
PLWD \\
IC Payment \\
IC Health Status \\
IC Dementia \\
Family History \\
IC Employment \\
Status \\
IC Training
\end{tabular} & \begin{tabular}{|l} 
Formal Caregiver \\
(FC) \\
Characteristics \\
FC N \\
FC Age (mean) \\
FC Sex (\% \\
female) \\
FC Race (\% \\
majority) \\
FC Education \\
(mean years)
\end{tabular} & $\begin{array}{l}\text { Formal } \\
\text { Caregiver (FC) } \\
\text { Char. RS } \\
\text { FC Health } \\
\text { Status } \\
\text { FC Training } \\
\text { FC Education } \\
\text { FC Position } \\
\text { FC Length of } \\
\text { Service }\end{array}$ & $\begin{array}{l}\text { Outcome } \\
\text { Timing(s) }\end{array}$ & \begin{tabular}{|l|} 
PLWD \\
Outcomes
\end{tabular} & $\begin{array}{l}\text { Caregiver } \\
\text { Outcomes } \\
\text { IC: } \\
\text { FC: }\end{array}$ \\
\hline
\end{tabular}




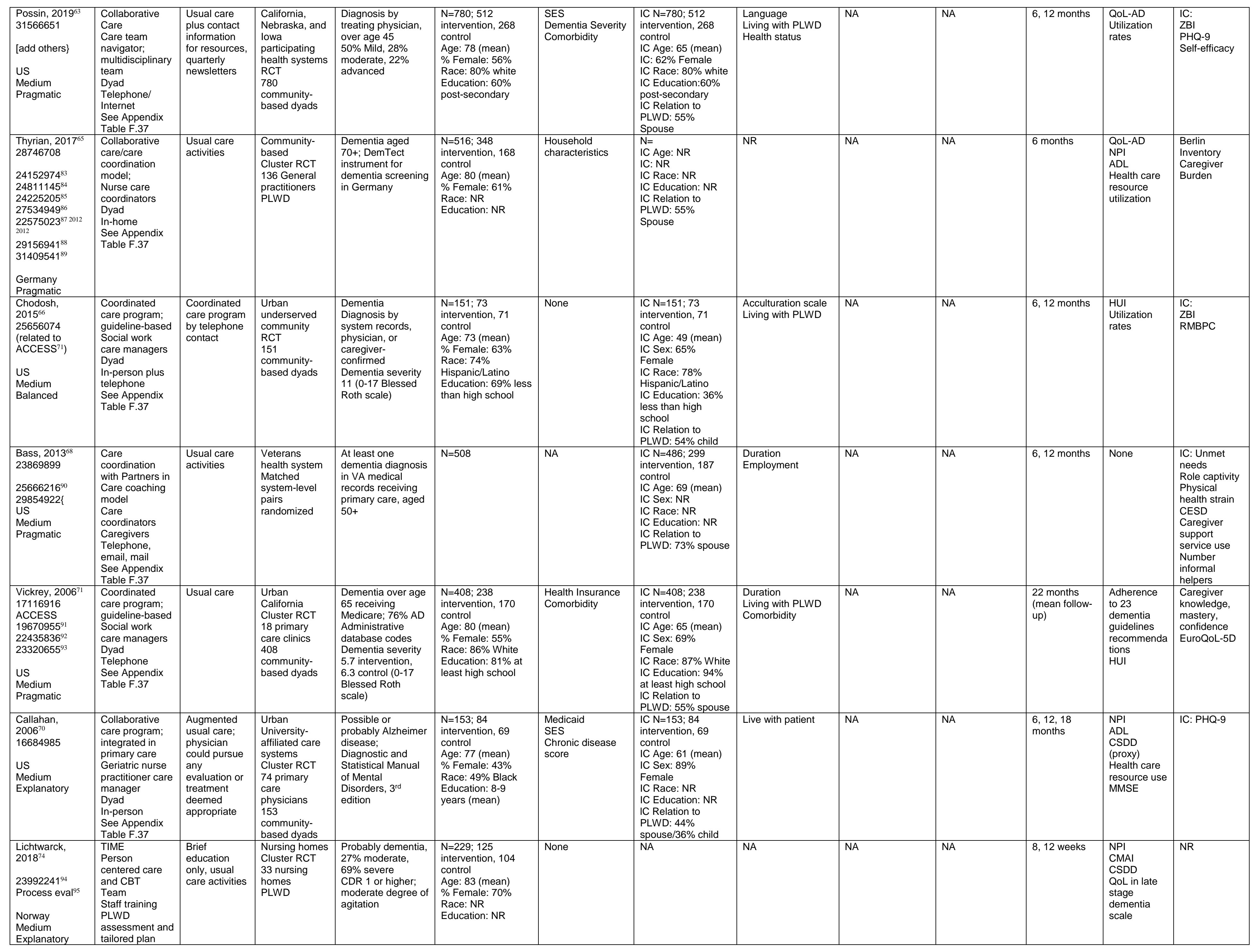




\begin{tabular}{|c|c|c|c|c|c|c|c|c|c|c|c|c|c|}
\hline $\begin{array}{l}\text { Ballard, 2018 } \\
29408901 \\
\text { Sustainability } \\
\text { Cost } \\
\text { effectiveness } \\
\text { UK } \\
\text { UK } \\
\text { Medium } \\
\text { Balanced }\end{array}$ & $\begin{array}{l}\text { WHELD } \\
\text { programme } \\
\text { person-centered } \\
\text { care and social } \\
\text { interaction } \\
\text { Team } \\
\text { PLWD } \\
\text { Training and } \\
\text { delivery of } \\
\text { WHELD } \\
\end{array}$ & $\begin{array}{l}\text { Usual care } \\
\text { activities }\end{array}$ & $\begin{array}{l}\text { Nursing homes } \\
\text { Cluster RCT } \\
69 \text { nursing } \\
\text { homes } \\
\text { PLWD }\end{array}$ & $\begin{array}{l}\text { Probably dementia, } \\
\text { 9\% moderate, } 60 \% \\
\text { moderately severe, } \\
\text { 21\% severe } \\
\text { CDR } 1 \text { or higher; } \\
\text { moderate degree of } \\
\text { agitation }\end{array}$ & $\begin{array}{l}\mathrm{N}=847 ; 404 \\
\text { intervention, } 443 \\
\text { control } \\
\text { Age: } 88 \text { (mean) } \\
\text { \% Female: } 69 \% \\
\text { Race: NR } \\
\text { Education: NR }\end{array}$ & None & NA & NA & $\mathrm{NR}$ & $\mathrm{NR}$ & 9 months & $\begin{array}{l}\text { DEMQOL } \\
\text { (proxy } \\
\text { CMAI } \\
\text { NPI } \\
\text { Serious } \\
\text { adverse } \\
\text { events } \\
\text { Cost } \\
\text { differences }\end{array}$ & NR \\
\hline $\begin{array}{l}\text { van de Ven, } \\
2013^{77} \\
23844003 \\
\\
\text { Netherlands } \\
\text { Medium } \\
\text { Pragmatic }\end{array}$ & $\begin{array}{l}\text { Dementia care } \\
\text { mapping, } \\
\text { training staff to } \\
\text { be certified } \\
\text { dementia-care } \\
\text { mappers, care } \\
\text { action planning }\end{array}$ & $\begin{array}{l}\text { Usual care } \\
\text { activities }\end{array}$ & $\begin{array}{l}\text { Nursing homes } \\
\text { Cluster RCT } \\
14 \text { care homes } \\
\text { PLWD }\end{array}$ & \begin{tabular}{|l|} 
Dementia, \\
Diagnostic and \\
Statistical Manual \\
of mental disorders \\
- IV criteria
\end{tabular} & $\begin{array}{l}\mathrm{N}=268 ; 102 \\
\text { dementia care } \\
\text { mapping, } 166 \\
\text { control } \\
\text { Age: } 84 \text { (mean) } \\
\text { \% Female: } 74 \% \\
\text { Race: } 97 \% \text { born in } \\
\text { Netherlands } \\
\text { Education: NR }\end{array}$ & None & NA & NA & NA & NA & 8 months & $\begin{array}{l}\text { CMAI } \\
\text { NPI } \\
\text { QUALID } \\
\text { EuroQQL 5D }\end{array}$ & GHQ-12 \\
\hline $\begin{array}{l}\text { Chenoweth, } \\
2009^{79} \\
19282246 \\
\\
(104505176)^{98} \\
(22078076)^{99} \\
\text { Australia } \\
\text { Medium } \\
\text { Balanced }\end{array}$ & $\begin{array}{l}\text { 11: person- } \\
\text { centered care } \\
\text { individualized } \\
\text { care program; } \\
\text { 12: dementia } \\
\text { care mapping }\end{array}$ & $\begin{array}{l}\text { Usual care } \\
\text { activities }\end{array}$ & $\begin{array}{l}\text { Nursing homes } \\
\text { Cluster RCT } \\
15 \text { care sites } \\
\text { PLWD }\end{array}$ & $\begin{array}{l}\text { Medical diagnosis } \\
\text { of dementia, } \\
\text { unspecified }\end{array}$ & $\begin{array}{l}\mathrm{N}=289 ; 109 \\
\text { dementia care } \\
\text { mapping, } 98 \\
\text { person-centered } \\
\text { care, } 82 \text { control } \\
\text { Age: } 84 \text { (mean) } \\
\% \text { Female: } 77 \% \\
\text { Race: } 69 \% \text { English } \\
\text { speakers } \\
\text { Education: NR }\end{array}$ & $\begin{array}{l}\text { Resident } \\
\text { classification }\end{array}$ & NA & NA & NA & NA & 8 months & $\begin{array}{l}\text { CMAI } \\
\text { QUALID } \\
\text { NPI }\end{array}$ & NR \\
\hline $\begin{array}{l}\text { Eloniemi- } \\
\text { Sulkava, 200980 } \\
(20121986) \\
\text { Finland } \\
\text { Medium } \\
\text { Explanatory }\end{array}$ & $\begin{array}{l}\text { Care } \\
\text { coordination; } \\
\text { coordinator } \\
\text { embedded in } \\
\text { multidisciplinary } \\
\text { team, includes } \\
\text { speer support } \\
\text { groups, } \\
\text { psychoeducatio } \\
\text { n } \\
\text { In-person }\end{array}$ & Usual care & $\begin{array}{l}\text { Community- } \\
\text { based } \\
\text { RCT } \\
\text { Dyads }\end{array}$ & \begin{tabular}{|l|} 
Community- \\
dwelling PLWD; \\
$87 \% \mathrm{AD}$, \\
predominately mild \\
to moderate \\
severity
\end{tabular} & $\begin{array}{l}\mathrm{N}=125 ; 63 \\
\text { intervention, } 62 \\
\text { control } \\
\text { Age: } 78 \text { (mean) } \\
\text { \% Female: } 77 \% \\
\text { Race: NR } \\
\text { Education: NR }\end{array}$ & None & $\begin{array}{l}\mathrm{N}=125 ; 63 \\
\text { intervention, } 62 \\
\text { control } \\
\text { Age: } 75 \text { (mean) } \\
\text { \% Female: } 72 \% \\
\text { Race: NR } \\
\text { Education: } 28 \% \\
\text { less than } 8 \text { years }\end{array}$ & None & NA & NA & $\begin{array}{l}20 \text { to } 24 \\
\text { months } \\
\text { (phased } \\
\text { recruitment) }\end{array}$ & $\begin{array}{l}\text { Time to } \\
\text { institutionaliz } \\
\text { ation } \\
\text { Use of } \\
\text { services } \\
\text { Service } \\
\text { expense }\end{array}$ & NA \\
\hline $\begin{array}{l}\text { Rovner, 199682 } \\
8537594 \\
\text { US } \\
\text { Medium } \\
\text { Explanatory }\end{array}$ & $\begin{array}{l}\text { Collaborative } \\
\text { dementia care } \\
\text { program } \\
\text { Unclear delivery } \\
\text { person } \\
\text { PLWD } \\
\text { In-person } \\
\text { See Appendix } \\
\text { Table F.37 }\end{array}$ & Usual care & $\begin{array}{l}\text { Urban Nursing } \\
\text { Home } \\
\text { RCT } \\
89 \text { PLWD }\end{array}$ & \begin{tabular}{|l|} 
Degenerative or \\
multi-infarct \\
dementia with \\
behavior disorder \\
Diagnostic and \\
Statistical Manual \\
of Mental \\
Disorders, $3^{\text {rd }}$ \\
edition
\end{tabular} & $\begin{array}{l}\mathrm{N}=81 ; 42 \\
\text { intervention, } 39 \\
\text { control } \\
\text { Age: } 81 \text { (mean) } \\
\% \text { Female: } 77 \% \\
\text { Race: } 83 \% \text { White } \\
\text { Education: NR }\end{array}$ & None & $\mathrm{NA}$ & NA & NA & NA & 6 months & $\begin{array}{l}\text { No useable } \\
\text { outcomes }\end{array}$ & $\mathrm{NA}$ \\
\hline
\end{tabular}

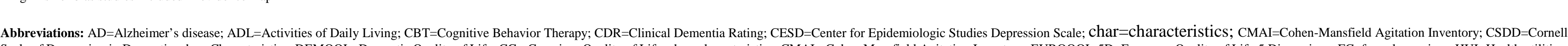

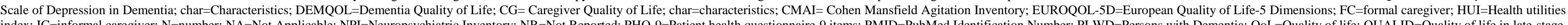

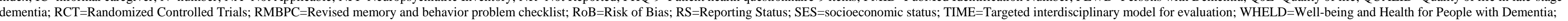
$\mathrm{ZBI}=$ Zarit burden inventory 
Table F-35. Intervention details for multidisciplinary integrated team collaborative care

\begin{tabular}{|c|c|}
\hline Study (PMID & Team \\
\hline $\begin{array}{l}\text { Possin, } 2019^{63} \\
31566651 \\
\text { HCB } \\
\text { Care Ecosystem }\end{array}$ & $\begin{array}{l}\text { Care team navigator (unlicensed; some bilingual } \\
\text { Spanish, Cantonese) APN, SW, pharmacist; } \\
\text { nurse-supervisor }\end{array}$ \\
\hline \begin{tabular}{|l|} 
Thyrian, $2017^{65}$ \\
28746708
\end{tabular} & $\begin{array}{l}\text { Nursing care coordinator embedded in German } \\
\text { health systems; general physician and social } \\
\text { service professionals; } \\
\text { initial weekly meeting with nursing scientist, } \\
\text { neurologist/ psychiatrist, psychologist, pharmacist } \\
\text { for initial assessment }\end{array}$ \\
\hline $\begin{array}{l}\text { Chodosh, 201566 } \\
\text { 25656074 } \\
\text { HCB } \\
\text { ACCESS }\end{array}$ & $\begin{array}{l}\text { Bilingual social work care managers from health } \\
\text { system; in-person included care manager from } \\
\text { local Association; } \\
\text { In-person included care manager from local } \\
\text { Association; Both used local resources from } \\
\text { participating organizations }\end{array}$ \\
\hline \begin{tabular}{|l|} 
Bass, $2013^{68}$ \\
23869899 \\
VA \\
Based on Partners in $\mathrm{C}$ \\
Care
\end{tabular} & Care coordinators, embedded in VA system \\
\hline $\begin{array}{l}\text { Vickrey, } 2006^{71} \\
17116916 \\
\\
\text { HCB } \\
\text { ACCESS }\end{array}$ & $\begin{array}{l}\text { Social work care managers from } 3 \text { health care } \\
\text { organizations, } 3 \text { local community agencies could } \\
\text { also have care managers; supervisor unclear }\end{array}$ \\
\hline $\begin{array}{l}\text { Callahan, 2006 } \\
16684985 \\
\text { HCB }\end{array}$ & $\begin{array}{l}\text { Geriatric nurse practitioner care manager, } \\
\text { Primary care physician; geriatrician, geriatric } \\
\text { psychiatrist, psychologist }\end{array}$ \\
\hline $\begin{array}{l}\text { Rovner, } 1996^{82} \\
8537594\end{array}$ & $\begin{array}{l}\text { Unclear; day program created within nursing } \\
\text { home; Psychiatrist, Creative arts therapist, } \\
\text { nursing aides }\end{array}$ \\
\hline
\end{tabular}

\begin{tabular}{|l|l|l|}
\hline Contact Frequency & Delivery & Caregiver Elements \\
\hline $\begin{array}{l}\text { Monthly by phone, } \text { email } \\
\text { mail also; CG-initiated for } \\
\text { problems }\end{array}$ & $\begin{array}{l}\text { Telephone/ internet, } \\
\text { some in-person }\end{array}$ & $\begin{array}{l}\text { Screen for problems, Standardized } \\
\text { education; personalized support, } \\
\text { manualized; ACP }\end{array}$ \\
\hline Monthly 1 hour home visits & In-home & $\begin{array}{l}\text { Included in PLWD contacts, if the } \\
\text { PLWD had an informal caregiver }\end{array}$ \\
$\begin{array}{l}\text { Minimum 7 contacts, } \\
\text { average 31 contacts in } \\
\text { telephone arm, 22 in in- } \\
\text { person arm }\end{array}$ & $\begin{array}{l}\text { In-person vs } \\
\text { telephone/ internet }\end{array}$ & $\begin{array}{l}\text { Protocolized problem assessment, } \\
\text { self-management counseling, } \\
\text { education, referrals and follow-up }\end{array}$ \\
$\begin{array}{l}\text { Minimum 1 contact per } \\
\text { month }\end{array}$ & $\begin{array}{l}\text { Telephone/ internet, } \\
\text { mail }\end{array}$ & $\begin{array}{l}\text { Protocolized initial assessment, } \\
\text { action plan, ongoing monitoring and } \\
\text { reassessment }\end{array}$ \\
\hline $\begin{array}{l}\text { Frequency based on as- } \\
\text { needed; } 6 \text { month } \\
\text { reassessments }\end{array}$ & Telephone & $\begin{array}{l}\text { Protocolized problem assessment, } \\
\text { self-management counseling, } \\
\text { education, referrals and follow-up }\end{array}$ \\
\hline $\begin{array}{l}\text { Bi-weekly initially, then } \\
\text { monthly, up to 12 months }\end{array}$ & In-person & $\begin{array}{l}\text { Manualized psychoeducation, } \\
\text { coping skills, group sessions, } \\
\text { legallfinancial advice, exercise } \\
\text { guidelines, printed caregiver guide, }\end{array}$ \\
\hline Daily & In-person & None \\
\hline
\end{tabular}

\begin{tabular}{|c|c|c|}
\hline PLWD Elements & Colocation & Other Supports \\
\hline $\begin{array}{l}\text { Medication review, monitor } \\
\text { health status }\end{array}$ & Yes & None \\
\hline $\begin{array}{l}\text { Initial assessment, } \\
\text { intervention task list }\end{array}$ & Unclear & $\begin{array}{l}\text { Intervention management } \\
\text { software system }\end{array}$ \\
\hline Unclear & $\begin{array}{l}\text { Unclear. Care manger } \\
\text { within County health } \\
\text { services with primary } \\
\text { care clinics }\end{array}$ & $\begin{array}{l}\text { Help cover cost of phone } \\
\text { minutes for informal } \\
\text { caregivers; } \\
\text { Steering committee for } \\
\text { cross-organization } \\
\text { collaboration }\end{array}$ \\
\hline None noted & Yes & Software system \\
\hline $\begin{array}{l}\text { Primary care provider } \\
\text { training }\end{array}$ & Unclear & $\begin{array}{l}\text { Software systems linked; } \\
\text { Steering committee for } \\
\text { cross-organization } \\
\text { collaboration }\end{array}$ \\
\hline $\begin{array}{l}\text { Medication, problem } \\
\text { assessments, non-drug } \\
\text { behavioral interventions; } \\
\text { PLWD group exercise } \\
\text { sessions }\end{array}$ & Unclear & Software tracking system \\
\hline $\begin{array}{l}\text { Activity program, medication } \\
\text { management, team } \\
\text { consulting }\end{array}$ & Unclear & None \\
\hline
\end{tabular}

Abbreviations: ACP= advance care practice; $\mathrm{APN}=$ Advanced practice nurse; $\mathrm{CG}=$ caregiver; $\mathrm{HCB}=$ Home and community-based; $\mathrm{NH}=$ Nursing Home; PMID=PubMed Identification Number; PLWD= Person With Dementia; $\mathrm{SW}=$ Social Worker; VA= Veterans Affair

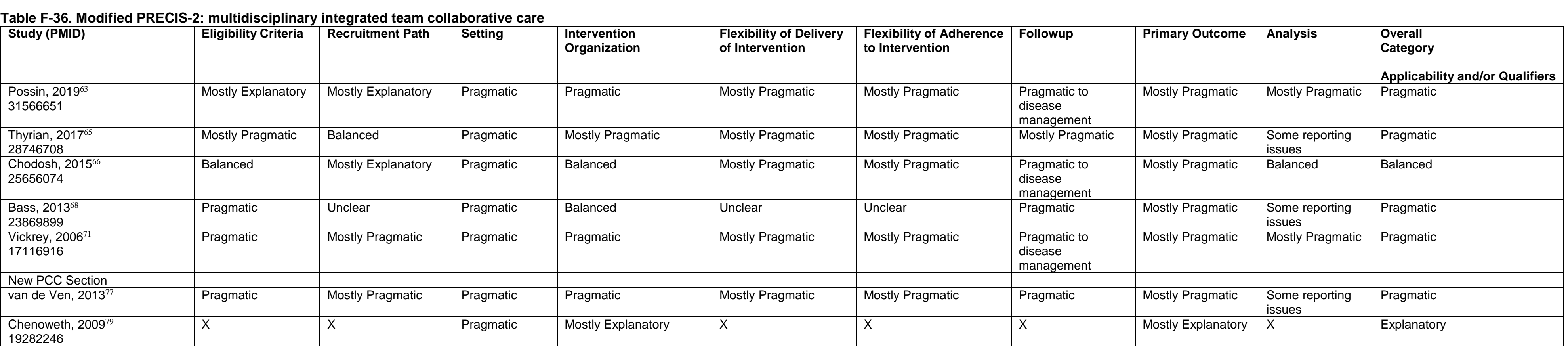

Abbreviations: NA=Not Assessed; PMID=PubMed Identification Number

Table F-37. PLWD outcomes summary low and medium risk of bias studies: multidisciplinary integrated team collaborative care

\begin{tabular}{|c|c|c|c|c|c|}
\hline $\begin{array}{l}\text { Study (PMID) } \\
\text { Comparison } \\
\text { RoB } \\
\text { Category* }\end{array}$ & $\begin{array}{l}\text { Outcome } \\
\text { Timing }\end{array}$ & Summary Finding & Intervention & Comparator & p-Value \\
\hline $\begin{array}{l}\text { Possin, } 2019^{63} \\
31566651 \\
\text { Medium } \\
\text { Pragmatic }\end{array}$ & $\begin{array}{l}\text { Unstandardized Beta }(\mathrm{Cl}) \\
\text { QoL-AD (proxy) (13-52; higher is better) } \\
12 \text { months }\end{array}$ & Favors intervention; 0.53 (0.25 to 1.30$)$ & NA & NA & 0.04 \\
\hline
\end{tabular}




\begin{tabular}{|c|c|c|c|c|c|}
\hline $\begin{array}{l}\text { Possin, 201963 } \\
31566651 \\
\text { Medium } \\
\text { Pragmatic }\end{array}$ & $\begin{array}{l}\text { Unstandardized Beta }(\mathrm{Cl}) \\
\text { ED visits (lower is better) } \\
12 \text { months }\end{array}$ & $\begin{array}{l}\text { Favors intervention; }-0.14(-0.29 \text { to }-0.01) \\
\text { NNT for single ED visit }=5 \\
\text { Prevented } 120 \text { visits (predicted based on based on usual care data) }\end{array}$ & NA & NA & 0.04 \\
\hline $\begin{array}{l}\text { Possin, 201963 } \\
31566651 \\
\text { Medium } \\
\text { Pragmatic }\end{array}$ & $\begin{array}{l}\text { Unstandardized Beta }(\mathrm{Cl}) \\
\text { Hospitalization (lower is better) } \\
12 \text { months }\end{array}$ & $\begin{array}{l}\text { No statistical difference; }-0.03(-0.18 \text { to } 0.12) \\
\text { Prevented } 13 \text { visits (predicted based on based on usual care data) }\end{array}$ & NA & NA & 0.71 \\
\hline $\begin{array}{l}\text { Possin, 201963 } \\
31566651 \\
\text { Medium } \\
\text { Pragmatic }\end{array}$ & $\begin{array}{l}\text { Unstandardized Beta }(\mathrm{Cl}) \\
\text { Ambulance use (lower is better) } \\
12 \text { months }\end{array}$ & $\begin{array}{l}\text { No statistical difference; - }-0.10(-0.23 \text { to } 0.03) \\
\text { Prevented } 16 \text { uses (predicted based on based on usual care data) }\end{array}$ & NA & NA & 0.12 \\
\hline $\begin{array}{l}\text { Possin, 201963 } \\
31566651 \\
\text { Medium } \\
\text { Pragmatic }\end{array}$ & $\begin{array}{l}\text { Mean cost estimates } \\
\text { Based on national estimates } \\
12 \text { months }\end{array}$ & $\$ 600$ per PLWD across ED, ambulance, and hospitalization, over latter 6 months. & NA & NA & NA \\
\hline $\begin{array}{l}\text { Thyrian, } 2017^{65} \\
28746708\end{array}$ & $\begin{array}{l}\text { Adjusted mean difference }(\mathrm{Cl}) \\
\text { QoL-AD (13-52; higher is better) } \\
12 \text { months }\end{array}$ & $\begin{array}{l}\text { No statistical difference } 0.02(-0.09 \circ 0.05) \\
\text { Effect size } 0.07\end{array}$ & NA & NA & 0.26 \\
\hline $\begin{array}{l}\text { Thyrian, } 2017^{65} \\
28746708\end{array}$ & $\begin{array}{l}\text { Adjusted mean difference }(\mathrm{Cl}) \\
\text { NPI } \\
12 \text { months }\end{array}$ & High risk of bias for missing data $36 \%$ of completers & NA & NA & NA \\
\hline $\begin{array}{l}\text { Thyrian, } 2017^{65} \\
28746708\end{array}$ & Daily living activities & No statistical difference, no data reported & NA & NA & NA \\
\hline $\begin{array}{l}\text { Thyrian, } 2017^{65} \\
28746708\end{array}$ & Institutionalization & No statistical difference, no data reported & NA & NA & NA \\
\hline $\begin{array}{l}\text { Chodosh, 2015 } \\
25656074 \\
\text { Medium } \\
\text { Balanced }\end{array}$ & $\begin{array}{l}\text { Adjusted mean difference (Cl) } \\
\text { HUI (proxy) (lower is better) } \\
6 \text { month } \\
\text { Grouped with QoL }\end{array}$ & No statistical difference; -0.064 (0.14 to 0.03 ) & NA & NA & 0.19 \\
\hline $\begin{array}{l}\text { Chodosh, 201566 } \\
\text { 25656074 } \\
\text { Medium } \\
\text { Balanced }\end{array}$ & 19 quality indicators & $\begin{array}{l}\text { All } 19 \text { indicators improved in both arms; Comparable proportional increases in quality } \\
\text { indicators for program, ACCESS (original protocol study) vs this study (Table 6, original } \\
\text { publication) }\end{array}$ & NA & NA & NA \\
\hline $\begin{array}{l}\text { Eloniemi-Sulkava, 200980 (20121986) } \\
\text { Medium } \\
\text { Balanced }\end{array}$ & Time to nursing home placement & $\begin{array}{l}\text { Favors intervention at } 1.6 \text { years, but no longer statistically significant at } 2 \text { years; Hazard } \\
\text { ration } 0.53 \mathrm{Cl}(0.23 \text { to } 1.19)\end{array}$ & 1.6 years $25.8 \%$ & 1.6 years $11.1 \%$ & 0.03 \\
\hline $\begin{array}{l}\text { Vickrey, 2006 } \\
17116916 \\
\text { Medium } \\
\text { Pragmatic }\end{array}$ & 23 quality indicators & All but 2 showed significant improvement. & NA & NA & $\leq 0.013$ \\
\hline $\begin{array}{l}\text { Vickrey, 2006 } \\
17116916 \\
\text { Medium } \\
\text { Pragmatic }\end{array}$ & \begin{tabular}{|l|} 
Adjusted mean difference $(\mathrm{Cl})$ \\
HUI \\
18 month \\
Grouped with QoL \\
\end{tabular} & $\begin{array}{l}\text { Favors intervention; } 0.06 \text { (0.005 to } 0.11) \\
\text { (MID } 0.03\end{array}$ & NA & NA & 0.034 \\
\hline $\begin{array}{l}\text { Callahan, 2006 } \\
16684985 \\
\text { Medium } \\
\text { Explanatory }\end{array}$ & $\begin{array}{l}\text { Adjusted mean difference }(\mathrm{Cl}) \\
\text { NPI (lower is better) } \\
12 \text { months }\end{array}$ & Favors intervention $-5.6(-9.9$ to -1.3$)$ & $8.0(12.0)$ & $16.1(19.4)$ & 0.01 \\
\hline $\begin{array}{l}\text { Callahan, 2006 } \\
16684985 \\
\text { Medium } \\
\text { Explanatory }\end{array}$ & $\begin{array}{l}\text { Adjusted mean difference }(\mathrm{Cl}) \\
\text { CSDD (lower is better) } \\
12 \text { months }\end{array}$ & No statistical significance $-1.0(-2.6$ to 1.5$)$ & $3.5(3.9)$ & $5.8(5.9)$ & 0.65 \\
\hline $\begin{array}{l}\text { Callahan, 2006 } \\
16684985 \\
\text { Medium } \\
\text { Explanatory }\end{array}$ & $\begin{array}{l}\text { Adjusted mean difference }(\mathrm{Cl}) \\
\mathrm{ADL} \text { (Higher is better) } \\
12 \text { months }\end{array}$ & No statistical significance 1.4 (-2.3 to 5.1$)$ & $48.6(17.7)$ & $44.6(17.0)$ & 0.44 \\
\hline $\begin{array}{l}\text { Callahan, 2006 } \\
16684985 \\
\text { Medium } \\
\text { Explanatory }\end{array}$ & $\begin{array}{l}\text { Nursing home placement } \\
12 \text { months }\end{array}$ & No statistical significance & $6 \%$ & $1.5 \%$ & 0.22 \\
\hline Lichtwarck, $2018^{74}$ & \begin{tabular}{|l|} 
Standardized mean difference \\
$\mathrm{NPI}-\mathrm{NH}-10$ \\
12 weeks
\end{tabular} & Favors intervention 0.25 & 31.1 (26.7 to 35.6$)$ & 41.4 (37.3 to 45.5$)$ & 0.053 \\
\hline Lichtwarck, 201874 & $\begin{array}{l}\text { Standardized mean difference } \\
\text { CMAl } \\
12 \text { weeks }\end{array}$ & Favors intervention 0.29 & 59.4 (55.2 to 63.6$)$ & $67.1(63.3$ to 70.9$)$ & 0.006 \\
\hline Lichtwarck, $2018^{74}$ & $\begin{array}{l}\text { Standardized mean difference } \\
\text { CSDD } \\
12 \text { weeks }\end{array}$ & Favors intervention 0.26 & 10.2 (8.7 to 11.7$)$ & 12.4 (10.9 to 13.8$)$ & 0.010 \\
\hline Lichtwarck, 201874 & $\begin{array}{l}\text { Standardized mean difference } \\
\text { QUALID } \\
12 \text { weeks }\end{array}$ & Favors intervention 0.17 & $27.2(25.3$ to 29.1$)$ & 29.6 (27.8 to 31.5$)$ & 0.044 \\
\hline $\begin{array}{l}\text { Ballard, } 2018^{73} \\
29408901\end{array}$ & $\begin{array}{l}\text { Mean difference }(\mathrm{Cl}) \\
\text { DEMQOL (proxy) } \\
9 \text { months }\end{array}$ & $\begin{array}{l}\text { Favors Intervention, } 2.54 \text { (0.81 to } 4.28) \\
\text { Cohen's D 0.24; NNT } 9\end{array}$ & & & 0.004 \\
\hline $\begin{array}{l}\text { Ballard, } 2018^{73} \\
29408901\end{array}$ & \begin{tabular}{|l|} 
Mean difference $(\mathrm{Cl})$ \\
CMAI \\
9 months \\
\end{tabular} & $\begin{array}{l}\text { Favors Intervention, } 4.27 \text { (-7.39 to -1.15) } \\
\text { Cohen's D 0.23; NNT } 6\end{array}$ & & & 0.008 \\
\hline $\begin{array}{l}\text { Ballard, } 2018^{73} \\
29408901\end{array}$ & $\begin{array}{l}\text { Mean difference (Cl) } \\
\mathrm{NPI}-\mathrm{NH} \\
9 \text { months }\end{array}$ & $\begin{array}{l}\text { Favors Intervention, } 4.55 \text { (-7.07 to }-2.02) \\
\text { Cohen's D 0.30; NNT } 9\end{array}$ & & & $<0.001$ \\
\hline
\end{tabular}




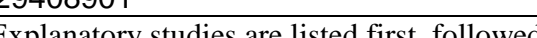

Abbreviations: ADL=Activities of Daily Living. CI=Confidence Interval· CMAI=Cohen-Mansfield Agitation Inventory; CSDD=Cornell Scale for Depression in Dementia; DEMQOL=Dementia Quality of Life; ED=Emergency Department; HUI=Health utilities index; N=Number; NPI=Neuropsychiatric Inventory; NPI-NH= Neuropsychiatric Inventory-Nursing Home; QoL-AD=Quality of Life-Alzheimer's disease; QUALID=Quality of Life in Late-stage Dementia; PMID=PubMed Identification Number; ROB=Risk of Bias 
Table F-38. Caregiver outcomes summary low and medium risk of bias studies: multidisciplinary integrated team collaborative care

\begin{tabular}{|c|c|c|c|c|c|}
\hline $\begin{array}{l}\text { Study (PMID) } \\
\text { Comparison } \\
\text { Caregiver Type } \\
\text { RoB } \\
\text { Category* }\end{array}$ & $\begin{array}{l}\text { Outcome } \\
\text { Timing }\end{array}$ & Summary Finding & Intervention & Comparator & p-Value \\
\hline $\begin{array}{l}\text { Possin, 201963 } \\
\text { 31566651 } \\
\text { Medium } \\
\text { Pragmatic }\end{array}$ & $\begin{array}{l}\text { Unstandardized Beta }(\mathrm{Cl}) \\
\text { PHQ-9 }(0-27 \text {; lower is better }) \\
12 \text { months }\end{array}$ & $\begin{array}{l}\text { Favors intervention; }-1.14(-2.15 \text { to }-0.13) \\
\text { NNT }=12\end{array}$ & NA & $\mathrm{NA}$ & 0.03 \\
\hline $\begin{array}{l}\text { Possin, 201963 } \\
\text { 31566651 } \\
\text { Medium } \\
\text { Pragmatic }\end{array}$ & $\begin{array}{l}\text { Unstandardized Beta }(\mathrm{Cl}) \\
\text { Self-efficacy (higher is better) } \\
12 \text { months }\end{array}$ & No statistical difference; 0.64 (0.14 to 1.41 ) & NA & NA & 0.11 \\
\hline $\begin{array}{l}\text { Possin, } 2019^{63} \\
31566651 \\
\text { Medium } \\
\text { Pragmatic }\end{array}$ & $\begin{array}{l}\text { Unstandardized Beta }(\mathrm{Cl}) \\
12 \text {-tem ZBI (0-48; lower is better) } \\
12 \text { months }\end{array}$ & Favors intervention; -1.90 (-3.89 to -0.08$)$ & NA & NA & 0.046 \\
\hline $\begin{array}{l}\text { Thyrian, } 2017^{65} \\
28746708\end{array}$ & $\begin{array}{l}\text { Adjusted mean difference }(\mathrm{Cl}) \\
\text { Berlin Inventory for Caregiver burden } \\
12 \text { months }\end{array}$ & $\begin{array}{l}\text { Favors intervention; }-0.50 \text { (-1.09 to } 0.08) \text {, one-sided analysis } \\
\text { Effect size }-0.18\end{array}$ & NA & NA & 0.045 \\
\hline $\begin{array}{l}\text { Bass, } 2013^{68} \\
23869899 \\
\text { Medium } \\
\text { Pragmatic }\end{array}$ & $\begin{array}{l}\text { Unstandardized B, no Cl provided } \\
\text { Unmet needs (lower is better, } 0-39 \text { items) } \\
6 \text { months }\end{array}$ & Favors intervention; -2.24 & NA & NA & 0.01 \\
\hline $\begin{array}{l}\text { Bass, } 2013^{68} \\
23869899 \\
\text { Medium } \\
\text { Pragmatic }\end{array}$ & $\begin{array}{l}\text { Unstandardized B, no Cl provided } \\
\text { Role captivity (lower is better, } 0-12 \text { ) } \\
6 \text { months }\end{array}$ & No statistical difference; 0.12 & NA & NA & NR \\
\hline $\begin{array}{l}\text { Bass, } 2013^{68} \\
23869899 \\
\text { Medium } \\
\text { Pragmatic }\end{array}$ & $\begin{array}{l}\text { Unstandardized B, no Cl provided } \\
\text { Physical health strain (lower is better, 0-12) } \\
6 \text { months }\end{array}$ & No statistical difference; -0.14 & NA & NA & NR \\
\hline $\begin{array}{l}\text { Bass, } 2013^{68} \\
2389899 \\
\text { Medium } \\
\text { Pragmatic }\end{array}$ & $\begin{array}{l}\text { Unstandardized B, no Cl provided } \\
\text { Relationship strain (lower is better, 0-24) } \\
6 \text { months }\end{array}$ & No statistical difference; 0.38 & NA & NA & NR \\
\hline $\begin{array}{l}\text { Bass, } 2013^{68} \\
2369899 \\
\text { Medium } \\
\text { Pragmatic }\end{array}$ & $\begin{array}{l}\text { Unstandardized B, no Cl provided } \\
11 \text { - item CESD (lower is better, 11-33) } \\
6 \text { months }\end{array}$ & $\begin{array}{l}\text { No statistical difference; }-0.69 \\
\text { (above from table, text reported beta }-0.08, p=0.047 \text { ) }\end{array}$ & NA & NA & NR \\
\hline $\begin{array}{l}\text { Bass, } 2013^{68} \\
2386899 \\
\text { Medium } \\
\text { Pragmatic }\end{array}$ & $\begin{array}{l}\text { Unstandardized B, no Cl provided } \\
\text { Number of informal helpers (higher is better) } \\
6 \text { months }\end{array}$ & No statistical difference; -0.32 & NA & $\mathrm{NA}$ & $\mathrm{NR}$ \\
\hline $\begin{array}{l}\text { Bass, } 2013^{68} \\
23869899 \\
\text { Medium } \\
\text { Pragmatic }\end{array}$ & $\begin{array}{l}\text { Unstandardized B, no Cl provided } \\
\text { Caregiver support service use (higher is better) } \\
6 \text { months }\end{array}$ & $\begin{array}{l}\text { Favors intervention; } 0.20 \\
\text { (above from table, text reported beta } 0.14, \mathrm{p}=0.008 \text { ) }\end{array}$ & NA & NA & NR \\
\hline $\begin{array}{l}\text { Chodosh, } 2015^{66} \\
25656074 \\
\text { Medium } \\
\text { Balanced }\end{array}$ & $\begin{array}{l}\text { Adjusted mean difference }(\mathrm{Cl}) \\
\mathrm{ZBI} \text { (lower is better) } \\
6 \text { month }\end{array}$ & No statistical difference; 1.8 (-3.46 to 7.05$)$ & NA & NA & 0.5 \\
\hline $\begin{array}{l}\text { Chodosh, } 2015^{66} \\
25656074 \\
\text { Medium } \\
\text { Balanced }\end{array}$ & $\begin{array}{l}\text { Adjusted mean difference }(\mathrm{Cl}) \\
\text { PHQ-9 (lower is better) } \\
6 \text { month }\end{array}$ & No statistical difference; 0.36 (-2.00 to 2.73 ) & NA & NA & 0.76 \\
\hline $\begin{array}{l}\text { Vickrey, } 2006^{71} \\
17116916 \\
\text { Medium } \\
\text { Pragmatic }\end{array}$ & $\begin{array}{l}\text { Adjusted mean difference }(\mathrm{Cl}) \\
\text { EuroQol-5D } \\
18 \text { month }\end{array}$ & No statistical difference; 0.02 (-0.01 to 0.06$)$ & NA & NA & 0.127 \\
\hline $\begin{array}{l}\text { Callahan, } 2006^{70} \\
16844985 \\
\text { Medium } \\
\text { Explanatory }\end{array}$ & $\begin{array}{l}\text { Adjusted mean difference }(\mathrm{Cl}) \\
\text { PHQ-9 (lower is better) } \\
12 \text { month }\end{array}$ & No statistical difference; -0.9 (-2.2 to 0.5$)$ & $3.1(3.9)$ & $4.6(5.6)$ & 0.50 \\
\hline $\begin{array}{l}\text { Callahan, 200670 } \\
16684985 \\
\text { Medium } \\
\text { Explanatory }\end{array}$ & $\begin{array}{l}\text { Adjusted mean difference (CI) } \\
\text { Caregiver NPI (lower is better) } \\
12 \text { month }\end{array}$ & Favors intervention; $-2.2(-4.2$ to -0.2$)$ & $3.5(5.8)$ & $7.7(8.7)$ & 0.03 \\
\hline
\end{tabular}

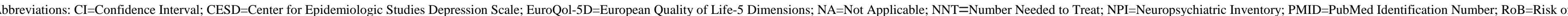
Bias; ZBI=Zarit Burden Interview 
Table F-39. Summary of strength of evidence for PLWD outcomes: multidisciplinary integrated team collaborative care

\begin{tabular}{|c|c|c|c|c|c|c|c|c|}
\hline $\begin{array}{l}\text { Comparison } \\
\text { Outcome }\end{array}$ & Timing & $\begin{array}{l}\text { \# Studies/ Design } \\
\text { (n analyzed) }\end{array}$ & Finding or Summary Statistic & Study Limitations & Consistency & Directness & Precision & Overall Grade/ Conclusion \\
\hline \begin{tabular}{|l} 
Collaborative care vs \\
usual care \\
QoL \\
\end{tabular} & 6-12 months & $\begin{array}{l}\begin{array}{l}4 \text { Pragmatic trials } \\
(\mathrm{n}=1,746)\end{array} \\
\end{array}$ & $\begin{array}{l}\text { QOL-AD benefit, no benefit } \\
\text { HUI benefit, no benefit }\end{array}$ & Medium & Consistent & Direct & Imprecise & $\begin{array}{l}\text { Low } \\
\text { (Weighted to larger, quality } \\
\text { pragmatic trials) }\end{array}$ \\
\hline $\begin{array}{l}\text { Collaborative care vs } \\
\text { usual care } \\
\text { NPI }\end{array}$ & 6-12 months & $\begin{array}{l}1 \text { Explanatory Cluster Trial } \\
(\mathrm{N}=152)\end{array}$ & One benefit & Medium & Unknown & Direct & Imprecise & Insufficient \\
\hline $\begin{array}{l}\text { Collaborative care vs } \\
\text { usual care } \\
\text { Utilization }\end{array}$ & 6-12 months & \begin{tabular}{|l}
1 Pragmatic Trial \\
$(\mathrm{n}=780)$
\end{tabular} & $\begin{array}{l}\text { ED visits benefit, hospitalization no difference, ambulance use } \\
\text { no difference }\end{array}$ & Medium & Unknown & Direct & precise & Low \\
\hline \begin{tabular}{|l} 
Colllaborative care vs \\
usual care \\
ADL \\
\end{tabular} & 6-12 months & $\begin{array}{l}1 \text { Pragmatic } \\
1 \text { Explanatory Cluster Trial } \\
(\mathrm{n}=560)\end{array}$ & 2 no difference & Medium & Consistent & Direct & Imprecise & Insufficient \\
\hline \begin{tabular}{|l} 
Collaborative care vs \\
usual care \\
Quality indicators \\
\end{tabular} & 6-12 months & $\begin{array}{l}2 \text { Pragmatic } \\
(n=559)\end{array}$ & Quality indicators, 2 benefit & Medium & Consistent & Direct & Imprecise & $\begin{array}{l}\text { Low } \\
\text { (Weighted to larger, quality } \\
\text { pragmatic trials) }\end{array}$ \\
\hline \begin{tabular}{|l} 
Collaborative care vs \\
usual care \\
Depression \\
\end{tabular} & 6-12 months & $\begin{array}{l}1 \text { Explanatory Cluster Trial } \\
\text { (N=152) }\end{array}$ & CSDD, no difference & Medium & Unknown & Direct & Imprecise & Insufficient \\
\hline \begin{tabular}{|l|} 
Collaborative care vs \\
usual care \\
Nursing home placement
\end{tabular} & 6-12 months & \begin{tabular}{|l}
1 Pragmatic \\
1 Explanatory Cluster Trial \\
$(\mathrm{n}=560)$
\end{tabular} & $\begin{array}{l}2 \text { no difference (not long enough for balance toward } \\
\text { mild/moderate) }\end{array}$ & Medium & Consistent & Direct & Imprecise & Insufficient \\
\hline
\end{tabular}

Abbreviations: ADL=Activities of Daily Living; CSDD=Cornell Scale for Depression in Dementia; ED=Emergency Department; HUI=Health utilities index; N=Number; NPI=Neuropsychiatric Inventory; QoL=Quality of Life; QoL-AD=Quality of Life-Alzheimer's disease

\begin{tabular}{|c|c|c|c|c|c|c|c|c|}
\hline $\begin{array}{l}\text { Comparison } \\
\text { Caregiver Type } \\
\text { Outcome }\end{array}$ & Timing & $\begin{array}{l}\text { \# Studies/ Design } \\
\text { (n analyzed) }\end{array}$ & Finding or Summary Statistic & Study Limitations & Consistency & Directness & Precision & Overall Grade/ Conclusion \\
\hline $\begin{array}{l}\text { Collaborative care vs } \\
\text { usual care } \\
\text { Informal } \\
\text { QoL }\end{array}$ & 6-12 months & $\begin{array}{l}1 \text { Pragmatic Trial } \\
(\mathrm{n}=408)\end{array}$ & EuroQol-5D no difference & Medium & Unknown & Direct & Imprecise & Insufficient \\
\hline $\begin{array}{l}\text { Collaborative care vs } \\
\text { usual care } \\
\text { Informal } \\
\text { Self-efficacy }\end{array}$ & 6-12 months & $\begin{array}{l}1 \text { Pragmatic Trial } \\
(\mathrm{n}=780)\end{array}$ & No benefit & Medium & Unknown & Direct & Imprecise & Insufficient \\
\hline $\begin{array}{l}\text { Collaborative care vs } \\
\text { usual care } \\
\text { Informal } \\
\text { Burden }\end{array}$ & 6-12 months & $\begin{array}{l}4 \text { Pragmatic } \\
(\mathrm{n}=1,719)\end{array}$ & $\begin{array}{l}\text { ZBI } 2 \text { Benefit, } 1 \text { Berlin no benefit; } \\
\text { Role captivity, } 2 \text { physical health strain, relationship strain, all no } \\
\text { benefit; Caregiver NPI no difference }\end{array}$ & Medium & Inconsistent & Direct & Imprecise & $\begin{array}{l}\text { Insufficient } \\
\text { (Weighted to larger, quality pragmatic } \\
\text { trials) }\end{array}$ \\
\hline $\begin{array}{l}\text { Collaborative care vs } \\
\text { usual care } \\
\text { Informal } \\
\text { Quality }\end{array}$ & 6-12 months & $\begin{array}{l}1 \text { Pragmatic Trial } \\
(\mathrm{n}=486)\end{array}$ & $\begin{array}{l}\text { Unmet needs, informal helpers, support service use } 2 \text { of } 3 \text { no } \\
\text { difference }\end{array}$ & Medium & Consistent & Direct & Imprecise & $\begin{array}{l}\text { Insufficient } \\
\text { (Weighted to larger, quality pragmatic } \\
\text { trials) }\end{array}$ \\
\hline $\begin{array}{l}\text { Collaborative care vs } \\
\text { usual care } \\
\text { Informal } \\
\text { Depression }\end{array}$ & 6-12 months & $\begin{array}{l}3 \text { Pragmatic, } 1 \\
\text { Explanatory } \\
(\mathrm{n}=1,570)\end{array}$ & 1 benefit, 3 No difference & Medium & Consistent & Direct & Imprecise & $\begin{array}{l}\text { Insufficient } \\
\text { (Weighted to larger, quality pragmatic } \\
\text { trials) }\end{array}$ \\
\hline
\end{tabular}

Abbreviations: EuroQoL-5D=N=Number; NPI=Neuropsychiatric Inventory; QoL=Quality of Life; ZBI=Zarit Burden Index

\section{Evidence Map: Multidisciplinary Integrated Team Collaborative Care}

\begin{tabular}{|c|c|c|c|c|c|c|c|c|c|c|c|c|c|}
\hline $\begin{array}{l}\text { Study (PMID) } \\
\text { Country } \\
\text { EM Reason }\end{array}$ & Intervention & Comparison & $\begin{array}{l}\text { Setting } \\
\text { and Design } \\
\\
\text { Setting } \\
\text { Design } \\
\text { Cluster N } \\
\text { Participants } \\
\text { Randomized } \\
\text { N }\end{array}$ & $\begin{array}{l}\text { PLWD } \\
\text { Dementia } \\
\text { Characteristics }\end{array}$ & $\begin{array}{l}\text { PLWD } \\
\text { Non-Disease Char } \\
\text { PLWD N } \\
\text { PLWD Age (mean) } \\
\text { PLWD Sex (\% } \\
\text { female) } \\
\text { PLWD Race (\% } \\
\text { majority) } \\
\text { PLWD Education } \\
\text { (mean years) }\end{array}$ & $\begin{array}{l}\text { PLWD } \\
\text { Non-Disease } \\
\text { Char Reporting } \\
\text { Status (RS) } \\
\\
\text { PLWD SES } \\
\text { PLWD Prior } \\
\text { Disability } \\
\text { PLWD } \\
\text { Household } \\
\text { Characteristics } \\
\text { PLWD Health } \\
\text { Insurance } \\
\text { PLWD Detailed } \\
\text { Race Information }\end{array}$ & $\begin{array}{l}\text { Informal Caregiver } \\
\text { (IC) } \\
\text { Characteristics } \\
\text { IC N } \\
\text { IC Age (mean) } \\
\text { IC Sex (\% female) } \\
\text { IC Race (\% } \\
\text { majority) } \\
\text { IC Education } \\
\text { (mean years) } \\
\text { IC Relation to } \\
\text { PLWD (\% majority) }\end{array}$ & $\begin{array}{l}\text { Informal Caregiver } \\
\text { (IC) Char. RS } \\
\text { IC Duration } \\
\text { IC Living With } \\
\text { PLWD } \\
\text { IC Payment } \\
\text { IC Health Status } \\
\text { IC Dementia } \\
\text { Family History } \\
\text { IC Employment } \\
\text { Status } \\
\text { IC Training }\end{array}$ & $\begin{array}{l}\text { Formal Caregiver } \\
\text { (FC) } \\
\text { Characteristics } \\
\text { FC N } \\
\text { FC Age (mean) } \\
\text { FC Sex (\% } \\
\text { female) } \\
\text { FC Race (\% } \\
\text { majority) } \\
\text { FC Education } \\
\text { (mean years) }\end{array}$ & $\begin{array}{l}\text { Formal } \\
\text { Caregiver (FC) } \\
\text { Char. RS } \\
\\
\text { FC Health } \\
\text { Status } \\
\text { FC Training } \\
\text { FC Education } \\
\text { FC Position } \\
\text { FC Length of } \\
\text { Service }\end{array}$ & $\begin{array}{l}\text { Outcome } \\
\text { Timing(s) }\end{array}$ & $\begin{array}{l}\text { PLWD } \\
\text { Outcomes }\end{array}$ & $\begin{array}{l}\text { Caregiver } \\
\text { Outcomes } \\
\text { IC: } \\
\text { FC: }\end{array}$ \\
\hline $\begin{array}{l}\text { Froggat } 2020^{100} \\
\text { UK } \\
\text { Pilot }\end{array}$ & $\begin{array}{l}\text { Namaste: } \\
\text { structured } \\
\text { personalized } \\
\text { care }\end{array}$ & $\begin{array}{l}\text { Usual care } \\
\text { activities }\end{array}$ & Nursing home & $\begin{array}{l}\text { Advanced } \\
\text { dementia }\end{array}$ & $\begin{array}{l}\text { N=32 } \\
\text { Age: Yes } \\
\text { Sex: Yes } \\
\text { Race: no } \\
\text { Education: No }\end{array}$ & None & NA & NA & NA & NA & 24 weeks & $\begin{array}{l}\text { QUALID } \\
\text { Comfort in } \\
\text { dying }\end{array}$ & NA \\
\hline
\end{tabular}




\begin{tabular}{|c|c|c|c|c|c|c|c|c|c|c|c|c|c|}
\hline $\begin{array}{l}\text { Halek 202062 } \\
(32062052) \\
\text { Germany } \\
\text { High ROB }\end{array}$ & $\begin{array}{l}\text { Two dementia- } \\
\text { specific Case } \\
\text { Conference } \\
\text { models }\end{array}$ & Usual care & $\begin{array}{l}\text { Nursing } \\
\text { homes } \\
\text { Cluster not } \\
\text { specified } \\
\mathrm{N}=413\end{array}$ & $\begin{array}{l}\text { Documented } \\
\text { diagnosed } \\
\text { dementia from } \\
\text { nursing charts, } \\
\text { FAS score }>1, \\
\text { living at least 15 } 15 \\
\text { days in the unit } \\
\end{array}$ & $\begin{array}{l}\mathrm{N}=224 \\
\text { Age: yes } \\
\text { Sex: yes }\end{array}$ & Low & $\mathrm{NA}$ & NA & $\mathrm{N}=189$ & Position: yes & $\begin{array}{l}15 \\
\text { days/unclear }\end{array}$ & & $\begin{array}{l}\text { Burnout risk } \\
\text { (CBI) }\end{array}$ \\
\hline $\begin{array}{l}\text { Chen, 2019101 } \\
\text { China } \\
\text { Pilot }\end{array}$ & $\begin{array}{l}\text { Interdisciplinary } \\
\text { care team }\end{array}$ & $\begin{array}{l}\text { Usual care } \\
\text { activities }\end{array}$ & $\begin{array}{l}\text { Community } \\
\text { based, China }\end{array}$ & Not specified & $\begin{array}{l}\text { N=148 } \\
\text { Age: Yes } \\
\text { Sex: Yes } \\
\text { Race: } n \text { no } \\
\text { Education: No }\end{array}$ & None & None & None & NA & NA & 6 month & $\begin{array}{l}\text { CDR } \\
\text { QoL } \\
\text { ADL } \\
\text { NPI }\end{array}$ & ZBI \\
\hline $\begin{array}{l}\text { Saxena, 2018 } \\
29193721 \\
\text { Singapore } \\
\text { Unable to } \\
\text { evaluate }\end{array}$ & $\begin{array}{l}\text { Primary care } \\
\text { Dementia Clinic } \\
\text { (PCDC): Each } \\
\text { visit consists of } \\
\text { a 15-min } \\
\text { consultation } \\
\text { with FP \& 15 } \\
\text { min consultation } \\
\text { with a nurse }\end{array}$ & $\begin{array}{l}\text { (1) Standard } \\
\text { primary care at } \\
\text { other National } \\
\text { healthcare } \\
\text { Group polyclinic } \\
\text { (2) Specialist } \\
\text { care at the MC }\end{array}$ & $\begin{array}{l}\text { Clinic } \\
\text { Quasi } \\
263 \text { PLWD }\end{array}$ & Not specified & $\begin{array}{l}\mathrm{N}=263 \\
\text { Age: Yes } \\
\text { Sex: Yes } \\
\text { Race: Yes } \\
\text { Education: No }\end{array}$ & Marital status & None & None & NA & NA & 12 months & & $\begin{array}{l}\text { Quality of life: } \\
\text { Qol-AD } \\
\text { CG } \\
\text { satisfaction: } \\
\text { Cost } \\
\text { effectiveness: }\end{array}$ \\
\hline $\begin{array}{l}\text { Mavandadi, } \\
2017^{103} \\
28134558 \\
\text { US } \\
\text { Pilot }\end{array}$ & $\begin{array}{l}\text { Dementia care } \\
\text { management: } \\
\text { Telephone-- } \\
\text { based patient- } \\
\text { and caregiver } \\
\text { (CG)-centered, } \\
\text { collaborative } \\
\text { care } \\
\text { management } \\
\text { program that } \\
\text { involves CG } \\
\text { education and } \\
\text { psychosocial } \\
\text { support }\end{array}$ & Usual care & $\begin{array}{l}\text { Community } \\
\text { RCT } \\
75 \text { Caregivers }\end{array}$ & $\begin{array}{l}\text { All types dementia } \\
\text { and stages of } \\
\text { severity }\end{array}$ & $\begin{array}{l}\text { N=75 } \\
\text { Age: Yes } \\
\text { Sex: no } \\
\text { Race: no } \\
\text { Education: no }\end{array}$ & None & $\begin{array}{l}\text { N=75 } \\
\text { Age: Yes } \\
\text { Sex: yes } \\
\text { Race: yes } \\
\text { Education: no } \\
\text { Relationship: yes }\end{array}$ & $\begin{array}{l}\text { Informal caregivers } \\
\text { of veterans }\end{array}$ & NA & NA & 6 months & $\begin{array}{l}\text { Activities of } \\
\text { daily living: } \\
\text { ADL; IADL }\end{array}$ & $\begin{array}{l}\text { Bother: } \\
\text { RMBPC } \\
\text { Distress: NPI- } \\
\text { Q, } \\
\text { Burden: ZBI } \\
\text { Cognitive } \\
\text { coping: } \\
\text { Perceived } \\
\text { mastery over } \\
\text { caregiving: }\end{array}$ \\
\hline $\begin{array}{l}\text { Laporte Uribe } \\
2017^{64} \\
(28249632) \\
\text { Germany } \\
\text { High RoB }\end{array}$ & $\begin{array}{l}\text { PLWD and } \\
\text { informal CG } \\
\text { using DCN }\end{array}$ & None & $\begin{array}{l}\text { Community- } \\
\text { dwelling } \\
\text { Quazi- } \\
\text { experimental } \\
560 \text { PLWD } \\
\text { and IC }\end{array}$ & $\begin{array}{l}\text { Dementia } \\
\text { diagnosis by } \\
\text { medical } \\
\text { professionals and } \\
\text { living with IC using } \\
\text { DCN }\end{array}$ & $\begin{array}{l}\text { N=389 } \\
\text { Age: Yes } \\
\text { Sex: Yes } \\
\text { Race: no } \\
\text { Education: no } \\
\text { Living with CG: Yes }\end{array}$ & None & $\begin{array}{l}\mathrm{N}=385 \\
\text { Age: Yes } \\
\text { Sex: Yes } \\
\text { Race: no } \\
\text { Education: no } \\
\text { Relationship Yes }\end{array}$ & & & & 12 months & $\begin{array}{l}\text { CMAI } \\
\text { IADL } \\
\text { EQ-5D L }\end{array}$ & BIZA-D \\
\hline $\begin{array}{l}\text { Samus, 2014 } \\
\text { US } \\
\text { Pilot }\end{array}$ & $\begin{array}{l}\text { Home-based } \\
\text { care } \\
\text { coordination: to } \\
\text { systematically } \\
\text { identiff \& } \\
\text { address } \\
\text { dementia } \\
\text { related care } \\
\text { needs- } \\
\text { Received the } \\
\text { written results of } \\
\text { the JHDCNA \& } \\
18 \text { months of } \\
\text { care } \\
\text { coordination }\end{array}$ & $\begin{array}{l}\text { Augmented } \\
\text { usual care: } \\
\text { Received the } \\
\text { written results of } \\
\text { the JHDCNA } \\
\text { following the BL } \\
\text { vist including } \\
\text { recommendatio } \\
\text { ns for each } \\
\text { identified unmet } \\
\text { need. }\end{array}$ & $\begin{array}{l}\text { Community } \\
\text { RCT } 303 \text { PLWD }\end{array}$ & $\begin{array}{l}\text { Type of dementia: } \\
\text { not specified } \\
\text { Severity: Mild - } \\
\text { severe }\end{array}$ & $\begin{array}{l}\text { N=303 } \\
\text { Age: Yes } \\
\text { Sex: Yes } \\
\text { Race: yes } \\
\text { Education: Yes } \\
\text { Living with CG: Yes }\end{array}$ & $\mathrm{NA}$ & NA & $\mathrm{NA}$ & $\mathrm{NA}$ & NA & 18 months & $\begin{array}{l}\text { Time to } \\
\text { transfer from } \\
\text { home: Study } \\
\text { partner } \\
\text { report } \\
\text { Unmet care } \\
\text { needs:JHDC } \\
\text { NA }\end{array}$ & None \\
\hline $\begin{array}{l}\text { Leontjevas, } \\
2013^{105} \\
23643110 \\
\text { Netherland } \\
\text { High ROB }\end{array}$ & $\begin{array}{l}\text { Act in Case of } \\
\text { Depression } \\
\text { (AiD) at } \\
\text { dementia } \\
\text { unit: A } \\
\text { multidisciplinary } \\
\text { care program } \\
\text { that involved 2- } \\
\text { step screening } \\
\text { \& diagnostic } \\
\text { procedure; } \\
\text { multidiscipline } \\
\text { treatment; \& } \\
\text { monitoring of } \\
\text { treatment } \\
\text { effects. }\end{array}$ & $\begin{array}{l}\text { AiD at somatic } \\
\text { unit: }\end{array}$ & $\begin{array}{l}\text { Nursing home } \\
\text { RCT (cluster) } \\
793 \text { PLWD }\end{array}$ & & $\begin{array}{l}\mathrm{N}=793 \\
\text { Age: Yes } \\
\text { Sex: Yes } \\
\text { Race: no } \\
\text { Education: no }\end{array}$ & NA & NA & NA & NA & $\mathrm{NA}$ & $?$ & $\begin{array}{l}\text { Depression: } \\
\text { CSDD }\end{array}$ & None \\
\hline
\end{tabular}




\begin{tabular}{|c|c|c|c|c|c|c|c|c|c|c|c|c|c|}
\hline $\begin{array}{l}\text { Chapman, } \\
2007^{69} \\
18232242 \\
\text { US } \\
\text { High ROB }\end{array}$ & \begin{tabular}{|l|} 
Advanced \\
illness care \\
teams (AlCTs): \\
holistic \\
approach that \\
focused on four \\
domains: (1) \\
medical, (2) \\
meaningul \\
activities, (3) \\
psychological, \\
and (4) \\
behavioral
\end{tabular} & $\begin{array}{l}\text { Usual care; } \\
\text { received all the } \\
\text { services } \\
\text { typically } \\
\text { provided by the } \\
\text { facility }\end{array}$ & \begin{tabular}{|l|} 
Nursing \\
homes \\
RCT \\
$118 \mathrm{NH}$ \\
residents
\end{tabular} & Advance dementia & \begin{tabular}{|l|} 
N=118 \\
Age: Yes \\
Sex: Yes \\
Race Yes \\
Education: no \\
Marital status: Yes
\end{tabular} & SES: Yes & NA & NA & NA & NA & 8 weeks & \begin{tabular}{|l} 
Agitated \\
behavior:CM \\
Al \\
Pain:FLACC \\
Depression: \\
CSDD
\end{tabular} & None \\
\hline $\begin{array}{l}\text { Bass, 2003 } 12606 \\
12604748 \\
14690867^{107} \\
\text { US } \\
\text { Pilot/ } \\
\text { demonstration }\end{array}$ & $\begin{array}{l}\text { Care } \\
\text { coordination } \\
\text { integrated within } \\
\text { health system; } \\
\text { average } 12 \\
\text { contacts per } \\
\text { year }\end{array}$ & \begin{tabular}{|l} 
Usual care \\
activities
\end{tabular} & $\begin{array}{l}\text { Community- } \\
\text { based } \\
\text { RCT } \\
157 \text { Dyads }\end{array}$ & $\begin{array}{l}\text { Dementia } \\
\text { diagnosis in health } \\
\text { records }\end{array}$ & $\begin{array}{l}\text { N=157 } \\
\text { Age: no } \\
\text { Sex: no } \\
\text { Race: no } \\
\text { Education: no } \\
\text { Marital status: no }\end{array}$ & Lives alone & NA & NA & NA & NA & 12 month & Utilization & $\begin{array}{l}\text { IC: } \\
\text { CES-D } \\
\text { Caregiver } \\
\text { strain }\end{array}$ \\
\hline $\begin{array}{l}\text { Person- } \\
\text { Centered Care }\end{array}$ & $\begin{array}{l}\text { And Dementia } \\
\text { Care Mapping }\end{array}$ & & & & & & & & & & & & \\
\hline $\begin{array}{l}\text { Surr, 201972 } \\
31056923 \\
\text { UK } \\
\text { High ROB }\end{array}$ & $\begin{array}{l}\text { Dementia care } \\
\text { mapping to } \\
\text { reduce agitation } \\
\text { in nursing } \\
\text { homes }\end{array}$ & $\begin{array}{l}\text { Usual care } \\
\text { activities }\end{array}$ & $\begin{array}{l}\text { Dementia } \\
\text { care homes } \\
\text { Cluster RCT } \\
50 \text { Care } \\
\text { homes }\end{array}$ & $\begin{array}{l}\text { Care home } \\
\text { residents }\end{array}$ & \begin{tabular}{|l|} 
N=726 \\
Age: yes \\
Sex: Yes \\
Race: $n$ o \\
Education: no \\
Marital status: no \\
\end{tabular} & None & NA & NA & $\begin{array}{l}\text { Information at care } \\
\text { home level }\end{array}$ & NA & 16 months & \begin{tabular}{|l} 
CMAI \\
Resident \\
behaviors \\
QoL
\end{tabular} & $\begin{array}{l}\text { System-level } \\
\text { outcomes: } \\
\text { Quality of } \\
\text { interactions } \\
\text { schedule }\end{array}$ \\
\hline $\begin{array}{l}\text { Li, } 2017^{108} \\
\text { US } \\
\text { Pilot }\end{array}$ & $\begin{array}{l}\text { Person- } \\
\text { centered } \\
\text { dementia care: } \\
\text { included in- } \\
\text { class staff } \\
\text { training plus } \\
\text { supervision and } \\
\text { support in } \\
\text { practice of } \\
\text { individualized } \\
\text { care } \\
\end{array}$ & \begin{tabular}{|l} 
Control : no \\
intervention
\end{tabular} & $\begin{array}{l}\text { Assisted } \\
\text { Living facility } \\
\text { RCT } \\
28 \text { PLWDs }\end{array}$ & $\begin{array}{l}\text { All types of } \\
\text { dementia except } \\
\text { those with } \\
\text { Parkinson \& in } \\
\text { severe stage }\end{array}$ & \begin{tabular}{|l}
$\mathrm{N}=22$ \\
Age: $y$ es \\
Sex: Yes \\
Race: Yes \\
Education: no \\
Marital status: Yes
\end{tabular} & None & NA & NA & NA & NA & $\begin{array}{l}3 \text { months } \\
1 \text { month post } \\
\text { intervention } \\
\text { follow up }\end{array}$ & \begin{tabular}{|l} 
Sleep-wake \\
pattern: \\
Actigraphy \\
social \\
engagement: \\
DCM
\end{tabular} & None \\
\hline $\begin{array}{l}\text { Ballard, 2016 } \\
26585409 \\
\\
\text { UK } \\
\text { Pilot }\end{array}$ & $\begin{array}{l}\text { Person } \\
\text { centered care } \\
\text { combined with } \\
\text { medication } \\
\text { review, social } \\
\text { interaction, and } \\
\text { exercise }\end{array}$ & & $\begin{array}{l}\text { Nursing } \\
\text { homes } \\
\text { Cluster } \\
\text { randomized } \\
\text { factorial } \\
\text { analysis } \\
16 \text { homes } \\
\end{array}$ & $\begin{array}{l}\text { Dementia } \\
\text { residents }\end{array}$ & \begin{tabular}{|l} 
N=187 \\
Age: yes \\
Sex: Yes \\
Race Yes \\
Education: no \\
Marital status: no
\end{tabular} & None & NA & NA & NA & NA & 9 months & $\begin{array}{l}\text { DEMQOL- } \\
\text { proxy } \\
\text { antipsychotic } \\
\text { use }\end{array}$ & None \\
\hline $\begin{array}{l}\text { Moyle, 2016 } \\
\text { Australia } \\
\text { Pilot }\end{array}$ & \begin{tabular}{|l} 
Capabilities \\
model based on \\
a person- \\
centered \\
approach
\end{tabular} & $\begin{array}{l}\text { Usual care } \\
\text { activities }\end{array}$ & $\begin{array}{l}\text { Nursing home } \\
\text { Quasi- } \\
\text { experimental }\end{array}$ & \begin{tabular}{|l} 
Confirmed \\
dementia \\
diagnosis
\end{tabular} & \begin{tabular}{|l|}
$\mathrm{N}=48$ \\
Age: no \\
Sex: no \\
Race: no \\
Education: no \\
Marital status: no \\
\end{tabular} & None & \begin{tabular}{|l|}
$\mathrm{N}=75$ \\
Age: Yes \\
Sex: yes \\
Race: no \\
Education: yes \\
Relationship: yes \\
\end{tabular} & Employment & $\begin{array}{l}\text { N=81 } \\
\text { Age: Yes } \\
\text { Sex: Yes } \\
\text { Race: } n \text { o } \\
\text { Education: Yes }\end{array}$ & Position & 6,12 months & QoL-AD & $\begin{array}{l}\text { Staff } \\
\text { experience o } \\
\text { working with } \\
\text { demented } \\
\text { residents } \\
\text { questionnaire }\end{array}$ \\
\hline $\begin{array}{l}\text { Dichter, } 2015^{75} \\
26138674 \\
\\
\text { Germany } \\
\text { High ROB }\end{array}$ & $\begin{array}{l}\text { Dementia Care } \\
\text { Mapping: } \\
\text { (A) DCM } \\
\text { applied since } \\
\text { 2009: received } \\
\text { two DCM cycles } \\
\text { per year until } \\
\text { 2009. } \\
\text { (B) DCM newly } \\
\text { introduced } \\
\text { during the } \\
\text { intervention } \\
\text { (C) received an } \\
\text { intervention } \\
\text { based on a } \\
\text { regular and } \\
\text { standardized } \\
\text { QoL rating that } \\
\text { was integrated } \\
\text { into the usual } \\
\text { care. }\end{array}$ & & $\begin{array}{l}\text { Nursing home } \\
\text { Quasi } \\
\text { 315 PLWD }\end{array}$ & $\begin{array}{l}\text { Types of } \\
\text { dementia: Not } \\
\text { specified }\end{array}$ & \begin{tabular}{|l|}
$\mathrm{N}=154$ \\
Age: yes \\
Sex: Yes \\
Race: No \\
Education: no
\end{tabular} & None & NA & NA & NA & NA & 18 months & \begin{tabular}{|l|} 
Quality of \\
life: QoL-AD \\
Challenging \\
behavior: \\
NPI-NH \\
Functional \\
ability: \\
PSMS
\end{tabular} & None \\
\hline
\end{tabular}




\begin{tabular}{|c|c|c|c|c|c|c|c|c|c|c|c|c|c|}
\hline $\begin{array}{l}\text { Rokstad, 201378 } \\
24022375 \\
\text { Norway } \\
\text { High ROB }\end{array}$ & $\begin{array}{l}\text { (1) Dementia } \\
\text { Care Mapping } \\
\text { (DCM): a } \\
\text { process to } \\
\text { develop the } \\
\text { care staff's skills } \\
\text { in delivering } \\
\text { PCC to the } \\
\text { patients } \\
\text { (2) VPM: a } \\
\text { weekly } \\
\text { consensus } \\
\text { meeting in the } \\
\text { nursing home } \\
\text { ward of } 45- \\
60 \text { min using } \\
\text { the indicators in } \\
\text { the VIPS } \\
\text { framework to } \\
\text { analyze a } \\
\text { challenging } \\
\text { patient-nurse } \\
\text { interaction. }\end{array}$ & $\begin{array}{l}\text { Control: } \\
\text { Education on } \\
\text { dementia: } \\
\text { received five } \\
\text { DVDs with } \\
\text { lectures (30 min } \\
\text { each) about } \\
\text { dementia. }\end{array}$ & $\begin{array}{l}\text { Nursing home } \\
\text { RCT } \\
\text { 624 PLWD }\end{array}$ & $\begin{array}{l}\text { All types and } \\
\text { stages of } \\
\text { dementia }\end{array}$ & $\begin{array}{l}\text { N=624 } \\
\text { Age: yes } \\
\text { Sex: Yes } \\
\text { Race: No } \\
\text { Education: no }\end{array}$ & $\begin{array}{l}\text { General physical } \\
\text { health: yes }\end{array}$ & $\mathrm{NA}$ & NA & NA & $\mathrm{NA}$ & 10 months & $\begin{array}{l}\text { Agitation: } \\
\text { BARS } \\
\text { NPIQ: } \\
\text { Quality of } \\
\text { life: QUALID }\end{array}$ & NA \\
\hline $\begin{array}{l}\text { Fossey, } 2006^{81} \\
16543297 \\
\text { UK } \\
\text { High ROB }\end{array}$ & $\begin{array}{l}\text { A training \& } \\
\text { support } \\
\text { intervention: } \\
\text { intervention } \\
\text { delivered to } \\
\text { nursing home } \\
\text { staff over } 10 \\
\text { months, } \\
\text { focusing on } \\
\text { alternatives to } \\
\text { drugs for the } \\
\text { management of } \\
\text { agitated } \\
\text { behavior in } \\
\text { dementia }\end{array}$ & $\begin{array}{l}\text { Treatment as } \\
\text { usual }\end{array}$ & \begin{tabular}{|l|} 
Nursing \\
homes \\
RCT (cluster) \\
349 PLWD
\end{tabular} & $\begin{array}{l}\text { Type of dementia } \\
\text { unspecified } \\
\text { Mild to severe }\end{array}$ & $\begin{array}{l}\mathrm{N}=349 \\
\text { Age: yes } \\
\text { Sex: Yes } \\
\text { Race: No } \\
\text { Education: no }\end{array}$ & None & NA & NA & NA & NA & 12 months & $\begin{array}{l}\text { Agitated } \\
\text { behavior: }\end{array}$ & NA \\
\hline $\begin{array}{l}\text { Chenoweth, } \\
2014^{76} \\
24666667 \\
\\
\text { Australia } \\
\text { High ROB }\end{array}$ & $\begin{array}{l}\text { (1) A person- } \\
\text { centered care } \\
\text { (PCC) } \\
\text { (2) Person } \\
\text { centered } \\
\text { environment } \\
\text { (PCE) } \\
\text { (3) PCE +PCC }\end{array}$ & $\begin{array}{l}\text { Usual care + } \\
\text { usual } \\
\text { environment }\end{array}$ & $\begin{array}{l}\text { Aged care } \\
\text { homes } \\
\text { RCT (Cluster) } \\
601 \text { PLWD }\end{array}$ & $\begin{array}{l}\text { Only AD \& } \\
\text { severe/very } \\
\text { severe were } \\
\text { specified in \% }\end{array}$ & $\begin{array}{l}\mathrm{N}=601 \\
\text { Age: yes } \\
\text { Sex: Yes } \\
\text { Race: No } \\
\text { Education: no }\end{array}$ & None & NA & NA & $\mathrm{NA}$ & NA & $\begin{array}{l}4 \text { months } \\
8 \text { months } \\
\text { follow up (4 } \\
\text { months post } \\
\text { intervention) }\end{array}$ & $\begin{array}{l}\text { Quality of } \\
\text { life: } \\
\text { DEMQOL } \\
\text { Agitation: } \\
\text { CMAI } \\
\text { Cost of } \\
\text { program: }\end{array}$ & NA \\
\hline
\end{tabular}

Abbreviations: ADL=Activities of Daily Living; AICT=Advanced illness care teams; AiD=Act in Case of Depression; BARS=Brief Agitation Rating Scale; BIZA-D= Berlin Inventory of Caregivers' Burden with of Dementia Patients; CDR=Clinical Dementia Rating; CESD= Center for

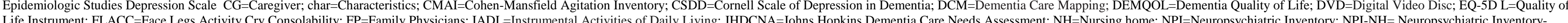

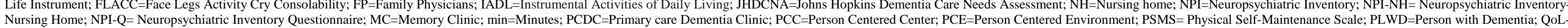
$\mathrm{AD}=$ Quality of Life-Alzheimer's Disease; QoL=Quality of Life; QUALID=Quality of Life in Late-Stage Dementia; RCT=Randomized Controlled Trial; RMBPC=Revised memory and behavior problem checklist; RoB=Risk of Bias; SES=Socioeconomic Status; VPM=V
$\mathrm{ZBI}=$ Zarit Burden Index

\section{Formal Caregiver Training}

\begin{tabular}{|c|c|c|c|c|c|c|c|c|c|}
\hline Study (PMID) & Outcome Timing & $\begin{array}{l}\text { Attrition Bias } \\
\text { Attrition \% }\end{array}$ & Selection Bias & Detection Bias & Performance Bias & Reporting Bias & Fidelity Bias & Funder & Overall Rating \\
\hline $\begin{array}{l}\text { Gozalo, 2014111 } \\
(24697702)\end{array}$ & 2 years & Medium & Low & Medium & High & Low & High & $\begin{array}{l}\text { Gov't } \\
\text { Developer }\end{array}$ & High \\
\hline Schindel Martin, 2016 ${ }^{11}(27659392)$ & 6 month & $\mathrm{x}$ & High & $\mathrm{x}$ & $\mathrm{x}$ & $\mathrm{x}$ & $\mathrm{x}$ & $\begin{array}{l}\text { Gov't } \\
\text { Developer }\end{array}$ & High \\
\hline $\begin{array}{l}\text { Galik, 2013 } \\
\text { (24092822) }\end{array}$ & 6 months & $\mathrm{x}$ & Medium & $\mathrm{x}$ & $\mathrm{x}$ & $\mathrm{x}$ & $\mathrm{x}$ & $\mathrm{X}$ & High \\
\hline
\end{tabular}

Abbreviations: PMID=PubMed Identification Number 


\section{Evidence Map: Formal Caregiver Training}

\begin{tabular}{|c|c|c|c|c|c|c|c|c|c|c|c|c|c|}
\hline $\begin{array}{l}\text { Study (PMID) } \\
\text { Country } \\
\text { EM Reason }\end{array}$ & Intervention & Comparison & $\begin{array}{l}\text { Setting } \\
\text { and Design } \\
\\
\text { Setting } \\
\text { Design } \\
\text { Cluster N } \\
\text { Participants } \\
\text { Random- } \\
\text { ized N }\end{array}$ & $\begin{array}{l}\text { PLWD } \\
\text { Dementia } \\
\text { Characteristics }\end{array}$ & \begin{tabular}{|l} 
PLWD \\
Non-Disease Char \\
PLWD N \\
PLWD Age (mean) \\
PLWD Sex (\% \\
female) \\
PLWD Race (\% \\
majority) \\
PLWD Education \\
(mean years)
\end{tabular} & \begin{tabular}{|l|} 
PLWD \\
Non-Disease \\
Char Reporting \\
Status (RS) \\
\\
PLWD SES \\
PLWD Prior \\
Disability \\
PLWD \\
Household \\
Characteristics \\
PLWD Health \\
Insurance \\
PLWD Detailed \\
Race Information \\
\end{tabular} & $\begin{array}{l}\text { Informal Caregiver } \\
\text { (IC) } \\
\text { Characteristics } \\
\text { IC N } \\
\text { IC Age (mean) } \\
\text { IC Sex (\% female) } \\
\text { IC Race (\% } \\
\text { majority) } \\
\text { IC Education } \\
\text { (mean years) } \\
\text { IC Relation to } \\
\text { PLWD (\% majority) }\end{array}$ & \begin{tabular}{|l} 
Informal Caregiver \\
(IC) Char. RS \\
IC Duration \\
IC Living With \\
PLWD \\
IC Payment \\
IC Health Status \\
IC Dementia \\
Family History \\
IC Employment \\
Status \\
IC Training
\end{tabular} & $\begin{array}{l}\text { Formal Caregiver } \\
\text { (FC) } \\
\text { Characteristics } \\
\text { FC N } \\
\text { FC Age (mean) } \\
\text { FC Sex (\% } \\
\text { female) } \\
\text { FC Race (\% } \\
\text { majority) } \\
\text { FC Education } \\
\text { (mean years) }\end{array}$ & $\begin{array}{l}\text { Formal } \\
\text { Caregiver (FC) } \\
\text { Char. RS } \\
\\
\text { FC Health } \\
\text { Status } \\
\text { FC Training } \\
\text { FC Education } \\
\text { FC Position } \\
\text { FC Length of } \\
\text { Service }\end{array}$ & \begin{tabular}{|l} 
Outcome \\
Timing(s)
\end{tabular} & \begin{tabular}{|l|} 
PLWD \\
Outcomes
\end{tabular} & $\begin{array}{l}\text { Caregiver } \\
\text { Outcomes } \\
\text { IC: } \\
\text { FC: }\end{array}$ \\
\hline $\begin{array}{l}\text { Williams, } 2017 \\
114 \\
(27048705) \\
\text { US } \\
\text { Small sample }\end{array}$ & $\begin{array}{l}\text { CHAT: } \\
\text { communication } \\
\text { training } \\
\text { designed to } \\
\text { alert nursing } \\
\text { staff to elder } \\
\text { speak } \\
\text { communication } \\
\text { and its negative } \\
\text { effects }\end{array}$ & \begin{tabular}{|l} 
Usual care \\
activities
\end{tabular} & $\begin{array}{l}\text { Nursing } \\
\text { Home } \\
\text { Cluster RCT } \\
13 \text { Nursing } \\
\text { homes }\end{array}$ & $\begin{array}{l}\text { Patients with } \\
\text { diagnosis of } A D\end{array}$ & $\begin{array}{l}\text { PLWD N=83 } \\
\text { Age: yes } \\
\text { Sex: yes } \\
\text { Race yes } \\
\text { Education: no }\end{array}$ & None & NA & NA & $\begin{array}{l}\mathrm{N}=130 \\
\text { Age: yes } \\
\text { Sex:: yes } \\
\text { Race: yes } \\
\text { Education: yes }\end{array}$ & \begin{tabular}{|l} 
Training \\
Position
\end{tabular} & 3 months & \begin{tabular}{|l}
$\begin{array}{l}\text { Resistance to } \\
\text { care }\end{array}$ \\
\end{tabular} & \begin{tabular}{|l|} 
FC: \\
Communicati \\
on
\end{tabular} \\
\hline $\begin{array}{l}\text { Conway, } \\
2016^{115} \\
(26821868) \\
\\
\text { Australia } \\
\text { Small sample }\end{array}$ & $\begin{array}{l}\text { MESSAGE } \\
\text { communication } \\
\text { intervention to } \\
\text { facilitate patient } \\
\text { support and } \\
\text { staff and patient } \\
\text { experience }\end{array}$ & $\begin{array}{l}\text { Usual care } \\
\text { activities }\end{array}$ & $\begin{array}{l}\text { Nursing } \\
\text { home } \\
\text { Cluster RCT } \\
12 \text { Nursing } \\
\text { homes }\end{array}$ & $\begin{array}{l}\text { Patients with } \\
\text { diagnosis of } A D \\
\text { with ability to } \\
\text { respond to direct } \\
\text { assessment }\end{array}$ & NR & NR & NA & NA & $\begin{array}{l}\mathrm{N}=38 \\
\text { Age: } y e s \\
\text { Sex: yes } \\
\text { Race: } n \text { o } \\
\text { Education: yes }\end{array}$ & $\begin{array}{l}\text { Training } \\
\text { Position }\end{array}$ & 3 months & \begin{tabular}{|l} 
Self-efficacy \\
M-NCAS \\
ADQ
\end{tabular} & \begin{tabular}{|l|} 
FC: \\
Communicati \\
on
\end{tabular} \\
\hline $\begin{array}{l}\text { Schindel Martin, } \\
2016^{112} \\
(27659392) \\
\\
\text { Canada } \\
\text { High ROB }\end{array}$ & \begin{tabular}{|l|} 
Standardized \\
dementia \\
education \\
training on \\
gentle \\
persuasion \\
approaches \\
\end{tabular} & $\begin{array}{l}\text { Usual education } \\
\text { supports }\end{array}$ & \begin{tabular}{|l|} 
Hospitals \\
Quasi- \\
experimental \\
12 clinical \\
areas at 2 \\
hospitals
\end{tabular} & AD diagnosis & NR & NR & NA & NA & \begin{tabular}{|l|}
$\mathrm{N}=745$ \\
Age: yes \\
Sex: yes \\
Race: $\mathrm{no}$ \\
Education: yes
\end{tabular} & \begin{tabular}{|l} 
Training \\
Length of service
\end{tabular} & 8 weeks & None & \begin{tabular}{|l|} 
FC: Self- \\
Perceived \\
Behavioral \\
Management \\
Self-Efficacy \\
Profile
\end{tabular} \\
\hline $\begin{array}{l}\text { Hattink, 2015116 } \\
\text { (26519106) } \\
\text { Netherlands \& } \\
\text { UK } \\
\text { Pilot }\end{array}$ & $\begin{array}{l}\text { Access to a } \\
\text { Web-based } \\
\text { portal consisting } \\
\text { of } 8 \text { modules \& } \\
\text { to online peer } \\
\text { and expert } \\
\text { communities for } \\
\text { support and } \\
\text { information } \\
\text { exchange } \\
\end{array}$ & Wait-list & $\begin{array}{l}\text { Community } \\
\text { setting } \\
\text { RCT } \\
\text { Caregivers }\end{array}$ & AD diagnosis & NR & NR & \begin{tabular}{|l|} 
N59 \\
Age: yes \\
Sex: yes \\
Race: no \\
Education: no \\
IC Relation to \\
PLWD: yes
\end{tabular} & \begin{tabular}{|l} 
IC Duration \\
IC Employment \\
status
\end{tabular} & $\begin{array}{l}\mathrm{N}=24 \\
\text { Age: } y e s \\
\text { Sex: yes } \\
\text { Race: no } \\
\text { Education: no }\end{array}$ & Length of service & 4 months & \begin{tabular}{|l|} 
ADKS \\
ADQ \\
IRI \\
QoL \\
Burden: \\
assessed \\
with 1 \\
question \\
SSCQ
\end{tabular} & $\begin{array}{l}\text { Usefulness of } \\
\text { training }\end{array}$ \\
\hline $\begin{array}{l}\text { Gozalo, 2014 } \\
(24697702) \\
\text { US } \\
\text { High ROB }\end{array}$ & $\begin{array}{l}\text { Education } \\
\text { program on } \\
\text { bathing patients } \\
\text { with dementia to } \\
\text { improve bating } \\
\text { experience of } \\
\text { patients }\end{array}$ & $\begin{array}{l}\text { Usual care } \\
\text { activities until } \\
\text { crossover }\end{array}$ & \begin{tabular}{|l|} 
Nursing \\
homes \\
Crossover \\
cluster RCT \\
6 Nursing \\
homes
\end{tabular} & $\begin{array}{l}\text { Dementia } \\
\text { unspecified }\end{array}$ & \begin{tabular}{|l|}
$\mathrm{N}=240$ \\
Age: yes \\
Sex: yes \\
Race: yes \\
Education: no
\end{tabular} & \begin{tabular}{|l|} 
Health insurance \\
Race Information
\end{tabular} & NA & NA & NR & NR & 2 years & \begin{tabular}{|l} 
Bath duration \\
Aggressive \\
activity
\end{tabular} & \begin{tabular}{|l} 
Usefulness of \\
BWAB in \\
reducing \\
aggressive \\
patient \\
behavior \\
during \\
bathing \\
\end{tabular} \\
\hline $\begin{array}{l}\text { O'Shea 2014117 } \\
\text { (24633858) } \\
\text { Ireland } \\
\text { Pilot }\end{array}$ & \begin{tabular}{|l} 
Dementia \\
Education \\
Program \\
Incorporating \\
Reminiscence \\
for staff \\
facilitated by \\
experienced \\
nurse \\
educators, \\
delivered over 3 \\
days ( 2 at the \\
beginning and 1 \\
six weeks later) \\
and augmented \\
by telephone \\
support and one \\
site visit \\
\end{tabular} & \begin{tabular}{|l} 
usual care \\
provided by \\
care staff who \\
did not receive \\
the structured \\
education \\
program
\end{tabular} & \begin{tabular}{|l|} 
Long term \\
care \\
Cluster RCT \\
18 \\
Residential \\
units \\
304 PLWD
\end{tabular} & $\begin{array}{l}\text { Dementia } \\
\text { diagnosis }\end{array}$ & \begin{tabular}{|l|}
$\mathrm{N}=304$ \\
Age: yes \\
Sex: yes \\
Race: no \\
Education: no
\end{tabular} & None & NR & $\mathrm{NR}$ & NR & $\mathrm{NR}$ & 18-22 weeks & \begin{tabular}{|l|} 
QoL-AD \\
CSDDD
\end{tabular} & $\begin{array}{l}\text { QoL-AD } \\
\text { ZBI }\end{array}$ \\
\hline
\end{tabular}




\begin{tabular}{|c|c|c|c|c|c|c|c|c|c|c|c|c|c|}
\hline $\begin{array}{l}\text { Galik, 2013113 } \\
(24092822) \\
\text { US } \\
\text { High ROB }\end{array}$ & $\begin{array}{l}\text { Function- } \\
\text { focused care } \\
\text { training }\end{array}$ & $\begin{array}{l}\text { Usual care } \\
\text { activities }\end{array}$ & $\begin{array}{l}\text { Nursing } \\
\text { home } \\
\text { Cluster RCT } \\
4 \text { Nursing } \\
\text { homes }\end{array}$ & \begin{tabular}{|l} 
Patients with \\
MMSE < 16
\end{tabular} & $\begin{array}{l}\mathrm{N}=103 \\
\text { Age: yes } \\
\text { Sex: yes } \\
\text { Race: yes } \\
\text { Education: } \mathrm{N}\end{array}$ & \begin{tabular}{|l|} 
Comorbidity \\
Marriage status
\end{tabular} & NA & NA & $\begin{array}{l}\mathrm{N}=77 \\
\text { Age: yes } \\
\text { Sex: yes } \\
\text { Ethnicity: yes } \\
\text { Education: yes }\end{array}$ & NR & 6 months & $\begin{array}{l}\text { Barthel ADL } \\
\text { CMAI } \\
\text { CSDD } \\
\text { Apathy } \\
\text { Inventory } \\
\text { Falls } \\
\text { ED transfers } \\
\text { Death }\end{array}$ & $\begin{array}{l}\text { Restorative } \\
\text { Care } \\
\text { Behavior } \\
\text { Checklist } \\
\text { Knowledge } \\
\text { Restorative } \\
\text { Care } \\
\text { Activities: } \\
\text { self-efficacy } \\
\text { and outcome } \\
\text { expectations } \\
\end{array}$ \\
\hline $\begin{array}{l}\text { Clare, 2013'118 } \\
\text { (22840185) } \\
\text { UK } \\
\text { Pilot }\end{array}$ & $\begin{array}{l}\text { Training in } \\
\text { observation of } \\
\text { awareness in } \\
\text { patients with } \\
\text { severe } A D\end{array}$ & No training & $\begin{array}{l}\text { Nursing } \\
\text { homes } \\
\text { Cluster RCT } \\
8 \text { Nursing } \\
\text { homes }\end{array}$ & $\begin{array}{l}\text { Participants with } \\
\text { AD moderate to } \\
\text { severe }\end{array}$ & $\begin{array}{l}\mathrm{N}=32 \text { residents } \\
\text { Age: yes } \\
\text { Sex: yes } \\
\text { Race: yes } \\
\text { Education: no }\end{array}$ & & NA & $\mathrm{N}$ & $\begin{array}{l}\mathrm{N}=63 \\
\text { Age: no } \\
\text { Sex: yes } \\
\text { Ethnicity: yes } \\
\text { Qualifications: yes }\end{array}$ & Training & 8 weeks & $\begin{array}{l}\text { Measures of } \\
\text { resident, } \\
\text { family and } \\
\text { staff quality } \\
\text { of life } \\
\text { Resident and } \\
\text { staff care } \\
\text { measures at } \\
\text { baseline and } \\
\text { follow-up }\end{array}$ & $\begin{array}{l}\text { Resident } \\
\text { quality of life } \\
\text { Secondary } \\
\text { outcome: } \\
\text { resident well- } \\
\text { being, } \\
\text { behavior and } \\
\text { cognition; } \\
\text { staff attitudes } \\
\text { and well- } \\
\text { being }\end{array}$ \\
\hline $\begin{array}{l}\text { Ghandehari, } \\
2013^{119} \\
(23457681) \\
\text { Canada } \\
\text { Pilot }\end{array}$ & $\begin{array}{l}\text { Education } \\
\text { program in pain } \\
\text { management }\end{array}$ & $\begin{array}{l}\text { Randomized } \\
\text { control trial }\end{array}$ & $\begin{array}{l}\mathrm{N}=131 \text { staff } \\
\text { Focus group } \\
\text { of } 28 \\
\text { participants } \\
\text { (16 nurses, } \\
12 \text { aides) } \\
29 \text { control } \\
\text { subjects (13 } \\
\text { nurses, } 16 \\
\text { aides } \\
\text { From 2 } \\
\text { health care } \\
\text { regions }\end{array}$ & Not reported & Not Reported & Not reported & NA & NA & Not reported & Training: yes & 2 weeks & $\begin{array}{l}\text { Evaluation of } \\
\text { training } \\
\text { session and } \\
\text { workshop } \\
\text { knowledge }\end{array}$ & $\begin{array}{l}\text { Assessment } \\
\text { of an expert- } \\
\text { based } \\
\text { education } \\
\text { program on } \\
\text { pain } \\
\text { management }\end{array}$ \\
\hline $\begin{array}{l}\text { Wenborn, } \\
2013^{120} \\
(23637069) \\
\text { UK } \\
\text { Pilot }\end{array}$ & $\begin{array}{l}\text { Assessment of } \\
\text { an occupational } \\
\text { therapy } \\
\text { program to } \\
\text { increase } \\
\text { provision of } \\
\text { activity in } \\
\text { residential care } \\
\text { home patients }\end{array}$ & $\begin{array}{l}\text { Randomized } \\
\text { control trial with } \\
\text { blinded } \\
\text { assessment }\end{array}$ & $\begin{array}{l}\text { Intervention } \\
\text { group of } 8 \\
\text { homes, } 104 \\
\text { residents } \\
\text { Control } \\
\text { group of } 8 \\
\text { homes, } 106 \\
\text { residents }\end{array}$ & \begin{tabular}{|l|} 
Patients with AD \\
with DSM_IV score \\
less than 25 \\
Age 60 or older, \\
residing at \\
residential home \\
for at least2 \\
months with no \\
other serious \\
physical or mental \\
health problems
\end{tabular} & $\begin{array}{l}\mathrm{N}=104 \text { intervention } \\
\text { group } \\
106 \text { control group } \\
\text { Age } 84.2 \\
\text { intervention group } \\
84.2 \text { control group } \\
\text { Sex } 66 \% \text { female } \\
\text { intervention group } \\
75 \% \text { control group } \\
\text { Race } 95 \% \text { white } \\
\text { intervention group } \\
88 \% \text { white control } \\
\text { group } \\
\text { Education: no }\end{array}$ & \begin{tabular}{|l} 
SES: no \\
Prior disability: no \\
Household \\
characteristics: no \\
Health insurance: \\
no \\
Race information: \\
yes
\end{tabular} & NA & NA & Not reported & Not reported & 12 weeks & $\begin{array}{l}\text { Measures of } \\
\text { resident, } \\
\text { family and } \\
\text { staff quality } \\
\text { of life } \\
\text { Resident and } \\
\text { staff care } \\
\text { measures at } \\
\text { baseline and } \\
\text { follow-up }\end{array}$ & $\begin{array}{l}\text { Effectiveness } \\
\text { of training in } \\
\text { promoting } \\
\text { activity } \\
\text { engagement } \\
\text { for patients } \\
\text { with } A D\end{array}$ \\
\hline $\begin{array}{l}\text { Leone, 2012 } \\
(22700526) \\
\text { France } \\
\text { Pilot }\end{array}$ & $\begin{array}{l}\text { Evaluation of } \\
\text { staff education } \\
\text { in managing } \\
\text { apathy in older } \\
\text { nursing home } \\
\text { patients with AD }\end{array}$ & $\begin{array}{l}\text { Randomization } \\
\text { of intervention } \\
\text { group and } \\
\text { control group by } \\
\text { nursing home } \\
\text { site; } 16 \text { total } \\
\text { sites }\end{array}$ & $\begin{array}{l}\mathrm{N}=119 \\
\text { patients, } 284 \\
\text { caregivers in } \\
\text { intervention } \\
\text { group } \\
111 \text { patients, } \\
279 \\
\text { caregivers in } \\
\text { reference } \\
\text { group }\end{array}$ & \begin{tabular}{|l|} 
Patients with \\
diagnosis of AD, \\
per medical \\
records \\
information, \\
MMSE<24 and \\
presence of apathy \\
per diagnostic \\
criteria
\end{tabular} & $\begin{array}{l}\mathrm{N}=119 \text { intervention } \\
\text { group } \\
111 \text { control group } \\
\text { Age } 87.83 \\
\text { intervention group } \\
88,82 \text { reference } \\
\text { group } \\
\text { Sex: } 72.3 \% \\
\text { intervention group } \\
87.4 \text { reference } \\
\text { group } \\
\text { Race: no } \\
\text { Education: no }\end{array}$ & Not reported & NA & NA & Not reported & Not reported & 17 weeks & $\begin{array}{l}\text { Measures of } \\
\text { resident } \\
\text { emotional } \\
\text { blunting, } \\
\text { interest, level } \\
\text { of initiative }\end{array}$ & $\begin{array}{l}\text { Effectiveness } \\
\text { of staff } \\
\text { training } \\
\text { measures in } \\
\text { reducing } \\
\text { patient } \\
\text { apathy in AD } \\
\text { patients }\end{array}$ \\
\hline $\begin{array}{l}\text { McCurry } 2012 \\
(22367233) \\
\text { US } \\
\text { Small sample }\end{array}$ & $\begin{array}{l}4 \text { training } \\
\text { sessions to } \\
\text { develop and } \\
\text { implement } \\
\text { individualized } \\
\text { PLWDD } \\
\text { behavioral sleep } \\
\text { plans }\end{array}$ & Usual care & $\begin{array}{l}\text { Adult family } \\
\text { homes } \\
\text { residents } \\
\text { RCT } \\
47 \text { PLWD }\end{array}$ & \begin{tabular}{|l|} 
Dementia \\
diagnosis by \\
primary care \\
physicians with \\
meantSD MMSE \\
score being \\
$8.1 \pm 7.6$ and has \\
one or more sleep \\
problems on the \\
Sleep Disorders \\
Inventory
\end{tabular} & $\begin{array}{l}\mathrm{N}=31 \\
\text { Age yes } \\
\text { Sex yes } \\
\text { Race: } \text { yes } \\
\text { Education yes }\end{array}$ & None & NA & NA & $\begin{array}{l}\mathrm{N}=37 \\
\text { Mean } \\
\text { Age: yes } \\
\text { Sex: yes } \\
\text { Race: yes } \\
\text { Education: yes }\end{array}$ & None & $\begin{array}{l}1 \text { month } \\
6 \text { months }\end{array}$ & $\begin{array}{l}\text { Actigraphy } \\
\text { CSDD } \\
\text { RMBPC } \\
\text { ESS }\end{array}$ & NA \\
\hline
\end{tabular}




\begin{tabular}{|c|c|c|c|c|c|c|c|c|c|c|c|c|c|}
\hline $\begin{array}{l}\text { Zimmerman, } \\
2010^{122} \\
\text { (No Id \#) } \\
\text { US } \\
\text { Training pilot }\end{array}$ & $\begin{array}{l}\text { Evaluation of } \\
\text { staff education } \\
\text { program on } \\
\text { dementia care } \\
\text { focused on } \\
\text { leadership, } \\
\text { knowledge } \\
\text { about AD and } \\
\text { pain reduction }\end{array}$ & \begin{tabular}{|l|} 
Randomization \\
of care centers \\
receiving \\
training vs \\
control group; \\
16 residential \\
care and \\
nursing home \\
sites in 4 states \\
\end{tabular} & \begin{tabular}{|l|}
$\mathrm{N}=213$ staff, \\
78 \\
supervisors \\
in 8 centers \\
for \\
intervention \\
group \\
278 staff, 93 \\
supervisors \\
for control \\
group \\
\end{tabular} & \begin{tabular}{|l|} 
Not reported \\
Intervention center \\
patients with $\mathrm{AD}=$ \\
$62 \%$ \\
Control center \\
patients with \\
$\mathrm{AD}=48 \%$
\end{tabular} & Not reported & Not reported & NA & NA & $\begin{array}{l}\mathrm{N}=490 \\
\text { Age: yes } \\
\text { Sex: yes } \\
\text { Race: yes } \\
\text { Education: yes }\end{array}$ & $\begin{array}{l}\text { Health Status :no } \\
\text { Training: yes } \\
\text { Education: yes } \\
\text { Position: yes } \\
\text { Length of } \\
\text { Service: yes }\end{array}$ & 3 months & \begin{tabular}{|l|} 
Measures of \\
supervisor \\
leadership \\
and \\
measures of \\
work stress, \\
work \\
satisfaction \\
and \\
knowledge
\end{tabular} & $\begin{array}{l}\text { Benefit of } \\
\text { training for } \\
\text { staff and } \\
\text { supervisory } \\
\text { personnel }\end{array}$ \\
\hline $\begin{array}{l}\begin{array}{l}\text { Deudon,2009123 } \\
(19370714)\end{array} \\
\text { France } \\
\text { Training pilot }\end{array}$ & $\begin{array}{l}8 \text { week, } 12 \\
\text { session staff } \\
\text { training on } \\
\text { dementia and } \\
\text { BPSD; } \\
\text { feedback } \\
\text { sessions }\end{array}$ & & \begin{tabular}{|l|} 
Nursing \\
homes \\
Cluster RCT \\
16 sites \\
306 PLWD
\end{tabular} & & $\begin{array}{l}\text { N=306 } \\
\text { Age: yes } \\
\text { Sex: yes } \\
\text { Race: no } \\
\text { Education: no }\end{array}$ & None & NA & NA & NA & NA & $\begin{array}{l}12 \text { week post } \\
\text { intervention }\end{array}$ & \begin{tabular}{|l|} 
NPI \\
CMAl \\
Observation \\
Scale (OS)
\end{tabular} & NA \\
\hline $\begin{array}{l}\text { Kuske, 2009124 } \\
(19193252) \\
\text { Germany } \\
\text { Training pilot }\end{array}$ & $\begin{array}{l}\text { Evaluation of a } \\
\text { staff education } \\
\text { program on } \\
\text { caregiver } \\
\text { knowledge and } \\
\text { competence; } \\
\text { evaluation } \\
\text { relaxation } \\
\text { training for staff }\end{array}$ & \begin{tabular}{|l|}
3 arm cluster \\
randomized \\
control trial \\
clustered in 6 \\
nursing homes
\end{tabular} & $\begin{array}{l}\mathrm{N}=68 \\
\text { patients, } 89 \\
\text { caregivers in } \\
\text { intervention } \\
\text { group } \\
68 \text { patients, } \\
90 \\
\text { caregivers in } \\
\text { relaxation } \\
\text { group } \\
74 \text { patients, } \\
94 \\
\text { caregivers in } \\
\text { control } \\
\text { group } \\
\end{array}$ & $\begin{array}{l}\text { Patients with AD, } \\
\text { per MMST and } \\
\text { Barthel Index } \\
\text { scores }\end{array}$ & $\begin{array}{l}\mathrm{N}=68 \text { intervention } \\
\text { group } \\
68 \text { patients } \\
\text { relaxation group } \\
74 \text { patients control } \\
\text { group } \\
\text { Age: yes } \\
\text { Sex: yes } \\
\text { Race: no } \\
\text { Education: no }\end{array}$ & Not reported & NA & NA & $\begin{array}{l}\text { Age: yes } \\
\text { Sex: yes } \\
\text { Race: no } \\
\text { Education: yes }\end{array}$ & $\begin{array}{l}\text { Health Status: } \\
\text { yes } \\
\text { Training: yes } \\
\text { Education: yes } \\
\text { Position: yes } \\
\text { Length of } \\
\text { Service: yes }\end{array}$ & 6 months & \begin{tabular}{|l|} 
Measures of \\
knowledge, \\
competence \\
and \\
emotional/he \\
alth status \\
\end{tabular} & $\begin{array}{l}\text { Benefit of } \\
\text { training on } \\
\text { quality of } \\
\text { care for AD } \\
\text { patients and } \\
\text { in reducing } \\
\text { caregivers' } \\
\text { burden in } \\
\text { caring for } \\
\text { patients }\end{array}$ \\
\hline $\begin{array}{l}\text { Chang, 2005'125 } \\
(16238764) \\
\text { Taiwan } \\
\text { Pilot }\end{array}$ & $\begin{array}{l}\text { Effects of a } \\
\text { training program } \\
\text { on feeding skills } \\
\text { for nursing } \\
\text { assistants (NA) } \\
\text { feeding patients } \\
\text { with AD } \\
\text { Pilot study }\end{array}$ & \begin{tabular}{|l|}
2 AD- \\
specialized long \\
term-care \\
treatment \\
centers \\
randomly \\
assigned as \\
training or \\
control group \\
\end{tabular} & $\begin{array}{l}\text { One patient } \\
\text { per NA pre } \\
\text { and post } \\
\text { training, not } \\
\text { matched }\end{array}$ & $\begin{array}{l}\text { Patients diagnosed } \\
\text { with AD and } \\
\text { evaluated by NA as } \\
\text { having eating } \\
\text { problems and } \\
\text { requiring } \\
\text { assistance }\end{array}$ & $\begin{array}{l}\mathrm{N}=31 \mathrm{NA} \text { in training } \\
\text { group } \\
36 \text { NA in control } \\
\text { group } \\
\text { Patient information } \\
\text { not reported; } 12 \\
\text { patients matched } \\
\text { pre and post training } \\
\text { to NA in training } \\
\text { group; } 8 \text { patients } \\
\text { matched in control } \\
\text { group }\end{array}$ & Not reported & NA & NA & $\begin{array}{l}\text { Age: yes } \\
\text { Sex: yes } \\
\text { Race: no } \\
\text { Education: no }\end{array}$ & $\begin{array}{l}\text { Health Status: no } \\
\text { Training: no } \\
\text { Education: no } \\
\text { Position: yes } \\
\text { Length of } \\
\text { Service: yes }\end{array}$ & $\begin{array}{l}\text { One post } \\
\text { training } \\
\text { assessment }\end{array}$ & \begin{tabular}{|l|} 
Assessment \\
of NA \\
knowledge, \\
using \\
checklist \\
Food intake \\
Feeding time \\
EdFED score
\end{tabular} & $\begin{array}{l}\text { Caregiver } \\
\text { skills and } \\
\text { attitude } \\
\text { developed } \\
\text { through } \\
\text { training } \\
\text { Effects of } \\
\text { caregiver } \\
\text { training on } \\
\text { patient eating } \\
\text { behavior }\end{array}$ \\
\hline $\begin{array}{l}\text { Sloane, 2004'126 } \\
(15507054) \\
\text { US } \\
\text { Small sample }\end{array}$ & $\begin{array}{l}\text { Techniques to } \\
\text { reduce } \\
\text { agitation, } \\
\text { aggression and } \\
\text { discomfort in } \\
\text { AD patients } \\
\text { 1) Training for } \\
\text { person-centered } \\
\text { showering or 2) } \\
\text { towel bath } \\
\end{array}$ & \begin{tabular}{|l|} 
Usual care \\
activities
\end{tabular} & \begin{tabular}{|l|} 
Nursing \\
homes, \\
Crossover \\
Cluster RCT \\
15 Cluster N \\
69 PLWD \\
37 FC
\end{tabular} & \begin{tabular}{|l} 
Patients 65 and \\
older with \\
diagnosis of $\mathrm{AD}$ or \\
related dementia \\
who required \\
assistance with \\
bathing
\end{tabular} & $\begin{array}{l}\mathrm{N}=69 \text { PLWD, } \\
\text { Age: yes } \\
\text { Sex: yes } \\
\text { Race: yes } \\
\text { Education: yes }\end{array}$ & \begin{tabular}{|l} 
SES: no \\
Prior disability: no \\
Household \\
characteristics: no \\
Health insurance: \\
no \\
Race information: \\
yes
\end{tabular} & NA & NA & $\begin{array}{l}\text { N: } 37 \\
\text { Age: yes } \\
\text { Sex: yes } \\
\text { Race: no } \\
\text { Education: no }\end{array}$ & $\begin{array}{l}\text { Health Status: no } \\
\text { Training: no } \\
\text { Education: no } \\
\text { Position: yes } \\
\text { Length of } \\
\text { Service: yes }\end{array}$ & 6 weeks & \begin{tabular}{|l|} 
Bathing- \\
associated \\
aggression, \\
agitation, and \\
discomfort
\end{tabular} & $\begin{array}{l}\text { Comparison } \\
\text { of bathing } \\
\text { behavior } \\
\text { difficulty of } \\
\text { non-trained } \\
\text { staff and } \\
\text { person- } \\
\text { centered vs } \\
\text { towel bath } \\
\text { intervention }\end{array}$ \\
\hline $\begin{array}{l}\text { Magai, 2002 } \\
(11395344) \\
\text { US } \\
\text { Pilot }\end{array}$ & $\begin{array}{l}\text { Training in } \\
\text { sensitivity to } \\
\text { nonverbal } \\
\text { communication, } \\
10-1 \mathrm{hr} \\
\text { sessions }\end{array}$ & $\begin{array}{l}\text { C1: Waitlist } \\
\text { C2: dementia } \\
\text { education } \\
\text { training }\end{array}$ & \begin{tabular}{|l|} 
Nursing \\
homes \\
Cluster RCT \\
3 Cluster N \\
\end{tabular} & \begin{tabular}{|l} 
Dementia \\
unspecified
\end{tabular} & $\begin{array}{l}\mathrm{N}=91 \\
\text { Age: yes } \\
\text { Sex: yes } \\
\text { Race: yes } \\
\text { Education: no }\end{array}$ & None & NA & NA & $\begin{array}{l}\mathrm{N}=21 \\
\text { Age: yes } \\
\text { Sex: yes } \\
\text { R: yes } \\
\text { Education: no }\end{array}$ & None & 12 weeks & \begin{tabular}{|l|} 
CDS \\
CMAI \\
BEHAVE-AD
\end{tabular} & BSI \\
\hline
\end{tabular}




\begin{tabular}{|c|c|c|c|c|c|c|c|c|c|c|c|c|c|}
\hline $\begin{array}{l}\text { Wells, 2000129 } \\
(10798473 \\
\text { Canada } \\
\text { Small sample }\end{array}$ & $\begin{array}{l}\text { Evaluation of } \\
\text { training program } \\
\text { for morning care } \\
\text { of nursing home } \\
\text { patients with AD }\end{array}$ & $\begin{array}{l}\text { Randomized } \\
\text { control study } \\
\text { with one } \\
\text { experimental } \\
\text { care unit and } 3 \\
\text { control units }\end{array}$ & $\begin{array}{l}\text { Four } \\
\text { cognitive } \\
\text { support units } \\
\text { in a large } \\
\text { geriatric care } \\
\text { center }\end{array}$ & \begin{tabular}{|l|} 
Patients with a \\
diagnosis of \\
dementia or AD \\
residing in the \\
memory assistance \\
care units for at \\
least 4 weeks; \\
MMSE <19; \\
Control group \\
selected for age \\
and MMSE \\
equivalency with \\
experimental group
\end{tabular} & $\begin{array}{l}\mathrm{N}=20 \mathrm{PLWD}, 16 \mathrm{FC} \\
\text { experimental group } \\
\text { 20PD, 28FC control } \\
\text { group } \\
\text { Age: yes } \\
\text { Sex: yes } \\
\text { Race: no } \\
\text { Education: no } \\
\text { Primary diagnosis: } \\
\text { yes }\end{array}$ & $\begin{array}{l}\text { SES: no } \\
\text { Prior disability: no } \\
\text { Household } \\
\text { characteristics: no } \\
\text { Health insurance: } \\
\text { no } \\
\text { Race information: } \\
\text { no }\end{array}$ & NA & NA & $\begin{array}{l}\text { N: yes } \\
\text { Age: yes } \\
\text { Sex: yes } \\
\text { Race: no } \\
\text { Education: yes }\end{array}$ & $\begin{array}{l}\text { Health Status: no } \\
\text { Training: yes } \\
\text { Education: yes } \\
\text { Position: yes } \\
\text { Length of } \\
\text { Service: yes }\end{array}$ & 6 months & $\begin{array}{l}\text { Measurement } \\
\text { s of resident } \\
\text { interaction } \\
\text { behavior, } \\
\text { level of } \\
\text { agitation, } \\
\text { perceived } \\
\text { ease of } \\
\text { caregiving, } \\
\text { level of stress }\end{array}$ & $\begin{array}{l}\text { Level of } \\
\text { resident } \\
\text { interaction } \\
\text { with } \\
\text { caregivers } \\
\text { with or } \\
\text { without } \\
\text { training }\end{array}$ \\
\hline $\begin{array}{l}\text { McCallion, } \\
1999^{130} \\
(10568079) \\
\text { US }\end{array}$ & $\begin{array}{l}\text { Evaluation pf an } \\
\text { education } \\
\text { program to } \\
\text { improve } \\
\text { effectiveness of } \\
\text { nursing home } \\
\text { assistants with } \\
\text { residents with } \\
\text { dementia }\end{array}$ & $\begin{array}{l}\text { Randomized } \\
\text { partial } \\
\text { crossover } \\
\text { control study; } \\
\text { control group } \\
\text { given training } \\
\text { after } 6 \text { month } \\
\text { assessment }\end{array}$ & $\begin{array}{l}\text { Two nursing } \\
\text { homes, } \\
\text { including } 2 \\
\text { care units } \\
\text { housing } \\
\text { patients with } \\
\text { dementia in } \\
\text { each }\end{array}$ & $\begin{array}{l}\text { Patients with } \\
\text { diagnosis of } \\
\text { dementia and } \\
\text { MMSE score } \\
\text { assessed against } \\
\text { educational level; } \\
\text { GDS of stage } 3 \text { or } \\
\text { above }\end{array}$ & $\begin{array}{l}\mathrm{N}=49 \mathrm{PLWD}, 39 \mathrm{FC} \\
\text { treatment group } \\
56 \text { PLWD, 49 FC } \\
\text { control group } \\
\text { Age: yes } \\
\text { Sex: yes } \\
\text { Race: no } \\
\text { Religion: yes } \\
\text { Education: yes } \\
\text { Primary diagnosis: } \\
\text { no }\end{array}$ & $\begin{array}{l}\text { SES: no } \\
\text { Prior disability: no } \\
\text { Household } \\
\text { characteristics: no } \\
\text { Health insurance: } \\
\text { no } \\
\text { Race information: } \\
\text { yes }\end{array}$ & NA & NA & $\begin{array}{l}\text { N: yes } \\
\text { Age: yes } \\
\text { Sex: yes } \\
\text { Race: yes } \\
\text { Education: yes }\end{array}$ & $\begin{array}{l}\text { Health Status: no } \\
\text { Training: yes } \\
\text { Education: yes } \\
\text { Position: yes } \\
\text { Length of } \\
\text { Service: yes }\end{array}$ & 9 months & $\begin{array}{l}\text { FC } \\
\text { interviews, } \\
\text { KAT, MHQ; } \\
\text { staff turnover } \\
\text { rate; } \\
\text { Resident } \\
\text { assessments } \\
\text { of signs of } \\
\text { and } \\
\text { symptoms of } \\
\text { depression } \\
\text { and } \\
\text { aggressive } \\
\text { behaviors }\end{array}$ & $\begin{array}{l}\text { Changes in } \\
\text { level of } \\
\text { behavioral } \\
\text { disturbances } \\
\text { in PLWD; } \\
\text { Changes in } \\
\text { FC } \\
\text { knowledge } \\
\text { and patient } \\
\text { management }\end{array}$ \\
\hline $\begin{array}{l}\text { Proctor, 1999131 } \\
(10406361) \\
\text { UK } \\
\text { Pilot }\end{array}$ & $\begin{array}{l}\text { Evaluation of } \\
\text { training and } \\
\text { education } \\
\text { intervention in } \\
\text { nursing and } \\
\text { residential care } \\
\text { facilities }\end{array}$ & $\begin{array}{l}\text { Randomized } \\
\text { control trial }\end{array}$ & $\begin{array}{l}\text { Twelve } \\
\text { matched } \\
\text { care } \\
\text { facilities, } \\
\text { selected } \\
\text { randomly as } \\
\text { intervention } \\
\text { or control } \\
\text { groups; } \\
10 \text { patients } \\
\text { with difficult } \\
\text { behavioral } \\
\text { problems } \\
\text { selected by } \\
\text { staff at each } \\
\text { facility }\end{array}$ & $\begin{array}{l}\text { Patients selected } \\
\text { by staff; Centers } \\
\text { paired by size and } \\
\text { accreditation status } \\
\text { and randomly } \\
\text { selected to } \\
\text { intervention or } \\
\text { control arm }\end{array}$ & $\begin{array}{l}\text { N=54 PLWD, } \\
\text { 51 PLWD control } \\
\text { group } \\
\text { Age: yes } \\
\text { Sex: yes } \\
\text { Race: no } \\
\text { Education: no } \\
\text { Primary diagnosis: } \\
\text { no } \\
\text { Counts for } \\
\text { intervention vs } \\
\text { control group not } \\
\text { reported }\end{array}$ & $\begin{array}{l}\text { SES: no } \\
\text { Prior disability: no } \\
\text { Household } \\
\text { characteristics: no } \\
\text { Health insurance: } \\
\text { no } \\
\text { Race information: } \\
\text { no }\end{array}$ & NA & NA & Not reported & Not reported & 6 months & $\begin{array}{l}\text { Measurement } \\
\text { s of patient } \\
\text { cognitive } \\
\text { impairment, } \\
\text { depression, } \\
\text { behavioral } \\
\text { disturbance } \\
\text { and } \\
\text { functional } \\
\text { ability }\end{array}$ & $\begin{array}{l}\text { Changes in } \\
\text { patient } \\
\text { depression } \\
\text { scores, level } \\
\text { of cognitive } \\
\text { impairment } \\
\text { and behavior }\end{array}$ \\
\hline
\end{tabular}

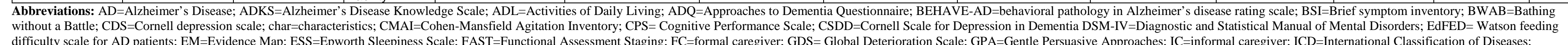

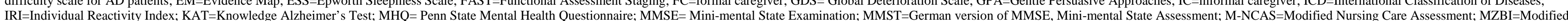
Zarit Burden Interview; N=number; NA=Not Applicable; NR=Not Reported; PMID=PubMed Identification Number; PLWD=Persons with Dementia; QOL-AD=Quality of Life - Alzheimer's Disease; RMBPC=Revised Memory and Behavior Problems Checklist; RS=Reporting Status;

\section{Informal Caregiver Training}

Table F-44. Risk of bias assessment: informal caregiver training

\begin{tabular}{|c|c|c|c|c|c|c|c|c|c|}
\hline \begin{tabular}{|l|} 
Study (PMID) \\
\end{tabular} & Outcome Timing & $\begin{array}{l}\text { Attrition Bias } \\
\text { Attrition \% }\end{array}$ & Selection Bias & Detection Bias & \begin{tabular}{|l} 
Performance Bias \\
\end{tabular} & Reporting Bias & Fidelity Bias & Funder & Overall Rating \\
\hline \begin{tabular}{|l|l} 
Hepburn, $2001^{132}$ (113477900) \\
\end{tabular} & 5 months & $\begin{array}{l}\text { Medium } \\
20 \%\end{array}$ & Low & Medium & High & Low & High & \begin{tabular}{|l|} 
Gov't \\
Developer \\
\end{tabular} & High \\
\hline \begin{tabular}{|l} 
Hepburn, 2006 \\
${ }^{133}(43539927)$
\end{tabular} & 6 month & Medium 21\% & Low & Medium & High & Low & High & \begin{tabular}{|l|}
$\begin{array}{l}\text { Gov't } \\
\text { Developer }\end{array}$ \\
\end{tabular} & High \\
\hline \begin{tabular}{|l|l} 
Hepburn, $2007^{134}(17378189)$ \\
\end{tabular} & 6 months & $\begin{array}{l}\text { High } \\
49 \% \\
\end{array}$ & Medium & $x$ & $x$ & $\mathrm{x}$ & $\mathrm{x}$ & $\mathrm{x}$ & High \\
\hline
\end{tabular}

$\mathrm{X}$ indicates that domain was not assessed due to high risk of bias.

Abbreviations: PMID=PubMed Identification Number 


\begin{tabular}{|c|c|c|c|c|c|c|c|c|c|c|c|c|c|}
\hline $\begin{array}{l}\text { Study (PMID) } \\
\text { Country } \\
\text { EM Reason }\end{array}$ & Intervention & Comparison & \begin{tabular}{|l|} 
Setting \\
and Design \\
\\
Setting \\
Design \\
Cluster N \\
Participants \\
Random- \\
ized N \\
\end{tabular} & \begin{tabular}{|l} 
PLWD \\
Dementia \\
Characteristics
\end{tabular} & $\begin{array}{l}\text { PLWD } \\
\text { Non-Disease Char } \\
\text { PLWD N } \\
\text { PLWD Age (mean) } \\
\text { PLWD Sex (\% } \\
\text { female) } \\
\text { PLWD Race (\% } \\
\text { majority) } \\
\text { PLWD Education } \\
\text { (mean years) }\end{array}$ & \begin{tabular}{|l|} 
PLWD \\
Non-Disease \\
Char Reporting \\
Status (RS) \\
\\
PLWD SES \\
PLWD Prior \\
Disability \\
PLWD \\
Household \\
Characteristics \\
PLWD Health \\
Insurance \\
PLWD Detailed \\
Race Information
\end{tabular} & \begin{tabular}{|l} 
Informal Caregiver \\
(IC) \\
Characteristics \\
IC N \\
IC Age (mean) \\
IC Sex (\% female) \\
IC Race (\% \\
majority) \\
IC Education \\
(mean years) \\
IC Relation to \\
PLWD (\% majority)
\end{tabular} & $\begin{array}{l}\text { Informal Caregiver } \\
\text { (IC) Char. RS } \\
\text { IC Duration } \\
\text { IC Living With } \\
\text { PLWD } \\
\text { IC Payment } \\
\text { IC Health Status } \\
\text { IC Dementia } \\
\text { Family History } \\
\text { IC Employment } \\
\text { Status } \\
\text { IC Training }\end{array}$ & $\begin{array}{l}\text { Formal Caregiver } \\
\text { (FC) } \\
\text { Characteristics } \\
\text { FC N } \\
\text { FC Age (mean) } \\
\text { FC Sex (\% } \\
\text { female) } \\
\text { FC Race (\% } \\
\text { majority) } \\
\text { FC Education } \\
\text { (mean years) }\end{array}$ & $\begin{array}{l}\text { Formal } \\
\text { Caregiver (FC) } \\
\text { Char. RS } \\
\text { FC Health } \\
\text { Status } \\
\text { FC Training } \\
\text { FC Education } \\
\text { FC Position } \\
\text { FC Length of } \\
\text { Service }\end{array}$ & $\begin{array}{l}\text { Outcome } \\
\text { Timing(s) }\end{array}$ & $\begin{array}{l}\text { PLWD } \\
\text { Outcomes }\end{array}$ & $\begin{array}{l}\text { Caregiver } \\
\text { Outcomes } \\
\text { IC: } \\
\text { FC: }\end{array}$ \\
\hline $\begin{array}{l}\text { Hattink, 2015116 } \\
\text { (26519106) } \\
\text { Netherlands \& } \\
\text { UK } \\
\text { Pilot }\end{array}$ & $\begin{array}{l}\text { Access to a } \\
\text { Web-based } \\
\text { portal consisting } \\
\text { of } 8 \text { modules \& } \\
\text { to online peer } \\
\text { and expert } \\
\text { communities for } \\
\text { support and } \\
\text { information } \\
\text { exchange }\end{array}$ & Wait-list & $\begin{array}{l}\text { Community } \\
\text { setting } \\
\text { RCT } \\
\text { Caregivers } \\
142\end{array}$ & \begin{tabular}{|l|} 
Dementia \\
unspecified
\end{tabular} & $\begin{array}{l}\mathrm{N}= \\
\text { Age: no } \\
\text { Sex: no } \\
\text { \% majority race: no } \\
\text { Education: no }\end{array}$ & None & $\begin{array}{l}\text { IC N=59 } \\
\text { IC Age: yes } \\
\text { IC Sex: yes } \\
\text { IC \% majority race: } \\
\text { no } \\
\text { IC Education: no } \\
\text { IC Relation to } \\
\text { PLWD: yes }\end{array}$ & $\begin{array}{l}\text { IC Duration } \\
\text { IC Employment } \\
\text { status }\end{array}$ & $\begin{array}{l}\text { FC N=24 } \\
\text { FC Age: yes } \\
\text { FC Sex: yes } \\
\text { FC Race: no } \\
\text { FC Education: no }\end{array}$ & Length of service & 4 months & $\begin{array}{l}\text { ADKS } \\
\text { ADQ } \\
\text { IRI } \\
\text { QoL } \\
\text { SSCQ }\end{array}$ & $\begin{array}{l}\text { FC: } \\
\text { Usefulness of } \\
\text { training } \\
\text { IC: Burden: } \\
\text { assessed with } \\
1 \text { question }\end{array}$ \\
\hline $\begin{array}{l}\text { Liddle, 2012135 } \\
\text { (23092595) } \\
\text { Australia } \\
\text { Small sample }\end{array}$ & $\begin{array}{l}\text { DVD-based } \\
\text { training for } \\
\text { memory and } \\
\text { communication } \\
\text { support skills }\end{array}$ & No training & \begin{tabular}{|l} 
Community \\
RCT \\
Caregivers
\end{tabular} & \begin{tabular}{|l|} 
Dementia \\
(Alzheimers, \\
vascular, \\
frontotemporal, not \\
otherwise \\
specified)
\end{tabular} & $\begin{array}{l}\mathrm{N}=29 \\
\text { Age: yes } \\
\text { Sex: yes } \\
\text { \% majority race: no } \\
\text { Education: yes }\end{array}$ & None & $\begin{array}{l}\mathrm{N}=29 \\
\text { Age: yes } \\
\text { Sex: yes } \\
\text { \% majority race: no } \\
\text { Education: yes } \\
\text { Relationship: yes }\end{array}$ & None & NA & NA & 3 months & None & $\begin{array}{l}\text { ZBI } \\
\text { PAC } \\
\text { RMBPC } \\
\text { CSDD }\end{array}$ \\
\hline $\begin{array}{l}\text { Klodnicka } \\
\text { Kouri,2011 } 136 \\
\text { ?? } \\
\text { Canada } \\
\text { Pilot }\end{array}$ & $\begin{array}{l}\text { Individual } \\
\text { communication } \\
\text { training, } 5 \\
\text { weekly 90-120 } \\
\text { minute session }\end{array}$ & $\begin{array}{l}\text { Printed } \\
\text { information }\end{array}$ & \begin{tabular}{|l|} 
Community \\
RCT \\
Caregivers
\end{tabular} & \begin{tabular}{|l} 
Mild probable \\
Alzheimer's \\
disease
\end{tabular} & $\begin{array}{l}\mathrm{N}= \\
\text { Age: no } \\
\text { Sex: no } \\
\text { \% majority race: no } \\
\text { Education: no }\end{array}$ & None & $\begin{array}{l}\mathrm{N}=50 \\
\text { Age: no } \\
\text { Sex: yes } \\
\text { \% majority race: yes } \\
\text { Education: no } \\
\text { Relationship: yes }\end{array}$ & $\begin{array}{l}\text { Duration; } \\
\text { Living with PLWD; } \\
\text { Employment }\end{array}$ & NA & NA & $\begin{array}{l}6 \text { weeks post } \\
\text { intervention }\end{array}$ & None & $\begin{array}{l}\text { IC: Caregiver } \\
\text { self-efficacy } \\
\text { scale; } \\
\text { RMBPC } \\
\text { communicatio } \\
\text { n knowledge } \\
\text { and skills }\end{array}$ \\
\hline $\begin{array}{l}\text { Neely, 2009137 } \\
(19294562) \\
\text { Sweden } \\
\text { Small sample }\end{array}$ & $\begin{array}{l}\text { Trained in and } \\
\text { practiced } \\
\text { strategies to } \\
\text { support } \\
\text { everyday } \\
\text { mnemonic and } \\
\text { occupational } \\
\text { performance, } \\
\text { home-based, } 8 \\
-1 \text { hour } \\
\text { sessions }\end{array}$ & No training & \begin{tabular}{|l|} 
Community \\
RCT \\
Dyad
\end{tabular} & \begin{tabular}{|l|} 
Mild to moderate \\
Alzheimer's \\
disease or with \\
vascular dementia
\end{tabular} & $\begin{array}{l}\mathrm{N}=30 \\
\text { Age: yes } \\
\text { Sex: yes } \\
\text { \% majority race: no } \\
\text { Education: no }\end{array}$ & None & $\begin{array}{l}\text { N=30 } \\
\text { Age: yes } \\
\text { Sex: yes } \\
\text { \% majority race: no } \\
\text { Education: no } \\
\text { Relationship: no }\end{array}$ & None & NA & NA & 8 weeks & None & $\begin{array}{l}\text { ZBI } \\
\text { Beck } \\
\text { depression }\end{array}$ \\
\hline $\begin{array}{l}\text { Hepburn, } \\
2007^{134} \\
(17378189) \\
\text { USA } \\
\text { High ROB }\end{array}$ & $\begin{array}{l}\text { Group } \\
\text { caregiving role- } \\
\text { training and } \\
\text { mastery- } \\
\text { focused } \\
\text { coaching } \\
6-2 \text { hour } \\
\text { sessions }\end{array}$ & Wait-list control & \begin{tabular}{|l} 
Community \\
RCT \\
Caregivers
\end{tabular} & \begin{tabular}{|l|} 
Dementia \\
unspecified
\end{tabular} & $\begin{array}{l}\mathrm{N}= \\
\text { Age: no } \\
\text { Sex: no } \\
\text { \% majority race: no } \\
\text { Education: no }\end{array}$ & None & $\begin{array}{l}\mathrm{N}=102 \\
\text { Age: yes } \\
\text { Sex: yes } \\
\text { \% majority race: yes } \\
\text { Education: yes } \\
\text { Relationship: yes }\end{array}$ & $\begin{array}{l}\text { SES } \\
\text { Duration }\end{array}$ & NA & NA & 5 months & None & $\begin{array}{l}\text { Relational } \\
\text { deprivation } \\
\text { Role captivity } \\
\text { Competence } \\
\text { Mastery } \\
\text { Loss of self- } \\
\text { Distress }\end{array}$ \\
\hline $\begin{array}{l}\text { Hepburn, } \\
2005^{133} \\
? ? \\
\text { USA } \\
\text { High ROB }\end{array}$ & $\begin{array}{l}\text { Group } \\
\text { caregiving role- } \\
\text { training and } \\
\text { mastery- } \\
\text { focused } \\
\text { coaching } \\
6 \text { weekly } 2 \text { hour } \\
\text { sessions }\end{array}$ & Wait-list control & $\begin{array}{l}\text { Community } \\
\text { RCT } \\
\text { NA } \\
\text { NA }\end{array}$ & $\begin{array}{l}\text { Dementia } \\
\text { unspecified }\end{array}$ & $\begin{array}{l}\mathrm{N}=215 \\
\text { Age: NR } \\
\text { Sex: NR } \\
\text { \% majority race: NR } \\
\text { Education: NR }\end{array}$ & NA & $\begin{array}{l}\text { IC N=215 } \\
\text { IC Age: yes } \\
\text { IC Sex: yes } \\
\text { IC \% majority race: } \\
\text { no } \\
\text { IC Education: yes } \\
\text { IC Relation to } \\
\text { PLWD: no }\end{array}$ & None & NA & NA & $\begin{array}{l}6 \text { and } 12 \\
\text { months }\end{array}$ & None & $\begin{array}{l}\text { BACS } \\
\text { remainder } \\
\text { were } \\
\text { development } \\
\text { of distress } \\
\text { measure }\end{array}$ \\
\hline $\begin{array}{l}\text { Martin-Cook, } \\
2005 \text { Martin- } \\
\text { Cook, 2005 } \\
\# 15808\} \\
(16136843) \\
\text { US } \\
\text { Small sample }\end{array}$ & $\begin{array}{l}\text { Individual } \\
\text { communication } \\
\text { training through } \\
\text { modeling and } \\
\text { feedback, } 4 \\
\text { sessions }\end{array}$ & Not specified & $\begin{array}{l}\text { Community } \\
\text { RCT } \\
\text { Caregivers }\end{array}$ & $\begin{array}{l}\text { Mild to moderate } \\
\text { dementia, primarily } \\
\text { Alzheimer's } \\
\text { disease }\end{array}$ & $\begin{array}{l}\mathrm{N}= \\
\text { Age: yes } \\
\text { Sex: yes } \\
\text { \% majority race: yes } \\
\text { Education: yes }\end{array}$ & None & $\begin{array}{l}\mathrm{N}=47 \\
\text { Age: no } \\
\text { Sex: yes } \\
\text { \% majority race: no } \\
\text { Education: yes } \\
\text { Relationship: yes }\end{array}$ & Duration & NA & $\mathrm{NA}$ & 17 weeks & $\begin{array}{l}\text { ILS/IIILS } \\
\text { ADCS } \\
\text { MMSE; } \\
\text { NPI; } \\
\text { GDS }\end{array}$ & $\begin{array}{l}\text { FMTCS; } \\
\text { GSE }\end{array}$ \\
\hline $\begin{array}{l}\text { Done, 2001138 } \\
\text { (11536349) } \\
\text { UK } \\
\text { Small sample }\end{array}$ & $\begin{array}{l}\text { Group } \\
\text { communication } \\
\text { training 1 } \\
\text { hour/week, } 2 \\
\text { weeks }\end{array}$ & $\begin{array}{l}\text { Information } \\
\text { booklet }\end{array}$ & \begin{tabular}{|l|} 
Community \\
Cluster RCT \\
Local groups \\
(day center \\
or local \\
home) \\
45 \\
Caregivers \\
\end{tabular} & \begin{tabular}{|l|} 
Dementia \\
unspecified
\end{tabular} & $\begin{array}{l}\mathrm{N}= \\
\text { Age: no } \\
\text { Sex: no } \\
\text { \% majority race: no } \\
\text { Education: no }\end{array}$ & None & $\begin{array}{l}\mathrm{N}=45 \\
\text { Age: no } \\
\text { Sex: no } \\
\text { \% majority race: no } \\
\text { Education: no } \\
\text { Relationship: no }\end{array}$ & None & NA & $\mathrm{NA}$ & 6 weeks & None & $\begin{array}{l}\text { IC: } \\
\text { The relatives } \\
\text { stress scale } \\
\text { TACI }\end{array}$ \\
\hline
\end{tabular}




\begin{tabular}{|c|c|c|c|c|c|c|c|c|c|c|c|c|c|}
\hline $\begin{array}{l}\text { Gormley, } \\
2001^{139} \\
(11395344) \\
\text { UK } \\
\text { High ROB }\end{array}$ & $\begin{array}{l}\text { Brief behavior } \\
\text { management } \\
\text { training } \\
\text { program, } 4 \\
\text { home sessions }\end{array}$ & $\begin{array}{l}\text { Attention } \\
\text { control: } \\
\text { sessions about } \\
\text { general care } \\
\text { questions }\end{array}$ & \begin{tabular}{|l|} 
Community \\
RCT \\
Caregivers
\end{tabular} & \begin{tabular}{|l|} 
Dementia \\
unspecified
\end{tabular} & $\begin{array}{l}\mathrm{N}= \\
\text { Age: yes } \\
\text { Sex: yes } \\
\text { \% majority race: no } \\
\text { Education: no }\end{array}$ & None & $\begin{array}{l}\mathrm{N}=62 \\
\text { Age: yes } \\
\text { Sex: yes } \\
\text { \% majority race: no } \\
\text { Education: no } \\
\text { Relationship: no }\end{array}$ & None & NA & NA & 8 weeks & $\begin{array}{l}\text { BEHAVE- } \\
\text { AD; } \\
\text { MMSE; } \\
\text { Blessed } \\
\text { Dementia } \\
\text { Rating } \\
\text { Scale; } \\
\text { Zarit Burden } \\
\text { Interview }\end{array}$ & None \\
\hline $\begin{array}{l}\text { Hepburn, } \\
2001^{132} \\
(11347790) \\
\text { USA } \\
\text { High ROB }\end{array}$ & $\begin{array}{l}\text { Group } \\
\text { caregiving role- } \\
\text { training and } \\
\text { mastery- } \\
\text { focused } \\
\text { coaching } \\
7 \text { weekly } 2 \text { hour } \\
\text { sessions }\end{array}$ & Wait-list control & \begin{tabular}{|l|} 
Community \\
RCT \\
Caregivers
\end{tabular} & \begin{tabular}{|l|} 
Dementia \\
unspecified
\end{tabular} & $\begin{array}{l}\mathrm{N}=117 \\
\text { Age: NR } \\
\text { Sex: NR } \\
\text { \% majority race: NR } \\
\text { Education: NR }\end{array}$ & None & $\begin{array}{l}\text { IC N=117 } \\
\text { IC Age: yes } \\
\text { IC Sex: yes } \\
\text { IC \% majority race: } \\
\text { yes } \\
\text { IC Education: yes } \\
\text { IC Relation to } \\
\text { PLWD: yes }\end{array}$ & IC: SES & NA & NA & 5 months & None & $\begin{array}{l}\text { BACS } \\
\text { Revised Zarit } \\
\text { burden scale } \\
\text { CESD }\end{array}$ \\
\hline $\begin{array}{l}\text { Burgener, } \\
1998^{140} \\
(9708136) \\
\text { US } \\
\text { Pilot }\end{array}$ & $\begin{array}{l}\text { Individual } \\
\text { education } \\
\text { I1: dementia } \\
\text { education only } \\
\text { 12: behavioral } \\
\text { education only } \\
\text { 13: Both I1 and } \\
\text { 12 training }\end{array}$ & No training & \begin{tabular}{|l|} 
Community \\
RCT \\
Caregivers
\end{tabular} & \begin{tabular}{|l|} 
Moderate to severe \\
Alzheimer's or \\
multi-infarct \\
dementia
\end{tabular} & $\begin{array}{l}\mathrm{N}= \\
\text { Age: yes } \\
\text { Sex: yes } \\
\text { \% majority race: no } \\
\text { Education: yes }\end{array}$ & None & $\begin{array}{l}\mathrm{N}=54 \\
\text { Age: yes } \\
\text { Sex: yes } \\
\text { \% majority race: no } \\
\text { Education: yes }\end{array}$ & Duration & NA & NA & $\begin{array}{l}6 \text { month post- } \\
\text { intervention }\end{array}$ & $\begin{array}{l}\text { Dementia } \\
\text { Behavior } \\
\text { Disturbance; } \\
\text { ADL }\end{array}$ & $\begin{array}{l}\text { Alzheimer's } \\
\text { Disease } \\
\text { Knowledge } \\
\text { Test; } \\
\text { Relative } \\
\text { Stress Scale }\end{array}$ \\
\hline $\begin{array}{l}\text { Robinson, } \\
\text { 1994141 } \\
(7993133) \\
\text { US } \\
\text { Small sample }\end{array}$ & $\begin{array}{l}\text { 11: Behavior } \\
\text { management } \\
\text { skill training } \\
\text { 12: Social skills } \\
\text { training to } \\
\text { mobilizing a } \\
\text { social network. } \\
\text { Both } 690 \text {-min } \\
\text { sessions over } 2 \\
\text { weeks; } \\
\text { individual }\end{array}$ & No training & $\begin{array}{l}\text { Community } \\
\text { RCT } \\
\text { Caregivers }\end{array}$ & \begin{tabular}{|l|} 
Dementia \\
unspecified
\end{tabular} & None & None & $\begin{array}{l}\mathrm{N}=33 \\
\text { Age: yes } \\
\text { Sex: no } \\
\text { \% majority race: no } \\
\text { Education: yes } \\
\text { Spouse/partner }\end{array}$ & None & NA & NA & $\begin{array}{l}1 \text { month post- } \\
\text { intervention }\end{array}$ & & $\begin{array}{l}\text { IC: } \\
\text { Montgomery } \\
\text { objective and } \\
\text { subjective } \\
\text { burden } \\
\text { Attitude } \\
\text { toward adult } \\
\text { day care, } \\
\text { asking for } \\
\text { help }\end{array}$ \\
\hline
\end{tabular}

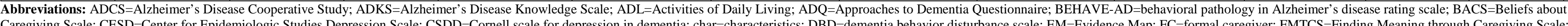

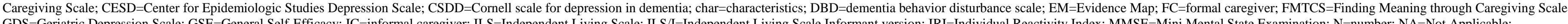

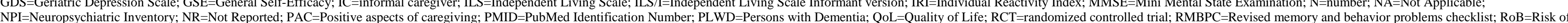
Bias; RS=Reporting Status; SES=socioeconomic status; SSCQ=Short Sense of Competence Questionnaire; TACI= Thomas Assessment of Communication Inadequacy; ZBI=Zarit caregiver burden interview 


\section{Family Involvement}

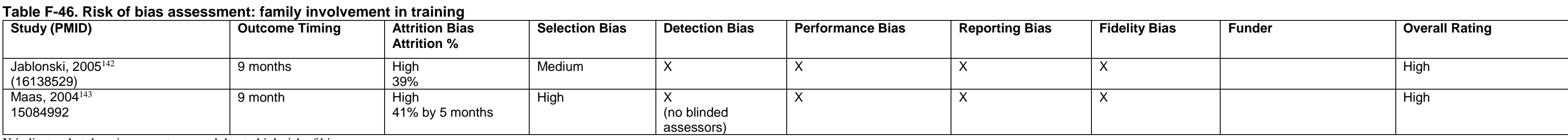

Abbreviations: PMID=PubMed Identification Number

\section{Evidence Map: Family Involvement}

\begin{tabular}{|c|c|c|c|c|c|c|c|c|c|c|c|c|c|}
\hline $\begin{array}{l}\text { Study (PMID) } \\
\text { Country } \\
\text { EM Reason }\end{array}$ & Intervention & Comparison & $\begin{array}{l}\text { Setting } \\
\text { and Design } \\
\\
\text { Setting } \\
\text { Design } \\
\text { Cluster N } \\
\text { Participants } \\
\text { Random- } \\
\text { ized N }\end{array}$ & $\begin{array}{l}\text { PLWD } \\
\text { Dementia } \\
\text { Characteristics }\end{array}$ & $\begin{array}{l}\text { PLWD } \\
\text { Non-Disease Char } \\
\text { PLWD N } \\
\text { PLWD Age (mean) } \\
\text { PLWD Sex (\% } \\
\text { female) } \\
\text { PLWD Race (\% } \\
\text { majority) } \\
\text { PLWD Education } \\
\text { (mean years) }\end{array}$ & $\begin{array}{l}\text { PLWD } \\
\text { Non-Disease } \\
\text { Char Reporting } \\
\text { Status (RS) } \\
\\
\text { PLWD SES } \\
\text { PLWD Prior } \\
\text { Disability } \\
\text { PLWD } \\
\text { Household } \\
\text { Characteristics } \\
\text { PLWD Health } \\
\text { Insurance } \\
\text { PLWD Detailed } \\
\text { Race Information }\end{array}$ & $\begin{array}{l}\text { Informal Caregiver } \\
\text { (IC) } \\
\text { Characteristics } \\
\text { IC N } \\
\text { IC Age (mean) } \\
\text { IC Sex (\% female) } \\
\text { IC Race (\% } \\
\text { majority) } \\
\text { IC Education } \\
\text { (mean years) } \\
\text { IC Relation to } \\
\text { PLWD (\% majority) }\end{array}$ & $\begin{array}{l}\text { Informal Caregiver } \\
\text { (IC) Char. RS } \\
\text { IC Duration } \\
\text { IC Living With } \\
\text { PLWD } \\
\text { IC Payment } \\
\text { IC Health Status } \\
\text { IC Dementia } \\
\text { Family History } \\
\text { IC Employment } \\
\text { Status } \\
\text { IC Training }\end{array}$ & $\begin{array}{l}\text { Formal Caregiver } \\
\text { (FC) } \\
\text { Characteristics } \\
\text { FC N } \\
\text { FC Age (mean) } \\
\text { FC Sex (\% } \\
\text { female) } \\
\text { FC Race (\% } \\
\text { majority) } \\
\text { FC Education } \\
\text { (mean years) }\end{array}$ & $\begin{array}{l}\text { Formal } \\
\text { Caregiver (FC) } \\
\text { Char. RS } \\
\text { FC Health } \\
\text { Status } \\
\text { FC Training } \\
\text { FC Education } \\
\text { FC Position } \\
\text { FC Length of } \\
\text { Service }\end{array}$ & $\begin{array}{l}\text { Outcome } \\
\text { Timing(s) }\end{array}$ & $\begin{array}{l}\text { PLWD } \\
\text { Outcomes }\end{array}$ & $\begin{array}{l}\text { Caregiver } \\
\text { Outcomes } \\
\text { IC: } \\
\text { FC: }\end{array}$ \\
\hline $\begin{array}{l}\text { Bramble, } \\
2011^{144} \\
(21702706) \\
\text { Australia } \\
\text { Pilot }\end{array}$ & $\begin{array}{l}\text { FIC: An } \\
\text { education } \\
\text { programme } \\
\text { delivered to } \\
\text { staff by the } \\
\text { research team. }\end{array}$ & Not reported & $\begin{array}{l}\text { Long term } \\
\text { care facility } \\
\text { Quasi } \\
58 \text { Caregiver } \\
\text { dyads }\end{array}$ & NA & NA & NA & $\begin{array}{l}\mathrm{N}=57 \\
\text { Age: Yes } \\
\text { Sex: Yes } \\
\text { Race: No } \\
\text { Education: Yes } \\
\text { Relationship: Yes }\end{array}$ & $\begin{array}{l}\text { Employment: Yes } \\
\text { Marital status: Yes }\end{array}$ & $\begin{array}{l}\text { N=58 } \\
\text { Age: Yes } \\
\text { Sex: Yes } \\
\text { \% majority race: } \\
\text { No } \\
\text { Education: Yes }\end{array}$ & $\begin{array}{l}\text { Occupation: Yes } \\
\text { Employment: Yes }\end{array}$ & 9 months & NA & $\begin{array}{l}\text { Staff } \\
\text { knowledge; } \\
\text { Stress; staff } \\
\text { attitude towards } \\
\text { family CGs; } \\
\text { Caregiving } \\
\text { roles; }\end{array}$ \\
\hline $\begin{array}{l}\text { Robison, } \\
2007^{145} \\
(17766671) \\
\text { USA } \\
\text { Pilot }\end{array}$ & $\begin{array}{l}\text { The partners in } \\
\text { caregiving in a } \\
\text { special care } \\
\text { environment } \\
\text { program: Staff } \\
\text { and family } \\
\text { received } \\
\text { training } \\
\text { sessions on } \\
\text { communication } \\
\text { and conflict- } \\
\text { resolution } \\
\text { techniques }\end{array}$ & Not reported & $\begin{array}{l}\text { Nursing } \\
\text { homes } \\
\text { Cluster RCT } \\
20 \text { nursing } \\
\text { homes }\end{array}$ & NR & $\begin{array}{l}\text { N=388 } \\
\text { Age: Yes } \\
\text { Sex: Yes } \\
\text { Race: Yes } \\
\text { Education: Yes }\end{array}$ & None & NA & NA & $\begin{array}{l}\text { N=384 } \\
\text { Age: Yes } \\
\text { Sex: Yes } \\
\text { Race: Yes } \\
\text { Education: Yes }\end{array}$ & None & $\begin{array}{l}2 \& 6 \text { months } \\
\text { follow up }\end{array}$ & NA & $\begin{array}{l}\text { IC: Frequency } \\
\text { of conflicts; } \\
\text { Staff empathy; } \\
\text { Negative staff } \\
\text { behavior; } \\
\text { Engagement in } \\
\text { NH activities; } \\
\text { Burden; } \\
\text { Depression } \\
\text { FC: Frequency } \\
\text { of conflicts; } \\
\text { Depression; } \\
\text { Job burnout; } \\
\text { Job satisfaction }\end{array}$ \\
\hline
\end{tabular}




\begin{tabular}{|c|c|c|c|c|c|c|c|c|c|c|c|c|c|}
\hline $\begin{array}{l}\text { Jablonski, } \\
2005^{142} \\
(16138529) \\
\text { USA } \\
\text { High RoB }\end{array}$ & $\begin{array}{l}\text { Family } \\
\text { involvement in } \\
\text { care protocol to } \\
\text { help family } \\
\text { negotiate a } \\
\text { partnership with } \\
\text { formal staff }\end{array}$ & Not reported & \begin{tabular}{|l|} 
Nursing \\
home \\
RCT \\
164 PLWD \\
\end{tabular} & Not reported & $\begin{array}{l}\mathrm{N}=164 \\
\text { Age: Yes } \\
\text { Sex: Yes } \\
\text { Race: Yes } \\
\text { Education: Yes }\end{array}$ & $\begin{array}{l}\text { Marital status: Yes } \\
\text { Occupation: Yes }\end{array}$ & NA & NA & NA & NA & $\begin{array}{l}\text { 2months; } 4 \\
\text { months, } 6 \\
\text { months }\end{array}$ & $\begin{array}{l}\text { Functional } \\
\text { abilities: } \\
\text { FAC }\end{array}$ & NA \\
\hline $\begin{array}{l}\text { Maas, 2004143 } \\
15084992 \\
\text { US } \\
\text { High ROB }\end{array}$ & $\begin{array}{l}\text { Family } \\
\text { involvement in } \\
\text { care protocol to } \\
\text { help family } \\
\text { negotiate a } \\
\text { partnership with } \\
\text { formal staff }\end{array}$ & Usual Care & \begin{tabular}{|l|} 
Nursing \\
home \\
Quasi- \\
experimental \\
Paired \\
nursing \\
homes \\
assigned
\end{tabular} & $\begin{array}{l}\text { PLWD in special } \\
\text { care units for } \\
\text { dementia }\end{array}$ & $\begin{array}{l}\text { N=185 } \\
\text { Age: no } \\
\text { Sex: no } \\
\text { Race: no } \\
\text { Education: no }\end{array}$ & Time in $\mathrm{NH}$ & $\begin{array}{l}\mathrm{N}=185 \\
\text { Age: Yes } \\
\text { Sex: Yes } \\
\text { \% majority race: yes } \\
\text { Education: Yes } \\
\text { Relationship: Yes }\end{array}$ & None & NA & NA & 9 months & None & $\begin{array}{l}\text { Family } \\
\text { Perceptions of } \\
\text { Caregiving } \\
\text { Role } \\
\text { Family } \\
\text { Perceptions of } \\
\text { Care Tool } \\
\text { Staff } \\
\text { Perceptions of } \\
\text { Caregiving } \\
\text { Role } \\
\text { Caregiver } \\
\text { Stress } \\
\text { Inventory } \\
\text { Attitudes } \\
\text { Toward } \\
\text { Families } \\
\text { Checklist }\end{array}$ \\
\hline $\begin{array}{l}\text { McCallion, } \\
1999^{146} \\
\text { US } \\
\text { Pilot }\end{array}$ & $\begin{array}{l}\text { Family training } \\
\text { in nonverbal } \\
\text { communication } \\
\text { and structuring } \\
\text { family visits }\end{array}$ & $\begin{array}{l}\text { Usual care } \\
\text { activities }\end{array}$ & $\begin{array}{l}\text { Nursing } \\
\text { home } \\
\text { RCT } \\
66 \text { dyads }\end{array}$ & $\begin{array}{l}\text { Moderate dementia } \\
\text { with behavioral } \\
\text { problems }\end{array}$ & $\begin{array}{l}\mathrm{N}=66 \\
\text { Age: Yes } \\
\text { Sex: Yes } \\
\text { Race: Yes } \\
\text { Education: no }\end{array}$ & None & $\begin{array}{l}\text { N=66 } \\
\text { Age: Yes } \\
\text { Sex: Yes } \\
\text { Race: yes } \\
\text { Education: Yes } \\
\text { Relationship: Yes }\end{array}$ & None & NA & NA & $\begin{array}{l}3 \text { and } 6 \\
\text { months }\end{array}$ & $\begin{array}{l}\text { MOSES } \\
\text { CSDD } \\
\text { CMAI } \\
\text { GIPB } \\
\text { medication } \\
\text { use }\end{array}$ & $\begin{array}{l}\text { IC: DMSS } \\
\text { CHS-M } \\
\text { Visit } \\
\text { satisfaction }\end{array}$ \\
\hline
\end{tabular}

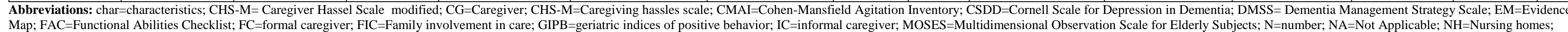
Map; FAC=Functional Abilities Checklist; FC=formal caregiver; FIC=Family involvement in care; GIPB=geriatric indices of positive behavior; IC=informal caregiver; MOSES=Multidimensional Ob
NR=Not Reported; PMID=PubMed Identification Number; PLWD=Persons with Dementia; RCT=Randomized Controlled Trial; RoB=Risk of Bias; RS=Reporting Status; SES=socioeconomic status

\section{Multitier Training Interventions}

\begin{tabular}{|c|c|c|c|c|c|c|c|c|}
\hline Study (PMID) & Outcome Timing & $\begin{array}{l}\text { Attrition Bias } \\
\text { Attrition \% }\end{array}$ & Selection Bias & Detection Bias & Performance Bias & Reporting Bias & Fidelity Bias & Overall Rating \\
\hline Kunik, 2017'147 (27743840) & $3,6,12$ month & Medium & Low & Low & Medium & Low & Low & Medium \\
\hline Teri $2005^{148}(16326662)$ & $\begin{array}{l}2 \text { months } \\
6 \text { months }\end{array}$ & $\begin{array}{l}\text { Low } \\
2 \text { months: } 12.6 \%\end{array}$ & Medium & Medium & Medium & Low & Low & Medium \\
\hline
\end{tabular}


Table F-49. Characteristics of included studies: caregiver focused training intervention

\begin{tabular}{|c|c|c|c|c|c|c|c|c|c|c|c|c|c|}
\hline $\begin{array}{l}\text { Study (PMID) } \\
\text { Country } \\
\text { RoB* } \\
\text { Type }\end{array}$ & $\begin{array}{l}\text { Intervention } \\
\text { Intervention } \\
\text { Focus } \\
\text { Theoretical } \\
\text { Model } \\
\text { Delivery } \\
\text { Person } \\
\text { Intervention } \\
\text { Target } \\
\text { Recipient } \\
\text { Mode } \\
\text { Components } \\
\text { Frequency } \\
\text { Duration }\end{array}$ & $\begin{array}{l}\text { Comparison } \\
\text { Target } \\
\text { Mode } \\
\text { Components } \\
\text { Frequency } \\
\text { Duration }\end{array}$ & $\begin{array}{l}\text { Setting } \\
\text { and Design } \\
\text { Setting } \\
\text { Design } \\
\text { Cluster N } \\
\text { Participants } \\
\text { Randomized } \\
\text { N }\end{array}$ & $\begin{array}{l}\text { PLWD } \\
\text { Dementia } \\
\text { Characteristics } \\
\text { Dementia Types } \\
\text { Dementia Severity } \\
\text { Diagnostic } \\
\text { Criteria } \\
\text { Age of Diagnosis }\end{array}$ & $\begin{array}{l}\text { PLWD } \\
\text { Non-Disease Char } \\
\text { PLWD N } \\
\text { PLWD Age (mean) } \\
\text { PLWD Sex (\% } \\
\text { female) } \\
\text { PLWD Race (\% } \\
\text { majority) } \\
\text { PLWD Education } \\
\text { (mean years) }\end{array}$ & $\begin{array}{l}\text { PLWD } \\
\text { Non-Disease } \\
\text { Char Reporting } \\
\text { Status (RS) } \\
\text { PLWD SES } \\
\text { PLWD Prior } \\
\text { Disability } \\
\text { PLWD } \\
\text { Household } \\
\text { Characteristics } \\
\text { PLWD Health } \\
\text { Insurance } \\
\text { PLWD Detailed } \\
\text { Race Information }\end{array}$ & $\begin{array}{l}\text { Informal Caregiver } \\
\text { (IC) } \\
\text { Characteristics } \\
\text { IC N } \\
\text { IC Age (mean) } \\
\text { IC Sex (\% female) } \\
\text { IC Race (\% } \\
\text { majority) } \\
\text { IC Education } \\
\text { (mean years) } \\
\text { IC Relation to } \\
\text { PLWD (\% majority) }\end{array}$ & $\begin{array}{l}\text { Informal Caregiver } \\
\text { (IC) Char. RS } \\
\text { IC Duration } \\
\text { IC Living With } \\
\text { PLWD } \\
\text { IC Payment } \\
\text { IC Health Status } \\
\text { IC Dementia } \\
\text { Family History } \\
\text { IC Employment } \\
\text { Status } \\
\text { IC Training }\end{array}$ & $\begin{array}{l}\text { Formal Caregiver } \\
\text { (FC) } \\
\text { Characteristics } \\
\text { FC N } \\
\text { FC Age (mean) } \\
\text { FC Sex (\% } \\
\text { female) } \\
\text { FC Race (\% } \\
\text { majority) } \\
\text { FC Education } \\
\text { (mean years) }\end{array}$ & $\begin{array}{l}\text { Formal } \\
\text { Caregiver (FC) } \\
\text { Char. RS } \\
\text { FC Health } \\
\text { Status } \\
\text { FC Training } \\
\text { FC Education } \\
\text { FC Position } \\
\text { FC Length of } \\
\text { Service }\end{array}$ & $\begin{array}{l}\text { Outcome } \\
\text { Timing(s) }\end{array}$ & $\begin{array}{l}\text { PLWD } \\
\text { Outcomes }\end{array}$ & $\begin{array}{l}\text { Caregiver } \\
\text { Outcomes } \\
\text { IC: } \\
\text { FC: }\end{array}$ \\
\hline $\begin{array}{l}\text { Teri 2005'148 } \\
(16326662) \\
\text { US } \\
\text { Medium } \\
\text { Exploratory } \\
\text { Teri 2005'149 } \\
(16199404) \\
\text { Teri 2012150 } \\
\text { (22247431) }\end{array}$ & $\begin{array}{l}\text { STAR-C: } 8 \\
\text { weekly sessions } \\
\text { between } \\
\text { community } \\
\text { consultants and } \\
\text { caregivers, } \\
\text { followed by four } \\
\text { monthly phone } \\
\text { calls; to train the } \\
\text { caregivers to } \\
\text { give consultants } \\
\text { the freedom to } \\
\text { use clinical } \\
\text { judgment and } \\
\text { modify the order } \\
\text { of session } \\
\text { topics in } \\
\text { response to a } \\
\text { caregiver's } \\
\text { needs }\end{array}$ & $\begin{array}{l}\text { Routine } \\
\text { medical car } \\
\text { (RMC)e }\end{array}$ & $\begin{array}{l}\text { resided in a } \\
\text { home setting } \\
\text { outside of a } \\
\text { nursing home } \\
\text { or assisted } \\
\text { living facility } \\
\text { RCT } \\
95 \text { PLWD and } \\
\text { caregivers }\end{array}$ & $\begin{array}{l}\text { Primary physician } \\
\text { diagnosis of } \\
\text { probable or } \\
\text { possible AD with } \\
\text { mean MMSE score } \\
\text { of } 14\end{array}$ & $\begin{array}{l}\mathrm{N}=95 \\
66 \% \text { Female } \\
80 \text { years } \\
\text { Race yes } \\
\text { Education yes }\end{array}$ & None & NA & $\begin{array}{l}\mathrm{N}=95 \\
70 \% \text { Female } \\
65 \text { years } \\
\text { Race yes } \\
\text { Education yes }\end{array}$ & NA & NA & $\begin{array}{l}2 \text { months } \\
6 \text { months }\end{array}$ & $\begin{array}{l}\text { NPI } \\
\text { RMBPC } \\
\text { QoL-AD; }\end{array}$ & \begin{tabular}{|l|} 
CES-D \\
HDRS \\
Caregiver \\
Sleep \\
Questionnaire \\
PSS \\
SSCQ
\end{tabular} \\
\hline
\end{tabular}

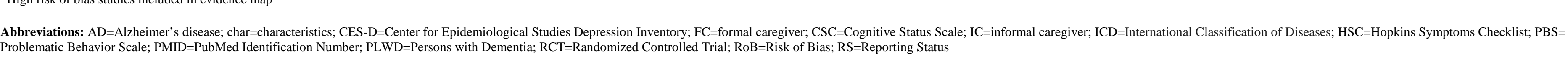


Table F-50. PLWD outcomes summary low and medium risk of bias studies: caregiver focused training intervention

\begin{tabular}{|c|c|c|c|c|}
\hline $\begin{array}{l}\text { Study (PMID) } \\
\text { Comparison } \\
\text { RoB } \\
\text { Category }\end{array}$ & $\begin{array}{l}\text { Outcome } \\
\text { Timing }\end{array}$ & \begin{tabular}{|l|} 
Intervention \\
\end{tabular} & Comparator & p-Value \\
\hline $\begin{array}{l}\text { Teri 2005' } 205^{148}(16326662) \\
\text { STAR vs RMC } \\
\text { Medium } \\
\text { Exploratory }\end{array}$ & $\begin{array}{l}\text { Mean difference (SD) } \\
\text { Caregiver depression } \\
\text { CES-D } \\
6 \text { months }\end{array}$ & $12.5(7.7)$ & $15.8(10.5)$ & 0.046 \\
\hline $\begin{array}{l}\text { Teri 2005'148 (16326662) } \\
\text { STAR vs RMC } \\
\text { Medium } \\
\text { Exploratory }\end{array}$ & $\begin{array}{l}\text { Mean difference (SD) } \\
\text { Caregiver depression } \\
\text { HDRS } \\
6 \text { months }\end{array}$ & $6.7(3.9)$ & $8.5(5.7)$ & 0.284 \\
\hline $\begin{array}{l}\text { Teri 2005'148 (16326662) } \\
\text { STAR vs RMC } \\
\text { Medium } \\
\text { Exploratory }\end{array}$ & $\begin{array}{l}\text { Mean difference (SD) } \\
\text { Caregiver burden } \\
6 \text { months }\end{array}$ & $21.4(12.5)$ & $25.8(13.7)$ & 0.011 \\
\hline $\begin{array}{l}\text { Teri 2005'148 (16326662) } \\
\text { STAR vs RMC } \\
\text { Medium } \\
\text { Exploratory }\end{array}$ & $\begin{array}{l}\text { Mean difference (SD) } \\
\text { Caregiver reaction } \\
6 \text { months }\end{array}$ & $21.9(15.6)$ & $23.4(14.5)$ & 0.024 \\
\hline $\begin{array}{l}\text { Teri 2005'148 (16326662) } \\
\text { STAR vs RMC } \\
\text { Medium } \\
\text { Exploratory }\end{array}$ & $\begin{array}{l}\text { Mean difference (SD) } \\
\text { Caregiver sleep questionnaire } \\
6 \text { months }\end{array}$ & $9.1(4.6)$ & $9.1(5.2)$ & 0.124 \\
\hline $\begin{array}{l}\text { Teri 2005'148 (16326662) } \\
\text { STAR vs RMC } \\
\text { Medium } \\
\text { Exploratory }\end{array}$ & $\begin{array}{l}\text { Mean difference (SD) } \\
\text { PLWD_QoL } \\
6 \text { months }\end{array}$ & $28.4(5.4)$ & $28.2(4.6)$ & 0.049 \\
\hline $\begin{array}{l}\text { Teri 2005'18 (16326662) } \\
\text { STAR vs RMC } \\
\text { Medium } \\
\text { Exploratory }\end{array}$ & $\begin{array}{l}\text { Mean difference (SD) } \\
\text { PLWD RMBPC-memory subscale } \\
6 \text { months }\end{array}$ & $2.8(0.8)$ & $3.1(1.0)$ & 0.070 \\
\hline
\end{tabular}

Abbreviations: CES-D=Center for Epidemiological Studies Depression Inventory; CI=Confidence Interval; NR=Not Reported; PMID=PubMed Identification Number; RoB=Risk of Bia

Table F-51. Summary of strength of evidence for PLWD outcomes: caregiver focused training intervention

\begin{tabular}{|c|c|c|c|c|c|c|c|c|}
\hline $\begin{array}{l}\text { Comparison } \\
\text { Outcome }\end{array}$ & Timing & $\begin{array}{l}\text { \# Studies/ Design } \\
\text { ( } \mathrm{n} \text { analyzed) }\end{array}$ & Finding or Summary Statistic & Study Limitations & Consistency & Directness & Precision & $\begin{array}{l}\text { Overall Gradel } \\
\text { Conclusion }\end{array}$ \\
\hline $\begin{array}{l}\text { Consultation vs Control } \\
\text { Nursing home admission }\end{array}$ & 12 months & $1 \mathrm{RCT}(\mathrm{n}=84)$ & $\begin{array}{l}\text { Nursing home admissions showed no difference between the } \\
\text { comparison groups. }\end{array}$ & Moderate & Unknown & Direct & Imprecise & Insufficient \\
\hline $\begin{array}{l}\text { Consultation vs Control } \\
\text { Symptom management } \\
\text { self-efficacy score }\end{array}$ & 12 months & $1 \mathrm{RCT}(\mathrm{n}=84)$ & $\begin{array}{l}\text { Symptom management self-efficacy scores were not different } \\
\text { between the comparison groups }\end{array}$ & Moderate & Unknown & Direct & Imprecise & Insufficient \\
\hline $\begin{array}{l}\text { Consultation vs Control } \\
\text { Support service self- } \\
\text { efficacy }\end{array}$ & 12 months & $1 \mathrm{RCT}(\mathrm{n}=84)$ & $\begin{array}{l}\text { Support service self-efficacy was not different between the } \\
\text { comparison groups }\end{array}$ & Moderate & Unknown & Direct & Imprecise & Insufficient \\
\hline $\begin{array}{l}\text { Consultation vs Control } \\
\text { CES-D score }\end{array}$ & 12 months & $1 \mathrm{RCT}(\mathrm{n}=84)$ & $\begin{array}{l}\text { CES-D scores were not different between the comparison } \\
\text { groups }\end{array}$ & Moderate & Unknown & Direct & Imprecise & Insufficient \\
\hline $\begin{array}{l}\text { Consultation vs Control } \\
\text { Zarit burden score }\end{array}$ & 12 months & $1 \mathrm{RCT}(\mathrm{n}=84)$ & $\begin{array}{l}\text { Zarit burden scores were not different between the comparison } \\
\text { groups }\end{array}$ & Moderate & Unknown & Direct & Imprecise & Insufficient \\
\hline $\begin{array}{l}\text { Consultation vs Control } \\
\text { Hopkins symptom } \\
\text { checklist score }\end{array}$ & 12 months & $1 \mathrm{RCT}(\mathrm{n}=84)$ & $\begin{array}{l}\text { Hopkins symptom checklist scores were not different between } \\
\text { the comparison groups }\end{array}$ & Moderate & Unknown & Direct & Imprecise & Insufficient \\
\hline
\end{tabular}

the 


\section{Evidence Map: Multitier Training Interventions}

\begin{tabular}{|c|c|c|c|c|c|c|c|c|c|c|c|c|c|}
\hline $\begin{array}{l}\text { Study (PMID) } \\
\text { Country } \\
\text { EM Reason }\end{array}$ & Intervention & Comparison & $\begin{array}{l}\text { Setting } \\
\text { and Design } \\
\text { Setting } \\
\text { Design } \\
\text { Cluster N } \\
\text { Participants } \\
\text { Random- } \\
\text { ized N }\end{array}$ & $\begin{array}{l}\text { PLWD } \\
\text { Dementia } \\
\text { Characteristics }\end{array}$ & $\begin{array}{l}\text { PLWD } \\
\text { Non-Disease Char } \\
\text { PLWD N } \\
\text { PLWD Age (mean) } \\
\text { PLWD Sex (\% } \\
\text { female) } \\
\text { PLWD Race (\% } \\
\text { majority) } \\
\text { PLWD Education } \\
\text { (mean years) }\end{array}$ & $\begin{array}{l}\text { PLWD } \\
\text { Non-Disease } \\
\text { Char Reporting } \\
\text { Status (RS) } \\
\text { PLWD SES } \\
\text { PLWD Prior } \\
\text { Disability } \\
\text { PLWD } \\
\text { Household } \\
\text { Characteristics } \\
\text { PLWD Health } \\
\text { Insurance } \\
\text { PLWD Detailed } \\
\text { Race Information }\end{array}$ & $\begin{array}{l}\text { Informal Caregiver } \\
\text { (IC) } \\
\text { Characteristics } \\
\text { IC N } \\
\text { IC Age (mean) } \\
\text { IC Sex (\% female) } \\
\text { IC Race (\% } \\
\text { majority) } \\
\text { IC Education } \\
\text { (mean years) } \\
\text { IC Relation to } \\
\text { PLWD (\% majority) }\end{array}$ & $\begin{array}{l}\text { Informal Caregiver } \\
\text { (IC) Char. RS } \\
\text { IC Duration } \\
\text { IC Living With } \\
\text { PLWD } \\
\text { IC Payment } \\
\text { IC Health Status } \\
\text { IC Dementia } \\
\text { Family History } \\
\text { IC Employment } \\
\text { Status } \\
\text { IC Training }\end{array}$ & 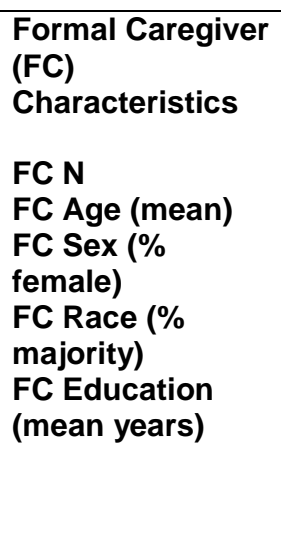 & $\begin{array}{l}\text { Formal } \\
\text { Caregiver (FC) } \\
\text { Char. RS } \\
\text { FC Health } \\
\text { Status } \\
\text { FC Training } \\
\text { FC Education } \\
\text { FC Position } \\
\text { FC Length of } \\
\text { Service }\end{array}$ & \begin{tabular}{|l|} 
Outcome \\
Timing(s)
\end{tabular} & $\begin{array}{l}\text { PLWD } \\
\text { Outcomes }\end{array}$ & $\begin{array}{l}\text { Caregiver } \\
\text { Outcomes } \\
\text { IC: } \\
\text { FC: }\end{array}$ \\
\hline $\begin{array}{l}\text { Livingston } \\
2019^{151} \\
\text { (30221615) } \\
\text { UK } \\
\text { Pilot }\end{array}$ & $\begin{array}{l}\text { DREAM-START } \\
\text { Intervention: } \\
\text { cognitive- } \\
\text { behavioral } \\
\text { components, } \\
\text { including } \\
\text { psychoeducatio } \\
\text { n, light therapy, } \\
\text { establishing a } \\
\text { new sleep- } \\
\text { wake schedule } \\
\text { (based on } \\
\text { actiwatch data), } \\
\text { behavioral } \\
\text { activation, } \\
\text { relaxation, and } \\
\text { coping skills for } \\
\text { families }\end{array}$ & $\begin{array}{l}\text { Treatment as } \\
\text { usual }\end{array}$ & $\begin{array}{l}\text { Community- } \\
\text { dwelling } \\
\text { RCT } \\
62 \text { PLWD }\end{array}$ & $\begin{array}{l}\text { Clinical diagnosis } \\
\text { of dementia and a } \\
\text { SDI item score } \geq 4\end{array}$ & $\begin{array}{l}\mathrm{N}=62 \\
\text { Age: yes } \\
\text { Sex: yes } \\
\text { Race: yes } \\
\text { Education: yes }\end{array}$ & None & $\begin{array}{l}\mathrm{N}=62 \\
\text { Age: yes } \\
\text { Sex: yes } \\
\text { Race: yes } \\
\text { Education: yes }\end{array}$ & None & NA & NA & 3 months & $\begin{array}{l}\text { referral rates; } \\
\text { follow-up rates } \\
\text { at three } \\
\text { months; all } \\
\text { psychotropic } \\
\text { medication } \\
\text { prescription (to } \\
\text { define rescue } \\
\text { medication's } \\
\text { role); reported } \\
\text { side effects: } \\
\text { co-morbid } \\
\text { physical } \\
\text { illnesses and } \\
\text { patient falls } \\
\text { sleep via } \\
\text { actigraphy } \\
\text { ESS, }\end{array}$ & $\begin{array}{l}\text { PSQI } \\
\text { SCl } \\
\text { HADS } \\
\text { ZBI } \\
\text { HSQ-12 }\end{array}$ \\
\hline $\begin{array}{l}\text { Kinnunen } \\
2018^{152} \\
(30538021) \\
\text { UK } \\
\text { Pilot } \\
\text { Livingston } \\
2018^{153} \\
(30221615)\end{array}$ & $\begin{array}{l}\text { DREAMS } \\
\text { START: } 6 \\
\text { sessions of } \\
\text { manual-based } \\
\text { intervention for } \\
\text { caregiver's } \\
\text { understanding } \\
\text { sleep and } \\
\text { dementia, } \\
\text { comprising a } \\
\text { cognitive- } \\
\text { behavioral } \\
\text { component and } \\
\text { light therapy }\end{array}$ & $\begin{array}{l}\text { Treatment as } \\
\text { usual }\end{array}$ & $\begin{array}{l}\text { Memory } \\
\text { service } \\
\text { Cluster RCT } \\
62 \text { PLWD }\end{array}$ & $\begin{array}{l}\text { Dementia } \\
\text { diagnosis (any type } \\
\text { and severity) and } \\
\text { sleep disturbances } \\
\text { (who scored } \geq 4 \text { on } \\
\text { at least one } \\
\text { question on SDI) } \\
\text { Sleep Disorders } \\
\text { Inventory) }\end{array}$ & $\begin{array}{l}\mathrm{N}=62 \\
\text { Age: yes } \\
\text { Sex: yes } \\
\text { Race: yes } \\
\text { Education: yes }\end{array}$ & $\begin{array}{l}\text { Annual } \\
\text { Income=yes }\end{array}$ & $\begin{array}{l}\mathrm{N}=62 \\
\text { Age: yes } \\
\text { Sex: yes } \\
\text { Race: yes } \\
\text { Education: yes }\end{array}$ & None & NA & NA & 3 months & $\begin{array}{l}\text { Feasibility } \\
\text { Acceptability } \\
\text { Referrara rate } \\
\text { Follow-up rate } \\
\text { Use of } \\
\text { psychotropic } \\
\text { medications } \\
\text { Adverse } \\
\text { effects and } \\
\text { comorbid } \\
\text { physical } \\
\text { illnesses }\end{array}$ & $\begin{array}{l}\text { Caregiver } \\
\text { burden } \\
\text { depression }\end{array}$ \\
\hline
\end{tabular}




\begin{tabular}{|c|c|c|c|c|c|c|c|c|c|c|c|c|c|}
\hline $\begin{array}{l}\text { Kunik 2017'147 } \\
(27743840) \\
\text { US } \\
\text { High RoB }\end{array}$ & $\begin{array}{l}\text { PAVeD: } 6 \text { to } 8 \\
\text { weekly sessions } \\
\text { of } 45 \text {-minute } \\
\text { home visits. To } \\
\text { give instruction } \\
\text { to caregivers on } \\
\text { recognizing } \\
\text { pain, enhancing } \\
\text { communication, } \\
\text { and making } \\
\text { daily activities } \\
\text { pleasant and } \\
\text { enjoyable, and } \\
\text { at least two } \\
\text { elective } \\
\text { sessions }\end{array}$ & $\begin{array}{l}\text { EU-PC: } 8 \\
\text { weekly } 15- \\
\text { minute phone } \\
\text { calls to query } \\
\text { symptom } \\
\text { severity, } \\
\text { ascertain needs } \\
\text { for immediate } \\
\text { psychiatric care, } \\
\text { and provide } \\
\text { minimal } \\
\text { support. }\end{array}$ & \begin{tabular}{|l|} 
Veterans' \\
Health \\
Administrati \\
on (VHA) \\
outpatient \\
database for \\
persons with \\
a diagnosis \\
of dementia \\
RCT \\
213 PLWD
\end{tabular} & \begin{tabular}{|l|} 
Mild-to-moderate \\
dementia (defined \\
using FAST 2-6 \\
Functional \\
Assessment \\
Staging validated \\
clinician-rated \\
measure of \\
functional decline \\
in dementia)
\end{tabular} & $\begin{array}{l}\mathrm{N}=203 \\
\text { Age: yes } \\
\text { Sex: yes } \\
\text { Race: yes } \\
\text { Education: yes }\end{array}$ & None & $\begin{array}{l}\mathrm{N}=203 \\
\text { Age: yes } \\
\text { Sex: yes } \\
\text { Race: yes } \\
\text { Education: yes }\end{array}$ & NA & NA of $\geq$ & NA & $\begin{array}{l}3 \text { months } \\
6 \text { months } \\
12 \text { months }\end{array}$ & $\begin{array}{l}\text { CMAI } \\
\text { PGPIS } \\
\text { GDS } \\
\text { PES-AD }\end{array}$ & $\begin{array}{l}\text { Caregiver } \\
\text { burden } \\
\text { Caregiver- } \\
\text { patient } \\
\text { relationship } \\
\text { Caregiver } \\
\text { satisfaction } \\
\text { and } \\
\text { perceptions } \\
\text { of } \\
\text { usefulness } \\
\text { of PAVeD }\end{array}$ \\
\hline $\begin{array}{l}\text { Suominen } \\
2015^{154} \\
(26482691) \\
\text { Finland } \\
\text { Small sample }\end{array}$ & $\begin{array}{l}\text { Tailored } \\
\text { nutritional } \\
\text { guidance on the } \\
\text { basis of the } \\
\text { food diaries, the } \\
\text { results of the } \\
\text { weight } \\
\text { measurement, } \\
\text { the home visits } \\
\text { and discussions } \\
\text { with the PLWD } \\
\text { and caregiver }\end{array}$ & $\begin{array}{l}\text { Got a written } \\
\text { guide about } \\
\text { nutrition in older } \\
\text { adults and all } \\
\text { community- } \\
\text { provided normal } \\
\text { care }\end{array}$ & $\begin{array}{l}\text { Community } \\
\text { dwelling } \\
\text { RCT } \\
\text { 99 PLWD }\end{array}$ & $\begin{array}{l}\text { Diagnosis criteria } \\
\text { for a probable AD } \\
\text { diagnosis based on } \\
\text { the NINCDS- } \\
\text { ADRDA } \\
\text { Alzheimer's criteria }\end{array}$ & $\begin{array}{l}\text { N=99 } \\
\text { Age: yes } \\
\text { Sex: yes } \\
\text { Race: no } \\
\text { Education: no }\end{array}$ & None & NA & NA & NA & NA & $\begin{array}{l}6 \text { months } \\
12 \text { months }\end{array}$ & $\begin{array}{l}\text { weight } \\
\text { change; } \\
\text { changes in } \\
\text { protein and } \\
\text { other nutrients } \\
\text { intake, QoL } \\
\text { and rate of } \\
\text { falls. }\end{array}$ & NA \\
\hline $\begin{array}{l}\text { McCurry } \\
2005^{155} \\
\text { (15877554) } \\
\text { US } \\
\text { Small Sample }\end{array}$ & $\begin{array}{l}\text { Written } \\
\text { materials } \\
\text { describing age- } \\
\text { and dementia- } \\
\text { related changes } \\
\text { in sleep and } \\
\text { standard } \\
\text { principles of } \\
\text { good sleep } \\
\text { hygiene. CG } \\
\text { also received } \\
\text { specific } \\
\text { recommendatio } \\
\text { ns for sleep } \\
\text { hygiene } \\
\text { program for the } \\
\text { dementia } \\
\text { patient. PLWDs } \\
\text { to walk daily } \\
\text { and increase } \\
\text { daytime light } \\
\text { exposure with } \\
\text { the use of a } \\
\text { light box }\end{array}$ & $\begin{array}{l}\text { Control PLWD } \\
\text { got general } \\
\text { dementia } \\
\text { education and } \\
\text { caregiver } \\
\text { support }\end{array}$ & $\begin{array}{l}\text { Community- } \\
\text { dwelling } \\
\text { RCT } \\
36 \text { PLWD }\end{array}$ & $\begin{array}{l}\text { PLWDs diagnosed } \\
\text { for probable or } \\
\text { possible AD with } \\
\text { mean MMSE } \\
\text { score } \pm \text { SD of } \\
11.8 \pm 8.4 \text { and at } \\
\text { least one sleep } \\
\text { problem on the } \\
\text { Neuropsychiatric } \\
\text { Inventory Nighttime } \\
\text { Behavior scale }\end{array}$ & $\begin{array}{l}\mathrm{N}=22 \\
\text { Age: yes } \\
\text { Sex: yes } \\
\text { Race: yes } \\
\text { Education: yes }\end{array}$ & None & $\begin{array}{l}\mathrm{N}=22 \\
\text { Age: yes } \\
\text { Sex: yes } \\
\text { Race: yes } \\
\text { Education: yes } \\
\text { Relationship: yes }\end{array}$ & NA & NA & NA & $\begin{array}{l}2 \text { months } \\
6 \text { months }\end{array}$ & $\begin{array}{l}\text { total night } \\
\text { sleep, } \\
\text { percentage of } \\
\text { time asleep, } \\
\text { number of } \\
\text { awakenings, } \\
\text { and duration } \\
\text { of time awake } \\
\text { Light exposure } \\
\text { outcomes } \\
\text { CESD } \\
\text { Daily sleep } \\
\text { logs } \\
\text { RMBPC }\end{array}$ & $\begin{array}{l}\text { sleep-wake } \\
\text { activity } \\
\text { PSQI } \\
\text { ESS } \\
\text { SDQ }\end{array}$ \\
\hline
\end{tabular}




\begin{tabular}{|c|c|c|c|c|c|c|c|c|c|c|c|c|c|}
\hline $\begin{array}{l}\text { McCurry } \\
2003^{156} \\
(14511168) \\
\text { US } \\
\text { Pilot }\end{array}$ & $\begin{array}{l}\text { Written } \\
\text { materials } \\
\text { describing age- } \\
\text { and dementia- } \\
\text { related changes } \\
\text { in sleep and } \\
\text { standard } \\
\text { principles of } \\
\text { good sleep } \\
\text { hygiene. CG } \\
\text { also received } \\
\text { specific } \\
\text { recommendatio } \\
\text { ns for sleep } \\
\text { hygiene } \\
\text { program for the } \\
\text { dementia } \\
\text { patient }\end{array}$ & $\begin{array}{l}\text { Control PLWD } \\
\text { got general } \\
\text { dementia } \\
\text { education and } \\
\text { caregiver } \\
\text { support }\end{array}$ & $\begin{array}{l}\text { Community- } \\
\text { dwelling } \\
\text { RCT } \\
22 \text { PLWD }\end{array}$ & \begin{tabular}{|l|} 
Dementia \\
diagnosis \\
according to \\
NINCDS-ADRDA \\
criteria for probable \\
or possible AD \\
confirmed in writing \\
by their primary \\
care physicians. \\
With mean MMSE \\
score \pm SD of \\
$10.7 \pm 7.8$ and at \\
least one sleep \\
problem on the \\
Neuropsychiatric \\
Inventory Nighttime \\
Behavior scale
\end{tabular} & $\begin{array}{l}\mathrm{N}=22 \\
\text { Age: yes } \\
\text { Sex: yes } \\
\text { Race: no } \\
\text { Education: no }\end{array}$ & None & $\begin{array}{l}\mathrm{N}=22 \\
\text { Age: yes } \\
\text { Sex: yes } \\
\text { Race: no } \\
\text { Education: no }\end{array}$ & NA & NA & NA & 2 months & $\begin{array}{l}\text { sleep-wake } \\
\text { activity } \\
\text { CESD } \\
\text { Daily sleep } \\
\text { logs }\end{array}$ & $\begin{array}{l}\text { sleep-wake } \\
\text { activity } \\
\text { PSQI } \\
\text { ESS }\end{array}$ \\
\hline
\end{tabular}

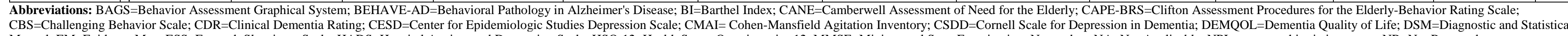
State Examination; N=number; NA=Not Applicable; NPI=neuropsychiatric inventory; NR=Not Reported

RAID=Rating for Anxiety in Dementia; RMBPC=Revised Memory

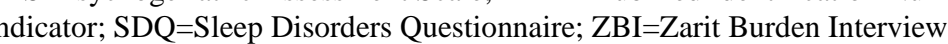




\section{References for Appendix F}

1. Fortinsky RH, Kulldorff M, Kleppinger A, et al. Dementia care consultation for family caregivers: collaborative model linkig
PMID: 19347683 .

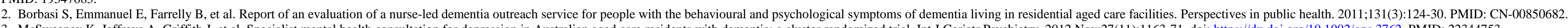
3. McSweeney K, Jeffreys A, Griffith J, et al. Specialist mental health consultation for depression in Australian aged care residents with dementia: a cluster randomized trial. Int J Geriatr Psychiatry. 2012 Nov;27(11):1163-71. doi: https://dx.doi.org/10.1002/gps.3762. PMID: 223447753.

4. Opie J, Doyle C, O'Connor DW. Challenging behaviours in nursing home residents with dementia: a randomized controlled trial of multidisciplinary interventions. Int J Geriatr Psychiatry. 2002 Jan;17(1):6-13. PMID: 11802224

5. Orrell M, Hancock G, Hoe J, et al. A cluster randomised controlled trial to reduce the unmet needs of people with dementia living in residential care. Int J Geriatr Psychiatry. 2007 Nov;22(11):1127-34. PMID: 17394129.

Xiao LD, De Bellis A, Kyriazopoulos H, et al. The Effect of a Personalized Dementia Care Intervention for Caregivers From Australian Minority Groups. Ain JAlzheimers Dis Other Demen. 2016 Feb;31(1):57-67. doi: https://dx.doi.org/10.1177/1533317515578256. PMID: 25805891.

7. MacNeil Vroomen J, Bosmans JE, van de Ven PM, et al. Commm

8. Chien WT, Lee IY. Randomized controlled trial of a dementia care programme for families of home-resided older people with dementia. J Adv Nurs. 2011 Apr;67(4):774-87. doi: https://dx.doi.org/10.1111/j.1365-2648.2010.05537.x. PMID: 21198803.

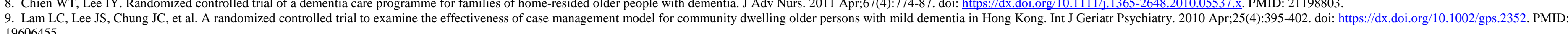

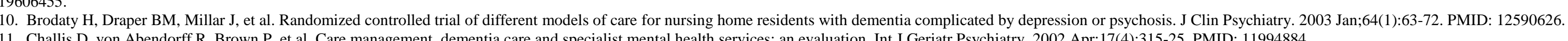

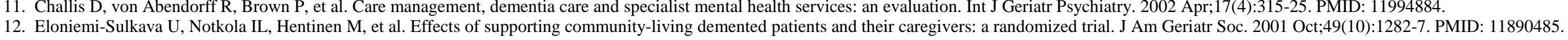

13. Weinberger M, Gold DT, Divine GW, et al. Social service interventions for caregivers of patients with dementia: impact on health care utilization and expenditures. J Am Geriatr Soc. 1993 Feb;41(2):153-6. PMID: 8426038.

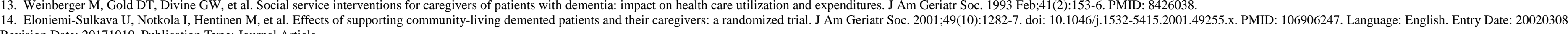
Revision Date: 20171010. Publication Type: Journal Article.

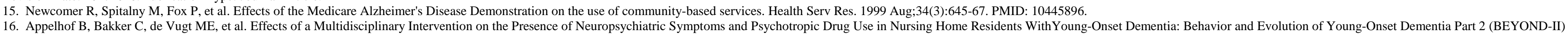
Study. Am J Geriatr Psychiatry. 2019 Jan 04;04:04. doi: https://dx.doi.org/10.1016/jijagg. 2018.12.032. PMID: 30799167.

17. Husebo BS, Ballard C, Aarsland D, et al. The Effect of a Multicomponent Intervention on Quality of Life in Residents of Nursing Homes: A Randomized Controlled Trial (COSMOS). Journal of the American Medical Directors Association. 2019. doi:

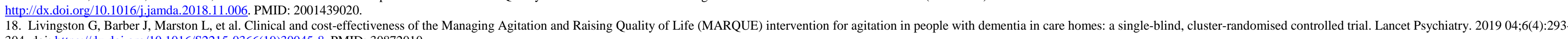
04. doi: https://dx.doi.org/10.1016/S2215-0366(19)30045-8. PMID: 30872010.

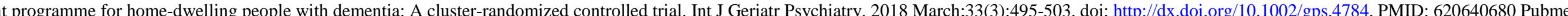

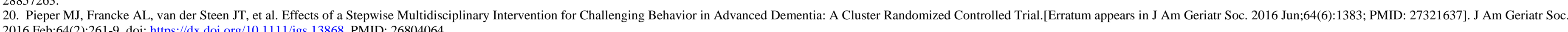
2016 Feb;64(2):261-9. doi: https://dx.doi.org/10.1111/jgs.13868. PMID: 26804064.

21. McCabe MP, Bird M, Davison TE, et al. An RCT to evaluate the utility of a clinical protocol for staff in the management of behavioral and psychological symptoms of dementia in residential aged-care settings. Aging Ment Health. 2015;19(9):799-807. doi:

22. Zwijsen SA, Smalbrugge M, Eefsting JA, et al. Coming to grips with challenging behavior: a cluster randomized controlled trial on the effects of a multidisciplinary care program for challenging behavior in dementia. J Am Med Dir Assoc. 2014 Jul;15(7):531.e1-.e10. doi:

23. Rapp MA, Mell T, Majic T, et al. Agitation in nursing home residents with dementia (VIDEANT trial): effects of a cluster-randomized, controlled, guideline implementation trial. J Am Med Dir Assoc. 2013 Sep;14(9):690-5. doi: https://dx.doi.org/10.1016/j.jamda.2013.05.017. PMID

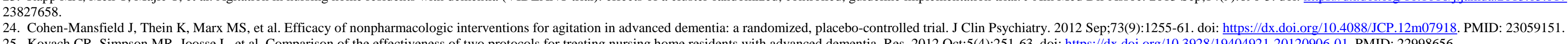

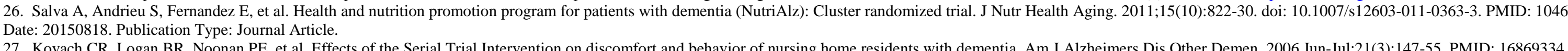

27. Kovach CR, Logan BR, Noonan PE, et al. Effects of the Serial Trial Intervention on discomfort and behavior of nursing home residents with dementia. Am J Alzheimers Dis Other Demen. 2006 Jun-Jul;21(3):147-55. PMID: 16869334.
28. Chen YH, Lin LC. Ability of the Pain Recognition and Treatment (PRT) Protocol to Reduce Expressions of Pain among Institutionalized Residents with Dementia: A Cluster Randomized Controlled Trial. Pain Manag Nurs. 2016 Feb;17(1):14-24. doi:

https://dx.doi.org/10.1016/j.pmn.2015.08.003. PMID: 26584896.

9. Pieper M, Francke A, Steen J, et al. Effects of a stepwise approach to behavioural problems in dementia: a cluster randomised controlled trial. In Nederlands tijdschrift voor geneeskunde

Z Zwijsen SA, Hekel B, Raba

Stud. 2015 Jan;52(1):68-74. doi: https:///dx.doi.org/10.1016/ji.jijurstu.2014.10.003. PMID: 25458804.

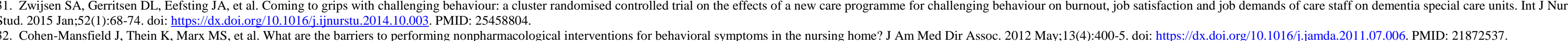

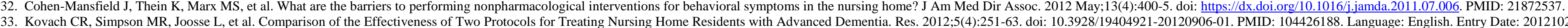

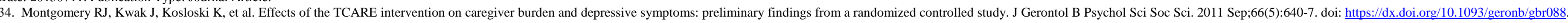
PMID: 21840840 .

35. Verkaik R, Francke AL, van Meijel B, et al. The effects of a nursing guideline on depression in psychogeriatric nursing home residents with dementia. Int J Geriatr Psychiatry. 2011 Jul;26(7):723-32. doi: https://dx.doi.org/10.1002/gps.2586. PMID: 21495077.

36. Cohen-Mansfield J, Libin A, Marx MS. Nonpharmacological treatment of agitation: a controlled trial of systematic individualized intervention. J Gerontol A Biol Sci Med Sci. 2007 Aug;62(8):908-16. PMID: 17702884.

37. Kovach CR, Cashin JR, Sauer L. Deconstruction of a complex tailored intervention to assess and treat discomfort of people with advanced dementia. J Adv Nurs. 2006 Sep;55(6): 678-88. PMID: 16925616.

38. Brazil K, Carter G, Cardwell C, et al. Effectiveness of advance care planning with family carers in dementia nursing homes: A paired cluster randomized controlled trial. Palliat Med. 2018 Mar;32(3):603-12. doi: https://dx.doi.org/10.1177/0269216317722413. PMID: 28786323. https://dx.doi.org/10.1001/jamainternmed.2018.1506. PMID: 29868778.

0. Cohen SM, Volandes AE, Shaffer ML, et al. Concordance Between Proxy Level of Care Preference and Advance Directives Among Nursing Home Residents With Advanced Dementia: A Cluster Randomized Clinical Trial. J Pain Symptom Manage. 2019 Jan;57(1):37-46.e1. doi:

https://dx.doi.org/10.1016/j.jpainsymman.2018.09.018. PMID: 30273717. 
42. Hanson LC, Song MK, Zimmerman S, et al. Fidelity to a behavioral intervention to improve goals of care decisions for nursing home residents with advanced dementia. Clin. 2016 Dec;13(6):599-604. PMID: 27271683.

43. Reinhardt JP, Chichin E, Posner L, et al. Vital conversations with family in the nursing home: preparation for end-stage dementia care. J Soc Work End Life Palliat Care. 2014;:10(2):112-26. doi: https://dx.doi.org/10.1080/15524256.2014.906371. PMID: 24835382.

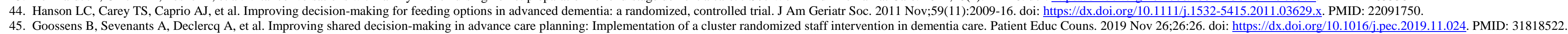
46. Goossens B, Sevenants A, Declercq A, et al. 'We DECide optimized' - training nursing home staff in shared decision-making skills for advance care planning conversations in dementia care: protocol of a pretest-postest cluster randomized trial. BMC geriatr. 2019 02 04;19(1):33. doi: https:///xx.doi.org/10.1186/s12877-019-1044-z. PMID: 30717700.

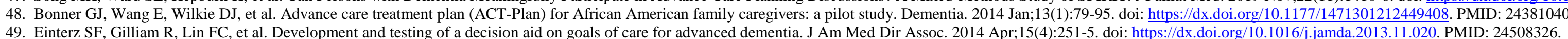

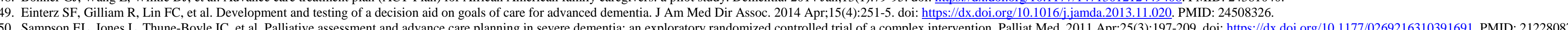
51. Vempson EL, Jones L, M Miste-Boyle IC, et al. Palliative assessment and advance care planning in severe dementia: an exploratory randomized controlled trial of a complex intervention. Palliat Med. $2011 \mathrm{Apr} ; 25(3): 197-209$. doi: https:///dx.doi.org/10.1177/0269216310391

hittps://dx.doi.org/10.1177/02692163177719588. PMID: 28731379,

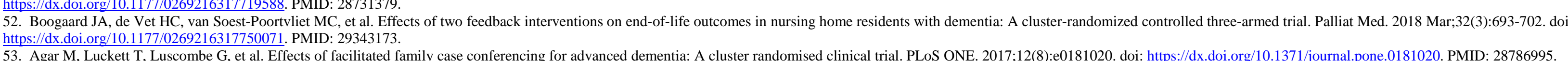

53. Agar M, Luckett T, Luscombe G, et al. Effects of facilitated family case conferencing for advanced dementia: A cluster randomised clinical trial. PLoS ONE. 2017;12(8)::0181020. doi: https://dx.doi.org/10.1371/journal.pone.0181020. PMID: 28786995.

.

56. Horvath KJ, Trudeau SA, Rudolph JL, et al. Clinical trial of a home safety toolkit for Alzheimer's disease. Int J Alzheimer Dis. 2013;2013:913606. doi: https:///dx.doi.org/10.1155/2013/913606. PMID: 24195007. 2018.10 .494 . PMID: 30342242.

57. Hanson LC, Gilliam R, Lee TJ. Successful clinical trial research in nursing homes: the Improving Decision-Making Study. Clin. 2010 Dec; $7(6): 735-43$. doi: https:///dx.doi.org/10.1177/17407774510380241. PMID: 20729251.

58. Snyder EA, Caprio AJ, Wessell K, et al. Impact of a decision aid on surrogate decision-makers' perceptions of feeding options for patients with dementia. J Am Med Dir Assoc. 2013 Feb;14(2):114-8. doi: https://dx.doi.org/10.1016/j.jamda.2012.10.011. PMID: 23273855. 59. Laver K, Liu E, Clemson L, et al. Does Telehealth Delivery of a Dyadic Dente

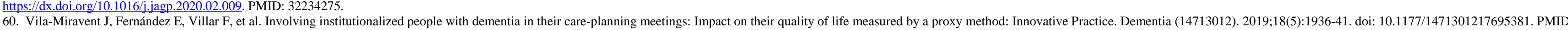

137592911. Language: English. Entry Date: 20190724. Revision Date: 20190724. Publication Type: Article.
61. Williams KN, Perkhounkova Y, Shaw CA, et al. Supporting Family Caregivers With Technology for Dementia Home Care: A Randomized Controlled Trial. Innov Aging. 2019 Jul;3(3):igz037. doi: https:///dx.doi.org/10.1093/geroni/igz037. PMID: 31660443. 62. Halek M, Reuther S, Muller-Widmer R, et al. Dealing with the behaviour of residents with dementia that challenges: A stepped-wedge cluster randomized trial of two types of dementia-specific case conferences in nursing homes (FallDem). Int J Nurs Stud. 2020 Apr; $104: 103435$. doi: hittp:://dx.doi.org/10.1016/j.ijnurstu.2019.103435. PMID: 32062052

https:///dx.doi.org/10.1001/jamainternmed.2019.4101. PMID: 31566651.

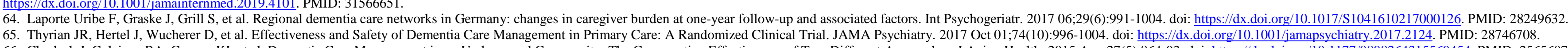

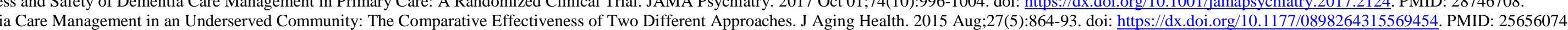

67. Leontjevas R, Teerenstra S, Smalbrugge M, et al. More insight into the concept of apathy: a multidisciplinary depression management program has different effects on depressive symptoms and apathy in nursing homes. Int Psychogeriatr. 2013;25(12):1941-52. doi:

68. Bass D, Judge K, Snow A, et al. Caregiver outcomes of partners in dementia care: effect of a care coordination program for veterans with dementia and their family members and friends. J Am Geriatr Soc. 2013;61(8):1377-86. doi: 10.1111/jgs.12362. PMID: CN-00875084.

69. Chapman DG, Toseland RW. Effectiveness of advanced illness care teams for nursing home residents with dementia. Soc Work. 2007 Oct;52(4):321-9. PMID: 18232242.

70. Callahan CM, Boustani MA, Unverzagt FW, et al. Effectiveness of collaborative care for older adults with Alzheimer disease in primary care: a randomized controlled trial_JJma 2006 May 10:295(18):2148-57. PMID: 16684985.

71. Vickrey BG, Mittman BS, Connor KI, et al. The effect of a disease management intervention on quality and outcomes of dementia care: a randomized, controlled trial.[SSummary for patients in Ann Intern Med. 2006 Nov 21;145(10):I31; PMID: 17116913]. Ann Intern Med. 2006 Nov

PMID: 31056923

73. Ballard C, Corbett A, Orrell M, et al. Impact of person-centred cae

作

75. Dichter M, Quasdorf T, Schwab C, et al. Dementia care mapping: effects on residents' quality of life and challenging behavior in German nursing homes. A quasi-experimental trial. Int Psychogeriatr. 2015;27(11):1875-92. doi: 10.1017/S1041610215000927. PMID: CN-01215190.

76. Chenoweth L, Forbes I, Fleming R, et al. PerCEN: a cluster randomized controlled trial of person-centered residential care and environment for people with dementia. Int Psychogeriatr. 2014 Jul;26(7):1147-60. doi: https://dx.doi.org/10.1017/S1041610214000398. PMID: 24666667.

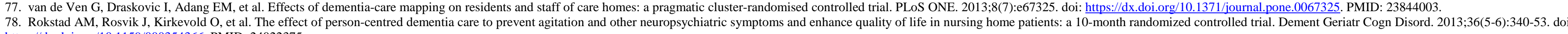
https://dx.doi.org/10.1159/000354366. PMID: 24022375.

79. Chenoweth L, King MT, Jeon YH, et al. Caring for Aged Dementia Care Resident Study (CADRES) of person-centred care, dementia-care mapping, and usual care in dementia: a cluster-randomised trial.[Erratum appears in Lancet Neurol. 2009 May;8(5):419]. Lancet neurol. 2009 5-6. PMID: 19282246.

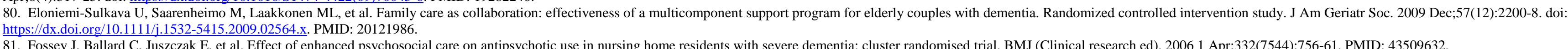

(

82. Rovner BW, Steele CD, Shmuely Y, et al. A randomized trial of dementia care in nursing homes. J Am Geriatr Soc. 1996 Jan;44(1):7-13. PMID: 8537594.

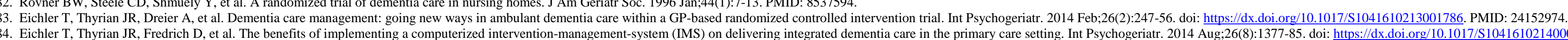

PMID: 24811445.
85. Fis T, Thyrian JR, Wucherer D, et al. Medication management for people with dementia in primary care: description of implementation in the DelpHi study. BMC geriatr. 2013 Nov 13;13:121. doi: https://dx.doi.org/10.1186/1471-2318-13-121. PMID: 24225205.

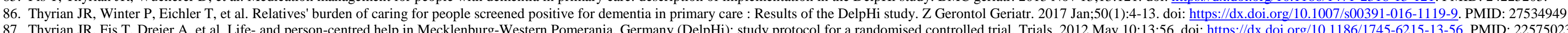

F-43 


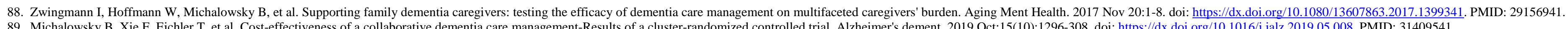
89. Michalowsky B, Xie F, Eichler T, et al. Cost-effectiveness of a collaborative dementia care management-Results of a cluster-randomized controlled trial. Alzheimer's dement. 2019 Oct;:15(10):1296-308. doi: https:///x.doi.org/10.1016/j.jalz.2019.05.008. PMID: 31409541.

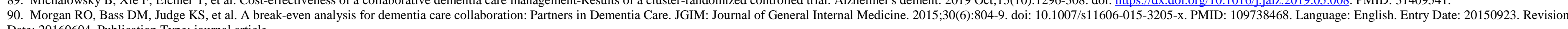
Date: 20160604. Publication Type: journal article.

Aluation of a coordinated care management intervention for dementia. Am J Manag Care. 2009 Aug;15(8):521-8. PMID: 19670955.

93. Brown AF, Vassar SD, Connor KI, et al. Collaborative care management reduces disparities in dementia care quality for caregivers with less education. J Am Geriatr Soc. 2013 Feb;61(2):243-51. doi: https://dx.doi.org/10.1111/igs.12079. PMID: 23320655.

94. Lichtwarck B, Selbaek G, Kirkevold O, et al. TIME - Targeted interdisciplinary model for evaluation and treatment of neuropsychiatric symptoms: protocol for an effectiveness-implementation cluster randomized hybrid trial. BMC Psychiatry. 2016 Jul 12;16:233. doi:

https:///dx.doi.org/10.1186/s12888-016-0944-0. PMID: 27406242.

作

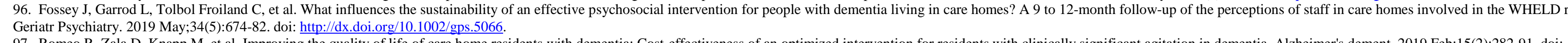

https://dx.doi.org/10.1016/j.jalz.2018.08.010. PMID: 30470592.

作

Type: Journal Article.
99. Jeon YH, Luscombe G, Chenoweth L, et al. Staff outcomes from the caring for aged dementia care resident study (CADRES): a cluster randomised trial. Int J Nurs Stud. 2012 May;49(5):508-18. doi: https://dx.doi.org/10.1016/ji.jinurstu.2011.10.020. PMID: 22078076.

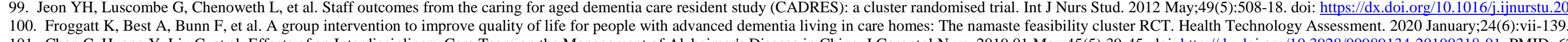

101. Chen C, Huang Y, Liu C, et al. Effects of an Interdisciplinary Care Team on the Management of Alzheimer's Disease in China. J Gerontol Nurs. 201901 May; 45 (5):39-45. doi: http://dx.doi.org/10.3928/00989134-20190318-01. PMID: 627579646.

102. Saxena N, George PP, Teo KW, et al. Evaluation of an integrated primary care-led dementia shared care program in Singapore: An effectiveness and cost-effectiveness study. Geriatr Gerontol Int. 2018 Mar;:18(3):479-86. doi: https://dx.doi.org/10.1111/ggi.13196. PMID: 29193721.

103. Mavandadi S, Wright EM, Graydon MM, et al. A End

104. Samus QM, Johnston D, Black BS, et al. A multidimension.

105. Leontjevas R, Gerritsen DL, Smalbrugge M, et al. A structural multidisciplinary approach to depression management in nursing-home residents: a multicentre, stepped-wedge cluster-randomised trial. Lancet. 2013 Jun 29;381(9885):2255-64. doi: https://dx.doi.org/10.1016/S0140-

6736(13)60590-5. PMID: 23643110.

106. Bass DM, Clark PA, Looman WJ, et al. The Cleveland Alzheimer's managed care demonstration: outcomes after 12 months of implementation. Gerontologist. 2003 Feb;43(1):73-85. PMID: 12604748.

107. Clark PA, Bass DM, Looman WJ, et al. Outcomes for patients with dementia from the Cleveland Alzheimer's Managed Care Demonstration. Aging Ment Health. 2004 Jan;8(1):40-51. PMID: 14690867.
108. Li J, Grandner M, Chang Y, et al. Person-centered dementia care and sleep in assisted living residents with dementia: a pilot study. Behav Sleep Med. 2017(2):97-113. doi: 10.1080/15402002.2015.1104686. PMID: CN-01380345.

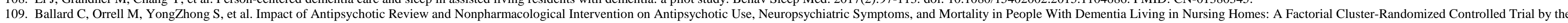

Well-Being and Health for People With Dementia (WHELD) Program. Am J Psychiatry. 2016 Mar 01;173(3):252-62. doi: https://dx.doi.org/10.1176/appi.ajp.2015.15010130. PMID: 26585409.

110. Moyle W, Venturato L, Cooke M, et al. Evaluating the capabilities model of dementia care: A non-randomized controlled trial exploring resident quality of life and care staff attitudes and experiences. Int Psychogeriatr. 2016 01 Jul;28(7):1091-100. do:

http://dx.doi.org/10.1017/S1041610216000296. PMID: 608901615.

111. Gozalo P, Prakash S, Qato DM, et al. Effect of the bathing without a battle training intervention on bathing-associated physical and verbal outcomes in nursing home residents with dementia: a randomized crossover diffusion study. J Am Geriatr Soc. 2014 May;62(5):797-804. doi:

112. Schindel ML, Gillies L, Coker E, et al. An Education Intervention to Enhance Staff Self-Efficacy to Provide Dementia Care in an Acute Care Hospital in Canada: a Nonrandomized Controlled Study. In American journal of alzheimer's disease and other dementis

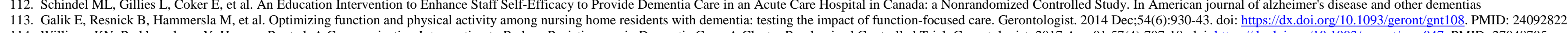

114. Williams KN, Perkhounkova Y, Herman R, et al. A Communication Intervention to Reduce Resistiveness in Dementia Care: A Cluster Randomized Controlled Trial. Gerontologist. 2017 Aug 01:57(4): $7077-18$. doi: hittps:////dx.doi.org /10.1093/geront/gnw047. PMID: 27048705.

115. Conway ER, Chenery HJ. Evaluating the MESSAGE Communication Strategies in Dementia training for use with community-based aged care staff working with people with dementia: a controlled pretest-post-test study. J Clin Nurs. 2016 Apr;25(7-8):1145-55. doi:

116. Hattink B. Meiland F, van der Roest $\mathrm{H}$, et al. Web-Ba

https://dx.doi.org/10.2196/imir.4025. PMID: 26519106.

118. Clare L, Whitaker R, Woods RT, et al. AwareCare: a pilot randomized controlled trial of an awareness-based staff training intervention to improve quality of life for residents with severe dementia in long-term care settings. Int Psychogeriatr. 2013 Jan; 25(1):128-39. doi

https://dx.doi.o 119 . Chandeh

Type: journal article.

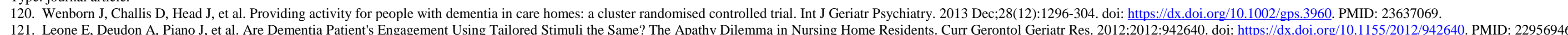

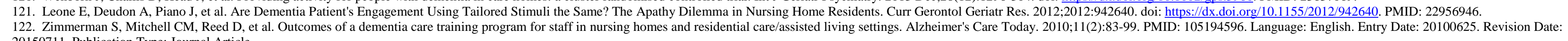

20150711. Publication Type: Journal Article.

123. Deudon A, Maubourguet N, Gervais X, et al. Non-pharmacological management of behavioural symptoms in nursing homes. Int J Geriatr Psychiatry. 2009 Dec;24(12):1386-95. doi: https://dx.doi.org/10.1002/gps.2275. PMID: 19370714.

124. Kuske B, Luck T, Hanns S, et al. Training in dementia care: a cluster-randomized controlled trial of a training program for nursing home staff in Germany. Int Psychogeriatr. 2009 Apr;21(2):295-308. doi: https://dx.doi.org/10.1017/S1041610208008387. PMID: 19193252.

125. Chang CC, Lin LC. Effects of a feeding skills training programme on nursing assistants and dementia patients. J Clin Nurs. 2005 Nov;14(10):1185-92. PMID: 16238764.

15507054.
127. Hoeffer B, Mitchell CM, et al. Effect of person-centered showering and the towel bath on bathing-associated aggression, agitation, and discomfort in nursing home
.

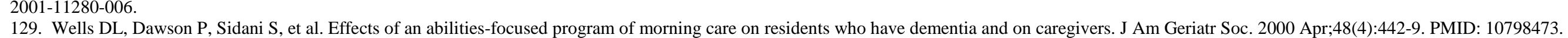

130. McCallion P, Toseland RW, Lacey D, et al. Educating nursing assistants to communicate more effectively with nursing home residents with dementia. Gerontologist. 1999 Oct;39(5):546-58. PMID: 10568079.

131. Proctor R, Burns

13. 
133. Hepburn KW, Lewis M, Narayan S, et al. Partners in caregiving: A psychoeducation program affecting dementia family caregivers' distress and caregiving outlook. Clin Gerontol. 2005;29(1):53-69. doi: http://dx.doi.org/10.1300/J018v29n01_05. PMID: 43539927. 134. Hepburn K, Lewis M, Tornatore J, et al. The Savvy Caregiver program: the demonstrated effectiveness of a transportable dementia caregiver psychoeducation program. J Gerontol Nurs. 2007 Mar;33(3):30-6. PMID: 17378189.

35. Liddle J, Smith-Conway ER, Baker R, et al. Memory and communication support strategies in dementia: effect of a training program for informal caregivers. Int Psychogeriatr. 2012;24(9):1927-42. doi: 10.1017/S1041610212001366. PMID: 104376604. Language: English. Entry Date:

Aug;10(3):435-53. doi: http:///dx.doi.org/10.1177/14711301211408124. PMID: 2011-16142-011.

137. Neely A, Vikstrom S, Josephsson S. Collaborative memory intervention in dementia: caregiver participation matters. In Neuropsychol

138. Done DJ, Thomas JA. Training in communication skills for informal carers of people suffering from dementia: a cluster randomized clinical trial comparing a therapist led workshop and a booklet. Int J Geriatr Psychiatry. 2001 Aug;16(8):816-21. PMID: 11536349,

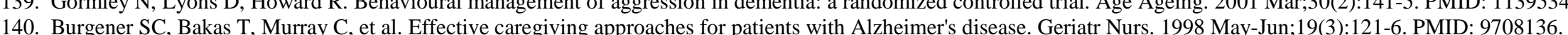

141. Robinson K, Yates K. Effects of two caregiver-training programs on burden and attitude toward help. Arch Psychiatr Nurs. 1994 Oct;:8(5):312-9. PMID: 7993133.

142. Jablonski RA, Reed D, Maas ML. Care intervention for older adults with Alzheimer's disease and related dementias: effect of family involvement on cognitive and functional outcomes in nursing homes. J Gerontol Nurs. 2005 Jun;31(6):38-48. PMID: 16138529.

143. Maas ML, Reed D, Park M, et al. Outcomes of tamily involvement in care intervention for caregivers of individuals with dementia. Nurs Res. 2004 Mar-Apr;53(2):76-86. PMID: 15084992.

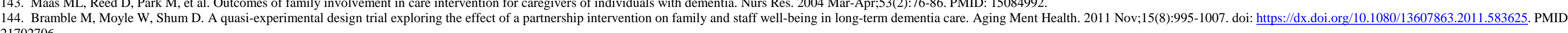

145. Robison J, Curry L, Gruman C, et al. Partners in caregiving in a special care environment: cooperative communication between staff and families on de
146. Mcallion P. Toseland RW, Freeman K. An evaluation of a family visit education program. J Am Geriatr Soc. 1999 Feb:47(2):203-14. PMID: 9988292 .

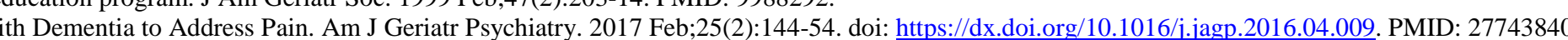

149. Teri L, McCurry SM, Logsdon R, et al. Training community consultants to help family members improve dementia care: a randomized controlled trial. Gerontologist. 2005 Dec;45(6):802-11. PMID: 16326662.

150. Teri L, McKenzie G, Logsdon RG, et al. Translation of two evidence-based programs for training families to improve care of persons with dementia. Gerontologist. 2012 Aug;:52(4):452-9. doi: https://dx.doi.org/10.1093/geront/gnr132. PMID: 22247431.

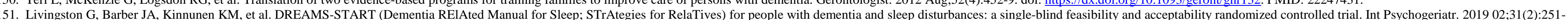
65. doi: https://dx.doi.org/10.1017/S1041610218000753. PMID: 30221615.

152. Kinnunen KM, Rapaport P, Webster L, et al. A manual-based intervention for carers of people with dementia and sleep disturbances: An acceptability and feasibility RCT. Health Technology Assessment. 2018 December;22(71):I-408. doi: http://dx.doi.org/10.3310/hta22710. PMID:

626134933

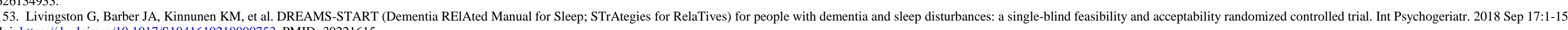
doi: https://dx.doi.org/10.1017/S1041610218000753. PMID: 30221615.

https://dx.doi.org/10.1007/s12603-015-0558-0. PMID: 26482691.

155. McCurry SM, Gibbons LE, Logsdon RG, et al. Nightime insomiad treatment and education for Alzheimer's disease: a randomized, controlled trial. J Am Geriatr Soc. 2005 May;53(5):793-802. PMID: 15877554.

156. McCurry SM, Gibbons LE, Logsdon RG, et al. Training caregivers to change the sleep hygiene practices of patients with dementia: the NITE-AD project. J Am Geriatr Soc. 2003 Oct;51(10):1455-60. PMID: 14511168. 